Digitized by the Internet Archive in 2007 with funding from Microsoft Corporation 



\section{A TEXT-BOOK OF \\ PRACTICAL PHYSICS}




\section{BY THE SAME AUTHOR.}

A Text-book of Physics. With 580 Diagrams and Illustrations and a Collection of Examples and Questions with Answers. Large crown $8 \mathrm{vo}$.

Intermediate Physics. With 311 Diagrams. 8vo.

Elementary Practical Physics.

With 124 Illustrations and 200 Exercises.

Crown 8vo.

LONGMANS, GREEN AND CO. LONDON, NEW YORK, BOMBAY, CALCUTTA, AND MADRAS 




\section{PREFACE TO THE THIRD EDITION}

British science is the poorer, as the result of the war, by many a distinguished name. Few have deserved more highly of their country, or done more useful work in defending our men against the scientific savagery of poison-gas shells, than Lt.-Col. Wiliiam Watson, as director of the Central Laboratory, B.E.F., from its establishment in June 1915 , soon after the first gas-attack, to the conclusion of 1918. The hazardous and exacting nature of this work, in the course of which he was frequently "gassed," no doubt largely contributed to his death, which took place, after two months in hospital, on 3rd March 1919, at the comparatively early age of fifty.

The work of revision of his books has thus fallen to other hands, but it is hoped that such changes as have been found necessary have been made strictly in accordance with the late author's ideas and in keeping with the character of the rest of the books. In the present text-book a number of new experiments have been added, notably those on the continuous flow calorimeter and on the viscosity of gases. Some of the tables and other constants have been brought up to date, while certain sections have been re-written. It is believed that the errors inevitable in a first edition of such a book have now been eliminated, but the editor will be grateful to receive a note of any that have escaped notice, or of any other suggestions for its improvement.

H. MOSS.

1922. 



\section{PREFACE}

THE following pages are intended to serve as a book of reference to the student working in a physical laboratory. The experiments described are not intended for a beginner, but are suited for a student who has already spent a little time in the laboratory and worked through a more elementary course of experiments, such as those described in the author's Elementary Practical Physics.

It is not intended, or expected, that any one class will work through all the experiments described in this book, but that the teacher will select those which he considers most suitable, being guided by the requirements of his pupils and the resources of his laboratory. It is, however, hoped that teachers and students will find that all the experiments which can be performed with advantage in a laboratory having the ordinary equipment are described.

In almost every case the descriptions and hints apply to any pattern of apparatus, no attempt being made to give elaborate instructions for working some particular form of instrument. It is hoped, however, that the figures will be found of assistance by teachers when making the apparatus needed to perform many of the experiments. The aim of the book is to draw attention to those points which require care, and to indicate the sources of error which are common to all the instruments which are likely to be employed. It is hoped that in this way the teacher will be relieved frum 
that constant repetition of warnings and elementary discussion of the errors with which the author has often found his time employed, and will thus have more leisure to investigate the peculiar difficulties of individual students, such as may be typified by the "loose contact" et hoc genus omne.

It is hoped that the series of tables at the end will be found of service, and every endeavour has been used to eliminate errors. But there, as well as in the body of the book, the author from bitter experience fears that mistakes will have crept in, and he will be much obliged to any readers who discover such if they will send him a note of them. 


\title{
CONTENTS
}

\author{
CHAPTER I
}

\section{METHODS USED IN THE REDUCTION AND DISCUSSION OF THE RESULTS OF PHYSICAL MEASUREMENTS}

81. Correct apportionment of the accuracy with which the different observations are to be made in an experiment. 2. On the application of corrections to observed quantities. 3 . Use of tables and curves of corrections when reducing observations. 4. Representation of a series of results. 5. To find an algebraical expression to represent a series of observations. 6. Mathematical tables. 7. The slide rule. 8. Arithmometers. 9. Determination of the area of plane figures. 10. The planimeter. 11. Harmonic analysis . . 1-40

\section{CHAPTER II}

\section{MEASUREMENTS OF LENGTH}

\$12. Standards of length. 13. The vernicr. 14. The micrometer screw. 15. The spherometer. 16. The micrometer screw gauge. 17. The travelling microscope. 18. Illumination of the divisions of a standard scale. 19. The comparator. 20. Correction of measurements of length to allow for the effects of temperature. 21. The cathetometer. 22. Meisurement of a vertical length by means of a separate scale and reading telescopes. 23. The optical lever. 24. To measure the radius of curvature of a spherical surface with the optical lever

\section{CHAPTER III}

\section{WEIGHING}

825. The balance. 26. Weighing by the method of oscillation and to compare the lengths of the arms of the balance. 27. Calibration of a set of weights. 28. Reductions of weighings to vacuo 


\section{CHA P T E I V}

\section{DENSITY}

829. Density. 30. The measurement of density-corrections for temperature of water and buoyancy of air. 31. Measurement of the density of a solid heavier than water by the method of Archimedes. 32. Measurement of the density of a solid lighter than water. 33. Measurement of the density of a solid in the form of small pieces-the pyknometer. 34. Measurement of the density of a liquid with a sinker. 35. Measurement of the density of a liquid with specific gravity bottle

\section{CHAPTER V}

\section{ELASTICITY OF SOLIDS}

\$ 36. Young's modulus by stretching a wire. 37. Young's modulus by stretching a wire, (Searle's apparatus). 38. Young's modulus by the flexure of a beam (1). 39. Young's modulus by the flexure of a beam (2). 40. Determination of Poisson's ratio. 41. Measurement of the period of an oscillating body by the eye and ear method-comparison of moment of inertias. 42. To calculate the simple rigidity of a wire from observations of the period of a torsional pendulum. 43. Simple rigidity by static determination of torsional couple. 44. Determination of Young's modulus and rigidity of a wire by Searle's method .

\section{CHAPTER VI}

\section{THE PENDULUM-MEASUREMENT OF " $g$ " AND RATING A CHRONOMETER}

$\S$ 45. The pendulum. 46. Determination of " $g$ " by means of a Borda pendulum. 47. To examine the laws of the compound pendulum. 48. Determination of " $g$ " by Kater's pendulum. 49. Determination of the rate of a clock by the occultation of a star by a terrestrial obstacle. 50. The sextant and its adjustment. 51. Determination of the error of a chronometer with the sextant . 114-134

\section{CHAPTER VII}

\section{SURFACE TENSION AND VISCOSITY}

§52. Measurement of surface tension by the elevation in a capillary tube. 53. Measurement of the surface tension of a soap film. 54. Measurement of the viscosity of a liquid by its rate of flow through a capillary tube. 55. Measurement of the viscosity of a gas. 56. Measurement of the logarithmic decrement of a vibrating body. 57. Measurement of the viscosity of a liquid by the oscillating disc method 


\section{CHAPTER VIII}

\section{THE BAROMETER}

\section{CHAPTER IX}

\section{THERMOMETRY}

59. Calibration of the tube of a mercury thermometer. 60. Determination of the upper fixed points of a mercury thermometer. 61. Determination of the lower fixed point of a mercury thermometer. 62. Application of the correction for fundamental interval. 63. Determination of the corrections to a thermometer by comparison with a standard. 64. Auxiliary fired pointstransition temperatures. 65. Correction of thermometer reading for emergent column. 66. Reduction of the readings of mercury thermometers to the hydrogen or air scales. 67. Changes in zero of mercurial thermometers. 68. The movable zero method of using a mercury thermometer. 69. Measurement of the depression in a mercury thermometer . . . . . 162-186

\section{CHAPTER X \\ EXPANSION OF SOLIDS AND LIQUIDS}

70. Linear expansion. 71. Measurement of the linear expansion of a rod. 72. Cubical expansion. 73. The weight thermometer or dilatometer. 74 . Measurement of the cubical expansion of glass with a simple form of weight dilatometer. 75. Measurement of the coefficient of expansion of a liquid with the volume dilatometer. 76 . The hydrostatic balance method of measuring the coefficient of expansion of a liquid. 77. Determination of the coefficient of apparent expansion of mercury in glass by means of a thermometer $\quad$. 157-202

\section{CHAPTER XI}

\section{THERMAL EXPANSION OF GASES}

78. The air thermometer. 79. Measurement of the coefficient of increase of pressure of air with the air thermometer. 80. Determination of the corrections to a mercury thermometer at high temperatures by means of the constant volume air thermometer. 81 . The constant pressure air thermometer ¿03-212

\section{CHAPTER XII \\ CALORIMETRY}

82. Calorimetry-the method of mixture. 83. Measurement of the specific heat of glass by the method of mixture. 84. Measurement of the specific heat of a liquid by the method of mixture. 85 . Measurement of the specific heat of a liquid by the method of cooling . . . . . . . . . 213-227 


\section{CHAPTER XIII}

\section{CALORIMETRY-LATENT HEAT}

§86. Measurement of the density of ice by Bunsen's method. 87. Bunsen's ice calorimeter. 88. Measurement of the latent heat of fusion of ice. 89. Measurement of the heat of vaporisation of water. 90. Measurement of the heat of vaporisation by Berthelot's method. 91. Joly's steam calorimeter. 92. Measurement of the heat of solution . . . . . . . . 228-244

\section{CHAPTER XIV}

\section{VAPOUR PRESSURE}

$\$ 93$. The measurement of vapour pressure. 94. Ramsay and Young's method of measuring vapour pressure. 95. Determination of the dew-point. 96. The wet and dry bulb thermometer. 97. The absorption hygrometer . . 245-252

\section{CHAPTER Xं \\ VAPOUR DENSITY-FREEZING AND BOILING POINTS OF SOLUTIONS}

§98. Measurement of vapour density by Victor Meyer's method. 99. Measurement of vapour density by Hofmann's method. 100. Measurement of the freezing point of a solution. 101. Calculation of molecular weight from the depression of the freezing point. 102. Correction of the readings of a thermometer in which the quantity of mercury is variable. 103. Measurement of the boiling point of a solution

\section{CHAPTER XVI}

\section{MELTING POINT-RATIO OF SPECIFIC HEATS- CONDUCTIVITY}

$\$ 104$. Determination of the melting point by the method of cooling. 105. Determination of the ratio of the specific heats for air by Clement and Desorme's method. 106. Measurement of the heat conductivity of copper. 107. Measurement of the heat conductivity of glass . . . . . 267-274

\section{CHAPTER XVII}

\section{$S O U N D$}

$\S 108$. Determination of the pitch of a tuning fork by means of a clock and a rotating drum. 109. Stroboscopic method of measuring the frequency of a fork. 110. Measurement of the pitch of a fork by means of a string. 111. Comparison of the frequency of two forks. 112. Measurement of the velocity of sound in air by resonance. 113. Measurement of the velocity of sound in a gas by Kundt's method. 114. To calculate the ratio of the specific heats of gases from measurements of the velocity of sound. 115. Measurement of the wave-length of a high note by interference . . . . . . 275-288 


\section{CHAPTER XVIII}

\section{REFRACTIVE INDEX}

116. Adjustment of a telescope for parallel rays. 117. Measurement of the angle of a prism with the spectrometer. 118. Refractive index of a solid or a liquid in the form of a prism. 119. Measurement of the refractive index of a liquid by total reflection, using an air film. 120. The measurement of refractive index by total reflection, using a prism (first method). 121. The measurement of refractive index by total reflection, using a prism (second method)

- 289-304

\section{CHAPTER XIX}

\section{DISPERSION AND WAVE-LENGTH MEASUREMENTS}

122. The measurement of dispersion-sources of light. 123. Light filters. 124. Calibration of a spectroscope by the use of lines of known wave-lengths. 125. Calibration of a spectroscope by interference fringes. 126. Measurement of wave-length with the diffraction grating $\quad . \quad . \quad . \quad .305-315$

\section{CHAPTER XX}

\section{IN TER FERENCE}

127. Measurement of the wave-length of light with Fresnel's biprism. 128. Measurement of the wave-length of light with Fresnel's biprism and a spectrometer. 129. Measurement of the wave-length of light by means of diffraction fringes. 130. On the localisation of interference fringes. 131. Michelson's interferometer. 132. Newton's rings, 133. Measurement of the wave-length of light by Newton's rings. 134. Diffraction through a slit and limit of resolution of a telescope. 135. Resolving power of a spectroscope. 136. Measurement of the resolving power of a prism spectroscope. 137. Measurement of the resolving power of a grating. 138. Rayleigh's refractometer.

\section{CHAPTER XXI}

\section{LENSES AND MIRRORS}

139. Measurement of the focal length of a thin lens. 140. Measurement of the radius of curvature of a concave surface. 141. Measurement of the radius of curvature of a convex surface. 142 . To test the flatness of the faces of a piece of glass. 143. Thick lenses and systems of lenses. 144. To find the focal length and the positions of the principal planes of a thick lens or system of lenses. 145. Test of a photographic lens. 146. Measurement of the magnifying power of a telescope. 147. Measurement of the magnifying power of a microscope . 


\section{CHAPTER XXII}

\section{POLARISED LIGHT}

8 148. Polarisation by reflection. 149 (a). Rotation of the plane of polarisation by optically active substances. $149(b)$. Methods of measuring the rotation of the plane of polarisation. 150. Measurement of the rotatory power of solutions of turpentine. 151. Measurement of the strength of a solution of sugar. 152. Elliptically and circularly polarised light. 153. Analysis of an elliptical vibration

\section{CHAPTER XXIII}

\section{PHOTOMETRY AND COLOUR VISION}

§154. Standards of light. 155. Photometers. 156. Determination of the connection between the candle-power of an incandescent lamp and the watts consumed for different voltages. 157. The spectro photometer. 158. Measurement of the absorption curve of a solution. 159. Colour mixture with the colour top. 160. Measurement of the luminosity of pigment colours. 161. Test for colour-blindness with Holmgren's wools . . . . . 382-395

\section{CHAPTER XXIV}

\section{MEASUREMENT OF THE EARTH'S MAGNETIC FIELD}

$\S 162$. Determination of the direction of the magnetic meridian. 163. Determination of the geographical meridian. 164. Measurement of the horizontal component of the earth's field. 165. Comparison of the values of " $H$ " at different parts of a building. 166. Measurement of " $H$ " with the Kewpattern unifilar magnetometer. 167. Determination of the temperature coefficient of a magnet. 168. Measurement of the dip with the Kew-pattern circle. 169. Comparison of the magnetic moments of two magnets . 396-419

\section{CHAPTER XXV \\ ADFUSTMENT AND USE OF GALVANOMETERS}

\$170. Measurement of angles of rotation by means of a mirror and scale. 171 . Adjustment of a telescope and scale to measure a rotation by means of a mirror. 172. Adjustment of a suspended needle reflecting galvanometer. 173. Adjustment of a suspended coil galvanometer. 174. Determination of the figure of merit of a galvanometer. 175. Comparison of electromotive forces with the galvanometer . . . . . . . . . 420-431 


\section{CHAPTER XXVI}

\section{MEASUREMENT OF RESISTANCE}

\$176. The Wheatstone's network of conductors. 177. The metre form of Wheatstone's bridge. 178. Calibration of a bridge wire by Carey Foster's method. 179. Calibration of a wire by means of a double circuit. 180. Calibration of a wire by Strouhal and Barus's method. 181. Comparison of two nearly equal resistances by Carey Foster's method. 182. To detcrmine the ratio of two nearly equal resistances. 183. The Post Office form of Wheatstone bridge. 184. The dial-pattern Wheatstone's bridge. 185. Calibration of a dial-pattern Wheatstone's bridge. 186. The Callendar and Griffiths pattern bridge. 187. Calibration of a Callendar-Griffiths bridge. 188. Measurement of high resistances. 189. Measurement of the insulation resistance of a plece of rubber covered cable. 190. Measurement of low resistances-Mathissen and Hocking's method of the projection of equal potentials. 191. Measurement of low resistance-the method of auxiliary conductors, or Kelvin's double bridge. 192. The differential galvanometer. 193. Comparison of two low resistances with the differential galvanometer. 194. The shunt potentiometer method of comparing two low resistances

\section{CHAPTER XXVII}

\section{THE RESISTANCE OF ELECTROLYTES •}

§195. Mance's method of measuring the resistance of a battery. 196. Beetz's method of measuring the resistance of a battery. 197. Measurement of the resistance of electrolytes. 198. Measurement of electrolytic resistance by the passage of a direct current. 199. Comparison of the specific resistances of electrolytes by the potentiometer method. 200. Measurement of electrolytic resistance by Kohlrausch's method with alternating currents. 201. The

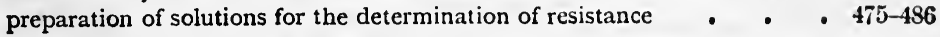

\section{CHAPTER XXVIII}

\section{MEASUREMENT OF ELECTROMOTIVE FORCE}

\$202. The construction of standard cadmium (Weston) cells. 203. The construction of the standard Clark cell. 204. Comparison of E.M.F.'s with the potentionmeter. 205. Potentiometers. 206. The capillary elecrometer. 207. To examine the relation between the surface tension of mercury and an electrolyte and the E.M.F. acting across the surface . . . . . 487-500 


\section{CHAPTER XXIX}

\section{RESISTANCE THERMOMETERS AND THERMO-ZUNCTIONS}

$\$ 208$. Determination of the constants of a platinum thermometer. 209. Measurement of the E.M.F. of a thermocouple. 210. Standardisation of a thermocouple.

\section{CHAPTER XXX}

\section{MEASUREMENT OF CURRENT}

\$211. The tangent galvanometer. 212. Adjustment of a tangent galvanometer: 213. The sine galvanometer. 214. Measurement of a current by the deposition of copper. 215. Calibration of an ammeter by copper deposition. 216. The silver voltameter. 217. The potentiometer method of measuring a current

\section{CHAPTER XXXI}

\section{THE BALLISTIC GALVANOMETER AND MEASUREMENT OF CAPACITY}

§ 218. The ballistic galvanometer. 219. Determination of the constant of a ballistic galvanometer. 220. Standardisation of a ballistic galvanometer by means of a solenoid inductor. 221. To compare the capacities of two condensers with the ballistic galvanometer. 222. Comparison of electromotive forces with the ballistic galvanometer. 223. Measurement of a magnetic field with the earth inductor. 224. Measurement of the strength of a magnetic field with an exploring coil. 225. Comparison of capacities by de Sauty's method. 226. Comparison of capacities by the method of mixtures. 227. Measurement of a capacity in electromagnetic units in terms of a resistance and a time . 518-535

\section{CHAPTER XXXII}

\section{MEASUREMENT OF SELF AND MUTUAL INDUCTION}

\$228. Measurement of the coefficient of self-induction of a coil by Rayleigh's method. 229. Use of a rotating commutator when making measurements of induction (Ayrton and Perry's secohmmeter). 230. Measurement of selfinduction by comparison with a capacity (Rimington's method). 23\%. Measurement of the self-induction of a coil by comparison with a capacity (Anderson's method). 232. Measurement of self-induction by means of a variable standard of self-induction. 233. To compare the mutual-induction between two coils with the self-induction of one of them. 234. Measurement of the coefficient of mutual-induction between two coils by means of a variable standard of selfinduction. 234a. Measurement of a mutual-induction by comparison with a capacity (Carey Foster's method). 235. 'The comparison of two mutualinductances. 236. The measurement of self-induction with alternating currents (Wien-Dolezalek method). 


\section{CHAPTER XXXIII}

\section{PERMEABILITY}

237. Measurement of the permeability of iron by the magnetometer method. 238. Correction on account of the finite length of the specimen. 23!). The ballistic method of measuring permeability. 240. To demagnetise a specimen of iron.

\section{CHAPTER XXXIV}

\section{THE QUADRANT ELECTROMETER}

241. The quadrant electrometer. 242. Determination of the capacity of a quadrant electrometer. 243. Determination of the saturation current. 244. Determination of an insulation resistance . . . . . . . 569-575

\section{CHAPTER XXXV}

THE MECHANICAL EQUIVALENT OF HEAT

245. Measurement of Joule's equivalent in mechanical units. 246. Measurement of Joule's equivalent in electrical units. 247. Measurement of Joule's equivalent by the continuous-flow calorimeter . . . . . . . 576-582

\section{APPENDIX}

1. Glass blowing. 2. Working fused silica-manufacture of quartz fibres. 3 . Making divided scales. 4. Silvering glass. 5. Mounting cross-wires in telescopes and microscopes. 6. On the use of manganine wire for the con-

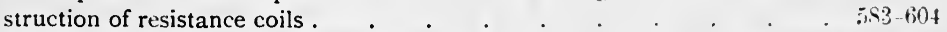

able I. Reduction of barometer readings to $0^{\circ} \mathrm{C}$. . . To face fage 604

II. Approximate formulie . . . . . . . . t to

III. Reduction of weighings to vacuo . . . . . . . Bi(16)

IV. Density of dry air at different temperatures and pressures $\quad . \quad 1 ; 07$

V. Density of water at various temperatures measured on the

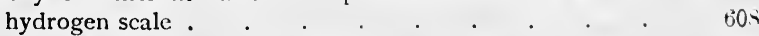

VI. Correction for buoyancy in density measurements . . . . $\quad 60 !$

VII. Moments of inertia . . . . . . . . . 60 ?

VIII. Reduction of periodic time to infinitely small arc, and correction on account of chrononeter rate . . . . . 6110

IX. Correction of the sun's or a star's apparent altitude. to allow for parallax and refraction . . . . . 5110

X. Reduction of barometer rendings to $0^{\circ} \mathrm{C}$. . . . . . 611 
Table XI. Correction to the height of the barometer to allow for the effect of capillarity . . . . . . . .

XII. Reduction of the volume of a gas to standard pressure
XIII. Values of $(1+a t)$ for reducing the volume of gases to standard temperature for values of $t$ from $0^{\circ}$ to $40^{\circ} \mathrm{C}$. . . .

$\mathrm{XIV}$. Tension of water vapour and mass of water in a cubic metre of saturated air. . . . . . . . .

XV. Boiling point of water $\quad \cdot \quad \cdot \quad \cdot \quad \cdot \quad \cdot$

XVI. Vapour pressure of liquids suitable for use in vapour jackets .

XVII. Vapour pressure of mercury . . . . . . .

XVIII. Depression of zero . . . . . . . . .

XIX. Corrections to rednce readings on mercury-in-glass thermometers to the hydrogen or air thermometer

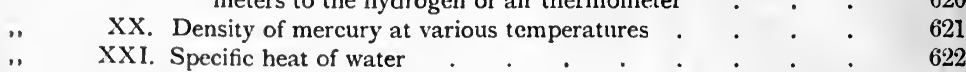

XXII. Refractive indices for sodium light . . . . . . 622

XXIII. Wave-lengths . . . . . . . . . . 623

" XXIV. Corrections to scale readings when a mirror and scale is used to measure a rotation $\quad . \quad . \quad 624$

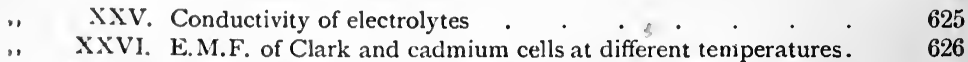

". XXVII. Connection between the units in the C.G.S. system and the

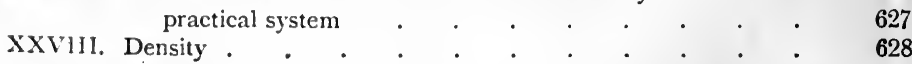

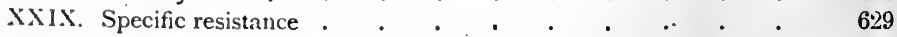

XXX. British standard wire gauge . . . . . . . . 629

INDEX . . . . . . . . . . . . . 630 


\section{A TEX'T-BOOK OF \\ PRACTICAL PHYSICS}

\section{CHAPTER I}

\section{METHODS USED IN THE REDUCTION AND DISCUSSION OF THE RESULTS OF PHYSICAL MEASUREMENTS}

1. Correct Apportionment of the Accuracy with which the different Observations are to be made in an Experiment.-When performing an experiment which involves the measurement of a number of different quantities in order to obtain the result which is sought, it is of the utmost importance that before starting on the experiments the student should satisfy himself as to the effect such errors as he is likely to make, in each of the individual measurements, will produce on the result. Having done this he will be able to adjust his observations so that he shall measure each of the quantities with an accuracy such that the possible error in the final result produced by each individual measurement shall be approximately equal. For example, suppose that in a determination of specific heat by the method of mixtures the rise of temperature of the calorimeter is about five degrees, while the thermometer used to measure this rise is only divided into half degrees. With such a therınometer it will be almost impossible to read the rise of temperature nearer than a twentieth of a degree, that is, to 1 per cent. of the total rise. Suppose that the calorimeter is made of copper and weighs about twenty grams, while it holds two hundred grams of water. The specific heat of copper being about $0 \cdot 1$, the water value of the calorimeter is only 1 per cent. of the weight of the water. Now it would be quite easy to weigh the calorimeter to within a milligram, that is, to within one part in 20,000 . Since, however, we only know the rise of temperature to one part in 100 , such refinement would be quite uscless, as the accuracy of the value obtained for the specific heat would in no way be increased thereby. It will be quite sufficient if we measure the weight of the calorimeter to within a gram, so that we know its water value to within $\cdot 1$ calory. In the same way the water need not be weighed to nearer than half a gram, that is, to within one part in 400 .

Not only does a careful consideration of the relative accuracy with 
which the different component measurements can be made tend very materially to increase the accuracy of the results obtained, by directing attention to those measurements which it is necessary to make with particular aocuracy, but also considerable time will in the long run be saved.

We are thus led to examine the extent to which a small error in the different magnitudes which are actually observed will affect the quantity which is being deduced.

Suppose that the quantity to be measured, $y$, is connected with the quantities $x_{1}, x_{2}, x_{8}, \&$ c., which are actually observed by the relation

$$
y=f\left(x_{1}, x_{2}, x_{3}, \& c .\right),
$$

and that the observed quantities receive small changes, so that $x_{1}$ becomes $x_{1}+\delta x_{1}, x_{2}$ becomes $x_{2}+\delta x_{2}$, \&c., and as a result $y$ becomes $y+\delta y$. Then it is shown in books on the differential calculus ${ }^{1}$ that

$$
\delta y=\frac{\delta f\left(x_{1}, x_{2}, x_{3}, \& c_{.}\right)}{\delta x_{1}} \delta x_{1}+\frac{\delta f\left(x_{1}, x_{2}, x_{3}, \& c .\right)}{\delta x_{2}} \delta x_{2}+\& c .
$$

where $\frac{\delta f\left(x_{1}, x_{2}, x_{3}, \& c_{.}\right)}{\delta x_{1}}$ means the partial differential coefficient of $f\left(x_{1}, x_{2}, x_{3}, \& c\right.$. $)$ with respect to $x_{1}$, and so on.

The above expression allows of our calculating what will be the effect on the quantity we are measuring of any given small error in any one of the observed quantities. Thus an error of magnitude $\delta x_{1}$ in $x_{1}$ will produce an error in $y$ of magnitude $\frac{\delta f\left(x_{1}, x_{2}, x_{3}, \& c_{.}\right)}{\delta x_{1}} \delta x_{1}$. The application of this method of estimating the effects of errors in the measured quantities un the result can most easily be studied by means of a few examples.

As a first case, let us suppose that we are measuring the volume of a circular cylinder by determining the length $l$ and diameter $d$. Here we have $y=\frac{\pi}{4} d^{2} l$. Hence

$$
\delta y=\frac{\pi}{4}\left\{d^{2} \delta l+2 l d \delta d\right\},
$$

or dividing each side by $y$ or its equivalent $\frac{\pi}{4} d^{2} l$,

$$
\frac{\delta y}{y}=\frac{\delta l}{l}+2 \frac{\delta d}{d}
$$

Let us now suppose that keeping $d$ fixed we change $l$ by 1 per cent., so that $\delta l$ is $01 l$. Then

$$
\frac{\delta y}{y}=\cdot 01
$$

1 Lamb, Infinitesimal Calculus, p. 138. 
i.e. $\delta y$ is 1 per cent. of $y$.' Next keeping $l$ fixed, let us change $d$ by 1 per cent. Then in the same way

$$
\frac{\delta y}{y}=02 \text {, }
$$

i.e. $\delta y$ is 2 per cent. of $y$. This shows that a given percentage error in $d$ will produce twice as great an error in the volume as would an equal percentage error in $l$. Thus if the cylinder has a length of $10 \mathrm{~cm}$. and a diameter of $1 \mathrm{~cm}$., and that we are only able to read the diameter on our measuring instrument to $0.0001 \mathrm{~cm}$., i.e. one part in 10,000 , then we need only measure the length to one part in 5000 , that is, to $0.002 \mathrm{~cm}$.

As a second example, let us suppose that we require to determine the moment of inertia of the above cylinder about an axis through its centre of gravity at right angles to the axis. In this case

$$
y=M\left(\frac{l^{2}}{12}+\frac{d^{2}}{16}\right)
$$

where $M$ is the mass of the cylinder.

$$
\begin{aligned}
& \text { Then } \quad \delta y=\left(\frac{l^{2}}{12}+\frac{d^{2}}{16}\right) \delta M+\frac{M l}{6} \delta l+\frac{M d}{8} \delta d \text {, } \\
& \frac{\delta y}{y}=\frac{\delta M}{M}+\frac{l \delta l}{6\left(\frac{l^{2}}{12}+\frac{d^{2}}{16}\right)}+\frac{d \delta d}{8\left(\frac{l^{2}}{12}+\frac{d^{2}}{16}\right)} \\
& \frac{\delta y}{y}=\frac{\delta M}{M}+\frac{8}{4+3 \frac{d^{2}}{l^{2}}} \frac{\delta l}{l}+\frac{6}{3+4 \frac{l^{2}}{d^{2}}} \frac{\delta d}{d} \text {. }
\end{aligned}
$$

In the present case, where $l=10$ and $d=1$, this equation reduces to

$$
\frac{\delta y}{y}=\frac{\delta M}{M}+\frac{800}{403} \frac{\delta l}{i}+\frac{6}{403} \frac{\delta d}{d}
$$

or quite nearly enough for our present purpose,

$$
\frac{\delta y}{y}=\frac{\delta M}{M}+2 \frac{\delta l}{l}+\cdot 015 \frac{\delta d}{d} \text {. }
$$

Here it is at once evident that an error in the diameter produces quite an insignificant effect compared to a proportional error in the length. If we can only measure $l$ to $0.001 \mathrm{~cm}$., i.e. to one part in 10,000 , we need not measure the diameter nearer than to about one part in 150 , i.e. to $0.07 \mathrm{~mm}$.

The above two examples illustrate the way in which a preliminary discussion helps us to concentrate our attention on those measurements which require making with especial care, for we see that when it is the volume we require we have to take particular pains over the measurement of the diameter, while to obtain the moment of inertia we must measure the length with special care.

2. On the Application of Corrections to Observed Quantities.-It is the exception rather than the rule that the actual measurements we make can be used without change in deducing the desired result. In 
general these measurements will require the application of certain small corrections. Thus where a length is measured by comparison with a standard scale, a correction will generally be necessary to allow for the fact that the distance between the two divisions of the scale is not what it purports to be, (1) on account of errors of graduation, (2) on account of the temperature of the scale at the time of comparison not being the same as it was when the scale was standardised. In the following pages we shall have numerous examples of the application of such corrections. Since, however, a considerable amount of time and labour may often be saved in the application of corrections by adopting certain devices, we shall consider the point at the present time. This saving in time is particularly the case where a number of observations of the same kind have to be reduced, the corrections in the various cases differing slightly.

When correcting observations, more particularly for temperature, mucb time will be saved by making use of the fact that when the corrections are small we may apply them by addition in place of multiplication. This can be shown as follows. Suppose the quantity $y$ which has to be determined is obtained from the observed quantities $a, b, c$ by means of the relation

$$
y=D \frac{a b}{c}
$$

where $D$ is some constant factor. If, now, the measured quantities $a, b, c$ have all to be reduced to what they. would be at some fixed temperature we should have

$$
y=D \frac{a\left(1+a t_{1}\right) b\left(1+\beta t_{2}\right)}{c\left(1+\gamma t_{3}\right)} .
$$

where $t_{1}, t_{2}$, and $t_{3}$ are the amounts by which the temperatures at which $a, b$, and $c$ were observed exceed the fixed temperatures, while $a, \beta$, and $\gamma$ are the coefficients which express the changes of the quantities $a, b$, and $c$ with temperature. If, as is almost always the case, $t_{1}, t_{2}$, and $t_{3}$ are not very large, while $\alpha, \beta$, and $\gamma$ are quite small quantities, we have, neglecting the squares and higher powers of the small quantities, ${ }^{1}$

$$
y=D \frac{a b}{c}\left(1+\alpha t_{1}+\beta t_{2}-\gamma t_{3}\right) .
$$

Hence by calculating the value of the expression within the brackets we can at once, by multiplying the uncorrected value of $y$, namely, $D \frac{a b}{c}$, by this quantity get the corrected value. When calculating the

1 In general if

$$
s=f(x+h, y+k, z+l, \& c .),
$$

we have by Taylor's theorem (Lamb, Infinitesimal Calculus, p. 595)

$$
s=f(x, y, z, \& \mathrm{c} .)+h \frac{\delta s}{\delta x}+k \frac{\delta s}{\delta s}+l \frac{\delta s}{\delta z}+\& \mathrm{c} .,
$$

where $\frac{\delta s}{\delta x}$ is the partial differential coefficient of $s$ with respect to $x$, and so on. 
value of the terms $a t_{1}, \beta t_{2}$, and $\gamma t_{3}$ it will generally be found sufficiently accurate to employ a slide rule, so that the evaluations of the correction factor can be rapidly performed.

To test whether the above approximation is sufficiently accurate in any given case, it will be as well to employ the formula (1) for one of the observations, and compare the two values obtained for $y$. If these do not differ by more than, say, a unit in the last significant figure which may fairly be retained in the value for $y$, then the approximation may be considered sufficiently good.

A list of approximate formulæ which may often be of service when applying corrections will be found in the Appendix ('Table 2).

3. Use of Tables and Curves of Corrections when reducing Observations. - When a number of reductions of the same kind have to be performed which involve the application of corrections to the observed quantities, a very considerable amount of time may often be saved by preparing suitable tables or curves. When the range of the quantity the variation of which causes the necessity for the correction-in most cases in practice this is the temperature-is great it is usually best to employ a curve, as interpolation is so much easier in the case of a curve than in the case of a table, unless the entries in the latter are very numerous.

When preparing such a curve it is necessary to exercise some discrimination, otherwise in order to obtain a sufficiently open scale the dimensions of the curve will be excessive. This point may best be illustrated by means of some examples.

When using a platinum thermometer to measure temperatures it is necessary to reduce the observations to the air thermometer scale. If the resistance of a piece of pure platinum wire at $0^{\circ} \mathrm{C}$. is $R_{0}$, its resistance $R_{t}$ at a temperature $t^{\circ}$ on the air thermometer may be expressed by a formula of the type

$$
R_{t}=R_{0}\left(1+a t+b t^{2}\right)
$$

Hence if $R_{t}$ and $R_{0}$ are measured, and we know the constants $a$ and $b$, it is possible to calculate the value of $t$. This calculation, however, would obviously be very laborious. Even if we calculated the values of $R_{t}$ for several values of $t$, and drew a curve through the points obtained, the result would not be satisfactory, as to obtain an accuracy of, say, $0^{\circ} \cdot 01$ it would be necessary to have a curve on such an enormous scale. Thus to be able to read all temperatures between $0^{\circ}$ and $100^{\circ}$, the curve would have to extend in both directions for about $200 \mathrm{~cm}$. We may, however, make use of the fact that the change in resistance is nearly proportional to the change in temperature, and as a first approximation assume that

$$
R_{t}=R_{0}(1+c t) \text {. }
$$

So that if $R_{100}$ is the resistance at $100^{\circ}$, we have, using $t p$ to represent the temperature as deduced on this assumption,

$$
t p=100 \frac{R_{t}-R_{0}}{R_{100}-R_{0}} .
$$


Now 'Callendar has shown that throughout a very wide range the deviation between the temperature on this platinum scale and the temperature on the air thermometer scale may be represented by

$$
t-t p=\delta\left\{\left(\frac{t}{100}\right)^{2}-\frac{t}{100}\right\}
$$

where $\delta$ is a constant for any particular sample of wire, and generally has a value very near 1.5 . The maximum value of $t-t p$ between $0^{\circ}$ and $100^{\circ}$ is less than $0^{\circ} \cdot 5$, and hence to be able to read the correction to $0^{\circ} \cdot 01$, a curve $5 \mathrm{~cm}$. high would be ample. That the values obtained by means of equations (2), (3), and (4) are the same as those given by (1) can be shown as follows :-

Substituting the value of $t p$ from (3) in (4), we have

But from (2)

$$
t-100 \frac{R_{t}-R_{0}}{R_{100}-R_{0}}=\delta\left(\left(\frac{t}{100}\right)^{2}-\frac{t}{100}\right) .
$$

Hence

$$
c=\frac{R_{100}-R_{0}}{100 R_{0}}
$$

or

$$
\begin{gathered}
t-\frac{R_{t}-R_{0}}{R_{0} c}=\delta\left\{\left(\frac{t}{100}\right)^{2}-\frac{t}{100}\right\} \\
R_{t}=R_{0}\left\{1+c\left(1+\frac{\delta}{100}\right) t-\frac{\delta c}{100^{2}} t^{2}\right\}
\end{gathered}
$$

which is the same as (1) if

$$
a=c\left(1+\frac{\delta}{100}\right) \text { and } b=-\frac{\delta c}{100^{2}} \text {. }
$$

As another example, suppose that a current has been measured by the potentiometer method, in which the drop of potential produced in a resistance $R$ when traversed by the current is balanced against the E.M.F. $E$ of a cadmium cell. If the temperature changes, then a correction will have to be applied on account of the variation of the resistance and of the E.M.F. of the cell. Suppose that the resistance has its nominal value at $15^{\circ}$ C., while the E.M.F. of the cell at a temperature $t_{e}$ is given by

$$
E_{t}=1 \cdot 0184\left\{1-0.000037\left(t_{c}-20\right)-0.00000064\left(t_{e}-20\right)^{2}\right\} .
$$

If the resistance is of manganine the temperature coefficient will be about +0.00003 , and for small ranges of temperature the change of resistance with temperature may be taken as linear, so that

$$
R_{t}=R_{15}\left\{1+\cdot 00003\left(t_{r}-15\right)\right\},
$$

where $t_{r}$ is the temperature of the coil. Now if the observations all take place at temperatures ranging from, say, $18^{\circ}$ to $22^{\circ}$, the term in $t^{2}$ in the expression for $E_{t}$ is negligible, and the current is given by

$$
\begin{gathered}
C=1.0184 R\left\{1-0.000037\left(t_{e}-20\right)\right\}\left\{1+0.00003\left(t_{r}-15\right)\right\} \\
=1.0184 R-1.0184 R \times 0.000037\left(t_{e}-20\right) \\
+1.0184 R \times 00003\left(t_{r}-15\right)+.
\end{gathered}
$$


Now in this expression the first term on the right is a constant, while the two other terms, which are small, vary with the temperature. Hence if two curves be drawn showing the values of these two terms for different temperatures, the corrections which have to be applied to allow for the fact that the resistance and the cell are not at their standard temperatures can easily be read off. In obtaining the curves only one point need be calculated in each case, say, for $10^{\circ}$ in the case of $E$ and $25^{\circ}$ in the case of $R$, for by the assumptions we have made the curves will both be straight lines, and one will cut the axis at a temperature of $20^{\circ}$ and the other at a temperature of $15^{\circ}$.

It will at once. be evident that if a number of observations have to be reduced, the above plan will be much quicker than correcting $E$ and $R$ separately for temperature and then finding the product of these two corrected values to give the value of the current.

' In 'such a case as that just considered, where the temperature coefficients are both very small, a table can easily be constructed which in some cases is more easily used than a curve. 'Thus suppose that the value of $R$ at $15^{\circ}$ is $1 \mathrm{ohm}$, then the following table gives the corrections over the range of ordinary room temperatures :-

\begin{tabular}{|c|c|c|}
\hline Temperature. & Correctión for Cell. & Correction for Resistance. \\
\hline $\begin{array}{r}15^{\circ} \\
16^{\circ} \\
17^{\circ} \\
18^{\circ} \\
19^{\circ}, \\
20^{\circ}, \\
21^{\circ}, . \\
22^{\circ},\end{array}$ & $\begin{array}{c}+.00019 \\
+.00015 \\
+.00014 \\
+.00008 \\
+.00004 \\
-.00004 \\
-.00008\end{array}$ & $\begin{array}{l}+.00003 \\
+\cdot 00006 \\
+\cdot 00009 \\
+\cdot 00012 \\
+\cdot 00015 \\
+.00018 \\
+\cdot 00021\end{array}$ \\
\hline
\end{tabular}

Thus if the cell has a temperature of $16^{\circ} \cdot 2$ and the resistance coil a temperature of $20^{\circ} \cdot 6$, the value of the current is

$$
\begin{aligned}
C & =1.0184+0.0001+0.0002 \\
& =1.0187 \text { amperes. }
\end{aligned}
$$

4. Representation of a Series of Results. - Frequently as a result of a physical investigation we obtain a series of values of the quantity which is being investigated for different values of one of the quantities on which it depends, as, for instance, we may have measured the rotation of the plane of polarisation by a magnetic field in water at different temperatures. In such a case the question arises as to the best manner of exhibiting the results. so as to show the connection between the two. Two methods are commonly employed for this purpose. In one of these a curve is plotted with the two variables as co-ordinates, and in the other an algebraical expression is sought which shall express the relation between them. We will first consider the representation of a series of 
measurements by means of a curve. The first thing to do is to choose a suitable scale on which to plot the curve and the kind of ruling to be used for the paper. For most purposes paper divided up into millimetre squares will be found most convenient. Such paper can now be obtained in sheets of almost any size and with very regular rulings at quite a moderate price. It is convenient to have the half centimetre lines slightly darker than the general ruling, and the centimetre lines slightly darker still.

When choosing the scale on which to plot the observations it will not do to use too small a scale, as then the points on the curve cannot be plotted with as great an accuracy as that with which the observations are taken, and thus we shall be failing to make use to the full of the time which has been spent in making the observations. On the other hand, it is possible to use too large a scale, for if such an exaggerated scale is employed then the unavoidable errors of observation will become so prominent that the points which represent the observations may appear so scattered that it will be difficult to draw a curve evenly between them.

As a general rule it will be found sufficient to so choose the scale that half a millimetre, or at most a millimetre, represents a unit in the last place which, from a discussion of the probable sources of error of our measurements, we consider we are justified in retaining. Occasionally it may happen that a very considerable change of one of the quantities only involves a comparatively small change in the other, in which case it is often sufficient to plot the quantity which changes rapidly on a considerably smaller scale than that given by the above rule. Thus suppose that we are plotting the refractive index of water at different temperatures. The refractive index at $0^{\circ}$ is 1.3341 and that at $100^{\circ}$ is 1.3184 , that is, for a difference of temperature of $100^{\circ}$ there is a change of 0257 . We will suppose that measurements of refractive index are such that we are not entitled to retain more than four places of decimals, while the temperature has been read to a tenth of a degree. According to our rule, we should take a millimetre to represent either one or two units in the fourth place of the refractive index and a tenth of a degree in the temperature. This scale would, however, unduly extend the temperature scale, for a unit in the last place of the refractive index corresponds to a change of temperature of nearly half a degree. Thus if we take a millimetre to represent a degree, or at most half a degree, we shall obtain a scale quite large enough.

Having fixed on the scale and written along the bottom and left-hand edge of the paper the values corresponding to the chief division lines, the points representing the observations must be inserted. The point corresponding to each observation must be indicated either by a small dot, which for distinctness may be surrounded by a circle of about 2 millimetres in diameter, or by the intersection of two short lines forming a cross, each arm of the cross being about 2 millimetres long.

A curve has now to be drawn which shall as nearly as possible pass through all the points, and yet shall not show any sudden twists and 
turns. Owing to the unavoidable errors to which all observations are liable it will probably be impossible to draw a regular curve so as to pass exactly through all the points. It must, however, be so drawn that at every point of the curve the points are distributed fairly evenly on the two sides of the line. The drawing of a curve to represent the results of a series of observations so that it shall give a truthful representation of the results is an operation of very considerable difficulty, and requires both practice and skill. The first thing to test is whether the points lie on a straight line, or at any rate whether a straight line can be drawn which shall pass evenly between the points. For this purpose a strip of glass or celluloid about 4 centimetres wide, along which a fine line has been ruled with a writing diamond or splinter of carborundum, will be found of great assistance. The glass is placed on the paper with the side on which the line is ruled downwards, and is moved about till the points appear evenly distributed on the two sides throughout the whole length. When this adjustment has been satisfactorily performed the readings where the line cuts the edge of the paper must be noted, and

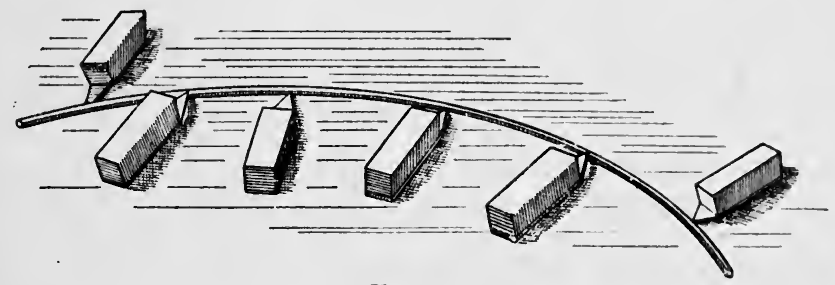

FIG. 1.

then by means of a straight-edge a line must be ruled joining these two points. It will be found very difficult to settle on the position of such a line by means of a straight-edge alone, as the ruler will eover all the points on one side and render it almost impossible to arrange that the points shall be evenly distributed on the two sides of the line.

If a straight line cannot be drawn to pass evenly through the points, it will be necessary to draw a curved line. The most convenient arrangement in such a case is that shown in Fig. 1. A thin flexible wooden lath or spline is bent so that one edge passes evenly between the points, being held in position by a number of lead weights in the manner shown. These weights are supplied with a wooden base, which is cut to a point, and they must, throughout that part of the spline where the curve has tc be drawn, be placed on the same side of the spline, otherwise they will prevent a pencil being passed along the edge of the spline when drawing the curve. Since half the points will be more or less hidden by the spline, it is rather difficult to adjust the spline. This difficulty is in a measure removed if the points are represented by a dot, and circles of equal size are drawn round the dots by means of a pair of compasses. Then, although the dot itself may be hidden by the spline, a portion of 
the circle may be visible and allow of the position of the dot being inferred. If an estimate has been made of the error which is possible in the measurement of the quantity being plotted it is a good plan to make the radius of ticese circles equal to this orror, so that if the curve passes everywhere through these circles it will at no point depart from the observed values by more than the possible errors of experiment. Hence if one or mare points are found to be. so placed that without introducing irregularities in the curve we cannot make the curve pass through the circles which surround them, it will ibe advisable to check the calculations made in reducing the observations corresponding to these points and to make certain that no error has been made in plotting. Particular care must be taken in adjusting the position of the spline at the ends of the curve, as there is a tendency to give undue weight to the end point.

Sets of splines are made of varying flexibility, and it is advisable to use a spline of such a thickness that, while it can be bent to follow the curve and be held in place by the weights, yet it is as stiff as possible, for by this means the chances of forcing the spline to take up an irregular form to pass through a point which, owing to errors of experiment, is somewhat displaced will be minimised. For use with curves in which the curvature is considerably greater at one end than at the other splines having a tapering section may be used.

As a check on the accuracy with which the curve has been drawn the ordinates of the curve corresponding to the abscissæ of the observed points should be taken, and a table drawn up showing the differences between these readings and the observed values. If the curve passes evenly between the points, then the sum of the positive differences ought to be numerically equal to the sum of the negative differences. There ought also to be no marked preponderance of differences of one sign at any part of the curve.

5. To find an Algebraical Expression to represent a Series of Observations.-It is often convenient to express the result of a series of measurements of the connection between two physical quantities in the form of an algebraical equation. The first thing to do is to discover the most suitable type of equation to employ - a matter often of no little difficulty-and then from the observed numbers we have to determine the values of the constants which appear in this equation. In some cases theory suggests the type of equation which must be employed, and in such a case we have only to determine the best values for the constants. For instance, suppose we have measured the distance a body falling freely traverses in different times. In this case we know that the space $s$ varies as the square of the time of fall $t$, so that

$$
s=a t^{2}
$$

where $a$ is some constant, the value of which we can deduce from the results of the measurements. If, however, theory does not suggest a suitable type, then the first thing to do is to plot the results on squared 
paper, as described in the preceding section. If the points lie uniformly about a straight line, then the connection between the two quantities can be represented by a linear equation of the form

$$
y=a+b x \text {. }
$$

where $a$ and $b$ are two constants, the values of which have to be deduced from the observations. To determine the values of these constants, read off two pairs of values of $x$ and $y$ from the straight line, the points chosen being near the extremities of the line so as to give as great an interval between the two points as possible, and then substituting first one set of values of $x$ and $y$ in the equation, and then the other, two simultaneous equations are obtained from which the values of $a$ and $b$ can be deduced in the ordinary manner.

The values of the constants $a$ and $b$ can also be obtained from the curve, for when $x=0, y=a$, so that $a$ is the value of $y$ where the curve cuts the $Y$ axis. Calling this value $y_{0}$, we have

$$
b=\frac{y-y_{0}}{x} \text {. }
$$

Now in the right-angled triangle, of which the co-ordinates of the angular points are $\left(0 y_{0}\right),\left(x y_{0}\right)$, and $(x y)$, the side parallel to the $X$ axis has a length $x$, and the side parallel to the $Y$ axis has a length $y-y_{0}$, the hypotenuse of the triangle being formed by the curve. Thus the tangent of the angle made by the straight line with the line through the point $\left(0 y_{0}\right)$ parallel to the axis of $X$ is $\frac{y-y_{0}}{x}$. Hence we see that the constant $b$ is the tangent of the angle the straight line through the observed points makes with the axis of $X$.

If a straight line cannot be drawn through the points, equations of other types must be tried. A hint as to a suitable form can often be obtained by plotting, not the actual values of the quantities, but their logarithms, or by plotting the value of one quantity against the logarithm of the other. In order to facilitate the plotting of these curres paper can be obtained in which, instead of the lines being equally spaced, they are spaced at distances proportional to the logarithms of the natural numbers to the base ten. A portion of such a sheet of logarithm paper is shown in Fig. 2.1 The distance from $\mathrm{A}$ to $\mathrm{B}$ or $\mathrm{A}$ to $\mathrm{C}$ being taken as the unit, the distance from $A$ to the point $D$, marked 2 , or 2 multiplied by some power of 10 , is equal to $\log 2$, i.e. $0 \cdot 301$ of the distance $\mathbf{A B}$. From $\mathbf{A}$ to $\mathbf{E}$ is equal to $\log 3$, and so on. It will be noticed that the spacing of the lines is periodically repeated. This is owing to the fact that the logarithm of two numbers, one of which is a power of ten times the other, differs by a whole number. Thus the logarithm of 20 is equal to $1+\log 2$, of 200 is $2+\log 2$, and so on.

Now suppose that two quantities $x$ and $y$ are related to one another in such a way that

$$
y=a x^{n} \quad . \quad . \quad . \quad . \quad . \quad . \quad .
$$

1 In this figure the lines for $1,1 \cdot 5,2,2 \cdot 5$, \&c., only are shown. 
where $a$ and $n$ are constants. Then taking logarithms, we get

$$
\log y=\log a+n \log x \text {. }
$$

Hence if instead of plotting $x$ and $y$ we plot $\log x$ and $\log y$, the resulting curve will be of the form

$$
y^{\prime}=c+n x^{\prime},
$$

and this represents a straight line. Of course we could plot such a curve on ordinary squared paper by looking out the values of the logarithms in a table and then plotting these values. Such a procedure would,

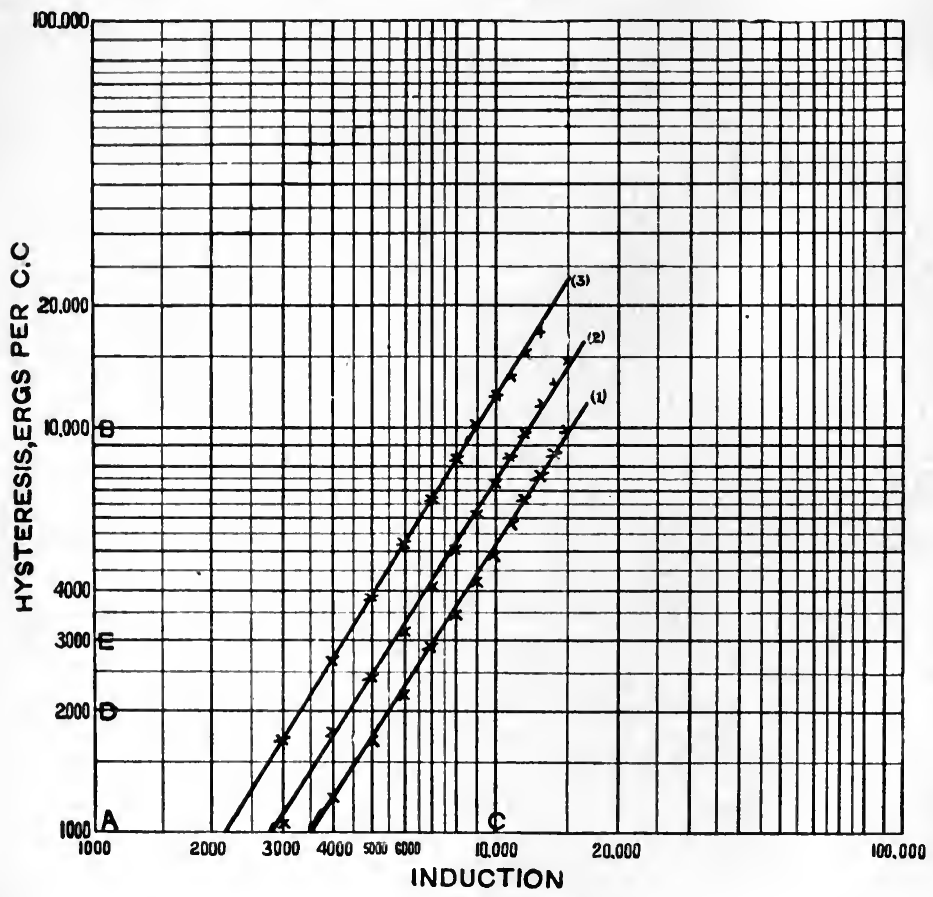

FIG. 2.

however, be somewhat lengthy, while by using logarithmically divided paper we can plot the points without the necessity of looking out the logarithms. As an example of the utility of logarithmic paper, we have plotted on Fig. 2 the results obtained by Ewing for the energy loss per cubic centimetre per cycle of three samples of iron for varying maximum inductions. It will, in the first place, be noticed that in each case a straight line passes very fairly between the various points; further, that all three straight lines are approximately parallel, that is, the con- 
stant $n$ has the same value for each sample of iron, for $n$ is equal to the tangent of the angle which the straight line makes with the axis of $X$.

To find the value of $a$ we notice that when $x=1, \log x=0$, and henc:

$$
\log y=\log a \text {. }
$$

Hence we have to find the value of $y^{\prime}$ corresponding to $x^{\prime}=1$. Now in Fig. 2 neither of the curves cuts the ordinate through the absciss $x^{\prime}=1$ on the diagram. They do, however, cut the ordinate through the point $x^{\prime}=10000$, and if $y^{\prime}$ is the ordinate at the point of intersection, we have

or

$$
\begin{aligned}
& y^{\prime}=\log a+4 n, \\
& \log a=y^{\prime}-4 n .
\end{aligned}
$$

Now from curve (3), when $x^{\prime}=10000, y=11800$ and $y^{\prime}=\log 11800$. Hence

$$
\begin{aligned}
\log a & =\log _{1}^{8} 11800-4 \times 1.58 \\
& =4.07-6.32 \\
& =3.75 \\
\therefore a & =0.0056 .
\end{aligned}
$$

Hence the observations corresponding to curve (1) can be represented by

$$
\eta=\cdot 0056 B^{1 \cdot 58},
$$

where $\eta$ is the energy loss due to hysteresis per cubic centimetre per cycle.

In the same way the other curves are represented by

$$
\begin{aligned}
& \boldsymbol{\eta}=.0035 B^{1.58}, \\
& \eta=.0024 B^{1.58} .
\end{aligned}
$$

Thus by plotting the results of these observations of Ewing's we are at once led to investigate an expression similar to Steinmetz's expression for the loss due to hysteresis, naniely,

$$
\eta=a B^{1 \cdot 6},
$$

where $a$ is a constant for any one sample of iron, but varies from ore sample to another.

If when the observations are plotted on logarithmic paper the curve drawn through the points does not depart greatly from a straight line for large values of the ordinate or abscissa, while for small values the curvature is considerable, it is advisable to try whether a formula of the type

$$
y+a=a(x+\beta)^{n} \text {. . . . . . . }
$$

will fit the observations; that is, we have to see whether we can find a constant $\alpha$ or $\beta$ which, added to $x$ or to $y$ respectively, will give a straight line on the logarithmic chart. To illustrate the method of procedure, 
suppose a series of measurements had given the results shown in the following table, and which are also shown plotted on the curve $\mathbf{A B}$ in Fig. 3 :-

\begin{tabular}{|c|c||c|c|}
\hline$x$ & $y$ & $x$ & $y$ \\
\hline 1.00, & 3.40 & $5 \cdot 79$ & $5 \cdot 87$ \\
1.50 & $3 \cdot 74$ & $6 \cdot 61$ & $6 \cdot 20$ \\
1.90 & $4 \cdot 00$ & $7 \cdot 70$ & $6 \cdot 60$ \\
2.80 & 4.50 & $8 \cdot 80$ & $6 \cdot 97$ \\
3.82 & $5 \cdot 00$ & $10 \cdot 00$ & $7 \cdot 35$ \\
4.90 & 5.50 & & \\
\hline
\end{tabular}

It will be noticed that for the higher values of $x$ the curve is very nearly a straight line, while for low values of $x$ the curve is distinctly

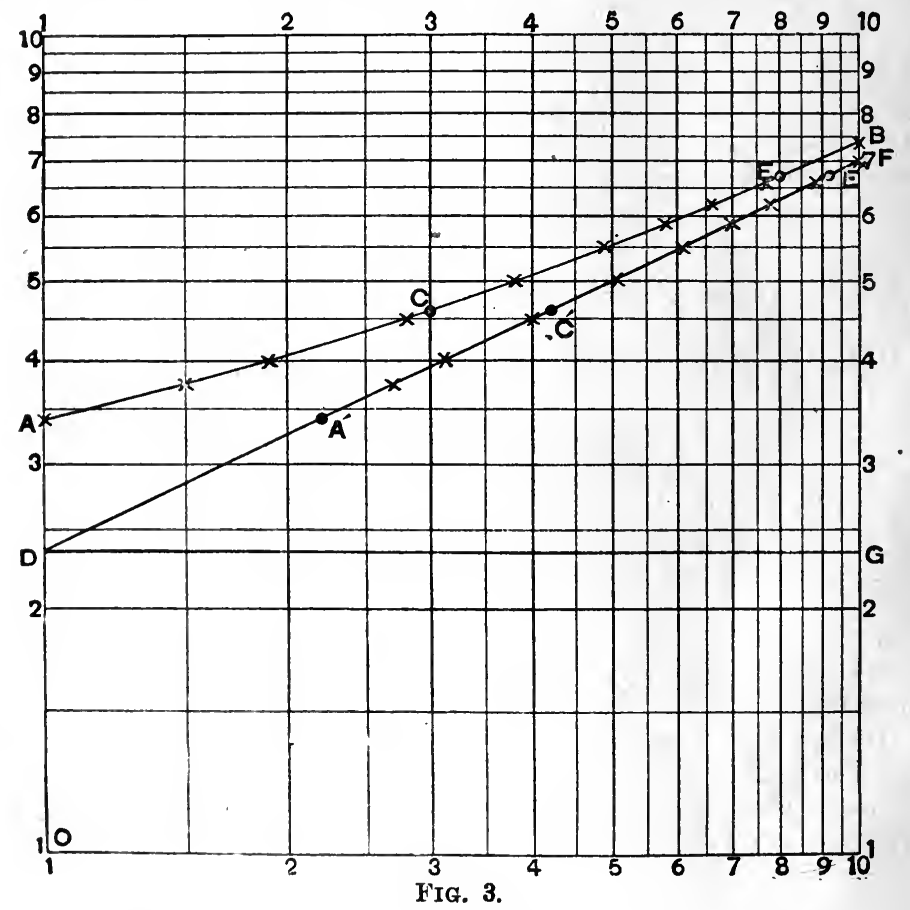

concave upwards. We will, therefore, try the effect of the addition of a constant quantity to $x$.

Selecting two points, $A$ and $E$, near the ends of the curve and a point $\mathbf{C}$ 
about half-way between, for convenience choosing points where the curve is cut by the thick vertical lines, take a straight-edge, or strip of glass with a line ruled down the centre, and lay it so that it passes through the points $A$ and E. Now move the straight-edge till it intersects the horizontal lines through $\mathbf{A}$ and $\mathrm{E}$ one division to the right of the points $\Delta$ and $\mathbf{E}$ respectively, and count how many divisions to the right the straight-edge intersects the horizontal line through the point $\mathrm{c}$. If this were one division, then we should have for the three points $\mathrm{ACE}$ that by adding $\cdot 1$ to $x$ the resulting points lie on a straight line, and we should then add 11 to the $x$ of each of the other points and see if all the fresh points lay on a straight line, or at any rate were evenly distributed about such a line. If the agreement at the three points does not occur, then move each end of the straight-edge through another division to the right and again try. Proceeding in this way in the case of the example, it will be found that when the $x$ has been increased by 1.2 the three points $A^{\prime} C^{\prime} E^{\prime}$ all line on a straight line DF. Adding $1 \cdot 2$ to the abscissæ of each of the other points, it will be found that all the points lie on or very near to the straight line DF. ${ }^{1}$ We therefore infer that the observations can be represented by an expression of the form

$$
y=a(x+1 \cdot 2)^{n}
$$

and it now remains to determine the values of the constants $a$ and $n$. Writing $z$ for $x+1 \cdot 2$, the expression takes the form

$$
y=a z^{n}
$$

or taking logarithms,

$$
\log y=\log a+n \log z .
$$

When $z$ is unity, $\log y=\log a$, that is, the point where the straight line DF cuts the $Y$ axis, gives the value of the constant $a$, which in the example is $2 \cdot 34$. Also, it can be at once shown, as on page 11 , that $n$ is equal to the tangent of the angle which the line DF makes with the axis of $X$, that is

$$
n=\frac{\overline{F G}}{\overline{D G}}
$$

which in the example gives $n=0.476$.

An approximate value of $\beta$ can be calculated from three points on the curve in the following manner. Let $x_{1} y_{1}, x_{2} y_{2}$, and $x_{8} y_{3}$ be the co-ordinates of the three points so chosen that

$$
\begin{aligned}
\log y_{2} & =\frac{1}{2} \log y_{1}+\frac{1}{2} \log y_{3}, \\
y_{2} & =\sqrt{y_{1} y_{3}} . \quad . \quad . \quad . . . .
\end{aligned}
$$

1 After plotting the points in this way it may often be seen that by slightly altering the value of $\beta$ a more uniform distribution of the points on the two sides of the straight line can be obtained, and that we can obtain a better value of $\beta$ by making successive approximations. 
then if the points obtained by adding $\beta$ to each of the $x$ 's are to be in a straight line we must have

$$
\begin{aligned}
\log \left(x_{2}+\beta\right) & =\frac{1}{2} \log \left(x_{1}+\beta\right)+\frac{1}{2} \log \left(x_{3}+\beta\right), \\
x_{2}+\beta & =\sqrt{\left(x_{1}+\beta\right)\left(x_{3}+\beta\right)} \\
\beta & =\frac{x_{2}^{2}-x_{1} x_{3}}{x_{1}+x_{3}-2 x_{2}} \text {. . . . . }
\end{aligned}
$$

or

that is

Thus the procedure is to choose two points $x_{1} y_{1}$ and $x_{3} y_{3}$, find from the curve a point $x_{2} y_{2}$ such that the condition expressed by $(4)$ is fulfilled: and then substituting the values of $x_{1}, x_{2}$, and $x_{3}$ in (5) calculate $\beta$.

If the connection between the observed quantities $x$ and $y$ is of the forms

or

$$
\begin{aligned}
& \begin{array}{l}
\left.\begin{array}{l}
y=a 10^{n x} \\
y=a \epsilon^{n x}
\end{array}\right\} . \\
y=\log a+n x,
\end{array} \\
& \log y=\log a+M n x \text {, }
\end{aligned}
$$

so that

or

where $M$ is the modulus to reduce Napierian logarithms to the base $\epsilon$ tc common logarithms to the base 10 (the value of $M$ is $\cdot 43429$ ), we may with advantage plot $\log y$ against $x$. In this case we get a straight line of which the equation is of the form

where $b$ and $c$ are constants.

$$
y^{\prime}=b+c x,
$$

To illustrate the advantages of this semi-logarithmic plotting we ma: take the observations made by Weber ${ }^{1}$ on the diffusion of zine sulphat through water given in the following table, where the concentration i measured in arbitrary units :-

\begin{tabular}{|c|c|c|c|}
\hline \multirow{2}{*}{$\begin{array}{c}\text { Time in } \\
\text { Iays. }\end{array}$} & \multicolumn{2}{|c|}{$\begin{array}{c}\text { Difference in Concentration be- } \\
\text { tween two given Points. }\end{array}$} & Difference. \\
\cline { 2 - 3 } & Observed. & Calculated. & \\
\hline & 871.5 & $883 \cdot 1$ & $-1 \cdot 6$ \\
0 & $717 \cdot 8$ & $720 \cdot 5$ & $-2 \cdot 7$ \\
1 & $589 \cdot 0$ & $587 \cdot 8$ & $+2 \cdot 8$ \\
2 & $483 \cdot 2$ & $480 \cdot 0$ & $+3 \cdot 2$ \\
3 & $394 \cdot 0$ & $391 \cdot 2$. & $+2 \cdot 8$ \\
4 & $320 \cdot 4$ & $319 \cdot 1$ & $+1 \cdot 1$ \\
5 & $260 \cdot 1$ & $260 \cdot 4$ & $-0 \cdot 3$ \\
6 & $212 \cdot 5$ & $212 \cdot 4$ & $+0 \cdot 1$ \\
7 & $173 \cdot 2$ & $173 \cdot 3$ & $-0 \cdot 1$ \\
8 & $141 \cdot 2$ & $141 \cdot 4$ & $-0 \cdot 2$ \\
9 & $115 \cdot 0$ & $115 \cdot 3$ & $-0 \cdot 3$ \\
10 & & & \\
\hline
\end{tabular}

If these numbers are plotted on ordinary paper, they will lie on 1 Wicd. Ann. (1879), vi. p. 485. 
curve. If, however, as shown in Fig. 4, we plot them on semi-logarithm paper, i.e. paper divided logarithmically in one direction and in equal parts in the other, all the points lie on a straight line $A B$.

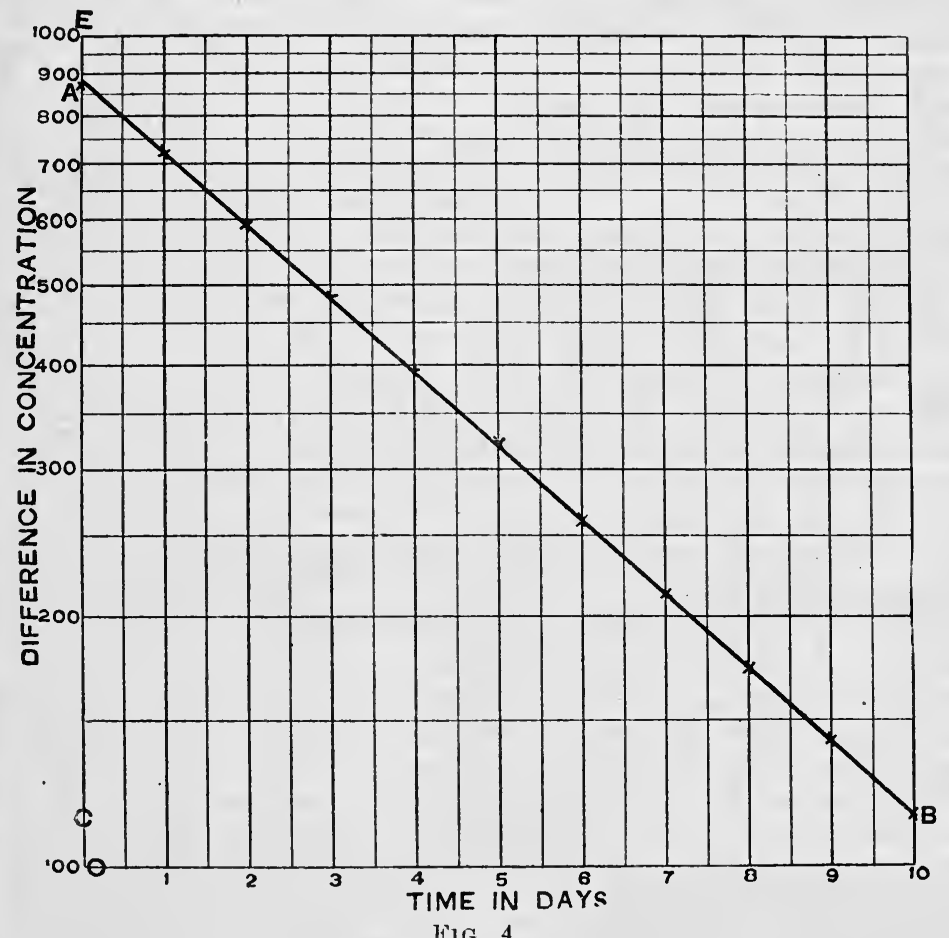

Fig. 4.

Calling $\log y y^{\prime}$, the equation to the straight line $\mathrm{AB}$ is

$$
y^{\prime}=b+n x
$$

where $b$ is a constant. Hence if $\Delta y^{\prime}$ is the change in $y^{\prime}$ produced by a change $\Delta x$ in $x$, we have

$$
n=\frac{\Delta y^{\prime}}{\Delta x} \text {. }
$$

Taking $\Delta x=10$, the corresponding $\Delta y^{\prime}$ is represented by $\overline{\mathbf{A C}}$. Since the logarithm of 10 is unity, the length $\overline{O E}$ represents unity on the scale on which $\overline{\mathbf{A C}}$ represents $\Delta y$. Hence by measuring up $\overline{\mathrm{AC}}$ and $\overline{\mathrm{OE}}$ we have

$$
\Delta y^{\prime}=\frac{\overline{A C}}{O E}=-\cdot 884,
$$

since $y^{\prime}$ decreases as $x$ increases. 
Thus

$$
n=-\frac{884}{10}=-\cdot 0884 \text {. }
$$

From the original equation

$$
y=a 10^{n x}
$$

we see that when $x=0, y=\alpha$. Hence $a=883$, which is the reading on the $y$ scale where the straight line cuts the axis of $y$.

The equation which represents the observation is therefore

$$
y=88310^{-0884 x} \text {. }
$$

or in terms of natural logarithms

$$
\begin{aligned}
y & =883 \epsilon^{-0884 \times \frac{1}{M} x} \\
& =883 \epsilon^{-203 x .}
\end{aligned}
$$

The values calculated by this expression are entered in the table, and it will be observed that they agree very fairly with the observed numbers.

When there is no reason for using any special form of function to give the relation between the two variables involved in a series of measurements, or where by plotting on ordinary and on logarithmic paper no such form of function has been suggested, it will often be found convenient to express the results of a series of measurements by an expression of the form

$$
y=a+b x+c x^{2}+d x^{3}+\& c .
$$

where $a, b, c, d, \&$ c., are constants. In practice it is hardly ever necessary to go beyond the term in $x^{3}$, and in fact very seldom beyond the term in $x^{2}$. We have now to consider how the values of the constants in the above expression can be deduced from the experimental numbers.

Suppose that $x_{1}, x_{2}, x_{3}$, and $y_{1}, y_{2}, y_{3}$ are three corresponding sets of values of $x$ and $y$, we then have, substituting these values in equation (7), and omitting the term in $x^{3}$,

$$
\begin{aligned}
& y_{1}=a+b x_{1}+c x_{1}{ }^{2} \\
& y_{2}=a+b x_{2}+c x_{2}{ }^{2} \\
& y_{3}=a+b x_{3}+c x_{3}{ }^{2} .
\end{aligned}
$$

By solving these three equations we could calculate the values of the three constants $a, b$, and $c$.

By taking any other three pairs of values of $x$ and $y$, we could in the same way calculate the values of $a, b$, and $c$; we should probably, however, find that the values now obtained do not exactly agree with the former values. This is owing to the fact that all measurements are subject to a certain error. If we only have three observations, then the solution of the equations gives the best values of the constants $a, b$, and $c$ we can deduce from the measurements. In general, however, there are more than three measurements, and, as we have mentioned above, any three will give a set of values for $a, b$, and $c$, but the different sets of three will give different values. We have, therefore, to consider how we may best use our available data to calculate the constants. Perhaps the - simplest way to proceed is to plot the observations on squared paper and 
to draw a curve evenly between the points. Then choosing three points, one near either end of the curve and one near the middle, read off the corresponding values of $x$ and $y$, and substitute these values in the three equations, and thence deduce the values of the constants. Having done this, proceed to calculate the values of $y$ according to the expression obtained for each value of $x$ corresponding to an observed point, and draw up a table in which there are four columns, the first column containing the value of $x$, the second the observed values of $y$, and the third the calculated values of $y$. In the fourth column enter the differences between the observed and calculated values of $y$.

As an example to illustrate the methods of deducing a formula, we may take the following series of values of the temperature $t$ and of a quantity $y$ which have been read off from a curve drawn through the observed points which themselves were not made at equal intervals of temperature :-

\begin{tabular}{|c|r|r|r|r|r|}
\hline \multicolumn{1}{|c|}{$t$} & $\begin{array}{c}y \\
\text { from Curve. }\end{array}$ & $\begin{array}{c}y \\
\text { calculated } \\
\text { from (A). }\end{array}$ & Difference. & $\begin{array}{c}y \\
\text { calculated } \\
\text { from (B). }\end{array}$ & Difference. \\
\hline 10 & $20 \cdot 8$ & $21 \cdot 11$ & $-\cdot 31$ & $20 \cdot 92$ & $-\cdot 12$ \\
20 & $34 \cdot 1$ & $34 \cdot 10$ & $\pm \cdot 00$ & $33 \cdot 91$ & $+\cdot \cdot 19$ \\
30 & $49 \cdot 2$ & $49 \cdot 11$ & $+\cdot 09$ & $48 \cdot 92$ & $+\cdot 28$ \\
40 & $65 \cdot 9$ & $66 \cdot 14$ & $-\cdot 24$ & $65 \cdot 95$ & $-\cdot 05$ \\
50 & $84 \cdot 8$ & $85 \cdot 17$ & $-\cdot 37$ & $84 \cdot 98$ & $-\cdot 18$ \\
60 & $106 \cdot 2$ & $106 \cdot 22$ & $-\cdot 02$ & $106 \cdot 03$ & $+\cdot 17$ \\
70 & $129 \cdot 1$ & $129 \cdot 27$ & $-\cdot 17$ & $129 \cdot 08$ & $+\cdot 02$ \\
80 & $154 \cdot 1$ & $154 \cdot 34$ & $-\cdot 24$ & $154 \cdot 15$ & -05 \\
90 & $180 \cdot 8$ & $181 \cdot 43$ & $-\cdot 63$ & $181 \cdot 24$ & $-\cdot 44$ \\
100 & $210 \cdot 5$ & $210 \cdot 52$ & $-\cdot 02$ & $209 \cdot 33$ & $+\cdot 17$ \\
\hline \multicolumn{2}{l|}{ Mean difference } & & $-\cdot 19$ & & $\cdot 00$ \\
\hline
\end{tabular}

Since it very much simplifies the calculations if the difference in $t$ between the first and second points chosen is equal to the difference between the second and third, we will choose the three values corresponding to the temperatures of $20^{\circ}, 60^{\circ}$, and $100^{\circ}$. Substituting these values in the equations, we get

$$
\begin{aligned}
34 \cdot 1 & =a+20 b+400 c \\
106 \cdot 2 & =a+60 b+3600 c \\
210 \cdot 5 & =a+100 b+10000 c .
\end{aligned}
$$

Subtracting

$$
\begin{aligned}
72 \cdot 1 & =40 b+3200 c \\
104 \cdot 3 & =40 b+6400 c .
\end{aligned}
$$

Subtracting

$$
c=\frac{32 \cdot 2}{3200}=\cdot 01006
$$


Hence

and

$$
\begin{aligned}
b & =\frac{72 \cdot 1-3200 \times \cdot 01006}{40} \\
& =\cdot 998
\end{aligned}
$$

$$
\begin{aligned}
a & =34 \cdot 1-20 \times \cdot 998-400 \times \cdot 01006 \\
& =10 \cdot 12 .
\end{aligned}
$$

Hence the expression for $y$ is

$$
y=10 \cdot 12+\cdot 998 t+\cdot 01006 t^{2}
$$

and the table shows the agreement between the numbers given by this expression and those read from the curve.

The mean of the differences, taking account of sign, is $-\cdot 19$, that is, the calculated values are on the whole too small, though of course they agree with the curve values at the temperatures $20^{\circ}, 60^{\circ}$, and $100^{\circ}$, which were used in deducing the constants $a, b$, and $c$. It would evidently be better, therefore, if we were to use slightly different values for the constants, so that on the whole the differences were evenly distributed both as to magnitude and as to sign. To see which of the constants $a, b$, or $c$ ought to be altered, the most convenient method is to plot the differences against the values of $t$ on squared paper. If the points thus obtained lie fairly evenly about a straight line parallel to the axis of $t$, then a change in $a$ is what is required. If the points lie evenly about a straight line inclined to the $t$ axis, but crossing this axis midway between the origin and the highest value of $t$, then a change in $b$ will be sufficient. If, however, the straight line does not pass through this mid point, then a change in both $a$ and $b$ will be necessary. The amount by which $b$ has to be changed can at once be obtained by dividing the difference between the ordinates at the two ends of the line by the difference in the corresponding abscissæ. If the line which lies most evenly between the points is curved, then a change in all three constants may be necessary. The sign of the change in $c$ can at once be seen by observing whether the curve is convex or concave to the axis of $t$. In most cases, however, it will save time to recalculate the values of $a, b$, and $c$ by the method which follows rather than to attempt to adjust the values of all three constants.

In the case before us the points lie fairly evenly on both sides of a straight line drawn parallel to the axis of $t$ corresponding to a difference of $-\cdot 19$. Thus we have to reduce the value of $a$ by this amount, so that the expression for $y$ becomes

$$
y=9 \cdot 93+\cdot 998 t+\cdot 01006 t^{2} .
$$

The values for $y$ calculated from this expression are entered in the fifth column of the above table and the differences in the sixth column, and it will be noticed how much more evenly the differences are distributed.

By taking any three other points and solving we should get different 
values for the constants $a, b$, and $c$. 'Thus taking the values of $y$ at $10^{\circ}$, $50^{\circ}$, and $90^{\circ}$, the expression

$$
y=9 \cdot 80+1 \cdot 000 t+\cdot 01000 t^{2}
$$

is obtained, and this would have to be adjusted by considering the differences. As any such adjustment is a matter of judgment, a method of combining all the observations when calculating the values of the constants, and in which the differences will necessarily be distributed as we require, is often a desideratum. Such a method we now proceed to describe.

Suppose that $y_{1}, y_{2}, \ldots y_{n}$, and $x_{1}, x_{2}, \ldots x_{n}$ are the observed values of $y$ and $x$, the calculated value of $y$ corresponding to $x_{1}$, namely

$$
a+b x_{1}+c x_{1}^{2}
$$

will differ from the observed value $y_{1}$. Let this difference be called $\delta_{1}$ and so on, then we shall have-

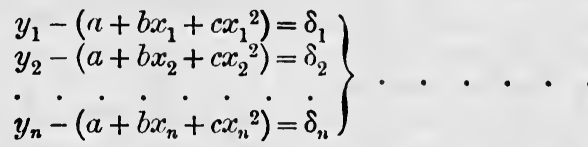

Now $\delta_{1}, \delta_{2}$, \&c., will be the differences between the observed and calculated values of $y$, and we require to so choose the constants $a, b$, and $c$ that these differences shall be as small as possible, but at the same time there shall be no preponderance of positive or negative values. Now in books on the method of least squares ${ }^{1}$ it is shown that these conditions are likely to be fulfilled if we so choose the constants that the sum of the squares of the differences is a minimum. Further, that this sum will be a minimum if we deduce the values of the constants in the following manner :-

Take the observational equations in the form

$$
\left.\begin{array}{l}
a+b x_{1}+c x_{1}^{2}-y_{1}=0 \\
a+b x_{2}+c x_{2}^{2}-y_{2}=0 \\
\dot{a}+\dot{b} x_{n}+\dot{c} \dot{x}_{n}^{2}-\dot{y}_{n}=0
\end{array}\right\} \quad \text {. . . . . . }
$$

and first ${ }^{2}$ add all the equations together. Then multiply each equation through by the coefficient of $b$ in that equation and add the equations thus obtained. Finally, multiply each equation through by the coefficient of $c$ in that equation and add the equation thus obtained. In this way the following equations, which are called normal equations, are obtained :-

$$
\left.\begin{array}{l}
\Sigma a+b \Sigma x+c \Sigma x^{2}-\Sigma y=0 \\
a \Sigma x+b \Sigma x^{2}+c \Sigma x^{3}-\Sigma x y=0 \\
a \Sigma x^{2}+b \Sigma x^{3}+c \Sigma x^{4}-\Sigma x^{2} y=0
\end{array}\right\}
$$

1 Merriman, A Text-book of Least Squares.

8. We assume that all the observations are made with the same accuracy. If the different observations have different weight, then the observational equation. will require adjustment before deducing the normal equation. 
where $\Sigma x$ means the sum of the $x$ 's, $\Sigma x^{2}$ the sum of the squares of the $x$ 's, and so on.

These three normal equations being solved will give values for the constants $a, b$, and $c$, which will fulfil the condition that the sum of the squares of the differences are a minimum. The calculation of the coefficients in the normal equations, if there are many observational equations, is a matter of very considerable labour. The work may, however, be considerably reduced if we have a series of values of $y$ for values of $x$ which differ by a constant quantity, for we may then take this constant quantity as the unit of $x$ while making the calculations. Further, it is advisable to take the first value of $x$ as the zero of $x$, and the first value of $y$ as the zero of $y$, so that in terms of these new coordinates, when $x=0, y=0$. In this case $\Sigma x$ is the sum of the first $n-1$ natural numbers, $\Sigma x^{2}$ the sum of the squares of the first $n-1$ natural numbers, and so on. ${ }^{1}$

As an illustration of the method, we proceed to apply it to the numbers used in the preceding pages.

In the first place, we take $10^{\circ}$ as our unit of $x$, and deduct 1 from each of the resulting numbers; thus on this new scale the first value for $x$ is zero. In the same way we deduct 20.8 from each of the values of $y$. We have then to calculate for each pair of values of $x$ and $y$ the quotients $x y$ and $x^{2} y$, and obtain the sums $\Sigma x y$ and $\Sigma x^{2} y$, as shown in the following table :-

\begin{tabular}{|c|c|c|c|}
\hline$x$. & $y$ & $x y$ & \multicolumn{1}{c|}{$x^{2} y$} \\
\cline { 1 - 2 } 0 & 0 & 0 & 0 \\
1 & $13 \cdot 3$ & $13 \cdot 3$ & $13 \cdot 3$ \\
2 & $28 \cdot 4$ & $56 \cdot 8$ & $113 \cdot 6$ \\
3 & $45 \cdot 1$ & $135 \cdot 3$ & $405 \cdot 9$ \\
4 & $64 \cdot 0$ & $256 \cdot 0$ & $1024 \cdot 0$ \\
5 & $85 \cdot 4$ & $427 \cdot 0$ & $2135 \cdot 0$ \\
6 & $108 \cdot 3$ & $649 \cdot 8$ & $3898 \cdot 8$ \\
7 & $133 \cdot 3$ & $933 \cdot 1$ & $6531 \cdot 7$ \\
8 & $160 \cdot 0$ & $1280 \cdot 0$ & $10240 \cdot 0$ \\
9 & $189 \cdot 7$ & $1707 \cdot 3$ & $15365 \cdot 7$ \\
\hline & & $5458 \cdot 7$ & $39728 \cdot 0$ \\
\hline
\end{tabular}

1 These quantities can be calculated from the following expressions :-

$$
\begin{aligned}
1+2+3+\quad+m & =\frac{\dot{m}(m+1)}{2} \\
1^{2}+2^{2}+3^{2}+\quad+m^{2} & =m(m+1)(2 m+1) / 6 \\
1^{3}+2^{3}+3^{3}+\quad+m^{3} & =\left(\frac{m(m+1)}{2}\right)^{2} \\
1^{4}+2^{4}+3^{4}+\quad+m^{4} & =\frac{m(m+1)(2 m+1)\left(3 m^{2}+3 m-1\right)}{30} .
\end{aligned}
$$


[\$ 5 REPRESENTATION OF A SERIES OF RESULTS

We have also to calculate

$$
\begin{aligned}
& \left(1^{2}+2^{2}+3^{2}+\quad+9^{2}\right)=285 \\
& \left(1^{3}+2^{3}+3^{3}+\quad+9^{3}\right)=2025 \\
& \left(1^{4}+2^{4}+3^{4}+\quad+9^{4}\right)=15333
\end{aligned}
$$

Since with the present values for the quantities $x$ and $y$, when $x=0$, $y=0$, the observational equations are of the form

$$
y=b x+c x^{2}
$$

and the normal equations are

$$
\begin{aligned}
& b \Sigma x^{2}+c \Sigma x^{3}-\Sigma x y=0 \\
& b \Sigma x^{3}+c \Sigma x^{4}-\Sigma x^{2} y=0
\end{aligned}
$$

Hence

$$
\begin{aligned}
& b=\frac{\Sigma x^{4} \cdot \Sigma x y-\Sigma x^{3} \cdot \Sigma x^{2} y}{\Sigma x^{2} \cdot \Sigma x^{4}-\left(\Sigma x^{3}\right)^{2}}, \\
& c=\frac{\Sigma x^{2} \cdot \Sigma x^{2} y-\Sigma x^{3} \cdot \Sigma x y}{\Sigma x^{2} \cdot \Sigma x^{4}-\left(\Sigma x^{3}\right)^{2}} .
\end{aligned}
$$

Substituting the values for the various sums, we have

$$
\begin{aligned}
b & =\frac{15333 \times 54587-2025 \times 39728}{285 \times 15333-(2025)^{2}}, \\
& =\frac{83698200-80449000}{4369900-4100620} \\
& =\frac{32490}{26928}=12.065, \\
c & =\frac{26861}{26928} \\
& =\cdot 9975 .
\end{aligned}
$$

Thus

$$
y=12 \cdot 065 t+\cdot 9975 t^{2}
$$

If, however, we change back to the original values of $x$ and $y$ we have

$$
\begin{aligned}
y-20 \cdot 8 & =12.065 \frac{t-10}{10}+.9975\left(\frac{t-10}{10}\right)^{2} \\
& =1.2065 t-12.065+.009975\left(t^{2}-20 t+100\right) \\
& =-11.068+1.0065 t+.009975 t^{2} \\
y & =9.732+1.0065 t+.009975 t^{2} . . .
\end{aligned}
$$


The following table exhibits the differences obtained by using this expression for $y$ :-

\begin{tabular}{|c|c|c|c|}
\hline$t$ & from Curve. & $\begin{array}{c}y \\
\text { calculated from (C). }\end{array}$ & Difference. \\
\cline { 1 - 3 } 10 & $20 \cdot 8$ & $20 \cdot 80$ & \pm 0 \\
20 & $34 \cdot 1$ & $33 \cdot 85$ & $+\cdot 25$ \\
30 & $49 \cdot 2$ & $48 \cdot 91$ & $+\cdot 29$ \\
40 & $65 \cdot 9$ & $65 \cdot 95$ & $-\cdot 05$ \\
50 & $84 \cdot 8$ & $85 \cdot 00$ & $-\cdot 20$ \\
60 & $106 \cdot 2$ & $106 \cdot 03$ & $+\cdot 17$ \\
70 & $129 \cdot 1$ & $129 \cdot 07$ & $+\cdot 03$ \\
80 & $154 \cdot 1$ & $154 \cdot 09$ & $+\cdot 01$ \\
90 & $180 \cdot 8$ & $181 \cdot 12$ & $-\cdot 32$ \\
100 & $210 \cdot 5$ & $210 \cdot 13$ & $+\cdot 47$ \\
\hline
\end{tabular}

When very large numbers of observational equations have to be dealt with, special schemes for conducting the necessary calculations are best adopted, for which see Kohlrausch's Physical Measurements, p. 21.

As has already been mentioned, to find an algebraical expression to represent the results of a series of observations, where theory does not indicate what the form of the expression should be, is often a matter of extreme difficulty, and there are no hard-and-fast rules which shall guide us in such a search. What has been said in the preceding pages, and the following list of expressions which may be tried, may be of some assistance :-

(1) $y=a+b x \ldots$ A straight line on squared paper.

(2) $y=a+b x^{2} \ldots\left\{\begin{array}{c}\text { A curved line on squared paper, the slope being } \\ \text { proportional to } x \text {. }\end{array}\right.$

(3) $y=a+b x+c x^{2}$.

(4) $y=a+b x^{n}$.

(5) $y=\frac{a+b x}{x} \cdot\left\{\begin{array}{c}\text { Plot } y \text { and } \frac{1}{x} \text { on squared paper, when a straight } \\ \text { line will be obtained. }\end{array}\right.$ (6) $y=\frac{a x}{1+b x} \cdot\left\{\begin{array}{l}\text { Plot } \frac{y}{x} \text { and } y \text { on squared paper, when a straight } \\ \text { line will be obtained. }\end{array}\right.$ (7) $y=\frac{a x^{2}}{1+b x^{2}} .\left\{\begin{array}{c}\text { Plot } \frac{y}{x^{2}} \text { and } y \text { on squared paper, when a straight } \\ \text { line will be obtained. }\end{array}\right.$

(8) $y=a(b+x)^{n}$.

(9) $\left.y=a 10^{n x}\right\}$. Plot $y$ and $x$ on semi-logarithm paper, when a

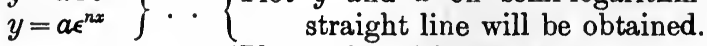

(10) $y=a x^{n} \ldots\left\{\begin{array}{c}\text { Plot on logarithm paper, when a straight line will } \\ \text { be obtianed. }\end{array}\right.$ 
6. Mathematical Tables.-The most generally useful aid to the calculations necessary in the laboratory is a table of the logarithins of the natural numbers and of the circular functions. For almost all the calculations necessary in the laboratory a table giving the logarithms to five places of decimals is sufficient, and it is ouly in very exceptional cases that it is necessary to employ six or seven figures. For many purposes, notably the calculation of corrections and the like, four figures are sufficient. Since a table of the logarithms of the numbers from 100 to 1000 to four places can be printed on a sheet about 9 inches by 6 inches, such a table is extremely handy, for no turning over of pages is involved, and thus much time is saved. ${ }^{1}$

The five-figure table ${ }^{2}$ ought to contain the logarithms of the numbers from 1 to 10000, and will occupy some twenty to thirty pages. It ought also to contain the logarithms of the sines, tangents, cosines, and cotangents of each minute of arc between 0 and $90^{\circ}$. A table of the natural sines and tangents proceeding by half degrees, and giving to three places of decimals, will also be found of service.

For such cases as demand more than five places, the book of sevenfigure logarithms published by Chambers will be found suitable.

Much time may sometimes be saved by the use of a table, such as that of Barlow (published by Spon), containing the squares, cubes, square roots, cube roots, and reciprocals of numbers from 1 to 10000 .

7. The Slide Rule.-In almost all calculations where an accuracy of about one in two or three hundred is sufficient, the most convenient method is to employ a slide rule. The theory of the slide rule is extremely simple, nevertheless it requires some practice to learn to use it with facility. In the physical laboratory, however, so much time can be saved by the use of the slide rule that all students will be well advised to acquire the necessary skill.

The most usual form of slide rule is shown in Fig. 5. A convenient size consists of a wooden body about 10 inches long, which carries two

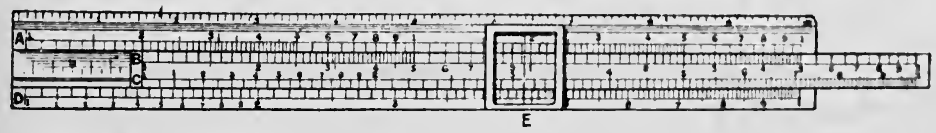

- FIG. 5.

divided scales, $\mathbf{A}$ and $\mathbf{D}$. Working in a groove in this base there is a slider which carries two scales, $B$ and $C$. The scales $A$ and $B$ are the same, while the scale $C$ is the same as the scale $D$. In the case of the

1 A four-figure table, printed on thin card, is issued hy Messrs. Vincent and Wilson, of Cambridge, price 6d. The Board of Education, South Kensington, also issue such a table as part of a set of examination tables, price one penny.

2 The most convenient five-figure table with which the author is acquainted is one by Dupuis, and is published by Hachette, Paris and London, price 2s. 2d. 
two upper scales the unit is equal to half the length of the rule, and the divisions are so spaced and numbered that the distance between the extreme left-hand division, which is marked 1 , and any division is equal to the logarithm of the number placed opposite this division. Thus the distance between the division 1 and the division 2 is equal to $\cdot 301$ of half the length of the rule, for $\cdot 301$ is the logarithm of 2 to the base ten. This scale is repeated twice on each of the scales $A$ and B, each half being numbered from 1 to 9 . The scales $\mathrm{C}$ and $\mathrm{D}$ are divided in the same logarithmic manner ; the unit taken, however, is the whole length of the scale ; that is, the unit in the case of these two lower scales is twice as great as the unit in the case of the two upper scales.

A light metal frame, called a cursor, moves in grooves in the body of the instrument, and is fitted with a plate of glass or celluloid, on which is engraved a fine line perpendicular to the length of the scales. This line serves as a reference mark, and also for obtaining the reading on the scale $\mathbf{D}$ corresponding to a reading on the scale $\mathbf{A}$.

Suppose, as shown in the figure, that the slide is drawn to the right so that the unit on the scale $B$ is opposite 2 on the scale $A$. Then opposite 3 on the scale $B$ we shall have that division of the scale $\mathbf{A}$ of which the distance from the zero is equal to the distance from 1 to 2 plus the distance from 1 to 3 , that is, the reading will represent the sum of these distances. But the distances represent the logarithms of 2 and 3 respectively, and therefore the sum of these distances will represent the logarithm of the product of 2 and 3 ; that is, the reading on the scale $\mathbf{A}$ opposite 3 on the scale $\mathbf{B}$ will be 6 , the product of the numbers 2 and 3 . In the same way, opposite each division on the scale B will be the number corresponding to the product of 2 into the reading corresponding to the division considered on scale $\mathbf{B}$.

In the same way, opposite any division of scale A will be found the quotient of the number corresponding to this division divided by 2 .

Hence by one setting of the slide we can directly read off the products or quotients of any number of numbers by some constant number. This property of the slide rule will be found of great value, for we often require to multiply or divide a column of figures by some common multiplier or divisor.

The manner in which the slide rule is used where there are more than two factors to be multiplied or divided is best explained by means of some examples.

1. Let us suppose that the fraction to be evaluated is

$$
\frac{980 \times 2 \cdot 43 \times 10.3}{27.5 \times 6.31}
$$

(1) Set $9 \cdot 8$ on the scale $\mathrm{A}$ opposite 2.75 on the scale $\mathbf{B}$.

(2) Set the black line on the cursor to $2 \cdot 43$ on B.

(3) Move the slide, the cursor remaining in place, till 6.31 on $B$ is opposite the line on the cursor. 
(4) Opposite 1.03 on $B$ read off the required result, namely, 1.415 on scale $\mathrm{A}$.

We thus obtain the value 1415 for the result, and the position of the decimal place remains to be fixed. Inspection of the fraction shows that its value must lie between 100 and 1000, hence we conclude that the true value is

\section{$141 \cdot 5$.}

This example could also be worked on the two lower scales in exactly the same manner, and owing to the more open nature of the scales the accuracy attainable would be increased. In the following two examples the advantage of having the scale duplicated will appear :-

\&.

$$
\frac{45.5 \times 31 \cdot 2}{12 \cdot 6}
$$

Suppose we use the lower scales, and set 1.26 on scale $\mathrm{c}$ opposite 4.55 on scale $\mathrm{D}$, then the result will be opposite $3 \cdot 12$ on scale c. This reading on $\mathrm{C}$ is, however, beyond the end of scale $\mathrm{D}$. To obtain the reading we must set the cursor on the left-hand end division of scale c, and then move the slide to the left till the right-hand end division on $\mathbf{c}$ coincides with the cursor line. The reading on scale D opposite 3.12 on scale $\mathrm{c}$ will give the required result, namely, $1 \cdot 125$, or adjusting the position of the decimal point,

\section{$112 \cdot 5$}

Here to obtain the required result it has been necessary to move the slider through its whole length, and to do this a setting of the cursor has been made independent of those necessary while manipulating the numbers. If the calculation be made by means of the two upper scales, owing to the repetition of the scales this movement of the slide will no longer be necessary, and hence some time will be saved. As, however, the scales are not so finely divided as the lower scales, the accuracy with which the settings can be made is not so great.

In certain cases, although it will not be necessary to move the slide, it becomes necessary to change from one half of the scales to the other, that is, to multiply or divide by ten. This is illustrated in the following example:-
3.
$68 \cdot 5 \times 3 \cdot 45 \times 95 \cdot 2$.

Putting the left-hand (1) division of scale B opposite 6.85 on scale A, move the cursor to 3.45 on scale $B$. If, now, the first division on the lefthand side of scale $B$ is placed at the line of the cursor it will be found that 9.52 on scale $\mathrm{B}$ is beyond the end of scale $\mathrm{A}$. If, however, the middle division (marked 1) on scale $B$ is placed so as to coincide with the mark on the cursor, then opposite the 9.52 division on $B$ will be found the required product, namely,

that is, the product is 22400 .

$$
2 \cdot 24 \text {, }
$$

The above examples will give some hints as to the methods which 
have to be adopted to perform simple calculations on the slide rule, and it is only for simple calculations that it will be in the long run found advisable to use the rule. In using the rule it is well to remember that whatever the relative positions of the two scales which are adjacent to one another, if $a_{1}, a_{2}$ are two readings on one scale and $b_{1}, b_{2}$ are the corresponding readings on the other, then

$$
\frac{a_{1}}{b_{1}}=\frac{a_{2}}{b_{2}} \text { or } \frac{a_{1}}{a_{2}}=\frac{b_{1}}{l_{2}} \text {. }
$$

Thus, suppose we require to find the quotient of $b / c$. Calling the quotient $a$, we have

$$
\frac{b}{c}=\frac{a}{1}
$$

or

$$
\bar{b}=\frac{c}{1} \text {, }
$$

the first of these expressions corresponds to the method of working described above, namely, $b$ on one scale is made to coincide with $c$ on the other, and the quotient is found opposite 1 on this latter scale. The second expression corresponds to the following method of working, which is often more convenient. Bring $c$ on one scale opposite the unit on the other, and then opposite $b$ on the first scale will be found the desired quotient $a$ on the second scale.

Squares and square roots may be directly read off by means of the cursor. Thus by setting the cursor to any reading on the $\mathrm{D}$ scale, the corresponding reading on the A scale will give the square. Conversely, by setting the cursor to a given reading on the $A$ scale where the division line of the cursor cuts the $\mathrm{D}$ scale will give the square root.

Cubes may be found by obtaining the square and then setting the unit division on scale $\mathrm{C}$ opposite the given number on scale $\mathrm{D}$, and then reading off the number on scale $\mathbf{A}$ opposite the given number on scale $\mathbf{B}$.

To obtain cube roots the slide must be taken ont and turned upside down, so that scale $\mathbf{c}$ is opposite scale $\mathbf{A}$. The unit division on scale $\mathbf{c}$ must then be placed opposite the given number on scale A, and scales B and $D$ examined for the point where the readings on the two scales which are opposite one another are the same. The reading corresponding to this coincidence is the required cube root. Thus if the cube root of 27 is required, the unit on scale $c$ is put opposite the left hand 2.7 of scale $\mathrm{A}$, when it is found that 3.0 of scale B coincides with 3.0 of scale $D$, and hence 3 is the required cube root. If the unit on scale $\mathrm{c}$ had been placed opposite the right hand 2.7 on scale $A$ it would be found that nowhere did the readings on scales $\mathbf{B}$ and $\mathbf{D}$ coincide.

The back of the slider of most rules carries three other scales; these are used for finding logarithms, sines, and tangents. They are seldom required in the laboratory, as it is generally more convenient to use tables. An examination of the rule will, however, at once indicate how they are to be used. 
8. Arithmometers. - There are certain calculations which, when a great mass of figures have to be dealt with, are most expeditiously performed by means of an instrument called an arithmometer. A common form of arithmometer is shown in Fig. 6. It is called the Thomas de Colmar pattern, and is very convenient to use, the only objections being the high price (£20 to $£ \check{\omega} 0$ ) and somewhat large size.

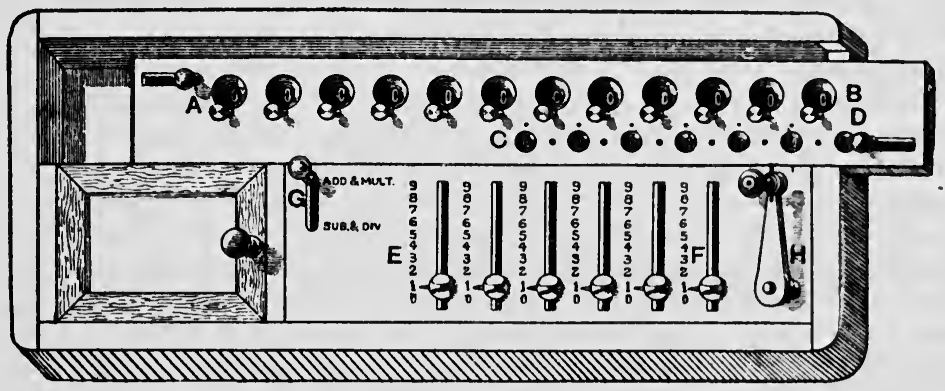

FIG. 6.

The instrument consists of a movable portion $\mathrm{AB}$, which on being raised slightly can be slid along to the right, and on closing always rests so that the upper line of perforations $A B$ lie opposite to the number slides EF on the fixed portion. Through each of the holes in the row $\mathbf{A B}$ can be seen one of the figures on a series of discs, each disc carrying the numbers from 0 to 9 . There is also a lower series of perforations CD ; each of these also allows one of the numbers on a disc similar to the upper row to be read. The sliders EF can be moved up grooves, and a small pointer attached to each slider moves on a scale which is numbered from 0 to 9 . A handle $\mathrm{g}$ can be put in two positions, one labelled "addition and multiplication," and the other "sultraction and division." A handle $\mathrm{H}$ can be rotated in the clockwise direction, and serves to set the mechanism working. Two levers, $\mathrm{A}$ and $\mathrm{D}$, when pulled to the left and right respectively after the slider is raised, serve to set all the discs in the two rows to zero.

If all the sliders EF are at zero, then turning the handle $\mathbf{~}$ produces no result on the upper row of figures If, however, any of the sliders are moved up, then on turning the handle through a complete turn the discs on the upper row $\mathbf{A B}$ turn so that the numbers showing through the windows are the same as the numbers to which the sliders which happen to be exactly opposite them are set. At the same time the disc on the lower row CD, which is opposite the handle $\mathrm{H}$, turns so that 1 appears, this representing the number of times the handle has been turned. If, now, the handle is given a second turn, then the readings on the upper row of discs will increase by an amount corresponding to the readings of the sliders $\mathrm{FF}$, while the fact that the handle has been 
turned twice will be indicated by 2 appearing on the disc of the lower row immediately opposite the handle. Now the number represented on the sliders having. been twice added to the reading (zero) on the upper row of discs, the number which is now shown on these discs will be twice the number shown on the sliders. In the same way, if the handle is turned three times, the number shown on the upper row of discs will be three times the number shown on the sliders, and so on. Let us suppose that the slider being as far to the left as it will go, and all the discs showing zero, we place the number 111111 on the sliders, that is, move each slider up till its pointer points to 1 on the scale. If, then, the handle is turned once the number 111111 will appear on the last six of the discs on the upper row, while 1 will appear on the last disc of the lower row, that is, on the disc opposite the handle. Next move the slider one notch to the right so that the second disc on the lower row is opposite the handle. If, now, the handle is turned once, each of the discs which is now opposite one of the sliders will have its reading increased by unity, that is, by the amount shown on the corresponding slider. The result now shown on the upper row of discs is not twice 111111 , as it would have been had the slider not been moved, but it is the product of 11 and 111111, for the machine has performed exactly the same sequence of operations that is used when doing multiplication by the ordinary method, namely, it has multiplied the multiplicand by the unit digit of the multiplier, then multiplied by the ten digit, displacing this product one place to the left, and then added the two results together. If the handle is turned twice when in the second position, the product of 111111 by 21 will appear on the upper row of discs, while the lower row will exhibit the multiplier 21. In the same way, by displacing the slider another place to the right and turning the handle once, we shall obtain the product of 111111 by 121 , and so on.

If the lever $\mathrm{G}$ is placed opposite the inscription "subtract or divide," then on turning the handle once the number represented on the slides will be subtracted from any number which may be exhibited on the upper row of discs. To perform division, the dividend is placed on the upper row of discs as near the left-hand end of the slider as possible. This can be done either by setting this number on the slides and then turning the handle once while the lever $\mathrm{g}$ is at "add," or, what is generally more convenient, by raising the slider and then turning, by means of a small stud which projects immediately below the window, each disc till the required digit appears in the window. The divisor is then placed on the slides, also as far to the left as possible. The slider is moved till the number shown on the upper row of discs is larger than that shown on the slides, the unit digit in the two cases being supposed to be vertically over one another. The lever $\mathrm{G}$ being at "subtract," the handle is turned till the number on the discs is smaller than that on the slides. The slider is then moved one place to the left, and the handle again turned till the number on the discs is less than that on the slides, and so on till the required number of 
places is obtained, when the quotient will appear on the lower row of discs. If, when performing this operation, the handle is accidentally turned once too often, this will be indicated by a number of nines appearing to the left of the numbers on the upper row of discs. In such a case the lever must be put at "add" and the handle given one turn, which will exactly annul the superfluous turn which has been given, and then again setting the lever at "subtract," the slider can be moved one step to the left and the division proceeded with. It will at once be evident that the machine proceeds in exactly the same manner as is done when doing long division by the ordinary method.

The arithmometer does not do division with anything like the rapidity with which it does multiplication. Hence if several numbers have to be divided by the same number, it will save time if the reciprocal of this number is obtained, either by means of the machine or from tables, and then each of the numbers is multiplied by this reciprocal in the manner described below.

When a number of numbers which do not differ much have to be multiplied by the same number, an operation which is of frequent occurrence in Physics, after performing the first multiplication it is not necessary to wipe out the numbers which appear on the discs before proceeding to the second multiplication. All that has to be done is by suitably manipulating the slide and the lever $G$, turning the handle when necessary of course, to change the reading on the lower set of discs till the second number appears there, when the product will be given on the upper row of discs. Thus, suppose the common multiplier is 64799 , and that two of the numbers to be multiplied are 17628 and 17635 . First set the common multiplier, 64799, on the slides. Then starting with the slider as far to the left as it will go, turn the handle eight times; move the slider one place to the right and turn the handle twice ; move slider another place to right and turn six times, and so on. The product is

\section{2 ,}

and as many digits of this as are required must be noted down. Next placing the slider so that the 8 of the number on the lower row of discs is opposite the handle and setting the lever $\mathrm{g}$ at "subtract," turn the handle three times, so that the 8 becomes 5 . Next move the slider one place to the right, so that the 2 comes opposite the handle. Place the lever $G$ at "add" and turn the handle once, so that the 2 becomes 3 . The number now shown on the lower row of discs is 17635 , and the product of this number by 64799 , namely,

\section{5 ,}

is shown on the upper row of discs. Thus we have obtained the second product by only turning the handle four times, while if we had started $a b$ initio the handle would require to have been turned twenty-two times.

The above arithmometer may be used for adding together long columns of figures. For this purpose each number is in turn placed on the slides, 
and the handle given one turn so as to add the number on the slides to the number already shown on the upper row of discs. The method is not a very rapid one, since the slides have to be manipulated each time, and, where much addition has to be performed, an instrument called a comptometer ${ }^{1}$ may with advantage be used. In this machine there are a number of keys, something like those of a typewriter, arranged in columns, each column containing keys numbered from 0 to 9 , and whenever a key is depressed the number on it is added to the number which appears at the top of that column on the recording dials.

9. Determination of the Area of Plane Figures.-A problem which occurs fairly frequently in Physics is the measurement of the area of a plane figure. Thus the work done by the gas in the cylinder of a steam or gas engine is obtained by measuring the area of the indicator diagram. This area can either be obtained by measuring a number of ordinates and using one of the rules given below, or an instrument called a planimeter can be employed.

The most convenient rule to employ when calculating the areas of a figure from the measurement of a number of ordinates is as follows :-

Draw two parallel lines touching the extremities of the figure, and a line at right angles to these two lines. Then divide the portion of this cross line intercepted between the parallel lines into an even number of parts (preferably 20,30,40), and through the 1st, 3rd, 5th, \&c., of these division points draw lines parallel to the first two lines. In this way half as many of these intermediate ordinates will be obtained as there were parts into which the cross line was divided, the interval between the end ordinates and the lines touching the boundaries of the figure being half the interval between the intermediate ordinates. The length of each of the intermediate ordinates intercepted between the boundaries of the figure is measured, and the mean value of this intercept is multiplied by the length of the cross line included between the two tangent lines, and the product is the area of the figure. The application of the method to an indicator diagram is shown in Fig. 7. Where a number of indicator diagrams have to be measured, it will save much time if a piece of glass be prepared with a scale, as shown in Fig. 7. This can be prepared either by ruling on the glass or by drawing the scale on paper, and then photographing it and printing a positive on a photographic plate.

Another rule frequently employed, and known as Simpson's rule, is as follows:-

Divide the area into an even number of strips by an odd number of equally spaced ordinates, the first and last touching the boundary of the figure. Measure the lengths of the ordinates intercepted by the figure, and then add together the sum of the first and last ordinates, four times the sum of the even ordinates, and twice the sum of the odd ordinates,

1 Made by Felt \& 'Tarrant Manufacturing Company, 52 Illinois Street, Chicago, U.S.A. Price, £25. 
omitting the first and last. This total must be multiplied by one-third the distance between consecutive ordinates to give the area.
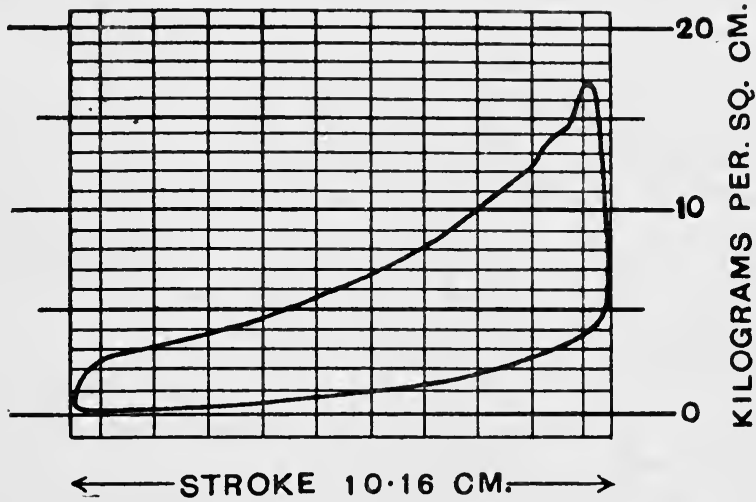

FIG. 7.

It will be seen that the first rule is considerably easier to apply, and the result is very little, if at all, less accurate than that given by Simpson's rule.

10. The Planimeter.-The commonest form of planimeter is that invented by Amsler, and shown in Fig. 8. It consists of two pivoted arms $A B$ and $C D$, with a sharp point at $D$, which, on being pressed into the paper, serves as a centre round which the instrument can turn. A small weight is generally attached above $D$ to keep this point in the hole

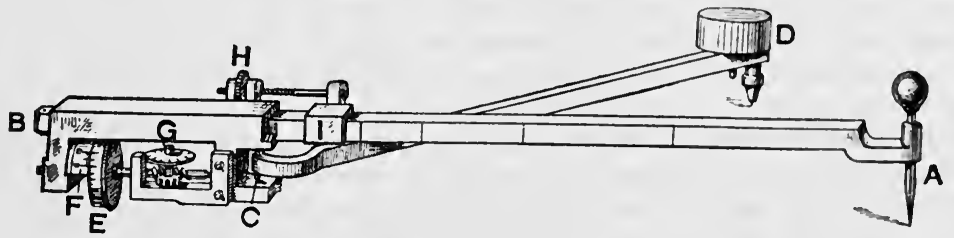

FIG. 8.

in the paper. At the end $\mathrm{A}$ of the other arm is a tracing-point, which is guided by hand round the perimeter of the figure of which the area is being measured. Attached to the arm $\mathrm{AB}$ is a wheel $\mathrm{E}$, which rests on the paper, and can turn about an axis prarallel to AB. The edge of this wheel is fairly sharp, and attached to the side of the wheel is a divided drum, by means of which, and a fixed vernier $r$, the angle through which the wheel has turned can be read. The whole turns of the wheel are registered by means of a divided disc $\mathrm{G}$, which is rotated by a worm attached to the spindle which carries the wheel $\mathrm{k}$. By means of a clamp 
I, and a fine adjustment $\mathrm{H}$, the distance between the point where the arm $\mathrm{OD}$ is pivoted and the tracing-point $\mathrm{A}$ can be varied. By varying this length the factor by which the change in reading on the wheel has to be multiplied to give the area can be altered. There are generally division lines on the arm $\mathbf{A B}$, so that when these are made to coincide with a fixed mark on the part which carries the pivot for the arm CD the readings on the wheel give the area in square centimetres or squar6 inches, as the case may be.

The position of the fixed point $\mathbf{D}$ having been so chosen that it is possible to trace the whole of the perimeter of the figure with the point $\mathbf{A}$, this pointer is brought to some marked position on the curve, and either the integrating wheel $\mathbf{E}$ is adjusted to zero, or the reading is noted. The tracing-point $A$ is then carried round the perimeter of the figure in the clockwise direction and brought back to the starting-point, and the reading of the wheel again noted. The difference in the readings of the wheel at the end and the beginning, when multiplied by the appropriate factor, as shown on the side of the arm $\mathbf{A B}$, will give the area of the figure.

If the figure is so large that the instrument will not stretch far enough to allow of the tracing-point being taken right round, the figure must be divided into parts, and the area of each part be determined separately.

It is only when the fixed point $\mathrm{D}$ is outside the area to be measured that the difference in the readings of the integrating wheel gives directly the area. If the fixed point lies within the area, then on tracing the perimeter of the figure the instrument will make a whole revolution round the fixed point. In such a case the area, as given by the difference in the readings of the wheel, must be added to the area of what is called the datum circle, the area of this circle being marked by the maker on the top of the arm AB. The datum circle is the circle swept out by the pointer when the two arms make such an angle with one another that the plane of the edge of the integrating wheel passes through the fixed point. It is evident if, while the arms make this angle, the instrument is rotated round the fixed point; since the wheel will always be moving in a direstion parallel to its axis, it will not rotate, and hence will not record the area swept out by the tracing-point. If the area of that portion of the figure which lies outside the datum circle is less than the difference in area between the remainder of the figure and the datum circle, then on tracing out the figure in the clockwise direction the reading on the integrating wheel will decrease. This decrease in the reading corresponds to a negative area, and hence to obtain the area of the figure this area, as read from the wheel, has to be subtracted from the area of the datum circle to obtain the area of the figure.

For the theory of the Amsler planimeter the student must refer to books on the integral calculus. A most interesting description of the theory of planimeters in general, and of Amsler's planimeter in particular, with a discussion of the errors to which it is liable, by Professor O. Hen. 
rici, will be found on p. 496 of the Report of the British Association for the Oxford Meeting of 1894 .

11. Harmonic Analysis.-According to Fourier's theory, any finite periodic function can be expressed as a series of terms, each of which is a simple harmonic function of this variable. Thus if $F$ is any periodic function of the time, according to Fourier's theory $F$ can, by suitably choosing the constants $A_{0}, A_{1}, A_{2}$, \&c., $B_{1}, B_{2}$, \&c., be expressed by a series of terms as follows :-

$$
\left.\begin{array}{rl}
F=A_{0} & +A_{1} \sin k t+A_{2} \sin 2 k t+A_{3} \sin 3 k t+d c . \\
& +B_{1} \cos k t+B_{2} \cos 2 k t+B_{3} \cos 3 k t+\& c .
\end{array}\right\} .
$$

This series, containing, as it does, terms involving both sines and cosines, is sometimes less convenient than the following form :-

$$
F=A_{0}+M_{1} \sin \left(k t+e_{1}\right)+M_{2} \sin \left(2 k t+e_{2}\right)+\& c_{.},
$$

where $M_{1}, M_{2}, M_{3}$, \&c., and $e_{1}, e_{2}, e_{3}$, \&c., are constants.

The connection between the constants which appear in the two series is as follows :-

$$
\begin{aligned}
M_{1}= & \sqrt{A_{1}{ }^{2}+B_{1}{ }^{2}} ; M_{2}=\sqrt{A_{2}^{2}}+\overline{B_{2}^{2}}, \& c ., \\
& \tan e_{1}=\frac{B_{1}}{A_{1}} ; \tan e_{2}=\frac{B_{2}}{A_{2}}, \text { \&c. }
\end{aligned}
$$

A problem which occurs in the study of alternating current machinery, in acoustics, and in meteorology, is that, given the form of the curve which expresses the connection between the function $F$ and the variable $t$, to deduce the values of the coefficients of the terms in the Fourier expansion. Although instruments have been devised for mechanically performing the analysis, yet owing to their great price and scarcity it is hardly necessary to describe them here. A large number of methods have boen devised for performing the analysis by calculation both with and without the aid of graphical methods.

The principles on which the methods of determining the values of the coefficients of a Fourier series to represent any given periodic curve are founded are as follows. By an easy application of the integral calculus it can be shown that the following definite integrals, taken over a whole period of the circular function, have the values shown, the constants $a$ and $b$ having any values, and $k$ being $2 \pi / T$, where $T$ is the period :-
(a) $\int_{0}^{T} \sin a k t \cdot d t=\int_{0}^{T} \cos a k t \cdot d t=0$,
(b) $\int_{0}^{T} \sin a k t \cdot \cos b k t . d t=0$,
(c) $\int_{0}^{T} \sin a k t \cdot \sin b k t . d t=0$, 

(d) $\int_{0}^{T} \cos a k t \cdot \cos b k t \cdot d t=0$,
(e) $\int_{0}^{T} \sin ^{2} a k t . d t=\int_{0}^{T} \cos ^{2} a k t \cdot d t=\frac{T}{2}$.

Suppose that we multiply both sides of the expression (1) given on p. 35 for the Fourier series by $\sin n k t$ and integrate between 0 and $T$, that is, over a complete period, we have

$$
\begin{gathered}
\int_{0}^{T} F \sin n k t . d t=A_{0} \int_{0}^{T} \sin n k t . d t+A_{1} \int_{0}^{T} \sin k t . \sin n k t . d t+\ldots \\
\quad+A_{n} \int_{0}^{T} \sin ^{2} n k t . d t \\
+B_{1} \int_{0}^{T} \cos k t . \sin n k t . d t+\ldots+B_{n} \int_{0}^{T} \cos n k t . \sin n k t . d t .
\end{gathered}
$$

Now the left-hand side of this equation does not necessarily vanish, for $F$ is a function of $t$. Of the terms on the right-hand side the only one which is not zero, according to the relations given above, is the term involving $A_{n}$, and the integral will have the value $T / 2$. Hence

But

$$
\begin{gathered}
A_{n}=\frac{2}{T} \int_{0}^{T} F \sin n t t . d t . \\
\frac{1}{T} \int_{0}^{T} F \sin n k t . d t
\end{gathered}
$$

is the mean value of the ordinates of the original curve each multiplied by the appropriate value of sin nkt.

Hence the coefficient $A_{n}$ is twice the mean value of the product of each ordinate corresponding to an epoch $k t$ multiplied by the sine of $n$ times this epoch. Similarly for the cosine terms we must multiply through by cos nkt and obtain the mean ordinate. Thus to analyse the curve we have to multiply each ordinate by $\sin k t$, $\cos k t$, sin $2 k t$, $\cos 2 k t$, and so on, and obtain the means. To perform this multiplication for many terms is very laborious; the calculation may, however, be considerably simplified by making use of the fact that as $k t$ increases from $0^{\circ}$ to $360^{\circ}$ the sine and cosine each pass four times through the same numerical value, twice positive and twice negative. Hence certain of the ordinates will have to be multiplied by the same value of the sine or cosine as the case may be, and therefore labour will be saved by adding or subtracting the ordinates first and then performing the multiplication on the sum or difference.

Various schedules ${ }^{1}$ have been prepared which give directions for

1 In addition to the methods described below, a series of schedules bas been prepared by General Strachey which are particularly applicable to meteorological phenomena of which we have either hourly observations throughout the day or daily observations throughout the year. 'These schedules and a number of tables to assist in the computations are printed in Part IV. of the Hourly Readings, 1884, published by the Meteorological Council (Eyre \& Spottiswoode). 
performing the necessary additions, subtractions, \&c., and of these those prepared by Professor C. Kiunge are particularly suited to the wants of the physicist. Runge ${ }^{1}$ gives two schemes, one in which twelve equidistant ordinates are used, and the other in which there are thirty-six ordinates. Since the scheme for thirty-six ordinates is somewhat complicated, and for most purposes sufficient accuracy can be obtained with twelve ordinates, the scheme for this latter will alone be given.

Let the values of $F$ taken at twelve equidistant points throughout the period be represented by $y_{1}, y_{2}, y_{3}, \ldots y_{12}$. These values must then be arranged in two horizontal lines, as shown in the schedule below, the lower line being written in the reverse direction. The sums $a_{1}, a_{2}, a_{3}, \& c$., and the differences $e_{2}, e_{3}, e_{4}$, \&c., of the various columns having been obtained, these numbers are written down in the order shown in Schedules 2 and 3, and the sums and differences of the columns of these tables obtained. These sums and differences are used to construct Schedules 4 and 5, and in one case the sums, and in the other the differences, are obtained.

When obtaining the sums and differences great care must be taken that the signs are correct. This is a matter of such vital importance that the author considers it advisable whenever any of the measured values of $F$ are negative to increase each of the values of $y$ by such an amount that all the numbers are positive. The only effect of this increase will be to increase the constant term $A_{0}$ by an equal amount.

The numbers obtained by these various operations must now be entered in the indicated places in the Schedule 6, each number having first been multiplied by the number given at the left-hand end of the horizontal row in which it is to be entered. Thus $f_{1}$ has to be multiplied by 0.5 and then entered at the top left-hand corner of the division which is headed 1 and 5 . It will be observed that the numbers in each of these divisions are not all arranged vertically under one another, but form two columns. The totals obtained by separately adding up the numbers in these columns are to be entered at the bottom of the divisions, the sum corresponding to the first column being placed vertically over that obtained from the second column. The sums and differences of these totals are then obtained, and these numbers when divided by 6 (in the case of $A_{0}$ divided by 12) give the values of the coefficients.

\begin{tabular}{|c|c|c|c|c|c|c|c|}
\hline $\left.\begin{array}{l}\text { alues of } \\
\text { rdinates }\end{array}\right\}$ & $y_{1}$ & $\begin{array}{l}y_{2} \\
y_{12}\end{array}$ & $\begin{array}{l}y_{3} \\
y_{11}\end{array}$ & $\begin{array}{l}y_{4} \\
y_{10}\end{array}$ & $y$ & & $y_{8}$ \\
\hline & & & $\begin{array}{l}a_{3} \\
e_{3}\end{array}$ & $e_{4}$ & & & $e_{e}$ \\
\hline
\end{tabular}

1 C. Runge, Zeitschrift für Mathematik und Physik(1903), 48, 443. 
(2)

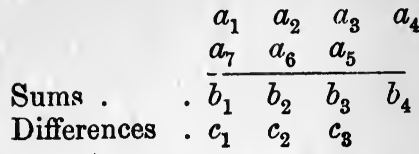

(4)
(3)

$\begin{array}{lll}e_{2} & e_{3} & e_{4}\end{array}$

\begin{tabular}{lll}
$e_{6}$ & $e_{5}$ \\
\hline$f_{1}$ & $f_{2}$ & $f_{3}$
\end{tabular}

$g_{1} g_{2}$

(5)

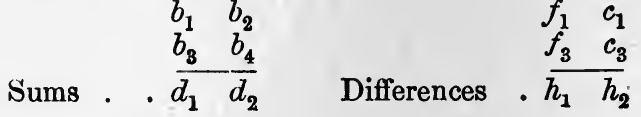

(6)

\begin{tabular}{|c|c|c|c|c|c|c|c|}
\hline \multirow{2}{*}{ Multipliers. } & \multicolumn{3}{|c|}{ Sine Terms. } & \multicolumn{4}{|c|}{ Cosine Terms. } \\
\hline & 1st. 5th. & 2nd. 4th. & 3rd. & 5th. & 2nd. 4th. & 3rd. & 6th. \\
\hline $1^{\cdot 5}$ & $\begin{array}{ll}f_{1} & \\
f_{3} & f_{2}\end{array}$ & $\begin{array}{ll}g_{1} & g_{2}\end{array}$ & $h_{1}$ & $\begin{array}{ll}c_{3} & \\
c_{1} & c_{2}\end{array}$ & $\begin{array}{cc}-b_{3} & b_{2} \\
b_{1} & -b_{4}\end{array}$ & $h_{2}$ & $d_{1}$ \\
\hline $\begin{array}{l}\text { Total 1st column } \\
\text { Total 2nd column }\end{array}$ & $\begin{array}{l}\cdots \\
\cdots\end{array}$ & $\begin{array}{l}\cdots \\
\cdots\end{array}$ & $\ldots$ & $\ldots$ & $\ldots$ & $\begin{array}{l}\ldots \\
\cdots\end{array}$ & $\ldots$ \\
\hline $\begin{array}{l}\text { Sums } \ldots \ldots \\
\text { Differences . . . }\end{array}$ & $\begin{array}{l}6 A_{1} \\
6 A_{5}\end{array}$ & $\begin{array}{l}6 A_{2} \\
6 A_{4}\end{array}$ & $\begin{array}{l}6 A_{3} \\
\ldots\end{array}$ & $\begin{array}{l}6 B_{1} \\
6 B_{5}\end{array}$ & $\begin{array}{l}6 B_{2} \\
6 B_{4}\end{array}$ & $\begin{array}{c}6 B_{3} \\
\ldots\end{array}$ & $\begin{array}{l}12 A_{0} \\
12 B_{6}\end{array}$ \\
\hline
\end{tabular}

It will be observed that since the multiplication by 0.5 and by 1 can

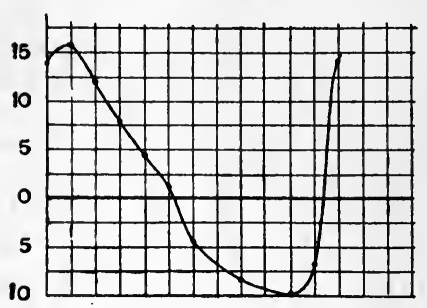

FIG. 9. be done as the figures are being copied down, there only remain four figures which have to be multiplied by 0.886 , that is, sine $60^{\circ}$, all the rest being addition and subtraction. The following example taken from Professor Runge's paper will show how the calculation ought to be set out, while in Fig. 9 is given the form of the curve which the numbers represent:-

\begin{tabular}{|c|c|c|c|c|c|c|c|}
\hline $\begin{array}{l}\text { Values of . } \\
\text { Ordinates. }\end{array}$ & $\begin{array}{c}14 \cdot 0 \\
\ldots\end{array}$ & $\begin{array}{r}15 \cdot 8 \\
-6 \cdot 8\end{array}$ & $\begin{array}{r}12.0 \\
-9.9\end{array}$ & $\begin{array}{r}7 \cdot 7 \\
-9 \cdot 3\end{array}$ & $\begin{array}{r}4 \cdot 3 \\
-8 \cdot 2\end{array}$ & $\begin{array}{r}1 \cdot 4 \\
-6.8\end{array}$ & $\begin{array}{r}-4 \cdot 6 \\
\ldots\end{array}$ \\
\hline $\begin{array}{l}\text { Sums }(a) \cdot \\
\text { Differences }(e)\end{array}$ & $\begin{array}{c}\overline{14} \cdot \overline{0} \\
\ldots\end{array}$ & $\begin{array}{r}+9 \cdot 0 \\
+22 \cdot 6\end{array}$ & $\begin{array}{r}+2 \cdot 1 \\
+21 \cdot 9\end{array}$ & $\begin{array}{r}-1 \cdot 6 \\
+17 \cdot 0\end{array}$ & $\begin{array}{r}-3.9 \\
+12.5\end{array}$ & $\begin{array}{r}-5 \cdot 4 \\
+8 \cdot 2\end{array}$ & $-4 \cdot 6$ \\
\hline & $\begin{array}{r}14 \cdot 0 \\
-4 \cdot 6\end{array}$ & $\begin{array}{r}9 \cdot 0 \\
-5 \cdot 4 \\
\end{array}$ & $\begin{array}{r}2 \cdot 1 \\
-3 \cdot 9\end{array}$ & $\begin{array}{c}-1 \cdot 6 \\
\ldots\end{array}$ & $\begin{array}{r}22 \cdot 6 \\
8 \cdot 2\end{array}$ & $\begin{array}{l}21 \cdot 9 \\
12 \cdot 5\end{array}$ & $17 \cdot 0$ \\
\hline $\begin{array}{l}\text { Sums }(b) \\
\text { Differences }(c)\end{array}$ & $\begin{array}{c}9 \cdot 4 \\
18 \cdot 6\end{array}$ & $\begin{array}{c}3 \cdot 6 \\
14 \cdot 4\end{array}$ & $\begin{array}{r}-1.8 \\
6.0\end{array}$ & $\begin{array}{r}-1 \cdot 6 \\
\ldots\end{array}$ & $\begin{array}{l}\text { (f) } 30 \cdot 8 \\
(g) 14 \cdot 4\end{array}$ & $\begin{array}{r}34 \cdot 4 \\
9 \cdot 4\end{array}$ & $17 \cdot 0$ \\
\hline
\end{tabular}



$9 \cdot 4 \quad 3 \cdot 6$
$30 \cdot 8 \quad 18 \cdot 6$
$-1 \cdot 8-1 \cdot 6$
Sums $(d)$
$7 \cdot \overline{6} 2 \cdot 0$
$17 \cdot 0 \quad 6 \cdot 0$
Differences $(h) \quad \overline{13 \cdot 8} \quad 12 \cdot 6$

\begin{tabular}{|c|c|c|c|c|c|c|c|}
\hline \multirow{2}{*}{ Multipliers. } & \multicolumn{3}{|c|}{ Sine Terms. } & \multicolumn{4}{|c|}{ Cosine Terms. } \\
\hline & 1st. 5th. & 2nd. 4th. & 3rd. & 1st. 5th. & 2nd. 4th. & 3rd. & 0. 6th \\
\hline $\begin{array}{l}0.5 \\
.866 \\
1.0\end{array}$ & ${ }_{17 \cdot 0}^{15 \cdot 4} 29 \cdot 8$ & $12.58 \cdot 1$ & $\cdot 13 \cdot 8$ & ${ }_{18 \cdot 6}^{3 \cdot 0} 12.5$ & $\mid \begin{array}{ll}0.9 & 1.8 \\
9.4 & 1.6\end{array}$ & $12 \cdot 6$ & $7 \cdot 6 \quad 2 \cdot 0$ \\
\hline $\begin{array}{l}\text { 1st column } \\
\text { 2nd column. }\end{array}$ & $\begin{array}{l}32 \cdot 4 \\
29 \cdot 8\end{array}$ & $\begin{array}{r}12 \cdot 5 \\
8 \cdot 1\end{array}$ & $\begin{array}{l}\cdots \\
\cdots\end{array}$ & $\begin{array}{l}21 \cdot 6 \\
12 \cdot 5\end{array}$ & $\begin{array}{r}10 \cdot 3 \\
3 \cdot 4\end{array}$ & $\begin{array}{l}\cdots \\
\cdots\end{array}$ & $\begin{array}{l}7 \cdot 6 \\
2 \cdot 0\end{array}$ \\
\hline $\begin{array}{l}\text { Sums . . . } \\
\text { Differences . }\end{array}$ & $\begin{array}{r}62 \cdot 2 \\
2 \cdot 6\end{array}$ & $\begin{array}{r}20 \cdot 6 \\
4 \cdot 4\end{array}$ & $\begin{array}{c}13 \cdot 8 \\
\ldots\end{array}$ & $\begin{array}{r}34 \cdot 1 \\
9 \cdot 1\end{array}$ & $\begin{array}{r}13 \cdot 7 \\
6.9\end{array}$ & $\begin{array}{c}12 \cdot 6 \\
\ldots\end{array}$ & $\begin{array}{l}9 \cdot 6 \\
5 \cdot 6\end{array}$ \\
\hline . & $\begin{array}{l}A_{1}=10.37 \\
A_{5}=0.43\end{array}$ & $\left\{\begin{array}{l}A_{2}=3.43 \\
A_{4}=0.73\end{array}\right.$ & $\begin{array}{c}A_{3}=2 \cdot 30 \\
\ldots\end{array}$ & $\left\{\begin{array}{l}B_{1}=5.68 \\
B_{5}=1.52\end{array}\right.$ & $\begin{array}{l}B_{2}=2 \cdot 28 \\
B_{4}=1 \cdot 15\end{array}$ & $\begin{array}{c}B_{3}=2 \cdot 10 \\
\ldots\end{array}$ & $\begin{array}{l}A_{0}=\cdot 80 \\
B_{6}=\cdot 47\end{array}$ \\
\hline
\end{tabular}

$$
\begin{aligned}
F= & 0.80+10.37 \sin k t+3.43 \sin 2 k t+2.30 \sin 3 k t \\
& +0.73 \sin 4 k t+0.43 \sin 5 k t \\
& +5.68 \cos k t+2 \cdot 28 \cos 2 k t+2 \cdot 10 \cos 3 k t \\
& +1.15 \cos 4 k t+1.52 \cos 5 k t+0.47 \cos 6 k t .
\end{aligned}
$$

Where only terms up to the third harmonic are required, Professor Silvanus Thompson has devised the following very simple method of performing the calculation. The values of the function are obtained every $60^{\circ}$, giving six values of $y$, and an additional ordinate must be measured at $90^{\circ}$; let this ordinate be called $y_{90}$. Then arrange as follows, and take the sums and differences :-

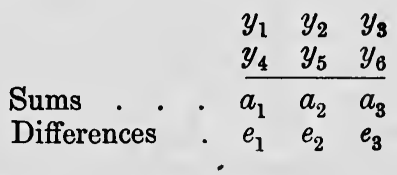

The values of the coefficients are then :-

$$
\begin{aligned}
& A_{0}=\left(a_{1}+a_{2}+a_{3}\right) \div 6 \\
& A_{1}=\left(e_{1}+e_{2}\right) \div 3 \cdot 464 \\
& A_{2}=\left(a_{1}-a_{2}\right) \div 3 \cdot 464 \\
& A_{3}=A_{0}+A_{1}-B_{2}-y_{90} \\
& B_{1}=\left(e_{1}-e_{2}-2 e_{3}\right) \div 6 \\
& B_{2}=\left(2 a_{3}-a_{1}-a_{2}\right) \div 6 \\
& B_{3}=\left(e_{2}-e_{1}-e_{3}\right) \div 6 .
\end{aligned}
$$

In the case of most of the curves which occur in the study of alternating currents, the two halves of the curve are always similar. In such 
a case the Fourier series contains only odd terms, and the analysis can be considerably simplified. Professor S. P. Thompson ${ }^{1}$ has modified Runge's method for use when only odd harmonics appear in the following manner :-

Draw the curve with the axis of $x$ halfway between the highest and lowest points, so that in the expression there will be no constant term. Also, take the origin where the curve crosses the axis of $x$ and measure the height of eleven ordinates, so spaced as to divide the half period into twelve equal parts, and call the values of these ordinates $y_{1}, y_{2}$, $y_{3}, \ldots y_{11}$.

Arrange these values of $y$ as under, and add and subtract :-

\begin{tabular}{|c|c|c|c|c|c|}
\hline & $\begin{array}{l}y_{1} \\
y_{11}\end{array}$ & $\begin{array}{l}y_{2} \\
y_{10} \\
\end{array}$ & $\begin{array}{l}y_{3} \\
y_{9} \\
\end{array}$ & $\begin{array}{l}y_{4} \\
y_{8}\end{array}$ & $\begin{array}{l}y_{5} \\
y_{7}\end{array}$ \\
\hline ums $_{\text {ifferences }}$ & $\begin{array}{l}a_{1} \\
e_{1}\end{array}$ & $\begin{array}{l}a_{2} \\
e_{2}\end{array}$ & $\begin{array}{l}a_{3} \\
e_{3}\end{array}$ & $\begin{array}{l}a_{4} \\
e_{4}\end{array}$ & $\begin{array}{l}a_{5} \\
e_{5}\end{array}$ \\
\hline
\end{tabular}

Group these numbers as follows to obtain the numbers $b_{1}, b_{2}, f:-$

$$
\begin{aligned}
& a_{1}+a_{3}-a_{5}=b_{1} \\
& a_{2}-a_{6}=b_{2} \\
& e_{1}-e_{3}-e_{5}=f .
\end{aligned}
$$

Next insert the numbers obtained in the following schedule, having first

\begin{tabular}{|c|c|c|c|c|c|c|}
\hline \multirow{2}{*}{ Multipliers. } & \multicolumn{3}{|c|}{ Sine Terms. } & \multicolumn{3}{|c|}{ Cosine Terms. } \\
\hline & 1st. 11th. & 3rd. 9th & 5th. 7th. & 1st. 11th. & 3rd. 9th. & 5th. 7 th \\
\hline $\begin{array}{l}0.259 \\
0.500 \\
0.707 \\
0.866 \\
0.966 \\
1.000\end{array}$ & $\begin{array}{ll}a_{1} & \\
a_{3} & a_{2} \\
& a_{4} \\
a_{5} & a_{6}\end{array}$ & $b_{1}$ & $\mid \begin{array}{cc}a_{5} & \\
& a_{2} \\
-a_{3} & \\
& -a_{4} \\
a_{1} & \\
& a_{6}\end{array}$ & $\begin{array}{ll}e_{4} & e_{5} \\
e_{2} & e_{3} \\
& e_{1}\end{array}$ & $-e_{4}$ & $\mid \begin{array}{cr}e_{4} & e_{1} \\
-e_{2} & -e_{3} \\
& e_{5}\end{array}$ \\
\hline $\begin{array}{l}\text { Total lst column } \\
\text { Total } 2 \text { nd column }\end{array}$ & $\begin{array}{l}\cdots \\
\cdots\end{array}$ & $\begin{array}{l}\cdots \\
\cdots\end{array}$ & $\cdots$ & $\cdots$ & $\begin{array}{l}\cdots \\
\cdots\end{array}$ & $\begin{array}{l}\cdots \\
\cdots\end{array}$ \\
\hline $\begin{array}{l}\text { Sums. . . . . . . } \\
\text { Differences . . }\end{array}$ & $\begin{array}{l}6 A_{1} \\
6 A_{11}\end{array}$ & $\begin{array}{l}6 A_{3} \\
6 A_{9}\end{array}$ & $\begin{array}{l}6 A_{5} \\
6 A_{7}\end{array}$ & $\begin{array}{l}6 B_{1} \\
6 B_{11}\end{array}$ & $\begin{array}{l}6 B_{3} \\
6 B_{9}\end{array}$ & $\begin{array}{l}6 B_{5} \\
6 B_{7}\end{array}$ \\
\hline
\end{tabular}
multiplied each by the multiplier given at the left-hand end of the line on which the product has to be entered :-

1 Proceedings of the Physical Society of London (1905), xix. p. 443. A further simplification is described by Thompson in the Proc. Phys. Soc., London (1911), xxiii. p. 334. 


\section{CHAPTER II}

\section{MEASUREMENTS OF LENGTH}

12. Standards of Length.- Since in almost every case the quantity which is actually observed when making any kind of physical measurement is a length, the consideration of this subject is one of particular importance. Thus, although the consideration of many arrangements, which are of special assistance in measuring the lengths which are required in certain measurements, are more conveniently dealt with in connection with the descriptions of these measurements, yet it will be convenient to describe at some length the arrangements more commonly employed at the very commencement. The student, at the same time, will find that the practice gained by performing the experiments given to illustrate the use of the various instruments and their adjustment a most valuable introduction to those more elaborate measurements which incidentally involve measurements of length.

We have to consider two things when dealing with the measurement of length, namely, the most suitable form to give the standard scale with which the distance to be measured is to be compared, and how it is to be graduated, and, secondly, the manner in which the distance to be measured is to be compared with the standard scale.

The conditions which a standard scale has to fulfil are as follows:(1) The bar of metal on which the divisions are traced must be so stiff that when a reasonable amount of care is taken in supporting the scale it shall not, owing to its weight, bend appreciably ; $(2)$ the material of which the scale is composed must be such that whenever the temperature has any given value the distance between any two divisions must be the same; (3) the division lines must be fine and of uniform thickness throughout the scale.

In addition, it is a great convenience if the coefficient of expansion of the material of which the scale is composed is small, so that the true distance between the graduations does not alter much with the temperature. It is also a great convenience if the graduations are so evenly spaced that, except in the case of measurements of the utmost refinement, we may take them as perfectly equal, and so do not have to apply a calibration correction. Although it is possible to obtain scales which are so accurately divided as to make the application of a calibration correction in most cases unnecessary, this accuracy cannot be taken for granted.

The cross-section adopted for accurate length standards of $50 \mathrm{~cm}$. 
and over is shown in Fig. 10. The divisions of the scale are engraved on the surface $\mathbf{A B}$ of the cross-bar of the н. The advantages of this shape are twofold. In the first place, this form of cross-section gives very great stiffness combined with lightness, so that the

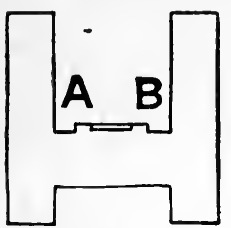

FIG. 10. bar does not bend owing to its own weight. This question of the bending of length standards under their own weight has been investigated by Chree, ${ }^{1}$ who has shown that a scale having this cross-section and supported at two points only, the points of support being at 0.22 of the length of the bar from the ends, does not bend sufficiently to alter the distance between any two divisions of the scale by an appreciable quantity. Secondly, the graduations being placed where they are, if the bar bends, that portion of the bar which carries the graduations is neither stretched nor compressed owing to the bending. The reason for this is that the scale occupies the position of the neutral fibres, so that, while the metal on one side is stretched, that on the other side is compressed. Incidentally this form of scale serves to protect the graduations from accidental injury.

Up to the last year or two the material of which almost all standard scales were constructed was brass, the divisions in the finer scales being engraved on a thin strip of silver, which was let into the upper surface of the scale. The disadvantage of brass is that its coefficient of expansion with rise of temperature is fairly large, namely, about 0.0000187 . Within the last few years Guillaume has carried on an important series. of investigations as to the best material to use for the construction of scales, and as a result has discovered an alloy which has a very much smaller coefficient of expansion than brass, and yet seems quite as unalterable. This alloy consists of about thirty-six parts of nickel to sixtyfour parts of iron, and its coefficient of thermal expansion (linear) is $0 \cdot 0000009$. This material takes a fine polish, so that the graduations can be made directly on the nickel alloy, and it is not necessary to insert a strip of silver to receive the divisions. It has the further advantage that it does not tarnish or rust when exposed to air.

Standard scales about a metre long are generally graduated in millimetres. In the case of very finely divided scales it is usual to subdivide a single millimetre at either end into tenths. It is also of importance to have an additional graduation put on at either end. Thus if the scale is intended to measure up to a metre an additional millimetre division ought to be placed at either end, so that the total length of the divisions is $1002 \mathrm{~mm}$. These additional divisions are used when standardising the scale.

The divisions of a good standard scale ought not to be more than two or three microns ${ }^{2}$ wide, and hence they are almost invisible to the naked

1 Proc. Physical Society of London (1901), xviii. ; and Philosophical Magazine (1901), 2, p. 532.

${ }^{2} \mathrm{~A}$ micron $(\mu)$ is a thousandth of a millimetre $=.001 \mathrm{~mm}$. 
eye. Some kind of auxiliary apparatus has, therefore, to be employed to read the scale. Again, the form of the scale does not permit of the object which has to be measured being placed alongside the divisions, so that some instrument is required to transfer the length from the object to the scale. Finally, it is generally necessary to make the measurements accurate to within a quantity which is considerably smaller than the smallest subdivision of the scale. We shall first consider the various methods which can be employed for the subdivision of the scale divisions, and in this connection shall for convenience deal not only with devices suitable for use with the form of scale described above, but also with other devices which are employed for this purpose, though they could not be applied to this particular form of scale.

13. The Vernier.-The vernier consists of a small auxiliary scale which can slide alongside the graduations of the main scale. 'The distance between the graduations of the vernier is either a little smaller or a little larger than the distance between the graduations of the scale. Thus, frequently, ten divisions of the vernier are equal to nine divisions of the scale, so that each division of the vernier is $\cdot 1$ of a division less than the divisions of the scale. Suppose, as in Fig. 11, the zero division of the vernier lies between the fourth and

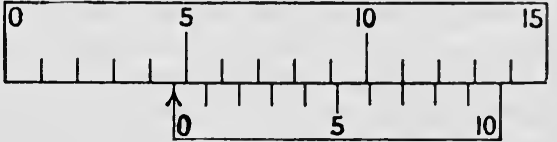

FIG. 11.

fifth divisions of the scale, the reading being $4+x$ divisions where $x$ is a fraction of a division. Then, since the length of a vernier division is less than the length of a scale division by a tenth of a scale division, the interval between the second division of the vernier and the fifth division of the scale is $x-\cdot 1$. In the same way the interval between the third division of the vernier and the sixth division of the scale is $x-\cdot 2$, and so on. But in the figure the interval between the seventh division of the vernier and the eleventh division of the scale is zero ; that is, we have

or

$$
\begin{gathered}
x-\cdot 7=0, \\
x=\cdot 7 .
\end{gathered}
$$

Hence by simply noting the division of the vernier which coincides with a division of the scale we get the fraction of a division by which the zero of the vernier is distant from the division of the scale immediately below. This result may be generalised as follows:-If $m$ divisions of the vernier are equal in length to $m+1$ divisions of the scale, and coincidence occurs at the $n$th division of the vernier, then the reading of the vernier, i.e. the distance between the zero of the vernier and the preceding division on the scale, is $n / m$ ths of a division of the scale.

Verniers are sometimes constructed so that $m$ divisions are equal in length to $m+1$ divisions of the scale. In such a case an argument similar to that given above shows that if the vernier is numbered in the 
reverse direction to the scale, the fiducial mark of the vernier being still at the end of the vernier nearest the zero of the scale, and coincidence occurs at the $n$th division, the distance between the fiducial mark of the vernier (i.e. the $m$ th division) and the preceding division of the scale is $n / m$ ths of a division. The advantage of this form of vernier is that the distance between the graduations is rather greater, i.e. the scale more open. It is, however, rather more confusing to read, and is seldom used on that account.

14. The Micrometer Screw.-When a screw such as A, Fig. 12, is rotated through a whole turn it advances through the nut $B$ by an amount equal to the distance, measured parallel to the axis, between consecutive threads. Thus, suppose that the screw has twenty threads to the centimetre, when the screw is rotated through a whole turn it advances through half a millimetre. By means of a divided head $o$ attached to the screw the fraction of a turn through which the screw has been rotated can be read. With a screw having a pitch of half a millimetre, which for most purposes is a convenient quantity, it is usual to divide the head into fifty parts, so that one division of the head corresponds to a motion of the end of the screw of $0.01 \mathrm{~mm}$. In order to determine the whole number of turns through which the screw has been turned a scale $\mathbf{D}$ is placed alongside the divided head. This scale is divided into half millimetres, and a circle engraved on the divided head or on the edge of the head serves to read the position of the screw to the nearest half millimetre, the fractions being obtained from the reading on the divided head opposite to the edge of the scale $D$. When using a micrometer screw for any but comparatively rough measurements it will not do to assume that the distance between the threads is exactly what it purports to be, nor that the threads are quite uniform, so that it becomes necessary to calibrate a micrometer screw. Various methods of performing such a calibration will be described.

In order to be able to adjust a micrometer screw to make a measurement, it is essential that it should move fairly easily in its nut. As a result, particularly when a screw has been used for some time, there will be a certain amount of play between the screw and the nut. Hence the position of the screw will vary slightly according as to whether the reading obtained has been approached by turning the screw in one direction or the other. This effect is referred to by the term backlash, and to ensure its not affecting the readings it is essential that the screw . should always be brought up to its final position by turning the head in the same direction, so that the threads of the screw shall always bear on the same side of the threads in the nut. If by accident the screw is turned too far, it must not simply be turned back till the reading is correct, but must be moved back about a quarter of a turn beyond the correct position and then gradually turned forward till the adjustment is complete.

Two simple instruments in which a screw is made use of to measure a length are the spherometer and the micrometer screw gauge, and it will be found advisable to obtain some practice in the use of a micrometer 
screw by making the measurements indicated in the following pages before proceeding to use a more complicated instrument.

15. The Spherometer.-The usual form given to the spherometer is shown in Fig. 12, from which the construction of the instrument will be clear. The screw is generally one having a pitch of half a millimetre, and the divided head $\mathrm{c}$ is divided into 500 parts, so that each division represents a movement of the screw parallel to its axis of $0.001 \mathrm{~mm}$. The hundreds divisions on the head are numbered $0 / 5,1 / 6,2 / 7$, \&c. This means that starting, say, from the zero when the division marked $0 / 5$ is opposite the zero of the fixed scale $D$, and turning the head till the division $1 / 6$ is reached, we have to take the upper number 1 , and the screw has advanced through $.01 \mathrm{~mm}$. When the division marked $0 / 5$ is reached we have now to take the lower number 5, and when we come to the next division the lower number 6 , and so on till we

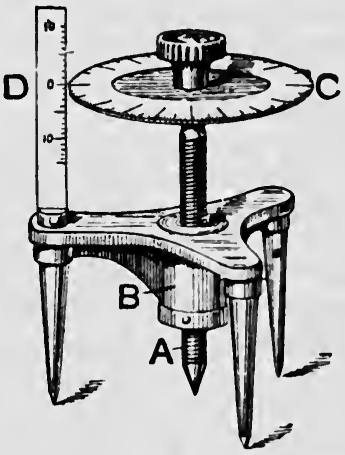

FIG. 12. get to the division $0 / 5$, when we start with the upper numbers again, but have a whole millimetre in addition to the fraction shown on the divided head. This whole millimetre will be shown on the scale $D$. This scale will also serve to show in any case whether we have to read the upper or the lower figure on the divided head, for if the reading on the fixed scale is between a whole millimetre division and the half millimetre division we have to take the upper figure, while if it is between the half millimetre division and the next whole millimetre then we have to take the lower figure. In some instruments the chief divisions on the head are simply numbered $1,2,3, \& c$., up to 5 , and 0.5 has to be added to the reading when the scale $\mathrm{D}$ shows that the reading ought to be between 0.5 and $1.0 \mathrm{~mm}$.

The first thing to do when making a measurement with a spherometer is to determine the reading of the micrometer head when the ends of the three fixed legs and of the micrometer screw are in the same plane. To make this test the instrument is generally provided with a dise of glass, one surface of which is ground to a plane surface. In the absence of such a disc a piece of plate glass, so long as it is not cut from near the edge of the original sheet of glass as supplied by the glass works, will do quite well. In fact, by selecting suitable pieces of plate glass by the method given in $\S 142$ it is possible to obtain pieces having surfaces much more nearly plane than are the ground surfaces ordinarily supplied with spherometers.

Place the piece of glass firmly on the table so that it does not rock, and on it stand the spherometer and roughly adjust the micrometer screw so that the four. legs are in contact at the same time. The final adjustment can be made in the following manner:- Lightly tap the 
spherometer in a horizontal direction in such a mauner as to tend to make it spin round the middle leg, when it will be found that if only the outside legs are in contact with the glass that the instrument will not turn. If, however, the middle leg is projecting beyond the others, then even a very light tap will be sufficient to make the instrument turn. Hence the adjustment is made by starting with the middle leg too short and then gradually turning the micrometer screw till the instrument just shows an inclination to turn when gently tapped. If by accident the micrometer is turned too far, it must be turned back and then brought up to the reading in the forward direction again. This adjustment must be made three or four times, being careful always to bring the screw up to its final position by turning it in the same direction, and the mean taken to represent the zero reading of the instrument.

Next use the spherometer to measure the thickness of a microscope cover glass. The upper surface of the glass plate used when determining the zero reading must be cleaned, particular care being taken that no specks of grit are left. The cover glass must then be cleaned in the same way by being drawn between two folds of a soft clean rag held between the thumb and first finger of the right hand. If the cover glass and the support are properly clean, on placing the cover glass on the support and gently pressing it down the cover glass will adhere quite firmly. Next place the spherometer on the plate and adjust the micrometer screw so that it just touches the upper surface of the cover glass, the three fixed legs resting on the glass plate. Repeat this adjustment three times, and then the difference between the mean of the readings and the zero reading will give the thickness of the cover glass.

The spherometer may be used to determine the curvature of a spherical surface, that is, the radius of the sphere of which the surface forms a part. The radius of the surface of a lens or spherical mirror can be measured in this way, and it is from its use to measure the curvature of such surfaces that the instrument derives its name.

When the three fixed legs rest on a spherical surface the plane passing through their tips will cut off a spherical cap ABC, Fig. 13,

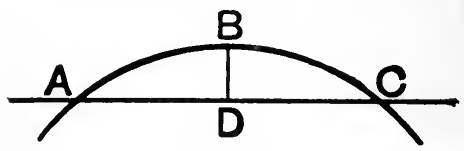

FIG. 13. and the radius of the circle bounding this cap will be equal to the distance between the outside legs and the middle leg of the instrument. When the micrometer is adjusted so that the central leg just touches the surface of the lens at the point $B$, the difference between the reading and the zero reading will give the length of the line BD. Calling this length $x$, the distance between the fixed legs and the central leg $r$, and the curvature of the surface of the lens, that is, the radius of the circle, $R$, then since 
$\mathrm{AD}=\mathrm{DC}=r$ and $x$ and $2 R-x$ are the two segments of the diameter of the circle which passes through $D$, we have

or

$$
\begin{gathered}
x(2 R-x)=\mathrm{AD} . \mathrm{DC}=r^{2} \\
R=\frac{x^{2}+r^{2}}{2 x}=\frac{x}{2}+\frac{r^{2}}{2 x} .
\end{gathered}
$$

In general the quantity $x$ will be very small, so that $x / 2$ will be negligible compared with $r^{2} / 2 x$, in which case we have

$$
R=\frac{r^{2}}{2 x}
$$

It will be noticed that to employ this method of measuring the curvature of a surface it is necessary that the surface should be so large that the three fixed legs of the spherometer can all rest on the lens at the same time.

Since the distance between the central leg and the outside ones is involved in the expression for the radius of curvature of the spherical surface, and further, since this length occurs to the second power, it is necessary to determine its value with accuracy. The maker generally attempts to make it exactly an inch or 2 centimetres, according as the screw has a pitch measured in English or metric units. A method of determining the distance between the central leg and the outside ones is to lay a sheet of thin and uncrumpled tin-foil on the glass plate used to determine the zero. The instrument is then placed on the tin-foil and lightly pressed down, so that all four legs mark the foil. The distance between the central dot thus obtained and the three outside dots is then measured with a travelling microscope (see $\S 17$ ). The mean of the three distances must be taken as the quantity $r$ in the formula. If the travelling microscope has sufficient overhang, the distances between the legs can be measured directly by placing the spherometer on the base of the microscope with the points of the legs turned upwards. Although it may be impossible to measure the distances from the central leg to the outside ones, the micrometer microscope will generally allow of the distances between the outside legs being measured. If $d$ is the mean of the three distances between the outside legs, then

$$
r=d / \sqrt{\mathbf{3}}
$$

16. The Micrometer Screw Gauge.-A common form of micrometer screw gauge is shown in Fig. 14. The cap $\mathrm{g}$ is attached to the screw, and it carries on its lower edge a series of division marks, by means of which the fractions of a turn of the screw can be read off. The number of whole turns made by the screw can be read off on the scale $\mathbf{F}$, which is engraved on the nut, and is uncovered by the cap a as the screw is turned. The usual pitch for the screw is half a millimetre, and the scale on the edge of the cap is generally divided into fifty parts, 
so that each division represents 0.02 of a complete turn, or a movement of the end of the screw of $0.01 \mathrm{~mm}$. The divisions on the cap are

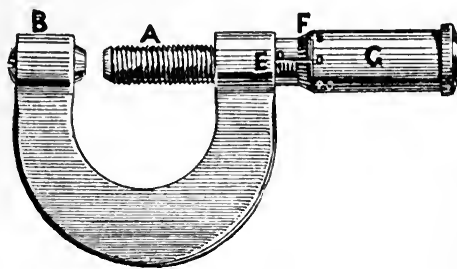

FIG. 14. usually numbered $0 / 5,1 / 6, \&$ c., as in the case of the spherometer, and the upper or lower figure has to be taken according as the screw has made an odd or even number of complete turns.

If the gauge is in exact adjustment, the scales will be at zero when the screw point is in contact with the stud B. In general, however, this adjustment is not quite correct, and the first thing to be done when making a measurement is to determine the amount and sign of the zero error. For this purpose, first clean the end of the screw and the stud, and then gently turn the screw till its end touches the stud. When screwing the micrometer screw up to the stud, or when making a measurement of thickness, it is of importance that it should be only lightly brought into contact with the object. For this reason the cap by which the screw is turned ought to be grasped very lightly between the thumb and first finger, so that whenever contact is secured the fingers may slip and so not strain the screw.

Having determined the zero reading and noted whether the quantity has to be added to or subtracted from the subsequent readings, the object to be measured can be placed between the jaws and the reading obtained when contact is secured. This reading, with the zero correction applied, will be the thickness of the object.

Determine by means of the gauge the thickness of the microscope cover glasses which were measured with the spherometer, and deter-

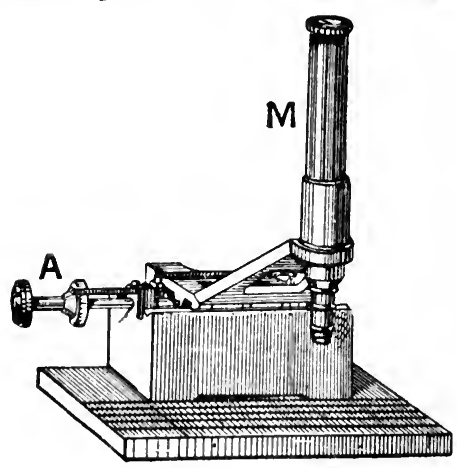

FIG. 15. mine whether there seems to be any consistent difference between the values obtained with the two instruments.

Determine with the gauge the diameter of a piece of steel or brass wire. The diameter ought to be measured at several points, while at each point the diameter should be measured in two directions at right angles. In this way ascertain whether the wire has a circular section, and also if its diameter is uniform throughout its length.

17. The Travelling Microscope.-

An instrument which is of very great service in making a large number of measurements of length, such as are constantly required in the physical laboratory, is shown in Fig. 15, 
and is sometimes called a travelling microscope. 'There are various forms of the instrument made, but they all consist essentially of a microscofie $\mathrm{M}$, Fig. 16, which is mounted on a frame so that it can be moved in a direction perpendicular to its length, and the distance through which it has been moved can be read off by means of a scale and vernier or a micrometer screw $\mathbf{A}$. The microscope can be moved up and down within the upright of the carriage so as to allow of the microscope being focused on the object which is being measured. Two cross-wires are fixed within the microscope, and the eye-piece can slide in and out so that these cross-wires can be focused. The object on which the measurement has to be made must be placed below the microscope, with the line of which the length has to be determined parallel to the direction in which the microscope traverses. To assist in making this adjustment it is useful to have a series of lines ruled on the base parallel to the slide along which the microscope moves. If these lines have not been put on by the maker they can be ruled by attaching a piece of pointed wire to act as a scriber and then traversing the microscope throughout the whole length of its travel, so that a line is ruled on the base by the point of the wire.

Determine by means of the travelling microscope the errors in the lengths of the divisions of a small scale ruled on glass. For this purpose the first adjustment to be made is to focus the cross-wires and microscope correctly, and as this is an adjustment which has constantly to be made, the following method had better be employed. Remove the microscope from the carriage, and directing it towards a window or some brightly illuminated white surface look into the eye-piece with the right eye. While doing this do not shut the left eye, but keep it fixed and focused on some object, such as a window bar, which is more than two yards away. With a little practice it will be possible to see the bright field of the microscope with the cross-wires, and apparently alongside them the window bar. The eye-piece must then be adjusted so that both the cross-wires and the window bar are sharply in focus at the same time. This method of focusing the eye-piece ensures that when the eye is focused on the cross-wires the accommodation muscles of the eye are not unduly strained. Unless some such device is employed it often happens that the eye-piece is so focused that although it is possible to see the cross-wires quite distinctly, yet to accomplish this one has unconsciously to strain the eye, so that if the instrument is used much in this condition very serious injury to the eyesight may ensue. Having focused the cross-wires in this way the microscope must be replaced in the instrument, being careful not to displace the eye-piece, and then by moving the microscope as a whole it must be focused on the divisions of the scale which has to be measured. When making this adjustment it is important that the image of the scale produced by the object-glass of the microscope should be in the same plane as the cross-wires. To see whether this adjustment is complete place the vertical cross-wire so that it appears immediately over one of the divisions of the scale, then 
move the eye from side to side as far as the aperture in the eye-piece will allow. If the image of the division on the scale and the cross-wires are in the same plane, they will not appear to alter their relative positions as the eye moves. If, however, the focus is not quite right, so that the image is nearer to the eye or farther away than the cross-wires, then, owing to parallax, there will appear to be relative motion when the eye is moved from side to side. The whole microscope must be moved up and down till there is no parallax between the image and the cross-wires. When this is secured, the focusing is complete. The reason that it is important to make this adjustment with great care is, that if parallax exists the setting of the cross-wires on a division of the scale when making a measurement will depend on the position of the eye with reference to the eye-piece. Hence as we cannot ensure that the eye occupies always exactly the same relative position when making the settings at the two ends of the object, an error of some considerable magnitude might be produced in this way.

Having adjusted the focus of the microscope, the next thing to be done is to arrange the scale which has to be measured on the bed of the instrument in such a way that the divided portion is parallel to the slide along which the microscope moves. To do this, move the microscope to the extreme right-hand end of its travel and adjust the scale so that one end of the division lines corresponds to the intersection of the crosswires. Then move the microscope to the other end of its slide, and again adjust the scale. By repeating this operation a few times it will be possible to arrange the scale so that, as the microscope is moved along, the ends of the division lines of the scale always coincide with the intersection of the cross-wires of the microscope, and hence the scale is parallel to the slide along which the microscope travels. Next turn the microscope, without altering its focus, so that one of the cross-wires is parallel to the division lines of the scale. The instrument is now in adjustment.

Take a reading of the micrometer screw when the cross-wire of the microscope coincides with each whole centimetre division of the scale, and draw up a table showing the difference between the nominal distance between the division lines of the scale and the distances as obtained with the micrometer microscope.

18. Illumination of the Divisions of a Standard Scale.-An important point when using scales with fine division lines is the illumination. In Figs. 16 and 17 are shown two ways in which the scale $\mathbf{D}$ can be illuminated. In Fig. 16 the light from a small incandescent lamp, or the light of a window reflected from a mirror, strikes the lens $\mathrm{c}$; it is then partly reflected downwards at the surface of a plane piece of unsilvered glass B, and having traversed the lenses of the object-glass $\mathrm{A}$ of the microscope, strikes the scale $\mathbf{D}$. The light, after reflection at the surface of the scale, passes through the object-glass and through the glass plate $\mathrm{B}$, and finally reaches the eye-piece of the microscope. In the second arrangement (Fig. 17), it is not necessary to alter the objective by cutting 
a hole in the side and by the insertion of a glass plate. To a small brass ring, which fits on the end of the objective, is attached a small ringshaped metallic mirror $B$, inclined at $45^{\circ}$ to the axis of the microscope. The rays of light from some source pass through the lens c, and are

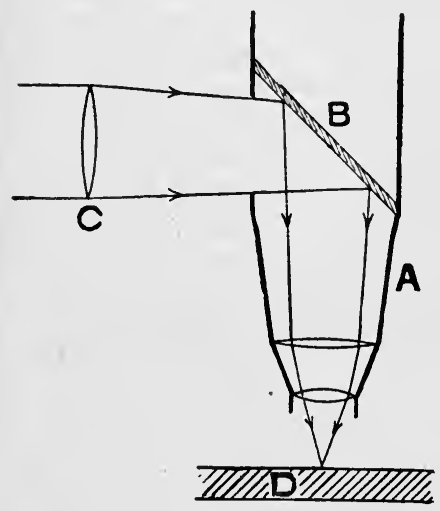

FIG. 16.

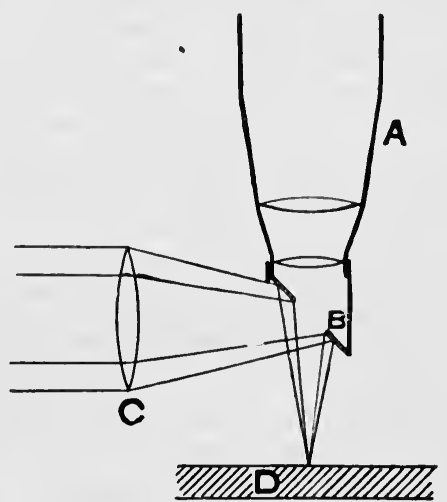

FIG. 17.

reflected by the mirror $\mathbf{B}$ on to the scale. After reflection at the scale they pass through the hole in the centre of the mirror $B$, and enter the microscope.

In both of the above arrangements the illumination of the graduations of the scale is symmetrical. If a source of light placed on one side of the microscope is used the illumination is asymmetrical, and the graduation being better illuminated on one side than the other an incorrect setting may result.

19. The Comparator.-The travelling microscope is unsuited for measuring lengths greater than about $5 \mathrm{~cm}$. When greater lengths, up to, say, a metre, have to be measured with an accuracy greater than the tenth of a millimetre, the principle of the comparator has to be employed. This instrument ${ }^{1}$ consists essentially of two micrometer microscopes attached to a rigid bed in such a way that the distance between the carriages which carry the microscopes can be varied. The object to be measured is placed on the bed of the instrument with the length to be measured parallel to the line along which the microscope carriages move, and the microscopes are so adjusted that their cross-wires coincide with the marks which define the ends of the line which has to be measured. The object is then removed, care being taken not to disturb the microscopes, and a standard scale placed in its place. The cross-wires of the

1 An interesting account of the comparators in use at the Bureau Interna. tional des Poids et Mesures at Paris will be found in a book by Guillaume, called La Convention du Metre, published by Gauthier-Villars, Paris. 
microscopes will not in general coincide with a division of the scale at either end. The divided heads of the micrometers having been read, the microscopes are moved till the cross-wires coincide with the nearest division line of the scale, when the micrometers are again read. The difference between the readings of the micrometers will give the amounts by which the object exceeds or falls short of the length between the two whole divisions of the scale. Unless the accuracy of the micrometer screws has been ascertained, it is as well to take the reading corresponding to two divisions of the scale, one on either side of the original position of the microscopes. In this way the amount by which the object exceeds the whole number of divisions of the scale can be obtained without involving the pitch of the micrometer screws. The following example will illustrate how the observations ought to be recorded, and the method of obtaining the fractions of a scale division from the micrometer readings :-

\begin{tabular}{|c|c|c|c|c|}
\hline \multicolumn{2}{|c|}{ Left-hand Microscope. } & \multicolumn{2}{|c|}{ Right-hand Microscope. } & \multirow{2}{*}{$\begin{array}{c}\text { Temperature } \\
18 \cdot 1 \\
18 \cdot 1 \\
18 \cdot 1\end{array}$} \\
\hline $\begin{array}{l}\text { Object } \\
0 \cdot 0 \text { of scale } \\
0 \cdot 1 \text { of scale }\end{array}$ & $\begin{array}{l}3 \cdot 1475 \mathrm{~cm} \text {. } \\
. \quad 3 \cdot 1251 ~ \\
.\end{array}$ & $\begin{array}{l}\text { Object } \\
50 \cdot 1 \text { of scale } \\
50.2 \text { of scale }\end{array}$ & $\begin{array}{l}.1 .7751 \mathrm{~cm} \\
.1 .7036 ” \\
. \quad 1.8048 ”\end{array}$ & \\
\hline $\begin{array}{r}0 \cdot 1 \text { of scale } \\
\text { Dif. } 0.0 \text { to ob } \\
0 \cdot 0 \\
\overline{0 \cdot 1}\end{array}$ & $\begin{aligned} & =0.1015 \mathrm{~cm} . \\
c t & =0.0224, \\
\frac{24}{15} & =0.02207,\end{aligned}$ & $\begin{array}{l}0.1 \text { of scale } \\
\text { Dif. object } \\
\begin{array}{r}50.2 \\
\quad \\
\quad 0.02\end{array}\end{array}$ & $\begin{array}{l}\text { to }=0.1012 \mathrm{~cm} . \\
\frac{97}{12}=0.0297,\end{array}$ & $\begin{array}{l}\cdots \\
\cdots \\
\cdots\end{array}$ \\
\hline
\end{tabular}

Length of object (uncorrected for scale error) $=50 \cdot 2-0.02207-0.02935$ $=50 \cdot 1486 \mathrm{~cm}$. at $18^{\circ} \cdot 1$.

20. Correction of Measurements of Length to Allow for the Effects of Temperature.-When making accurate measurements of length it is of importance to note the temperature of the body being measured, as also of the scale, for, owing to the fact that bodies change their length with change of temperature, the length will differ at different temperatures. It is only at some fixed temperature that the divisions of a standard scale have the length they profess to have. In the case of a scale divided in metric measure the divisions are generally correct at $0^{\circ} \mathrm{C}$. Scales divided in English measure are generally correct at $62^{\circ} \mathrm{F}$., or $16^{\circ} .67 \mathrm{C}$. If, when making a measurement, the temperature of the scale is higher than the temperature at which it is correct, each of the divisions will be too long by an amount which depends on the coefficient of expansion of the material of which the scale is composed and the amount by which the temperature exceeds the standard temperature. If the scale is of brass it will, except in cases where the utmost refinement is required, be sufficiently accurate to assume that the coefficient of expansion is 
0.0000187 ; while if the scale is made of steel, the coefficient of expansion may be taken as 0.000011 . The method of correcting a length measurement for the temperature of the scale is given below, where the measurement given on p. 52 is reduced.

The excess of the length of the scale over its nominal value at 50.1 cm., as derived from the tabulated set of corrections, is -0.0028 , that is, the true distance between the zero graduation and 50.1 at a temperature of $0^{\circ} \mathrm{C}$. is 50.9972 . Hence, applying this correction, the length of the object becomes $50 \cdot 1458$. The temperature coefficient of the scale is 0.0000187 . Hence the actual distance between the zero and 50.1458 at a temperature of $18^{\circ} \cdot 1$ is

or

$$
\begin{aligned}
& 50 \cdot 1458(1+0 \cdot 0000187.18 \cdot 1), \\
& 50 \cdot 1628 \text {, }
\end{aligned}
$$

The above results would be entered thus-

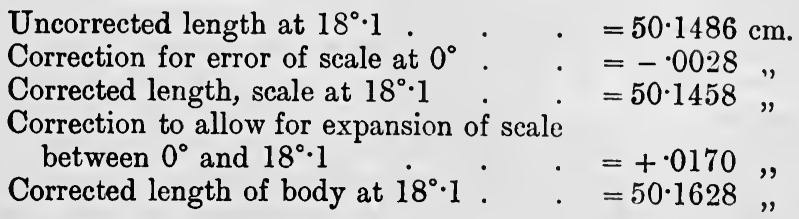

If we required the length of the body at any fixed temperature, say, $0^{\circ} \mathrm{C}$, , then a knowledge of the coefficient of expansion would be necessary. The body measured in the above example being made of brass, we may assume its coefficient of expansion as 0.0000187 . The length at $0^{\circ}$ would then be given by

$$
50 \cdot 1628\{1-0 \cdot 0000187 \times 18 \cdot 1\} \text {. }
$$

When applying corrections for temperature, particularly when they have to be applied to the standard, great care must be taken that the correct sign is used.

21. The Cathetometer.-The comparator is only suitable for measuring lengths when the object can be rested in a horizontal position on the base of the instrument. In many cases, however, the length to be measured is vertical, as, for instance, the height of a column of liquid in a tube. In such a case an instrument called a cathetometer is often employed, though of late years the arrangenent described on p. 58 is frequently used for this purpose, since it avoids many of the errors and troublesome adjustments which are involved in the use of the cathetometer.

Cathetometers are of many patterns, though they all consist of an upright slide along which a telescope can be moved, the axis of the telescope being horizontal. One form of cathetometer is shown in Fig. 18. An upright metal pillar $\mathrm{AB}$ is fixed to a heavy metal stand in such a way that it can be made to rotate about a vertical axis, the rotation being limited when necessary by two adjustable stops. The pillar has a scale engraved 
along one face. The telescope $\mathbf{T}$ is supported by a carriage which can slide along the pillar, a second carriage $\mathrm{D}$ sliding along the same pillar being connected with the carriage which supports the telescope by a micrometer screw E. This carriage $\mathrm{D}$ can be clamped to the pillar, and then by turning the screw $\mathrm{E}$ the telescope can be moved up or down

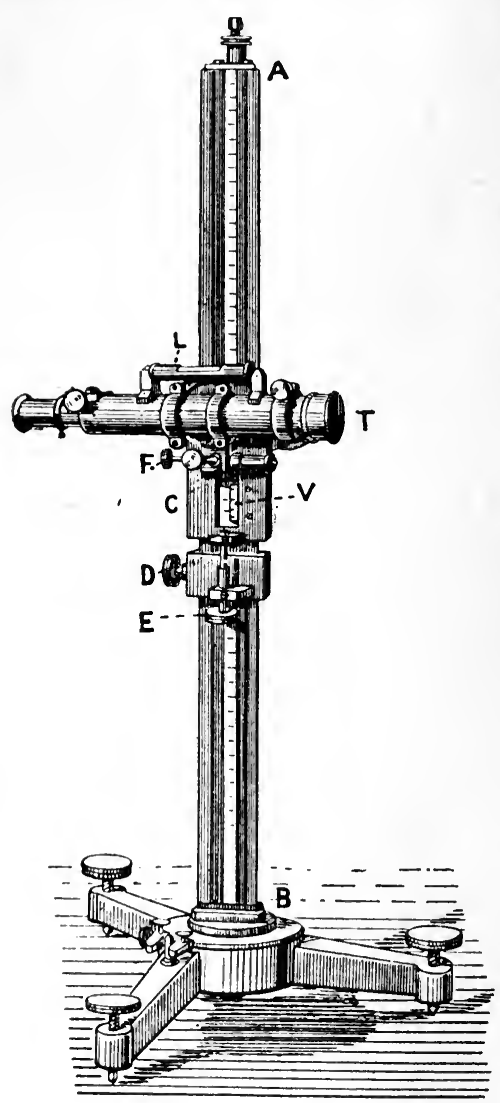

FIG. 18. through a small distance and so its position adjusted. The position of the carriage $c$ can be read off on the scale on the pillar by means of a vernier v. A spirit-level $\mathbf{L}$ serves to show when the axis of the telescope is horizontal, and a screw $\mathrm{F}$ serves to slightly tilt the telescope, and thus permits of its axis being adjusted.

The cross-wires of the telescope are attached to a ring, which ring is supported by four screws, as shown in Fig. 19. These screws pass through holes in the side of the telescope tube and are threaded into the ring, so that by slackening off one screw and tightening the opposite one the position of the ring, and hence also of the cross-wires, can be altered.

The line joining the centre of the object-glass to the intersection of the cross-wires is called the optic axis of the telescope, and the object in having the crosswires adjustable is to allow of the optic axis being made parallel to the axis of the tube of the telescope. This adjustment, which is generally referred to as the collimation adjustment, is performed by turning the telescope to some fairly distant object having a sharp horizontal edge or line, and bringing the horizontal wire into coincidence with the image of this edge. The telescope is then turned about its own axis through $180^{\circ}$, the springs or clamps which hold it to the carriage having been loosened if necessary. If the horizontal wire still coincides with the image of the edge, this wire is correctly adjusted ; if not, the cross-wire must be moved half-way between its position and the image of the edge. It will generally be necessary to repeat the operation two or 
three times before the adjustment is complete. The vertical wire must be adjusted in the same manner. When both wires are adjusted, then on rotating the telescope the intersection of the wires ought not to move relatively to the image formed in the telescope. When the collimation of the telescope is correct we are then certain that if we arrange that the axis of the tube of the telescope is horizontal, then the optic axis of the telescope will also be horizontal.

When being used to measure a vertical distance a cathetometer has to fulfil the following conditions :(1) The vertical column which carries the scale, and on which the telescope slides, must be truly vertical; (2) as the telescope is moved up and down the column the axis of the telescope must

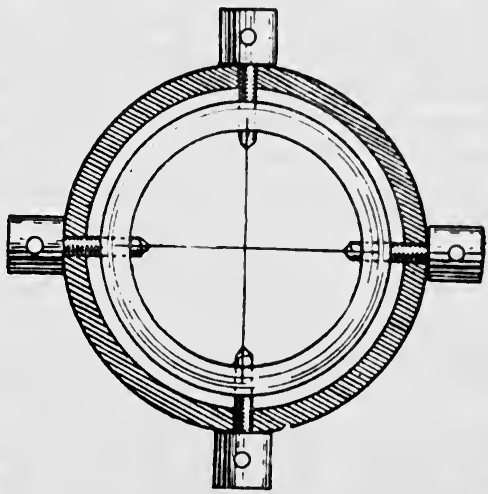

FiG. 19. remain parallel to some fixed direction. The fixed direction chosen is a horizontal plane.

As an exercise in the use of the cathetometer, hang up a standard metre scale or other finely divided scale. By means of a plumb line attached to the stand which carries the scale, adjust the scale so that it is accurately vertical. Find, by moving backwards and forwards a piece of paper on which there is some writing, the shortest distance at which the cathetometer telescope will focus an object. Place the cathetometer at a distance from the suspended scale slightly greater than this distance, and in such a position that the scale on the cathetometer can be conveniently read.

The first adjustment to make is to set the axis of the telescope parallel to the axis of the level. To do this adjust the inclination of the telescope, either by means of the levelling screws at the base or by the screw F, till the bubble is at the centre of the scale. Then remove the level, and leaving the telescope untouched turn the level end for end. If the bubble is not now at the centre of the scale bring it half-way back by tilting the telescope by the screw $F$, and the other half by means of the adjusting screw attached to the level itself. It will generally be found necessary to repeat the reversal two or three times before the adjustment is complete.

Great care must be taken when reading or adjusting the level not to heat the tube, either by breathing on it or by touching it with the hand. The extent to which a trifling amount of heat can affect the reading can be tested by lightly touching the tube near one end of the bubble for a few seconds. The bubble will be found to move towards the heated end, and it will be some little time before it returns to its correct position. 
The next adjustment to be made is to set the column of the cathetometer vertical. To do this turn the column till the telescope is parallel to the line joining two of the levelling screws of the base, and by turning these screws bring the bubble of the telescope level to the centre of the scale. Next turn the column through $180^{\circ}$, and if the bubble is not at the centre of the scale bring it half-way back by turning the levelling screws, and the other half-way by turning the screw F (Fig. 18). Now turn the column so that the telescope is at right angles to the line joining the two levelling screws that have been used up to now, and bring the bubble back to the central position by turning the remaining levelling screw. It will generally be necessary to repeat the adjustment, as moving the third screw will often slightly throw out the adjustment of the others. When the adjustment is complete the level bubble ought to remain at the zero of its scale, whatever the direction in which the telescope is turned.'

The theory of this adjustment is as follows:-Suppose AB (Fig. 20) represents the position of the vertical axis, and CD that of the telescope.

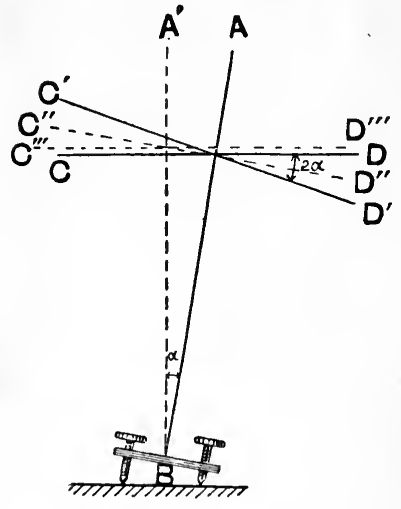

FIG. 20.

On turning the: instrument through $180^{\circ}$ the vertical axis remains unaltered, but the telescope takes up the position $\mathbf{c}^{\prime} \mathbf{D}^{\prime}$. Now if $a$ is the angle the vertical axis makes with the vertical, then the angle between $\mathbf{O}^{\prime} \mathbf{D}^{\prime}$ and the horizont-i? is $2 \alpha$. Hence if the bubble of the telescope level is brought half-way back by moving the vertical axis through an angle $\alpha$, the telescope would be in the position $\mathbf{C}^{\prime \prime} \mathbf{D}$, making an angle $a$ with the horizontal. Hence when the other half of the movement of the bubble is taken out by the screw which tilts the telescope, the axis of this latter takes up the horizontal position $\mathbf{c}^{\prime \prime \prime} \mathbf{D}^{\prime \prime \prime}$.

The eye-piece of the telescope must now be focused on the cross-wires and the telescope on the scale by the method given on p. 49 , so that there is no parallax. The adjustments of the instrument being complete, set the telescope so that the horizontal cross-wire coincides with the lower division of the suspended scale, and then read the vernier on the cathetometer scale. Next move the telescope up so that the horizontal cross-wire coincides with the image of the upper division of the scale. If the level bubble is found to be exactly at the zero of its scale, the vernier can now be read. If the telescope is not exactly level adjust its position by means of the screw F, and then again set the cross-wire to coincidence with the upper division of the scale, and if the telescope has still remained level read the vernier. The difference between the two readings of the vernier will give the distance between the two divisions of the scale in terms of the cathetometer scale. As a check on the results 
the adjustment of the cathetometer ought to be thrown out and then made again and the measurement repeated.

To obtain some idea of the accuracy which a careful observer may expect to get, examine the vernier and find the smallest movement which could be read. Twice this amount will be the largest error in the distance between the two positions of the telescope which ought to be obtained in any setting. This distance, however, does not necessarily equal the distance between the two divisions of the scale which was measured, for the cathetometer scale may not have been vertical, or, what is of very much greater importance, the axis of the telescope may not have been exactly parallel when making the two readings at the two ends of the object. To ascertain what influence on the final result a possible error in this adjustment may make, we require to know through what angle the telescope has to be tilted to cause the level bubble to move through one division of its scale, and, further, within what fraction of a division of the level we are able to read and adjust the position of the bubble. The angle through which the telescope has to be tilted so that the bubble may move through one division may be determined in the following manner:-By means of the screw $F$ tilt the telescope so that the bubble is near one end of the scale, and then read the division of the suspended scale corresponding to the horizontal cross-wire of the telescope and the position of the bubble. Next tilt the telescope in the opposite direction, and again read the scale and bubble. Let the difference of the readings on the suspended scale be $s$, and the difference in the positions of the bubble, expressed in divisions of the level scale, be $b$. Measure the distance between the pivot about which the telescope turns when tilted by the screw $F$ and the suspended scale, and call it $D$. Then if a is the angle through which the telescope has been tilted we have

$$
\frac{8}{2} / D=\tan \frac{\alpha}{2}
$$

or since $\alpha$ is very small

$$
\alpha=\frac{8}{D} \text {. }
$$

Hence one division of the level scale corresponds to a tilt of $a / b \mathrm{ex}$ pressed in circular measure, or to $206300 a / b$ seconds of arc.

If, now, an examination of the level indicates that we are not able to fix the reading to less than $1 / n$th of a division of the scale, this means that the two positions of the telescope may have been inclined to one another when measuring the scale by $1 / n$th of the angle found for one division. Hence, knowing the distance of the telescope from the scale, the uncertainty of the length of the scale corresponding to this uncertainty of level can immediately be calculated.

22. Measurement of a Vertical Length by Means of a Separate Scale and Reading Telescopes.-The cathetometer is by no means a satisfactory instrument with which to measure a vertical distance. In 
the first place, in order to give results which are at all accurate it is necessary that the instrument should be constructed with very great care as to its design and workmanship, which makes the instrument very costly. Secondly, the adjustments which have to be made are troublesome and require repeating at each reading, so that it takes a very considerable time to make a measurement. Thirdly, unless the instrument is supported on a masonry pillar the weight of the observer will bend the floor sufficiently to alter the level of the telescope, and hence influence the readings. Fourthly, the presence of the observer's

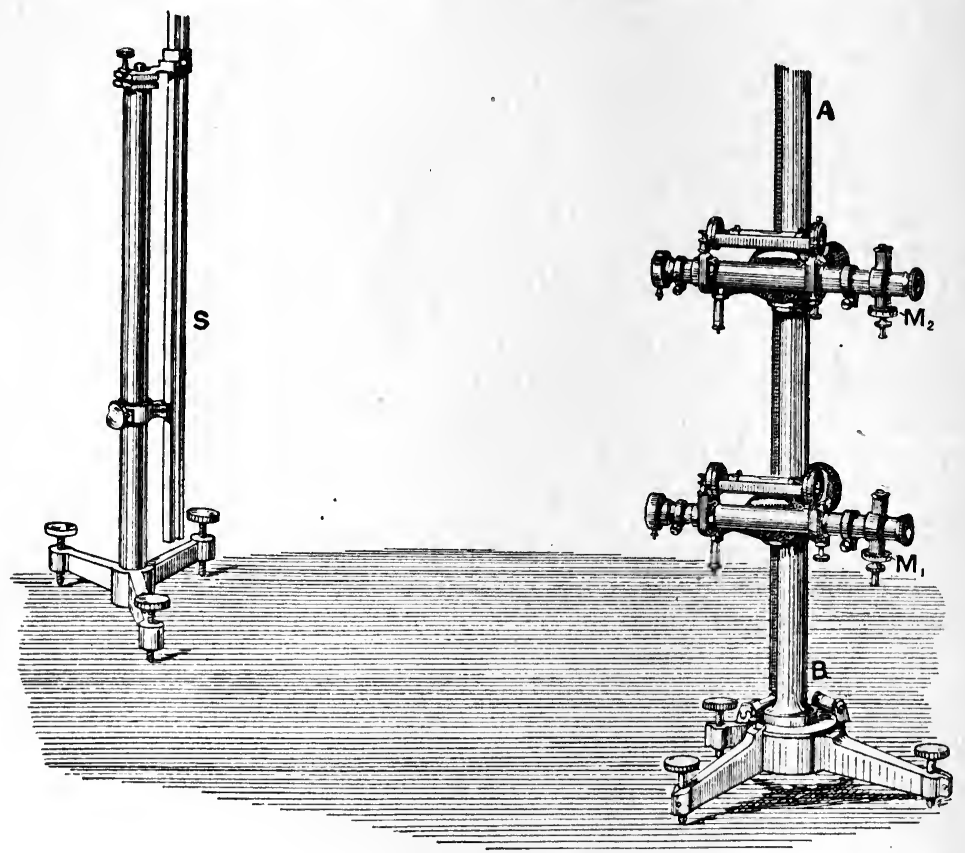

FIG. 21.

body in the immediate neighbourhood of the divided scale on the cathetometer column causes a very considerable uncertainty as to what is the actual temperature of this scale, and hence the actual distance between the divisions. These objections may in a great measure be obviated by separating the scale with which the object is to be compared from the telescope used in making the comparison and placing this scale as near to the object as possible, while the telescope simply serves to transfer the length from the object to the scale. The method of measuring thus resembles that used with the comparator, except that in place of moving the object and replacing it by the scale while the microscopes are kept 
fixed, the object and scale are here kept fixed, and the rertical column which carries the telescopes is rotated about a vertical axis.

The method of supporting the scale $s$, and one form of upright for carrying the telescopes, is shown in Fig. 21. The telescopes are each provided with a micrometer screw $\mathbf{M}_{1}$ and $\mathbf{M}_{2}$, by means of which the horizontal cross-wire can be moved in a vertical direction, the amount of the movement being read off on the divided head of the screw. When making an observation the scale must be placed as near to the object as is convenient, and at the same distance from the telescopes as the object. Using a plumb line as a guide, the scale must be made vertical. The axis about which the column $\mathbf{A B}$, which carries the telescopes, turns must be set accurately vertical. This is done in exactly the same way as was described in the case of the cathetometer (p. 56). Next the axes of the telescopes must be made horizontal, which is also done by the method previously described ( $p$. 55), namely, by first turning the telescopes on their V's and adjusting the cross-wires so that the line of collimation is parallel to the tube on which the level rests and which itself rests in the $\nabla$ 's, and secondly by reversing the level till the reading of the bubble is the same in either position.

The telescopes and scale being in adjustment, bring the cross-wires of the telescopes to coincide with the upper and lower marks on the object, and read the two micrometers $\mathbf{M}_{1}$ and $\mathbf{M}_{2}$. Now turn the column carrying the telescopes so that the scale is seen in the telescopes, and take the readings of each of the micrometers when the cross-wires coincide in turn with each of the division lines of the scale on either side of the positions they occupied after being adjusted on the object. From these readings the fractions of a scale division can be immediately deduced, exactly as was described in the case of the comparator $(\$ 19)$.

In cases where an accuracy of a tenth or twentieth of a millimetre is sufficient, one of the simplest and most satisfactory methods of measuring a length, whether horizontal or vertical, so long as the scale can be brought within about $2 \mathrm{cms}$. of the object, is to use a scale divided on looking-glass. The glass used must be not less than $7 \mathrm{~mm}$. thick. Such glass, silvered on the back, can be obtained in strips of about 6 or $8 \mathrm{cms}$. wide, and up to about a metre long, from most makers of pier glasses. These strips are the wastage when the glass is cut to size to fit the frames. The method of dividing such a scale is described in the Appendix.

As an example of the application of such a mirror scale to the measurement of a length we may take the measurement of vapour density described in $\S 99$.

23. The Optical Lever.-The optical lever consists of a lozenge-shaped metal plate A (Fig. 22), having four needlo-points projecting from the under side,

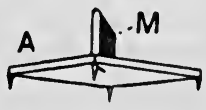

FIG. 22. and a mirror $M$ attached to the upper side. The two middle needlepoints project a very little farther than the end ones, so that when the lever is placed on a plane surface it can rock slightly about the middle points. In addition to the lever, a stand with a piece of good 
plate glass cemented to the top will be required, also a reading telescope with a vertical scale. A convenient form of reading telescope is shown in Fig. 23. The telescope can be moved up and down along the vertical rod, and clamped by means of the screw $\mathbf{A}$.

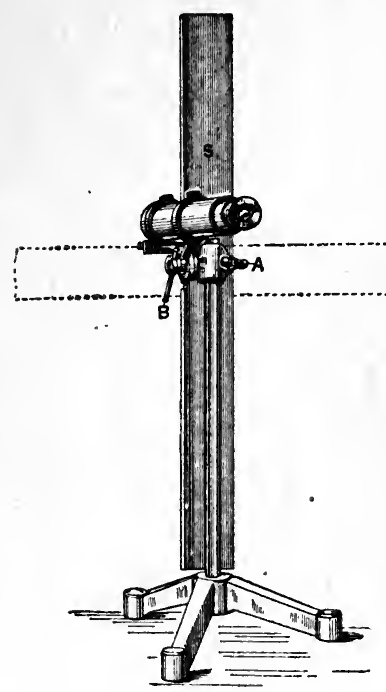

FIG. 23. The telescope can also be moved about a horizontal axis, and clamped by the screw $B$. A scale $\mathrm{s}$ can be placed either vertical when measuring rotations about a horizontal axis, or horizontal when measuring rotations about a vertical axis.

Set up the stand for the lever on a shelf attached to the wall, or on a very firm table, and place the telescope at a distance of about a metre from the stand, and focus on the scale as seen reflected in the mirror of the lever.

The readings of the scale must now be taken when the lever is tilted, so as to rest first on the front leg and then on the back, and let the difference of the readings be $d$. We will suppose that the thickness of a piece of microscope cover glass has to be measured. The cover glass having been well cleaned is placed on the stand in such a way that the central legs of the lever rest on the cover glass, but the end legs are clear of the edge and rest on the plate glass. The readings of the scale, as the lever rests first on one end leg and then on the other, must again be taken, the difference now being $D$.

The thickness of the plate placed beneath the central legs is given by

$$
\frac{l(D-d)}{4 L}
$$

where $l$ is half the distance between the points of the end legs, and $L$ is the distance between the mirror and the scale.

Suppose that when the lever is placed on a plane surface the angle through which it rocks is $a$. This also is the angle through which the mirror turns, and since a beam of light reflected from a mirror turns through twice the angle through which the mirror turns (Watson's Physics, p. 441), it follows that the angle included between the two rays MG and MF (Fig. 24) is $2 \alpha$. But if $\alpha$ is small, we have $2 \alpha=\overline{\mathrm{GF}} / \overline{\mathrm{MS}}=d / L$. Now $\overline{\mathrm{DE}}$ is the amount by which the central legs project beyond the end legs. From the triangle $A D E$ we have

or

$$
\begin{aligned}
& \overline{D E} / \overline{A D}=\tan D A E=\tan \alpha / 2, \\
& \overline{D E}=l \tan \alpha / 2=l \alpha / 2,
\end{aligned}
$$

since $\alpha$ is small. 
Hence, substituting for $\alpha$ the value already obtained,

$$
\overline{D E}=\frac{d l}{4 L} \text {. }
$$

When the lever is placed with its central legs on a plate of thickness

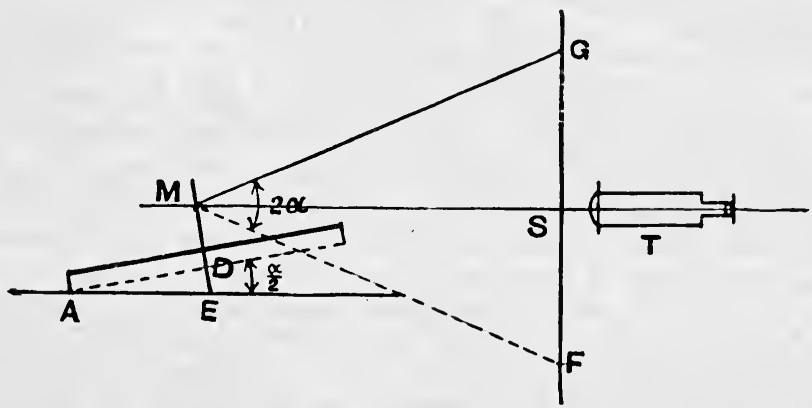

FIG. 24.

$t$, and the difference in the readings on the scale is $D$, we have in the same way

Hence

$$
t+\overline{D E}=\frac{D l}{4 L}
$$

$$
t=\frac{(D-d) l}{4 L}
$$

It will be noticed that in obtaining the above formula we have supposed that the angle through which the lever is tilted is small, hence the central legs must not project more than one or two tenths of a millimetre beyond the end legs, and the lever must only be used for measuring thin plates.

24. To Measure the Radius of Curvature of a Spherical Surface with the Optical Lever.-The optical lever can be employed to measure the radius of a spherical surface, such as a lens or mirror, so long as the radius of curvature is large and the surface of sufficient size to allow all the legs of the lever to rest on the surface. The method of making the measurement is exactly the same as that described in the preceding section, except that the lens will require supporting in such a way that it will not rock as the lever rocks, which may be done by attaching it with some soft wax.

If $D$ is the difference in the scale readings when the lever is placed on the spherical surface, then the radius of curvature $R$ of the surface is given by

$$
R=\frac{2 L}{D-d}\left(l-\frac{b^{2}}{l}\right),
$$


where $b$ is half the distance between the central legs. To deduce this formula, let the end legs touch the lens at the points $\mathrm{AC}$ (Fig. 25, a), and the centre legs at the points EE (Fig. 25, b). Through the mid

(a)

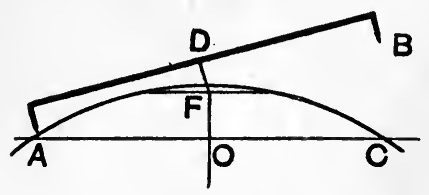

(b)

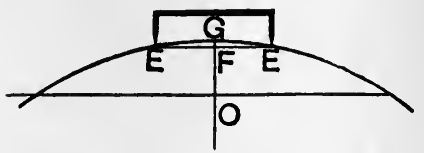

FIG. 25.

point $\mathrm{o}$ of $\mathrm{AC}$ draw the perpendicular oFG. Then if $R$ is the radius of the sphere of which the surface is a part, that is, the radius of the circle AGC, we have (Euclid, III. 35)

$$
\overline{O G}(2 R-\overline{O G})=\overline{A O^{2}} \text {. }
$$

But if $R$ is large, $\overrightarrow{\mathrm{AO}}$ is very nearly equal to the distance between the end and middle legs.

Hence, neglecting the square of the small distance $\overline{O G}$, we have

$$
2 R . \overline{O G}=2 R(\overline{G F}+\overline{F O})=l^{2} .
$$

But by tilting the lever we measure the distance $\overline{\text { Fo, }}$, so that

$$
\overrightarrow{F O}=l(D-d) / 4 L \text {. }
$$

Also from Fig. $25(b)$, we see in the same way that

Hence

$$
2 R \cdot \overline{G \bar{F}}={\overline{E F^{2}}}^{2}=b^{2} \text {. }
$$

$$
b^{2}+2 R l(D-d) / 4 L=l^{2}
$$

or

$$
R=\frac{2 L}{D-d} \frac{l^{2}-b^{2}}{l}=\frac{2 L}{D-d} \cdot \frac{(l+b)(l-b)}{l} .
$$




\section{CHAPTER IIJ}

\section{WEIGHING}

25. The Balance.-The theoretically perfect balance beam would be one in which the three knife-edges were in the same plane, and were parallel to one another. Further, they would remain so whatever the load in the pans. Practically, however, it is impossible to secure the exact fulfilment of the above conditions, although in a well-made balance they are so nearly fulfilled as to produce practically negligible errors. It can easily be shown that if the central knife-edge is below the plane containing the terminal knife-edges then the balance will be more sensitive with a large load than with a small one. In the same way, if the central knife-edge is above the terminal ones the sensitiveness will decrease as the load increases. Thus if the relative position of the knife-edges did not alter as the load was varied we could, by measuring the change of sensitiveness with change of load, determine the relative positions of the knife-edges. Owing, however, to unavoidable flexure of the beam with increasing loads the relative positions of the knife-edges alters as the load is increased. Makers generally arrange that the central knife-edge is a little below the terminal ones when there is no load in the pans. Thus at first the sensitiveness increases as the load increases. When, owing to the flexure of the beam, all three knife-edges are in the same plane, then for small changes of load in this neighbourhood the sensitiveness will not vary. If the load is increased above this point the terminal knife-edges will be below the central one, and so the sensitiveness will decrease as the load is further increased.

If the terminal knife-edges are not parallel to the central one, then, if the scale pan stirrup were so constructed that by altering the position of the weight placed in the pan we could cause either one or other end of the terminal knife-edge to carry the weight, we should alter the arm of the lever at which the pan and its contents works, and so change the position of equilibrium of the balance. In order that this may not occur in practice, owing to the slight unavoidable errors in the adjustment of the knife-edges, the pan supports are made with one or more movable links, such as that shown in Fig. 26, so that wherever in the pan the weight is placed the weight may be distributed uniformly along the knife-edge, or at any rate the distribution of the weight along the knife-edge may remain unaltered.

The accuracy of the adjustment of the terminal knife-edges to parallelism to the central knife-edge may be tested by doing away with 
the freedom to swing about the point $A$ of the scale pan, for which purpose some balances have a set screw, B, provided. Place a weight of about a quarter the maximum the balance is supposed to carry at the edge of the pan farthest from the front of the balance-case, and counterpoise by placing weights in the other pan. Then

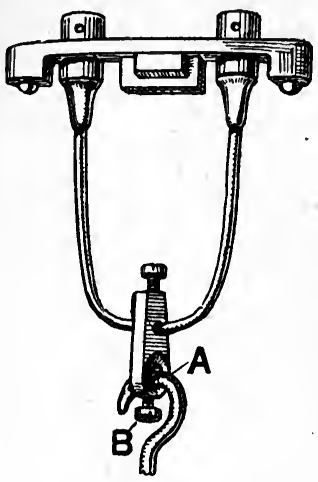

Fra. 26. move the weight in the clamped pan to the odge nearest the front of the case, and see whether the balance is still in equilibrium. In practice, however, the more important thing to test is whether, when the pans are not clamped but are allowed all the freedom that the construction permits, on changing the position of the weights in the pan the equilibrium of the balance is affected. If any measurable difference is found it shows that the knife-edges are out of adjustment, and that the pan supports are not sufficiently free to eliminate the error thus introduced. If cleaning the links, or whatever arrangement is used to secure the freedom of the pans, does not remedy the matter the balance must be sent back to the makers to have the knife-edges readjusted, since this adjustment is one which no one but a practised balance-maker can perform satisfactorily. When using a balance it is always advisable to be careful to place the weights, \&c., in the pans in such a way that the pans hang in about the same position that they occupy when no load is in the pans.

The weights of the pans and their supports ought to be the same, though their exact equality is not essential. To test whether they are of equal weight, remove the two pans and adjust the vane of the beam so that the pointer is at the zero of the scale. Then attach the pans and read the position of the pointer. Interchange the pans and supports and again read the position of the pointer. The readings corresponding to the two positions of the scale pans and supports ought to be the same. If they are different, they can be adjusted to equality by carefully filing the bottom of the heavier pan or adding a piece of platinum wire to the lighter.

The pans having been adjusted to approximate equality, the beam may be brought to the horizontal position, that is, the pointer to the middle of the scale, by adjusting the vane on the beam. As it is not a good thing to be continually readjusting the vane, unless the pointer, when there is no load on the pans, comes to rest at some distance from the centre of the scale, it is better not to alter the vane, but to take the reading corresponding to no load as the zero.

Balances nowadays are made with very much shorter beams than they used to be, the requisite sensitiveness being obtained by using a long pointer and reading lens or microscope. The advantage of the short beam is that the time of vibration of the balance is considerably 
reduced, while the beam itself can be made much lighter without loss of rigidity. In some balances a mirror is attached to the beam, and the deflections are read either with a telescope and scale or with a lamp and scale, as described in $\S 170$.

On account of the shortness of the beam, if the rider used weighed a centigram, and, in order to obtain the milligrams and fractions of a milligram, were moved from the centre of the beam to the end, the graduations of the beam would need to be very close, rendering it difficult to obtain the exact position of the rider For this reason in

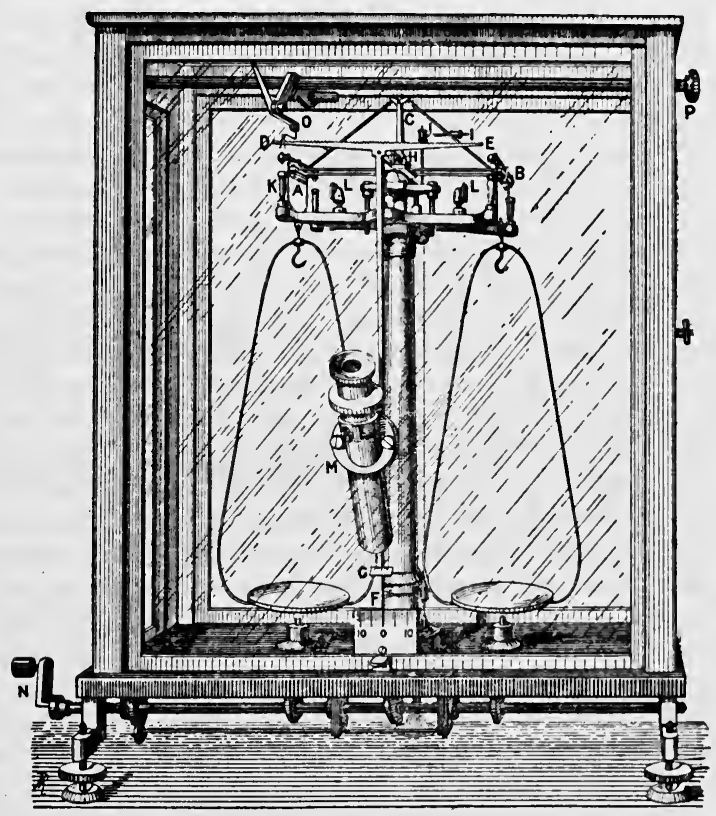

FIG. 27.

short beam balances it is usual to employ a rider which weighs only half a centigram and to attach to the front of the beam a light scale DE, Fig. 27, the graduated portion being of the same length as the distance between the end knife-edges. The rider is then placed on this scalc, and the vane, by means of which the beam is balanced, is so adjusted that when the rider is at the extreme left-hand edge (i.e. at the zero of the graduations) the pointer of the balance is at zero. When the rider is at the extreme right-hand end of the scale the effect is as if half a centigram had been moved to the end of an arm twice as long as the balance arms, that is, as if the weights in the right-hand pan had been increased by 1 centigram. Thus the rider scale is twice as open as it 
would be with the old arrangement. The separate scale to carry the rider has a further great advantage in that it allows of the rider being placed as near as we like to its zero position; while in the old arrangement, owing to the presence of the gravity bob, it was usually impossible to place the rider very near the centre of the beam.

26. Weighing by the Method of Oscillation and to Compare the Lengths of the Arms of the Balance.-If a balance is to be sensitive it is necessary to reduce the friction between the knife-edges and the planes to the minimum, so that a good balance, unless some specia] device is provided for damping the oscillations, will make a great number of vibrations before the beam comes to rest. Thus if we had to wait in every case till the pointer came to rest before we could take a reading, the operation of weighing would take a very long time. It is, however, unnecessary to wait till the balance comes to rest, for we can, by observing the readings of the scale corresponding to the turning points of the pointer as the balance swings, calculate what would be the reading corresponding to the position of rest. This method of performing a weighing, which must always be used when we are attempting to perform the weighing with the utmost accuracy with which the balance is capable, is called the method of oscillation.

Confusion and possible error will be saved if the scale of a balance with which the method of vibrations is to be used is numbered from the left, the extreme left being called 0 , the middle division 50 , and the extreme right-hand division 100 .

The first thing to do is to determine the zero. For this purpose gently raise and lower two or three times the beam from off the arrestors, to ensure that the planes have settled down into their correct positions. Next raise the beam in such a way that the pointer swings over between five and ten divisions on either side of the zero, and after the beam has made two or three swings to get into a state of regular motion, read the

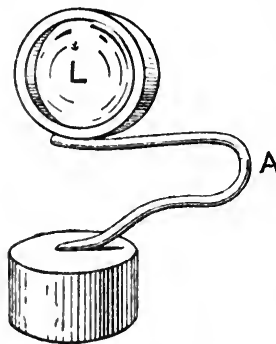

FIG. 28. turning points of the pointer on the scale. The turning points must be estimated to within a tenth of a division of the scale, and if the divisions are at all fine a lens fixed to the front of the balance case will be found of great assistance. Since a lens will often be used for reading a scale, it is convenient to have several having a focal length of about $5 \mathrm{~cm}$. mounted in such a way that they can be placed in any desired position. One of the simplest, and at the same time most convenient, forms of mounting is to have the lens fixed in a brass hoop, this hoop being soldered to a short length of lead wire A (Fig. 28). The lower end of the lead wire is attached to a small base piece made of lead, and by bending the lead wire the lens can be adjusted to any desired position.

$\mathrm{As}$ in the case of most balances the pointer swings in front of the scale, care must be taken to avoid parallax. For this purpose the pointer ought to be bent so that it only just clears the surface of the 
scale. If a lens is used, then parallax can be avoided by arranging that the eye always occupies the same position relative to the lens. This can be secured by sticking a piece of paper to the front glass of the balance case with a small hole in such a position that when the eye is placed opposite the hole the scale can be focused.

When taking the turning points of the pointer of the balance, readings must be taken on both sides of the position of rest of the pointer and an odd number of readings must be made, so that the first and last turning points observed are on the same side of the zero. It will in general be found best to take five readings, two on one side and three on the other. Then take the mean of the three readings corresponding to the turning points in one direction and the mean of the two in the other, and the mean of these two means will be the scale reading corresponding to the position of rest. The reason that an odd number of elongations have to be observed is that the arc over which the pointer is swinging is continually growing less, and thus if we only took two observations, say, one to the left and then one to the right, we should obtain a position of rest too far to the left. This will be at once apparent from a study of Fig. 29. In this figure the abscissæ represent

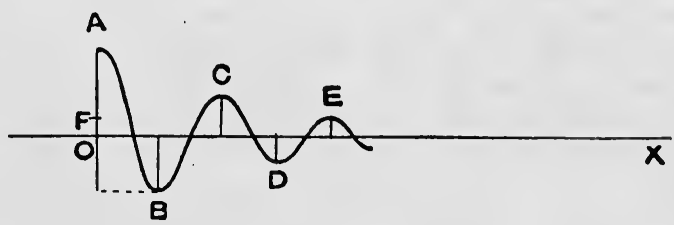

FIG. 29. time, and the ordinates the position of the pointer measured from the position of rest, which is represented by the axis $0 \mathrm{x}$. The elongations are represented by the ordinates through the points $\mathrm{A}, \mathrm{B}, \mathrm{C}, \mathrm{D}, \mathrm{E}$. The mean of the first two elongations would give the point $\mathrm{F}$ as the position of rest, which is considerably above the true position of rest. The mean of any odd number of elongations obtained as above will, so long as the decrease in the successive elongations is not very rapid, represent the position of rest with a degree of accuracy quite sufficient for the purpose in hand.

The determination of the position of rest ought to be repeated three or four times, the beam being arrested between each observation. If the balance is working satisfactorily, the values obtained for the position of rest ought not to differ by more than a few tenths of a scale division. If larger discrepancies than a tenth of a division occur, it indicates that the balance beam is not swinging freely, or that there are air currents within the balance case which are causing the beam to swing unevenly. To remedy the first of these defects, the pan supports and the beam must be removed, and the knife-edges and planes be gently rubbed with a clean piece of pith or soft cork and then dusted with a camel's hair pencil. If this cleaning does not remove the irregularity, and, further, the rate at which the elongations decrease is small, then probably air currents are the cause of the irregularity. These air currents may be produced by the heat from a neighbouring gas flame, or from the balance case being placed too near a window. 
Having determined the position of rest when there is no load in the pans, proceed to determine the ratio of the lengths of the arms of the balance. For this purpose place two equal weights in the pans. These weights ought to be about half the maximum load the balance will carry, so that for a balance intended to carry 200 grams a weight of 100 grams ought to be placed in each pan. Adjust the position of the rider so that it is at the nearest milligram division ${ }^{1}$ to the point it would have to occupy to bring the pointer to the zero position, and determine by the method of vibrations the position of rest $(\Delta)$. Then move the rider so as to alter the weight on one side by 1 milligram, and again determine the position of rest (B). The weights in the pans must now be interchanged, and the readings ( $C$ and $D)$ for two positions of the rider determined as before.

From the two sets of readings before and after changing the weight by 1 milligram calculate the number of scale divisions which correspond to 1 milligram, or, what is for most purposes more convenient, the increase of weight which will produce a deflection of one scale division. Let $W$ and $W^{\prime}$ be the true weights of the weights placed in the pans, which true weights may, and probably will, differ from their nominal values. When $W$ is in the right-hand pan and $W^{\prime}$ in the left, calculate the weight $w$ which has been added to the one which appears to be lighter, say, $W$, to produce equilibrium. The whole number of milligrams in $w$ will be obtained from the position of the rider on the beam, and the tenths and hundredths will be obtained by multiplying the difference between the scale reading for the zero and the scale reading corresponding to the position of rest (A) above by the weight corresponding to a scale division. In the same way calculate the weight $w^{\prime}$ which must be added to $W$ when $W$ is in the left-hand pan to bring the balance to the zero position. The quantity $w^{\prime}$ may be either positive or negative.

Let the length of the right-hand arm of the beam be called $R$, and that of the left $L$. Then in the first position of the weights we have $(W+w) R$ $=W^{\prime} L$, and in the second position $W^{\prime} R=\left(W+w^{\prime}\right) L$. Multiplying these two equations together we get $W^{\prime}(W+w) R^{2}=W^{\prime}\left(W+w^{\prime}\right) L^{2}$.

Thus

$$
\begin{aligned}
\frac{R}{L} & =\left(\frac{W+w^{\prime}}{W+w}\right)^{\frac{1}{2}}=\left\{\frac{1+\frac{w^{\prime}}{W}}{1+\frac{w}{W}}\right\}^{\frac{1}{2}} \\
& =\left(1+\frac{w^{\prime}}{2 W}+\right)\left(1-\frac{w}{2 W}+\right) \\
& =1+\frac{w^{\prime}-w}{2 W} .
\end{aligned}
$$

1 In the case of a very sensitive balance the position of the rider can be adjusted to the nearest division in the scale which carries the rider, while the change in the position of the rider made to determine the sensitiveness will have to be less than 1 milligram. 
For as $w$ and $w^{\prime}$ are small compared with $W$ or $W^{\prime}$, we may neglect the terms in squares and higher powers of $\frac{w}{W}$ and $\frac{20^{\prime}}{W}$

If the arms are found to be unequal it will be necessary either to correct all weighings by multiplying them by the ratio of the arms determined in this way, a process which is not very satisfactory if great accuracy is desired, since owing to unequal heating of the two arms this ratio may not be constant; or we may so conduct the weighings that the ratio of the lengths of the arms is eliminated from the result. There are two methods by which this may be performed. One method, called the method of double weighing, or Gauss's method, is to weigh the body first when it is placed in the right-hand pan, and then when it is placed in the left-hand pan. Let the apparent weights be $w$ and $w^{\prime}$; then if $W$ is the true weight, we have

$$
\begin{aligned}
& W R=w L \text { and } W L=w^{\prime} R . \\
& \frac{W R}{w^{\prime} R}=\frac{w L}{W L}, \\
& W=\sqrt{w w^{\prime}} .
\end{aligned}
$$

Hence

or

Since $w$ and $w^{\prime}$ are always very nearly equal, the arithmetical mean $\frac{1}{2}\left(w+w^{\prime}\right)$ will not differ appreciably from the geometrical mean $\sqrt{ } w w^{\prime}$. Thus since the arithmetical mean is so very much easier to calculate, the true weight of the body is taken as the mean of the weights obtained when it is placed in the two pans. The other method is called Borda's method, or the method of substitution. In this method the body, say, is placed in the left-hand pan, and weights are added to the right-hand pan till equilibrium is obtained. Then, leaving these weights in the right-hand pan, the body is removed and weights are added to the lefthand pan till the balance is again in its zero position. The sum of the weights in the left-hand pan will then be equal to the weight of the body, independent of any inequality in the lengths of the balance arms.

Of these two methods of making the weighing independent of the lengths of the balance arms, the first, or the method of double weighing, is the better, as in addition to being somewhat quicker and involving only the use of a single set of weights, it is slightly more accurate, since two weighings of the body are made, and the probable error of the final value is smaller than in the method of substitution, where the body is weighed and then the weights are weighed.

When two very nearly equal weights have to be compared, as occurs when determining the value of a weight from that of a standard, or when calibrating a set of weights, the method of double weighing is employed. Since in general the differences of the weights will be very small, instead of adding weights to one side till the balance comes back to zero, the position of rest is noted, first, when the weight $W$, say, is in the right pan, and, second, when $W$ is in the left pan. If $\theta_{1}$ and $\theta_{2}$ are these 
readings on the scale, we have, if $R$ and $L$ are the lengths of the balance arms,

$$
\begin{aligned}
& W R-W^{\prime} L=\delta \theta_{1} \\
& W^{\prime} R-W L=\delta \theta_{2}
\end{aligned}
$$

where $\delta$ is a constant depending on the position of the centre of gravity of the beam, weight of the beam, length of pointer, \&c. \&c.

Subtracting (2) from (1)

or

$$
\begin{gathered}
W(R+L)-W^{\prime}(R+L)=\delta\left(\theta_{1}-\theta_{2}\right), \\
W-W^{\prime}=\frac{\delta}{R+L}\left(\theta_{1}-\theta_{2}\right),
\end{gathered}
$$

or since $R$ and $L$ are very nearly equal, and the whole difference $W-W^{\prime}$ is small compared to either, we may take

$$
W-W^{\prime}=\frac{\delta}{2 R}\left(\theta_{1}-\theta_{2}\right) \text {. }
$$

If, now, the sensitiveness $(s)$ of the balance (that is, the deflection produced by one milligram) is determined, we have

or

$$
\begin{gathered}
1 \times R=\delta s, \\
\frac{R}{\delta}=s .
\end{gathered}
$$

Hence substituting this value in (3)

$$
W-W^{\prime}=\frac{\theta_{1}-\theta_{2}}{2 s} \text { milligrams. }
$$

The following example will illustrate the way of entering results when using the method of vibrations, also the manner in which the readings are arranged when comparing two nearly equal weights. The numbers were obtained when comparing a 1 gram weight (indicated by $1_{.}$) with a standard 1 gram weight $\left(1_{\text {s.k. }}\right)$ which has itself been compared with the standards at the Bureau International at Sèvres.

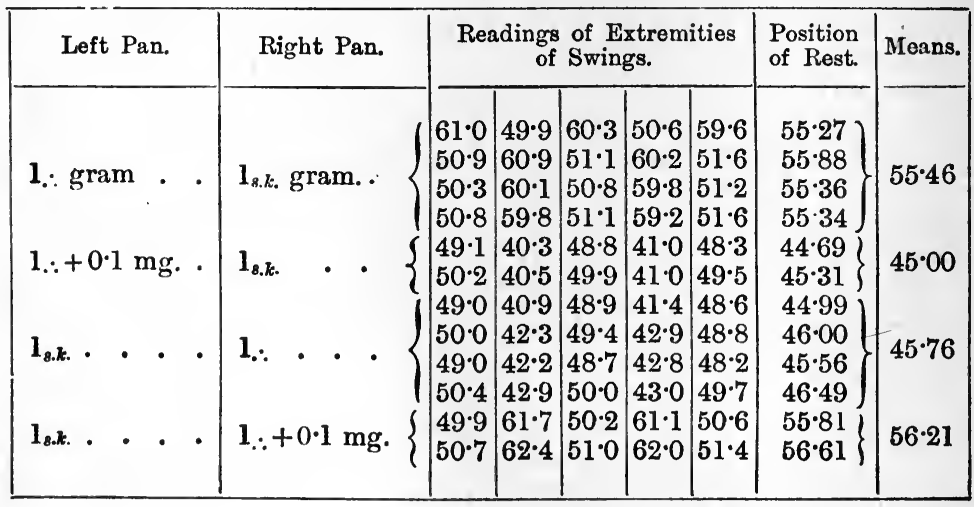


Deflection for $0 \cdot 1 \mathrm{mg} .=55 \cdot 46-45 \cdot 00=10 \cdot 46$ scale divisions

$$
" \quad=56.21-45 \cdot 76=10.45 \quad, \quad "
$$

Sensitiveness (deflection for $1 \mathrm{mg}$.) $=104.5$ scale divisions.

Difference of reading on reversing weights $=2\left\{1_{. .}-1_{a . k}\right\}$ $=55 \cdot 46-45 \cdot 76=9 \cdot 70$ scale divisions.

Hence $1_{\therefore}-1_{\text {s.k. }}=\frac{4 \cdot 85}{104 \cdot 5}=0 \cdot 0464$ milligrams.

But the standard $1_{\text {8.k. }}$ is really 1 gram $+0.083 \mathrm{mg}$.

Hence the true weight of $1 . \therefore$ is 1.000129 grams.

27. Calibration of a Set of Weights.-Although a set of weights by a good maker when new will be wonderfully correct, yet when making really accurate measurements it is necessary to determine carefully the amounts by which the individual weights differ from their nominal values, and, further, if the weights are much used it will be necessary to repeat this calibration from time to time. To obtain the absolute values of the weights it will be necessary to have a standard weight the absolute value of which is known, and in order to obtain accurately the values of the other weights of the set it is essential that this standard weight should have the same nominal value as one of the largest weights of the set which is being tested. Thus if the set to be tested consists of weights from 100 grams downwards the standard ought to be a 100 gram weight, though a 50 gram weight would do fairly well. It would be quite impossible to determine the errors of such a set of weights with any reasonable accuracy by comparing them with a standard 1 gram weight. If no standard weight is available then we can still determine the errors in the ratios which the several weights bear to one another, and for many purposes this is all that is necessary. Thus when making a chemical analysis, or determining a density, the absolute values of the weights is immaterial ; all that we wish to know is the relation the weights bear one to the others.

A set of weights from 100 grams downwards generally consists of the following pieces:- $100,50,20,10,10,5,2,1,1,1, \cdot 5, \cdot 2, \cdot 1, \cdot 1, \cdot 05$, $\cdot 02, \cdot 01, \cdot 01$, and a rider weighing $\cdot 01$ gram or $\cdot 005$ gram. For purposes of calibration, however, a more convenient arrangement is the following : $-100,50,20,20,10,5,2,2,1,1, \cdot 5, \cdot 2, \cdot 2, \cdot 1, \cdot 05, \cdot 02, \cdot 02, \cdot 01, \cdot 01$, and a centigram or a half centigram rider. We shall describe two methods of conducting the calibration, the first a comparatively short and simple one, applied to the first of the above arrangements of weights, and the second a more elaborate and accurate method, which has been worked out by M. René Benoît of the Bureau International, and arranged to suit a set of weights having the second arrangement.

Unless the weights in the set which have the same denomination have received distinguishing marks it will be necessary to provide such marks. The best way of doing this is to take a sharp-pointed sewing- 
needle and immediately after the figure denoting the denomination of the weight make a small dot or set of dots by gently pressing the point of the needle vertically down on the weight. Thus the 1 gram weights would be marked $1 ., 1 \ldots, 1 . \therefore$. This method of marking the weights is preferable to attempting to scratch letters or any such device on the weights, as being more legible and less likely to remove any of the metal.

The first operation to be performed is to compare the 50 gram weight with the sum of the 20 gram to 1 gram weights inclusive. This comparison must be performed by the method of reversal, in which the 50 gram weight is first placed in the left-hand pan and the others in the right, and then the loads in the pans are interchanged, the calculation being performed as in the example given in the last section. If the weights differ much from their nominal values, it will sometimes be necessary to add a small weight to one pan or the other to produce equilibrium. In a balance having each of the arms graduated into a hundred parts and using a centigram rider, this would involve moving the rider from side to side or using two riders. In that form of balance in which the whole beam from one end to the other is divided into a hundred parts, and a half centigram rider is used, it is impossible by means of the rider to add a small weight to the left-hand side. Hence in either case it is advisable to place a weight of half a centigram in the left-hand pan and keep it there throughout the weighings. By this means the rider, when no other weights are placed in the pans, will be half-way along the scale, and hence by moving it to one side or other of this position we are able to increase the load in either pan.

Place the 50 gram weight in the left-hand pan and the others in the right, and find the position of the rider which will most nearly bring the pointer to the zero of the scale. Then determine very carefully by the method of vibrations the scale reading corresponding to the position of rest. Next reverse the weights and repeat. The results should be entered as in the example given in the last section.

In the same way perform the comparisons indicated in the following table, in which the expressions (20), \&c., represent the actual weight of the weight whose nominal weight is 20 grams, and the quantities $a, b, c$, $\& c$., are the differences deduced from the weighings, and which may be either positive or negative :-

$$
\begin{aligned}
& (50)-\{(20)+(10 .)+(10 . .)+(5)+(2)+(1 .)+(1 . .)+(1 . .)\}=a(1) \\
& (20)-\{(10 .)+(10 . .)\}=b \\
& (10 . .)-(10 .)=c \\
& (10 .)-\{(5)+(2)+(1 .)+(1 . .)+(1 . .)\}=d \\
& (5)-\{(2)+(1 .)+(1 . .)+(1 \therefore)\}=e \\
& (2)-\{(1 .)+(1 . .)\}=f \text {. } \\
& (1 .)-(1 \ldots)=g \\
& (1 . .)-(1 . .)=h
\end{aligned}
$$


Consider, first, the first four weighings, and using the symbol ( $\Sigma 10)$ for the true weight of the five smaller weights, we have

From (4)

From (3) and

$$
(10 .)=(\Sigma 10)+d
$$

From (2) and

From (1) and (11)

$(10 .)=.(\Sigma 10)+d+$

(9)

(10..) $=(\Sigma 10)+d+c$

$(20)=2(\Sigma 10)+2 d+c+b$.

$(50)=5(\Sigma 10)+4 d+2 c+b+a$

or

$$
(\Sigma 10)=\frac{1}{5}(50)-\frac{1}{6}\{4 d+2 c+b+a\} \text {. }
$$

Or if we assume for the present that the 50 gram weight is correct, and write

$$
\begin{aligned}
S & =\frac{1}{5}\{4 d+2 c+b+a\} \\
(\Sigma 10) & =10-S \\
(10 .) & =10-S+d \\
(10 \ldots) & =10-S+d+c \\
(20) & =20-2 S+2 d+c+b \\
(50) & =50-0 .
\end{aligned}
$$
above

Next to obtain the errors of the units we have from $(5,6,7$, and 8$)$

$$
\begin{aligned}
(1 . .) & =(1 \therefore)+h \\
(1 .) & =(1 \therefore)+h+g \\
(2) & =2(1 \therefore)+2 h+g+f \\
(5) & =5(1 \therefore)+4 h+2 g+f+e,
\end{aligned}
$$

adding

$$
\Sigma(10)=10(1 \therefore)+8 h+4 g+2 f+e \text {, }
$$

or since

$$
\Sigma(10)=10-S
$$

If we call

$$
(1 \therefore)=1-\frac{1}{10}\{S+8 h+4 g+2 f+e\} \text {. }
$$

then

$$
\frac{1}{10}\{S+8 h+4 g+2 f+e\}=8
$$

$$
\begin{aligned}
(1 . .) & =1-s \\
(1 . .) & =1-s+h \\
(1 .) & =1-s+h+g \\
(2) & =2-2 s+2 h+g+f \\
(5) & =5-5 s+4 h+2 g+f+e .
\end{aligned}
$$

In this way the errors of the weights taken with respect to the 50 gram weight are obtained, and for many purposes this will be sufficient. Occasionally, however, as, for instance, when a current is measured by the weight of metal deposited in a voltameter, it is necessary to know the actual errors of the weights. To obtain these it is necessary to compare the weights with a standard weight, the value of which is known in terms of the standard kilogram kept at Sèvres. In order to obtain the errors of the whole set of weights with an accuracy comparable with the accuracy with which two weights can be compared on the balance used, it is necessary that the standard weight should be at least equal to the 
largest of the weights in the set being tested. If this is not done, and, say, we attempt to standardise a set of weights up to 50 grams by comparison with a standard 1 gram weight, the error of the standard and of the comparison of the standard with one of the 1 gram weights of the set will be magnified fiftyfold in the case of the 50 gram weight. Thus, in the case before us, it is necessary to have either a 50 or a $100 \mathrm{gram}$ standard. Suppose that we have a standard 100 gram weight, then we compare this with the sum of all the weights from 50 to $1 \mathrm{gram}$. This observation must be repeated several times, and the mean of the differences taken to represent the quantity $x$ in the expressions below. Then

$$
(\Sigma 100)=(100)+x \text {, }
$$

where (100) represents the actual weight of the standard as given on its certificate.

Let $\Sigma y$ be the algebraical sum of all the errors of the weights from 20 to 1 gram obtained when the 50 gram was taken as correct. Then

$$
(50)=(100)-50-\Sigma y+x \text {, }
$$

from which the error of the 50 gram weight can at once be obtained, and thence the errors of the other weights.

When comparing the standard weight it is important to ascertain whether its density is the same as that of the weights being tested. If not, a correction for buoyancy will have to be made, as described in $\S 28$. The density of the standard weight will be given on its certificate, since it will have been measured before it was standardised. The density of the weights being calibrated will have to be measured by weighing one of them, say, the 50 gram weight, first in air, and then in water (see $\S 31$ ). This, however, cannot be done if the weight is not made in a single piece of metal as it ought to be, that is, if it has the knob at the top screwed into the main portion. In such a case, the only thing will be to take the density as being equal to that of the particular metal of which the weights are supposed to be composed, as given in Table 28. In any case, it is a good thing to compare the weight of the weight on which the measurement of density has been made both before and after being immersed in water. If the weight has increased, it will indicate that there is some hollow in the weight into which the water has penetrated, and the weight will require readjusting.

The errors of the fractions of the gram and of the rider can be determined in the same manner. To obtain accurate results it will, however, be necessary to use a lighter and more sensitive balance than was used for the larger weights.

The following account of the method he uses for calibrating a set of weights is due to $M$. René Benoit, Director of the International Bureau at Sèvres, and has been communicated to the author in a letter :-

The set of weights is supposed to consist of the following :-100, 50, $20,20 ., 10,5,2,2 ., 1,1$, and so on, and the 100 gram weight is sup- 
posed to have been compared with the standards, the error of comparison being negligible.

The $(100)$ is then compared with the $(\Sigma 100)$, the operation being performed three or four times, the mean result being that

$$
(50)+(20)+(20 .)+(10)=M \quad . \quad . \quad . \quad \text {. }
$$

The additional comparisons in the following equations are then made; in each case the weights to which a + sign are prefixed being compared with those to which a - sign is prefixed :-

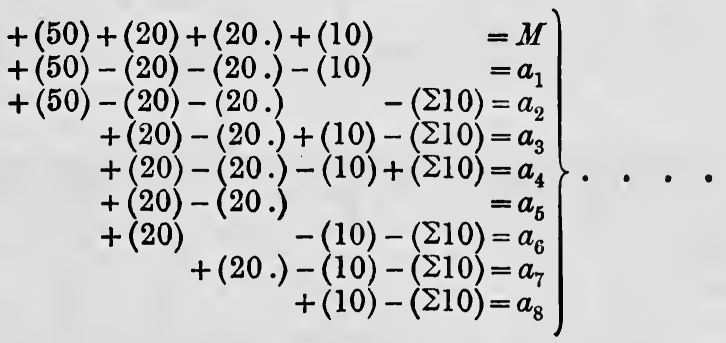

Here we have nine equations and only five unknowns, namely, the values of the five weights $(50),(20),(20),.(10),(\Sigma 10)$, the sum of the units being considered as a single weight. The equations must, therefore, be reduced to five normal equations. The rule founded on the principle of least square is to multiply each equation through by that coefficient of one of the unknowns which occurs in that equation, and to add all the equations thus obtained to form a new equation, which is called a normal equation. Thus in the case of the (20) we have to multiply the first equation through by +1 , the second and third equations through by -1 , and the next four equations through by +1 , while the last two equations, as they do not contain (20), i.e. we multiply through by 0 , vanish. Then adding, we get

$$
-(50)+7(20)+(10)=M-a_{1}-a_{2}+a_{3}+a_{4}+a_{5}+a_{6} .
$$

Proceeding in this way the following normal equations are obtained :-

$$
\begin{aligned}
3(50)-(20)-(20 .)+(10)-(\Sigma 10) & =M+A_{1} \\
-(50)+7(20)+7+A_{2} & =M+A_{3} \\
-(50)+7(20 .)+(10) & =M+A_{3} \\
-(50)+(20)+(20 .)+7(10)-(\Sigma 10) & =M+A_{4} \\
-(10)+6(\Sigma 10) & =
\end{aligned}
$$

where

$$
\left.\begin{array}{l}
A_{1}=+a_{1}+a_{2} \\
A_{2}=-a_{1}-a_{2}+a_{3}+a_{4}+a_{5}+a_{6} \\
A_{3}=-a_{1}-a_{2}-a_{3}-a_{4}-a_{5}+a_{7} \\
A_{4}=-a_{1}+a_{3}-a_{4} \quad-a_{6}-a_{7}+a_{8} \\
A_{5}=\quad-a_{2}-a_{3}+a_{4}-a_{6}-a_{7}-a_{8}
\end{array}\right\}
$$


Solving the set of simultaneous equations (3) we obtain

$$
\begin{aligned}
& \text { (50) }=\frac{1}{2} M+\frac{1}{28}\left\{7 A_{1}-A_{4}+A_{5}\right\} \\
& \text { (20) }=\frac{1}{5} M+\frac{1}{35}\left\{A_{1}+5 A_{2}-A_{4}\right\} \\
& \text { (20.) } \left.=\frac{1}{6} M+\frac{1}{35}\left\{A_{1}+5 A_{3}-A_{4}\right\}\right\} \\
& \text { (10) }=\frac{1}{10} M+\frac{1}{140}\left\{7 A_{1}+23 A_{4}+5 A_{5}\right\} \\
& \left.(\Sigma 10)=\frac{1}{10} M+\frac{1}{140}\left\{7 A_{1}+3 A_{4}+25 A_{5}\right\}\right\}
\end{aligned}
$$

Calling now the correction to $(\Sigma 10) M_{1}$, so that

$$
\text { (5) }+(2)+(2 .)+1=M_{1} \text {, }
$$

we may proceed in exactly the same manner to obtain the corrections to these weights, and then having obtained the correction to the 1 gram weight, the corrections to the fractions can be obtained by a similar process.

M. Benoit has shown that by combining the comparisons in the above manner there is no accumulation of errors, and that any probable error made in comparing the $(\Sigma 100)$ with the standard becomes of less and less influence as the weights of lower denomination are reached.

In the event of a standard 100 gram weight not being available, the ratios of the weights can be obtained by the above process, for if we put $M=0$ in equations (5), we shall obtain the corrections to the weights on the supposition that the sum of the weights $50+20+20++10$ is correct.

28. Reductions of Weighings to Vacuo.-The downward force exerted by a body on a scale pan in air is slightly less than the weight of the body owing to the buoyancy of the air, the actual pressure on the scale pan being equal to the weight of the body less the weight of the air displaced by the body. Thus, unless the volume of the body being weighed and that of the weights is the same, the amounts by which their weights appear to be diminished on account of the buoyancy of the air will be different, and hence, although they may be in equilibrium when placed in the opposite pans of a balance, they have not exactly the same weight. If, however, the weighings were made in vacuo, there would be no such difference depending on the difference between the density of the body and of the weights. Since it would be very inconvenient to perform weighings in vacuo, we have to apply a correction to allow for the buoyancy of the air, an operation called reducing the weighings to vacuo.

Let $W$ be the true weight of the body, i.e. its weight in vacuo, and $w$ the true weight of the weights which counterpoise the body. Then if $D$ is the density of the body, $\delta$ that of the weights, and $\sigma$ that of the air at the time of the weighing, we have that the volume of the body is $W / D$, and that of the weights is $w / \delta$. Hence the weight of the air displaced by the body is $\sigma W / D$, and of that displaced by the weights is $\sigma w / \delta$, and therefore

$$
W-\sigma W / D=w-\sigma w / \delta \text {. }
$$


Hence

$$
W=\frac{w(1-\sigma / \delta)}{1-\sigma / D},
$$

or since $\sigma$ is generally small compared with $D$ or $\delta$,

$$
W=w+v o \sigma\left(\frac{1}{\delta}-\frac{1}{D}\right) \cdot . \cdot . \cdot . \cdot
$$

From this expression it will be seen that when the density of the weights is greater than that of the body, we have to slightly increase the apparent weight of the body to get the true weight, while if the density of the weights is less than that of the body, we have to reduce the apparent weight.

The density of the air, $\sigma$, varies slightly from day to day, and where the utmost accuracy is desired it must be calculated from the expression?

$$
\sigma=\frac{0.001293}{1+0.00367 t} \frac{B-3 h / 8}{760} . . . \quad .
$$

where $B$ is the height of the barometer reduced to $0^{\circ}, h$ is the tension of aqueous vapour present in the air, and $t$ is the temperature. Where no measurements of the hygrometric state have been made, it will generally be best to assume that the air is half saturated at a temperature equal to that inside the balance case. ${ }^{2}$ Of course if the interior of the case is very carefully dried by means of some desiccating agent, $h$ may be taken as equal to zero. For most measurements made in the physical laboratory it is quite accurate enough to take a mean value for the density of air, and the number generally employed is 0.0012 . In the same way we may take the density of the weights if of brass as $8 \cdot 4$, if of platinum as 21.5 , if of quartz or aluminium as $2 \cdot 65$. Assuming the above values for the densities of air and the weights, it is possible to calculate a table to facilitate the correction of weighings for the buoyancy of the air.

Table 3 has been worked out on these assumptions, and contains in the first column the density of the body being weighed, and in the second, third, and fourth columns the value of the expression $.0012\left(\frac{1}{D}-\frac{1}{\delta}\right) \times 1000$, that is, the correction in milligrams to the weight of the body for each gram of this weight. For instance, if a body of density 3.0 is found to weigh 12.3456 grams when brass weights are used, then the buoyancy correction is $+12.35 \times 0.26=+3.21$ milligrams, so that the weight of the body in vacuo would be $12 \cdot 3488$ grams.

The correction on account of buoyancy in the case of water when, as is usual, brass weights are used is very appreciable. Thus in the case of 10 grams of water the correction amounts to $10 \cdot 6$ milligrams. Thus when water is used to gauge the volume of a vessel, the correction for buoyancy must be made if results correct to 1 in 1000 are required.

1 The values of $\frac{0.001293}{1+0.00367 t}$ for various temperatures are given in Table 4.

${ }^{2}$ For the tensions of aqueous vapour at different temperatures, see Table 14. 
It is important to remember that weighings have to be reduced to vacuo not only when the absolute weight of a body is required, but also in some cases when we have to do with the ratio of the weights of two bodies, as, for instance, in a chemical analysis. Thus if two of the constituents of the body being analysed have very different densities in the form in which they are weighed, the ratio of their apparent weights in air will not be the same as the ratio of their true weights. Thus, suppose that the true weight of one of the constituents is $W$, its density $D$, and that its apparent weight is $w$, the density of the weights being $\delta$, while let the same letters dashed represent the corresponding quantities for the second constituent. Then we have the true ratio of the weights. of the bodies given by

$$
\begin{aligned}
\frac{W}{W^{\prime}} & =\frac{w\left\{1+\sigma\left(\frac{1}{D}-\frac{1}{\delta}\right)\right\}}{w^{\prime}\left\{1+\sigma\left(\frac{1}{D^{\prime}}-\frac{1}{\delta}\right)\right\}} \\
& =\frac{w}{w^{\prime}}\left\{1+\sigma\left(\frac{1}{D}-\frac{1}{D^{\prime}}\right)\right\},
\end{aligned}
$$

since $\sigma$ is small compared with $D, D^{\prime}$, or $\delta$. It will be seen from this expression that we have not to correct for the air displaced by the weights used in performing the weighings if, as we have supposed, weights of the same density are used in the two cases.

Thus, suppose a mass of sea water (density 1.026) is weighed, the apparent weight being $w^{\prime}$, and the apparent weight of the silver chloride formed when the chlorides are precipitated by adding silver nitrate is $w$. Then, since the density of silver chloride is $5 \cdot 6$, the true ratio of the weight of the silver chloride to the weight of water is

$$
\frac{W}{\bar{W}^{\prime}}=\frac{w}{w^{\prime}}\left\{1+\cdot 0012\left(\frac{1}{5 \cdot 6}-\begin{array}{c}
1 \\
1 \cdot 026
\end{array}\right)\right\}={ }_{w^{\prime}}^{w}\{1-\cdot 0011\}
$$

so that the correction amounts to about 1 in 1000 . 


\section{CHAPTER IV}

\section{DENSITY}

29. Density.-By the density of a substance is meant the mass of unit volume. Thus in the C.G.S. system the density of a body is the mass in grams of a cubic centimetre. Since, however, the cubic centimetre is defined not as the volume of a centimetre cube, but as the thousandth part of a litre, and a litre as the volume of a kilogram of water at a temperature of $4^{\circ} \mathrm{C}$., it follows that the density of a body is really the ratio of the mass of any given volume of the substance to that of an equal volume of water taken at a temperature of $4^{\circ} \mathrm{C}$. The term specific gravity is sometimes taken to represent the ratio of the mass of any given volume of the substance to the mass or weight of an equal volume of water taken at the same temperature as the substance. Since different bodies expand with heat at different rates, the change in density which takes place as the temperature rises is not the same for all substances, and hence the specific gravity as defined above is not a constant, but depends on the temperature at which the comparison is made. The following ratios are sometimes used to express the density or specific gravity of substances; their use, however, has nothing to recommend them :-

$$
\begin{aligned}
& \text { Mass of substance at } 0^{\circ} \\
& \text { Mass of equal volume of water at } 0^{\circ} \\
& \text { Mass of substance at } 15^{\circ} \\
& \text { Mass of equal volume of water at } 15^{\circ}
\end{aligned}
$$

Throughout this book, when we use the term density of a substance we shall imply the following definition :-

The density of a substance at a temperature $t$ is the ratio of the mass or weight of a given volume of this substance taken at the temperature $t$ to the mass or weight of an equal volume of water taken at $4^{\circ} \mathrm{C}$.

Of course the observations need not necessarily be made in water at $4^{\circ} \mathrm{C}$., since elaborate experiments have been made to determine the density of water, as above defined, at all temperatures. Table 5 gives the density of water at temperatures between $0^{\circ}$ and $35^{\circ} \mathrm{C}$.

30. The Measurement of Density-Corrections for Temperature of Water and Buoyancy of Air.-The determination of the density of a substance, whether it be a solid, a liquid, or a gas, consists of two parts. In the first place, we determine the weight of a given volume of the substance, and, secondly, we determine, directly or indirectly, the weight of an equal volume of water at a temperature of $4^{\circ} \mathrm{C}$. Both the 
weights will require the application of certain corrections, and as these are the same whatever the method employed, it will be convenient to consider these corrections once for all before proceeding to give detailed instructions for performing the measurements.

Let $w$ be the apparent, i.e. not corrected for buoyancy of the air, weight of the body in air, or, if we are determining the density of a liquid by weighing a solid sinker in it, the apparent loss of weight of the sinker as it is transferred from air to the liquid. Let $w^{\prime}$ be the uncorrected weight of a volume of water at temperature $t$ and density $\Delta$ equal to the volume of the body. Lastly, let $\sigma$ be the density of the air at the time of the observations, which we may in general take as being equal to 0.0012 .

In the first place, we may note that as we shall throughout be dealing with ratios of weights it will be unnecessary to correct for the air displaced by the weights if we use weights of the same density throughout (see p. 78).

If the volume of the body is called $v$, then the weight of air it displaces is $v \sigma$, and thus its weight in vacuo will be $w+v \sigma$. If, however, the substance is a fluid, and we obtain the weight of a given volume $v$ by weighing a sinker of this volume first in air and then in the fluid, the apparent weight of the sinker in air will be the true weight less $v \sigma$, and its apparent weight in the fluid will be its true weight less the true weight of the volume $v$ of the fluid. Hence the loss of weight as the sinker is moved from air to the fluid will be the true weight of the volume $v$ of the fluid less the weight of air it displaced; that is,

Loss of weight of sinker = true weight of sinker $-v \sigma$

- true weight of sinker - true weight of $v$ c.c. of liquid\}.

Thus the true weight of the volume $v$ of the fluid will be the loss of weight plus $v \sigma$. Therefore, whatever the method employed for determining the weight of the body being measured, we have that the true weight is $w+v \sigma$.

Next we have to consider the weight of an equal volume of water at $4^{\circ} \mathrm{C}$. Suppose, in the first place, that we actually weigh the volume $v$ of water in the air, as when we use a specific gravity bottle. Then the true weight of the water will be $w^{\prime}+v \sigma$. Next, if we obtain the weight of the water by wcighing the body being measured in the water by the method of Archimedes, then, as explained above, the observed loss of weight will be less than the weight of water displaced by the amount $v \sigma$. Hence in this case also the weight of the water of density $\Delta$, which occupies a volume equal to that of the body, is $w^{\prime}+v \sigma$. Thirdly, if we have weighed a sinker, first, in the given fluid, and, secondly, in water, we have as before to add to the loss the quantity $v \sigma$ to get the true weight of the water which occupies a volume equal to that of the sinker, and hence equal to the volume considered of the given fluid.

Now, what we require is not the weight of the volume $v$ of water at a temperature $t$, but the weight of the same volume of water at $4^{\circ} \mathrm{C}$. 
Now at $4^{\circ} \mathrm{C}$. the density of the water is 1 , while at $t$ its density is $\Delta$. Hence to obtain the weight of water which will fill any given volume, we have to divide the weight of water at a temperature $t$, which will fill the same volume, by $\Delta$.

Thus whatever the method used for measuring the density, the weight of water at $4^{\circ} \mathrm{C}$. which would occupy the same volume as the body is given by $\left(w^{\prime}+v \sigma\right) / \Delta$.

But we have seen that the true weight of the body is $v+v \sigma$. Hence the density is given by

$$
D=\frac{w+v \sigma}{v v^{\prime}+v \sigma} \Delta
$$

We have now to find an expression for $v$ in terms of the measured quantities.

The volume $v$ is equal to the weight of water, $w^{\prime}+v \sigma$, which occupies the volume $v$, divided by the density of the water $\Delta$. Hence

or

$$
\begin{aligned}
& v=\frac{w^{\prime}+v \sigma}{\Delta}, \\
& v=\frac{w^{\prime}}{\Delta-\sigma} .
\end{aligned}
$$

Substituting this value for $v$, we get

$$
\begin{aligned}
D & =\frac{w+\frac{w^{\prime} \sigma}{\Delta-\sigma}}{w^{\prime}\left(1+\frac{\sigma}{\Delta-\sigma}\right)} \Delta \\
& =\frac{w+\frac{w^{\prime} \sigma}{\Delta-\sigma}\left(1-\frac{\sigma}{\Delta-\sigma}+\text { terms in higher powers of } \sigma /(\Delta-\sigma)\right) \Delta .}{w^{\prime}} .
\end{aligned}
$$

Since $\sigma$ is small compared to $\Delta$, we may neglect squares and higher powers of $\frac{\sigma}{\Delta-\sigma}$. Hence

$$
D=\frac{w}{w^{\prime}} \Delta+\frac{\sigma \Delta}{\Delta-\sigma}-\frac{w}{w^{\prime}} \frac{\sigma \Delta}{\Delta-\sigma}+.
$$

Now the two last terms on the right are both small compared to the first term, so that we may in these small terms neglect $\sigma$ compared to $\Delta$, so that the expression reduces to

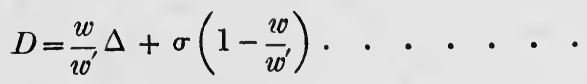

If $D^{\prime}$ is the density, uncorrected for buoyancy, that is, $D^{\prime}=\frac{w}{w^{\prime}} \Delta$, then since $\Delta$ is always nearly unity, we may substitute $D^{\prime}$ for $w / w^{\prime}$ in the buoyancy correction term. Hence

$$
D=D^{\prime}+\sigma\left(1-D^{\prime}\right) \text {. . . . . . }
$$


When correcting observations of density by this expression, it will generally be quite sufficient to take the mean value 0.0012 for the density of the air. Thus $\sigma$ being constant, we may construct a table of the values of the correction $\sigma\left\{1-\frac{w}{w^{\prime}}\right\}$ for different values of $w / w^{\prime}$, that is, of the uncorrected density. Such a table is given in the Appendix (Table 6). It will be seen from the table, as is also at once evident from the formula, that the correction vanishes when the density of the body is unity. For bodies of density less than unity the correction is positive, while for densities greater than unity it is negative.

31. Measurement of the Density of a Solid heavier than Water by the Method of Archimedes.-Suppose we have to measure the density of a solid body which is not soluble in water and has a density.

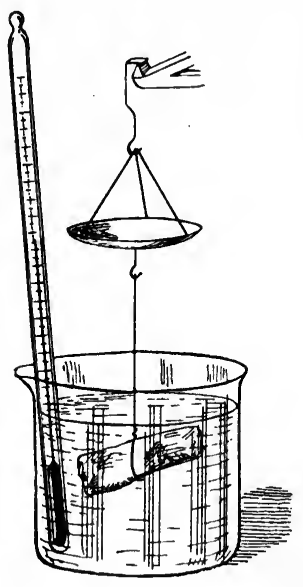

(a)

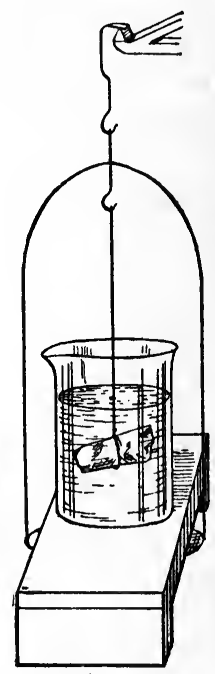

(b)

Fig. 30. greater than unity, such as a piece of quartz or glass. The operations to be performed is to determine the weight of the body, first in air and, secondly, when suspended so that it is totally immersed in water at a known temperature. In order to be able to place a vessel containing the water so that the body when suspended from the arm of the balance may hang in the water, either a short pan support, as shown in Fig. $30(a)$, may be used, or we may use a small wooden stand to bridge over the pan, as shown in Fig. $30(b)$. Generally this latter arrangement will be found the most convenient. When being weighed in water the body will require to be suspended in some manner. The usual arrangement is to hang it by means of a fine fibre of unspun silk or a fine platinum wire. Cotton must on no account be used, since the water would be sucked up along the cotton fibres, and would thus make the weight when weighing in water appear greater than it should be. Of course with this arrangement what is really measured is the mean density of the body and a certain amount of the silk fibre, so that it is essential that the fibre should be as fine as possible. When the utmost accuracy is desired, and whenever a large number of measurements of the density of solids have to be made, it is much preferable to use the arrangement shown in Fig. 31. A light metal pan, preferably of platinum, is suspended 
by a fine platinum wire as shown, and throughout the measurements remains immersed in the water. When determining the weight in air the body is placed in the upper pan, and when measuring the weight in water in the lower. Care must be taken always to bring the pointer of the balance to the same division of the scale and to keep the level of the water constant, so that the same amount of the suspension wire is immersed in the water, when the loss of weight on the body being transferred to the lower pan will be due alone to the loss of weight in the body when transferred from air to water.

Owing to the effects of capillarity at the point where the suspension wire cuts. the surface of the water, the sensitiveness and accuracy of a balance is appreciably less when used to weigh a body in water. Hence it is advisable, whenever possible, to use a fairsized piece of the material which is being tested.

Kohlrausch ${ }^{1}$ has shown that the effect of capillarity in producing irregularities,

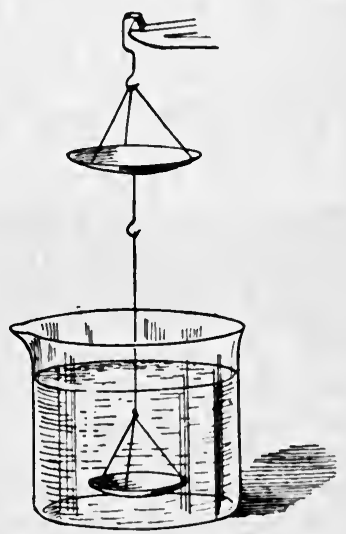

FIG. 31. where a platinum suspension fibre cuts the surface of the water, can be very much reduced if the wire is treated in the following manner:-The wire is used as the negative electrode in an electrolytic cell containing a dilute solution of platinic chloride ( 1 part platinic chloride, 30. parts water, and 0.008 part lead acetate, i.e. a trace), and an E.M.F. of about 4 volts is applied. In this way, after washing and drying, a black mat surface is obtained. The wire is then heated to redness in a small Bunsen flame, so that the surface turns grey. Care must be taken not to dirty the portion of the wire which will cut the surface of the water, even by touching it with a clean finger.

Take a piece of glass rod weighing about 10 grams, and determine its weight in air. Then tie a silk fibre round the middle and suspend it from the support which carries the pan, and again weigh. The difference in the weights will give the weight of the fibre. Next take a beaker and nearly fill it with distilled water, and, having well stirred the water, place the beaker on a stand so that the glass is immersed in the water and does not touch the sides of the beaker. Also place a thermometer in the water. Examine the glass very carefully to see whether there are any air bubbles adhering. If so, these must be removed. This can best be done by means of a small camel's hair pencil. Next determine the weight of the glass as it hangs in the water, and then read the thermometer.

In very accurate measurements it is necessary to take account of the effect of the suspension fibre. To do this, estimate what proportion 
of its total length is immersed in the water, and then from the weight of the whole fibre and its density (platinum is generally used in such delicate measurements, density $21 \cdot 5$ ) calculate the volume of the fibre which was immersed in the water. The weight of this volume of water must then be subtracted from the total weight of the fibre, and the difference subtracted from the weight of the substance in water.

If the weight of the body in air is $w$, and its loss of weight in water is $w^{\prime}$, the density of the water at the temperature of observation as obtained from Table 5 being $\Delta$, and the density of the air at the time of observation $\sigma$, then the density of the body $D$ is given (see p. 81) by

$$
D=\frac{w}{w^{\prime}} \Delta+\sigma\left\{1-\frac{w}{w^{\prime}}\right\}
$$

32. Measurement of the Density of a Solid lighter than Water.If the density of the solid is less than unity, it will float in water. Hence to determine the weight of water it will displace we have to attach a weight to act as a sinker. A piece of lead or copper, the weight of which need only be just sufficient to sink the body, must be weighed in water, and let the weight be $s$. Next weigh the body in air, and let its weight be $w$. The sinker must be attached to the body and the weight of the two determined in water, and let the weight be $x$. Then the weight of the body alone in water will be given by

$$
x-s \text {. }
$$

Thus the apparent weight $w^{\prime}$ of the water displaced by the body is given by

$$
w^{\prime}=w-x+s,
$$

and this value substituted in the expression given at the end of the last section will give the density of the body.

If a number of determinations of density of bodies lighter than water have to be made, it will be worth while to make a small cylindrical cage of wire gauze, as shown in Fig. 32, and to suspend this by

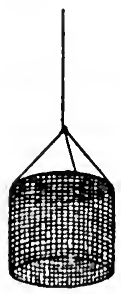
a fine wire so that it is immersed in water. The weight $s$ necessary to counterpoise the cage when immersed in water is first determined. Then when measuring the density of the body it is first weighed when placed in the scale pan, the cage being immersed in water, and the difference between the weight and $s$ gives the weight of the body in air. The body is then transferred to the cage and the weight determined, which has to be added to the right-hand pan to produce balance. The difference between the weights Fia. 32. on the right-hand pan when the body is in the pan and in the cage gives the apparent weight of the water displaced, $w^{\prime}$.

Bodies which are soluble in water, or on which water acts, must be weighed in some fluid in which they are unacted upon. The density of this fluid must be measured by one of the methods given later if it 
is not already known. The quantity $\Delta$ in the formula in this case must be taken as representing the density of the liquid employed.

33. Measurement of the Density of a Solid in the form of Small Pieces-The Pyknometer.-If the solid, the density of which is to be determined, is in the form of small pieces, the previous methods are inapplicable, and we must use a specific gravity bottle or pyknometer. Two forms of specific gravity bottle' are shown in Fig. 33. The bottle figured at $(a)$ is fitted with a carefully ground-in stopper, which stopper is pierced with a fine hole. The bottle is always filled to the top, and then the stopper is replaced, and the excess water which escapes through the hole in the stopper is removed by wiping the bottle with a clean duster or filter paper. The bottle shown at (b) has a fine mark etched round the neck, and in use is always filled up to this mark.

First weigh the bottle empty and carefully dried. Then fill the bottle full of water which has been boiled for a short time to get rid of dissolved air and has then been

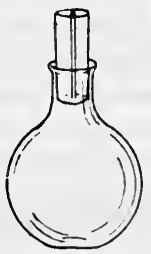

(a)

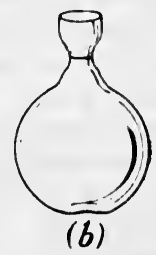

Fig. 33. allowed to cool. The bottle must then be stood in a beaker of water which is at the temperature of the room or a degree or two hotter, and must remain there for at least twenty minutes, the water being kept well stirred the while. A thermometer placed in the water is read just before the bottle is removed, and this will give the temperature of the water. In the case of the bottle with the mark on the neck, the quantity of water in the bottle must be adjusted to the mark before the bottle is removed from the water. For this purpose a piece of glass tube, the end of which is drawn out into a fine capillary, or a small twist of filter paper, may be employed. After carefully drying the outside, the bottle must be again weighed. If the pattern of bottle with the mark on the neck is employed, it will be found best to fill it at a temperature of $0^{\circ} \mathrm{C}$. For this purpose, first fill it with water at the temperature of the room to a little above the mark, and then place the bottle in a mixture of pounded ice and distilled water so that the mark is just flush with the upper surface of the ice, and allow it to stand for about half-an-hour. Having allowed the water in the bottle to acquire the temperature of the ice, adjust the surface of the water to the mark on the neck.

From the difference between the weight of the bottle empty and full of water the volume of the bottle can be calculated.

The bottle having been emptied and dried is now about three parts filled with the solid, say, sand, and then weighed. The difference between this weight and the weight of the bottle empty is the apparent weight of the sand.

The bottle has now to be filled up to the mark with water. This operation is often one of considerable difficulty, owing to the way in 
which air bubbles cling to a finely divided solid. If an air-pump is available the bottle may be nearly filled up to the mark, or at any rate well above the surface of the sand, and then placed beneath the receiver of the pump. On working the pump the air will be extracted from between the grains of sand. In the absence of a pump the bottle, after partly filling with water, must be heated till the water just boils. In this way the air will be expelled. After the expulsion of the air the bottle must be filled a little above the mark, and then by immersion in water or ice, as the case may be, brought to a constant temperature, which must be noted, and then, after drying outside, be again weighed.

In the first place, let us suppose that the temperature at which the bottle has been weighed partly filled with the sand and partly filled with the water is the same as the temperature at which the bottle was filled entirely with water, so that as far as the expansion of the bottle is concerned the volume was the same in the two cases. If $\Delta$ is the density of water at this temperature and the following are the results of the weighings -

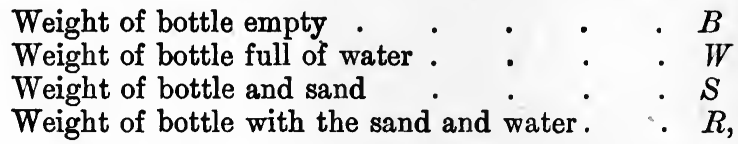

then the apparent weight of the sand is $S-B$. The apparent weight of the water, which occupies the same volume as the sand, is given by

$$
w^{\circ}=W+S-B-R \text {. }
$$

Hence the density, uncorrected for the reduction of the weighings to vacuo, is given by

$$
D^{\prime}=\frac{S-B}{W+\frac{S-B-R}{S}} \Delta .
$$

Correction (1).- - To reduce the weighings to vacuo we must add the term $\sigma\left(1-D^{\prime}\right)$, so that if $D$ is the corrected density,

$$
D=D^{\prime}+\sigma\left(1-D^{\prime}\right) \text {. }
$$

The value of the correction is at once obtained from Table 6 .

Correction (2).- If the temperature at which the bottle is filled with water when it contains the sand is not the same as that at which it was filled with water when its volume was being determined, a correction will have to be applied to allow for the fact that, owing to the expansion of the glass, the volume changes with temperature. To calculate the value of this correction we must know the value of the coefficient of cubical expansion of the glass of which the bottle is made. The manner in which this quantity may be determined is described in $\S 73$, but as the measurements of density made with the spccific gravity bottle are always made at temperatures not far removed from the temperature at which the bottle was gauged, it is generally sufficient to take the value 0.000023 for the coefficient of cubical expansion of glass. 
Suppose that when the bottle is filled with water the temperature is $t_{0}$ and that the density of water at this temperature is $\Delta_{0}$, while when the bottle contains water and sand the temperature is $t_{1}$ and the density of the water is $\Delta_{1}$. The volume of the bottle at $t_{1}$ is $(W-B) / \Delta_{0}$, and hence if $\gamma$ is the coefficient of cubical expansion of the glass, the volume at $t_{1}$ is

$$
\frac{W-B}{\Delta_{0}}\left\{1+\gamma\left(t_{1}-t_{0}\right)\right\} \text {. }
$$

Thus the weight of water which would fill the bottle at the temperature $t_{1}$ is given by

$$
(W-B)\left\{1+\gamma\left(t_{1}-t_{0}\right)\right\}{\stackrel{\Delta}{\Delta_{0}}}_{0}
$$

The actual weight of water contained in the bottle, in addition to the sand, is $R-S$. Hence the weight of the water displaced by sand is

$$
(W-B)\left\{1+\gamma\left(t_{1}-t_{0}\right)\right\} \frac{\Delta_{1}}{\Delta_{0}}-R+S .
$$

This expression may be simplified by writing $1+\left(\Delta_{1}-1\right)$ for $\Delta_{1}$ and $1+\left(\Delta_{0}-1\right)$ for $\Delta_{0}$ and dividing, when, since $\Delta_{1}$ and $\Delta_{0}$ are each very nearly equal to unity, we get

$$
(W-B)\left\{1+\gamma\left(t_{1}-t_{0}\right)\right\}\left\{1+\left(\Delta_{1}-\Delta_{0}\right)\right\}-R+S .
$$

Also since $\gamma$ and $\left(\Delta_{1}-\Delta_{0}\right)$ are each small, the expression reduces to

$$
(W-B)\left\{1+\gamma\left(t_{1}-t_{0}\right)+\Delta_{1}-\Delta_{0}\right\}-R+S .
$$

The above expression is the value of $w^{\prime}$ corrected for the temperature, and it must now be substituted in the expression

$$
D=\frac{S-B}{w^{\prime}} \Delta_{1} .
$$

To obtain the density reduced to vacuo the correction $\sigma(1-D)$ must be added.

34. Measurement of the Density of a Liquid with a Sinker.-If the liquid is not too volatile and can be procured in sufficient quantity, one of the most convenient ways of measuring its density is to determine the weight of a suitable sinker when suspended, first, in water at a known temperature, and, secondly, in the liquid. A convenient form of sinker is shown in Fig. 34, and consists of a glass bulb partly filled with shot so that it will sink in the densest liquid likely to be measured. It is made by blowing a bulb on the end of a piece of glass tubing, introducing the shot, and then drawing off the neck and at the same time forming a small hook by means of which the bulb is attached to the suspension wire.

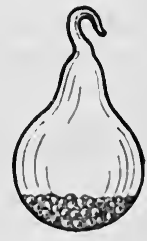

FIG. 34.

Suspend the bulb from the left-hand arm of a balance by means of a silk fibre or fine wire, and determine the weight of the bulb in air 
Next weigh the bulb when immersed, first in water and, secondly, in the given liquid. The temperature of the water and of the liquid must' be noted in each case, and care must be taken to immerse the same length of the suspension fibre each time. This can be secured if the level of the upper surface of the liquid is always brought to the same height above the surface of the balance case. An allowance will have to be made for the weight of that portion of the suspension fibre above the surface of the liquid. This can most conveniently be done by placing a piece of the same kind of fibre or wire equal in length to that of the portion of the suspension fibre which is above the surface of the liquid in the right-hand pan of the balance and keeping it there throughout the weighings.

Let the weight of the sinker in air be $B$, and its weight in water, of which the temperature is $t_{0}$ and the density $\Delta_{0}$, be $W$, while its weight in the liquid is $S$, and first we will suppose that the temperature of the liquid is also $t_{0}$. Then the apparent volume of the sinker is $(B-W) / \Delta_{0}$, and the apparent weight of this volume of the liquid is $B-S$. Hence the density, without reduction to vacuo, is given by

$$
D^{\prime}=\frac{B-S}{B-W} \Delta_{0}
$$

Correction (1).-Reduced to vacuo the correction $\sigma\left(1-D^{\prime}\right)$ has to be added to the uncorrected value given above.

Correction (2). - If the temperature of the liquid is not the same as that of the water, but is, say, $t_{1}$, then a correction must be applied for the expansion of the sinker with increase of temperature. Let $\gamma$ be the coefficient of cubical expansion of the material of which the sinker is made (it will in the case of a glass sinker generally be sufficiently accurate to take the value 0.000023 for $\gamma$ ). Then the volume of water at $t_{0}$ displaced by the sinker is $(B-W) / \Delta_{0}$. The volume of the sinker at a temperature $t_{1}$ is therefore

$$
\frac{(B-W)\left\{1+\gamma\left(t_{1}-t_{0}\right)\right\}}{\Delta_{0}} .
$$

Hence correcting for change in temperature we get

since $\gamma$ is small.

$$
\begin{aligned}
D & =\frac{(B-S) \Delta_{0}}{(B-W)\left\{1+\gamma\left(t_{1}-t_{0}\right)\right\}} \\
& =\frac{B-S}{B-W} \Delta_{0}\left\{1-\gamma\left(t_{1}-t_{0}\right)\right\}
\end{aligned}
$$

The correction to reduce to vacuo is as before, $\sigma(1-D)$.

Kohlrausch has pointed out that when using a glass float to determine the density of water solutions it is advantageous to observe at a temperature of between $5^{\circ}$ and $7^{\circ}$, for over this range of temperature the coefficient of expansion of water is practically the same as that of glass. 
If a silver float is employed, the temperature ought to be somewhere between $7^{\circ}$ and $10^{\circ}$.

35. Measurement of the Density of a Liquid with Specific Gravity Bottle.-When only a comparatively small quantity of liquid is available, the specific gravity bottle may be used for measuring the specific gravity of a liquid. Either of the forms of bottle shown in Fig. 33 may be used, but on the whole the form with a mark on a narrow neck is to be preferred to that which has a removable stopper. If the liquid is volatile, then it will be necessary to employ some means of closing the top. For this purpose either a glass stopper may be ground into the

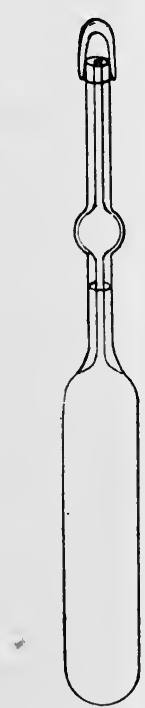

(a)

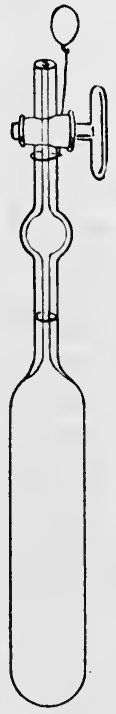

(b)

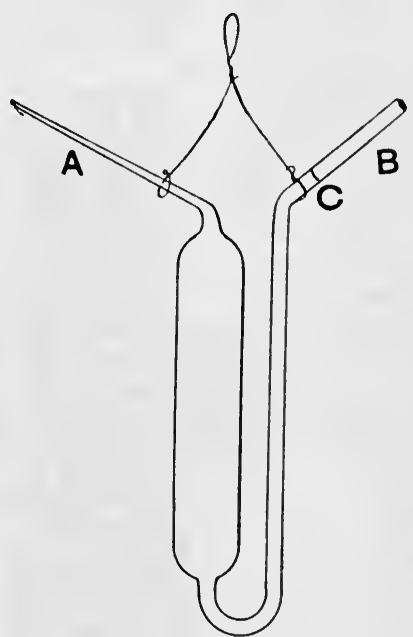

(c)

Fig. 35.

top of a bottle of the form shown in Fig. $35(a)$, or a bottle of the form shown at $(b)$, with a tap above the mark on the neck, may be employed. As no grease must be used on the stopper or tap, care must be taken not to force these in too tightly or they will stick, and great difficulty may be experienced in removing them.

The method of measuring the density of a liquid with the specific gravity bottle consists in first measuring the volume of the bottle by weighing the water which it will contain. If, then, the weight of any other liquid which fills it up to the mark is determined, we can immediately calculate the density of the liquid.

When the liquid to be experimented upon is valuable, as, for instance, a sample of some specially purified chenical compound, the filling of the 
bottle in such a way that there shall be no chance of the liquid being lost or contaminated is of great importance. A piece of apparatus which may be used in such a case is shown in Fig. 36. It consists of two glass vessels $A B$, one of which $A$ is fixed to an upright board, and the other $B$ is movable, these being connected by a length of rubber tube c. The vessel $\mathbf{B}$ can be placed in two positions, a loop attached to the top being

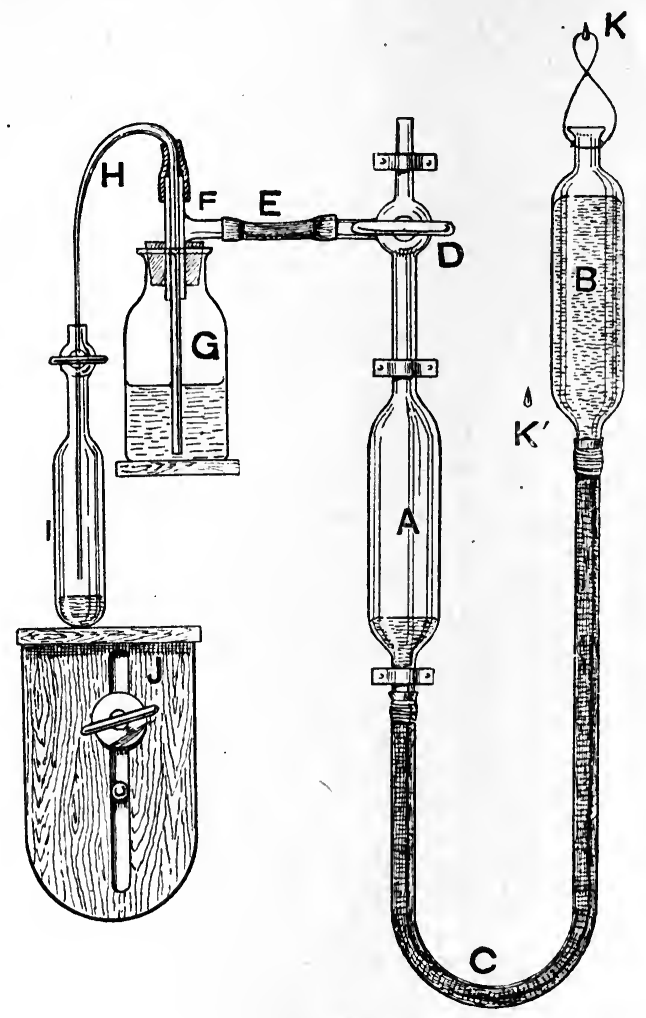

Fig. 36.

hooked on either of the screws $\mathbf{K}$ or $\mathbf{K}^{\prime}$. The vessel A communicates, by means of a three-way tap, with the open air or a side tube $E$. This side tube is connected by rubber tubing with the side tube of a small glass T-piece F. The lower arm of the $T$-piece passes through a cork which fits into the neck of the bottle $a$, in which the liquid to be- used is stored. Through the vertical arm of the T-piece passes a capillary tube B bent into a $U$, a small length of rubber tubing being employed to make an air-tight joint. The other leg of the capillary passes down into 
the pyknometer $\mathrm{I}$, which is supported on a shelf $\mathrm{J}$, the height of which is adjustable.

The vessels $A$ and $B$ being partly filled with dry mercury, when the vessel $\mathbf{B}$ is hung on the peg $\mathbf{K}$ the air in $\mathbf{A}$ is compressed, and by suitably turning the tap $D$ the pressure is communicated to the bottle $\mathrm{G}$, forcing the liquid over into the pyknometer. To withdraw the liquid from the pyknometer the vessel $\mathbf{A}$ is made to communicate with the open air, B is raised till $A$ is very nearly full of mercury, and then the tap $D$ is closed. B is then lowered, and the tap turned so as to put $A$ into communication with the bottle $\mathrm{G}$. In this way a reduction of pressure is produced in the bottle $G$, and hence the liquid in the pyknometer is forced over by the atmospheric pressure.

A form of pyknometer which can easily be filled, and has the further advantage that it can easily be cleaned and dried, is shown at (c) in Fig. 35. The capillary $\mathrm{A}$ being dipped below the surface of the liquid, the liquid is drawn up into the bulb by applying a gentle suction to $B$ through a piece of rubber tubing. The bottle is filled till the surface of the liquid is above the mark c. The bottle is then immersed in ice so that the ends of the capillaries project above the ice, and when the temperature of the liquid has fallen to zero the instrument is tilted so that the liquid flows to the end of the capillary $\Delta$. Then by holding a piece of filter paper against A, so much liquid is removed that the surface of the liquid in the capillary B coincides with the mark c. When a volatile liquid is being used, both capillaries must be closed by caps.

When great accuracy is desirable, it is almost essential that the bottle should be filled with water and with the liquid at a temperature of $0^{\circ} \mathrm{C}$. Even when a comparatively rough result is aimed at, yet, since the temperature at which the filling takes place will have to be determined, it will generally be found to save time to work at the temperature of melting ice, since this temperature is so easily maintained and reproduced. The bottle must be filled slightly above the mark, and then placed in a mixture of pounded ice and distilled water so that the mark is on a level with the top surface of the ice. After the bottle has been in the ice for between ten and fifteen minutes the surface of the liquid must be adjusted to the mark by means of a pipette, made by drawing a piece of glass tube down to a fine capillary, or with filter paper if the bottle is of the double capillary pattern. The outside having been carefully dried, the bottle is then weighed.

To remove the water from a bottle with a single neck the instrument shown in Fig. 36 may be employed, or holding the bottle upside down, blow air into it through a capillary glass tube attached to a piece of india-rubber tubing. Having removed as much as possible of the water, the bottle must be rinsed out twice with a small quantity of absolute alcohol and then once with ether. To remove the ether, blow air through a capillary into the bottle by means of a foot blow-pipe. The mouth must on no account be used to blow air through the bottle, since the water vapour in the breath would condense in the bottle. If no 
absolute alcohol is available for washing out the bottle, it may be dried by heating over a flame and blowing a current of air through it with a bellows. This will have to be continued for some time, and the heating must be very thorough, care being taken not to crack the bottle by too sudden heating.

Next fill the bottle with the liquid to be measured, using the same precautions as in the case of the water, and again weigh.

If, as directed above, the bottle has been filled in both cases at the same temperature, namely, that of melting ice, its volume will be the same when filled with the liquid and with water, and no correction for the expansion of the glass will be necessary. If the weight of the empty bottle is $B$, that of the bottle when full of water $W$, and when full of the liquid $S$, the value of the density, not reduced to vacuo, is

$$
D=\frac{S-B}{W-B} \Delta_{0}
$$

where $\Delta_{0}$ is the density of water at $0^{\circ}$.

Correction (1).-To reduce to vacuo, the correction $\sigma(1-D)$ must be added.

Correction (2).-When the temperature at which the bottle is filled with the liquid is different from that at which it was filled with water, a correction has to be applied for the expansion of the bottle with rise of temperature. If $t_{0}$ and $\Delta_{0}$ are the temperature and density of the water, and $t_{1}$ is the temperature of the liquid, while the coefficient of expansion of the glass of which the bottle is made is $\gamma$, then the volume of the bottle at $t_{0}$ is given by $\frac{W-B}{\Delta_{0}}$.

Hence the volume of the bottle at $t_{1}$ is

$$
\frac{W-B}{\Delta_{0}}\left\{1+\gamma\left(t_{1}-t_{0}\right)\right\} \text {. }
$$

Thus the density of the liquid is given by

$$
\begin{aligned}
D & =\frac{S-B}{W-B} \Delta_{0} \frac{1}{1+\gamma\left(t_{1}-t_{0}\right)} \\
& =\frac{S-B}{W-B} \Delta_{0}\left\{1-\gamma\left(t_{1}-t_{0}\right)\right\}_{,}
\end{aligned}
$$

and to allow for buoyancy a further correction of $\sigma(1-D)$ must be added. 


\section{CHAPTER V}

\section{ELASTICITY OF SOLIDS}

36. Young's Modulus by Stretching a Wire.-If a wire of length $L$ and radius $r$ becomes elongated by an amount $e$ when a tension $F$ is applied, then since

$$
\text { Young's modulus }=\frac{\text { stress }}{\text { strain }}
$$

and the stress is $F /$ (area of cross-section) and the strain is the elongation per unit length, we have

$$
Y=\frac{F L}{\pi r^{2} e}
$$

A simple piece of apparatus for measuring the elongation of a wire under the influence of a stretching force is shown in Fig. 37. It consists of a scale $\Delta$ divided into half millimetres, which is attached to the lower end of a wire of the material to be tested. A second wire of the same material carries the vernier $B$, which is capable of sliding along the edge of the scale with very little friction. The upper ends of the two wires are soldered or clamped to the same support immediately alongside one another, so that if, owing to the load applied to one wire, the support bends, the point of support of both wires will drop to exactly the same amount, and hence the relative positions of the vernier and scale will not alter. This method of supporting the scale also eliminates any correction for changes in temperature affecting the lengths of the wire being tested; for as the wires are of the same material and are suspended close together they will have the same temperature, and so whenever one expands the

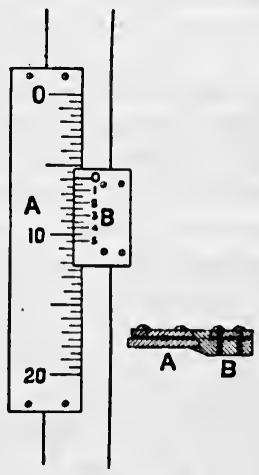

FIG. 37. other will expand to an equal extent, so that the reading on the scale is unaltered on this account.

The wire which carries the scale has a weight attached to its lower end, which serves to keep the wire taut and free from kinks. The other wire carries a scale pan, so that by placing weights in this pan the wire can be stretched.

First ascertain the breaking weight of the wire. To obtain this 93 
quantity measure the diameter of the wire and calculate the area of crosssection in square centimetres. Then multiply this area by the breaking stress for the substance given in the following table. This will give the breaking weight with sufficient accuracy for the present purpose. Having ascertained the breaking weight, care must be taken never to load the wire to more than half this amount :-

Breaking STtrhss.

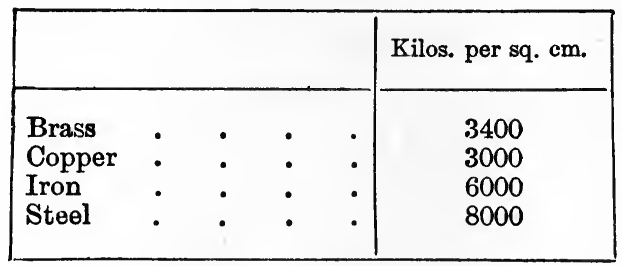

Place the maximum load permissible in the scale pan and allow the wire to remain stretched for some minutes, and then remove the greater part of the weight, leaving, however, enough to keep the wire taut and free from kinks. Read the position of the vernier, and then increase the weight in the pan by equal amounts so chosen that ten readings will be obtained before the maximum load is reached. Repeat the readings as the weights are removed. The readings obtained while putting on and taking off the weights ought to agree within a fifth of a millimetre. If they do not, either the wire was slightly kinked, and as the weights were added the kinks straightened out, or the maximum load was too great, so that permanent elongation has been produced.

The readings must be entered in a table, as in the following example, and the mean elongation worked out by subtracting the first reading from the sixth, the second from the seventh, and so on, and then taking the mean of these differences :-

\begin{tabular}{|c|c|c|c|c|}
\hline \multirow{2}{*}{ Load in Pan. } & \multicolumn{2}{|c|}{ Vernier Reading. } & \multirow{2}{*}{ Mean. } & \multirow{2}{*}{$\begin{array}{l}\text { Elongation } \\
\text { for } 5 \text { kilos. }\end{array}$} \\
\hline & Load Increasing. & Load Decreasing. & & \\
\hline $\begin{array}{l}3 \text { kilos. } \\
4 \\
5 \\
6 \\
7 \\
8 \\
9 \\
10 \\
11 \\
12\end{array}$ & $\begin{array}{l}4 \cdot 65 \mathrm{~mm} . \\
6 \cdot 00 \\
7 \cdot 30 \\
8 \cdot 65 \\
10 \cdot 00 \\
11 \cdot 35 \\
12 \cdot 75 \\
14 \cdot 05 \\
15 \cdot 40 \\
16 \cdot 75\end{array}$ & $\begin{array}{r}4 \cdot 70 \mathrm{~mm} . \\
6 \cdot 05 \\
7 \cdot 35 \\
8 \cdot 70 \\
10 \cdot 00 \\
11 \cdot 40 \\
12 \cdot 75 \\
14 \cdot 10 \\
15 \cdot 45 \\
16 \cdot 80\end{array}$ & \begin{tabular}{|l|}
$4 \cdot 67 \mathrm{~mm}$. \\
$6 \cdot 02$ \\
$7 \cdot 32$ \\
$8 \cdot 67$ \\
$10 \cdot 00$ \\
$11 \cdot 37$ \\
$12 \cdot 75$ \\
$14 \cdot 07$ \\
$15 \cdot 42$ \\
$16 \cdot 77$ \\
\end{tabular} & $\begin{array}{l}\ldots \\
\ldots \\
\ldots \\
\ldots \\
6 . \\
6.70 \mathrm{~mm} \\
6 \cdot 73 \\
6.75 \\
6.75 \\
6 \cdot 77 \\
\end{array}$ \\
\hline 12 & & & Mean . & $6 \cdot 74 \mathrm{~mm}$ \\
\hline
\end{tabular}


Elongation for 1 kilo (981000 dynes) $=\cdot 1348 \mathrm{~cm}$.

$\begin{aligned} & \text { Mean diameter : : }: \quad: \quad=06224 \mathrm{~cm} . \\ & \text { Length . : }=837.5 \mathrm{~cm} .\end{aligned}$

$$
\begin{aligned}
\text { Young's modulus } & =\frac{981000 \times 837.5}{\pi \times \cdot 1348 \times(\cdot 03112)^{2}} \\
& =2.003 \times 10^{12} \text { dynes per sq. } \mathrm{cm} .
\end{aligned}
$$

Measure the diameter of the wire by means of a micrometer screw gauge. The diameter must be measured at a number of points along the length of the wire, and at each point a measurement must be made along two diameters of the wire at right angles to one another. In this way errors due to want of uniformity in the wire may be reduced to a minimum. To obtain the length of the wire a steel tape may be suspended from the support which carries the upper ends of the wires, and the readings taken corresponding to the point of attachment of the wires and the point where the upper screw which clamps the vernier to the wire touches the wire. If a steel scale is not available the length may be measured by using a long wooden lath on which marks are made corresponding to the upper and lower ends of the wire, which length is then determined by measurement with a millimetre scale.

The accuracy which it is necessary to attain in the measurement of the total length of the wire may be calculated in the following manner :Let $L$ be the length of the wire and $r$ its radius, and the mean elongation as obtained above be e. The expression for calculating Young's modulus is

$$
Y=\frac{F L}{\pi r^{2} e},
$$

and we wish to arrange that each of the factors which enter into this expression, namely, $F, L, r^{2}$, and $e$, are measured with the same percentage accuracy. If the vernier reads to $1 / 20 \mathrm{~mm}$., the percentage accuracy to be expected of a single reading of the elongation is about $\cdot 5 / e$, since $e$ is supposed to be given in centimetres. If the screw gauge reads to $001 \mathrm{~cm}$., the percentage accuracy of a single setting is about $0 \cdot 1 / 2 r$, which corresponds to a percentage accuracy of $0 \cdot 1 / r$ in $r^{2 .}$. Since a number of measures of the above two quantities are made, we may in general suppose that the means are correct to about a fifth of the number given above, so in calculating the accuracy with which we have to know $L$ we may suppose that we know $e$ to $1 / e$ per cent., and $r^{2}$ to $02 / r$ per cent. Then if $\delta L$ is the magnitude of the error we can allow in measuring $L$ we have to arrange so that

$$
\delta L=100 \text { is not greater than } \cdot 1 / e \text { or } \cdot 02 / r \text {. }
$$

In the same way, if $\delta W$ is the error of the weights $W$ employed, 
$100 \delta W / W$ must not be greater than $1 / e$ or $\cdot 02 / r$. In the example given above, $e=\cdot 13 \mathrm{~cm}$., $r=\cdot 031, L=837 \mathrm{~cm}$., $W=1000$ grams. Hence

and

$$
\frac{1}{e}=\frac{\cdot 1}{\cdot 13}=\cdot 76 \text { per cent., }
$$

$$
\frac{\cdot 02}{r}=\frac{\cdot 02}{.031}=\cdot 64 \text { per cent. }
$$

Hence we see that the error to be expected owing to our method of measuring $r$ is slightly greater than that due to $e$. Further, $\frac{100 \delta L}{L}$ must not be greater than 6 , and therefore $\delta L$ must not be greater than $5 \mathrm{~cm}$. Hence if we measure the total length of the wire to within a centimetre or two, it will be quite accurate enough. In the same way, if the weights are correct to 2 or 3 grams in the kilogram, they are sufficiently accurate.

The above investigation illustrates the care which has to be exercised to avoid spending a lot of time and trouble in measuring with great accuracy some one of the factors which enter into the final calculation

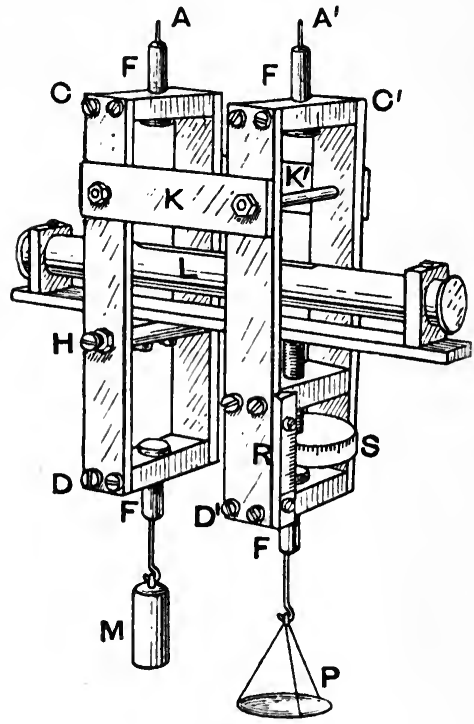

FIG. 38 . when, owing to unsurmountable difficulties in making some other measurement or want of uniformity in the quantity measured, the accuracy of the final result is almost entirely governed by the accuracy with which we can measure this latter quantity. Thus if we are able to use a wire of the length considered above, a vernier which reads to one-twentieth of a millimetre will measure the elongation with a percentage accuracy greater than that with which we are able to measure the radius of the wire. If, however, it is for some reason impossible to use such a long wire, or if measurements have to be made on wires of such a large diameter that the elongation which can conveniently be obtained with the weights at our disposal is much smaller than that considered above, then some more refined method of measuring the elongation becomes necessary. In the case of the short wire, we shall also have to measure the length with greater accuracy. This last measurement is not a matter of very great difficulty, since it is easier to measure shorter lengths than lengthe of about 8 metres. 
37. Young's Modulus by Stretching a Wire (Searle's Apparatus). A device for measuring the elongation of a short wire, designed by Mr. G. F. C. Searle, ${ }^{1}$ is shown in Fig. 38. The two wires of the same material $\mathbf{A}, \mathbf{A}^{\prime}$ are fastened at the top to the same support, and at the bottom to two metal frames $C D$, $C^{\prime} D^{\prime}$. These frames are connected together by two links $\mathrm{K}, \mathbf{K}^{\prime}$, which allow of their free relative movement in a vertical direction. One end of a sensitive level $L$ is pivoted to the frame CD, while the other end rests on the point of a micrometer screw $\mathrm{s}$. From the lower parts of the frames hang a stretching weight $\mathrm{m}$ and $\mathrm{a}$ scale pan $P$. When using the instrument, a sufficient weight having been put in the pan to keep the wire straight, the bubble of the level is brought to the centre of the scale by turning the screw $s$, and the reading on the scale $\mathbf{R}$ and divided head $\mathbf{s}$ are recorded. A weight is then added to the

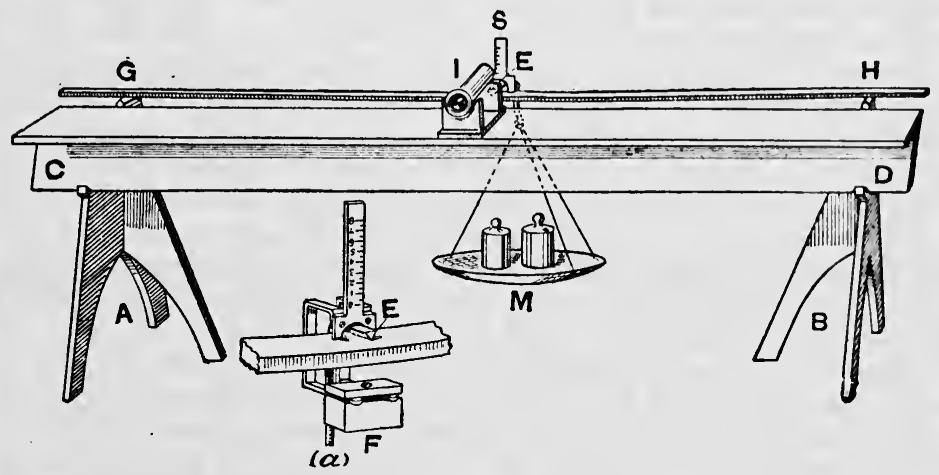

FIG. 39.

pan, and the bubble being brought back to the centre of the scale, the micrometer is again read. The difference in the micrometer readings of course gives the elongation produced by the additional load.

38. Young's Modulus by the Flexure of a Beam (1).-A convenient arrangement for measuring the flexure of a loaded beam is shown in Fig. 39. The beam rests on the upper edges of two cast iron stands $\mathbf{A}$ and B, which are filed up to a blunt knife-edge. These uprights also carry a wooden board $\mathrm{CD}$, which supports a microscope $\mathrm{M}$, used to measure the flexure. This board is not permanently attached to the uprights but simply rests on two small projections, so that the distance between the uprights, and hence the length of the beam, can be altered. The microscope has a horizontal cross-wire in the eye-piece, and is focused on a small scale $\mathrm{s}$, which rests on the beam. This scale is shown on an enlarged scale at $(a)$. It may conveniently be divided into quarter millimetres, with every tenth division numbered. The scale pan in which the

1 Proceedings of the Cambridge Philosophical Society (1900), vol. x. p. 318. 
weights are placed is attached to a hook $\mathrm{E}$, which rests on the upper surface of the beam at its mid point, passing between the feet of the scale.

With a pencil make a mark at the mid point of the beam, and then draw transverse lines at either side at distances of, say, 30, 40, and 50 centimetres from the middle. Place the uprights at a distance of $100 \mathrm{~cm}$. apart and adjust the beam so that the two marks at $50 \mathrm{~cm}$. from the middle are immediately over the knife-edges, and also place the scale and scale pan in position at the middle of the beam, taking care that the scale is vertical. Focus the microscope on the scale, and take the reading corresponding to the cross-wire of the microscope. Then gradually add weights to the scale pan, and take for each load the reading on the scale. Repeat the readings while removing the load, ${ }^{1}$ and enter the results as in the example given in $\S 36$. Repeat the measurements with the supports at 80 and $60 \mathrm{~cm}$., also with the beam turned so that what was the depth becomes the breadth, and vice versa.

Measure the depth and breadth of the bar at ten points at equal intervals along its length, and take the means. Since the depth occurs to the third power, it is important that this quantity should be measured with care. The measurement is best made with a micrometer screw gauge. If, however, the dimensions of the bar are such that the micrometer will not open sufficiently wide, a pair of vernier callipers must be used.

As the length also occurs to the third power, this will also require measuring with care. It can be measured by placing a steel metre scale divided into millimetres across the uprights, with the face which carries the divisions vertical, and reading the distance between the knife-edges.

The method to be adopted in recording and reducing the observations is the same as that used in the preceding sections.

If $e$ is the deflection of the mid point of the beam produced by a load of $P$ dynes, the length, breadth, and depth of the beam being $L, b$, and $d$ respectively, then Young's modulus $Y$ is given by

$$
Y=\frac{P L^{3}}{4 b d^{3} e}
$$

From this expression it will be seen that if $P$ is constant, the quotient $L^{3} / e$ is a constant for different lengths. Hence use the mean value of $L^{3} / e$ for the different lengths of beam when calculating the value of Young's modulus.

39. Young's Modulus by the Flexure of a Beam (2).-In place of measuring the depression at the centre of a beam supported at the ends and loaded in the middle, we may measure the inclination of the ends

1 If the readings taken when removing the load differ appreciably, it generally indicates that the beam has been loaded beyond its elastic limit, and a smaller maximum load should be tried. 
of such a beam. If an inclination $\phi$ is produced by a load $P$ in a beam of length $L$, depth $d$, and breadth $b$, then we have

$$
Y=\frac{3}{4} \frac{L^{2} P}{d^{3} b \tan \phi} \cdot \cdot . \cdot . \quad . \quad . \quad .
$$

In order to measure $\phi$ two mirrors $\mathrm{M}_{1}$ and $\mathrm{M}_{2}$ are attached to the bar beyond the knife-edges which support the bar in such a way that the scale $\mathrm{s}$ (Fig. 40) is seen in the telescope $\mathrm{T}$ after reflection in both

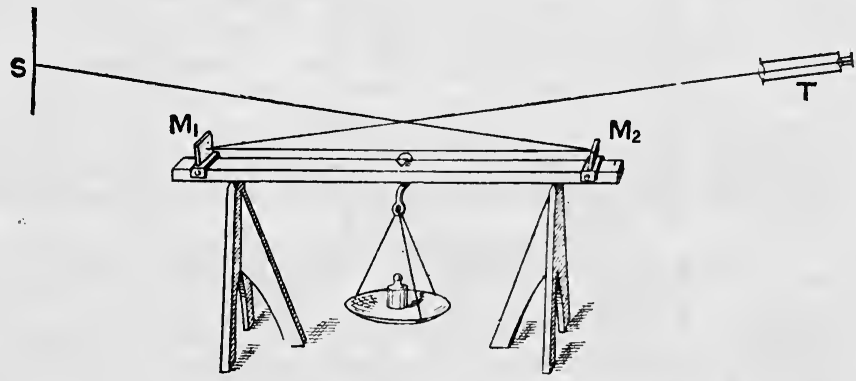

FiG. 40.

mirrors. Then if the reading on the scale corresponding to the crosswire of the telescope changes by $x \mathrm{~cm}$. when the bar is loaded and the distance between the scale and the mirror $M_{2}$ is $D$, and that between the two mirrors is $a$, we have to a first approximation, if $\phi$ is the angle through which each of the mirrors turns,

$$
\phi=\tan \phi=\frac{x}{4 D+2 a} .
$$

Hence substituting this value for $\tan \phi$ in (1) above

$$
Y=\frac{3(2 D+a) L^{2} P}{2 a^{3} b x}
$$

To obtain the expression for $\phi$ in terms of $x$, let A and B (Fig. 41) be the mirrors, $\mathbf{T}$ the telescope, and $\mathrm{C}$ the reading on the scale before the beam is bent. The path of the axial pencil of rays from $c$ to the telescope is along CВАт. Next suppose the mirror A alone rotated in the clockwise direction through an angle $\phi$, an incident ray TA would now be reflected along $\mathbf{A} \mathbf{B}^{\prime}$, the angle $\mathbf{B} \Delta \mathbf{B}^{\prime}$ being $2 \phi$. If $\alpha$ is the angle $\mathbf{T A B}$, or CBA, the ray $\mathbf{A B}$ will be incident on $\mathbf{B}$ at an angle $a / 2$, while the ray $\Delta B^{\prime}$ will be incident at an angle of $\alpha / 2-2 \phi$. If, now, the mirror $B$ be rotated through an angle $\phi$ in the anti-clockwise direction, the angle of incidence of the ray $\mathbf{A B}^{\prime}$ will be $a / 2-3 \phi$. Hence if $\mathbf{B}^{\prime} \mathbf{C}$ is the reflected 
ray, the angle $A B^{\prime} C^{\prime}$ is $a-6 \phi$. If $B^{\prime} D^{\prime}$ is a line through $B^{\prime}$ parallel to $B A$, the angle $\triangle B^{\prime} D^{\prime}$ is $2 \phi$. Hence the angle $C^{\prime} B^{\prime} D^{\prime}$ is $\alpha-4 \phi$.

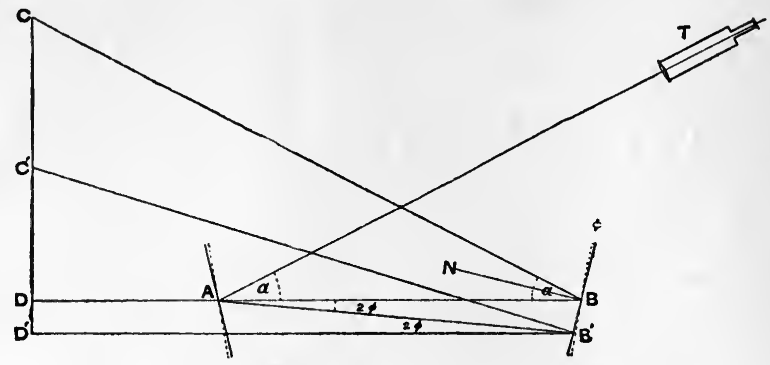

Fra. 41.

If, now, the scale $\mathbf{C c}^{\prime}$ is placed so that it is at right angles to the line $\Delta B$, we have

$$
C D=D \tan a \quad C^{\prime} D^{\prime}=D \tan (\alpha-4 \phi) \quad D D^{\prime}=d \tan 2 \phi .
$$

Hence

$$
x=C C^{\prime}=D \tan a-D \tan (\alpha-4 \phi)+d \tan 2 \phi .
$$

If both $\alpha$ and $\phi$ are so small that the angles may be put equal to the tangents, we get

$$
x=4 D \phi+2 d \phi, \text { or } \phi=\frac{x}{4 D+2 d} .
$$

In order to ensure that this approximation is permissible, the scale should be placed at a considerable distance, and the telescope adjusted so that the angle $\alpha$ is as small as is compatible with clearing the tops of the mirrors.

By replacing the mirrors by two right-angled prisms, as shown in plan in Fig. 42, $a$ can be made rigorously zero. When prisms are used, the

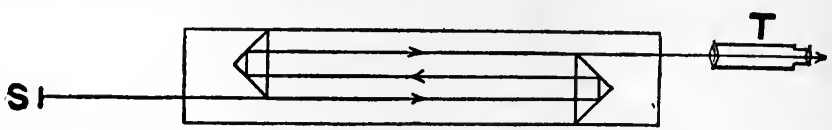

FIG. 42.

quantity $D$ in the formula is the distance between the scale and the hypotenuse face of the further prism $+\delta / \mu$, where $\mu$ is the refractive index of the glass, and $\delta$ is the height of the prism measured from the hypotenuse face. In the same way $d$ is the distance between the hypotenuse faces of the two prisms $+2 \delta / \mu$.

The advantages of the reflection method are that, owing to the sensitiveness of the method employed for measuring the deformation of the beam, smaller loads can be used, and therefore the chance of passing the elastic limit of the material is reduced. In the second place, the fact that, as the load increases, the supporting knife-edges 
slightly sink into the material has no effect on the readings, as is the case when the deflection at the centre is measured.

40. Determination of Poisson's Ratio.-Elongation of a material by tension is accompanied by a lateral contraction and compression by lateral expansion, the ratio of lateral strain to longitudinal strain being Poisson's Ratio. When a beam is bent as in $\S 38$, the lower surface is extended longitudinally and therefore contracts laterally, while the upper surface contracts longitudinally and expands laterally. The shape of the cross-section thus changes from $a b c d$ to $a^{\prime} b^{\prime} c^{\prime} d^{\prime}$ (Fig. $43(a)$ ). If a beam is bent uniformly it can be shown that the ratio of the curvature of the lateral dimensions to the curvature of the longitudinal dimensions is equal to Poisson's Ratio. The experiment can be performed with the apparatus of Fig. 39 . The uprights $A$ and $B$ are placed about $50 \mathrm{cms}$. apart, and a long beam of 2 or $3 \mathrm{~cm}$. width and small depth is bent upwards by means of equal weights hung on the ends at equal distances from and outside the supports. The bending moment at all points between the supports is thus constant, and a uniform curvature is obtained over this length. The radius of longitudinal curvature can be obtained from the deflection measured by the scale $\mathrm{s}$ and the distance between the supports. The radius of lateral curvature is obtained by the arrangement shown in Fig. $43(b)$. At the edges of the central cross-section $a b c d$ two binding screws are soldered vertically, and in them two long vertical rods, $P$ and $Q$, are firmly clamped. When the beam is bent by the addition of weights at the ends, the lateral section will be curved with concavity upwards and the upper ends of the rods will approach one another. A light graduated scale is fixed horizontally at the top of the scale $P$ and a pointer attached to $Q$, giving the relative motion of the upper ends of the two rods. If the added weights produce a deflection $e$ of the centre point of the bar, the distance apart of the uprights being $L$, the radius of longitudinal curvature $R_{1}=L^{2} / 8 e$. If the length of the rols $P$ and $Q$ is $l$ and the relative movement of the upper ends is $f$, then the radius of lateral curvature $R_{2}=b l / f$. Poisson's Ratio is then given by $R_{2} / R_{1}$.

A possible error arises in the method just described, owing to the 
bending of the uprights $P$ and $Q$ when they are out of the vertical position. This can be avoided by using the method of $\S 39$ to obtain the lateral curvature. In place of the two binding screws, two short uprights are soldered to the edges of the bar, and two mirrors are fixed on them at a small inclination to the vertical. The radius of curvature can then be obtained from the change in the angle between the mirrors and the distance between them.

\section{Measurement of the Period of an Oscillating Body by the Eye} and Ear Method-Comparison of Moment of Inertias.-You are supplied with a light metal cradle a (Fig. 44) suspended by a phosphor bronze wire, and having a mirror $M$ attached, by means of which an image of a scale is reflected into a telescope. The upper end of the suspension

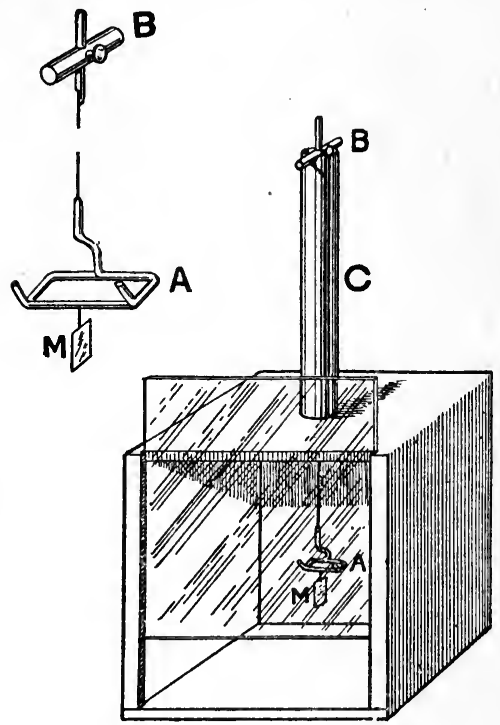

FIG. 44. wire is soldered to a metal rod, which is itself firmly clamped to a cross-bar $B$ that rests in two v's attached to an upright tube c, which is itself carried by a box with glass sides. This box serves to protect the suspended part of the apparatus from draughts. Metal cylinders of various shapes are provided, which can be placed in the v's of the cradle. The object of the experiment is to determine the period of the cradle alone, and also when it carries each of the metal cylinders in succession. From these periods we shall be able to compare the moments of inertia of the cylinders, and also, if we know the moment of inertia of one of the cylinders, determine the torsional rigidity of the suspension wire.

Adjust the telescope and scale so that when the cradle is empty and at rest the vertical cross-wire of the telescope coincides with a whole division near the centre of the scale. It will generally be found advisable to mark this division in some conspicuous manner, so that it catches the eye when the mirror is vibrating. For this purpose a small pointed piece of black paper may be attached to the scale, so that the point coincides with the upper end of the central division line but does not cover the line.

Next set the cradle vibrating by slightly twisting it, and then releasing it. Care must be taken not to produce pendulum vibrations during this process. If the cradle is set swinging like a pendulum, this motion may be stopped without greatly checking the torsional vibrations by lightly 
touching the suspension wire just above the cradle with the finger or with a feather or brush.

Determine the time occupied by fifty complete vibrations by means of a stop-watch, and suppose that the value of the period determined in this way is $T_{0}$.

In order to determine the period accurately, some more reliable method of measuring the time than the stop-watch will be required. For this purpose a chronometer or good clock, which ticks every half second, will be required. If a clock which ticks every second is employed, a very slight modification in the procedure will be required, which modification will be quite apparent, and in what follows we shall suppose that a chronometer beating half seconds is used.

Since it is impossible to watch the chronometer and look through the telescope at the same time, we have to make use of the ear to follow the chronometer, while the eye is used to follow the movement of the image of the scale as seen through the telescope after reflection in the mirror. There are various methods in use for counting the number of seconds which elapse between the instant when the chronometer is looked at and the instant when the event which is being timed occurs, but the author has found that in his experience the following is least likely to lead to a mistake in the counting:-At each tick corresponding to a half second say tick, while at each tick corresponding to a whole second say the number of the second. Thus starting at the whole minute one counts in time with the beats of the chronometer as follows: tick, one, tick, two, tick, three, \&c. Up to the twelfth second this is quite simple, but from thirteen up to sixty the numbers consist of more than one syllable, and the question arises, which of the syllables should be made to coincide with the beat of the chronometer. The author is in the habit of strongly accenting the first syllable of those numbers which have more than one syllable, and making this first syllable coincide with the beat of the chronometer. The syllables after the first are hardly sounded at all, and in the three-syllable numbers the middle syllable is almost entirely slurred. This method of counting may perhaps be indicated in the following manner, in which the asterisk indicates the instant of the chronometer beat: tick, thir-teen, tick, four-teen, . . . tick, twen-ty, tick, twen-t'one, tick, twen-t'two, \&c. The above may at first seem somewhat complicated, but a very little practice will enable the reader to count on for several minutes, if need be, without making any mistake, and practically without mental effort. When a little practice has been gained it will not be necessary to count out loud, though at first this will be found an assistance. It is important to practise taking the time from the chronometer and start counting the seconds at any beat, so as not to have to wait till the end of the minute. Thus if when you look at the chronometer it has just ticked on the eighth second, you must with the next beat say tick, and then go on, nine, tick, ten, tick, \&c.

Returning to the suspended cradle: this, without any metal cylinder 
will generally have a period of about a second, and the following will be found a convenient method of measuring such a period with accuracy :Set the cradle swinging over pretty well the whole range of the scale, then, taking the time from the chronometer, count on while you watch the movements of the scale in the telescope. Go on watching till, exactly on a beat of the chronometer, the zero mark of the scale crosses the cross-wire of the telescope, apparently moving from left to right. Immediately write down the time at which this transit occurred, which will be a complete whole or half second. Then take up the time again and watch till the zero mark sweeps across the cross-wire exactly on a beat, but this time moving from right to left. Write this time down in a column alongside the first, as shown in the example given below. Shortly before a minute after the first observation again take up the time, and determine the time when the zero crosses at a beat, moving from left to right. Then make an observation as the scale swings from right to left, and so on, making an observation of transit each way about every minute till the amplitude of the oscillations have so far died down as to make the exact determination of the instant of transit doubtful.

From the times obtained in this way we are able, by means of the approximate value of the period which we have already determined, to calculate the number of vibrations which have occurred between any two observations of the time of transit. The method employed can most easily be explained by means of an example. The following figures refer to the oscillations of a cradle, such as that shown in Fig. 44 :-

Approximate period: 100 vibrations took 2 minutes 4.5 seconds. Hence period is $1 \cdot 245$ seconds.

The times of transit given on the next page were determined with a chronometer, and were entered in the two columns $A$ and $B$ as the vibrations proceeded.

The columns $\mathbf{C}$ and $\mathbf{D}$ contain the differences between successive transits in the same direction. By dividing these intervals by the approximate time of vibration we obtain the number of vibrations which occur between the transit observations, and these numbers are entered in columns $\mathbf{E}$ and $\mathbf{F}$. It will be found very convenient to use a-slide rule for dividing the intervals in columns $\mathrm{C}$ and $\mathrm{D}$ by the approximate period, for if the 1 in the bottom, or $\mathbf{D}$ scale, is put opposite the approximate time of oscillation on the third or $\mathrm{c}$ scale, then opposite any interval between transits on the $\mathrm{c}$ scale will be found the number of vibrations on the $\mathrm{D}$ scale and a single setting of the slide will do for all the intervals.

We now have to calculate the number of vibrations between the first transit taken as 0 and each of the others. This is at once obtained from the numbers in the columns $\mathbf{E}$ and $\mathbf{F}$ by addition. As, however, we shall only require the first and last five, the sums of the numbers included by the brackets can be obtained once for all, and used to calculate the last five. 
\$41] COMPARISON OF MOMENT OF INERTIAS 105

\begin{tabular}{|c|c|c|c|c|c|c|c|c|c|c|c|}
\hline \multicolumn{3}{|c|}{ A. } & \multirow[b]{2}{*}{ 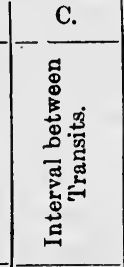 } & \multirow[b]{2}{*}{ 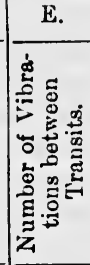 } & \multirow[b]{2}{*}{ 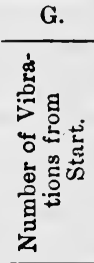 } & \multicolumn{3}{|c|}{ B. } & \multirow[b]{2}{*}{ 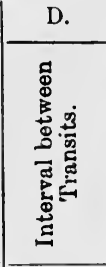 } & \multirow[b]{2}{*}{ 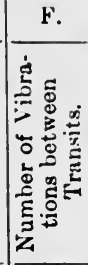 } & \multirow[b]{2}{*}{ 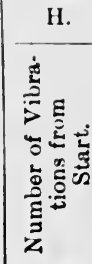 } \\
\hline & $\begin{array}{l}\text { Time } \\
\text { Tran }\end{array}$ & & & & & \multicolumn{3}{|c|}{$\begin{array}{l}\text { Time of } \\
\text { Transit. }\end{array}$} & & & \\
\hline H. & M. & s. & Seconds. & & & & M. & s. & Seconds. & & \\
\hline & & & 60 & 48 & & & & & 61 & 49 & v \\
\hline 3 & 43 & $25 \cdot 0$ & & & 48 & 3 & 43 & $38 \cdot 0$ & & & 49 \\
\hline 3 & 44 & $26 \cdot 0$ & 01 & 49 & 97 & 3 & 44 & $39^{\circ} 0$ & 0. & 10 & 98 \\
\hline 3 & & & 61 & 49 & 146 & 3 & & $39 \cdot 0$ & 60 & 48 & 146 \\
\hline 3 & & & 61 & 49 & 195 & 3 & & 40.0 & 61 & 49 & 195 \\
\hline 3 & & $28 \cdot 0$ & 60 & 48 & & & & & 61 & 49 & \\
\hline 3 & & $29 \cdot 0$ & 61 & 49 & & & & & 61 & 49 & \\
\hline 3 & 49 & $30 \cdot 0$ & 61 & 49 & & & & $42 \cdot 0$ & 60 & 48 & \\
\hline 3 & 50 & $30 \cdot 0$ & 60 & 48 & & & & 43.0 & 61 & 49 & \\
\hline & & & 61 & 49 & $\stackrel{\mathscr{2}}{0}$ & & & & 61 & 49 & ప \\
\hline 3 & 51 & $31 \cdot 0$ & 61 & 49 & 莺 & & 51 & $44^{\circ} 0$ & 60 & 48 & 焉 \\
\hline 3 & 52 & $32 \cdot 0$ & 60 & & $\frac{0}{7}$ & 3 & 52 & $44 \cdot 0$ & 61 & 49 & $\frac{2}{7}$ \\
\hline 3 & 53 & $32 \cdot 0$ & 00 & To & 我 & 3 & 53 & $45 \cdot 0$ & 01 & & 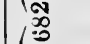 \\
\hline .3 & 54 & $33 \cdot 0$ & 61 & 49 & प्त & 3 & 54 & 46.0 & 01 & & $\widetilde{0}$ \\
\hline 3 & 55 & $34 \cdot 0$ & & 49 & 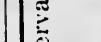 & 3 & & $47 \cdot 0$ & 61 & 49 & $\sum_{2}^{\infty}$ \\
\hline & 56 & $35 \cdot 0$ & 61 & 49 & $\bar{\Xi}$ & 3 & 56 & $48 \cdot 0$ & 61 & 49 & $\Xi$ \\
\hline & & & 60 & 48 & & & & & 60 & 48 & \\
\hline 3 & 57 & $35 \cdot 0$ & 61 & 49 & & 3 & & 48.0 & 61 & 49 & \\
\hline 3 & 58 & 36.0 & 61 & 49 & & 3 & 58 & $49 \cdot 0$ & 61 & 49 & \\
\hline 3 & 59 & $37 \cdot 0$ & 60 & 48 & & 3 & 59 & $50 \cdot 0$ & 60 & 48 & \\
\hline 4 & 0 & $37 \cdot 0$ & & & 876 & 4 & 0 & $50 \cdot 0$ & 61 & 49 & 877 \\
\hline 4 & 1 & $38 \cdot 0$ & & 20 & 925 & 4 & 1 & $51 \cdot 0$ & 61 & 49 & 926 \\
\hline 4 & 2 & $39 \cdot 0$ & & & 974 & 4 & 2 & $52 \cdot 0$ & & & 975 \\
\hline 4 & 3 & $39 \cdot 0$ & 60 & 48 & 1022 & 4 & 3 & $52 \cdot 0$ & & & 1023 \\
\hline 4 & 4 & $40 \cdot 0$ & 01 & $\mathbf{4 Y}$ & & & 4 & $53 \cdot 0$ & & & $10 \pi 2$ \\
\hline
\end{tabular}


We can now obtain five intervals corresponding to the scale moving in either direction, the number of vibrations being known, as shown below :-

\begin{tabular}{|c|c|c|c|c|c|c|c|c|}
\hline \multicolumn{5}{|c|}{ Time. } & \multirow{2}{*}{\multicolumn{2}{|c|}{ Interval. }} & \multirow{2}{*}{$\begin{array}{l}\text { Number of } \\
\text { Vibrations. }\end{array}$} & \multirow{2}{*}{ Period. } \\
\hline \multicolumn{3}{|c|}{ At Start. } & \multicolumn{2}{|c|}{ At End. } & & & & \\
\hline 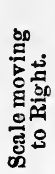 & $\left\{\begin{array}{l}M . \\
42 \\
43 \\
44 \\
45 \\
46\end{array}\right.$ & $\begin{array}{c}\text { s. } \\
25 \cdot 0 \\
25 \cdot 0 \\
26 \cdot 0 \\
27 \cdot 0 \\
28 \cdot 0\end{array}$ & $\begin{array}{l}M . \\
60 \\
61 \\
62 \\
63 \\
64\end{array}$ & $\begin{array}{r}\mathbf{8 .} \\
37 \cdot 0 \\
38 \cdot 0 \\
39 \cdot 0 \\
39 \cdot 0 \\
40 \cdot 0\end{array}$ & $\begin{array}{l}\text { M. } \\
18 \\
18 \\
18 \\
18 \\
18\end{array}$ & $\begin{array}{r}8 . \\
12 \cdot 0 \\
13 \cdot 0 \\
13 \cdot 0 \\
12 \cdot 0 \\
12 \cdot 0\end{array}$ & $\begin{array}{l}876 \\
877 \\
877 \\
876 \\
876\end{array}$ & $\begin{array}{l}1 \cdot 2466 \\
1 \cdot 2463 \\
1 \cdot 2463 \\
1 \cdot 2466 \\
1 \cdot 2466\end{array}$ \\
\hline \multirow[t]{2}{*}{ 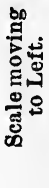 } & $\left\{\begin{array}{l}42 \\
43 \\
44 \\
45 \\
46\end{array}\right.$ & $\begin{array}{l}37 \cdot 0 \\
38 \cdot 0 \\
39 \cdot 0 \\
39 \cdot 0 \\
40 \cdot 0\end{array}$ & $\begin{array}{l}60 \\
61 \\
62 \\
63 \\
64\end{array}$ & $\begin{array}{l}50 \cdot 0 \\
51 \cdot 0 \\
52 \cdot 0 \\
52 \cdot 0 \\
53 \cdot 0\end{array}$ & $\begin{array}{l}18 \\
18 \\
18 \\
18 \\
18\end{array}$ & $\begin{array}{l}13 \cdot 0 \\
13 \cdot 0 \\
13.0 \\
13 \cdot 0 \\
13 \cdot 0\end{array}$ & $\begin{array}{l}877 \\
877 \\
877 \\
877 \\
877\end{array}$ & $\begin{array}{l}1 \cdot 2463 \\
1 \cdot 2463 \\
1 \cdot 2463 \\
1 \cdot 2463 \\
1 \cdot 2463\end{array}$ \\
\hline & & & & & & & Mean & $1 \cdot 2464$ \\
\hline
\end{tabular}

Having determined the period of the cradle alone, that of the cradle loaded with one of the metal cylinders has to be determined. Before placing the cylinder in the cradle, adjust the horizontal cross-wire of the telescope so that it coincides with what appears as the upper edge of the division lines of the scale. Then when placing the cylinder on the cradle, adjust its position till the horizontal cross-wire again coincides with the upper ends of the division lines. In this way you can ensure that the cradle hangs in the same position with reference to the axis of suspension as it did when empty, and hence that its moment of inertia about this axis is unaltered. At the same time it ensures that the centre of gravity of the cylinder is on the axis of rotation, an important matter when we come to calculate its moment of inertia from its dimensions.

The period with the cylinder will be considerably greater than that of the empty cradle, and a slightly different procedure will be required to measure it.

Determine the time taken to make about fifty vibrations with a stopwatch, and calculate the approximate period.

Next set the arrangement swinging over almost the whole of the scale, and taking the time from the chronometer determine the time of transit of the zero across the cross-wire when moving from left to right. By noting the position of the cross-wire on the scale with reference to the zero at the tick immediately preceding and following the transit it will be found possible, after a little practice, to determine the time of transit to within one- or two-tenths of a second. Having written down the time of transit, again take the time from the chronometer and determine in the same way the time of transit when the scale is travelling from right to left. When nearly a minute has elapsed from the first transit again determine the time of transit from left to right, and proceed in this way 
till five transits in each direction have been recorded, as in the example below. It will now be sufficient to record a transit in either direction every two or even four minutes till about twenty minutes after the first transit. Then note the times' of five transits in either direction at intervals of a minute, as at the start.

The reason why observations are only made every two or four minutes during the middle portion is, that these observations are only used to count the number of vibrations made in this interval. The only thing we have to be careful to secure is that the interval during which we allow the instrument to vibrate without making a reading of the time of transit is not of such a length that when we divide by the approximate time of vibration there should be a doubt as to what the number of vibrations ought to be. If the interval is too long then, although if we knew the correct period this would divide into the interval almost exactly a whole number of times, thus giving the number of vibrations in the interval, yet the approximate period might divide into this interval a different number of times. Thus if the quotient obtained by dividing the interval by the approximate period consists of a whole number, $n$ plus 4 or over, there will be considerable doubt as to whether we ought to take $n$ or $n+1$ as the number of vibrations in the interval.

Suppose that the true period is $T$ and that the approximate period may differ from this by $\delta T$, then the number of vibrations, $n$, allowed to elapse between consecutive observations of transit must be such that $n . \delta T$ is not greater than about $T / 3$. In practice it will generally be found advisable to make the observations of transit at least twice as frequently as indicated by this expression, since then if for any reason one of the transit observations is incorrect, owing to a mistake in taking the time or other cause, it will be possible to neglect this observation and still be able to calculate the number of vibrations in the interval from the transits on either side of the one which has been neglected. When taking the time of vibration with a stop-watch the possible error in the interval taken will be quite 0.5 second, so that if we suppose that no errors have been made in counting we have, in the case of the example given above for the carriage only, that $\delta T$ ' is 005 . Thus the limiting value which it is safe to take for $n$ is given by

$$
n=\frac{1 \cdot 2}{.005 \times 3}=80 \text {. }
$$

A short calculation such as the above will always give the safe interval between successive transits, and ought always to be made when using this method of counting the number of vibrations performed by a body of which the period is being determined. The method of reducing the observations of period made with the cylinder in the cradle will be at once evident from the following example :-

Determination of approximate period : 40 vibrations took 136.7 seconds. Hence the period is 3.418 seconds. 


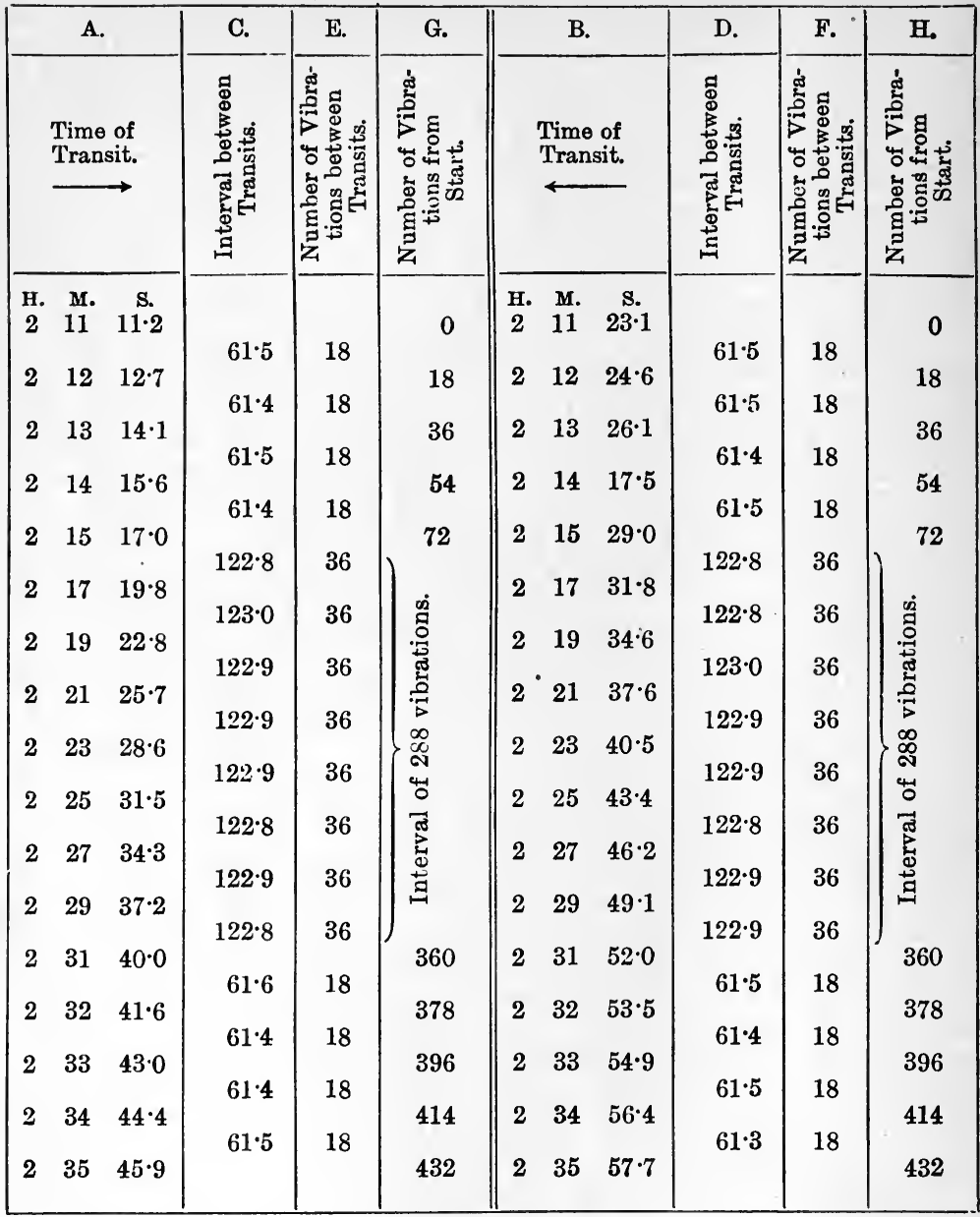

From these numbers we obtain the following values for the interval occupied by 360 vibrations :

\section{Scale moving to Right.}

$\begin{array}{rc}\text { M. } & \text { s. } \\ 20 & 28 \cdot 8 \\ 20 & 28 \cdot 9 \\ 20 & 28 \cdot 9 \\ 20 & 28 \cdot 8 \\ 20 & 28 \cdot 9\end{array}$

Scale moving to Left.

$$
\begin{array}{lr}
\text { M. } & \text { s. } \\
20 & 28 \cdot 9 \\
20 & 28 \cdot 9 \\
20 & 28 \cdot 8 \\
20 & 28 \cdot 9 \\
20 & 28 \cdot 7
\end{array}
$$

Hence

Mean 20 minutes $28 \cdot 85$ seconds.

Period $=3 \cdot 4137$ seconds. 
If $t_{0}$ is the time of vibration of the cradle only, and if $k$ is the moment of inertia of the cradle, we have

or

$$
\begin{aligned}
& t_{0}=2 \pi \sqrt{\frac{k}{c}} \\
& k=\frac{t_{0}{ }^{2} c}{4 \pi^{2}} .
\end{aligned}
$$

where $c$ is a constant depending on the length, diameter, and nature of the suspension wire. This constant $c$ represents the couple which, if applied at one end of the suspension wire while the other end is held fixed, would twist the end of the wire through one radian.

If $t_{1}$ is the time of vibration when a cylinder of which the moment of inertia is $K$ is placed in the cradle we have

or

$$
t_{1}=2 \pi \sqrt{\frac{K+k}{c}}
$$

Substituting from (1) above

$$
K+k=\frac{t_{1}^{2} c}{4 \pi^{2}} \text {. }
$$

$$
\begin{aligned}
& k=K \frac{t_{0}^{2}}{t_{1}^{2}-t_{0}^{2}}, \\
& c=\frac{4 \pi^{2} K}{\left(t_{1}^{2}-t_{0}^{2}\right)^{\bullet}}
\end{aligned}
$$

Hence if we calculate the value of $K$ from the dimensions of the cylinder, we can from our observations deduce both $k$ and $c$.

The moment of inertia of a solid cylinder about an axis passing through its centre of gravity and perpendicular to the axis is given by (see Table 7)

$$
K=M\left\{\frac{l^{2}}{12}+\frac{r^{2}}{4}\right\}
$$

where $M$ is the mass of the cylinder, $l$ its length, and $r$ its radius.

Measure the length, diameter, and weight of each of the cylinders which have been used in the vibration experiment, and calculate the moment of inertia of each. Then using these calculated moments of inertia and the periods determined, calculate as many values of $k$ and $c$ as there are experiments with cylinders, and enter the results in a table.

If the shape of a body is such that we are not able to obtain the moment of inertia by calculation, or if the density ${ }^{1}$ of the body is not

1 All the expressions for the moment of inertia given in Table 7 only hold if the density of the body is quite uniform. It is rather the exception than the rule for a mass of metal to have uniform density throughout, and so in accurate measurements involving the moment of inertia to test for the uniformity of the density is most important. 
uniform throughout, the moment of inertia $K$ can be obtained by determining the period $t_{1}$ when it is placed in the cradle, and then measuring the period $t_{2}$ when a body of known moment of inertia $K_{2}$ is placed in the cradle. For we have

$$
\begin{aligned}
& t_{0}=2 \pi \sqrt{\frac{\bar{k}}{c}} \\
& t_{1}=2 \pi \sqrt{\frac{\overline{K+k}}{c}} \\
& t_{2}=2 \pi \sqrt{\frac{K_{2}+k}{c}}
\end{aligned}
$$

whence

$$
K=K_{2} \frac{t_{1}^{2}-t_{0}^{2}}{t_{2}^{2}-t_{0}^{2}}=K_{2} \frac{\left(t_{1}-t_{0}\right)\left(t_{1}+t_{0}\right)}{\left(t_{2}-t_{0}\right)\left(t_{2}+t_{0}\right)}
$$

the second form of the expression being more convenient for use when logarithms are employed.

42. To Calculate the Simple Rigidity of a Wire from Observations of the Period of a Torsional Pendulum.-The quantity $c$, which is the torsional couple called into play when the end of the wire used in the last section is twisted through one radian, depends on the length $l$ of the wire, its radius $r$, and on the material of which the wire is composed. It can be shown that

$$
c=\frac{\pi r^{4} n}{2 l}
$$

where $n$ is called the simple rigidity of the material of which the wire is composed. The simple rigidity of a material is a constant for that material. It must be observed, however, that the simple rigidity of a wire depends in a great measure on the state of hardness of the wire. Thus annealing a wire often alters its rigidity very considerably, while stretching a wire so as to give it a permanent set will also alter the rigidity.

Measure the length and diameter of the wire used to suspend the carriage in the previous exercise, and by means of the values obtained for $c$ calculate the simple rigidity of the wire. Since in the expression for the rigidity the radius of the wire occurs raised to the fourth power, care must be taken to measure $r$ with the utmost possible accuracy. Since a wire is seldom perfectly cylindrical, the diameter must be measured at a number of points along its length, and at each point two measurements must be taken in directions at right angles to one another. As

$$
\begin{gathered}
c=\frac{4 \pi^{2} K}{t_{1}^{2}-t_{0}^{2}}=\frac{\pi r^{4} n}{2 l} \\
n=\frac{8 \pi K l}{\left(t_{1}^{2}-t_{0}^{2}\right) r^{4}}
\end{gathered}
$$


43. Simple Rigidity from Static Determination of Torsional Couple.-The value of the simple rigidity of a wire may also be obtained by a static experiment giving directly the value of $c$. If the couple required to twist the wire through $\theta^{\circ}$ is $C$, then $c=180 C / \theta \pi$, and $\quad n=\frac{360 C l}{\theta \pi^{2} r^{4}}$

The determination may be conveniently performed with the apparatus of Fig. 45. The wire under experiment is clamped in the binding screw $\mathbf{A}$ at the top of the supporting frame, and is kept straight and vertical by attaching a heavy weight $w$ at the bottom. On the top of the weight is fixed a large horizontal pulley or thick disc $\mathrm{K}$, on the upper surface of which is a circular scale $\mathrm{s}$, and the twist of the wire is measured by the mean reading of two pointers $P$ fixed to the frame at the opposite ends of a diameter of the scale. The twisting couple is produced by two threads $T$, shown also in plan in Fig. $45(a)$. The threads are attached to the edge of the disc and passed round it in opposite directions and then over the pulleys $B$, one at the front and one at the back of the frame. Scale pans are attached to the threads and the twist produced by the addition of equal masses $M$ in the pans is obtained. If $d$ is the diameter of the disc, the twisting couple $C$ is then $M g d$ cm. dynes. The twist $\theta$ produced by gradually increasing masses $M$ should be measured, and

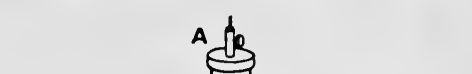
it will be found that for moderate twists $\theta \propto M$, and $n$ is given by

$$
n=360 \mathrm{Mg} d l / \theta \pi^{2} r^{4} \text {. }
$$

44. Determination of Young's Modulus and Rigidity of a Wire by Searle's Method. - A method of measuring Young's modulus of a wire which only requires a comparatively small length of the wire, and in 
which the measurement of an elongation or of deflection is replaced by a measurement of periodic time, has been devised by Searle. ${ }^{1}$

The arrangement used is shown in Fig. 46. In consists of two metal bars $\mathrm{AB}$ and $\mathrm{CD}$, each about $30 \mathrm{~cm}$. long, and of either square or circular

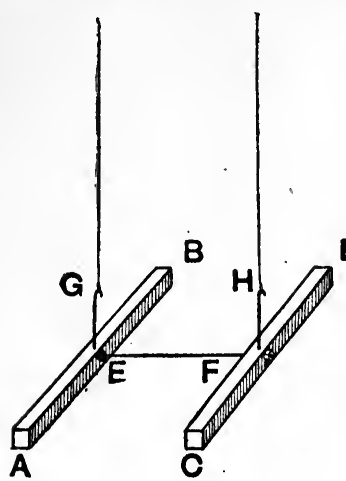

Fig. 46. cross-section, the area of crosssection being about 1.5 sq. $\mathrm{cm}$. These bars are connected at their mid points by the wire which is under test, the method by which the wire is attached being shown at $(a)$. The wire is soldered into a hole drilled in the head of a screw $J$, which is held in place by a nut $k$, this nut being of such a size that it would just fill up the hole left in the bar when the screw is in place. Two light wire hooks are attached to the bars at their mid points, (a) and serve for the attachment of two pieces of thread about a metre long. The upper ends of these threads are fixed to a support, so that the threads are parallel and the bars lie in the same horizontal plane.

The bars are caused to vibrate in a horizontal plane, each about its mid point, and if $t_{1}$ is the period, Young's modulus for the wire is given. by

$$
Y=\frac{8 \pi K l}{t_{1}^{2} r^{4}} \cdot \text {, . . . . . . }
$$

where $r$ and $l$ are the radius and length of the wire $\mathrm{EF}$, and $K$ is the moment of inertia of one of the bars.

The bars are then unhooked from the suspension fibres, and while one bar is held in a clamp, the period of the other, acting as a torsion pendulum, is determined. If $n$ is ridigity of the material of the wire, we have, as in the last section,

$$
n=\frac{8 \pi K l}{t_{2}^{2} r^{4}} \cdot \text {. . . . . . . . }
$$

$K$ having the same value as before, since the bars are either of square or circular cross-section.

If the mass of the wire is negligible compared to that of the bars, in the first experiment the wire will be bent into a circular arc as the bars vibrate. If $\rho$ is the radius of the arc into which the wire is bent, 
the bending moment is $\frac{\pi r^{4} Y}{4 \rho}$. Further, if $\phi$ is the angle either har makes with its position of rest $\rho=l / 2 \phi$, so that the bending moment is $\frac{\pi r^{4} Y \phi}{2 l}$.

Since the bending moment of the wire is equal to the couple acting on either bar tending to bring it back to its position of rest, the equation of motion of either of the bars is

and hence

$$
\begin{aligned}
& K \frac{d^{2} \phi}{d t^{\prime \prime}}=-\frac{\pi r^{4} Y \phi}{2 l}, \\
& t_{1}=2 \pi \sqrt{\frac{2 K l}{\pi r^{4} Y}}, \\
& Y=\frac{8 \pi K l}{t_{1}{ }^{2} l^{4}} \cdot \cdot \cdot \cdot \cdot \cdot \cdot \cdot
\end{aligned}
$$

To perform the experiment, the dimensions of the bars are measured and the moment of inertia $K$ is calculated. The bars having been suspended by the threads, a losp of cotton is tied round the ends of the bars, so that they are slightly drawn together. The bars are then started into vibration by burning through the cotton, and the period measured either with a stop-watch or by the eye and ear method, using a chronometer. The determination of the period as a torsion pendulum is performed as in $\S 41$.

- See Minchin's Statics, vol. ii. p. 424. 


\section{CHAPTER VI}

\section{THE PENDULUM-MEASUREMENT OF " $g$ " AND RATING A CHRONOMETER}

45. The Pendulum.-It can be shown (see Watson's Physics, p. 123) that so long as the arc over which a simple pendulum swings is very small, the time of vibration $t$ is given by

$$
t=2 \pi \sqrt{\frac{l}{g}}
$$

where $l$ is the length of the pendulum, and $g$ is the acceleration of

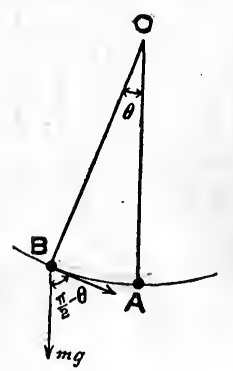

FIG. $4 \pi$. gravity. Since at any one place $g$ is constant, it. follows that $t^{2} / l$ must also be constant.

If the angle through which the pendulum swings is greater than one or two degrees, then the observed time of vibration will be appreciably greater than what it would be supposing the amplitude were very small, and so a correction will have to be applied on this account.

Thus let OA (Fig. 47) be the position of rest of the pendulum, and OB the position occupied at a given instant $t$, then the force acting on the bob in the direction of its motion is $-m g$ sin $\theta$, where $m$ is the mass of the bob. The acceleration with which the bob is moving is $\frac{d^{2} 8}{d t^{2}}$, where $d s$ is an element of the circular arc in which the bob moves. Hence

$$
\text { Force acting in bob }=m \frac{d^{2} s}{d t^{2}}=-m g \sin \theta .
$$

Now if $l$ is the length of the pendulum, $s=l \theta$. Thus

$$
\frac{d s}{d t}=l \frac{d \theta}{d t} \text { and } \frac{d^{2} s}{d t^{2}}=l \frac{d^{2} \theta}{d t^{2}} \text {. }
$$

Therefore

$$
l \frac{d^{2} \theta}{d t^{2}}=-g \sin \theta . . . . . .
$$

If both sides are multiplied by $2 d \theta$ and we integrate, then

$$
l\left(\frac{d \theta}{d t !}\right)^{2}=\underset{114}{2 g \cos \theta+a \text { constant } . . . . ~ . ~}
$$


If we measure $t$ from the instant of maximum elongation $\theta_{0}$, so that when $t=0, \theta=\theta_{0}$, and since at maximum elongation the velocity $\frac{d \theta}{d t}$ is zero, we have from (2)

$$
0=2 g \cos \theta_{0}+\text { const. }
$$

Hence substitute this value for the constant in (2)

or

$$
\begin{gathered}
\left(\frac{d \theta}{d t}\right)^{2}=\frac{2 g}{l}\left(\cos \theta-\cos \theta_{0}\right), \\
d t=\mp \sqrt{\frac{l}{2 g}}\left(\overline{\left.\cos \theta-\cos \theta_{0}\right)^{l}}\right.
\end{gathered}
$$

This may be written

$$
d t=\mp \sqrt{\frac{\bar{l}}{4 g}} \frac{1}{\sin \left(\theta_{0} / 2\right)} \frac{d \theta}{\left(1-\sin ^{2}(\theta / 2) / \sin ^{2}\left(\theta_{0} / 2\right)\right)^{\prime}} .
$$

Take a new variable $\phi$ such that

$$
\sin \phi=\frac{\sin (\theta / 2)}{\sin \left(\theta_{0} / 2\right)},
$$

which is alwas $\tilde{3}$ possible, since $\theta_{0}>\theta$. Differentiating

$$
\begin{aligned}
\cos \phi d \phi & =\frac{1}{2} \frac{\cos (\theta / 2) d \theta}{\sin \left(\theta_{0} / 2\right)}, \\
d \theta & =\frac{2 \sin \left(\theta_{0} / 2\right) \cos \phi d \phi}{\cos (\theta / 2)} \\
& =\frac{2 \sin \left(\theta_{0} / 2\right) \cos \phi d \phi}{\left\{1-\sin ^{2}\left(\theta_{0} / 2\right) \sin ^{2} \phi\right\}^{\circ}} .
\end{aligned}
$$

and

Substituting in (3)

$$
d t=\mp \sqrt{\frac{l}{g}} \frac{d \phi}{\left\{1-\sin ^{2}\left(\theta_{0} / 2\right) \sin ^{2} \phi\right\}^{\natural}}
$$

To find the time taken to reach the equilibrium position, i.e. the quarter period, we have to integrate between $\theta=\theta_{0}$ and $\theta=0$, that is, between $\phi=\pi / 2$ and $\phi=0$.

Hence

$$
\frac{T}{4}=\sqrt{\frac{l}{g}} \int_{0}^{\frac{\pi}{2}} \frac{d \phi}{\left(1-\sin ^{2}\left(\theta_{0} / 2\right) \sin ^{2} \phi\right)^{3}} \quad . \quad .
$$

The integral is a complete elliptic integral of the first kind, and its value can be obtained from tables. We may, however, expand 
$\left(1-\sin ^{2}\left(\theta_{0} / 2\right) \sin ^{2} \phi\right)-1$ by the binomial theorem, and integrate the terms separately.

Thus

or

$$
\begin{aligned}
\frac{T}{4}= & \sqrt{\frac{l}{g}} \int_{0}^{\frac{\pi}{2}}\left\{1+\frac{1}{2} \sin ^{2}\left(\theta_{0} / 2\right) \sin ^{2} \phi+\frac{1.3}{2.4} \sin ^{4}\left(\theta_{0} / 2\right) \sin ^{4} \phi+\right\} d \phi \\
= & \sqrt{\frac{l}{g}}\left\{\frac{\pi}{2}+\left(\frac{1}{2}\right)^{2} \frac{\pi}{2} \sin ^{2}\left(\theta_{0} / 2\right)+\left(\frac{1.3}{2.4}\right)^{2} \frac{\pi}{2} \sin ^{4}\left(\theta_{0} / 2\right)^{\prime}+\right\} \\
& T=2 \pi \sqrt{\frac{l}{g}}\left\{1+\frac{1}{4} \sin ^{2}\left(\theta_{0} / 2\right)+\frac{9}{64} \sin ^{4}\left(\theta_{0} / 2\right)+\right\}
\end{aligned}
$$

If $T_{0}$ is the time of vibration of the pendulum when the amplitude is very small, we may write the expression for $t$ as follows :-

or

$$
\begin{aligned}
T & =T_{0}\left(1+\frac{1}{4} \sin ^{2} \frac{\theta_{0}}{2}\right) \\
T_{0} & =T\left(1-\frac{1}{4} \sin ^{2} \frac{\theta_{0}}{2}\right) \\
& =T\left(1-\frac{\theta_{0}^{2}}{16}\right) . . . . . . . . . .
\end{aligned}
$$

so long as $\theta_{0}$ is not very great.

When making an actual determination of period, the amplitude gradually decreases during the experiment, and having observed the initial amplitude $\theta$ and the final amplitude $\theta^{\prime}$, we require to calculate the period with infinitely small arc from the observed period $t$. When the amplitudes are not very large, we may employ the following approximate formula ${ }^{1}$

$$
t_{0}=t\left(1-\frac{\theta \theta^{\prime}}{16}\right)
$$

In Table 8 the values of the correction term for different values of the initial and final amplitudes are given, and this table will be found of use in reducing observations of period, both in the present case and in others which we shall consider later, as, for instance, in determining the period of a magnet oscillating in the earth's field.

Next suppose in place of an ideal simple pendulum we have a compound pendulum, that is, a rigid body of any shape capable of rotation about a horizontal axis. Let $r$ be the distance of a given point $P$ of the body from the axis about which it can rotate (the axis of suspension), and let $y$ be the perpendicular distance of the point from a vertical plane through the axis. Thus if $\omega$ is the angular velocity with which the body is moving, the linear velocity of the point $P$ is $\omega r$, and the acceleration

$1 \theta$ and $\theta^{\prime}$ are supposed to be measured in radians. 
is $r \frac{d \omega}{d t}$. If $m$ is the mass of the particle at $P$, the force acting on $P$ is $m r \frac{d \omega}{d t}$, and the moment of this force about the axis is $m r^{2} \frac{d \omega}{d t}$. Hence for the whole body the moment of the force acting about the axis is $\frac{d \omega}{d t} \Sigma\left(m r^{2}\right)$. Now the only force acting on the particle $m$ is the attraction of gravity, and the moment of this force about the axis is myy, and for the whole body the moment is $\Sigma(m g y)$; but this is equal to the moment of the whole mass $M$ of the body, supposed concentrated at the centre of gravity, about the axis. Hence if $h$ is the distance of the centre of gravity from the axis, and $\theta$ is the angle through which the body has turned from the equilibrium position, we have

Moment of forces acting on the body $=g M h \sin \theta$.

Hence equating the two expressions for the moment of the forces, we get

$$
\frac{d \omega}{d t} \Sigma\left(m r^{2}\right)=-g M h \sin \theta .
$$

Now

$$
\omega=\frac{d \theta}{d t} \text {. }
$$

Hence

$$
\frac{d^{2} \theta}{d t^{2}} \Sigma\left(m r^{2}\right)=-g M h \sin \theta .
$$

The quantity $\Sigma\left(m r^{2}\right)$ is the moment of inertia of the body about the axis. Hence if $R$ is the radius of gyration about a parallel axis through the centre of gravity,

$$
\Sigma\left(m r^{2}\right)=M\left(R^{2}+h^{2}\right)
$$

Thus

$$
\begin{aligned}
& \frac{d^{2} \theta}{d t^{2}}=-\frac{g h}{h^{2}+R^{2}} \sin \theta, \\
& \frac{d^{2} \theta}{d t^{2}}=-\frac{g}{h+\frac{R^{2}}{h}} \sin \theta .
\end{aligned}
$$

In the case of a simple pendulum we have

$$
\frac{d^{2} \theta}{d t^{2}}=-\frac{g}{l} \sin \theta
$$

Hence if

$$
l=h+\frac{R^{2}}{h} \cdot \cdot \cdot \cdot \cdot \cdot \cdot \cdot \cdot
$$

the simple pendulum of length $l$ will have the same period as the compound pendulum.

If the body is now supported by an axis in the same plane as the 
first axis and the centre of gravity, and parallel to the first, and at a distance $l$ from it, we get similarly

$$
l^{\prime}=h^{\prime}+\frac{R^{2}}{h^{\prime}},
$$

where $h^{\prime}$ is the distance of the new axis from the centre of gravity. But $h^{\prime}=l-h=R^{2} / h$. Hence

$$
l^{\prime}=\frac{R^{2}}{h}+h=l
$$

that is, the period about the new axis will be the same as about the old. The point where the first axis cuts the vertical plane drawn through the centre of gravity perpendicular to the axis being called the centre of suspension, the point where the second axis cuts this plane is called the centre of oscillation.

In the case of a heavy sphere of radius $r$ suspended by a fine wire of negligible weight, and length $\lambda$, we have (see Table 8)

$$
R^{2}=\frac{2}{5} r^{2} \text {. }
$$

Hence the length of the equivalent simple pendulum is given by

$$
l=h+\frac{R^{2}}{h}=\lambda+r+\frac{\frac{2 r^{2}}{5}}{\lambda+r} . \quad . \quad . \quad . \quad . .
$$

Hence the periodic time of such a pendulum is

$$
T=2 \pi\left(1+\frac{\theta \theta^{\prime}}{16}\right) \sqrt{\frac{\lambda+r+\frac{2 r^{2}}{5(\lambda+r)}}{g}} \text {. . . }
$$

46. Determination of " $g$ " by Means of a Borda Pendulum.-The expression found in the last section for the period of a heary bob suspended by a fine wire may be employed for measuring $g$. For (10) may be written

$$
g=\frac{4 \pi^{2}}{T^{2}}\left(1+\frac{\theta \theta^{\prime}}{16}\right)\left\{\lambda+r+\frac{2 r^{2}}{5(\lambda+r)}\right\}^{\prime} . \quad \text {. }
$$

A piece of apparatus suitable for determining the value of $g$ from the period of such a pendulum is shown in Fig. 48. It consists of a bracket A, to the upper surface of which a piece of plate glass is attached. This glass serves as a plane on which the knife-edge which supports the pendulum rests. A slot in the glass allows the suspension wire to pass. The bob consists of a brass sphere having a diameter of about $5 \mathrm{~cm}$., to which the suspension wire is attached by being soldered into a fine hole bored radially into the sphere. The upper end of the wire passes through a knife-edge $\mathrm{B}$, and is held by being clamped by the screws at $\mathrm{C}$. 
A scale $\mathbf{D}$ is attached to the wall in such a way that the wire passes just clear of its surface, and is used to measure the initial and final amplitude of the pendulum. A screw $\mathbf{E}$ serves, as will be explained later, to assist in measuring the length of the pendulum.

A good clock beating seconds or a chronometer will be required to measure the time of oscillation, also a metal rod having a diameter of about a centimetre, and about $105 \mathrm{~cm}$. long, will be required to assist in measuring the length. The ends of this rod must be carefully turned so as to be planes perpendicular to the axis of the rod. If the rod has not been exactly adjusted to $105 \mathrm{~cm}$. long, or if its length is not known, it will require to be measured, which can be done with the comparator ( $\$ 19$ ).

The first operation to be performed is to adjust the knife-edge so that without the suspension wire it will have a period of two seconds. The period can be adjusted by screwing the weight $w$ up or down. Since the length of the

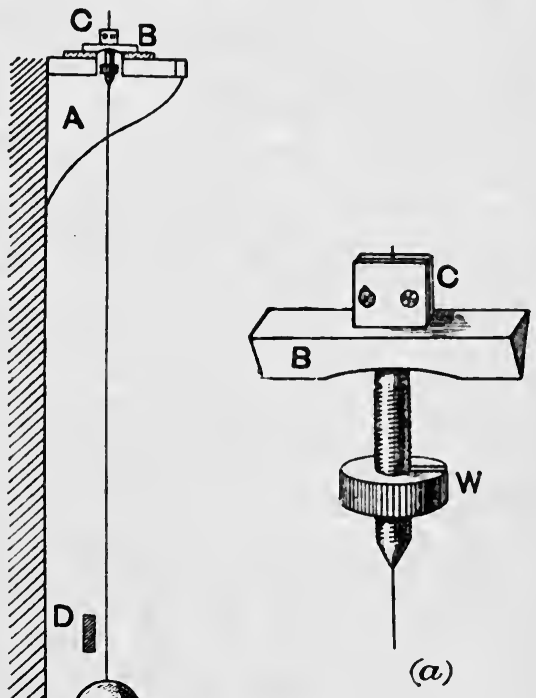
pendulum will be adjusted so that the period is nearly two seconds, when the knife-edge is adjusted in this way it will have no appreciable influence on the period of the pendulum. Of course it is only when the natural periods of the pendulum and knife-edge are exactly equal that the knife-edge has no influence whatever on the period of the pendulum. Since, however, the knife-edge is purposely made as light as possible, any slight departure from isochronism will not appreciably affect the result.

Having adjusted the period of the knife-edge, pass the suspension wire through the hole, and adjust the length of the suspension wire so that the distance between the centre of the bob and the knife-edge is about $99.5 \mathrm{~cm}$. Place the knife-edge on the glass plate, and steady the bob so that the pendulum comes to rest.

Set up a telescope at a distance of about 2 metres in front of the scale $D$, and focus it on this scale. The suspension wire will be seen 
crossing the scale, and the reading corresponding to the point where it crosses the scale must be taken. This will give the position of rest of the pendulum, and this point must be marked on the scale by a small blackened pointer.

The pendulum must now be set in vibration, so that the bob moves through about $8 \mathrm{~cm}$. on each side of the zero. The best way of starting the swings without setting up irregular vibrations is to tie a loop of cotton round the bob, and by means of a piece of cotton attached to this loop to draw the bob aside, and fasten it in this position by attaching the cotton to a stand. Then by burning the eotton the bob will be released and the pendulum started. Having noted the readings on the scale corresponding to the end of the swing on either side of the zero, the period must be determined, and, finally, a second reading made of the scale corresponding to the extreme positions. To determine the period, the method given in $\S 41$ is employed, that is, we note the time when the pendulum crosses its position of rest exactly when the clock or chronometer gives a tick.

Taking the time from the chronometer, or clock, watch the movement of the suspension thread through the telescope till it is seen to cross the zero mark, apparently going from left to right, exactly on a tick. Having written this time down, again take up the time, and from the chronometer note when the pendulum crosses from right to left on a tick. Continue observing the coincidences in either direction for at least half-an-hour. The interval between consecutive coincidences will depend on the relative periods of the pendulum and the clock or chronometer; the nearer these agree in period, the longer the coincidence period. The coincidence period ought not to be less than two minutes. After the first pair of coincidences have been observed it will not be necessary to watch the pendulum, as the time when to expect the next coincidence can be readily calculated and the time can be taken up, and the watching started, about twenty seconds before the expected time.

A note must be made as to whether the pendulum or the clock is going the faster. This can at once be seen by watching the next few transits after a coincidence. If on the ticks following a coincidence the pendulum appears to lag, so that it does not reach its zero position on the tick, the clock is going faster than the pendulum, and vice versa.

We have now to calculate how many vibrations have been made by the pendulum between the first and last coincidence in either direction.

Let us suppose that the pendulum is vibrating quicker than the clock or chronometer. By this we mean that while the pendulum makes one vibration the clock pendulum has not quite completed one vibration, that is, it has not made two ticks, or that the chronometer has not quite made four ticks. The periodic time of the pendulum is very nearly two seconds, so let its period be called $2-x$. Then starting from the instant when there is coincidence, at its next transit the time will be $2-x$, at the next transit $2(2-x)$, and so on till after $n$ vibrations of the pendulum the time will be $n(2-x)$ or $2 n-n x$. Now, as the 


\section{§ 46] DETERMINATION OF “ $g$ ” BY A BORDA PENDULUM}

pendulum vibrates it gradually has fallen behind the ticks of the clock, till finally it will have fallen behind the clock by a whole tick when the next coincidence will take place. This occurs when $n x$ is equal to the interval between the ticks. Hence we have

and

$$
\begin{aligned}
& n x=1 \text { for the clock beating seconds, } \\
& n x=-5 \text { for the chronometer beating half seconds. }
\end{aligned}
$$

But the coincidence interval is equal to $2 n-n x$ seconds. Hence calling the coincidence interval $c$, we have

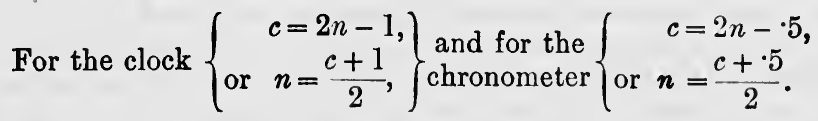

If the pendulum is moving slower than the clock, then by an exactly similar argument we can show that

$$
\text { In the clock }\left\{\begin{array}{c}
c+1=2 n, \\
\text { or } n=\frac{c-1}{2},
\end{array}\right\} \text { and for the }\left\{\begin{array}{l}
c+5=2 n, \\
\text { chronometer } n=\frac{c-5}{2} .
\end{array}\right.
$$

Hence, knowing $c$, we can immediately calculate the number of vibrations, $n$, which the pendulum has made between the coincidences. Proceeding in this way we can obtain the total number of vibrations made by the pendulum during the whole time of vibration, and then from the times of the first and last transits observed (or the first two

\begin{tabular}{|c|c|c|c|c|c|c|c|c|c|c|c|}
\hline \multicolumn{6}{|c|}{ Pendulum moving to Right. } & \multicolumn{6}{|c|}{ Pendulum moving to Left. } \\
\hline \multicolumn{3}{|c|}{$\begin{array}{c}\text { Time of } \\
\text { Coincidence. }\end{array}$} & \multicolumn{2}{|c|}{$\begin{array}{l}\text { Coincidence } \\
\text { Interval } \\
\text { (Pendulum } \\
\text { gaining). }\end{array}$} & \multirow{3}{*}{$\begin{array}{c}\text { Number of } \\
\text { Vibrations. } \\
76\end{array}$} & \multicolumn{3}{|c|}{$\begin{array}{c}\text { Time of } \\
\text { Coincidence. }\end{array}$} & \multicolumn{2}{|c|}{$\begin{array}{l}\text { Coincidence } \\
\text { Interval } \\
\text { (Pendulum } \\
\text { gaining). }\end{array}$} & \multirow{2}{*}{$\begin{array}{l}\text { Number of } \\
\text { Vibrations. }\end{array}$} \\
\hline $\begin{array}{l}\text { н. } \\
10\end{array}$ & $\begin{array}{c}\text { M. } \\
7\end{array}$ & $\begin{array}{l}\text { s. } \\
23\end{array}$ & & s. & & & 8 & $\begin{array}{r}\text { s. } \\
39\end{array}$ & м. & s. & \\
\hline & 9 & 54 & 2 & 31 & & & 11 & 12 & 2 & 33 & 77 \\
\hline & 12 & 27 & 2 & 33 & 77 & & 13 & 43 & 2 & 31 & 76 \\
\hline
\end{tabular}
or three and last two or three) we can calculate the period of the pendulum. The following scheme shows the method of recording such a set of observations :-

To measure the length of the pendulum, the screw E (Fig. $4 s$ ) is adjusted till it just touches the bottom of the bob. By allowing the bob to swing over a small arc while moving the screw, it will at once be seen when the point of the screw touches the bob. When 
making this adjustment, it is important to see that the point of the screw is vertically below the point of support of the pendulum. This can be secured by bringing the pendulum to rest, and then moving the knife-edge on the plane till the bob appears to be arranged symmetrically with regard to the screw.

Having completed the adjustment of the screw, remove the pendulum and stand the metal rod on the point of the screw with its upper end in the slot of the glass plate on which the knife-edge rested. To determine the amount by which the length of the pendulum differs from the length of the rod, determine the zero reading of a spherometer and then the reading when the centre leg touches the end of the rod and the three fixed legs rest on the surface of the glass. The difference between the spherometer readings will give the amount by which the length from the point of support to the bottom of the bob exceeds or falls short of, as the case may be, the length of the rod. To obtain the quantity $l$, we have to decrease this length by the radius of the bob. Measure the diameter of the bob with the vernier callipers, and thus obtain the radius.

The only thing that remains is to calculate the initial and final amplitudes in minutes of arc, so that we may apply the correction to reduce to infinitely small arc. If $a$ and $a^{\prime}$ are the readings on the scale $\mathrm{D}$ at the commencement and end of the observation of period, and $D$ is the distance of the scale from the plane on which the knife-edge rests, we have

$$
\text { Initial amplitude }=\tan ^{-1} a / D=a / D \text { radians, }
$$

since the angle is always very small. But a radian contains 3438 minutes of arc. Hence the initial amplitude in minutes is given by

$$
\theta=3438 \frac{a}{D}
$$

Similarly, calculate the final amplitude.

The time of vibration will require correction for the rate of the clock or chronometer. This can be obtained by comparison with a standard clock, of which the rate is known from astronomical observations. In the absence of such a clock, one of the methods described in $\$ \S 49,51$ may be employed to rate the clock or chronometer.

47. To Examine the Laws of the Compound Pendulum.-The apparatus used for this experiment consists of a uniform brass bar $90 \mathrm{~cm}$. long, the cross-section being a rectangle, of which the sides are $2 \mathrm{~cm}$. and $0.5 \mathrm{~cm}$. respectively. Holes $6 \mathrm{~mm}$. in diameter are bored at equal intervals throughout the length of the bar, which allow of its being suspended from a knife-edge which passes through the hole. This knifeedge consists of a piece of hard steel ground to a sharp edge and driven into the edge of a piece of wood. This wooden base has two fixed legs in front, formed by the heads of two round-headed screws, and an adjustable levelling screw behind. The object of the levelling screw in 
to allow of the knife-edge being made horizontal, so that the bar will swing regularly without twisting as it swings.

Determine with a stop-watch the period of the bar when suspended from each of the holes in turn, by observing the time taken to make from 50 to 100 vibrations. It will be found, when the centre of the bar is approached, that the period gets very long, and only a few vibrations can be observed. At the centre hole the period will be too long to observe, and the bar will probably refuse to vibrate steadily. If the knife-edge were placed exactly at the mid point of the bar, instead of at a distance from the middle point equal to the radius of the hole, the bar when deflected would not tend to come back into its original position, that is, its period would be infinite.

Next measure the distance from one end of the bar to the point of support in each case, and plot the results on a piece of squared paper,

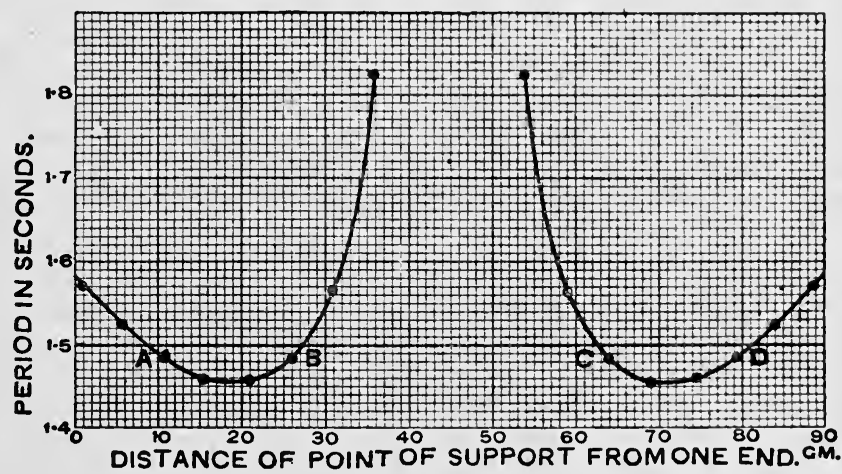

FIG. 49.

taking the lengths measured from one end of the bar as abscissa, and the times of a single vibration as ordinates.

The curve drawn through the observed points will be similar to that shown in Fig. 49, and consists of two branches, which ought, as the pendulum is uniform, to be exactly similar. If we draw any horizontal line, such as ABCD, it will cut the curve four times, which means that there are four points of suspension from which the bar would have a period equal to $1 \cdot 5$.

Now it was shown in $\S 45$ that if we can find two points on a body which are not symmetrically placed with reference to the centre of gravity, such that the period when suspended from either of these points is the same, then the distance between these points is equal to the length of the simple pendulum which would have the same period as has the body. These points are called the centre of suspension and the centre of oscillation or percussion respectively. From a consideration of the curve in 
Fig. 49, we see that the pairs of points $\mathrm{A}$ and $\mathrm{C}$ and $\mathrm{B}$ and $\mathrm{D}$ fulfil the above conditions, so that the length $\overline{\mathrm{AO}}$ or $\overline{\mathrm{BD}}$ will be the length of the simple pendulum, which would have the period 1.5 seconds.

On your curve draw a horizontal line through the first observed point, and determine the length of the equivalent simple pendulum from the curve. Then fit up a simple pendulum of this length by suspending a small lead bullet by a silk fibre, and prove experimentally that it has the same period as the rod when suspended from the end hole.

Since we can obtain from the curve the length of the equivalent simple pendulum which would have a period equal to the ordinate of the straight line which is drawn parallel to the axis of $X$, we can immediately calculate the value of $g$ by means of the formula $g=4 \pi^{2} l / t^{2}$.

Measure off the lengths of the simple pendulum for three or four lines drawn parallel to the $X$ axis, and for each calculate the value of $g$.

48. Determination of " $g$ " by Kater's Pendulum.-The method of deducing the length of the equivalent simple pendulum from the distance between the centre of suspension and the centre of oscillation was first used by Kater in 1818, and it remains to this day the most accurate method for measuring $g$. . Various forms of Kater's pendulum have been devised, but they all consist essentially of a metal rod weighted at one end so that the centre of gravity is much nearer one end than the other and having two knife-edges, one near either end. In addition, there is some method of adjusting the periods to equality when the pendulum is suspended from either knife-edge. In one form, while one of the knife-edges is fixed the other can be moved through a small distance along the stem of the pendulum. This form has the disadvantage that it is difficult to ensure that the movable knife-edge is always parallel to the fixed knife-edge. There is the further disadvantage that every time the movable knife-edge is moved the distance between the knife-edges alters and has to be re-measured. In the other form the knife-edges are fixed at almost the correct distance apart, and the final adjustinent is performed by sliding a weight along the stem of the pendulum. In this form, since the distance between the knife-edges remains the same, all the measurements made of this distance can be combined together and the mean taken as the length used in reducing the different observations of period which are made.

Whichever the form of pendulum used, it will be found a very tedious operation to attempt to adjust the periods about the two knife-edges to exact equality. This exact adjustment is also unnecessary, for, as we shall see, if the periods are very nearly the same we can calculate a correction to allow for the want of equality in the periods. Suppose that the periods about the two knife-edges are $t_{1}$ and $t_{2}$, and that the distances of the knife-edges from the centre of gravity of the pendulum are $h_{1}$ and $h_{2}$ respectively. Then if $R$ is the radius of gyration of the pendulum about an axis passing through its centre of gravity and parallel to the edges of the knife-edges, and $M$ is the mass of the pendulum, we have 
that the moment of inertia about the knife-edges is $M\left(R^{2}+h_{1}{ }^{2}\right)$ and $M\left(R^{2}+h_{2}^{2}\right)$. Hence the periods are given by

$$
\begin{aligned}
& t_{1}=2 \pi \sqrt{\frac{R^{2}+h_{1}^{2}}{h_{1} g},} \\
& t_{2}=2 \pi \sqrt{\frac{R^{2}+h_{2}^{2}}{h_{2} g}}
\end{aligned}
$$

Squaring and multiplying across we get

and

$$
\begin{aligned}
& h_{1} g t_{1}{ }^{2}=4 \pi^{2}\left(R^{2}+h_{1}{ }^{2}\right) \\
& h_{2} g t_{2}{ }^{2}=4 \pi^{2}\left(R^{2}+h_{2}^{2}\right) .
\end{aligned}
$$

Eliminating $R$ by means of these two equations we obtain

$$
\begin{aligned}
g\left(h_{1} t_{1}{ }^{2}-h_{2} t_{2}{ }^{2}\right) & =4 \pi_{-}^{2}\left(h_{1}{ }^{2}-h_{2}{ }^{2}\right), \\
\frac{4 \pi^{2}}{g} & =\frac{h_{1} t_{1}{ }^{2}-h_{2} t_{2}{ }^{2}}{h_{1}{ }^{2}-h_{2}{ }^{2}}, \\
& =\frac{t_{1}{ }^{2}+t_{2}{ }^{2}}{2\left(h_{1}+h_{2}\right)}+\frac{t_{1}{ }^{2}-t_{2}{ }^{2}}{2\left(h_{1}-h_{2}\right)} .
\end{aligned}
$$

Since $\left(h_{1}+h_{2}\right)$ is the distance between the knife-edges, the first term on the right above can be at once calculated. Also, since the periods about the two knife-edges are always very nearly equal, so that $t_{1}{ }^{2}-t_{2}{ }^{2}$ is small, the second term is small compared to the first. Hence we need only determine approximately the distances of the knife-edges from the centre of gravity. The position of the centre of gravity can be determined with sufficient accuracy by balancing the pendulum.

If the pendulum is one in which the knife-edge is movable, then a slightly different procedure to avoid having to adjust to strict equality of period is possible. Having approximately determined the position of the movable knife-edge for equality, determine the period

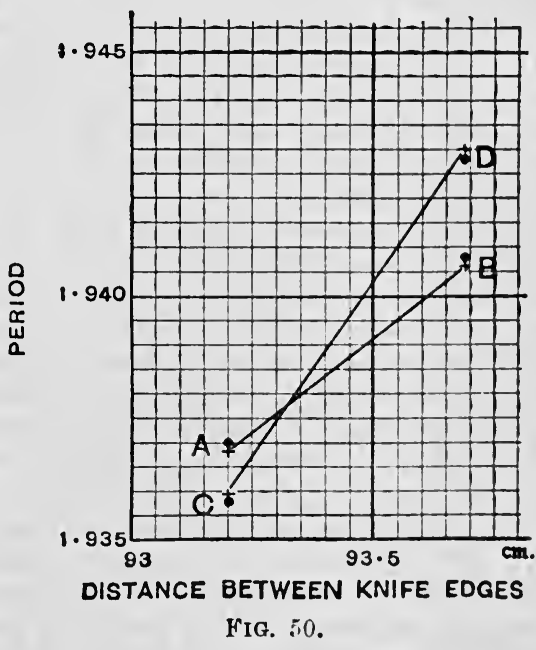
about both knife-edges; and suppose the distance slightly smaller than that corresponding to equality of periods. Then move the kuife-edge so that it is 
approximately as far beyond the correct position as it was short of it when the above measurements were made, and again determine the period about both knife-edges. Next plot the observed times and distances between the knife-edges on squared paper, as is done in Fig. 50, the points A and $\mathrm{B}$ corresponding to the heavy bob being uppermost and the points $\mathrm{C}$ and $\mathrm{D}$ to the bob being below, and connect the points representing the period about each of the knife-edges by straight lines $\mathbf{A B}$ and $\mathrm{CD}$. Then the length and period corresponding to the point $P$, where these straight lines intersect, will give the distance between the knife-edges and the corresponding period which would be found if the knife-edge were adjusted for strict equality in the periods. Thus in the example the length of the equivalent simple pendulum is $93.33 \mathrm{~cm}$., and its period is 1.9378 seconds.

To determine the period, the method of coincidences must be employed. Since the conduct of this part of the experiment differs in no manner from that described in $\$ 4$, it will not be again described, but reference must be made to that section. The distance between the knife-edges is a measurement which is of very considerable difficulty. By placing the pendulum on a horizontal table, and supporting a steel scale alongside so that the divided surface of the scale is on a level with the edges of the knife-edges, it is possible with care, and by using a reading microscope, to obtain the distance to within one- or two-tenths of a millimetre. To obtain greater accuracy it will be necessary to employ a vertical comparator, in which the microscopes are first focused on the knife-edges as the pendulum hangs, and then, replacing the pendulum by a standard scale, the distance between the cross-wires of the microscopes is obtained. It will in general be unnecessary in an ordinary physical laboratory experiment to go to this refinement, since to justify it we should have to take many precautions and make corrections which would be outside the range of the means ordinarily at the disposal of the student. Thus the slightest flexure of the stand under the influence of the swinging pendulum would have to be measured and allowed for, and a correction, the magnitude of which can only be obtained by swinging the actual pendulum used in a vacuum, would have to be applied for the effect of the surrounding air on the period of the pendulum. Special precautions would have to be taken to allow of the temperature being kept constant throughout the measurements, and so on.

The effect of air friction may be made the same, whichever end of the pendulum is uppermost, by fitting a dummy bob at the end remote from the real bob. A form of pendulum which Fig. 51. satisfies this requirement is shown in Fig. 51. The brass cylinder $\mathbf{A}$ is filled with lead, while the similar brass cylinder B is empty. The rod connecting these consists of a brass tube, to which are fixed the knife-edges $\mathrm{D}$ and $\mathbf{E}$. The weight $\mathrm{C}$ slides on the tube, and can be clamped by means of a set-screw. The rod is graduated 
in millimetres to allow of the position of $\mathrm{c}$ being identified. The pendulum is supported from a bracket, such as shown at $\mathrm{A}$ in Fig. 48. Two small wires $\mathrm{F}$ and $\mathrm{G}$ serve to read the amplitude of movement on a scale.

49. Determination of the Rate of a Clock by the Occultation of a Star by a Terrestrial Obstacle.-A simple method of rating a clock or chronometer, which is capable of giving results sufficiently accurate for many purposes, and does not involve any elaborate instrument, is as follows:-Attach a metal plate, in which there has been bored a small hole (about $3 \mathrm{~mm}$. in diameter), to the top of a wall or the side of a house in such a position that, looking through the hole in a southerly direction, some object, such as a lightning conductor, which has a smooth vertical edge, can be seen at a considcrable elevation. A chimney is not a suitable object to select, owing to the heat causing the air in the neighbourhood to shimmer. In order to rate the chronometer look through the hole in the metal plate, and by the eye and ear method determine the time when a fairly bright star vanishes behind the lightning conductor. The instant of occultation can be determined with very considerable accuracy, since the disappearance of the star is most surprisingly sudden. ${ }^{1}$ The time at which the same star is occulted on the following night must be determined in the same way. The interval between the two occultations will be equal to one sidereal day, that is, to a mean solar day less 3 minutes 55.91 seconds, and by comparing this interval with the interval shown by the clock or chronometer the rate of the latter can be obtained.

50. The Sextant and its Adjustment.-The usual form of sextant is shown in Fig. 52. It consists of a triangular framework ABC, having a graduated circular arc AB. An arm cv, called the index arm, is pivoted at the centre of the arc, and carries a vernier $v$. This arm is supplied with a clamp screw and tangent screw, by means of which the position can be adjusted. A mirror I, called the index glass, is fixed to the arm $\mathrm{Cv}$, the plane of the mirror being perpendicular to the plane of the circular arc, which we may call the plane of the instrument. A second mirror $\mathbf{B}$, called the horizon glass, is fixed with its plane perpendicular to the plane of the instrument. The lower half only of the horizon glass is silvered, the upper half being left clear. A telescope $\mathrm{T}$ is mounted so that its optic axis is parallel to the plane of the instrument, and passes through the centre of the horizon glass. Two sets of coloured glasses, E and $\mathrm{F}$, are provided for use when observing the sun, while a lens $\mathrm{G}$ is provided for reading the vernier $\nabla$.

When using the sextant to mcasure the angle subtended at the observer by two distant objects, the telescope is directed so that one object is seen through the clear part of the horizon gliss. The index arm $\mathrm{CV}$ is then moved, the instrument being so held that its plane pisses through the two objects till an image of the second object, formicd by reflection in the mirrors $I$ and $H$, appears in the field of view of the telescope. The index arm is then clamped and the tangent screw adjusted

1 It is advisable to note the times of occultation of three or four stars. 
till the two images appear to coincide. The angle which the index arm now makes with its position when the two mirrors I and $\mathrm{H}$ are parallel, is half the angle subtended by the two objects at the mirror I (sec Watson's Physics, § 336). Since the arc $\mathbf{A B}$ is so graduated that the zero reading corresponds to the position of the index arm when the mirrors $I$ and $\mathrm{H}$ are parallel, and each half degree is numbered as a degree, the arc as read off will give directly the angle between the two objects.

In order that the angle as measured with the sextant should be correct, the following adjustments must be made :-

(1) The plane of the index glass I must be perpendicular to the plane of the instrument. To test whether this adjustment is complete, set the

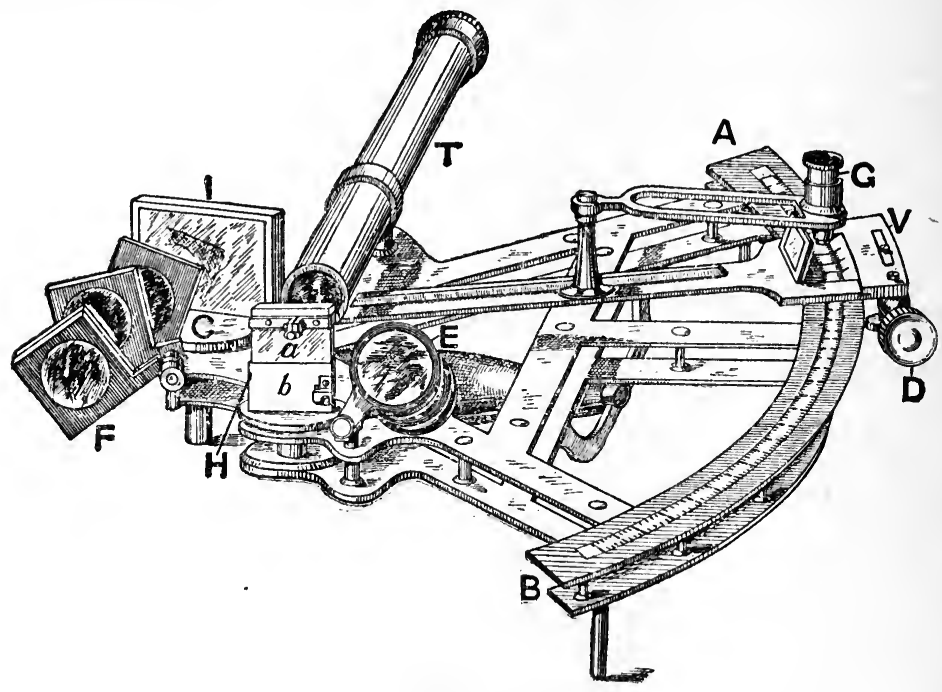

FIG. 52.

radius bar to about the middle of the arc. Then with the eye near the index glass look obliquely into the glass, so as to see at the same time part of the arc direct and part after reflection in the mirror. If the two portions of the arc appear in the same plane, the mirror is in adjustment. If not, then the screws at the back of the mirror must be moved till the adjustment is complete.

(2) The plane of the horizon glass must be perpendicular to the plane of the instrument. Direct the instrument so as to view some small welldefined distant object, and move the radius arm till two images of the object appear in the field of view. If by adjusting the radius arm the two images can be made to exactly coincide, then the mirror $\mathrm{H}$ is parallel to the mirror $\mathrm{I}$, and hence is perpendicular to the plane of the instrument. If turning the radius arm will not bring the two images into exact 
coincidence, the screw at the back of the mirror $\mathrm{n}$ must be adjusted till complete coincidence of the images can be secured.

(3) The line of collimation of the telescope must be parallel to the plane of the instrument. Turn the eye-piece of the telescope so that one of the wires, or two of the wires if there are four wires, is parallel to the plane of the instrument.

The images of two stars at a distance of between $90^{\circ}$ and $120^{\circ}$ are then brought to coincidence on the wire farthest from the plane of the instrument, or, if there is only one wire, near the edge of the field. If on tilting the sextant so that the images are near the other wire, or near the other edge of the field, they remain superposed, then the axis of the telescope is correctly adjusted. If the images scparate, then the telescope must be adjusted by the screws which hold the collar.

Another way of testing this adjustment is to prepare two L-shaped pieces of brass, the upright part being of such a height that when they are placed in the arc of the sextant the upper edges are at the same level as the optical axis of the telescope. The heights of these two pieces of brass must be very carefully adjusted to equality. This may be tested by first carefully levelling a small table by means of a delicate spirit-level, then placing the L's on the table and resting the level on the tops. These L's are then placed on the arc of the sextant as far apart as possible, and, the instrument being placed on a firm table, a sight is taken over the tops of the L's on some distant object. The telescope is then adjusted so that the sighted point on the distant object appears in the centre of the field as defined by the cross-wires.

Whenever the sextant is used to measure an angle, the images must be brought into contact at the centre of the field. If contact is secured near the edge of the field, then the angle as measured will be too great.

(4) The zero of the scale is supposed to coincide with the position of the index' arm when the two mirrors are parallel. This adjustment is seldom exactly correct, and hence the correction (called the index correction) which has to be applied must be determined.

If the reading on the vernier when the mirrors are parallel is $e$, then the index correction is $-e$, and this quantity has to be added to all the measured angles. The mirrors will be parallel when the images of a very distant object, or a star, coincide. The reason why a very distant object must be chosen is that we suppose that the lines drawn from the object to the mirrors $I$ and $H$ are parallel, and this is only strictly true if the object is at an infinite distance. An easy calculation will show at what distance the object must be so that the error produced may not be appreciable on the scale of the instrument. With sextants of the ordinary size the angle subtended by the distance between the two mirrors at half a mile is about 20 seconds. Hence if the sextant reads to 10 seconds, the object chosen must be more than a mile away. Having brought the images of such a distant object into coincidence, read the vernier, and let the reading be $e$. If $e$ is positive, that is, if the reading is on the side of the zero towards B (Fig. 5:J), then the quantity $e$ has to be subtracted from all the readings, and vice versa. 
The sextant is most often employed to measure the altitude of the sun or stars, and in such a case, on land at any rate, what is called an artificial horizon is necessary. This consists of a brass or gun-metal

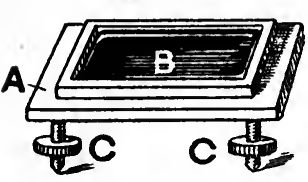

FIG. 53.

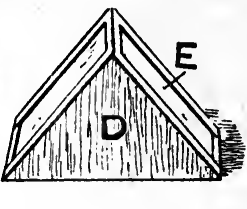

stand A (Fig. 53) fitted with levelling screws c. On the upper surface is a shallow recess $B$, in which is placed a small quantity of mercury. The bottom of the recess $B$ is amalgamated by filling it with a solution of mercuric nitrate. In this form of

artificial horizon accidental tremors die down very rapidly, while in the ordinary form in which a deep pool of mercury is used it is quite impossible to observe in a town, since the mercury surface is never sufficiently still.

The surface of the mercury will become dull owing to the formation of an amalgam, but this skin may be removed before taking an observation by drawing a piece of paper or card across the surface. The levelling screws must be adjusted so that the film of mercury (about $1 \mathrm{~mm}$. thick) extends all over the recess. If observations are to be made in a wind the mercury surface must be protected by a small cover D, which has two windows, one of which is shown at $\mathbf{E}$. These windows may be closed by pieces of "cabinet" mica.

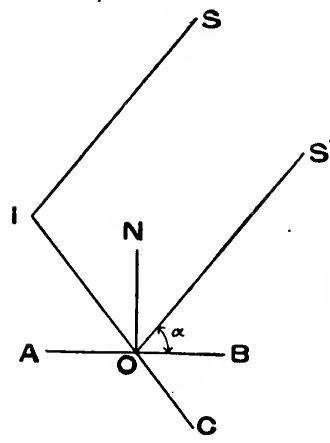

FIG. 54. If glass is used, it is necessary to use optically worked glass in which the surfaces are strictly parallel.

When determining the altitude of a heavenly body, say, a star, the telescope of the sextant is turned so as to view directly the image of the star formed by reflection in the mercury. Then the radius bar is turned till the image formed by reflection in the two mirrors, but without reflection in the mercury, coincides with the image seen by reflection in the mercury. When this adjustment is complete, the angle read off on the sextant is twice the altitude of the star. For if $\mathrm{AB}$ (Fig. 54) is the horizontal surface of the mercury, $\mathrm{N}$ being the normal to the surface, the angle measured is the angle sro. But the star being at such a great distance, SI is parallel to s'o, and therefore if Io is produced to c, the angle given by the sextant is the angle s'oc. Now, since the angle of incidence is equal to the angle of reflection, the angle $s^{\prime} O N$ is equal to the angle ION, and hence the angle $\mathrm{s}^{\prime} \mathrm{OB}$ is equal to the angle IOA or to the angle Boc. Thus the angle $s^{\prime} O{ }^{\prime}$ is equal to twice the angle $s^{\prime} \mathrm{OB}$, that is, the measured angle is equal to twice the altitude $\mathrm{s}^{\prime} \mathrm{OB}$ of the star.

When using the sextant to measure altitudes with the artificial 
horizon it will be found a great assistance to hold the sextant in a clamp attached to a retort stand.

51. Determination of the Error of a Chronometer with the Sextant.-The two methods suitable for determining time with a sextant are by (1) single altitude, and (2) equal altitudes of the sun or a star. Since it is in general more convenient to observe during the day, we shall assume, in what follows, that the sun is used.

Since in both these methods the time is deduced from the altitucie, it is of importance to observe when the altitude is changing as rapidly as possible. It can be shown that the altitude is changing most rapidly when the sun or star is crossing the prime vertical, that is, is due east or west of the observer. Further, errors in the assumed values for the latitude will produce the least effect when the star is near the prime vertical. The hour angle, that is, the time which will elapse, or has elapsed, before the sun or star crosses the observer's meridian when the sun or star is crossing the prime vertical, is given in the following table :-

Hour Angle of the Sun or a Star on the Prime Vertical.

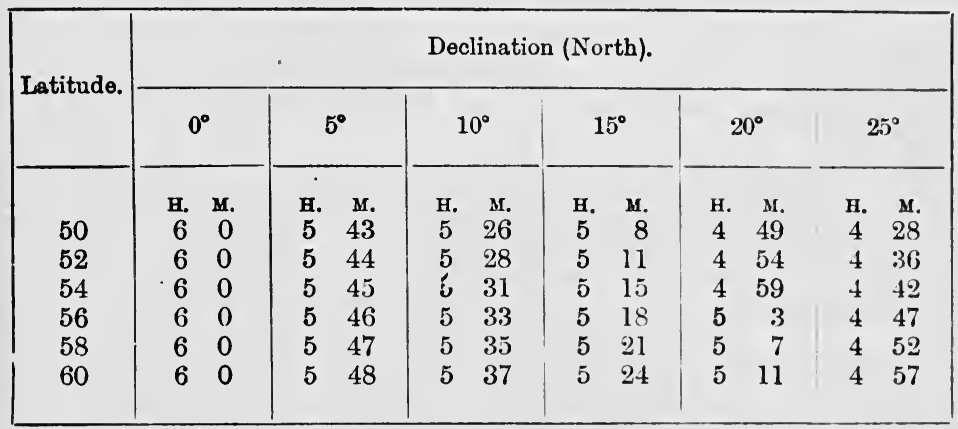

It will often be impossible to observe when the sun is crossing the prime vertical, since with the artificial horizon it is impossible to observe altitudes much less than $20^{\circ}$. Hence it may be necessary to observe some time after the sun has crossed the prime vertical. Observations ought not, however, to be made within about two hours of noon. Thus in the winter, when in the latitude of Great Britain the altitude of the sun does not become sufficiently high till near noon, it will be necessary to use a star, one with northerly declination being chosen.

(1) Time by Single Altitude.- In this method the altitude of the sun at a time noted on the chronometer is measured by means of a sextant and artificial horizon.

The artificial horizon having been set up on a steady support and levelled so that the mercury covers the dish, the sextant is adjusted so that two images of the sun are seen, one after reflection in the mercury, and the dark glasses are so adjusted that the two inages appear equally bright. 
If the observations are being made before noon, move the index arm till the images are separated slightly; if a reversing telescope is used, the image which moves with the movement of the index arm is to be uppermost. Then set the vernier to the next whole ten minute division, and taking the time from the chronometer, note when the two images just touch. Next move the vernier forward ten minutes, and again note the time of contact. Repeat this observation so as to obtain five readings of the scale and chronometer, that is, of the double altitude of the sun's upper limb at certain observed times.

Next move the radius arm so that the images of the sun overlap, and note the times when the images separate, obtaining, as before, five observations. These observations give the double altitudes of the sun's lower limb at the observed times.

If the observations are being made in the afternoon, then the lower limb should be observed first.

Enter the observations as in the following example, applying the correction for index error. The apparent altitude will require correction for refraction and parallax, the amount of which two corrections can be obtained from Table 9, the correction in every case being subtractive:-

Date, 3 April 1906.

Latitude of station, $51^{\circ} 29^{\prime} 48^{\prime \prime}$.

Longitude of station, $0 \mathrm{~h} .0 \mathrm{~m} .41 \cdot 5 \mathrm{~s} . \mathrm{W}$.

Approximate error of chronometer (G.M.T.) 10 s. fast.

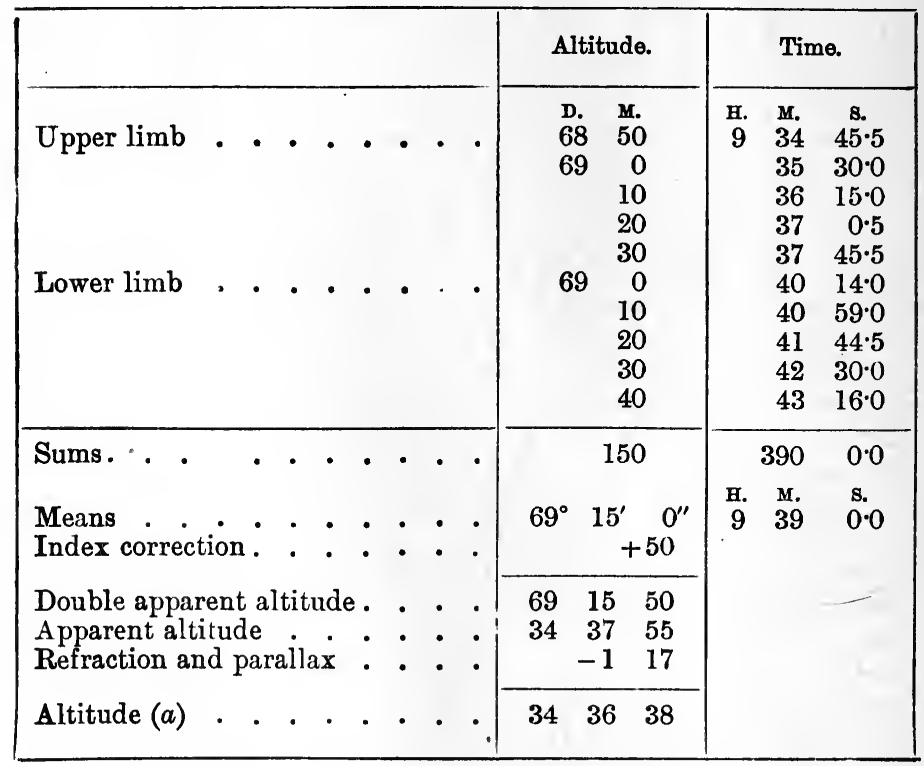




\begin{tabular}{|c|c|c|c|}
\hline \multicolumn{2}{|c|}{ Declination. } & \multicolumn{2}{|c|}{ Equation of Time. } \\
\hline At Noon. & Variation. & At Noon. & Variation. \\
\hline \multirow[t]{3}{*}{$\begin{array}{rl}5^{\circ} 3^{\prime} & 58^{\prime \prime} \cdot 6 \mathrm{~N} \\
-2 & 16 \cdot 4\end{array}$} & $\begin{array}{r}57 \cdot 55 \\
\cdot 2 \cdot 37\end{array}$ & $\begin{array}{cc}\text { M. } & \text { s. } \\
+3 & 32 \cdot 8 \\
& -1 \cdot 8\end{array}$ & $\begin{array}{l}\cdot 75 \\
2 \cdot 4\end{array}$ \\
\hline & $\begin{array}{r}115 \cdot 10 \\
17 \cdot 26 \\
4 \cdot 03\end{array}$ & & $\begin{array}{r}1 \cdot 50 \\
\cdot 30 \\
\end{array}$ \\
\hline & 136.39 & & 1.80 \\
\hline $\begin{array}{lll}5^{\circ} & 1^{\prime} & 42^{\prime \prime} \cdot 2\end{array}$ & & +3310 & \\
\hline \multicolumn{4}{|c|}{$84^{\circ} 58^{\prime} 17^{\prime \prime} \cdot 8=$ Polar distance $\left(90^{\circ}-\right.$ declination $)$} \\
\hline
\end{tabular}

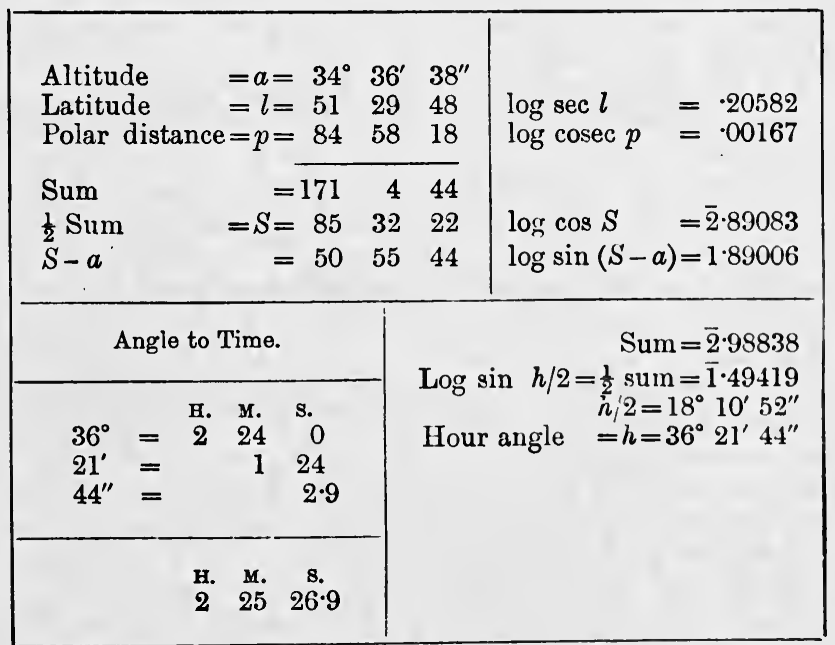

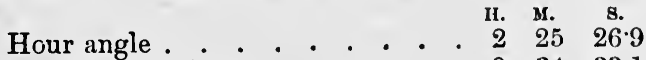

Apparent local time. . . . . . . $9 \begin{array}{ccc}9 & 34 & 33 \cdot 1\end{array}$

Equation of time. . . . . +331.0

Mean local time . . . . . . . . 938 (38

Longitude (W.) . . . . . . . $+41 \cdot 5$

G.M.T. . . . . . . . . . . 938

\begin{tabular}{llllll} 
Time by chronometer . . . . . & 9 & 39 & 000 \\
\hline
\end{tabular}

Chronometer error (fast) . . . . 
The formula employed to deduce the hour angle $h$ is

$$
\sin \frac{h}{2}=\sqrt{\frac{\cos S \sin (S-a)}{\cos l \sin p}},
$$

where $l$ is the latitude, $p$ the polar distance of the sun (i.e. $90^{\circ}-$ sun's declination), $a$ is the observed altitude, and $S$ is written for

$$
\frac{a+l+\underline{p}}{2} \text {. }
$$

The declination and equation of time are obtained from the Nautical Almanac for noon, and the quantities at the actual time of the observations are calculated from the change in one hour given in the almanac. The method of carrying out the calculation is clearly indicated in the example, and it will be found advantageous to set out the different steps of the calculation as shown there.

(2) In the method of equal altitudes the time the sun or star has the same altitude is observed on the two sides of the meridian. If the declination of the heavenly body observed remained constant, then the mean of the two observed times will give the time of meridian passage on the chronometer, and the G.M.T. of this passage can at once be obtained from the Nautical Almanac if the longitude of the observing station is known.

In the case of the sun the declination alters appreciably during the time which must elapse between the two altitude observations, and hence a correction, called the equation of -equal altitudes, must be applied. For the method of calculating this correction, reference may be made to Martin's Navigation (Longmans), p. 345. When only a few observations have to be reduced, the simplest procedure is to treat.separately each of the two sets of altitude observations made before and after noon, as in the case of single altitudes, and then take the mean of the two chronometer corrections deduced.

The advantage of having the two sets of observations is that errors in the adjustment of the sextant, and in the values assumed for the refraction, latitude, and declination, are in a great measure eliminated.

When the rate only of the chronometer is desired, then if the time at which the same star has any given altitude on successive nights is observed, the interval observed on the chronometer compared with a sidereal day $(23 \mathrm{~h} .56 \mathrm{~m} .4 \cdot 1 \mathrm{~s}$.) will give the rate. When making the observations, five or ten altitudes ought to be observed on each night, a star being chosen as near the prime vertical as possible.

For methods of determining time involving the use of a transit instrument or a theodolite, reference must be made to works on practical astronomy. 


\section{CHAPTER VII}

\section{SURFACE TENSION AND VISCOSITY}

52. Measurement of Surface Tension by the Elevation in a Capillary Tube.-Take three lengths of thermometer tubing having different bores ranging from $0.5 \mathrm{~mm}$. to $1.5 \mathrm{~mm}$., the lengths of the pieces being about $15 \mathrm{~cm}$. The bores of these tubes must be very carefully washed, ${ }^{1}$ first - with sulphuric acid, then with a strong solution of caustic soda, and finally rinsed out by passing a stream of water from the tap through them for some minutes. It is best to use tap water for this purpose, as distilled water is very apt to have a minute trace of grease, which would be quite fatal to the accuracy of the measurements. If the tap is allowed to run freely for a minute or so before the tubes are placed in the stream, any grease which may be on the tap will be carried away by the surface of the water as it streams out. The fact that the tap water may contain a small quantity of dissolved salts is of less importance, as far as its surface tension is concerned, than the presence of grease or dirt of a greasy nature. When waiting to be used the tubes must be kept immersed in a beaker of tap water drawn off with the precautions observed above, and the top of the beaker must be kept covered with a clock glass.

A convenient instrument for measuring the elevation of a liquid in a capillary tube is the small cathetometer microscope shown in Fig. 55. When this, or a similar type of instrument, is not available, a scale engraved on glass may be employed. Since it is difficult to focus the microscope on the surface of the liquid contained in the beaker, ${ }^{2}$ a needle is fixed to the tube in the manner shown. A convenient form of clip may be made out of a small piece of sheet brass, and an ordinary stout sewing-needle is soldered to the brass, as shown on a large scale at $(a)$. An india-rubber ring serves to hold the clip against the tube. The needle cannot be placed close alongside the tube, since, owing to capillarity, the water is raised up where the tube cuts the surface. When making a measurement the needle is adjusted so that its point just touches the surface of the liquid, and then the distance between the top of the

1 Instead of using old tubes, which require very careful cleaning, new capillaries may be prepared by drawing out pieces of clean glass tube. The ends of these capillaries must be sealed up to exclude dust till the tube is required.

2 Glass vessels with plate glass sides, through which good definition can be obtained, are supplied by Leybold's Nachfolger, Köln, Germany. 
needle and the surface of the liquid in the capillary is measured. By adding the length of the needle to this distance the capillary elevation is obtained.

When a glass scale is employed, the arrangement used by Quinke, shown in Fig. 56, will be found convenient. The bent pointer $B$ is cemented to the glass scale $\mathbf{A}$, to which the capillary tube is also attached

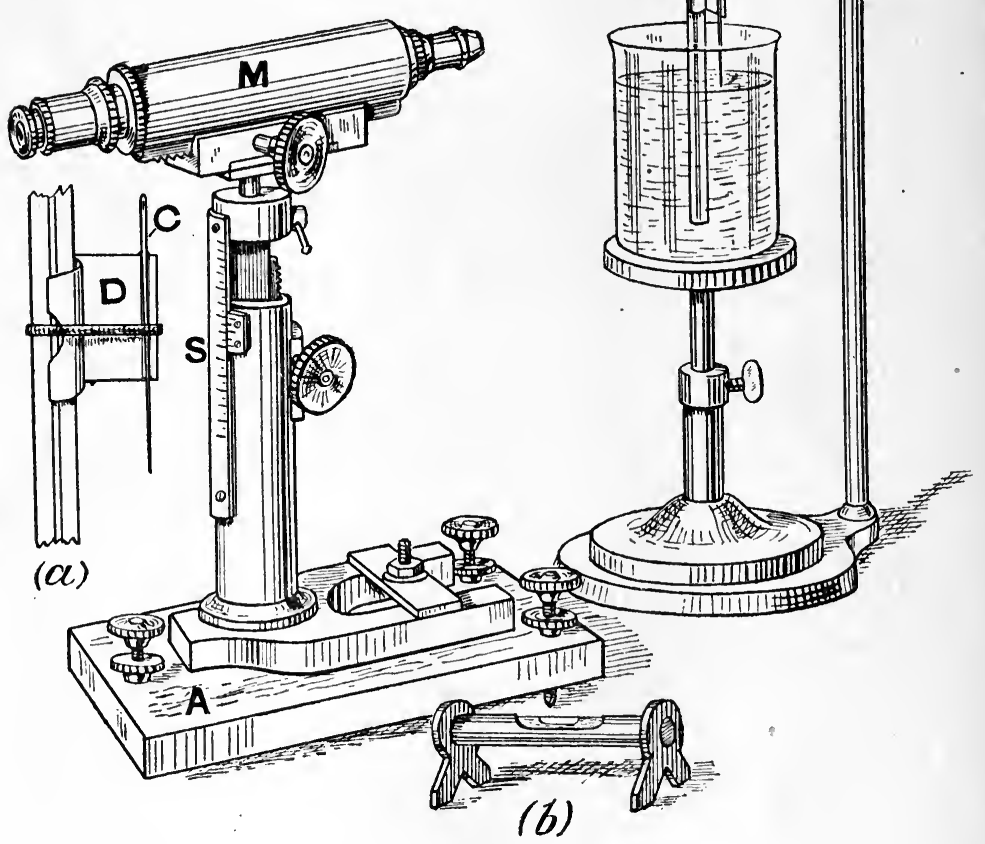

FIG. 55.

by two india-rubber bands. The scale is either clamped to the side, if a square vessel is employed to contain the liquid, or is held in the clip of a retort stand. The reading on the scale corresponding to the tip of the pointer B can be obtained by means of a small set-square, and the difference between this reading and that corresponding to the meniscus in the capillary tube gives the capillary elevation.

To make a measurement of the surface tension of water, clean out 
a small beaker or rectangular glass trough by the method used in cleaning the capillaries, and fill the beaker with water from the tap. Take a capillary tube from the water in which they have been stored, and having attached a short length of rubber tubing to one end, fix it in a clamp c (Fig. 55) so that its lower end dips into the water, and make it vertical by means of a plumb line. Attach a needle as shown, and adjust the needle so that its point just touches the surface of the water. Set up the table cathetometer at such a distance that the capillary tube can be focused, and by means of a striding level on the microscope make the axis about which the microscope turns vertical. To allow of this adjustment the base of the cathetometer is provided with levelling screws. The microscope is first turned parallel to the line joining two of the screws $A$ and B, say, and the level bubble brought to zero. The microscope being turned through a right angle, the level is brought to zero by means of the third screw c. After repeating this adjustment once

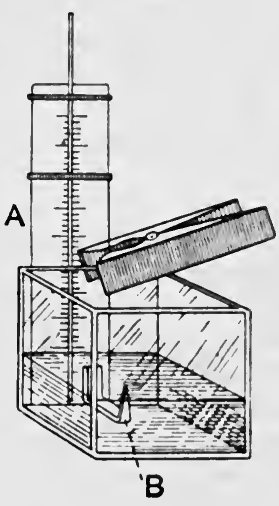

FIG. 56. or twice the level will remain at zero in whatever direction the microscope is turned.

The needle must be placed so that it is at the same distance from the vertical axis of the cathetometer as is the bore of the capillary tube, so that having focused the microscope on the meniscus in the tube, and turned the microscope about the vertical axis so that the needle appears in the field, the image seen may be sharply defined.

Having first slightly raised the water in the tube by pinching the rubber tube in two places, ${ }^{1}$ measure the height of the water surface ${ }^{2}$ above the top of the needle. Place a mark on the outside of the tube at the point occupied by the surface of the raised liquid column, and then raise the tube through about half a centimetre, and having again adjusted the needle, measure the elevation. Make a series of measurements in this way, raising the tube half a centimetre at a time till observations have been made with the surface at a number of points ranged along about 3 centimetres of the tube, and mark the lowest point occupied by the surface. We have now to measure the radius of the capillary

1 Never raise the liquid by sucking the tube, as doing so is almost certain to contaminate the surface.

2 It is best to measure to the lowest part of the concave surface. A correction must be applied to allow for the weight of the liquid above the lowest point of the surface. In fairly narrow tubes we mar a sume that the surlace is a hemisphere, so that the volume of liquid in question is the difference between the volume of a crlinder of height $r$ and radius $r$ and a hemisphere of radius $r$, that is, $\pi r^{3}-\dot{2} / 3 \pi r^{3}$ or $1 / 3 \pi r^{3}$. Hence if we increase the measured height $h$ by an amount $r / 3$, we shall allow for the weight of the liquid above the point to which $h$ was measured. 
tube. The elevation of the water depends on the radius at the point occupied by the water surface, and not at all on the radius either above or below this point. The measurement of the diameter at a point can only be made by cutting the tube and using a microscope, and it is difficult to obtain any great accuracy in this way. By determining the weight of a quantity of mercury which occupies a measured length of the tube, we can calculate the mean radius of the tube in the part which the column occupied when we measured its length. If, then, we weigh a column of mercury which occupies approximately the portion of the tube between the two marks made when making the observations of elevation, we can obtain the mean radius of this portion of the tube, and this mean can be used with the mean of the elevations to calculate the surface tension.

Having carefully dried the tube by drawing hot air through it, care being taken not to displace the marks, introduce a column of mercury of such a length that it will approximately extend from one mark to the other. The mercury may be introduced by attaching a piece of indiarubber tubing to the tube, when by pinching this tube in two places the mercury may be drawn up. Measure with a vernier microscope the length of the mercury column when it is arranged symmetrically with respect to the marks, and then run the mercury into a weighed watch-glass and weigh.

Then if $D$ is the density of the mercury at the temperature at which the length of the column was measured, $l$ the measured length, ${ }^{1}$ and $w$ the weight of the mercury, we have

$$
r=\sqrt{\frac{w 0}{\pi l D}}
$$

Knowing the elevation $h$, the radius $r$, and taking the density $\rho$ of the liquid at the temperature of the experiment from a table, we can now calculate the surface tension $T$ on the supposition that the liquid wets the walls so that the angle of contact is zero by the expression ${ }^{2}$

$$
T=\frac{h r \rho g}{2},
$$

where $g$ is the acceleration of gravity.

Repeat the experiments, using the other tubes, and enter your results as deduced from the various tubes in a table such as that below :-

\begin{tabular}{|c|c|c|c|}
\hline Tube. & $\begin{array}{c}\text { Radius } \\
r .\end{array}$ & $\begin{array}{c}\text { Elevation } \\
h .\end{array}$ & $\begin{array}{c}\text { Surface Tension } \\
T .\end{array}$ \\
\hline & & & \\
\hline
\end{tabular}

1 A correction must be applied for the hemispherical ends of the mercury column; see note on previous page.

'Watson's Physics, p. 187. 
53. Measurement of the Surface Tension of a Soap Film.-The pressure inside a soap bubble of radius $R$ exceeds the external pressure by an amount $p$, which is given by the relation ${ }^{1}$

$$
p=\frac{4 T}{R} \text {. }
$$

where $T$ is the surface tension of the soap solution. Hence if we measure $p$ and $R$ we can calculate the surface tension.

A piece of apparatus suitable for performing this experiment is shown in Fig. 57 (a). It consists of a wooden box $\mathrm{A}$, the front and back being

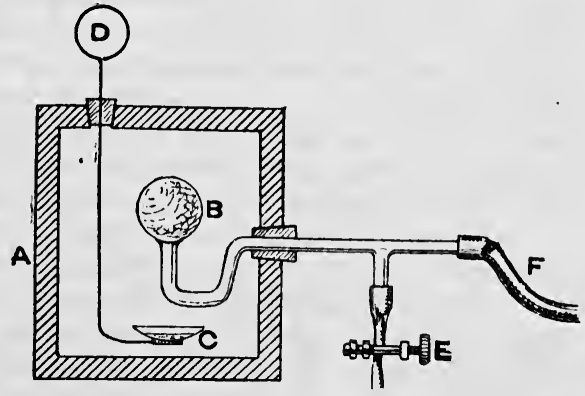

(a)

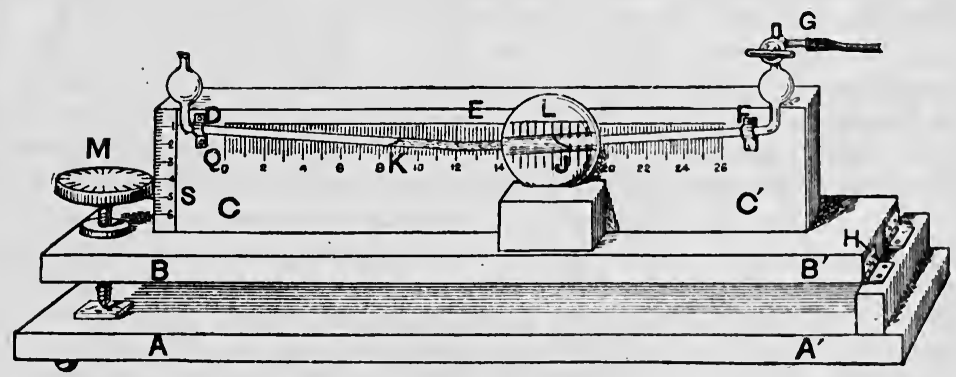

(b)

FIG. 57.

closed by pieces of plate glass. A glass tube $\mathrm{B}$ passes through the side of the box, and is connected by a rubber tube to a manometer. A small watch-glass $\mathrm{C}$ is supported by a wire which passes through a cork in the top of the box, and can be moved up and down by the handle D. This watch-glass serves to contain the soap solution, which by raising the handle $D$ and by rotating the tube $B$ can be brought up to the end of the tube. 
Since the pressure inside a bubble of sufficient size to be satisfactorily measured is about equal to the pressure exerted by a column of water of 2 millimetres in height, a special form of manometer will be required.

A suitable manometer is shown in Fig. 57.(b). It consists of a baseboard A, to which a second board B is hinged at $\mathrm{H}$, the other end of this board being supported by a micrometer screw $\mathbf{M}$, the point of which bears on a small glass plate cemented to the base-board.

An upright piece of wood cc' supports a bent glass tube DEF, the two portions $\mathrm{DE}$ and $\mathrm{EF}$ being inclined at equal angles to the board B. The tube has a bore of about 3 millimetres, and the turned-up ends expand into small bulbs. One end is attached to a three-way cock, by means of which the air in the tube DF may either be put in communication with the air, or through a rubber tube may be connected to the soap bubble through the tube F (Fig. $57(a)$ ). In the bent tube DF is a thread of xylene about 10 centimetres long, and a scale attached to the upright c enables the horizontal distance between the ends of the thread to be measured. A lens L attached to a cork, which rests on the upper surface of $B$, serves to assist in reading the position of the meniscus.

To obtain a reading of pressure with this manometer, first place the tube DF in communication with the air, and adjust the board B approximately horizontal. Then read and record the positions of the two ends of the liquid thread, and also note the reading on the scale $s$ and the divided head of the micrometer $\mathrm{m}$.

The tap a having been turned so as to put the instrument in connection with the vessel in which the pressure is to be measured, the liquid column will be moved to the left or right according as the pressure to be measured is greater or less than atmospheric pressure. Suppose it is greater, so that the liquid moves to the left. The screw $\mathbf{M}$ must be turned so as to raise the end $\mathbf{B}$ of the board $\mathbf{B B}^{\prime}$ till the liquid column comes back to the original position. When this adjustment is complete the micrometer is again read, the difference in the readings giving the height $\mathrm{H}$, through which the end of the board $\mathrm{B}$ has been raised.

If $l$ is the distance between the ends of the column of liquid $\mathrm{KJ}$, as measured on the scale $Q, \rho$ the density of the liquid, and $h$ the vertical height of the meniscus $\dot{K}$ above the meniscus $\mathrm{J}$, the pressure being measured exceeds the atmospheric pressure by an amount $p$, which is given by

$$
p=h \rho g \text {. }
$$

But if $\theta$ is the angle through which the board $\mathbf{B B}^{\prime}$ has been tilted, $h=l \sin \theta$.

Further, if $L$ is the distance between the micrometer screw and the hinge $\mathrm{H}$, we have

$$
\frac{H}{L}=\tan \theta
$$


Now in practice $\theta$ is always small, and hence we may take $\sin \theta=\tan \theta$.

Thus

$$
p=\frac{H l \rho g}{L} \text {. }
$$

Hence, if we know the density $\rho$ of the xylene, the measured quantities allow us to calculate the pressure.

To measure the pressure in a soap bubble some soap solution ${ }^{1}$ is placed in the watch-glass c (Fig. $57(a)$ ), and the tube B is turned so that the open end is downwards. Then by raising $D$ the end of $B$ is made to dip in the solution. The tap $\mathrm{g}$ (Fig. $57(b)$ ) being turned so as to cut off communication with the manometer, a bubble is formed by blowing into the tube $\mathbf{E}$. The glass tube $\mathrm{B}$ is then turned into the position shown in the figure, and the bubble put in communication with the manometer, and the pressure is measured as described above.

To measure the diameter of the bubble, a convex lens having a focal length of about $30 \mathrm{~cm}$. is arranged in a stand, so as to form an image of the bubble on a scale divided on cardboard. A gas flame placed behind the box containing the bubble serves to illuminate the bubble. The distance $u$ from the bubble to the lens, and that, $v$, from the lens to the scale, must be measured. Then if 0 is the diameter of the image, the radius of the bubble is given by

$$
R=\frac{u 0}{2 v}
$$

The measurement ought to be repeated, using bubbles of varying size, the value of the surface tension being calculated in each case by means of the equation

$$
T=\frac{p R}{4}
$$

The surface tension of a plane film may be obtained approximately by suspending a wire shaped thus $\square$ from the arm of a balance in a manner similar to that shown in Fig. 30, so that when counterpoised the pointed ends are just in contact with the surface of the soap solution contained in a beaker below it. The wire is first drawn down until it dips into the solution. When released, a vertical film will be formed bounded by the wire and the surface of the solution, and the tension

1 The soap solution can be prepared as follow's :-Fill a clean bottle (about a quart) three-quarters full of distilled water, and add one-fortieth of the weight of the water of pure fresh oleate of sola. After the oleate has dissolved fill up the bottle with Price's glycerine, and well shake. Laave the hottle to stand for a week in a dark place, and then by means of a syphon draw off the clear liquid and add one or two drops of strong ammonia to every pint of the liquid. This solution must be preserved in a well-stoppered buttle, preferably in a dark place, and will be found to improve with keeping. When making the solution do not warm the liquid or filter it. Exposure to the air for any length of time will injuriously affect the solution. 
along the intersection of the two faces of the film with the surface will give an increase in the weight required for balance. The film is allowed to drain and the weight required to bring the points to the surface is found. The film is then broken and balance again found, giving the weight of wire and film. If $w$ is the difference in the two weights due to the surface tension, and $a$ the distance between the two points of the wire, then $2 \alpha T=v g$,

In a second method, a horizontal wire about $2 \mathrm{~cm}$. long is suspended by vertical threads about $8 \mathrm{~cm}$. long at its ends from a second and longer horizontal wire held in a clamp at one end. A vertical rectangle is thus formed by the two wires and the two threads. A shallow dish of soap solution is brought up to the arrangement which is dipped into it and the solution removed. A film is thus formed bounded by the wires and threads, and the latter now take the form of arcs of circles. Measurements are made of the distance between the threads at the points where they are fixed to the wires $(2 a)$, the distance between them at their centres where they are nearest together $(2 b)$, and the vertical distance now between the wires $(2 c)$. A watch-glass is placed underneath the film and the threads are cut at their centres, so that the lower wire, the lower halves of the threads and the solution adhering, fall into the glass and are weighed. If this weight be $w$ grams, it can be shown that

$$
w g=\frac{2 T\left(c^{2}+a^{2}-b^{2}\right)}{a-b}
$$

54. Measurement of the Viscosity of a Liquid by its Rate of Flow through a Capillary Tube. - When a liquid flows through a tube, at any rate so long as the liquid wets the wall of the tube, the layer of liquid in immediate contact with the wall of the tube remains at rest. The speed with which the liquid moves increases as we go from the surface of the tube up to a maximum along the axis of the tube. Hence if we imagine the liquid to consist of a number of hollow cylinders coaxial with the tube, the fluid within each of these cylindrical shells will be moving with the same speed, but will be moving more slowly than that in the shell immediately inside, and more quickly than that in the shell immediately outside. This relative motion of adjacent layers of the liquid is resisted by what is called the viscosity of the liquid. The viscosity of a liquid is measured by the tangential force exerted on unit area of either of two planes which are parallel to the direction of motion of the fluid and at unit distance apart, one plane moving in its own plane with a velocity greater than the other by unity, the velocity of the liquid being supposed to change at a uniform rate as we pass from one plane to the other.

As long as the velocity with which the liquid is forced through the tube does not exceed a certain value, depending on the bore of the tube and the viscosity of the liquid, there is the following relation between the coefficient of viscosity $\eta$, the radius of the tube $R$, its length $l$, the 
difference of pressure $p$ acting on the fluid at the two ends of the tube, and the volume $V$ of fluid which passes through in the time $t,^{1}$

$$
V=\frac{\pi p R^{4} t}{8 l \eta}
$$

Thus by measuring the volume of liquid which flows through a tube of known dimensions under a measured head, we can calculate the coefficient of viscosity.

An arrangement suitable for measuring the coefficient of viscosity of a liquid such as water is shown in Fig. 58. The capillary tube $\mathbf{E}$ is held in a cork inserted in the end of the glass tube $\mathrm{D}$. This tube is itself connected by means of a cork with a metal cylinder A, which has a height of about $50 \mathrm{~cm}$. , and a diameter of about $10 \mathrm{~cm}$. A glass tube 1 passes through a second tubulure on the vessel $A$, and serves as an overflow, and ensures that the head remains constant so long as the supply

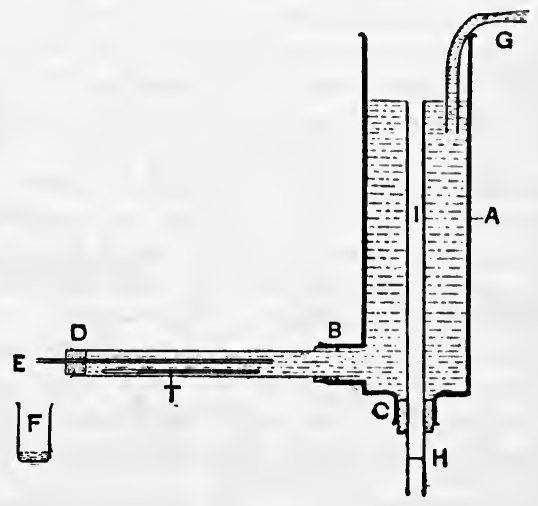

FIG. 58. of water through $G$ is at a greater rate than that of the escape through the capillary. A thermometer $\mathrm{r}$ serves to give the temperature of the water passing through the capillary. When the capillary is mounted in this way its temperature is kept constant by being surrounded by a considerable bulk of water, and, further, tubes of different lengths can be used without altering the arrangement of the apparatus. 'The water as it escapes is collected in a weighed beaker $F$. If in place of dropping from the end of the tube, the water, owing to capillarity, runs back along the outside of the capillary, a little vaseline should be smeared on the outside of the capillary tube near the end. Care must, however, be taken not to get any vaseline in the bore of the tube.

To obtain the head of liquid a mark $\mathbf{H}$ is made on the outflow tube, and the distance from this mark to the top of the tube is measured. Then, when making an experiment, the difference in height of this mark and the end $\mathrm{E}$ of the capillary tube is measured. This difference in height is most easily obtained by measuring the height of $\mathrm{H}$ and $\mathrm{E}$ above the top of the table and then taking the difference.

A tube about $30 \mathrm{~cm}$. long and with a bore of about $1 \mathrm{~mm}$. must be selected, and the uniformity of its bore tested by introrlucing a column of mercury about $5 \mathrm{~cm}$. long, and measuring the length 
occupied by the mercury in different parts of the tube. If the lengths of the column differ by more than about 5 per cent., the bore is too irregular, and another tube must be tried. A suitable tube having been selected, a column of mercury must be introduced, extending as nearly as possible the whole length of the tube, and the length of this column and the weight of the mercury must be measured in the manner described in $\S 52$. And as described in that section, the length having been corrected for the curvature of the ends of the column, the mean radius must be calculated. The total length of the tube having been measured, it must be fitted up as shown in Fig. 58.

A method of allowing for small variations in the radius of the tube and of obtaining an average value of $R^{4} / l$ is given in $\S 55$ on the determination of the viscosity of a gas.

A series of experiments should be made using different heads, which can be done by altering the height of the outflow tube $I$ in the reservoir. A second series should then be made using the same head but gradually shortening the capillary tube.

In the case of tubes where the velocity of efflux is considerable, a correction will have to be made to allow for the fact that part of the pressure is employed, not in overcoming the viscosity of the fluid, but in imparting to the fluid the kinetic energy with which it escapes from the end of the tube. The amount by which the pressure is reduced on this account is $\frac{\rho V^{2}}{\pi^{2} t^{2} R^{4}}$, where $\rho$ is the density of the liquid, so that the expression for the coefficient of viscosity becomes

$$
\eta=\frac{\pi p t R^{4}}{8 V l}-\frac{\rho V}{8 \pi l t}
$$

In general, if long narrow tubes are used, the second term on the right is negligible.

The expression for the quantity of liquid which flows through a tube can be obtained in the following manner:-Let the speed with which the liquid is moving parallel to the axis at, a distance $r$ from the axis be $v$. Then the stress due to viscosity parallel to the direction of motion acting on unit area is given by $\eta \frac{d v}{d r}$. Hence if we consider unit length of the tube, the stress over the cylindrical surface of radius $r$ is

$$
2 \pi r \eta \frac{d v}{d r}
$$

The stress over a cylindrical surface of radius $r+d r$ will be

$$
2 \pi \eta(r+d r) \frac{d v}{d(r+d r)}
$$

Now, if we write $f(r)$ for $r \frac{d v}{d r}$, we have by Taylor's theorem

$$
f(r+d r)=f(r)+d r \frac{d}{d r} f(r)+\& \mathrm{c} .
$$


or, neglecting terms in $(d r)^{2}$, we have

$$
(r+d r) \frac{d v}{d(r+d r)}=r \frac{d v}{d r}+\frac{d}{d r}\left(r \frac{d v}{d r}\right) d r+
$$

Hence the difference in the tangential forces acting on the two surfaces of the cylindrical shell of thickness $d r$ is

$$
-2 \pi \eta \frac{d}{d r}\left(r \frac{d v}{d r}\right) d r \quad . \quad \cdot \quad . \quad . \quad . \quad .
$$

Now, if $p$ is the difference in pressure between the two ends of the tube of length $l$, the difference in pressure between the ends of the shell will be $p / l$, which we will call $a$. Hence, since the area of the end of the annulus is $2 \pi r d r$, the resultant force acting on the anuulus, owing to the difference of the pressures, is

$$
2 \pi r a d r \text {. . . . . . . . }
$$

As the motion of the liquid is supposed to be steady, this force must be equal and opposite to the resistance due to viscosity, or

$$
-2 \pi \eta \frac{d}{d r}\left(r \frac{d v}{d r}\right) d r=2 \pi r a d r
$$

Hence integrating

$$
-\eta \cdot \frac{d v}{d r}=\frac{r^{2} \alpha}{2}+C
$$

where $C$ is a constant.

Dividing by $r$ and again integrating, we get

$$
-\eta v=\frac{r^{2} \alpha}{4}+C \log r+C^{\prime}
$$

where $C^{\prime}$ is a second constant. We have now to evaluate the values of the constants $C$ and $C^{\prime}$. In the first place, we see that since $\log 0=\infty$, if the constant $C$ is not zero, then $v$ must be infinite when $r=0$, i.e. along the axis of the tube. This of course is impossible, and hence $C$ is zero. To obtain $C^{\prime}$ we note that experiment has shown that at the surface of the tube $v=0$. Hence if $R$ is the radius of the tube we get

$$
\frac{R^{2} a}{4}=-C^{\prime}
$$

and hence

$$
\eta v=\frac{a}{4}\left(R^{2}-r^{2}\right) \text {. . . . . . . . }
$$

The volume of liquid which in a time $t$ passes through the annulus is

$$
2 \pi r d r . v t \text {. }
$$

The total volume which passes across any section of the tube is therefore

$$
\begin{aligned}
V & =2 \pi t \int_{0}^{R} v r d r \\
& =\frac{\pi a t}{2 \eta} \int_{0}^{R} r\left(R^{2}-r^{2}\right) d r \\
& =\frac{\pi a t R^{4}}{8 \eta},
\end{aligned}
$$


or

$$
\eta=\frac{\pi p t R^{4}}{8 V l}
$$

If we have to allow for the kinetic energy acquired by the liquid, then it can be shown ${ }^{1}$ that

$$
\eta=\frac{\pi p t R^{4}}{8 V l}-\frac{\rho V}{8 \pi l t}
$$

where $\rho$ is the density of the liquid.

55. Measurement of the Viscosity of a Gas.-If a gas is caused to flow through a capillary tube by the application of a constant pressure difference between the ends, the density of the gas diminishes and its velocity increases as it passes towards the end at the lower pressure. If the difference of pressure between the ends of an element $d l$ of the length of the tube is $d p$, then

$$
8 V \eta d l=\pi t R^{4} d p
$$

where $V$ is measured at the pressure $p$ at the element. Now if $p_{1}$ and $V_{1}$ be the pressure and volume at the entrance to the tube, while $p_{0}$ and $v_{0}$ are the corresponding quantities at the exit, $p V=p_{1} V_{1}=p_{0} V_{0}$. Hence

$$
8 p_{1} V_{1} \eta d l=\pi t R^{4} p d p .
$$

Integrating

$$
\begin{aligned}
8 p_{1} V_{1} \eta l & =\pi t R^{4}\left(p_{1}^{2}-p_{0}^{2}\right) / 2 \\
\eta & =\frac{\pi t R^{4}\left(p_{1}^{2}-p_{0}^{2}\right)}{16 p_{1} V_{1} l} .
\end{aligned}
$$

The viscosity of a gas may thus be found by measuring the volume of gas forced through a capillary tube, if the absolute pressures at the ends of the tube are known.

In the case of air or of any gas of which a considerable quantity is available, the following simple method may be used. A gas-tight vessel of considerable capacity is fitted with a rubber stopper through which three tubes are fixed. One tube is fitted with a tap and through it the gas is pumped into the vessel. The second tube is also fitted with a tap of wide bore, to the exit of which is fixed a long capillary tube. The third tube leads to a water pressure gauge. The tap leading to the capillary tube being closed, gas is pumped into the vessel until a pressure of about $30 \mathrm{~cm}$. of water is shown on the gauge. The apparatus is then left until it attains the temperature of its surroundings as shown by the gauge remaining stationary. The exit tap is then opened and readings are taken of the time and gauge reading as the gas flows out through the capillary tube until the excess pressure has fallen

${ }^{1}$ See Couette, Ann. de Chim. Phys. (1890), [6] xxi. 433. 
to about half the original excess pressure. Applying the above formula, $p_{0}$ is the atmospheric pressure, while $p_{1}$ is the pressure within the vessel which is equal to the sum of the atmospheric pressure and that shown by the gauge, and which gradually diminishes during the course of the experiment.

Let $Q$ be the volume of the vessel and let the pressure $p_{1}$ fall by amount $d p_{1}$ in time $d t$. The volume of the gas which has escaped, if measured at pressure $p_{1}$, would be $V_{1}=Q d p_{1} / p_{1}$.

Hence

$$
\begin{aligned}
& -16 \eta Q l \quad d p_{1}=\pi R^{4}\left(p_{1}^{2}-p_{0}^{2}\right) d t \\
& -\frac{1}{p_{1}^{2}-p_{0}^{2}} d p_{1}=\frac{\pi R^{4}}{16 \eta Q l} d t .
\end{aligned}
$$

If at times $t$ and $t^{1}$, the values of the gauge pressures are $P_{1}$ and $P_{1}^{1}$, the initial and final values of $p_{1}$ are $p_{0}+P_{1}$ and $p_{0}+P_{1}^{1}$. Integrating the equation and inserting these limits,

$$
\frac{1}{p_{0}} \log _{e}\left(\frac{P_{1}^{1}+2 p_{0}}{P_{1}+2 p_{0}} \cdot \frac{P_{1}}{P_{1}^{1}}\right)=\frac{\pi R^{4}}{8 \eta Q l}\left(t^{1}-t\right) .
$$

Professor Rankine has devised a neat and very simple method of determining the viscosity of a gas, which may be used even if only very small quantities are available. The apparatus required is shown in Fig. 59, and cunsists of two straight pieces of glass-tubing, each about $50 \mathrm{~cm}$. long, joined at the top and bottom by similar pieces of rubber-tubing. One of the tubes should be a fine capillary, $0.2 \mathrm{~mm}$. in bore, while the other should have an internal diameter of between 2.5 and $3 \mathrm{~mm}$. If a small quantity of clean mercury is placed in the wider tube, the circuit closed, and the tubes placed vertically, it will be found that the mercury pellet remains intact and slowly descends, forcing the gas below it

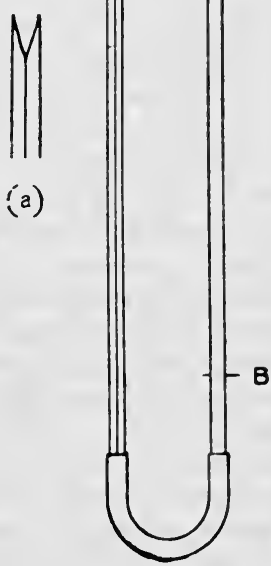

FIG. 59. through the capillary tube into the splace above it. It can be shown ${ }^{1}$ that, provided the space swept out by the pellet is symmetrically placed, the viscosity of the gias is given by the same formula as for a liquid

$$
\eta=\frac{\pi R^{4} p t}{8 l V}
$$

1 Proc. Roy. Soc., vol. Ixxxiii, 1910. 
where $p$ is the difference of pressure below and above the mercury and $V$ is the volume swept through in time $t$. The pressure $p$ is less than that due to the weight of the mercury over the crosssection of the tube, owing to the capillary effect between the upper and lower surfaces of the pellet and the sides of the tube.

It is particularly important that the wider tube should be quite clean, or the mercury thread will stick and descend intermittently. The tube should be washed with a weak solution of caustic soda, thoroughly rinsed with distilled water, and finally dried by drawing through it pieces of clean cotton wool. The bore of the capillary tube should be blown out into a cone at each end, as shown in Fig. $59(a)$, in order to avoid the formation of eddies. The rubber tubes should be of equal bore and length, and the connections should be airtight. Two marks $A$ and $B$ are made on the wider tube about $10 \mathrm{~cm}$. from each end. The experiment, in the first place, may be performed with air, the use of other gases necessitating a $T$-piece and tap at each end for evacuating and filling.

The dimensions of the tubes may be found in the usual manner by obtaining the weight of mercury required to occupy a measured length of the tubes. In the case of the wider tube, a capillary glass tap is attached to one end by means of a short piece of clean rubber tubing, thus forming a temporary burette. With this, several determinations of the mass of mercury which occupies the space between $A$ and $B$ are made. From the density of mercury at the temperature of experiment and the distance between the marks, the volume $V_{0}$ and the mean sectional area $a$ may be found. In the case of the capillary tube, the following method will give a mean value of $l / R^{4}$ and also allow for the effect of the conical ends, which, of course, will be small. Introduce into the tube a short thread ( 1 or $2 \mathrm{~cm}$. long) of mercury. Place the tube horizontally under a travelling microscope, and starting with one end of the thread well into the coned end, obtain readings of the two ends of the thread. By blowing or sucking, move the thread into the tube until the outer end coincides with the former position of the inner end and again take readings. Repeat until the whole length of the tube has been divided into sections of volume equal to that of the thread. If $v$ is the volume of the thread and $\lambda$ its length at a point where the radius is $r$,

$$
\begin{aligned}
v & =\pi r^{2} \lambda \\
r^{4} & =\frac{v^{2}}{\pi^{2} \lambda^{2}}
\end{aligned}
$$

and for the section of tube

$$
\frac{l}{R^{4}}=\frac{\lambda \cdot \pi^{2} \lambda^{2}}{v^{2}}=\frac{\pi^{2} \lambda^{3}}{v^{2}}
$$


For the whole tube

$$
\frac{l}{R_{4}}=\frac{\pi^{2}}{v^{2}} \sum \lambda^{3}
$$

The volume $v$ may be found by weighing the mercury threarl, but as it will be very small it may be found more conveniently as follows:The volume of the whole of the capillary tube (excluding the coned ends) is found from the weight of a mercury column required to fill it, and the length of the column noted. By simple proportion, the volume of a thread may be obtained equal in length to the average found previously (also excluding the ends).

Having obtained the dimensions of the two tubes, the wider one should, if necessary, be cleaned again, and one end joined by rubber tubing to the capillary. Into the other end about 5 grams of clean mercury is introduced and the circuit is completed by the second piece of rubber tubing. The tubes are fastened to a piece of wood or cardboard, so that they can easily be turned upside down. Several observations are made of the time occupied by the mercury pellet in describing the symmetrical interval between $\mathbf{A}$ and $\mathbf{B}$, when the tubes are alternately in the two vertical positions. The time between the passage of the tail of the pellet over one mark and the passage of the head over the other mark should be noted for correct symmetry. The experiment is then repeated with various smaller weights of mercury, the mass in each case being noted. Now the capillary effect being due to the ends alone, may be represented as a diminution of the weight which is the same for all the threads of mercury used. Thus if $m$ is the mass of a thread, $p=\frac{(m-e) g}{a}$ where $e$ is an unknown constant and

$$
\eta=\frac{\pi R^{4} g}{8 l a} \cdot \frac{(m-e) t}{V} .
$$

$V$ varies for the different pellets used, being the volume between $\Delta$ and $\mathrm{B}$ less the volume of the pellet. For the various pellets $(m-e) t / V$ should be constant, i.e. $m=k \frac{V}{t}+e$. By plotting $m$ against $V / t$ for the pellets, the value of the constant $k$ is given by the slope of the curve and

$$
\eta=\frac{\pi R^{4} g}{8 l a} k \text {. }
$$

56. Measurement of the Logarithmic Decrement of a Vibrating Body.- On account of the resistance of the air and the viscosity of the suspension fibre, the arnplitude of the vibrations of a torsion pendulum gradually decreases. In such a case it can be shown that if the resistance experienced is proportional to the velocity with which the vibrating body is moving, then the ratio of the amplitude of any vibration to the 
amplitude of the succeeding vibration is constant. Now, experiment has shown that in most cases of vibrating bodies this equality of the ratios of the amplitudes of successive vibrations holds, so that to a near approximation we may suppose that the resistance experienced by the vibrating body is proportional to the velocity.

Suppose that the vibrations of a torsion pendulum are being watched by observing the image of a scale seen reflected in a mirror attached to the pendulum, and that the readings on the scale corresponding to the cross-wire of the telescope at the extremities of the swings are $x_{1}, x_{2}, x_{3}$, $x_{4}$, \&c., where $x_{1}, x_{3}, x_{5}$, \&c., are the swings to the right, and $x_{2}, x_{4}, x_{6}$, $\& c$. , those to the left. If, further, the zero of the scale is at one end so that all the readings are positive and the reading corresponding to the position of rest is $x_{0}$, the amplitudes of the successive swings are

$$
x_{1}-x_{0}, x_{0}-x_{2}, x_{3}-x_{0}, x_{0}-x_{4}, \text { \&c. }
$$

Calling these amplitudes $a_{1}, a_{2}, a_{3}, a_{4}$, \&c., we have

$$
\frac{a_{1}}{a_{2}}=\frac{a_{2}}{a_{3}}=\frac{a_{3}}{a_{4}}=\quad=\mathrm{a} \text { constant }=\rho, \text { say. }
$$

Taking logarithms, we get

$$
\begin{aligned}
\log _{e} a_{1}-\log _{e} a_{2} & =\log _{e} a_{2}-\log _{e} a_{3}=\log _{e} a_{3}-\log _{e} a_{4}= \\
& =\text { a constant }=\lambda \text {, say . . . . }
\end{aligned}
$$

The quantity $\lambda$, which is the difference between the logarithms of successive amplitudes, or the logarithm of the constant ratio of successive amplitudes, is called the logarithmic decrement. The logarithms considered above are natural or Napierian logarithms. Hence if $l$ is the difference of the logarithms to the base ten of the successive amplitudes

$$
\lambda=2 \cdot 3026 l \text {. }
$$

In order to obtain the quantities $a_{1}, a_{2}, a_{3}$, \&c., it is necessary to know the scale reading corresponding to the position of rest. As in practice this is a decided inconvenience, the following change is advisable. Since if

$$
\frac{a_{1}}{a_{2}}=\frac{a_{2}}{a_{3}}=\frac{a_{3}}{a_{4}}=\frac{a_{4}}{a_{5}}=\quad \quad \quad=\rho .
$$

Then

Hence

$$
\frac{a_{1}+a_{2}}{a_{2}+a_{3}}=\frac{a_{2}+a_{3}}{a_{3}+a_{4}}=\frac{a_{3}+a_{4}}{a_{4}+a_{5}}=\quad=\rho \text {. }
$$

$$
\log _{e}\left(a_{1}+a_{2}\right)-\log _{e}\left(a_{2}+a_{3}\right)=\log _{e}\left(a_{2}+a_{3}\right)-\log _{e}\left(a_{3}+a_{4}\right)=\quad=\lambda .
$$

But

$$
\begin{aligned}
& a_{1}+a_{2}=x_{1}-x_{0}+x_{0}-x_{2}=x_{1}-x_{2}=A_{1}, \text { say, } \\
& a_{2}+a_{3}=x_{0}-x_{2}+x_{3}-x_{0}=x_{3}-x_{2}=A_{2}, \text { say, tc. }
\end{aligned}
$$


Not $x_{1}-x_{2}$ is the distance between the first extreme right-hand clongation and the next extreme left-hand elongation, and so on, and the determinations of these quantities does not involve the necessity of knowing the position of rest.

Hence for practical purposes $\lambda$ is derived from

$$
\lambda=\log _{e} A_{1}-\log _{e} A_{2} ; \lambda=\log _{e} A_{2}-\log _{e} A_{3} ; \lambda=\log _{e} A_{n-1}-\log _{e} A_{n} .
$$

Hence if we were to observe $n$ readings of extremities of swing ${ }^{1}$ we should have, adding the above equations together,

or

$$
\begin{aligned}
(n-1) \lambda & =\log _{e} A_{1}-\log _{e} A_{n} \\
\lambda & =\frac{\log _{e} A_{1}-\log _{e} A_{n}}{n-1} . \quad . \quad . \quad . \quad . \quad .
\end{aligned}
$$

where as before the logarithms are natural logarithms.

If; however, we employ this formula the accuracy of the result will depend on the accuracy. with which we observe the first and last elongations. Thus if the vibrations are fairly rapid, so that it is difficult to make accurate readings of the scale at the extremities of the swing, considerable error may result. Suppose, however, we start by observing an even number ( $2 r$ ) extremities of swing and obtain the amplitudes

$$
A_{1}, A_{3}, A_{5} \ldots A_{2 r-1} \text {, }
$$

then after a time, when the amplitude has fallen to about a third of its initial value, again observe $2 r$ extremities of swing and obtain the amplitudes

Now

$$
A_{n}, A_{n+2}, A_{n+4} \cdots A_{n+2 r-1} \text {. }
$$

Hence

$$
\frac{A_{1}}{A_{n}}=\frac{A_{3}}{A_{n+2}}=\ldots=\frac{A_{2 r-1}}{A_{n+2 r-1}^{2 r}}
$$

Thus

$$
\frac{A_{1}}{A_{n}}=\frac{A_{1}+A_{3}+A_{5} \ldots A_{2 r-1}}{A_{n}+A_{n+2}+A_{n+4} \cdots A_{n+2 r-1}} .
$$

$(n-1) \lambda=\log _{e}\left\{A_{1}+A_{3}+A_{5}++A_{2^{r-1}}\right\}-\log _{e}\left\{A_{n}+A_{n+2}++A_{n+2 r-1}\right\}$

and we make use of a number of scale readings of extremities of swing at either end of the series of observations.

If the vibrations die down fairly rapidly, so that only a comparatively small number of elongations can be olserved, then it will be advisable to note all the elongations till the amplitude has died down to about a quarter of its initial value. Then divide the observations into two groups and treat them by the method indicated by (3) above; $n$ being in this case equal to $2 r$, and one group following immediately after the other. In cases where the logarithmic decrement is very sinall, so that the 
amplitude only decreases very slowly, the above method of observation would be very tedious, since in order to get a sufficiently great decrease in elongation to give an accurate value of $\lambda$ would involve making a very large number of readings. In such a case it is best to determine the period $T$ ' of the vibrations, then observe, say, ten or twenty turning points so as to allow of the calculation of the elongations $A_{1}, A_{8}, A_{5}$ \&c. Then start a stop-watch, or note the time on a chronometer. After the vibrations have died down to about a third of their initial value ${ }^{1}$ stop the watch or note the time, and then again observe ten or twenty turning points. Now from a knowledge of the period and the interval which has elapsed we can at once calculate how many vibrations took place between the two sets of observations of turning points. As it will in general be found inconvenient to start the watch or read the time at the same instant as make the last observation of turning point, it is better to count one or two vibrations after the last reading before starting the watch or taking the time. In the same way, at the end count one or two vibrations after stopping the watch before beginning to read the turning points. Of course the number of swings thus counted will have to be added to the number deduced from the time as indicated by the watch. The method of making and recording the observations will be clear from the following example :-

\section{Determination of Logarithmic Decrement.}

\begin{tabular}{|c|c|c|c|c|c|}
\hline \multicolumn{2}{|c|}{ Turning Points. } & \multirow{2}{*}{ Elongation. } & \multicolumn{2}{|c|}{ Turning Points. } & \multirow{2}{*}{ Elongation. } \\
\hline Left. & Right. & & Left. & Right. & \\
\hline $\begin{array}{r}9 \cdot 50 \\
9 \cdot 55 \\
9 \cdot 60 \\
9 \cdot 70 \\
9 \cdot 75 \\
9 \cdot 80 \\
9 \cdot 90 \\
10 \cdot 00 \\
10 \cdot 05 \\
10 \cdot 10\end{array}$ & $\begin{array}{l}43 \cdot 80 \\
43 \cdot 70 \\
43 \cdot 65 \\
43 \cdot 55 \\
43 \cdot 50 \\
43 \cdot 40 \\
43 \cdot 35 \\
43 \cdot 30 \\
43 \cdot 20 \\
43 \cdot 15(a)\end{array}$ & $\begin{array}{lll}34 \cdot 30 & A_{1} \\
34 \cdot 15 & A_{3} \\
34 \cdot 05 & A_{5} \\
33 \cdot 85 & A_{7} \\
33 \cdot 75 & A_{9} \\
33 \cdot 60 & A_{11} \\
33 \cdot 45 & A_{13} \\
33 \cdot 30 & A_{15} \\
33 \cdot 25 & A_{17} \\
33 \cdot 05 & A_{19}\end{array}$ & $\begin{array}{l}13 \cdot 90(b) \\
14 \cdot 00 \\
14 \cdot 00 \\
14 \cdot 10 \\
14 \cdot 15 \\
14 \cdot 20 \\
14 \cdot 25 \\
14 \cdot 30 \\
14 \cdot 35 \\
14 \cdot 40\end{array}$ & $\begin{array}{l}39 \cdot 30 \\
39 \cdot 25 \\
39 \cdot 20 \\
39 \cdot 15 \\
39 \cdot 10 \\
39 \cdot 05 \\
39 \cdot 00 \\
38 \cdot 95 \\
38 \cdot 90 \\
38 \cdot 85\end{array}$ & $\begin{array}{l}25 \cdot 40 A_{143} \\
25 \cdot 25 A_{145} \\
25 \cdot 20 A_{147} \\
25 \cdot 05 \text { \&c. } \\
24 \cdot 95 \\
24 \cdot 85 \\
24 \cdot 75 \\
24 \cdot 65 \\
24 \cdot 55 \\
24 \cdot 45\end{array}$ \\
\hline \multicolumn{3}{|c|}{ Sum . . 336.75 } & \multicolumn{3}{|c|}{ Sum . . 249.10 } \\
\hline
\end{tabular}

Approximate period $=6 \cdot 76$ seconds.

Interval by watch $=6$ minutes 46 seconds.

Number of whole vibrations $=\frac{406}{6 \cdot 76}=60$.

1 If the logarithmic decrement is excessively small, then it will generally be unnecessary to wait till the amplitude has decreased so much as this. 
There was one half-vibration between the reading marked $(a)$ and starting the watch, and two half-vibrations after stopping the watch before the reading marked $(b)$.

\section{Hence}

$$
n=20+120+3=143 \text {. }
$$

Thus

and

$$
\begin{aligned}
142 l & =\log _{10} 336 \cdot 75-\log _{10} 249 \cdot 10 \\
& =2 \cdot 52731-2 \cdot 39637 \\
& =\cdot 13094 \\
l & =\frac{\cdot 13094}{142}=0.000922 \\
\lambda & =0.000922 \times 2.3026=0.002123 .
\end{aligned}
$$

57. Measurement of the Viscosity of a Liquid by the Oscillating Disc Method. - It has been shown by O. E. Meyer ${ }^{1}$ that when a thin disc oscillates in its own plane, in a liquid of which the coefficient of viscosity is $\eta$, under the influence of the torsion of the suspending wire, then

$$
\eta=\frac{16 M^{2}}{\pi \rho T\left(R^{4}+2 R^{3} \delta\right)^{2}}\left\{\frac{\lambda_{1}-\lambda_{0}}{\pi}+\left(\frac{\lambda_{1}-\lambda_{0}}{\pi}\right)^{2}+. \cdot\right\}^{2}
$$

where the symbols have the following meanings :-

$M=$ moment of inertia of the disc and its supports.

$R$ and $\delta=$ the radius and thickness of the disc.

$T=$ the time of vibration of the disc when vibrating in air.

$\lambda_{0}$ and $\lambda_{1}=$ the logarithmic decrements of small oscillations of the disc in air and in the liquid respectively.

$\rho=$ the density of the liquid.

The theoretical deduction of the above formula is not above criticism ; it may, however, be regarded as an empirical formula which gives results agreeing very fairly with those obtained by other methods.

A piece of apparatus suitable for performing this experiment is shown in Fig. 60. It consists of a wooden box, the sides of which can be closed by sheets of glass sliding in grooves, and having an upright brass tube with a $V$ notch at the top. The disc $\mathrm{A}$ hangs from a rod $\mathbf{B}$, which carries a mirror $\mathbf{M}$. This rod is soldered to the buttom of a fine steel wire, the top end of the wire being soldered to the rod c, which is clamped to a cross-bar D. This cross-bar rests in the notch at the top of the tube. A window $\mathrm{E}$ allows the vibrations of the disc to be watched, a telescope and scale being used in the ordinary manner (sce $\$ 170$ ). The liquid to be experimented upon is contained in a glass dish $\mathrm{F}$, which must be of such a size as to allow at least $3 \mathrm{~cm}$. clear all round the disc. 
To perform the experiment, first set the disc vibrating, and having damped out any pendulum vibrations by lightly touching the bottom of

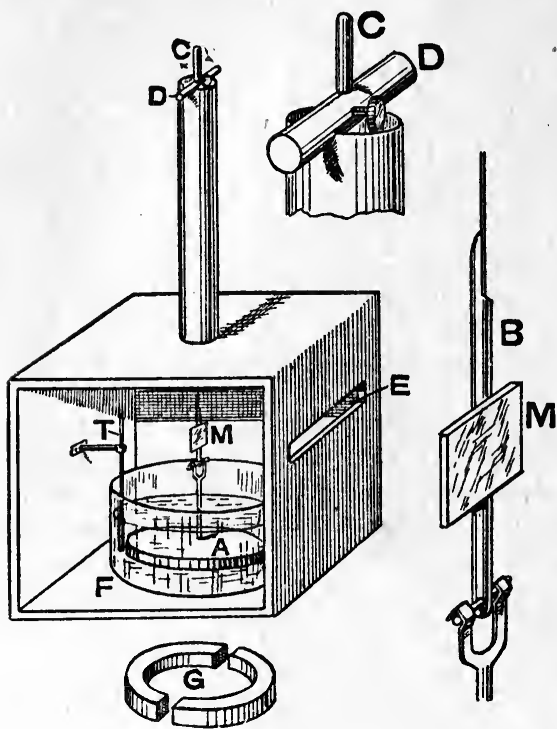
the suspending fibre, determine the period of the disc in air by the method given in $\S 43$. Next determine the period when the disc is loaded with the brass ring $G$, the outside diameter of which is equal to the diameter of the disc. Having determined the moment of inertia of the ring by calculation from its dimensions, calculate the moment of inertia of the disc and the supporting rod and mirror by the method used in $\S 43$.

After removing the ring, determine the logarithmic decrement of the disc by the method described in the last section.

Having filled the vessel $\mathbf{F}^{-}$ with water, stir it well, then start the disc vibrating, and read the temperature as Fig. 60. shown by the thermometer $\mathrm{T}$. Having determined the logarithmic decrement, again read the temperature. The mean of the two temperature readings may be taken as the temperature for which the viscosity has been determined. Since the viscosity changes very rapidly with temperature, it is important either to work at the room temperature, or, if observing at other temperatures, to protect the vessel from loss or gain of heat as much as possible by surrounding it with a layer of felt or other bad conductor of heat. 


\section{CHAPTER V I I I}

\section{THE BAROMETER}

58. Measurement of the Atmospheric Pressure.-In practice the value of the atmospheric pressure is always obtained by reading the height of the barometer, that is, by measuring the height of the column of mercury which will exert a pressure equal to that of the atmosphere. The form of barometer generally employed is that known as Fortin's cistern barometer (see Watson's Physics, \$133).

The chief difficulty with the Fortin form of barometer is setting the surface of the mercury to coincide with the tip of the ivory point which marks the zero of the scale. When the barometer is quite new, and therefore the surface of the mercury in the cistern is perfectly clean, this adjustment can be made with considerable accuracy, particularly if a lens having a focal length of about $5 \mathrm{~cm}$. is used. Griffiths recommends that the sides of the cistern be covered with black paper, small windows being left in front and behind the point. A fine black wire is stretched across the back window, and the position of the reading lens is so adjusted that an image of the line is seen formed by rays reflected in the mercury near the point. - Directly the point touches the mercury, the image will become distorted. The mercury must always be brought up to the point when making a setting whatever method is employed, since if the surface is lowered after having been raised too high it will be found that the mercury is apt to cling to the point, being raised up above the general level.

After some time it will be found that the surface of the mercury becomes more or less dirty, and then the difficulty of making a setting becomes very great. The condition of the surface can sometimes be temporarily improved by lowering the mercury in the cistern as much as possible and then bringing it back to its proper level. During this process some of the scum on the surface of the mercury is left clinging to the walls of the reservoir, and a fresh and clean surface is produced near the point.

By replacing the ivory point by one made of platinum iridium and having an insulated platinum wire dipping in the mercury of the cistern, an electrical method of noting when the point touches the mercury can be employed. The case of the barometer and the insulated wire are joined, through a fairly high resistance and a telephone, to a Leclanche cell, when a distinct click will be heard in the telephone when the mercury touches the point. 
A form of Fortin barometer which can be made at a small fraction of the cost of the ordinary pattern is shown in Fig. 61. The tube $\mathbf{A}$ has a bore of about a centimetre at the top and bottom, and is bent at the bottom as shown, a fine point of black glass

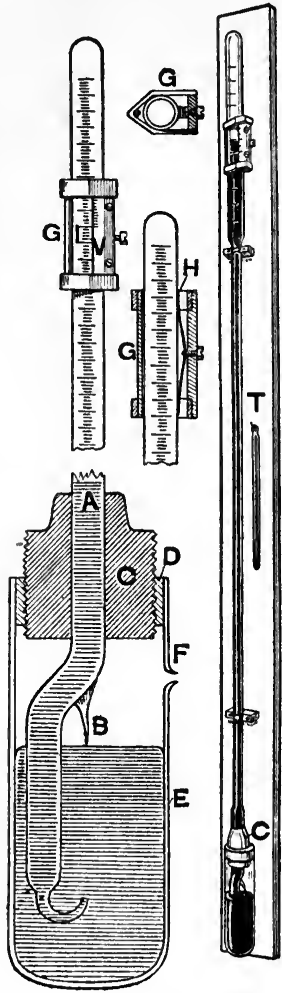

FIG. 61 . being attached at the bend, the extremity of the point lying on the prolongation of the axis of the upper part of the tube. A steel collar $\mathbf{c}$ is cemented to the tube just above the bend. A fine screw is cut on this collar, and a steel nut which engages with this screw is cemented inside a glass thimble E. This thimble has a small hole $\mathrm{F}$ in the side, which may be kept lightly plugged with cotton wool in order to exclude dust as much as possible. The surface of the mercury can be brought into coincidence witb the point $\mathrm{B}$ by turning the thimble. The upper part of the tube is graduated in millimetres, the divisions being numbered as if the scale started at the end of the point $B$. If it is only required to obtain the height of the barometer to within one- or two-tenths of a millimetre, then the scale may be read by eye. When, however, it is desired to obtain closer readings, the small slider shown at $G$ can be used. There is a spring $H$ in the slider which keeps the v's on one side pressed against the tube. Two openings $I$, one in front and the other behind, serve to view the mercury column, the upper edges being brought into line with the top of the mercury meniscus. A vernier $\nabla$ engraved on the slider is used to read the scale. This vernier has ten divisions, equal to eleven divisions of the scale, and is numbered downwards (see p. 43). The barometer is mounted on a board as shown, and a thermometer $\mathrm{T}$ is attached to the board, the bulb being enclosed in a piece of the same tube used to form the barometer. This piece of tube is filled with mercury, so that the thermometer bulb may, as far as possible, be subjected to the same conditions as it would if enclosed within the mercury column.

To take a reading of the height of a Fortin barometer we must proceed in the following manner :-

1. Read the temperature of the barometer as given by the attached thermometer. The temperature as read will generally require correcting for the error of the thermometer.

The reason for reading the thermometer before the barometer is, that the presence of the observer's body will most likely cause the reading of 
the thermometer to go up, though the heat will not have time to reach the column of mercury, and so affect the height, before the reading is made.

2. Having gently tapped the tube of the barometer near the upper surface of the column to"prevent sticking of the mercury to the sides of the tube, adjust the level of the mercury in the cistern so that the point, which corresponds to the zero of the scale, just touches the surface. The surface is raised till the point and its image, seen by reflection in the mercury surface, just appear to touch, and yet there is no depression of the mercury surface as indicated by the irregularity in the reflected image of some straight object, such as a wire, the reflection taking place alongside the point.

3. Adjust the vernier so that the bottom appears to be a tangent to the convex surface of the mercury. To make this adjustment satisfactorily a piece of white paper or milk glass placed behind the tube must be brightly illuminated. In order to avoid parallax in making the setting, the movable tube which carries the vernier is trimmed so that the back edge and the front edge, which corresponds to the zero of the vernier, are in the same horizontal plane. Thus if when making the adjustment the eye is placed so that the front and back edges coincide and further appear to touch the top of the mercury meniscus, then the vernier will be set correctly. When the adjustment is correct it should just be possible to see some light at the edges of the tube, but on slightly moving the eye up and down no light should appear at the middle. The vernier attached to the slider must then be read.

To the reading obtained as described above a number of corrections will have to be applied, and we now proceed to consider these in detail.

1. Correction for Temperature.-Since the pressure exerted by a column of liquid of given height depends on the density of the liquid, and that the density of mercury varies with the temperature, a correction will have to be applied on this account. Further, a correction will have to be applied for the expansion with rise of temperature of the scale which has been used to measure the height of the column, for it is only at one temperature that the distance between the divisions of the scale are what they profess to be. Hence we have to reduce the observed height $h_{t}$ to what it would be if the temperature of the mercury had some standard value. for which purpose $0^{\circ} \mathrm{C}$. is always taken, and further correct for the expansion of the scale. If $a$ is the coefficient of linear expansion of the material of which the scale is composed, and it is correct at $0^{\circ}$, then the actual length at a temperature $t$ corresponding to the observed length $h_{t}$ is $h_{t}(1+a t)$. Since the height of a column of liquid which will exert a given pressure varies inversely as the density of the liquid. If $\rho_{t}$ is the density of mercury at $t_{0}$ and $\rho$ the density at $0^{\circ}$, we have

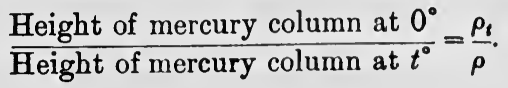


But if $v$ and $v_{t}$ are the volumes of unit mass of mercury at $0^{\circ}$ and $t^{\circ}$ respectively, and $\delta$ is the coefficient of cubical expansion of mercury, then

$$
\frac{\rho_{t}}{\rho}=\frac{v}{v_{t}}=\frac{v}{v(1+\delta t)}=\frac{1}{1+\delta t}
$$

Hence the corrected height $H$ of the mercury column is given by

$$
H=\frac{1+\alpha t}{1+\delta t} h_{t} \text {. . . . . . . }
$$

which may be written

$$
\begin{aligned}
H & =\frac{1+\delta t-\delta t+a t}{1+\delta t} h_{t} \\
& =\left\{1-\frac{\delta-a}{1+\delta t} t\right\} h_{t}
\end{aligned}
$$

that is, the correction to be applied to the observed height $h_{t}$ is

$$
-\frac{\delta-a}{1+\delta t} t h_{t}
$$

In most cases it will be sufficiently accurate ${ }^{1}$ to neglect $\delta t$ compared to unity, when the correction takes the simplified form

$$
-(\delta-a) t . h_{t} \text {. }
$$

If the scale instead of being correct at a temperature of $0^{\circ}$ is correct at a temperature $t_{0}$, then the true length corresponding to an observed length $h_{t}$ at a temperature $t$ is $h_{t}\left\{1+a\left(t-t_{0}\right)\right\}$. Hence the reduced height of the barometer in this case is given by

$$
\begin{aligned}
H & =\frac{1+a\left(t-t_{0}\right)_{h_{t}}}{1+\delta t} . . . . . . \\
& =\left\{1-\frac{\delta-a}{1+\delta t} t-\frac{a t_{0}}{1+\delta t}\right\} \quad . \quad . . . .
\end{aligned}
$$

or approximately, if we neglect $\delta t$ compared to unity,

$$
H=\left\{1-(\delta-a) t-a t_{0}\right\} \text {. }
$$

The quantity $\delta$ is the same for all mercury barometers, and is equal to 0.0001819 when the temperature is measured in degrees Centigrade. The value of $a$, however, depends on the material which has been used for the scale. If the scale is of brass, then we may take $a$ as being

1 In the case of a brass scale, if $h_{t}$ is $760 \mathrm{~mm}$. and the temperature is $30^{\circ} \mathrm{C}$. we have $\frac{\delta-a}{1+\delta t} t h_{t}=3.707 \mathrm{~mm}$. and $(\delta-a) t h_{t}=3 \cdot 728$, thus the difference amounts to $0.021 \mathrm{~mm}$. Hence if it is required to obtain the height correct to a hundredth of a millimetre, the more accurate expression must be employed. 
0.0000184 . Thus for a brass scale $\delta-\alpha$ is 0.0001635 . If the scale is of glass (verre dur) $\alpha=0.0000087$ and $(\delta-\alpha)=\cdot 0001732$. It is convenient to calculate a table which shall, for different temperatures in the neighbourhood of ordinary room temperatures and for different values of $h_{t}$ between about 65 and $79 \mathrm{~cm}$., give the value of the correction to reduce the height of the barometer to $0^{\circ} \mathrm{C}$.; that is, we tabulate for different values of $t$ and of $h_{t}$ the values of $h_{t} \frac{(\delta-\alpha) t}{1+\delta t}$. See Table 10.

A very handy graphical method of obtaining the barometer correction, which is capable of giving results correct to about $01 \mathrm{~mm}$., has been described by Melhmke. ${ }^{1}$ A diagram drawn by his method is given in the Appendix. A straight line from the observed height on the scales to the left or right, according as the barometer has a brass or a glass scale, to the temperature on one of the two middle scales will intersect the corresponding inclined scale at the required correction, which, for all temperatures shown, has to be subtracted from the observed height. The most convenient way of reading off the correction is by means of a straight line ruled on a sheet of glass or of celluloid.

In the case of some English barometers the scale is divided into inches, and the thermometer reads in degrees Fahrenheit. The scale in such a case is correct, not at the temperature of melting ice $\left(32^{\circ} \mathrm{F}\right.$.), but at $62^{\circ} \mathrm{F}$. The reduction formula in this case becomes

$$
\begin{aligned}
H & =h_{t}^{\prime}\left\{1-\left(\delta^{\prime}-\alpha^{\prime}\right)\left(t_{F}-32\right)-\alpha^{\prime}(62-32)\right\} \\
& =h_{t}\left\{1-\left(\delta^{\prime}-\alpha^{\prime}\right) t_{F}^{\prime}+32 \delta^{\prime}-62 \alpha^{\prime}\right\} .
\end{aligned}
$$

Here $\alpha^{\prime}$ and $\delta^{\prime}$ are the coefficients of linear expansion of the scale and of cubical expansion of mercury respectively in the Fahrenheit scale. For brass $a^{\prime}=0.00001022$, for glass $\alpha^{\prime}=0.00000483$, and for mercury $\delta^{\prime}=0: 0001010$.

Hence, substituting these values for $\alpha^{\prime}$ and $\delta^{\prime}$ in (6) we get

$$
\begin{aligned}
& \text { Brass scale } \ldots H=h_{t}\left\{1.002598-0.0000908 t_{t}\right\} . \\
& \text { Glass scale } \ldots H=h_{t}\left\{1.002933-0.0000962 t_{F}\right\} .
\end{aligned}
$$

It will be observed that at a temperature of $28^{\circ} \cdot 6 \mathrm{~F}$. the expression within the brackets is unity in the case of a brass scale. Hence at this temperature the barometer reading is correct. In the case of the glass scale, the temperature at which the barometer reading is correct is $30^{\circ} \cdot 5 \mathrm{~F}$.

2. Correction for Capillarity and Index Error.-Owing to the effect of capillarity at the surface of the mercury in the tube the column is shorter than it ought to be. The smaller the bore, the greater is this capillary depression. For any given tube it also depends to a certain extent on whether the mercury at the time of observation is rising or 
falling, that is, on the height of the meniscus. Tables have been prepared showing the amount of the correction for tubes of different bores with different heights of meniscus. See Table 11.

Since the value of the capillary depression depends very much on the cleanliness of the mercury and the state of the walls of the tube, it is better, when the height of the barometer is to be measured accurately, to employ a barometer with so wide a tube that the correction is negligible. Such is the case when the diameter of the bore of the tube is greater than $2.5 \mathrm{~cm}$. In the case of a barometer having a narrower bore, the best way of obtaining the capillary depression is to compare its reading with a standard barometer having a wide bore.

The index correction is to allow for the point of the index not being exactly at the zero of the scale. This also is best determined by comparing the reading of the barometer with that of a standard. In this way a single correction is obtained which includes both the capillary correction and the correction for index error. Such a comparison is generally performed at some observatory, and in England accurate barometers are sent to the Kew Observatory, which issues a certificate showing the correction.

3. Reduction to Sea-level and Latitude $45^{\circ}$.-By the application of the corrections considered above we obtain the height of the mercury column which at $0^{\circ} \mathrm{C}$. would exert a pressure at the place of observation equal to the atmospheric pressure. Since, however, the value of gravity varies from place to place, the same atmospheric pressure would not be represented by columns of mercury of the same height at every station, but only so long as the value of gravity is the same at all stations. Hence to obtain a number which shall represent the pressure and shall not involve in any way any local peculiarity of the station at which the mercury column was measured, we have either to allow for the actual value of gravity at the station and reduce the pressure to dynes per square centimetre, or calculate what would be the height of the mercury column suppose gravity had some particular fixed value. Since the value of gravity varies with the latitude of the place of observation, and also with the height of the station above the sea-level, the reduction consists of two parts to allow for these two effects. The standard value of gravity which is adopted is that at latitude $45^{\circ}$ and at the sealevel.

According to Helmert, the value of gravity at a station of which the latitude is $\phi$ and the height above sea-level is $l$ metres is given by

$$
g=g_{45}-2.593 \cos 2 \phi-0.0003086 l \text {. }
$$

The standard value of $g_{45}$ adopted by the Conférence des Poids et Mésures, Paris, 1913 , is $980.665 \mathrm{~cm}$. $/ \mathrm{sec}^{2}$., so that

$$
g=g_{45}(1-0.00265 \cos 2 \phi-0.00000031 l) .
$$

Since the height of the mercury column which will exert a given 
pressure is inversely as the value of gravity, if $H$ is the height reduced to $0^{\circ} \mathrm{C}$., and $H_{0}$ is the height corresponding to the standard value of gravity, we have

$$
H_{0}=H\{1-0.00265 \cos 2 \phi-0.00000031 l\} .
$$

The labour involved in the application of this correction can be considerably reduced by tabulating the values of the term $0.00265 \cos 2 \phi$ for different values of $\phi$, and of the term $0.00000031 l$ for different values of $l$, in each case for different heights of the barometer. Such tables will be found in the Smithsonian Physical Tables, and in Landolt and Bornstein's Tables. Since, however, for any given station both $\phi$ and $l$ are constant, it is quite easy for that place to make a table of the corrections to be applied to different heights of the barometer, or a curve may be drawn giving the correction for any height. 


\section{CHA P TER IX}

\section{THERMOMETRY}

59. Calibration of the Tube of a Mercury Thermometer.-A degree Centigrade on the mercury-in-glass thermometer is defined as such that when the temperature of a thermometer which is graduated on this scale is raised through 1 degree, the apparent increase in the volume of the mercury is $1 / 100$ th of the apparent decrease which takes place when the thermometer is cooled from the temperature of the steam given off by water boiling under standard conditions to the temperature of melting ice. If the tube of the thermometer is perfectly cylindrical, and the space between the marks corresponding to $0^{\circ}$ and $100^{\circ}$ is divided into a hundred equal parts, then the volume of the bore between each of these divisions and the next will be exactly a hundredth of the volume between the $0^{\circ}$ and the $100^{\circ}$ marks. With a thermometer having such a perfectly cylindrical tube, the temperature could be obtained directly by reading the position of the end of the mercury column. In practice, however, it is found that the bores of the glass tubes of which thermometers are made are never perfect cylinders ; in other words, the bore of all tubes is found to vary from point to point. Thus if the space between the $0^{\circ}$ and the $100^{\circ}$ marks is divided into a hundred equal parts, it will not follow that when the end of the mercury column advances through one of these divisions that the temperature of the thermometer has been raised through 1 degree, for if the bore between the two divisions considered happens to be wider than the mean bore between the fixed marks, then the volume of the part of the bore intercepted between these divisions will be greater than 1/100th of the volume of the bore between the fixed marks. Thus the thermometer will have been heated through more than 1 degree, and to obtain the true temperature from the reading given by such a thermometer we shall have to apply a correction, the magnitude of this correction being different at different parts of the scale. The process of determining the values of the corrections to allow for this irregularity of the bore of the tube is called calibrating the tube. In the case of all thermometers which are required for accurate work the space between the fixed marks is divided into divisions of equal length, and then the corrections which have to be applied are determined. In the case of thermometers which are only required for rough measurements, the maker sometimes roughly examines the bore of the tube, and then instead of dividing the stem into divisions of equal length he attemits to so regulate the spacing of the divisions that the volume of 
the bore included between any two adjacent divisions should be the same. This adjustment of the scale is in general a very rough affair, so that there are residual errors which have to be allowed for which necessitate the preparation of a table of corrections. Further, although the changes in the diameter of the bore are gradual, the maker, instead of adjusting the length of the divisions in the same gradual manner, a process which cannot be easily performed with a dividing engine, is satisfied with dividing the scale into a number of portions, throughout each of which he makes the divisions of equal length, the change from divisions of one length to the next taking place suddenly.

In order to determine the corrections which have to be applied to the readings of a thermometer to allow for the irregularities of the bore a thread of mercury is broken off the end of the mercury column, and the length of this thread is measured when it occupies different portions of the tube. Since the volume of the thread is constant, it follows that in a perfectly cylindrical tube, or in the case of a thermometer where the maker had perfectly adjusted the divisions, the length of the portion of the thread would be everywhere the same. In general, however, the length will vary as the thread is moved from one part of the tube to the other, and from the variations in the length we can calculate the corrections which have to be applied to different parts of the scale.

We will suppose that it is desired to calibrate the tube of a thermometer which reads from $0^{\circ}$ to $100^{\circ}$, and that it has been decided that it will be sufficient to determine the corrections at every 10 degrees between the two fixed marks. Suppose that a length of the mercury column about equal to 10 degrees has been separated, and that, starting with one end of this column near the zero and the other end at or near the $10^{\circ}$ mark, we measure the length of the column when it lies between $0^{\circ}$ and $10^{\circ}$, between $10^{\circ}$ and $20^{\circ}$, and so on. Let the lengths thus measured be $l_{1}, l_{2}, l_{3}, \& c$., and let the mean of all the lengths, namely, $\frac{1}{10}\left(l_{1}+l_{2}+\ldots+l_{10}\right)$, be $L$. If the bore were perfectly uniform, the length of the column would be everywhere the same and equal to $L$. Now $L$ represents the number of degrees which the column would occupy if it were placed in a tube having a perfectly uniform bore, and such that a length of it equal to the length of the tube being calibrated between the $0^{\circ}$ and $100^{\circ}$ marks would have the same volume as the volume of the tube between these marks. If, then, we suppose that we had two tubes -the actual one and the corresponding one having a uniform bore-and that they contained a thread of the same volume; when the ends of the two threads are both at $0^{\circ}$ the other end of the thread in the uniform tube will be at $L$, and that of the thread in the actual tube at $l_{1}$. Now the position of the thread in the uniform tube gives the position which ought to be occupied by the end of the thread in the actual tube. Hence the correction which has to be applied at $10^{\circ}$ is $L-l_{1}$, say $\delta_{1}$, for if we add $\delta_{1}$ to $l_{1}$ we get $L$, which is what the reading ought to be. Now move both threads so that they have one end at the $10^{\circ}$ marks and the other ends at or near the $20^{\circ}$ marks. The reading for the end in 
the case of the uniform tube will be $10^{\circ}+L$, while that for the actual tube will be $10^{\circ}+l_{2}$. Thus if we call $L-l_{2} \delta_{2}$, and suppose for the moment that the $10^{\circ}$ mark on the actual tube is correct, the correction near $20^{\circ}$ would be $\delta_{2}$. But the $10^{\circ}$ is not correct, for, as we have already seen, the correction there is $\delta_{1}$. If, then, the end of the thread instead of being at $10^{\circ}$ were placed at the reading which really corresponds to $10^{\circ}$, namely, $10^{\circ}+\delta_{1}$, its other end would also be moved through the distance $\delta_{1}$; in other words, the reading corresponding to this end would now be $10+L+\delta_{1}+\delta_{2}$, while the reading corresponding to the same end of the thread in the uniform tube would be $10+L$. Thus the correction to the tube at $20^{\circ}$ is $\delta_{1}+\delta_{2}$. In the same way it can be shown that the correction at $30^{\circ}$ is $\delta_{1}+\delta_{2}+\delta_{3}$, and so on.

Since we have assumed that the scale is correct at $100^{\circ}$, it follows that the correction at $100^{\circ}$ obtained in this way, namely, $\delta_{1}+\delta_{2}+\ldots$. $+\delta_{10}$, must be zero, and we shall thus have a test of the accuracy with which we have made our calculations. The fact that this sum is zero is, however, no test of the accuracy of the observations.

In the process above described it is important to note that since each of the measurements of thread length we make is subject to a possible observational error the results will be affected by these errors, and that the magnitude of the error at any of the points at which we have determined the correction depends on the number of times we have measured the length of the thread between the extreme points, that is, on the number of places along the scale at which we have determined the correction. Thus it is quite possible that by taking a very short thread, and thus determining the corrections at a great number of points of the scale, we may obtain a less accurate correction curve than if we had been content to use a longer thread and to obtain the corrections at the intermediate points by interpolation, say, by plotting the corrections obtained on squared paper and then drawing a curve freehand between these points. The probable error of the corrections is a maximum at the middle point of the scale, ${ }^{1}$ and hence if the tube is so irregular that a large number of points have to be observed on the correction curve, it is best to determine this curve by two sets of experiments. In the first set a comparatively long thread, say, $\frac{1}{2}, \frac{1}{3}$, or $\frac{1}{4}$ of the length between the extreme points, is used to determine the correction at 1,2 , or 3 equidistant points between the extremes, and then by means of a short thread the corrections at points intermediate between these principal points are obtained.

Unless the glass of which the thermometer is made is either "verre dur" or- Jena normal glass, any very great refinement in the determination of the calibration curve will be thrown away, owing to the fact that different glasses expand at different rates at the different temperatures.

1 For a discussion of the probable error and a description of this and other methods of calibrating a tube, see the Report of the Committee on the Calibration of Mercurial Thermometers, British Association Report for 1882 (Southampton Meeting). 
This fact is illustrated in Fig. 62, which shows the corrections ${ }^{1}$ which have to be applied to the readings of thermometers made of different kinds of glass to reduce the temperatures measured on these thermometers to the hydrogen scale. It must be borne in mind that all these curves refer to thermometers which have been calibrated to allow for irregularities in the bore. Practically, unless the glass is undoubtedly either one of the Jena glasses or "verre dur," we shall be uncertain of the temperature on account of the properties of the glass alone to about $0^{\circ} \cdot 05$

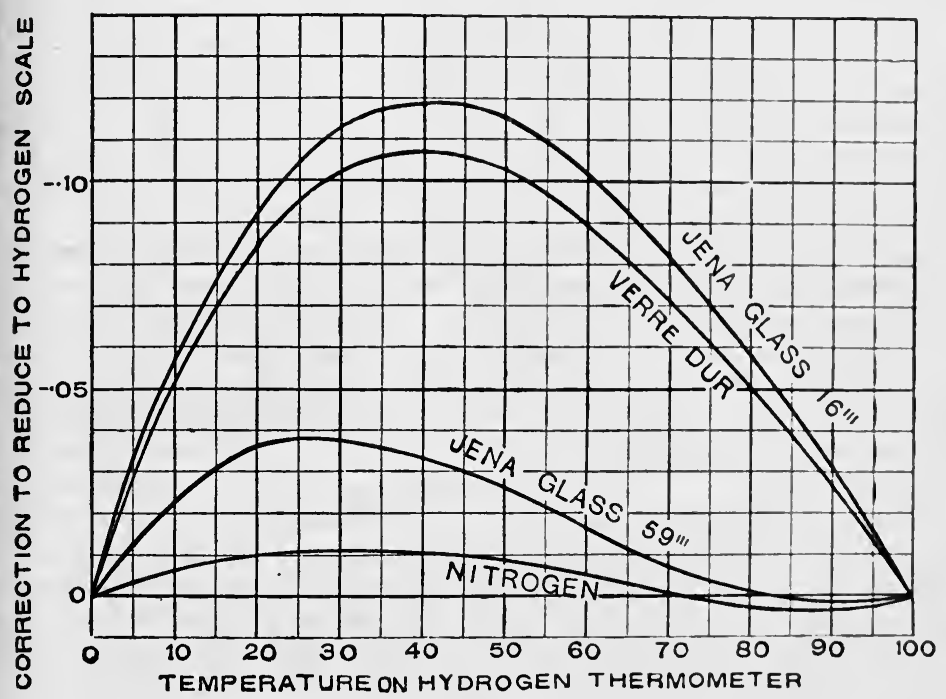

FIG. 62.

Centigrade, and hence it is unnecessary to determine the calibration corrections to less than this quantity.

When making a calibration, the first process to be performed is to detach a portion of the mercury column of suitable length from the rest. We will suppose that the corrections have to be determined for erery tenth degree of a thermometer which reads from $0^{\circ}$ to $100^{\circ}$ We have thus to separate a thread of mercury which shall occupy very nearly 10 degrees on the scale of the thermometer. The method aduited at the Bureau International at Sèvres, and given by Guillaume, is as follows :The thermometer is held in a vertical position, with the bulb upjermost and the other end resting on the tip of the index finger of the right hand. By giving the thermometer slight jars in an up-and-down direction the mercury is caused to run down the tube, and then by a

1 These curves have been plotted from numbers given by Chree, Mhil. Mag., March 1898, p. 218. 
rapid movement of the right hand the thermometer is reversed, and by striking it transversely against the left hand the mercury column is caused to separate at the junction of the stem and bulb. The thread of mercury left in the tube is then used to separate a thread of the desired length on account of the property of fine mercury columns of separating at the point of the bore where a column, such as has now been separated, is allowed to rejoin the rest of the column. The thermometer is placed in a horizontal position, and the lower part of the detached thread is brought to some noted division on the scale, say, the $15^{\circ}$ division, and then by gently heating the bulb in the left hand, or over a very small flame, the column is made to join on to the detached thread. Then the bulb is allowed to cool till the desired length of thread is beyond the division at which the junction took place, that is, in the case we have taken, when the extreme end of the mercury column is at $25^{\circ}$. When this point is reached the thermometer is given a slight jar in the longitudinal direction, when the column separates at the point of the tube where the junction took place. The separation is caused by the fact that when the junction took place a very minute bubble of air was left sticking to the side of the tube. The presence of the air is due to the fact that it is never possible to entirely deprive the mercury used to fill a thermometer of air.

The above method of separating a thread of the mercury is by no means easy, and requires a very considerable amount of skill and practice. The following method is much easier, but involves heating a portion of the tube of the thermometer to the temperature at which mercury boils.

A very small gas flame is obtained by using as burner a finely drawnout piece of glass tube (Fig. 63). The gas must be turned down till

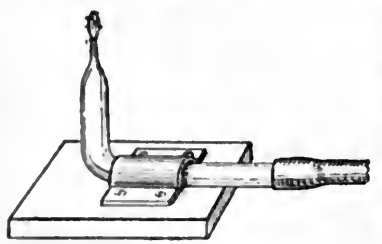

FIG. 63. the flame is about 4 millimetres high and is non-luminous. The thermometer is then held in a horizontal position, and the tube is heated at a point which intercepts a thread of the desired length. The tube must be brought near the flame slowly and kept in continuous rotation, so that it shall be heated uniformly all round. The heating is continued till the column separates. The separated thread is then run up to the top of the tube, and the bulb heated so that the mercury column passes over the heated part, and thus collects all the globules of mercury which will have condensed on the sides of the tube.

If reasonable care is used in the heating, the second method is quite safe. It is not, however, applicable to thermometers of the German pattern, in which the stem of the thermometer consists of a capillary tube placed alongside a milk glass scale, and enclosed together with this scale in an outside glass tube. Thermometers of this form necessitate the use of the first method given above.

Having separated a thread of the desired length, we have now to 
measure its length at different points along the tube. The length of the column has to be measured in terms of the scale of the thermometer, and hence it is necessary to measure in each position of the thread the amount by which the ends of the thread project beyond some whole number of divisions. To do this we may either estimate the fractions of a division by eye, aided if necessary by a lens, or we may employ an instrument, such as that shown in Fig. 64, called a vernier microscope. The chief objection to the use of a microscope for the purpose of measuring the thread length is that it is impossible to focus on the thread and the graduations of the scale at the same time. Unless the slide in which the microscope moves is very well made, it is not safe to focus first on the thread and then move the microscope so that it is in focus on the graduations. If the microscope is of fairly long focus, it will generally be advisable to so arrange matters that the thread and the divisions of the scale are about equally clearly in focus. In this case there is some danger of errors arising owing to parallax if the eye is not always placed

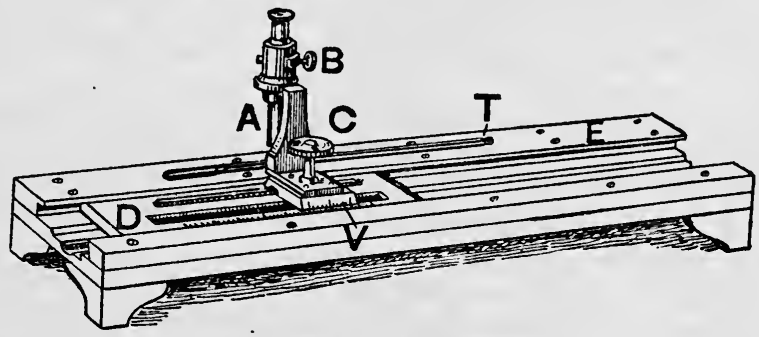

FIG. 64.

in exactly the same position with reference to the eye-piece. This may to a certain extent be obviated in the case where the diameter of the eye-hole is greater than the diameter of the pupil of the eye by fixing a piece of black card pierced with a hole the size of the pupil over the eye-hole of the eye-piece.

When using the vernier microscope three readings will have to be made at each end of the thread, one reading on the end of the thread, and the others on the division marks between which the end of the thread lies. The difference between these latter readings will give the length of the division in terms of the scale of the microscope, and from this the fraction of a division of the scale of the thermometer by which the thread projects beyond the division can immediately be calculated.

It will in general be found sufficient if the length of the thread is measured by eye, using a low power lens. In order to avoid parallax the thermometer must be placed on a sheet of mirror glass, and when taking a reading the eye must be so placed that the image of the eye in the mirror is alongside the position of the end of the mercury thread which is being observed, or the instrument shown in Fig. 67 may be 
employed. It will generally be found advisable to adjust the position of one end of the thread so that it lies as nearly as possible on one of the division marks. Having noted the length of the thread when the lower end approximately coincides with the $0^{\circ}, 10^{\circ}, 20^{\circ}, \&$ c., divisions, the measurements ought to be repeated, starting with the upper end in coincidence with the $100^{\circ}$ division, then with the $90^{\circ}$ division, and so on. The means of the lengths obtained in the two sets of measurements will then have to be used in the calculations. The object in thus beginning first at one end and then at the other is to vary to a certain extent the fractions which have to be estimated, and also to allow for any steady change in temperature which may take place during the measurements. Such a change in temperature will cause a change in the length of the thread. A change of temperature of 1 degree Centigrade will cause the thread to expand by 0.0002 of its length.

The numbers obtained are to be entered in a table, as in the following example :-

\begin{tabular}{|c|c|c|c|c|c|}
\hline \multicolumn{2}{|c|}{$\begin{array}{l}\text { Readings for Ends of } \\
\text { Thread. }\end{array}$} & \multicolumn{2}{|c|}{ Length of Thread. } & $\begin{array}{l}\text { Difference from } \\
\text { Mean. }\end{array}$ & Corrections. \\
\hline A. & B. & & $\begin{array}{l}\text { Means } \\
=l, \text { \&c. }\end{array}$ & $L-l, \& c$ & \\
\hline $\begin{array}{r}0.02 \\
10.01 \\
19.98 \\
30.00 \\
4000 \\
50.03 \\
611.02 \\
69.97 \\
80.00 \\
90 \cdot 01 \\
90 \cdot 12 \\
8007 \\
70.08 \\
60.09 \\
50.12 \\
40.08 \\
3002 \\
2009 \\
10.24 \\
0.11\end{array}$ & $\begin{array}{r}9 \cdot 86 \\
19 \cdot 79 \\
29 \cdot 90 \\
39 \cdot 98 \\
49 \cdot 92 \\
59 \cdot 89 \\
69 \cdot 86 \\
79 \cdot 87 \\
89 \cdot 96 \\
99 \cdot 91 \\
100 \cdot(12 \\
90 \cdot 01 \\
79 \cdot 98 \\
69 \cdot 95 \\
60 \cdot 00 \\
50 \cdot(10 \\
40 \cdot 00 \\
30 \cdot 03 \\
20 \cdot 04 \\
9 \cdot 97\end{array}$ & $\begin{array}{l}9 \cdot 84 \\
9 \cdot 78 \\
9 \cdot 92 \\
9 \cdot 98 \\
9 \cdot 92 \\
9 \cdot 86 \\
9 \cdot 84 \\
9 \cdot 90 \\
9 \cdot 96 \\
9 \cdot 90 \\
9 \cdot 90 \\
9 \cdot 94 \\
9 \cdot 90 \\
9 \cdot 86 \\
9 \cdot 88 \\
9 \cdot 92 \\
9 \cdot 98 \\
9 \cdot 94 \\
9 \cdot 80 \\
9 \cdot 86\end{array}$ & $\begin{array}{c}9.85 \\
9 \cdot 79 \\
9.93 \\
9.98 \\
9.92 \\
9.87 \\
9.85 \\
9.90 \\
9.95 \\
9.90 \\
\text { Sum }=94 \\
\text { Mean }=9.89 \\
=L\end{array}$ & $\begin{array}{l}+\cdot 04 \\
+\cdot 10 \\
-.04 \\
-.09 \\
-.03 \\
+\cdot 02 \\
+.04 \\
-.01 \\
-.06 \\
-.01\end{array}$ & $\begin{array}{l}+\cdot 04 \\
+\cdot 14 \\
+\cdot 10 \\
+\cdot 01 \\
-\cdot 02 \\
+\cdot 04 \\
+\cdot 08 \\
+\cdot 07 \\
+\cdot 01 \\
\pm \cdot 00\end{array}$ \\
\hline
\end{tabular}

In order to determine the corrections at intermediate points of the scale the observed points must be plotted on squared paper, the abscissæ 
being the readings on the thermometer scale, and the ordinates the corrections, taken above the zero line if they are positive, and below if they are negative (see Fig. 70). A curve must then be drawn passing as evenly as possible through all the points, remembering that the correction is zero at $0^{\circ}$ and $100^{\circ}$. A suitable scale for the ordinates will be one on which 2 centimetres represent the smallest division on the scale of the thermometer. Thus if the thermometer is divided in half degrees, 2 centimetres would be taken as representing half a degree, while if the thermorneter is divided in tenths of a degree, each centimetre would represent a twentieth of a degree.

If on plotting the observations it is found that the corrections vary considerably from point to point so that a curve passing through the points would be very wavy, it indicates either that the observations are not sufficiently accurate, the irregularities of the curve being due to the errors of experiment, or that the bore of the tube is very irregular, and we are not justified in filling in the parts of the curve between the observed points. A consideration of the individual readings will generally indicate which of the above is the true explanation. In particular, if the corrections are calculated independently for the readings made, starting from either end of the scale, the agreement or otherwise between the curves obtained will indicate the divergence to be expected on account of errors of observation.

60. Determination of the Upper Fixed Points of a Mercury Thermometer.-The upper fixed point $\left(100^{\circ}\right)$ is defined as the temperature of the steam given off from water boiling under a pressure of a standard atmosphere. A standard atmosphere is the pressure equivalent to the weight of a column of mercury $76 \mathrm{~cm}$. high at the sea-level and at latitude $45^{\circ}$, the temperature of the mercury being that of melting ice.

The reason that in the above definition the temperature of the steam is taken rather than that of the water is, that it is found that the temperature of the water depends to a slight extent on the nature of the vessel in which ebullition takes place. A more important reason, however, is that slight impurities, in the form of dissolved salts, in the water affect the temperature of the water to a considerable extent. The temperature of the steam given off from water containing dissolved salt is, however, the same as that of the steam given off from pure water. Thus by immersing the thermometer in the steam we avoid the necessity of employing perfectly pure water.

Since the temperature at which water boils depends on the pressure, we have to measure the pressure to which the water is subjected at the time when the reading of the thermometer is made. For this reason the barometric height must be read immediately before the thermometer is read. The barometric height, as given by the reading, will have to be reduced to what it would be if the scale employed were at the temperature at which it is correct and the mercury were at the temperature of melting ice $\left(0^{\circ}\right)$. Further, the height will have to be reduced 
to what it would be at sea-level and latitude $45^{\circ}$. The method of making these corrections has already been described in $\S 58$.

Knowing the pressure to which the water was subjected while determining the boiling point, the actual temperature of the steam is obtained from tables giving the boiling point of water for different temperatures. Such a table is given in the Appendix (Table 15). The difference between the temperature corresponding to the barometric

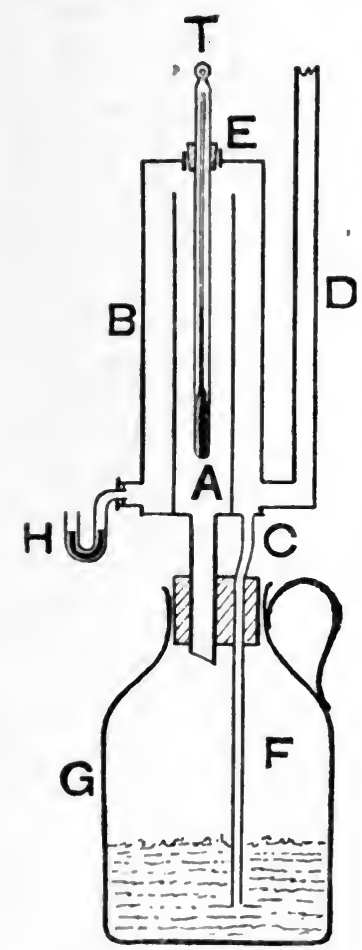

k'IG. 65. pressure and the reading of the thermometer will give the correction to the upper fixed point of the scale.

The thormometer of which the upper fixed point has to be determined is placed within a double walled steam-jacket, such as is shown in Fig. 65. It is held in place by means of a cork $\mathrm{E}$, or is suspended from a retort stand in such a way that the $100^{\circ}$ mark is just showing above the cork which closes the steam-jacket. The upright tube D serves as a condenser, so that no steam escapes into the air. The escape of steam in to the air of a laboratory is often objectionable, and, further, if the steam is not condensed and the water returned to the boiler, this latter is apt to run dry and be damaged. The tube $\mathrm{F}$ by which the condensed water is returned to the boiler must be long enough to dip below the surface of the water in the boiler. The $U$ tube $\mathrm{H}$ has a little water at the bend, and serves as a manometer to show whether the pressure inside the steam-jacket is the same as the atmospheric pressure. It is of importance that the pressure inside the apparatus should not exceed the external pressure, for it is this external pressure which is measured by a barometer, and we assume, when calculating the actual temperature at which the water boils, that this external pressure is the pressure to which the boiling water is subjected. If the manometer shows that the pressure inside the apparatus is higher than the external pressure, this shows either that the exit tube $\mathrm{D}$ is stopped up, or that the boiler is being heated too much, so that steam is being developed at such a rate that it is unable to escape as quickly as it is being generated. The flame under the boiler ought to be adjusted so that while the lower part of the tube $\mathrm{D}$ is quite hot, yet no steam is seen to issue from the top.

The thermometer ought to be exposed to the steam for about ten 
minutes, a reading should then be taken, and the height of the barometer noted, together with the temperature of the barometer. The thermometer must then be again read, and the reading should be the same as before. The barometer is then again read, including a readjustment of the level of the mercury in the cistern. Finally, the thermometer must be read a third time. The mean of the three readings of the thermometer is taken as the reading for the boiling. point, while the mean of the two barometer readings is used in calculating the actual temperature of the steam.

While the thermometer is in the steam, preparation must be made for the determination of the lower fixed point, so that immediately after the last reading in steam the thermometer may be removed, and after the reading has fallen to about $50^{\circ}$ it may be placed in the ice.

In order to avoid parallax, the thermometer ought to be read by means of a telescope. The telescope should be capable of focusing the scale of the thermometer when placed at a distance of about $40 \mathrm{~cm}$. Care must be

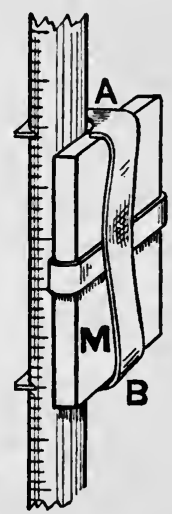

Fig. 66. taken that the thermometer is held vertical, while by means of a level the axis of the telescope is set horizontal. As these two adjustments are often troublesome where, as here, the greater part of the stem of the thermometer is hidden, the following modification, which may often be used when reading a temperature, may be employed :-A small piece of mirror glass $\mathrm{M}$ (Fig. 66), to which is attached a metal clip $\mathbf{A B}$, as shown, is fixed to the thermometer, so that the lower edge of the mirror is below the $100^{\circ}$ mark. The telescope is then adjusted and focused so that an image of the object glass, seen by reflection in the mirror, occupies the centre of the field. The telescope being firmly clamped in this position, the draw-tube is carefully pulled out till the scale and thread are in focus. By this means we adjust the axis of the telescope normal to the mirror, and since the thermometer is held by
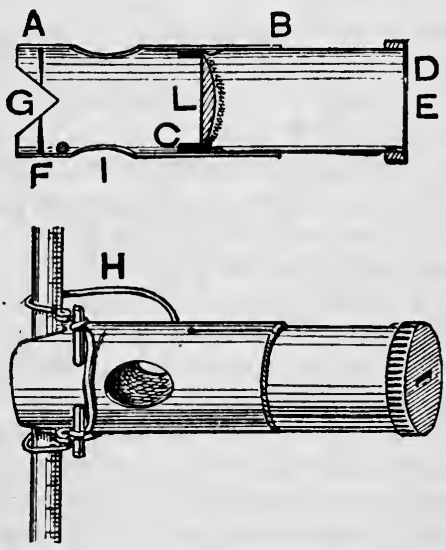

FIG. 67. the clip against the surface of the mirror, the axis of the telescope must be perpendicular to the stem of the thermometer.

Another method of avoiding parallax when reading a thermometer is to make use of the small instrument shown in Fig. 67.1 It consists

1 These thermometer microscopes may be obtained from Messrs. Griffin, Kingsway, London, W.C. 
of a short metal tube $\mathrm{AB}$, which has two $\mathrm{V}$ notches $\mathrm{G}$ and a spring $\mathrm{H}$, by means of which the tube is held against

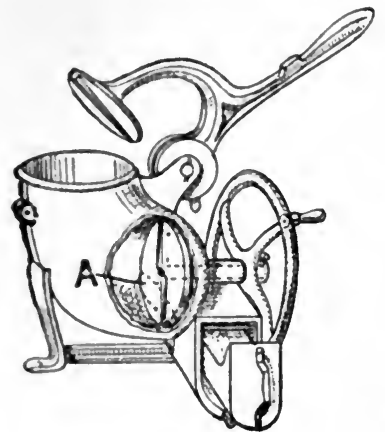

FiG. 68. the thermometer. A second tube CD slides within the first, and has a planoconvex lens $\mathrm{L}$ of about 5 centimetres focus at one end, and an eye-hole $\mathbf{E}$ at the othe end. Two metal pointers $\mathbf{F}$ are fixed on a diameter of the tube at right angles to the two notches $G$. The pointers are always brought opposite the point at which the reading is to be made, and hence the line of sight, that is, the line joining $\mathrm{E}$ to the line joining the pointers, is always perpendicular to the thermometer stem. The illumination is somewhat improved by having a hole $I$ cut in the side of the tube $\mathbf{A B}$.

61. Determination of the Lower Fixed Point of a Mercury Thermometer.-The lower fixed point on the Centigrade scale, or $0^{\circ} \mathrm{C}$., is the temperature of fusion of pure ice. Pressure has such a small influence on the temperature of fusion of ice that within the range of pressure ordinarily met with no appreciable variation takes place.

Since the presence of even a small quantity of impurity in the ice will appreciably lower the temperature of fusion it is most important that only pure ice should be employed, and that the vessel in which it is placed should be clean and the distilled water used free from all dissolved salts. The ice, having been washed in running water to remove the dirt on the outside of the lump, must be either pounded till the individual lumps are not larger than a pea, or, what is moro convenient, cut into shavings with the machine shown in Fig. 68 . The finely powdered ice is introduced into a glass funnel $\mathrm{A}$ (Fig. 69) capable of holding from two to three

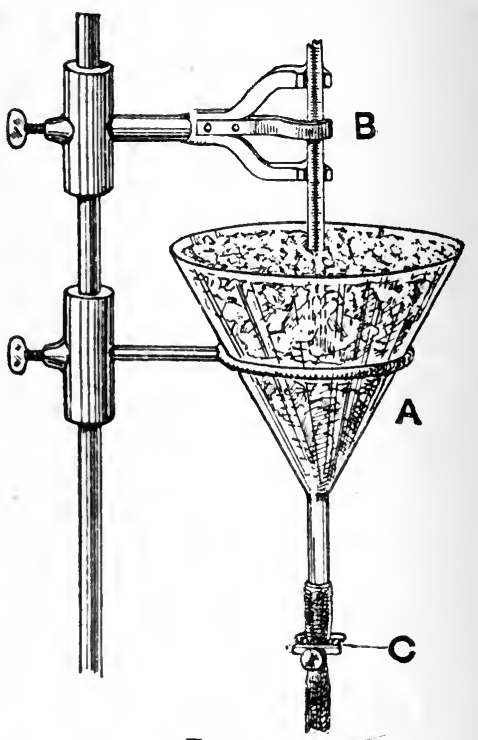

FIG. 69. litres, and fitted at the bottom with a length of india-rubber tubing, which can he closed by means of a pinch-cock $\mathrm{c}$. The ice is saturated with distilled water, and by means of a piece of wood the ice is firmly pressed down, 
additional ice being added so as to completely fill the funnel. The water having been run off the funnel is again filled with distilled water, the ice again pressed well down, more ice being added if necessary. A little of the water is then run off till the upper surface of the ice begins to become dry. A small hole is then produced in the ice by means of a glass rod held in the clip B. This hole must not go beyond the centre of the funnel.

The thermometer having been removed from the steam in which the upper fixed point was determined is allowed to cool till the temperature indicated is between $45^{\circ}$ and $50^{\circ}$. It is then introduced into the ice, being supported by the clip B, with the zero mark just above the surface of the ice. The zero reading should be taken immediately the mercury ceases to fall, a telescope being with advantage used to read the scale, as in the case of the boiling point. The reason the freezing point is determined as rapidly as possible after the boiling point is that there is a progressive rise in the zero, which sets in as soon as the temperature commences to fall (see $\S 67$ ). This rise is, however, small, and, in the case where we are only attempting to read the temperature to within a tenth of a degree, need hardly be considered.

62. Application of the Correction for Fundamental Interval. Suppose that by the methods described in the last two sections it has been found that the correction to a thermometer at $0^{\circ}$ is $\Delta_{0}$ and at $100^{\circ}$ it is $\Delta_{100}$; that is, that the reading on the thermometer when placed in melting ice at $0^{\circ}$ is $-\Delta_{0}^{\circ}$, while the reading when in steam at the standard pressure is $1\left(10-\Delta_{100}{ }^{\circ}\right.$. Thus the interval between the fixed points, or the fundamental interval as it is called, instead of being 100 degree divisions is $\left(100-\Delta_{100}+\Delta_{0}\right)$ divisions. In other words, the interval between the fixed points, in place of being divided into 100 degrees, is really divided into $\left(100-\Delta_{100}+\Delta_{0}\right)$ degrees. Each degree division on the thermometer therefore really corresponds to $\frac{100-\Delta_{100}+\Delta_{0}}{100}$ degree. Thus when the thermometer reads $t$ degrees the

true temperature will be obtained by adding to the correction at the zero of the scale, namely, $\Delta_{0}$, the correction due to the part of the scale between the zero and the division $t$. Now the correction to be applied on account of the error between the zero of the scale and $t$ consists of two parts. In the first place, there is the calibration correction $\delta_{t}$ to allow for the irregularity in the bore in the tube, which has been obtained on the supposition that the scale was correct at the two fixed points. Secondly, a correction has to be applied on account of the error in the fundamental interval. Since each degree on the scale really corresponds, as we have seen above, to $\left(100-\Delta_{100}+\Delta_{0}\right) / 100$, the correction on $t$ degrees on account of the fundamental interval amounts to $\frac{\left(\Delta_{100}-\Delta_{0}\right)}{100} t$.

Thus the total correction to be applied to the reading $t$ is

$$
\delta_{i}+\Delta_{0}+\frac{\Delta_{100}-\Delta_{0}}{100} t \text {. }
$$


By a slight addition to the curve (Fig. 70) on which the calibration corrections are plotted, we are able to read off directly the whole correction. Thus suppose on the calibration curve we take a point $\mathrm{A}$ on the

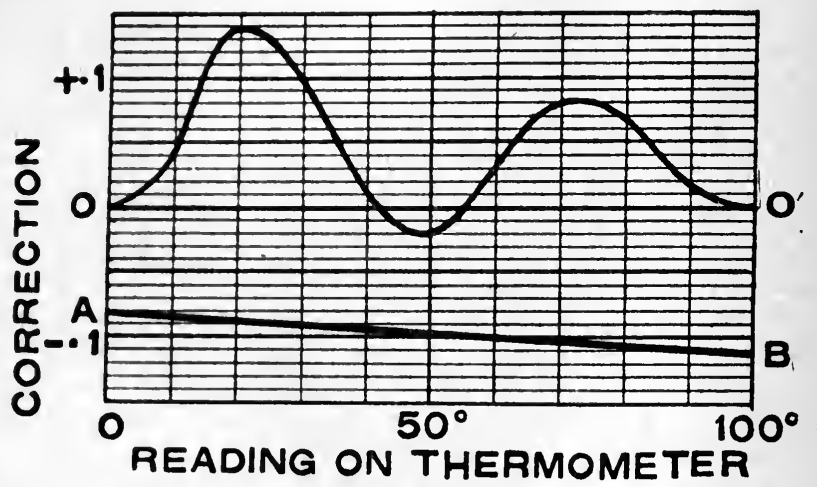

Fra. 70.

ordinate through the zero such that $\mathrm{OA}$ is equal to $-\Delta_{0}$. Also at the $100^{\circ}$ ordinate take a point B such that its distance from the axis of temperature is $-\Delta_{100}$. Then if the points $\mathrm{A}$ and $\mathrm{B}$ are joined by a straight line, the ordinate drawn through any temperature $t$ intercepted by this line will be equal to

$$
-\Delta_{0}-\frac{\Delta_{100}-\Delta_{0}}{100} t
$$

But the ordinate through $t$ of the calibration curve is $\delta_{t}$. Hence the difference of the two ordinates will be equal to

$$
\delta_{\mathrm{t}}+\Delta_{0}+\frac{\Delta_{100}-\Delta_{0}}{100} t
$$

Thus if we take as a new axis the line $A B$, then the readings on the calibration curve measured from this new axis will give the whole correction to be applied.

Since it is not very convenient to read from such an inclined axis as $A B$, it will be convenient to construct a new curve, such as that given in Fig. 71, where the ordinates measured from the line ox are equal to the corresponding ordinates measured from the axis $\mathrm{AB}$ in Fig. 70. The values of the fixed point corrections for the thermometer for which the calibration curve is drawn were $\Delta_{0}=+0.08, \Delta_{100}=+0.11$. Hence oA is taken downwards, i.e. towards the negative direction, and made equal to $-\cdot 08$; $\mathrm{o}^{\prime} \mathrm{B}$ in same way is $-\cdot 11$.

63. Determination of the Corrections to a Thermometer by Comparison with a Standard.-In most cases in the laboratory it is most 
convenient to use a thermometer of comparatively limited range, but which includes the temperatures to be measured. Thus to measure the temperature of, say, a resistance coil, a thermometer reading from $0^{\circ}$ to $30^{\circ}$ will have ample range, and without being unwieldy it can be given a much more open scale than if it were capable of reading from $0^{\circ}$ to $100^{\circ}$. It is, however, important that every thermometer should be capable of registering for one or two degrees on both sides of one or other of the fixed points. For simplicity in checking, and to avoid heating the thermometer to a high temperature, it is preferable that the point chosen should be the freezing point. If the thermometer is to read, say, between $15^{\circ}$ and $25^{\circ}$, it would render the stem unduly long to make it read from $0^{\circ}$ to $25^{\circ}$, so what is usually done is to blow a small auxiliary bulb, so arranged that when the thermometer is at zero the end of the mercury column is between the main bulb and this auxiliary bulb, and when the thermometer is at $15^{\circ}$ the

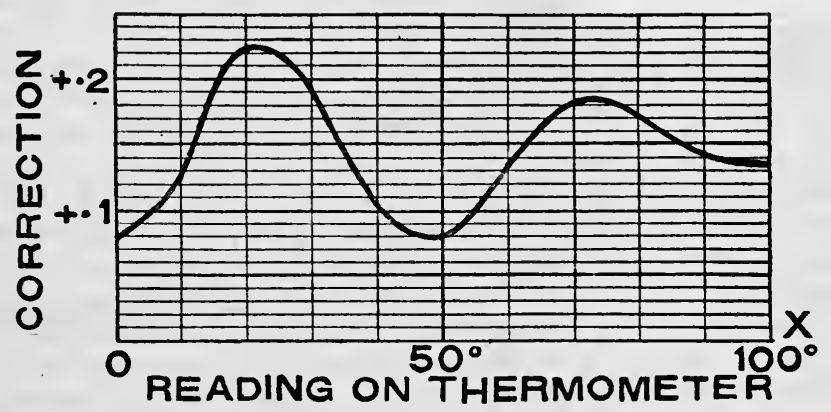

Fig. 71.

end of the column just projects above the auxiliary bulb. The reason for having one of the fixed points on the thermometer is to allow of the secular rise of the zero being determined.

Such thermometers as the above cannot conveniently have their corrections determined by a process of calibration, so the method employed to determine their errors at different parts of the scale is to compare their readings with those of a thermometer which has itself been calibrated. It will in general be quite sufficient if the thermometers are compared at every 5 degrees throughout the scale of the short-range instrument, since modern thermometer makers are able to obtain tubes in which sudden irregularities of bore are very seldom met with.

The thermometer or thermometers to be compared must be placed together with the standard in a position in which the temperature can be maintained constant for some time at all points where the thermometers are to be compared. For temperatures between the air tempera- 
ture and about $40^{\circ}$ a water bath will be found suitable. A suitable vessel for containing the water is a copper box in the form of a hollow cube open at the top. This box can be placed in a wooden box which is large enough to allow a space of about $5 \mathrm{~cm}$. on all sides, which space can be packed with cotton wool. The most convenient arrangement for stirring consists of a small propellor c (Fig. 72) driven by a cord, which

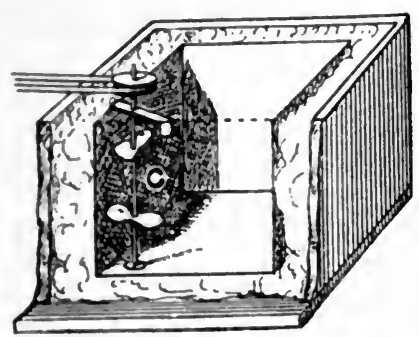

FIG. 72. is itself driven by a small electric motor. In order to heat the liquid in the bath, by far the most convenient arrangement is a small coil of fine manganine wire which has been treated with shellac varnish in the manner described in the Appendix. This coil is placed in series with an adjustable carbon resistance and a storage cell. Owing to the passage of the current heat is developed in the coil, and the rate of heat evolution can be adjusted with great ease by adjusting the resistance.

Another method of heating the water is to use an L-shaped piece of stout copper bar, of which one arm dips in the water and the other arm is horizontal and projects over the edge of the wooden box. By placing a Bunsen burner under the projecting portion of the copper bar heat is conveyed by conduction through the copper to the water, and moving the flame to different distances along the bar allows of the supply of heat being adjusted with very considerable delicacy.

The thermometers to be compared must be suspended in such a manner that the tops of the mercury threads can be seen over the edge of the box and so that all their bulbs are close together. ${ }^{1}$ It is generally best to arrange a separate reading telescope to read each of the thermometers, as in this way there is little delay when taking a set of readings between reading the several thermometers.

The resistance in the heating circuit is so adjusted that the temperature of the bath becomes practically stationary at the different temperatures at which a comparison is to be made. When the temperature is stationary, the rate at which heat is developed within the coil is exactly equal to the rate at which the bath is losing heat by radiation, conduction, and evaporation, \&c. The reason the temperature has to be pretty well stationary when a comparison is made, is, that unless the thermometers are all exactly alike the quickness with which the thermometers follow the changes in temperature of the bath will be different, and hence their readings will differ from what they would be if the temperature of the bath were stationary by different amounts. If it is found impossible to arrange for the temperature of the bath being stationary, the effects of the different heat capacities of the different bulbs can in a great measure be got over by immersing all the bulbs of

I It may be necessary to apply corrections for emergent column. See $\$ 65$. 
the thermometers in a small vessel of mercury supported in the middle of the bath.

For temperatures over $40^{\circ}$ a water bath is not suitable, owing to the rapid loss of heat which goes on owing to evaporation, and also to the moisture which condenses on the stems of the thermometers. By replacing the water by glycerine or oil and heating the bath below by means of a flame, the comparison can be continued above $40^{\circ}$. It is, however, necessary that the bath should contain a very considerable volume of liquid and be very well stirred. Owing to the difficulty of adjusting the supply of heat so that the temperature may remain stationary while the thermometers are read the experiment is very tedious, and unless a number of thermometers have to be compared with a standard at the same time, the instrument described below will be found much more convenient and accurate.

The action of the instrument depends on the fact that the temperature of the vapour given off by a pure liquid boiling under a constant pressure is constant, and hence if the thermometers to be compared are surrounded by such a vapour, their temperature must be the same. Further, by merely altering the pressure we can alter the temperature of the vapour.

A general view of the instrument is shown in Fig. 73. A glass tube $A B$, closed at the top and open below, about $76 \mathrm{~cm}$. long and $2.5 \mathrm{~cm}$. in diameter, passes up inside a second glass tube of about the same length and $4.7 \mathrm{~cm}$. in diameter. The space between these two tubes is closed at the bottom by an india-rubber cork c (Figs. 73 and 74),

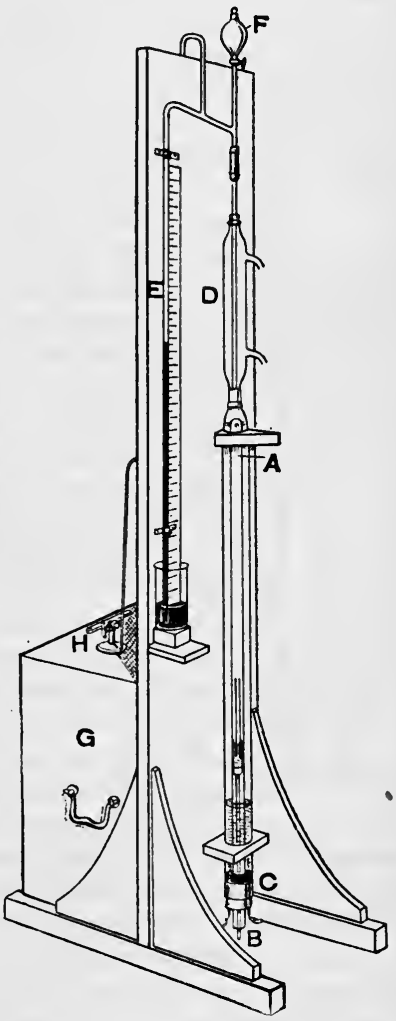

F1G. 73. while at the top the inner tube is kept in place by three small pieces of glass rod fused on and forming a triradiate star. The upper end of the outside tube is fused on to a Liebeg condenser $D$, the upper end of the condenser being connected by a rubber joint fitted with a glycerine seal to a manometer E, a stoppered funnel F, and a large glass bottle which acts as a reservoir. This bottle is packed round with cotton wool, and is enclosed in a wooden box $\mathrm{G}$. The air can be exhausted from or admitted to this bottle by means of a three-way tap н. Some mercury is placed on the 
top of the cork c (Fig. 74) to prevent the liquid used to form the vapour touching the cork. The liquid is heated by means of a spiral of fine uncovered platinum wire, the terminals being fused into glass tubes which pass down through the mercury and the cork.

The vapour of the boiling liquid rises between the inside and outside tube, is condensed in the condenser $\mathrm{D}$, and runs down to the bottom again.

The thermometers are fastened together, their bulbs resting in a small glass vessel $\mathrm{k}$ (Fig. 74), which is filled with mercury. This vessel is supported by a glass rod which rests on the table on

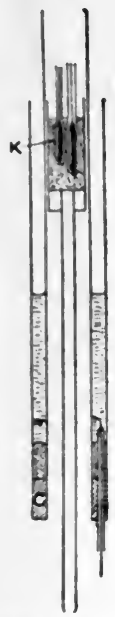
which the instrument stands. To remove the thermometers the instrument is drawn to the edge of the table till this rod can be lowered, and with it the thermometers. The scales are read by means of two telescopes, it being quite easy to see through the vapour if suitable liquids are used; carbon bisulphide $\left(20^{\circ}\right.$ to $\left.46^{\circ}\right)$, ethyl alcohol $\left(46^{\circ}\right.$ to $\left.79^{\circ}\right)$, and chlorobenzene $\left(79^{\circ}\right.$ to $\left.120^{\circ}\right)$ do very well. Water cannot be used, as it forms drops on the inside of the glass so that the thermometers cannot be read.

In order that the india-rubber cork may form a thoroughly air-tight joint it must be well cleaned with benzene, coated with india-rubber solution, and put in place while the solution is wet. When the solution has got thoroughly dry, which takes four or five days, the joint will be quite air-tight.

With such an arrangement the thermometers can easily be maintained at a temperature constant to within $0^{\circ} .01$ for three or four hours together. The manometer is only used to adjust the temperature to the desired point, a table of vapourpressures being employed. In about half-an-hour after alterNig. 74. ing the pressure, and hence the temperature, the thermometer readings become quite constant. The instrument once started can be left entirely to itself; the only thing the observer has to do is to read the thermometers, then let a little more air in to get the next higher temperature, and so on.

64. Auxiliary Fixed Points-Transition Temperatures. - It is sometimes convenient to have some other fixed temperatures besides $0^{\circ}$ and $100^{\circ}$, and thus to directly determine the error of a thermometer at these points. For high temperatures the fixed points used are generally the boiling points of sulphur, mercury, and the freezing points of some metals. Since, however, mercury thermometers cannot satisfactorily be used at temperatures much over $200^{\circ}$, the description of the methods of using such fixed points will be postponed till the sections dealing with platinum resistance thermometers and thermocouples.

It is, however, very convenient to have a fixed point somewhere near the ordinary air temperature. Richards ${ }^{1}$ has shown that such a fixed point is supplied by the temperature at which hydrated sodium sulphate $\left(\mathrm{Na}_{2} \mathrm{SO}_{4}, 10 \mathrm{H}_{2} \mathrm{O}\right)$ melts. There is only one temperature, the pressure

${ }^{1}$ Proc. American Acad. (1902), xxsviii. p. 431. 
being kept constant, at which anhydrous sodium sulphate $\left(\mathrm{Na}_{2} \mathrm{SO}_{4}\right)$, hydrated sodium sulphate $\left(\mathrm{Na}_{2} \mathrm{SO}_{4}, 10 \mathrm{H}_{2} \mathrm{O}\right)$, the saturated solution and the vapour can coexist in a state of equilibrium. This temperature is called the transition temperature. If a mixture of the above substances is placed in an environment at a higher temperature than the transition temperature part of the hydrated salt will go into solution, a process which, owing to the latent heat of the solid, involves an absorption of heat. If the surrounding temperature is below the transition temperature, then some of the saturated solution will form crystals of the hydrated salt and heat will be evolved. In either case, therefore, the temperature of the mixture will remain constant till either all the crystals of the hydrated salt are melted, or till all the solution has crystallised.

The sodium sulphate crystals must be pure, and they can be prepared by recrystallising two or three times the commercial "pure" salt. The crystallising ought to be continued till the transition temperature remains constant.

The arrangement used in testing a thermometer at the transition temperature of sodium sulphate is shown in Fig. 75. The thermometer $T$ passes through a cork in the mouth of the glass boiling-tube $\mathrm{A}$, the sodium sulphate being contained in this tube. The tube $\mathrm{A}$ itself passes through a cork in the outer glass tube $\mathrm{B}$, the interspace forming an air-jacket to prevent the too rapid heating of the sulphate. The outer tube $\mathbf{B}$ is placed in a water bath $\mathrm{c}$, this bath being kept well stirred and at a temperature of $33^{\circ} \mathrm{C}$. The necessary supply of heat can most easily be provided by a coil of German silver wire immersed in the water, an electric current being passed through the wire and, by means of a variable resistance in the circuit, the current being so adjusted that the

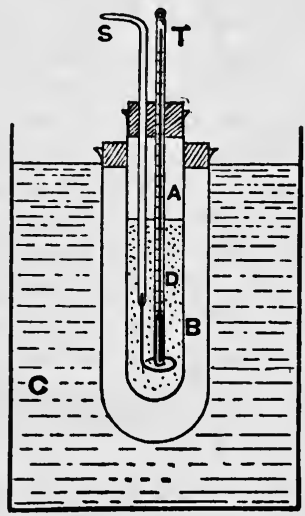

FIG. 75. temperature remains constant.

The sodium sulphate crystals are powdered, and if necessary some anhydrous sodium sulphate added. The efflorescence which naturally occurs when the hydrated crystals are kept is in general quite sufficient to provide the necessary quantity of anhydrous salt. The reason for adding the anhydrous salt is to be certain that there is no water present besides the water of crystallisation. The crystals having been introduced the thermometer is put in place, and the tube $\mathbf{A}$ is heated till, by the melting of some of the crystals, a pasty mass results. The tube $\mathbf{A}$ is then placed within the tube B, and the whole placed in the water bath. The crystals are kept well stirred, and the thermometer read at intervals. When the thermometer reading becomes constant, the temperature is the transition temperature, which Richards has found to be 
on the hydrogen scale. By comparing the reading of the thermometer with the above value, the error of the thermometer is obtained.

If the temperature of the bath is maintained between $32^{\circ} .5$ and $33^{\circ}$, the temperature of the sodium sulphate will be found to remain constant for two or three hours. The accuracy with which this temperature can be reproduced is about the same as that with which the freezing point can be determined, and is greater than that with which the boiling point can be measured unless very accurate pressure measurements are made. The use of this fixed point has another recommendation in that it avoids the necessity of heating a thermometer, which is ordinarily only used at temperatures near that of the air, to such a high temperature as $100^{\circ}$, and hence producing a serious depression of the zero (see $\S 67){ }^{1}$

65. Correction of Thermometer Reading for Emergent Column.In many cases where a mercury thermometer is used, it is not possible to arrange so that the temperature of the thread is the same as that of the bulb. If, as is usually the case, the temperature of the thread is less than that of the bulb the reading will be too low, for the mercury in the cold part of the thread will not have expanded so much as it would, supposing the temperature had been the same throughout.

By making the assumption that the bulb and stem, up to the division corresponding to the temperature $t_{0}$, is at one temperature, and that the rest of the stem is at a temperature $t^{\prime}$, then we can calculate the correction which must be applied to the observed reading $t$ to obtain the true temperature to which the bulb and lower part of the scale is subjected. Let $v$ be the volume of 1 degree of the capillary, then the volume of the mercury in the emergent portion of the column is $v\left(t-t_{0}\right)$, and if this volume of mercury were heated to a temperature $t$, its apparent increase in volume would be

$$
(\gamma-c) v\left(t-t_{0}\right)\left(t-t^{\prime}\right)
$$

where $\gamma$ is the coefficient of expansion of mercury, and $c$ the coefficient of cubical expansion of the glass. The number of degrees corresponding to this apparent increase of volume is

$$
(\gamma-c)\left(t-t_{0}\right)\left(t-t^{\prime}\right)
$$

and hence this quantity expresses the correction for emergent column. For most kinds of thermometer glass $(\gamma-c)$ is $\frac{1}{6400}$.

The correction calculated in this way is not of much value, since the assumptions we have made are certainly erroneous. Thus the transition from the portion of the stem, which is at a temperature $t$, to that at a temperature $t^{\prime}$ is gradual, and not sudden, as has been assumed.

An elaborate scries of experiments has been made by Rimbach ${ }^{2}$ to determine experimentally the correction for the emergent cool column, and tables have been drawn up embodying the results. The following table gives the correction in the case of (1) the ordinary solid stem thermometers, and (2) the German pattern, in which a fine capillary is enclosed in an outer glass tube. In the table $t$ is the temperature as shown by

1 The transition temperature of $\mathrm{MnCl}_{2} .4 \mathrm{H}_{2} \mathrm{O}$, viz., $58^{\circ} .089 \mathrm{C}$., is another useful fixed temperature.

2 Zeitsch. für Instrumentenkunde (1890), x. 153. 
§ 65] CORRECTION FOR EMERGENT COLUMN

the thermometer, and $t^{\prime}$ the temperature shown by an auxiliary thermometer, of which the bulb is on a level with the mid point of the exposed thread, and at a distance of $10 \mathrm{~cm}$. from it. This auxiliary thermometer must be screened from the direct action of any source of heat used to heat the vessel in which the main thermometer is placed. The quantity $n$ is the length of the exposed thread measured in 1 degree divisions of the thermometer. The correction, when the temperature of the thread is below that of the bulb, is additive. The tables are for thermometers of Jena glass having the degree intervals from 9 to $1 \cdot 1 \mathrm{~mm}$. long.

Thermometer with Enclosed Scale (German Pattern).

\begin{tabular}{|c|c|c|c|c|c|c|c|c|c|c|}
\hline \multirow{2}{*}{$n$} & \multicolumn{9}{|c|}{$t-t^{\prime}$} & \multirow{2}{*}{$n$} \\
\hline & $70^{\circ}$ & $80^{\circ}$ & $90^{\circ}$ & $100^{\circ}$ & $110^{\circ}$ & $120^{\circ}$ & $140^{\circ}$ & $160^{\circ}$ & $180^{\circ}$ & \\
\hline 10 & $\cdot 01$ & $\cdot 01$ & $\cdot 03$ & .04 & .06 & $\cdot 07$ & $\cdot 10$ & $\cdot 13$ & $\cdot 17$ & 10 \\
\hline 20 & .08 & $\cdot 12$ & $\cdot 14$ & $\cdot 19$ & .23 & .25 & .28 & .32 & $\cdot 40$ & 20 \\
\hline 30 & $\cdot 25$ & $\cdot 28$ & $\cdot 32$ & $\cdot 36$ & $\cdot 39$ & $\cdot 42$ & $\cdot 48$ & $\cdot 54$ & $\cdot 66$ & 30 \\
\hline 40 & $\cdot 30$ & $\cdot 35$ & $\cdot 41$ & $\cdot 48$ & $\cdot 54$ & $\cdot 60$ & 67 & $\cdot 77$ & .92 & 40 \\
\hline 50 & $\cdot 41$ & $\cdot 46$ & .52 & .59 & $\cdot 70$ & $\cdot 79$ & $\cdot 89$ & $\cdot 98$ & $1 \cdot 16$ & 50 \\
\hline 60 & $\cdot 52$ & $\cdot 60$ & $\cdot 68$ & $\cdot 79$ & $\cdot 89$ & .99 & $1 \cdot 11$ & $1 \cdot 23$ & $1 \cdot 46$ & 60 \\
\hline 70 & $\cdot 63$ & $\cdot 74$ & $\cdot 85$ & $\cdot 98$ & $1 \cdot 11$ & $1 \cdot 20$ & 1.32 & $1 \cdot 45$ & $1 \cdot 70$ & 70 \\
\hline 80 & $\cdot 75$ & 87 & $1 \cdot 01$ & $1 \cdot 15$ & 1.28 & $1 \cdot 38$ & 1.53 & $1 \cdot 70$ & 1.98 & 80 \\
\hline 90 & $\cdot 87$ & .99 & $1 \cdot 13$ & $1 \cdot 28$ & 1.45 & $1 \cdot 62$ & $1 \cdot 82$ & 1.94 & $2 \cdot 25$ & 90 \\
\hline 100 & .98 & $1 \cdot 12$ & $1 \cdot 29$ & 1.47 & $1 \cdot 65$ & 1.82 & $2 \cdot 03$ & $2 \cdot 20$ & 2.55 & 100 \\
\hline 120 & $\ldots$ & $\ldots$ & $\ldots$ & $1 \cdot 88$ & $2 \cdot 10$ & $2 \cdot 28$ & $2 \cdot 49$ & $2 \cdot 68$ & $3 \cdot 13$ & 120 \\
\hline 140 & $\ldots$ & $\ldots$ & $\ldots$ & $\ldots$ & $\ldots$ & $2 \cdot 75$ & 2.97 & $3 \cdot 22$ & $3 \cdot 75$ & 140 \\
\hline 160 & $\ldots$ & $\ldots$ & $\ldots$ & $\ldots$ & $\ldots$ & $\ldots$ & $3 \cdot 35$ & $3 \cdot 80$ & $4 \cdot 35$ & 160 \\
\hline
\end{tabular}

Thermometers with solid Stem, Scale on Stem.

\begin{tabular}{|c|c|c|c|c|c|c|c|c|c|c|}
\hline \multirow{2}{*}{$n$} & \multicolumn{9}{|c|}{$t-t^{\prime}$} & \multirow{2}{*}{$n$} \\
\hline & $70^{\circ}$ & $80^{\circ}$ & $90^{\circ}$ & $100^{\circ}$ & $110^{\circ}$ & $120^{\circ}$ & $140^{\circ}$ & $160^{\circ}$ & $180^{\circ}$ & \\
\hline 10 & $\cdot 02$ & $\cdot 03$ & $\cdot 05$ & $\cdot 07$ & $\cdot 09$ & $\cdot 11$ & $\cdot 17$ & $\cdot 21$ & $\cdot 27$ & 10 \\
\hline 20 & $\cdot 13$ & $\cdot 15$ & $\cdot 18$ & $\cdot 22$ & $\cdot 26$ & $\cdot 29$ & $\cdot 38$ & $\cdot 46$ & $\cdot 53$ & 20 \\
\hline 30 & $\cdot 24$ & $\cdot 28$ & $\cdot 33$ & $\cdot 39$ & $\cdot 44$ & $\cdot 48$ & $\cdot 59$ & $\cdot 70$ & .78 & 30 \\
\hline 40 & $\cdot 35$ & $\cdot 41$ & $\cdot 48$ & 56 & $\cdot 62$ & $\cdot 68$ & $\cdot 82$ & 94 & 1.04 & 40 \\
\hline 50 & $\cdot 47$ & $\cdot 53$ & $\cdot 62$ & $\cdot 72$ & $\cdot 81$ & $\cdot 88$ & $1 \cdot 03$ & $1 \cdot 17$ & $1 \cdot 31$ & 50 \\
\hline 60 & $\cdot 57$ & $\cdot 66$ & 77 & $\cdot 89$ & 1.00 & 1.09 & $1 \cdot 25$ & 1.42 & $1 \cdot 58$ & 60 \\
\hline 70 & $\cdot 69$ & $\cdot 79$ & $\cdot 92$ & 1.06 & $1 \cdot 19$ & $1 \cdot 30$ & $1 \cdot 47$ & 1.67 & $1 \cdot 86$ & 70 \\
\hline 80 & $\cdot 80$ & 91 & $.1 \cdot 05$ & $1 \cdot 21$ & $1 \cdot 37$ & 1.52 & $1 \cdot 71$ & 1.94 & $2 \cdot 15$ & 80 \\
\hline 90 & $\cdot 91$ & 1.04 & $1 \cdot 19$ & $1 \cdot 38$ & 1.56 & 1.73 & 1.96 & $2 \cdot 20$ & $2 \cdot 42$ & 90 \\
\hline 100 & 1.02 & $1 \cdot 18$ & $1 \cdot 35$ & 1.56 & 1.79 & 1.97 & $2 \cdot 18$ & $2 \cdot 45$ & $2 \cdot 70$ & 100 \\
\hline 120 & $\cdots$ & $\cdots$ & $\cdots$ & 1.98 & $2 \cdot 23$ & $2 \cdot 43$ & $2 \cdot 69$ & $2 \cdot 95$ & $3 \cdot 26$ & 120 \\
\hline 140 & $\ldots$ & $\ldots$ & $\ldots$ & $\ldots$ & $\ldots$ & $2 \cdot 92$ & $3 \cdot 22$ & $3 \cdot 47$ & $3 \cdot 86$ & 140 \\
\hline 160 & $\ldots$ & $\ldots$ & $\ldots$ & $\ldots$ & $\ldots$ & $\cdots$ & $\cdots$ & 4.00 & $4 \cdot 46$ & 160 \\
\hline
\end{tabular}


66. Reduction of the Readings of Mercury Thermometers to the Hydrogen or Air Scales.-As has already been mentioned, the scales of mercury thermometers, made of different kinds of glass, do not agree, the differences amounting, as will be seen from Fig. 62, to nearly a tenth of a degree at $50^{\circ}$, even in the case of the standard glasses. Whenever, therefore, an accuracy of a tenth of a degree is aimed at, it is always advisable to express all temperature measurements on the scale of either the hydrogen or the air thermometer. For all temperatures below $100^{\circ}$ the hydrogen thermometer is taken as the standard, but for temperatures above $100^{\circ}$ it is usual to employ either the nitrogen or the air scale.

In Table 19 are given the corrections which must be applied to the readings of thermometers made of the standard glasses to reduce to either the hydrogen or the air scale.

67. Changes in Zero of Mercurial Thermometers.-After a mercury thermometer has been filled, it is in general kept for some time before being graduated, so that the bulb may in a measure recover from the effects of the extreme heating to which it was subjected in the process of manufacture. It is, however, found that the bulb goes on slowly contracting, as indicated by a gradual rise in the zero reading, for many years. This slow rise of the zero is called the secular rise.

The secular rise is much more rapid at high than at low temperatures, and hence it is advisable to keep a thermometer after filling, and before graduation, at a temperature of about $300^{\circ}$ for a day or so, and then to very gradually reduce the temperature. This annealing process has been elaborated at the Jena Glass Works, where in certain cases the glass is cooled at a regular rate from $465^{\circ}$ to $370^{\circ}$, the whole operation occupying four weeks. Glass so treated is found remarkably free from strain, and is said to have been "fine annealed."

All thermometers ought to be made of one of the standard glasses. These are (1) verre dur, as used by the French thermometer maker Tonnelot; (2) the normal thermometer glass made at Jena, and designated by the number $16^{\prime \prime \prime}$; and (3) the Jena borosilicate glass, designated $59^{\prime \prime \prime}$. The Jena glass, $16^{\prime \prime \prime}$, is the most commonly met .with, and it may be known by the presence of a thin violet line near the surface.

In the case of the glasses used more than ten years ago the secular rise was very considerable, and went on for many years. In the case of the French verre dur and the Jena glasses (16"' and $\left.59^{\prime \prime \prime}\right)$ the secular rise is comparatively small, amounting to about 0.04 in four years, after which time the rise is very small. The secular rise can be taken into account in the case of thermometers made of either of the above glasses by making occasional observations of zero, and then assuming that the rise has taken place at a uniform rate between the determinations.

In addition to the secular rise, which is probably due to the recovery of the glass from a state of strain produced during the process of manufacture of the thermometer, there are other and more troublesome changes in zero produced when the thermometer is heated to quite moderate temperatures. Thus if after a thermometer has been kept at a tempera- 
ture of zero for some time its zero is determined, and the thermometer is then heated to $100^{\circ}$ for about half-an-hour, and as soon after as possible the zero is again tested, it will be found that the zero is now lower than it was before the heating to $100^{\circ}$. The depression obtained in this way by heating a thermometer to $100^{\circ}$ and then immediately determining the zero is called the depression constant of the thermometer. The depression constants of the three standard thermometer glasses are shown in the following table:-

\begin{tabular}{|c|c|c|}
\hline \multicolumn{2}{|l|}{ Glass. } & Depression Constant. \\
\hline Verre dur & - $\quad$. & $0 \cdot 11$ \\
\hline Jena glass $\left(16^{\prime \prime \prime}\right)$ & - $\quad$. & 0.08 \\
\hline Jena glass $\left(59^{\prime \prime \prime}\right)$ & . & 0.03 \\
\hline
\end{tabular}

At ordinary room temperatures the depression takes two or three days to die out, at higher temperatures the recovery is more rapid. The time the heating at $100^{\circ}$ has to be continued to produce the full depression is only. two or three minutes in the case of the standard glasses. With ordinary crystal glass, however, it requires about half-an-hour's heating to produce the full depression, which may amount to half a degree. The fact that the standard glasses reach their full depression rapidly makes their use in hypsometric thermometers advisable, even if there were not other reasons for their employment in this case.

If a thermometer is maintained for a long time at a high temperature it is found that there is a progressive rise in the zero. Thus in the case of a verre dur thermometer, examined by Guillaume, heating to $200^{\circ}$ for about six hours produced a rise of about $0^{\circ} \cdot 4$, while heating for thirty hours to $370^{\circ}$ produced a rise of about $3^{\circ} \cdot 1$. In the case of the Jena glasses Wiebe found that heating a thermometer of $16^{\prime \prime \prime}$ to $300^{\circ}$ for twenty hours produced a rise of about $1^{\circ} \cdot 6$. In the case of $59^{\prime \prime \prime}$, heating to $300^{\circ}$ for thirty hours produced a rise of $3^{\circ} \cdot 9$. If, however, the thermometer had been subjected to the fine annealing process referred to on p. 182, then the rise was only $0^{\circ} \cdot 2$.

The rise produced by prolonged heating to high temperatures appears to be permanent, and further exposure to a slightly lower temperature produces less and less effect as the period of the heating is prolonged. If a thermometer of Jena glass, $16^{\prime \prime \prime}$, is heated to $300^{\circ}$ for twenty-four hours before it is graduated, then for temperatures up to, say, about $250^{\circ}$ the rise produced is for most purposes inappreciable.

68. The Movable Zero Method of Using a Mercury Thermometer. - The phenomenon of the depression of the zero of mercury in glass thermometers indicates that when such a thermometer is heated, even to temperatures below $100^{\circ}$, then, in addition to the reversible expansion 
which disappears when the bulb is cooled, there is an irreversible expansion which does not at once vanish when the thermometer is cooled, and which produces the depression of the zero. This irreversible effect introduces a complication when we come to consider the definition of the scale of the mercury-in-glass thermometer. When the zero of the thermometer is determined immediately after the boiling point, then, since it is found that the irreversible effect persists for some time, the fundamental interval obtained corresponds to the reversible effect only. Suppose, now, the fundamental interval is divided into degrees of equal volume, the scale, therefore, corresponding to the reversible effect only, and that after the thermometer has been preserved for some time at a temperature not much above zero it is heated to $50^{\circ}$ as indicated by the scale. Now the temperature will not be $50^{\circ}$, for, in the first place, if $D$ is the depression constant of the thermometer before the thermometer was heated, the zero will have risen by an amount $D$, so that if there were no irreversible effect produced by heating to $50^{\circ}$ the true temperature corresponding to the reading $50^{\circ}$ would be $50^{\circ}-D$. There is, however, an irreversible expansion produced by the heating to $50^{\circ}$, which we may call $d$, and on this account the thermometer will read too low by an amount $d$, so that the actual temperature corresponding to the reading $50^{\circ}$ is $50^{\circ}-(D-d)$. Now, if after taking the reading at $50^{\circ}$ we had immediately plunged the thermometer into melting ice and determined the zero, the reading would have been $D-d$. If, then, we apply this reading as a correction to the reading at $50^{\circ}$, that is, take the zero as found immediately after the $50^{\circ}$ reading as the zero corresponding to that reading, we shall have allowed for the irreversible expansion of the glass. This method of referring every reading of a mercury thermometer to a special zero taken immediately after the reading is called the movable zero method of using a thermometer.

It is quite obvious that in many cases it would be impossible to interrupt a series of temperature measurements to take a zero reading after every reading of the thermometer. With a view of overcoming this difficulty various observers have made determinations of the depressions at different temperatures for thermometers made of the standard glasses, and have drawn up tables giving these depressions. The results obtained have not been very concordant, so that in Table 18 the means of the results obtained by the different observers are given; and these are probably correct to one or two hundredths of a degree.

In order to use the tables of depression of zero the thermometer to be used must be kept at zero for some considerable time so that the depression produced by previous heating has time to disappear, which will be indicated by the zero becoming constant. Let the zero reading obtained in the way be $D$. Then when the reading of the thermometer is $t$, the temperature having been kept at $t$ for such a time that the glass has reached the permanent condition corresponding to this temperature, the true temperature is $t-\left(D-d_{t}\right)$, where $d_{i}^{i}$ is the depression corresponding to the temperature $t$ as obtained from the tables. 
For example, suppose that the thermometer for which Fig. 70 gives the correction curve after being kept in ice for two or three days reads $+0^{\circ} \cdot 03$, and when placed in a given enclosure reads $78^{\circ} \cdot 32$, to find the temperature of the enclosure. From the correction curve we have that the correction at $78^{\circ} \cdot 32$ is $+0^{\circ} \cdot 13$. If the thermometer is of verre dur the depression at $78^{\circ} .32$ is $0^{\circ} .08$, that is, if the zero had been determined immediately after heating to $78^{\circ} .32$ the depression would have been 0.08 , and, therefore, the zero reading $+.03-.08$ or $-0^{\circ} .05$. Hence the correction for the irreversible effect is $+0^{\circ} .05$, and the true temperature is

$$
78 \cdot 32+0 \cdot 13+0 \cdot 05
$$

or $78^{\circ} .50$ on the scale of the verre dur thermometer. To reduce the hydrogen scale a correction -0.05 (see Table 19) has to be applied, so that in the hydrogen scale the temperature was $78^{\circ} \cdot 45$.

For a description of the corrections which must be applied and the precautions to be taken if attempts are made to read temperatures with mercury thermometers nearer than $0^{\circ} \cdot 01$, reference must be made to Guillaume's Traité de la Thermométrie de Precision.

69. Measurement of the Depression in a Mercury Thermometer.Take a thermometer graduated in tenths, or at least fifths, of a degree and keep it in melting ice for two or three days so as to allow any depression existing to disappear. The thermometer used ought not to have been heated above the ordinary room temperature for at least a week before the experiment.

Determine the zero as described in $\S 61$, and then heat the thermometer for ten minutes to a temperature of $50^{\circ}$, and again determine the zero as quickly as possible. The difference between the zero now found and that obtained before the heating will give the depression for $50^{\circ}$.

Next place the thermometer in a boiling point apparatus, and read the boiling point every minute for half-an-hour, or till it becomes constant. The barometer must be read between each thermometer reading to make certain that the differences obtained are not due to variations of atmospheric pressure. Having applied, if necessary, a correction for the effects of variation in pressure, draw up a table showing the rapidity with which the thermometer takes up its final condition.

Remove the thermometer from the steam, and as soon as safe plunge it into ice and take a zero reading. The difference between the first zero reading and the one obtained after heating to $100^{\circ}$ will be the depression constant of the thermometer.

A zero reading should then be taken every day for a few days to determine how long the thermometer takes to recover from its heating to $100^{\circ}$.

To a near approximation the depression $d_{t}$ at any temperature $t$, below $100^{\circ}$, may be calculated on the supposition that the depression 
is proportional to the temperature to which the thermometer has been raised. Thus

$$
d_{t}=\frac{D t}{100}
$$

Resistance and thermoelectric methods of measuring temperature are described in the later chapters, where the electrical phenomena on which they depend are considered. 


\section{CHAP'TER X}

\section{EXPANSION OF SOLIDS AND LIQUIDS}

70. Linear Expansion.-If the distance between two marks on a solid body at the temperature $0^{\circ}$ is $L_{0}$, and when the temperature is raised to $t$ the length between the marks is $L_{t}$, then, so long as $t$ is not too great, it is in general possible to express $L_{t}$ by an equation of the form

$$
L_{t}=L_{0}\left(1+a t+b t^{2}\right) \quad \text {. . . . . . . }
$$

where $a$ and $b$ are constants. If the range of temperature $t$ is small, then the term in $t^{2}$ can generally be neglected, so that the increase in length is directly proportional to the increase in temperature, and we have

$$
L_{t}=L_{0}(1+a t) \text {. }
$$

In these circumstances it is usual to call $a$ the coefficient of linear expansion of the solid.

If the lengths of the solid at two temperatures $t_{1}$ and $t_{2}$ are $L_{1}$ and $L_{2}$, then we have

$$
\begin{aligned}
& L_{1}=L_{0}\left(1+a t_{1}+b t_{1}^{2}\right) \\
& L_{2}=L_{0}\left(1+a t_{2}+b t_{2}^{2}\right) . \\
& \frac{L_{2}}{L_{1}}=\frac{1+a t_{2}+b t_{2}^{2}}{1+a t_{1}+b t_{1}^{2}},
\end{aligned}
$$

Hence

Since $a$ is small and $b$ even smaller, we may neglect terms in $a b, a^{3}, l^{2}$ and higher powers. Thus.

$$
L_{2}=L_{1}\left\{1+a\left(t_{2}-t_{1}\right)+b\left(t_{2}^{2}-t_{1}^{2}\right)-a^{2} t_{1}\left(t_{2}-t_{1}\right)\right\} . .
$$

If the difference of temperature $\left(t_{2}-t_{1}\right)$ is small, this reduces to

$$
L_{2}=L_{1}\left\{1+a\left(t_{2}-t_{1}\right)\right\} \text {. }
$$

Hence if $L_{2}$ and $L_{1}$ are the lengths measured at the two temperatures $t_{2}$ and $t_{1}$, we can from this expression calculate $a$.

If the term in $t^{2}$ in the expression (1) above is not negligible, then the value obtained for $a$ at different temperatures, i.e. for different values of the mean temperature $\left(\begin{array}{c}t_{2}-t_{1} \\ 2\end{array}\right)$, will not be the same, or, in other words, the coefficient of expansion defined by (2) above will 
be a variable. In such a case the coefficient of linear expansion, at a temperature $t$ is defined by the expression

$$
a_{t}=\frac{\delta L}{L \delta t}
$$

where $L$ is the length at a temperature $t$, and $L+\delta L$ is the length at a temperature $t+\delta t, \delta t$ being small.

Leaving out of account methods depending on the use of levers, which are not suitable for accurate measurements, methods for measuring the coefficient of linear expansion may be divided into two classes. In one of these a comparatively long piece of the solid (about a metre) is used, and the elongation measured by means of a micrometer screw or a microscope with an eye-piece scale, and in the other a short length of 2 or 3 centimetres is used, and the elongation is measured by various devices depending on the interference of light.

If the length of a solid at a temperature $t$ is $L$, and when the temperature increases by $\Delta t$ the length becomes $L+\Delta L$, then the coefficient of expansion between these limits of temperature is

$$
\frac{\Delta L}{L \cdot \Delta t}
$$

This shows that to determine the coefficient of expansion we have to measure the increase of length $\Delta L$, the rise in temperature $\Delta t$, and the original length $L$. As all these quantities appear in the expression to the first power, we ought to measure them all with the same accuracy. Since $\Delta L$ is small, the most difficult part of the measurement consists in determining the increase in length, the measurement of the original length, and of the increase in temperature being in general much less difticult.

71. Measurement of the Linear Expansion of a Rod.-An arrangement suitable for measuring the coefficient of thermal expansion of a

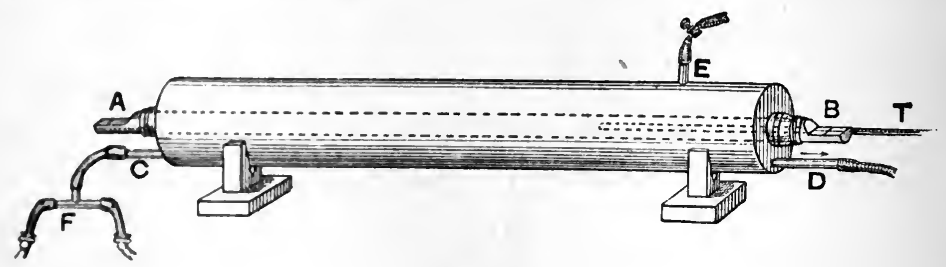

FIG. 76.

metal rod about a metre long is shown in Fig. 76. The expansion is read by means of two reading microscopes which are clamped at a fixed distance apart, the expansion being read off on eye-piece scales or by means of a micrometric eye-piece. In order to obtain an accurate measure of the expansion, it is essential that two very fine lines should 
be ruled on the metal rod to act as fiducial marks between which the expansion shall be measured. To give a suitable surface for these marks, half the thickness of the rod is filed away for a short distance at either end, as shown. The flat surface must then be very carefully polished, first with fine emery paper, and finally with rouge. While performing the final stages of the polishing, it is essential that the rubbing should take place in a direction parallel to the length of the rod. When the polishing is complete, a line parallel to the axis of the rod must be ruled on the polished surface. This can be done by means of a pair of dividers, one leg being held against the side of the rod. This longitudinal line need not be very fine, but may be made so as to be easily seen by the naked eye. The transverse line, on the other hand, must be so fine as to be practically invisible except with a lens. A very sharp needle is selected by examining the points of a number of needles under a low power microscope, and then a transverse line is ruled with this needle, a very light touch being employed. A small square resting against the side of the rod can be used to guide the needle. It is advisable after ruling the line, and before moving the square, to make with another needle a somewhat coarse mark as a prolongation of either end of the fine line. This coarse mark will be found of great assistance when adjusting the apparatus.

Another way of making a suitable mark is to file a notch at each end of the rod, as shown in Fig. 77, and then solder a very fine metallic wire across the notch. The wire ought to have a diameter of about $05 \mathrm{~mm}$., and is preferably of platinum, as it does not then corrode.

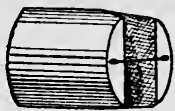

FIG. 77. In the absence of sufficiently fine wire a fine thread of glass may be used, but the attachment presents some difficulty. If the rod is not going to be heated above a temperature of $100^{\circ}$, a little melted shellac does fairly well. When making the measurements, one of the edges of the wires is used as the mark.

The rod is enclosed, except for about 2 centimetres at either end, in a metal tube about 6 centimetres in diameter. At one end A (Fig. 76) the rod passes through a cork which fits tightly; at the other end it passes through a short tube soldered to the disc which closes the end of the jacket. The joint between the tube and the rod is made water-tight by slipping a short length of india-rubber tubing over the rod and the projecting tube. This joint will allow of the free expansion or contraction of the rod as the temperature is changed.

A thermometer passes through a small hole at either end, and is held in place by a small cork. Two side tubes $\mathrm{C}$ and $\mathrm{D}$ allow of a stream of steam or cold water being passed through the jacket. A rubber tube from one of these leads to a sink, while the other is connected to a T-piece F. The other arms of this T-piece are connected to the water supply and a can in which water can be boiled respectively. By clamping one or other of these tubes, water or steam may be passed in succession 
through the jacket, and the change from one to the other can be made without disturbing the jacket and the rod. A small vertical tube $\mathbf{r}$ having a rubber tube attached which can be closed by means of a pinchcock is placed at the top of the jacket. This tube allows of the escape of the air when filling the jacket with water. The jacket is supported on two v's at such a height that the microscopes can be focused on the marks on the rod.

To perform the experiment the two microscopes are clamped firmly down on a steady table, or preferably on a stone or slate shelf, and the rod is adjusted so that the transverse marks are in focus and the longitudinal mark is near the centre of the field.

The microscopes must be so adjusted that when the rod is cold the transverse marks on the rod are near the ends of the scales nearest to the centre of the rod, otherwise when the rod is heated it may expand so much that the marks would go out of the field of view. Of course if either of the microscopes can be moved parallel to the length of the rod by a sufficiently fine micrometer screw this adjustment is of little or no importance.

Water is passed through the jacket till the readings of the thermometers become constant, when the reading on the two microscope scales are taken, as well as the readings of the thermometers. The water is then run out and steam passed through the jacket till again the temperature is constant. If, owing to the expansion, the mark at the end $\mathrm{B}$ is beyond the scale, the jacket and rod must be moved through the 's till the mark comes into view again. The readings of the microscopes and thermometers are then taken. Cold water is again passed through the jacket, and the readings at the low temperature repeated. The two sets of readings at the low temperature ought to agree. If they do not the microscopes have probably been moved, and the whole set of readings must be repeated.

It is a good thing to take a number of readings of the microscopes at each temperature, and, to prevent being biased by the readings already taken, to move the rod longitudinally in the v's between each reading. In this way the readings of the microscope scales will be different, but if the scales both read in the same direction the sum of the two readings ought to be constant.

The values of the scale divisions of the micrometer scales of the microscopes must be determined by means of a fine scale ruled on glass, on which each microscope is focused in turn.

To determine the distance between the two marks on the rod a divided metre scale can be supported in place of the rod, and the readings on the microscope scales obtained between any two of the divisions of the scale. Then from the readings of the microscopes corresponding to the marks on the rod the distance between these marks can be immediately determined.

If the material of which the coefficient of thermal expansion is to be measured is in the form of a tube, then it will be possible to obtain a very 
fairly accurate measure by alternately passing cold water and steam through the tube itself, the outside of the tube, except near the ends where the marks are made, being wrapped round by some non-conductor of heat, such as felt or cotton wool.

72. Cubical Expansion. - Suppose that the volume of a body at $0^{\circ} \mathrm{C}$. is $V_{0}$, then the volume $V_{t}$ at a temperature $t$ is given by an expression of the form

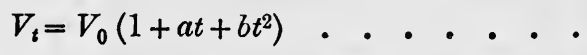

where $a$ and $b$ are constants. Some experimenters have expressed their results by a formula involving the cube of $t$, such as

$$
V_{t}=V_{0}\left(1+a t+b t^{2}+c t^{3}\right),
$$

but it has been found that a formula involving only the first and second powers of $t$ is sufficient to express the iresults given by experiment to an accuracy as great as that of the measurements, and hence it is unnecessary to include the term in $t^{3}$.

If $\rho_{0}$ and $\rho_{t}$ are the density of the substance at $0^{\circ}$ and $t$ respectively, then

$$
\rho_{t} / \rho_{0}=V_{0} / V_{t}=1 /\left(1+a t+b t^{2}\right) \text {. }
$$

Now in general $a^{2}$ and $b$ are of about the same magnitude, and so long as $\boldsymbol{t}$ is not very great we may neglect all terms involving higher powers of $a$ and $b$, such as $a^{3}, a b, b^{2}$, \&c. Hence, $1 /\left(1+a t+b t^{2}\right)=1-a t-\left(b-a^{2}\right) t^{2}$, so that

$$
\rho_{t}=\rho_{0}\left(1-a t-\left(b-a^{2}\right) t^{2}\right) \cdot . \cdot . \cdot .
$$

If we are given the volume $V_{t}$ at a temperature $t$ and require to find the volume at a temperature $t^{\prime}$ we have

$$
\begin{aligned}
& V_{t}=V_{0}\left(1+a t+b t^{2}\right) \\
& V_{t^{\prime}}=V_{0}\left(1+a t^{\prime}+b t^{\prime 2}\right) .
\end{aligned}
$$

Hence

$$
\begin{aligned}
V_{t} & =V_{t} \frac{1+a t^{\prime}+b t^{\prime 2}}{1+a t+b t^{2}} \\
& =V_{t}\left(1+a t^{\prime}+b t^{\prime 2}\right)\left(1-a t-b t^{2}\right) \\
& =V_{t}\left\{1+a\left(t^{\prime}-t\right)+b\left(t^{\prime 2}-t^{2}\right)-a^{2} t\left(t^{\prime}-t\right)\right\} . .
\end{aligned}
$$

if as before we neglect terms involving higher powers than $a^{2}$ and $b$.

If we define the mean coefficient of expansion between any two temperatures $t$ and $t^{\prime}$ as the change of volume per degree of unit volume of the body at the lover temperature $t$, or in symbols as

$$
\frac{V_{t^{\prime}}-V_{t}}{V_{t}\left(t^{\prime}-t\right)},
$$


we get from (3) that the mean coefficient of expansion

$$
\begin{aligned}
\beta & =\frac{V_{t}-V_{t}}{V_{t}\left(t^{\prime}-t\right)}=a+\frac{b\left(t^{\prime 2}-t^{2}\right)}{t^{\prime}-t} \\
& =a+b\left(t^{\prime}+t\right) . . . .
\end{aligned}
$$

if $a$ and $b$ are small.

If, on the other hand, we define the mean coefficient of expansion between $t$ and $t^{\prime}$ as the change in volume per degree of unit volume of the body at $0^{\circ} \mathrm{C}$., or in symbols as

$$
\frac{V_{t \prime}-V_{t}}{V_{0}\left(t^{\prime}-t\right)}
$$

we have that the mean coefficient of expansion $\beta^{\prime}$ is given by

$$
\begin{aligned}
& \beta^{\prime}=\frac{V_{t^{\prime}}-V_{t}}{V_{0}\left(t^{\prime}-t\right)}=\frac{V_{0}\left\{a\left(t^{\prime}-t\right)+b\left(t^{\prime 2}-t^{2}\right)\right\}}{V_{0}\left(t^{\prime}-t\right)} \\
& \beta^{\prime}=a+b\left(t^{\prime}+t\right) .
\end{aligned}
$$

Hence we see that when $a$ and $b$ are so small that the squares and higher powers of these quantities can be neglected, the value of the mean coefficient of expansion is the same whichever of these definitions we employ.

The true coefficient of expansion at any given temperature $t$ is the rate at which the volume varies at that temperature per unit volume, or, if we like, is the mean coefficient of expansion between two temperatures taken one on either side of $t$, and so near $t$ that the expansion does not change appreciably over the range of temperature considered. Thus the true coefficient of expansion at $t$ is obtained by making $t^{\prime}$ coincide with $t$ in the above expressions. for $\beta$, and we thus obtain that the true coefficient of expansion at $t$ is

$$
a+2 b t
$$

We may also obtain this expression by noting that by definition the trae coefficient of expansion is

$$
\frac{1}{V_{0}} \frac{d V_{t}}{d t}
$$

which from (1) is $a+2 b t$.

In the case of liquids and solids, $a$ and $b$ are so small that the approximations we have made above are sufficiently close. In the case of gases, however, this is not the case, and therefore to prevent confusion we shall for gases always adopt the definition of the coefficient of expansion in terms of the volume at $0^{\circ} \mathrm{C}$., that is, in symbols

$$
\beta=\frac{V_{t^{\prime}}-V_{t}}{V_{0}\left(t^{\prime}-t\right)}
$$


In the case of an isotropic solid, if the length at $t$ is $L_{t}$, and that at $0^{\circ} L_{0}$, we have

$$
\begin{aligned}
\frac{L_{t}}{L_{0}}=\frac{\sqrt[3]{V_{t}}}{\sqrt[3]{V_{0}}} & =\sqrt[3]{\left(1+a t+b t^{2}\right)} \\
& =1+\frac{a}{3} t+\frac{b}{3} t^{2}+\ldots . . .
\end{aligned}
$$

The determination of the absolute coefficient of cubical expansion of a liquid is a problem which can only be solved by means of elaborate experiments, such as those of Regnault, by the method of balancing columns. We can, however, measure the relative expansion of a liquid and a solid containing vessel, and then, if we know the absolute expansion of the solid, we can at once deduce the absolute coefficient of expansion of the liquid. To obtain the coefficient of expansion of the envelope, we may make use of the fact that the expansion of mercury and of water have been determined with great accuracy. Thus an experimental determination of the value of the coefficients of apparent expansion of mercury or water when enclosed in this envelope will allow us to deduce the coefficient of expansion of the envelope.

The methods generally employed for measuring the apparent expansion of a liquid are (1) the weight thermometer, (2) the volume dilatometer, and (3) the hydrostatic balance.

73. The Weight Thermometer or Dilatometer.-The simplest form of weight thermometer is shown in Fig. 78. It consists of a glass bulb with a capillary neck, the neck being generally bent round as shown. The dilatometer having been weighed is filled with the liquid at a tem-

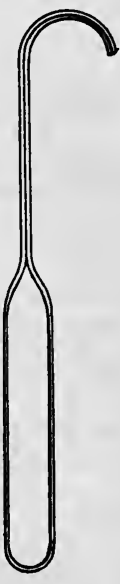

FIG. 78. perature $t_{1}$, and again weighed. The difference in the weights gives the weight $v$ of the contained liquid. The dilatometer is then heated to $t_{2}$, some of the liquid escaping. By again weighing, the weight $w_{2}$ of liquid which fills the dilatometer at $t_{2}$ is obtained.

If $v_{1} \rho_{1}$ and $v_{2} \rho_{2}$ are the volumes of unit mass and density respectively of the liquid at the two temperatures, and $a$ is the true coefficient of expansion of the liquid, we have by definition

$$
a=\frac{1}{t_{2}-t_{1}} \frac{v_{2}-v_{1}}{v_{1}}=\frac{1}{t_{2}-t_{1}}\left\{\frac{v_{2}}{v_{1}}-1\right\}
$$

If $V_{1}$ and $V_{2}$ are the volumes of the dilatometer at the temperatures $t_{1}$ and $t_{2}$, and $\beta$ is the coefficient of cubical expansion of the material of which the dilatometer is constructed, then

Now

$$
\begin{aligned}
& V_{2}=V_{1}\left\{1+\beta\left(t_{2}-t_{1}\right)\right\}_{.} \\
& w_{1}=V_{1} \rho_{1} \text { and } w_{2}=V_{2} \rho_{2} .
\end{aligned}
$$


Hence

$$
\begin{aligned}
& \frac{v_{2}}{v_{1}}=\frac{\rho_{1}}{\rho_{2}}=\frac{w_{1}}{w_{2}} \cdot \frac{V_{2}}{V_{1}} \\
& \frac{v_{2}}{v_{1}}=\frac{w_{1}}{w_{2}}\left\{1+\beta\left(t_{2}-t_{1}\right)\right\} .
\end{aligned}
$$

Substituting this value for $v_{2} / v_{1}$ in $(1)$, we get

$$
\begin{aligned}
& a=\frac{1}{t_{2}-t_{1}}\left\{\frac{w_{1}}{w_{2}}+\frac{w_{1}}{w_{2}} \beta\left(t_{2}-t_{1}\right)-1\right\} \\
& a=\frac{1}{t_{2}-t_{1}}\left\{\frac{w_{1}-w_{2}}{w_{2}}\right\}+\frac{w_{1}}{w_{2}} \beta .
\end{aligned}
$$

If $d$ is the weight of liquid which escapes at the higher temperature, so that $w_{1}-w_{2}=d$, then (2) may be written

$$
a-\beta=\frac{d}{w_{1}}\left\{\frac{1}{t_{2}-t_{1}}+a\right\} \text {. . . . . . . }
$$

a form sometimes convenient.

Thus if either $\alpha$ or $\beta$ is known, the other can immediately be calculated. The coefficients of expansion of water and mercury can be obtained from Table 20, so that for these liquids $\alpha$ is known, and $\beta$ can therefore be calculated.

The coefficients of expansion of the standard thermometer glasses have been measured with considerable care, and thus if a dilatometer is constructed of either of these glasses the value of $\beta$ is known, and the experiment with mercury or water can be omitted.

The following are the values ${ }^{1}$ of the coefficients of cubical expansion, that is, the coefficients $\beta$ and $\beta^{\prime}$ in the equation

$$
v_{\bar{i}}^{-}=v_{0}\left(1+\beta t+\beta^{\prime} t^{2}\right):-
$$

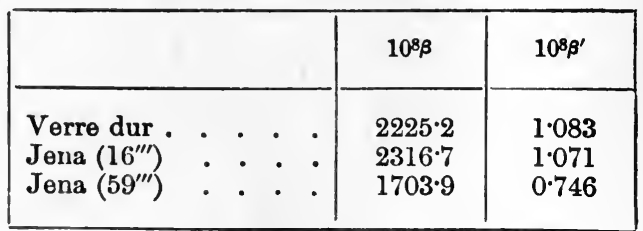

In the expression (2) the factor $w_{1} / w_{2}$ is often very nearly unity. If we assume that it is so nearly unity that we may put it equal to 1 , then (2) reduces to

$$
a-\beta=\frac{1}{t_{2}-t_{1}} \frac{w_{1}-w_{2}}{w_{2}} \text {. . . . . . }
$$

This simplified formula must, however, never be used unless we have satisfied ourselves by actual calculation that the assumption is justified.

1 Thiesen, Scheel, and Sel], Zeit. für Instrumentenkunde (1896), xvi. p. 54. 
The magnitude of the error which may be caused by this assumption is shown in the following example :-

Weight of dilatometer empty . . . $=3.0510$ grams.

Then

$" \quad$ full of mercury at $29^{\circ} \mathrm{C} .=76 \cdot 0747 "$ " $" \quad 98^{\circ} \mathrm{C}=75 \cdot 2925 "$

Taking the coefficient of expansion of mercury between $29^{\circ}$ and $98^{\circ}$ as $\cdot 0001818$ we get

$$
\alpha=\cdot 00002446 \text {. }
$$

If, however, we had put the coefficient of $a$ as unity, we should obtain

$$
\alpha=\cdot 0000249 \text {, }
$$

and hence have produced an error of about 2 per cent.

74. Measurement of the Cubical Expansion of Glass with a Simple Form of Weight Dilatometer.-Take a piece of glass tube having a bore of about a centimetre and close one end, carefully removing any blob of glass produced, so that the thickness of the glass is the same at the end as at the sides. Then about 5 centimetres from the closed end heat the glass in the blowpipe flame. During the heating keep the glass in continuous rotation, but do not draw the tube out. In this way the glass will become thickened at the point heated. When the bore has been reduced to about half, remove the glass from the flame, and then slowly draw out the heated portion till the capillary has a bore of about a millimetre. In this way the walls of the capillary will be almost as thick as the rest of the bulb, and by gently heating in a small flame the capillary can easily be bent into the form shown in Fig. 78 .

Having carefully cleaned the outside of the bulb, weigh it.

The bulb has now to be filled with clean mercury. This operation is one of some little difficulty. The bulb is supported, as shown in Fig. 79, being suspended from a retort stand by some copper wire, the stand itself being placed in a mercury tray to catch the mercury in case the bulb breaks. The

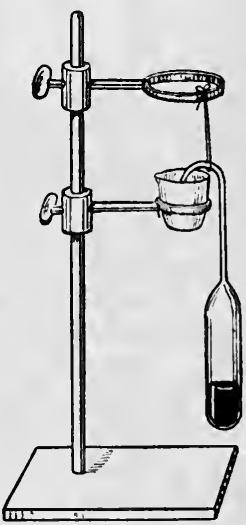

FIG. 79. mercury is placed in a porcelain crucible, the end of the capillary dipping below the surface. The bulb and the mercury are gently heated with a Bunsen flame, expelling some of the air. On allowing the bulb to cool some of the mercury will be driven back into the bulb. The whole of the dilatometer, together with the mercury in the crucible, must then be heated up to the boiling point of mercury, and nearly the whole of the mercury in the bulb allowed to boil away. On removing the flame the mercury vapour will condense, and the mercury 
will be driven over into the bulb. It is most important that the mercury in the crucille should be kept hot, otherwise when the mercury commences to pass over into the bulb the glass will be cracked owing to the cooling produced by the cold mercury. When the mercury is quite cold, it will generally be found that there is a small bubble of air left. By shaking the dilatometer this bubble should be made to move as far up the capillary as possible. Then placing the end of the capillary below the surface of the mercury, gently heat the bulb so as to drive the bubble out of the capillary.

Next suspend the dilatometer so that the bulb and as much of the neck as possible is immersed in water at a temperature a degree or so above that of the air of the laboratory, the end of the capillary still dipping below the surface of the mercury in the crucible. An arrange-

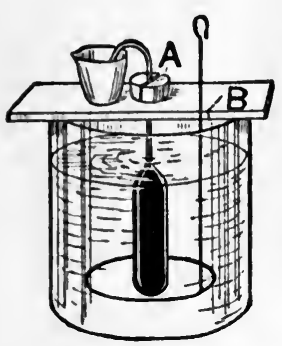

FIG. 80. ment for supporting the dilatometer is shown in Fig. 80. The cork is split, and fits tightly into a hole in the wooden cross-bar B. Keep the water stirred, and allow the dilatometer to remain for at least a quarter of an hour. Then read the temperature of the water, and remove the dilatometer from the water and from the mercury in the crucible. Carefully dry the dilatometer and weigh. While drying the dilatometer and transferring it to the balance case be careful not to warm it, otherwise some of the mercury will be expelled owing to expansion. It was for this reason that the temperature of the water was taken slightly above that of the air.

The dilatometer must now be suspended in a beaker of boiling water so that it is entirely immersed, capillary and all, and the water kept boiling for at least twenty minutes. The temperature having been read, remove the dilatometer, dry, and again weigh.

As a check on the weighings transfer the mercury which has escaped into the beaker in which the dilatometer has been heated to a watch glass, and weigh. The weight of this mercury ought to be equal to the difference in the weights of the dilatometer.

If the only delicate balance available will not carry the weight of the full dilatometer, then weigh the dilatometer empty and full at the lower temperature on a less sensitive balance which will carry this load, and thus obtain the quantity $w_{1}$. Then weigh the globule of mercury which escapes when the dilatometer is heated to $100^{\circ}$ on the sensitive balance, and thus obtain $d$. By this method of procedure the quantities $w_{1}$ and $d$, which enter into the expression for calculating the coefficient of expansion of the glass, can be determined with about the same percentage accuracy; while if the less sensitive balance had been used to weigh the dilatometer at both temperatures, and the quaritity $d$ deduced from the difference of the weighings, this quantity would not be known with anything like the same percentage accuracy as is $w_{1}$. 
By taking the lower temperature, when part of the capillary has to project outside the bath in which the dilatometer is immersed, nearly equal to the room temperature, the error produced on account of the temperature of the mercury in the capillary not being the same as that of the bulb is reduced to a minimum. It is, however, sometimes necessary to work with the lower temperature differing from that of the room. For instance, it may be desired to measure the mean coefficient of cubical expansion of a liquid between $0^{\circ}$ and $100^{\circ}$. In such a case it is evident that it will be impossible to weigh the bulb when it has been filled at $0^{\circ}$, since when it is warmed up in the balance case some of the liquid would escape. It is, therefore, best, having filled the dilatometer, to pack it round with melting ice, leaving as little of the capillary projecting as possible, and allow the end of the capillary to dip below the surface of some of the liquid contained in a small crucible. When the bulb has taken up the temperature, the crucible is removed and weighed. The end of the capillary having been again dipped in the liquid in the weighed crucible, the bulb of the dilatometer is placed in boiling water. When the temperature has had time to become steady, the crucible is removed and again weighed. The difference in the weights of the crucible gives the quantity $d$. The dilatometer is then removed from the boiling water, and when it has cooled, the capillary in this case no longer dipping below the surface of the liquid, the dilatometer is weighed, and from this weighing, and that of the dilatometer empty, the quantity $w_{2}$ is deduced. Then $w_{1}=w_{2}+d$.

Having measured the coefficient of cubical expansion of the glass, the weight dilatometer may be used to measure the true coefficient of expansion of any other liquid. The procedure to be adopted is the same as that described above, and then since we now know $\beta$, equations (2) or (3) of the last section will allow of our calculating $\alpha$.

75. Measurement of the Coefficient of Expansion of a Liquid with the Volume Dilatometer.-A simple form of volume dilatometer is shown in Fig. 81 . It consists of a bulb $\mathrm{A}$, with a capillary stem BC, on which a scale has been etched. When using the instrument to measure the expansion of volatile liquids, and also for keeping out dust when the instrument is not in use, it is a good thing to have a tap D

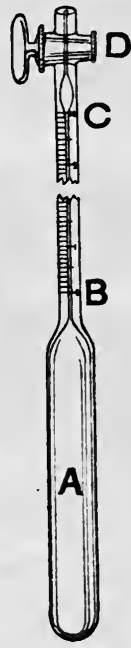

FIG. 81. at the top. There also ought to be a small auxiliary bulb at c. This form of dilatometer suffers from the same objections as the form of weight thermometer shown in Fig. 78, in that it is difficult to fill and clean, but to a somewhat smaller degree. Owing to the fact that the capillary is straight, it is possible to fill the bulb by means of a tube drawn down to a fine capillary and the arrangement shown in Fig. 36, also since the dilatometer can be plunged into a water bath till only a few millimetres of the liquid column project above the surface of the water, the correction for cool column can be rendered practically negligible. 
In order to reduce the measurements with the volume dilatometer it is necessary to know the volume of the bulb up to the zero graduation of the scale, and also the volume of the capillary from the zero of the graduations up to any division. These quantities can be determined by means of mercury. First the weight $w$ of the dilatometer when empty is

- determined, then the weight $W x$ when filled with mercury up to a point $(x)$ near the top of the scale, the temperature being that of melting ice. Then the volume up to the division $x$ at $0^{\circ} \mathrm{C}$. is given by

$$
\frac{W x-w}{\Delta_{0}},
$$

where $\Delta_{0}$ is the density of mercury at $0^{\circ}$.

Next some of the mercury is removed till, when the whole is at a temperature of melting ice, the end of the mercury column is near the zero of the scale, say at the division $y$, and let the weight be $W y$. Then the volume up to the $y$ division is

$$
\frac{W y-w}{\Delta_{0}},
$$

and the volume of one division of the scale is

$$
\frac{W x-W y}{(x-y) \Delta_{0}} \cdot \cdot \cdot \cdot \cdot \cdot \cdot
$$

Hence the volume up to the zero is

$$
\frac{W y-w}{\Delta_{0}}-\frac{W x-W y}{(x-y) \Delta_{0}} y \quad \text {. . . . . . . }
$$

We have, in the above, supposed that the bore of the capillary is cylindrical. This must always be tested by measuring the length of a thread of mercury when it occupies different parts of the scale. If the length varies from one part of the scale to another, indicating that the bore is irregular, then the scale will have to be calibrated in the same way as the tube of a thermometer, and a table of corrections prepared, by means of which the volume from the zero up to any division can be obtained.

To determine the coefficient of expansion of the glass, an experiment must be performed with mercury.

Let the volume, as read off on the scale, at a temperature $t_{1}$ be $V_{1}$, and the volume at a temperature $t_{2}$ be $V_{2}$. Then the volume of the liquid at $t_{2}$ is $V_{1}\left\{1+\beta\left(t_{2}-t_{1}\right)\right\}$, where $\beta$ is the coefficient of cubical expansion of the liquid.

The volume occupied in the dilatometer is apparently $V_{2}$ but owing to the expansion of the glass this volume is really $V_{2}\left\{1+a\left(t_{2}-t_{1}\right)\right\}$, where $a$ is the coefficient of cubical expansion of the glass. 
Hence the increase in volume of the liquid is

$$
V_{2}\left\{1+a\left(t_{2}-t_{1}\right)\right\}-V_{1},
$$

and this must be equal to $V_{1} \beta\left(t_{2}-t_{1}\right)$.

$$
\begin{aligned}
& \therefore V_{1} \beta\left(t_{2}-t_{1}\right)=V_{2}\left\{1+a\left(t_{2}-t_{1}\right)\right\}-V_{1}, \\
& \quad \beta=\frac{V_{2}}{V_{1}} a+\frac{V_{2}-V_{1}}{V_{1}\left(t_{2}-t_{1}\right)} \text {. . . . . . }
\end{aligned}
$$

Thus knowing $\beta$, in the case of mercury, we can calculate $a$, and then in the case of any other liquid we can use this value of $\alpha$ to calculate the coefficient of cubical expansion of the liquid.

The form of volume dilatometer shown in Fig. 81, but without the tap, can easily be made by the student, the stem being formed by a piece of wide bore thermometer tube. In the abrence of a tap, the top can be closed by about a centimetre of rubber tube and a small piece of glass rod. The graduations on the stem can be made by the method described in the Appendix.

If an air-pump is available, then the arrangement shown in Fig. 82 will be found to save an enormous amount of time. It consists of a small bottle with a well-fitting india-rubber cork. This cork is pierced by two holes. The dilatometer stem passes through one, and a piece of bent glass tube through the other. The liquid is placed in the bottle, and the bent tube is connected by means of a length of thick-walled rubber tube to the air-pump. To fill the dilato. meter the pump is worked, and the bulb of the dilatometer is heated with a Bunsen

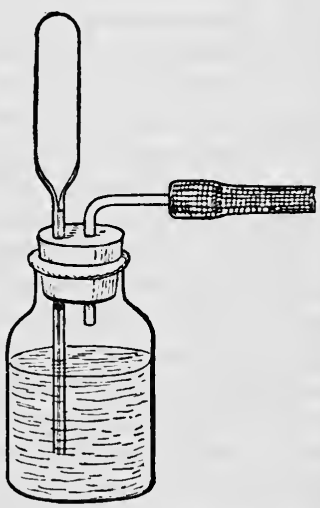

FIG. 82. flame to drive off any moisture. On admitting air from the pump the liquid is driven into the bulb. By admitting the air very slowly, and when enough liquid has entered to fill the dilatometer up to the required height in the capillary, raising the dilatometer through the cork, the advent of any further liquid can be prevented.

If the dilatometer is fitted with a tap, then the cork must be split on one side.

Having weighed the dilatometer empty, fill it with mercury up to a little above the top graduation, and place it in melting ice prepared as described in $\S 61$. When the temperature has become stationary, read the position of the top of the mercury column, and then again weigh. Next heat the dilatometer so that some of the mercury escapes, and hence when the instrument is placed in ice the mercury meniscus is near the bottom of the scale. When the temperature is again $0^{\circ}$, read the position of the 
meniscus, and again weigh the dilatometer. From the readings and weighings calculate the volume up to the zero of the scale and the volume of one division of the capillary.

The dilatometer being placed in steam read the position of the meniscus, also the barometer. Having calculated the temperature of the steam from the barometer reading in the manner described in $\S 60$, calculate by (3) the coefficient of cubical expansion of the glass.

To remove the mercury, replace the dilatometer in the filler (Fig. 82), and produce as good a vacuum as possible. If the mercury does not flow out, gently heat the bulb.

The procedure when measuring the expansion of a liquid is practically the same as the above, namely, the bulb is filled so that at $0^{\circ}$ the meniscus is near the bottom of the scale. The position of the meniscus is then read off when the instrument is maintained at different temperatures, and the coefficient of expansion calculated by (3).

76. The Hydrostatic Balance Method of Measuring the Coefficient of Expansion of a Liquid.-This method consists in determining the loss of weight of a piece of glass, or other solid, when immersed in the liquid at different temperatures. It is only applicable when a comparatively large volume of the liquid is available. In order to obtain the absolute expansion of the liquid, we require to know the coefficient of cubical expansion of the solid used. This solid generally consists of a cylindrical piece of glass tube, closed at the ends, with a small hook formed at one end for the attachment of the suspending wire. A little mercury inside the glass serves to ensure its sinking.

To determine the expansion of the glass, an experiment can be made with a liquid of known coefficient of expansion. Since mercury cannot be used for this purpose, on account of its great density, water must be employed. The expansion of the glass can also be measured by the method of the weight thermometer, after which the neck of the thermometer is fused off so that the bulb forms the sinker.

Let $V_{1}$ be the volume of the sinker at a temperature $t_{1}$ and $V_{2}$ the volume at $t_{2}$, these volumes being obtained by weighing in water, the formula used being

$$
V_{1}=\frac{w^{\prime}-w^{\prime \prime}}{\Delta-\sigma}
$$

where $w^{\prime}$ is the weight of the sinker in air, and $w^{\prime \prime}$ its weight when immersed in water at a temperature $t_{1}, \Delta_{1}$ is the density of the water as given in Table 5, and $\alpha$ is the density of the air (see $\S 30$ ).

Then

or

$$
\begin{gathered}
V_{2}=V_{1}\left\{1+\beta\left(t_{2}-t_{1}\right)\right\}, \\
\beta=\frac{V_{2}-V_{1}}{V_{1}} \cdot \frac{1}{t_{2}-t_{1}},
\end{gathered}
$$

where $\beta$ is the coefficient of cubical expansion of the glass.

If the loss of weight of the sinker when immersed in a liquid at 
temperatures $t_{1}$ and $t_{2}$ is $w_{1}$ and $w_{2}$ respectively, then the coefficient of cubical expansion of the liquid between these temperatures is given by formulæ (2) and (3) on p. 194.

The sinker is suspended by means of a fine platinum wire (diameter about $\cdot 004 \mathrm{~cm}$.), the upper end of the wire being attached to the pan of a balance, which must be one in which there are no pan arrestors. A hole must be bored through the base of the balance to allow of the passage of the wire, and the wire ought to be at least $40 \mathrm{~cm}$. long, so that the heated liquid is not near the balance. For this reason also it is as well to allow the wire to pass through a small hole in the table which carries the balance, the liquid being supported on a stand under the table. It is not necessary for the whole of the wire to be of platinum. Only the portion below the surface of the liquid and for about $10 \mathrm{~cm}$. above the surface need be of this metal; the rest can be of fine copper wire.

In order to reduce the disturbance produced by capillarity where the wire cuts the surface of the liquid, it is almost necessary that the surface of the platinum wire should be treated as described on p. 83 .

Having suspended the sinker, obtain its weight in air. Next immerse the sinker in the liquid, the ressel containing the liquid being packed round with non-conducting material, so that the rate of loss of heat is small. The liquid must be well stirred just before an observation, and then, when the currents set up by the stirring have subsided, the balance must be read, as well as a thermometer in the liquid. The bulb of this thermometer must be placed as near the sinker as possible.

When the temperature of the liquid is higher than that of the surrounding air, it is as well to adjust the weights so that the pointer is slightly deflected towards the side indicating that the sinker is too heary. As the liquid cools its density will increase, and the loss of weight of the sinker therefore will also increase, and so the balance will gradually come to its zero position. When the pointer passes the zero, read the thermometer, and take this reading as giving the temperature corresponding to the weights in the balance pan.

77. Determination of the Coefficient of Apparent Expansion of Mercury in Glass by means of a Thermometer.-A method of obtaining the mean coefficient of apparent expansion $(e)$ of mercury in glass when a calibrated thermometer of suitable range is available, has been used at the Reichsanstalt. ${ }^{1}$ Suppose that we have a thermometer reading from $-100^{\circ} \mathrm{C}$. to $+100^{\circ}$, the bore having been calibrated, and that the volume of the bulb up to the zero division is equal to $n$ times the volume between two consecutive degree divisions on the stem. If, now, the thermometer is immersed first in steam at $100^{\circ} \mathrm{C}$. and then in ice at $0^{\circ}$, and the number of degrees of the scale between these two fundamental points is $f$, we have

$$
e=\frac{f}{100 n} \cdot \text { • } \cdot \text {. . . . . . . }
$$

1 Wissen. Abhandlungen, i. 102 ; see also Chree, Philosophical Magazine [5] (1898), ×'v. 322. 
Next run some of the mercury into the bulb at the top of the stem, and repeat the observations at $0^{\circ}$ and $100^{\circ}$. If the mercury run into the top bulb would at $0^{\circ}$ fill $a$ degrees, and if the fundamental interval is now $f_{1}$, we have

$$
e=\frac{f_{1}}{100(n-a)} \text {. . . . . . . . }
$$

Next repeat the observation when a different quantity $(b)$ of mercury is run into the top bulb, so that we have

$$
e=\frac{f_{2}}{100(n-b)} \cdot \text { • • • . . . . }
$$

From equations (1) and (2) or (1) and (3) we can deduce both $n$ and $e$. In this experiment $a$ ought to correspond to about $50^{\circ}$ and $b$ to about $100^{\circ}$ in order to obtain a sufficiently great variation in $f$ to give a satisfactory measure of $e$. 


\section{CHAPTER XI}

\section{THERMAL EXPANSION OF GASES}

78. The Air Thermometer.-There are two forms of air thermometer. In one the volume of the gas is supposed to remain constant throughout, and the temperature is deduced from the change in pressure. In the other form the pressure is kept constant, and it is the change in volume of the gas which is used to give the temperature. In practice, however, it is found impossible to keep the volume in the one case and the pressure in the other rigorously constant. Hence it is advantageous to obtain expressions for the temperature when both the pressure and volume are supposed simultaneously to vary. These expressions can then be modified to suit the case when either the volume only changes slightly or the pressure only changes slightly, as the case may be.

\section{Let}

$V_{t}=$ volume of bulb, and that part of the capillary which is heated, at a temperature $t$;

$v_{t}=$ volume of gas in the remainder of the capillary, the manometer, \&c., i.e. in the dead space when the bulb is at a temperature $t$, the temperature of this air being $t^{\prime}$; Also let

$p_{t}=$ the pressure to which the gas is subjected at a temperature $t$.

$V_{0}, v_{0}$, and $p_{0}$ be the corresponding quantities at a temperature of $0^{\circ} \mathrm{C}$. ; and

$V_{1}, v_{1}$, and $p_{1}$ at the temperature of steam when the barometeric pressure is $B$.

Then if $\rho_{0}$ is the density of air at $0^{\circ}$ and standard pressure, the density of the air at a temperature $t$ and pressure $p_{t}$ is

$$
\frac{\rho_{0} p_{t}}{(1+a t) 76}
$$

where $a$ is the coefficient of expansion of air. Hence the mass of air contained in the bulb, of which the volume is $V_{b}$ is

$$
\frac{\rho_{0} p_{t}}{(1+a t) 76} V_{t}
$$

and the mass of air contained in the dead space is

$$
\frac{\rho_{0} p_{t}}{\left(1+a t^{\prime}\right) 76} v_{t} \text {. }
$$


Hence the total mass $m$ of air contained in the thermometer is given by

or

$$
\begin{gathered}
m=\left\{V_{t} /(1+a t)+v_{t} /\left(1+a t^{\prime}\right)\right\} \frac{\rho_{0} p_{t}}{76}, \\
\left\{V_{t} /(1+a t)+v_{t} /\left(1+a t^{\prime}\right)\right\} p_{t}=\frac{76 m}{\rho_{0}}=\text { constant ... }
\end{gathered}
$$

This is the fundamental equation of gas thermometry. It may be written in a slightly different form. Thus if $\theta$ is the temperature measured from the absolute zero corresponding to $t$, we have

$$
\theta=t+\frac{1}{a}=\frac{1+a t}{a}
$$

Hence equation (1) can be written

$$
\left\{\frac{V_{t}}{\theta}+\frac{v_{t}}{\theta^{\prime}}\right\} p_{t}=\text { constant }=k \text { (say) . . . . . }
$$

where $\theta^{\prime}$ is the absolute temperature corresponding to $t^{\prime}$.

If an observation is made at $0^{\circ} \mathrm{C}$. or $\theta_{0}$ on the absolute scale, we have

$$
\left\{\frac{V_{0}}{\theta_{0}}+\frac{v_{0}}{\theta_{0}^{\prime}}\right\} p_{0}=k . . . \cdot \cdot \cdot \cdot \cdot
$$

Further, if an observation is made in steam at a pressure $B$, the temperature of the steam at this pressure being $100^{\circ}+b$ or $\theta_{0}+100+b$ on the absolute scale, we have

$$
\left\{\frac{V_{1}}{\theta_{0}+100+b}+\frac{v_{1}}{\theta_{1}^{\prime}}\right\} p_{1}=k \text {. . . . . . }
$$

If $\beta$ is the coefficient of cubical expansion of the material of which the bulb is constructed, then

$$
V_{t}=V_{0}(1+\beta t)=V_{0}\left\{1+\beta\left(\theta-\theta_{0}\right)\right\} \quad . \quad \text {. . . }
$$

These equations will serve to perform all the calculations necessary when using air thermometers, whether of the constant volume or the constant pressure type. In the constant volume type $v_{t}$ remains very nearly constant, while $p_{t}$ varies; while in the constant pressure type $v_{t}$ varies considerably, while $p_{t}$ is kept as nearly as possible constant.

79. Measurement of the Coefficient of Increase of Pressure of Air with the Air Thermometer.-A simple form of constant volume air thermometer is shown in Fig. 83. The bulb $\mathbf{A}$ is connected to the manometer by a fine capillary tube, a three-way tap c being included. The glass reservoir $\mathrm{K}$ has a black glass point $\mathrm{D}$ attached near the top, and the surface of the mercury is always brought to coincide with this point. The object of the reservoir is that when the bulb is heated the air may not be driven over into the manometer tube, even if the pressure is not 
increased. The lower part of the reservoir $\mathrm{K}$ is connected by means of a three-way cock with the manometer tube EF and a length of thick-walled rubber tubing, which is attached to the mercury reservoir G. This mercury reservoir can be raised or lowered by means of a cord which, after passing over a pulley near the top of the apparatus, is wound on a drum. This drum can be rotated by a tangent screw actuated by a

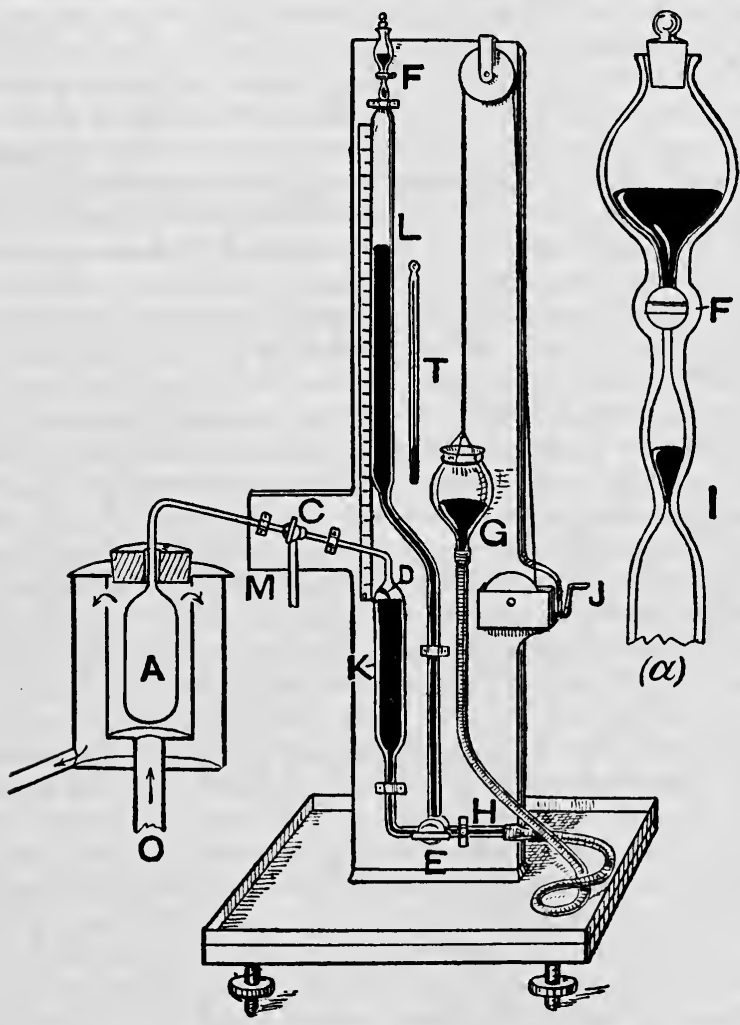

Fra. 83.

handle J. The manometer tube EF is designed to measure the whole pressure to which the gas is subjected, that is, it is not necessary to read an auxiliary barometer. The top of the tube is fused to a well-ground tap $F$ and a small separating funnel. Just below the tap the tube is constricted, as shown at I on the enlarged drawing $(a)$.

The manometer tube is filled in the following manner:-The tap $F$ being open, and the tap $\mathbf{E}$ turned so as to cut off connection with $\mathrm{K}$, the reservoir $G$ is raised till the mercury flows into the funnel. The tap $F$ is 
then closed, and the reservoir lowered as far as possible. In this way a Torricellian vacuum will be left in the upper part of the tube EF. Owing, however, to moisture condensed on the sides of the tube the vacuum will generally be bad. To get rid of this moisture the tube must be heated, the mercury reservoir remaining at its lowest. Then by raising the reservoir, and when it is at its highest point opening the tap $F$, the vapour can be driven out. By repeating this operation several times it will be possible to get quite a good vacuum. The object of the constriction I is that owing to capillarity the mercury in the small bulb above the constriction will remain, and if any air leaks in at the tap $F$ this will at once be indicated by the mercury in this bulb being forced down. Thus as long as there is mercury in the bulb we can feel confident that the vacuum remains good.

When the tap $\mathbf{E}$ is turned so as to connect $\mathbf{K}$ with the manometer, and the surface of the mercury is adjusted to coincide with the end of the point $D$, then the height of the mercury meniscus $L$ above the point $D$ will give the pressure to which the gas in the bulb is subjected. The height of this mercury column is read off on a divided scale fastened alongside the tube, the zero of the scale being adjusted to coincide with the point $\mathbf{D}$.

The bulb a has now to be filled with dry air. To do this the side tube $\mathrm{M}$ is connected, through some drying tubes containing phosphorus pentoxide, with an air-pump. The mercury surface in the reservoir $\mathbf{k}$ having been lowered as much as possible, the tap $\mathrm{E}$ is turned so as to shut off $\mathrm{K}$ from the manometer and reservoir $\mathrm{G}$, and then the bulb is exhausted. While the vacuum is kept as good as possible, the bulb A, the connecting tubes, and the vessel $\mathrm{K}$ are warmed with a flame so as to drive off all moisture. Air is then admitted through the drying tubes. The operation of exhaustion and admission of air, which latter operation ought to be performed very slowly, is repeated several times. In this way the bulb can be filled with dry air, and the film of moisture which always clings to the surface of glass can in a great measure be removed.

Having either adjusted and filled the instrument as described above, or ascertained that it has been so filled, place the bulb $\mathrm{A}$ inside a funnel which is supported on a retort stand, and pack with pounded ice, treated in the manner described in $\S 61$. When the bulb has been in the ice for a few minutes, adjust the surface of the mercury to coincide with the end of the point $D$, using the reflection of the point in the mercury surface to assist in making the adjustment. If after two or three minutes the adjustment is still complete, read the thermometer $\mathrm{T}$ and the position of the mercury meniscus $\mathrm{L}$.

The bulb must now be placed either in boiling water, the temperature of which is taken by a thermometer of which the boiling point error is known, or in a steam-jacket, such as is shown in the figure. The wooden lid $\mathbf{s}$ is split in halves so that it may be fitted round the bulb stem. Steam is passed in at $o$, and the position of the meniscus roughly adjusted to coincide with the point $\mathrm{D}$. After the steam has been passing for about ten minutes, the adjustment of the mercury meniscus is com- 
pleted, and the thermometer $\mathbf{T}$ and the position of the meniscus $\mathrm{L}$ are again read. The height of the barometer must also be noted, so as to allow of the temperature of the steam being obtained.

The height of the mercury column in the manometer will have to be reduced to what it would be at $0^{\circ}$ (and in very accurate measurements to standard gravity) in the manner described in $\S 58$. The temperature of the steam must also be calculated as in $\$ 60$.

The end of the point $\mathrm{D}$ may not exactly coincide with the zero of the scale used to read the height of the column. This point may be tested, and the correction, together with that for the effect of capillarity (\$57), can be obtained if a standard barometer is available. The tap $\mathrm{c}$ being turned so as to connect the upper part of $\mathrm{K}$ with the open air, the mercury meniscus is adjusted to the point $\mathrm{D}$, and the temperature and height of the mercury column are read. The barometer being also read, the difference between the values for the barometric pressure obtained after reducing to $0^{\circ}$ will give the correction to be applied to the readings of the manometer.

It is possible to obtain a formula by means of which the coefficient of increase of pressure $a$ is expressed in terms of the observed quantities. Such an expression, however, is very complicated and quite unsuited to computation. It is much easier to perform the reduction by successive steps, using the equations given in the last section. We have from equations (3), (4), and (5), since in this experiment $v_{1}=v_{0}$,

$$
\left(\frac{V_{0}}{\theta_{0}}+\frac{v_{0}}{\theta_{0}^{\prime}}\right) p_{0}=\left\{\frac{V_{0}(1+100 \beta)}{\theta_{0}+100+b}+\frac{v_{0}}{\theta_{1}^{\prime}}\right\} p_{1},
$$

or

$$
\left\{\frac{1}{\theta_{0}}+\frac{1}{\theta_{0}^{\prime}} \frac{v_{0}}{V_{0}}\right\} p_{0}=\left\{\frac{1}{\theta_{0}+100+b}+\frac{100 \beta}{\theta_{0}+100+b}+\frac{1}{\theta_{1}^{\prime}} \cdot \frac{V_{0}}{v_{0}}\right\} p_{1} .
$$

Neglecting the effect of the dead space and the expansion of the bulb, this gives as a first approximation for $\theta_{0}$

$$
\frac{p_{0}}{\theta_{0}}=\frac{p_{1}}{\theta_{0}+100+b}
$$

or

$$
\theta_{0}=\frac{p_{0}(100+b)}{p_{1}-p_{0}}
$$

We may now employ this approximate value of $\theta_{0}$ to calculate the values of $\theta_{0}^{\prime}$ and $\theta_{1}^{\prime}$ from the observed temperatures given by the thermometer T. Further, to obtain the values of the correction terms in (6) we require to know the values of $\beta$ and of $v_{0} / V_{0}$. These quantities do not require to be known with any very great degree of accuracy, since they are only involved in small correction terms.

(1) If some of the glass of which the bulb $\mathbf{\Lambda}$ is made is available, then $\beta$ may be measured by using this glass to make a weight dilato- 
meter, and observing in the manner described in $\S 74$. For most purposes it will be sufficiently accurate to assume the value 0.0000232 for $\beta$.

(2) The quantity $v_{0} / V_{0}$, which is the ratio of the volume $v_{0}$ of the dead space to the volume $V_{0}$ of the bulb, may be determined in the following manner:-The bulb being immersed in water, which has stood for some time so as to acquire the temperature of the air, the mercury is adjusted to the point $\mathrm{D}$, and the height $\pi_{1}$ of the mercury column is read. The reservoir $G$ is then very slowly raised till the mercury has almost reached the bend in the capillary just above the bulb, and the pressure $\pi_{2}$ is read off. We then have

or

$$
\begin{gathered}
\pi_{1}(V+v)=\pi_{2} V, \\
\frac{v}{V}=\frac{\pi_{2}-\pi_{1}}{\pi_{1}} .
\end{gathered}
$$

The values of the various quantities are then inserted in equation (6), which then becomes a simple equation involving the single unknown $\theta_{0}$, and from it we therefore deduce this quantity, and then $\alpha=1 / \theta_{0}$.

80. Determination of the Corrections to a Mercury Thermometer at High Temperatures by means of the Constant Volume Air Thermometer.-Determine the pressure of the air thermometer when the bulb is in melting ice and steam respectively, as in the preceding section. Then place the bulb in a beaker containing glycerine, the top of the beaker being closed by a wooden disc similar to that used to close the steam-jacket in Fig. 83. There must be two additional holes in the wooden disc, one for the passage of a mercury thermometer reading to $300^{\circ}$, and the other for the handle of a stirrer. The beaker must rest on a sheet of asbestos card, and it is an advantage to wrap the sides of the beaker with asbestos cloth, so as to reduce the loss of heat from the sides. Place a small Bunsen burner below the beaker, and adjust the size of the flame so that the temperature of the glycerine becomes stationary at about $150^{\circ}$. Then read the thermometer and the height of the mercury column in the manometer, the meniscus having been adjusted to the standard position. Then increase the size of the flame, and in the same way obtain readings at $200^{\circ}, 250^{\circ}$, and $300^{\circ}$.

Having first reduced all the manometer readings to $0^{\circ}$, proceed as in the previous section to calculate the value of $\theta_{0}$. Then from equations $(2)$, (3), and (5), § 78 , we have

$$
\frac{1+\beta t}{\theta} p_{t}+\frac{v_{0} p_{t}}{V_{0} \theta^{\prime}}=\frac{p_{0}}{\theta_{0}}+\frac{v_{0} p_{0}}{V_{0} \theta_{0}^{\prime}}
$$

in which all the quantities except $\theta$ are known; for in the term $\beta t$ we may, without causing appreciable error, take $t$ as the temperature of the glycerine bath as given by the mercury thermometer, while $v_{0} / V_{0}$ can be obtained as in the last section. Then the temperature $t_{\alpha}$ of the bath on the scale of the air thermometer is given by

$$
t_{a}=\theta-\theta_{0} \text {. }
$$


§ 81] CONSTANT PRESSURE AIR THERMOMETER 209

The difference between the reading of the mercury thermometer and $t_{a}$ gives the correction to the thermometer.

81. The Constant Pressure Air Thermometer.-A form of constant pressure air thermometer which is fairly simple is represented in Fig. 84. It is slightly modified from that described by Callendar and Griffiths. ${ }^{1}$ The thermometer bulb $\mathbf{A}$ is connected through a capillary to a second bulb B, and also to an oil manometer c. The lower part of the bulb B has two tubes attached, each of which is furnished with a stop-cock.

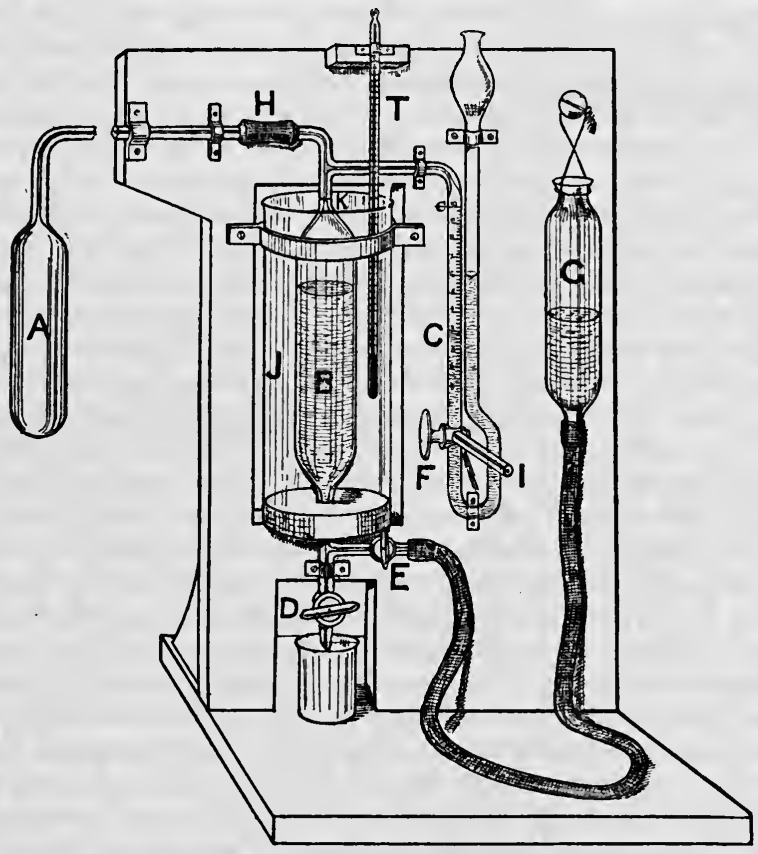

FIG. 84 .

Through E mercury can be passed into $\mathbf{B}$ from the reservoir $\mathbf{G}$, and through $\mathbf{D}$ mercury can be run out of $\mathbf{B}$ into a weighed beaker placed to receive it. The bulb B is surrounded by water contained in a glass cylinder $\mathrm{J}$, the temperature of the water being given by a finely divided thermometer T. The manometer $\mathrm{C}$ is fitted with a three-way cock $\mathrm{F}$, 'and the limb nearest the bulb is graduated in millimetres. The bulb $\mathbf{A}$ may either be connected to the rest of the apparatus by a continuous length of capillary, or, what is generally much more convenient, the bulb $\mathrm{B}$ and manometer $\mathrm{C}$ may be made in one piece, and this be joined

1 Philosophical Transactions of the Royal Society(1891), vol. clxxxii. p. 119. 
to the capillary attached to the bulb $\mathrm{A}$ by a joint $\mathrm{H}$. This joint is made by drawing down a piece of glass tube till it makes a tight fit on the outside of the capillary, then cutting off about 2 centimetres of this tube, slipping it over the ends of the capillaries, and having well heated the glass, running in sealing-wax between the glass sleeve and

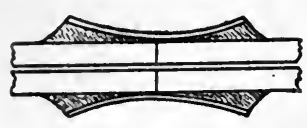

FIG. 85. the capillaries. A section of the joint is shown on a large scale in Fig. 85.

Before the instrument is mounted on the stand and the bulb attached it will be necessary to measure the volume of the bulb up to a point about 2 centimetres beyond the bend in the capillary, up to which point the heating will in each case be made. This volume may be determined with sufficient accuracy by weighing the bulb empty, and then when filled up to the given point, which should be indicated by a mark on the capillary, with water at $0^{\circ}$. The water can be removed by connecting the end of the capillary to a water-pump, and then heating the bulb to a temperature over $100^{\circ}$. The volume of the capillary between the mark and the end where it is to be joined to the rest of the instrument must also be determined. This can be done by drawing up a thread of mercury hy first.warming and then cooling the bulb, and then weighing the thread.

Finally, the volume of that part of the capillary between the joint $\mathrm{H}$, the zero of the scale on the manometer, and a mark $\mathrm{K}$ on the top of the bulb must be determined, as well as the volume of unit length of the divided portion of the manometer. These volumes can be determined by weighing the mercury which fills the various portions of the tube.

To fill the bulb with dry air a pump is connected to $\mathrm{I}$, and the tap $\mathrm{D}$ is connected to some drying tubes, and the air is alternately exhausted and readmitted through the drying tubes, the whole apparatus being kept warm and the bulb $\Delta$ heated to a fairly high temperature. Dry and clean mercury is then run in through $\mathbf{E}$ till the surface reaches almost up to where the manometer joins the other capillary. Some oil, such as is used in the Fleuss pump, is then sucked in through the tap I, the tap being so turned that connection is cut off from the bulbs $\mathbf{A}$ and $\mathbf{B}$. The $\operatorname{tap} \mathrm{F}$ is then turned so that the side tube I is closed, and by slightly lowering the mercury in $\mathbf{B}$ the oil is caused to stand at the same level in the two limbs of the manometer. To prevent the oil from being sucked over into the bulbs, owing to the pressure in the bulbs falling below that of the atmosphere, it is necessary to take care that, except when taking a reading, the $\operatorname{tap} F$ is turned so as to cut off the two halves of the manometer from one another.

To perform an experiment, place the bulb $\mathrm{A}$ in melting ice, and by raising the reservoir $\mathrm{G}$ bring the surface of the mercury to the mark $\mathrm{k}$. When the temperature of the bulb has had time to reach $0^{\circ}$, run off mercury by the tap $\mathrm{D}$ into a weighed heaker till the manometer shows that the pressure within the instrument is equal to that of the atmosphere. 
Immediately read the temperature $t^{\prime}$ on the thermometer $\mathrm{T}$ and the barometric height. Let the weight of mercury run out be $w_{0}$.

Next heat the bulb in steam, and determine the weight of mercury $w_{1}$ which has to be run out so as to make the pressure inside equal to the external pressure, and read the temperature $t^{\prime}$ on the thermometer $T$ and the barometric height. It is not essential that the pressure shall be always adjusted to exactly atmospheric pressure, but if this adjustment is not quite exact, then the difference in the levels of the oil in the two limbs of the manometer, as well as the reading on the scale $\mathbf{c}$, must be noted. These readings allow of the pressure to which the gas is subjected being calculated, as well as the volume between the zero of the scale $c$ and the top of the oil column.

The method of reducing the observations is very much the same as that adopted in the case of the constant volume instrument. The dead space here, however, may be considered as consisting of three portions. The first portion consists of the volume $\nu$ of the capillary up to the mark $\mathrm{K}$, and the zero of the scale $\mathrm{c}$. This quantity remains constant throughout the observations, and has been determined once for all before the instrument was mounted. Secondly, there is the volume $v^{\prime}$ of the space between the zero of the scale $c$ and the top of the oil column. The value of this volume is obtained from the reading of the scale c and the calibration of the manometer tube made before mounting. Thirdly, there is the volume $v^{\prime \prime}$ of the space occupied by the air between the top of the mercury in the bulb $\mathbf{B}$ and the mark $\mathrm{K}$. The volume of this space is obtained from the weight of mercury run out. Thus if $\rho$ is the density of mercury at the temperature $t^{\prime}$ of the water bath $\mathrm{J}$, the value of $v^{\prime \prime}$ when the bulb was in ice is given by $v_{0}^{\prime \prime}=w_{0} / \rho$, while when the bulb was in steam we have $v_{1}{ }^{\prime \prime}=\left(w_{0}+w_{1}\right) / \rho$. If, however, the temperature of the water bath $J$ changes, then a correction will have to be applied to allow for the expansion of the bulb $\mathrm{B}$ and the mercury remaining in the bulb. The magnitude of this correction can easily be obtained; it is, however, simpler if the conditions are such as to cause the temperature of $J$ to vary to fill the jacket with melting ice, so that the temperature of the bulb $\mathbf{B}$ is always the same, namely, $0^{\circ}$.

The general equation (2), p. 204, may in this case be written

$$
\left\{\frac{V_{t}}{\theta}+\frac{v}{\theta^{\prime}}+\frac{v^{\prime}}{\theta^{\prime}}+\frac{v^{\prime \prime}}{\theta^{\prime}}\right\} p_{t}=k
$$

where we assume that the whole dead space has the same temperature $\theta^{\prime}$ as the jacket $J$.

In the ice experiments we have

$$
\left\{\frac{V_{0}}{\theta_{0}}+\frac{\nu}{\theta_{0}^{\prime}}+\frac{v_{0}^{\prime}}{\theta_{0}^{\prime}}+\frac{v_{0}^{\prime \prime}}{\theta_{0}^{\prime}}\right\} p_{0}=k,
$$

and in steam at the temperature $\theta_{1}$

$$
\left\{\frac{V_{1}}{\theta_{1}}+\frac{\nu}{\theta_{1}^{\prime}}+\frac{v_{1}^{\prime}}{\theta_{1}^{\prime}}+\frac{v_{1}^{\prime \prime}}{\theta_{1}^{\prime}}\right\} p_{1}=k .
$$


We may then evaluate the various terms, as was done in the case of the constant volume air thermometer, and deduce a value of $\theta_{0}$, just as in that case.

If we assume that we know the value of $\theta_{0}\left\{272^{\circ} \cdot 2\right\}$, then the observations in ice and steam will allow of $V_{0}$, the volume of the bulb at $0^{\circ}$, being calculated.

The method of using this thermometer to calibrate a mercury thermometer is exactly similar to that described in the last section, and the method of reducing the results will be obvious from what has been said above. 


\section{CHAPTER XI 1}

\section{CALORIMETRY}

82. Calorimetry-The Method of Mixture.-In the method of mixtures for determining the specific heat of a body, the body is heated to a known high temperature, and is then introduced into a mass of water contained within a vessel called the calorimeter, and from the rise in temperature of the water the specific heat of the body is calculated. Although the principle of the method is very simple, yet to obtain accurate results it is necessary to take several important precautions and to introduce corrections, some of which, at any rate, are by no means easy to determine.

Since the heat given out by the hot body is employed not only in heating the water in the calorimeter but also in heating the calorimeter itself, a correction has to be applied to allow for this heat, and hence it is of importance to make the mass of the calorimeter as small as possible. Further, since to calculate the heat given to the calorimeter we shall have to multiply its mass by its specific heat and by the rise in temperature, it is important that the calorimeter should be at the same temperature throughout. Now, it being impossible to fill the calorimeter quite full of water, unless the material of which the calorimeter is constructed is a good conductor of heat, the portions of the calorimeter above the surface of the water will be at a different temperature to the rest, and hence the change in temperature of the water within the calorimeter will not be the same as the change in temperature of the whole of the calorimeter. For this reason it is important to make the calorimeter of a good conductor of heat, and always to take care that it is nearly filled with water. The materials best suited for the construction of calorimeters are copper and silver, for both these metals are very good conductors of heat. If a liquid has to be used in the calorimeter which would corrode either of these metals a glass calorimeter must be employed, but in this case it is essential that the glass should be very thin and that the liquid should fill the calorimeter almost up to the top, so that the mass of glass above the surface of the liquid should be as small as possible.

Since if the temperature of the calorimeter is different from that of the surrounding air it will either lose or receive heat at a rate dependent on its surface and the difference in temperature between its surface and the surrounding air, it is of importance to make the surface of the calorimeter (including the free surface of the water) as small as possible, 
so that if the calorimeter is cylindrical in form the height ought to be made equal to the diameter of the base, for in this case for a given volume the ratio of the volume to the surface will be greatest. This shape of calorimeter has one disadvantage, namely, that the free surface of the liquid in the calorimeter is considerable, and hence if the liquid being experimented upon is at all volatile, the evaporation may introduce considerable error. This is particularly the case with such a liquid as water, which has a very large latent heat of evaporation. Hence it is generally advisable to make the height about 1.25 times the diameter. The evaporation may be checked by providing the calorimeter with a lid. Such lid ought to be made of the thinnest possible metal, and must be pierced with a slot to allow of its being removed for the introduction of the hot body without disturbing the thermometer.

An important adjunct to the calorimeter is the stirrer, for it is only by keeping the contents of the calorimeter well stirred that we can ensure that they are at a uniform temperature. The conditions aimed at in the design of a stirrer are that it should be light, and that when in use it should thoroughly mix the liquid without requiring to be worked at a very great rate. Where possible, it is also an advantage to use a form of stirrer which, when being worked, does not move in

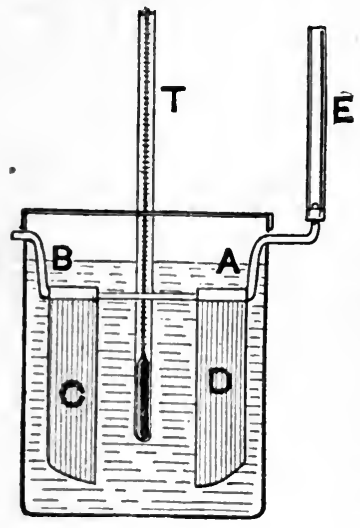

FiG. 86. such a way that the handle moves in and out of the water, for such a motion of the handle must necessarily encourage the evaporation of the liquid.

Since it is important to reduce the rate of loss of heat from the outside surface of the calorimeter to a minimum the outside of the calorimeter ought to be polished, for the radiation from a polished surface is less than that from a black surface. Also to prevent loss of heat by conduction the calorimeter ought to be suspended by three fine strings, or supported on three small cones made of cork. For most purposes the cork supports will be found the more convenient, since with them it is so much easier to remove the calorimeter. The bases of the three cork cones ought to be glued to a disc of brass or copper which will just fit inside the water-jacket referred to below.

In order to render the loss of heat from the outside of the calorimeter regular, and hence allow of a correction being calculated, it is necessary to keep the calorimeter during the observations within a vessel which is itself kept at a constant temperature. The best method of securing such a constant temperature surrounding is to have a doublewalled metal vessel, the space between the walls being filled with water. A form of calorimeter which fulfils the conditions mentioned above 
is shown in Fig. 86. It consists of a cylindrical copper vessel $10 \mathrm{~cm}$. in diameter and $12 \mathrm{~cm}$. high. Two small notches are cut in the top edge at opposite ends of a diameter. The stirrer consists of a thin horizontal copper rod $\mathrm{AB}$, bent as shown in the figure, and resting in the notches. Two thin copper plates CD are soldered to this rod, while a small glass handle is cemented to the outside. By giving this handle a to-and-fro motion the paddles $\mathrm{C}$ and $\mathrm{D}$ are moved backwards and forwards through the liquid, and thus stir it. The thermometer $\mathbf{T}$ is supported in a clip, so that its bulb is near the centre of the calorimeter. A simpler form of stirrer consists of a copper annulus of such a diameter

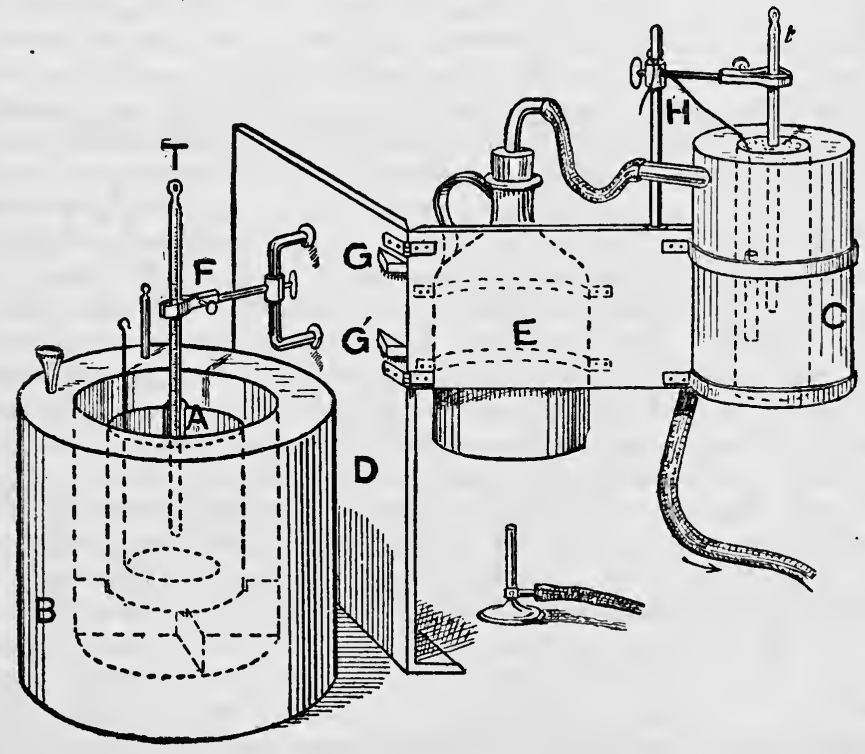

FIG. 87.

that it loosely fits inside the calorimeter, and having a short length of copper wire soldered to the upper surface. A piece of glass tube is cemented to this wire, and forms a handle by which the annulus can be moved up and down through the liquid, the bulb of the thermometer passing through the hole in the centre. This form of stirrer suffers from the objection that as it is used part of the stem moves in and out of the liquid, and thus promotes evaporation.

The general arrangement of the apparatus when measuring the specific heat of a solid by the method of mixtures is shown in Fig. 87. The calorimeter $\mathbf{A}$ is placed inside a double-walled copper vessel $\mathbf{B}$, the space between the walls being filled with water. The thermometer $\mathbf{T}$ is 
supported by a clip attached to an arm $\mathrm{F}$, which is itself movable on a vertical rod attached to the upright board D. This board serves as a screen to protect the calorimeter from heat radiated from the heater $\mathbf{c}$. The heater consists of a double-walled copper vessel, so that steam can be passed through the space between the walls. ${ }^{1}$ The heater is carried by a hinged arm $\mathbf{E}$, and the position of the calorimeter is so adjusted that when the heater is swung round till the arm E touches two stops GG, the heater is vertically over the calorimeter. The substance of which the specific heat is to be determined is suspended within the heater alongside the bulb of the thermometer $t$, the top and bottom of the inner part of the heater being closed by plugs of cotton wool. By swinging the heater over the calorimeter and releasing the string $\mathrm{H}$ the substance can be dropped into the liquid contained in the calorimeter, the heater being immediately swung back into its position behind the screen $\mathbf{D}$.

The thermometer $\mathrm{T}$ used in the calorimeter must be divided into at least tenths of a degree, since, otherwise, the comparatively small rise in temperature which takes place on the introduction of the hot body will not be measured with reasonable accuracy. The range of the thermometer need not be very great, say, from $15^{\circ}$ to $30^{\circ}$. Since it is important to be able to quickly read the thermometer, it is advisable to use a thermometer in which the thread can be distinctly seen. Further, since it is not very convenient to use a reading telescope, it is advisable to have the divisions as near the thread as possible, so as to avoid errors due to parallax. For this reason the German pattern of thermometer has decided advantages, for the capillary having a thin wall, the scale is nearer the mercury thread than in the ordinary English pattern, where the stem has to be made thick to give strength.

If $M$ is the mass of the body of which the specific heat is being. measured, $W$ the mass of the water in the calorimeter, and $t_{1}$ is the initial temperature of the body, $t_{2}$ the initial temperature of the water, and $t_{3}$ the final temperature of the water and body, then to a first approximation the specific heat $s$ of the body is given by

$$
\boldsymbol{s}=\frac{W\left(t_{3}-t_{2}\right)}{M\left(t_{1}-t_{3}\right)}
$$

The result obtained in this way will require correction to allow for a number of things.

1. The heat given out by the body has been expended, not only in raising the temperature of the water in the calorimeter, but also in raising the temperature of the calorimeter itself, of the stirrer, and of the portion of the thermometer which dips in the water. If we assume that the whole of the calorimeter and the copper part of the stirrer are

1 Another form of heater is shown in Fig. 94. This heater can be easily. brought over the calorimeter and the body tipped into the water by inverting the heater. 
always at the same temperature as the water, which we inay expect if the calorimeter is very nearly filled and only a small part of the stirrer projects beyond the calorimeter, then we can at once calculate the amount of heat spent in heating these. Thus if $w$ is the mass of the calorimeter and stirrer (without the glass handle), and $\sigma$ is the specific heat of the material of which the calorimeter and stirrer are made, the heat used up in raising their temperature is $\omega \sigma\left(t_{3}-t_{2}\right)$. The quantity $20 \sigma$, which represents the quantity of heat necessary to raise the temperature of calorimeter and stirrer through 1 degree, is called the water value of the calorimeter and stirrer. If $w$ is small compared to the weight of water $W$, and the calorimeter is made of copper, then it will in general be quite sufficiently accurate to take the value $\cdot 1$ for $\sigma$.

The determination of the water value of the immersed portion of the thermometer is a matter of much greater difficulty. For most purposes it will, however, be sufficiently accurate to proceed in the following manner :-The density of mercury being $13 \cdot 6$, and its specific heat 0.0333 , the quantity of heat required to raise the temperature of unit volume of mercury through 1 degree is $13.6 \times 0.0333$, or $\cdot 453$. The density of Jena glass $16^{\prime \prime \prime}$ is 2.58 , and its specific heat is 0.199 . Hence the quantity of heat necessary to raise the temperature of unit volume of this glass 1 degree is $2.58 \times 199$, or $\cdot 513$. It will thus be seen that approximately it requires the same quantity of heat to raise the temperature of unit volume of mercury and glass through the same range. Hence if we measure the volume of the portion of the thermometer which is immersed in the calorimeter, and assume that the whole of this volume is filled with mercury, we shall not be much in error. If $v$ is the volume of the immersed part of the thermometer, then the water value of the thermometer is given by $\cdot 45 v$.

The quantity $v$ can be measured by immersing the thermometer, as far as it is immersed in the calorimeter, in the water contained in a graduat tube, such as a burette, and reading off the volume corresponding to the rise of the surface of the water.

2. Some heat will have been lost during the transfer of the body from the heater to the calorimeter. It being practically impossible to estimate the amount of this loss, we are constrained to reduce it as much as possible. It is for this reason that the heater is made to swing over the calorimeter, so that the body may drop straight down into the water. The importance of the error caused by this loss of heat will become less as the mass of the body is increased, so that it is always advisable to use as much of the substance as the heater will conveniently hold. In this case, as in nearly all the errors which are met with in performing a determination of specific heat by the method of mixtures, the error is approximately as the surface of the body or calorimeter, as the case may be, while the quantity affected by the error varies as the volume. Hence the percentage error produced decreases as the volume increases, for the increase in the volume is more rapid than the increase in the surface. 
3. Between the time when the hot body is introduced and the instant when the temperature of the water in the calorimeter, as indicated by the reading of the thermometer, reaches its maximum, there will in general be a loss of heat by radiation and convection from the surface of the calorimeter, so that the final temperature attained is not so high as it ought to be. There are various ways of allowing for this loss of heat by radiation (and conduction).

In Rumford's method the initial temperature of the water is taken below that of the surrounding water-jacket, so that during the first half of the rise the calorimeter will gain heat from the surroundings, while during the second half it will lose heat. If the rate of rise of temperature in the calorimeter were even approximately constant, then a fairly good compensation could be secured by Rumford's method. In practice, however, the temperature rises at first very rapidly, and the maximum is only reached slowly, so that the interval during which the calorimeter is losing heat is greater than that during which it is gaining heat, if the initial cooling is such as to make the mean between the initial and final temperatures equal to the temperature of the surroundings. To allow for this effect, it has been proposed to cool the water, so that its initial temperature is twice as much below that of the surroundings as its final temperature is above. It is, however, much preferable to measure the loss of heat due to radiation and to make a correction on this account, as described below.

Regnault's method of performing an experiment so as to correct for radiation is as follows :-

The experiment is divided into three periods. During the first period the temperature of the calorimeter is noted at intervals during three or four minutes, the temperature of the jacket being also noted. In the second period the hot body is introduced at a noted time, and the temperature of the calorimeter is read every fifteen or thirty seconds till the maximum reading is obtained. During the third period the temperature of the calorimeter is read every minute for about ten minutes. The temperature of the jacket must also be noted.

By plotting the numbers obtained during the first period.against the time, the temperature of the calorimeter at the instant when the hot body was introduced can be obtained by a slight extrapolation.

From the numbers obtained in the third period, calculate the fall of temperature per second when the calorimeter has its highest temperature. For this purpose it will be best to take the fall of temperature during four or five ininutes and divide by the number of seconds in the interval. Then on a piece of squared paper, taking the abscissæe as the temperatures of the calorimeter, and as ordinates the fall of temperature per second, plot the point $Q$ (Fig. 88) to correspond to the value of the rate. of fall of temperature at the high temperature obtained above. Also take the point $P$ on the axis of temperatures corresponding to the temperature of the water-jacket surrounding the oalorimeter, for when the temperature of the calorimeter is equal to that of the surroundings, the rate of fall 
of temperature will be zero. If, then, we assume that the rate of fall of temperature is proportional to the excess of the temperature of the calorimeter over the temperature of the jacket, the straight line joining $\mathbf{P}$

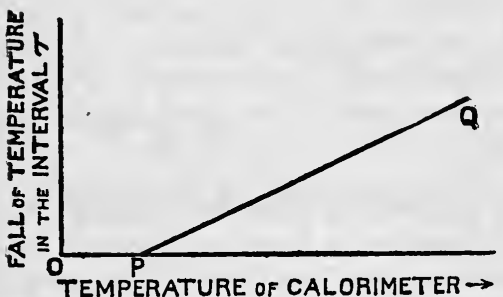

FIG. 88. and $Q$ will give the rate of fall of temperature for all temperatures of the calorimeter. The assumption made above, namely, that Newton's law of cooling holds, is quite justified so long as the excess of temperature of the calorimeter does not exceed a fow degrees, which differencein temperature must never be exceeded if anaccuratedetermination of specific heat is being made.

Next, the numbers obtained in the second period must be plotted, taking the times as abscissæ and the temperatures of the calorimeter as ordinates, and in this way a curve $A B C D E$ (Fig. 89) is obtained.

Divide the curve into segments $\mathrm{AB}, \mathrm{BC}, \mathrm{CD}, \& \mathrm{c}$., which are approximately straight. Let the temperatures corresponding to the points $A$ and B be $\theta_{1}$ and $\theta_{2}$, so that the mean temperature during the interval oM is $\frac{\theta_{1}+\theta_{2}}{2}$. From the cooling curve (Fig. 88) obtain the rate of cooling corresponding to this mean temperature, and niultiply by the time oM. The product, which we may call $t_{1}$, will be the amount by which the temperature at the end of the interval om will be below what it would have been suppose there had been no loss of heat due to radiation, \&c. Hence if we increase the ordinate $\mathrm{MB}$ by an amount $\mathbf{B B}^{\prime}$ equal to $t_{1}, \mathbf{M B}^{\prime}$ will represent what would have been the temperature of the calorimeter at the end of the interval oM if there had been no loss of heat. In the same way, read off the mean temperature for the interval

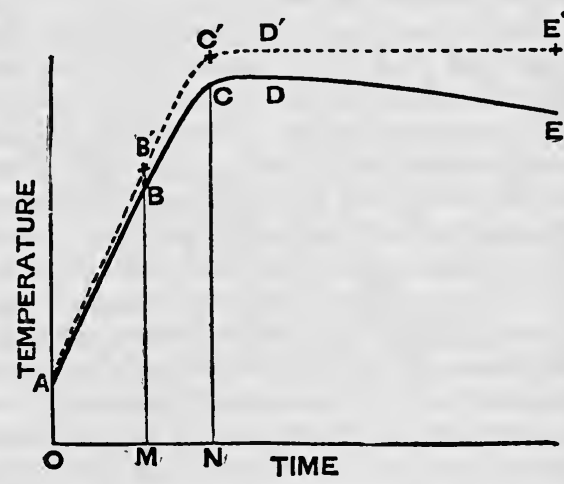

FIG. 89. MN, and multiply the rate of cooling for this temperature by the interval, and obtain the correction $t_{2}$ for loss of heat during the second interval. If the ordinate NC is then increased by an amount $\mathrm{Cc}^{\prime}$ equal to $t_{1}+t_{2}, \mathrm{Nc}^{\prime}$ will represent what the temperature would be at the end of the second interval if there had been no loss of heat, for the loss of heat at the end of the 
second interval is equal to the sum of the losses during the first and second intervals. Proceeding in this way a new curve $A^{\prime} C^{\prime} D^{\prime} E^{\prime}$ is obtained, which represents what would have been the temperature of the calorimeter supposing there had been no loss of heat due to radiation, conduction, and convection. The part $D^{\prime} E^{\prime}$ of this curve ought to be horizontal, since we are correcting for the fall of temperature due to los? of heat, and if there is no loss of heat, then the temperature of the calorimeter would remain constant. The final temperature to be used in calculating the specific heat is that corresponding to the horizontal part of the curve, viz. $\mathrm{D}^{\prime} \mathrm{E}^{\prime}$.

If the temperature of the jacket surrounding the calorimeter varies during the experiment, then when obtaining the cooling curve and calculating the corrections the difference between the temperature of the calorimeter and that of the jacket must be used.

The importance of the correction for radiation increases as the time which elapses between the introduction of the hot body and the temperature reaching the maximum gets greater. Thus it is more important in the case of a bad conductor of heat, such as glass, which only slowly parts with its heat, than in the case of a good conductor, such as a metal. In order to reduce the time for equilibrium to be set up it is advisable, where possible, to use the solid in the form of small pieces, which may be contained within a cage made of thin copper gauze. The water value of the cage will have to be calculated, and the heat conveyed by it to the calorimeter allowed for in the calculations. When the cage is suspended in the heater, the bulb of the thermometer ought to be surrounded by the pieces of solid contained in the cage. When the cage is in the calorimeter it can be used as the stirrer, being moved round and round by the string by which it was suspended.

Regnault's method of correcting for the loss of heat due to radiation is unsatisfactory in one particular. It assumes that the temperature of the thermometer follows exactly the temperature of the water in the calorimeter, and, further, that the temperature of the outside of the calorimeter does not lag behind that of the contents. If the calorimeter is very thin and well polished outside, this last assumption is probably justified. The question of the lag of the thermometer is, however, more important. Since the rise to be measured is small the thermometer requires to be very sensitive, and hence the bulb has to be large, and it is quite certain that when the temperature is varying rapidly that the temperature of the mercury in the thermometer will be less than the temperature of the surrounding water.

The correction for lag of the thermometer can be obtained in the following manner :- Having the calorimeter filled with water at the same temperature as the jacket, cool the thermometer to about 6 degrees lower, and then introduce it into the calorimeter, and, keeping the water stirred, take the reading of the thermometer every fifteen seconds till the readings become constant. Then plot the readings of the thermometer 
against the time and obtain a curve, such as oPQ (Fig. 90). If we draw a tangent at any point $P$ of this curve, and call the thermometer reading $r$ and the corresponding time $t$, the rate at which the thermometer reading is changing is $\frac{d r}{d t}$, and if $\theta$ is the angle the tangent makes with the axis of time we have $\frac{d r}{d t}=\tan \theta$. Now at the point $P$ the lag of the thermometer behind the temperature of the surrounding water is represented by $\overline{\mathrm{MN}}$, so that when the temperature of the thermometer is changing at a rate represented by $\tan \theta$, the lag is $\mathrm{MN}$.

Suppose, then, we require to find the lag when the reading is changing at the rate of half a degree in twenty seconds. Take a point $\mathrm{s}$ such that os represents twenty seconds, and take $\overline{S R}$ equal to half a degree, and join $\mathrm{OR}$. Then the tangent of the angle Ros is equal to the rate of change of the thermometer reading. Thus if we draw a line AP tangential to the curve and parallel to or, the lag will be equal to $\overline{\mathbf{M N}}$. To apply the corrections to the specific heat observations, the lag must becalculated for each reading of the thermometer and added to the reading to give the actual temperature of the water in the calorimeter. The readings thus obtained corrected for thermometer lag must then be plotted on a curve, and the radiation correction applied as described above.

We have hitherto tacitly assumed that the specific heat of water is a constant, so

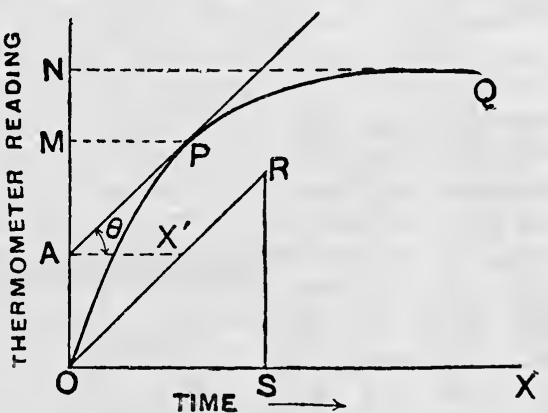

FIG. 90. that the heat required to heat a gram of water between any two temperatures is numerically equal to the difference in the temperatures. This assumption is, however, not correct, for the quantity of heat necessary to heat a gram of water through 1 degree is different at different temperatures. Hence it is necessary in the first place to choose some temperature, and to say that at that temperature the specific heat of water is unity, and then determine the specific heat of water at other temperatures in terms of this unit. Various temperatures have been suggested for the standard temperature. Perhaps the most frequently used is $15^{\circ} \mathrm{C}$., a calorie being defined as the heat required to raise the temperature of $1 \mathrm{gram}$ of water from $14^{\circ} .5$ to $15^{\circ} .5$. Callendar has advocated the employment of a mean calorie taken between $15^{\circ}$ and $25^{\circ}$, this mean calorie being practically the same as the calorie at $20^{\circ}$.

When we come to consider the variations of the specific heat of water, the results obtained by different observers differ very considerably. 
The following equation represents the results obtained by Callendar by the continuous flow method of mixtures, ${ }^{1}$ and by the electrical continuous flow method $(\S 247)$, in terms of the calorie at $20^{\circ} \mathrm{C}$. It will be seen that the specific of water is a minimum at about $38^{\circ} \mathrm{C}$, where it has a value 0.9985 ; while at $0^{\circ} \mathrm{C}$. and $100^{\circ} \mathrm{C}$. the values are 1.0106 and 1.0070 respectively.

$$
s=0.98536+\frac{0.504}{t+20}+0.0084 \frac{t}{100}+0.0090\left(\frac{t}{100}\right)^{2}
$$

The values of the specific heat of water calculated by this formula are given in Table 21, from which the mean specific heat over any range can at once be calculated. It is the mean specific heat of the water over the range used in the experiment which must be employed when calculating out the specific heat of a body, if the accuracy aimed at in the experiment is sufficiently great to necessitate a correction on account of the variation of the specific heat of the water.

83. Measurement of the Specific Heat of Glass by the Method of Mixture. - Make a bundle of pieces of fine-bore glass tube, each piece being about 6 centimetres long, using a weighed length of copper wire to fasten the glass together. Suspend the bundle in the steam heater, and fix the bulb of a thermometer reading to $100^{\circ}$ alongside. Pass steam from a boiler through the heater, using rubber tube to conduct the steam to the heater and to lead away the excess steam The glass must be left for at least half-an-hour in the heater, and if convenient an hour is a distinct advantage.

While the glass is being heated weigh the calorimeter and stirrer, and calculate their water value. Then fill the calorimeter about two-thirds full of water, and again weigh. Place the calorimeter in the waterjacket, and having adjusted the position of the thermometer, place over the top of the calorimeter a card with a slit cut to allow of the passage of the thermometer. The thermometer used in the calorimeter ought to be one having a very open scale divided into tenths of a degree, each degree corresponding to about a centimetre. With such a thermometer hundredths of a degree can be read with a very fair degree of accuracy.

When the glass has been in the heater long enough to have acquired the temperature of the steam, read the calorimeter thermometer every half minute for a few minutes. Then read the thermometer in the heater, and exactly at a whole minute swing the heater round and lower the glass into the calorimeter. The glass must be lowered quickly, but not in such a way as to risk spilling the water by splashing. Immediately after the introduction of the glass swing the heater back, and keeping the water stirred read the thermometer as quickly as possible, and continue taking a reading every twenty seconds till the maximum has been

1 See Watson's Text-Book of Physics, § 202 ; Phil. Trans. R. S. (1912), vol. cexii. (A). 
passed. Continue reading the thermometer every minute for about five minutes.

Note how much of the thermometer has been immersed in the calorimeter, and determine the volume in cubic centimetres of this part of the thermometer, and multiply this volume by 0.45 to get the water value of the thermometer (see $\S 82$ ).

From your numbers calculate the specific heat of the glass by the method described in the previous section.

84. Measurement of the Specific Heat of a Liquid by the Method of Mixture.-The method of mixtures may be employed for measuring the specific heat of a liquid. One method of experiment is to use a solid of which the specific heat has already been measured, preferably a metal having a high thermal conductivity, such as copper. The solid is heated and then transferred to the liquid which replaces the water in the calorimeter, the method of performing the experiment being exactly the same as that described in the preceding section.

The above method is only applicable when a fairly large volume of the liquid is available. If only a small quantity of the liquid is available, then it is introduced into a weighed glass tube shaped like a weight dilatometer, but with a straight capillary. The liquid must only about three-quarters fill the bulb, otherwise when the temperature is raised there is danger of the bulb being burst. The end of the capillary having been closed in the blow-pipe flame, the tube is bent round to form a hook by which the bulb may be suspended. By again weighing the bulb, the weight of the liquid is obtained.

If the liquid does not boil below $100^{\circ} \mathrm{C}$., then the bulb can be suspended within a steam heater; otherwise the bulb must be placed, together with a thermometer, in a test-tube, which is itself immersed in water which is maintained at a temperature a little below the boiling point of the liquid.

The heated bulb is introduced into the water contained in the calorimeter, and the experiment proceeds as in the preceding section, an allowance being of course made for the water value of the glass of the bulb.

In place of sealing up the liquid in a glass bulb, Berthelot recommends that the liquid of which the specific heat is to be measured be contained in a small bottle made of platinum capable of containing from 50 to 100 cubic centimetres. Since, however, such a platinum bottle is seldom available, we may

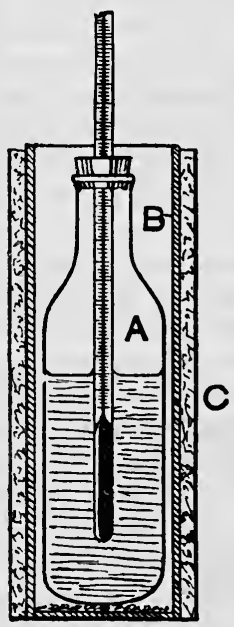

Fig. 91. use a thin-walled glass bottle of the shape shown in Fig. 91, and made by drawing down a thin-walled glass boiling tube. A sensitive thermometer similar to that used in the calorimeter is fixed in the mouth of this bottle by means of a tight-fitting cork. 
Berthelot's method of conducting the experiment is as follows:The bottle is weighed and its water value, together with that of the thermometer, is calculated. The water value of the cork can in general be neglected. The bottle is then filled about two-thirds full of the liquid and again weighed, the difference in the two weights giving the weight of the liquid. The bottle is then placed inside a fairly tight-fitting metal cylinder B, the side of which is wrapped round with a layer of asbestos cloth c. The cylinder is rested in a sand-bath or over a sheet of asbestos cardboard, and a small flame is placed beneath. While the liquid is heating the calorimeter is filled with water and weighed, and after placing inside a water-jacket the thermometer is read every minute in the manner described in $\S 82$.

By occasionally shaking the cylinder containing the bottle the heating of the liquid may be kept uniform. When the temperature of the liquid is sufficiently high, remove the cylinder and the contained bottle to the neighbourhood of the calorimeter, and having well shaken, read the thermometer very carefully and immediately remove the bottle from the cylinder, using the thermometer as a handle, and immerse in the calorimeter, being careful to keep the cork about a centimetre above the surface of the water.

Keeping the water in the calorimeter well stirred by moving the bottle about, using the thermometer as a handle, read both the thermometers every half minute. When the reading of the thermometers are within a degree of one another give a final good shake and carefully read both thermometers, noting the time, and then remove the bottle. Next observe the fall of temperature in the calorimeter for five minutes.

Although the water value of the calorimeter and its contents during the last period is less than it was during the middle period when the bottle was immersed, yet it will in general be found quite sufficiently accurate to assume that the rate of fall of temperature deduced from the observations when the bottle is removed applies to the case when the bottle is in the calorimeter, and then to calculate the correction to the maximum reading of the calorimeter thermometer in the manner described in $\S 82$. Knowing the water value of the calorimeter and its contents and the rise in temperature, the amount of heat acquired can at once be calculated. In the same way the heat given out by the bottle and its contents can be calculated in terms of the specific heat of the liquid, and equating the two quantities of heat, the specific heat of the liquid can be deduced.

85. Measurement of the Specific Heat of a Liquid by the Method of Cooling.-The specific heats of two liquids may be compared by noting the times taken by a calorimeter filled, first with the one and then with the other, to cool from a temperature $t_{1}$ to a temperature $t_{2}$, the conditions as to the temperature, \&c., of the surroundings being kept the same in the two cases.

Let $w$ be the water value of the calorimeter, thermometer, and stirrer, and $W$ the weight of liquid of specific heat $s$ placed in the calorimeter. 
If by some means or other the calorimeter were maintained at the temperature $t_{1}$, the temperature of the surroundings being $t_{0}$, then during a second the calorimeter and its contents would luse a quantity of heat which would depend (1) on the nature and extent of the surface of the calorimeter, and (2) on the difference between the temperatures $t_{1}$ and $t_{0}$. Since the surface and the surroundings are kept constant throughout, we only have to consider the effect of the difference between the temperature of the calorimeter and of the surroundings on the rate of loss of heat. Thus we may represent the rate of loss of heat at the temperature $t_{1}$ by $f\left(t_{1}\right)$, where $f\left(t_{1}\right)$ is some function of the temperatures $t_{1}$ and $t_{0}$ the value of which we do not require to know. Next suppose that instead of keeping the temperature constant we allow the calorimeter to cool, and we notice the time $\delta_{1} T$ which is taken to cool from the temperature $t_{1}$ to the temperature $t_{1}-\delta t$, where $\delta t$ is very small. Since the change of temperature is very small, we may without appreciable error suppose that the rate of loss of heat remains unchanged. The heat lost during the time the temperature is falling by the amount $\delta t$ is therefore $\delta t f\left(t_{1}\right) \delta_{1} T$. The heat lost by the calorimeter is given by $(W s+v) \delta t$. Hence

or

$$
\begin{aligned}
& (W s+w) \delta t=\delta t f\left(t_{1}\right) \delta_{1} T, \\
& \frac{\delta_{1} T}{(W s+w)}=\frac{1}{f\left(t_{1}\right)} \cdot . \quad . \quad . \quad . \quad . \quad .
\end{aligned}
$$

Next suppose that the time taken for the temperature to fall from $t_{1}-\delta t$ to $t_{1}-2 \delta t$ to be $\delta_{2} T$, we then get in the same way

$$
\frac{\delta_{2} T}{(W s+w)}=\frac{1}{f\left(t_{1}-\delta t\right)} \quad . \quad . \quad . \quad . \quad . \quad .
$$

Proceeding in this way till the temperature has fallen to $t_{2}$, and adding all the expressions similar to (1) and (2), we get

$$
\frac{\delta_{1} T+\delta_{2} T+\delta_{3} T+\& c .}{(W s+w)}=\frac{1}{f\left(t_{1}\right)}+\frac{1}{f\left(t_{1}-\delta t\right)}+\frac{1}{f\left(t_{1}-\ddot{-} \delta t\right)}+\& c .
$$

But $\delta_{1} T+\delta_{2} T+\delta_{3} T+\& c$. is the total time $T$ taken for the calorimeter to cool from $t_{1}$ to $t_{0}$. Hence

$$
\frac{T}{(W s+v)}=\Sigma \frac{1}{f\left(t_{1}\right)} \quad \cdot \quad \cdot \quad \cdot \quad . \quad . \quad .
$$

where the sign $\Sigma$ indicates the sum of the terms like $1 / f\left(t_{1}\right)$.

If, now, we repeat the experiment, using a weight $W^{\prime}$ of a liquid of specific heat $s^{\prime}$ in the calorimeter, starting at the same temperature $t_{1}$ and finishing at the same temperature $t_{2}$. we obtain in the same way as before, if $T^{\prime}$ is the total time taken to cool from $t_{1}$ to $t_{2}$,

$$
\frac{T^{\prime}}{\left(W s^{\prime}+w\right)}=\longleftarrow \frac{1}{f\left(t_{1}\right)} \cdot \text {. . . . . . }
$$


Now in the expressions (3) and (4) the parts on the right-hand side are the same, since they only depend on the surface of the calorimeter, the temperature range, and the temperature and nature of the surroundings Hence equating the two left-hand expressions we get

$$
s^{\prime}=\frac{W s T^{\prime}+w\left(T^{\prime \prime}-T\right)}{W^{\prime} T}=\frac{1}{W^{\prime}}\left\{(W s+w) \frac{T^{\prime}}{T}-w\right\} . .
$$

Hence if one of the liquids is water, or some other liquid of known specific heat, we can immediately obtain the specific heat of the other.

In the practical application of this method it is important to secure the rigorous fulfilment of the conditions we have postulated above. Thus the outer surface of the calorimeter must remain exactly the same during the two experiments. For this reason it is not safe to heat the liquid up to the initial temperature in the calorimeter itself, as the flame or other source of heat would be likely to alter the surface. The liquid must be heated in a beaker or flask, and then poured into the calorimeter. The weight of liquid used can be found by weighing the calorimeter after the end of the cooling experiment. In order to secure that the surrounding conditions remain the same, the calorimeter must be placed inside a hollow double-walled copper vessel, the space between the walls being filled with water or melting ice.

The calorimeter itself must be made of some good conducting material such as copper or silver, the walls being as thin as possible. The reason for this is, that we assume that the temperature of the outside is the same as the temperature of the liquid. As any evaporation of the liquid during the course of the experiment would vitiate the result, owing to the latent heat of the vapour formed, the calorimeter must be fitted with a lid. This lid must be pierced with holes for the thermometer and stirrer. The stirrer must be a rotary one, for an up-and-down stirrer would cause a portion of the stem of the stirrer to move in and out of the liquid, and thus encourage evaporation. This effect may easily produce a material error, since the stirring has to be vigorous in order to ensure that the temperature remains uniform throughout the liquid. The best shape for the calorimeter is a cylinder, of which the length is about four times the diameter, and the lid must fit tightly, so that the vessel may be filled with liquid almost up to the top without risk of any of the liquid escaping.

When performing the experiment clean out the calorimeter and black the outside with dead black varnish, formed by mixing vegetable black with methylated spirits and adding a few drops of shellac varnish. Then weigh the calorimeter, stirrer, and thermometer. Heat the liquid to a temperature of about $50^{\circ}$, if this can be done without the vapour tension becoming too high. Pour the liquid into the calorimeter and place it in the water-jacket. Stir the liquid well, and read the temperature every half minute till the temperature has fallen to a little more than half-way to the temperature of the water-jacket. Then remove the calorimeter and weigh. 
Having cleaned the calorimeter, being careful not to touch the outside more than is absolutely necessary, repeat the experiment using water, starting and finishing at about the same temperatures as before.

Two curves must now be plotted showing the relation between the temperature and the time for the two experiments. From these curves the times corresponding to equal initial and final temperatures can be obtained. The initial temperature must not be taken till a minute or two after the observations are started, so as to allow time for a regular state of affairs to have set in. The final temperature ought to be about half-way between the initial temperature and the temperature of the surroundings.

If the temperature of the water-jacket varies between the two experiments, then so long as the variation is not more than a degree or two we may allow for this effect by taking our initial and final temperatures such that there is the same difference between the temperature of the calorimeter and of the jacket in the two experiments.

It is extremely difficult to obtain accurate measurements by this method. The errors are chiefly caused by change in the condition of the surface of the calorimeter and evaporation of the liquid. The method, however, being simple, it is often convenient where an approximate value of the specific heat of a liquid is required. It is quite inapplicable to solids, even when good conductors, and in the form of powder, the temperature not remaining sufficiently uniform throughout the solid. 


\section{CHAPTER XIII}

\section{CALORIMETRY-LATENT HEAT}

86. Measurement of the Density of Ice by Bunsen's Method.Take a piece of glass tube a little over a centimetre in diameter and about $30 \mathrm{~cm}$. long, and having well cleaned it, dry it thoroughly. Near one end thicken the glass by rotating it before a small blow-pipe flame,

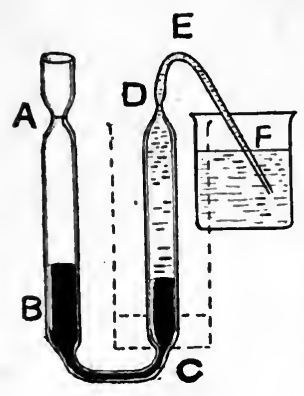

FIG. 92. and draw it down so as to make a narrow and short neck as at A (Fig. 92). Near the other end thicken the glass, and then draw out into a narrow capillary. Where this capillary leaves the main tube form a slight neck, as shown at $D$. Finally heat the middle of the tube in a fairly large flame, and allow the glass to get quite thick. During this process the ends of the tube may be very slightly forced together. When the glass is thoroughly thickened and uniformly hot, withdraw from the flame and then very slowly draw out till a neck about $8 \mathrm{~cm}$. long is formed, and while the glass is still hot bend round so that the two parts of the tube are parallel. Finally, bend the capillary, as shown at $\mathbf{E}$ in the figure, and make a fine mark on the neck $\mathbf{A}$ with a wire file.

The U-tube thus formed must be filled about half full with pure dry mercury, and then weighed carefully.

Now fix the tube in a retort clamp, so that the end of the capillary dips into a beaker containing distilled water. The beaker must stand on some wire gauze and the water be boiled. After the water has boiled for some minutes tilt the U-tube so that the mercury runs into the arm Dc and nearly fills it and drives out the air. On tilting the tube back into the vertical, water will be drawn over into the arm DC. Again tilt the tube, driving out the remaining air and most of the water. Repeat the process of drawing in water and forcing it out again two or three times, the water in the beaker being kept boiling all the time. In this way the upper half of the arm DC will be filled with water which is almost free from dissolved air. Having fixed the $U$-tube in the vertical position, direct a small flame from a hand blow-pipe against the tube at $D$, and when the glass becomes soft draw off the capillary, leaving the end of the arm DC sealed. The operation of sealing off a tube filled with 
water in this way is really not a matter of any difficulty. What is chiefly required is boldness. This, together with a little practice on odd pieces of glass, will enable the operation to be performed without cracking the glass.

Carefully dry the portion of the capillary removed, and weigh the $U$-tube and this portion of glass. The difference in the two weighings will give the weight of the water which has been introduced. Next fill up the arm AB a little above the mark at A with mercury, and immerse the whole up to $\mathrm{A}$ in melting ice, prepared as described in $\S 61$. After about an hour the water and mercury will have attained a temperature of zero. The excess of mercury above the mark $A$ must then be removed with a small pipette having a capillary tube.

Over the closed limb must now be placed a wide glass tube, shown dotted in the figure, and closed below by a cork. This tube is filled with pounded ice, and then a small cone of paper must be placed round the extreme end of the closed limb, and some freezing mixture, consisting of a mixture of pounded ice and crystallised calcium chloride, placed in the cone. In this way the extrome end of the water column will become frozen. When this occurs, pull up the outer glass tube till the cork is just below the frozen part, and fill the tube with the freezing mixture. By slowly moving the outside tube down, the water may then be entirely frozen, the freezing proceeding regularly from top to bottom. The reason for freezing in this way is that if the water at the bottom became frozen first, when the upper portions froze the expansion would burst the tube. When the whole of the water has been frozen, remove the outer tube and carefully wipe the U-tube and introduce it into the pure melting ice again. After it has been in the ice for an hour, carefully remove the mercury which is above the mark A with a pipette, and place this mercury in a weighed watch-glass and weigh.

Let the weight of the mercury expelled by the freezing of the water be $w$, and $d$ the density of mercury at $0^{\circ}$. Then the increase in volume of the water in freezing is $w / d$. If $W$ is the weight of the water, and $D$ is the density of the water at $0^{\circ}$, then the volume of the water at $0^{\circ}$ is $W / D$. Hence the volume of the ice at $0^{\circ}$ is $W / D+w / d$. Thus if $\delta$ is the density of ice at $0^{\circ}$, we have

$$
\begin{gathered}
\frac{W}{\delta}=\frac{W}{D}+\frac{w}{d}, \\
\delta=\frac{W D d}{W d+v D} .
\end{gathered}
$$

The value of $D$ is 0.999868 , and that of $d$ is 13.5955 .

It has been found that the density of ice varies very considerably according to the method used to freeze the water. The value obtained by this method is, however, probably the one to use when reducing the observations taken by the ice calorimeter. 
87. Bunsen's Ice Calorimeter.-In Bunsen's ice calorimeter the heat given out by a body as it cools from a given temperature to $0^{\circ} \mathrm{C}$., is measured by obtaining the quantity of ice which will be melted by this heat. In order to measure the quantity of ice melted, use is made of the change in volume which takes place during fusion.

The increase in volume in 1 gram of water when it becomes ice at $0^{\circ} \mathrm{C}$. is 0.0907 c.c. Thus the communication of one calorie will cause

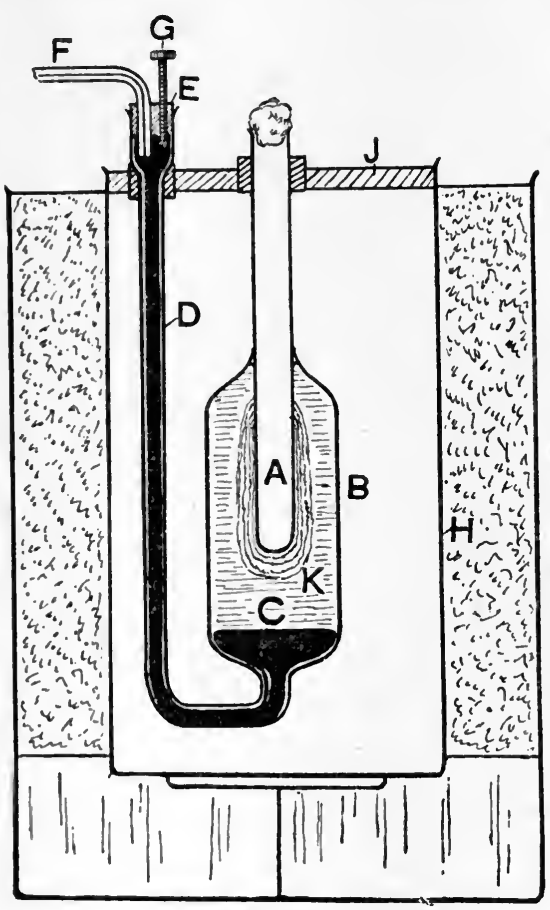

FIG. 93. a change of volume of about 0.00113 c.c.

The usual form of calorimeter consists of an inner glass tube A (Fig. 93) closed at the bottom, which serves to receive the body of which the specific heat is being measured. The lower part of this tube is surrounded by a glass bulb $\mathbf{B}$, which is connected to a side tube D. A piece of fine-bore thermometer tubing $\mathrm{F}$ about a metre long is connected to $\mathrm{E}$ by being cemented into an ebonite stopper E. An iron screw $G$ passing through the stopper serves to adjust the position of the mercury in the tube $\mathbf{F}$. The upper part of the bulb $B$ is filled with air-free distilled water, while the lower part, the tube $\mathrm{D}$, and part of $\mathrm{F}$ are filled with mercury. By a process to be described later, a cap of ice $\mathrm{K}$ is formed round the lower part of the tube $\Delta$, and when a hot body is' introduced into $\mathrm{A}$ its heat melts some of the ice, and the contraction which takes place causes the mercury meniscus to travel along the tube $\mathrm{F}$ towards the instrument. If we know the cross-section of the tube we can calculate, from the distance traversed by the meniscus, the diminution of volume, and hence, knowing the densities of water and ice at $0^{\circ} \mathrm{C}$., obtain the weight of ice melted, and therefore, knowing the latent heat, the quantity of heat given out by the boòy placed in the instrument. This method of obtaining the result not only involves a knowledge of the change of volume which takes place when ice melts, a quantity which seems to depend in some measure on the conditions under which the ice was 
formed, but also the latent heat of ice. Further, a correction would have to be applied to allow for the contraction of the mercury which Hows from the tube $F$, where it is at the temperature of the room, into the tube $\mathrm{D}$, where it is at $0^{\circ} \mathrm{C}$. For these reasons it is usual to calibrate the instrument by introducing a known weight of water at a measured temperature, and to note the range through which the mercury meniscus moves. In this way the quantity of heat which has to be communicated to $\mathrm{A}$ to cause the mercury to move through 1 centimetre is obtained, and can be used to reduce the observations when bodies of unknown specific heat are used.

In the ordinary manner of performing the experiment, the instrument, as described above, is placed in a vessel containing pounded ice or snow, the top of the tube $\mathrm{A}$ and the tube $\mathrm{F}$ alone projecting above the ice. It is, however, found that there is always a small difference in the freezing point of the ice in the instrument and that of the ice outside. If the temperature of the outside ice is the higher, then there will be a slow melting of the ice in the instrument, which will cause a continuous creep of the mercury meniscus towards the instrument. If the freezing point of the ice outside is lower than that of the ice in the instrument, then there will be a slow freezing of the water in the instrument, causing the meniscus to creep away from the instrument. This creep generally amounts to 2 or 3 centimetres per hour, and is sufficient to make it very difficult to obtain trustworthy measurements. A slight addition ${ }^{1}$ to the instrument will almost eliminate the creep, reducing it to about a tenth. This addition is shown in Fig. 93, and consists in placing the instrument in an empty vessel $\mathrm{H}$, the top of which is closed by a cork through which the tubes $A$ and $D$ pass. This vessel is surrounded by the pounded ice. Owing to air being a bad conductor of heat, heat can now only very slowly pass between the instrument and the surrounding ice. Another method of securing the same conditions has been employed by Callendar, who uses a second glass bulb surrounding the bulb $\mathrm{B}$ and produces a vacuum in the space between the two bulbs. The vacuum being a worse conductor of heat than air, the passage of heat from the surrounding ice to the instrument is in this way reduced yet more. When this device is used, when originally cooling the instrument down to zero the process will take a considerable time, unless a stream of ice-cool water is passed through the tube $\mathbf{A}$.

Since when a body has once been placed in the tube $A$ it is very difficult to extract it, it will be found of advantage to place a thin-walled test-tube inside A. This test-tube must be a fairly good fit, and a little glycerine can be placed in A so as to fill the interspace, and thus facilitate the passage of heat from the hot body to the ice. When it is required to remove a body from the calorimeter, the test-tube is removed, and the body can then be tilted out.

The filling of the Bunsen calorimeter with water and mercury is an operation of some delicacy. The following method is recommended by

1 Boys, Philosophical Magazine (1887), xxiii. 214. 
Wadsworth. ${ }^{1}$ The required quantity of mercury is first introduced by means of a funnel with the stem drawn out into a fine tube capable of reaching down to the bend of the tube $\mathrm{D}$. The calorimeter is then inverted, the mercury running to the top of the bulb $\mathrm{B}$, and immersed upside down in a beaker full of distilled water. The water must cover the entire calorimeter. The water is then heated to boiling and then cooled. When the bulb $\mathrm{B}$ is partly filled with water, the beaker is again heated and the water allowed to boil for some considerable time. In this way the remaining air in the bulb $\mathrm{B}$ will be replaced by water vapour, and, on allowing the whole to cool, the water will be forced into the bulb. When the whole is cool enough to handle the end of the tube $\mathrm{D}$ is closed with the finger, and the calorimeter removed and turned into the upright position. More-mercury must now be introduced by means of the funnel till the side tube is entirely filled with mercury.

The screw $\mathrm{g}$ having been withdrawn from the stopper, the stopper with the tube $\mathbf{F}$ attached must be placed in the end of the side tube and cemented in place with a little shellac varnish or india-rubber cement. On replacing the screw, the mercury will be forced up the tube $F$.

Before freezing the cap of ice, the whole instrument must be cooled down to a temperature of about $2^{\circ} \mathrm{C}$. by immersing it in a beaker full of water containing some ice. To freeze the cap of ice round $\mathbf{A}$ a freezing mixture, consisting of finely powdered ice and crystalline calcium chloride, must be prepared, and introduced within the tube $\mathbf{A}$. This freezing mixture will require renewing from time to time till the ice cap formed extends about half-way from the inside tube to the outside bulb. The freezing being complete, the inside of the tube $\mathrm{A}$ must be rinsed out with ice-cold water, and dried by means of a roll of blotting-paper. A thin test-tube which fits fairly well inside $\Delta$ must be weighed and then put in place, and the instrument must then be introduced in the ice-box and allowed to stand for several hours before an experiment is made. The reason is that the temperature of the ice after its formation by the freezing mixture will be below zero, and it must have time to reach this temperature, also the surrounding water must have time to cool down to zero.

When the instrument has been in the ice for an hour or two, the mercury meniscus must be adjusted to near the far end of the tube $\mathbf{F}$ by means of the screw G. By watching the movement of the end of the column it will then be possible to judge when the temperature of the instrument becomes constant. When the movement becomes small ( 2 or 3 millimetres per hour), the rate of movement must be carefully observed.

Some water having been heated to a temperature of about $40^{\circ}$, the temperature must be carefully read, and then enough of the hot water poured into the test-tube to fill it about two-thirds of the way up to the top of the ice cap. The movement of the mercury meniscus is then again observed, the observations being continued till the rate of move.

1 American Journal of Science (1897), iv. 282. 
ment is the same as it was before the introduction of the hot water. The test-tube is then removed and again weighed, and in this way the weight of water is obtained. Knowing this weight and the initial temperature, the heat given out by the water can at once be calculated.

The movement of the mercury meniscus consists of two parts, that due to the ice melted by the hot water, and that due to the slow creep which occurs when no heat is being supplied to the tube $A$, due to the introduction of a hot body. The time the experiment lasted being observed, the amount of the creep can be calculated, and this amount must be added to or subtracted from the observed displacement of the meniscus, according as the creep takes place away from or towards the instrument. The corrected displacement divided by the number of calories given up by the water gives the displacement per calorie, and this quantity is used when measuring the specific heat of other bodies.

The test-tube having been dried, a small pellet of cotton wool is placed at the bottom, and the tube is replaced in the instrument, and after about half-an-hour the rate of movement of the meniscus is again observed. The body of which the specific heat is to be measured is placed in the test-tube $\Delta$ of the heater shown in Fig. 94. This heater consists of an outer tube B, closed at either end by corks. Through the upper cork the test-tube A passes, while through the lower cork there pass the tubes $\mathrm{C}$ and $\mathrm{D}$. These tubes are attached to two pieces of rubber tubing about 2 feet long. Steam is passed in through the tube $\mathrm{F}$, and escapes through E. The heater is wrapped round with felt. This felt serves the double purpose of decreasing the loss of heat, and of allowing the heater being taken in the hand. To transfer the heated body to the calorimeter, the thermometer $\mathbf{T}$ is read and then

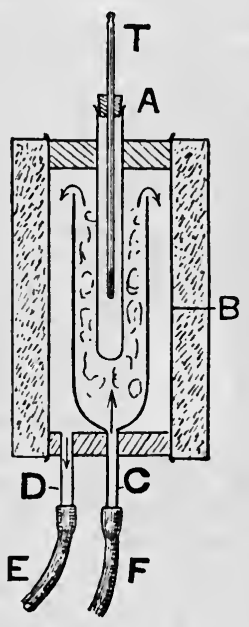

FIG. 94. the cork is removed, and, the heater being inclined, the hot body is tilted into the ice calorimeter.

The movement of the meniscus is observed as in the case of the water, and a correction having been applied for the steady creep, the total displacement divided by the displacement per calorie obtained from the water experiment gives the heat given out by the hot body. Hence, knowing the mass and initial temperature of the hot body, the specific heat can at once be calculated.

When using the ice calorimeter for accurate measurements, it is necessary to calibrate the capillary tube. This can be done by means of a mercury thread in the manner described in $\$ 59$.

88. Measurement of the Latent Heat of Fusion of Ice.-The chief difficulty to be overcome in determining the latent heat of fusion of ice consists in introducing blocks of ice into the calorimeter which, while 
having a temperature of $0^{\circ}$, shall be entirely free from attached water. Further, it is important that during the time necessary for melting that the ice should be kept below the surface of the liquid in the calorimeter, otherwise the gain of heat from surrounding objects will be considerably increased.

Although by taking the ice in the form of small fragments we can make the time occupied by the melting short, and hence reduce the radiation correction, yet it is hardly advisable to do so on account of the difficulty of removing the adherent water from the large surface of the ice. Better results will be obtained by taking the ice in one piece of such a shape that it can easily be freed from attached water. If the calorimeter has a capacity of about 500 c.c., a cylinder of ice about $3 \mathrm{~cm}$. in diameter and $5 \mathrm{~cm}$. long will produce a suitable fall of temperature (about $5^{\circ}$ ). Such cylinders of ice may be prepared by freezing water in a test-tube of the appropriate size by means of a freezing mixture. By slightly warming the test-tube, the block of ice can be removed.

Until required, the cylinder of ice may be wrapped inside a roll of blotting-paper and placed in a large test-tube, the test-tube being immersed in melting ice. The ice must be left for at least an hour, so as to allow it to acquire the temperature of $0^{\circ}$.

In order to hold the ice below the surface of the water in the calorimeter, the stirrer has the form shown in Fig. 95. It consists of a cup formed of copper gauze soldered to the handle B. The ice having been thrown into the water, the stirrer is raised and the ice imprisoned within the gauze

FIG. 95. cup, and the melting is facilitated by the motion of the stirrer through the water.

In order to allow for radiation the experiment is divided into three parts, as in the case described on p. 219. In the first part, the calorimeter, having been filled with water and weighed, is introduced within the water-jacket, and the course of the thermometer watched during about five minutes. At a noted time the ice is then introduced, and the thermometer read every half minute till all the ice is melted. Finally, the course of the thermometer is watched during another five minutes. The correction for radiation is calculated exactly as previously described. The weight of ice melted is obtained by weighing the calorimeter and its contents at the end of the experiment.

By packing the cylinder of ice round with blotting-paper as described, it is generally quite dry; if not, then it can be dried in the following way :-A strip of clean blotting-paper is cut, having a length of about $50 \mathrm{~cm}$. and a width slightly greater than the length of the ice cylinder. The cylinder of ice is rolled up in this strip of paper, and by folding in the ends the attached water is sucked up. Then, holding the paper cylinder over the calorimeter between the finger and thumb of the left hand, the ice is forced out by means of a piece of glass rod held in the right hand 
The magnitude of the radiation correction can be very considerably reduced by using Rumford's device. ${ }^{1}$ Having roughly calculated the drop of temperature which may be expected from the size of the ice cylinder and the capacity of the calorimeter, heat the calorimeter to a temperature higher than that of the surrounding water-jacket by half this amount. It will be found impossible to secure exact compensation between the heat lost by radiation during the first half of the experiment and that gained during the second, and the observed drop of temperature will still require a certain amount of correction. By reducing the amount of the correction, however, we minimise the effects of slight errors in determining the rate of cooling.

Using the following symbols for the quantities entering into the calculations :-
$W=$ weight of water in the calorimeter.
$w=$ water value of the calorimeter, thermometer, and stirrer.
$x=$ the weight of ice melted.
$t_{1}=$ the initial temperature of the water.
$t_{2}=$ the final temperature of the water.
$\delta t=$ the correction to the final temperature to allow for radiation.
$L=$ the latent heat of ice.

Then the following is the thermal equation-

or

$$
x L+x\left\{t_{2}+\delta t\right\}=\{W+w\}\left\{t_{1}-\left(t_{2}+\delta t\right)\right\},
$$

$$
L=\frac{W+w}{x}\left\{t_{1}-\left(t_{2}+\delta t\right)\right\}-\left(t_{2}+\delta t\right)
$$

from which the latent heat can be calculated.

Further correction.-The thermometer readings may be corrected for the effect of lag as described on p. 221. The results will require correction to allow for the variation of the specific heat of water. Thus, if $s_{1}$ is the mean specific heat of water between $0^{\circ}$ and $t_{2}$, as given in Table 21, the heat required to raise the temperature of the water formed by meltirg the ice is $s_{1} x\left\{t_{2}+\delta t\right\}$. In the same way, if $s_{2}$ is the mean specific heat of water between $t_{2}$ and $t_{1}$, the heat given out by the water in the calorimeter is $W s_{2}\left\{t_{1}-\left(t_{2}+\delta t\right)\right\}$. It will in general be found that the accuracy of the results obtained does not warrant the application of this correction.

89. Measurement of the Heat of Vaporisation of Water.-The determination of the heat of vaporisation of a liquid is a matter of very great difficulty if an accuracy greater than about 1 per cent. is desired. The usual method is to pass the vapour of the liquid into a vessel which is immersed in a calorimeter and is at a temperature lower than the boiling point of the liquid. The weight of vapour condensed is obtained

1 In this experiment the rate at which the temperature of the calorimeter falls is approximately constant, and so Rumford's method is applicable. See p. 218. 
by weighing the vessel before and after the experiment, while the heat liberated by the condensation of the vapour and the cooling of the liquid formed to the temperature of the calorimeter is obtained from the rise of temperature of the calorimeter.

The chief sources of error when performing an experiment such as outlined above consist of-(1) Heat communicated to the calorimeter by conduction through the tube through which the vapour is conducted from the boiler into the calorimeter. (2) The vapour is very apt to

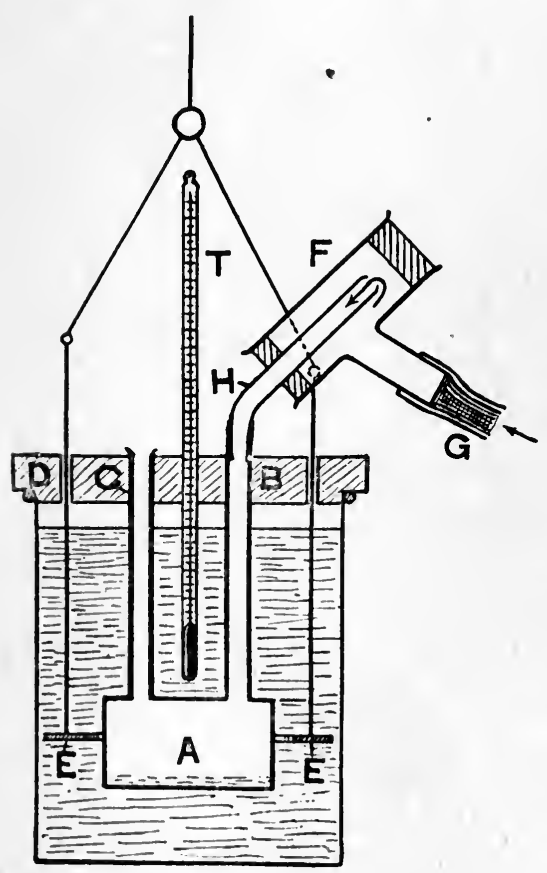

Fig. 96. carry over into the calorimeter small particles of the liquid. The presence of this liquid in the vapour may be due either to spirting, owing to the boiling being too rapid, or to the fact that some of the vapour has been condensed into liquid owing to loss of heat during the passage of the vapour through the tube connecting the boiler with the calorimeter. It is a very difficult matter to estimate the magnitude of the corrections to be applied to allow for these two errors, or even to ensure that the magnitude of the errors remains the same from one experiment to the next.

The manner in which the above difficulties can in a measure be overcome, where time is no object, and it is possible to work with large quantities of material, will be found in the account Regnault gives of his determination of the latent heat of steam.1

An arrangement by means of which the latent heat of steam can be measured to within 1 or 2 per cent. is shown in Fig. 96. The copper drum A has two copper uprights, one of which B serves for the admission of the steam, and the other c communicates with the air. This vessel is attached to a thick disc of varnished wood $D$, which serves to form a lid

1 See Regnault, Relation des expériences, dec., vol. i. p. 635; also Cours de Physique, by Jamin and Bouty, vol. ii. p. 159. 
to the calorimeter. The uprights attached to a stirrer $\mathbf{E}$ and a ther mometer also pass through the lid.

The steam is conducted from the boiler, through a short length of rubber tube, to the side tube $G$ of a small separator, which serves to catch any condensed steam which would otherwise be carried over into the calorimeter.

To conduct an experiment with this apparatus, first weigh the calorimeter, stirrer, thermometer, the internal vessel $\Delta$, and the cover. Having filled the calorimeter with water to within about $2 \mathrm{~cm}$. of the top, again weigh. The difference in the weights will give the weight of water in the calorimeter. Place the calorimeter within a double-walled vessel (see p. 214), and watch the changes in temperature during five minutes, keeping the water stirred all the time. Having allowed the steam to pass through the trap $F$ for a few minutes, at a noted time place the end of the glass tube $\mathrm{B}$ into the upper end of the upright $\mathrm{B}$, so that the steam passes into the calorimeter. Keep the water in the calorimeter well stirred, and read the thermometer every thirty seconds till the temperature has risen about 10 degrees. At a noted time withdraw the tube $\mathrm{H}$, and continue stirring and reading the thermometer for another five minutes, then weigh the calorimeter and its contents, and thus obtain the weight of steam condensed.

The rise in temperature of the calorimeter will have to be corrected for loss of heat, due to radiation and conduction, in the manner described on p. 219. It will also be necessary to obtain the water value of the calorimeter, \&c., which can be done either by calculation, if the specific heats of the constituents are known, or it may be determined experimentally by pouring water at a known temperature (about $100^{\circ}$ ) into $\mathbf{A}$, and proceeding as in the method of mixtures.

The method of calculating the value of the latent heat is exactly the same as that employed in the previous section.

90. Measurement of the Heat of Vaporisation by Berthelot's Method.-Berthelot has devised a form of apparatus which is designed for the determination of the latent heat of a vapour when only a small quantity of the material is available. His form of the apparatus is described in Watson's Physics, p. 240, and possesses the great defect that it is almost impossible to aroid the vapour becoming superheated, owing to the proximity of the tube leading the vapour from the boiler to the calorimeter to the flame used to heat the boiler. The presence of this flame also causes a considerable difficulty in screening the calorimeter and thermometer. Finally, the liquid is very apt to boil with bumping. A modification of Berthelot's apparatus, in which the above objections are avoided, has been used by Kahlenberg. ${ }^{1}$ In this form, which is shown in Fig. 97, the heating is electrical. The liquid is contained in a test-tube shaped tube $A$, which is closed by a cork $D$. Two glass tubes $\mathbf{E}$ pass through this cork, and have pieces of stout platinum wire fused at their bottom ends. A coil of fairly fine platinum wire $\mathbf{F}$ is 
attached to these stout platinum wires. These tubes are partly filled with mercury, and the wires from the battery dip into the mercury. The vapour produced by the boiling of the liquid passes down an upright tube $\mathrm{c}$ into a glass worm in the calorimeter. The worm terminates in

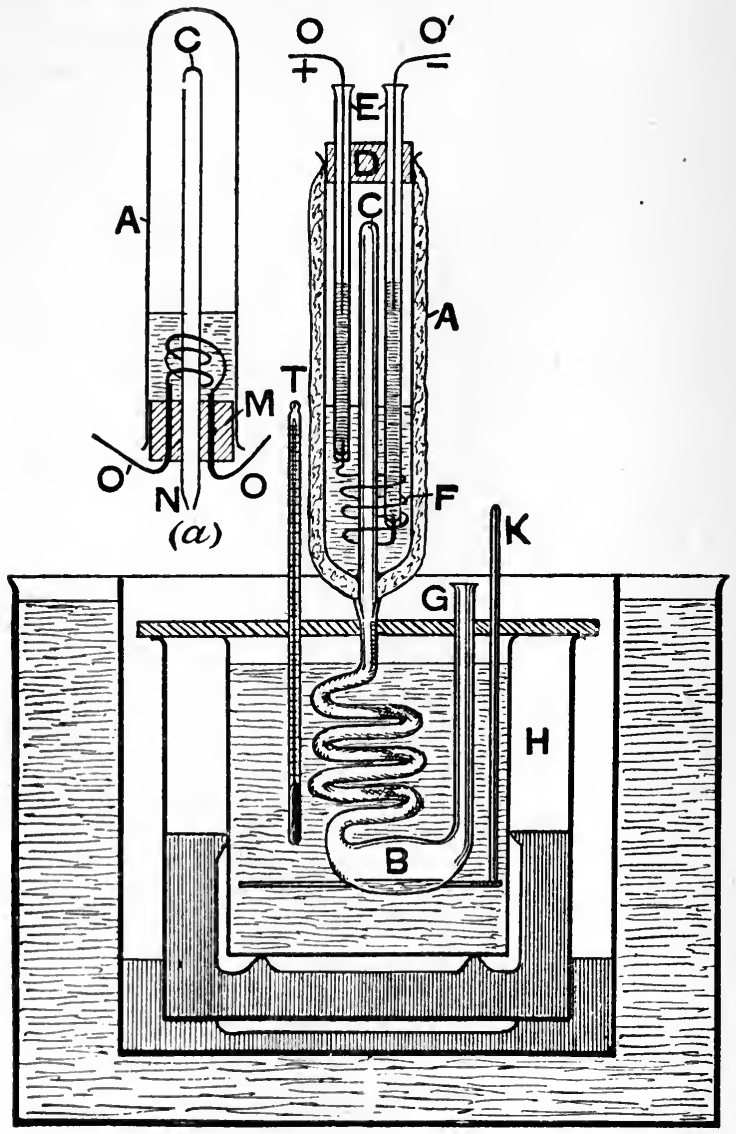

FIG 97.

an enlargement $\mathrm{B}$, in which the liquid accumulates, and an upright tube G, which is open to the air. The boiler A is not permanently attached to the worm, but the lower part of the tube $\mathrm{c}$ fits loosely into the expanded upper part of the worm, the joint being made tight by a ring of cork or of rubber tube. The calorimeter is closed by a thick wooden 
or ebonite cover, and a thermometer and stirrer pass through this cover. The boiler $\mathbf{A}$ is wrapped round with a close fitting cover of felt, so as to check the loss of heat due to radiation and conduction.

A simpler form of the boiler is shown in Fig. $97(a)$. Here the boiler consists of a test-tube $\mathbf{A}$, closed by a cork $\mathbf{M}$, through which pass a tube $\mathrm{c}$, and the wires $\mathrm{o}$ attached to the platinum spiral. To introduce the liquid the cork is removed, the required quantity of liquid introduced into the test-tube, and the cork replaced. The whole is then inverted into the position shown in the figure.

When performing an experiment, the worm is carefully dried and weighed. The calorimeter is weighed first empty, and also full of water. The liquid having been introduced into the boiler, the apparatus is put together, and the thermometer read at intervals for five minutes. The current is then switched on and the vapour allowed to pass into the calorimeter till the temperature has risen about 5 degrees, the thermometer being read every thirty seconds. The current is then stopped, the boiler removed, and the march of the thermometer followed for five minutes. The worm is then removed from the calorimeter, dried outside, and weighed. If the liquid is at all volatile, corks must be placed in the open ends of the worm directly the boiler is removed.

The rise of temperature of the calorimeter will re-

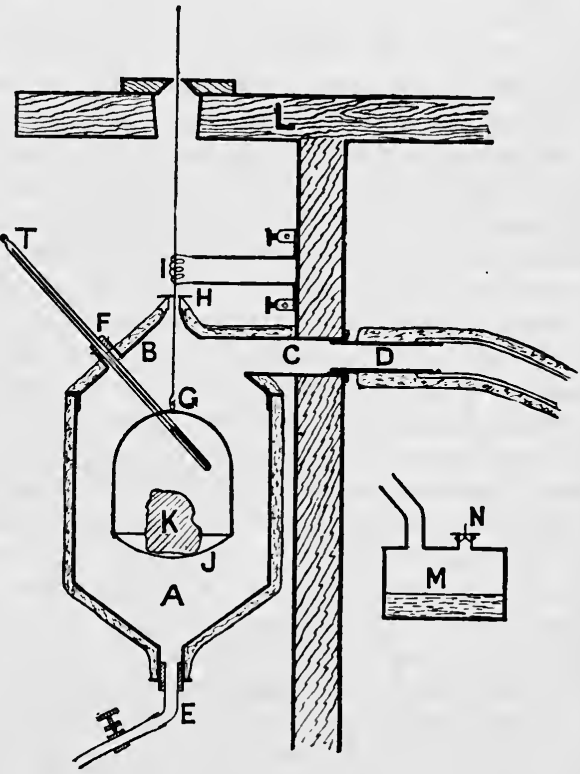

FIG. 98. quire correction for gain or loss of heat by radiation in the ordinary manner.

91. Joly's Steam Calorimeter. ${ }^{1}$-In the steam calorimeter the heat necessary to raise the temperature of a body from the air temperature to $100^{\circ}$ is measured by determining the weight of steam which must be condensed into water at $100^{\circ}$ to supply this heat. The instrument is somewhat troublesome to use, but in the hands of a careful observer it gives very accurate results.

The body $\mathrm{k}$ (Fig. 98) is supported on a light platinum or copper

1 Proceedings of the Royal Society (1889), xlvii. 219. 
dish $\mathrm{J}$, which is suspended by a fine platinum wire $\mathrm{G}$ from one of the pans of a balance, which rests on the wooden shelf $L$. The pan hangs within a vessel $A$, made of thin brass and covered with felt. The upper part B of this vessel is attached to the stand by means of the tube $c$, which also serves for the admission of the steam. The lower part can be removed to get at the pan, and is attached to the top by a simple bayonet joint. A drain pipe $\mathbf{E}$ allows the superfluous steam to escape: This pipe can be closed when necessary by closing the rubber tube by means of a pinch-cock. The suspension wire passes through a light metal disc $\mathrm{B}$, which is pierced with a hole having a diameter of about two-thirds of a millimetre, which rests lightly on the upper opening of the calorimeter. As the pan swings about after placing the body in it the suspension wire moves this disc, and when the vibrations die out the disc is left in such a position that the wire passes through the hole without touching the edge. To prevent the steam condensing on the suspension wire and on the disc $\mathrm{B}$ an electric current is passed through a coil of fine platinum wire I, which it heats to redness. The steam is brought from a boiler through a wide bore piece of india-rubber tube to a short length of brass tube $\mathrm{D}$, which can be fitted into the side tube c. The steam is generated in a boiler, which is shown on a smaller scale at M. In addition to the bent tube to which the rubber tube leading to the calorimeter is attached, there is a short upright tube with a sharp edge. This tube is closed by a light sheet of mica, and acts as a safety-valve to allow the steam to escape when the tube leading to the calorimeter is closed. To prevent the mica being shot off, a fine upright wire passes loosely through a hole in the mica.

The procedure adopted when making a measurement is as follows :The empty scale pan having been counterpoised, the body is placed on the pan, and the lower part of the calorimeter having been put in place, the weight is determined which has to be added to the other pan of the balance to produce equilibrium. Thus the weight of the body is obtained. The body must be left in the calorimeter till its temperature, as shown by the thermometer T, has become constant, and even in the case of good conductors of heat, at least half-an-hour must be allowed. During this time the drain tube at the bottom of the calorimeter must be closed. The tube from the boiler must also be removed, and a cork inserted into the opening to the tube $c$. While the temperature of the body is becoming constant the steam is raised in the boiler, care being taken to preserve the calorimeter from radiation from the burner or boiler by interposing screens. The steam must be allowed to pass freely through the tube $\mathrm{D}$, so as to. heat it.

The temperature having been read, the thermometer $\mathrm{T}$ must be withdrawn, and the hole $\mathbf{F}$ closed with a cork. The current having been switched on to the spiral $\mathrm{r}$, the drain tube must be fully opened and the steam pipe connected to the calorimeter, so as to allow the steam to enter the calorimeter suddenly. Some of the steam will be condensed on the body, and will be caught in the pan J. After from one to four minutes 
the body will have reached the temperature of the steam, and it will be possible to adjust the balance. Till the body reaches the temperature of the steam the weight will increase steadily, but when the body is completely heated, the weight will only increase very slowly, about half a milligram in five minutes. Finally, the barometric height must be read.

A similar experiment will have to be made with the empty pan to allow for the increase in weight due to the steam condensed while raising the temperature of the pan and stirrup to the temperature of the steam.

If $t_{2}$ is the temperature of the steam as deduced from the barometric height, $t_{1}$ the initial temperature of the body, $S$ its specific heat, $W$ its weight, $w$ the weight of steam condensed, and $L$ the latent heat of steam, while $w^{\prime}$ is the weight of steam condensed by the pan alone when its temperature is raised from $t_{1}{ }^{\prime}$ to $t_{2}{ }^{\prime}$, we have

$$
W S\left(t_{2}-t_{1}\right)=\left\{w-\frac{t_{2}-t_{1}}{t_{2}^{\prime}-t_{1}}, w^{\prime}\right\} L .
$$

A correction will have to be applied to allow for the fact that the density of steam at $100^{\circ}$ is about half that of air at ordinary temperatures, and so the buoyancy correction in steam is considerably less than in air at the lower temperature. The deduction from the weight $w$ on this account will be equal to the product of the volume of the body into the difference in density between the air at the lower temperature and that of steam at the higher. Of course the actual density of the steam will vary with its temperature, that is, with the height of the barometer. For most practical purposes, however, it is sufficient to take a mean value for the density of steam, and the following table, which gives the relative density of air at various temperatures to steam at $100^{\circ}$, will serve for the calculation of the correction :-

$\begin{array}{cc}\text { Initial } & \text { Correction } \\ \text { Temperature. } & \text { Factor. } \\ 0^{\circ} & 0 \cdot 00069 \\ 5^{\circ} & 0 \cdot 00066 \\ 10^{\circ} & 0 \cdot 00064 \\ 15^{\circ} & 0.01062 \\ 20^{\circ} & 0 \cdot 00060\end{array}$

The volume of the body must be multiplied by the most suitable of the above factors, and the product deducted from $w$ to give the actual weight of steam condensed.

92. Measurement of the Heat of Solution.-If two substances which are soluble are taken at the same temperature and mixed, then in general the act of solution is accompranied by a change in temperature. The thermal change may indicate either an absorption or an evolution of 
heat, according to the nature of the substances. The quantity of heat which has to be communicated or abstracted from the substances to maintain the temperature constant during the solution of 1 gram of the solute in a given weight of the solvent is called the heat of solution of the solute for the given concentration.

If $p$ grams of a salt, of which the molecular weight is $m$, are dissolved in $q$ grams of a solvent, the heat developed during solution being $H$, then $m H / p$ is called the molecular heat of solution for the solution containing $\frac{100 p}{p+q}$ per cent. of the solute. With strong solutions the molecular heat of solution in general decreases as $p$ increases.

In the practical determination of heats of solution it is not possible to keep the temperature constant during solution and to measure the heat which has to be abstracted or communicated. What is done is to prevent as far as possible all exchange of heat with surrounding bodies, and to measure the change in temperature which occurs on solution. From this change in temperature we can calculate the heat of solution if we know the specific heat of the solution.

Let $s$ be the specific heat of the solution, and $w$ the water value of the calorimeter, thermometer, and stirrer. Then if $t_{0}$ is the initial temperature of the salt and the solvent, and $t_{1}$ is the final temperature after solution is complete, the heat of solution is given by

$$
Q=\frac{1}{p}\{(p+q) s+w\}\left(t_{1}-t_{0}\right) \quad \text {. . . . . }
$$

The heat of solution at any temperature having been measured, we can calculate the heat of solution at any other temperature if we know the specific heats of the salt, of the solution, and of the solvent. For suppose we start with the salt and solvent at the lower temperature $t_{1}$, we may obtain a solution at the higher temperature $t_{2}$ in two ways. In the first way we dissolve the salt at $t_{1}$, the heat of solution being $Q_{t_{1}}$. We then heat the solution to $t_{2}$, during which we have to supply an amount of heat

$$
(p+q) s\left(t_{2}-t_{1}\right)
$$

Next we may heat the salt and solvent separately to $t_{2}$, during which we shall have to supply a quantity of heat which, if $s_{p}$ and $s_{q}$ are the specific heats of the salt and of the solvent respectively, is equal to

$$
\left(p s_{p}+q s_{q}\right)\left(:-t_{1}\right)
$$

and then dissolve the salt at $t_{2}$, the heat of solution being $Q_{t_{2}}$.

If we neglect the external work due to the very small changes ir 
volume which take place, the quantities of heat required in the two cases must be the same. Ḧence

$$
\begin{aligned}
& Q_{t_{1}}+(p+q) s\left(t_{2}-t_{1}\right)=Q_{t_{2}}+\left(p s_{p}+q s_{q}\right)\left(t_{2}-t_{1}\right), \\
& Q_{t_{1}}-Q_{t_{2}}=\left\{p s_{p}+q s_{q}-(p+q) s\right\}\left(t_{2}-t_{1}\right) \\
&=\left(\frac{p s_{p}+q s_{q}}{p+q}-s\right)(p+q)\left(t_{2}-t_{1}\right) .
\end{aligned}
$$

But $\frac{p s_{p}+q s_{q}}{p+q}$ is the mean specific heat of the constituents, thus if this is greater than $s$, the specific heat of the solution, the heat of solution decreases with rise of temperature, and vice versa.

A convenient form of calorimeter for use when measuring heats of solution is shown in Fig. 99. A thin beaker B, which would hold about half a litre, is supported on a cardboard cross within a larger beaker $\mathbf{A}$. A wood or ebonite cover c is pierced with three holes, one of which serves to give passage to a thin-walled test-tube $\mathrm{D}$. A delicate thermometer $\mathrm{T}$ and $\mathrm{a}$ stirrer $\mathrm{s}$ pass through the other holes. The stirrer is best made of glass rod, since in many cases the solutions would act upon metal.

As an example of the measurement of the heat of solution of a salt, we may take the case of sodium chloride in water. The salt must be well dried over a water bath and finely powdered. The beaker B having been weighed, it must be about three-quarters filled with distilled water, and then again weighed.

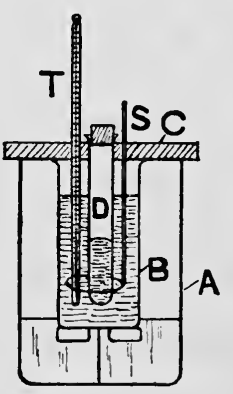

FIG. 99. The test-tube $\mathrm{D}$ must also be weighed, and sufficient of the salt put in to form a solution of the required strength. The test-tube containing the salt having been introduced within the calorimeter, it must be left there for at least half-an-hour, to allow the salt to acquire the same temperature as the water. During the last ten minutes the water must be kept stirred, and the temperature read at intervals of a minute or half a minute. The temperature of the water and salt being the same, the bottom of the test-tube must be broken by pushing down a glass rod, and the solution of the salt assisted by vigorous stirring. The thermometer must be read every half minute during the time solution is taking place, and then every minute for five minutes after all the salt has dissolved.

The observed fall of temperature will have to be corrected for gain of heat by radiation exactly in the manner described in $\S 82$. The water value of the beaker, test-tube, thermometer, and stirrer must be obtained either by calculation from their weights and the specific heat of glass, or by experiment. If the specific heat of the solution is not 
known, ${ }^{1}$ it will have to be measured by one of the methods previously described (\$ 84, 85). The heat of solution is then calculated by equation (1) above.

1 Where no very great accuracy is required. we may assume that the solution requires the same quantity of heat to raise its temperature $1^{\circ}$ as the water it con. tains alone would require. The magnitude of the error produced by this assumption will be apparent from the following table, in which a few ot Berthelot's results are given :-

Composition of Solution.

\begin{tabular}{|c|c|c|c|c|c|c|c|c|}
\hline \multirow{2}{*}{\multicolumn{2}{|c|}{3600 grams $\mathrm{H}_{2} \mathrm{O}$}} & & & & & & & \\
\hline & & +80 & $\operatorname{ram}$ & $\mathrm{SO}_{3}$ & & & & 3595 \\
\hline ," & $"$ & 63 & $"$ & $\mathrm{HNO}_{3}$. & & ${ }^{\circ}$ & . & 3597 \\
\hline$"$ & $\because$ & $36 \cdot 5$ & ", & $\mathrm{HCl}$. & • & - & - & 3561 \\
\hline " & " & $40 \cdot 0$ & ", & $\mathrm{NaHO}$ & - & • & - & 3578 \\
\hline " & , & 58.5 & ", & $\mathrm{NaCl}$. & - & . & . & 3578 \\
\hline , & ", & $74 \cdot 6$ & ", & $\mathrm{KCl}$ & . & . & . & 3565 \\
\hline " & $"$ & $53 \cdot 5$ & $"$ & $\mathrm{NH}_{4} \mathrm{Cl}$ & . & • & - & 3588 \\
\hline
\end{tabular}

Heat in Calories required to raise Temperature $1^{\circ} \mathrm{C}$.

3595

3561

3578

3565

3588 


\section{CHAPTER XIV}

\section{VAPOUR PRESSURE}

93. The Measurement of Vapour Pressure.-The methods in use for measuring vapour pressure may be divided into two classes. In the first class, called the statical methods, a limited portion of the liquid to be experimented upon is introduced into a confined space, and evaporation is allowed to go on till this space is saturated with the vapour. The temperature and pressure are then measured. In the second class, called the dynamical methods, the liquid is caused to boil under a constant pressure, and the temperature of ebullition gives the temperature at which the vapour pressure is equal to the pressure to which the boiling liquid is subjected. Both of these methods were used by Regnault in his classical researches on the vapour pressure of water. More recent experiments have, however, shown that the statical method is in general unreliable. The reason is that it is practically impossible to obtain absolutely pure liquids. In the statical method any trace of impurity will, owing to the small quantity of liquid evaporated, be liable to produce quite a considerable variation in the pressure exerted by the vapour of the liquid.

94. Ramsay and Young's Method of Measuring Vapour Pressure.A very convenient and accurate method of measuring the vapour pressure of a liquid by the dynamical method has been worked out by Ramsay and Young. ${ }^{1}$ Their arrangement is shown in Fig. 100, and consists of a glass boiling tube $\mathrm{A}$, with a side tube $\mathbf{B}$. A thermometer $\mathrm{T}$ and the stem of a tap funnel c pass through a cork, which closes the top of the tube $A$, the joint being made quite air-tight. The bulb of the thermometer is wrapped round with some porous material, such as cotton wool or asbestos, and the drawn-out end of the tap funnel is bent round so as to allow the liquid to drop on the bulb. The side tube $B$ is connected to a small bottle $\mathrm{D}$ in the manner shown on the figure, while this bottle is connected through a three-way tap with a large bottle $F$ and a tube $\mathrm{H}$ leading to a manometer. A side tube $\mathrm{G}$, which can be closed with a tap, is connected with an air-pump, by means of which the pressure of the air in the apparatus can be altered. All the joints must be made air-tight by being painted, if necessary, with. india-rubber cement. The bottle $\mathrm{F}$ must be protected from change in temperature by being packed round with cotton wool or inmersed in a bucket of water. The function of this bottle is to act as an air reservoir, and so

1 Journal of the Chemical Society (1885), xlvii. 42. 
reduce the effects of any slight leak, or variation in the rate of formation of vapour, on the pressure.

When performing the experiment, the liquid to be measured is placed in the funnel $\mathbf{c}$, and the tube $\mathrm{A}$ is placed in a bath, which is heated to a temperature slightly higher than the boiling point of the liquid under the pressure at which the experiment is to be made. By means of the air-pump the air is exhausted till this pressure is reached, when the tap $\mathrm{G}$ is closed. The bottle $\mathrm{D}$ is placed in a mixture of ice and water, or ice and calcium chloride, according to the volatility of the liquid, and serves to catch the vapour as it passes over from the tube $\mathbf{A}$.

The liquid is allowed to flow freely till the bulb of the thermometer

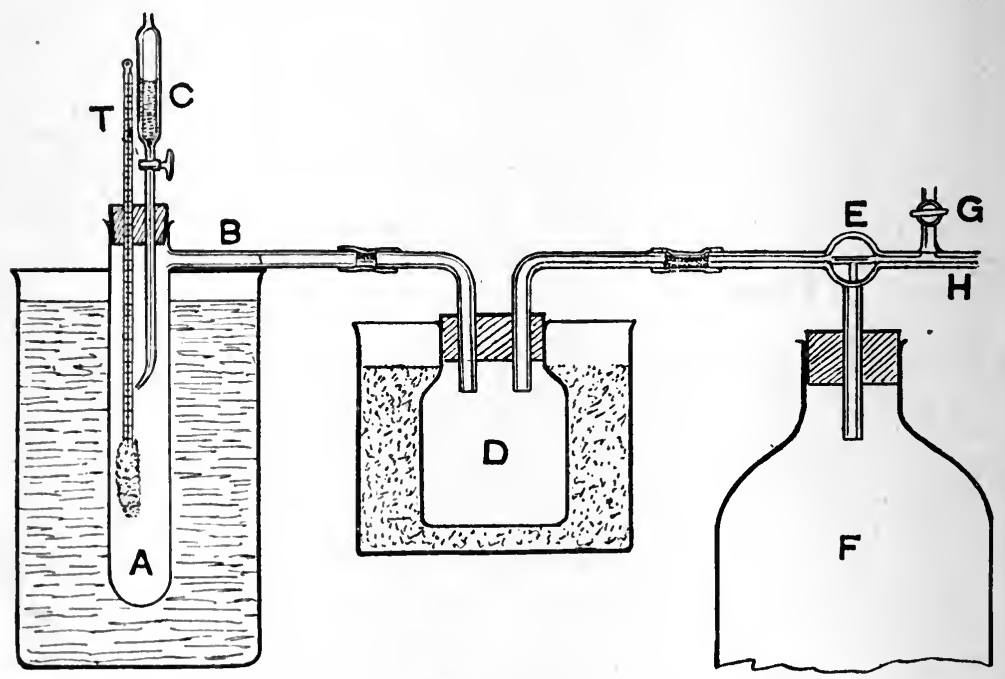

FiG. 100.

is thoroughly moist, and then the tap on the funnel is turned off to such an extent that the supply of liquid is just sufficient to allow for the evaporation which takes place as the vapour is drawn off through the tube B. When the reading of the thermometer becomes constant, say, at $t$, the pressure as indicated by the manometer is read as well as the height of the barometer. From these readings the pressure at which the vapour was formed can be at once calculated, the usual corrections for the temperature of the mercury columns, \&c., being applied. This pressure will be the vapour tension of the liquid at the temperature $t$.

By altering the pressure in the apparatus, a series of measurements of vapour tension at different temperatures can be made. 
95. Determination of the Dew-Point.-One of the most convenient instruments for measuring the dew-point is Regnault's hygrometer (see Watson's Physics, § 220). The aspirator should be placed at least 2 metres from the instrument, and preferably at a lower level, the connection being conveniently made by a length of lead or "compo" tubing. The silver thimbles must be very well polished by rubbing with a soft leather and polishing powder (jewellers' rouge) till the surface looks almost black. As the presence of the observer in the neighbourhood of the instrument is certain to alter the hygrometric state of the air it is best to use two telescopes, one to view the silver thimble, and the other to read the thermometer in the cooled thimble. If it is impossible to observe by means of telescopes, then a sheet of glass at least 50 centimetres in each direction must be held between the observer and the instrument. The thimble which is going to be cooled must be so placed that a bright light, such as a window or a gas flame, is seen reflected in the silver.

To perform an experiment, fill the thimble to which the aspirator is attached with ether well above the thermometer bulb. To assist the other thermometer taking up the temperature of the air it is advisable to place some liquid, such as xylene or toluene, in the other thimble. Water is not a good liquid to use for this purpose, since it has such a high specific heat. Neither is ether, since it is so volatile, and if any evaporation takes place it will cause the temperature to fall, owing to the latent heat of the liquid.

Start the aspirator, and watch the reflection of the light as seen in the silver, and as soon as any dulness appears reduce the current of air through the ether till the dew on the silver disappears, at the same time noting the temperature. Having in this way roughly determined the dew-point, slightly increase the rate of flow of the water, and immediately any sign of dew appears read the thermometer. Decrease the flow of water, and read the air temperature. Then watch the dew, and as soon as it vanishes, again read the thermometer. The mean of the temperatures corresponding to the deposition and disappearance of the dew is taken as the dew-point. The observation should be repeated several times.

From a table of the vapour pressures of water (Table 14) the vapour pressures corresponding to the dew-point and the temperature of the air can be obtained. The ratio of the actual pressure as deduced from the dew-point to the maximum pressure corresponding to the temperature of the air is the hygrometric state.

If the actual weight of water contained in the unit volume, or what is more usual in a cubic metre, be required, a small correction will have to be applied to the number corresponding to the dew-point obtained from Table 14.

Let the weight of water which is required to saturate a cubic metre of air at the temperature of the dew-point $\tau$ be $w$. Now if we start with 
a cubic metre of unsaturated air at the temperature of the air $t$, and cool it down to the dew-point $\tau$, the volume will be

$$
\frac{273+\tau}{273+t} \text { cubic metres. }
$$

But $w$ is the water contained in a cubic metre at $\tau$. Hence a cubic metre of the air at the air temperature $t$ will contain less than $w$ grams of water, the true weight of water being

$$
\frac{273+\tau}{273+t} w \text { grams. }
$$

96. The Wet and Dry Bulb Thermometer.-A convenient way of obtaining an approximate value for the hygrometric state of the air is to measure the cooling of a thermometer, which is kept moist with water, owing to the evaporation of the water. Two similar thermometers are generally fixed on a stand, the one being used to give the temperature of the air. The bulb of the other is surrounded with muslin, which is kept moist by a piece of lamp wick which dips in a vessel containing water.

The drier the air, the more rapid the evaporation of the water on the wet bulb, and hence the greater the difference in the readings of the two thermometers. The rate of evaporation of the water, however, does not depend exclusively on the hygrometric state of the air, but also on the nature of the surroundings of the thermometers, and more particularly whether the air in the neighbourhood of the wet bulb is in motion or not.

If $t_{w}=$ the temperature given by the wet bulb thermometer,

$t_{d}=$ the temperature of the air as given by the dry bulb thermometer,

$F_{w}=$ the maximum tension of water at the temperature $t_{w o}$

$B=$ the height of the barometer in millimetres,

then the actual tension of the water vapour present in the air is given by

$$
f=F_{w}-c B\left(t_{d}-t_{v}\right),
$$

where $c$ is a constant depending on the nature of the surroundings. The folluwing values of $c$ may be used in the conditions mentioned :-

In the open air with slight wind $c=0.0008$.

In the open air with no wind $c=0.0009$.

In a small closed room $\quad c=0.001$.

Owing to the uncertainty as to the correct value for $c$ to use on any given occasion, it is generally quite sufficient to take for $B$ a mean value, such as $750 \mathrm{~mm}$.

Somewhat more consistent results are obtained if the wet bulb thermometer is kept swinging backwards and forwards for a minute or two before taking a reading. In such a case $c$ may be taken as equal to 
0.0007 . The motion may be communicated by attaching the thermometer to a heavy pendulum, which is set swinging.

97. The Absorption Hygrometer.-The most aciurate method of determining the quantity of moisture contained in a cubic metre of air is to draw a measured volume of the air through some tubes in which are placed drying materials, such as strong sulphuric acid or phosphorus pentoxide. The difference between the weight of these drying tubes before and after the passage of the air gives the weight of the moisture present in the volume of air passed through.

A convenient form of absorption tube for use in this experiment is shown in Fig. 101, two tubes being generally ample to absorb all the moisture. The test tubes are about $10 \mathrm{~cm}$. long and $2 \frac{1}{2} \mathrm{~cm}$. wide, while the glass tubes through which the air passes have a bore of about $5 \mathrm{~mm}$. These tubes are filled with pumice saturated with strong sulphuric acid. In order to prevent leakage at the corks these are pushed right

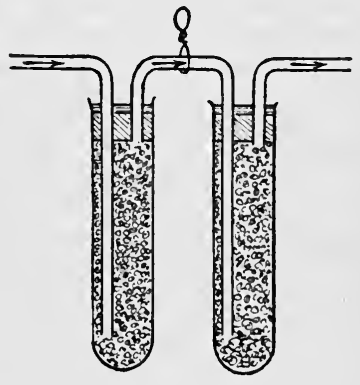

FIG. 101. into the top of the test tubes, and then a layer of melted paraffin wax is poured in.

The pumice is prepared in the following manner:-Having been broken into lumps about the size of a pea, the dust is removed and the remainder is heated to redness in a crucible, and then thrown, while still red-hot, into strong ("pure redistilled") sulphuric acid. The superfluous sulphuric acid having been poured off, the pumice is preserved in a wellstoppered bottle.

When the drying tubes are not in use, the ends of the tubes must be closed by means of a short length of rubber tubing and a piece of glass rod. These stoppers are weighed with the tubes. During the weighing the tubes may be suspended by means of a loop of wire attached to the bent tube joining the two test-tubes.

As it is necessary to know the

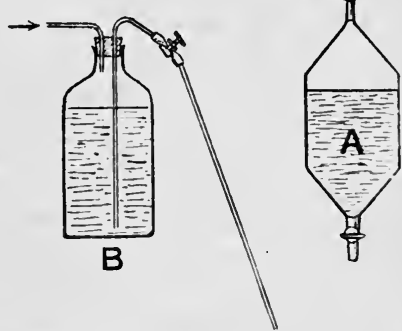

FIG. 102. volume of air which passes through the drying tubes, some form of aspirator which is capable of giving this volume must be used. The usual form is shown at A (Fig. 102). The vessel is filled with water and the upper tube connected with the drying tubes through a length of rubber tubing. On opening the tap on the bottom tube the water runs out and the air is drawn in to take its place. The volume of the vessel, and hence that of the air, is obtained by 
weighing the vessel empty and full of water. Another form of aspirator, which can readily be set up, is shown at $\mathbf{B}$, and its construction will be obvious from the figure.

When making an experiment the aspirator must be placed at a distance from the point where the air is drawn into the drying tubes, otherwise the water used in the aspirator will influence the hygrometric state of the air. It is best not to have a long tube between the intake and the drying tubes owing to the errors which might be caused by the evaporation from, or deposition of moisture on, the tube. A long length of tubing, so long as it is air-tight, has, however, no ill effect when placed between the drying tubes and the aspirator.

It will be advisable to include a third drying tube, which need not be weighed, between the two weighed tubes and the aspirator, to prevent any moisture from the aspirator finding its way to these tubes.

The tubes having been weighed, connect them to the aspirator and start the current of air. The air should pass at the rate of about a litre per minute. If the current is too rapid, all the aqueous vapour may not be removed in the drying tubes; while if the current is too slow, the experiment will take an unduly long time. A thermometer, hung with its bulb freely exposed to the air in the neighbourhood of the intake, must be read at regular intervals during the course of the experiment. After about 15 to 20 litres of air have been drawn through the apparatus the drying tubes must be again weighed. The increase in weight divided by the volume of air, in cubic metres, which has passed gives the weight of water contained in a cubic metre. The weight of moisture which would be required to saturate the air is obtained from the air temperature by means of Table 14 .

Corrections.-The volume $V$, which is the volume of water which has been run out of the aspirator, will require a slight correction to obtain the exact volume of air drawn into the drying tubes. Thus if $t$ is the temperature of the air, and $t^{\prime}$ that of the water in the aspirator, $f$ is the tension of the water vapour actually present in the air, and $f^{\prime}$ the maximum tension of water at $t^{\prime}$, while the barometric height is $B$. Then since the air in the aspirator becomes saturated owing to its contact with the water, the pressure of the $d r y$ air would be $B-f^{\prime}$; that is, if the water vapour were removed, the volume remaining $V$, the pressure would fall to $B-f^{\prime}$. If, further, the temperature were reduced to zero, the dry air would have a volume $V /\left(1+a t^{\prime}\right)$, the pressure being $B-f^{\prime}$.

Now when drawn into the drying tubes the air was at a temperature $t$, and at this temperature its volume, the pressure being $B-f^{\prime}$, would be $\frac{V(1+a t)}{1+a t^{\prime}}$. The actual pressure to which the dry air was subjected was, however, $B-f$. Hence the volume of the air before it entered the drying tubes was

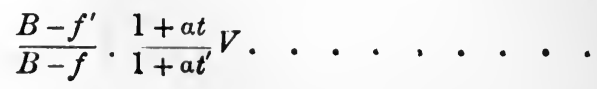


From the weight of moisture $w$ contained in a cubic metre of air we may calculate the tension of the aqueous vapour $f$. Thus if $a$ is the mass of a cubic metre of air at $760 \mathrm{~mm}$. pressure and $0^{\circ} \mathrm{C}$., the mass of a cubic metre of air at a pressure $f$ and a temperature $t$ is $\frac{a f}{760(1+a t)}$. If, then, the density of aqueous vapour in terms of that of dry air at the same pressure and temperature is $\delta$, the mass of water vapour contained in a cubic metre of the moist air is given by

$$
w=\frac{a f \delta}{760(1+a t)}
$$

Hence

$$
f=\frac{760(1+a t) w}{a \delta} \text {. . . . . . . }
$$

Now Regnault has shown that if $\delta$ be taken as a constant and equal to 0.6221 , the above expression gives the correct value for $f$, so that we are justified in using this value for $\delta$. The value of $a$ is 1292.8 grams per cubic metre, and $a$ is 0.00367 . Hence substituting these values we get

$$
f=0.9449(1+0.00367 t) w \text {. . . . . }
$$

Thus if $W$ is the total increase in the weight of the drying tubes when $V$ cubic metres of water are run out of the aspirator we have, combining (1) and (3),

or

$$
\begin{aligned}
f & =\frac{0.9449(1+a t) W}{\frac{\overline{B-f^{\prime}} \cdot 1+a t}{B-f} \cdot \frac{1+a t^{\prime}}{1+},} \\
\frac{f}{B-f} & =\frac{0.9449\left(1+0.00367 t^{\prime}\right) W}{\left(B-f^{\prime}\right) V} \text {. . . . . }
\end{aligned}
$$

The above method of conducting the experiment takes some considerable time and requires constant attention, since the aspirator has to be refilled and the temperature of the air read. We cannot make use of a water pump, since we require to know the volume of air which has passed through the apparatus. By arranging the experiment, however, so that we directly determine the weight of water contained in a given volume of air, and also the weight of water contained in the same volume of air saturated at the temperature of the air, then it will be unnecessary to know either the volume of the air or its temperature in order to obtain the hygrometric state.

The air is first passed through two drying tubes, such as those shown in Fig. 101. It then passes through a series of tubes, which are freely exposed to the air so that they and their contents are at the air temperature. These tubes contain water, and during its passage through the water the air becomes saturated. Next the air passes through a second 
set of drying tubes. The increase of weight in the first set of drying tubes gives the weight of water actually contained in the volume of air drawn through the apparatus, while the increase of weight of the second set gives the weight of water contained in this volume of air when saturated with water at the air temperature. The hygrometric state is then obtained by dividing the first weight by the second. The tubes used to saturate the air with moisture may consist of three or four large $U$-tubes packed with glass wool, sufficient water being added to saturate the wool and fill the bend for about 5 centimetres. After passing through these $U$-tubes the air is passed through a tube lightly packed with cotton wool to prevent any drops of water being mechanically carried into the drying tubes by the passing air.

The air may be sucked through the apparatus by means of a water pump, and by allowing the pump to work slowly we may continue the experiment for twenty-four hours, and thus obtain the mean hygrometric state of the air during the day. 


\section{CHAPTER XV}

\section{VंAPOUR DENSITY-FREEZING AND BOILING POINTS OF SOLUTIONS}

\section{Measurement of Vapour Density by Victor Meyer's Method.-}

A convenient method of measuring the vapour density of a body which can be volatilised below a temperature of about $400^{\circ} \mathrm{C}$., and where an accuracy of something less than 5 per cent. is sufficient, is that devised by Victor Meyer. A slightly modified form of the apparatus is. shown in Fig. 103. The wide tube A contains at the bottom a liquid which has a higher boiling point than the substance of which the vapour pressure has to be measured. The substance is vaporised in the bulb $\mathbf{B}$, and in doing so displaces its own volume of air, which is driven up the tube $\mathrm{c}$ through a length of thickwalled india-rubber tubing attached to the side tube $\mathbf{D}$ and into a graduated burette $\mathbf{E}$. This burette is supported in the manner shown within a glass cylinder $F$, the bottom of the cylinder being closed either by being drawn down as shown, or with a cork and a drainage tube attached, which can be closed by a pinch-cock $\mathrm{G}$. The liquid to be experimented upon is contained either in a small bottle with a glass stopper, or in a thinwalled glass bulb, which at the commencement of the experiment is hung from a wire $J$, which passes through the cork which closes the

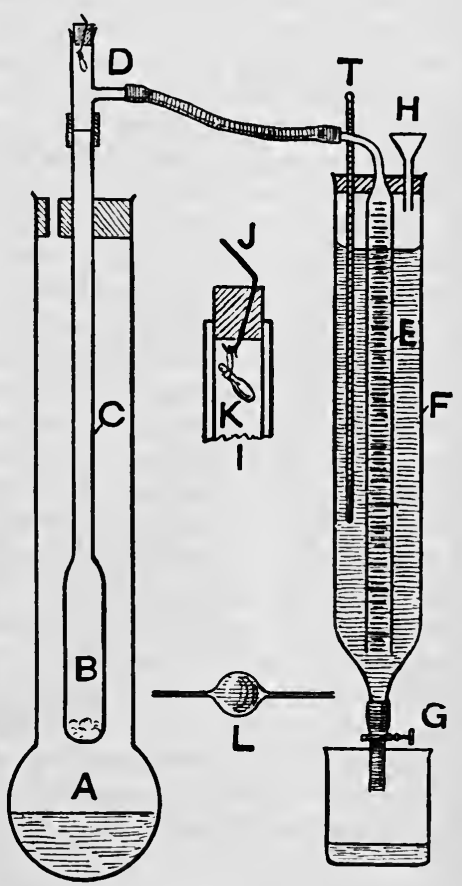

FIG. 103. top of the tube c. The form of this wire is shown on a large scale at I. In the position shown the bottle hangs from the lower end of the wire, a loop of wire being attached to the neck of the bottle for the purpose. On turning the wire through 
two right angles by means of the handle $J$, the wire loop slips off the hook, and the bottle falls down the tube c. A little asbestos placed in the bulb B serves to prevent the bottle breaking the tube in its fall.

The following liquids may be used in the heater, and will serve. for substances which vaporise at temperatures below those given opposite each liquid :-

Liquid in Heater.

Water

Water $\quad \cdot \quad \cdot \quad \cdot \quad$
Aniline
Methyl salicylate
Sulphur $\quad: \quad:$

Maximum Boiling

Point of

Substance.

$80^{\circ}$

$150^{\circ}$

$200^{\circ}$

$400^{\circ}$

To conduct an experiment, the small bottle or glass bulb which is to contain the liquid is weighed. It is then filled with liquid, and again weighed. If a glass bulb is used, after the introduction of the liquid the ends of the capillaries used in filling the bulb, which may have the shape shown in Fig. 103 at $L$, must be sealed off. This may readily be done by holding the ends in the edge of a Bunsen flame.

The flame used to heat the tube $\mathbf{A}$ is adjusted so that the vapour of the liquid used extends to within about a third of the length of the tube from the upper end. As it is of importance that the temperature of the various parts of the tube $\mathrm{c}$ should remain constant throughout the experiment, it is important to screen the flame and the boiling tube from draughts by means of an asbestos or cardboard tube. After the boiling liquid in $\mathbf{A}$ has reached a steady state, the bottle containing the substance is attached to the hook and the cork inserted in c. The level of the water in the tube $F$ is then adjusted so that the surfaces of the water in the burette and in the tube $\mathrm{F}$ are exactly at the same level, and the reading of the water surface in the burette is noted. The pin $J$ is then turned, allowing the bottle to fall into the bulb B. Owing to the heat the stopper is forced out of the bottle or the bulb is burst, as the case may be, and the liquid vaporises, driving air over into the burette $\mathrm{E}$. By allowing water to run out, the level of the water inside and out is kept the same, and as soon as the volume becomes constant the reading on the burette is noted. Immediately after the thermometer $T$ is read, and also the height of the barometer.

After taking the second reading of the burette, the cork closing the tube $\mathrm{c}$ should be removed to prevent any chance of the water in the burette being forced over into the bulb $\mathrm{B}$.

The difference between the two readings of the burette gives the volume which the vapour would occupy at the temperature shown by the thermometer $\mathbf{T}$ and at the pressure corresponding to the barometric height, subject to a correction to allow for the volume occupied by the water vapour in the upper part of the burette.

Let $V$ be the volume as read off on the burette corrected for any graduation error which may exist, $T$ the temperature, and $B$ the height 
of the barometer. Then if $F$ is the tension of the water vapour at the temperature $T$, the actual pressure of air in the burette is $H-F$. Thus the volume of the vapour at the temperature $T$ and the pressure $H-F$ is $V$. If the volume at $0^{\circ} \mathrm{C}$. and the standard pressure of $76 \mathrm{~cm}$. is $V_{0}$, we have

or

$$
\begin{gathered}
76 V_{0}=\frac{V(H-F)}{1+a T}, \\
V_{0}=\frac{V(H-F)}{76(1+a T)} .
\end{gathered}
$$

But the difference between the weight of the bottle full and empty is the weight $W$ of the vapour. Hence the density $D$ is given by

$$
D=\frac{76 W(1+a T)}{V\left(H-F^{\prime}\right)}
$$

To obtain the molecular weight of the vapour we have to calculate the density in terms of that of hydrogen taken as 2 . Now 1 cubic centimetre of hydrogen at $0^{\circ}$ and $76 \mathrm{~cm}$. pressure weighs 0.0000900 grams. Hence the molecular weight $M$ is given by

$$
M=\frac{152 W(1+a T)}{0.0000900 V(H-F)} .
$$

99. Measurement of Vapour Density by Hofmann's Method.-A more accurate method of determining vapour density than that described in the previous section is that devised by Hofmann. A glass tube A (Fig. 104) having a diameter of about $2 \mathrm{~cm}$. and a length of about $90 \mathrm{~cm}$., and closed at the top, is filled with dry mercury sind inverted in a trough $\mathrm{D}$ containing mercury. This tube is surrounded by a wide glass tube, $B$, which serves as a vapour jacket for heating the inner tube. This outer tube also dips in the mercury in the trough, resting in a shallow groove cut in the bottom of the trough and is held at the top by a loosely fitting clamp $\mathbf{H}$, which is attached to a wooden upright.

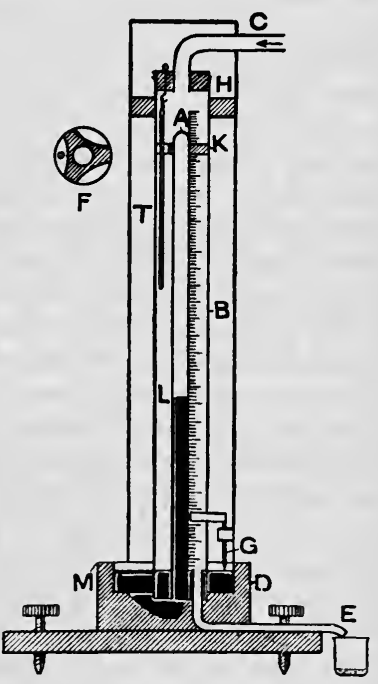

FIf. 104. The upper end of the tube $A$ is held in a central position by a ring of cork $\mathbf{E}$, which is cut away in the manner shown at $\mathbf{F}$ to allow of the passage of the vapour used to heat the jacket. A glass tube $\mathrm{E}$, bent in the manner shown, has its upper end ground flat, and is cemented into the trough, so that when the mercury just comes to the top there is a layer of about $2 \mathrm{~cm}$. of mercury in the trough. This tube serves as a drain tube to carry 
away the liquid which condenses in the jacket. The heating vapour enters the jacket through a tube $\mathrm{c}$, which passes through the cork which closes the top of the vapour jacket. A thermometer $T$ serves to give the temperature of the vapour.

When performing an experiment the tube $\mathbf{A}$ is thoroughly cleaned and well dried by driving a current of hot air up to the closed end through a glass tube. The tube is then filled with dry mercury, all air bubbles attached to the side being removed by running a bubble of air up the tube. This removal of the air can further be facilitated by placing the lower end of the tube in the mercury bath, so that a Torricellian vacuum is formed. If, then, the tube is slowly inclined till the mercury reaches the top, the air which has collected will appear as a bubble, which may be run up to the open end and removed. The tube filled with mercury is then introduced into the jacket, and the whole inverted over the mercury bath. The liquid to be observed is introduced in a small stoppered bottle or glass bulb, as in the previous method. In order to facilitate the introduction of the liquid, the bottom of the mercury bath has a groove cut below the end of the tube A, as shown in the figure.

The vapour of the substance which is to be used to heat the jacket is passed in through the tube c. Since if too large a quantity of liquid is not used the vapour will exist under a pressure less than one atmosphere, the jacket need not be heated to a temperature higher than the boiling point of the substance. In fact, by using a wide tube and a small quantity of the substance, so that the pressure within the tube is very small, the temperature of the jacket may well be very considerably lower than that of the boiling point under atmospheric pressure.

When the vapour used to heat the jacket has been issuing from the tube $\mathbf{E}$ for some time, so that the mercury column has had time to reach the temperature of the jacket, the volume of the vapour and the height of the mercury column must be read. In some forms of the apparatus the tube $\mathrm{A}$ is divided along its whole length in millimetres. In this case the readings corresponding to the mercury meniscus at $L$ and at $M$ must be made, and to facilitate this measurement it is well to provide the mercury trough with plate glass at front and back. In the other arrangement a millimetre scale engraved on mirror glass is attached to the wooden stand immediately behind the jacket, and in order to facilitate the reading of the position of the mercury surface in the trough a small metal rod $G$ with a pointed end is attached to the upright. The lower point of this rod is brought into contact with the surface of the mercury, and the position of the upper end is read on the scale. Then by measuring the length of the rod we can immediately obtain the reading corresponding to the surface of the mercury. When ine tube is not graduated, it will be necessary not only to take the reading on the scale corresponding to the meniscus $\mathrm{L}$, but also to read the position of the top of the tube $\mathrm{K}$, or of a fine line engraved on the side of the tube.

To obtain the volume of the vapour it is next necessary to determine 
the volume of the tube $\mathrm{A}$ from the closed end down to the position occupied by the mercury meniscus when the vapour was present. For this purpose the tube must be cleaned and dried, and fixed in an upright position with the closed end downwards. Mercury must be poured in till the meniscus occupies the position it did in the experiment. If the tube itself is graduated, this position can be identified from the graduations. If the tube is not graduated, then the position must be determined by measuring from the top or the fixed mark, as the case may be. The volume of the mercury is then determined by pouring the mercury into a weighed beaker and weighing.

A correction will have to be applied to allow for the fact that the curvature of the mercury meniscus is in the opposite direction when measuring the volume from what it was when the vapour was present. Thus if the readings have been made, as they should, to the top of the meniscus, the volume obtained from the weight of mercury will have to be increased by the volume of the shaded part shown in

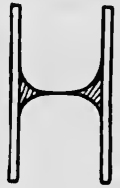

Fia. 105. Fig. 105. The amount of the corrections which have to be applied with tubes of different diameters have been determined, and are given below.

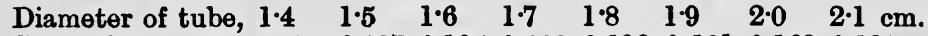

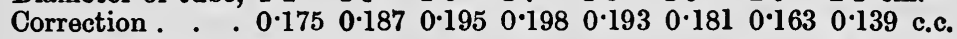

The correction in each case is the volume which has to be added to the volume as deduced from the weight of mercury to give the volume of the vapour. ${ }^{1}$

The volume as determined above will require correction to allow for the expansion of the glass. In calculating this correction it will be sufficiently accurate to take a mean value, 0.0000232 , for the coefficient of cubical expansion of glass. If $v$ is the volume of the tube as measured at a temperature $t$, and $T$ is the temperature at which the vapour was measured, we have that the volume $V$ of the vapour is given by ${ }^{2}$

$$
V=v\{1+0.0000232(T-t)\} \text {. }
$$

To find the pressure to which the vapour is subjected, we must deduct from the height of the barometer the pressure due to the column of mercury in the tube $\mathbf{A}$. Let the height of this column be $h$; then the height $H$ if the temperature of the mercury were $0^{\circ}$ would be given by ${ }^{3}$ $H=h\{1-0.000182 T\}$. Thus the pressure within the tube is equal to $B-H$, where $B$ is the height of the barometer at the time of the experiment, reduced of course to $0^{\circ}$. Theoretically, these heights ought to be reduced to what they would be at the sea-level and at latitude $45^{\circ}$, but in practice this is hardly necessary.

1 A table of the volume of a mercury meniscus for different diameters of tube and heights of the meniscus will be found in Ann. d. Physik, xxxiii. p. 291 (1910); also in Science Abstracts, A (1910), No. 1563.

2 If the length of the tube occupied by the vapour is measured by a scale outside the jacket the correction is obtained by taking of of $\gamma$ for the coefficient of expansion.

8 See $\$ 58$. 
The pressure inside the tube is, however, not entirely due to the vapour, for the mercury has a vapour tension, and so part of the pressure is due to the presence of mercury vapour, and hence at temperatures above $100^{\circ}$ a correction becomes necessary on this account. If $e$ is the vapour tension of mercury at the temperature $T$, as given in Table 17, the actual pressure exerted by the vapour is

$$
B-e-h\{1-0.000182 T\} \text {. }
$$

Hence the density of the vapour is given by

$$
D=\frac{76 W(1+\alpha T)}{V(B-e-h\{1-0.000182 T\})},
$$

where $W$ is the weight of liquid vaporised.

In accurate determinations an allowance will have to be made for the volume of the bottle or bulb in which the liquid was contained. This can at once be calculated from the weight, taking the value 2.5 for the density of glass.

100. Measurement of the Freezing Point of a Solution.-A very convenient piece of apparatus for determining the lowering in the freezing point produced by the solution of a given

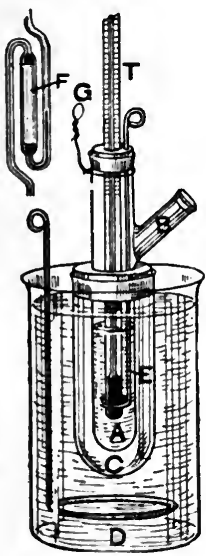

FIG. 106. substance has been devised by Beckmann, and is shown in Fig. 106. It consists of a glass test-tube $\Delta$ provided with a side tube B. This side tube may in many cases be dispensed with, and a simple boiling tube used. The tube $\mathrm{A}$ is fitted, by means of a cork, within a slightly wider tube $\mathrm{c}$, which is itself immersed in water or some freezing mixture contained in a beaker $\mathrm{D}$, the liquid in this beaker being maintained at a temperature of a few degrees below the freezing point of the solution placed in $\dot{A}$. A delicate thermometer $\mathbf{T}$ passes through the cork which closes the top of $\mathrm{A}$, as also does a glass rod which carries a loop of stout platinum wire at its lower end and acts as a stirrer.

In order to allow of its being used with solvents which have freezing points at different temperatures the thermometer is fitted at the top with an auxiliary bulb, shown on a large scale at F. By allowing more or less of the mercury to flow into this bulb, and then detaching it from the rest of the mercury column, we can adjust matters so that when the bulb is at the freezing point of the solvent being examined, the top of the mercury thread is near the top of the thermometer scale.

The quantity of mercury in the thermometer is adjusted in the following manner:- The bulb of the thermometer is placed in some water heated to a temperature 1 or 2 degrees above the freezing point 
of the solvent, and when the thermometer has had time to acquire the temperature of the water, by a sudden jerk the mercury column is caused to break at the top of the bulb $F$. The thermometer is then cooled to the melting point of the solvent, and if the end of the mercury column is within about a degree of the top of the scale, the adjustment is complete. If not, the operation must be repeated. The mercury in the bulb $\mathrm{F}$ can be made to join up with the rest of the mercury by heating the bulb till the mercury reaches the top of the bulb $F$, and then inverting the thermometer so that the mercury at the bottom of the bulb joins on to that at the end of the column.

Having adjusted the thermometer, the next operation is to determine the freezing point of the pure solvent. The tube A, with the thermometer, stirrer, \&c., is weighed, and then enough of the solvent is intro duced to well cover the bulb of the thermometer. By again weighing, the weight of solvent is obtained. About 20 grams of solvent ought to be employed, so that a balance which will weigh to within about 0.02 gram will be sufficiently accurate. During the weighing the apparatus can be suspended from the hook which carries the balance pan by means of a wire sling $G$.

The tube $A$, without the air jacket $c$, is immersed in the outside bath till the solvent commences to freeze. The outside of $\mathbf{A}$ having been wiped, it is placed within the air jacket, and the contents are allowed to cool slightly below the freezing point, if this can be done without the whole mass of the solvent becoming solid. By working the stirrer the solidification of a portion of the supercooled solvent is caused, and the reading of the thermometer first rises and then remains constant. This constant temperature is the freezing point of the solvent. If the solvent is not quite pure there may be a slow fall of the thermometer reading while solidification goes on, owing to the change in the composition of the fluid portion of the liquid, due to one constituent of the liquid freezing out more rapidly than the other.

Having determined the freezing point of the pure solvent, the tube $\Delta$ is removed and a weighed quantity of the substance is introduced through the side tube B. The solidified portion of the solvent is allowed to melt, and the solution of the substance promoted by stirring. When solution is complete the tube $A$ is replaced in the air jacket, and the temperature allowed to fall a little below the freezing point. Freezing is then started in the supercooled liquid by stirring, when the thermometer rises slightly, becomes constant, and then slowly falls. If the supercooling has not been too great, the maximum temperature corresponds to the freezing point of the solution of the strength used.

It is sometimes found difficult to start solidification, the solution becoming much supercooled. In these circumstances some of the solvent must be placed in a test-tube immersed in the outside bath and caused to solidify. A glass rod is dipped into this test-tube and allowed to remain till the end has become thoroughly cold. The side tube $\mathbf{B}$ is then uncorked and the stirrer $\mathbf{E}$ raised and touched with 
the end of the glass rod, to which some of the solidified solvent must be attached. On coming in contact with the solidified solvent the solution attached to the rod will solidify, and then on stirring the solution solidification of part of the solvent will be set up.

Correction 1.- Owing to the fact that some of the solid solvent has to separate out from the solution to cause the rise of temperature which occurs when solidification takes place, the strength of solution, for which the maximum temperature shown by the thermometer is the freezing point, is less than that of the original solution. A correction will have to be applied to the results to allow for this effect. Of course the correction becomes less and less as the amount of the supercooling of the solution is decreased. Hence it is advisable to reduce the amount of the supercooling as much as possible, and not to attempt to start solidification by increasing the supercooling, but to use a trace of the solid solvent in the manner described above. If $c$ is the specific heat, $w$ the weight of the solution, $L$ the latent heat of the solvent, and $\Delta$ the amount by which the temperature just before solidification starts is below the maximum reading given by the thermometer, so that $\Delta$ is the supercooling, then the heat necessary to raise the temperature of $w$ grams of the solution $\Delta$ degrees is cwo $\Delta$. This quantity of heat has to be supplied by the latent heat of the solidified solvent. Hence if $x$ is the weight of solvent which solidifies, we have

or

$$
\begin{aligned}
x L & =c \cos , \\
x & =\frac{c v \Delta}{L} .
\end{aligned}
$$

Hence when working out the strength of the solution we must deduct the quantity $x$ from the weight of solvent in order to obtain the strength of the solution which has the observed freezing point.

Correction 2.-A further correction has to be applied, if very accurate measurements are to be made, to allow for the temperature of the freezing solution being affected by exchange of heat with the surroundings and heat developed by the stirrer. Owing to these causes the liquid in the tube A, supposing no "freezing" take place, will settle down to a temperature differing from that of the external vessel containing the freezing mixture, this temperature being called by Nernst and Abegg the convergence temperature. Thus when the solution has been supercooled and freezing takes place we shall have two effects going on. In the first place, there will be a supply of heat: owing to the freezing of the solvent causing the temperature to approach the true freezing point of the solution. At the same time the temperature will, owing to radiation, conduction, and heat development from stirring be approaching the convergence temperature. The actual maximum temperature reached will be intermediate between the true freezing point and the convergence temperature. Hence it is of advantage to make the convergence temperature as nearly equal to the 
freezing point as possible. For this reason the temperature of the freezing mixture should not be more than 1 or 2 degrees below the freezing point of the solution. If $T_{0}$ is the observed freezing point, $T$ the true freezing point, and $T_{c}$ the convergence temperature, then

$$
T=T_{0}+A\left(T_{0}-T_{c}\right),
$$

where $A$ is a constant the value of which depends on the circumstances of the experiment, and is equal to the change in the observed freezing point produced by a change of $1^{\circ}$ in the convergence temperature. For Beckmann's form of apparatus, the value of $A$ is approximately 0.0005 in the case of water solutions.

For a discussion of the corrections to be applied to the readings of a Beckmann thermometer, see $\S 102$.

101. Calculation of Molecular Weight from the Depression of the Freezing Point.-If a dilute solution containing $s$ grams of the solute in $S$ grams of the solvent has a freezing point $\Delta$ degrees below that of the pure solvent, then the molecular weight of the solute is given by

$$
\mathrm{M}=\kappa \frac{\boldsymbol{8}}{\mathrm{S} \Delta},
$$

where $\kappa$ is a constant, the value of which depends on the nature of the solvent.

\begin{tabular}{|c|c|c|}
\hline Solvent. & Freezing Point. & * \\
\hline $\begin{array}{l}\text { Water, } \mathrm{H}_{2} \mathrm{O}_{1} \\
\text { Benzene, } \mathrm{C}_{6} \mathrm{H}_{6} \\
\text { Nitro-benzene, } \mathrm{C}_{6} \mathrm{H}_{6} \mathrm{NO}_{2} \\
\text { Naphthalene, } \mathrm{C}_{10} \mathrm{H}_{8} \\
\text { Phenol, } \mathrm{C}_{6} \mathrm{H}_{6} \mathrm{OH}^{\circ} \\
\text { Acetic acid, } \mathrm{CH}_{3} \mathrm{CO}_{2} \dot{\mathrm{H}}\end{array}$ & $\begin{array}{l}0^{\circ} \\
5^{\circ} \cdot 5 \\
5^{\circ} \cdot 3 \\
80^{\circ} \cdot 1 \\
39^{\circ} \\
17^{\circ}\end{array}$ & $\begin{array}{l}1850 \\
5010 \\
7050 \\
6850 \\
7300 \\
3900\end{array}$ \\
\hline
\end{tabular}

The values of $\kappa$ for some solvents are given in the following table :-

In the cases of solvents other than water, besides the series of substances which give normal depressions of the freezing point, there is in general a series of other substances which give depressions which are only about half the normal. These abnormally small depressions are supposed to be due to the formation of aggregates of two molecules of the solute, so that in the solution the molecular weight is double the normal value. Such polymerisation appears in the case of solutions of ethyl alcohol, methyl alcohol, phenol, \&c., in benzene, and with sulphuric and hydrochloric acid in acetic acid. 
In the case of solutions in water, in addition to the normal series it is found that in the case of electrolytes the depression is generally twice as great as the normal. This effect is supposed to be due to dissociation of the solute into simpler molecules. (See Watson's Physics, §§ 225, 543.)

102. Correction of the Readings of a Thermometer in which the Quantity of Mercury is Variable.-Thermometers of the Beckmann ${ }^{1}$ type, described in $\$ 100$, are so very useful wherever small differences of temperature are to be measured, that it becomes necessary to study a correction which must be applied to allow for the fact that the quantity of mercury in the bulb varies according to the temperature at which the instrument has been adjusted. Since practically all the Beckmann thermometers in the market are made of Jena normal glass $\left(16^{\prime \prime \prime}\right)$, it will simplify the description if we only consider a thermometer made of this glass. The coefficient of apparent expansion of mercury in Jena glass $\left(16^{\prime \prime \prime}\right)$ is 0.000157 , or $1 / 6370 .^{2}$ Hence if we have a thermometer such that when placed in melting ice the end of the mercury column is at the $0^{\circ}$ division of the scale, and take as our unit of volume the volume of $1^{\circ}$ of the capillary, the volume of the mercury will be 6370. For if the temperature is raised $1^{\circ}$, the apparent increase in volume of the mercury will be $6370 \times 1 / 6370$, or 1 , i.e. 1 degree.

Next suppose that enough mercury to fill $n$ degrees is passed over into the auxiliary bulb. The mercury will now be at the $0^{\circ}$ of the scale at a temperature $n$. If, now, the temperature is raised to $n+1$ degrees C., the apparent increase in volume of the mercury will be $\frac{6370-n}{6370}$, or $1-\frac{n}{6370}$. In other words, when the temperature is increased $1^{\circ}$ the reading will only increase $1-n / 6370$ degrees, that is, the value of a degree on the scale will be greater than $1^{\circ} \mathrm{C}$. Thus suppose $n=10^{\circ}$, then each degree on the scale of the thermometer now corresponds to $1 \div\{1-10 / 6370\}$, or $1^{\circ} .0016$, quite an appreciable correction, since such thermometers are generally divided into $0^{\circ} 01$.

We have in the above supposed that when the thermometer was placed in melting ice it read exactly $0^{\circ}$, and further that when heated to $1^{\circ}$ it read exactly $1^{\circ}$ on the scale, and that the bore of the capillary was cylindrical. These, however, are points which have to be tested experimentally. In the first place, the uniformity of the bore has to be tested by a calibration performed as described in $\S 59$. When performing this

1 This form of thermometer was really devised by Walferdin, who called them metastatic thermometers. They are so widely known, however, as Beck. mann thermometers, that it seems hardly possible to designate them by any othes name without risking confusion. $1 / 6080$.

${ }^{2}$ The coefficient of apparent expansion of mercury and Jena glass $59^{\prime \prime \prime}$ is 
calibration the $0^{\circ}$ and, say, the $6^{\circ}$ are assumed correct, and the interval is subdivided into portions of equal volume.

In order to test whether, when all the mercury is in the main bulb, the thermometer reads correctly, resource must be had to a standard thermometer (preferably made of the same kind of glass) of which the errors are known. The Beckmann thermometer, having had its zero determined in the ordinary manner, must be compared with the standard at a temperature near the top of the scale, preferably near the division used as a fixed point when performing the calibration. In this way the error at the upper fixed point of the calibration is determined, and then a curve showing the errors at the intermediate points can be drawn up, as described in $\S 62$. When any mercury is placed in the upper bulb, then the difference in the readings, corrected from the curve, and multi plied by $\frac{6370}{6370-n}$, will give the difference in temperature on the scale of the mercury thermometer. To reduce to the hydrogen thermometer, we must remember that when using a Beckmann thermometer we are only concerned with the accurate measurement of a difference of temperature; in other words, it is only the difference in the corrections to reduce to the hydrogen scale at the upper and lower points which has to be considered. For example, if the correction to reduce to the hydrogen scale was the same at the upper and the lower temperatures, it is evident that the difference in temperature obtained with a mercury thermometer would be the same as that given by a hydrogen thermometer. If the correction to reduce to the hydrogen thermometer, as given in Table 19, at the lower temperature $t_{0}$, as measured on the mercury scale, is $\delta_{0}$, and that at the higher temperature $t_{1}$ is $\delta_{1}$, then the true difference of temperatures is

$$
\begin{aligned}
& t_{1}+\delta_{1}-\left(t_{0}+\delta_{0}\right), \\
& t_{1}-t_{0}+\delta_{1}-\delta_{0},
\end{aligned}
$$

that is, the correction to be applied to the difference of temperature as read on the thermometer is $\delta_{1}-\delta_{0}$. This correction may become quite appreciable for those temperatures where the curves given in Fig. 62 are steep. Thus suppose $t_{0}=0^{\circ}$ and $t_{1}=5^{\circ}$, so that for Jena glass $\left(16^{\prime \prime \prime}\right) \delta_{0}=0$ and $\delta_{1}=-.03$, then the true difference of temperature on the hydrogen scale is $5^{\circ} \cdot 00-0^{\circ} \cdot 03$, or $4^{\circ} \cdot 97$, the correction amounting to nearly 1 per cent.

Another correction which may often be very considerable is that due to emergent column. This point has been examined in the case of Beckmann's pattern of thermometer by Grützmacher. ${ }^{1}$ His results are given in the following table:- 
Corrections for Emergent Column in Beckmann's Thermometer (the whole column is supposed outside the bath, as in Fig. 106) :-

\begin{tabular}{|r|c|c|}
\hline $\begin{array}{c}\text { Temperature Interval } \\
\text { Measured. }\end{array}$ & $\begin{array}{c}\text { Mean Temperature of } \\
\text { Column. }\end{array}$ & $\begin{array}{c}\text { Correction per } 1^{\circ} \text { for } \\
\text { Effect of Cool Column. }\end{array}$ \\
\hline$-35^{\circ}$ to $-30^{\circ}$ & $0^{\circ}$ & $-0^{\circ} .005$ \\
$0^{\circ}$ to $+5^{\circ}$ & $15^{\circ}$ & $-0^{\circ} .002$ \\
$45^{\circ}$ to $50^{\circ}$ & $26^{\circ}$ & $+0^{\circ} .004$ \\
$95^{\circ}$ to $100^{\circ}$ & $32^{\circ}$ & $+0^{\circ} .011$ \\
$145^{\circ}$ to $150^{\circ}$ & $38^{\circ}$ & $+0^{\circ} .018$ \\
$195^{\circ}$ to $200^{\circ}$ & $44^{\circ}$ & $+0^{\circ} 025$ \\
$245^{\circ}$ to $250^{\circ}$ & $50^{\circ}$ & $+0^{\circ} .031$ \\
\hline
\end{tabular}

Thus if in the case considered above the room temperature was such that the mean temperature of the column was $15^{\circ}$, only the bulb being

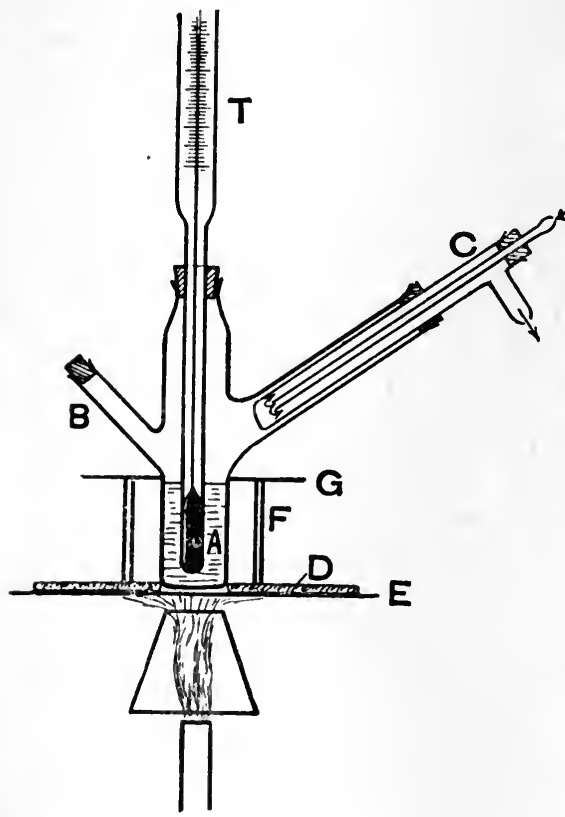

FIG. 107. immersed in the liquid, the true difference of temperature would be $4.97(1-.002)$, or $4^{\circ} \cdot 96$.

103. Measurement of the Boiling Point of a Solution. - The accurate determination of the boiling point of a solution is rendered difficult owing to the readiness with which almost all solutions become superheated. The form of apparatus ordinarily adopted is that devised by Beckmann, and shown in Fig. 107. The three-necked flask $\Delta$ is filled about a third full of glass beads or garnets, while a piece of very thick platinum wire is fused through the bottom. These two devices are used to secure regular ebullition and prevent superheating. A thermometer $T$ passes through the cork which closes the middle neck, while a condenser $\mathbf{c}$ is attached to one of the side necks. The third neck $\mathrm{B}$ serves for the addition of the substance, the effect of which on the boiling point of the solvent is being measured. The instrument is stood on a sheet of thick 
asbestos card $\mathrm{D}$, which is pierced with a hole. This card rests on a sheet of wire gauze $\mathbf{E}$. A short glass cylinder F rests on the upper side of the card $a$, leaving an air space all round the bulb. There is a plate of mica or asbestos $\mathrm{G}$ used to close the upper end of this air jacket. The height of the flame is adjusted so that about five drops a minute fall from the end of the condenser. This form of apparatus is by no means free from superheating, and Beckmann has devised a more complicated form, in which the inner bulb containing the solution is jacketed, except at the bottom, with the vapour of the solvent boiling in a separate vessel.

The only really satisfactory method of avoiding superheating is to use electrical heating, and as no modern physical laboratory is without a supply of accumulators, there is no reason why this method should not be used exclusively. By this means, not only is regular boiling secured, and hence superheating avoided, but owing to the ease with which the supply of heat can be regulated and kept constant the time necessary for the experiment can be materially reduced.

A convenient form of apparatus, which is a very slight modification of that described by Christensen, ${ }^{1}$ is shown in Fig. 108. It consists of a glass test-tube A closed by a cork, through which passes the thermometer T, two thin glass tubes $B$, and two other

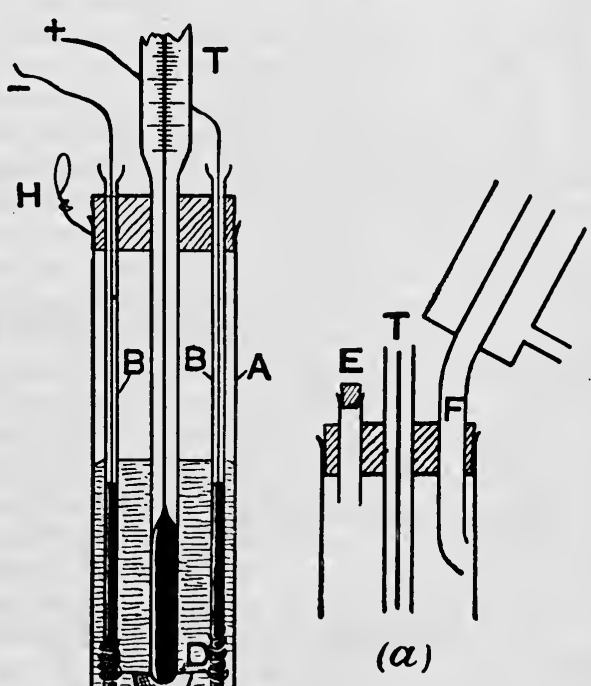
tubes $\mathrm{E}$ and $\mathrm{F}$ (shown at $a$ ).

The bottom of the tubes $\mathrm{B}$ are fused round a short length of stout platinum wire $\mathrm{G}$, while a spiral of thin platinum wire $\mathrm{C}$ is attached to these pieces of wire either by autogeneous soldering or with pure gold. The tubes B are partly filled with mercury, and are connected to a battery of three or four accumulators and an adjustable resistance by copper wires, which dip in this mercury. A tube $\mathrm{E}$, which is normally closed with a cork, serves for the introduction of the substance. The end of a small condenser $\mathrm{F}$, or, in the case of a solvent with a high boiling point, simply an air condenser passes through the cork in the top of the tube $\mathbf{A}$.

I J. C. Christensen, Journal of Physical Chemistry (1900), iv. 585. 
The end of the condenser is slightly bent as indicated, and cut off at an angle, so that by holding the tube $\Delta$ slightly inclined the condensed solvent may run down the side of the outer tube, and not come in contact with the thermometer. To prevent any chance of the thermometer being affected by the rising bubbles of superheated vapour a small thimbleshaped glass dish $D$ is supported in the position shown by two pieces of platinum wire, which are fused to the edge of the glass and wound round the tubes $\mathbf{B}$. When making an experiment the tube $\mathbf{A}$ is packed round with cotton wool or asbestos cloth, according to the temperature at which the solvent boils. ${ }^{1}$

When making an experiment the tube $A$, without the cork and the pieces of apparatus attached, is weighed, the solvent introduced, so that it will quite cover the bulb of the thermometer, and the whole again weighed. During this weighing the tube may be suspended from the hook attached to the stirrup of the balance by means of a wire sling $\mathbf{H}$. If the weights are determined to within $0.01 \mathrm{gram}$, it will be quite sufficient. The thermometer, condenser, \&c., are now attached, and the tube packed round with some non-conducting material, and tho current started. The resistance in the circuit must be adjusted, so that when boiling has become regular the vapour only extends a very short distance up the condenser tube.

The boiling must be continued, the thermometer being read every five minutes till the temperature becomes constant. This in general takes about ten minutes. Then the temperature must be read, as also the height of the barometer. A weighed quantity of the substance is then introduced through the tube $\mathrm{E}$, the current having been turned off before removing the cork. The current having been switched on, the reading of the thermometer must be made every five minutes till the temperature becomes constant, when the thermometer and barometer are again read.

The solution of which the boiling point is actually determined is slightly more concentrated than would appear from the weighings, owing to some of the solvent during the ebullition being absent from the solution, since it is in the form of vapour in the upper portion of the tube, and of liquid in the condenser and on the sides of the tube $A$. The exact evaluation of the amount which has to be deducted from the weight of solvent on this account is difficult, and so it is important to reduce the correction to a minimum by adjusting the rate of boiling so that the vapour only just reaches the condenser, and using as large a volume of the solvent as the apparatus will allow.

1 The instrument may be placed inside a Dewar vacuum vessel. 


\section{CHAPTER XVI}

\section{MELTING POINT-RATIO OF SPECIFIC HEATS-CONDUCTIVITY}

104. Determination of the Melting Point by the Method of Cooling.Since when a liquid solidifies heat is evolved, the rate of fall in the temperature of a liquid placed in a region at a lower temperature than the melting, point will be decreased, or even become zero, during the time when solidification is taking place. Hence by noting the temperature at which this change in the rate of decrease of temperature takes place, the melting point can be determined.

The substance to be experimented upon is contained in a thin-walled test-tube. This test-tube passes through a cork which is placed in the mouth of a wider test-tube, this outer tube being placed in a beaker of water. In this way the rate of loss of heat is made fairly regular. A thermometer passes through the cork which closes the inner tube.

Sufficient of the solid substance having been placed in the inner tube to well cover the bulb of the thermometer, the solid is melted and its temperature raised about 10 degrees above the melting point by placing the inner tube in hot water or over a flame. During this operation it is advisable to remove the thermometer.

The inner tube having been replaced in the outer, and the whole immersed in water at a temperature considerably below the melting point, the reading of the thermometer is taken every half minute till a temperature below the melting point is reached. The temperatures and times are then plotted on squared paper and a curve is drawn through the points. The temperature or temperatures at which the curve becomes distinctly more nearly parallel to the axis of time correspond to the melting points of the various constituents of the substance.

If the changes in the slope of the cooling curve are very gradual, so that it is difficult to determine the temperature which corresponds to the melting point, it probably means that the outside layers of the liquid have solidified before the temperature of the inside portions has fallen to the melting point. This effect will be found to be particularly well marked if a somewhat wide test-tube is employed with substances, such as paraffin wax, which are very bad conductors of heat, and have only a small latent heat. In such a case a very much sharper inflection in the curve will be obtained if the liquid is kept well stirred during the time it is cooling.

105. Determination of the Ratio of the Specific Heats for Air by Clement and Desormes' Method. - Suppose that the air within a vessel 
is suddenly slightly compressed, so that the pressure rises from the atmospheric pressure $B$ to $p_{1}$. Since the compression which takes place in the air within the vessel is rapid, there will be little transference of heat between the air and the walls of the vessel while the compression is going on, so that the compression is adiabatic. Hence if $v_{0}$ and $v_{1}$ are the initial and final volumes of unit mass of the air within the vessel, and $k$ is the ratio of the specific heats, we have ${ }^{1}$

or

$$
\begin{aligned}
& p_{1} v_{1}^{k}=B v_{0}^{k}, \\
& \left(\frac{v_{1}}{v_{0}}\right)=\frac{B}{p_{1}} .
\end{aligned}
$$

After a time the heat which has been caused by the adiabatic compression of the air will be dissipated by conduction, \&c., to the walls of the vessel, till finally the temperature regains its initial value. Owing to this fall in the temperature of the air within the vessel, the pressure will fall, say, to $p_{2}$. Now, since the total volume of the gas is the same as it was at the end of the adiabatic compression, the volume of unit mass is the same as at the end of the compression, namely, $v_{1}$. Further, since the temperature is now the same as the start, we have, from Boyle's law,

or

$$
p_{2} v_{1}=B v_{0}
$$

$$
\frac{v_{1}}{v_{0}}=\frac{B}{p_{2}} \text {. }
$$

Hence, substituting this value in equation (1) above, we get

$$
\left(\frac{B}{p_{2}}\right)^{k}=\frac{B}{p_{1}}
$$

or, taking logarithms of both sides,

$$
\begin{aligned}
& k\left(\log B-\log p_{2}\right)=\log B-\log p_{1} \\
& k=\frac{\log B-\log p_{1}}{\log \frac{B-\log p_{2}}{B}} \text {. . . . . . }
\end{aligned}
$$

A form of apparatus suitable for applying this method of determining the ratio of the specific heats is shown in Fig. 109.

It consists of a glass flask $\mathrm{A}$, about 30 centimetres in diameter, which is placed inside a wooden box and packed round with cork dust, cotton wool, or other bad conductor of heat. The neck of the flask is closed by a well fitting rubber cork, which is pierced by three holes through which pass three glass tubes. One of these, $\mathrm{k}$, is closed by a tap, another $\mathbf{F}$ is attached to a manometer $G$, while the third $c$ is of at least 1 centimetre in bore, and is connected by a short length of wide bore rubber

${ }^{1}$ See Watson's Physics, § 259. 
tubing with the mercury reservoir $\mathbf{H}$. This rubber tube can be closed by a pinch-cock $I$. Two small shelves $J$ and $K$ are attached to the side of the box, and each has a slot of such a size as to allow the flange on the neck of the reservoir $\mathrm{B}$ to catch.

The quantity of mercury in the apparatus is so adjusted that when the reservoir is on the lower shelf $K$ the surface of the mercury.in the flask covers the cork to the depth of about 1 centimetre. A small quantity of concentrated sulphuric acid is then introduced through the tube $E$ so as to form a layer $L$ on the surface of the mercury. This sulphuric acid serves to keep the air within the flask dry, a matter of great importance. Some oil, such as is used in the Fleuss pump, is introduced into the manometer tube, and the apparatus is then ready for use.

To perform an experiment in which the air is suddenly compressed, place the mercury reservoir on the lower shelf, and open the pinch-cock and

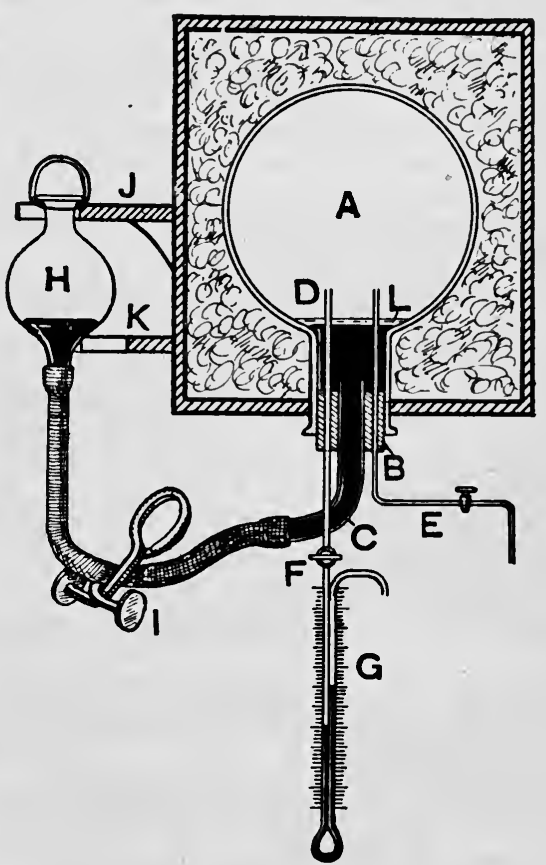

FIG. 109.

the tap E. Then close the pinch-cock, and raise the mercury, reservoir on to the upper shelf. After allowing the apparatus to stand for a few minutes, so that the temperature of the air in the flask may become uniform, close the tap E. Then open the tap $\mathrm{F}$ on the manometer, and fully open the pinch-cock, thus allowing the mercury to flow into the flask and compress the air. Watch the manometer, and as soon as the reading is a maximum close the tap $F$. By this preliminary experiment the liquid in the manometer has been adjusted almost to the position which it will occupy at the end of the compression. Leaving F closed, lower the mercury reservoir and allow the mercury to run out from the flask, close the pinch-cock, and raise the reservoir on to the upper shelf. Open the tap $\mathrm{E}$ and allow the apparatus to stand for a few minutes, then close E. Next open the pinch-cock, and when the mercury has nearly all run into the flask open the tap F, and carefully note the highest reading given by the manometer. If the preliminary adjustment of the manometer has been properly performed there will only be a small movement 
of the column, and the maximum reading will be attained without oscillations being set up.

As the heat developed owing to the compression of the air is dissipated, the pressure will fall and the reading of the manometer when this fall is complete, which will occur after three or four minutes, must be recorded.

If $h_{1}$ and $h_{2}$ are the differences in the level of the oil in the manometer at the two readings, and $d$ is the density of the oil, and $B$ the barometer height, then we have

$$
p_{1}=B+\frac{d h_{1}}{13 \cdot 6} \text { and } p_{2}=B+\frac{d h_{2}}{13 \cdot 6}
$$

and substituting these values in (3) the value of $k$ is obtained.

If the compression is small, so that $\frac{d h_{1}}{13 \cdot 6}$ is small, then the formula can be very much simplified, for

$$
\begin{aligned}
\operatorname{og}{Y_{1}}_{1} & =\log B+\log \left(1+\frac{d h_{1}}{13 \cdot 6 B}\right) \\
& =\log B+\frac{d h_{1}}{13 \cdot 6 B}-\frac{1}{2}\left(\frac{d h_{1}}{13 \cdot 6 B}\right)^{2}+\& c .
\end{aligned}
$$

Thus if we may neglect the square and higher powers of $\frac{d h_{1}}{13 \cdot 6 B}$ we get

$$
\log p_{1}=\log B+\frac{d h_{1}}{13 \cdot 6 B}
$$

Similarly

$$
\log p_{2}=\log B+\frac{d h_{2}}{13 \cdot 6 B}
$$

and hence substituting in (3)

$$
k=\frac{h_{1}}{h_{2}}
$$

an expression which does not involve a knowledge of the density of the oil in the manometer.

Experiments should also be made in which the air in the instrument is first compressed by raising the mercury reservoir to $\mathrm{J}$, then closing the pinch-cock $\mathrm{I}$, and lowering the mercury reservoir to $\mathrm{K}$. On opening the pinch-cock the air expands and becomes cooled. The pressure before expansion, immediately after expansion, and after the air has regained its initial temperature, are determined by means of the manometer in a manner similar to that described above.

106. Measurement of the Heat Conductivity of Copper.-The measurement of the conductivity of a solid is in general rendered difficult owing to the loss of heat from the surface of the rod or bar used, the 
evaluation of this loss being a matter of some difficulty. In the case of a good conductor, such as copper, we may, however, so arrange the experiment that the proportional loss of heat from the surface may be negligible. A piece of apparatus designed with this view is shown in Fig. 110 The copper rod $\mathrm{AB}$ has a total lf:ngth of 15 centimetres and a diameter of 3 centinetres, and has a copper box $\mathrm{C}$ soldered on one end. Steam is passed into this box through the tube $D$, the excess escaping through the tube $\mathrm{E}$. A second copper box $\mathrm{F}$ is soldered to the other end of the rod, and is fitted with an annular partition which compels the water that enters at $G$ and leaves at $I$ to flow in the manner indicated by the arrows. Two thermometers $T_{1}$ and $T_{2}$ serve to indicate the temperature of the incoming and escaping water. Both the boxes

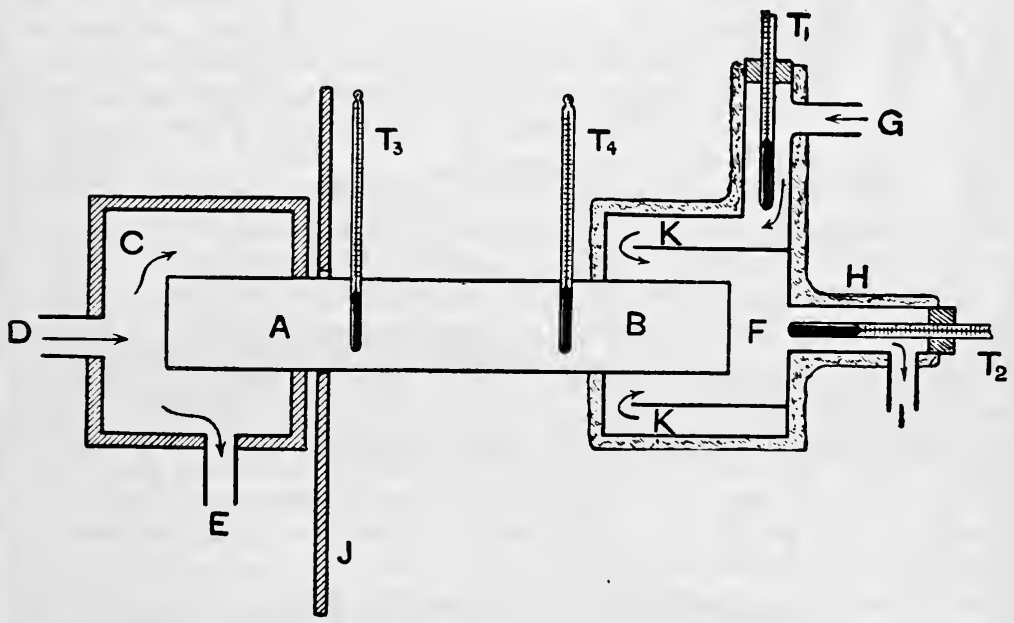

Fig. 110.

are carefully lagged with felt, so as to protect them as much as possible from external loss of heat.

Two narrow holes are drilled almost right through the copper rod at a distance of 8 centimetres apart, and in these holes are inserted the bulbs of two small thermometers $\mathrm{T}_{3}$ and $\mathrm{T}_{4}$. The holes ought to be of such a size that the bulbs of the thermometers are a fairly good fit. The surface of the copper rod between the two boxes may with advantage be lightly packed round with cotton wool.

Water is passed through the instrument by connecting the side tube a to the rubber tube D of a pressure tank A (Fig. 111). 'This tank consists of a small metal vessel having three tubes soldered through the bottom. One of these tubes is connected through the rubber tube B with the water supply. The middle tube extends about two-thirds up 
the vessel and serves as an overflow, while the third tube is connected to the conductivity apparatus. The supply tap is turned on so that a little water always escapes through the tube $c$, and hence

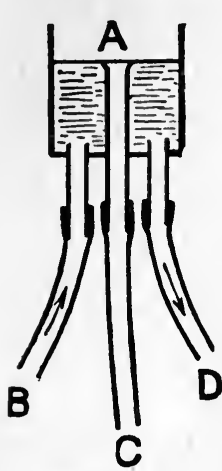

FIG. 111. the head of water always remains the same, and so the rate of flow through the apparatus is constant.

Steam being passed into the box c, adjust the flow of water through the box $F$ by means of a pinch-cock on the supply tube, so that the rise of temperature of the water is about 10 degrees, and read the four thermometers at intervals till the readings become constant. When this is the case, read and record the temperatures indicated by the thermometers, and place, at a noted instant, a weighed beaker below the water outlet I. Continue collecting the water for about five minutes, and at a noted instant remove the beaker and again read the thermometers. By weighing the beaker determine the quantity of water $W$ which has passed in the time $T$.

If the mean temperatures indicated by the thermometers $\mathbf{T}_{1}, \mathbf{T}_{2}, \mathbf{T}_{3}$, and $\mathrm{T}_{4}$ are $t_{1}, t_{2}, t_{3}$, and $t_{4}$, then the heat which has been conducted along the bar to the water is $W\left(t_{2}-t_{1}\right)$. Hence if $l$ is the distance between the thermometers $\mathrm{T}_{2}$ and $\mathrm{T}_{3}$, and $s$ is the cross-section of the copper rod, the conductivity $k$ is given by

$$
k=\frac{W\left(t_{2}-t_{1}\right) l}{s\left(t_{3}-t_{4}\right) T} .
$$

Correction.- Since most of the stem of the thermometers $T_{3}$ and $T_{4}$ are not heated to the temperature of the bulb, the correction for emergent column may be considerable. The magnitude of the correction may be determined experimentally, or the corrections given in the tables in $\$ 65$ may be used.

107. Measurement of the Heat Conductivity of Glass.-In the case of a bad conductor of heat, such as glass, the following method of measuring the conductivity is capable of giving results which are accurate to within about 5 per cent. :-

The glass is taken in the form of a tube $\mathrm{AB}$ (Fig. 112), having a bore of about 1.5 centimetres. The tube is enclosed for the greater part of its length in a larger tube c, which forms a steam jacket, the steam entering at $\mathrm{D}$ and leaving at $\mathbf{E}$. Two T-pieces are connected to the ends of the tube $\mathrm{AB}$, and each encloses a thermometer, $T_{1}$ and $T_{2}$, by means of which the initial and final temperature of a stream of water which traverses the tube $\mathrm{AB}$ can be determined.

The $T$-piece $G$ is connected by the rubber tube $H$ to the constant head reservoir shown in Fig. 111. The tube $A B$ is inclined so that the water flows upwards, and in this way any air evolved from the water owing to the rise in temperature is swept out. 
Since it is important that the temperature of the water at any crosssection of the tube $\mathbf{A B}$ shall be the same, the water is caused to traverse the tube spirally. For this purpose a piece of rubber tube is wound in a very open spiral round a thin brass rod, and the spiral is placed within the tube AB.

When making an experiment steam is passed through the jacket, and the stream of water adjusted so that the temperature of the water rises about $10^{\circ}$. When the thermometer readings have become constant, the water which passes in a noted time is collected in a weighed beaker. If

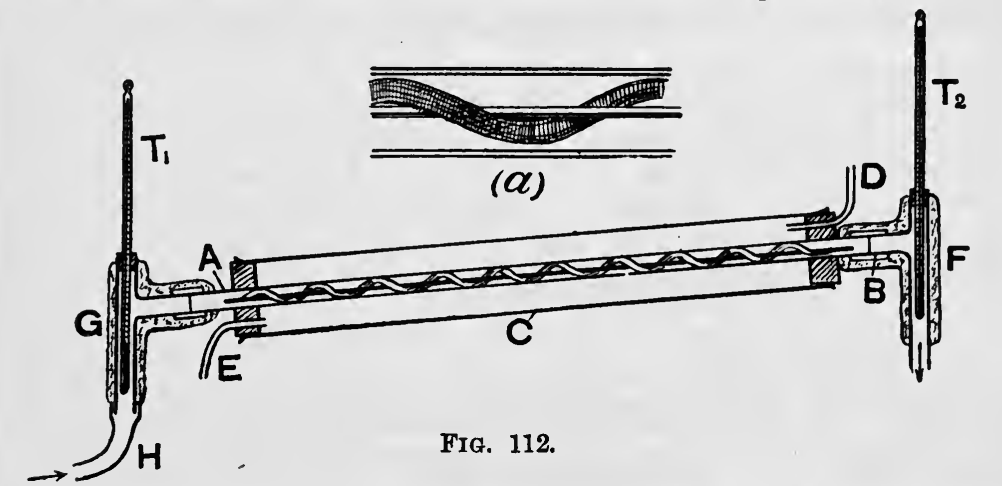

$W$ is the weight of water which passes in a time $T$, and $t_{1}$ and $t_{2}$ are the initial and final temperatures, then the quantity of heat which has passed through the glass is $W\left(t_{2}-t_{1}\right)$.

The temperature $\theta_{2}$ of the external surface of the glass can be obtained from the barometric height and Table 15. The temperature, $\theta_{1}$, of the internal surface may be taken as the mean of $t_{1}$ and $t_{2}$.

If $l$ is the length of the glass tube $\mathrm{AB}$ exposed to the steam, and $r_{1}$ and $r_{2}$ are the radii ${ }^{1}$ of the internal and external surfaces of the tube, then

1 If $r$ and $r+d r$ are the radii of the surfaces of a thin cylindrical shell in the glass, and the difference in temperature between these surfaces is $d \theta$, since the quantity of heat $Q$ must pass in a time $T$ through this cylinder, and also through every similar cylindrical shell into which we may imagine the glass subdivided, we have

or

$$
\begin{aligned}
& Q=\frac{2 \pi r k d \theta \cdot T}{d r}, \\
& d \theta=\frac{Q d r}{2 \pi k T r} .
\end{aligned}
$$

Iutegrating between the inner and outer surfaces of the glass tube

$$
\begin{aligned}
& \int_{0_{1}}^{\theta_{2}} d \theta=\frac{Q}{2 \pi k T} \int_{r_{1}}^{r_{2}} \frac{d r}{r}, \\
& \theta_{2}-\theta_{1}=\frac{Q}{2 \pi k T} \log _{e} \frac{r_{2}}{r_{1}} .
\end{aligned}
$$


the quantity of heat which passes through the glass in a time $T$ is given by

$$
Q=\frac{2 \pi k l T\left(\theta_{2}-\theta_{1}\right)}{\log _{e} \frac{r_{2}}{r_{1}}} \text {. . . . . . }
$$

Since $Q$ is known, this expression enables us to calculate $k$, the conductivity of the glass.

If the glass is thin, so that $r_{2}-r_{1}$ is small, we may expand $\log _{e} \frac{r_{g}}{r_{1}}$ and replace it by the first term, namely

$$
2 \frac{\frac{r_{2}}{r_{1}}-1}{\frac{r_{2}}{r_{1}}+1} \text { or } 2 \frac{r_{2}-r_{1}}{r_{2}+r_{1}}
$$

and hence

$$
Q=\frac{2 \pi \cdot \frac{r_{2}+r_{1}}{2} \cdot l k T\left(\theta_{2}-\theta_{1}\right)}{r_{2}-r_{1}} .
$$

Now $2 \pi \frac{r_{2}-r_{1}}{2} l$ is the mean, $A$, of the areas of the external and internal surfaces of the glass, and $r_{2}-r_{1}$ is the thickness, $d$, of the glass, so that we have

$$
Q=\frac{A k T\left(\theta_{2}-\theta_{1}\right)}{d} \text {. . . . . . . }
$$

The external radius is obtained by measuring the diameter of the glass tube in a number of places with callipers and taking the mean. To obtain the mean internal radius two corks are fitted into the ends of the tube, and the whole is weighed. One cork is then removed, and the tube filled with water. After replacing the cork, the distance between the inver surfaces of the corks is measured, and the tube is again weighed. If $w$ is the increase in weight, that is, the weight of the water column, and $L$ is its length, the mean radius, $r_{1}$, is given by

$$
r_{1}=\sqrt{\frac{w}{\pi L \Delta}}
$$

where $\Delta$ is the density of the water at the temperature of the experiment. 


\section{CHAPTER XVII}

\section{SOUND}

108. Determination of the Pitch of a Tuning Fork by means of a Clock and a Rotating Drum.-One method of measuring the number of vibrations a tuning fork makes in a second is to cause the fork to trace a wavy line on a smoked drum by attaching a fine style to one of the prongs, and at the same time making a trace alongside with a second style attached to a small electro-magnet, the circuit of this electro-magnet being broken once every second by the movement of the pendulum of a clock.

Since the attachment of a style will slightly lower the pitch of the fork, it is important to use as light a style as possible. The most convenient form of style is a fairly fine pig's bristle, attached to the prong with the

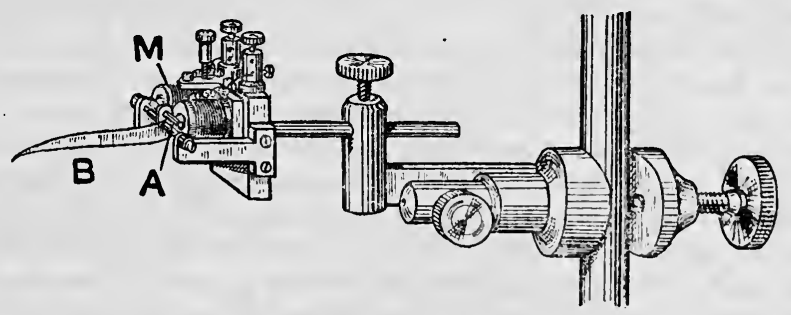

Fra. 113.

minimum quantity of soft wax which will make it stick. The drum must be so mounted that as it rotates it is traversed through a distance of between 1 and $2 \mathrm{~cm}$. for each revolution. If the drum is rotated mechanically, and has a diameter of $20 \mathrm{~cm}$., it ought to make a revolution in two seconds, though, of course, the minimum speed to give a legible trace varies with the pitch of the fork.

The electro-magnetic style (Fig. 113) must be placed so that its tracing point lies alongside the style attached to the fork, so that the two traces are about half a centimetre apart. If the clock is not fitted with an arrangement for making or breaking an electric circuit every second, such an arrangement may be prepared as follows:-A short and fairly stout piece of platinum wire is fixed to the bottom of the pendulum, and, if the pendulum rod is of wood or other non-conductor, a fine wire must be run 
from this platinum point along the pendulum and attached to the clamp which is fixed to the suspension spring. The platinum point makes contact with a thread of mercury, B (Fig.

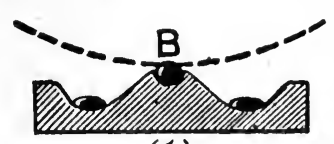

(b)

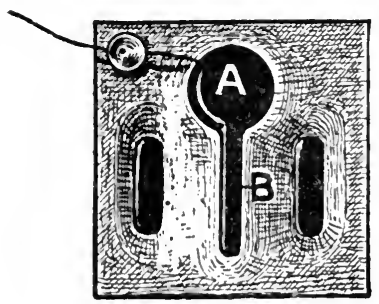

(a)

Fig. 114 114) contained in a narrow channel cut in a wooden block. This block is shown in plan at $(a)$, and in section at $(b)$. The groove widens out into a circular pool $\mathbf{A}$, which serves to contain a reserve of mercury. Owing to capillarity the mercury in the groove stands up above the wood, and when the platinum point sweeps through the mercury the circuit containing the electro-magnetic style and a battery is completed, one terminal being connected to the metal frame of the clock, and the other to the mercury in A. If it is found that the platinum point does not make contact, heat the point to redness and plunge it into mercury, thus amalgamating the point. Then heat the point gently so as to drive off the mercury and replace the wire, being careful not to touch the point with the fingers, and so grease the wire.

The fork must be held firmly in a clamp in such a position that the end of the style just touches the surface of the cylinder. The surface of the cylinder may itself be blacked, or a sheet of glazed paper may first be stretched over the surface, the edge being pasted down. To smoke the surface the cylinder must be slowly revolved over the flame of a paraffin lamp, or over some burning turpentine. A thin but uniform coating of soot is to be aimed at.

Having bowed the fork, so that it is vibrating strongly, start the drum, and continue the motion as long as the fork gives a trace of sufficient amplitude to be read. Then draw a line parallel to the axis of the cylinder through the point where the first time mark occurs, also through the third or fifth, seventh, \&c., according to the length of the trace, and count the number of vibrations which the fork has made between these two marks. The reason for using only every other mark is that the time marks used at the beginning and end should be made by the pendulum when passing through the contact maker in the same direction, for unless the contact is made rigorously at the middle of the swing the intervals between successive passages will not be equal, though the interval between alternate passages will be so. By dividing the number of vibrations by the time the frequency of the fork with the style attached will be obtained, and it will then be necessary to determine the correction which has to be made to allow for the effect of the style. For this purpose obtain a second fork of the same frequency as that being tested, and determine the number of beats made by the two forks in five or six 
seconds. ${ }^{1}$ Also, by slightly loading the auxiliary fork with wax, determine which fork is the sharper. In this way the difference in the pitches of the two forks is obtained. Next remove the style from the fork, and again determine by means of beats the difference in the frequencies. Thus the effect of the style is obtained, and by adding the number of vibrations per second, due to the addition of the style, to that obtained by means of the drum, the frequency of the fork without the style is obtained.

The correction obtained in this manner for the effect of the style does not take account of any influence which may be exerted on the period of the fork owing to friction of the style on the drum. If care is taken that the style is very pliable, and that it only rests very lightly on the surface, such an influence on the period will be very small. This point may be investigated by determining the number of beats made by the fork when the style rests against the surface of the drum, the drum being rotated as in the actual experiment, and comparing this number with the number obtained when the style does not touch the surface of the drum.

Since the frequency of a fork depends on the temperature, a note of the temperature, $t$, must be taken when the determination of frequency is made. The frequency at any other temperature, $t^{\prime}$, can be obtained by means of the following expression, which holds for forks made of ordinary steel :-

$$
n_{t}^{\prime}=n_{t}\left\{1-0.000112\left(t^{\prime}-t\right)\right\} \text {. }
$$

A correction would have also to be applied for the rate of the clock if it were excessive, but such rates as ordinarily occur will hardly affect the results obtained, unless extreme accuracy is aimed at. Thus a rate of five seconds a day will necessitate a correction amounting to one part in 17300.

A modification of this method which does not necessitate the use of an electro-magnetic style, and a clock with a make-and-break device, has been devised by Koenig. A second fork is taken, which is also provided with a style, and by loading its prongs it is made to give about four beats per second with the fork to be measured, and the number of beats (a) given per second are accurately determined. The two forks are then caused to record alongside on the rotating drum, and by counting the waves the ratio $(b)$ of the frequencies of the two forks is determined. If, then, $n_{1}$ is the frequency of one fork, and $n_{2}$ that of the other, we have

Thus

$$
\begin{gathered}
n_{1}-n_{2}=a, \text { and } n_{1} / n_{2}=b . \\
n_{1}=\frac{a b}{b-1} .
\end{gathered}
$$

109. Stroboscopic Method of Measuring the Frequency of a Fork. -Two small and light plates, ${ }^{2} \mathrm{C}$ and $\mathrm{D}$ (Fig. 115), are attached to the

1 See also $\$ 111$.

2 These plates may be made of thin card or aluminium foil. 
ends of the prongs $\mathbf{A}$ and $\mathrm{B}$ of the fork. Each of these plates is pierced by a narrow slit, and the plates are so placed that when the fork is at rest the two slits are opposite each other. Thus, when the fork is

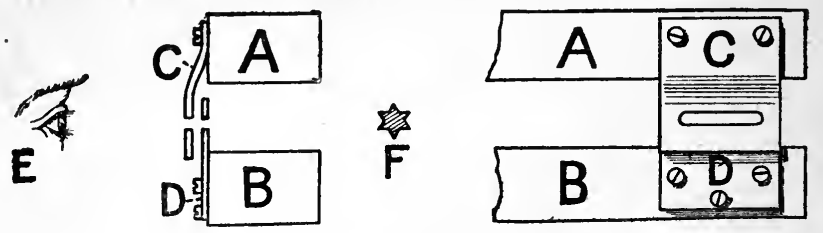

Fig. 115.

sounding, light can only pass through the two slits when the prongs are passing through their position of rest. Hence if $n$ is the frequency of the fork, light will pass through the slits $2 n$ times per second.

$A$ disc on which are painted a number of rows of equidistant dots,

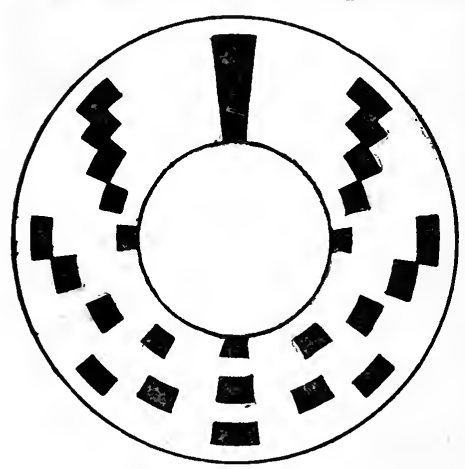

FIG. 116. as shown in Fig. 116, is mounted on the axle of a small electric motor, and a worm-wheel attached to the motor axle works against the edge of a wheel which has a hundred teeth cut in its circumference. A pin attached to this wheel completes an electric circuit containing a battery and an electric bell once during each revolution of the wheel, that is, for every 100 revolutions of the disc.

The disc is placed behind the fork, and the surface is brightly illuminated, and is looked at through the slits attached to the prongs of the fork. An adjustable resistance is placed in the circuit of the motor, so that by altering this resistance the speed of the motor can be varied.

Start the fork and set the disc in rotation, and looking at the disc through the slits, gradually increase the speed of the motor till the dots in one of the rings on the disc appear stationary. By adjusting the resistance, or lightly touching the axle of the motor with the finger, keep the speed constant, so that the dots appear at rest, and with a stop-watch or a chronometer beating half seconds determine the time the disc takes to make 500 or 1000 revolutions, that is, the interval corresponding to five or ten strokes of the bell, and from the result obtain the number of revolutions $N$ the disc makes in one second.

Unless the fork is maintained electrically, it will be necessary to bow it lightly every now and then in order to keep up the vibrations. Koenig has shown that such bowing does not affect the phase of the vibrations. 
If there are $m$ dots in the ring which appeared stationary, then in one second $N m$ dots have passed any given point placed near the edge of the disc. Now the reason the row of dots appears stationary is that each time the disc is seen any given dot has moved on, so that it occupies the place previously occupied by the preceding dot. Thus as a dot has moved on one place each time the disc has been seen, it follows that the disc must have been seen $m N$ times in a second. But if $n$ is the frequency of the fork, the disc is seen $2 n$ times in a second. Hence

$$
n=\frac{m N}{2}
$$

The measurement should be repeated, varying the speed of the motor, so that different rings of dots appear stationary.

It is important to make certain that the dots when they appear stationary are at the same distance apart as when the disc is at rest, for if the disc is going at half the right speed the dots will appear stationary, but there will seem to be twice the right number of dots. This is due to the fact that, owing to persistence of vision, we are unable to detect that we only see a dot in each position every alternate time we we the disc. By placing a piece of card near the edge of the disc, and making two marks on this card at a distance apart equal to the distance between the dots, it will be at once apparent if the disc is turning too slowly. Similarly, if the disc is turning twice or three times too fast the dots will still appear stationary, for now between each view of the disc each dot will have moved up two, three, \&c., places. By watching the disc as the motor gains speed, however, it is easy to make certain of picking out the lowest speed at which the right number of dots is seen.

A correction will have to be applied to allow for the effect of the metal plates attached to the prongs if the fork is to be used without these plates. The method of determining the correction is similar to that described in the last section. The temperature of the fork must also be noted.

A modification of the above method, which does not involve attaching anything to the prongs of the fork, consists in replacing the disc with dots by a disc with a number of equally spaced narrow radial slits near the circumference. This disc is placed near the fork, and the extremity of the prong is examined through the slits in the disc, which is rotated by a motor as in the previous method. When the interval between the passage of successive slits past the eye is equal to the periodic time of the fork, the fork, though sounding, will appear to be at rest. Thus by noting the time taken by the disc to make, say, 500 revolutions, the frequency of the fork can at once be deduced.

110. Measurement of the Pitch of a Fork by means of a String.When a string of length $l$ is stretched by a tension $T$, the mass of unit 
length of the string being $m$, the frequency of the note produced when the string is sounding its fundamental is given by

or

$$
\begin{aligned}
& n=\frac{1}{2 l} \sqrt{\frac{T}{m}}, \\
& n=\frac{1}{2} \sqrt{\frac{T}{l M}},
\end{aligned}
$$

where $M$ is the mass of a length $l$ of the string.

When using a string to measure the pitch of a note, it is better to use a monochord which can be hung against the wall than one which is

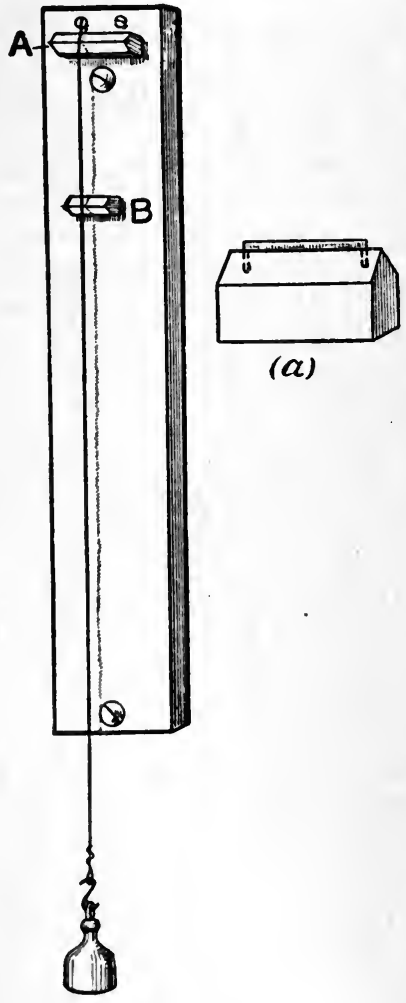

FIG. 117. placed horizontal and in which the string passes over a pulley, since, owing to the friction of the pulley, the tension in the horizontal portion of the string is never the same as that in the vertical portion. A form of monochord which can be easily made in the laboratory is shown in Fig. 117. The bridge $A$ is fixed, and the movable bridge $B$, shown on a larger scale at $(a)$, is about 2 millimetres higher than $A$. The tops of the bridges are formed of a piece of brass wire formed into a staple and driven into the wood. This wire is then filed to a knife-edge.

Take a length of steel piano wire having a diameter of about $0.5 \mathrm{~mm}$., and soften about 2 inches at either end by heating in a flame. Turn the softened end into a loop, and attach one loop to the pin of the monochord, and hang a weight of. about 10 kilos from the other. Adjust the movable bridge till the note given by the wire is in unison with the fork, and measure with a scale the distance between the two bridges. Repeat the observation several times, and then alter the weight, and again repeat. While the weight is still attached to the wire make two light file marks on the wire at a distance apart about equal to that used when in unison with the fork, and then, having measured the distance between the two marks, cut the wire at these points and weigh, thus obtaining the weight of unit length of the wire. The tension 
of the wire is obtained by multiplying the stretching weight in grams by $g(981)$. Inserting the values for $m, l$, and $T$, calculate the frequency of the fork by the formula given above.

When tuning the string to unison with the fork the length of the wire should be varied till beats are heard, and then the position of the movable bridge adjusted till these beats vanish. The position of unison should be approached both by lengthening the wire and by shortening it. When unison is attained, on striking the fork and bringing the end of the stem in contact with the base of the monochord the wire will be set into violent motion, owing to resonance, and if difficulty is found in finding the position for unison by ear, this resonance effect may be used to detect the point at which unison is attained.

111. Comparison of the Frequency of Two Forks.-The method of comparing the frequency of two forks by tracing curves on a smoked drum has already been described on p. 277. This method is applicable, however large the interval between the forks. When the frequencies of the forks do not differ from each other by more than about four vibrations per second, then this difference may most conveniently be deduced from the number of beats.

Take two forks which give about four beats per second, and having fixed them firmly in a stand, bow them, and count the number of beats in twenty or thirty seconds, and thus obtain the number of beats per second, that is, the difference in the frequency of the forks. To ascertain which fork has the lower frequency attach a very small pellet of wax to the prong of one fork, and again determine the number of beats per second. If there are more beats than before the attachment of the wax, it follows that the loaded fork was that of lower pitch, since further lowering its pitch by loading has increased the difference between the pitches of the forks. If loading reduces the number of beats, the loaded fork was the higher.

If the forks to be compared are fitted with mirrors, then Lissajous' figures form a convenient method of comparing the frequency of the forks. This method can be applied when the ratios of the frequencies of the forks do not differ from the ratio of any two simple whole numbers by more than about four vibrations per second.

The forks are supported in clamps, with their mirrors turned towards each other and their planes of vibration at right angles. A small hole is pierced in a piece of card and placed before a source of light, and the image of this hole, seen after reflection in succession in the two mirrors, is examined by means of a low powered telescope. On setting the forks in motion the spot of light seen in the telescope will trace out the figure corresponding to the ratio of the frequencies of the forks, and if the ratio is not exactly correct, the figure will in turn take all the forms corresponding to this ratio, according as the relative phases of the two simple harmonic motions alters. The time taken to go through a complete series of changes is the interval in which the phase of the quicker fork has gained a whole vibration on the lower. Thus suppose that the 
figures traced out are those shown in Fig. 118, that is, that the frequencies of the forks are nearly as 2 to 3 . If, then, the whole series of figures is gone through in $x$ seconds, and if $n_{1}$ is the frequency of the higher fork, and $n_{2}$ that of the lower, so that $n_{1} / n_{2}$ is very nearly $3 / 2$,

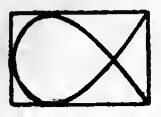

(a)

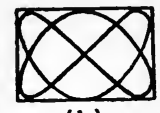

(b)

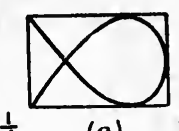

(c)

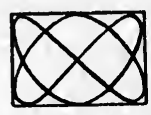

(d)

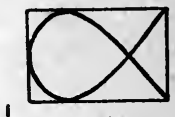

(e)

FIG. 118.

then during the interval $x$ one fork will make $n_{1} x$ vibrations, and the other $n_{2} x$ vibrations. During this interval the higher fork has either made one more vibration or one less vibration than $3 / 2$ times the number made by the slower. Hence we have

$$
\begin{aligned}
& \frac{n_{1} x \pm 1}{n_{2} x}=\frac{3}{2}, \\
& \frac{n_{1} \pm \frac{1}{x}}{n_{2}}=\frac{3}{2} .
\end{aligned}
$$

In order to determine whether to take the plus or minus sign, slightly load the higher fork, and thus lower its frequency. If $x$ increases, this shows that reducing $n_{1}$ brings the forks more nearly to the interval $3 / 2$, and hence we must take the plus sign, and vice versa.

This method is very convenient for adjusting the pitches of two forks to give any desired interval, so long as this interval can be expressed by the ratio of two whole numbers, neither of which differ much from unity.

If a vibration microscope ${ }^{1}$ is available, then this method can be applied to compare the frequency of the fork of the microscope with that of any other fork, and it will not be necessary to attach a mirror or style to the fork, and thus alter its period.

112. Measurement of the Velocity of Sound in Air by Resonance. -If $\lambda$ is the wave-length in air of the sound produced by a fork of frequency $n$, then, on bringing the fork near the mouth of a tube closed at the other end, there will be strong resonance if the length of the tube is equal to $\frac{\lambda}{4} ; \frac{3 \lambda}{4} ; \frac{5 \lambda}{4}$, \&c. ; subject to a small corrction to allow for the fact that in such a tube the first loop is not exactly at the open end of the tube. Since, however, if we use tubes of the same diameter, the correction $x$ for the end will be the same for two tubes having lengths 
of $\lambda / 4-x$ and $3 \lambda / 4-x$ respectively, the difference in the two lengths will be equal to $\lambda / 2$, and the unknown correction for the end will be eliminated. A convenient arrangement for applying this method of measuring the wave-length in air of the note given by a fork is shown in Fig. 119. It consists of an upright metal tube a supported on a firm base, and fitted at its upper end with a glass window B. A narrower brass tube $\mathrm{C}$, which is open at both ends, is supported within the tube $\Delta$ by means of three cords, which, after passing over pulleys $D$, are attached to a lead counterpoise $\mathrm{E}$. The tube $A$ is filled with water up to about the middle of the window, the water serving to close the lower end of the tube $\mathrm{c}$. By holding the sounding-fork over the open end of $\mathrm{c}$ and moving this tube up and down, so as to alter the length of the column of air contained within the tube, the positions at which the resonance is a maximum can be determined. The lengths of the tube corresponding to these positions can be read off on a scale divided in millimetres which is engraved on the outside of the tube $c$, the top end of this tube being at the zero of the scale. By

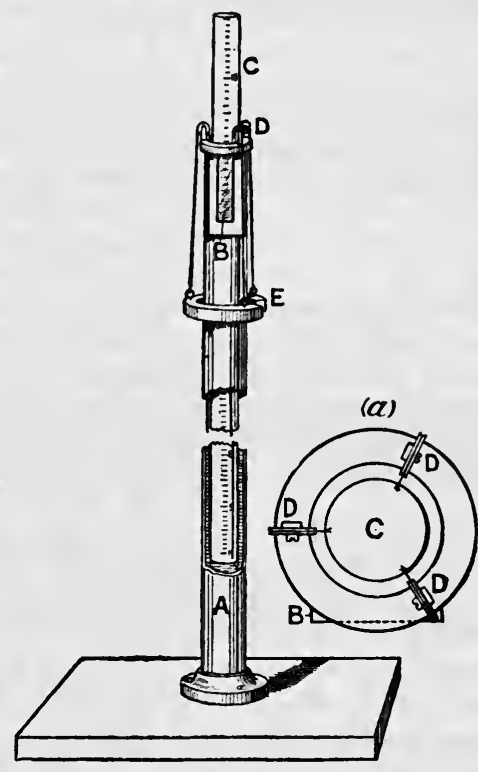

FIG. 119. deducting the reading of the scale corresponding to the shortest column which is in resonance with the fork from the readings corresponding to the other positions of resonance, the lengths of $\lambda / 2, \lambda, 3 \lambda / 2$, \&c., as the case may be, will be obtained, and the mean value of $\lambda$ can be calculated. Knowing the wave-length, the velocity of sound in the air at the temperature of the experiment is given by $v=\lambda N$.

To obtain the velocity in dry air at $0^{\circ} \mathrm{C}$., this result will have to be corrected (1) to allow for the effect of the moisture present on the density, and (2) to reduce to $0^{\circ}$.

To apply the correction for moisture, we require to know the humidity of the air. In the experiment as described above, we may suppose that the air is saturated and at the temperature of the water. ${ }^{1}$ If $\delta_{0}$ is the density of dry air at $760 \mathrm{~mm}$. pressure and at the temperature $t$ of the experiment (Table 4), and $e$ is the vapour pressure of water at the same

1 If the air is not saturated, then the quantity $e$ in equation (1) will be the actual vapour pressure of the moisture present in the air. 
temperature (Table 14), then the density of the moist air at $t$ and $a$ pressure of $760 \mathrm{~mm}$. is given by

$$
\frac{\delta_{t}}{\delta_{0}}=\frac{760-0 \cdot 378 e}{760}
$$

But if $v_{0}$ is the velocity of sound in dry air at $0^{\circ}$, and $v_{t}$ is the velocity in air, of which the density is $\delta_{t}$, we have (see next section)

$$
v_{\mathrm{o}}=v_{t} \sqrt{\frac{\delta_{t}}{\delta}} \cdot \cdot \cdot \cdot \cdot \cdot \cdot \cdot \cdot \cdot \cdot
$$

Hence the observed velocity $v_{t}$ can be reduced to $0^{\circ}$, and dry air by means of equations (1) and (2).

113. Measurement of the Velocity of Sound in a Gas by Kundt's Method.-The apparatus used in this experiment consists of a glass tube CD (Fig. 120), having a diameter of about 4 centimetres and a length of about 2 metres, closed at one end by a tightly fitting cork D. A glass tube

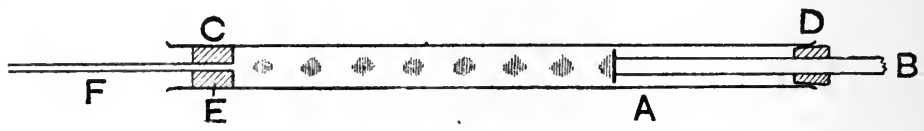

FIG. 120.

about 180 centimetres long passes through this cork, so that it is supported at its mid point. The end $\mathrm{A}$ of this tube is furnished with a small card disc, which has a diameter a few millimetres less than the inside of the tube CD. The end $\mathrm{c}$ of the large tube can be closed by a piston E, consisting of a cork, round the outside of which a strip of chamois leather is glued; this piston can be moved in and out by the $\operatorname{rod} \mathbf{F}$.

The tube CD must be thoroughly dried by heating it over a flame and blowing air through with a bellows, and then a narrow streak of welldried lycopodium powder is poured down the tube. This can be done by inclining the tube and pouring the lycopodium from the blade of a knife, the tube being tapped to cause the powder to run down. The tube is then rested in a horizontal position on two $\mathrm{V}$ blocks, and the rod $\mathrm{AB}$ is fixed in place. Starting with the piston $\mathrm{E}$ at the extremity $\mathrm{C}$ of the tube, the $\operatorname{rod} A B$ is caused to vibrate longitudinally by stroking with a damp cloth, while the piston is gradually moved in till strong vibrations are set up in the air inside the tube, which is manifest by the lycopodium being strongly agitated, and the formation of groups of characteristic ridges. When this resonance takes place, the piston $\mathrm{E}$ will be at a node and the disc $\mathbf{A}$ at a loop of the stationary vibrations set up in the gas in the tube, while the transverse ridges will be at the loops, the distance between consecutive loops being equal to half the wave-length of the note produced by the rod $\mathrm{AB}$ in the gas. Measure the distance between the end 
groups of ridges, and by dividing by the number of intervals get the mean value for half a wave-length, twice this quantity being the wavelength. The temperature of the gas must also be noted, for which purpose a thermometer is placed alongside the tube.

To obtain the velocity, we require to know the frequency of the waves as well as the wave-length. To measure the frequency of the note given by the rod, determine the length of the wire of a monochord in unison with the rod, and also that in unison with a fork of known pitch, and from the relation that the frequencies of two notes given by a stretched string, the tension being constant, are inversely proportional to the lengths, calculate the frequency $n$ of the note given by the rod. Then the velocity $v$ of sound in the gas at the temperature of the experiment is given by

$$
v_{t}=n \lambda
$$

the velocity at $0^{\circ}$ being given by

$$
v_{0}=v_{t}(1-0.00183 t) \text {. }
$$

When measuring the velocity in gases other than air, the gas can be fed into the apparatus through a tube which acts as the handle for moving the piston $\mathrm{E}$, while an exit tube is fitted through the cork which closes the end $\mathbf{D}$ of the outside tube. Unless the gas is quite dry it will be necessary to pass the gas through a series of drying tubes before pasiing into the instrument, as a minute trace of moisture is sufficient to prevent the lycopodium powder moving freely, and thus indicating the position of the loops.

If it is only wished to compare the velocity of sound in two gases, then it will not be necessary to measure the frequency of the rod $A B$, for the velocities will be proportional to the wave-lengths in the two gases.

By surrounding the tube DC with a larger tube, and passing a current of steam through the space between the two tubes, it is possible to determine the ratio of the velocity of sound at $100^{\circ}$ to that at ordinary temperatures, and thus obtain the rate at which the velocity changes with temperature.

The change of velocity with temperature may, however, be at once calculated from the coefficient of expansion of the gas. If $v_{t}$ is the velocity of sound in a gas at a temperature $t$, the density at this temperature being $\rho_{t}$, and $k$ is the ratio of the specific heats, we have

$$
\boldsymbol{v}_{\boldsymbol{t}}=\sqrt{\frac{p k}{\rho_{t}}}
$$

If $v_{0}$ and $\rho_{0}$ are the values of the velocity and density at $0^{\circ}$, then

$$
v_{0}=\sqrt{\frac{p k}{\rho_{0}}} \text {, and } \rho_{t}=\rho(1-a t)
$$


where $a(\cdot 00366)$ is the coefficient of expansion of a gas. Hence

$$
v_{t}=\sqrt{\frac{p}{\rho_{0}(1-a t)}}=\frac{v_{0}}{\sqrt{1-a t}}
$$

or approximately,

$$
v_{t}=v_{0}\left(1+\frac{a}{2} t\right)
$$

114. To Calculate the Ratio of the Specific Heats of Gases from Measurements of the Velocity of Sound.-If $v$ is the velocity of sound in a gas, the density of which at the temperature of observation and at a pressure $p$ is $\rho$, then

$$
v=\sqrt{\frac{k p}{\rho}},
$$

where $k$ is the ratio of the specific heats.

Having measured the velocity of sound in air, carbon-dioxide, hydrogen, and ammonia by the method described in the last section, calculate for each gas the value of $k$.

115. Measurement of the Wave-Length of a High Note by Interference.-The most convenient source of sounds of high frequency is a

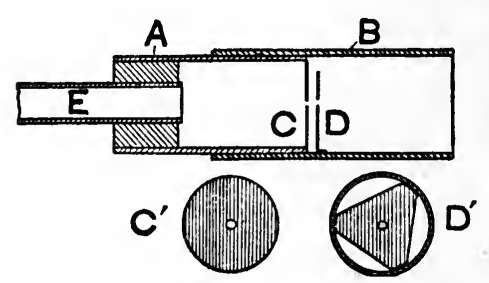

FIG. 121. kind of bird-call. This consists of two brass tubes, $\mathrm{A}$ and $\mathbf{B}$ (Fig. 121), which can slide one over the other. The end of one of these tubes is closed by a flat disc of thin copper foil, at the centre of which a hole having a diameter of about $1.5 \mathrm{~mm}$. is bored. It is important that this hole should be quite central, and, further, that the edge of the hole be sharp and smooth. A triangular piece $D$ of the same copper foil is soldered in the tube $\mathrm{B}$, and has an equal-sized hole bored at the middle, so as to be exactly opposite the hole in the disc $\mathrm{c}$. By sliding the tube $\mathbf{B}$ over the tube $A$ the distance between the copper plates can be varied, and thus to a certain extent the pitch of the note produced altered. Air at a pressure of about 15 centimetres of water is forced into the tube $\mathrm{A}$ through a tube $\mathrm{E}$. The air must be contained in a gas bag, and the desired pressure obtained by weighting the pressure boards. As it is essential, if the pitch of the note is to remain constant, that the pressure should be kept the same, it is desirable to attach a water manometer, consisting of a U-tube, to a $\mathrm{T}$-piece forming part of the air supply tube.

To detect the vibrations of high pitch produced by the bird-call a sensitive flame is used. Such a flame is obtained by forcing coal-gas 
from a gas bag under a pressure of about 25 centimetres of water through a pin-hole burner. This burner consists of a plug of steatite attached to the end of a metal tube, the plug being pierced by a hole a little less than a millimetre in diameter. The gas is supplied from a gas bag, and care must be taken that there are no obstructions in the supply tube near the burner. The quantity of gas must be adjusted by a stop-cock near the bag, and a fair length of rubber tube used to convey the gas to the burner. It is sometimes rather difficult to obtain a thoroughly satisfactory burner, the reason probably being that the holes are not quite smooth and regular. In general, such a burner gives a flame which is much more sensitive to waves coming in one direction than to waves coming in other directions. The sensitive side of the burner ought therefore to be determined, and this side turned towards the direction in which the waves are coming.

Fix a flat surface, such as a sheet of glass or a drawing-board, perpendicular to the table, and in front and at a distance of about 2 metres place the bird-call, the call being about 30 centimetres above the surface of the table. Between the call and the reflecting surface a board is placed on the table. This board has a scale divided into millimetres attached to one edge, and the sensitive burner is attached to a block of wood which rests with one side against the scale. A mark on the base of the burner enables the distance through which the burner is moved to be read off on the scale.

Light the gas and adjust the pressure so that a flame about 40 centimetres high is obtained, but the flame does not flare. Then start the bird-call, so adjusting the air pressure that a clear note, as distinct from a hissing noise, is produced. Starting with the burner as near to the reflecting surface as possible, slowly move it towards the call, noting the readings on the scale at the points where the flame does not flare. The points where the flame does not flare are the nodes; that is, the points of maximum changes in pressure of the stationary waves produced by the direct and reflected systems of wares. Since the distance between consecutive nodes is equal to half the wave-length, the wave-length is at once obtained from the readings on the scale.

In place of the sensitive flame the ear may be used to detect the positions of the nodes and loops of the stationary waves. For this purpose the pin-hole burner is replaced by a piece of glass tube having a bore of about $5 \mathrm{~mm}$. This tube is held on a block of wood similar to that which carried the burner, with its upper end on the level with the call, while the lower end is connected by means of a length of thin indiarubber tubing with a piece of tube which is fitted into the ear. On moving the stand carrying the glass tube backwards and forwards maxima and minima of sound will be heard, and the distances between consecutive maxima or minima will be equal to half the wave-length. In this case the minima of sound occur at the loops, while the maxima occur at the nodes, exactly the opposite to what occurred in the case of the flame. The reason is that the flame is sensitive to changes in motion 
of the air, which is a maximum at the loops, while the maximum effect. as far as producing waves in the tube is concerned occurs when the pressure of the air at the open end of the tube varies most.

Having measured the wave-length of the sound given by the bird-call, then, from the velocity of sound in air at the temperature of the experiment, it is possible to calculate the frequency of the note given by the call. 


\section{CH A P TER X V III}

\section{REFRACTIVE INDEX}

116. Adjustment of a Telescope for Parallel Rays.-An adjustment which has frequently to be made is to focus a telescope so that a pencil of parallel rays falling on the objective is brought to a focus in the plane of the cross-wires. The first thing to be done is to focus the eye-piece on the cross-wires. The best way of doing this is to turn the telescope towards some fairly brightly lighted object, such as a white wall, which is at a distance of at least 2 metres. Then adjust the object glass so that the wall is not at all in focus, and so that on looking into the telescope a bright field of view is seen, but no image. If, now, one eye is used to look into the eye-piece, and the other is kept open and turned towards the wall, the appearance presented will be that the crosswires of the telescope seem to cross the wall. The position of the eyepiece is then adjusted till the cross-wires appear quite distinct as seen with one eye, while with the other eye the wall is also seen distinctly.

In this way we ensure that whenever the cross-wires are seen distinctly the eye is adjusted for seeing a distant object, which for most persons is an unstrained condition. In the case of a short-sighted person the wall may be replaced by the open page of a book, supported at such a distance from the eye-piece that with the unaided eye the print can be seen distinctly, and then proceeding to adjust the eye-piece so that the print can be seen distinctly with the unaided eye at the same time as the cross-wires with the other eye.

If the telescope is to be continually used by the same observer it will be well, having once fixed the correct position of the eye-piece, to make a light scratch corresponding to the end of the tube in which the eyepiece fits, so that the eye-piece can always be placed in the correct position without having to go through the above described procedure.

The eye-piece having been focused, the telescope must be supported in a clamp, so that it may be focused on some distant object. The object chosen ought to have a clearly defined outline, and a lightning arrestor or a telegraph wire will be found suitable. If the telescope is placed inside a room the window must be opened, since window glass is so irregular that it will be found impossible to obtain a sharp focus if the rays have passed through the glass. The telescope must then be adjusted till the image formed by the object glass is in the plane of the cross-wires. This will be secured when, on moving the eye from side to 
side as far as the occular of the telescope will allow, the cross-wires do not seem to move relatively to the image.

Another method of focusing a telescope for parallel rays is described on p. 291.

117. Measurement of the Angle of a Prism with the Spectrometer. -When making a measurement of the angle of a prism with the spectrometer, the following adjustments have to be made :-

1. The optical axes of the telescope and collimator have to be made perpendicular to the axis about which the telescope and table turn.

2. The telescope focused for parallel rays.

3. The collimator focused for parallel rays.

4. The prism adjusted so that the faces which include the angle which is to be measured are both parallel to the axis about which the telescope and table turn.

1. If the instrument is provided with a Gauss eye-piece, that is, with an eye-piece with a side opening between the eye-piece lenses and a plate

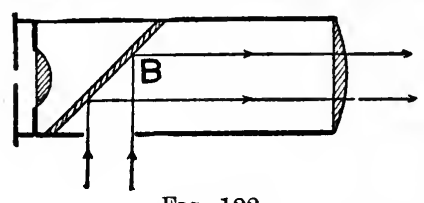

FIG. 122. of unsilvered glass B (Fig. 122) inclined at $45^{\circ}$ to the axis, the following method can be employed:-When a light is placed opposite the opening the rays are reflected by the glass along the axis of the telescope, and if, after they leave the object glass, they are reflected back by a plane mirror, an image of the cross-wires will be seen alongside the cross-wires themselves. If the telescope is focused so that the image is in the same plane as the cross-wires, as shown by the absence of parallax, then it must be in adjustment for parallel light. A piece of plate glass is mounted on the table with its sides vertical, the telescope is focused and the glass adjusted so that the image of the cross-wires formed by reflection at the surface of the glass coincides with the crosswires themselves. The telescope is then turned through $180^{\circ}$, so that the light is now reflected back into the telescope from the other surface of the glass. If the image of the cross-wires now coincides with the wires, the axis of the telescope is perpendicular to the axis about which the telescope turns. If the image does not coincide with the wires, it must be brought half-way back by adjusting the telescope, ${ }^{1}$ and halfway by adjusting the table on which the plate glass is supported. This adjustment must, if necessary, be repeated till the image in both positions coincides with the wires. The adjustment will not be absolutely correc 1 unless the two sides of the plate glass are exactly parallel. In general however, the angle included between the two sides is so small as tc produce no material error.

If the telescope is not fitted with a Gauss eye-piece, we may assum.

1 This adjustment is made by tilting the telescope about a horizontal axis witj reference to the movable arm which carries the telescope. The arrangements fo making this adjustment vary in different instruments, but an examination will shor the manner in which the adjustment is to be made in the case of any particula instrument. 
that the optic axis of the telescope is parallel to the telescope tube, and proceed as follows:-A striding level, that is, a level provided with a $\mathbf{V}$ at either end, which V's can rest on the upper surface of the telescope tube, is placed on the telescope. The telescope is then turned so that it lies parallel to the line joining two of the levelling screws of the instrument, and by adjusting these screws the bubble is brought to the centre. The telescope is then turned through $180^{\circ}$, and if the bubble is no longer at the centre, it is brought half-way back by adjusting the levelling screws, and the other half by adjusting the telescope in its support. It will generally be found necessary to repeat the adjustment two or three times before the bubble remains exactly at the centre as the telescope is turned.

The principle on which this method of adjusting is made is the same as that described on p. 56 with reference to the cathetometer.

To adjust the collimator, a thin wire must be stretched across the middle of the slit, and the screws which attach the collimator altered till the image of this cross-wire, as seen in the telescope, coincides with the intersection of the cross-wires in the telescope.

2. The telescope may be focused for parallel rays in the manner described in $\S 116$ or $\S 117$ (1), but if no Gauss eye-piece is fitted, a convenient and accurate way in the case of a spectrometer is as follows:Place a prism on the table, and turn the telescope so as to see the refracted image of the slit, which must be illuminated by monochromatic light. This light is most conveniently produced by holding in the flame of a Bunsen burner a piece of asbestos soaked in a strong solution of sodium chloride. The prism is then rotated till it is in the position of minimum deviation. The telescope having then been turned to a position corresponding to a greater deviation than the minimum, it will be found that there are two positions of the prism for which a refracted image of the slit will be seen in the telescope. In one of these positions $(a)$ the angle of incidence of the light on the first face will be greater than for minimum deviation, and in the other position $(b)$ less. Turn the prism into position $(a)$, and focus the telescope till the imagc of the slit appears sharp. Then turn the prism into the position $(b)$, and focus the collimator till the image is sharp. Turn back into position $(a)$ and again focus the telescope, and so on. After one or two trials the image will remain in good focus for both positions of the prism. When this is the case, both the collimator and the telescope are focused for parallel rays. In practice it is quite unnecessary to try and remember the order in which the telescope and collimator are to be focused, since after one or two alternations, i.e. focusing the telescope in one position of the prism and the collimator in the other position of the prism, the adjustment will either get very much better, indicating that the right order has been adopted, or the adjustment will get worse, when the order must be reversed.

4. The prism must now be mounted on the table with its centre o (Fig. 123) over the centre of the table, and with one of the faces which includes the angle to be measured, A, perpendicular to a line joining two of the table levelling screws. To assist in placing the prism in this 
position, it is an advantage to have a series of lines scribed on the table top, as indicated in the figure.

The telescope having been turned so that its axis makes an angle of about $90^{\circ}$ with the axis of the collimator, the prism is turned till an

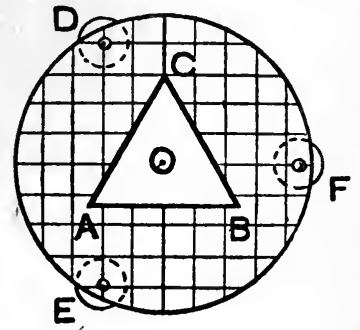

FIG. 123. image of the slit is seen produced by light reflected from the face $\mathbf{A B}$, i.e. the face which is perpendicular to the line joining two of the levelling screws, and the levelling screws adjusted so that the image of the wire stretched across the slit coincides with the intersection of the cross-wires. The prism is then turned so that the light reflected from the face $\Delta \mathrm{c}$ enters the telescope, and the image of the wire is made to coincide with the intersection of the cross-wires by turning the levelling screw $F$. Since altering the screw $F$ will move the face $A B$ in its own plane, by proceeding in this way the adjustment of this face will not be thrown out when adjusting the face $\mathbf{A C}$, as it would if the prism had not been placed in this particular position on the table. The adjustment of the prism is now complete, and we may proceed to the measurement of the angle.

There are two methods which may be used. If, however, the table is not provided with verniers; by means of which its rotation can be measured with as great an accuracy as that with which the rotation of the telescope can be read, then only the second method is available.

Method 1.-Keeping the telescope in the position in which it was placed when adjusting the prism, and the centre of the prism being over the centre of the table, turn the table till the image reflected from the face $A B$ coincides with the intersection of the cross-wires, and read the verniers attached to the table. Then turn the table till the reflection from the face AC coincides with the intersection of the cross-wires, and again read the vernier. The difference between the two readings subtracted from $180^{\circ}$ will give the angle $\mathrm{CAB}$.

Method 2.-Place the prism on the table so that the angle at $\mathbf{A}$ is turned towards the collimator and vertically over the axis of the instrument, and obtain the readings on the verniers attached to the telescope when the intersection of the cross-wires coincides with the image of the slit when seen reflected first from the side $A B$, and then from the side $A C$, the prism remaining fixed. The difference between these readings will give twice the angle CAB.

If the instrument is so constructed that the collimator can be moved to different parts of the divided circle, the measurements ought to be repeated with the collimator making angles of $90^{\circ}, 180^{\circ}$, and $270^{\circ}$ with its original position, in order to eliminate errors of graduation of the circle as much as possible.

If the difference between the angle, as measured by these two methods, 
is greater than would be expected from the accuracy with which the circle can be read, it most probably is due to the fact that the collimator has not been correctly adjusted for parallel rays, and this adjustment must be repeated.

A study of the effect of want of adjustment of the collimator on the observed angles by the two methods is instructive. Consider, in the first place, the first method, in which the prism is turned and the telescope is kept fixed. Let $\mathrm{ABC}$ (Fig. 124) be the position of the prism when the image of the slit reflected in the face $A B$ is on the cross-wires of the telescope, and let $o$ be the centre of the divided circle, that is, the centre about which the prism is turned. If the collimator is not correctly adjusted, then the image of the slit it produces will not be at infinity; thus, suppose that this image is virtual and situated at $\mathrm{s}$, then the image formed by reflection in the face $A B$ will be situated at $s_{1}$, and the axis of the telescope will be directed along the straight line $\operatorname{Tos}_{1}$.

Next suppose the prism rotated into the position $\mathbf{A}^{\prime} \mathbf{B}^{\prime} \mathbf{C}^{\prime}$, so that the image of the slit reflected in the side $\mathrm{BC}$ is seen on the cross-wire of the telescope, i.e. this image is situated on the axis of the

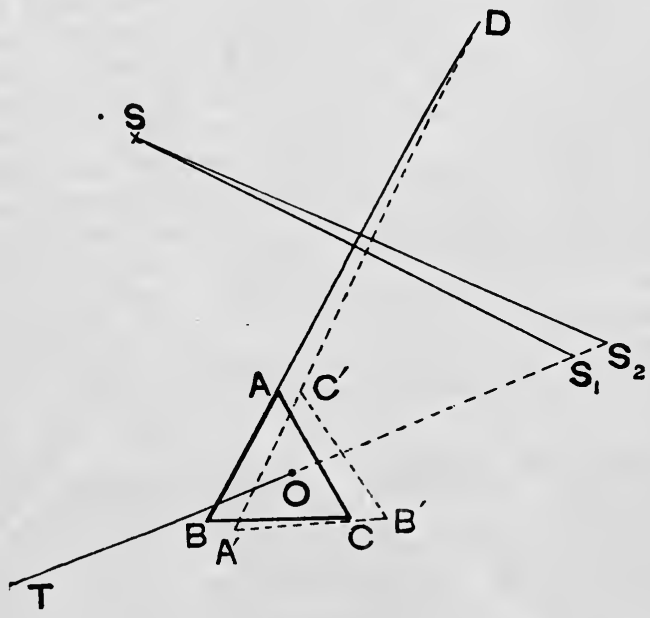

FTG. 124. telescope, and therefore somewhere on the line $\operatorname{Tos}_{1}$, say, at $s_{2}$. Now, unless the centre 0 , about which the prism turns, is at the centre of the triangle $A B C$, when the prism has been turned so that $A^{\prime} C^{\prime}$ is parallel to $B A$, the perpendicular distance of $\mathrm{s}$ from $\mathrm{A}^{\prime} \mathrm{C}^{\prime} \mathrm{D}$ will be different from the perpendicular distance of $\mathrm{s}$ from the line $\mathrm{BAD}$, and hence the images $\mathrm{s}_{1}$ and $\mathrm{s}_{2}$ will not coincide, but in the case shown in the figure we must turn the prism through a further angle $\mathrm{BDA}^{\prime}$ or $\mathrm{s}_{1} \mathrm{Ss}_{2}$ before the image $\mathrm{s}_{2}$ comes on the line $\operatorname{Tos}_{1}$. In other words, the angle through which the prism has been turned is $180^{\circ}+\angle \mathrm{BDA}^{\prime}-<\mathrm{BAC}$. Hence to obtain a correct result the angle $\mathrm{BDA}^{\prime}$ or $\mathrm{S}_{1} \mathrm{SS}_{2}$ must vanish. This occurs (1) when $s$ is at infinity, or (2) when o coincides with the centre of the triangle $\triangle B C$. Hence when using this method adjust the collimator as well as possible, and then to reduce the effect of any outstanding 
error set the prism so that its centre is over the axis about which the table turns.

Next consider the second method, in which the prism is kept fixed and the telescope is rotated. Thus let ABC (Fig. 125) represent a section of the prism, the centre of the divided circle being at 0 , and the image of the slit formed by the collimator in place of being at infinity being at $\mathrm{s}$. Then after reflection in the face $\mathbf{A B}$ the light proceeds as if it came from

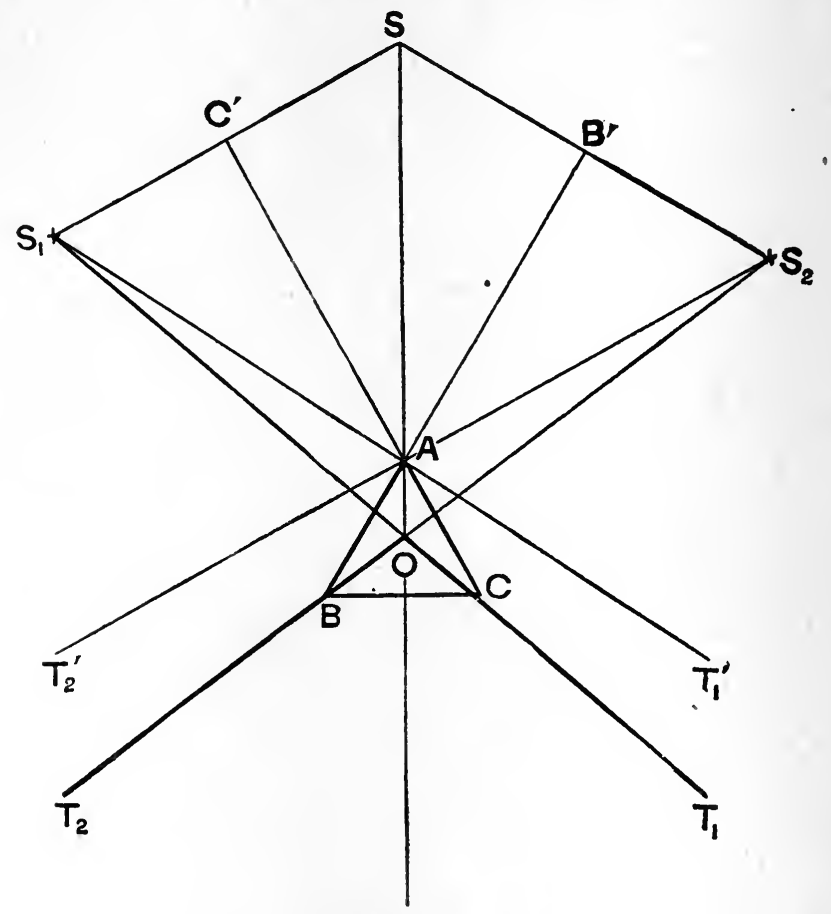

Frg. 125

$\mathbf{s}_{2}$, which is the image of $\mathrm{s}$ formed by reflection in the face AB. Similarly, $\mathrm{s}_{1}$ is the image of $\mathrm{s}$ formed by reflection in the face $\mathrm{AC}$. Then it is at once evident from the similar triangles $\mathrm{SAC}^{\prime}, \mathrm{S}_{1} \mathrm{AC}^{\prime}$, and $\mathrm{SAB}^{\prime}, \mathrm{S}_{2} \mathrm{AB}^{\prime}$ that the angle $\mathrm{S}_{1} \mathrm{AS}_{2}$ is twice the angle $\mathrm{BAC}$, that is, the angle $\mathrm{T}_{1}{ }^{\prime} \mathrm{AT}_{2}{ }^{\prime}$ is twice the angle $\mathrm{BAC}$.

Since, however, the telescope turns about the point o as centre, the angle measured is the angle $\mathrm{T}_{1} \mathrm{OT}_{2}$ or $\mathrm{S}_{1} \mathrm{OS}_{2}$, and this angle differs from twice the angle $\mathrm{BAC}$ by the sum of the angles $\mathrm{OS}_{1} \mathrm{~A}, \mathrm{OS}_{2} \mathrm{~A}$. Hence the condition that the angle measured by the rotation of the telescope shall be twice the angle of the prism is, that the angles ${ }^{\circ} s_{1} A$ and ${ }_{0} s_{2} A$ shall vanish. This condition is fulfilled if either 0 coincides with $\Delta$ or if $s_{1}$ and 
$s_{2}$ are at an infinite distance from $o$, that is, if $s$ is at infinity, and there fore the collimator is correctly adjusted. Since it is impossible to be absolutely certain that the adjustment of the collimator is quite accurate, the effect of this want of adjustment should be rendered as small as possible by placing the angle $\mathrm{A}$ vertically over the centre of the divided circle.

An objection to the second method is that at most half the objective of the telescope is used. for either reading, and it is not the same half which is used in the two cases. Thus errors may be caused by spherical aberration in the lenses of the telescope. In a well constructed telescope, however, the error from this cause will not be important.

Both the telescope and the table ought to be provided with two verniers at $180^{\circ}$ apart, for unless the centre of the divided circle exactly coincides with the axis of rotation of the telescope or table, then the angle read off by means of a single vernier may differ from the true angle. The mean, however, of the angles of rotation as read off on the two verniers is free from error on account of excentricity of the circle.

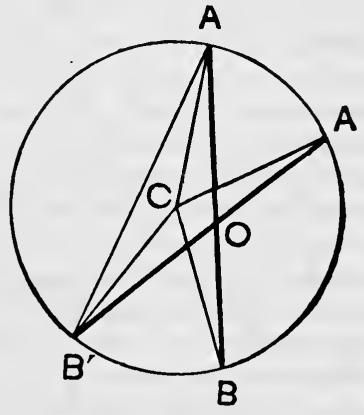

FIG. 126. For suppose c (Fig. 126) is the centre of the divided circle, and o is the centre about which the telescope rotates. Then if $\mathbf{A B}$ and $\mathbf{A}^{\prime} \mathbf{B}^{\prime}$ are two positions of the verniers, the angle through which the telescope has actually been rotated is $\mathrm{AOA}^{\prime}$ or $\mathrm{BOB}^{\prime}$, while the angles read off on the verniers are $\mathbf{A C A}^{\prime}$ and $\mathbf{B C B}^{\prime}$. But since the angle subtended by the arc $\mathbf{A A}^{\prime}$ at the centre $\mathbf{C}$ is twice the angle it subtends at the circumference of the circle, we have

also

$$
A C A^{\prime}=2 A B^{\prime} A^{\prime}=2\left\{A O A^{\prime}-B^{\prime} A B\right\},
$$

Hence

$$
B C B^{\prime}=2 B^{\prime} A B \text {. }
$$

or

$$
\begin{aligned}
& A C A^{\prime}=2 A O A^{\prime}-B C B^{\prime}, \\
& \frac{A C A^{\prime}+B C B^{\prime}}{2}=A O A^{\prime},
\end{aligned}
$$

that is, the mean of the angles read off on the verniers is equal to the true angle.

118. Refractive Index of a Solid or a Liquid in the form of a Prism.-If the angle of the prism is $\theta$, and the angle of minimum deviation $\delta$, then the refractive index $\mu$ is given by ${ }^{1}$

$$
\mu=\frac{\sin \frac{1}{2}(\theta+\delta)}{\sin \frac{1}{2} \theta} .
$$

Hence to determine the refractive index of the material of which a prism 1 Watson's Physics, § 349. 
is composed, we have to measure the refracting angle by one of the methods given in the preceding section, and then determine the angle of minimum deviation for light of some definite wave-length.

To determine the angle of minimum deviation the prism is placed on the table of the spectrometer with its centre over the centre of the table, and the faces which include the refracting angle which is to be used are adjusted parallel to the vertical axis of the instrument in the manner described on p. 292. Then turn the refracting edge of the prism to the right and adjust the telescope so that the refracted image is seen, and turning the prism in such a way that the deviation decreases, follow the movement of the refracted image with the telescope till the image commences to turn back, and by trial adjust the telescope so that when the prism is turned the image just reaches the intersection of the crosswires and then moves back. Read the verniers attached to the telescope, and turning the prism so that the refracting edge is turned towards the left, repeat the adjustment for minimum deviation. The difference between the readings for the telescope verniers in the two positions will be twice the angle of minimum deviation.

Since the refractive index changes with temperature, a note of the temperature must be made. For this purpose a thermometer can be suspended with its bulb near the prism, care being taken when adjusting the prism for minimum deviation not to handle the prism itself, but to turn it by means of the table.

If the prism is in the form of an equilateral triangle, so that all the angles are very nearly equal to $60^{\circ}$, it will be unnecessary to measure the angles if measurements of minimum deviation are made with all the angles in turn, and the mean is taken as the deviation corresponding to a refracting angle of $60^{\circ} .1$

In the case of a liquid, a hollow glass prism has to be employed to contain the liquid. The sides of the prism are formed by two plates of glass with optically worked surfaces, so as to be plane and parallel. By taking a little trouble it is possible to select pieces of plate glass which shall be as good as any but the very best, and therefore very expensive, optically worked glass. The manner in which a suitable piece can be selected is described in $\S 142$. These glass sides are either cemented ${ }^{2}$ to the body of the prism-which is the most convenient arrangement when a series of liquids of the same nature have to be examined, so that the same cement is unattacked by all the liquids-or the plates are simply held in place by india-rubber bands, the plates having been made such a good fit that the liquid will not escape. In this case care must be taken that the prism is not left on the spectrometer table longer than is necessary to take a reading. To protect the table in case some of the liquid does escape, a shallow glass dish may be cemented to the top of the table, and the prism placed in this dish. Since the refractive index

1 See Proceedings of the Royal Society, vol. 1xx. p. 330.

2 For water and solutions in water, marine glue can be used as a cement. For liquids such as alcohol and carbon bisulphide glycerine glue is suitable. 
of a liquid changes rapidly with temperature, a small thermometer must pass through the cork which closes the opening at the top of the prism, the bulb being immersed in the liquid. This thermometer must be read immediately after each setting for minimum deviation.

When measuring the angle of the hollow prism, unless the faces of the plates of glass which form the sides are exactly parallel, two images of the slit will be seen in each position, one formed by reflection at the outside surface of the glass plate, and the other at the inside surface. To ascertain which image is formed by the inside surface, fill the prism with liquid, when the brightness of one of the reflected images will be decreased. The image in which this decrease in brightness takes place is the image formed by light reflected at the inside surface of the face of the prism. When measuring the angle of the prism, the images formed by reflection from the outside surfaces must be used. To allow for the effect of the prismatic form of the faces, two sets of observations of angle and of minimum deviation must be made. In the second set the prism faces must be rotated in their own plane through an angle of $180^{\circ}$, that is, the edge of the glass plate which was nearest the refracting angle in the first observation must be turned so as to be nearest the base in the second observation. The mean of the values obtained for the refracting angle and the angle of minimum deviation must be employed when calculating the refractive index.

119. Measurement of the Refractive Index of a Liquid by Total Reflection, using an Air Film. ${ }^{1}$ - If two parallel sided plates of glass

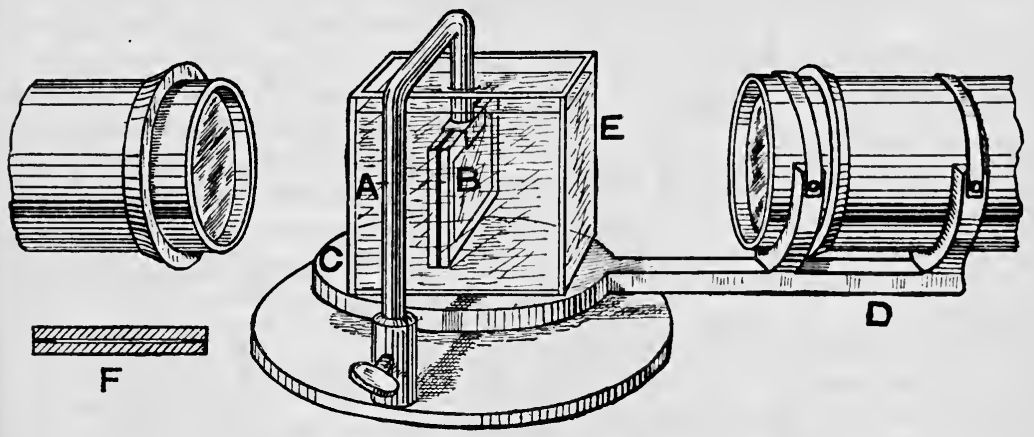

FIG. 127.

separated by an air film are immersed in a liquid contained in a glass sided cell, which is placed between a collimator and a telescope, and the two positions of the glass plates are determined for which the light is just

1 Watson's Physics, § 360. 
cut off owing to total internal reflection at the air film, the refractive index from air to the liquid is given by

$$
\mu=\frac{1}{\sin a^{\prime}}
$$

where $2 \alpha$ is the angle through which the glass plates have to be turned to pass from one position of extinction to the other.

An arrangement by means of which an ordinary spectrometer can be used to measure the refractive index of a liquid by total reflection is shown in Fig. 127. It consists of an upright A carried by the prism table, which is bent twice at right angles, and supports the two glass plates with the enclosed film of air. A small metal plate $\mathbf{c}$ is carried on a bracket $\mathrm{D}$ attached to the telescope or collimator, and on this plate is placed a box with plate glass sides, $\mathrm{k}$, in which the liquid is contained.

When performing an experiment the liquid is placed in the glass trough, and the double plate $\mathbf{B}$ is introduced and set approximately perpendicular to the incident light. The telescope is then turned so that the image of the slit is seen to coincide with the intersection of the crosswires. The prism table, carrying with it the double plate, is then turned till the image of the slit is extinguished, and the readings of the verniers taken. The prism table is then turned in the opposite direction till

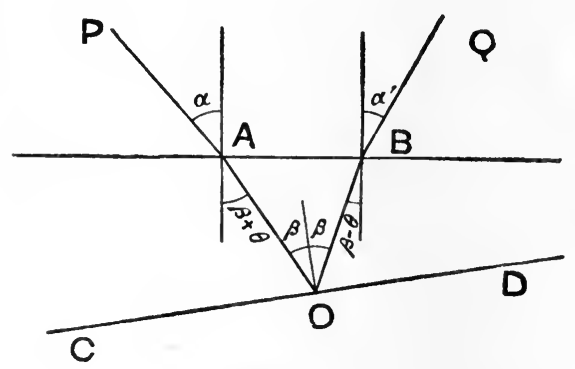

FIG. 128. the image again vanishes, and the verniers are read. The difference between the readings gives twice the critical angle from air to the liquid. A thermometer ought to be placed in the liquid, and the temperature read both before and after the measurements.

This method requires a considerable volume of the liquid, and hence in many cases, where only a small quantity of liquid is available, it is inapplicable. The methods described in the following sections, since only a few drops are required, may be used in all cases.

Corrections. - The formula given above assumes that the sides of the plate of glass which encloses the air film on the side next the collimator are parallel. If they are not parallel, then a correction must be applied. Suppose the two sides AB, CD (Fig. 128) make an angle $\theta$, and that PA and QB are the directions of the incident rays when the refracted rays in the glass are incident on the surface $\mathrm{CD}$ at the critical angle $\beta$. If, then, 
$\boldsymbol{\mu}$ is the refractive index from air to the liquid in which the rays PA and QB are incident we have, as in $\S 360$ of Watson's Physics,

or

$$
\begin{aligned}
& \mu=\frac{\sin (\beta+\theta)}{\sin \alpha \sin \beta}=\frac{\sin (\beta-\theta)}{\sin \alpha^{\prime} \sin \beta}, \\
& \mu=\frac{\sin \beta \cos \theta+\cos \beta \sin \theta}{\sin \alpha \sin \beta}=\frac{\sin \beta \cos \theta-\cos \beta \sin \theta}{\sin \alpha^{\prime} \sin \beta} .
\end{aligned}
$$

But since $\theta$ is small, we may put $\sin \theta=\theta$, and $\cos \theta=1$.

Hence

$$
\mu=\frac{\sin \beta+\theta \cos \beta}{\sin \alpha \sin \beta}=\frac{\sin \beta-\theta \cos \beta}{\sin \alpha^{\prime} \sin \beta},
$$

or

$$
\mu \sin \alpha=1+\theta \cot \beta \text {, }
$$

and

$$
\mu \sin \alpha^{\prime}=1-\theta \cot \beta \text {. }
$$

Hence adding

$$
\mu\left(\sin \alpha+\sin \alpha^{\prime}\right)=2
$$

or

$$
\begin{aligned}
\mu & =\frac{2}{\sin a+\sin a^{\prime}} \\
& =\frac{1}{\sin \frac{1}{2}\left(\alpha+\alpha^{\prime}\right) \cos \frac{1}{2}\left(\alpha-\alpha^{\prime}\right)} .
\end{aligned}
$$

Hence the correction to allow for the effect of the glass plate is to multiply by the factor $1 / \cos \frac{1}{2}\left(a-a^{\prime}\right)$. To obtain the magnitude of this correction it is necessary to measure the difference of the angles $a$ and $a^{\prime}$.

120.-The Measurement of Refractive Index by Total Reflection, using a Prism (First Method).-A very convenient and accurate method of measuring the refractive index of a liquid or solid which is available if we possess a prism having a higher refractive index than that to be measured is as follows :- Suppose that one side BC (Fig. 129) of a prism is unpolished, and that this side is illuminated by monochromatic light. We may then consider each point on the side BC as being selfluminous, sending rays into the prism in all directions. Let $P$ be such a point, and $\mathbf{P Q}$ be the ray that strikes the surface $\mathbf{A B}$ at an angle of incidence just greater than the critical angle. This ray will be reflected along QR, and after refraction at the surface AC, will travel along RS, and will be very little diminished in intensity during the process. Similarly, a ray such as $\mathbf{P Q}^{\prime}$ will reach $\mathbf{s}^{\prime}$ with little loss. It will, however, be quite otherwise with a ray such as $\mathbf{P Q}^{\prime \prime}$ which strikes the face $\mathbf{A B}$ at an angle of incidence less than the critical angle, for part of the light will be refracted along $Q^{\prime \prime} T$, and only part will be reflected along $Q^{\prime \prime} R^{\prime \prime}$, and so the ray $\mathbf{R}^{\prime \prime} \mathbf{s}^{\prime \prime}$ 
will be much more feeble than the rays $R S$ or $R^{\prime} \mathrm{s}^{\prime}$. If, then, a telescope is placed so as to look into the face $\mathbf{A C}$, with its axis parallel to $\mathrm{SR}$, the field of view will be seen divided into two parts, one half being much brighter than the other, the line of demarcation corresponding to the direction SR of the ray for $\mathbf{P}$, which has fallen on $\mathbf{B A}$ at the critical angle.

An exactly similar argument would apply to the rays sent out from any other point on the face $\mathbf{B C}$, and the direction of the rays which leave

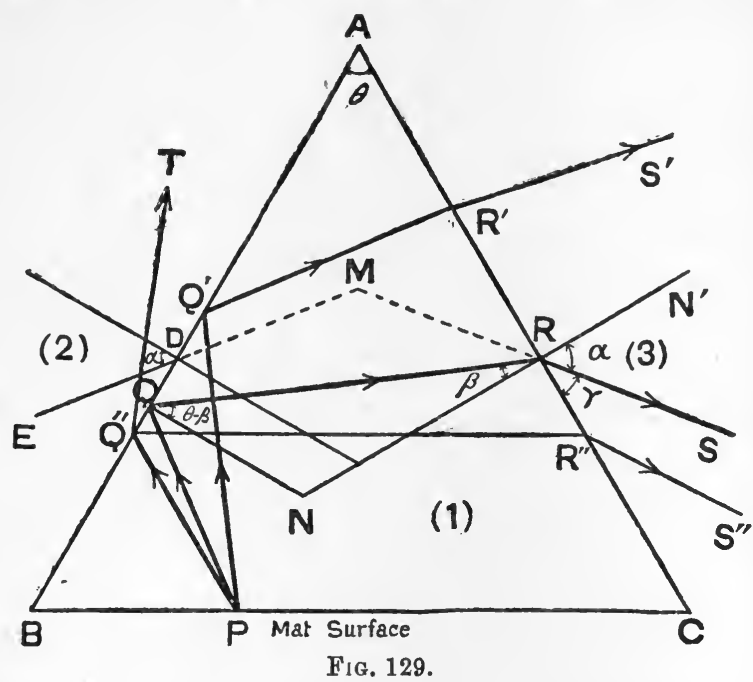

the face $A C$ and limit the brighter part of the field would all be parallel to RS. Hence if the telescope is focused for parallel rays, all these rays would be focused along a line parallel to the edge $\boldsymbol{\Lambda}$ of the prism.

Let $a$ be, the angle RS makes with the normal $\mathrm{RN}^{\prime}$ to the face $\mathrm{CA}, \theta$ the angle of the prism, and $\beta$ the angle QRN.

Further, for generality, let us suppose that the medium (2) outside the face $A B$ is not the same as the medium (3) (air) outside the face $A C$. Then using the symbol ${ }_{2} \mu_{1}$ for the refractive index from the medium (2) to the glass of the prism, ${ }_{3} \mu_{1}$ for the refractive index from air to the glass of the prism, and ${ }_{3} \mu_{2}$ for the refractive index from air to the medium (2), we have, since the angle $\mathrm{NQR}$ is the critical angle from medium (1) to medium (2), and the angle $\mathrm{NQR}=\theta-\beta$,

$$
\begin{aligned}
& { }_{2} \mu_{1}=\frac{1}{\sin (\theta-\beta)} \text {. . . . . . . } \\
& { }_{3} \mu_{1}=\frac{\sin \alpha}{\sin \beta} \text {. } \\
& { }_{3} \mu_{2}=\frac{{ }_{3} \mu_{1}}{{ }_{2} \mu_{1}} \text {. (See Watson's Physics, } \S 345 \text { ) } \\
& { }_{3} \mu_{2}={ }_{3} \mu_{1} \sin (\theta-\beta) \text {. } \\
& \text { and }
\end{aligned}
$$

Substituting from (2) we get$$
{ }_{3} \mu_{2}={ }_{3} \mu_{1} \sin \left\{\theta-\sin ^{-\frac{1}{2}} \frac{\sin \alpha}{{ }_{3} \mu_{1}}\right\} \text {. }
$$ 
The formula may be written in another form in cases where the method is used to measure the refractive index of the prism.

Substituting from (2) we get

$$
{ }_{3} \mu_{2}={ }_{3} \mu_{1} \sin (\theta-\beta)={ }_{3} \mu_{1}\{\sin \theta \cos \beta-\cos \theta \sin \beta\} .
$$

$$
{ }_{3} \mu_{2}=\sin \theta \sqrt{ }\left({ }_{3} \mu_{1}^{2}-\sin ^{2} a\right)-\cos \theta \sin a \text {. . . . . }
$$

If the angle $\theta$ is $90^{\circ}$, then this formula reduces to

$$
{ }_{8} \mu_{2}=\sqrt{ }\left({ }_{3} \mu_{1}^{2}-\sin ^{2} a\right) \text {. . . . . . . }
$$

In the case where medium (2) is air, then ${ }_{3} \mu_{2}=1$, and equation (4) reduces to

$$
{ }_{8} \mu_{1}^{2}=1+\left\{\frac{\cos \theta+\sin \alpha}{\sin \theta}\right\}^{2} \text {. . . . . . }
$$

a formula which may be employed to measure the refractive index of the prism.

The angle $\theta$ of the prism must not be greater than a certain limiting value, or the ray $\mathbf{Q R}$ will suffer total internal reflection at $\mathbf{R}$. The limiting value for $\theta$ is such that the ray Rs is parallel to the face, i.e. that $\sin \beta=\frac{1}{{ }_{3} \mu_{1}}$. But from (1) $\theta=\sin ^{1} 1 /{ }_{2} \mu_{1}+\beta$. Hence the limiting condition is that

$$
\theta<\sin ^{-1} \frac{1}{{ }_{2} \mu_{1}}+\sin ^{-1} \frac{1}{{ }_{3} \mu_{1}} . \quad . \quad . \quad . \quad . .
$$

If the medium (2) is air, this reduces to

$$
\theta<2 \sin ^{-1} \frac{1}{{ }_{3} \mu_{1}} \text {. . . . . . . . . }
$$

and it is evident that the smaller the refractive index of the glass, the larger may be the angle. For a glass having a refractive index of 1.5 the limiting value of $\theta$ is $83^{\circ} 38^{\prime}$. If the refractive index of the glass is 162 , the limiting angle is $76^{\circ} 14^{\prime}$. If with this glass $\theta=90^{\circ}$, then the method is not applicable to finding the refractive index of the glass. It may, however, be used to measure the refractive index of a liquid or solid, so long as (1) the refractive index of this substance is less than 1.62 and greater than a value which we can at once deduce from equation (7). We have, since ${ }_{2} \mu_{1}=\frac{3}{8} \mu_{1} \mu_{2}$, in the limiting case

$$
\begin{aligned}
90^{\circ} & =\sin ^{-1} \frac{{ }^{3} \mu_{2}}{1 \cdot 62}+\sin ^{-1} \frac{1}{1.62} \\
{ }_{8} \mu_{2} & =\sin \left\{90^{\circ}-38^{\circ} 7^{\prime}\right\} 1.62 \\
& =1.27 .
\end{aligned}
$$

Thus the method may be used for substances having refractive indices 
between $1 \cdot 3$ and $1 \cdot 6$. In the same way the limits may be obtained for a prism of any other angle and refractive index.

The prism to be used ought to have a high refractive index, since the method presupposes that the refractive index of the prism is higher than that of the medium (2) of which the refractive index is to be measured.

(a) To measure the refractive index of the prism by this method, the prism is mounted on the table of a spectrometer with its centre over the axis of the instrument, and the faces $A B, A C$ (Fig. 129) are adjusted parallel to the axis by the method described in $\S 117$, and the telescope is focused for parallel rays. The collimator is not employed, but the source of monochromatic light, such as a Bunsen flame coloured with salt, is placed opposite the unpolished face BC at a distance of 10 to $20 \mathrm{~cm}$. The telescope is then turned to look into the face $\mathrm{AC}$, and adjusted till the line separating the bright from the dark portion of the field is on the cross-wires. The position of the telescope having been read on the divided circle, the telescope is turned to look into the face $\mathbf{A B}$, the prism remaining fixed, and the cross-wires are again adjusted to coincide with the line of separation between the bright and dark portions of the field, this reading corresponding to the direction ED (Fig. 129). The difference, $\delta$, between the readings for the position of the telescope gives the angle EMs. But the angle

Hence

$$
E M S=2 \pi-(\pi-\theta)-2 a .
$$

$\delta=\pi+\theta-2 a$

or

$$
a=\frac{\pi+\theta-\delta}{2} \text {. }
$$

Hence substituting this value of $\alpha$ in equation (6), and knowing the value $\theta$ (see $\S 117$ ), the refractive index of the prism can be calculated.

(b) To measure the refractive index of a liquid, which must be less than that of the prism, a drop of the liquid is placed on the face $\mathbf{A B}$, and a microscopic cover glass placed over. The cover glass is either kept in place by capillarity, or a little wax may be used.

The telescope having been adjusted on the line of separation between the light and dark parts of the field, the verniers are read. In this case it is not convenient to turn the telescope to face the other face of the prism, since this would involve cleaning off the liquid from the face $\mathbf{A B}$ and putting it on the face AC, during which operation the prism might accidentally get moved. The best way is to employ a Gauss eye-piece

- (see Fig. 122), and having illuminated the cross-wires, turn the telescope till the image of the wires, as seen reflected in the face $\mathrm{AC}$, coincides with the wires themselves. The reading of the verniers then gives the direction of the normal $\mathbf{R N}^{\prime}$, and the difference in the vernier readings for the two positions of the telescope gives directly the value of $a$, and from this, together with the value of the angle of the prism and 
its refractive index, the refractive index of the liquid can be obtained by equation (4) above.

(c) To measure the refractive index of a solid. If a plate of some solid is placed against the face $\mathbf{A B}$, a layer of a liquid of higher refractive index than the solid being interposed, then we may proceed exactly as described in (b) above and obtain the refractive index of the solid. A suitable liquid to use is mono-bromnapthaline, which has a refractive index of 1.65 for sodium light.

121. The Measurement of Refractive Index by Total Reflection, using a Prism (Second Method).-A modification of the method described in the last section consists in using monochromatic light at grazing incidence on the face $\mathbf{A B}$ (Fig. 129), the source of the light being in the plane of the face, and nearer B. In this case the portion of the field of the telescope, directed along $\mathbf{S R}$, on the side next $\mathbf{A}$ will appear bright, while the other side of the field will be dark, as is evident from a study of the figure. The formulæ (4), (5), and (6) of the last section apply to this case also. The advantage of the method lies in the fact that in place of having to set the cross-wire of the telescope on the line separating two regions, the brightness of which do not differ greatly, in this case one portion of the field is quite dark, and so the line of separation is much more easily seen.

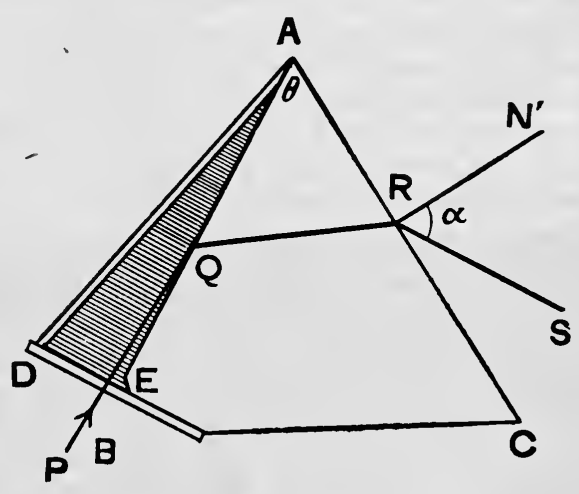

Fig. 130.

\section{When an extended source} of light, such as a Bunsen flame coloured with some salt, is employed, the source can be placed at a distance of from 10 to $30 \mathrm{~cm}$. from the prism, so that the centre of the flame is in the plane of the face BA. If a small source of light, such as a vacuum tube, is employed, then it is advisable to use a lens of about $10 \mathrm{~cm}$. focal length between the source and the prism, the position of the lens being adjusted so as to give a slightly convergent beam of light.

When measuring the refractive index of the prism no difficulty will be met with, particularly if small cardboard screens are placed so as to cut off stray light from the telescope. When applying the method to measuring the refractive index of liquids and solids, however, some points have to be attended to, or it will be found impossible to obtain a sharp line of separation between the two halves of the field.

Thus when only a drop of the liquid is used, the cover glass employed must have a straight edge, and this edge must be adjusted to 
lie parallel to the edge of the prism where the light enters. If more than a drop of the liquid is available, then the form of prism shown in Fig. 130 may advantageously be employed. The angle $\mathbf{B}$ of a prism of dense glass is ground off by a plane perpendicular to the face BA, and then two pieces of glass, BD, DA, are cemented as shown. These pieces of glass, together with a plate of glass cemented to the bottom, enclose a prism-shaped space in which the liquid is contained, the angle at $\mathbf{D}$ serving to allow the introduction of the bulb of a thermometer. The light is incident parallel to $\mathbf{P Q}$, and the limiting rays are parallel to $Q R$, $R S$. If the corner at $\mathrm{E}$ is slightly bevelled off as shown, so that none of the cement used to attach the plate DB interferes with the light being incident at $90^{\circ}$, the field of view is very sharply divided, and very accurate settings can be made.

The angle BAC of the prism must be measured before the glass plates are cemented in place.

The advantages possessed by this arrangement over the hollow prism are as follows:-(1) Less liquid is required. (2) The plates of glass used to enclose the liquid need not be optically worked, and the fact that their faces are not parallel produces no effect. (3) The angle of the glass prism has only to be measured, and this can be done once for all, while with the hollow prism the measurement of the angle has to be repeated each time the side plates are removed. 


\section{CHAPTER XIX}

\section{DISPERSION AND WAVE-LENGTH MEASUREMENTS}

122. The Measurement of Dispersion - Sources of Light. - The dispersion of a medium can be determined by measuring the refractive index by one of the methods previously described for light of different wave-lengths. For this purpose it is necessary to have sources of light which shall give light of some definite and known wave-length. Since such sources of light of definite wave-length will be required for several other measurements, it will be convenient to consider them here in detail, and under different heads.

(a) Flames coloured with metallic salts.-A source of light which is often convenient is a Bunsen flame coloured with some metallic salt. Since the brightness of the coloration depends on the temperature of the flame being high, it is of importance that the support used to introduce the salt into the flame should not be such as to cool down the flame more than is absolutely necessary. If the light is only required for a short time, a piece of fine platinum wire dipped in a solution of the salt and held in the outside portion of a Bunsen flame about $2 \mathrm{~cm}$. from the bottom of the flame will be found convenient. As, however, the wire soon loses all salt, this arrangement is not suitable when the light is required for any time. In such a case, a small scoop of thin platinum foil fused to the end of a short platinum wire will be found convenient for containing the salt. A small shred of asbestos or a piece of wood charcoal which has been dipped into a strong solution of the salt can also be used, being held in the edge of the flame.

A much brighter light than can be obtained with the Bunsen burner is pro-

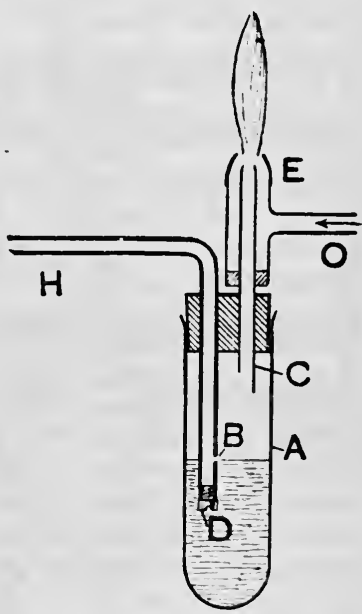

FIG. 131. duced by means of the arrangement shown in Fig. 131. It consists of a strong glass test-tube A, which contains a solution of the salt. A thick copper tube $\boldsymbol{H}$ is connected to a steel bottle of compressed hydrogen gas, such as is used in connection with the lime light 'This tube is connected 
direct to the bottle without the intervention of any pressure regulator, so that the full pressure of the gas can act. The lower end of the tube is closed by a screw $D$, while a very fine hole is pierced through the side of the tube at B. The gas escaping through this hole, under great pressure, churns the solution into a fine spray, which is carried up the tube c. A concentric tube $\mathbf{E}$, connected with a side tube o, serves for feeding oxygen gas round the burning hydrogen. The oxygen need not be supplied at any great pressure, so that it is best to use a regulator in connection with the bottle in which this gas is stored.

If possible, it is always advisable to place the flame under a hood, so that the fumes may not escape into the room. This is particularly necessary in the case of salts of thallium, which are very poisonous.

The chief difficulty when using this burner is to prevent the fine hole becoming clogged up with dirt. A small plug of cotton wool placed in the tube $\mathrm{H}$ will to a certain extent prevent dirt carried over by the gas reaching the nozzle.

The following table gives the salts which can be used in a Bunsen flame to give light of definite wave-length.

\begin{tabular}{|c|c|c|c|}
\hline Salt to be Used. & $\begin{array}{l}\text { How Introduced into } \\
\text { Flame. }\end{array}$ & $\begin{array}{l}\text { Wave-length in } \\
10^{-8} \mathrm{~cm} \text {. }\end{array}$ & $\begin{array}{l}\text { Colour of } \\
\text { Light. }\end{array}$ \\
\hline Potassium nitrate & On platinum wire & $\left\{\begin{array}{l}7668 \cdot 5 \\
7701 \cdot 0\end{array}\right.$ & Red \\
\hline Lithium chloride & On charcoal & $\left\{\begin{array}{l}6708 \cdot 2 \\
6703 \cdot 8\end{array}\right.$ & Red. \\
\hline Sodium chloride & On charcoal & $\left\{\begin{array}{l}5890 \cdot 2 \\
5896 \cdot 9\end{array}\right.$ & Orange \\
\hline Thallium chloride & On platinum wire & $\begin{array}{l}5896 \% 2 \\
5350 \cdot 7\end{array}$ & $\begin{array}{l}\text { (aouble). } \\
\text { Green. }\end{array}$ \\
\hline Strontium chloride & On charcoal & $4607 \cdot 5$ & Blue. \\
\hline Calcium chloride & On charcoal & $4226 \cdot 9$ & Blue. \\
\hline Potassium nitrate & On platinum wire & $\left\{\begin{array}{l}4044 \cdot 3 \\
4047 \cdot 4\end{array}\right.$ & $\begin{array}{l}\text { Violet } \\
\text { (double). }\end{array}$ \\
\hline Rubidium chloride & On platinum wire & $\left\{\begin{array}{l}4202 \cdot 0 \\
4215 \cdot 7\end{array}\right.$ & $\begin{array}{l}\text { Violet } \\
\text { (double). }\end{array}$ \\
\hline
\end{tabular}

(b) A source of light which is in many ways more convenient than a flame coloured by metallic salts, since it does not require the constant attention this latter requires, consists of a tube fitted with electrodes, and filled with certain gases under reduced pressure. On passing an electric discharge through such a tube by means of an induction coil the contained gas will become luminous, the spectrum produced consisting of lines.

The commonest form of such a vacuum tube is shown in Fig. 132. It consists of two wider portions $\mathbf{A}$ and $\mathbf{B}$, connected by a narrow tube 0 having a bore of about $2 \mathrm{~mm}$. The electrodes consist of short aluminium wires fused on to short pieces of platinum wire. The platinum passes through the glass and terminates outside in a small loop, to which the 
wires from the induction coil are attached. On passing a discharge through the tube, the gas within the capillary tube $\mathrm{c}$ glows brightly. The disadvantage of this form of tube is that the bright part of the tube can only be used sideways, since the electrodes prevent the light which passes along the axis of the tube being used. A better form of tube is that shown in Fig. 133. Here the capillary tube c is so placed that nothing interferes with the light which passes axially along the tube. This is an advantage, since the light given along the axis is very much brighter than that given at right angles to the axis. It is also an advantage to have electrodes of

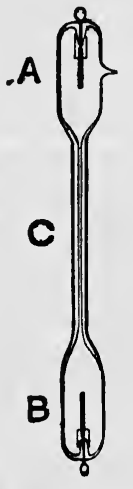

Fig. 132.

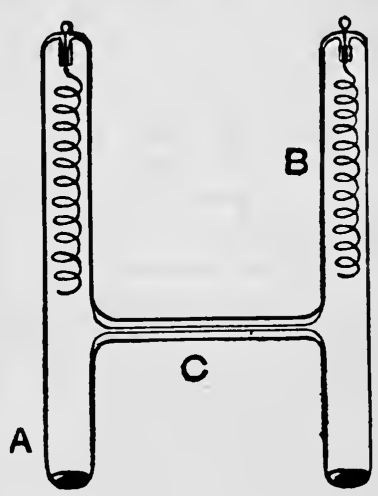

Fig. 133. considerable size, and hence the aluminium wire is coiled into a spiral.

The following table gives a list of the more useful gases for use in vacuum tubes, with the wave-lengths of the principal lines in air at $15^{\circ}$ and $760 \mathrm{~mm}$. pressure :-

\begin{tabular}{|c|c|c|}
\hline , Gas. & Wave-length in $10^{-8} \mathrm{~cm}$. & Colour. \\
\hline $\begin{array}{l}\text { Hydrogen . } \\
\text { Helium . . }\end{array}$ & $\begin{array}{l}6563 \cdot 0 \\
4861 \cdot 5 \\
4340 \cdot 7 \\
7065 \cdot 2 \\
6678 \cdot 1 \\
5875 \cdot 6 \\
5015 \cdot 7 \\
4921 \cdot 9 \\
4713 \cdot 2 \\
4471 \cdot 5\end{array}$ & $\begin{array}{l}\text { Red. } \\
\text { Blue-green. } \\
\text { Violet. } \\
\text { Red. } \\
\text { Red. } \\
\text { Yellow. } \\
\text { Green. } \\
\text { Blue. } \\
\text { Blue. } \\
\text { Violet. }\end{array}$ \\
\hline
\end{tabular}

Vacuum tubes containing gases are found to deteriorate fairly rapidly, and for almost all purposes the light given out by mercury vapour, caused to glow by the passage of an electric discharge, is more convenient. There are two distinct types of tubes for giving the mercury spectrum. Where a small source of light with a fair amount of brilliancy is sufficient, a tube such as that shown in Fig. 133, and in which a little mercury has been enclosed, can be used with the discharge from an induction coil. In order to fill the tube with mercury vapour, it is 
- necessary to heat the tube over a small Bunsen flame. A small shield of asbestos cardboard placed over the tube will be found useful in keeping off draughts and securing uniform heating.

Where, however, the electric light is available, what is called a mercury, or Cooper-Hewitt, lamp is by far the most convenient arrangement to use, since it does not require an induction coil, and gives a very brilliant light. A very convenient form is the Bastian ${ }^{1}$ lamp, which is shown in Fig. 134. The exhausted tube $\mathbf{A B}$ through which the discharge passes, is bent into a zig-zag, and there is a small mercury reservoir at the end $A$. The electrodes are connected, through a resistance adjusted to suit the voltage of the supply, to an ordinary incandescent electric light socket. The lamp with its resistance is

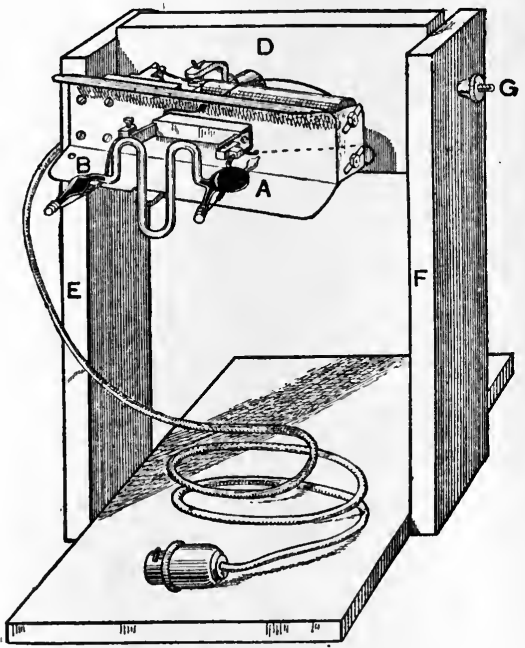

FIG. 134.

screwed to a board $D$, which is pivoted on a horizontal axis between the uprights EF.

To start the lamp, the board $\mathrm{D}$ is turned so that the lamp is facing downwards, and then the tube is tilted so that the mercury flows from the reservoir A and fills the tube, thus completing the electric circuit. On releasing the lamp, the tube tilts so that the mercury flows back into the reservoir $A$, and in doing so breaks the circuit in the tube, and the small arc formed at the point of the rupture vaporises some of the mercury, and the discharge continues to pass through this mercury vapour. When the lamp is started, the board $\mathrm{D}$ can be turned up into a vertical plane, in which positions the vertical portions of the tube form convenient sources of light for use with a spectrometer.

1 A similar type of lamp, though on a larger scale, is made by the Westing. house Company. 
The light given by the mercury lamp contains a number of lines but in the following table only the more conspicuous ones are given :-

Wave-Lengths in Air at $15^{\circ}$ and 760 mm. Pressure of the Chief Lines given by the Mercury Lamp.

\begin{tabular}{|c|c|c|c|}
\hline Colour. & & Wave-length in $10^{-8} \mathrm{~cm}$. & Remarks. \\
\hline $\begin{array}{l}\text { Red . } \\
\text { Yellow : } \\
\text { Yellow : } \\
\text { Green · : } \\
\text { Green-blue } \\
\text { Blue · · } \\
\text { Blue · : } \\
\text { Violet. : } \\
\text { Violet. . }\end{array}$ & 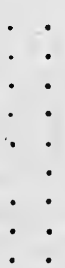 & $\begin{array}{l}6232 \\
5790 \cdot 7 \\
5769 \cdot 6 \\
5460 \cdot 7 \\
4959 \cdot 7 \\
4916 \cdot 4 \\
4358 \cdot 3 \\
4078 \cdot 1 \\
4046 \cdot 8\end{array}$ & $\begin{array}{l}\text { Fairly bright. } \\
\text { Very bright. } \\
\text { Very bright. } \\
\text { Very bright. } \\
\text { Fairly bright. } \\
\text { Faint. } \\
\text { Bright. } \\
\text { Bright. } \\
\text { Faint. }\end{array}$ \\
\hline
\end{tabular}

The light given by cadmium or zinc, when the vapour of these metals is rendered incandescent by the passage of an electric discharge, is often useful. These metals require to be heated to a considerable temperature before they vaporise, and it is found that the vacuum tubes in which they are used deteriorate rather rapidly. A form of tube which can easily be made by even an inexperienced glassblower has been devised

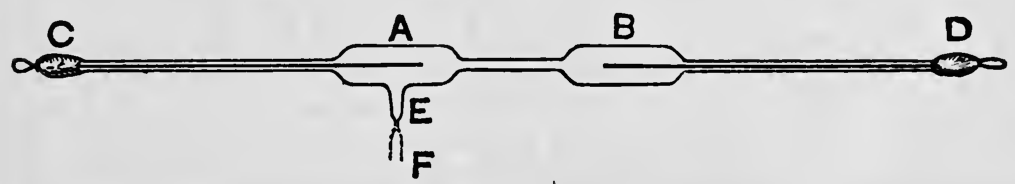

FIG. 135.

by Lord Rayleigh, and is shown in Fig. 135. The central part of the tube is similar to that shown in Fig. 132. Since, however, the insertion of the electrodes through the glass is a matter of some difficulty, the glass showing a marked inclination to crack at the point where the platinum wire is inserted when the tube is heated, the aluminium wires are inserted through two long tubes $\mathrm{CA}$ and $\mathrm{BD}$, the joints at $\mathrm{C}$ and $\mathrm{D}$ being made air-tight with sealing-wax. The tubes $\mathrm{CA}, \mathrm{BD}$ are each about 20 centimetres long, and have a bore of 1.5 millimetre. A little zinc or cadmium is introduced, and the tube is then connected to the pump at $\mathbf{r}$, and when exhaustion is complete is sealed off at $\mathbf{E}$. When in use the central part $A B$ of the tube is enclosed in a little metal box, in which a hole is pierced so that the light may escape, and this box is heated over a flame. The tubes $\mathrm{CA}$ and $\mathrm{BD}$ project beyond the box, and are so long 
that the ends do not become sufficiently hot to soften the wax. After the tubes have been in use for a few hours they will require re-exhaustion.

The wave-lengths of the chief lines in air at $15^{\circ}$ and $760 \mathrm{~mm}$. pressure are :-

\begin{tabular}{|c|c|c|c|}
\hline \multicolumn{2}{|c|}{ Metal. } & Wave-length in $10^{-8} \mathrm{~cm}$. & Colour. \\
\hline $\begin{array}{l}\text { Cadmium } \\
\text { Zinc. }\end{array}$ & $\begin{array}{l}\cdot \quad \cdot \\
\text {. }\end{array}$ & $\begin{array}{l}6438 \cdot 47 \\
5085 \cdot 82 \\
4799 \cdot 91 \\
6362 \cdot 34 \\
4810 \cdot 53 \\
4722 \cdot 16 \\
4680 \cdot 14\end{array}$ & $\begin{array}{l}\text { Red. } \\
\text { Green. } \\
\text { Blue. } \\
\text { Red. } \\
\text { Blue. } \\
\text { Blue. } \\
\text { Blue. }\end{array}$ \\
\hline
\end{tabular}

123. Light Filters.-When using such a source of light as a mercury lamp, which gives light of several wave-lengths, with a spectrometer, no inconvenience is caused, for the prism itself sorts out the rays of the different colours. For small deviation this is also true when a grating is being used. If, however, a grating of high dispersive power is used, or if spectra of a high order are to be employed, then inconvenience will be caused by the overlapping of the light of the wave-length being investigated by light of another colour, and corresponding to a spectrum of a

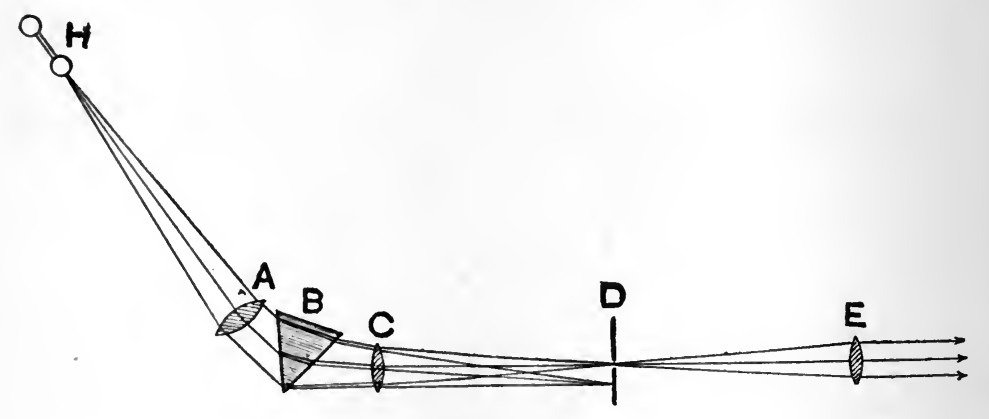

Fig. 136.

different order. In the same way, when using the mercury light with interferometers the interference fringes due to the different wave-lengths would overlap and obscure each other. In such a case we have to use some piece of auxiliary apparatus to sift out the light of the wave-length we are using from the other light. A convenient arrangement for this purpose is shown in Fig. 136. It consists of a mercury lamp В, which is at the principal focus of the collimation lens $A$, a prism B, which need not be of any very dispersive glass, and a lens $\mathrm{c}$, at the principal focus of 
which is placed a slit of adjustable width D. Finally, in many cases, it is advisable to use a third lens $\mathbf{E}$, with the slit at its principal focus. With this arrangement a beam of parallel light falls on the prism, and, if the lenses are so arranged that the deviation is a little greater than minimum, any line of the spectrum formed in the plane of the slit by the lens $\mathrm{c}$ can be brought on the slit by slightly rotating the prism. In the cases where a beam of parallel monochromatic light is required, this can be obtained by means of the lens $\mathrm{E}$. The light of wave-lengths other than that which is required is cut off by the slit. When used with a grating spectroscope the slit $\mathbf{D}$ and lens $\mathbf{E}$ are unnecessary, since the collimator of the instrument with its slit perform the functions of this slit and lens. In such a case the spectrum formed by the prism and lens $\mathrm{C}$ is projected on the slit of the instrument.

124. Calibration of a Spectroscope by the Use of Lines of known Wave-lengths.-Owing to the fact that the deviation produced by a prism is not proportional to the wave-length, when using a prism spectroscope to measure wave-lengths it is necessary to calibrate the spectroscope, that is, to draw a curve giving the deviation in terms of the wave-length. One way of obtaining-such a curve is to measure the deviation for a number of different lines of known wave-length fairly uniformly distributed throughout the spectrum, and then to draw a uniform curve through the points obtained.

To obtain such a curve, having adjusted the spectroscope in the manner described in $\S 117$, set the prism to minimum deviation for some known line. For this purpose the more refrangible of the two sodium lines will be found convenient. Then keeping the prism fixed, determine the deviation for a number of lines throughout the spectrum, using as source of light one of those described in the previous section. It will be necessary, if great refinement is required, to either always work at the same temperature, or by observing at different temperatures to determine how the deviation for each of the standard lines varies with the temperature.

Having obtained the readings for the deviations, plot a curve having the wave-lengths for ordinates and the corresponding deviations for abscissæ. When using the spectroscope to measure the wave-length of some unknown line, the prism must be set for minimum deviation for sodium light, and the deviation corresponding to the unknown line measured. Then from the curve the wave-length corresponding to this deviation can be immediately read off.

The curve obtained by plotting wave-lengths as ordinates and deviations as abscissæ is approximately a rectangular hyperbola, and since the curvature is very considerable, unless there are a large number of observations, it will be found difficult to draw an accurate curve. If, however, in place of plotting wave-lengths we plot frequencies, or what comes to the same thing, the number of waves in 1 centimetre, against the deviations, the curve obtained is much less curved, and hence can be more accurately drawn. A curve which is very nearly straight can be 
obtained by plotting $1 / \lambda^{2}$ against the deviations, and where great accuracy is desired, the extra arithmetical labour is quite worth while.

Where the wave-length of a single line is desired, it is sometimes convenient to avoid the trouble of making a whole series of observations so as to plot the complete curve. In such a case two lines may be observed, one on either side of the unknown line, and the wave-length of this line obtained by arithmetical interpolation.

If the two known lines are very near the unknown line, we may interpolate by proportional parts. In general, however, such a procedure would lead to a very erroneous result. Since, however, the deviations are very nearly proportional to the inverse square of the wavelengths, we may use this property for interpolation without causing any very great error.

Let $\lambda_{1}$ and $\lambda_{2}$ be the wave-lengths of the two known lines, $\lambda$ the wave-length of the unknown line, and $n_{1}, n_{2}$, and $n$ the corresponding deviations. Then

or

$$
\begin{gathered}
\frac{\frac{1}{\lambda^{2}}-\frac{1}{\lambda_{1}^{2}}}{\frac{1}{\lambda_{2}}-\frac{1}{\lambda_{1}^{2}}}=\frac{n-n_{1}}{n_{2}-n_{1}} \\
\frac{1}{\lambda^{2}}=\frac{1}{\lambda_{1}^{2}}+\left\{\frac{1}{\lambda_{2}^{2}}-\frac{1}{\lambda_{1}^{2}}\right\} \frac{n-n_{1}}{n_{2}-n_{1}},
\end{gathered}
$$

from which $\frac{1}{\lambda^{2}}$, and hence $\lambda$ can at once be calculated.

125. Calibration of a Spectroscope by Interference Fringes. ${ }^{2}-A$ method of calibrating a spectroscope, which is particularly convenient when the spectrum is photographed, is to make use of interference fringes. If a very thin film of air is enclosed between two optically plane pieces of glass and placed immediately before the slit of a spectrometer, then on illuminating the slit with a slightly convergent beam of white light the spectrum will be crossed by a number of dark bands. These bands are due to the interference between the light which has passed straight through the air film and that which, owing to internal reflection, has traversed the air film three times. The number and breadth of the bands depends on the thickness of the air film, being more numerous the thicker the film. In order to render the bands quite dark, it is necessary to partly silver the glass surfaces which enclose the film so as to strengthen the portion of the light which undergoes reflection within the film. The silvering can be performed by the process described in the Appendix, and must be carried on till about threequarters of the incident light is reflected at the silvered surface. The following is the method recommended by Edser and Butler for preparing

1 Edser and Butler, Phil. Mag. [5] (1898), vol. xlvi. p. 207, Proc. Physical Society of London (1898), xvi. 207. 
the air film:-Having selected two pieces of plate glass which, when tested by the method given in $\S 142$, show broad and straight interference fringes, silver them on one side. Place a little soft red wax round the edges of the plates, and press them firmly together while looking through them at a bright spot of light. A long train of images will be seen, due to multiple reflections. Squeeze the glass till only a single image is obtained, when on looking through the glasses at a sodium flame interference fringes will be seen. Adjust the glasses till these fringes are as broad as possible. When the adjustment is nearly complete it will be necessary to look at the fringes from a little distance, owing to the fact that with a parallel air film viewed normally the bands are formed at an infinite distance in front of the film.

Having prepared an air film, illuminate the slit of the spectroscope with a slightly convergent beam of light from an arc, and place the air film as near the slit as possible. The spectrum will then be found to consist of bright lines separated by almost dark intervals.

To take a photograph, half of the slit can be covered while the interference bands are being photographed, while, when the spectrum is being photographed, this half of the slit can be exposed, that part previously uncovered being now covered. In this way a photograph of the interference bands will appear alongside the photograph of the spectrum.

If $d$ is the thickness of the air film, the length of the path traversed by the twice reflected part of the light will exceed that of the light which has gone straight through by $2 d$, and there will be a bright band in the spectrum wherever the wave-length goes exactly an integral number of times into $2 d$; that is, if

$$
\lambda_{0}, \lambda_{1}, \lambda_{2}, \lambda_{3} \ldots \lambda_{m}
$$

are the wave-lengths corresponding to the bright bands, then

$$
2 d=n \lambda_{0}=(n+1) \lambda_{1}=(n+2) \lambda_{2}=(n+3) \lambda_{3}=\ldots=(n+m) \lambda_{m},
$$

where $n$ is some integer. Suppose that the wave-lengths $\lambda_{0}$ and $\lambda_{m}$, corresponding to two of the bands, are known, then $n$ can be determined from the equation

$$
n=\frac{m \lambda_{m}}{\lambda_{0}-\lambda_{m}}
$$

where $\lambda_{m}$ is the wave-length of the $m$ th band from that corresponding to $\lambda_{0}, \lambda_{0}$ being the band nearer the red end of the spectrum. The wavelength $\lambda_{r}$, corresponding to the $r$ th band, counting from $\lambda_{0}$ in the same way, is given by

$$
\lambda_{r}=\frac{n \lambda_{0}}{n+r}
$$

Thus if we know the wave-lengths of any two bands sufficiently far apart so as to be able to calculate the value of $n$, we can, by simply 
counting the number of bands from the first line up to any given line, determine the wave-length corresponding to this line.

126. Measurement of Wave-length with the Diffraction Grating. -If a parallel beam of monochromatic light of wave-length $\lambda$ falls normally on a grating, either transparent or reflecting, having $N$ lines to the centimetre, then if $\theta$ is the angle between the direct light and the deviated light in the first spectrum on either side, we have (see Watson's Physics, § 377)

$$
\lambda=\sin \theta / N .
$$

In the second spectrum, in the same way

$$
\lambda=\sin \theta^{\prime} / 2 N,
$$

and so on for the spectra of higher orders. Hence if we know $N$ and measure $\theta$, we can immediately calculate $\lambda$.

When performing the experiment, having adjusted the telescope and collimator for parallel rays, as described in 116,117 , the first thing is to take the reading $\phi_{0}$ corresponding to the direct light. We have then to set up the grating on the spectrometer table so that it is normal to the incident light. Place the grating on the table so that the ruled surface is immediately over the centre of the circle and parallel to the line joining two of the levelling screws (see p. 292), and adjust the plane of the grating parallel to the axis of the circle by the method given on p. 290. Next set the telescope so that the readings on the attached verniers are $\phi_{0}+90^{\circ}$, that is, so that the axis of the telescope is at right angles to the direction of the rays of light proceeding from the collimator. Turn the grating till the image, which is simply reflected from the surface of the grating and not diffracted, coincides with the cross-wires of the telescope, and read the verniers attached to the table. The plane of the grating now makes an angle of $45^{\circ}$ with the incident light. Add or subtract, as the case may be, $45^{\circ}$ to the reading for the table, and set it to this new angle. The plane of the grating will now be perpendicular to the incident light.

The final adjustment is to set the lines of the grating parallel to the axis about which the telescope turns. To do this, having adjusted a wire across the slit, illuminate the slit with monochromatic light, say, sodium light, and adjust the table levelling screw which lies in the plane of the grating, till the intersection of the cross-wires coincides with the horizontal wire across the slit in the case of all the diffracted images which can be observed on both sides. When this adjustment is complete, the rulings on the grating are parallel to the axis of the instrument. The slit should then be made very narrow, and, having placed the telescope so as to observe one of the diffracted images, the slit must be turned so that the image is as sharp as possible. In this way the slit is made parallel to the rulings on the grating.

Having arranged a source of light to give the lines required, determine the readings on the circle corresponding to the diffracted images 
corresponding to the spectra of each order. The difference between the readings corresponding to the diffracted images on the two sides correponding to any given order will be equal to twice the angle $\theta$.

If the number of lines to the centimetre is not known, it can at once be determined by making a series of measurements with light of known wave-length. For this purpose sodium light will be found as convenient as any. It is sometimes convenient, when using a reflection grating, to place the grating in such a way that the incidence is not normal. If the angle of incidence of the light on the grating is $\phi$, and $\theta$ is the angle between the axis of the telescope and collimator for a line in the spectrum of the $n$th order, it can at once be shown that

$$
n \lambda=\{\sin (\theta-\phi)-\sin \phi\} \frac{1}{N} .
$$




\section{GHAPTER XX}

\section{INTERFERENCE}

127.-Measurement of the Wave-length of Light with Fresnel's Biprism.-Fresnel's biprism is a method of obtaining interference fringes between waves of light which, having started from the same point of the scurce, traverse slightly different paths and then meet. The theory of the biprism follows at once from a consideration of Fig. 137. If $\mathrm{P}$ is the source of light, then after passing through the biprism .AB the light which. has passed through the two halves of the prism will travel as if it came

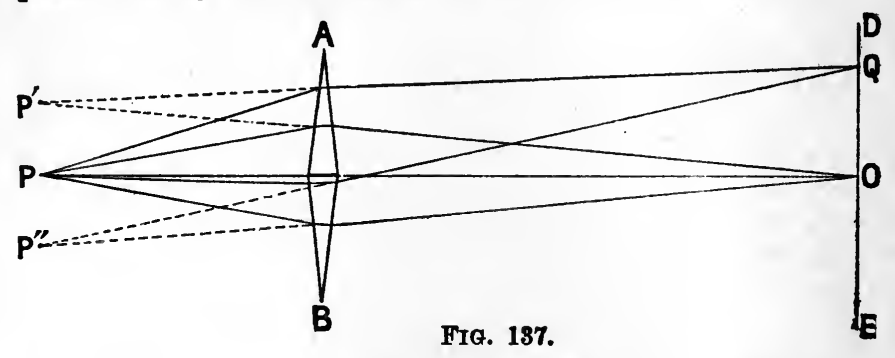

from the two virtual images $\mathrm{P}^{\prime}$ and $\mathrm{P}^{\prime \prime}$ respectively. If a screen is placed at $\mathrm{DE}$, then at the point $\mathrm{o}$, which is on the straight line drawn through the source of light and the centre of the biprism, the two trains of waves will always be in the same phase, and will therefore strengthen each other. At a point $Q$ at a distance $x$ from $o$ there will be interference, owing to the two trains of wares reaching $Q$ in opposite phases, if $P^{\prime \prime} Q$ exceeds $P^{\prime} Q$ by an odd number of half wave-lengths, that is, if

$$
\overline{P^{\prime \prime} Q}-\overline{P^{\prime} Q}=(2 n+1) \frac{\lambda}{2} \text {. }
$$

Calling the distance $\overline{P O}$ between the source and the screen $\mathrm{D}$, and the distance $\overline{\mathbf{P}^{\prime} \mathbf{P}^{\prime \prime}}$ between the images $2 d$, we have

and

$$
\overline{P^{\prime \prime} Q^{2}}=D^{2}+(x+d)^{2},
$$

Hence

$$
\begin{aligned}
& \overline{P^{\prime} Q^{2}}=D^{2}+(x-d)^{2} . \\
& \overline{P^{\prime \prime} Q}=D \sqrt{1+\left(\frac{x+d}{D}\right)^{2}},
\end{aligned}
$$


or since $x+d$ is always very small compared to $\mathrm{D}$

In the same way

$$
\overline{P^{\prime \prime} Q}=D\left(1+\frac{1}{2}\left(\frac{x+d}{D}\right)^{2}\right) \text {. }
$$

Hence

$$
\overline{P^{\prime} Q}=D\left(1+\frac{1}{2}\left(\frac{x-d}{D}\right)^{2}\right) \text {. }
$$

or if there is interference at $Q$

$$
\overline{P^{\prime} Q}-\overline{P^{\prime} Q}=\frac{2 l x}{D},
$$

$$
\begin{aligned}
& (2 n+1) \frac{\lambda}{2}=\frac{2 d x}{D}, \\
& x=(2 n+1) \frac{\lambda}{2} \frac{D}{2 d} .
\end{aligned}
$$

or

Now the distance between consecutive interference bands is obtained by giving to $n$ the values of two consecutive integers, and obtaining the difference in $x$. Calling the distance between the bands $y$ we get

or

$$
\begin{aligned}
& y=\lambda D_{l}^{\prime} 2 d, \\
& \lambda=\frac{2 d}{D} y .
\end{aligned}
$$

Thus to obtain the wave-length we have to measure the distance between the bands, the distance between the source of light and the screen, and the distance between the virtual images formed by the biprism. To carry out the measurement we shall require a narrow slit to form the source of

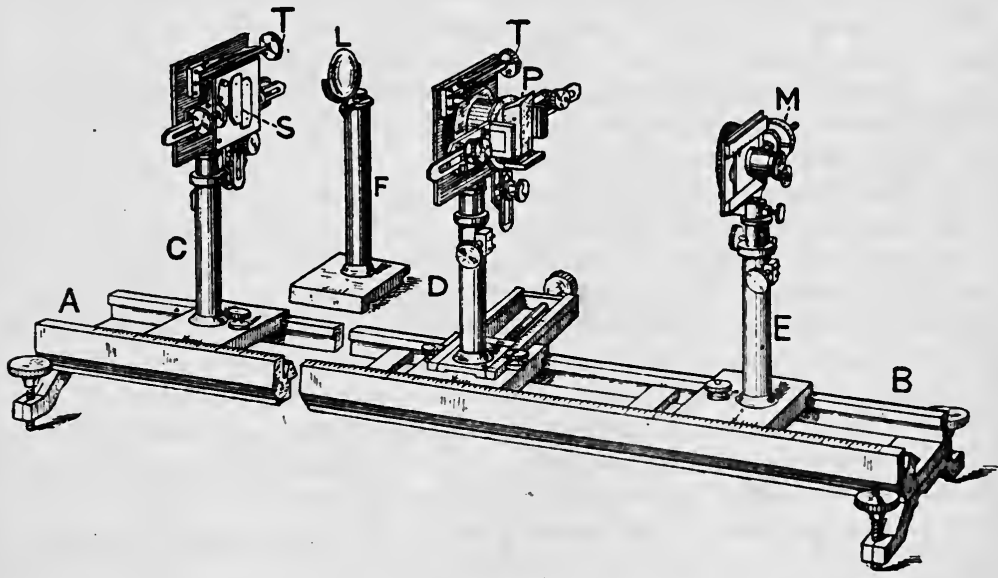

FrG. 138.

light, an adjustable upright to carry the biprism, and a micrometer eyepiece to measure the distance between the bands. In addition, a convex 
lens will be required to assist in measuring the distance between the images. A form of optical bench suitable for this purpose is shown in Fig. 138. It consists of a strong metal bed $A B$, along which can slide a number of uprights which are used to carry the slit, biprism, \&c. There is a scale attached to this bed, and verniers attached to the uprights serve to read the positions of these uprights. Two of the uprights, $c$ and $D$, are fitted with metal jaws, which serve to clamp the slit, biprism, \&c., while a third upright $\mathbf{E}$ carries a micrometer $\mathbf{M}$. This micrometer consists of a vertical cross-wire and a positive or Ramsden's eye-piece. The eye-piece and the cross-wire can be moved in a direction at right angles to the length of the bed by means of a micrometer screw. There is a fourth upright $\mathrm{F}$, which carries the convex lens $\mathrm{L}$. The upright $\mathrm{D}$, which carries the biprism $P$, has a slide and screw by means of which it can be moved in a direction at right angles to the bed. made :-

When making a measurement, the following adjustments have to be

1. Focus the eye-piece on the cross-wire, and turn it till it is vertical. Then place the lens between the slit and the eye-piece, and adjust till an image of the slit is seen in the eye-piece. Turn the slit about a horizontal axis till the slit is parallel to the cross-wire. To allow of this adjustment being made, the jaws which carry the slit (as also those which carry the biprism) can be rotated about a horizontal axis by means of a tangent screw T.

2. Move the eye-piece up to the slit and adjust them to the same vertical height, at the same time adjusting the micrometer slide perpendicular to the length of the bed.

3. Place the biprism in the jaws of the upright, and adjust so that the prism is at the same height as the slit, and the plane face is at right angles to the length of the bed.

4. Having illuminated the slit, which should be narrow, with sodium light, on looking through the eye-piece indistinct bands will probably be seen. If no bands are visible, move the upright carrying the biprism at right angles to the bed till the bands appear. Turn the biprism about a horizontal axis by means of the tangent screw till the bands appear as distinct as possible. When this occurs, the refracting edge of the biprism will be parallel to the slit.

5. Draw the upright which carries the eye-piece away from the biprism, and observe whether the bands appear to travel across the field of view. If they do, adjust the positions of the biprism and the crosswire in directions at right angles to the bed till such a transverse motion on moving back the eye-piece no longer takes place. When this adjustment is complete, the line joining the slit to the reiracting edge of the biprism will be parallel to the scale along the bed.

The preliminary adjustments are now complete, but before proceeding to measure the distance between the bands it will be convenient to determine the distance between the slit and the focal plane of the eye-piece, i.e. the plane of the cross-wire. For this purpose remove the upright 
which carries the biprism, taking care not to disturb the adjustments of this latter. Also remove the cap which carries the cross-wire, leaving the eye-piece in place. Then hold one end of a metal rod about $20 \mathrm{~cm}$. long against the middle of the slit, and move the upright which carries the eye-piece till the other end of the rod is in focus when seen through the eye-piece. Measure the length $a$ of the rod, and take the readings on the scale corresponding to the uprights which carry the slit and eye-piece. If the difference between these readings is $b$, then the correction which has to be added to the distance as given by the readings on the scale to obtain the true distance will be $a-b$.

Having replaced the upright which carries the biprism and readjusted the cross-wire, it will be necessary to adjust the width of the slit so that the bands may be as distinct as possible. If the slit is too wide, only a few bands of varying width will be seen; these are diffraction bands, and at present we are not concerned with them. On narrowing the slit the diffraction bands will be less obvious, while the interference bands due to the biprism will become more distinct. If, however, the slit is made too narrow, the light will be so feeble that the bands will be difficult to see. To obtain good measurements it is necessary to use a bright source of light. It will be found a great advantage to mount a convex lens of about $4 \mathrm{~cm}$. diameter and $3 \mathrm{~cm}$ : focal length so as to form an image of the flame on the slit.

Take the reading on the micrometer corresponding to every fifth or tenth bright band right across the field, and by dividing the readings into two groups in the method described on p. 100, calculate the mean distance between consecutive bands. The distance between the bright bands is the same as that between the dark bands, and since it is easier to see the cross-wire when it is at the centre of a bright band, it will be found best to measure the distance between the bright bands.

To measure the distance between the images place the convex lens ${ }^{1}$ between the biprism and the eye-piece, taking care not to alter the relative positions of the slit and biprism. The eye-piece having been removed to a distance from the slit greater than four times the focal length of the lens there will be found two positions for the lens, in each of which a sharp pair of images of the slit are formed. The distance between the images in either position must be measured with the micrometer. Let these distances be $c_{1}$ and $c_{2}$. Then the distance between the virtual images formed by the biprism is given by

$$
2 d=\sqrt{c_{1} c_{2}} \text {. }
$$

This follows at once, for if in the first position of the lens the distance

1 It will be found of advantage to cover the lens with a disc of paper having a hole of about $5 \mathrm{~mm}$. diameter pierced in the centre, so that only the central part of the lens is used. In this way, and using monochromatic light, good images will be obtained with a single piece lens. Care must be taken that the axis of the lens is parallel to the bed. 
between the lens and the slit is $d_{1}$, and the distance between the lens and the cross-wires is $d_{2}$, we have

$$
\frac{2 d}{c_{1}}=\frac{d_{1}}{d_{2}}
$$

In the second position, in the same way

Hence

$$
\frac{2 d}{c_{2}}=\frac{d_{2}}{d_{1}}
$$$$
\frac{2 d}{c_{1}}=\frac{c_{2}}{2 d}
$$

or

$$
2 d=\sqrt{c_{1} c_{2}} \text {. }
$$

128. Measurement of the Wave-length of Light with Fresnel's Biprism and a Spectrometer.-In the absence of an optical bench, such as that described in $\S 127$, we may employ a spectrometer in its place. Having adjusted the collimator to give parallel light, mount the biprism. on the table with the refracting edge perpendicular, and its plane face in the plane which passes through the vertical axis of the instrument and one of the levelling screws of the table. The final adjustment of the edge in the plane of the biprism can be left, and the plane face can be set parallel to the axis of the spectrometer by any of the methods described in $\S 117$. The biprism can also be set with its plane face perpendicular to the axis of the collimator by the method employed in the case of the diffraction grating $(\S 126)$.

Turn the telescope so that the vertical cross-wire is half-way between the two images of the slit, and unscrew the object glass. On illuminating the slit with monochromatic light, interference fringes will be seen in the field of view of the eye-piece. Rotate the biprism in its own plane by using the levelling screw of the table which is immediately under the prism, till the fringes are the most distinct, i.e. till the refracting edge of the prism is parallel to the slit. The angle $\theta$ subtended by the interval between two adjacent fringes has now to be determined. This is best obtained by taking the reading corresponding to every tenth fringe right across the system of bands, and then obtaining the mean width of a band in the manner described on p. 100 . If the circle of the spectrometer is not very finely divided, say, to read to $10^{\prime \prime}$, it will be better to attach a small plane mirror to the telescope, and to read the rotation by means of a scale and telescope, as described in $\S 170$.

Having replaced the object glass of the telescope, measure the angle $a$ subtended by the two images of the slit. The distance $d$ between the focal plane of the eye-piece and the axis of the spectrometer must also be measured.

The distance $y$ between consecutive fringes is then given by $y=d \theta$, 
where $\theta$ is the angle subtended by consecutive bands measured in circular measure. The wave-length of the light is obtained from

$$
\lambda=2 y \sin \alpha / 2 .
$$

This formula can be obtained as follows :-Let AB (Fig. 139) be the biprism, and oP the direction of the incident light. Then the wavefront, such as GH, before reaching the prism is at right angles to oP. After passing through the prism let the wave-fronts be $\mathrm{Co}$ and $\mathrm{OD}$, which

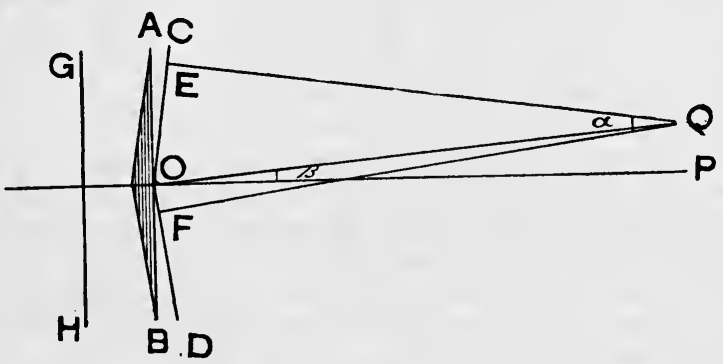

Frg. 139.

from symmetry are equally inclined to the line op. At $P$ the waves will always be in the same phase, and if QE and QF are drawn from any point $Q$ normal to the wave-fronts, then the waves will reach $Q$ in the same phase if

$$
\overline{F Q}-\overline{E Q}=n \lambda \text {. }
$$

But

$$
\overline{F Q}=\overline{O Q} \sin Q O F,
$$

and

$$
\overline{E Q}=\overline{O Q} \sin Q O E \text {. }
$$

Hence

$$
\begin{aligned}
\overline{F Q}-\overline{E Q} & =\overline{O Q}(\sin Q O F-\sin Q O E) \\
& =2 \overline{O Q}\left\{\sin Q O P \cos \frac{D O C}{2}\right\} \\
& =2 \overline{O Q} \frac{\overline{P Q}}{\overline{O Q}} \sin \frac{\alpha}{2},
\end{aligned}
$$

where $\alpha$ is the inclination of the normals to the two wave-fronts EO and oD. Calling $\overline{P Q} x$, there will be a bright band at $\mathrm{Q}$ if

$$
n \lambda=2 x \sin \frac{\alpha}{2} .
$$


Hence if $y$ is the distance between consecutive bands,

$$
\lambda=2 y \sin \frac{a}{2}
$$

The distance $y$ between consecutive bands is given by $\frac{\lambda}{2} \operatorname{cosec} \frac{a}{2}$, and where $a$ is the inclination of the wave-fronts after leaving the prism, and this angle depends on the angle of the biprism $\delta$, and the refractive index of the glass of which it is made. Thus to increase the distance between the bands it is necessary to decrease $a$ by using a biprism having a refracting angle very nearly equal to $180^{\circ}$. When used in the optical bench with divergent light the distance between the bands could be increased by either decreasing the distance between the slit and the biprism, or increasing the distance between the biprism and the eye-piece. For this reason the optical bench is on the whole the more convenient arrangement. By doing away with the collimator lens, so as to use divergent light, and placing the slit fairly near the axis of the spectrometer, the width between the bands can be increased. The formula to be used in this case can at once be obtained.

129. Measurement of the Wave-length of Light by means of Diffraction Fringes.-The optical bench described in $\S 127$ can be used for determining the wave-length of light from measurements made on diffraction fringes. The object, edge, wire or slit, as the case may be, is mounted in place of the biprism, and the distance between the fringes is measured with the micrometer.

1. Diffraction at a Straight Edge.-A metal plate with a carefully worked straight edge is mounted on the upright D (Fig. 138), the edge being vertical, and in such a position that the line joining the slit to the edge is parallel to the bench. The edge is then adjusted parallel to the slit by turning the plate in its own plane till the diffraction bands are as distinct as possible.

If $x$ is the distance of a band from the geometrical shadow of the edge, then it can be shown ${ }^{1}$ that if $a$ is the distance of the edge from the slit, and $b$ the distance of the focal plane of the eye-piece from the edge, then for a bright band

$$
x^{2}=\frac{b(a+b)}{a}(2 n+1) \lambda .
$$

and for a dark band

$$
x^{2}=\frac{b(a+b)}{a} 2 n \lambda
$$

where $n$ is an integer.

1 For an elementary discussion of diffraction bands the student is recommended to read Chapter V. of Schuster's T'heory of Optics (Arnold). 
Taking the case of the bright bands, and giving $n$ in succession the values $0,1,2, \& c$., we get the following values :-

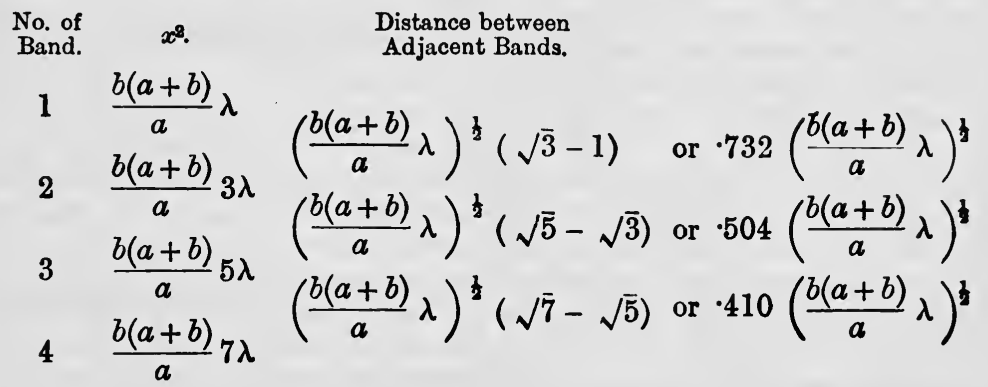
\&c. \&c.

Measure by means of the micrometer the distances between the different bands, and by means of the above expressions deduce values for the wave-length of the light, and show that within the limits of the accuracy of the experiment the above expressions are correct.

To obtain from the measurements the best value for the wave-length, measure the distance between the second band and the farthest visible, say, the $m$ th ; if this distance is $d$, we have

$$
\left.d=\left(\frac{b(a+b)}{a} \lambda\right)^{\frac{1}{2}} \sqrt{(2 m-1)} \cdot-\sqrt{ } 3\right),
$$

from which $\lambda$ can be calculated.

2. Diffraction by a Wire.-Mount a wire having a diameter of about $\cdot 4 \mathrm{~mm}$. in a vertical position, and with its length parallel to the slit, and at a distance of about $6 \mathrm{~cm}$.

Three systems of fringes will be seen, two series outside the geometrical shadow, in which the spacing of the bands is unequal, and which are similar to those produced by a single edge considered above, and a third set within the geometrical shadow, which are approximately equally spaced. ${ }^{1}$

If $b$ is the distance between the wire and the focal plane of the eyepiece, and $c$ is the width of the wire, the distance between consecutive bands of the internal set is

$$
\frac{b}{c} \lambda
$$

Measure the distance between as many bands of the internal set as possible, and deduce the wave-length of light.

1 With a very narrow obstacle these internal bands spread ont beyond the limits of the geometrical shadow. 
The inverse operation may, however, often be more convenient; that is, knowing the wave-length of the light, we may use the bands to measure the diameter of the wire. This is the principle on which Young's Eriometer depends.

3. Diffraction by a Slit.-Using one slit as a source, mount a second slit at a distance of about $60 \mathrm{~cm}$., and examine the fringes with the eyepiece at different distances. When the slit is fairly broad, and the eye-piece is near the slit, a system of unequally spaced bands will be seen within the geometrical image. There is also an external system, so long as the slit is not too broad, in which the bands are equally spaced. If the slit is made very narrow the internal system will disappear, while the external system will be more widely separated.

If $c$ is the width of the slit, and $b$ the distance between the slit and the focal plane of the eye-piece, the distance between consecutive bands is

$$
\frac{b \lambda}{c}
$$

Since it is very difficult to accurately measure the width of the slit, these bands can hardly be used to measure the wave-lengths. They do, however, form a means of measuring the width of the slit, which may on occasion be of service.

A convenient way of observing and measuring the diffraction bands due to a slit is to use a spectrometer. Focus the telescope and collimator for parallel light, and mount an adjustable slit on the table with its length parallel to the slit of the collimator. Using sodium light, measure the angle subtended by five or six of the diffraction bands, and dividing this angle by the number of intervals obtain the angle between two consecutive bright bands or, what is the same thing, between two consecutive dark bands. It can at once be shown that this angle is equal to the angle $\theta$ between the central bright band and the first dark band. Calculate from your result and the wave-length of sodium light the width of the slit, and compare the number obtained with the width as determined by direct measurement with a micrometer microscope. Repeat the measurements for different widths of slit.

130. On the Localisation of Interference Fringes.-Since interference fringes are often employed for the purpose of measuring small lengths, it will be worth while considering the position such fringes appear to occupy in some detail ; that is, supposing we have a set of fringes produced by some arrangement, and it is desired to examine the fringes in a telescope, on what point are we to focus the telescope? From the point of view of the measurement of length the fringes which are of importance are those which are produced by a thin film, which in practice is always an air film between two glass surfaces.

The following method of investigating the problem is due to Edser : ${ }^{1}$

1 Light for Students, by E. Edser (Macmillan). 
-Let $\mathrm{AB}$ and $\mathrm{CD}$ (Fig. 140) be the surfaces of the air film, these surfaces being inclined to one another at an angle $\theta$. Then if IM is a ray incident on the first surface at an angle $\alpha$, it will be partly reflected

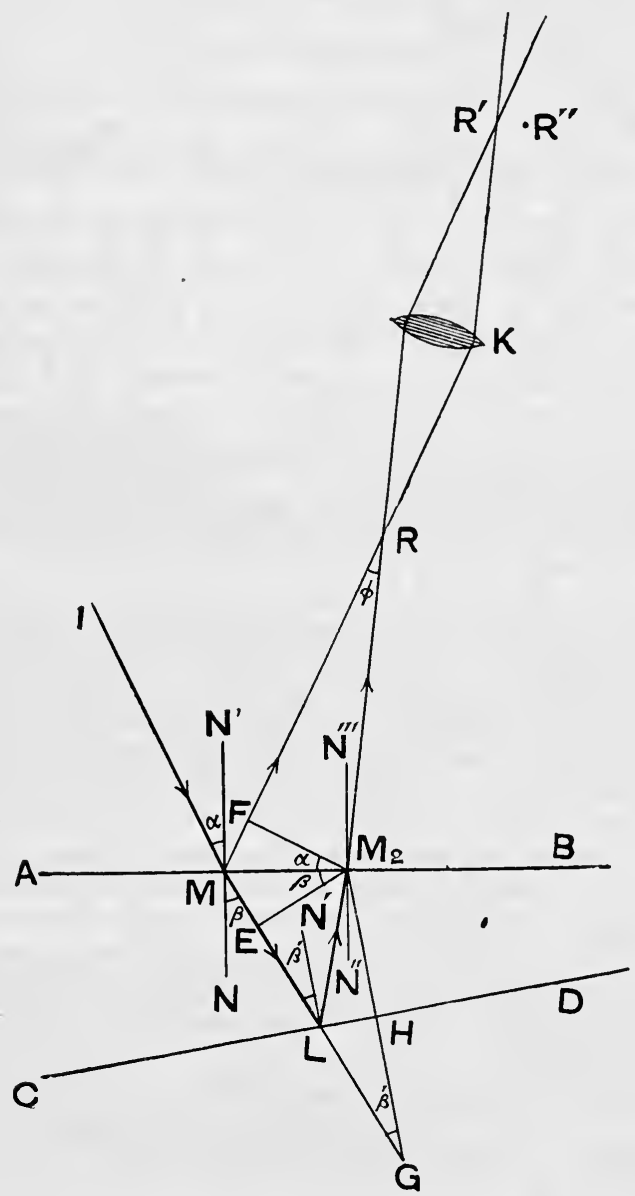

FIG. 140.

along MR and partly refracted along $M L$, the angle NML being $\beta$. This refracted ray is reflected at $\mathrm{L}$, refracted at $\mathrm{M}_{2}$, and finally proceeds along $\mathbf{M}_{2}$ R. From $M_{2}$ draw $M_{2} F$ perpendicular to $M R, M_{2} E$ perpendicular to $M L$, and $\mathrm{M}_{2} \mathrm{HG}$ perpendicular to $\mathrm{CD}$, and produce $\mathrm{ML}$ to meet this line 
at $G$. Calling the angle of incidence at $\mathrm{L} \beta^{\prime}$, and the refractive index of the material above $A B \mu$, we have

$$
\begin{gathered}
\overline{M F}=\overline{M M_{2}} \sin \alpha \\
\overline{M E}=\overline{M M_{2}} \sin \beta, \\
\sin \beta=\mu \sin \alpha . \\
\overline{M E}=\mu . \overline{M F},
\end{gathered}
$$

and

Hence

and thus $\overline{M E}$ and $\overline{M F}$ are equal optical paths. Thus the difference in optical length of the paths $\mathrm{MLM}_{2} \mathrm{R}$ and $\mathrm{MR}$ is $\overline{\mathrm{EL}}+\overline{\mathrm{LM}}_{2}$. But the triangles $\mathrm{M}_{2} \mathrm{LH}$, GLH being equal, $\overline{\mathrm{LG}}$ is equal $\overline{\mathrm{LM}}_{2}$, and hence the difference in optical length is $\overline{\mathrm{EG}}$. But $\overline{\mathrm{EG}}=\overline{\mathrm{M}_{2} \mathrm{G}} \cos \mathbf{M}_{2} \mathrm{GE}$, and the angle $\mathrm{M}_{2} \mathrm{GE}$ is equal to $\beta^{\prime}$, since $\mathrm{M}_{2} \mathrm{G}$ and $\mathrm{N}^{\prime} \mathrm{L}$ are each perpendicular to CD. Thus if we call the thickness $\overline{\mathrm{M}_{2} \mathrm{H}}$ of the film $T$, we have that the difference in optical length of the two paths which meet at $R$ is

$$
2 T \cos \beta^{\prime} \text {. }
$$

Taking account of the fact that there is a change of phase ${ }^{1}$ of $\lambda / 2$ at the reflection at $M_{1}$, we shall get an interference band at $R$ if

$$
\begin{gathered}
2 T \cos \beta^{\prime}+\frac{\lambda}{2}=(2 n-1) \lambda / 2 \\
2 T \cos \beta^{\prime}=n \lambda .
\end{gathered}
$$

and we may consider that this relation fixes the value of $\beta^{\prime}$ for any given band, and the values of $\beta^{\prime}$ for the different bands are obtained by giving to $n$ the values $1,2,3,4, \&$ c.

If we now suppose $\beta^{\prime}$ given, we may obtain an expression for the position of $R$. Let $\phi$ be the angle $\mathbf{M R M}_{2}$, and $\mathrm{D}$ the distance $\mathbf{M}_{2} \mathrm{R}$, then we have from the triangle $\mathbf{M M}_{2} \mathrm{R}$

$$
\frac{\overline{M_{2} R}}{\overline{M M_{2}}}=\frac{\sin (\pi / 2-\alpha)}{\sin \phi}=\frac{\cos \alpha}{\sin \phi},
$$

and from the triangle $\mathrm{MM}_{2} \mathrm{G}$

Hence

$$
\frac{\overline{G M_{2}}}{\overline{M M_{2}}}=\frac{\sin (\pi / 2-\beta)}{\sin \beta^{\prime}}=\frac{\cos \beta}{\sin \beta^{\prime}} .
$$

$$
D=\overline{M_{2} R}=2 T^{\prime} \frac{\cos \alpha \sin \beta^{\prime}}{\sin \phi \cos \beta} .
$$


But in practice the angles $\alpha, \beta, \beta^{\prime}$, and $\phi$ are all small, hence approximately

$$
D=\frac{2 T \beta^{\prime}}{\phi}
$$

But

$$
\phi=N^{\prime} M R-N^{\prime \prime \prime} M_{2} R,
$$

and

$$
\sin N^{\prime \prime \prime} M_{2} R=\mu \sin N^{\prime \prime} M_{2} L=\mu \sin \left(\beta^{\prime}-\theta\right),
$$

or since the angles are small

$$
\begin{aligned}
& N^{\prime \prime \prime} M_{2} R=\mu\left(\beta^{\prime}-\theta\right), \\
& \phi=\alpha-\mu\left(\beta^{\prime}-\theta\right), \\
& \alpha=\beta / \mu=\left(\beta^{\prime}+\theta\right) / \mu \\
& \phi=2 \theta / \mu .
\end{aligned}
$$

and hence

or since

Hence

$$
D=\frac{\mu \beta^{\prime} T}{\theta} \text {. }
$$

Remembering that $\beta^{\prime}$ is given by equation (1), equation (2) shows that if $T$ is very small, i.e. the air film is very thin, then $\mathrm{D}$ is very small also, unless $\theta$ is exactly zero; that is, the interference bands apparently coincide with the film, and to see them an observer's eye, or a telescope, should be focused on the film. If $\theta=0$, and $T$ is not infinitely small, then $\mathrm{D} \doteq \infty$, and the bands appear to be at an infinite distance, and the observing telescope should be focused for parallel rays. If $\theta$ is small, while $T$ is considerable, the value of $\mathrm{D}$ will vary rapidly with $\beta^{\prime}$. Now, if $\mathrm{K}$ is the object-glass of the observing telescope, and $\mathrm{R}^{\prime}$ is the conjugate focus of $R$, any phase difference which exists at $R$ will be reproduced at $R^{\prime}$, and hence if there is interference at $R$ there will be interference at $R^{\prime}$, and $\mathrm{R}^{\prime}$ will be a point on one of the interference bands seen in the telescope. If the neighbouring band is seen at $\mathrm{R}^{\prime \prime}$, then by drawing a similar figure, in which the difference in phase is now $\lambda$ greater than that at $R$, we should find that the rays which interfere are incident on $\mathbf{A B}$ and CD at a different angle, i.e. $\beta^{\prime}$ would be different. But if $\beta^{\prime}$ is different, $\mathrm{D}$ will be different, and hence all the interference bands cannot be in focus at the same time.

Again, suppose that while $\theta$ remains constant $T$ is varied, as is the case in the Michelson interferometer ( $\$ 131)$, then $\mathbf{D}$ will also vary, and, therefore, the focus of the observing telescope will have to be continually altered unless $\theta$ is zero ; that is, the film of air is parallel sided.

131. Michelson's Interferometer.-The principle on which this instrument depends is as follows :-A ray of light is incident in the direction Io (Fig. 141) on a parallel sided plate of glass $G_{1}$. Part of the light passes straight through this plate and through a similar plate $G_{2}$, and is reflected back at a plane mirror $\mathbf{m}_{1}$. On again reaching the 
surface of the plate $G_{1}$ part of this light will be reflected in the direction $\mathrm{OR}_{1}$. Part of the incident beam, instead of passing through the plate $\mathbf{G}_{1}$, will be reflected at its second surface. and will reach the mirror $\mathbf{M}_{2}$. Here it will be reflected, and part passing through the plate $G_{1}$ will travel in the direction $\mathbf{R}_{2}$. Thus the incident ray of light has been split up into two rays which, having traversed different paths, are finally brought

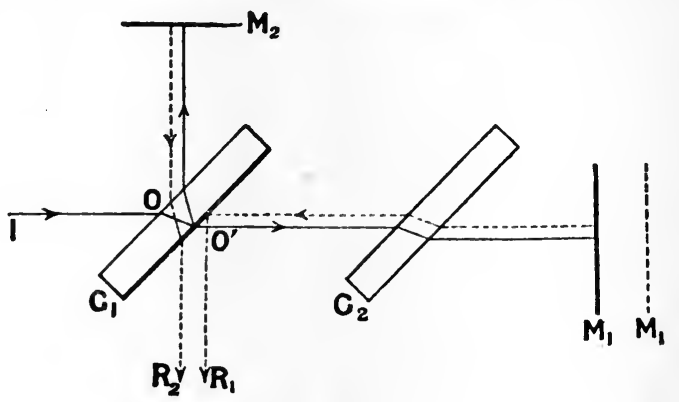

FIG. 141.

back to traverse the same path. In the figure the reflected rays from the mirrors $\mathbf{M}_{1}$ and $\mathrm{M}_{2}$ are shown slightly displaced so as to avoid confusion. In reality the rays $R_{1}$ and $R_{2}$ coincide. The object of the plate $G_{2}$ is that by its introduction the two rays traverse exactly the same thickness of glass, otherwise the light reflected from the mirror $\mathbf{M}_{\mathbf{1}}$ would only traverse the glass plate once, while that reflected in the mirror $\mathbf{M}_{2}$ would do so three times.

The two systems of waves, which have traversed the two paths, are in such a condition that they can interfere, and thus a system of interference bands will be seen on looking in the direction $R_{1} \mathbf{O}^{\prime}$. If one of the mirrors, say, $\mathbf{M}_{1}$, can be moved parallel to itself the bands will appear to move, and it is evident that a movement of the mirror through a distance of $\lambda / 2$ will cause the displacement of each band into the position previously occupied by the adjacent band.

A form of Michelson interferometer is shown in Fig. 142. One of the mirrors, $\mathbf{M}_{1}$, is carried on a carriage $\mathbf{\Delta}$ which moves along carefully worked ways planed on the bed. This carriage is moved by means of a fine micrometer screw. A divided head $B$ is attached to the screw, and a tangent screw $\mathrm{c}$ allows of the micrometer screw being rotated slowly; at the same time a divided head attached to the tangent screw serves for the determination of the fractions of a division of the scale on $\mathbf{B}$. The second mirror, $\mathbf{s}_{2}$, is capable of slight rotation about a vertical and horizontal axis by means of the screws $\mathrm{F}$ and $\mathrm{E}$. The points of these screws do not press directly against the mirror or its frame, but against two small springs which are attached to the frame. The frame itself is carried by a small horizontal metal pillar, which is screwed to an upright attached to 
the bed. The pressure of the screws against the springs cause this pillar to bend slightly, and thus the plane of the mirror is moved. This arangement allows of a very delicate adjustment of the plane of the mirror, for, owing to the elasticity of the springs, the frame does not move through anything like the distance through which the points of the screws are moved. The glass plate $G_{2}$ is also carried on a pillar attached

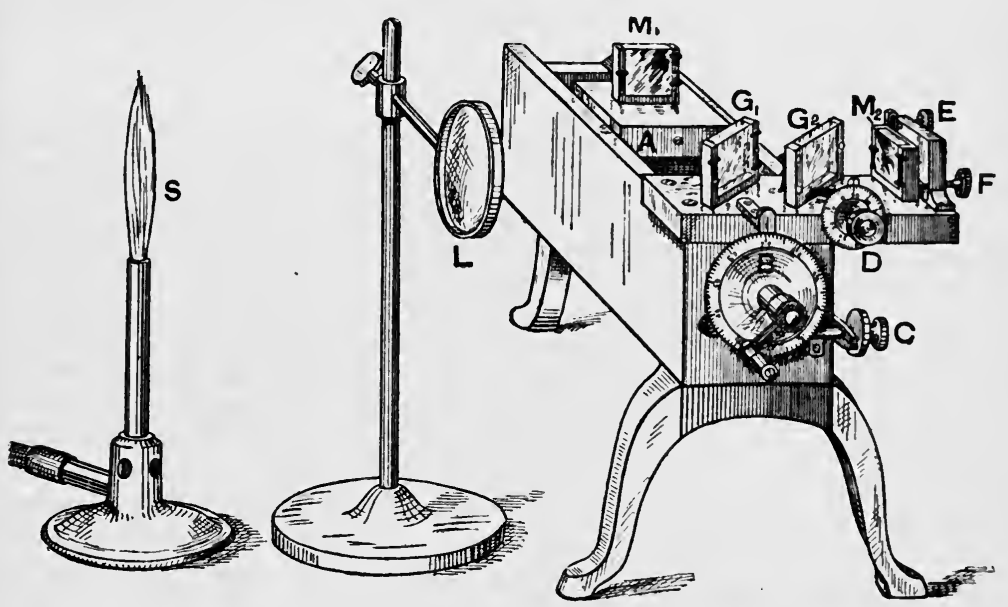

FIG. 142.

to the bed, and a screw $\mathrm{D}$, with a divided head acting on a spring attached to the support, serves to give this glass plate a small rotation about a vertical axis.

Adjustment of the Interferometer.-By means of a scale or pair of dividers adjust the movable mirror $M_{1}$ so that it is at the same distance from $G_{1}$ as is the fixed mirror $M_{2}$.

Place either a bright sodium flame $\mathrm{s}$ or a white light at the principal focus of the lens $\mathrm{L}$, and fix a fine needle point between the lens and $\mathrm{G}_{1}$. Four images of the needle point will in general be seen, two formed by reflection in each of the mirrors $M_{1}$ and $\mathbf{M}_{2}$. This is owing to the fact that light is reflected from both sides of the glass plate $G_{1}$, the way in which the four images are formed being indicated in Fig. 143. Interference can take place when either of the reflected beams (1) and (2) coincide with either (3) or (4). When (2) coincides with (3) the interfering beams have traversed equal thicknesses of glass. When, however, (1) coincides with (4), one beam (4) has traversed five times the thick- 
ness of glass traversed by the other (1). Hence it is (2) and (3) that should be brought together, and the screws at the back of the mirror $\mathbf{M}_{2}$ must be adjusted till these two images are exactly superposed. It is easy by screening either of the mirrors with a card to see which pair belong to the adjustable mirror. When this adjustment has been carefully made, monochromatic light must be used at $\mathrm{s}$, when the fringes ought to be visible. If they are not visible, and a slight alteration in the adjustment of $\mathrm{M}_{1}$ does not bring them into view, the setting with the needle point must be repeated.

We may look upon Michelson's instrument as being an arrangement in which interference takes place in the film of air inclosed between the mirror $\mathbf{M}_{1}$ and the image of the mirror $\mathbf{M}_{2}$ formed by reflection in the

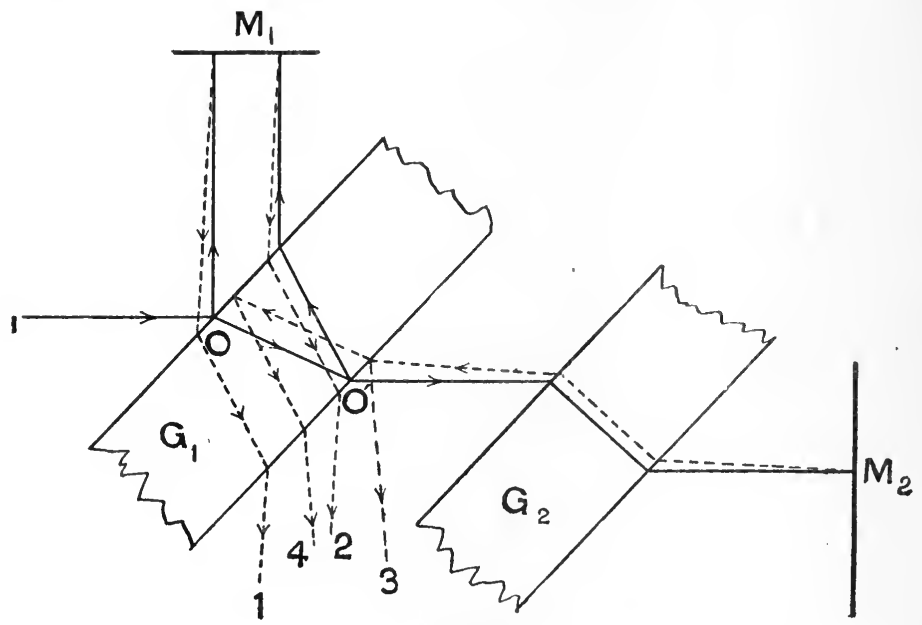

FIG. 143.

glass plate $G_{1}$. Now, when the mirror $M_{2}$ is adjusted so that its image is parallel to $\mathbf{M}_{1}$, the interference fringes will be circles, with their centres at the foot of the perpendicular drawn from the observer's eye to the mirror $\mathrm{M}_{1}$. The air film having parallel sides, it follows, from what has been said in the preceding section, that the fringes are at infinity, and hence an observing telescope should be adjusted for parallel rays.

If the image of $\mathrm{M}_{2}$ is inclined at a finite angle $\theta$ to $\mathrm{M}_{1}$, then the fringes are approximately arcs of circles, having their centres on a line perpendicular to the line of intersection of the image and mirror. The greater the value of $\theta$, the narrower will be the bands. In this case the apparent position of the bands will depend on $\theta$ and the thickness of the air film, and will vary as this thickness varies. Hence as $M_{1}$ is 
moved, the focus of the observing telescope will need to be continually altered if the bands are to appear as sharp as possible. For this reason it is advisable whenever possible to use the circular bands corresponding to $\theta=0$. By altering the adjusting screws at the back of the mirror $\mathbf{M}_{2}$, any desired form of bands can be obtained.

Since with white light there are only a few fringes formed, these will only appear when the optical length of the two paths are exactly equal. The most convenient way of finding the position of the movable mirror for which the paths are equal is to adjust $\mathbf{M}_{2}$ so that the fringes are arcs of very large circles. It will then be found, as the carriage is moved backwards and forwards, that the direction of the curvature of the fringes changes when the carriage passes through a certain position. Note the reading on the tangent screw and on the scale $B$ when the fringes are just perceptibly curved, first in one direction and then in the other. Having placed a source of white light at $s$, move the carriage very slowly from one of the positions just found to the other, when the interference bands will be seen to sweep across the field. The movement must be very slow, otherwise the bands will pass across so rapidly as not to be noticed. For this reason it is not a bad plan to leave the sodium flame in place, putting the source of white light just behind it. By watching the motion of the sodium fringes, the speed at which the tangent screw is turned can then be regulated, and, what is also of importance, the eye can be kept focused on the bands. When making measurements with the interferometer, using straight fringes, it will be necessary to observe the fringes with a low-power telescope fitted with a cross-wire, so as to provide some fixed point with reference to which the movement of the fringes can be noted.

(a) To determine the pitch of the screw of the interferometer in terms of the wave-length of sodium light. Adjust the mirror to give circular fringes, and move the carriage towards $G_{1}$ till the fringes begin to get indistinct, and read the position of the scale $B$ and the tangent screw. Then slowly move the carriage away from $G_{1}$, counting the number of fringes which vanish at the centre till the screw has made about one whole turn, and again read the scale and the tangent screw. If $n$ fringes have vanished, the distance through which the carriage has moved is $n \lambda / 2$. Hence if the screw has made $x$ turns, as given by the reading at the commencement and end, the pitch of the screw is $n / 2 x$ times the wave-length of sodium light.

(b) A stage micrometer divided, say, into tenths and hundredths of a millimetre can be calibrated by use of the interferometer by attaching the micrometer to the carriage and clamping a microscope to the bed so that it can be focused on the scale. Then by counting the number of fringes which vanish while the scale is moved, so that the cross-wire of the microscope coincides in turn with each of the divisions of the scale, the distance between successive divisions in terms of the wave-length of sodium light is at once obtained.

By using a mercury tube or lamp and the arrangement shown in 
Fig. 136, the wave-lengths of the mercury lines can be used in place of the sodium.

(c) The interferometer may be used to examine whether a given source of light produces strictly monochromatic light, or whether, say, light of two nearly identical wave-lengths, as also to determine in such a case the difference in the wave-lengths of the two lines. Either the yellow lines of mercury or the sodium lines may be employed for this purpose. Suppose that we adjust the instrument to give approximately straight bands, and start with the two paths of the interferometer of equal length, that is, with the central band produced with white light on the cross-wire, and that we use a source of light the spectrum of which consists of two neighbouring lines $A$ and $B$, having wave-lengths $\lambda_{a}$ and $\lambda_{b}$. Each of these kinds of light will produce a set of interference bands, and these two sets will coincide in the position in which the paths are equal. If, now, we increase one of the paths by moving back the movable mirror, since the wave-length of the two lights are different, so that the bands due to the smaller wave-length are nearer together than those due to the other, it follows that the agreement between the two sets of fringes will gradually be lost. Thus, after a certain time, a bright band for one system will coincide with a dark band for the other, and hence, if the two lines are equally bright, one set of fringes will exactly destroy the other, and no fringes will be seen. On further increasing the difference in the two paths, the fringes will gradually reappear and get more and more distinct, till finally there is exactly one whole wave-length more of the system $A$ than of the system $B$ in the distance by which the one path has been increased. Hence if the movable mirror has been moved through a distance $d$ between two maxima of distinctness of the bands the increase of path is $2 d$, and the number of waves of the system $A$ in this distance is $2 d / \lambda_{a}$, and the number of waves in the system $B$ is $2 d / \lambda b$.

Hence as there is one more wave in the system $A$ than in the system $B$ we have

$$
\begin{aligned}
& \frac{2 d}{\lambda_{a}}-\frac{2 d}{\lambda b}=1 ; \\
& \frac{\lambda_{a}}{\lambda_{b}}=1-\frac{\lambda_{a}}{2 d} .
\end{aligned}
$$

Examine by this method the yellow light given by a mercury tube, and determine the distance through which the carriage has to be moved between consecutive maxima of the bands, and taking the value of $\lambda_{a}$ from the table on p. 309 , calculate $\lambda_{b}$. Repeat the experiment, using sodium light.

132. Newton's Rings.-If a convex lens, having a radius of curvature of $R$, is placed in contact with a plane surface of glass, and the point of contact examined, there will be seen a system of rings both when the transmitted light and the reflected light is examined. If white light is 
used, only a few rings, and these coloured, will be visible. With monochromatic light, however, a large number of rings are visible. These rings are due to the interference produced in the air film between the two glass surfaces. The theory of these rings is given in books on light (see Watson's Physics, § 379), and it is shown that in the case of the transmitted light, which falls normally on the plane surface, the radii $r$ of the successive dark rings are given by

$$
r_{n}{ }^{2}=(2 n+1) \frac{\lambda^{\prime} R}{2}
$$

where $\lambda^{\prime}$ is the wave-length of the light in the medium between the surfaces, and $n$ is any whole number. If $\lambda$ is the wave-length in air, and the medium between the surfaces has a refractive index $\mu$, then

$$
\mu=\lambda / \lambda^{\prime} \text {. }
$$

Hence for the dark rings we have

$$
r_{n}^{2}=(2 n+1) \frac{\lambda R}{2 \mu} .
$$

Similarly the radii of the bright rings are given by

$$
r_{n}^{2}=\frac{n \lambda R}{\mu}
$$

In general the medium between the surfaces is air, so that $\mu=1$. Hence knowing $R$ we can determine the wave-length of the light by measuring the diameters of the rings, or conversely, knowing $\lambda$ we can obtain $R$.

In the case of reflected light, the angle of incidence being $\beta$, we have

for dark rings

$$
\boldsymbol{r}_{n}^{\prime}=\frac{n \lambda R}{\cos \beta}
$$

for bright rings

$$
r_{n}^{\prime}=\frac{(2 n+1) \lambda R}{2 \cos \beta},
$$

where the symbols have the same meaning as before.

If in place of using a plane surface a concave surface of radius $R^{\prime}$ greater than $R$ is employed, then the radii of the dark rings are given by

Transmitted light

$$
\boldsymbol{r}_{n}^{2}=\frac{2 n+1}{2} \lambda\left\{\frac{R^{\prime}-R}{R^{\prime} R}\right\}
$$

Reflected light

$$
r_{n}^{2}=\frac{n \lambda}{\cos \beta}\left\{\frac{R^{\prime}-R}{R^{\prime} R}\right\} \text {. }
$$

The formulæ given above are not in general applicable, since it is impossible to be certain (1) that the lens and plate are in contact at the centre ; and (2) if they are in contact, that there is no deformation of the surfaces 
at the point of contact due to the pressure employed to keep them in contact. This difficulty is got over by measuring the diameters of two rings, say, the $x$ th and the $(x+n)$ th. In the case of the dark rings by transmitted light we then have, if $d_{0}$ and $d_{n}$ are the diameters,

$$
\begin{aligned}
& \left(\frac{d_{o}}{2}\right)^{2}=(2 x+1) \frac{\lambda R}{2}, \\
& \left(\frac{d_{n}}{2}\right)^{2}=(2 x+2 n+1) \frac{\lambda R}{2} .
\end{aligned}
$$

Hence

$$
\frac{d_{n}^{2}-d_{0}^{2}}{4}=n \lambda R
$$

in which expression it is no longer necessary to know the number of the rings being used, but only the number of rings between the two of which the diameters have been measured.

Similarly for reflected light we have

$$
\frac{d_{n}^{2}-d_{v}^{2}}{4}=\frac{n \lambda R}{\cos \beta},
$$

and for the case of two lenses in contact

Transmitted

$$
n \lambda=\frac{d_{n}^{2}-d_{0}^{2}}{4}\left(\frac{1}{R}-\frac{1}{R^{\prime}}\right)
$$

Reflected

$$
n \lambda=\frac{d_{n}^{2}-d_{0}^{2}}{4}\left(\frac{1}{R}-\frac{1}{R^{\prime}}\right) \cos \beta
$$

133. Measurement of the Wave-length of Light by Newton's Rings.-Either the transmitted or reflected system of rings can be used to deduce the wave-length of the light. Since, however, it is difficult to measure the angle of incidence $\beta$ if the reflected system is used, an arrangement allowing normal incidence to be employed is advisable. The advantage in using the reflected system is that the dark rings are much darker than is the case in the transmitted system, for in this latter, owing to the light which has suffered two reflections being very much more feeble than that which has passed straight through the film, the interference is only partial, and the rings are by no means black.

An arrangement which will allow of the reflected system being examined with normal incidence is shown in Fig. 144. It consists of a small wooden box A open on two sides and the top. A piece of plate glass B, selected by the method described in $\S 142$, is cemented into a groove cut in one of the sides so as to be at an angle of $45^{\circ}$ with the horizontal. 
The inside of the box is blacked, and the plane plate of glass $\mathrm{o}$ and the lens $\mathrm{D}$ are placed on the bottom. A Bunsen flame $\mathrm{s}$, coloured with common salt, and a lens $\mathrm{L}$ serve to supply the light, which is reflected downwards by the glass plate $\mathrm{B}$. A travelling microscope M serves to measure the diameters of the rings. The lens $D$ may be a plano - convex lens having a focal length of about 100 centimetres.

When performing an experiment, first measure the radius of curvature of the lens D, either with a spherometer (\$ 15), an optical

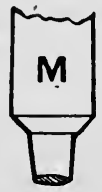
lever ( $\$ 23)$, or by one of the optical methods given in $\S 141$. Then very carefully clean the surface of the lens and of the piece of plate glass $c$, and having placed the lens in contact with the glass, frx them together with some soft wax so that the centre of the rings seen is near the centre of the lens.

Since in this case the thickness of the air film used is small, and the angle between the two surfaces enclosing the film is not zero, or even very small, the rings appear as if located within the film ( $\$ 130)$. Hence if the microscope $M$ is focused on a speck of dust or mark on the upper surface of the plate $\mathrm{c}$ the rings will be seen, and then the focus can be adjusted till they appear most distinct. It will generally be found advisable to place a screen to cut off stray light, and the lens I may with advantage be placed over a hole in this screen.

Measure the diameters of one of the broad rings near the centre which can easily be identified. Then count out either ten or twenty rings and measure the diameter of this ring, and obtain the value of the wave-length of the light by the formula given in the preceding section.

134. Diffraction through a Slit and Limit of Resolution of a Telescope. - Suppose that a slit of breadth $A B$ (Fig. 145) equal to $a$ is placed before the object glass $\mathrm{L}$ of a telescope, and that a parallel beam of monochromatic light falls on the slit, the axis of the telescope op being parallel to the direction of the incident light. Then if a screen is placed at the focal plane $\mathbf{Q P Q}^{\prime}$ of the lens, since all parts of the wave-front $\mathbf{A B}$ will reach $P$ in the same phase, there will be a bright band formed passing through P. On either side of this bright band, which represents the image of the slit of the collimator used to produce the parallel beam of light, there will be a series of alternate dark and bright bands. Suppose that $Q$ is the centre of the first dark band. Then if фом is drawn 
through the centre of the lens, and $\mathbf{A B ^ { \prime }}$ is drawn through $\mathbf{A}$ perpendicular to $M Q$, if $A_{B^{\prime}}$ were a wave-front all parts of this wave would reach $Q$ in the same phase. Hence if instead of being a wave-front so that all parts of $\mathbf{A} \mathbf{B}^{\prime}$ are in the same phase the different parts of $\mathbf{A \mathbf { B } ^ { \prime }}$ are in different phases, then the disturbances which reach $Q$ from $\mathbf{A B}^{\prime}$ will be in different phases, but the relation between the phases at $Q$ will be the same as that which exists between the phases of the different parts of $\mathbf{A B}^{\prime}$. If, now, $\mathbf{B B}^{\prime}$ is

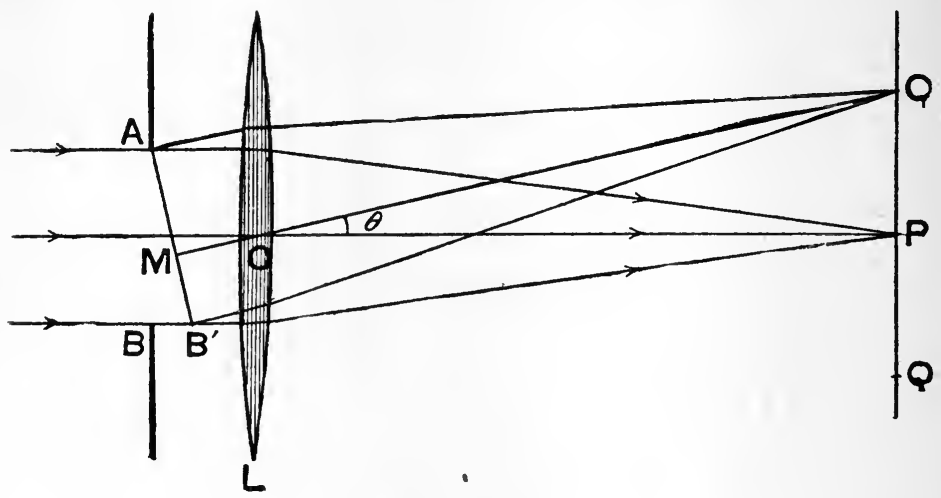

FIG. 145.

equal to $\lambda$, where $\lambda$ is the wave-length of the light, the disturbances which start from $\mathbf{A}$ and $\mathbf{B}^{\prime}$ will be in the same phase, but the disturbances which leave the intermediate points will differ from the phases of these two points by amounts varying from 0 to $2 \pi$. Hence by dividing $\mathbf{A B}$ into two parts there will always be an element in one half which sends a disturbance to $Q$, which is exactly opposite in phase to the disturbance sent to $Q$ by the corresponding element of the other half, so that these disturbances will interfere at $Q$, and so a dark band will pass through this point. Now the triangles $\mathbf{A B B}^{\prime}$ and $\mathbf{O P Q}$ are similar. Hence

$$
\frac{\overline{B B^{\prime}}}{\overline{A B}}=\frac{\overline{P Q}}{\overline{O P}} \text {. }
$$

Thus calling the distance QP between the first dark band and the central bright band $x$, and the focal length $\overline{\mathrm{OP}}$ of the lens $F$, we have

$$
\overline{B B}^{\prime}=\lambda=\frac{a x}{F^{\circ}}
$$

If we call the angle qop $\theta$, this relation may be written

$$
\lambda=a \theta \text {. }
$$

If in place of having a single beam of parallel light we have two beams 
inclined at a small angle $\alpha$, then each will produce its own central bright image bordered on either side by diffraction bands. Now if $\theta$ is the angle subtended at the centre of the lens by the interval between the centre of the central bright band and that of the first dark band, it is evident that if $\alpha$ is less than $\theta$, the central images due to the two sets of incident waves will overlap, and it will be impossible to distinguish the two images. If $\alpha$ is equal to $\theta$, then the central images will just touch; while if $\alpha$ is greater than $\theta$, the central bright images will be separated by one or more dark bands, and thus it will be possible to distinguish the two images, and hence detect the fact that the incident light consists of two distinct systems of waves. In other words, when $\alpha$ is greater than $\theta$ we are able to detect the fact that we are observing a double source. If $a$ is less than $\theta$, however, we shall be unable to detect the fact that the source is double. The condition

$$
a=\theta=\frac{\lambda}{a} \cdot . \cdot . \cdot . \cdot .
$$

is called the limit of resolution, and it will be observed that it depends alone on the width of the slit placed before the telescope objective and the wave-length of the light.

The resolving power of a telescope with apertures of different sizes can be obtained by setting up a piece of fine wire gauze containing about twenty meshes to the centimetre before a source of light, which to avoid complications, owing to imperfect achromatism of the lenses, may consist of a sodium flame, and having fitted a cap over the objective with an aperture of the desired size, determining the distance at which the individual wires of the gauze just cease to be distinguishable. If a rectangular aperture is used, the gauze must be set up with one set of wires parallel to the longer edge of the aperture, and it is the distance at which these wires cease to be distinguishable which has to be obtained. As the telescope is moved away from the gauze, the focus must be continually adjusted so that the object is seen as distinctly as possible.

If $d$ is the distance between adjacent wires of the gauze, and $\mathrm{D}$ is the distance between the objective of the telescope and the gauze when resolution ceases, the angle $a$ subtended by two adjacent sources is given by $\alpha=d / D$. When the aperture used in front of the objective is a slit of width $a$, it has been shown above that resolution will cease for a value of $a$ given by $a=\lambda / a$.

Determine experimentally the value of $\alpha$ for slits of different widths, and compare the results with the values calculated, that is, with the angle subtended by the wave-length of the light at a distance equal to the width of the slit. Slits $2 \mathrm{~mm}$. and $4 \mathrm{~mm}$. wide will be found suitable.

Perform the same experiment, using circular apertures having diameters of $5 \mathrm{~mm}$. and $10 \mathrm{~mm}$.

With a point source, such as a star, the radius of the first dark ring in 
the case of a lens of diameter $D$ and focal length $F$ has been calculated by Airy to be

$$
\frac{1 \cdot 22 F \lambda}{D} \text {. }
$$

Hence two point sources, which subtend an angle $\theta$ at the object glass will be distinguishable as two sources when

$$
\theta>\frac{1 \cdot 22 \lambda}{D} \text {. }
$$

It is of importance when making measurements on the resolving power of telescopes to make certain that all the light which passes through the object glass enters the pupil of the eye. Since the width of the pupil is about $3 \mathrm{~mm}$., if $\mathrm{D} \mathrm{cm}$. is the diameter of the object glass, the magnifying power $M$ of the telescope must be greater than $\mathrm{D} / \cdot 3$ (see $\S 146$ ). If the magnifying power is less than this, then the iris acts as a diaphragm, and the peripheral portions of the object glass are not used, and thus the effective aperture is reduced. (See Watson's Physics, § 357.)

135. Resolving Power of a Spectroscope.-(a) Prism Spectroscope. -Let $\triangle B C$ (Fig. 146) represent a section of a prism by a principal plane,

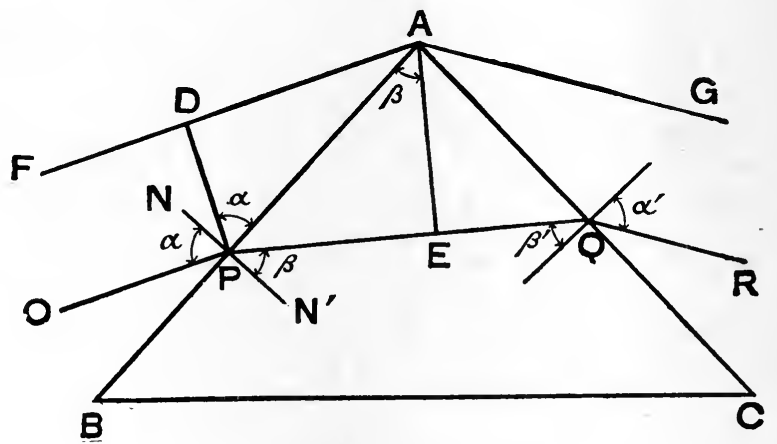

FIG. 146.

and FAG and OPQR the limiting rays of a rectangular pencil of parallel rays traversing the prism. If the refracting angle is $\theta$, and the angles of incidence and refraction at $\mathrm{P}$ are $\alpha$ and $\beta$, the corresponding angles at $Q$ being $\alpha^{\prime}$ and $\beta^{\prime}$, we have the following three equations :-

$$
\left.\begin{array}{l}
\mu=\frac{\sin \alpha}{\sin \beta} \\
\mu=\frac{\sin \alpha^{\prime}}{\sin \beta^{\prime}} \\
\theta=\beta+\beta^{\prime}
\end{array}\right\}, .,, ., \quad,
$$


Suppose, now, keeping a constant, we change the wave-length of the incident light from $\lambda$ to $\lambda+d \lambda$, as a result $\beta, \beta^{\prime}, \alpha$, and $\mu$ will all vary. Hence let us calculate the change $d a^{\prime}$ in the angle between the emergent beam and the normal. To do this we differentiate the equations (1) above, regarding $\alpha$ and $\theta$ as constants. Thus we obtain

$$
\begin{aligned}
0 & =\mu \cos \beta . d \beta+\sin \beta . d \mu \\
\cos \alpha^{\prime} . d \alpha^{\prime} & =\mu \cos \beta^{\prime} . d \beta^{\prime}+\sin \beta^{\prime} . d \mu \\
0 & =d \beta+d \beta^{\prime} .
\end{aligned}
$$

By eliminating $d \beta$ and $d \beta^{\prime}$ between these three equations, we get

$$
\begin{aligned}
\cos \alpha^{\prime} \cdot d \alpha^{\prime} & =\left(\frac{\sin \beta \cos \beta^{\prime}+\cos \beta \sin \beta^{\prime}}{\cos \beta}\right) d \mu \\
& =\frac{\sin \left(\beta+\beta^{\prime}\right) d \mu}{-\cos \beta}=\frac{\sin \theta \cdot d \mu}{\cos \beta} .
\end{aligned}
$$

Hence

$$
d \alpha^{\prime}=\frac{\sin \theta}{\cos \alpha^{\prime} \cos \beta} d \mu \text {. . . . . . . . . }
$$

When the prism is at minimum deviation $\alpha=\alpha^{\prime}, \beta=\beta^{\prime}$, and $\theta=2 \beta$.

Hence (2) reduces to

$$
d \alpha^{\prime}=\frac{\sin 2 \beta}{\cos \alpha \cos ^{1} \beta} d \mu=\frac{2 \sin \beta}{\cos \alpha} d \mu . . . .
$$

Now if PD is drawn perpendicular to the incident light, and the width of the incident or emergent beam is called $a$, so that $\mathrm{PD}=a$, we have $\cos a=a / \overline{P A}$. Further, if $A E$ is drawn perpendicular to $P Q$, and $P Q$ is called $t$, we have $\sin \beta=t / 2 \overline{\mathrm{PA}}$. Hence (3) reduces to

$$
d a^{\prime}=\frac{t}{a} d \mu \quad . \quad . \quad . \quad . \quad . \quad . \quad .
$$

which gives an expression for the separation, after passing through the prism, between the two beams corresponding to wave-lengths $\lambda$, and $\lambda+d \lambda$.

Now we have seen in $\S 134$ that if two beams of parallel rays of wavelength $\lambda$, and inclined at an angle $d \phi$, the section of the beams being rectangular and of breadth $a$, are received in the object glass of a telescope, the images formed will be separated if

$$
d \phi=\frac{\lambda}{a} \cdot \text {. . . . . . . . }
$$

Thus the slit of the collimator being of course adjusted to a very narrow width so that (5) applies, we shall be able to distinguish the images 
corresponding to the wave-lengths $\lambda$ and $\lambda+d \lambda$, i.e. the line will appear double, if $d a^{\prime}$ is greater than $d \phi$, that is, the limit is reached when

$$
d a^{\prime}=d \phi, \text { or } \frac{t}{a} d \mu=\frac{\lambda}{a} .
$$

Here $d \mu$ is the change in refractive index of the prism for a change of wave-length $d \lambda$. It is often convenient to replace $d \mu$ by $d \lambda$ in the expression for the limit of resolution. To do this we require to know how the refractive index changes with wave-length. In general it will be sufficiently accurate to take Cauchy's formula

$$
\mu=A+\frac{B}{\lambda^{2}}
$$

Differentiating

$$
d \mu=-\frac{2 B}{\lambda^{3}} d \lambda
$$

Substituting in (6)

$$
\frac{\lambda}{d \lambda}=-\frac{2 t B}{\lambda^{3}} \cdot \text {. . . . . . . . }
$$

The quantity $\lambda / d \lambda$ is called the resolving power of the spectroscope, and it depends on the wave-length of the light, the difference $t$ in the length of the path of the extreme rays in the prism, and a constant $B$ depending on the kind of glass of which the prism is made.

The dispersion $D$ of a prism is the ratio of the change in deviation to the change in wave-length, or since the change in deviation is equal to $d a^{\prime}$

$$
D=\frac{d a^{\prime}}{d \lambda}
$$

or substituting the value of $d a^{\prime}$ from (4)

$$
D=\frac{t}{a} \frac{d \mu}{d \lambda} \cdot \text {. . . . . . . }
$$

Hence the dispersion varies inversely as the width of the beam. If the prism is not at minimum deviation, then the above equation (8) still holds if $a$ is the width of the beam after its passage through the prism. Hence if a prism is placed so that the deviation is not a minimum, then in one position the dispersion will be greater, and in the other less, than at minimum deviation, since although $t$ will remain practically the same, $a$ will vary, as can easily be seen by drawing a figure. There will, however, be very little change in resolving power, for this is equal to $\frac{t d \mu}{d \lambda}$ and $t$ does not change much. Using a prism at greater than minimum deviation may therefore give greater angular separation between 
the centres of two lines, but it does not give greater definition, the lines themselves being broadened.

(b) Resolving Power of a Grating.-If there are $N$ lines per centimetre in the grating, and we observe in the $m$ th order spectrum, thc light being incident normally, we have

$$
\sin \theta=N m \lambda \text {. }
$$

If, now, the wave-length of the light changes from $\lambda$ to $\lambda+d \lambda$, and the angle $\theta$ changes to $\theta+d \theta$, we obtain the connection between $d \theta$ and $d \lambda$ by differentiating, $N$ and $m$ being constant. Thus

$$
d \theta=\frac{N m}{\cos \theta} d \lambda . . . \quad . \quad . \quad . \quad . \quad . \quad .
$$

If $b$ is the width of the grating, the width $a$ of the diffracted beam of light is given by $a=b \cos \theta$. Hence if the object glass of the telescope is wider than $a$, the smallest angle $d \phi$ between two heams of light which shall produce images which are visibly separated is given by

$$
d \phi=\frac{\lambda}{a}=\frac{\lambda}{b \cos \theta} .
$$

Hence the limit of resolution is reached when $d \theta=d \phi$, or

$$
\frac{\lambda}{d \lambda}=b N m \quad \text {. . . . . . . }
$$

that is, the resolving power of the grating is given by

$$
R=b N m \text {. . . . . . . }
$$

But $b N$ is the total number of lines in the grating, and $m$ is the order of the spectrum. Hence the resolving power is directly proportional to the total number of lines in the grating, and to the order of the spectrum.

The dispersion $D$ is defined, as in the case of the prism, by

$$
D=\frac{d \theta}{d \lambda}
$$

Hence from (9)

$$
D=\frac{N m}{\cos \theta} \cdot \text {. . . . . . . }
$$

that is, the dispersion is directly proportional to the order of the spectrum, and directly proportional to the number of lines per centimetre.

Hence while the resolving power depends on the total number of lines, and not on their closeness, the dispersion depends on the closeness of the lines, and not on the total number.

136. Measurement of the Resolving Power of a Prism Spectroscope.-Measure the refractive index of the prism for light of two or 
more known wave-lengths, and then calculate the value of the constant $B$ in Cauchy's formula

$$
\mu=A+\frac{B}{\lambda^{2}}
$$

from the observations taken in pairs.

Having adjusted the prism to minimum deviation for sodium light, adjust two cards so as to cut down the aperture of the object glass of the telescope till the two sodium lines just cease to be resolved. Make certain that the beam of light which passes through the prism entirely fills the portion of the object glass left uncovered, and then measure the width of the rectangular aperture left between the cards. This width gives the quantity $a$ (see previous section), and $t$ can be calculated by the formula

$$
t=\frac{2 a \sin \frac{\theta}{2}}{\cos \alpha},
$$

where $\theta$ is the angle of the prism. Then calculate the resolving power from equation (7), and compare your results with the value of $\frac{d \lambda}{\lambda}$ for the two sodium lines.

Repeat the experiment, using the two yellow mercury lines.

Also calculate the full resolving power of the instrument, using the full aperture of the prism. Two cases may occur. (1) The width of the beam may be limited by the prism. In this case, if $c$ is the width of the face of the prism, then

$$
t=2 c \sin \theta / 2 \text {. }
$$

(2) If the beam of light which passes through the prism entirely fills the object glass of the telescope, then it can be shown ${ }^{1}$ that

$$
t=82 \frac{2 d \sin \theta / 2}{\cos \alpha}
$$

where $d$ is the diameter of the object glass.

137. Measurement of the Resolving Power of a Grating.-Having mounted and adjusted the grating as described in $\$ 126$, bring the sodium lines in the first spectrum into the field, and by means of two cards reduce the width of the part of the grating being used till the sodium lines just cease to be resolved. Then calculate the resolving power by equation (10) of $\S 135$.

Repeat the experiment, using the second order spectrum, and compare your results with the value of $\lambda / d \lambda$ for the sodium lines.

Calculate the resolving power of the whole grating, and find for what value of $t$ the prism used in the last section would have the same resolving power.

1 Verdet, Legons d'optique physique, vol. i. p. 301. 
138. Rayleigh's Refractometer.-Suppose that before the object glass L (Fig. 147) of a telescope we place a screen pierced by two parallel slits, each of width $a$, separated by a distance $b$, and that a beam of parallel monochromatic light falls on the slits, the direction of the incident light being parallel to the axis of the telescope. If $\mathrm{QPQ}^{\prime}$ is a screen placed at the focal plane of the lens $L$, the two systems of waves which pass through the slits $B C$ and $D E$ will both reach $P$ in the same phase, and hence a bright band will be formed at this point. There

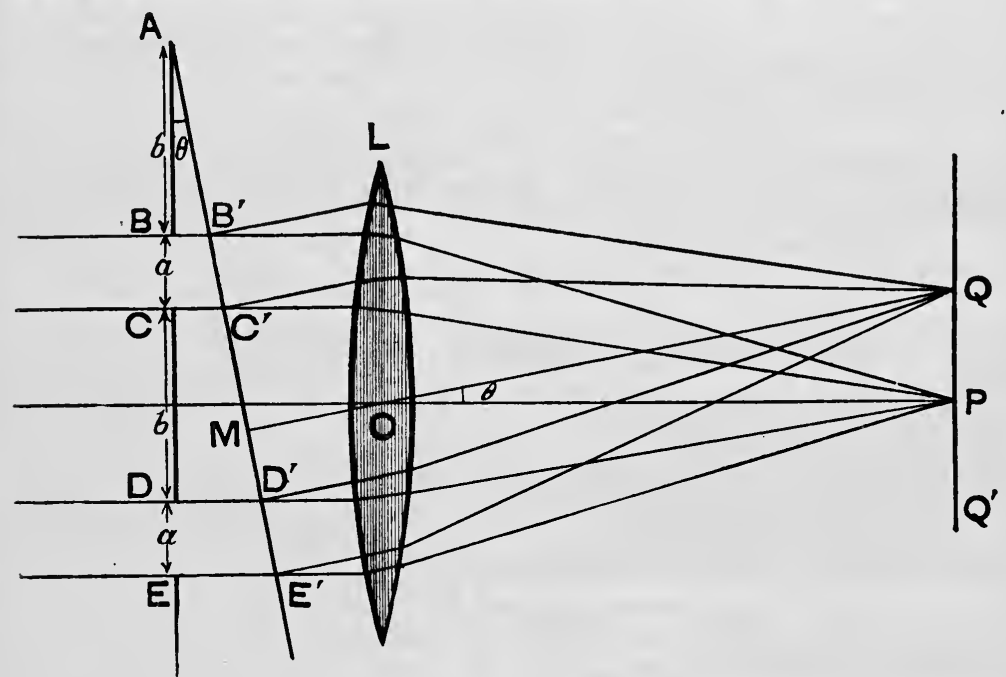

Fig. 147.

will also be produced diffraction bands by each of the slits, just as in the case of the single slit considered in $\S 134$. In addition, however, there will be a series of interference bands due to the mutual action of the disturbances which have traversed the two slits. We now proceed to determine the conditions under which these interference bands are produced.

Let us consider the illumination at a point $\mathrm{Q}$ in the focal plane at a distance $x$ from the central bright band at P. Draw the straight line QOM, and from a point $A$, such that $A B$ is equal to the distance $b$ between the slits, draw $\mathbf{A E}^{\prime}$ at right angles to $\mathbf{Q M}$. Then since $\overline{\mathbf{A E}}$ is equal to $2(a+b)$, we have that $\overrightarrow{\mathrm{EE}^{\prime}}=\overrightarrow{2 \mathrm{Co}^{\prime}}$ and $\overrightarrow{\mathrm{DD}^{\prime}}=2 \overrightarrow{\mathrm{BB}^{\prime}}$, and if we divide each of the two slits into an equal number of elements, this relation holds for each pair of elements. Hence if $\overline{\mathrm{EE}^{\prime}}=\lambda, \overline{\mathrm{co}^{\prime}}=\frac{\lambda}{2}$, and the disturbance at the points $\mathrm{E}^{\prime}$ and $\sigma^{\prime}$ will be in opposite phases, and each element of $\mathrm{k}^{\prime} \mathbf{D}^{\prime}$ 
will be in the opposite phase to the corresponding element of $\mathbf{c}^{\prime} \mathbf{B}^{\prime}$. The same will occur when

while if

$$
\overline{E E^{\prime}}=3 \lambda, 5 \lambda \ldots \text { or }(2 n+1) \lambda \text {, }
$$

$$
\overline{E E^{\prime}}=2 \lambda, 4 \lambda \ldots \text { or } 2 n \lambda,
$$

the phases of corresponding elements of $\mathrm{E}^{\prime} \mathrm{D}^{\prime}$ and $\mathrm{C}^{\prime} \mathbf{B}^{\prime}$ will agree.

Hence, since any phase difference which exists along $\mathbf{B}^{\prime} \mathbf{C}^{\prime}, \mathbf{D}^{\prime} \mathbf{E}^{\prime}$ will still exist when the disturbances reach $Q$, we see that there will be a minimum of illumination at $Q$ if

$$
\overline{E E^{\prime}}=(2 n+1) \lambda
$$

and a maximum of illumination at $Q$ if

$$
\overline{E E^{\prime}}=2 n \lambda \text {. }
$$

Now

$$
\frac{\overline{E E^{\prime}}}{\overline{E A}}=\frac{\overline{P Q}}{\overline{O P}}=\frac{x}{F^{\prime}}=\theta,
$$

where $F$ is the focal length of the lens, and $x$ is so small that $\theta=\tan \theta$.

Hence for a minimum of illumination at $Q$

$$
\begin{gathered}
x=\frac{F(2 n+1) \lambda}{2(a+b)}, \\
\theta=\frac{(2 n+1) \lambda}{2(a+b)},
\end{gathered}
$$

or

and for a maximum of illumination at $\mathrm{Q}$

or

$$
\begin{gathered}
x=\frac{F 2 n \lambda}{2(a+b)}, \\
\theta=\frac{2 n \lambda}{2(a+b)} .
\end{gathered}
$$

Thus the distance between consecutive bands, whether bright or dark, is given by

or

$$
\begin{gathered}
x=\frac{\lambda F}{a+b}, \\
\theta=\frac{\lambda}{a+b} .
\end{gathered}
$$

The distance between the bands being inversely proportional to the distance between the centres of the slits, and the wave-length of light being very small, it is evident that unless the slits are very near together the bands will be excessively fine.

Lord Rayleigh ${ }^{1}$ has employed the bands produced by two slits to

1 Collected Works, vol. iv. p. 364. This arrangement had previously been used by Arago, Euvres Completes, x. p. 321. 
compare the refractive index of two gases. The form of apparatus he used is shown in Fig. 148. A collimator c, with a very fine slit, s, is placed before a bright source of white light $\mathrm{L}$. The slit of the collimator may be made by making a fine scratch with a sharp knife on a piece of silvered glass, so as to remove the silver along a fine line. The gases to be compared are contained in two tubes $A$ and $B$, which are of the same length, and closed by two pieces of selected plate glass. A single piece of glass is used at either end to close the two tubes, and this glass projects slightly above the tubes, as shown at $\mathrm{F}$. The two slits are attached to a cap which fits on the telescope $T$, and are at such a distance apart that the light which enters through the lower part of one slit traverses the tube $A$, while that which enters through the lower part of the other traverses the tube B. ${ }^{1}$ The light which passes over the tops of the tubes

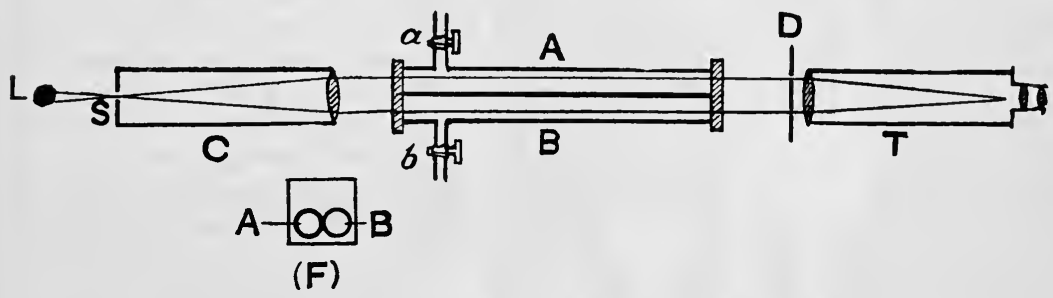

Fig. 148.

enters the upper parts of the slits and gives a series of bands, the position of which does not depend on the refractive index of the gases within the tubes, and thus serves as a reference mark.

Since the bands produced are very fine, the magnification produced by an ordinary eye-piece is not sufficient, and hence Lord Rayleigh used as eye-piece a small cylindrical lens. This lens consisted of a cylinder of glass having a diameter of $4 \mathrm{~mm}$. fixed to a cork which replaced the ordinary eye-piece of the telescope. The axis of the cylinder is adjusted to be parallel to the slit $s$ and the slits $\mathrm{D}$, the final adjustment being performed by turning the eye-piece till the bands are most distinct. One of the tubes $\mathbf{A}$ and $\mathbf{B}$ is filled with the gas to be tested and the other with air. The air tube is connected to a manometer, and an arrangement by

1 The tubes $\mathrm{A}$ and $\mathrm{B}$ can have a diameter of about 1 centimetre, the diameter of the object glass of the telescope and collimator being about 3 centi. metres. The slits $D$ will then be 1 centimetre apart, and may have a width of from 2 to 3 millimetres. The width of the field on which the bands are seen is inversely proportional to the width of the individual slits. Thus if very narrow slits are used a considerable field covered with bands may be seen, but the light will be so feeble as to render measurement difficult. These slits can be made by removing the silver from a piece of glass. Although Lord Rayleigh says that he used cheap telescopes, the author has found that it is only with fairly good lenses that satisfactory bands can be obtained. 
means of which the pressure of the air can be adjusted. Such an arrangement is shown in Fig. 149, the tube A being connected to the tube $(a)$, Fig. 148. The pressure is adjusted by raising or lowering the mercury

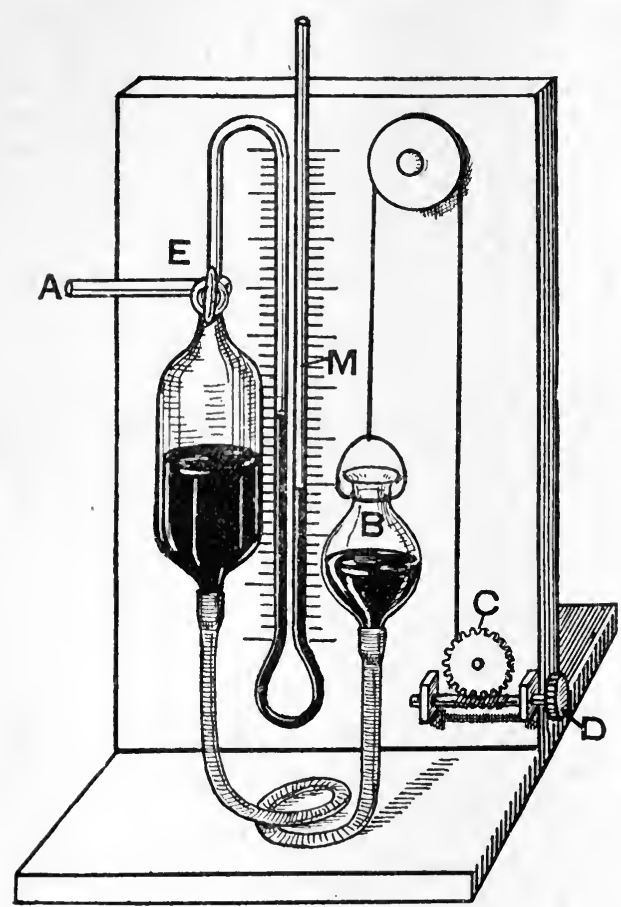

FIG. 149. reservoir B. This is done by means of a string which passes round a drum c, the drum being rotated by a tangent screw $D$. The pressure is read off on the manometer $\mathbf{M}$.

Two series of bands will be seen, one set formed by light which has passed over the top of the tubes, and the other by light which has passed through the tubes. The upper series is used as a reference mark, and by adjusting the pressure in the air tube the lower series of bands are brought to form continuations of the upper bands. This can be performed since, owing to a white source of light being used, the central band of either system is the only one which is free from colour, and hence can be identified.

Suppose the gas which is being experimented upon is at atmospheric pressure, and when the bands are in their central position the pressure in the air tube exceeds the external pressure by an amount $p$, the height of the barometer being $B$, and the temperature $t$. Then since the refractive index of the gas is equal to that of air at a pressure $B+p$, if $\mu_{a}$ is the refractive index of air at a pressure $B$ and temperature $t$, we have that the refractive index $\mu$ of the gas at this pressure and temperature is given by

$$
\mu-1=\frac{B+p}{B}\left(\mu_{a}-1\right) .
$$

For in the case of a gas we have

$$
\frac{\mu-1}{\rho}=\text { constant, }
$$

where $\rho$ is the density. Hence if $\rho^{\prime}$ is the density of air at a pressure 
$B+p$, and $\rho$ the density at a pressure $B$, and $\mu$ the refractive index at a pressure $B+p$, we have

$$
\frac{\mu-1}{\mu_{a}-1}=\frac{\rho^{\prime}}{\rho}=\frac{B+p}{B} .
$$

In the above we have supposed that the two tubes are of exactly the same length, and when both are filled with air at the same pressure the central band in the lower part of the field coincides with the central band in the upper part of the field. This assumption may be avoided by having a manometer and pressure-changing device connected with the tube containing the gas which is being tested, and making two comparisons, one at a pressure nearly atmospheric, and the other at a considerably different pressure.

Calling $\mu-1$ the refractivity, let the refractivity of air at a pressure $p_{a}$ be $r_{a}$, the density being $\rho_{a}$, and the corresponding quantities at a pressure $p_{a}^{\prime}$ be $r_{a}^{\prime}$ and $\rho_{a}^{\prime}$. Also let the corresponding quantities for the gas be indicated by $p_{g}, r_{g} \rho_{g}$ and $p_{g}^{\prime} r_{g}^{\prime} \rho_{g}^{\prime}$. Then since the central band is brought back to the same position when the refractivities are $r_{a} r_{g}$ and $r_{a}^{\prime} r_{g}^{\prime}$ we have

But

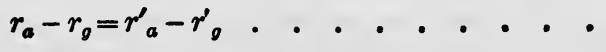

But

$$
\frac{r_{a}}{r_{a}^{\prime}}=\frac{\rho_{a}}{\rho_{a}^{\prime}} \text { and } \frac{r_{g}}{r_{g}^{\prime}}=\frac{\rho_{g}}{\rho_{g}^{\prime}} \text {. }
$$

Hence substituting in (1)

$$
\begin{gathered}
r_{a}-r_{g}=\frac{\rho_{a}^{\prime}}{\rho_{a}} r_{a}-\frac{\rho_{g}^{\prime}}{\rho_{g}} r_{o} \\
\frac{r_{g}}{r_{a}}=\frac{\rho_{a}-\rho_{a}^{\prime}}{\rho_{g}-\rho_{g}^{\prime}} \cdot \frac{\rho_{g}}{\rho_{a}}=\frac{p_{a}-p_{a}^{\prime}}{p_{g}-p_{g}^{\prime}} \frac{p_{g}}{p_{a}} .
\end{gathered}
$$

Since $r_{g}$ and $r_{a}$ are measured at different pressures, to get the ratio of the refractivities we must reduce one to the pressure of the other. If $r_{0}$ is the refractivity of the gas at a pressure $\mu_{a}$, we have

$$
\frac{r_{0}}{r_{g}}=\frac{p_{a}}{p_{q}}
$$

and hence

$$
\frac{r_{0}}{r_{a}}=\frac{p_{a}-p_{a}^{\prime}}{p_{g}-p_{g}^{\prime}}
$$

that is, the ratio of the refractivity of the gas to that of air is as the change in pressure in the air tube is to the change in pressure in the gas tube. 


\section{CHAPTER XXI}

\section{LENSES AND MIRRORS}

139. Measurement of the Focal Length of a Thin Lens.-It is shown in books on optics (Watson's Physics, § 352) that the focal length $f$ of a lens is connected with the distances $u$ and $v$ of the conjugate foci by the relation ${ }^{1}$

$$
\frac{1}{f}=\frac{1}{v}-\frac{1}{u}
$$

Hence by measuring the distances from the lens to any pair of conjugate foci we can calculate the focal length of the lens. Thus if we place an object on the axis of a lens at a measured distance from the centre of the lens, and then determine the position of the image of the object, we have

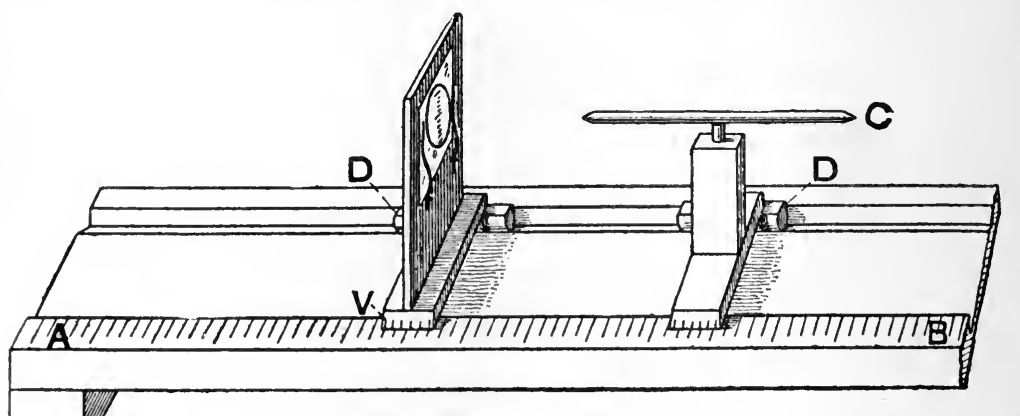

FIG. 150.

the requisite data for obtaining the focal length. The chief practical difficulty in carrying out this experiment is to determine the exact position of the image. The usual method employed is to use as the object a brightly illuminated and sharply defined body, and to move a screen about till the image formed on the screen appears most sharply defined. In practice, however, it is often extremely difficult to tell the exact position of the screen for which the definition is the best, the image appearing equally sharp over a considerable range of the position of the screen. $\bar{A}$ very much better method is to make use of the parallax method to deter-

1 We suppose all distances measured from the lens, and that the positive direction is opposite to that in which the light is travelling. 
mine the position of the image. The parallax method has the further great advantage that it does not necessitate the use of a dark room.

As object, a scale consisting of fine lines on clear glass may be used. Such a scale ought to have division lines about half a millimetre apart, and can easily be prepared by photographing a suitable scale divided, say, in tenths of an inch, by means of a camera. The image screen consists of a plate of plate glass on which a fine vertical line has been ruled. The object scale, the lens, and the glass to locate the image, are mounted on the uprights of an optical bench, such as that shown in Fig. 150.1 These uprights can be moved along a wooden base, to which is attached a scale $\mathbf{A B} 2$ metres long. The base-plates which carry the uprights have a short cylinder $D$ attached at one side, which slides in a groove in the board. The other end of the base rests on the scale, and is fitted with a vernier $v$, by means of which the position of the carriages can be read. A metal rod $\mathrm{c}$ with blunt points, and exactly $20 \mathrm{~cm}$. long, is supported in a horizontal position by a similar carriage, and serves for measuring the distance between the different pieces of apparatus. The scale used as an object is illuminated by placing behind it a flame, or even a brightly illuminated sheet of white paper. If a very accurate measurement is being made, since in non-achromatised lenses the focal length depends on the wave-length of the light, a source of monochromatic light, such as a sodium flame, should be employed.

(a) Convex Lens. - First Method.-In the case of a convergent lens the eye is placed behind the glass plate having the single vertical line, when an image of the scale will be seen on suitably moving the glass and the eye. The surface of the glass which carries the vertical line, which must be that surface which is turned towards the lens, is then adjusted by the method of parallax so that it lies in the same plane as the image; that is, the position of the glass must be adjusted till on moving the eye from side to side the line on the glass does not appear to move with relation to the division lines of the image.

If the image is so small that it is difficult, with the unaided eye, to see the division lines, then a short focus lens or positive eye-piece ${ }^{2}$ may be employed to examine the image and fiducial line.

When measuring a lens which is uncorrected for spherical aberration, it will be found an advantage to stop down the lens so that the light can only pass through the central portion, as in this way the image will be much improved in definition.

Having fixed the position of the image, the distance between the scale and the lens and between the lens and the image must be measured. For this purpose the readings corresponding to the three carriages are taken. Suppose that the reading for the carriage which carries the scale is $a$, that for the one which carries the lens $b$, and for the glass

1 The lens, \&c., are held against the uprights by two small spring clips. Each npright is pierced with a hole about $3 \mathrm{~cm}$. in diameter, the centres of all the holes being at the same height.

2 The thermometer reading glass shown in Fig. 67 is suitable for this purpose. 
plate c. Next place one end of the rod o against the scale, and bring the upright which carries the lens up till the point of the rod rests against the surface of the lens, and let the reading for this carriage be $b^{\prime}$. Next bring the surface of the lens and the glass plate on which the image was formed against the ends of the rod, and take the readings for the positions of the carriages $b^{\prime \prime}$ and $c^{\prime}$, say. Finally, measure the thickness of the lens, and let it be $t$. Now, when the difference between the readings on the scale for the object and the lens was $b^{\prime}-a$, the true distance was $20 \mathrm{~cm}$., i.e. the length of the rod c.

\section{Hence}

$$
20-\left(b^{\prime}-a\right)+t / 2
$$

will be the correction to be added to the difference between the readings for the two carriages to give in any case the true difference between the object and the middle of the lens. In the same way the quantity $20-\left(c^{\prime}-b^{\prime \prime}\right)+t / 2$ has to be added to the difference of the readings for the lens carriage and the image carriage to give the true distance between the middle of the lens and the image. Applying these corrections to the readings, obtain the values of $v$ and $u$, and hence calculate the value of the focal length of the lens. Repeat the measurements for different positions of the lens, and take the mean for the true value of the focal length.

When employing this method it is advisable to choose the distance between the object and image such that there is no very great difference between the distances of the image and object from the lens. If one of these distances is very much less than the other, then errors in measuring the smaller distance will cause a larger proportional error in the focal length deduced than would be the case if the two lengths measured were nearly equal.

Second Method.-If the screen on which the image is formed is placed at a distance greater than four times the focal length of the lens from the object, then there will be two positions of the lens in which a sharp image will be formed. Let $u$ and $u^{\prime}$ be the two distances of the lens from the object when a sharp image is formed on the screen, and $v$ and $v^{\prime}$ the corresponding distances from the lens to the screen, then

$$
\begin{aligned}
& \frac{1}{f}=\frac{1}{v}-\frac{1}{u} \\
& \frac{1}{f}=\frac{1}{v^{\prime}}-\frac{1}{u^{\prime}} .
\end{aligned}
$$

But from symmetry it is evident that, neglecting for the moment the signs,

$$
u=v^{\prime} \text { and } v=u^{\prime} \text {. }
$$

Also, if $D$ is the distance between the object and image screen, and $d$ is the distance between the two positions of the lens

$$
u=\underset{2}{\stackrel{D-d}{\longrightarrow}}
$$

and

$$
-v=\frac{D+d}{2} \text {. }
$$


Hence

$$
\begin{aligned}
\frac{1}{f} & =\frac{1}{\frac{D-d}{2}}+\frac{1}{\frac{D+d}{2}} \\
& =\frac{4 D}{D^{2}-d^{2}} .
\end{aligned}
$$

Thus by measuring the distance through which the lens has been moved, which can be directly obtained by noting the difference in the readings for the vernier attached to the support which carries the lens, and the distance from the object to the screen, we can calculate the focal length of the lens. As it is easier to measure the distance through which the lens has been moved than to measure distances up to the lens, there are distinct advantages in using this method.

Third Method.-In the case of a very long focus lens, both of the preceding methods would involve the use of an optical bench having a length at least equal to four times the focal length, and hence might not be available. In such a case we may make use of the fact that if a luminous object is placed at the principal focus of a convex lens, and the rays of light after passing through the lens are reflected back through the lens by means of a plane mirror, then they will form an image of the object at the principal focus, that is, alongside the object.

To apply this method the lens is mounted on the optical bench, and a plane mirror is mounted on a second stand and placed immediately behind the lens. A convenient form of object to use will be a needle point mounted in one of the carriages, so that the point is on a level with the centre of the lens. If the eye is placed on the side of the needle remote from the lens an inverted image of the needle will be seen, and by moving the carriage which supports the needle backwards and forwards till there is no parallax between the needle point and its image, the point can be adjusted to occupy the principal focus of the lens. When making this adjustment it is advisable to so adjust the plane mirror that the point of the image seems just opposite the point of the needle, when any parallax will easily be detected. The distance between the needle and the lens must then be measured by means of the measuring rod, and this distance is the focal length of the lens.

(b) Concave Lens.-The most generally convenient method of measuring the focal length of a concave lens is to combine it with a convex lens of shorter focal length, so that the combination acts as a convex lens capable of giving a real image, and determining by one of the methods considered above the focal length $F$ of the combination, and also the focal length $f^{\prime}$ of the convex lens alone. The focal length $f$ of the concave lens is then given by

$$
\frac{1}{f}=\frac{1}{F^{\prime}}-\frac{1}{f^{\prime}}
$$

Another way of performing this experiment, which does not necessitate 
the concave and convex lenses being placed in contact is to arrange the convex lens on the optical bench so as to give a real image at the point o (Fig. 151), then to insert the concave lens at $\mathbf{B}$, and determine the position of the image I. Having obtained the reading for the measuring rod when in contact with the. screen at $I$ and the lens $B$, this lens is removed, and without disturbing the position of the convex lens $\Delta$, the position of the image formed at $\mathrm{o}$ is determined. Then by obtaining the

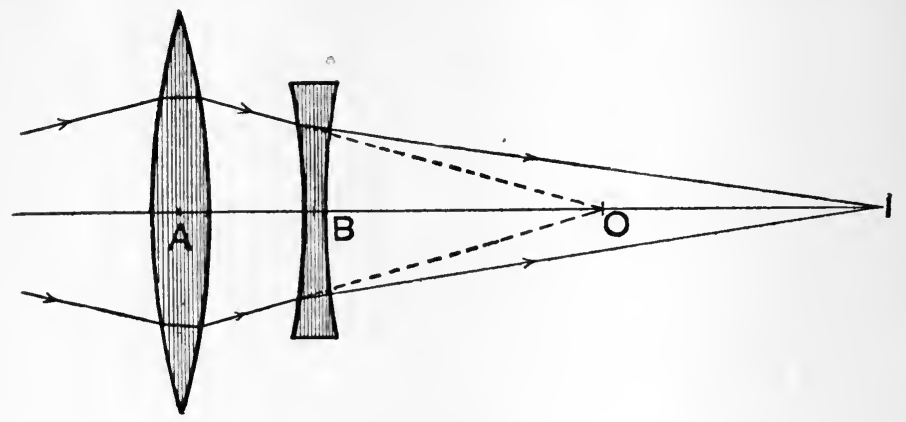

FIG. 151.

reading for the measuring rod when in contact with the screen at 0 , the distances Bo and BI are at once deduced. Since $O$ and I are conjugate foci for the concave lens, the focal length can be calculated by means of the formula

$$
\frac{1}{f}=\frac{1}{v}-\frac{1}{u}
$$

140. Measurement of the Radius of Curvature of a Concave Surface. - The concave surface of which the radius of curvature has to be measured is mounted on one of the carriages of the optical bench, while a needle is mounted on another carriage, the point of the needle being at the same level as the centre of the reflecting surface. On looking towards the surface from beyond the needle an inverted image of the latter will be seen. The needle is then moved till the needle and its image are in the same plane, as tested by the parallax method. The ease with which this adjustment can be made will be very much increased if the position of the needle is so adjusted that the image appears to just touch the point of the needle. A short focus lens or eye-piece may be used to assist in seeing when the image is in the same plane as the object, that is, when the needle point is at the centre of curvature of the reflecting surface. The distance between the needle point and the centre of the reflecting surface must then be measured by the measuring rod, and this distance is the radius of curvature of the surface.

When measuring, by this method, the radius of curvature of the 
surface of a lens, there will often be seen two images, one formed by reflection at the front surface, and one at the back. It is generally easy to distinguish which is which from the form of the two surfaces. If, however, there is -any ambiguity, by covering the back surface with a piece of blotting paper, moistened with glycerine, or even water, the intensity of the image formed by the back surface will be so much reduced as to render it easily recognisable.

In place of the parallax method of determining when the object and image are in the same plane, the following is sometimes more convenient, especially when dealing with large mirrors, or when it is desired to examine the accuracy with which the surface has been polished to a true sphere :- A small metal plate $\mathbf{A B}$ (Fig. 152) has a small circular hole
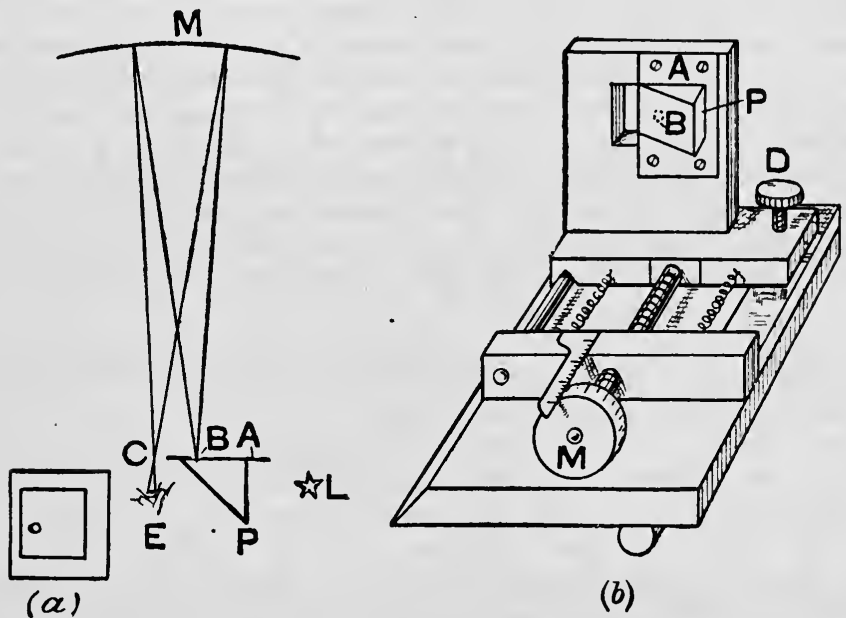

FIG. 152.

pierced at $B$, and a right-angled prism $P$ or piece of mirror is placed so as to reflect the light from a flame $L$ through this hole. The edge next $B$ of the metal plate is ground to a smooth and sharp edge. The metal plate with the prism is mounted on a stand as shown, and is placed on the axis of the nirror $\mathbf{M}$, so that the hole is a little to the right-hand side of the axis. In this way an image of the hole will be formed alongside the edge of the metal plate c. An eye placed at $\mathrm{E}$ will see the whole surface of the mirror illuminated. If, now, the metal plate is gradually moved to the left by turning the screw $\mathrm{D}$, the image at $\mathrm{c}$ will approach the edge, and, if the image is in the plane of the edge, as the light is intercepted by the edge, the mirror surface will appear to darken uniformly all over. If, however, the edge is nearer to, or farther away from, the mirror than the image, then the darkening of the surface of the 
mirror will appear to sweep gradually across the mirror. If the edge is nearer to the mirror than the image, then the darkening will appear to sweep across the surface of the mirror in the same direction as that in which the edge is being moved, while if the edge is farther away from the mirror than the image the contrary will be the case, namely, the darkening will appear to sweep across in the opposite direction to that in which the edge is moved. The distance of the plate from the mirror is therefore adjusted, by means of the micrometer screw $\mathrm{m}$, till the mirror seems to darken uniformly as the light is intercepted by the edge of the plate. When this adjustment is complete, the edge of the plate and the hole are both at the centre of curvature of the mirror.

If the edge of the metal plate, having been adjusted to lie at the centre of curvature of the mirror, is very slowly moved so as to intercept the light, then if the surface of the mirror is a true sphere, the surface will appear of a uniform tint throughout. If, however, the surface is not truly spherical, then the want of truth will be apparent just as the greater part of the surface appears to become dark, for the irregularities will appear bright. This method of testing the curvature of a spherical surface, particularly in the case of a mirror of large radius of curvature, is an excessively delicate one, and merely warming a portion of the mirror by touching it with the tip of the finger will, owing to the expansion caused by the heat communicated to the mirror, cause the figure to alter by an amount which is at once apparent; the spot which has been touched appearing bright and in marked relief.

In the case of a parabolic mirror, the radii of curvature of the various zones can be measured by this method, cardboard screens being used so that only the zone being tested is exposed.

141. Measurement of the Radius of Curvature of a Convex Surface.-In the case of a convex surface the image of a real object is virtual, and hence the methods described in the last section are not

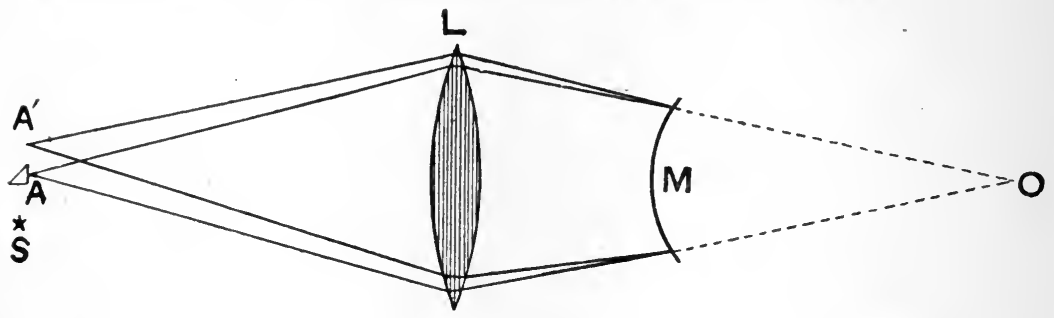

FIG. 153.

applicable. If, however, a virtual object is used, then a real image may be formed, and can be received upon a screen.

Place the stand carrying the metal plate with the prism shown in Fig. 152 on the optical bench at A (Fig. 153), and on another upright place a convex lens L. The convex mirror $\mathbf{M}$ is mounted on a third upright, and 
the positions of $\mathbf{L}$ and $M$ are adjusted so that the image is formed at $\mathbf{A}^{\prime}$ in the plane of the metal edge. Then, being careful not to disturb A or $\mathrm{L}$, take the reading on the optical bench scale corresponding to the mirror $\mathbf{M}$. Next remove $M$, and determine the position of the image o formed by the lens $L$; the distance between $O$ and $M$ will be the radius of curvature of the mirror ; for when the mirror was in place, the image at $\Lambda^{\prime}$ was alongside the object, that is, the rays of light which struck the mirror were reflected straight back, that is, they were incident normally on the mirror. But such normally incident rays would, if produced, pass through the centre of curvature of the mirror, and when so produced, or when the mirror is removed, they intersect at $o$, and hence $o$ is the centre of curvature of the mirror.

The radius of curvature of the surface of a thin convex lens can be measured by adjusting the position of the lens so that the light reflected from the back surface of the lens forms an image alongside the object. If $f$ is the focal length of the lens, and $D$ the distance between the lens and the object and image, the radius of curvature is given by

$$
r=\frac{f D}{f+D}
$$

We may obtain this expression as follows:- Since the object and image are alongside, it follows that the rays of light are incident normally on the back surface of the lens at which they are reflected.

Hence the rays which are refracted at the first surface of the lens proceed in the glass as if they would intersect (either actually or when produced backwards) at the centre of curvature of the back surface. Hence rays proceeding from an object at a distance $D$ from the front surface proceed in the glass after refraction at this surface as if they came from a point at a distance $r$; i.e. $D$ and $r$ are conjugate as far as refraction in a single spherical surface are concerned. Hence if $r^{\prime}$ is the radius of the surface, and $\mu$ the refractive index, we have, by the known formula for refraction at a single spherical surface,

$$
\frac{\mu}{r}-\frac{1}{D}=\frac{\mu-1}{r^{\prime}}
$$

which may be written

$$
\frac{1}{r}-\frac{1}{D}=\mu\left(\frac{1}{r^{\prime}}-\frac{1}{r}\right)-\left(\frac{1}{r^{\prime}}-\frac{1}{r}\right) .
$$

But if $f$ is the focal length of the lens

$$
\frac{1}{f}=(\mu-1)\left(\frac{1}{r^{\prime}}-\frac{1}{r}\right) .
$$


Hence

$$
\frac{1}{r}-\frac{1}{D}=\frac{1}{f}
$$

or

$$
r=\frac{f D}{f+D}
$$

142. To Test the Flatness of the Faces of a Piece of Glass.It is often necessary to test whether the surface of a plate of glass or a mirror is flat, and the following method will be found a convenient and expeditious one :-Prepare an artificial star by piercing a hole about half a millimetre in diameter in a screen of metal or cardboard, and placing a gas flame behind the screen. This star should be fixed up at as great a distance as possible from the table on which observations are to be made. Lay the reflecting surface flat on the table, and fix a telescope having a fairly large objective in a retort stand with its object glass quite near to the reflecting surface. Turn the telescope to view the artificial star directly, and focus carefully. Then turn the telescope so that an image is seen after reflection in the surface. If the image now seen in the telescope is as sharply defined as when seen by direct light, then the reflecting surface is plane. If the definition is impaired, this may be due - to two causes. In the first place, the surface, although it is not plane, may be a regular surface of revolution, such as a sphere, in which case the original definition can be regained by altering the focus of the telescope. If, however, no alteration in the focus of the telescope suffices to restore the definition, this indicates that the surface is irregular. In this case we may test whether parts of the surface are plane by covering the surface by a sheet of paper with a hole about a centimetre in diameter pierced in the middle. The paper screen is moved about so as to expose each part of the surface in turn, and by watching the image in the telescope it will at once be seen whether any part of the surface is plane.

In the case of a plate of glass the reflection at the second surface may cause some confusion as to which is the image corresponding to the upper surface. By placing a piece of wet blotting paper against the back surface, the intensity of the light reflected at this surface will be so much reduced that there will be no difficulty in distinguishing the two images.

If a surface which is known to be optically plane is available, the planeness of the surface of a glass plate can most quickly be tested by means of interference fringes formed in the air film enclosed between the two surfaces when they are placed in contact. Having cleaned the standard surface and that to be tested, place them in contact, and examine the interference fringes seen when a sodium flame is observed reflected from the adjacent surfaces. If the surfaces are both plane, the interference fringes will be straight and parallel throughout. If, however, either of the surfaces is at all irregular, then the fringes will be curved. By making a drawing of the fringes we obtain a contour map of the 
surface of the plate, the distance between adjacent contour planes being equal to half a wave-length of sodium light.

By examining in this way a number of pieces of thick plate glass it is generally possible in the course of a few minutes to pick out a few pieces which have at least one surface as nearly plane as expensively worked surfaces, and these selected pieces of plate glass will be found suitable for use in many optical experiments, such as in Michelson's interferometer.

It is sometimes necessary to measure the angle which the two plane faces of a piece of glass make with one another. This can conveniently be done by mounting the glass on the table of a spectrometer, the telescope and collimator having been adjusted for parallel light. If the two sides are exactly parallel, then only a single image will be seen when the light from the collimator is reflected by the surface of the glass into the telescope. If, however, the faces are not parallel, then two images

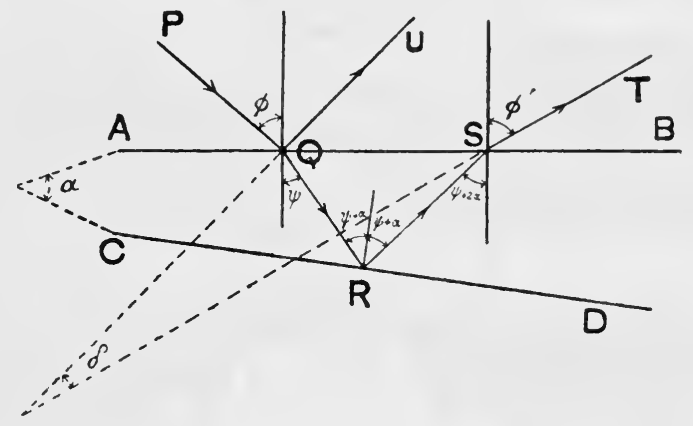

FrG. 154.

will be seen. To measure the angle, turn the glass round in its own plane till the two images of the slit are as widely separated as possible, and then by adjusting the telescope so that the intersection of the crosswires coincides first with one image and then with the other, and reading the spectrometer circle, measure the angle $\delta$ between the two images. If $\phi$ is the angle of incidence, and $\mu$ is the index of refraction of the glass, the angle $\alpha$ included between the two faces is given by

$$
a=\frac{\delta \cos \phi}{2 \mu \cos \psi}
$$

where $\delta$ and $a$ are supposed so small that we may take the angle as equal to the sine and $\psi$ is the angle, such that

$$
\sin \phi=\mu \sin \psi \text {. }
$$

This formula can at once be obtained, for if $\mathrm{AB}, \mathrm{CD}$ (Fig. 154) are the traces of the faces enclosing the glass plate, and PQU and PQRST are 
two of the rays forming the two images, we have, using the notation given in the figure for the angles

From (1)

$$
\begin{aligned}
& \sin \phi^{\prime}=\mu \sin (\psi+2 \alpha) . \quad . \quad . \quad . \quad . \quad . \\
& \delta=\phi^{\prime}-\phi \text {. . . . . . . . . }
\end{aligned}
$$

or

$$
\begin{aligned}
\sin \phi^{\prime} & =\mu(\sin \psi \cos 2 \alpha+\cos \psi \sin 2 \alpha), \\
2 \alpha & =\frac{\sin \phi^{\prime}-\mu \sin \psi}{\mu \cos \psi},
\end{aligned}
$$

if $\alpha$ is so small that we may take $\sin 2 \alpha=2 \alpha$ and $\cos 2 \alpha=1$.

From (2) in the same way

$$
\begin{aligned}
\sin \phi^{\prime} & =\sin (\delta+\phi) \\
& =\sin \delta \cos \phi+\cos \delta \sin \phi,
\end{aligned}
$$

or since $\delta$ is small

$$
\sin \phi^{\prime}=\delta \cos \phi+\sin \phi,
$$

also

$$
\begin{aligned}
\mu \sin \psi & =\sin \phi, \\
\therefore \quad \alpha & =\frac{\delta \cos \phi}{2 \mu \cos \psi} .
\end{aligned}
$$

143. Thick Lenses and Systems of Lenses.-Let $\mathrm{FF}^{\prime}$ (Fig. 155) be the axis of a thick lens or system of coaxial lenses, then if the media on

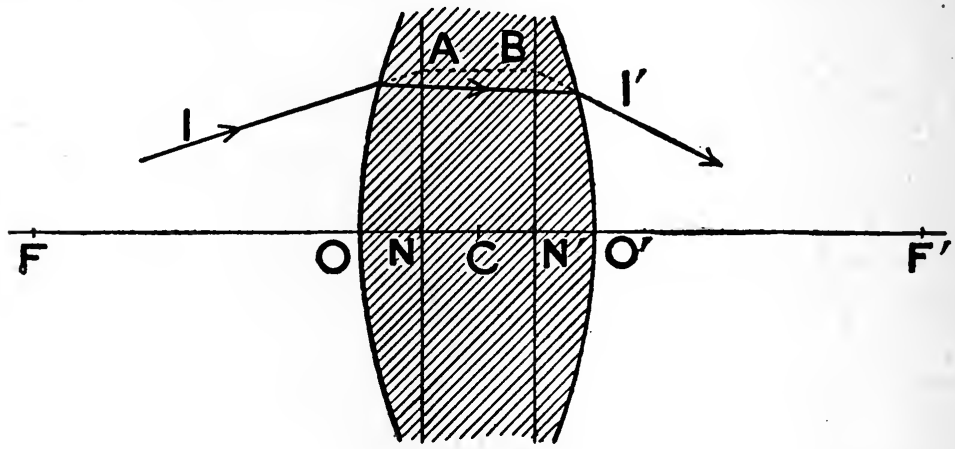

Fig. 155.

the two sides of the lens have the same refractive index, the following are the cardinal points of the lens :-

1. The principal focal points $F$ and $F^{\prime}$ are such that a plane wave incident on the lens at $O$ is brought to a focus at $F^{\prime}$, while a plane wave incident at $o^{\prime}$ is brought to a focus at $F$. Planes drawn through $F$ and $\mathbf{F}^{\prime}$ perpendicular to the axis are called the principal focal planes. 
2. The points $\mathrm{NN}^{\prime}$, called the principal points, which in the case where there is the same medium on the two sides of the lens, coincide with the nodal points. These points are such that if the direction of an incident ray passes through one nodal point, then the direction of the ray after passing through the lens will pass through the other nodal point, and, further, will be parallel to the incident ray. These points are conjugate foci ; that is, if an incident pencil is converging on $\mathrm{N}$, then the emergent pencil will diverge from $N^{\prime}$.

The distances $\mathrm{FN}$ and $\mathrm{F}^{\prime} \mathrm{N}^{\prime}$ are equal, and are called the focal length of the lens or system. Planes drawn through $N$ and $\mathbf{N}^{\prime}$ perpendicular to the axis of the lens are called the principal planes of the lens, and have the property that, if an incident ray meets the first principal plane at a point $\mathrm{A}$, then the corresponding emergent ray $\mathrm{I}$ meets the second principal plane at a point $B$, such that the line joining $A$ and $B$ is parallel to the axis of the lens.

The distance $u$ of an object from one of the principal points $\mathrm{N}$, and the distance $v$ of the image from the other principal point $\mathrm{N}^{\prime}$, are connected with the focal length $f$ by the relation

$$
\frac{1}{f}=\frac{1}{v}-\frac{1}{u} \text {. }
$$

Further, the angle subtended by the object at the point $\mathrm{N}$ is equal to the angle subtended by the image at the point $\mathrm{s}^{\prime}$.

If $U$ is the distance of the object from the first principal focus, and $V$ is the distance of the image from the second principal focus, then the following relation between these quantities and the focal length $f$ will sometimes be found of use

$$
U V=f^{2} \text {. }
$$

144. To find the Focal Length and the Positions of the Principal Planes of a Thick Lens or System of Lienses. - Method 1.In the case of a convex lens, or converging system, the following method of determining the focal length, since it does not involve the use of a micrometer, is often convenient :-

If the image and object are of the same size, and $u$ and $v$ are the distances of the object and image from the first and second nodal points respectively, we have

$$
\frac{\text { image }}{\text { object }}=-1=\frac{v}{u} \text {. }
$$

Hence substituting in the general equation

$$
\frac{1}{v}-\frac{1}{u}=\frac{1}{f}
$$

we get

$$
\frac{v}{f}=2 \text {, }
$$

or

$$
v=2 f \text {, }
$$

also

$$
u=-2 f \text {, }
$$


that is, the image and object are in either case $3 .$, a distance equal to twice the focal length from the corresponding nodal point.

If, now, we readjust the lens system so that the image is twice the size of the object, and $u^{\prime}$ and $v^{\prime}$ are the new distances of object and image from the nodal points, we get

$$
\frac{\text { image }}{\text { object }}=-2=\frac{v^{\prime}}{u^{\prime \prime}}
$$

and hence

$$
\frac{v}{f}=3 \text {, }
$$

or

$$
v=3 f
$$

and

$$
u=-\frac{3}{2} f
$$

Thus in the second case (Fig. 156, b) the lens system has been moved through a distance $f$ away from the image $\mathbf{P}^{\prime}$, and the object $\mathrm{P}$ has been
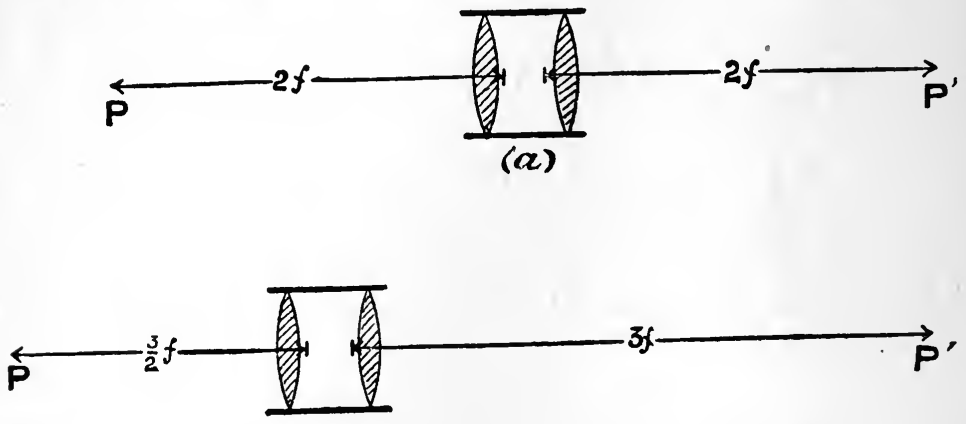

(6)

FTG. 156.

moved through a distance $f / 2$. Hence if the distance through which the lens system has been moved is measured this gives the focal length, the image being formed in either case at the same point, $\mathbf{P}^{\prime}$.

To carry out the experiment it is necessary to have two transparent scales having equal divisions. Such scales can easily be obtained by photographing a suitably divided scale and then taking two positives on a lantern plate from the negative. These two scales are mounted on two of the uprights of the optical bench, with the film sides turned towards each other, and the lens or lens system is mounted on a third carriage between them. It is evident that the optical bench must have a length at least four and a half times as great as the focal length of the lens system. Having placed a light behind one of the scales, so that it is well illuminated, the position of this scale and the lens must be adjusted till the image is in the same plane as the other scale, and the divisions of the image are of the same size as those of the scale. 
It will be found an assistance when making the adjustments to fix a low power microscope or reading lens immediately behind the scale on which the image is to be received, and to focus this microscope on the divisions of the scale.

The positions of the lens and the object having been so adjusted, the position of the fiducial mark on the carriage which supports the lens is recorded.

The object and the lens are then moved farther from the image scale, care being taken not to disturb the position of this latter till the divisions of the image are twice as far apart as those of the scale. The fact that the image and the scale are in the same plane being tested in this case, as in the previous measurement, by the parallax method. The reading for the carriage which supports the lens is again taken, and the difference. between the readings for the two positions gives the focal length of the system.

Determine by this method the focal length of a photographic objective, a microscope or telescope eye-piece, and a microscope objective.

Having measured the focal length of the system, the positions of the principal planes can now be found. To do this replace the lens and object scale in the first position, namely, that in which the image and object have the same size. In this position the focal planes are at a distance of $2 f$ from the object and image respectively. Hence if a distance $2 f$ is measured from the object, the position of the principal plane with reference to the mount which supports the lens is at once determined. It will generally be found convenient to measure the distances between the object or image and some well-defined point on the lens system, say, one edge of the mount, and then the differences between these distances and $2 f$ will give the distances of the principal planes from this point on the mount.

Method 2.-If when the distances of the image and object from the nodal points are $u$ and $v$ respectively the magnification is $m_{1}$, while when the distances are $u^{\prime}$ and $v^{\prime}$ the magnification is $m_{2}$, we have

$$
\frac{v}{u}=m_{1} \text { and } \frac{v^{\prime}}{u^{\prime}}=m_{2} ;
$$

hence substituting in the general equation we get

and

$$
\begin{aligned}
& 1=\frac{1}{m_{1}}-\frac{u}{f^{\prime}} \\
& 1=\frac{1}{m_{2}}-\frac{u^{\prime}}{f^{\prime}} .
\end{aligned}
$$

Thus

$$
\frac{1}{m_{1}}-\frac{u}{f}=\frac{1}{m_{2}}-\frac{u^{\prime}}{f}
$$

or

$$
f=\frac{u^{\prime}-u}{\frac{1}{m_{2}}-\frac{1}{m_{1}}} \text {. }
$$


Now $u^{\prime}-u$ is the difference in the distance between the object and the first nodal point in the two positions of the lens, and its measurement does not involve a knowledge of the position of the nodal point. Hence if, in addition, we measure the two magnifications, we can calculate the focal length.

In order to measure the magnification a micrometer similar to that used in $\S 127$ will be required, and also a transparent scale to act as the object. The lens system being mounted on the optical bench, it is placed fairly close to the object, and the size of the image corresponding to a convenient number of graduations of the scale is measured with the micrometer. Before measuring the size of the image, care must be taken that the image is formed in the same plane as the cross-wire of the micrometer. The position of the carriage which supports the lens is read, and the measurement repeated with the lens at a considerably greater distance from the object. The distance through which the carriage which supports the lens has been moved gives the quantity $u^{\prime}-u$, while the magnifications $m$ and $m^{\prime}$ are obtained by dividing the sizes of the images by the corresponding size of the object. If the scale which is used for an object is not one in which the distance between consecutive divisions is known, it will be necessary to determine this quantity by means of a micrometer microscope.

A modification of the above method consists in keeping the distance between the object and the image fixed, the distance being chosen considerably greater than four times the focal length, and measuring the size of the image in the two positions of the lens which give an image in the plane of the micrometer cross-wire.

If 0 is the size of the object, and $I$ and $I$ are the sizes of the image in the two positions while the lens is moved through a distance l between its two positions, we have

$$
m_{1}=\frac{0}{I} \text { and } m_{2}=\frac{0}{\Gamma} \text {. }
$$

But from symmetry $m_{1}=1 / m_{2}$. Hence $0=\sqrt{I \Gamma}$

and

$$
m_{1}=\sqrt{\frac{I}{I}} \text { and } m_{2}=\sqrt{\frac{I}{T}} \text {. }
$$

Substituting these values in the expression used in the first method. we get

$$
\begin{aligned}
f & =\frac{l}{\sqrt{\frac{I}{I}}-\sqrt{\frac{I}{I_{1}}}} \\
& =\frac{l \sqrt{I I^{\prime}}}{I^{\prime}-I},
\end{aligned}
$$

which does not involve a knowledge of the size of the object, and from which the focal length can be calculated. 
Method 3.-In the case of a lens or system of short focal length, if we take an object at a considerable distance, the image will be formed practically at the principal focus. Thus in the case of a lens having a focal length of a centimetre, if the object is at a distance of a metre the image will be at only a tenth of a millimetre from the principal focal plane. Hence if 0 is the size of the object, and $u$ is its distance from the first principal plane, and $I$ is the size of the image, we have

$$
\frac{I}{f}=\frac{0}{u} \text {. }
$$

Now if $u$ is large, it will not appreciably alter the result if in place of measuring $u$ to the principal plane we measure it to the centre of the lens. Thus the focal length is given by

$$
f=\frac{I u}{0} \text {. }
$$

To apply this method, a brightly illuminated scale, or object of known magnitude, is placed at a considerable distance from the lens, and the size of the image is measured with a micrometer microscope.

If the lens is of such a long focus that it is not possible to place the object at a sufficiently great distance, a collimator may be employed, that is, the object is placed at the principal focus of an auxiliary lens. A convenient arrangement is to use the collimator of a spectrometer which, after being adjusted for parallel rays by one of the methods given in $\S 117$, has the slit opened wide. The angle subtended by the two edges of the slit must then be measured with the spectrometer telescope in the ordinary manner. Let this angle be $\theta$. The collimator must then be removed and used as an object, while the size of the image, i.e. the width of the image of the slit, must be measured with a micrometer microscope. If the size of this image is $I$, then

$$
\begin{aligned}
& \frac{I}{f}=\tan \theta, \\
& f=I \cot \theta .
\end{aligned}
$$

Another method of measuring the focal length of a system of lenses is given in the next section.

145. Test of a Photographic Lens.-The following method of testing photographic lenses, which was developed by Major L. Darwin ${ }^{1}$ for the Kew Observatory, is both convenient and instructive. The instrument used in making the tests is shown in Fig. 157, and can easily be constructed in an ordinary laboratory workshop. The lens $\mathbf{L}$ to be tested is mounted on a wooden block, and this block can be moved backwards or forwards by a rack and pinion $\mathrm{s}$. The lens and its mounting can also be rotated about a horizontal axis, the amount of the rotation being read by means of a pointer and divided circle. The

1 Proceedings of the Royal Society (1892), vol. lii. p. 403. 
upright B, which carries the above lens fittings, is screwed to a wooden board $\mathrm{c}$, this board being pivoted at a point vertically under the horizontal axis, about which the lens can turn. The board c moves over a T-shaped base DE, and the amount of the rotation can be read off on a scale of degrees engraved on the curved edge of the base. A clamp serves to fix $\mathrm{c}$ to the base, while two adjustable stops, $\mathrm{D}$ and $\mathrm{E}$, serve to limit the rotation.

On the board $\mathbf{c}$ slides a carriage $\mathrm{G}$, which can be clamped in place by the screw $\mathrm{H}$. On the top of the carriage a second plate I can be traversed through a distance of about $3 \mathrm{~cm}$. by means of a micrometer screw M. This plate I carries a divided scale $\mathrm{J}$, the divisions (mm.) being engraved on ground glass. The image formed by the lens is received on this ground glass, and the position of the glass is roughly

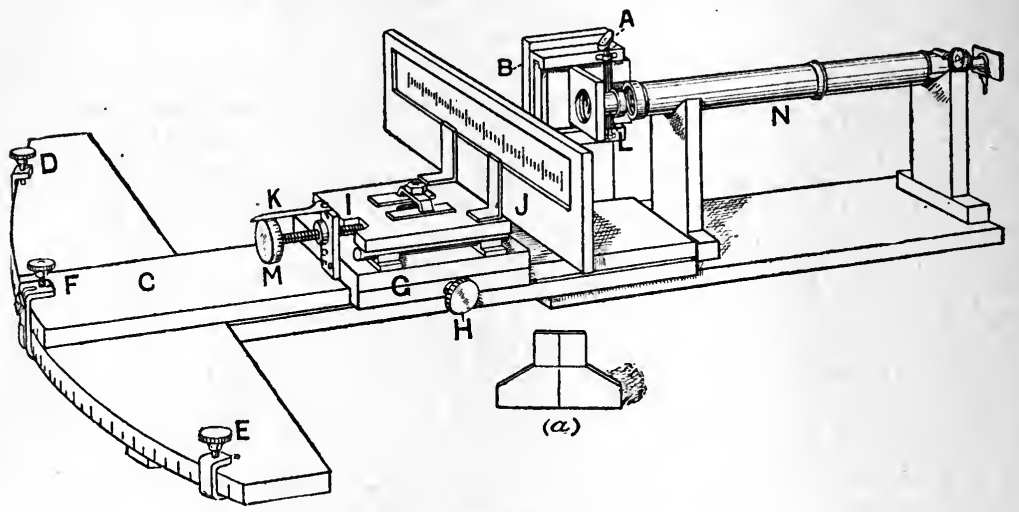

FIG. 157.

adjusted by moving $\mathrm{G}$, the final adjustment being performed by means. of the micrometer screw. A collimator $\mathrm{N}$ can be placed on two V's, which are carried on an extension of the bed $\mathrm{DE}$. The scale $\mathrm{J}$ can be removed when desired, and replaced by a metal plate $(a)$ in which a small hole is pierced.

The first adjustment to be made is to move the lens backwards or forwards by the pinion A till the horizontal axis about which the lens can be turned, and hence also the vertical axis about which the board $\mathrm{c}$ can turn, passes through the second nodal point of the lens. To make this adjustment, focus the collimator $\mathrm{N}$ for parallel rays by the method given in $\$ 117$. Then set the collimator in place with the slit horizontal, and having set up a bat's-wing burner behind the slit, focus the image on the ground glass scale $J$ by moving the carriage $G$. Next rotate the lens about the horizontal axis, and move it backwards or forwards by the pinion $\mathrm{A}$ till the image remains fixed in position. If the image moves in the opposite direction to that in which the lens is rotated, then 
move the lens nearer the scale, and vice versa. When this adjustment is complete, the axis about which the lens rotates passes through the second nodal point, as will be at once evident from what has been said in $\S 143$.

1. Determination of the Effective Aperture of the Stops.-Having focused the lens on the ground glass, using the collimator as object, replace the scale by the metal plate with a hole about a millimetre in diameter, and place a flame behind the plate. The light from this hole, which passes through the lens, will now issue as a parallel beam, and the diameter of the beam will be the effective aperture of the stop which is in place in the lens. This is at once evident, for if we imagine the direction of the light reversed, it is only light within a cylinder of this diameter which will be brought to a focus at a given point of the image. For each of the stops provided with the lens measure the diameter of the beam of parallel rays, using for the purpose the scale engraved on the ground glass, which must be placed in the position previously occupied by the collimator.

2. Determination of the Angle of the Cone of Ilumination with the Stops.-Having replaced the collimator, look through the hole in the metal plate, and turn the lens about a horizontal axis till all light is cut off by the mount. Take the difference in the readings of the circle attached to the horizontal axis when the lens is rotated first in one direction and then in the other. Half the difference will give the angle of the cone which includes all the rays which traverse the lens. Since the illumination at the edges of this outer cone falls off very much, it is advisable to measure the angle of the inner cone, which is limited by the condition that we stop rotating the lens when the edge of the mount is first seen to cut off the light. By measuring this inner cone for the different stops, determine the largest plate which can be used with each stop, so that the illumination should not fall off at the edge, due to the plate extending beyond the inner cone.

3. Determination of the Focal Length.Using the collimator as object, put the ground glass scale in place, and having focused with

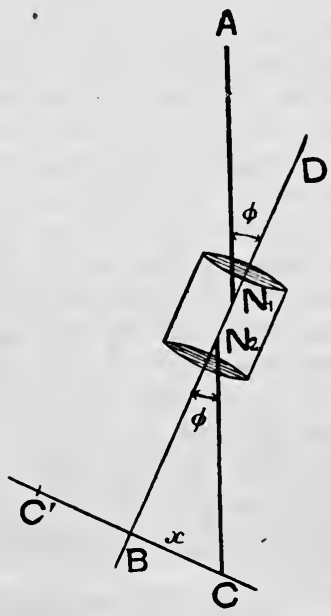

Fig. 158. the board $\mathrm{c}$ in the central position, turn it through an angle $\phi\left(\right.$ say, $\left.20^{\circ}\right)$, and take the reading on the scale. Then rotate the board $\mathrm{c}$ through an angle $\phi$ on the opposite side, and again read the position of the image on the scale. If $x$ is the mean distance of the image from the mid point of the scale, then

$$
F=x \cot \phi .
$$

For if $\mathrm{N}_{1} \mathrm{~N}_{2}$ (Fig. 158) are the nodal points of the lens system, and when 
the axis of the lens is rotated into the position $\mathrm{DB}$, the axis of the collimator lies along $\mathrm{AN}_{1}$, then the axis of the emergent pencil forming the image will be along $\mathrm{N}_{2} \mathrm{C}$, which is parallel to $\mathrm{AN}_{1}$. But the lens has been rotated about the point $\mathrm{N}_{2}$, and $\mathrm{N}_{2} \mathrm{~B}$ is the focal length $F$, while the scale reading $x$ is $\mathbf{B C}$. Hence from the right-angled triangle $\mathrm{CBN}_{2}$ (the scale $\mathrm{CBC}^{\prime}$ remains perpendicular to $\mathrm{N}_{2} \mathrm{~B}$ throughout) we have

$$
F=\overline{B C} \cot \phi .
$$

4. Determination of the Curvature of the Principal Focal Plane.The image of the slit of the collimator is focused as carefully as possible when the axis of the lens is parallel to the axis of the collimator, and the reading on the micrometer screw $\mathbf{M}$ is noted. The board $\mathrm{c}$ is then rotated through $10^{\circ}, 20^{\circ}, 30^{\circ}, \& c$, and in each position the focus is adjusted, and the micrometer screw is read. By plotting the difference between the central reading and the peripheral readings against $F \tan \phi$, where $\phi$ is the angle through which the board $\mathrm{c}$ has been rotated, the shape of a meridian section of the principal focal "plane" will be obtained.

5. Determination of the Distortion of the Image of a Straight Line near the Margin of the Plate.-From the dimensions of the plate the lens is intended to cover, calculate the angle $\beta$ which half the breadth subtends at a distance equal to the focal length of the lens. Place the slit of the collimator horizontal, and turn the lens about the horizontal axis through an angle $\beta$, and measure the distance $a$ between the image and the horizontal line engraved on the scale. Next turn the board $c$ through such an angle that the image travels along the scale a distance equal to half the length of the plate, and again measure the distance $b$ of the image from the horizontal line on the scale. Finally determine the distance $c$ of the image from the line when the board $c$ is rotated to an equal amount on the other side of the axis. In this experiment we have, by the rotation of the lens, obtained the same effect as if we had used a horizontal linear source, and the image of the two ends and the middle of this line are formed at distances $b, c$, and $a$ from the straight line ruled on the scale. Hence if the image is linear we must have

$$
a=\frac{b+c}{2},
$$

and the amount by which $a$ differs from $(b+c) / 2$ measures the distortion.

6. To Test the Achromatism of the Lens.-To test the achromatism it will be necessary to use a distant object, as it would not do to employ the collimator, for any want of achromatism in its lens would vitiate the result. As object a slit may be used, placed as far off as convenient. The position of the focus is then determined when a red, yellow, and blue glass is placed in succession behind the slit, a bright gas flame being used as source. In this way any difference in focus for red, yellow, and blue rays will be detected. 
7. To Examine the Lens for Astigmatism.-A point source of light is obtained by using the light reflected from a small glass bulb filled with mercury or a silvered bead, when the bulb or bead is illuminated by a small flame placed at a distance of about a metre. When the board $\mathrm{c}$ is in the central position, the image will be a small circular disc. When, however, the lens is tilted by rotating the board c, it will generally be found that by altering the position of the ground glass the image in one position is a fine radial, i.e. horizontal, line, and in another a fine line at right angles to the first. Half-way between the position in which these focal lines are formed the image is approximately circular, but the diameter is greater than is the case when the image is on the axis of the lens.

146. Measurement of the Magnifying Power of a Telescope.The magnifying power of a telescope is the ratio of the angle which the distant object subtends at the eye to the angle subtended by the image as seen through the telescope. The most usual method adopted to measure the magnifying power of a telescope is to place a scale at some distance from the telescope, and to focus the telescope on the scale. The distance of the scale from the telescope must be so great that the length of the telescope can be neglected compared to this distance. In addition to the divisions, which must be of such a size that they can be seen clearly by the telescope, two distinct marks must be attached to the scale of such a nature that they can be distinctly seen with the naked eye from the position where the telescope is set up. Looking through the telescope with one eye, and at these two marks with the other unaided eye, the two images will appear superposed. The number $(n)$ of divisions of the scale, which appear to correspond to the distance between the marks, must be noted. If the distance between the marks is $N$, then the magnifying power $(m)$ of the telescope is given by

$$
m=\frac{N}{n}
$$

The angle subtended by the $n$ divisions at the telescope is $n / D$, where $D$ is the distance of the scale from the telescope, while the angle subtended by the $N$ divisions at the eye is $N / D$, and these appear equal.

Hence

$$
\begin{aligned}
\frac{n}{D} \times m & =\frac{N}{D}, \\
m & =\frac{N}{n} .
\end{aligned}
$$

When making the comparison, the telescope must be so focused that the two images do not appear to move relatively to one another when the eye which receives light through the telescope is moved slightly from side to 
side, which of course means that the image formed by the telescope is at the same distance from the eye as the scale. In the case of a shortsighted person, the spectacles habitually used when observing distant objects must be worn when making the measurement.

When it is not convenient to place a scale at a sufficient distance, the following modification of the method may be employed :- Having focused the telescope for a very distant object, a thin convex lens having a focal length of about 2 metres is fixed immediately in front of the object glass of the telescope. The focal length of this lens need not be known, and a spectacle lens does quite well. Care being taken not to disturb. the adjustment of the focus of the telescope for distant objects, the telescope is then placed at such a distance from a scale that the divisions appear quite distinct. Then looking through the telescope with one eye, and at the scale with the other eye, the number, $n$, of divisions of the scale, as seen through the telescope, which appear to coincide with $N$ divisions as seen with the naked eye, must be noted. Then if $d$ is the distance from the scale to the object glass of the telescope, and $D$ is the distance from the scale to the eye, the magnifying power is given by

$$
m=\frac{N d}{D n}
$$

Since the telescope is focused for parallel rays, it follows that light issuing from any given point on the scale after passing through the auxiliary lens must form a parallel pencil. The angle included between the two parallel pencils, which leave the two points of the scale at a distance $n$ apart, is the same as the angle subtended by these two points at the centre of the auxiliary lens. Hence this angle is $n / d$. The angle subtended by the two divisions at a distance $N$ apart at the eye is $N / D$. and the two angles are the same, and hence

or

$$
\begin{aligned}
\frac{n}{d} m & =\frac{N}{D}, \\
m & =\frac{N d}{D n}
\end{aligned}
$$

147. Measurement of the Magnifying Power of a Microscope.The magnifying power of a microscope is the ratio of the angle subtended by the image of a given object as seen through the microscope to the angle which the same object would subtend if placed at the distance of most distinct vision. This distance may be taken as $25 \mathrm{~cm}$. A finely divided scale is placed on the stage of the microscope, and a scale divided in millimetres is supported at a distance of $25 \mathrm{~cm}$. below the eye-piece of the microscope. Then by: looking through the microscope with one eye, and at the other scale with the other, if it is found that the two divisions of the scale as seen through the microscope, which are at 
\$ 147] MAGNIFYING POWER OF A MICROSCOPE 369 distance $n$ apart, appear to coincide with $N$ divisions of the scale seen with the naked eye, then the magnifying power is given by

$$
m=\frac{N}{n} .
$$

The comparison is nade easier if a camera lucida is available, for then the scale and the image, as seen through the microscope, can be seen at the same time with a single eye. 


\section{CHAPTER XXII}

\section{POLARISED LIGHT}

148. Polarisation by Reflection.-The refractive index of a plate of glass can be obtained by a determination of the angle at which the light reflected from its surface undergoes maximum polarisation. In a laboratory experiment a plate of black glass may be employed in order that the light transmitted into it may be absorbed. The plate of glass under experiment is fixed with its surface vertical on the table of a spectrometer, and sodium light is allowed to fall on it from the collimator in the usual manner, at any angle of incidence, and is received into the telescope of the spectrometer. In front of the object lens of the telescope is fixed a Nicol prism or other analyser for polarised light, which is capable of rotation about the axis of the telescope. The position of the Nicol can be determined by means of a pointer working on a circular fixed scale. For a given angle of incidence of the light

- on the glass the Nicol can be rotated until the reflected light has minimum intensity. Other angles of incidence are then used, and it will probably be found that for a certain range of incident angles the analyser can be rotated to a position giving total extinction of the reflected light as far as the eye can distinguish. If the experiment is performed in a very dark room and the eye givell time to become accustomed to the dark field of view seen in the telescope, the range of incident angles over which total extinction can be obtained may be reduced to two or three degrees, depending on the sensitivity of the eye of the observer and the transparency of the Nicol. At the centre of the range it will be possible to rotate the analyser a few degrees before any light is visible, and the number of degrees will be less and less for angles of incidence further from the centre of the range. By plotting a curve between the angle of incidence and the number of degrees of rotation of the analyser giving total extinction, the angle of incidence can be found at which maximum polarisation takes place. The consistency of the observations will depend entirely on a systematic method of taking them, and the eye must always be given sufficient time to distinguish weak intensities of the reflected light. If apparent total extinction is not obtained for any angle of incidence, it is necessary to judge that angle at which the minimum intensity obtained by rotation of the analyser is the nearest to extinction. The determination should be repeated several times and a mean value obtained. If the angle of incidence giving maximum polarisation is $\theta$, then, by Brewster's Law, $\mu-\tan \theta$.

149a. Rotation of the Plane of Polarisation by Optically Active Substances.-If a beam of plane polarised light is passed through a plate 
of quartz cut perpendicular to the optic axis, or through a solution of cane sugar, the emergent light will be plane polarised. The plane of polarisation of the emergent light will not, however, be the same as that of the incident light. During the passage of the light through these substances the plane of polarisation undergoes a rotation, which, for any given substance, is proportional to the length of the substance traversed. Substances which are capable of rotating the plane of polarisation are said to be optically active. The rotation is said to be positive or righthanded if, looking towards the source of light, the rotation takes place in the direction of the hands of a clock. If the rotation takes place in the opposite direction, the substance is said to possess negative or lefthanded rotatory power.

The rotation produced by a decimetre of a pure substance divided by the density is called the specific rotation of the substance, and varies with the wave-length of the light, being greater for light of short wave-length than for light of longer wave-length.

If a solution of an active substance in an inactive solvent is used, the rotation produced by a decimetre of the solution divided by the weight of the active substance in unit volume of the solution is called the specific rotation of the dissolved substance. Thus, suppose that $x$ grams of a substance are dissolved in an inactive solvent so that the volume of the solution is 100 c.e., and that a length of $20 \mathrm{~cm}$. of this solution rotates the plane of polarisation through $\theta$ degrees, then the specific rotation of the dissolved body is given by

$$
a=\frac{100 \theta}{2 x} \text {. }
$$

The specific rotation is not a constant, for not only does it depend upon the wave-length of the light used and on the temperature, but it also varies with the concentration of the solution used and the nature of the inactive solvent. The variations are, however, small, and may often be neglected.

The specific rotation multiplied by the molecular weight of the active substance is called the molecular rotation of the substance.

The following table gives the specific rotation of some substances for sodium light and a temperature of $20^{\circ}$, the concentration being $c$ grams of the salt in 100 c.c. of the solution :-

\begin{tabular}{|c|c|c|}
\hline Active Sabstance. & Solvent. & Spectfio Rotation. \\
\hline $\begin{array}{l}\text { French turpentine }\left\{\mathrm{C}_{10} \mathrm{H}_{6}\right\} \\
\text { Gthyl tartrate }\left\{\left(\mathrm{C}_{2} \mathrm{H}_{5}\right)_{2} \mathrm{C}_{4} \mathrm{H}_{4} \mathrm{O}_{6}\right\} \\
\text { Potassium tartrate }\left\{\mathrm{K}_{2} \mathrm{C}_{4} \mathrm{H}_{4} \mathrm{O}_{6}\right\} \\
\text { Tartaric acid }\left\{\mathrm{C}_{4} \mathrm{H}_{6} \mathrm{O}_{6}\right\} \\
\text { Potassium sodium tartrate } \\
\left\{\mathrm{KNaC} \mathrm{H}_{4} \mathrm{O}_{6}\right\} \\
\text { Camphor }\left\{\mathrm{C}_{10} \mathrm{H}_{10} \mathrm{O}\right\}: . .\end{array}$ & $\begin{array}{l}\text { None } \\
\text { None } \\
\text { Water } \\
\text { Water } \\
\text { Water } \\
\text { Ethyl alcohol }\end{array}$ & $\begin{aligned} & -37^{\circ} \cdot 01 \\
& +8 \cdot 31 \\
+27 \cdot 14 & +0.0992 c-0.000938 c^{2} \\
+15.06 & =0.1310 \\
& +29 \cdot 73-0.0078 c \\
+ & 41.982+0.11824 c\end{aligned}$ \\
\hline
\end{tabular}


In the case of cane sugar $\left(\mathrm{C}_{12} \mathrm{H}_{22} \mathrm{O}_{11}\right)$ dissolved in water, the specific rotation for sodium light at a temperature $t$ is given by 1

$$
[a]_{\mathrm{D}}^{t}=66^{\bullet} \cdot 5-0.0184(t-20) \text {. }
$$

For quartz, the rotation produced by 1 millimetre for different temperatures is given by

$$
[a]_{\mathrm{D}}^{t}=21^{\bullet \cdot 72}[1+0.000147(t-20)] \text {. }
$$

In the case of invert sugar $\left(\mathrm{C}_{12} \mathrm{H}_{24} \mathrm{O}_{12}\right)$ the specific rotation changes with the concentration much more than in the case of cane sugar. The effect of temperature is also greater. Landolt gives the following expressions for obtaining the specific rotation of invert sugar where $c^{\prime}$ is the weight of invert sugar contained in 100 c.c. of solution, which holds for values of $c^{\prime}$ between 2 and 30 and from $0^{\circ} \mathrm{C}$. to $30^{\circ}$.

$$
[a]_{\mathrm{D}}^{t}=19^{\circ} \cdot 657+0.0361 c^{\prime}-0.304(t-20) \text {. }
$$

If a solution is made up which contains $c$ grams of cane sugar in 100 c.c., and then the cane sugar is converted into invert sugar, since the molecular weights of the two sugars are not the same there will not be c grams of invert sugar in 100 c.c. The molecular weight of caue sugar is 342 , and that of invert sugar is 360 . Hence the concentration $c^{\prime}$ of the invert sugar solution is given by

$$
c^{\prime}=\frac{360}{342} c \text {. }
$$

149b. Methods of Measuring the Rotation of the Plane of Polarisation.-Theoretically, the simplest method of determining the plane of polarisation of a beam of light is to examine it with a Nicol prism, for when the prism is so turned that its principal plane is perpendicular to the plane of polarisation of the light, no light will pass through the prism. In practice, however, such a simple prism forms a very insensitive arrangement for determining the plane of polarisation, for it is impossible to tell exactly when the light is exactly cut off, the field appearing dark while the Nicol is being rotated through an appreciable angle. On this account various arrangements have been devised to assist the setting of the Nicol so that its principal plane is inclined at a definite angle to the plane of polarisation of the incident light. Most of these arrangements consist in the use of an auxiliary piece of apparatus, by means of which, instead of using a beam of light which is polarised throughout in the same direction, the incident beam is divided into two parts, and the light in these two parts is polarised in planes inclined at a small angle $\beta$ to one another. When the analysing Nicol is so placed that its principal plane is perpendicular to the plane of polarisation of half the field, that half of the field will appear black. If, then, the analysing Nicol is turned through an angle $\beta$, so that its plane is now perpendicular to the plane of polarisa-

1 Schönrock, Zeit. Instrumentenkunde (1900), xx. 97. 
tion of the other half of the field, this half will now appear black. When the principal plane of the Nicol is equally inclined to the planes of polarisation of the two halves of the field, the whole field will appear of a uniform tint. Now it is found that the eye can tell when the two halves of the field are equally bright very much better than it can tell when the field is quite dark. Since turning the analyser through an angle $\beta$ changes from one half of the field being quite dark to the other half being quite dark, the smaller we make $\beta$, the smaller the angle through which the analyser can be turned between these two limits, and it might at first sight appear that by making $\beta$ very small the accuracy of setting could be indefinitely increased. This is not the case, for if $\beta$ is very small, then the intensity of the light in the two halves of the field is so small that the eye is not able to judge whether the illumination is equal. The smallest value of $\beta$ which it is advisable to use depends on the intensity of the light which enters the analyser, that is, on the intensity of the source of light and on the transparency of the substance being investigated. Hence, since the intensity of the light varies in different cases, it is an advantage to be able to adjust the value of $\beta$ so that maximum sensitiveness may be obtained in any given condition.

The principal methods employed for producing the difference in the plane of polarisation of the two halves of the field are as follows:-

1. A small plate of quartz cut parallel to the axis is placed in half the beam of light, the thickness being such that during the passage of the light through the plate the ordinary ray gains in phase over the extraordinary ray by $180^{\circ}$, or $\lambda / 2$, where $\lambda$ is the wave-length of the light used. Let $\triangle B C$ (Fig. 159) represent the quartz plate covering half the field, the optical axis of the quartz being parallel to the edge $\mathbf{A B}$. Let the plane of polarisation of the light from the polarising Nicol be at right angles to $\mathrm{OP}$, so that the vibrations in the incident light take place along OP. Then in the quartz the incident vibration is resolved into two components, one taking place along $O \Delta$, and the other at right angles to the optic axis, i.e. along oc. On emergence from the quartz one of these vibrations, namely, that in the direction $\mathbf{O A}$, will be retarded by $180^{\circ}$ more than that along oc, and hence when on leaving the quartz they recombine to produce a single

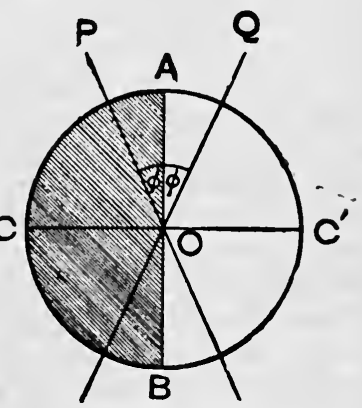

Fig. 159. plane polarised beam, the vibration will take place along $O Q$, which makes the same angle with the axis on as does the original direction of vibration op, that is, the plane of polarisation of the light which has traversed the quartz will be inclined to the original plane of polarisation at an angle equal to twice the angle AOP (see $\$ 152)$. By altering the angle $\triangle O P$, that is, by altering the angle included between 
the principal plane of the polarising Nicol and the optical axis of the quartz plate, the angle between the planes of polarisation of the two halves of the field can thus be altered. It will be noticed that such a half wavelength plate of quartz can only be used with one kind of monochromatic light, since it is only for light of a single wave-length that the difference in phase between the two waves which have traversed the plate will be exactly equal to $180^{\circ}$, and hence it is only for such light that on recombination we shall obtain a beam of plane polarised light. If white light were used with a half wave-length plate of such a thickness that a difference of phase of $180^{\circ}$ is produced with yellow light, then the yellow part of the spectrum would on emergence be polarised in a plane making an equal angle with the axis of the quartz to that made by the original plane of polarisation. For red light, on the other hand, the difference of phase would be less than $180^{\circ}$, and hence the emergent light would be elliptically polarised, and would not be completely extinguished for any position of the analysing Nicol. This same remark applies to the green and blue, so that the field would appear coloured, and no satisfactory match of the two halves could be obtained.

2. Poynting has devised a simple arrangement for producing the field having two halves polarised in slightly different directions, which consists of a small tube, the two ends of which are closed by two plane glass plates A and B (Fig. 160). A semicircular plate of glass $\mathbf{C}$ is enclosed in the cell, and the remainder of the cell is

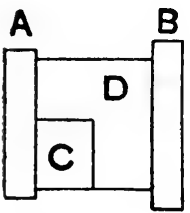

FIG. 160. filled with a solution of sugar. The sugar solution rotates the plane of polarisation, and the amount of the rotation is greater in the case of the light which traverses the upper part of the cell than in the case of the light which passes through the lower part of the cell where part of the path consists in the glass plate c.

3. One or more auxiliary Nicols can be placed after the analyser so as to partly cover the field, their principal planes being inclined at a small angle to the principal plane of the polariser. Lippish uses two auxiliary Nicols $B$ and $\mathrm{c}$ (Fig. 161). Thus the field, as shown at (a), consists of three parts. The central part A corresponds to light which has passed through the analyser only while the two outer parts B and c correspond to light which has passed in addition through the two auxiliary Nicols. The principal planes of the auxiliary Nicols are parallel, but slightly inclined to the principal plane of the analyser. It is found that using a field divided into three parts is an advantage, as it facilitates judging when the whole field is of a uniform brightness.

4. An arrangement called a biquartz may be used. This consists of two semicircular plates of quartz of equal thickness cut with the optic axis perpendicular to the semicircular faces. One half of the quartz is right-handed, and the other half left-handed. When plane polarised white light is passed through the biquartz, the plane of polarisation of the 
light in the two halves is rotated in the opposite directions, and the different colours are rotated to a different extent. If the thickness of the quartz plates is about $3 \mathrm{~mm}$., then the yellow light in each half is rotated through $90^{\circ}$. Hence if the principal plane of the analysing Nicol is turned so as to be perpendicular to the plane of polarisation of the incident light, and therefore parallel to the plane of polarisation to the yellow rays, the yellow rays will be extinguished on either side, and both halves of the field will appear a greyish-violet colour, called the tint of passage. On slightly rotating the analysing Nicol the colour cut out will on one side be nearer the blue, and on the other side nearer the red end of the spectrum, and
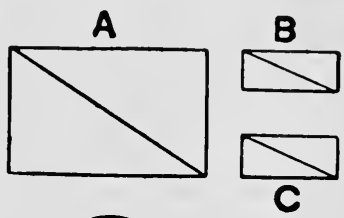
hence one half of the field will appear of a red tinge, and the other half of a blue tinge. This change is fairly sudden, and hence it is possible to adjust the analysing Nicol to the tint of passage with considerable accuracy. The rotation measured when using the tint of passage corresponds to yellow light near the Fraunhofer line $E$ of the solar spectrum.

The general arrangement of the optical parts of most polarimeters, as instruments for measuring the rotation of the plane of polarisation are called, is shown in Fig. 162 . The source of light 8 is placed at a distance from the lens $L_{1}$ equal to its focal length, so that an approximately parallel pencil of rays enters the polarising Nicol $\kappa_{1}$. The half wave-length plate $Q$ is attached to a ring, so that the angle the optical axis of the quartz makes with the principal plane of the Nicol can be varied. The analysing Nicol $\mathrm{N}_{2}$ and a low-power telescope, consisting of an object glass $\mathrm{L}_{2}$ and eye-piece $\mathbf{H}$, are attached to a divided circle, by means of which the angle through which they are rotated can be measured. The substance to be investigated is placed between the two parts of the instrument. In the case of a liquid it is enclosed in a tube
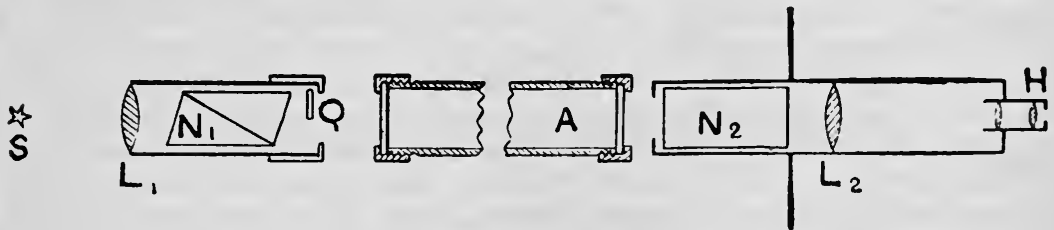

FIG. 162.

A, which is closed with plates of optically worked glass. These plates are held against the ends of the tube with metal caps, which screw on to the ends of the tube. If it is desired to make accurate measurements of rotation, it is essential that it should be possible to rotate the analysing 
Nicol completely round so as to be able to make two settings, the Nicol being rotated through $180^{\circ}$ between the two.

In some forms of saccharimeter instead of directly measuring the angle throuyh which the plane of polarisation is rotated by means of a divided circle attached to the

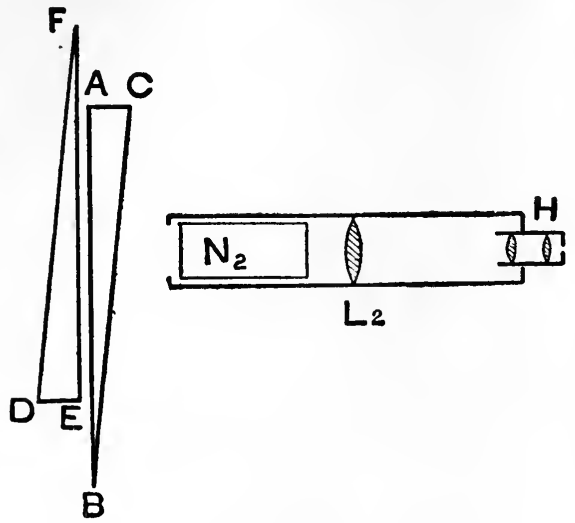

Fig. 163. analysing Nicol, the rotation is measured by determining what thickness of some substance which rotates the plane in the opposite direction must. be interposed to neutralise the rotation produced by the body under investigation.

Soliel's compensator consists of two wedges of quartz, $A B C$ and DEF (Fig. 163). These wedges have equal angles, and they are both cut so that the optical axis of the quartz is perpendicular to the faces $A B$ and $F E$. These wedges are fixed in frames, which by means of a rack

and pinion can be caused to move one in one direction and the other in the opposite, the amount of movement being indicated by a scale. In this way the thickness of quartz which the light traverses before it reaches the $\mathrm{Nicol}_{2}$ can be varied. The azimuth of the principal plane of the Nicol $\mathrm{N}_{2}$ is so adjusted with reference to the plane of polarisation of the polarising Nicol, that when the wedges are placed immediately one behind the other the two halves of the field appear equally dark. The reading on the scale is then zero. When an optically active body is placed in the instrument the wedges are moved so as either to increase or decrease the thickness of quartz till the two halves of the field again appear equally dark. Then from a previous calibration of the scale the rotation produced by the substance is deduced.

150. Measurement of the Rotatory Power of Solutions of Turpentine.-As an illustration of the measurement of the rotatory power of a solution we may take the case of solutions of turpentine in paraffin.

The first operation is to obtain the zero reading of the instrument. Focus ${ }^{1}$ the eye-piece so that the line dividing the field into two parts is seen sharply, and in the case of instruments using a biquartz adjust for the sensitive tint. Then determine the reading when the two halves of the field are equally illuminated or of the same tint, as the case may be.

1 When making this adjustment, it is advisable to place the tube filled with water in the instrument. In this way the focus will probably be correct when the liquid under observation is used. 
Having carefully cleaned the tube and the glass plates which are used to close the ends, place one of these plates on one end of the tube, then an india-rubber ring, and finally the cap, which must not be screwed down too tight. The reason for the introduction of the rubber ring between the outside of the glass plate and the cap is that if glass is strained it becomes birefringent, and would thus affect the plane of polarisation of the light. The effect of strain is generally to produce elliptic polarisation, so that the field appears patchy. Next fill the tube with pure turpentine quite to the top, and slide on the other glass plate, and having wiped off any superfluous liquid, fix the plate in place with a rubber washer and the cap.

Place the tube in the instrument, and allow it to stand there for some time so that the temperature may become uniform, and thus the striæ due to differences of density may disappear. Since the rotatory power changes with temperature, it is necessary to measure the temperature of the substance. For this reason polarimeters which are intended for accurate measurements are provided with water-jackets, which resemble Liebig condensers, to go round the tube, the temperature of the liquid being obtained by measuring the temperature of the water which is passed through the jacket. In the absence of such a jacket a thermometer must be placed alongside the tube, and time be allowed for the thermometer and liquid within the tube to come to the same temperature.

Several settings of the instrument must be made for the analyser in the two positions, separated by about $180^{\circ}$, for which the field is equally bright on either side. The mean of these, less the zero reading, will give the rotation.

In the case of substances which have a large rotatory power, there may be an uncertainty as to whether the rotation is to the right or to the left. In such a case observations must be made with a shorter tube or with a weaker solution, and these will at once show the sign of the rotation.

Having determined the rotation produced by pure turpentine, make up solutions containing different percentages of turpentine mixed with paraffin oil. The easiest way of obtaining these solutions is to weigh a 50 or 100 c.c. measuring flask empty, and then introduce about the right quantity of turpentine, and again weigh. Finally, fill the flask up to the mark with the paraffin.

Carefully measure the length of the tube, and then calculate the rotatory power of the turpentine when pure and when in solution, and see to what extent the rotatory power is constant.

151. Measurement of the Strength of a Solution of Sugar.-To determine the quantity of cane sugar in a solution, which may also contain other substances which rotate the plane of polarisation, we proceed as follows :-

Take about 15 grams of the sugar to be tested and introduce it into a weighed 100-c.c. flask, and again weigh. Then fill the flask up to its mark with distilled water. Measure off 50 c.c. of this solution, and add 
5 c.c. of strong hydrochloric acid, and warm this mixture to a temperature of $70^{\circ} \mathrm{C}$., keeping it at this temperature for about ten minutes, and then allow to cool to the room temperature. By the action of the acid the cane sugar will be converted into invert sugar.

The rotations produced by the original solution, and that in which the sugar has been converted into levulose, must then be measured. It must be remembered that 10 per cent. by volume of hydrochloric acid has been added to one portion of the solution. Hence if $v$ is the weight of sugar originally taken, the unacted-upon solution contains $w$ grams of sugar in 100 c.c. of the solution. The other portion, however, contains only $10 w / 11$ grams of the original sugar in 100 c.c. of the solution. Thus if the same tube is used in the two measurements, the rotations with the converted sugar must be multiplied by $1 \cdot 1$ to convert them to what they would have been had not the solution been diluted with the hydrochloric acid. To save having to make this correction two tubes are sometimes supplied with saccharimeters, the length of one tube being $20 \mathrm{~cm}$., and that of the other $22 \mathrm{~cm}$. The weaker solution being placed in the longer tube, the extra length compensates for the weakening of the solution. The temperature of the solutions must be noted when measuring the rotation, this being especially necessary in the case of the invert sugar.

If $\alpha$ and $\alpha^{\prime} 1$ are the rotations produced by the two solutions, and $t$ the temperature at which the observations were made, and $l$ the length of the tube, we have $(\S 148)$

$$
\alpha=\{66 \cdot 5-0.0184(t-20)\} \frac{c l}{100}+\beta,
$$

where $c$ is the weight of cane sugar in $w$ grams of the sugar being tested, and is the quantity it is required to find, while $\beta$ is the rotation produced by substances other than cane sugar which may be present.

Also $\alpha^{\prime}=-\left\{19.657+0.0361 c^{\prime}-0.304(t-20)\right\} \frac{c^{\prime} l}{100}+\beta$,

where

$$
c^{\prime}=\frac{360}{342} c \text {. }
$$

Hence

$$
\begin{aligned}
a-a^{\prime}= & \frac{c l}{100}\{66.5-0.0184(t-20)\} c+\{19.657 \\
& +0.0380 c-0.304(t-20)\} 1.0526
\end{aligned}
$$

from which $c$ can be calculated.

It will generally be most convenient to obtain the approximate value for $c$, neglecting the term $0 \cdot 0380 c$, and then introduce this approximate value in the above term to get the final value for $c$. In the case of fairly pure samples it is even sufficiently accurate to substitute $w$ in place of $c$ in this small term.

${ }^{1} a^{\prime}$ is the rotation corrected for the effect of the dilution by the acid which has been added. 
152. Elliptically and Circularly Polarised Light.-Suppose that we have a plate of a doubly refracting crystal cut parallel to the optic axis, and that the principal planes of this plate are parallel to the directions ox and oy (Fig. 164), while a beam of plane polarised light traverses the plate normally, the plane of polarisation of the incident light being parallel to $\mathrm{OQ}$. Then the vibrations in the incident light will take place in a direction perpendicular to $O Q$, that is, parallel to op. The incident vibration having an amplitude equal to op will give rise to a vibration of amplitude on or $A \cos a=a$ (say) parallel to $o x$, and a vibration of amplitude om or $A \sin a=b$ (say) parallel to or. At the face of incidence

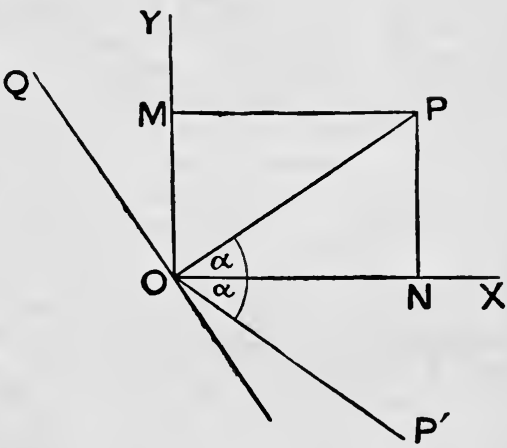

FIG. 164. these two vibrations will be in the same phase, but as they traverse the crystal plate with different velocities, they will differ in phase when they emerge. If the difference in the optical paths is $\delta$, the period of the incident light being $T$, and its wave-length in air $\lambda$, while the incident light vibration be represented by $A \cdot \cos 2 \pi \frac{t}{T}$, then the vibrations of the two components when they reach the second face of the plate will be represented by

respectively.

$$
\begin{aligned}
& x=a \cos 2 \pi \frac{t}{T} \\
& y=b \cos 2 \pi\left(\frac{t}{T}+\frac{\delta}{\lambda}\right)
\end{aligned}
$$
obtain

From these two equations the time $t$ can be eliminated, when we

$$
\frac{x^{2}}{a^{2}}+\frac{y^{2}}{b^{2}}-\frac{2 x y \cos 2 \pi \frac{\delta}{\lambda}}{a b}=\sin ^{2} 2 \pi \frac{\delta}{\lambda} \text {. . . . . }
$$

which represents the resultant vibration on emergence. This expression is the equation to an ellipse described in a rectangle having its sides parallel to $\mathrm{xx}$ and oy, and of length $2 a$ and $2 b$ respectively.

If $\delta=0$, then $\sin 2 \pi_{\bar{\lambda}}^{\delta}=0$, and $\cos 2 \pi_{\bar{\lambda}}^{\delta}=1$. Thus the vibration is represented by

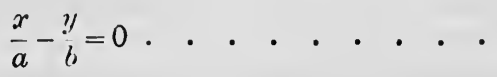


which is the equation to a straight line parallel to $\mathrm{OQ}$. Thus the emergent light is plane polarised parallel to the incident light.

If $\delta=\frac{\lambda}{2}$, then $\sin 2 \pi \frac{\delta}{\lambda}=0$, and $\cos 2 \pi \frac{\delta}{\lambda}=-1$, and therefore

$$
\frac{x}{a}+\frac{y}{b}=0 \text {. . . . . . . . . }
$$

This represents plane polarised light, but the plane of vibration is now parallel to $O P^{\prime}$, where $O P^{\prime}$ is inclined at the same angle to $O X$ as is. $O P$, but on the opposite side of ox.

When $\delta=\frac{\lambda}{4}$, then $\sin 2 \pi \frac{\delta}{\lambda}=1$, and $\cos 2 \pi \frac{\delta}{\lambda}=0$, and therefore

$$
\frac{x^{2}}{a^{2}}+\frac{y^{2}}{b^{2}}=1
$$

which represents an ellipse with its axes parallel to the principal axes of the crystal.

If $a=45^{\circ}$, that is, if the plane of polarisation of the incident light is equally inclined to the principal axes, the resultant vibration reduces to

$$
x^{2}+y^{2}=a^{2} \quad \text {. . . . . . . }
$$

which represents a circle, and thus the emergent light is circularly polarised.

It follows from the above that if we resolve an elliptic vibration into components along the axes of the ellipse, then the difference in phase of the components is $\frac{\pi}{2}$ or $\frac{3 \pi}{2}$. Hence if by any means we reduce the difference of phase between the components by $\frac{\pi}{2}$ or $\lambda / 4$, they will be in the same phase, or differ in phase by $180^{\circ}$, and will, if then recombined, produce plane polarised light. If the angle which the vibrations in the plane polarised light make with the major axis of the ellipse be $a$, then the ratio of the axes of the ellipse is given by

$$
\frac{a}{b}=\cot a \text {. . . . . . . . . }
$$

153. Analysis of an Elliptical Vibration.-The apparatus required for this experiment is a spectrometer to which can be attached three vertical graduated circles. Two of these fit over the object glasses of the collimator and telescope, and the third fits in the eye-piece end of the telescope. The circles on the collimator and at the eye-piece end of the telescope are fitted with Nicol prisms; arms are also attached, by means of which the angles through which the Nicols are rotated can be read off on the circles. A quarter wave-length plate is mounted on the circle attached to the object glass of the telescope. 
The quarter wave-length plate is a plate of crystal such that incident plane polarised light is separated into two components polarised at right angles, and the phase of one component is retarded by $\lambda / 4$ over that of the other. Generally the plate is adjusted for sodium light. As has been shown in the last section, when a difference of phase of $\lambda / 4$ is introduced in the components into which elliptically polarised light can be split, then these components will combine to form plane polarised light. Hence by turning the quarter wave-length plate so that the directions in which the vibrations within the plate take place are parallel to the axes of the ellipse, the emergent light will be plane polarised, and can be extinguished by the eye-piece Nicol. The position of the principal plane of the quarter wave-length plate will then give the direction of the axes of the elliptic vibration, and the cotangent of the angle the principal plane makes with the principal plane of the eye-piece Nicol will give the ratio of the diameters of the ellipse.

The telescope and collimator having been focused for parallel light, the readings on the circle when the principal plane of the eye-piece Nicol is horizontal must be obtained. To do this remove the polarising Nicol and mount a piece of plate glass vertically on the prism table, or use the face of a prism, and turn it so that the angle of incidence $\phi$ of the light from the collimator is the polarising angle, i.e. $\tan \phi=\mu$. Place the telescope so as to receive the reflected light, and turn the eye-piece Nicol till the light is completely extinguished. The principal plane of the Nicol is then horizontal, and the reading on the circle must be noted.

Next replace the Nicol on the collimator, and turn it till it is crossed with the eye-piece Nicol, and hence its principal plane is vertical. The quarter wave-length plate is then put in place and turned till the field is dark, in which position its principal plane is either horizontal or vertical, and the reading on the circle must be recorded.

The instrument is now in adjustment, and as an exercise a thin sheet of mica may be introduced between the polariser and the quarter wavelength plate. Unless the principal plane of the mica is horizontal or vertical, elliptically polarised light will be produced.

To analyse this elliptically polarised light rotate the quarter wavelength plate and the analysing Nicol till no light is transnritted. Then read the circles for the plate and the analyser. The difference between the two readings for the plate will give the angle made by the axes of the elliptic vibration with the horizontal and vertical directions. The difference in the two readings for the analyser will be the angle whose tangent is equal to the ratio of the seni-diameters of the ellipse. 


\section{CHAPTER XXIII}

\section{PHOTOMETRY AND COLOUR VISION}

154. Standards of Light. - The legal standard of light in Great Britain is the sperm candle, which burns 120 grains (7.776 grams) of spermaceti per hour, such a standard candle being said to give a luminous intensity of one candle-power. Numerous investigations have, however, shown that as a standard of light the candle is unsuitable and unreliable. As alternative standards there have been a number suggested, of which the following are the chief :-

1. The Carcel standard, which consists of a colza lamp of fixed form originally designed by Regnault and Dumas. This standard is used in France.

2. The Amyl Acetat lamp, which is a lamp burning amyl acetate under standard conditions, invented by von Hefner-Alteneck, and is used as a standard in Germany.

3. A lamp burning normal pentane, invented by Vernon Harcourt, and now used as the official standard for testing gas in London.

4. The light given out normally by a square centimetre of molten platinum at its solidifying temperature. This unit of light was proposed by Violle.

5. The light emitted by a square centimetre of solid platinum heated by the passage of an electric current to such a temperature that 10 per cent. of the radiation which it emits, as measured by a bolometer, can pass through a layer of water 2 centimetres thick contained in a cell with quartz sides. This standard was suggested by Lummer and Kurlbaum, and is in use at the Reichsanstalt at Berlin.

As a secondary standard, that is, one of which the value is determined once for all by comparison with one of the standards mentioned above, Fleming has recommended the use of a special form of carbon incandescent electric lamp working under fixed electrical conditions.

Of these various standards, the molten platinum and the LummerKurlbaum incandescent platinum strip standards require such delicate manipulation that their description is beyond the scope of this book. The Carcel is not a standard which in convenience in handling or accuracy much exceeds the standard candle. The Hefner-Alteneck lamp suffers from the defect that the colour of the light is much redder than the light given, say, by an incandescent electric lamp. A description of this lamp will be found in Elektrotechnische Zeitschrift, vol. iii. p. 445, and vol. v. p. 20. It must be noted that the light given by this standard varies very considerably with the quantity of water vapour in the air and the proportion of carbon dioxide present. A correction has also to be applied for the effects of changes in the barometric pressure.

A description of the Vernon Harcourt pentane lamp will be found in the Notification of the Gas Referees for the Year 1904, published by Eyre \& Spottiswoode. The light given by this standard also varies with 382 
the moisture and carbon dioxide present in the air, and with the barometric pressure.

The Fleming-Ediswan standard glow-lamp consists of a $U$-shaped carbon filament which has been run in an exhausted bulb for fifty hours at about 5 per cent. above its normal voltage. The filament was then removed and sealed into a new bulb, which is very mucb larger than the bulb of an ordinary incandescent lamp. Fleming ${ }^{i}$ finds that such lamps, if not run above their marked voltage, and not very much used, remain constant for a long time.

The candle-powers of sources of light are always compared by finding at what distances the illumination they produce on a surface are equal. If the source is of small dimensions the illumination at a distance $d$, from a source of candle-power $I$, is $I / d^{2}$. Hence if the candle-powers of two sources are $I_{1}$ and $I_{2}$, and they produce equal illumination at distances $d_{1}$ and $d_{2}$ respectively, we have

$$
\frac{I_{1}}{I_{2}}=\frac{d_{1}^{2}}{d_{2}^{2}} .
$$

It is important to remember that the inverse square law only holds for sources of small extent, or at any rate at such distances that the angle subtended by the largest dimension of the luminous body at the photometer is small. If the source is a circular disc of unit radius, the inverse square law holds within 1 per cent. at all distances greater than 10 .

155. Photometers.-(1) The Bunsen Photometer.-In its original form the Bunsen photometer consisted of a disc of unglazed paper, with a circular grease spot at the centre. The grease renders the paper more translucent than the untreated portion of the disc. Hence when the disc is placed between two lights practically no light is transmitted through the ungreased portion of the paper, and the illumination of this part of the disc depends on the light received from the source on that side. The illumination of the spot, however, depends on the light which is reflected at its surface, together with the light which traverses the spot from the other side. If the illumination of the spot seems the same as that of the surrounding paper, then just as much light traverses the spot from right to left as is transmitted from left to right, so that the light transmitted just makes up for the light which, in place of being reflected at the surface, has passed through the paper. But if the amounts transmitted by the spot in the two directions are equal, it means that the illumination received on the two sides of the disc are equal. Hence if we measure the distances of the sources of light from the disc, since the illuminations at the disc are equal, we can immediately calculate the ratio of the quantities of light given by the two sources in the directions considered.

When using the Bunsen photometer it is necessary to be able to see the two sides of the disc simultaneously; hence it is usual to arrange two mirrors inclined at $45^{\circ}$ to the plane of the disc, and to examine the images of the two sides of the disc as seen reflected in these two mirrors.

In practice it is found impossible to find a position of the disc at

1 Proceedings of the Institution of Electrical Engineers (1903), xxxii. p. 1. 
which both sides of the disc seem quite uniformly illuminated. This is particularly the case if the sources which are being compared do not give light of exactly the same colour. This difficulty has been in a measure reduced by Dibdin, who replaced the simple grease spot by a sheet of tissue paper, placed between two sheets of white card in which starshaped openings have been cut. The two stars are placed exactly opposite one another, and the tissue paper which stretches across the openings plays the part of the grease spot.

(2) The Lummer-Brodhun Photometer.-This photometer consists in a system of reflecting prisms, arranged so as that the field of view of a telescope is divided into two parts, one of which is illuminated by the light diffused from one side of a slab of magnesium carbonate which is exposed to the light from one of the sources, while the rest of the field is illuminated by light received from the other side of the slab, which is exposed to the second source of light. ${ }^{1}$ The arrangement of prisms is shown in Fig. 165. The essence of the instrument consists in the double prism CD, shown on a larger scale at $(a)$. This prism consists of two right-angled prisms placed with their hypotenuse faces in contact. The hypotenuse face of the prism $\mathrm{c}$ is slightly ground away, except for a

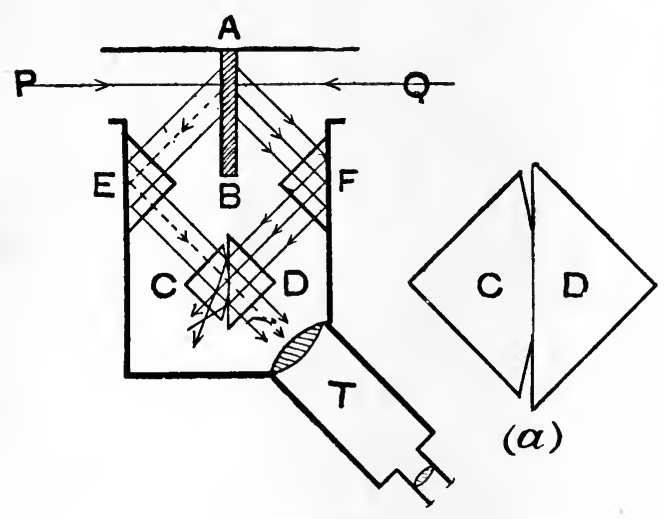

FIG. 165. circular portion in the centre. The two prisms are then cemented together so that at the centre light can pass through the central part from one prism to the other, whatever the angle at which it is incident on the common face. $\quad \mathbf{A B}$ is the magnesia slab, and light from the two sides is reflected by means of the prisms $\mathbf{E}$ and $F$ into the double prism in the manner shown by the arrows. Of the light which reaches the prism $\mathrm{c}$ from the left-hand surface of the slab, only that portion which falls on the part of the prism in contact with the prism $\mathrm{D}$ will be able to pass through and reach the telescope $\mathrm{T}$. The rest of the light will be reflected at the ground away portions of the hypotenuse face of the prism c. Of the light which comes from the right-hand surface of the slab, that which falls on the central part of the hypotenuse face of the prism $D$ will pass right through the prism $C$, and hence will not reaci the

1 Owing to the appreciable thickness of the slab $\mathrm{AB}$, the distances from the sources of light must be measured each op to the nearest surface of the slab. Hence each light is placed at a distause equal to half the thickness of the slab beyond the end of the photometer scale. 
telescope. The light which falls on the outside portions of the hypotenuse face of the prism $D$ will, however, be totally reflected in to the telescope. Hence the central part of the field of the telescope will be illuminated by light from the left-hand surface of the slab only, while the surrounding portions of the field will be illuminated by light coming from the right-hand surface of the slab. It will be seen that both portions of light have traversed equal thicknesses of glass, so that absorption within the glass will affect them equally. Thus if the whole field of the telescope appears equally bright, it follows that the two sides of the slab AB are equally illuminated. Arrangements are made so that the whole instrument may be rotated through $180^{\circ}$ with reference to the sources of light, thus interchanging the surfaces of the slab exposed to the two lights, so that any inequality in the surfaces may be eliminated.

The Lummer-Brodhun photometer is probably the most accurate form of photometer at present in use, and with it comparisons of light, which are of the same colour, can be made correct to about one-fifth per cent.

(3) 'The Rumford Photometer.-In this photometer, which ought with justice to be called the Lambert photometer, the two sources of light throw shadows of a rod on a white screen, and the distances of the sources from the screen are adjusted till the shadows appear equally dark. The distance of the rod which casts the shadow from the screen is immaterial; the edges of the shadow which it casts must, however, be sharp, so that in the case of sources of light of considerable extent the rod must be placed fairly close to the screen, and the lights at some distance. The lights and rod must also be so adjusted that the two shadows just touch, as in this case it is much easier to tell when the shadows are equally bright. This photometer has the merit of extreme simplicity, and since the necessary apparatus can be prepared in a few minutes, it is very convenient'for making rough measurements of the relative intensity of two sources of light.

(4) The Jolly Paraffin Block Photometer.-This

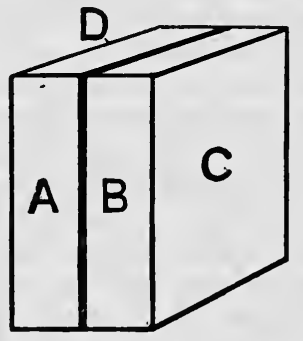

FIG. 166. photometer consists of two blocks of clear paraffin wax $\Delta$ and B (Fig. 166), which are separated by a sheet of tin-foil. The faces $\mathrm{C}$ and $\mathrm{D}$ are turned towards the sources of light, while the compound block is looked at from the side $\mathbf{A B}$. When the illumination of the faces $\mathbf{C}$ and $\mathbf{D}$ are equal, the two halves of the block will appear equally bright.

(5) The Photoped.-This photometer is used by the Gas Referees when testing the illuminating power of London gas. It consists of a metal screen A (Fig. 167), pierced at the centre with a square hole B, over which is stretched a piece of thin white paper. A tube $\mathrm{C}$ is attached to the screen, and within this tube slides a second tube $\mathbf{D}$. A diaphragm $\mathbf{E}$ having a rectangular aperture is attached to $D$. The distance of the diaphragm from the paper $\mathrm{B}$ is so adjusted that the two patches of light due to the lights $s_{1}$ and $s_{2}$ just touch. When the two patches appear equally bright, the illumination at $\mathbf{B}$, due to the two lights, is equal. 
(6) The Ritchie Photometer.-In this photometer two white surfaces are inclined at equal angles to the light coming from the two sources. The two surfaces meet along an edge, and the observer looks towards the edge,

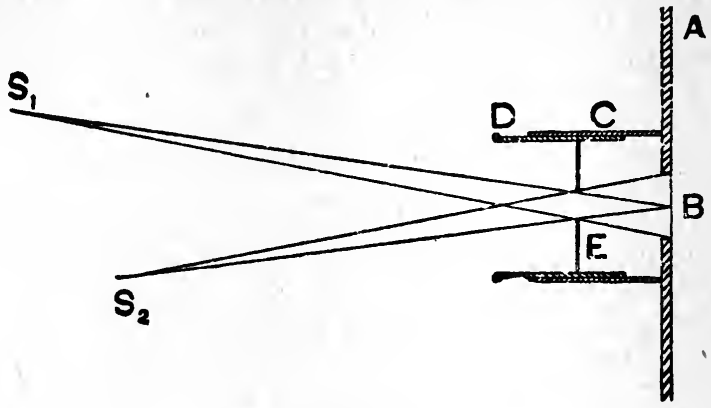

Fra. 167.

thus seeing the two surfaces alongside. The sources of light are then adjusted so that the two surfaces appear equally bright. In order to

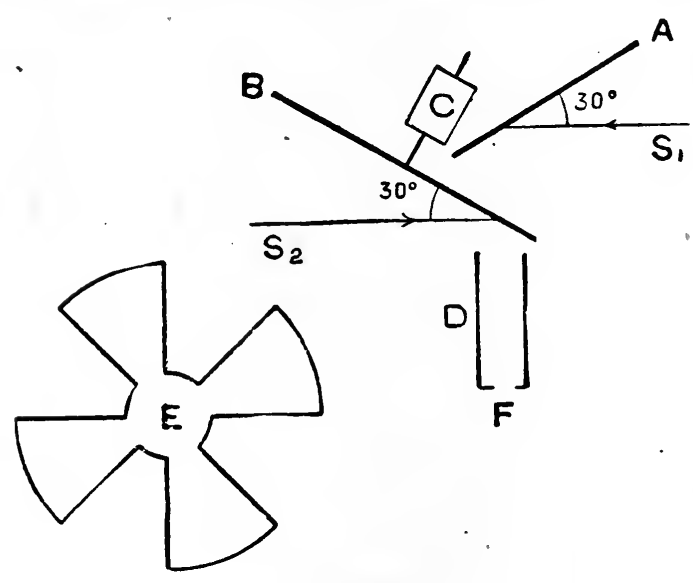

FIG. 168. make an accurate setting, it is of great importance that the edge, where the two surfaces meet, should be sharp.

(7) The Flicker Photometer.-This photometer consists of a white screen A (Fig. 168) placed at an angle of $30^{\circ}$ to the direction of the light coming from one of thelightss $s_{1}$. Asecond screen $B$ is mounted on the axle of a small electric motor. This screen is in the form of a Maltese cross, as shown at $\mathbf{E}$, the arms of the cross and the intervals being of equal width. The plane of this cross makes an angle of $30^{\circ}$ with the light from the second source $s_{2}$. The observer's eye is placed at $F$, and looks down a blackened tube $\mathrm{D}$, and as the cross rotates he alternately sees the face of the cross illuminated by the source $s_{2}$, and the screen $A$ illuminated by the source $s_{1}$. If the two illuminations are not equal, then when the cross rotates a sense of flicker will be produced, which will vanish when the illuminations are equal. This form of photometer is of special use when 
comparing two sources of different colours, since the fact that the lights are of different colours does not interfere with the vanishing of the sensation of flicker when the intensities of the lights are suitably adjusted.

It is essential when making photometrical measurements that all reflected light should be prevented from reaching the screen or photometer. Thus the walls of the room in which the photometer is placed must be painted a dead black, and the photometer itself placed at some distance from the walls, for even such substances as black velvet reflect an appreciable amount of light at large angles of incidence. Fleming recommends that the minimum size of room should be 2.5 metres wide, 2.5 metres high, and 6 metres long, with the photometer bar running down the middle of the room. ${ }^{1}$ The photometer bar consists of a long optical bench, with two carriages to support the lights, and a third carriage to support the photometer. If a flame standard is used, it will be necessary to provide means, by a fan or otherwise, to renew the air within the room, otherwise, owing to the accumulation of carbon dioxide and moisture in the air, the light given by the standard will decrease.

156. Determination of the Connection between the Candle-power of an Incandescent Lamp and the Watts consumed for Different Voltages.-The lamp to be investigated is mounted on the carriage of the photometer bar, and is connected through an ammeter and a variable resistance with a source of E.M.F. slightly greater than the marked voltage of the lamp. The terminals of the lamp are also connected to a voltmeter. On the other carriage is mounted another lamp, which will be taken as a standard. This lamp must either be permanently connected to a voltmeter, or a switch must be arranged by means of which a voltmeter can be connected to one or other of the lamps at will. An adjustable resistance must also be placed in series with this standard lamp. Adjust the resistance in series with the comparison lamp till the E.M.F. between its terminals has the value for which the lamp is marked, or some whole number of volts a little below this value. Also adjust the resistance in series with the lamp to be tested so that it is at its marked voltage, and then by moving the photometer or one of the lamps, secure uniformity of illumination on the two sides of the photometer. Immediately this balance is secured, read off the volts and amperes corresponding to the lamp under test. The setting should be repeated sereral times, and the mean taken. When adjusting the position of the photometer for equality of illumination, it will be found advantageous to oscillate it backwards and forwards fairly rapidly over a few millimetres, and to gradually reduce the amplitude of the movement till finally a position of adjustment is arrived at.

Repeat the observations, using gradually decreasing voltages on the lamp to be tested, but keeping the voltage on the comparison lamp constant. Then plot a curve showing the connection between the voltage and the light given by the lamp, taking the light given by the conparison lamp as unit. Also calculate the watts consumed by the lamp, that is,

1 If a number of blackened card screens, perforated with a central bole, are placed between the sources and the photometer, it is possible to completely screen the photometer from all reflected light, and thus obtain trustwortby measurements even in a room which has white walls. 
the product of the volts into the amperes, and divide the watts by the intensity of the light, and plot the quotient, that is, watts per candle, against the voltage.

157. The Spectro-photometer.-It is sometimes necessary to compare the intensities of the light given by two sources for light of the various wave-lengths taken throughout the spectrum. For this purpose it is necessary to form a spectrum with the light from each source, and then to compare the brightness of corresponding parts of the spectra. Such a comparison is made by means of a spectro-photometer. An ordinary spectrometer may be converted into a spectro-photometer in the following manner :-In front of the lens of the collimator AB (Fig. 169) is placed a double image prism c. This prism consists of a prism of calcite or quartz, cut with its refracting edge parallel to the optic axis of the crystal, cemented to a second prism of equal angle made of the same material, but cut with the refracting edge perpendicular to the optic axis. A ray of

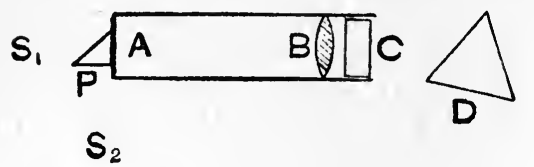

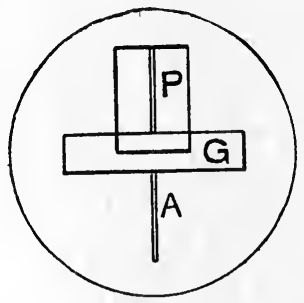

(a)

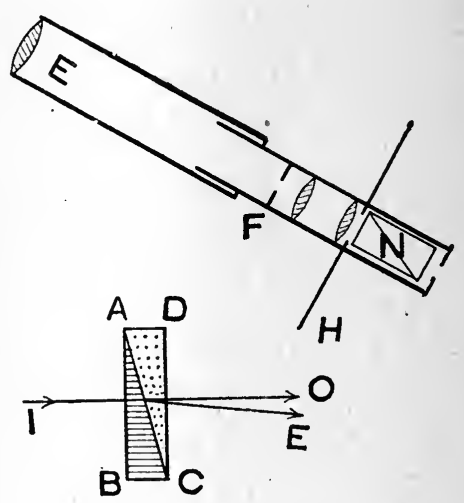

(b)

FIG. 169.

light I (Fig. 169, b) will pass through the prism $\triangle \mathrm{BC}$, in which the refracting edge is perpendicular to the optic axis, parallel to the optic axis, and therefore the ordinary and the extraordinary rays will coincide in direction. On reaching the prism $\triangle C D$, in which the refracting edge is parallel to the optic axis, the ordinary ray o will be undeviated, while the extraordinary ray $\mathbf{E}$ will be deviated, and thus after passing through the double prism the ordinary and extraordinary rays will proceed in directions inclined at a small angle. Thus the incident unpolarised light by its passage through the prism is divided into two rays of equal intensity which are plane polarised, the planes of polarisation in the two rays being at right angles.

The double image prism being placed in front of the collimator. 
object glass, on turning the telescope to view the slit, the prism $\mathbf{D}$ having been removed, two images of the slit will be seen. The double image prism must then be rotated till these two images form a single line. The two images will overlap at the centre, and a piece of paper G (Fig. 169, $a$, must be pasted over the central part of the slit, and its width so adjusted that of the four images now formed the two middle oncs just touch but do not overlap. Thus in the centre of the field there will be two adjacent images of the slit, one formed by light which has passed through the upper portion of the slit, and the other by light which has passed through the lower portion, and since one of these will correspond to an ordinary ray, and the other to an extraordinary ray, they will be polarised in perpendicular planes. ${ }^{1}$ The uppermost and lowest of the four images must then be cut out by a screen placed in the telescope in the plane of the cross-wires.

The Nicol N (Fig. 169), with its divided circle, is then attached to the telescope, and the Nicol is turned till one of the images vanishes, when the reading of the circle is noted. This will give the zero reading, and a rotation of the Nicol through $90^{\circ}$ from this position will cause the other image to vanish.

On placing the prism $\mathrm{D}$ on the table two spectra will be seen, one formed with light from each half of the slit, and these spectra will be polarised in planes at right angles.

Suppose, then, that the lower part of the slit is illuminated by a source of light $s_{1}$, while, by means of a small totally reflecting prism $P$, the light from a second source $s_{2}$ is caused to illuminate the upper part of the slit, then the two spectra will be due to the two lights. To compare the brightness for different colours a diaphragm $F$, with a fairly narrow vertical slit, is placed at the focal plane of the eye-piece of the telescope so as to cut out all the spectrum except the portions which are to be compared. The Nicol is then turned till the two portions of the spectra appear equally bright. Let the angle through which the Nicol has to be turned from its zero position be $\theta$. Then

$$
\frac{I_{2}}{I_{1}}=\tan ^{2} \theta,
$$

where $I_{1}$ and $I_{2}$ are the intensities of the two sources for light of the wave-length being compared.

Let $\triangle О В$ (Fig. 170) represent the zero position of the Nicol, that is, the position of its plane of polarisation when the upper

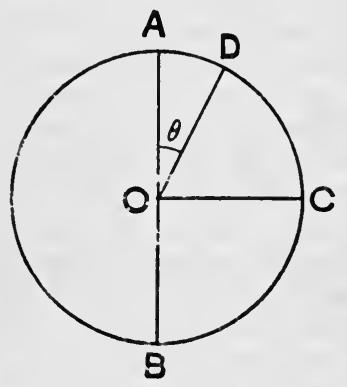

Fig. 170. images is cut off, i.e. when the light from the source $s_{1}$ is extinguished. Then oc will be the position of the plane of polarisation of the Nicol when the light from the source $s_{2}$ is

1 Owing to the amount of light lost by reflection at the face of the prism depending on the plane of polarisation of the light, the expression given above may gire results which are erroneous by as much as 10 per cent. 
extinguished. When the plane of polarisation of the Nicol is parallel to oD, the intensity of the light from source $s_{1}$ transmitted will be $I_{1} \sin ^{2} \theta$, while the intensity of the light due to the source $s_{2}$ will be $I_{2} \cos ^{2} \theta$. Hence since the intensities of the light transmitted by the Nicol from the two sources are equal, we have

$$
I_{1} \sin ^{2} \theta=I_{2} \cos ^{2} \theta \text {. }
$$

As an exercise in the use of the spectro-photometer, compare the light given by an ordinary bat's-wing burner with that given by a Welsbach incandescent mantle. It will, in the first place, be necessary to draw a curve giving the deviations produced by the prism D (Fig. 169) in terms of the wave-length by one of the methods described in $\S 124$.

Next place the two flames in such positions that they illuminate the slit, one directly, and the other after reflection in the prism P. The exact equality of the distances of the lights from the slit is not important, so long as each of them entirely fills the angle obtained by producing the lines drawn from the edge of the collimator lens through the slit.

Then determine the position of the. Nicol when equality of illumination is secured for a number of points taken through the spectrum, and plot a curve showing the connection between $\tan ^{2} \theta$ and the wave-length. It will appear from this curve that the Welsbach is relatively richer in the light of smaller wave-length.

158. Measurement of the Absorption Curve of a Solution.-A small glass cell containing a dilute solution of cyanine in alcohol is placed over half of the slit of the spectro-photometer, and then the light from a bright source of light, such as the filament of a Nernst lamp, is allowed to pass -directly through half ${ }^{\circ}$ of the slit, and after passing through the solution, through the other half of the slit. Then determine the readings of the Nicol when the different portions of the two spectra are equally bright. If $\theta$ is the reading for the Nicol, then the ratio of the light transmitted by the solution to the original light is given by $\tan ^{2} \theta$. Plot a curve showing the connection between the proportion of light transmitted and the wave-length.

Similar curves may be obtained for solutions of permanganate of potash and for various coloured glasses. When measuring the absorption with a cell containing the solution before part of the slit there will be a certain amount of loss of light, owing to reflection at the glass surfaces, as well as owing to absorption within the glass itself. Hence it is often advisable to use a second cell with a smaller thickness of solution before the other half of the slit, so that both beams of light pass through a cell, but the thickness of solution in one case is greater than in the other. In place of using two cells a single cell may be used, but a plate of glass of measured thickness, say, $1 \mathrm{~cm}$., may be placed in the lower part of the cell, and the light which enters through the lower half of the slit allowed to traverse this plate of glass, while the light which enters through the upper part of the slit traverses the whole width of the cell in the solution. The differences which are obtained in the intensities of the transmitted light then correspond to the absorption 
of a layer of the solution having a thickness equal to the thickness of the glass plate.

If $I_{0}$ is the intensity of the incident light, and $I_{t}$ is the intensity of the light after passing through a thickness $t$ of the substance, it is found that the following relation holds,

$$
I_{t}=I_{0} e^{-a t},
$$

where $a$ is a constant depending on the colour of the light and the nature of the substance, and $e$ is the base of the Napierian system of logarithms. Taking the Napierian logarithms of both sides, we have,

$$
a=-\frac{1}{t} \log _{e} \frac{I_{t}}{I_{0}}
$$

In the case of logarithms to the base ten, then we have a similar relation, namely,

$$
A=-\frac{1}{t} \log _{10} \frac{I_{t}}{I_{0}}
$$

where $A=a / 2 \cdot 30$. The quantity $A$ is called the absorption coefficient of the substance.

By making a series of observations with different thicknesses of solution, and plotting on semi-logarithmic paper the relation between $I_{t} / I_{0}$ and the thickness, a straight line will be obtained, proving the correctness of the expression assumed above for the relation between the intensities of the incident and transmitted light.

159. Colour Mixture with the Colour Top.-The apparatus used for this experiment consists of a small electric motor, to the spindle of which is attached a circular plate and a milled clamping nut. The nut allows of coloured cardboard discs being clamped against the face of the plate. There are also cardboard dises of two sizes, coloured black (lamp black), white (zinc white), red (vermilion), yellow (chrome yellow), green (emerald green), and blue (French ultramarine). The larger disc of each colour has a diameter of about $14 \mathrm{~cm}$., while the smaller discs have a diameter of about $8 \mathrm{~cm}$. The discs have a hole punched at the centre, ${ }^{1}$ so that they will pass over the screwed end of the motor spindle. Each disc is also slit radially, so that by arranging these discs in the manner illustrated in Fig. 171, and then slipping round one disc with reference to the other, the relative amounts of each colour exposed can be altered.

Taking any five of the six colours, it will be found possible to match the colour obtained by blending two of them taken in suitable proportions with a blend of the other three. Thus, take a black and a white disc of the larger size, and take smaller discs of the three colours,

1 It is essential that these coloured discs should be uniformly coated with the paint, and further, that they should be cut truly circular and be accurately centred. If the discs are not centred correctly, then when rotated a coluured line will appear at the edge of the smaller discs, which will be found to make the adjustment to an exact match very difticult. 
red, green, and blue, and mount them all on the spindle of the motor, and place the motor facing a window which has a northerly aspect. Connect the motor to a switch, an adjustable carbon resistance, and a sufficient number of accumulators, so that when the motor is rotating the coloured discs appear to blend into one, and no sense of flicker is apparent.

Starting with about a quarter of the white disc exposed, set the motor in rotation, and observe whether the inner discs blend into a grey tinge. If the tinge observed seems purple, increase the green sector; if yellow, increase the blue; while, of course, if the appearance is reddish or greenish, the red or green, as the case may be, is to be reduced. Having adjusted the inner discs to produce a grey, adjust the outer discs so that the luminosity of the grey they form is the same as that of the inner discs.

When making the final match, it is advisable to observe the discs from a distance of 2 or 3 metres, and not to stare at the discs too

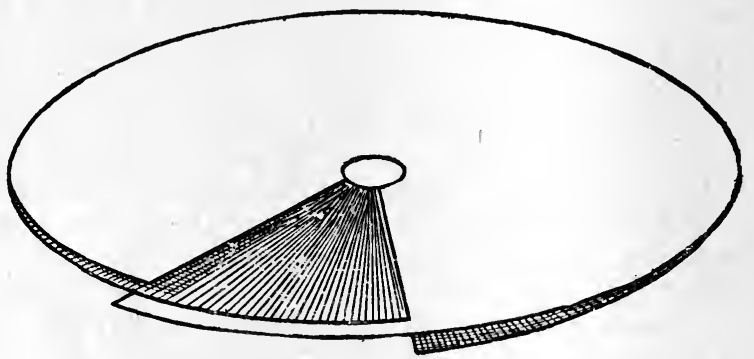

FIG. 171.

long. If you cannot make up your mind as to whether the match is complete, look away for a few seconds, and then, when the eyes are a bit rested, look back at the discs. The quantities of the various colours required to make a match depend on the character of the light by which the discs are illuminated. Thus it will be found impossible to make a consistent set of measurements if the light varies much, say, by clouds drifting over a blue sky. For this reason.it is often advisable to use the light from an electric arc, the quality of which is much more constant than is that of day-light.

To obtain the amounts of the different colours exposed, and which form a match, a card annulus, of which the inner diameter is a trifle less than the diameter of the smaller discs, and the external diameter is less than the diameter of the larger discs, is used. The outside and inside edges of this annulus are graduated in 100 parts, and the annulus being placed over the discs, the angular aperture of each colour can at once be read off.

Having made several settings with the above five discs, next take 
large discs of white, black, and yellow, and small discs of green and red, and adjust the green and red sectors till they blend into a yellow. Then adjust the yellow, white, and black discs till they blend into a yellow which matches the inner discs both as to colour and luminosity.

Then proceed to match the black and white against the green, yellow, and blue; the black, white, and red against the yellow and blue; the black, yellow, and blue against the white and green; and finally, the white, red, and green against the yellow and blue.

By the above experiments, six sets of matches will have been obtained between five of the six colours. Now, according to Maxwell's experiments, a match can be obtained between any four colours. The reason we have had to take five colours in each of the above experiments is that the rotating disc method of blending colours involves a special condition, namely, that the whole circumference of both the circles must be filled up by the colours. This is the reason for the inclusion of the black disc, the effect of the black being to alter the luminosity of the tint formed by the blending of the other colours with which it is combined.

Using the symbols $R, Y, G, B, W$, and $X$ to represent the colours red, yellow, green, blue, white, and black, we may write the result of the first match in the form of an equation as follows:

$$
r_{1} R+g_{1} G+b_{1} B=w_{1} W+x_{1} X . \quad \text {. . . . }
$$

where $r_{1}, g_{1}, b_{1}$, \&c., are the sizes of the sectors of the different colours when a match is secured.

In the same way, for the second match we should obtain an equation of the form

$$
r_{2} R+g_{2} G=w_{2} W+x_{2} X+y_{2} Y \quad \text {. . . . . }
$$

In the same way, the third match will give

$$
g_{3} G+b_{3} B+y_{3} Y=w_{3} W+x_{3} X
$$

Now if we multiply equation (1) through by $r_{2}$, and equation (2) by $r_{1}$ and subtract, we obtain

$$
\left(r_{2} g_{1}-r_{1} g_{2}\right) G+r_{2} b_{1} B+r_{1} y_{2} Y=\left(r_{2} w_{1}-r_{1} w_{2}\right) W+\left(r_{q_{1}} x_{1}-r_{1} x_{2}\right) X
$$

that is, we get a relation between green, yellow and blue, and white and black. But this is exactly what we have obtained by experiment as expressed by equation (3). Proceeding in this manner, we can from any two of the colour equations calculate the other four equations.

Choosing two of the matches which you consider the most accurate, deduce from these the colour equations of the other combinations, and compare the numbers obtained with those given by experiment.

In place of arbitrarily choosing two of the matches, and using these to calculate the others, we may take the six colour equations obtained 
experimentally and calculate two normal equations between the six quantities $R, Y, G, B, W$, and $X$ by the method described in $\S 5$. Then from these two equations calculate the colour equations corresponding to the six matches, when a comparison of the numbers thus obtained with the experimental numbers will give a measure of the accuracy with which the experiments have been carried out.

In the case of colour-blind observers, the matches obtained may differ very considerably from those made by a normal-eyed observer.

160. Measurement of the Luminosity of Pigment Colours.-To measure the luminosity of the light reflected from different pigments the piece of apparatus shown in Fig. 136 is arranged with an arc lamp as the source of light, so that by rotating the prism a beam of monochromatic light of different wave-lengths is obtained beyond the lens. The pigment to be tested is painted on a card disc about $8 \mathrm{~cm}$. in diameter, and together with larger discs of white and black is mounted on the axle of the motor used in the last section. The rotating discs are then illuminated by the monochromatic light, and the white and black discs are adjusted till the central and peripheral parts of the rotating disc appear equally bright. When this adjustment is complete, the number of divisions of the white sector gives the luminosity of the pigment for the given coloured light in terms of the luminosity of the white disc taken as 100 .

Using discs of the colours employed in the last section, determine the luminosity for different coloured lights taken throughout the spectrum. By placing salts, such as lithium, sodium, and magnesium chlorides, in the carbons of the arc, the lines corresponding to these metals will be seen in the spectrum formed in the plane of the slit. Hence if the prism is so adjusted that the red lithium line, the yellow sodium lines, the green magnesium lines, and the blue lithium line are in turn on the slit, observations may be made with light of these known wave-lengths, and a curve may be plotted for each pigment showing the luminosity in terms of the wave-length of the incident light.

A study of these curves, in connection with the character of the light given by different sources, such as a gas flame, a sodium flame, \&c., will explain the different appearances of the different pigments when examined by the light of these various sources.

161. Test for Colour-Blindness with Holmgren's Wools.-Holmgren's wool test for colour-blindness consists in asking the candidate to match certain test skeins by selecting from a large number a different coloured skein. The skeins from which a selection has to be made consist of several shades of red, orange, yellow, yellowish-green, green, blue-green, blue, violet, purple, pink, brown, and grey. Varieties of pinks, blues, violets, and light greys should be particularly well represented. The test skeins are three in number. The first is a very light green, and is chosen of such a colour as to match the spectrum colour which a red or green colour-blind person cannot distinguish from white or grey (see Watson's Physics, § 401). A colour-blind person will 
therefore select, in addition to correct matches, some light greys or drabs.

The second test skein is a light pink, which is very nearly a complementary colour to the green of the first test. This colour in the green-blind excites both the blue and the red sensations, as do also greys and greens. Hence a green-blind person will select as matches greys and greens in addition to the correct colours. To a red-blind person the pink only excites the blue sensation, and hence he will include in his selection blues and violets.

The third test skein is a vivid red containing a little blue. The green-blind person will select shades of red slightly lighter than the test skein. The red-blind, however, since he does not see the red, will match this test against dark green, dark blue, and dark brown.

To carry out the test, the skeins are arranged in a heap on a sheet of white paper in a good light, and the candidate is handed the first test skein and asked to select from the heap all the skeins which are of the same colour as the test skein. $\mathrm{He}$ is told to include not only skeins which appear to him exactly the same colour, but also skeins which appear to be slightly lighter and darker shades of the same colour. If no greys, drabs, or light pinks are included in the selection, the candidate has normal colour vision.

The second test skein is then treated in the same way, the skeins selected during the first test having been returned to the heap. If, after the correct matches have been removed from the selected skeins, the prevalent colour of the incorrect skeins is blue, the candidate is red colour-blind. If in addition to purple he selects greys and greens, he is green colour-blind.

The third test is made in the same manner as the first two, and the character of the mistakes will confirm the results given by the second test.

A person with normal colour vision may obtain an idea of the selection which will be made by a colour-blind person by making a selection, using either a blue-green or a purple glass before the eyes. Using a blue-green glass he will make the mistakes made by a red colour-blind person, while with the purple glass he will make the same mistakes as a green colour-blind person.

For further particular's as to testing for colour-blindness, reference may be made to the Proceedings of the Royal Society (1892), vol. li. p. 376. 


\section{CHAPTER XXIV}

\section{MEASUREMENT OF THE EARTH'S MAGNETIC FIELD}

162. Determination of the Direction of the Magnetic Meridian.When determining the direction of the magnetic meridian it is not sufficient simply to determine the direction in which the geometrical axis of a freely suspended magnet points, since the magnetic axis of the magnet seldom exactly coincides with the geometrical axis. If, however, after noting the direction of the geometrical axis when the magnet is suspended in one position, the magnet is turned through an angle of $180^{\circ}$ about its geometrical axis, suspended in this new position, and the direction of the geometrical axis again noted, then the bisection of the angle between the two positions occupied by the magnet is the magnetic meridian.

In the Kew pattern magnetometer the magnet consists of a cylinder of steel A (Fig. 172). This cylinder is fixed in a brass collar, to which

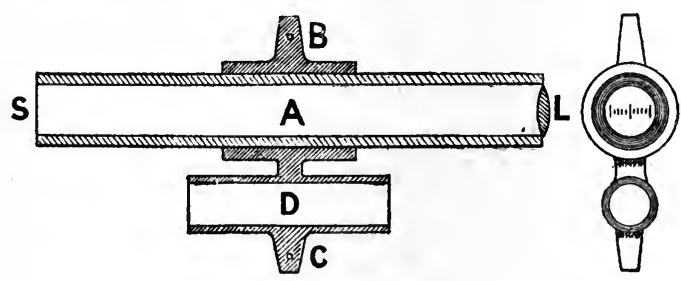

FIG. 172. are attached two pegs B and c. Either of these pegs fits into a brass collar which is attached to the end of the silk suspension fibre. A short hollow cylinder $\mathrm{D}$, forming part of the brass attachment, serves for the support of a cylindrical bar when determining the moment of inertia of the magnet. At one end of the hollow magnet. is placed a convex lens $L_{\text {, while at }}$ the other end is placed a disc of plane glass, on which is engraved a fine scale. The focal length of the lens is equal to the distance between the lens and the scale, so that the rays of light starting from any point on the scale leave the lens as a parallel pencil. The line joining the centre of the scale to the optical centre of the lens forms the geometrical axis of the magnet, and this does not usually differ by more than about five minutes of arc from the magnetic axis.

Since the magnetic axis of the suspended magnet will only point in the direction of the magnetic meridian if the suspension thread is entirely free from torsion, care has to be taken to remove as far as possible all torsion from the silk suspension, and to allow for the effect of any residual 
torsion. The suspension fibre usually consists of a bundle of unspun silk fibres, and since the torsion of such a bundle of fibres is found to vary with their dampness, it is advisable to ensure that they are always damp by moistening the fibre with a little glycerine. To remove the torsion the magnet is replaced by a plummet having the same weight as the magnet, and if the fibre is new, the plummet is allowed to hang for several hours. The plummet having come to rest, the torsion head, from which the fibre hangs, is turned so that the clip is in such a position that when the magnet is put in place the fibre will be free from torsion.

To make a measurement of declination with the Kew-pattern of magnetometer, the instrument is set up on a firm tripod stand or pillar and levelled. The wooden box with the glass tube carrying the torsion head is then attached, and the plummet suspended. The torsion having been removed, the magnet is suspended in such a way that the scale, as seen in the telescope, appears right side up. The motion of the magnet having been checked with the arrestor, the whole instrument is turned till the magnet swings approximately equally on the two sides of the zero of the scale. The motion of the magnet must then be reduced to a fraction of a scale division by alternately approaching and removing a fecbly magnetised piece of steel, such as the screw-driver supplied with the instrument. Having finally adjusted the instrument so that the vertical cross-wire coincides with the middle division of the scale, the time is noted, and the two verniers on the horizontal circle of the instrument are read. The magnet is then reversed, so that the scale appears inverted, and two settings are made, the time and the readings of the verniers being noted in each case. Finally, a second setting is made with the magnet in its original position. The mean of all the vernier readings gives the reading on the azimuth circle of the instrument corresponding to the magnetic meridian at the mean time of the observations.

To obtain the declination, it is necessary to know the reading of the azimuth circle corresponding to the geographical north. In the case of a fixed observing station it is usual to have some fixed mark, the azimuth of which is determined once for all. In the absence of such a fixed mark, it will be necessary to make observations on the sun or stars to determine the geographical meridian (see next section). In the case of the fixed mark, the instrument is turned till the vertical cross-wire bisects the mark, and the readings of the azimuth circle are taken. Then from the known azimuth of the mark the reading corresponding to the geographical north is obtained, and the difference between this reading and that obtained for the magnetic meridian gives the declination.

163. Determination of the Geographical Meridian.-If the geographical meridian is to be obtained by ohservations of the sun, then it will be necessary to know (1) the latitude and longitude of the place of observation, which may be obtained with sufficient accuracy from the Ordnance map, and (2) the error of a chronometer.

Since in the Kew-pattern magnetometer the telescope is fixed in a horizontal position, in order to obtain an image of the sun in the telescope 
a small mirror is used which can turn about a horizontal axis in two V's. The axis about which this mirror turns must fulfil three conditions: (1) it must be exactly horizontal, (2) it must be parallel to the plane of the mirror, and (3) it must be perpendicular to the optical axis of the telescope. To perform the first adjustment a small striding level is provided, and one of the V's is adjustable in a vertical direction. This adjustable V must be moved till, on reversing the level, the bubble remains in the same position; when this is the case, the axle of the mirror is horizontal. To allow of the second and third adjustments being made, the eye-piece of the telescope is provided with a lateral opening and an annular mirror set at $45^{\circ}$ to the axis. By means of this annular mirror the cross-wires are illuminated, and if a blackened tube or other screen is placed between the mirror and the telescope, so as to cut off stray light, on turning the mirror an image of the cross-wires will be seen. The table which carries the mirror V's must be turned about a vertical axis till the vertical crosswire coincides with its image. The mirror must then be reversed in its $V$ 's, and if the image still coincides with the vertical cross-wire, the mirror is in adjustment. If there is not coincidence, then the image must be brought half-way back by rotating the table which carries the V's, and the remainder by adjusting the mirror with reference to the axle by means of a small screw placed at the back. In this way the adjustment of the mirror can be made fairly accurately. Since, however, the observations are so arranged as to allow of a correction being applied, so long as the adjustment is not very bad, it is not necessary to attempt to make the adjustment perfect.

The times of first and second contacts of the image of the sun with the vertical cross-wire for four transits are then taken, a coloured glass being interposed so as to reduce the brightness of the image. For the two first direct transits the observer faces the sun, the mirror being reversed in its V's between the two transits, while for the second pair, or back transits, the instrument is turned in azimuth through $180^{\circ}$, so that the observer has his back to the sun, the mirror being again reversed between the two transits. After each transit the readings on the horizontal azimuth circle are recorded as in the following example.

To calculate from the times of transit and the circle readings what would be the reading if the telescope were pointed due geographical north, it will be necessary to have a copy of the Nautical Almanac. The method of performing the reduction will be seen by studying the example in conjunction with the following notes :- 
Observation of Sun's Azimuth. Date 23rd April 1891. Station, Kew. Lat. $51^{\circ} 28^{\prime} 6^{\prime \prime}$. Long. $0^{\circ} 18^{\prime} 46^{\prime \prime} \cdot 5 \mathrm{~W} .=m$. \& Chronometer, Dent. Error at Station, $45^{\circ} 0$ seconds fast.

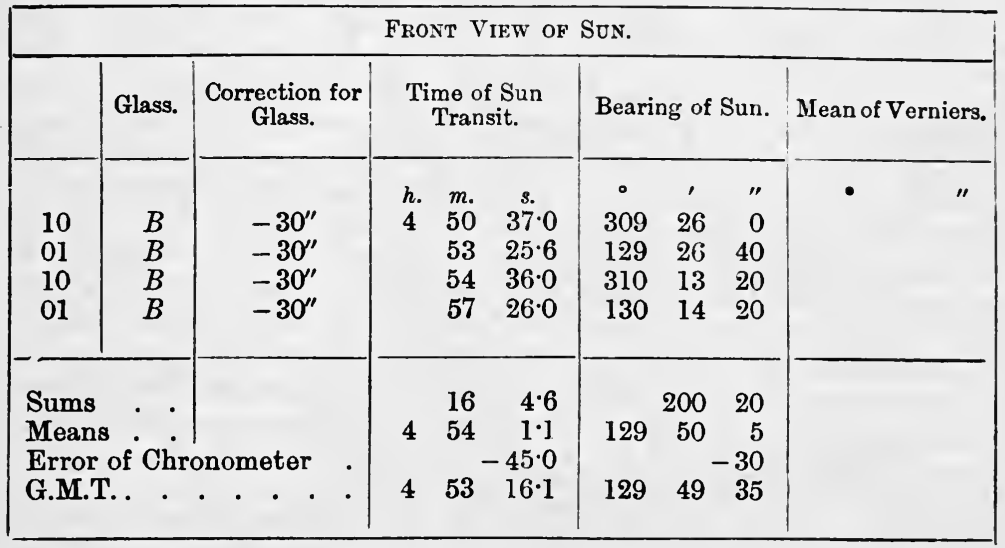

BACK VIEW OF SUN.

\begin{tabular}{|c|c|c|c|c|c|c|c|}
\hline \multirow{2}{*}{$\begin{array}{l} \\
01 \\
10 \\
01 \\
10\end{array}$} & \multirow{2}{*}{$\begin{array}{c}\text { Glass. } \\
\\
B \\
B \\
B \\
B\end{array}$} & \multirow{2}{*}{\begin{tabular}{|c}
$\begin{array}{c}\text { Correction for } \\
\text { Glass. }\end{array}$ \\
\\
$-30^{\prime \prime}$ \\
$-30^{\prime \prime}$ \\
$-30^{\prime \prime}$ \\
$-30^{\prime \prime}$
\end{tabular}} & \multicolumn{3}{|c|}{$\begin{array}{l}\text { Time of Sun } \\
\text { Transit. }\end{array}$} & Bearing of Sun. & Mean of Verniers. \\
\hline & & & $\begin{array}{l}h . \\
4 \\
5 \\
5 \\
5\end{array}$ & $\begin{array}{r}m . \\
59 \\
1 \\
\mathbf{3} \\
\mathbf{5}\end{array}$ & $\begin{array}{r}s \cdot 5 \\
7 \cdot 5 \\
56 \cdot 5 \\
6 \cdot 0 \\
55 \cdot 3\end{array}$ & \begin{tabular}{rrr}
$\bullet$ & $\prime$ & \multicolumn{1}{c}{} \\
131 & 7 & 10 \\
311 & 6 & 0 \\
131 & 53 & 50 \\
311 & 52 & 40
\end{tabular} & • ' \\
\hline \multicolumn{3}{|c|}{$\begin{array}{l}\text { Sums . . } \\
\text { Means : } \\
\text { Error of Chronometer } \\
\text { G.M.T.. . . . . . . . }\end{array}$} & & $\begin{array}{r}10 \\
2 \\
1\end{array}$ & $\begin{array}{r}5 \cdot 3 \\
31 \cdot 3 \\
-45 \cdot 0 \\
46 \cdot 3\end{array}$ & $\begin{array}{rrr} & 119 & 40 \\
131 & 29 & 55 \\
& & -30 \\
131 & 29 & 25\end{array}$ & \\
\hline & $\begin{array}{l}\text { Co-Lati } \\
\text { nd } C^{\prime}= \\
\text { nd } Q= \\
\text { nd } A^{\prime}= \\
180^{\circ}-\end{array}$ & $\begin{array}{l}\text { de of place o } \\
\text { our angles of } \\
\text { agles, such th } \\
\text { zimuths of } S\end{array}$ & & $\begin{array}{l}\text { un a } \\
-Q=\end{array}$ & $\begin{array}{l}\text { on. } \\
\text { the } t \\
\text { A. } \\
\text { orth at }\end{array}$ & $\begin{array}{l}(a+b) \\
\text { of direct and } \\
\text { e of directand }\end{array}$ & $\begin{array}{l}\text { ck observations. } \\
\text { ack observations. }\end{array}$ \\
\hline
\end{tabular}

Observer, w. w. 


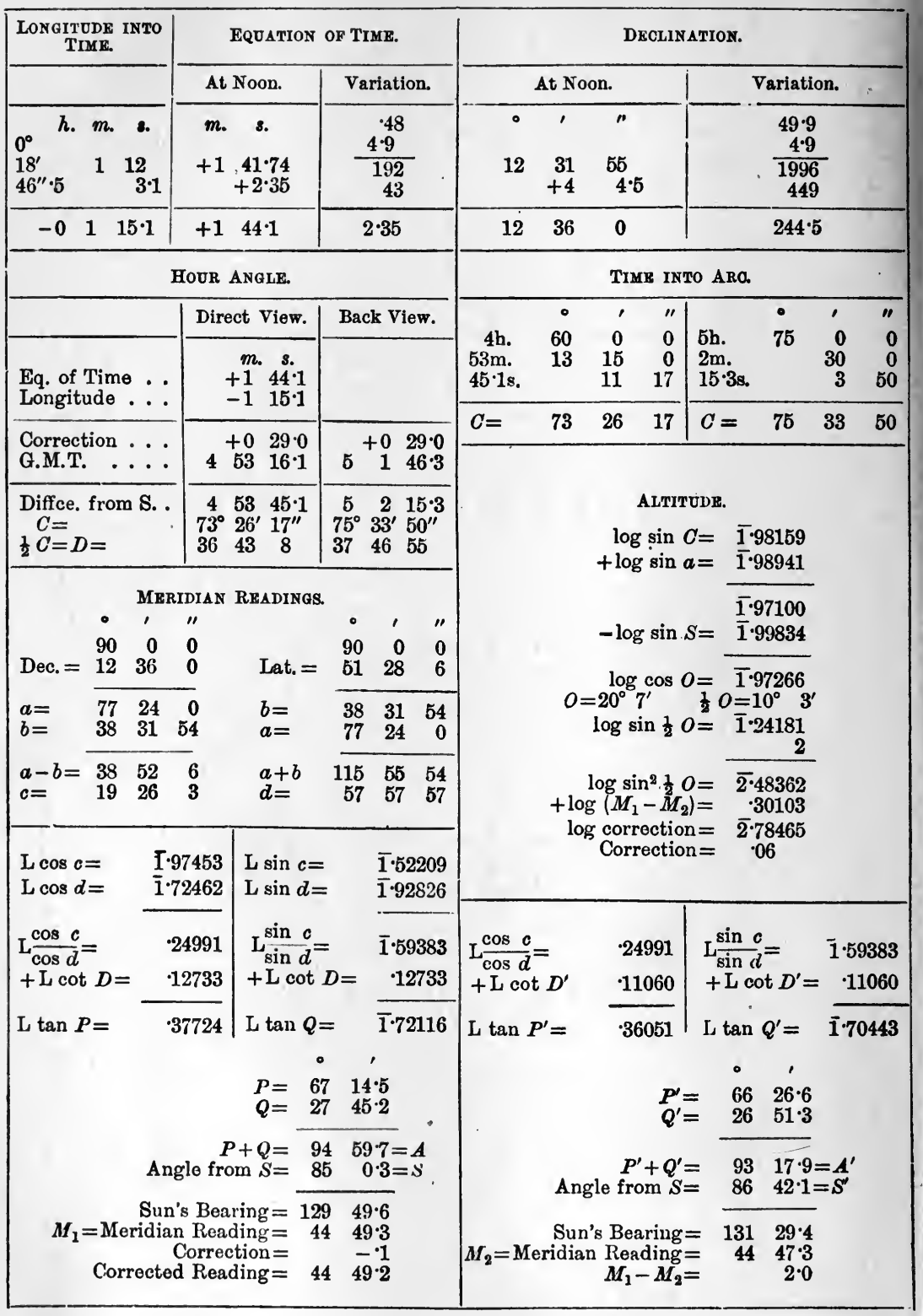


Take the mean of the four times of contact for the "front" transits, and apply the chronometer correction so as to obtain the Greenwich mean time corresponding to the mean of the times of contact. Also take the mean of the vernier readings, applying where necessary a correction to allow for any error due to the dark glass employed being prismatic. The value of this correction is generally supplied with the instrument. It can easily be obtained by looking with the telescope at a bright spot of light, and seeing how much the telescope has to be rotated to bring the image back on the cross-wire when the glass is interposed.

Obtain the same means for the back observations.

Convert the longitude into time, allowing four minutes for each degree ${ }^{1}-i . e$. calculate how long after noon at Greenwich, noon will occur at the place of observation. Of course if the station is east of Greenwich, noon will occur earlier than at Greenwich.

We have next to allow for the fact that owing to the eccentricity of the earth's orbit the true sun is not in general on the meridian at noon by mean time. The time before or after mean noon when the sun crosses the meridian is called the equation of time, and is given for noon of each day in the Nautical Almanac. What is required is the equation of time at the mean time of the "front" observations of transit. This is obtained by multiplying the variation of the equation of time per hour by the number of hours between the mean time of transit for the front observations and noon. The value of the sun's declination at the mean time of transit for the front observation is also calculated from the quantities given in the Almanac.

Let o (Fig. 173) be the position of the observer, $p$ the north pole of the earth, and $\mathbf{P P}^{\prime}$ the axis about which the earth turns, cutting the celestial sphere at the points $P$ and $\mathbf{P}^{\prime}, \mathrm{z}$ the zenith, and $\Sigma$ the sun.

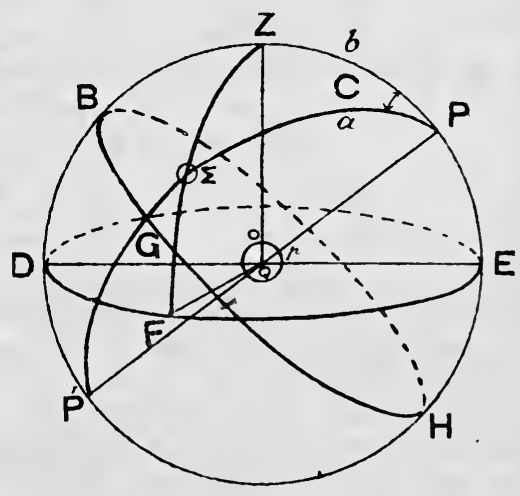

FIG. 173. Then the great circle HGB is the celestial equator, and the great circle DFE is the observer's horizon, and the angle to be calculated is DQF, for the mean of the circle readings corresponds to the point $F$, where the vertical circle through the sun at the time of the transit observations cuts the horizon. To obtain this angle we must first calculate the hour angle $\mathrm{ZP} \Sigma$, which is the angle the great circle $\mathrm{P}^{\mathrm{S}} \mathrm{P}$ ' through the sun makes with the meridian PZP'.

To obtain this angle we have to calculate what is the local solar time

${ }^{1} 1^{\circ}=4$ minutes; $1^{\prime}=4$ seconds $; 1^{\prime \prime}=1$ second. Tables for this conversion are given in Mathematical Tables, $c f$. Chambers, p. 434. 
corresponding to the mean time of the front transition observations. Now this time will differ from Greenwich mean time (G.M.T.) on account (1) of the equation of time, and (2) of the longitude of the place being different from the longitude of Greenwich. The correction for equation of time has already been calculated, and is positive if the true sun is ahead of the imaginary mean-time sun. In the example this is the case, for on April 23 the true sun crosses the meridian at Greenwich before noon, as indicated by a mean-time clock. Hence solar time is ahead of mean time, and we have to add the equation of time.

If the station is east of Greenwich, the sun will cross the meridian before noon, as indicated by a clock keeping Greenwich solar time, and hence the correction for longitude is positive. If the station is west of Greenwich, the correction for longitude is negative, as in the example.

Applying the sum of these two corrections, we get the local solar time corresponding to the time of transit for the mean of the front observations, and if the back observations were only a few minutes later, the same correction may be used in their case. The difference between this corrected time and noon gives the angle $\mathrm{ZP}_{\mathrm{P}} \mathrm{i}$ in time. This has to be converted into degrees, \&c., by means of the table given in the Nautical Almanac, two small spaces on the form being set apart for the purpose.

We now know two sides, and the included angle of the spherical triangle $Z \mathrm{P} \Sigma$, and have to calculate the value of the angle $\mathrm{PZ} \Sigma$. The side $\Sigma \mathrm{P}$, or the polar distance of the sun, is $90^{\circ}-\mathrm{G} \Sigma$, that is, $90^{\circ}-$ sun's declination, the declination being taken plus when the sun is above the celestial equator. The side $\mathrm{ZP}$ is the co-latitude of the place of observation, i.e. $90^{\circ}$-latitude. Calling the polar distance of the sun $a$, the co-latitude $b$, the hour angle $C$, the angle $\mathrm{Pz} \Sigma A$, and the angle $\mathrm{P} \Sigma \mathrm{z} B$, we have, by the usual formulæ for the solution of spherical triangles,

and

$$
\begin{aligned}
& \tan \frac{A+B}{2}=\frac{\cos \frac{a-b}{2}}{\cos \frac{a+b}{2} \cot \frac{C}{2}} \\
& \tan \frac{A-B}{2}=\frac{\sin \frac{a-b}{2}}{\sin \frac{a+b}{2}} \cot \frac{C}{2}
\end{aligned}
$$

Calling $\frac{A+B}{2} P$ and $\frac{A-B}{2} Q$, we get from the above equations the values of $P$ and $Q$, and then

$$
A=P+Q
$$

Now, when making the declination observations the telescope looks at the $N$ end of the needle. Hence what we require is the reading on 
the horizontal circle when the telescope points due south, that is, we require the angle $\mathrm{Dz} \Sigma$, which is given by

$$
S=180^{\circ}-A .
$$

This gives the actual angle through which the telescope must be turned from its position when the transits were taken to that in which it points due south. If the transits take place before noon, then this angle must be added to the "sun's bearing," i.e. the mean of the vernier readings corresponding to the transits, and in the afternoon subtracted from this bearing. The result is the meridian reading of the circle. Two values of this meridian reading are obtained, one corresponding to the front observations, and the other to the back. If these agree to within a minute, then the mirror was in such good adjustment that no correction is necessary, the value given by the front observations being alone used when calculating the declination. It can be shown that the correction to be applied to the front reading is the difference between the front and back readings multiplied by the square of the sine of half the sun's altitude. This correction has to be applied so as to bring the corrected result nearer the result given by the back observations. The method of calculating the sun's altitude and the correction is sufficiently clearly indicated in the example.

\section{Measurement of the Horizontal Component of the Earth's} Field.-In order to measure the horizontal component of the earth's field, we have to determine the period of vibration of a suspended magnet, and then determine the deflection which this magnet gives when placed at a known distance from an auxiliary suspended magnet.

Suitable magnets may be made by taking lengths of Stubb's drawn steel, having a diameter of about $3 \mathrm{~mm}$. and a length of about $10 \mathrm{~cm}$., and carefully squaring off the ends. A number of such pieces are bound together with iron wire and then heated in a furnace to a bright red heat, and, holding them in a vertical position, they are plunged into cold water. Those of the steel rods which remain straight after the hardening are then heated in boiling water for eight or ten hours, and are then magnetised to saturation by placing them inside a coil through which an electric current is sent. After magnetisation the magnets are again maintained at a temperature of $100^{\circ}$ for about five hours. In this way magnets are obtained which only lose their magnetism very slowly. Care, however, must be taken not to jar the magnets or allow them to touch one another or any piece of iron. Also they must be handled with care, as glass-hard steel is very brittle.

One of the magnets is suspended by a length of about $30 \mathrm{~cm}$. of unspun silk of such a thickness that it will just safely carry the weight of the magnet. The upper end of the fibre is made into a single loop and the lower into a double loop, the method of tieing such a double loop being shown in Fig. 174. The upper loop is put on a hook, and the magnet rests in the double loop, so that its axis is horizontal. The 
magnet will have to be protected from the disturbing effects of draughts by enclosing it within a box with glass sides, such as that shown in Fig. 46 .

Having removed all other magnets and iron from the neighbourhood of the suspended magnet, this magnet is brought to rest, and then two

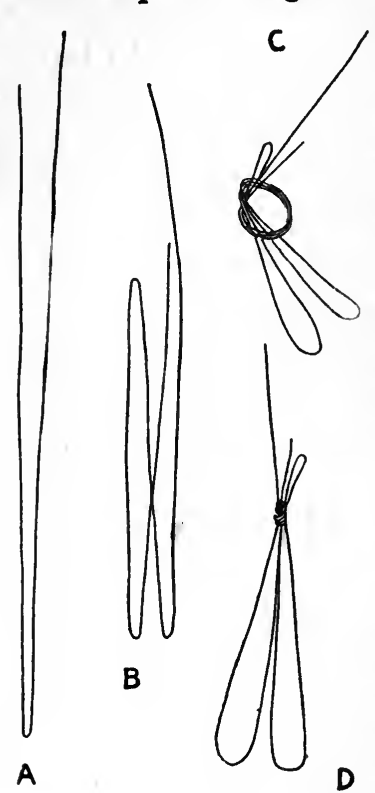

FIG. 174. small strips of paper are gummed to the front and back of the box, so that they lie on the prolongation of the axis of the magnet, or a telescope may be set up at a little distance, and in such a position that the end of the magnet, when at rest, appears to coincide with the vertical cross-wire.

The magnet having been set swinging through an arc not greater than $10^{\circ}$ by bringing near another magnet, and this deflecting magnet having been removed, the period of the suspended magnet must be determined either by timing 100 vibrations with a stop-watch, or by the eye and ear method described in $\S 43$.

A convenient form of magnetometer to use in the deflection experiment is shown in Fig. 175. It consists of a boxwood scale $\mathbf{A}$, 1 metre long, which is attached to a circular base of wood $\mathrm{H}$. An upright of $\operatorname{wood}$ в has a groove in one face, in which the suspended system hangs. This system consists of two short magnetised pieces of watch spring

D cemented on opposite sides of a piece of mica c. This piece of mica also carries a light galvanometer mirror $\mathbf{E}$. The front of the groove in which the suspended system hangs is closed by a piece of plate glass G. A notch is cut in the boxwood scale so that the suspended magnets can be placed at a level with the upper surface of the scale, while a $\mathrm{V}$ groove cut along the upper edge of the scale permits of the deflecting magnet being supported on the scale. The mica to which the magnets and mirror are attached serves as a damper to check the vibrations of the system.

A line having been drawn in the east and west magnetic direction through the position occupied by the magnet in the vibration experiment, either by suspending a long magnetised needle or with a prismatic compass, the magnetometer is placed with the scale immediately over this east and west line. A telescope and scale (Fig. 23) are then set up, and the image of the scale seen by reflection in the magnetometer is focused, care being taken that the telescope scale is at right angles to the scale of the magnetometer.

The magnet used in the vibration experiment is then placed on the west side of the magnetometer needle, with its centre at $30 \mathrm{~cm}$. from the 
needle, and the reading on the telescope scale is noted. The magnet is then reversed, and the reading again taken. Two similar readings are then taken with the magnet placed with its centre at $30 \mathrm{~cm}$. on the east side of the magnetometer needle. The differences between the pair of readings taken on either side gives twice the angle through which the needle has

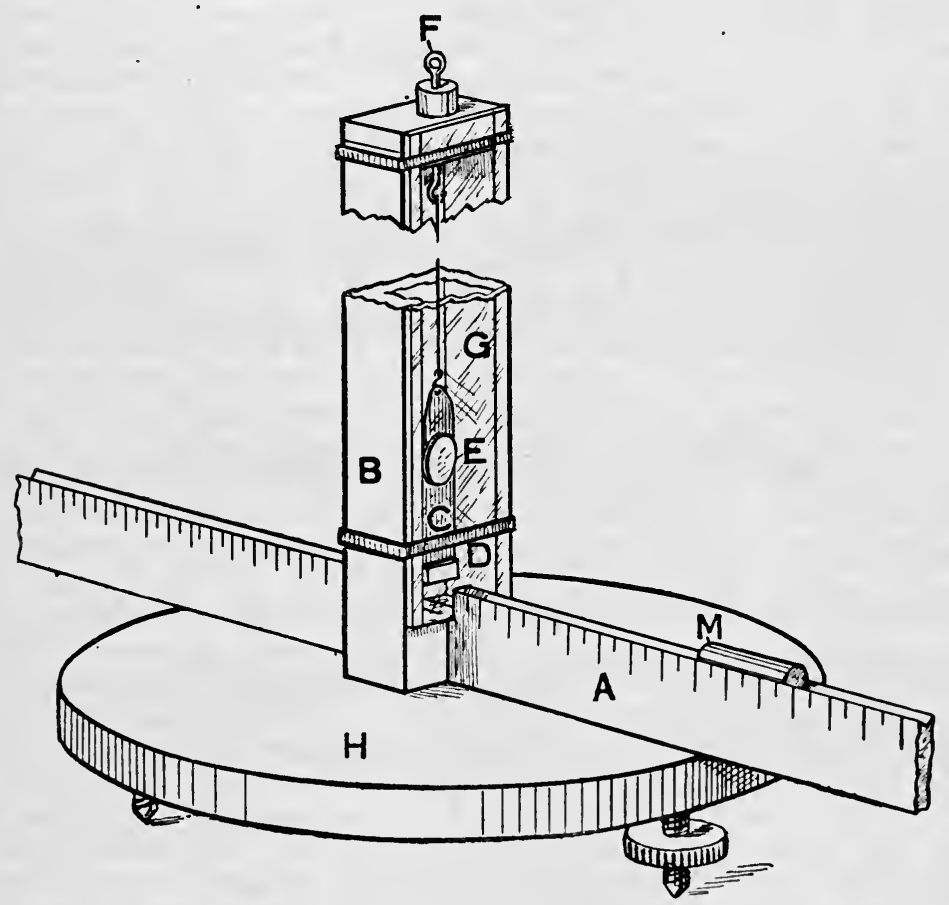

FIG. 175.

been deflected, and the mean of the two values obtained on the two sides is taken when calculating $\mathbf{B}$ from the results. If this mean at any given distance is $d$, while the distance between the mirror and the telescope scale is $S$, the angle $\theta$ through which the needle has been deflected is given by

$$
\tan 2 \theta=\frac{d}{S}
$$

Similar sets of deflections must be made with the centre of the deflecting magnet at 35 and $40 \mathrm{~cm}$. from the magnetometer needle.

To reduce the vibration observations it is necessary to know the moment of inertia of the magnet. This quantity can be calculated if the 
length $l$, diameter $2 r$, and mass $m$ of the magnet are measured, when the moment of inertia is given by

$$
K=m\left(\frac{l^{2}}{12}+\frac{r^{2}}{4}\right)
$$

Special care must be taken when measuring the length, as the proportional error produced by an error in the length is much greater than that produced by an error in the diameter.

If $T$ is the period of the magnet, and $M$ its magnetic moment, then (see Watson's Physics, $\$ 430,431$ )

$$
\begin{gathered}
M H=\frac{\pi^{2} K}{T^{2}} \\
\frac{M}{H}=\frac{\left(D^{2}-L^{2}\right)^{2}}{2 D} \tan \theta=\frac{D^{3}}{2}\left(1-\frac{L^{2}}{D^{2}}\right)^{2} \tan \theta,
\end{gathered}
$$

where $D$ is the distance between the centre of the deflecting magnet and the deflected needle, and $2 L$ is the distance between the poles of the deflecting magnet. This distance may be taken as $2 / 3$ of the length of the magnet.

Now if the length of the magnet is $10 \mathrm{~cm}$., so that $L=5$, and the smallest value of the distance $D$ employed in the experiments is $30 \mathrm{~cm}$., then we may expand the factor $\left(1-\frac{L^{2}}{D^{2}}\right)^{2}$ by the binomial theorem and neglect all the terms but the first two, so that the formula reduces to

$$
\frac{M}{\bar{H}}=\frac{D^{3}}{2}\left(1-\frac{2 L^{2}}{D^{2}}\right) \tan \theta
$$

a form which is more convenient for calculation, since the correction term $2 L^{2} / D^{2}$ can easily be evaluated on a slide rule.

165. Comparison of the Values of " $H$ " at different parts of a Building. - It is sometimes necessary to measure the ratio of the values of the horizontal component of the earth's field at two spots in the same room or building, that is, in two neighbouring points. This may be done most readily by determining the times of vibration of a suspended magnet in the two positions. The method of making the observation is similar to that adopted when making the vibration experiment described in the previous section. Having made a determination of period at $\mathrm{A}$, and noted the temperature of the inside of the box in which the magnet was suspended, a similar determination is made at B. A second determination is then made at $B$, and then a second at $A$. In this way the mean time of the observations at the two stations is the same, and thus the effects of diurnal change and disturbances are minimised. Since the horizontal force is, owing to diurnal change, near a minimum at 11 A.M., it is advisable not to time the observations so that the observations at station $\mathrm{A}$ are one before and the other after 11 A.M. If two observers 
are available, then the effects of all changes in $\mathbf{H}$ during the course of the comparison can be eliminated by the two observers making determinations of period simultaneously with two marnets, one at either station, and then interchanging stations.

If the temperature differs at the two stations, then a correction will have to be applied, since the moment of a magnet changes with temperature. The temperature coefficient of the moment of the magnet can be determined in the manner described in $\S 167$. If $T_{1}$ and $T_{2}$ are the periods of the magnet at the two stations, the temperature at one station being $t_{1}$ and that at the other $t_{2}$. Then if $\alpha$ is the temperature coefficient of the magnet, and $M$ the moment at the lower temperature, say, $t_{1}$, the moment at $t_{2}$ is $M\left\{1-\alpha\left(t_{2}-t_{1}\right)\right\}$. Hence

$$
T_{1}^{2}=\frac{\pi^{2} K}{M H_{1}}
$$

and

$$
T_{2}^{2}=\frac{\pi^{2} K}{M\left\{1-a\left(t_{2}-t_{1}\right)\right\} H_{2}} \text {. }
$$

Therefore

$$
\frac{H_{1}}{H_{2}}=\frac{\left\{1-\alpha\left(t_{2}-t_{1}\right)\right\} T_{2}^{2}}{T_{1}^{2}} \text {. }
$$

If a really accurate comparison is required, then further corrections to allow for the rate of the chronometer, effect of temperature on the moment of inertia, \&c., must be taken into account, as described in $\S 166$.

166. Measurement of " $\mathrm{H}$ " with the Kew-pattern Unifilar Magnetometer.-The determination of the horizontal component of the earth's magnetic field with the Kew-pattern unifilar magnetometer consists in two distinct operations. One of these is the determination of the period of the collimator magnet which was used in determining the magnetic meridian when it is vibrating in a horizontal plane, and the other is the determination of the angle through which an auxiliary suspended magnet is deflected by the collimator magnet. The first experiment gives the product $M H$, where $M$ is the magnetic moment of the collimator magnet, and the other the quotient $M / H$, so that from the two observations both $H$ and $M$ can be calculated.

1. Vibration Experiment.-The collimator magnet is suspended as in the declination experiment, and the vertical cross-wire brought into coincidence with the centre of the scale. By means of the magnetised screw-driver the magnet is then deflected, so that it vibrates over the whole extent of the scale. The temperature as indicated by the thermometer attached to the box containing the magnet, and the number of divisions of the scale over which the magnet is swinging, are then noted, and the observations of times of transit commenced. The method employed is the eye and ear method described in $\S 43$, a chronometer beating half seconds being used. The time of transit of the centre division of the scale when moving from left to right is noted down. The magnet being of such size that where $H$ has a value of about 0.18 a 
half vibration takes place in about four seconds, about twenty seconds after the first transit the magnet will have completed five half vibrations, and will then transit, moving from left to right. Thus about fifteen seconds after the first transit the time is again taken up from the chronometer, and the time of the transit from left to right, which occurs about twenty seconds after the first transit, is noted. This is repeated till the times of six transits in either direction have been noted, the results being entered as in the following example. The difference between the times of transit of vibrations 0 and 50 and 5 and 55 respectively give the time occupied by fifty half vibrations. These times added to the times of the 50th and 55th vibrations give the times to expect the 100th and 105th transits. Just before the time at which the 100th transit is expected the time is taken up from the chronometer, and the observation of every fifth transit continued up to the 150th and 155th transit. In this way twelve independent observations of the time of 100 half vibrations are obtained, and the mean is taken in deducing the time of a single vibration. Immediately after the observation of the time of the last transit the amplitude of the vibrations must be noted as well as the temperature.

Since the suspension fibre is never so thin as to possess negligible rigidity, the observed period has to be corrected to allow for the fact that it is smaller than it would be supposing the fibre were entirely free from rigidity. In order to be able to apply such a correction, it is necessary to determine the magnitude of the couple brought into play when the magnet is deflected due to the torsion of the fibre. To obtain the necessary data, the number of scale divisions through which the magnet is deflected when the torsion head, from which the suspension fibre is supported, is turned through $180^{\circ}$ is observed. The deflection is noted when the torsion head is turned first in one direction and then in the other, and the mean is taken.

The observed period has to be corrected to allow for (1) the rate of the chronometer, (2) the fact that the arc of swing is not infinitely small, (3) the torsion of the fibre, (4) the variation of the magnetic moment of the magnet with temperature, and (5) the increase of the moment of the magnet owing to the inductive action of the earth's field. To assist in the application of these different corrections, a series of tables is supplied with the instrument.

If the chronometer gains $s$ seconds a day, one second as given by the chronometer is really $1-s / 86400$ true seconds, and hence the observed period has to be multiplied by $(1-s / 86400)$. If the chronometer loses 8 seconds a day, the factor will be $(1+s / 86400)$. The values of the factor for different rates are given in Table 8 .

To reduce to infinitely small arc, the initial and final arcs must be reduced from scale divisions to minutes of arc. The value of a scale division in minutes is given in the table of corrections supplied with the instrument. If it is not known, then it can be obtained by noting the angle through which the instrument has to be turned to cause the vertical 
cross-wire of the telescope to pass from one end of the scale to the other. If $\alpha$ and $\alpha^{\prime}$ are the initial and final amplitudes in radians the correction is $\frac{a a^{\prime}}{16}$, so that to correct for both rate and finite arc the observed time has to be multiplied by $\left(1-\frac{8}{86400}-\frac{a \alpha^{\prime}}{16}\right)$. The values of $\frac{\alpha \alpha^{\prime}}{16}$ for different values of $\alpha$ and $a^{\prime}$ are given in Table 8 .

If $F$ is the couple called into play owing to the rigidity of the suspension fibre when one end is twisted through an angle of one radian, then if a deflection of the magnet of $d$ (radians) is produced when the torsion head is turned through $90^{\circ}$, the couple due to the torsion is $F(\pi / 2-d)$. Since $d$ is small, the couple due to the earth's field tending to bring the magnet back into the meridian is $M H d$. Hence

$$
F\left(\frac{\pi}{2}-d\right)=M H d, \text { or } \frac{F}{M H}=\frac{d}{\frac{\pi}{2}-d} .
$$

Thus the force of restitution for a small rotation $\theta$ is $(M H+F) \theta$, or $M H\left(1+\frac{F}{M H}\right)$. Hence the time of vibration $T_{1}$ is given by $T_{1}{ }^{2}=4 \pi^{2} K / M H\left(1+\frac{F}{M H}\right)$. If there were no torsion the time of vibra. tion, $T_{1}$ would be given by $T^{2}=4 \pi^{2} K / M H$. Therefore $T^{2}=T_{1}^{2}\left(1+\frac{F}{M H}\right)$. So that the correction factor to reduce the square of the periods is $1+\frac{F}{M H}$. The values of this factor for different values of $d$ are given in the following table :-

Values of $1+\frac{F}{M H}$ For different Values of the Deflection Produced by a Twist of $90^{\circ}$ of the Suspension Fibre.

\begin{tabular}{|c|c|c|c|}
\hline $\begin{array}{l}\text { Effect of } 90^{\circ} \\
\text { Twist. }\end{array}$ & $1+\frac{F}{M I H}$ & $\begin{array}{l}\text { Effect of } 90^{\circ} \\
\text { Twist. }\end{array}$ & $1+\frac{F}{M H}$ \\
\hline $\begin{array}{l}1^{\prime} \\
2^{\prime} \\
3^{\prime} \\
4^{\prime} \\
5^{\prime} \\
6^{\prime} \\
7^{\prime}\end{array}$ & $\begin{array}{l}1 \cdot 00019 \\
1 \cdot 00037 \\
1 \cdot 00056 \\
1 \cdot 00074 \\
1 \cdot 00093 \\
1 \cdot 00111 \\
1 \cdot 00130\end{array}$ & $\begin{array}{r}8^{\prime} \\
9^{\prime} \\
10^{\prime} \\
11^{\prime} \\
12^{\prime} \\
13^{\prime} \\
14^{\prime}\end{array}$ & $\begin{array}{l}1 \cdot 00148 \\
1 \cdot 00167 \\
1 \cdot 00185 \\
1 \cdot 00204 \\
1 \cdot 00223 \\
1 \cdot 00241 \\
1 \cdot 00260\end{array}$ \\
\hline
\end{tabular}

If the temperature of the magnet were the same during the vibration and the deflection experiments, then the value obtained for $H$ would be independeut of the temperature of the magnet. In general, however, 
there is a small difference in temperature between the two experiments, and hence a correction has to be applied on this account. It is usual to reduce the period and the deflection to what they would be if the temperature of the magnet were $0^{\circ} \mathrm{C}$. A table of the values of the magnetic moment at different temperatures, the moment at $0^{\circ} \mathrm{C}$. being taken as unity, is supplied with each magnetometer, and serves for this reduction. Since the magnetic moment decreases with rise of temperature, this correction is negative for temperatures above $0^{\circ} \mathrm{C}$.

Finally, the magnetic moment has to be corrected for the increase in the moment produced by the inductive action of the earth's field. On this account we have to add a quantity which is the product of a constant factor $\mu$, which is determined once for all, into $H / M$. The value of $H / M$ is obtained from the deflection experiment, while that of $\mu$ is given in the tables of corrections.

The manner of applying the various corrections is shown in the example which follows. The corrected value of the square of the period has to be divided into $\pi^{2} K$ to give the value of $M H$. Since the moment of inertia of the magnet varies with the temperature, the value of $K$ varies with the temperature, and a table for different temperatures is given amongst the constants of the instrument.

\section{Observations of Vibration. April 23, 1891.}

Station, Kew.

Lat. ' ". Long. ' ".

Chronometer, Dent. Error at Station $=$. Daily Rate $(s)=+1 \cdot 1$.

Magnet $(70 \mathrm{~A})$ suspended. $q=\quad$ Log. $\mu=\overline{1} \cdot 74630$.

Effects of $90^{\circ}$ of Torsion $=1 \cdot 2$. Div. $=2^{\prime} \cdot 2$. One Div. of Scale $=1^{\prime} \cdot 8$. At Commencement \} Mean Time $\{11 \mathrm{~h} .28 \mathrm{~m} \text {. S Semiarc }\}^{\prime} 8^{\prime}$ Temp. of $\left\{12^{\circ} \cdot 7\right\}$

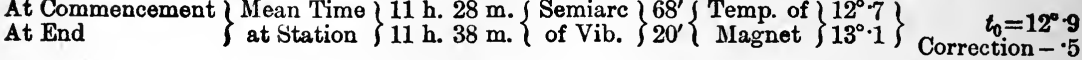

$12 \cdot 4$

\begin{tabular}{|c|c|c|c|c|c|c|c|c|c|}
\hline \multicolumn{5}{|c|}{ Scale moving apparently to the Right. } & \multicolumn{5}{|c|}{ Scale moving apparently to the Left. } \\
\hline $\begin{array}{l}\text { No. } \\
\text { of } \\
\text { vib. }\end{array}$ & $\begin{array}{c}\text { Time of } \\
\text { Centre } \\
\text { passing Wire. }\end{array}$ & $\begin{array}{c}\text { No. } \\
\text { of } \\
\text { Vib. }\end{array}$ & $\begin{array}{c}\text { Time of } \\
\text { Centre } \\
\text { passing Wire. }\end{array}$ & $\begin{array}{c}\text { Time } \\
\text { of } \\
100 \text { Vib. }\end{array}$ & $\begin{array}{l}\text { No. } \\
\text { of } \\
\text { vib. }\end{array}$ & $\begin{array}{c}\text { Time of } \\
\text { Centre } \\
\text { passing Wire. }\end{array}$ & $\begin{array}{l}\text { No. } \\
\text { of } \\
\text { vib. }\end{array}$ & $\begin{array}{c}\text { Time of } \\
\text { Centre } \\
\text { passing Wire. }\end{array}$ & $\begin{array}{c}\text { Time } \\
\text { of } \\
100 \text { Vib. }\end{array}$ \\
\hline $\begin{array}{r}0 \\
10 \\
20 \\
30 \\
40 \\
50\end{array}$ & $\begin{array}{rcc}h . & \text { m. } & \text { s. } \\
11 & 28 & 19 \cdot 0 \\
28 & 58 \cdot 9 \\
29 & 38 \cdot 9 \\
30 & 18 \cdot 8 \\
30 & 58 \cdot 7 \\
31 & 38 \cdot 7\end{array}$ & $\begin{array}{l}100 \\
110 \\
120 \\
130 \\
140 \\
150\end{array}$ & $\begin{array}{cc}\text { m. } & s . \\
\mathbf{3 4} & \mathbf{5 8 \cdot 5} \\
\mathbf{3 5} & 38 \cdot 5 \\
\mathbf{3 6} & \mathbf{1 8} \cdot 6 \\
36 & \mathbf{5 8 \cdot 5} \\
\mathbf{3 7} & \mathbf{3 8} \cdot 5 \\
\mathbf{3 8} & \mathbf{1 8 \cdot 5}\end{array}$ & $\begin{array}{r}m . \quad s . \\
69 \\
\cdot 6 \\
\cdot 7 \\
7 \\
\cdot 8 \\
\cdot 8\end{array}$ & $\begin{array}{r}5 \\
15 \\
25 \\
35 \\
45 \\
55\end{array}$ & $\begin{array}{rcc}h . & m . & \text { s. } \\
11 & 28 & 39 \cdot 0 \\
29 & 18 \cdot 9 \\
29 & 58 \cdot 8 \\
30 & 38 \cdot 7 \\
31 & 18 \cdot 7 \\
31 & 58 \cdot 7\end{array}$ & $\begin{array}{l}105 \\
115 \\
125 \\
135 \\
145 \\
155\end{array}$ & $\begin{array}{lc}m . & 8 \cdot \\
3 \tilde{3} & 18 \cdot 5 \\
35 & 58 \cdot 5 \\
36 & 38 \cdot 6 \\
37 & 18 \cdot 5 \\
37 & 58 \cdot 5 \\
38 & \mathbf{3 8} \cdot 5\end{array}$ & $\begin{array}{r}m . \\
6.5 \\
-6 \\
.8 \\
.8 \\
.8 \\
.8\end{array}$ \\
\hline $\begin{array}{l}\text { Diff. } \\
\text { for } 50\end{array}$ & $319 \cdot 7$ & & & 41 & $\begin{array}{l}\text { Diff. } \\
\text { for } 50\end{array}$ & $319 \cdot 7$ & & & 43 \\
\hline 100 at & $3458 \cdot 4$ & & $\operatorname{Mean}(1)=$ & $639 \cdot 68$ & 105 at & $3518 \cdot 4$ & & Mean $(2)=$ & $639 \cdot 72$ \\
\hline
\end{tabular}




$$
\begin{aligned}
& T_{1}=T_{0}\left\{1-\frac{s}{86400}-\frac{a a^{\prime}}{16}\right\} ; T_{2}=T_{1}^{2}\left\{1+\frac{F}{M H}-q\left(t_{0}-t\right)+\mu \frac{H_{0}}{M_{0}}\right\} ; M H=\frac{\pi^{2} K}{T^{2}} . \\
& 1 \frac{8}{86400}=1 \cdot 00000 \\
& \text { Mean (1) for } 1 \text { Vib. }=3.9968 \\
& -\frac{a a^{\prime}}{16}=\cdot 00001 \\
& \text { " (2) } . \quad \text { " = } 72 \\
& 1=\frac{8}{86400}-\frac{a a^{\prime}}{16}=\overline{1 \cdot 00001} \\
& 1+\frac{F}{M H}=1 \cdot 00039 \\
& -q\left(t_{0}-t\right)=\cdot 00503 \\
& +\mu \div \frac{M_{0}}{H_{0}}=\cdot 00110 \\
& T_{0}=3 \cdot 9970 \quad \log =\cdot 60173 \\
& \text { - . . . } \begin{array}{r}
\log =\overline{1} \cdot 99999 \\
T_{1} \log =\frac{.60172}{2}
\end{array} \\
& T_{12} \log =\overline{1 \cdot 20344} \\
& 1+\frac{F}{M H}-q\left(t_{0}-t\right)+\mu \div \frac{M_{0}}{H_{0}}=.99646 \\
& \mu \log =\overline{\mathbf{1}} \cdot 74630 \\
& \frac{M_{0}}{H_{0}} \log =2 \cdot 70491 \\
& \mu \div \frac{M_{0}}{H_{0}}=\cdot 00110 \mathrm{Log}=\overline{\mathbf{3}} \cdot 04139 \\
& \text { Obsertation of Torsion, } \\
& \text { Circle turned. Scale. Mean. Diff. }
\end{aligned}
$$

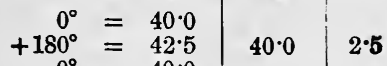

$$
\begin{aligned}
& \begin{array}{rl|l|l}
-180^{\circ} & =40^{\circ} \cdot 6 \\
0^{\circ} & 40.0
\end{array} \quad 40.0 \quad 2.5 \\
& 0^{\circ}=40.0 \\
& \text { - . . . } \log =\overline{1} \cdot 99846 \\
& \begin{array}{r}
T^{2} \log =\overline{1 \cdot 20190} \\
\pi^{2} K \log =3 \cdot 42247 \\
M H=\frac{\pi^{2} K}{T^{2}} \log =2 \cdot 22057
\end{array} \\
& \begin{array}{r}
T^{2} \log =\overline{1 \cdot 20190} \\
\pi^{2} K \log =3 \cdot 42247 \\
M H=\frac{\pi^{2} K}{T^{2}} \log =2 \cdot 22057
\end{array} \\
& \begin{array}{r}
T^{2} \log =\overline{1 \cdot 20190} \\
\pi^{2} K \log =3 \cdot 42247 \\
M H=\frac{\pi^{2} K}{T^{2}} \log =2 \cdot 22057
\end{array} \\
& 90^{\circ}=\overline{1 \cdot 2 \text { divisions. }}
\end{aligned}
$$

Observer, W. W.

2. Deflection Experiment.-When making the deflection experiment the wooden box which surrounded the vibration magnet is removed, the glass suspension tube being screwed into the metal base of the instrument, and a small auxiliary magnet, fitted with a mirror, is suspended. A metal bar, having divisions on one surface, fits immediately below the compartment in which the mirror magnet hangs. This bar serves to support a small carriage, on the V's of which the deflecting magnet is placed. This carriage is placed on the right-hand side of the mirror magnet when looking towards the face of the mirror, and with its centre at a distance of $30 \mathrm{~cm}$. from the centre of the instrument. A small sighting tube is then placed on the V's of the carriage, and the mirror magnet adjusted so that its centre lies along the prolongation of this sighting tube, so that the centre of the magnet will lie in the same horizontal plane as the deflecting magnet when placed in the carriage.

To determine the position of the mirror magnet a small telescope with an ivory scale attached is screwed to the base below the telescope 
used in the vibration experiment, and when making a deflection experiment the cross-wire of this telescope is brought to the middle division of the scale, as seen after reflection in the mirror attached to the magnet. A thermometer is attached to the bar which supports the magnet, the bulb being placed as near the magnet as possible.

The collimator magnet having been placed in the carriage with its north end pointing towards the east, the whole instrument is turned till the cross-wire corresponds with the centre division of the scale. The temperature and time are then noted, and the verniers attached to the azimuth circle are read. The carriage is then moved to $40 \mathrm{~cm}$., and the deflected reading obtained. The magnet is then reversed in the carriage, and another deflection is obtained. Another deflection is then obtained with the carriage at $30 \mathrm{~cm}$., but with the magnet in the reverse position to that when the first deflection was obtained. Finally, an exactly similar series of deflections are obtained with the magnet placed on the other side of the mirror magnet. The order of making the observations, as well as the method of recording them, will be clear from a study of the example given.

Observations of Deflection. April 23, 1891.

Station, Kew.

Lat. ' ". Long.

Mean Time at Station; commencing 11 h. 57 m., ending 12 h. $23 \mathrm{~m}$.

Magnet $(70 \mathrm{~A})$ deflecting ; $(70 \mathrm{C})$ suspended. One Div. of Scale $=60^{\mathrm{N}}$.

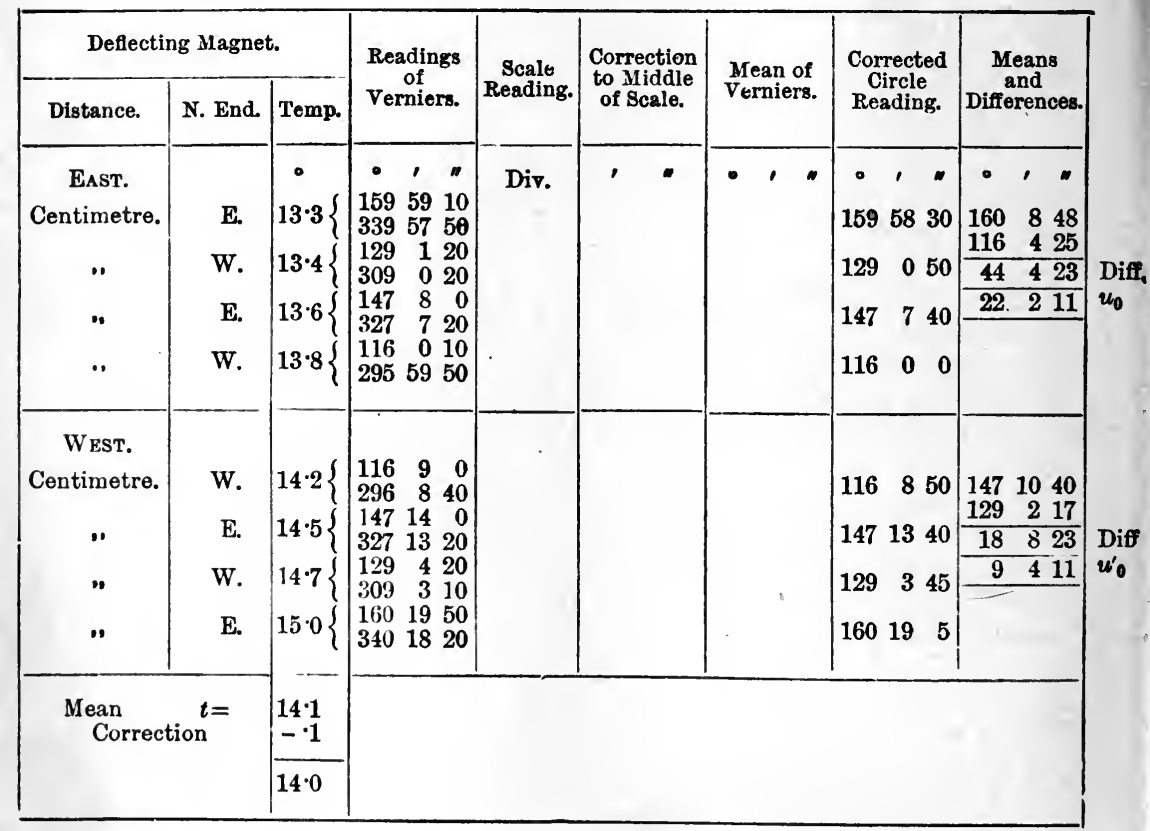




$$
\begin{aligned}
& \frac{M_{0}}{H_{0}}=\frac{1}{2} r^{3} \sin u_{0} ; \frac{M}{H^{\prime}}=\frac{M_{0}}{H_{0}}\left\{1+\frac{2 \mu}{r_{0}^{3}}+q\left(t_{0}-t\right)\right\} ; \frac{M}{H}=\frac{M^{\prime}}{\bar{H}^{\prime}}\left(1-\frac{P}{r_{0}^{2}}\right) \text {. } \\
& r=30 \quad r^{\prime}=40 \\
& r=30 \quad r^{\prime}=40 \\
& 1+\frac{2 \mu}{r_{0}^{3}}=1.00041 \mid 1.00017 \\
& \begin{array}{r|r}
+\left(t_{0}-t\right) q=\frac{.00571}{1+\frac{2 \mu}{r_{0}{ }^{3}}+\left(t_{0}-t\right) q}=\frac{.00571}{1.00612} & 1.00588
\end{array} \\
& \frac{1}{2} r^{3} \log =4 \cdot 13066 \mid 4 \cdot 50548 \\
& 1-\frac{P}{r_{0}^{2}}= \\
& \text { Sin } u_{0} \log =\overline{1} \cdot 57425 \quad \overline{1} \cdot 19765 \\
& \overline{M_{0}} \log =\overline{3 \cdot 70491} \overline{3 \cdot 70313} \\
& \log =\cdot 00265 \quad \cdot 00254 \\
& \frac{M^{\prime}}{H^{\prime}} \log =\overline{3 \cdot 70756} \overline{3 \cdot 70567} \\
& \log =9 \cdot 99547 \quad 9 \cdot 99746
\end{aligned}
$$

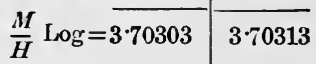

$$
\begin{aligned}
& M H \log =2 \cdot 22057 \quad 2 \cdot 22057 \\
& M H \div \frac{M}{H}=H^{2} \log =\overline{\overline{2} \cdot 51754} \overline{\overline{2} \cdot 51744} \\
& \begin{array}{r|r}
H \log =\overline{1} \cdot 25877 & \mathrm{i} \cdot 25872 \\
H & =\cdot 18145
\end{array} \\
& \log M=2 \cdot 96180 \quad 2 \cdot 96185 \\
& \begin{array}{l|l}
M=915 \cdot 80 & 915 \cdot 90
\end{array}
\end{aligned}
$$

Observer, W. W

To reduce the observations, the means of the vernier readings in each position are first entered. Then the mean of the two deflections at $30 \mathrm{~cm}$. in the anti-clockwise direction is deducted from the mean of the two deflections in the clockwise direction, the difference being twice the deflection $u_{0}$. A similar operation with the deflections taken with the magnet at $40 \mathrm{~cm}$. gives the deflection $u_{0}^{\prime}$ at this distance.

The values of $\log r^{3} / 2$ for different temperatures is given in the table of constants, and this is looked out and written down. Then $\log \sin u_{0}$ is added. Corrections have now to be applied for induction and temperature. These are looked out in the tables, added together, and their logarithm added to $\log r^{3} / 2 . \sin u_{0}$.

The next correction has to be applied to allow for the fact that the formula $\frac{H}{M}=\frac{1}{2} r^{3} \sin u_{0}$ only holds if the magnets concerned are infinitely short. To allow for the finite length of the magnets, the value obtained for $\frac{M}{H}$ has to be multiplied by $\left(1-P / r^{2}\right)$, where $P$ is obtained from the values of $\log M^{\prime} / H^{\prime}$ obtained at the two distances by means of the following formula : ${ }^{1}$ -

$$
P=\frac{A-A^{\prime}}{\frac{A}{r^{2}}-\frac{A^{\prime \prime}}{r^{\prime 2}}}
$$

where $A$ and $A^{\prime}$ are the values of $M^{\prime} / H^{\prime}$ at the distances $r$ and $r^{\prime}$.

1 For a graphical method of calculating $P$ and $\log \left(1-P / r^{2}\right)$, see Terrestricl Magnetism (1901), vol. vi. p. 70. 
To obtain a satisfactory value for $P$, it is necessary to take the mean of a number of observations and use this mean value when reducing all the observations. Since the value of $P$ only changes very slowly with the age of the magnets, it is generally sufficient to use a mean value for $P$ obtained from all the observations taken in any year.

Having added $\log \left(1-P / r^{2}\right)$ to the value of $\log M^{\prime} / H^{\prime}$, the value of $\log M H$ is copied down from the vibration experiment, and the values of $H$ and of $M$ are deduced. The value of $H$ corresponds to the mean of the values of this quantity during the vibration and the deflection experiments, and would require to be corrected for diurnal variation and any accidental disturbance by means of the record of a self-recording magnetograph, such as those set up at a magnetic observatory.

167. Determination of the Temperature Coefficient of a Magnet.The magnetometer described on p. 404 may be used in measuring the change in the moment of a magnet with change in temperature. - The magnet is supported on V's within a glass or porcelain vessel, and is placed due north or south of the magnetometer, with its axis east and west, and at such a distance that the deflection produced is nearly the full extent of the telescope scale. The magnet must be firmly fixed in the V's, and the vessel fixed in position with the magnet in the same horizontal plane as the magnetometer needle.

The vessel containing the magnet having been filled with a mixture of ice and water, the magnet is allowed to take up the temperature, and then the deflection is read off on the scale. The water is then syphoned off and replaced by water at about $20^{\circ} \mathrm{C}$. and the deflection again read, the temperature being read on a thermometer placed in the water, which must be kept well stirred. By gradually syphoning off the water and adding hot water a series of readings are thus obtained every $20^{\circ}$ up to nearly $100^{\circ} \mathrm{C}$. Then by adding first cold water and then ice a second series at as nearly the same temperatures as the first set, but in the reverse order, is taken, and finally the magnet is removed and a second zero is obtained. The means of the temperatures and deflections at each temperature are then taken, and the values of their deflections are plotted on a curve against the temperature. If the points obtained lie on a straight line, then the expression for the moment $M$ at a temperature' $t$ is given by an equation of the form

$$
M_{t}=M_{0}(1-a t)
$$

in which the value of the quantity $a$ can at once be deduced from the straight line drawn on the curve (see $\S 5$ ). In general, the moment decreases a little faster at high temperatures than at low, and an expression of the form

$$
M_{t}=M_{0}\left(1-\alpha t-\beta t^{2}\right)
$$

has to be employed. In such a case the values of the constants $a$ and $\beta$ 
must be obtained from the curve by one of the methods given in $\S 5$.

168. Measurement of the Dip with the Kew-pattern Circle.The Kew-pattern dip circle is shown in Fig. 176. It consists of a metal base on levelling screws, the upper part of the instrument being capable of rotation about a vertical axis with reference to this base, the azimuth being indicated by a divided circle and vernier $\mathrm{H}$. The needle $\Delta B$ rests on two agate knife-edges $K, K^{\prime}$, and the position of the ends of the

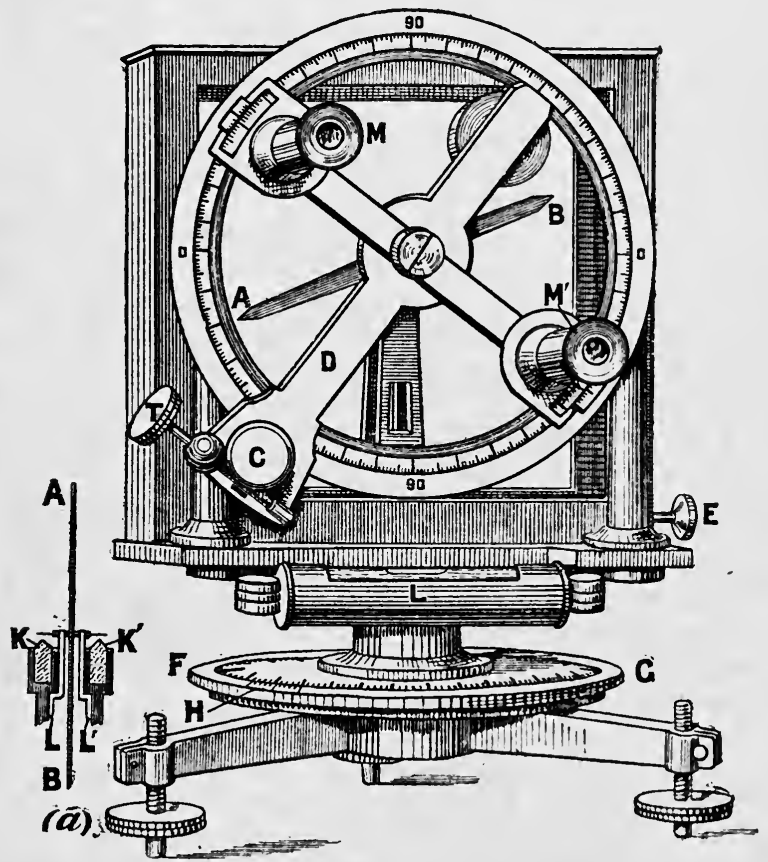

FIG. 176.

needle are observed by two small microscopes $\mathbf{M}, \mathbf{M}^{\prime}$ and the attached verniers which read on the vertical circle. These microscopes and verniers can be slowly moved by means of the tangent screw T. Two V's L, L' (Fig. 176, a), actuated by a screw head E, serve to lift the needle from the knife-edges and replace it so that the axis coincides with the axis of the vertical circle. A level $\mathrm{L}$ serves to show when the vertical axis of the instrument is vertical, in which position the agate knife-edges are horizontal. A small wooden block, with a clip to protect the axle of the needle, is used to support the needle while it is being magnetised.

The circle having been set up and levelled, the needle is placed in the 
magnetising clip, and taking one of the magnets supplied in each hand, that in the right hand having the north pole downwards, and that in the left the south pole downwards, the needle is stroked ten times, starting at the middle and drawing the two magnets apart till they are past the ends of the needle. The needle is then reversed in the clip, and the other side stroked ten times in the same manner. In this way the needle is magnetised. Since it is of importance that the needle should be magnetised to the same extent each time, it is essential that the stroking should be performed in exactly the same manner throughout.

The needle having been removed from the clip, in which operation, and on all other occasions when the needle is handled, care being taken not to touch the axles, the axles must be cleaned by pressing them into a piece of cork or dry pith. They must then be dusted with a clean camel'shair brush. The agate knife-edges having been rubbed lightly with the pith are also dusted, and then the needle is placed in the instrument, with the side marked AB turned away from the vertical circle. The two verniers are then adjusted to read $90^{\circ}$, that is, the line joining the two wires of the microscopes is vertical, and the whole instrument is turned in azimuth till the upper end of the needle coincides with the wire of the upper microscope. The needle, while this adjustment is being made, should be raised and lowered several times by means of the screw $\mathrm{F}$, and the adjustment made so that the end appears to swing equally on the two sides of the wire. It is always best to have the needle swinging over a small arc, as in this way the effect of dust on the axle or knifeedges is more easily observed, when, if necessary, the axle can again be cleaned in the manner already employed. The adjustment being complete, the vernier on the azimuth circle is read and recorded. A similar reading is then obtained when the lower end of the needle is made to coincide with the wire of the lower microscope.

The whole upper part of the instrument is then turned through $180^{\circ}$, and the settings for the upper and lower ends of the needle repeated. The needle is then reversed, so that the face lettered $\mathrm{AB}$ is turned towards the vertical circle, and a setting for each end of the needle made, first with the instrument in its present position, and then when turned through $180^{\circ}$. The mean of all the readings obtained gives the azimuth reading when the axle of the needle points north and south, that is, when the plane in which the needle swings is perpendicular to the magnetic meridian. The azimuth circle is so divided that if the vernier is set to this reading in the next quadrant, then the plane in which the needle swings will be that of the magnetic meridian.

The instrument having been turned with its face towards the east, and having been set to the correct azimuth reading, a stop attached to the base plate is adjusted, so that after rotation the instrument can be replaced in the same azimuth. The needle having been set swinging, the upper microscope is adjusted on the upper end of the needle and the corresponding vernier is read. Two independent settings are then made with the lower microscope, the needle being raised from the agates 
between the two, and then another setting with the upper. If these settings differ by more than two minutes of arc, then both the axle of the needle and the knife-edges must be cleaned, and this must be repeated till the readings agree to within this limit. The instrument is then turned through $180^{\circ}$, the vernier $\mathrm{H}$ being adjusted to the correct azimuth reading, and a second stop is fixed in position, and then readings are made for the two ends of the needle as before. The needle is then reversed in the V's and another set of readings taken, first with the instrument facing west, and then when it faces east.

Next the needle is removed and remagnetised with its polarity reversed, and a similar set of readings obtained. The mean of all the readings gives the dip at the time of observation.

The series of readings is so arranged as to eliminate errors due to the centre of gravity of the needle not coinciding with the centre of the axle, and the agate planes not being exactly horizontal, or the line joining the $90^{\circ}$ marks on the vertical circle truly vertical (see Watson's Physics, $\S 434$ ). The example on p. 418 illustrates the method of recording the observations.

169. Comparison of the Magnetic Moments of Two Magnets.1. Deflection Method.-The magnetic moments of two magnets may be compared by means of the magnetometer shown in Fig. 175. The magnetometer having been set up as described on p. 404, the two magnets are placed on the cross-bar, one on either side of the needle, and with like poles towards one another. Their distances are then so adjusted that the needle is undeflected. The positions of the centres of the magnets having been noted, the magnets are turned through $180^{\circ}$, and leaving one of the magnets, say, A, at the same distance as before, the other is moved till the deflection is again reduced to zero. The two magnets are then interchanged, and keeping the magnet $\mathbf{A}$ at a fixed distance from the needle, the position of the other magnet is adjusted to give no deflection, first, when the two north poles are turned towards the needle, and secondly, when the two south poles are so turned. The mean of all the readings for the centre of magnet $\mathrm{B}$ is then taken, when the ratio of the moments is obtained by means of the expression

$$
\frac{M_{1}}{M_{2}}=\frac{D_{1}^{3}}{D_{2}^{3}}
$$

where $D_{1}$ and $D_{2}$ are the distances of the centres of the magnets from the needle.

If the two magnets differ much in length, or if the distances from the needle at which they have to be placed differ considerably, then account will have to be taken of the distance between the poles of the magnets in deducing the ratio of their moments. It will generally be sufficient if the distance between the poles is taken as $2 / 3$ of the total length of the magnets, and the following expression, in which $L_{1}$ and $L_{2}$ are the distances 


\section{Magnetic Dip.}

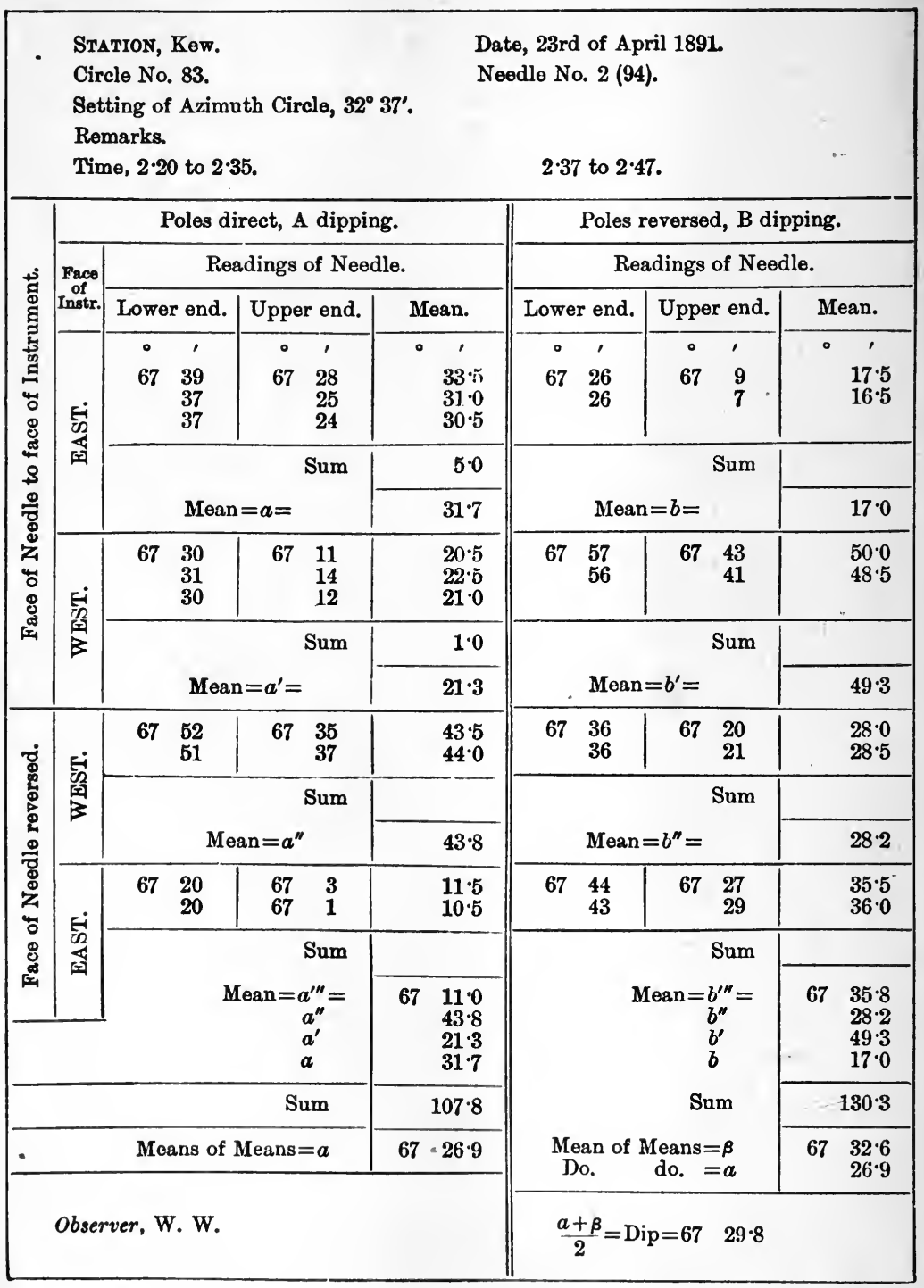


between the poles, is immediately deduced from that given in $\S 429$ of Watson's Physics:-

$$
\frac{M_{1}}{M_{2}}=\frac{D_{1}{ }^{3}}{D_{2}^{3}} \frac{\left(1-\frac{L_{1}{ }^{2}}{D_{1}{ }^{2}}\right)^{2}}{\left(1-\frac{L_{2}{ }^{2}}{D_{9}{ }^{2}}\right)^{2}}
$$

or since $\frac{L_{1}}{D_{1}}$ and $\frac{L_{2}}{D_{2}}$ are generally fairly small,

$$
\frac{M_{1}}{M_{2}}=\frac{D_{1}^{3}}{D_{2}^{3}} \frac{1-2 \frac{L_{1}{ }^{2}}{D_{1}^{2}}}{1-2 \frac{L_{2}^{2}}{D_{2}^{3}}} .
$$

2. Vibration Method.-If the moments of inertia of the magnets are known, or can be calculated, then the ratio of the moments can be obtained by determining the periods of vibration when they are in succession suspended by a fine silk thread at the same spot. A determination of period with magnet A should first be made, then two with magnet $B$, and finally a second with magnet $A$, so that the mean time of the experiment may be the same for each magnet, and thus to a certain extent the effects of alterations in the earth's field eliminated. If $T_{1}$ and $T_{2}$ are the mean periods, and $K_{1}$ and $K_{2}$ the moments of inertia, we have

$$
\frac{M_{1}}{M_{2}}=\frac{K_{1}}{K_{2}} \cdot \frac{T_{2}^{2}}{T_{1}^{2}}
$$




\section{CHAPTER XXV}

\section{ADJUSTMENT AND USE OF GALVANOMETERS}

170. Measurement of Angles of Rotation by means of a Mirror and Scale.-A method much used in Physics of measuring the angle through which a body has rotated is to attach a mirror to the body and observe the movement of a beam of light reflected from the mirror. There are two arrangements commonly employed. In one an image of an illuminated slit is formed by the mirror on a divided scale, and the rotation of the mirror is deduced from the displacement of the spot of light. In the

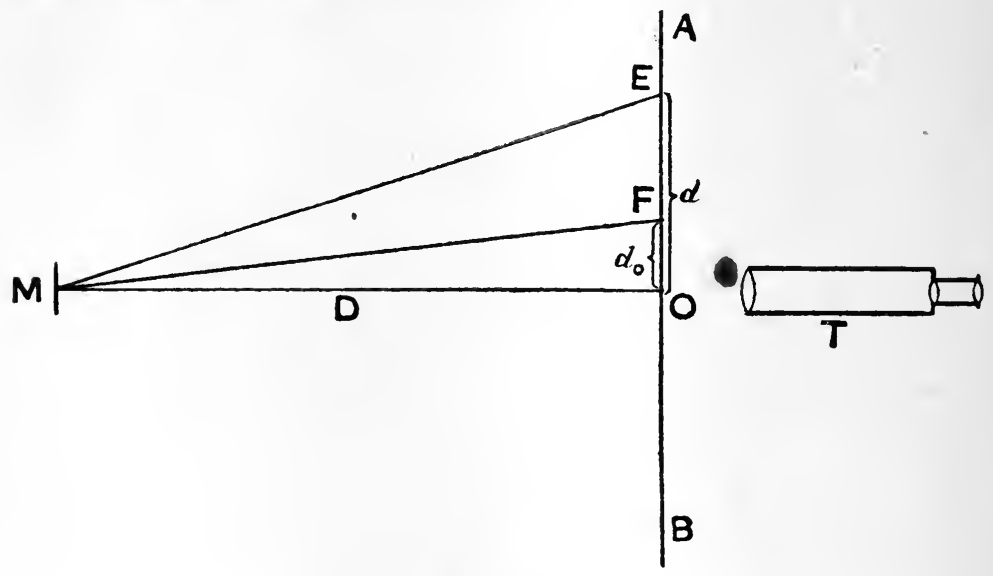

Fia. 177.

other arrangement a telescope is used, and an image of a scale seen by reflection in the mirror is observed, the rotation being deduced from the change in the reading of the scale corresponding to the cross-wire of the telescope.

The only advantage of the lamp and slit is that the image on the scale can be examined by more than one observer at a time. The disadvantages are that the room has to be partly darkened in order to allow of the image being seen, further it is difficult to read the position of the image, and to focus the image it is in general necessary to move 
the scale, a procedure which will upset the adjustment of this latter. With the telescope, of course, only one person can observe; this limitation, however, is generally of no importance. Since the focusing is done by the telescope independent of the scale, the focus can always be adjusted without disturbing the scale. With a telescope of about an inch aperture, and a reasonably good mirror, it is possible to read the position of the cross-wire on a scale placed at a metre from the mirror to within 05 of a millimetre, while with the lamp and scale to read to $2 \mathrm{~mm}$. is difficult. Hence where it is of importance to read the deflection accurately, the telescope method should always be employed.

We will first suppose that the plane of the mirror M (Fig. 177) is parallel to the axis about which the rotation takes place. Let $A B$ be the scale, and $o$ the foot of the perpendicular from the axis about which the mirror turns to the scale, the perpendicular distance between the mirror and the scale being $\mathrm{D}$. We will suppose that when the plane of the mirror is parallel to the scale, the image of the point o on the scale is on the vertical cross-wire of the telescope. Then if the mirror turns through an angle $\theta$ so that the image of the point $\mathbf{E}$ of the scale now coincides with the vertical cross-wire, and we call the distance of $d$, we have, since the angle through which the reflected ray is turned is twice the angle through which the mirror turns,

$$
\tan 2 \theta=\frac{d}{D}
$$

from which $\theta$ can be calculated.

When $\theta$ is fairly small, it is possible to obtain an expression for $\theta$ and the other functions of $\theta$ commonly required, such as $\tan \theta, \sin \theta$, and $\sin \theta / 2$, in terms of powers of $\frac{d}{D}$. Thus using the symbol $\rho$ for $d / D$, we have, by Gregory's series, ${ }^{1}$

$$
\theta=\frac{\rho}{2}\left\{1-\frac{1}{3} \rho^{2}+\frac{1}{5} \rho^{4}-\frac{1}{7} \rho^{6}+\right\} . . . . .
$$

If $\rho$ is not greater than $\cdot 2$, i.e. if we are dealing with deflections $d$ less than 200 scale divisions when the distance between the mirror and scale is equal to 1000 scale divisions, the term in $\rho^{6}$ in the bracket is negligible. For values of $\rho$ less than $\cdot 1$, in the same way the term in $\rho^{4}$ is negligible, while for values of $\rho$ less than 01 the term in $\rho^{2}$ does not affect the results to more than one part in 30,000 . If we are able to read the deflection on the scale only to one part in 500 , then $\rho$ may be as great as .05 without it becoming necessary to consider terms in $\rho^{2}$ or higher powers; this corresponds to a deflection of 50 scale divisions when the distance between the mirror and scale amounts to 1000 divisions.

1 The value obtained for $\theta$ is in circular measure. To reduce to degrees we must multiply the value obtained for $\theta$ by $57^{\circ} \cdot 296$, or $57^{\circ} 17^{\prime} 45^{\prime \prime}$. 
In Table 24 are given for different values of $\rho$ the values of the terms $\frac{1}{3} \rho^{2}-\frac{1}{5} \rho^{4}$, so that the correction to be applied in any given case can be obtained by interpolation. Thus if $\rho=\cdot 15$, the table gives 0.00740 as the value of the correction terms, so that

$$
\theta=\frac{d}{2 D}\{1-0.00740\} \text {. }
$$

By inserting the value of $\theta$ given by equation (1) in the expressions for the sine, tangent, \&c., we obtain

$$
\begin{gathered}
\sin \theta=\frac{\rho}{2}\left\{1-\frac{3}{8} \rho^{2}+\frac{31}{128} \rho^{4}-\right\} \\
\tan \theta=\frac{\rho}{2}\left\{1-\frac{1}{4} \rho^{2}+\frac{1}{8} \rho^{4}-\frac{5}{64} \rho^{6}+\right\} \\
\sin \theta / 2=\frac{\rho}{4}\left\{1-\frac{11}{32} \rho^{2}+\frac{431}{2048} \rho^{4}-\right\},
\end{gathered}
$$

and the corresponding corrections are given in Table 24 .

If the reading on the scale in the undeflected position does not coincide with the foot of the perpendicular drawn from the axis of rotation on to the scale, but to some point such as F (Fig. 177), then calling of $d_{0}$, we have

$$
\tan E M O=d / D \text {, and } \tan F M O=d_{0} / D .
$$

Hence

$$
\text { angle } E M O=\frac{d}{D}\left\{1-\frac{1}{3}\left(\frac{d}{D}\right)^{2}+\frac{1}{5}\left(\frac{d}{D}\right)^{4}-\& \mathrm{c} .\right\}
$$

and

$$
\text { angle } F M O=\frac{d_{0}}{D}\left\{1-\frac{1}{3}\left(\frac{d_{0}}{D}\right)^{2}+\frac{1}{5}\left(\frac{d_{0}}{D}\right)^{4}-\& \mathrm{c} .\right\}
$$

And $\theta$, the angle through which the mirror has turned, is given by

$$
\begin{aligned}
\theta & =\frac{1}{2}\{E M O-F M O\}=\frac{1}{2}\left\{\frac{d-d_{0}}{D}-\frac{1}{3} \frac{d^{3}-d_{0}^{3}}{D^{3}}+\frac{1 d^{5}-d_{0}^{5}}{5}-\& c .\right\} \\
& =\frac{d-d_{0}}{2 D}\left\{1-\frac{1}{3} \frac{d^{2}+d d_{0}+d_{0}^{2}}{D^{2}}+\frac{1}{5} \frac{d^{4}+d^{3} d_{0}+d^{2} d_{0}^{2}+d d_{0}^{3}+d_{0}^{4}}{D^{4}}\right\}
\end{aligned}
$$

Now, in practice the adjustment of the apparatus is always so nearly correct that $d_{0}$ is small, and hence the terms involving $d_{0}$ within the bracket may be neglected, so that

$$
\theta=\frac{d-d_{0}}{2 D}\left\{1-\frac{1}{3}\left(\frac{d}{D}\right)^{2}+\frac{1}{5}\left(\frac{d}{D}\right)^{4}=\& c .\right\},
$$

and in the correction terms an approximate value for $d$ will give suffciently accurate results, the value of the correction being obtained from Table 24. 
If the plane of the mirror is not parallel to the axis about which it turns, then a correction will have to be applied. If $F$ is the height above the horizontal plane through the mirror of the point of intersection of the axis of the telescope with the vertical plane through the scale, and $S$ is the height above the same horizontal plane of the scale, then the distance between the mirror and scale to be used in computing the deflection is

$$
D+\frac{(S+F)(S-F)}{4 D},
$$

where $D$ is the horizontal distance between the mirror and the vertical plane through the scale. For the proof of the formula, the reader must refer to a paper by F. Kohlrausch in Wiedemann's Annalen (1887), vol. xxxi. p. 96.

The following corrections, in addition to those considered above, have to be made when great accuracy is aimed at :-

(a) For the thickness of the glass window. If the mirror is enclosed in a box which is provided with a plate glass window of thickness $t$, the refractive index of the glass being $\mu$, then the distance between the mirror and scale must be reduced by the amount

$$
\frac{t(\mu-1)}{\mu},
$$

where in general $\mu$ may be taken as $3 / 2$, so that the correction amounts to $t / 3$.

The above expression is obtained as follows:-Let MB (Fig. 178) be a reflected ray incident on the glass plate at $\mathbf{B}$. This ray is refracted along $\mathbf{B C}$, and after leaving the glass travels along Cr, meeting the scale $O A$ at $F$. If there had been no glass plate, the ray would have met the scale at $\mathrm{A}$. If $\mathrm{FF}^{\prime}$ is drawn perpendicular to the scale, then the reading on a scale $o^{\prime} F^{\prime}$, if there were no glass plate, would be the same as the actual reading on the scale

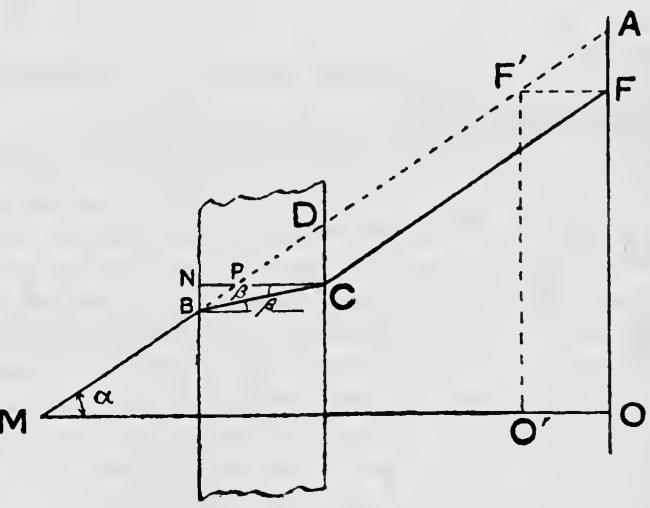

FIG. 178. oA when the plate is present, so that we virtually have to do with a scale at a distance $\mathrm{so}^{\prime}$ from the mirror, and $\mathrm{o}^{\prime} \mathrm{o}$ is the correction to allow for the effect of the glass. 
If $\mathrm{CN}$ is drawn normal to the surface of the glass, CP is equal to $F^{\prime} \mathbf{P}$ or o'o. But if $a$ is the angle BMO, the angle of incidence at $\mathbf{B}$ is $a$. Hence calling the angle of refraction at $\mathrm{B} \beta$, we have

$$
\sin \alpha=\frac{N B}{\bar{B} \bar{P}} ; \sin \beta=\frac{N B}{B C} ; \text { and } \mu=\frac{B C}{B P} .
$$

But if, as is always the case, $\alpha$ is small, BP is very nearly equal to NP, and NC very nearly equal to BC. Thus

But

$$
\mu=\frac{N C}{N P}
$$

Hence

$$
P C=N C-N P \text {. }
$$

$$
P C=N C-\frac{N C}{\mu}
$$

Thus

$$
O^{\prime} O=t\left(1-\frac{1}{\mu}\right)=t\left(\frac{\mu-1}{\mu}\right) .
$$

If the mirror consists of a piece of glass silvered on the back, then the distance between the front surface of the mirror and the scale must be increased by $\delta / \mu$, where $\delta$ is the thickness of the glass of the mirror. This formula follows immediately from the discussion given above.

171. Adjustment of a Telescope and Scale to measure a Rotation by means of a Mirror.-The telescope and scale shown in Fig. 23 can be used for measuring rotations, and if, as is generally the case, the axis about which the rotation takes place is vertical, the scale is placed horizontally. The numbers on the scale are printed as follows :-

\section{.9 .8 .7 .6 .5 .4 .3 .2 .1}

so that when seen by the telescope, which is not fitted with an erecting eye-piece, after reflection in the mirror they appear the right way up.

For definiteness suppose the body of which the rotation is to be measured is the needle of a galvanometer, a thin plane mirror being attached to the needle system.

Having focused the eye-piece of the telescope on the cross-wires, and adjusted one of these vertical, focus the telescope on an object at twice the distance at which the scale is to be placed from the galvanometer. Then hang a small plummet in front of the object glass of the telescope so that it bisects the object glass, and having placed the telescope and scale in front of the galvanometer at the desired distance, which may conveniently be a metre, adjust the position till an image of the scale is seen in the field. Then move the telescope and scale till the image of the thread of the plummet where it crosses the scale coincides with the vertical cross-wire. 
It is next required to adjust the scale so that it is at right angles to the axis of the telescope, that is, that the perpendicular from the mirror on the scale shall intersect the thread of the plummet. In the case of the telescope and scale shown in Fig. 23, the axis of the telescope is permanently fixed perpendicular to the scale, so that when the adjustment described above is complete the scale must necessarily be perpendicular to the line joining the mirror to the centre of the object glass of the telescope.

In those cases where the telescope and scale are capable of relative motion, the easiest way of making the adjustment is to attach a small piece of plane mirror to the scale behind the plummet and push in the draw-tube of the telescope till an image of the galvanometer is seen. This image is formed by reflection in the mirror attached to the scale and the galvanometer mirror. Then rotate the scale about a vertical axis till the image of the galvanometer mirror is bisected by the vertical cross-wire. If when the telescope is again focused on the scale the image of the plummet thread no longer coincides with the vertical wire, the first adjustment must be repeated, and then the sccond, till both adjustments are complete.

Having in this way completed the adjustment, it remains to measure the distance between the mirror and the scale. To do this support a metre scale in a retort stand at a higher level than the mirror and scale, and by means of a level adjust it horizontal. Then hang two plummets, and adjust one so that it just 'grazes the surface of the scale, and the other so that it hangs in front of the galvanometer. A small pointed rod of brass about $10 \mathrm{~cm}$. long must then be held in a clamp, and carefully moved till the point just touches the surface of the mirror. Having then adjusted the plummet nearer the galvanometer so that its thread just touches the other extremity of this piece of brass, read the metre scale where the two threads of the plummets cross. Then the difference of these readings plus the length of the brass rod will give the distance between the mirror and the scale.

If necessary, this distance must be corrected to allow for the effect of the thickness of the mirror and the cover glass enclosing the galvanometer mirror, as described in the last section.

172. Adjustment of a Suspended Needle Reflecting Galvanometer.- First remove the controlling magnet, and then, if it is not already known, determine whether the magnetism of the astatic system is such that the instrument requires to be set up facing east or west. Suppose that it requires to face the east. By means of a prismatic compass or a long suspended magnetised needle draw a line north and south, and then draw a line through the position to be occupied by the galvanometer at right angles to this north and south line. Adjust the galvanometer so that the coils are parallel to the magnetic meridian, and level the instrument till the suspension hangs quite free. Whether the suspension is free can usually be discovered by lightly tapping the case, for if any part of the suspended system is touching the case, the system will be seen to give a sudden jump when the case is tapped. 
In order to observe the rotation of the suspended system, use may be made either of a lamp and scale, or of a telescope and scale. The telescope and scale is in general to be preferred, both on account of its being the more accurate, and because it does not necessitate a partly darkened room. Place the telescope and scale on the east and west line, so that the scale is at some definite distance, such as 100 centimetres, from the mirror of the galvanometer, and adjust its position in the manner described in the last section.

The next adjustment which has to be made is that of setting the magnets of the suspended system parallel to the coils, and of so placing the directing magnet that the resultant couple, due to the earth's field and the field of this magnet, when the needle is deflected should be sufficiently small so that the required sensitiveness shall be attained. The suspended system is generally so magnetised that the upper magnet, in the case where the directing magnet is above, and the lower when the directing magnet is below the galvanometer, shall be the stronger of the astatic pair or sets of magnets. Thus to weaken the resultant couple the directing magnet must be turned so that its north pole points towards the north. The sensitiveness of the galvanometer being proportional to the square of the period of the suspended system, we may make use of observations of period to judge how the adjustment for sensitiveness is proceeding. It will in general be found advisable to use a period of about ten seconds, since a much longer period makes working with the instrument tedious, and often involves changes of zero. The desired sensitiveness having been attained, the director magnet is turned till the central division of the scale coincides with the vertical cross-wire. For most purpases we may now assume that the magnets of the suspended system are parallel to the plane of the coils, since the suspended system is made with the magnets and mirror in the same plane. In some cases, however, it is necessary to arrange that the axis of the magnet is accurately parallel to the galvanometer coil. This is best

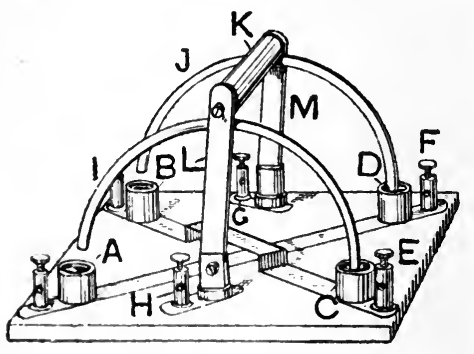

FIG. 179. done by setting up a circuit consisting of a constant cell, such as a Daniell or a small accumulator, a very high resistance, a commutator, and the galvanometer. A suitable form of commutator is the Pohl commutator, shown in Fig. 179. It consists of a base made of some insulating material, such as ebonite, on which are fixed four mercury cups A, B, C, D. Two metal uprights $L, M$ pivot about metal blocks attached to the base, and to these uprights are soldered two semicircular pieces of strong copper wire I and $\mathrm{J}$, while a piece of glass or ebonite $\mathbf{K}$ connects the upper ends of the uprights. The mercury cups $A$ and $D$ are 
connected together by a copper wire, as are also the cups $\mathrm{B}$ and $\mathrm{c}$. Four binding screws E, F, G, H are connected as shown, and serve for joining the commutator to the circuit. If the battery terminals are connected to $G$ and $H$, and the ends of the circuit containing the resistance and the galvanometer are connected to $\mathbf{E}$ and $F$, then when the movable part is rocked into the position shown, $\mathrm{H}$ is connected to $\mathbf{E}$, and $\mathbf{G}$ to $\mathbf{F}$. If, however, the movable part is rocked towards the left, then $\mathrm{H}$ is connected to $\mathrm{F}$, and $\mathrm{G}$ to $\mathrm{E}$, that is, the direction of the current in the galvanometer is reversed.

A simpler form of commutator, which can be made in a few minutes, but which is not quite so convenient, is shown in Fig. 180. It consists of a block of ebonite or paraffin wax, with four small hollows filled with mercury, which are either connected with binding screws as shown, or into which the ends of the wires forming the different parts of the circuit simply dip. The mercury cups can be connected by two small U-shaped pieces of copper-wire, which are attached to a cross bar of ebonite $\mathrm{k}$, as shown in the figure. $A$ and $D$ being connected to the battery, and $\mathrm{C}$ and $\mathrm{B}$ to the circuit, then by putting the connector as shown in the figure $\mathrm{A}$ is joined to $\mathrm{C}$ and $\mathrm{D}$ to $\mathrm{B}$. On rotating the connector through $90^{\circ} \mathrm{A}$ is joined to $\mathrm{B}$ and $\mathrm{D}$ to c, thus reversing the direction of the current in the circuit.

To adjust the needle parallel to the coils, the resistance in the circuit must be adjusted so that the deflection is about 10 centimetres, and the directing magnet rotated till on reversing the direction of the current the deflections on the two sides of the zero are equal. In the case of a galvanometer without controlling magnet, it would be necessary to rotate the whole galvanometer when making this adjustment, and hence it should be performed before the final adjustments of the scale are made.

173. Adjustment of a Suspended Coil Galvanometer.-In places where the earth's field is disturbed by the neighbourhood of moving masses of magnetised matter, such as lifts or dynamos, or by the presence of stray electric currents, such as exist in the vicinity of electric railways or tramways, the ordinary form of suspended needle galvanometer cannot be used, owing to the continual disturbance of zero which is produced. In such a case a suspended coil galvanometer will be found of service. In this type of galvanometer the current to be measured is passed through a small coil which is suspended between the poles of a strong permanent magnet, so that when undeflected the plane of the coil lies parallel to the lines of force due to the magnet. The current is led into and out from the coil by means of two thin ribbons of phosphor bronze. The upper ribbon is generally straight, and serves as a suspension fibre for the coil, while the lower ribbon is wound into the form of a helix, so as to reduce its torsional rigidity to a minimum. 
The azimuth in which such a galvanometer is set up is immaterial, a point which is sometimes of importance.

The instrument having been levelled, so that the coil hangs freely, the adjustment of the telescope and scale, or of the lamp and scale, is made as in the case of the needle galvanometer. The sensitiveness, however, cannot be varied by altering the field strength, unless the permanent magnet is replaced by an electromagnet, in which case the sensitiveness can be altered within certain limits by altering the strength of the current in the electromagnet.

When using a suspended coil galvanometer it is advisable to have a key by means of which the terminals of the galvanometer can be connected together, and thus the suspended coil short-circuited. This short-circuiting key serves to bring the coil to rest when it has been set swinging, for, owing to the movement of the coil in the magnetic field, currents are induced which rapidly damp out the oscillations of the coil. On open circuit, however, the E.M.F. induced in the coil, owing to its motion in the magnetic field, cannot produce a current, and hence very little damping is produced, and the coil continues swinging for some time. If the coil is of fairly high resistance, it is advisable to insert some resistance in series with the short-circuiting key, as otherwise the motion of the coil is so much damped that a very considerable time elapses before the zero position is reached. The best results are attained when the coil just passes beyond its position of rest and then comes to rest at the second passage.

174. Determination of the Figure of Merit of a Galvanometer.By the figure of merit of a galvanometer is meant the current in amperes required to produce a deflection of one division of the scale. Since the sensitiveness depends ' $n$ the resultant field in which the suspended system swings, in the case of needle galvanometers the figure of merit will depend on the position of the directing magnet. It will also depend on the distance at which the scale is placed. For this reason it is usual to calculate the figure of merit corresponding to a period of the needle of ten seconds and for a scale distance equal to 1000 scale divisions, that is, in the case of a scale divided into millimetres for a distance of 1 metre. Further, since the strength of the deflecting field produced by the coils, when the galvanometer is traversed by a current, depends not only. on the strength of the current, but also on the number of turns in the coils, and on the position of these turns with respect to the needles, it is evident that a galvanometer having a very great number of turns will, for a given current, be likely to give a larger deflection than another containing a few turns, and this although the latter may be the better galvanometer.

The only really satisfactory way of comparing the performance of different galvanometers, of which the resistance varies, is to compare the amount of electrical energy which has to be supplied to the galvanometer when the period is ten seconds and the scale is at 1000 scale divisions to maintain a deflection of one scale division. The electrical power is obtained by multiplying the resistance of the galvanometer by the square 
of the current required to give a deflection of one division under standard conditions.

In order to find the figure of merit of a galvanometer it is connected up with a battery and a series of resistances, as shown in Fig. 181. Here $B$ is the battery, which should preferably consist of a single accumulator, which after being fully charged has been allowed to partly discharge through a resistance. $K$ is a make-and-break key, and may consist of a piece of copper wire, the ends of which dip into two mercury cups scooped in a piece of paraffin wax. $P$ and $Q$ are two resistance boxes, and, if one is available, it is of advantage that $P$ should be capable of variation by tenths of an ohm. $\mathbf{R}$ is a high resistance, generally 10,000 or 100,000 ohms, while $G$ is the galvanometer. The sum of the two resistances $P$ and $Q$ is kept constant, and may conveniently be made a thousand times the

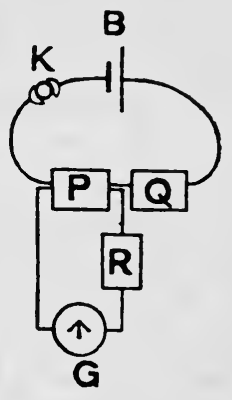

FIG. 181. E.M.F. of the battery. Thus if an accumulator is used, $P+Q$ may be $2100 \mathrm{ohms}$; while if a Daniell cell is used, their sum may be 1080 ohms.

If $E$ is the E.M.F. of the battery, and $B$ its resistance, and $P, Q, R$, and $G$ represent the resistances of the various parts of the circuit, the E.M.F. between the terminals of the resistance $P$ when the galvanometer circuit is broken is given by

$$
\frac{E P}{P+Q+B}
$$

If $P$ is very small compared to $R+G$, as is generally the case, then this E.M.F. when the galvanometer circuit is closed will be sensibly the same. Hence the current in the galvanometer is

$$
\frac{E P}{P+Q+B} /(R+G) \text {. . . . . . . }
$$

Generally $B$ is so small compared to $P+Q$, that the expression for the current may be written

$$
\frac{E P}{(P+Q)(R+G)}
$$

If we take into account the effect of the galvanometer circuit in reducing the resistance between the terminals of the resistance $P$, since the resistance of $P$ and $R+G$ in parallel is

$$
\begin{aligned}
& P(R+G) \\
& \bar{P}+\bar{R}+\bar{G}
\end{aligned}
$$


the E.M.F. between the terminals of $P$ is

$$
\frac{E\left(\frac{P(R+G)}{P+R+G}\right)}{\frac{P(R+G)}{P+R+G}+Q+B} \text {. . . . . . }
$$

and the current in the galvanometer is this quantity divided by $(R+G)$, which is the same as the expression (1), where $P(R+G) /(P+R+G)$ has been written in place of $P$. Now $P(R+G) /(P+R+G)$ may be written $P\left(1-\frac{P}{P+Q+R}\right)$, so that unless $P /(P+G+R)$ is greater than, say, 001 , we need not employ the more cumbersome expression for finding the current in the galvanometer.

Determine for different positions of the directing magnet the period and the current required to produce a deflection of, say, 5 or $6 \mathrm{~cm}$. In performing this experiment, adjust the resistance $P$ so that a deflection of about the desired amount is produced, and then read the deflection. The accuracy will be slightly increased if a commutator is included in the galvanometer circuit, and deflections on either side of the zero are taken.

Plot on squared paper the current required to produce a deflection of one division against the square of the period, and see how nearly the points lie on a straight line.

Also determine the currents necessary for producing different deflections right up to the end of the scale, and plotting current against deflection test whether the two are proportional.

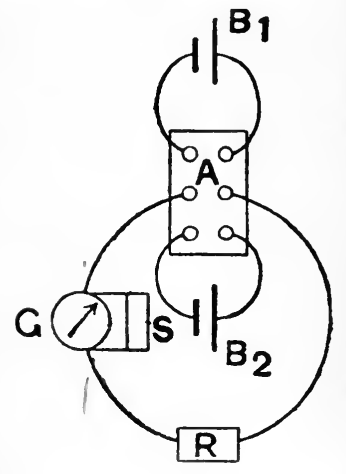

FIG. 182.

175. Comparison of Electromotive Forces with the Galvanometer.-The two cells of which the E.M.F.'s are to be compared, $B_{1}$ and $B_{2}$ (Fig. 182), are connected in the manner shown to a set of mercury cups in a block of paraffin, the two middle cups being connected to the galvanometer $\mathrm{G}$ and a high resistance $\mathbf{R}$. A resistance $s$ is placed as a shunt on the galvanometer, so that the sensitiveness may be reduced if required. The resistance $\mathrm{k}$, together with that of the galvanometer, ought to be at least $10,000 \mathrm{ohms}$.

The shunt resistance is adjusted till with the cell of higher electromotive force the deflection is almost to the end of the scale, and then the deflections when first one and then the other cell is connected with the galvanometer circuit are read. The deflection experiment should be repeated several times, and the mean taken. Also by slightly altering the shunt resistance, readings can be taken with different deflections. If $D_{1}$ and $D_{2}$ are the mean deflections. then the ratio of the electromotive forces, so long as the resistance ir 
the galvanometer circuit is very high compared to the resistance of either of the cells, is given by

$$
\frac{E_{1}}{E_{2}}=\frac{D_{1}}{D_{2}}
$$

If $C_{1}$ and $C_{2}$ are the currents in the circuit in the two cases, we have

and

$$
C_{1}=\frac{E_{1}}{R+G+B_{1}}
$$

$$
C_{2}=\frac{E_{2}}{R+G+B_{2}}
$$

where $B_{1}$ and $B_{2}$ are the resistances of the cells.

Hence if the deflections are proportional to the currents 1

$$
\frac{D_{1}}{D_{2}}=\frac{C_{1}}{C_{2}}=\frac{E_{1}}{E_{2}} \cdot \frac{R+G+B_{2}}{R+G+B_{1}}
$$

If $B_{1}$ and $B_{2}$ are both small compared to $R+G$, the factor wbich multiplies $E_{1} / E_{2}^{\prime}$ is sensibly unity, and hence

$$
\frac{D_{1}}{D_{2}}=\frac{E_{1}}{E_{2}}
$$

To test whether we are justified in making the above assumption, we have

$$
\frac{R+G+B_{2}}{R+G+B_{1}}=1+\frac{B_{2}-B_{1}}{R+G+B_{1}}
$$

Hence if $\frac{B_{2}-B_{1}}{R+G+B_{1}}$ is negligible compared to unity, the approximation is sufficiently accurate.

1 The experiments made in the preceding section will show whether this assumption is justified. If the currents are not proportional to the deflections, then the value of the ratio $C_{1} / C_{2}$ deduced from the curve obtained in $\$ 174$ must be used in place of $D_{1} / D_{2}$. 


\section{CHAPTER XXVI}

\section{MEASUREMENT OF RESISTANCE}

176. The Wheatstone's Network of Conductors. - That particular net-

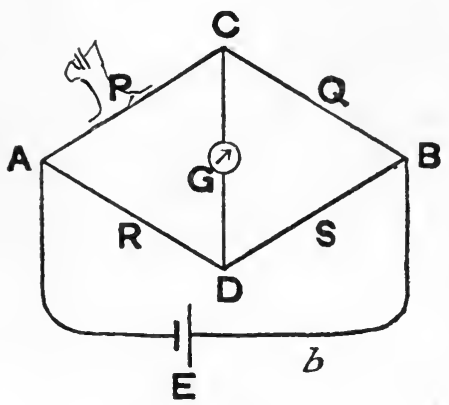

FIG. 183. work consisting of six conductors, joined up as shown in Fig. 183, which is called a Wheatstone's network or bridge, will be found of such wide application in the following pages that a few words as to its general properties may be of service. Let $\mathrm{P}, \mathrm{Q}, \mathrm{R}, \mathrm{S}, \mathrm{G}$, and $b$ be the resistances of the arms $\mathrm{AC}, \mathrm{CB}, \mathrm{AD}, \mathrm{DB}, \mathrm{DC}$, and $\mathrm{AB}$ respectively. If we suppose that there is a battery of E.M.F. E placed in the arm $\mathrm{AB}$, while the resistances $P, Q, R$, and $\mathrm{s}$ are so adjusted that a galvanometer placed in the arm $\mathrm{CD}$ is undeflected, then it at once follows that $\mathbf{P S}=\mathbf{R Q}$. For the fact that there is no current in the arm DC shows that the points $\mathrm{D}$ and $\mathrm{C}$ are at the same potential. Hence

$$
\frac{\text { drop of potential along } A D}{\text { drop of potential along } D B}=\frac{\text { drop of potential along } A C}{\text { drop of potential along } C B} \text {. }
$$

But as there is no current in the arm DC, the current through $\mathrm{AD}$ is the same as the current through DB. Hence the drop of potential along $\mathbf{A D}$ is to the drop of potential along $\mathrm{DB}$ as the resistance of $\mathrm{AD}$ is to the resistance of $\mathbf{D B}$. Similarly the drop of potential along $\mathrm{AC}$ and $\mathrm{CB}$ are to one another as the resistances of $\mathbf{A C}$ to $\mathbf{C B}$. Hence

$$
\frac{P}{Q}=\frac{R}{\bar{S}}
$$

In exactly the same way we can prove that the condition that when an E.M.F. is placed in the arm $C D$ there shall be no current in $A B$ is

$$
\frac{P}{\bar{Q}}=\frac{R}{S}
$$

that is, the same condition as before. The two arms $A B$ and $C D$, such 
hat an E.M.F. in one produces no current in the other, are said to be conjugate.

An important property of conjugate arms is that if we insert an E.M.F. in any other arm of the network, then the current produced by this E.M.F. in one of the conjugate arms is independent of the resistance of the other. For suppose that an E.M.F. inserted in one of the arms, say, AC, produces a current in $\mathbf{A B}$. Then we may, if we please, introduce an additional E.M.F. in the arm $\mathbf{A B}$, and adjust its magnitude till the current it sends is just equal and opposite to the current in $\mathbf{A B}$, due to the E.M.F. in the arm $\triangle C$. Now the E.M.F. introduced in $A B$ will produce no current in the arm $C D$, since $A B$ and $C D$ are conjugate, and hence the introduction of the E.M.F. in $\mathbf{A B}$ will not have affected the current in $\mathbf{C D}$; its effect, however, has been to reduce the current in $\mathbf{A B}$ to zero, and hence we may make the resistance of the arm $A B$ whatever value we like without affecting the current in CD.

Hence to calculate the current in CD, produced by an E.M.F. $e_{r}$ introduced in the arm $A C$, we may take the resistance of the arm $A B$ as being infinite. This being the case, the current in $\Delta \mathrm{C}$ is

$$
\frac{e_{r}}{P+R+\frac{(Q+S) G}{Q+S+G}}
$$

Hence the current in $\mathrm{CD}$ is

$$
\frac{e_{r}}{P+R+\frac{(Q+S) G}{Q+S+G}} \cdot \frac{Q+S}{Q+S+G^{\prime}}
$$

or

$$
\frac{e_{r}(Q+S)}{(P+R)(Q+S+G)+(Q+S) G} \text {. }
$$

But the resistances $P, Q, R, S$ are subject to the condition $P S=R Q$. Hence the current in CD is

$$
\frac{e_{r} S\left(1+\frac{P}{R}\right)}{R\left(1+\frac{P}{R}\right)\left(\frac{P S}{R}+S+G\right)+S\left(1+\frac{P}{R}\right) G}=\frac{e_{r} S}{S(P+R)+G(R+S)}
$$

Exactly the same expression would be obtained for the current in $\mathrm{CD}$, due to the introduction of an E.M.F. $e_{p}$ in the arm $A D$.

Next suppose that we introduce an E.M.F. $e_{a}$ in the arm DB. The current produced in $\mathrm{CD}$ is

$$
\frac{e_{s}}{S+Q+\frac{(P+R) G}{P+R+G}} \times \frac{P+R}{P+R+G}
$$


and making use of the relation $P S=R Q$ this reduces to

$$
\frac{e_{s} R}{S(P+R)+G(R+S)} \cdot \cdot \quad \cdot \quad \cdot \quad \cdot \quad .
$$

which also gives the current in CD produced by an E.M.F. $e_{\mathrm{g}}$ in the arm CB. It will be noticed that the denominators in the expressions (2) and (3) are the same.

If the current sent through the arm CD, when an E.M.F. $e_{r}$ is placed in $\mathrm{AC}$ or $\mathrm{AD}$, is the same as that sent when an E.M.F. $e_{s}$ is placed in $\mathrm{CB}$ or BD, then

$$
\frac{e_{r}}{e_{s}}=\frac{R}{S}=\frac{P}{Q} \cdot \cdot \cdot \cdot \quad \cdot \quad \cdot
$$

Since whether the galvanometer is connected between $\mathrm{C}$ and $\mathrm{D}$, and the battery between $\mathbf{A}$ and $\mathrm{B}$, or the galvanometer connects $\mathbf{A}$ and $\mathbf{B}$, and the batteries $\mathrm{C}$ and $\mathrm{D}$, there will be no current in the galvanometer if $P / Q=R / S$. It follows that in any given case we have a choice as to the way in which the battery and galvanometer are connected up, and it becomes necessary to decide which is the better arrangement. If the resistances are all of about the same magnitude, then it is quite immaterial which way the connection is made. If, however, the resistances are of very different magnitudes, then if $R$ and $S$ are the two larger, and, as is usually the case, the resistance of the galvanometer is greater than that of the battery (including the battery leads and any resistance which may be inserted in series with the battery to reduce the magnitude of the currents sent through the resistances), the galvanometer ought to be connected to the point where the resistances $R$ and $S$ meet, and to the point where the two lower resistances $P$ and $Q$ meet. If the resistance of the battery is greater than that of the galvanometer, then the battery should connect the point where the two higher resistances meet to the point where the two lower ones meet.

Since the passage of the currents through the resistances heats the resistances, and hence alters their resistance, if any of the resistances are of small section, or constructed of a material of which the resistance changes rapidly with temperature, then considerations of heat development, rather than the above, ought to settle the manner in which the battery is connected up.

Let us suppose that the values of the resistances are $P=100 \mathrm{ohms}$, $Q=50 \mathrm{ohms}, R=10 \mathrm{ohms}$, and $S=5$ ohms. Then when the battery is connected, as in Fig. $184(a)$, we have

$$
\frac{c_{2}}{c_{1}}=\frac{P+Q}{R+S}=\frac{150}{15}=10
$$

If, however, the battery is connected, as shown in Fig. $184(b)$, and 
$c_{1}^{\prime}$ is the current in the arm $\mathrm{CAD}$, and $c_{2}^{\prime}$ that in the arm $\triangle B D$, we have

$$
\frac{c_{2}^{\prime}}{c_{1}^{\prime}}=\frac{P+R}{Q+S}=\frac{110}{55}=2 .
$$

Thus supposing $S$ is the resistance which is liable to be heated, we see that the first arrangement is much more unfavourable than the second, for a much greater proportion of the current passes through $S$ in the first case. Thus althuugh the arrangement shown in Fig. 184(a)
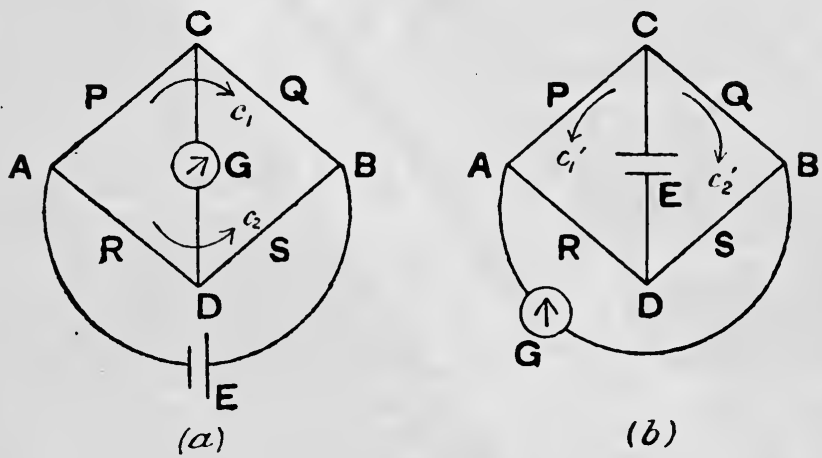

FIG. 184.

is theoretically the more sensitive when, as is almost always the case, the galvanometer resistance is larger than the battery resistance, yet owing to the excessive development of heat, due to the fact that such a large proportion of the current passes through one pair of resistances, the other arrangement is much to be preferred.

177. The Metre Form of Wheatstone's Bridge.-A form of bridge which is frequently employed ${ }^{1}$ is shown in Fig. 185. It consists of a uniform wire AC, the ends being soldered to two thick copper strips $P$ and $\mathrm{H}$, while attached to the same wooden base are three other copper strips, and binding screws are soldered to these in the positions shown. The gaps at $\mathrm{M}$ and $\mathrm{N}$ can, when required, be closed by two thick copper connectors, as shown in the figure. The battery is connected to the terminals $\mathbf{B}_{1}$ and $\mathbf{B}_{2}$, while one galvanometer terminal is attached to $\mathrm{I}$, and the other to a movable slider D. On depressing the knob of this slider, contact is made with the stretched wire by means of a thin metal plate, which has its length placed at right angles to the wire. Immediately above this contact plate a fiducial line is engraved on the

1 This form of bridge is in many ways inconvenient and inaccurate. It is, however, useful as an exercise in the various methods of using a Wheatstone's bridge, and is comparatively cheap. 
slider by means of which the position of the contact is read off on the scale.

In the simplest method of using this form of bridge, the two resistances to be compared are connected to the terminals $P_{1} P_{2}$ and $\mathbf{Q}_{1} \mathbf{Q}_{2}$. Balance is obtained by moving the slider $\mathrm{D}$, and then if the wire is

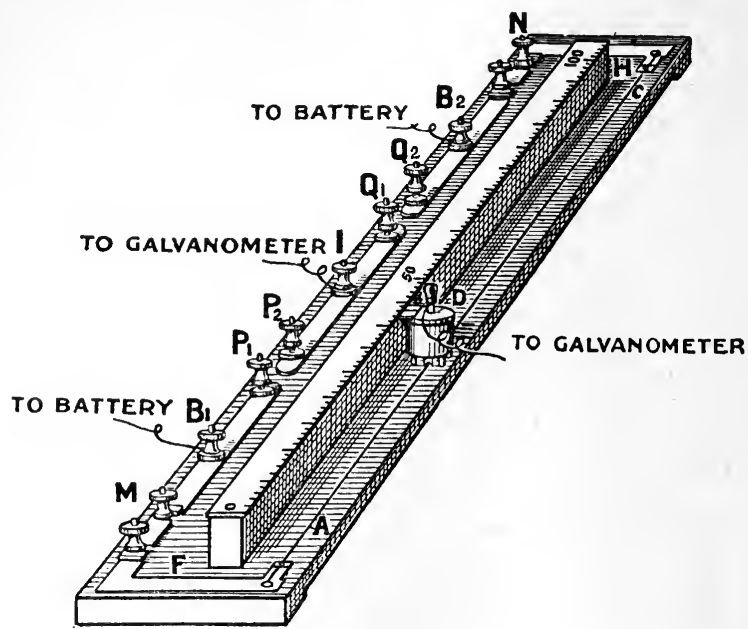

FIG. 185.

uniform, and the resistance of the connecting pieces $F$ and $H$ can be neglected, and the slider divides the wire into portions of length $p$ and $q$ respectively, we have

$$
A / B=p / q,
$$

where $A$ is the value of the resistance between $\mathrm{P}_{1}$ and $\mathrm{P}_{2}$, and $B$ that between $Q_{1}$ and $Q_{2}$.

If the resistances of the end connections of the wire, including the soldered junction, are not negligible, suppose that the added resistance at one end is equivalent to the resistance of a length $\lambda_{p}$ of the wire, the corresponding quantity for the other end being $\lambda_{q}$, then the expression giving the ratio of the two resistances which are being compared is

$$
\frac{A}{B}=\frac{p+\lambda_{p}}{q+\lambda_{q}}
$$

In order to determine the values of the end corrections $\lambda_{p}$ and $\lambda_{q}$, two resistances, the ratio of which are known, are inserted in the gaps $\mathrm{P}$ and Q. Let the ratio of these two resistances be $n$, so that $B / A=n$. Then

$$
q+\lambda_{q}=\left(p+\lambda_{p}\right) r
$$


Next reverse the two resistances, placing $A$ in the $Q$ gap and $B$ in the $P$ gap, and let the point of balance divide the wire into lengths of $\boldsymbol{p}^{\prime}$ and $q^{\prime}$. Then

$$
q^{\prime}+\lambda_{q}=\left(p^{\prime}+\lambda_{p}\right) \frac{1}{n} .
$$

Subtracting one of the equations from the other

$$
q-q^{\prime}=n\left(p+\lambda_{p}\right)-\frac{1}{n}\left(p^{\prime}+\lambda_{p}\right)
$$

or

$$
\lambda_{p}=\frac{n\left(q-q^{\prime}\right)-\left(n^{2} p-p^{\prime}\right)}{n^{2}-1} .
$$

But since the whole length of the wire is $1000,:=100-p$, and $q=1000-p^{\prime}$, therefore

Similarly

$$
\lambda_{p}=\frac{p^{\prime}-n p}{n-1}
$$

$$
\lambda_{q}=\frac{q-n q^{\prime}}{n-1} .
$$

When performing the experiment, $n$ should be large, say, about 100 . A convenient arrangement is to make one resistance $1 \mathrm{ohm}$, and the other 101 ohms. Then $n=101$, and $n-1$, which occurs in the denominator of each of the expressions, is 100 .

178. Calibration of a Bridge Wire by Carey Foster's Method.Since the resistance of a unit of length of a wire is never quite uniform throughout the whole length of a wire, it becomes necessary to calibrate the wire of a Wheatstone's bridge, that is, to determine the manner in which the resistance per unit length varies at different parts of the length. The resistance of the whole of the bridge wire is either measured by means of another bridge, or it may be obtained sufficiently accurately in the following way:-Two approximately equal resistances, say, $1 \mathrm{ohm}$ each, are placed in the gaps $P$ and $Q$, while a $10-0 \mathrm{hm}$ coil is placed in each of the gaps $\mathrm{M}$ and $\mathrm{N}$, a box of coils being arranged as a shunt on the coil in the $M$ gap. The value of the shunting resistance is then altered till a point of balance is obtained near the end $\mathrm{c}$ of the wire. This point of balance having been determined, the coils in and $\mathbf{N}$; together with the shunt, are interchanged, and the point of balance near the end $A$ of the wire is determined.

If $r_{1}$ is the value of the resistance in one gap, and $r_{2}$ the value of that in the other, while the length of the wire between the two points of balance is $d$, the resistance of unit length of the wire is given by

$$
\frac{r_{1}-r_{2}}{d}
$$

The proof of this expression is given in $\$ 181$. 
Suppose that it is desired to calibrate the wire by twenty steps, that is, to divide the wire into twenty segments of equal resistance. A gauge piece is prepared, consisting of a short length of thick German silver or manganin wire, having a resistance equal to, or very slightly less than, that of $50 \mathrm{~mm}$. of the bridge wire, soldered to two copper tags suitable for clamping in the gaps of the bridge. If the resistance of the gauge is made slightly less than the desired value, it can be adjusted by filing so as to reduce the diameter of the wire.

A stretched wire, which may conveniently be the wire of a second bridge, is connected to the bridge in the manner shown in Fig. 186.

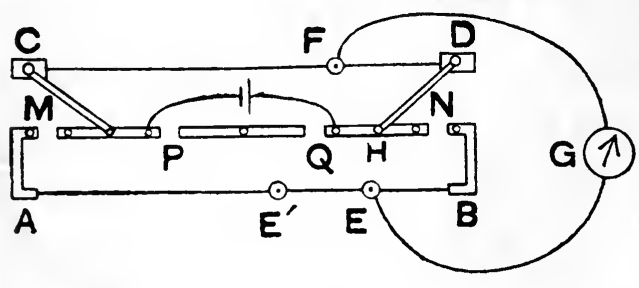

FIG. 186.

A battery is connected to $\mathrm{C}$ and $\mathrm{D}$, while the galvanometer is connected to the movable contact $\mathrm{F}$ on the wire of the bridge, and to a second movable contact $F$ on the auxiliary wire.

The copper connector belonging to the bridge being placed in the gap $\mathrm{N}$, and the gauge in the gap $\mathbf{M}$, the slider $\mathbf{E}$ is placed as near the end of the wire as possible, and slider F moved till, on completing the galvanometer circuit, no deflection is produced. The reading for the slider $\mathbf{E}$ having been recorded; the gauge and connector are interchanged, and the slider $F$ remaining fixed in position, balance is obtained by moving the slider $\mathbf{x}$. The gauge is then replaced in $\mathrm{N}$, and balance obtained by moving the slider $\mathrm{F}$, and so on till the end of the wire $A B$ is reached. When the gauge is in the gap $\mathbf{M}$, the slider $\mathbf{E}$ is moved to obtain balance, while, when the gauge is in $\mathrm{N}$, the slider $F$ on the auxiliary wire is moved.

The portions of the wire $\mathrm{AB}$ (as also of the auxiliary wire), between the successive positions of the slider, have resistances equal to the difference between the resistance of the gauge and the connector used to bridge the other gap. That this is the case is at once evident, for, suppose that when the gauge is in $\mathrm{N}$, the positions of balance are as shown in Fig. 186. Then the fact that there is no current in the galvanometer shows that the two points $\mathbf{E}$ and $\mathbf{F}$ are at the same potential. When the gauge is in $\mathbf{M}$, let the position of balance be at $\mathbf{E}^{\prime}$, that is, the points $\mathbf{E}^{\prime}$ and $\mathrm{F}$ are now at the same potential. We then have that the resistances of the gauge, the strip of copper at the end of the bridge, and the portion $\mathrm{BE}$ of the wire is to the resistance of the connector, the strip of copper at the end $\mathrm{A}$ and the portion $\mathrm{AE}$ of the wire as the resistance of the connector, the copper strip at $\mathrm{B}$, and the portion $\mathrm{BE}^{\prime}$ of the wire is to the resistance of the gauge, the strip of copper at $\mathbf{A}$, and the portion $\mathbf{A} \mathbf{E}^{\prime}$ of the wire; that is, if $\mathrm{G}$ and $\mathrm{C}$ are used to indicate the resistances of the gauge and connector respectively, while $\lambda_{B}$ is the resistance of the copper 
connecting strips at the end $B$, that is, the resistance of the strips between the point $\mathrm{B}$, where the battery circuit divides, and the end $\mathrm{B}$ of the wire, exclusive of the resistance in the gap $N, \lambda_{A}$ being the corresponding quantity at the other end, and the resistances of the portions of the wire are indicated by $\mathrm{BE}$, \&c., we have

$$
\frac{G+\lambda_{B}+B E}{C+\lambda_{A}+A E}=\frac{C+\lambda_{B}+B E^{\prime}}{G+\lambda_{A}+A E^{\prime}},
$$

or, subtracting the denominators from the numerators for new numerators, and adding denominators and numerators for a new denominator,

$$
\frac{G-C+\lambda_{B}-\lambda_{\Lambda}+B E-A E}{G+C+\lambda_{\Delta}+\lambda_{B}+B E+A E}=\frac{C-G+\lambda_{B}-\lambda_{A}+B E^{\nu}-A E^{\prime \prime}}{G+C+\lambda_{\Delta}+\lambda_{B}+B E^{\prime}+A E^{\prime \prime}}
$$

But

$$
B E+A E=B E^{\prime}+A E^{\prime} \text {. }
$$

Hence

$$
2(G-C)=B E-B E^{\prime}+A E-A E^{\prime}=2 E^{\prime \prime}
$$

or

$$
E E^{\prime}=G-C
$$

In the same way, it can be shown that the steps on the auxiliary wire have each a resistance equal to $G-C$.

When performing the experiment, care must be taken to protect the wires and movable contacts from becoming heated by the presence of the observer, otherwise thermo-currents will affect the results. The effects of thermo-currents at the two movable contacts $\mathrm{E}$ and $\mathrm{F}$ may be eliminated by having a key in the battery circuit, and then, having closed the two contacts at $\mathrm{E}$ and $\mathrm{F}$, and waited till the galvanometer deflection becomes constant, closing the battery key, and noting whether any permanent change of the deflection takes place. There may be a slight kick on closing the battery circuit owing to induction in the circuits, but this will in general be so small as to be unobservable. The effects of thermocurrents at the ends of the wires can be eliminated by having a commutator in the battery circuit, and obtaining the point of balance with the current in either direction.

If $l_{1}, l_{2}, l_{3}$, \&c., are the lengths of the bridge wire intercepted between consecutive points of balance, then each of these represents the length of the wire having a resistance equal to the difference in the resistances of the gauge and connector. Then if the difference between the resistance of the gauge and the connector is $r$, the mean resistance of unit length of the wire over the first step is $r / l_{1}$, that over the second step $r / l_{2}$, and so on. The value of $r$ can be at once obtained from the observations, if the resistance $R$ of the whole wirc has been determined. 
For if $\Sigma l$ represents the sum of the lengths of the steps, and $L$ is the total length of the wire, there being $n$ steps, we have

$$
\frac{n r}{R}=\frac{\Sigma l}{L}
$$

from which $r$ can be obtained, and hence the mean resistance per unit length at the different parts of the bridge wire calculated. For the purposes of interpolation, it is advisable to draw a curve in which the ordinates are the readings on the bridge wire, and the abscissæ are the resistances of unit length. This curve is obtained by plotting the values for the mean resistance of unit length at the reading of the wire corresponding to the middle point of each step, and drawing an even curve through the points thus obtained. Instead of plotting the actual resistances per unit length, in the case where great accuracy is required, paper may be saved by plotting the differences between the actual resistance of unit length at the different parts of the wire, and the mean resistance of unit length taken over all the wire, that is, the quantity $R / L$.

The objection to the above method of calibration lies in the fact that the positions of the gauge and connector are continually being changed, and hence if there are any differences in the resistances of the contacts between the terminals of the gauge, or the connector, and the binding screws of the bridge, errors will be produced. For this reason it is necessary to make the terminals of the gauge of a flat piece of copper, with a slot cut in it, so as to fit the screw

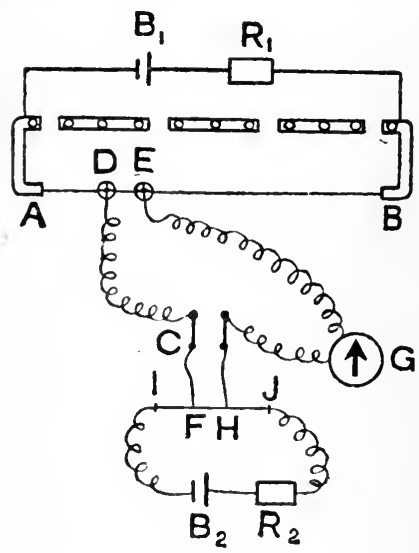

FIG. 187. of the terminals, and to carefully clean these copper plates as well as the binding screws.

179. Calibration of a Wire by means of a Double Circuit. - The following method of calibrating a wire due to Griffiths ${ }^{1}$ has the advantage over Carey Foster's method that the only contacts which are movable are potential contacts, that is, points between which the potential is compared, no current being allowed to flow through the contact. In such a case the resistance of the contact has no effect on the position of the point of balance.

The wire to be treated $\mathbf{A B}$ (Fig. 187 ) is connected in series with a resistance $R_{1}$ and a storage cell $\mathbf{B}_{1}$. A second similar cell $\mathrm{B}_{2}$ is connected to an auxiliary circuit, consisting of a portion of stretched wire, IJ, of thickness at least as great as that of the wire $A B$, and a resistance $R_{2}$. The two cells must have a considerable capacity, and after being fully charged they must be partly discharged before the measurements are 1 Proceedings of the Cambridge Philosophical Society, vol. vii. p. 269. 
commenced. The resistances $R_{1}$ and $R_{2}$ must be adjusted so that the resistances of the two circuits are approximately equal, and such that the current sent by each cell is not greater than a tenth of an ampere. If these precautions are taken, it will be found that the E.M.F. of the cells varies very little, and what variation there is takes place at the same rate in the two cells, and thus produces no effect.

Two contact makers are placed on the wire $\Delta B$, at a distance approximately equal to the step which is to be employed in the calibration. One of these contact makers $\mathbf{E}$ is connected to a high resistance galvanometer $G$, and the other terminal of the galvanometer is taken to a point $\mathbf{B}$ on the wire IJ, where it is soldered in place. The other contact maker D must then be connected to a second point $\mathrm{F}$ on the wire IJ, and the point $F$ moved till the galvanometer remains undeflected. The contact at F should then be soldered, so that accidental movement is impossible.

Starting with one contact near the end $A$ of the wire, the other is moved till there is no deflection of the galvanometer. The left-hand contact $\mathrm{D}$ is then moved to the right of $\mathrm{E}$, and the commutator $\mathrm{C}$ reversed. Then keeping $\mathrm{E}$ fixed, the position of $\mathrm{D}$ is altered till there is no galvanometer deflection. When this is the case, the resistance of the portion of the wire included between the two contacts is equal to the resistance of that portion of the wire which was included between the two contacts in their previous position. The contact $\mathbf{D}$ is then left in place, and $\mathbf{E}$ moved over to the right of $\mathrm{D}$, the commutator is reversed and the position of no deflection obtained as before, and this process is continued till the end B of the wire is reached. The whole proceeding is then repeated, starting from B. The wire having been divided into steps having equal resistance, the calibration curve may be prepared in the manner described in the preceding section.

This method lends itself to the performance of a rapid test of the uniformity of a wire, which is sometimes necessary when choosing a wire for use in a wire bridge or a potentiometer. For this purpose the two contacts $\mathbf{D}$ and $\mathbf{E}$ consist of two sharpened pieces of metal cemented to a piece of ebonite at a convenient distance apart. They are placed in contact with the wire to be tested, and the position of the contacts $\mathrm{F}$ and $\mathbf{H}$ on the auxiliary wire adjusted in the manner already described. Then the ebonite carrying the two contacts is moved along the wire, and if the wire is perfectly uniform, the galvanometer will remain undeflected, and any deflections obtained will indicate the extent of the departure of the wire from uniformity.

180. Calibration of a Wire by Strouhal and Barus's Method.-A convenient arrangement to use when a number of similar wires have to be calibrated, or where, owing to possible wear, it will be necessary to frequently recalibrate the same wire, is that used by Strouhal and Barus. ${ }^{1}$ Ten approximately equal resistances, each about a tenth of the resistance of the wire, are prepared. These resistances may with advantage consist of the same kind and gauge of wire as that to be tested, when they may be adjusted by simply measuring off the required length

1 Wied. Annalen (1880), x. p. 326. 
of wire. If the wire to be calibrated is $100 \mathrm{~cm}$. long, then cut off ten pieces of the same wire each $12 \mathrm{~cm}$. long, and solder the end centimetre of each pipce to a strip of copper A (Fig. 188), bent at right angles as shown. These strips of copper are screwed to a small block of wood $c$, which has been soaked in melted paraffin wax, and then the ends of the
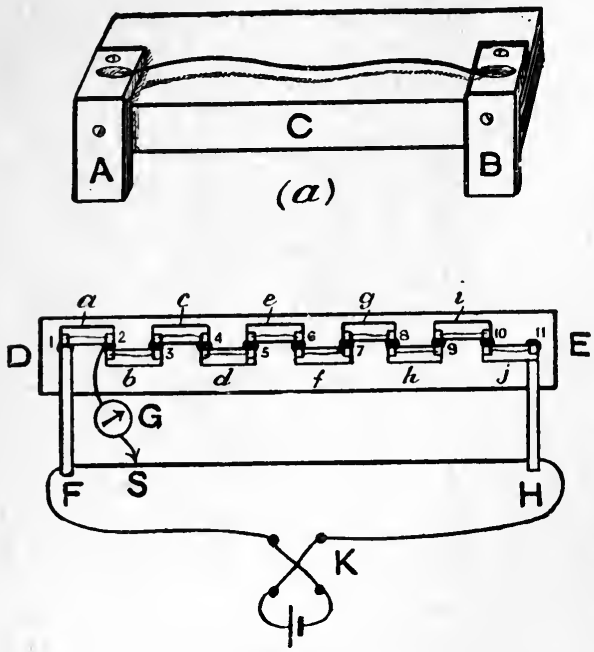

F1G. 188. copper which project below the wood are amalgamated. A row of eleven holes, at a distance apart equal to the distance between $\mathbf{A}$ and $B$, are then bored in a piece of wood, each hole being about a centimetre deep. These holes are filled half full with mercury, and into these holes the terminals of the resistances dip, as shown in Fig. 188 . Mercury cups 1 and 11 are connected to the ends of the wire FH by thick copper straps, while a battery is connected to $\mathrm{F}$ and $\mathrm{H}$. One terminal of a galvanometer $\mathrm{G}$ is then placed in mercury cup 1 , and the position of the slider $\mathrm{s}$ on the wire is adjusted till the galvanometer is undeflected. The position of $\mathbf{s}$ having been recorded, the galvanometer terminal is moved to mercury cup 2, and the position of $\mathbf{s}$ for no galvanometer deflection again determined. The resistances $a$ and $b$ are then interchanged, and the positions of $\mathrm{s}$ on the wire determined for which the galvanometer is undeflected when connected in turn to mercury cups 2 and 3 . The length of wire now intercepted has the same resistance as that previously intercepted between the readings for $\mathrm{s}$ when the resistance $a$ was between the cups 1 and 2 . The resistances $a$ and $c$ are next interchanged, and so on till the resistance $a$ is finally placed between the cups 10 and 11 . In this way the wire $F_{H}$ is divided into ten segments which have equal resistances, and the calibration curve can be calculated, as in $\S 178$.

181. Comparison of Two nearly equal Resistances by Carey Foster's Method.-Let two nearly equal resistances $P$ and $Q$ be inserted in the $P$ and $Q$ gaps of the bridge (Fig. 185), while the two resistances $X$ and $Y$, which have to be compared, are inserted in the gaps at $M$ and $N$. Then when $X$ is in the gap $\mathbf{M}$, the condition for balance is

$$
\frac{P}{Q}=\frac{X+\lambda_{p}+p}{Y+\lambda_{q}+q}
$$

the symbols having the same meaning as in $\S 177$. 
If, now, the resistances $X$ and $Y$ are interchanged, the new condition for balance is

$$
\frac{P}{Q}=\frac{Y+\lambda_{p}+p^{\prime}}{X+\lambda_{q}+q^{\prime \prime}}
$$

From these equations we have

$$
\frac{P-Q}{P+Q}=\frac{X-Y+\lambda_{p}-\lambda_{q}+p-q}{X+Y+\lambda_{p}+\lambda_{q}+p+q}=\frac{Y-X+\lambda_{p}-\lambda_{q}+p^{\prime}-q^{\prime}}{X+Y+\lambda_{p}+\lambda_{q}+p^{\prime}+q^{\prime}},
$$

or since

$$
\begin{aligned}
& p+q=p^{\prime}+q^{\prime}, \text { and } p-p^{\prime}=q^{\prime}-q, \\
& X-Y=p-p^{\prime}=q^{\prime}-q .
\end{aligned}
$$

Thus the difference of the resistance of $X$ and $Y$ is equal to the resistance of the portion of the wire intercepted between the points of contact in the two positions, and since the resistance of this portion of the wire is known from the calibration, we thus obtain the difference in the resistance of the two coils. It will be observed that, as the resistance of the coils placed in the gaps $\mathbf{P}$ and $\mathbf{Q}$ are not involved in the expression for the difference in the resistance of the two coils being measured, we do not require to know their resistance. What is, however, necessary is that their ratio should remain constant throughout the measurement. Hence their temperature must either remain quite constant, or, if they are made of the same material, their temperature, although it may vary, must at every instant be the same. For this reason these two coils are often wound on the same bobbin, and hence, as the wires lie side by side, their temperature will always remain the same.

A form of bridge suitable for comparing resistance coils with a standard by the above method is illustrated in Fig. 189, in which the lettering is similar to that adopted in Fig. 185. The bridge wire $\mathrm{AC}$ is only 10 centimetres long, and is connected to the rest of the bridge by thick copper wires dipping into two mercury cups $\mathrm{L}$. Thus bridge wires of different thicknesses can be employed, according to the difference in the resistances of the two coils being compared. The two coils to be compared have thick copper terminals which dip in the mercury cups $\mathbf{M}$ and $\mathrm{N}$. The ratio coils $(a)$ are wound on the same bobhin, and their terminals, which are also copper rods, dip in the mercury cups $\mathbf{P}$ and $\mathbf{Q}$.

Each of the copper blocks which carry the mercury cups $\mathbf{M}, \mathbf{N}, \mathbf{P}, \mathbf{Q}$, and $\mathrm{L}$ has a second mercury cup, and over these cups there is an ebonite disc, fitted with four copper connectors, F, H, J, and $\mathrm{K}$, as shown. 'The ends of these connectors dip in the mercury cups, and the arrangement forms a commutator, by means of which the coils in the gaps $\mathbf{M}$ and $\mathbf{N}$ can be interchanged with reference to the rest of the bridge when they are being compared by Carey Foster's method. When the comnutator is in the position shown, the coil in $\mathbf{M}$ is connected between $\mathbf{C}$ and $\mathbf{B}_{1}$. When, however, the commutator is rotated through $180^{\circ}$, so that the copper connectors occupy the positions shown dotted, the coil $N$ is now 
between $\mathrm{C}$ and $\mathbf{B}_{1}$. Thus the interchange of the coils is obtained without having to move them, a great convenience, since, to prevent errors due to changes of temperature, the coils have to be immersed in well stirred

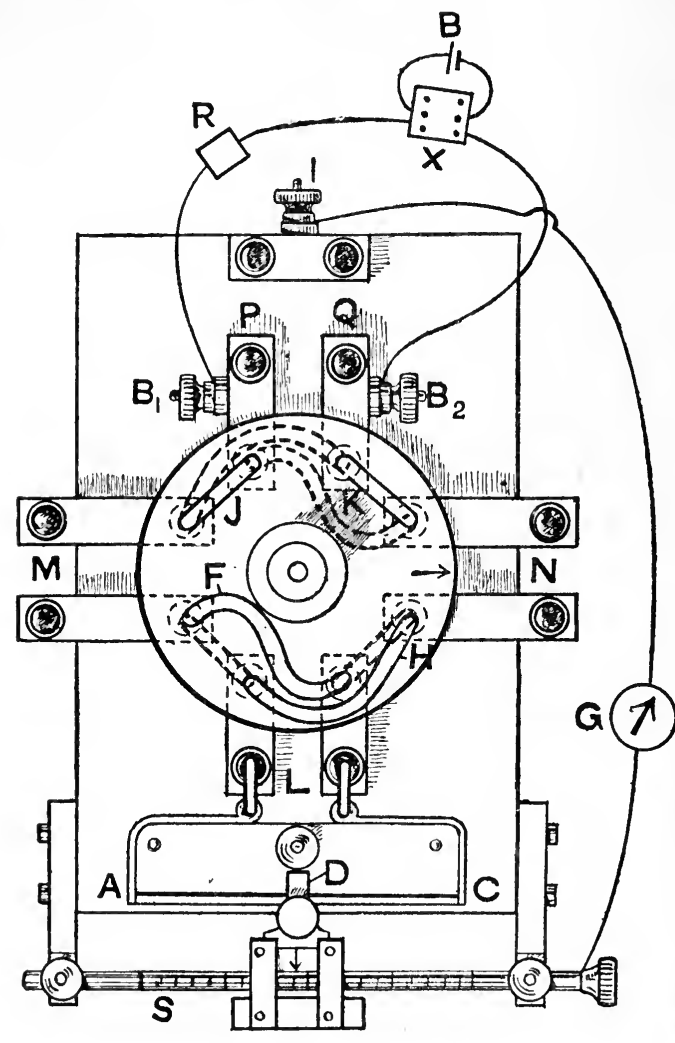

FIG. 189.

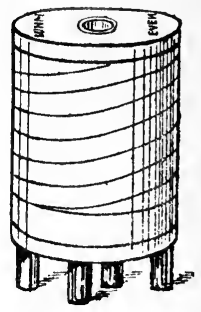

(a)

oil baths, and either lifting the coils out of the baths or interchanging the baths is an awkward operation.

The galvanometer contact $\mathbf{D}$ moves on a divided $\operatorname{rod} \mathbf{s}$, on which a scale is engraved, and the length of wire, of which the resistance is equal to the difference in the resistances of the coils in $M$ and $N$, is read off on this scale.

Generally three pairs of proportional coils to go in the gaps $\mathbf{P}$ and $\mathbf{Q}$ are supplied, having resistances of 1,10 , and $100 \mathrm{ohms}$ respectively. 
The pair should be chosen of which the resistance most nearly approaches the resistance of the coils to be compared.

Since the difference in the resistance of the coils is obtained in terms of the resistance of a portion of the bridge wire, it is necessary to measure the resistance of unit length of the wire, and, where great accuracy is desired, calibrate the wire.

Having carefully amalgamated the terminals of the proportional coils to be used and those of two standard resistances, set up the bridge with the standard coils in oil baths. An accumulator can with advantage be used as battery, but if the coils to be compare are 1 or $10 \mathrm{ohm}$ coils, some resistance $R$ must be included in the battery. circuit. It is also advantageous to place a commutator $X$ in the battery circuit. Determine which of the coils $X$ and $Y$ in the gaps $M$ and $\mathbf{N}$ has the higher resistance by seeing in which direction the galvanometer contact has to be moved when the coils are interchanged by rotating the bridge commutator. Then connect a resistance box in parallel with the coil of higher resistance, say, $X$, using thick copper leads which dip in the same mercury cups. Now adjust this resistance so that the galvanometer contact, when balance is obtained, is near the centre of the wire, and let the shunting resistance be $S_{1}$. Then reverse the resistances by rotating the commutator, and let the distance through which the galvanometer contact has to be moved to preserve the balance be $b_{1}$. Next adjust the shunt so that the point of balance is near the end of the wire, and let the new value of the shunting resistance be $S_{2}$, and the change in reading for the galvanometer contact on reversal be now $b_{2}, b_{2}$ corresponding to nearly the whole length of the wire. If, then, $r$ is the resistance of unit length of the wire, we have

$$
\begin{aligned}
& r b_{1}=\frac{X S_{1}}{X+S_{1}}-Y \\
& r b_{2}=\frac{X S_{2}}{X+S_{2}}-Y .
\end{aligned}
$$

Hence, subtracting

$$
r\left(b_{2}-b_{1}\right)=X\left\{\frac{S_{2}}{X+S_{2}}-\frac{S_{1}}{X+S_{1}}\right\}
$$

from which $r$ can be calculated. In general, however, this expression may be much simplified, for if $X$ and $Y$ are very nearly equal, and $S_{1}$ is very large, also if, as usual, the wire has a small resistance per unit length, then $S_{2}$ will also be large compared to $X$. Thus

$$
r\left(b_{2}-b_{1}\right)=X\left\{\frac{1}{X / S_{2}+1}-\frac{1}{X / s_{1}+1}\right\}=X^{2}\left\{\begin{array}{l}
1 \\
s_{1}
\end{array}-\frac{1}{S_{2}}\right\} .
$$

When making the balances, thermo-currents at the galvanometer contact $D$ are liable to give trouble. In such a case it will be found 
advisable to make the contact at $\mathrm{D}$, and after the galvanometer has come to rest, to reverse the battery current and note whether the galvanometer deflection remains unaffected.

A note must be made of the temperature of the wire, which may be obtained by placing a small thermometer alongside. A piece of cotton wool placed over the wire and thermometer will be found of service in protecting both from the heat radiated from the hand when adjusting the contact.

The procedure when comparing two coils will be evident from what has been said above. It is of great importance that the temperatures of the coils should be known accurately, particularly in the case of coils wound with platinum-silver, which has a very high temperature coefficient. If the temperature of the room is very constant, and care is taken that the testing current is not too large, and is kept on for only a short time, then the temperature of the oil baths in which the coils stand may be taken as giving the temperatures of the coils themselves. If, however, the temperature is changing, or the testing current has to be kept on for some time, then the baths must be kept well stirred. This can best be accomplished by using a small propeller in each bath, driven by a small electric motor by means of a light cord (see Fig. 72).

182. To Determine the Ratio of Two nearly equal Resistances. -It is frequently necessary to determine the ratio of two nearly equal resistances. The two coils $P$ and $Q$ to be compared are placed in the $\mathbf{P Q}$ gaps of the bridge (Fig. 185), while in the two outer gaps $\mathbf{M}$ and $\mathrm{N}$ are placed two known resistances $\mathbf{A}$ and $\mathbf{B}$, which are approximately equal. If the resistances of the two parts of the bridge wire on either side of the slider, when there is no current through the galvanometer, are $p$ and $q$, while the resistance of the whole wire is $\rho$, we have

$$
\frac{P}{Q}=\frac{\lambda_{p}+A+p}{\lambda_{q}+B+q}=\frac{\lambda_{p}+A+p}{\lambda_{q}+B+\rho-p} .
$$

If, now, $P$ and $Q$ are interchanged, and the new point of balance divides the wire into portions having resistances of $p^{\prime}$ and $q^{\prime}$, we have

$$
\frac{P}{Q}=\frac{\lambda_{q}+B+q^{\prime}}{\lambda_{p}+A+p^{\prime}}=\frac{\lambda_{q}+B+\rho-p^{\prime}}{\lambda_{p}+A+p^{\prime}} .
$$

Adding the denominators for a new denominator and the numerators for a new numerator on either side of these two equations, we get

$$
\begin{aligned}
\frac{P}{Q} & =\frac{\lambda_{p}+\lambda_{q}+A+B+\rho+\left(p-p^{\prime}\right)}{\lambda_{p}+\lambda_{q}+A+B+\rho-\left(p-p^{\prime}\right)} \\
& =\frac{R+\delta}{R-\delta}
\end{aligned}
$$

where $R$ is written for $\left(\lambda_{p}+\lambda_{q}+A+B+\rho\right)$, and $\delta$ for the resistance of tile portion of the bridge wire between the two points of balance. When 
$\delta$ is small compared to $R$, that is, when $P$ and $Q$ are nearly equal and $A$ and $B$ are fairly large, we have as a very near approximation

$$
\frac{P}{Q}=1+\frac{2 \delta}{R}
$$

Since the maximum value we can have for $\delta$ is $\rho$, that is, the two points of balance are at the extreme ends of the bridge wire, the maximum ratio of $P$ to $Q$ which it is possible to measure by this method is

$$
\bar{Q}=\frac{R+\rho}{R-\rho}
$$

or since $\lambda_{p}$ and $\lambda_{q}$ are always small compared to $\Delta$ and $\mathbf{B}$, the maximum ratio is given by

$$
\frac{P}{Q}=\frac{A+B+2 \rho}{A+B}=1+\frac{2 \rho}{A+B} .
$$

Suppose, then, that the total resistance of the bridge wire is $1 \mathrm{ohm}$,

\begin{tabular}{|c|c|c|}
\hline Value of $A$ or $B$ & $\begin{array}{l}\text { Maximum Ratio } \\
\text { of } P \text { to } Q \text {. }\end{array}$ & $\begin{array}{l}\text { Alteration in the Ratio } \frac{P}{Q} \\
\text { Corresponding to an Error } \\
\text { of } 1 \mathrm{~mm} \text {. in the Wire } \\
\text { in Measuring } \delta \text {. }\end{array}$ \\
\hline $\begin{array}{l}1 \\
5 \\
10 \\
100\end{array}$ & $\begin{array}{l}2 \\
1 \cdot 2 \\
1 \cdot 1 \\
1 \cdot 01\end{array}$ & $\begin{array}{l}.0007 \\
.0002 \\
.0001 \\
.00001\end{array}$ \\
\hline
\end{tabular}
and that we calculate the maximum ratio of $P$ to $Q$ for $A$ and $B$ being each equal to $1,5,10,100 \mathrm{ohms}$, we obtain the following numbers :-

Thus by increasing the value of the resistances placed in the end gaps of the bridge, we rapidly limit the range of departure from equality in $P$ and $Q$ which we are able to measure. Although the range gets more and more limited as the resistances placed in the end gaps get greater and greater, the accuracy with which we are able to read the value of the ratio on the wire increases. Thus in the last column of the above table is given the alteration in the ratio of $\mathbf{P}$ to $\mathbf{Q}$ corresponding to an error of one scale division (that is, $1 \mathrm{~mm}$. in a wire $1000 \mathrm{~mm}$. long) in the determination of $\delta$. When there is no resistance in the end gaps, a millimetre corresponds to an error in the ratio of $P$ to $Q$, if $P$ and $Q$ are nearly equal, of about 004 , that is, about 4 parts in 1000. When A and B are each 100 ohms, a millimetre, however, corresponds to 00001 , or 4 in 400000 .

Of course we are unable to make use of the increased sensitiveness 
obtained with high resistances in the end gaps, unless the galvanometer is sufficiently sensitive to indicate a readable departure from the correct position of balance. Increased sensitiveness may be obtained by increasing the battery power, but care must be taken not to use currents of such a magnitude as to appreciably heat the resistances. For this reason the current should not be kept on longer than necessary. Further, if $\mathbf{A}$ and $\mathbf{B}$ are either much larger or smaller than $\mathbf{P}$ and Q, then the positions of the battery and galvanometer should be so chosen that the current divides approximately equally between the two arms of the bridge (see p. 434).

183. The Post Office form of Wheatstone Bridge.-A convenient form of bridge for rapidly making measurements of resistance where no very great degree of accuracy is aimed at is shown in Fig. 190, and is known as the Post Office form of resistance box. It consists of a
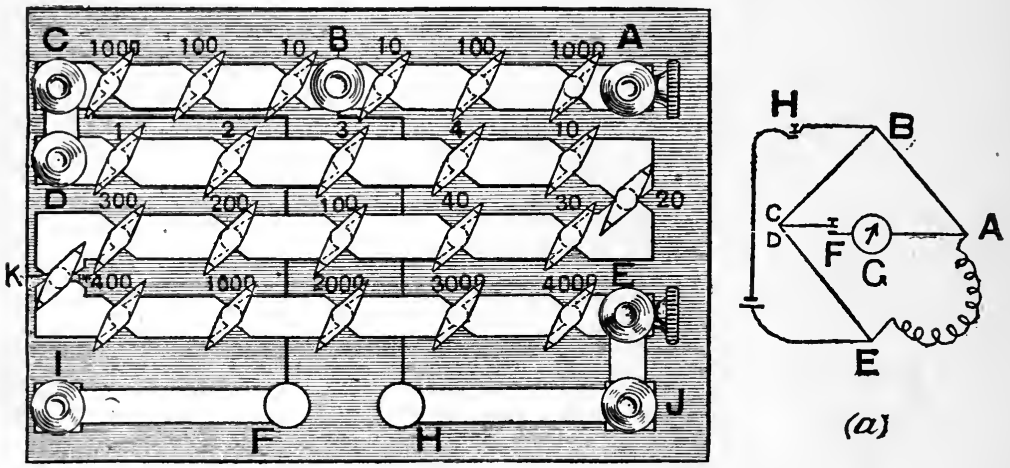

FIG. 190.

number of resistance coils wound on bobbins placed under an ebonite top, and connected to brass blocks screwed to the upper surface of the ebonite. By means of brass plugs which fit into conical holes between the blocks, the resistance coil which connects the two blocks can be short-circuited. The resistances of the different coils are indicated by the numbers placed alongside the holes for the plugs. The resistance to be measured is connected to the two binding screws $\mathbf{A}$ and $\mathbf{E}$, which are generally marked "line" and "earth" respectively, as this form of box was originally designed for testing the resistance of telegraph lines. The battery is connected to the two binding screws 2 and $\mathrm{J}$, while the galvanometer is connected to $\mathrm{A}$ and $\mathrm{I}$. By means of a wire beneath the ebonite top, when the key $\mathrm{H}$ is depressed connection is made with the point $\mathbf{B}$, while on depressing the key $\mathrm{F}$ connection is made with the point c. The resistances between $A$ and $B$ and $B$ and $\mathbf{C}$ consist of coils of 10,100, and 1000 ohms respectively, while by removing the necessary plugs the 
resistance between $\mathrm{c}$ and $\mathrm{E}$ can be made of any integral value between 0 and 10,000 ohms.

The way in which this arrangement of coils forms a Wheatstone's bridge is indicated diagrammatically in Fig $190(a)$, where the different branches are lettered similarly to what they are in the figure of the bridge. If the resistances in the branches $\mathbf{A B}$ and $\mathbf{B C}$ are equal, then to obtain balance the resistance of the branch DE must be equal to the resistance being measured. If, however, the resistance of the branch $\mathbf{A B}$ is made ten times the resistance of the branch BC, the resistance in DE required to produce balance is only a tenth of the resistance which is being measured. Finally, if the resistance of the branch $\mathrm{BC}$ is ten times the resistance of the branch $\mathrm{AB}$, the resistance in $\mathrm{CE}$ required to produce balance is ten times the resistance which is being measured. Similarly; by making the ratio arms $\mathrm{AB}$ and $\mathrm{BC} 1$ to 100 or 100 to 1 , the resistance in the branch $\mathrm{ce}$ will be 100 times or one hundredth respectively of the resistance being measured.

The following is the procedure to be adopted when measuring a resistance with the Post Office bridge :-The resistance having been connected to the terminals $\mathrm{A}$ and $\mathrm{E}$, the plugs corresponding to $10 \mathrm{ohms}$ are taken out of each of the proportional arms $\mathbf{A B}$ and $\mathbf{B C}$. The battery key $\mathbf{H}$ having been depressed, the galvanometer key $\boldsymbol{H}$ is momentarily depressed, and the direction of the resulting galvanometer throw noted. If no deflection is produced, it probably indicates that there is a break in the battery circuit. The infinity plug, marked "inf.," is then removed, and the direction of the galvanometer deflection again noted, which should be opposite to that which occurred previously. These tests will have indicated the direction of the deflection corresponding to the resistance in the branch CE being too small. Resistance is then gradually unplugged in the branch $\mathrm{CE}$ till two values are discovered, which differ from one another by $1 \mathrm{ohm}$, and for which the galvanometer is deflected in opposite directions, indicating that the resistance being measured lies between these two values. Suppose that in this way it is found that the resistance lies between 14 and 15 ohms. If, now, $100 \mathrm{ohms}$ is unplugged in the arm BC, we shall have to increase the resistance in the arm $\mathrm{CE}$ to at least $140 \mathrm{ohms}$, since it will be ten times the resistance being measured. Proceeding as before, it is found that $146 \mathrm{ohms}$ is too small and $147 \mathrm{ohms}$ is too large, showing that the resistance lies between $14 \cdot 6$ and $14 \cdot 7$ ohms. Next making the resistance in the arm $\mathrm{BC} 1000 \mathrm{ohms}$, it was found that the resistance which had to be unplugged in $\mathrm{CE}$ was between 1462 and $1463 \mathrm{ohms}$, indicating that the resistance being measured lies between 14.62 and 14.63 ohms. To obtain a nearer value to the resistance, recourse must be had to galvanometer deflections. Thus when the resistance in CE was $1462 \mathrm{ohms}$ the galvanometer was deflected through thirteen divisions to the right, while when the resistance was 1463 the deflection was twenty-five divisions to the left. Thus a change in the resistance in $\mathrm{CE}$ of $1 \mathrm{ohm}$ produces a deflection of thirty-eight divisions, while to produce equilibrium we should have to increase the resistance in 
CE so as to produce a deflection of thirteen divisions; that is, we should have to increase the resistance by $13 / 38$ of an ohm, or $0.34 \mathrm{ohm}$. Hence the resistance in the arm CE required to give balance is 1462.34 ohms, and the resistance being measured is therefore $14.6234 \mathrm{ohms}$.

If the resistance to be measured is greater than 11,111 ohms it will be impossible to obtain a balance with equal ratio arms, and the resistance of $\mathbf{A B}$ must be made 10 or 100 times the resistance of $\mathbf{B C}$.

It will sometimes be found more sensitive to use a 1 to 1 or a 10 to 1 ratio for the ratio arms rather than a 10 to 1 or a 100 to 1 ratio as the case may be, and to deduce the fractions of an ohm by galvanometer deflections. In such a case we have a choice as to the way in which we make up the desired ratio. Thus in the case of equal ratio arms we may make the resistance in either of the branches $\mathbf{A B}$ or $\mathbf{B C} 10,100$, or 1000 ohms. The best value to take in any particular case is that which will make the resistance of each of the ratio arms most nearly equal to the resistance being measured.

Since the resistance of the wire of which the coils are constructed varies with the temperature, it is only at some fixed temperature that the coils of the bridge have the value assigned to them. The temperature at which the coils have their nominal values is generally marked on the box by the makers. When the temperature of the box is different from this value a correction will be required, the magnitude of which depends on the material of which the coils are made. The following table gives the temperature coefficients of the alloys which are commonly employed for the construction of resistance coils. It must, however, be remembered that the temperature coefficient of different samples of what is nominally the same alloy differ considerably, so that this table must be used with circumspection, and in every case where accurate measurements are being made either care must be taken to observe with the coils at the temperature at which they were compared with a standard, or in each case the actual temperature coefficient of the wire used in the coils must be measured.

\begin{tabular}{|c|c|c|c|}
\hline Alloy. & $\begin{array}{l}\text { Approximate Composition } \\
\text { (parts by weight). }\end{array}$ & $\begin{array}{c}\text { Specific } \\
\text { Resistance. }\end{array}$ & $\begin{array}{l}\text { Temperature } \\
\text { Coefficient. }\end{array}$ \\
\hline $\begin{array}{l}\text { Platinum silver . } \\
\text { Platinum iridium } \\
\text { German silver }\end{array}$ & $\begin{array}{l}2 \mathrm{Ag} ; 1 \mathrm{Pt} \\
\mathrm{Pt} \text { with trace of } \mathrm{Ir}\end{array}$ & $\begin{array}{l}31 \times 10^{6} \\
27 \times 10^{-6}\end{array}$ & $\begin{array}{l}.00026 \\
.0017\end{array}$ \\
\hline Platinoid. . \{ & $\begin{array}{l}\text { German silver with trace } \\
\text { of tungsten }\end{array}$ & $34 \times 10^{-6}$ & $\cdot 0002$ \\
\hline $\begin{array}{l}\text { Manganin . : } \\
\text { Constantan . }\end{array}$ & $\begin{array}{l}84 \mathrm{Cu} ; 12 \mathrm{Mn} ; 4 \mathrm{Ni} \\
60 \mathrm{Cu} ; 40 \mathrm{Ni}\end{array}$ & $\begin{array}{l}42 \times 10^{-6} \\
49 \times 10^{-6}\end{array}$ & $\begin{array}{c}\cdot 00004 \\
-\cdot 000017\end{array}$ \\
\hline
\end{tabular}

184. The Dial-pattern Wheatstone's Bridge.-Although the Post Office pattern of bridge is very compact and fairly inexpensive to make, 
considering the great range of resistances which it is capable of measuring, for accurate work it suffers from one very serious defect; this is, that in the arm $\mathrm{DE}$ (Fig. 190, p. 448) there are always a large number of plug contacts, and the resistances of these contacts are neither negligible nor are they constant. This inconstancy is not only due to the fact that the cleanliness of the plugs and of the holes into which they fit varies, but also to the fact that the insertion of any one plug affects the fit of the plugs in the neighbouring holes. This is due to the displacement of the brass blocks, either owing to a slight give in the screws by which they are attached, or owing to bending of the ebonite top of the box. It is thus practically impossible to allow for the resistance of the plugs, and all we can do is to reduce the resistance of these contacts to a minimum by using well-fitting plugs ${ }^{1}$ and keeping them as clean as possible.

To reduce the plug difficulty to a minimum, the form of box shown in Fig. 191 is used. This pattern of box is known as the dial pattern, and

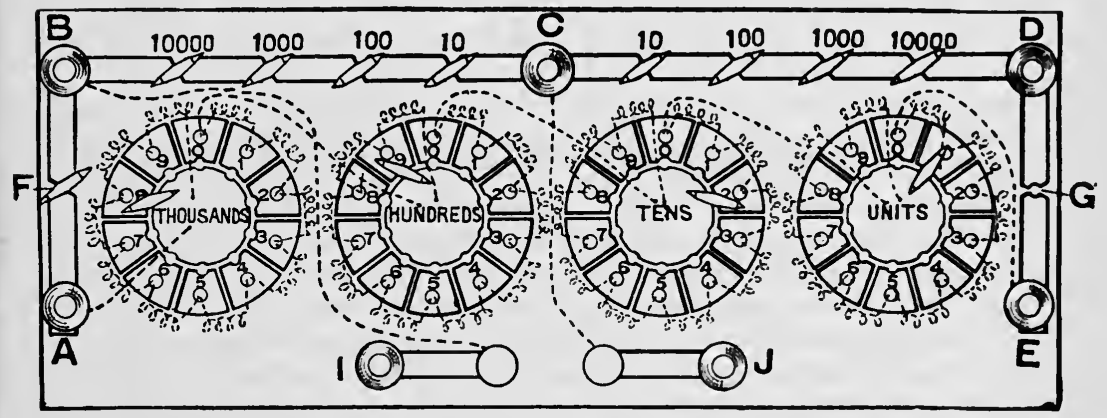

FIG. 191.

the example shown in the figure contains ten coils of $1 \mathrm{ohm}$ eack, ten of $10 \mathrm{ohms}$ each, ten of $100 \mathrm{ohms}$ each, and ten of $1000 \mathrm{ohms}$ each, the manner in which the coils are connected up being indicated by the dotted lines. There is a single plug in each of the dials, and it will be seen that if all the plugs are opposite the blocks marked $o$, then none of the coils are included between the terminals $\mathrm{A}$ and $\mathrm{E}$, the only resistance between these two points consisting of the connecting pieces between the dials and the resistance corresponding to four plug contacts. If, now, the plug in the units dial is moved to the hole marked 1 , the resistan e included between the terminals $\mathrm{E}$ and $\mathrm{A}$ will consist of $1 \mathrm{ohm}$, together with the resistance of the connecting wires and the four plug contacts. If the units plug is moved to the hole marked 2 , there will be 2 ohms included

1 As the plugs belonging to various boxes, although these boxes are of the same pattern, are generally not interchangeable, all plugs belonging to any one box ought to bear a distinguishing mark, such as a letter stamped on the head. This mark should be stamped on the top of the box, and care be taken that the plugs are always replaced in their own box. 
In addition to the connecting and plug resistances. Proceeding in this way, it will be seen that it is possible to obtain a resistance consisting of any whole number of ohms up to $11,110 \mathrm{ohms}$, and whatever the resistance there will be only four plug contacts. Further, the insertion of a plug has no influence on the fit of any of the other plugs, and so it is possible to keep the resistance of the plug contact very much more constant than in the case where the plugs are arranged as shown in Fig. 190. In addition to the dials, a set of ratio coils are provided in the arms BC, CD.

The resistance to be measured is connected between $\mathbf{A}$ and $\mathbf{B}$, the plug at $\mathbf{F}$ being removed and that at $\mathrm{G}$ inserted, or between $\mathbf{E}$ and $\mathbf{D}$; the plug at $\mathrm{a}$ being removed and that at $\mathrm{F}$ inserted.

It will be observed that the tenth coil in each of the dials, that is, the coil between the block marked 9 and the central block, is not absolutely necessary in order to obtain any combination up to 10,000 ohms. These additional coils are, however, of much use when testing the box, since with them the sum of the resistances in any dial is equal to the resistance of a single coil in the dial next above. Hence when testing the box the unit coils are first compared together, then the sum of these coils is compared with one of the tens. The tens are then compared amongst themselves, and their sum is compared to the first hundred coil, and so on.

When using the bridge, if the ratio arms are taken equal, any error due to their ratio not being exactly unity can be eliminated by placing the resistance to be measured first between $A$ and $B$ and then between $D$ and $\mathbf{E}$ and taking the mean of the two values obtained.

185. Calibration of a Dial-pattern Wheatstone's Bridge.-The dial bridge is connected by thick copper straps to a mercury cup $a$ (Fig. 192) and one terminal of a resistance box $r$. The other terminal of this

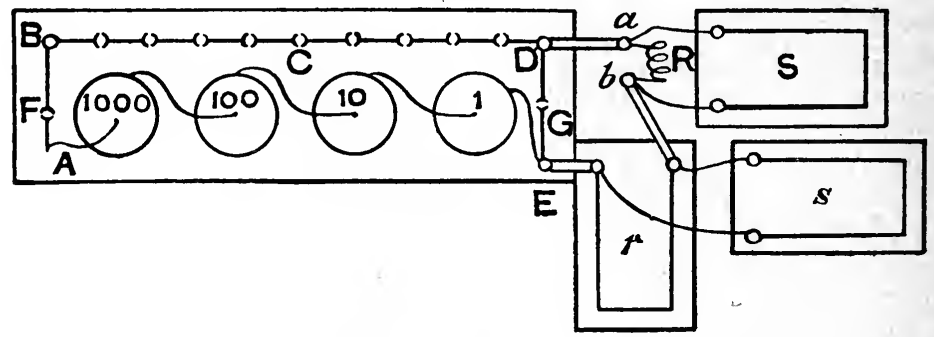

FIG. 192.

box is connected to a second mercury cup $b$. Two other resistance boxes, $s$ and $s$, are connected as shunts on the mercury cups and the resistance box $r$ respectively. Finally, a resistance $\mathrm{R}$ of $1 \mathrm{ohm}$ is placed connecting the mercury cups, and a thick U-shaped piece of copper is provided by means of which the mercury cups can be connected, and thus the resistance $\mathbf{R}$ short-circuited. 
The resistance $\mathrm{R}$ must be very slightly larger than $1 \mathrm{ohm}$, and may consist of a thick piece of manganine wire soldered to two stout copper strips, the mercury cups $a$ and $b$ consisting of holes made in these strips. To adjust the value of $R$, the mercury cup $b$ should be connected direct to $\mathrm{E}$ by means of a copper strap, and using $10 \mathrm{ohms}$ in each of the arms $\mathbf{B C}$ and $\mathrm{CD}$, and $1 \mathrm{ohm}$ in the dial arm, the resistance of $\mathbf{R}$ is adjusted till the bridge is very nearly balanced. It will be found most convenient to adjust the resistance of $\mathrm{R}$ by means of the soldered junctions till its resistance is a little too small. Then by filing the wire the resistance can be made a little greater than $1 \mathrm{ohm}$.

Next, placing the plug at 1 in the units dial, and still using $10 \mathrm{ohms}$ in each of the arms BC, CD, adjust the shunt $s$ till the bridge is balanced. Then if $R$ and $S_{a}$ are the resistances of $\mathrm{R}$ and the shunt $\mathrm{s}$, and $a$ is the resistance of the first unit coil, including the resistance of the wires joining the different dials, we have

$$
a=\frac{R S_{a}}{R+S_{a}},
$$

but since $R$ is very nearly unity and $S_{a}$ is large, we may, without appreciable error, replace $R$ in the denominator by 1 . Then

$$
a=\frac{S_{a}}{1+S_{a}} R \text {. • • • • • • . }
$$

Next place the U-shaped connector between the cups $a$ and $b$ and connect up the resistance boxes $r$ and $s$ in parallel between $b$ and $\mathrm{E}$, as shown in the figure. Keeping the plug in the units dial at 1 , adjust $r$ and $s$ till the bridge is balanced. To obtain this balance make $r 1 \mathrm{ohm}$ and $s$ infinity. If the resistance between $D$ and $E$ is now too small, replace the copper strap joining $\mathbf{E}$ to $r$ by a short piece of thick copper wire, and thus make the resistance in this arm too great. Then a balance can be obtained by adjusting the resistance of the shunt $s$, and since $r$ is only a little too great, the resistance unplugged in $s$ will be large, and thus the adjustment can be made with accuracy.

Next remove the connection between the mercury cups and move the dial plug to 2 , and then adjust the bridge by altering the shunt $\mathrm{s}$. If $h$ is the resistance of the second dial coil, and $S_{b}$ the value of $s$ when balance is secured, we now have

$$
b=\frac{R S_{b}}{R+S_{b}},
$$

in which, as before, we may put $R=1$ in the denominator, and thus

$$
b=\underset{1+S_{b}}{S_{8}} R
$$

Proceeding in this way we obtain the resistance of each of the coils of the unit dial in terms of the resistance $R$. Or if we like we may express them all in terms of the resistance $a$ of the first coil. 
To compare the coils of the tens dial, we put the plug at 10 in the units, i.e. remove the plug altogether (see Fig. 191), and adjust the resistances $r$ and $s$ (the connector being inserted between $a$ and $b$ ) till the bridge is balanced. Then place the tens plug at 1, i.e. at $10 \mathrm{ohms}$, and the unit plug at 0 , and adjust $s$ till the bridge is again balanced. Then let $s_{1}$ be the value of $s$ when the $10 \mathrm{ohm}$ coil is in, $s_{2}$ the value when the ten $1 \mathrm{ohm}$ coils are in, $r$ the value of $r, a_{10}$ the resistance of the $10 \mathrm{ohm}$ coil, and $\Sigma$ the sum of the resistances of the units coils. Thus

$$
\begin{aligned}
a_{10}-\Sigma & =\frac{s_{1} r}{s_{1}+r}-\frac{s_{2} r}{s_{2}+r} \\
& =\left(\frac{s_{1}}{s_{1}+r}-\frac{s_{2}}{s_{2}+r}\right) r \text {. . . . . }
\end{aligned}
$$

Next remove the units plug and balance by altering $r$ and $s$, and let the value of these quantities be $r^{\prime}$ and $s_{1}^{\prime}$. Then move the tens plug to 2 and the unit plug to 0 , and balance by altering $s$, and let the new value be $s_{2}^{\prime}$. If, then, $b_{10}$ is the resistance of the second $10 \mathrm{ohm}$ coil, we have

$$
b_{10}-\Sigma=\left\{\frac{s_{1}^{\prime}}{s_{1}^{\prime}+r^{\prime}}-\frac{s_{2}^{\prime}}{s_{2}^{\prime}+r^{\prime}}\right\} r^{\prime} . . . . .
$$

Proceeding in this way, we obtain the difference between the resistance of each of the $10 \mathrm{ohm}$ coils and the sum of the $1 \mathrm{ohm}$ coils, and since we have found the resistances of these in terms of the resistance $a$ of the first coil, we can express the resistance of the $10 \mathrm{ohm}$ coils in terms of the resistance of the coil $a$.

Proceeding in the same way, each of the hundred coils is compared with the resistance of the sum of the $10 \mathrm{ohm}$ coils, and each of the thousand coils with the sum of the $100 \mathrm{ohm}$ coils.

Having in this way expressed each of the coils in terms of the resistance of $a$, we now require to determine the value of this quantity. To do this the mercury cup $b$ is connected direct to $\mathrm{E}$, and a standard resistance coil is placed between the mercury cups. If possible, this coil ought to be a $1000 \mathrm{ohm}$ coil. The bridge is then balanced, and the resistance of the coils used on the dials expressed in terms of $a$ is equated to the resistance of the standard, and thus the value of $a$ is deduced. If only a $1 \mathrm{ohm}$ coil is available, then the plug in the units dial being at 1 , balance must be obtained by either altering the shunt $\mathrm{s}$, or if the resistance in the arm is already too low, a resistance box must be connected as a shunt on the dials, and balance be obtained by adjusting this shunt. In this way the resistance of $a$ is directly obtained in terms of the value of the unit and the shunt resistance.

The same method as that described above can be employed to calibrate an ordinary Post Office pattern resistance box, or, by using two auxiliary coils as ratio arms, a simple box of resistance coils. 
It is of importance when making such a calibration as that described above that the temperature should be fairly constant, particularly if the material of which the coils are wound has a large temperature coefficient.

186. The Callendar and Griffiths Pattern Bridge.-A form of Wheatstone's bridge which is particularly well suited for measuring the change of resistance of platinum resistance thermometers has been devised by Callendar and Griffiths. ${ }^{1}$ A simple form of this bridge, which is suitable for measurements in which the greatest accuracy is not aimed at, is shown somewhat diagrammatically in the upper part of Fig 193, the scheme of connections being shown in the lower part of the figure. The
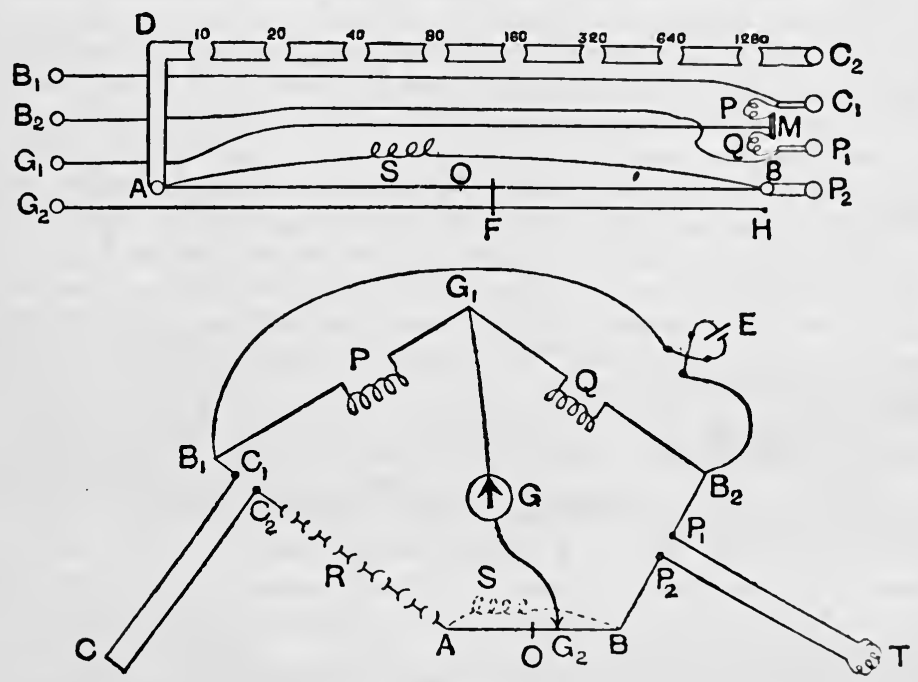

FIG. 193.

bridge wire $A \mathbf{B}$ is stretched alongside a second wire $G_{2} \mathbf{H}$ of the same material, which is connected to the galvanometer, and a movable slider carries a cross-piece $\mathbf{F}$ of the same wire, which serves to make contact between the bridge wire and the galvanometer. The object of making the two wires and the cross-piece all of the same wire is to reduce the thermo-electromotive forces at the contacts, for if the contacts are between exactly the same materials, then differences of temperature will not produce thermo-electromotive forces. The effect of any thermo-electromotive forces that may be present is eliminated in the usual way, viz. by using as the criterion of balance the fact that reversing the current should produce no change of deflection on the galvanometer. One end

1 For a discussion of the best arrangement of resistances in a bridge for use with a platinum thermometer see Callendar, I'hil. IFag., April 1910, p. 539. Other brilge arrangements suitable for use with a platinum thermometer are described by F. E. Smith in Phil: Mag., Oct. 1912, p. $5+1$. 
$\Delta$ of the wire is connected to a series of resistance coils $\mathrm{DC}_{2}$. The first of these has a resistance equal to that of $20 \mathrm{~cm}$. of the bridge wire, the next to 40, and so on, the resistance of each coil being double that of the preceding one. For a reason that will appear later, however, the coils are marked in numbers of half this value, i.e. their resistances are given in units equal to $2 \mathrm{~cm}$. of bridge wire.

Connected to the binding screw $c_{1}$ is one terminal of the ratio coil $P$, the other ratio coil $Q$ being connected to the binding screw $P_{1}$. The two battery terminals $B_{1}$ and $B_{2}$ are connected to the points $C_{i}$ and $P_{1}$, while the galvanometer terminal $G_{1}$ is connected to the point between the ratio coils. The ratio coils are wound together so as to always have the same temperature, and they are adjusted to equality.

The thermometer $T$ with its leads is connected to the screws $P_{1} P_{2}$, and the compensating leads are connected to $c_{1}$ and $c_{2}$. This pair of leads are joined together at the end, and are of exactly the same resistance as the thermometer leads, and are placed alongside these latter.

Let $r$ be the resistance of the thermometer leads or the compensator leads, $T$ the resistance of the thermometer coil, and $R$ the resistance unplugged in the coils, and let the galvanometer contact be at $G_{2}$ in the lower figure when balance is secured. If $\rho$ is the resistance per centimetre of the bridge wire, we have, since $P=Q$,

or

$$
\begin{gathered}
T+r+\rho \overline{B G_{2}}=\rho \overline{A G_{2}}+R+r, \\
T+\rho\left(\overline{O B}-\overline{\left.O G_{2}\right)}=\rho\left(\overline{A O}+\overline{O G_{2}}\right)+R,\right.
\end{gathered}
$$

where $o$ is the centre of the bridge wire. Hence

$$
T=R+2 \rho \cdot \overline{O G_{2}} \text {. }
$$

Now if $(R)$ is the value of the resistance coils unplugged in units of $2 \mathrm{~cm}$. of wire, as marked, then $R=(R) 2 \rho$. Hence

$$
\begin{aligned}
T & =(R) \cdot 2 \rho+\overline{O G_{2}} \cdot 2 \rho \text { ohms } \\
& =(R)+\overline{O G_{2}} \text { units. }
\end{aligned}
$$

Thus the resistance of the thermometer is equal to the marked value of the coils unplugged plus the length of the part of the bridge wire intercepted between the galvanometer contact and the centre of the wire. If the galvanometer contact is to the left of $o$, then the length of the portion of the wire intercepted between the contact and the centre must be subtracted from the resistance unplugged.

It will be observed that any change in the resistance of the thermometer letds, so long as the same change takes place in the compensating leads, will not affect the point of balance in the wire.

It is very convenient to arrange the resistance of the bridge wire so that 1 centimetre has a resistance of $\frac{1}{200}$ of an ohm. In this case each centimetre that the galvanometer contact is away from the centre of the bridge wire represents 01 of an ohm. The first coil, since it has a resistance equal to that of $20 \mathrm{~cm}$. of the wire, has therefore a resistance of 0.1 of an ohm. The remaining coils have resistances of $\cdot 2, \cdot 4, \cdot 8,1 \cdot 6$, 
$3 \cdot 2,6 \cdot 4$, and $12 \cdot 8$ ohms. Thus the bridge will measure up to $25 \cdot 6 \mathrm{ohms}$. If a platinum thermometer is used of which the resistance in ice is about $12.8 \mathrm{ohms}$, then its change in resistance when heater from $0^{\circ}$ to $100^{\circ}$ (its fundamental interval, see $\S 208$ ) will be $5 \mathrm{ohms}$, and hence $1 \mathrm{~cm}$. on the bridge wire will represent one-fifth of a degree. Such a thermometer is suitable for work at comparatively low temperatures. For high temperatures a thermometer having a fundamental interval of $1 \mathrm{ohm}$ may be used, in which case each centimetre of the bridge wire represents a tenth of a degree. From what has been said above, it is apparent that the adjustment of the resistance of the bridge wire to a particular resistance per centimetre is of considerable practical convenience. As, however, it is difficult to draw a wire such that its resistance per centimetre has any preiletermined value, Professor Callendar has adopted the device of shunting the wire. A wire is chosen of which the resistance per centimetre is slightly greater than the value required, and then a resistance $\mathrm{s}$ (Fig. 193) is placed as a shunt on the wire, the magnitude of the resistance being so chosen that the combined resistance of the wire and $s$ in parallel divided by the total length of the wire has the desired value, viz. 1/200 of an ohm. The presence of the shunt has no effect on the reading of the bridge, except that the resistance of the wire must now be assumed to be that of the wire and shunt in parallel. For if $c_{s}$ is the current passing in the shunt, and $c_{w}$ that in the wire, $l$ the total length of the wire, and $\rho$ its resistance per centimetre, then

$$
\frac{c_{w}}{c_{s}}=\frac{S}{\rho l} \text { or } \frac{c_{w}}{c_{w}+c_{s}}=\frac{S}{S+\rho l} \cdot \text {. . . . . . }
$$

The difference of potential between $\mathrm{B}_{1}$ and $\mathrm{G}_{2}$ is $\left(c_{w}+c_{s}\right)(r+R)+c_{\mathrm{v}} \overline{A G_{2}}$, where $r$ is the resistance of the compensating leads. The difference of potential between $\mathrm{G}_{2}$ and $\mathrm{B}_{2}$ is $\left(c_{v}+c_{s}\right)\left(r+T^{\prime}\right)+c_{w} \rho \overline{G_{2} B}$. Hence since the ratio coils $P$ and $Q$ are equal, these two differences of potential are equal. Thus

$$
\begin{gathered}
\left(c_{w}+c_{s}\right)(r+R)+c_{w} \rho\left(\overline{A O}+\overline{O G_{2}}\right)=\left(c_{w}+c_{s}\right)(r+T)+c_{u} \rho\left(\overline{A O}-\overline{O G_{2}}\right) \\
T=R+\frac{c_{w}}{c_{v v}+c_{s}} \rho .2 \overline{U \dot{i}_{2}} .
\end{gathered}
$$

Substituting from (2) we get $T=R+\frac{S \rho}{S+\rho l} \cdot 2 \overline{U G} \quad . \quad . \quad$.

But $\frac{S \rho l}{S+\rho l}$ is the resistance of the wire and shunt in parallel, and this quantity livided by $l$ is the equivalent resistance per centimetre of the shunted wire. Hence calling this equivalent resistance per centimetre $\rho^{\prime}$, we have $T=R+2 \rho^{\prime} . \overline{U G}$.

the same expression as before ol,tained, except that $\rho$ ' replaces $\rho$.

187. Calibration of a Callendar-Griffiths Bridge. - The culihration of the bridge described in the last section consists of (1) determination of the electrical mid-point of the bridge wire; (2) determination of the 
ratio of the resistances of the two ratio coils; (3) calibration of the bridge wire; (4) determination of the resistance of the coils and the bridge wire in terms of a unit which gives the coil of highest resistance its nominal value, and which is approximately the resistance of $2 \mathrm{~cm}$. of bridge wire; and (5) if the bridge is to be used for other purposes besides platinum thermometry, a determination of the value of the bridge unit in ohms.

(1) To determine the centre of the bridge wire the gaps $c_{1} C_{2}$ and $\mathbf{P}_{1} \mathbf{P}_{2}$ (Fig. 193) are closed by copper strips, and the position of the slider is adjusted till there is no galvanometer deflection. The reading for this point gives the mid-point 0 of the wire.

(2) Next two nearly equal resistances, $X$ and $Y$, are placed in the gaps $\mathbf{P}_{1} \mathbf{P}_{2}, C_{1} \mathbf{C}_{2}$. These resistances ought to be about 10 ohms each: Let the reading on the wire when the resistance $X$ is in the gap $\mathbf{c}$ be $x_{1}$, and that when the resistance $X$ is in the gap $P$ be $x_{2}$. If the ratio coils $P$ and $Q$ are equal, then $\left(x_{1}+x_{2}\right) / 2$ will be the same as the reading for the point 0 . The difference between $\left(x_{1}+x_{2}\right) 2$ and 0 is a measure of the difference of the resistances $P$ and $Q$, and the question arises whether such a difference will appreciably affect the measurements made with the bridge. If the bridge is to be used exclusively for platinum thermometer work, then such differences as are likely to be found between $P$ and $Q$ will probably not appreciably affect the measurements. The reason for this is that if for the moment we suppose that the thermometer leads and compensating leads have zero resistance, then all the resistance measurements will require to be multiplied by the same factor $(P / Q)$, and hence in the expression ased in platinum thermometry, namely (see $\S 208$ ),

$$
p t=\frac{R_{t}-R_{0}}{R_{1}-R_{0}} \times 100
$$

we may divide out the numerator and denominator by this correction factor.

A slight error will, however, be produced if the leads have appreciable resistance. This error will in general be negligible, for the resistance of the leads is small compared to the resistance of the thermometer, and the correction on account of the error in the ratio coils is a small correction on this small resistance, i.e. is of the second order.

If the bridge is to be used for other measurements besides thermometric measurements, then it will be advisable to adjust $P$ and $Q$ to exact equality. This may be done by moving the point м (Fig. 193) where the galvanometer lead is attached, the adjustment being tested by reversing the coils $\mathrm{x}$ and $\mathrm{y}$ and secing whether the mean of the points of balance coincides with the point of balance when the gaps are closed with copper connectors.

(3) The calibration of the bridge wire is performed by placing two pieces of copper strip $A$ and $B$ (Fig. 194) in the terminals $C_{1}$ and $C_{2}$. These strips have two mercury cups $\mathrm{D}$, formed by cementing small 
pieces of ebonite having a central hole on the top of the copper. A piece of wire $\mathbf{E}$, preferably of the same material as the bridge wire, is soldered to the copper strips, and its resistance is adjusted so as to be equal to the resistance of 2 centimetres of the bridge wire. In the gap $\mathbf{P}_{1} \mathbf{P}_{2}$ are placed two resistance boxes connected in parallel, one of which ought if possible to be variable by tenths of an ohm.

A copper connector being placed between the mercury cups $D$, the coils 10,320 , and 640 are unplugged (hence $R=9 \cdot 7 \mathrm{ohms}$ ), and $10 \mathrm{ohms}$ is taken out of one of the boxes in the gap P. The galvanometer contact is then moved to

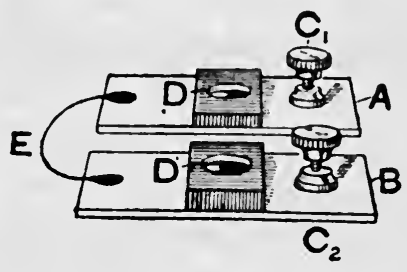

FIG. 194. near the extreme right-hand end of the wire, and balance ${ }^{1}$ is obtained by altering the resistance unplugged in the other box in $P$. The position of the slider having been noted, the copper connector between the mercury cups $\mathrm{D}$ is removed, and the slider moved till the balance is restored. The difference between the slider readings gives the length of bridge wire which corresponds to the resistance of the wire $\mathrm{E}$, i.e. has a resistance half that of $\mathrm{E}$. The connector is then placed between the mercury cups, and balance obtained by altering the resistance box in the gap $P$. The connector is then removed and balance restored by $\mathrm{m}$ jving the slider, and so on. In this way the bridge wire is divided up into segments of equal resistance. When balancing by altering the resistance in the gap $P$, it will be found best to get approximate balance by altering the resistances and to obtain the final balance by moving the slider. In this way the end of one segment into which the wire is divided will not exactly coincide with the commencement of the next, but so long as the part left out, or the overlap, as the case may be, is not very great, then no appreciable error will arise on this account. The reduction of the numbers is performed just as in the case of the calibration of a mercury thermoneter described in $\$ 59$.

(4) Calibration of the Coils. - The gap c being closed by a copper connector, and the coil 1280 unplugged, sufficient resistance is inserted in the gap $P$ to bring the balance point about $5 \mathrm{~cm}$. to the right of the centre of the wire. The coil 1280 is then cut out and all the remaining coils unplugged and balance is then again found. Twice the movement of the slider will give the difference between the 1280 coil and the sum of the remaining coils. Let the movement of the slider be $Z_{1}$. Coil 640 alone is then unplugged and the resistance in $P$ adjusted to give balance near the original point, $5 \mathrm{~cm}$. to the right of the centre. The coil 640 is then cut out and the remaining coils of lesser value unplugged, and balance again obtained. Twice the movement of the slider will now give the difference between the 640 coil and the sum of the coils

1 The final balance can if necessary be made by slightly moving the position of the galvanometer contact. 
of smaller value. Let the movement of the slider be $Z_{2}$. If (1280) represents the value of the 1280 coil in the units used,

$$
\begin{aligned}
(1280) & =(640)+(320)+(160)+\ldots+(10)+2 Z_{1} \mathrm{~cm} . \text { of wire. } \\
(640) & \left.=(320)+(160)+\dot{Z}_{1}\right)+(10)+2 Z_{2} \mathrm{~cm} . \text { of wire. } \\
\therefore(640) & =\frac{1}{2}(1280)+\left(Z_{2}-Z_{1}\right) \text { of wire. }
\end{aligned}
$$

Now although the resistance of the 1280 coil, which is 1280 units, may not be exactly that of $2560 \mathrm{cms}$. of wire, the error in assuming this for the small length $Z_{2}-Z_{1}$ will be negligible, and therefore

$$
(640)=640+\frac{1}{2}\left(Z_{2}-Z_{1}\right) \text { units. }
$$

The value of the 640 coil being now known, that of the 320 can be obtained, and so on down to the 10 coil. A final determination consists in finding the necessary movement of the slider when balance is obtained with 10 unplugged and when no coils are unplugged. Twice this movement gives the 10 coil directly in $\mathrm{cm}$. of bridge wire, and as the previous readings will have given the 10 coil in terms of the unit, the value of the unit in cms. of wire can be found. This will probably be so nearly $2 \mathrm{cms}$. that it will usually be unnecessary to correct the length $\overline{O G}_{2}$ in a resistance determination.

By arranging that $Z_{1}, Z_{2}, Z_{3}$, \&c., all correspond to nearly the same portion of the bridge wire, errors in the calibration have very little influence on the results.

(5) To determine the value of the resistance of the unit used in the above calibration, a resistance of known value must be inserted in the

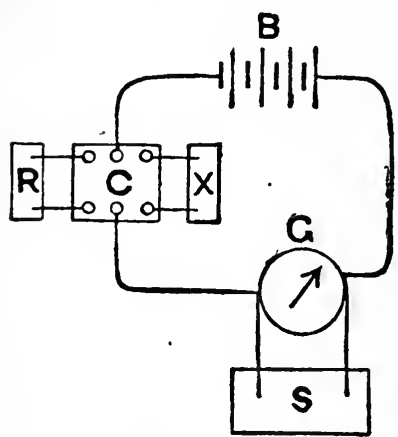

FIG. 195. gap $P$, and its value in terms of the bridge resistances measured. Then since the coils are all expressed in terms of the bridge unit, the value of this unit can at once be determined.

188. Measurement of High Resistances.- - In the case of resistances of a megohm $\left(10^{6} \mathrm{ohms}\right)$ and over the Wheatstone bridge method is generally not applicable, and a direct deflection method is usually adopted, that is, the current a battery of given E.M.F. can send through a known high resistance is measured by means of a galvanometer and compared with the current the same battery can send through the unknown resistance.

A convenient arrangement is shown in Fig. 195, where $\mathbf{B}$ is a battery, $\mathrm{G}$ a high resistance galvanometer, and $\mathrm{C}$ a commutator, consisting of six small mercury cups scooped out in a block of paraffin. The - known resistance $R$, and the resistance to be measured $X$, are connected to these mercury cups as shown, so that by means of two U-shaped 
pieces of copper one or other can be included in the circuit. A resistance $S$ is connected so as to act as a shunt on the galvanometer.

Suppose that the E.M.F. of the battery is $E$, and that the resistance $S$ being infinite, that is, the galvanometer unshunted, the deflection produced with the resistance $X$ in series is $d_{1}$. Then we have

$$
\frac{E}{X+B+G}=K d_{1} \quad \cdot \quad \cdot \quad \cdot \quad \cdot \quad \cdot \quad . \quad .
$$

where $K$ is a constant depending on the galvanometer, and expresses the factor by which the deflection has to be multiplied to give the value of the current passing through the galvanometer.

The resistance $R$ is then introduced into the circuit, and the resistance of the shunt altered till the deflection is of about the same magnitude as in the previous case. If the deflection is $d_{2}$, we have

$$
\frac{E}{R+\frac{G S}{G+S}+B} \times \frac{S}{G+S}=d_{2} K \text {. . . . . . }
$$

From the expressions (1) and (2) we can eliminate $E$ and $K$, when we get

$$
X=\frac{d_{2}}{d_{1}}\left\{\frac{(R+B)(G+S)}{S}+G\right\}-G-B . . .
$$

In general, the battery resistance $B$ is small compared to $R$ and $G$, and may be neglected, so that the expression for $X$ becomes

$$
X=\frac{d_{2}}{d_{1}}\left\{\frac{R(G+S)}{S}+G\right\}-G \text {. . . . . }
$$

The reason for reducing the galvanometer deflection in the second experiment to nearly the same value as in the first, is that in general the currents are not proportional to the galvanometer deflections, i.e. $K$ is not a constant for all deflections. If, however, the deflections are nearly the same, we are justified in assuming that the same value of $K$ is applicable in the two cases.

189. Measurement of the Insulation Resistance of a Piece of Rubber Covered Cable.-The coil of cable E (Fig. 196) is placed either in a metal tub, or, if a wooden or earthenware vessel is used, a sheet of zinc $\mathrm{D}$ is placed at the bottom. The tub is filled with water to well over the cable, except the two ends which project above the water, and which may be conveniently clamped between two picces of wood which rest on the rim of the tub. The insulation must then be pared away at the two ends of the cable with a clean and sharp knife so as to leave a conical, fresh-cut surface of the rubber, as shown at $(a)$. The copper core of the cable is connected by a wire to one of the terminals $F$ of the galvanometer, while the metal plate in the tub is connected to one 
pole of a battery of cells, the other terminal of the battery being connected to the other terminal of the galvanometer. The wire joining c and $\mathrm{F}$ should be an air-wire, that is, it should not touch any body between $\mathrm{c}$ and $\mathrm{F}$. A piece of fine uncovered copper wire is then wound round the middle of the conical, fresh-cut portions of the insulation and connected to the terminal $\mathbf{r}$ of the galvanometer. The object of this wire is to allow of the electricity which leaks over the surface of the insulation to pass back to the battery without going through the galvanometer. In the absence of this guard-wire, the use of which is due to Price, ${ }^{1}$ the deflection of the galvanometer would be due to the sum of the current which passes through the rubber coating of the cable, and the current which passes from the water along the surface of the rubber to the copper core at A or c. Owing to the fact that a film of moisture almost immediately forms, even on a freshly cut surface, this surface leakage has quite an
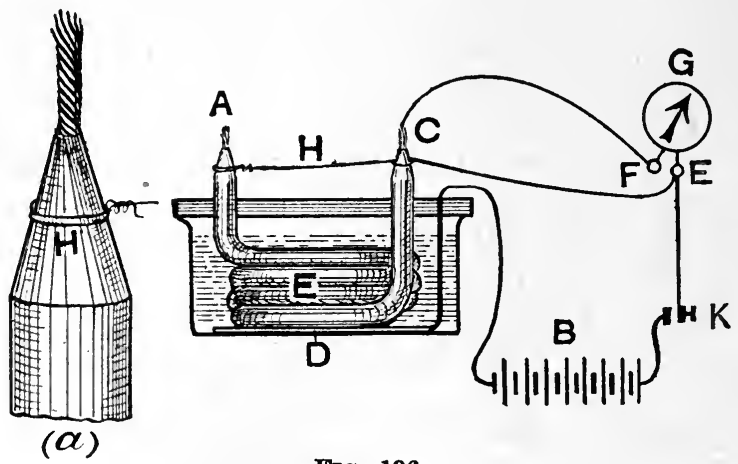

Fig. 196.

appreciable magnitude compared to the current which passes through the insulating sheath when this sheath is composed of a good insulator. Since the wire $\mathrm{H}$ and the core of the cable are very nearly at the same potential, there is no appreciable leakage over the portion of the cleaned rubber between the wire $H$ and the core. There will be some leakage between the water and the wire $\mathrm{H}$ along the surface of the sheath, but this leakage current, since it does not pass through the galvanometer, will have no influence on the galvanometer deflection.

The procedure is the same as in the last section, the value of the galvanometer constant $K$ being derived from an experiment made with a known high resistance, the galvanometer heing shunted.

190. Measurement of Low Resistances-Mathiessen and Hockin's Method of the Projection of Equal Potentials.-When the conductor of which the resistance has to be determined has a very low resistance, the Wheatstone bridge method is not applicable, owing to the fact that

1 Electrical Review (1895), vol. xxxvii. p. 702. 
the resistances at the places where the conductor is connected to the bridge would bear an appreciable ratio to the resistance to be measured. For this reason, methods of measuring the resistance have to be employed in which the contacts which are employed are potential contacts, and hence variations in the resistance of these contacts will not affect the result.

Suppose that it is required to determine the resistance of a certain length, say, $50 \mathrm{~cm}$., of a piece of thick copper wire. The wire CD (Fig. 197 ) is stretched straight, and two contact pieces, $\mathbf{E}$ and F, are slipped on the wire and placed at a distance of $50 \mathrm{~cm}$. apart. The construction of one of the contact pieces is shown at $(a)$. It consists of a wooden block, with a V groove cut in its lower face. In a slot in the middle of the block is placed a brass disc c, pierced with a circular hole, through which the rod B, which is being measured, passes. To the upper edge of the disc is soldered a screwed upright which carries three nuts. The lower of these, $\mathbf{E}$, serves to clamp the

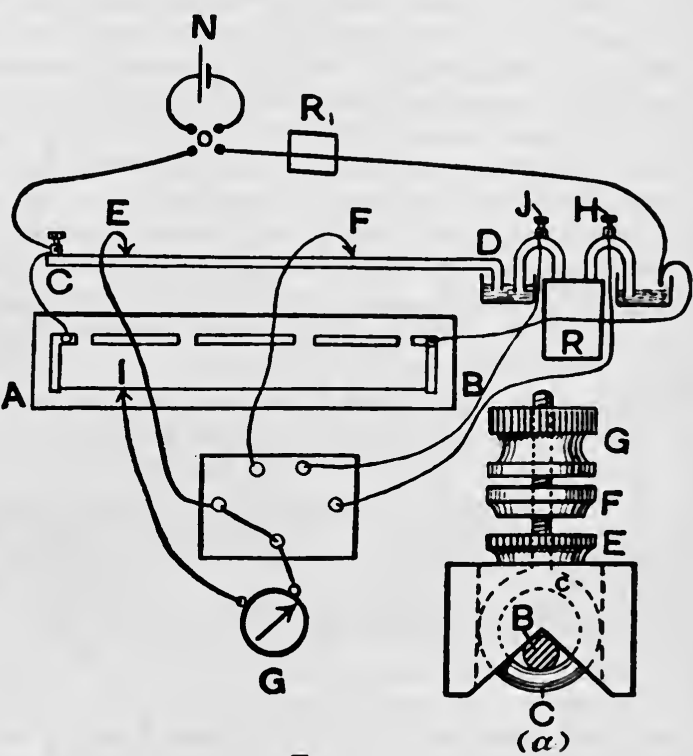

FrG. 197. contact maker down

on the rod by drawing the disc o upwards. The other two, $\mathrm{F}$ and $\mathrm{G}$, serve to clamp the connecting wire which goes to the galvanometer. In addition a calibrated wire, such as the wire of a metre bridge, must be placed alongside the copper rod CD. A standard resistance coil of $0.01 \mathrm{ohm}$ will be required. In the usual form of such a coil there are two thick copper terminals with binding screws $J$, $\mathbf{H}$, so placed that when a current is passed through the coil, entering at the end of one of the terminals and leaving by the end of the other, then the resistance between the points where these binding screws are attached is exactly $0.01 \mathrm{ohm}$.

The manner in which the copper rod, the bridge wire, and the resistance coil $\mathbf{R}$ are connected to a battery $B$ (preferably a storage cell) is 
shown in the figure. Connecting wires are run from the points E, F, J, B to four mercury cups in a block of varnished wood or paraffin, while one terminal of a low resistance galvanometer $\mathrm{G}$ is connected to a fifth cup, and by means of a $U$-shaped copper connector can be connected to either of the other cups. The second terminal of the galvanometer is connected to the slider I on the bridge wire. A resistance, $R^{\prime}$, of an ohm or two will generally be found necessary in the battery circuit to prevent the cell sending too great a current.

The battery circuit having been closed by means of the key 0 , the galvanometer is connected to the point $\mathrm{E}$, and the slider $\mathrm{I}$ moved till the galvanometer is undeflected. The galvanometer is then connected in turn with the points $F, J$, and $B$, and the positions of the slider for no deflection determined. When making these adjustments it is as well to roughly determine the positions for balance of all the points, and then going through the different points again to determine the exact positions, keeping the battery current on only just long enough to see whether there is a permanent deflection after each adjustment of the slider. The reason for not keeping the current on longer than is absolutely necessary is that in order to obtain sensitiveness it is necessary to use a fairly large current, and hence if this current is allowed to flow for any length of time, the conductors will become appreciably heated.

If the length of the wire included between the points which are at the same potential as $\mathrm{E}$ and $\mathrm{F}$ is $l_{1}$, while the length between the points at the same potential as the points $\mathrm{J}$ and $\mathrm{H}$ is $l_{2}$, while the resistance of the coil $\mathrm{R}$ is called $R$, the resistance of the rod $\mathrm{CD}$ between the points $\mathbf{E}$ and $\mathbf{F}$ is given by

$$
X=\frac{l_{1}}{l_{2}} R
$$

In this expression the lengths $l_{1}$ and $l_{2}$ are the reduced lengths of the corresponding portions of the wire ; that is, they are the lengths, deduced from the calibration curve, of the mean uniform wire, which would have the same resistance as the actual portion of the wire used.

The above expression for the resistance of the portion of the rod can at once be obtained as follows:-If $C$ is the current in the rod, and $c$ that in the wire $\mathrm{AB}$, the difference of potential between $\mathrm{E}$ and $\mathrm{F}$ is $X C$, while the difference of potential between $\mathrm{J}$ and $\mathrm{H}$ is $R C$. Also, the difference of potential between the two points of balance on the wire corresponding to $\mathrm{E}$ and $\mathrm{F}$ is $l_{1} r c$, and that corresponding to the points $\mathrm{G}$ and $\mathrm{H}$ is $l_{2} r c$, where $r$ is the resistance of unit length of the bridge wire Hence we have the two equations

$$
\begin{gathered}
X C=l_{1} r c \\
R C=l_{2} r c, \\
X=\frac{l_{1}}{\bar{R}} .
\end{gathered}
$$


If the mean diameter of the rod between the points $\mathbf{E}$ and $\mathbf{F}$ is $d$, while the distance between $\mathbf{E}$ and $\mathbf{F}$ is $L$, the specifie resistance $\rho$ of the material of which the rod is composed is given by

$$
\rho=\frac{\pi d^{2} X}{4 L} \text {. }
$$

This method may also be used for comparing the resistance of two rods, and hence comparing the specific resistances of the materials of which the rods are composed. For this purpose it is only necessary to replace the resistance coil $\mathbf{R}$ by the second rod fitted with contact pieces similar to those at $\mathbf{E}$ and $\mathbf{F}$, proceeding exactly as before. It is important to make the junction between the two rods in such a way that the resistance of the junction does not vary during the course of the experiment, otherwise the positions of the points of balance on the bridge wire will also vary, and it will be impossible to obtain satisfactory measurements. A satisfactory way of joining the rods is either to solder them together or to bend about an inch at the end of either rod at right angles, amalgamate these bent portions, and then bind them together with uncovered copper wire, and dip the bent portions into a mercury cup.

191. Measurement of Low Resistance-The Method of Auxiliary Conductors, or Kelvin's double Bridge. - The two conductors which are to be compared are stretched out straight and connected together at c (Fig. 198), either by soldering or with a mercury cup, as described in the last section. Two contacts will be required on each rod; those on one rod, as they can be kept at a fixed distance apart, may be botli attached to a wooden rod, and the distance between the points of contact determined once for all. The contact makers are connected,

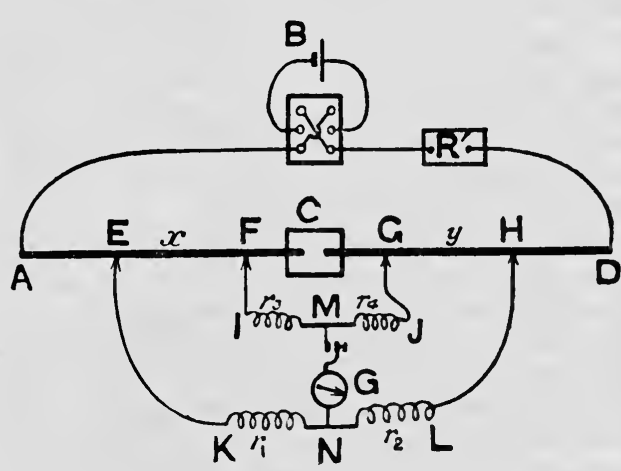

FiG. 198. as shown in the figure, to two resistances IJ and $\mathrm{KL}$. These resistances consist of resistance coils having a resistance of 2 or $3 \mathrm{ohms}$, and means is provided for connecting the middle point of each resistance to the galvanometer $G^{\prime}$. The two resistance coils are placed close alongside in one box, so that they shall always have the same resistance, and the ends and the mid-point may conveniently be soldered to three binding screws, as shown in Fig. 199. 
The contacts on one of the rods, say, $\mathrm{G}$ and $\mathrm{H}$, being kept fixed in position, the distance between the contacts on the other rod is varied till

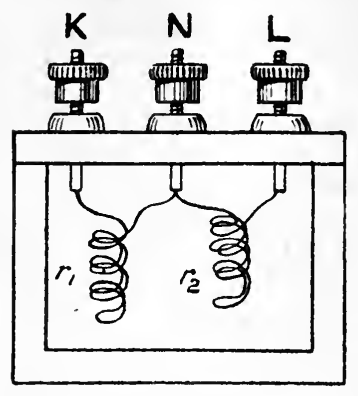

FIG. 199. there is no deflection of the galvanometer.

When making the adjustment the battery circuit must only be closed for the time that is absolutely necessary, so as to reduce the heating effect of the current to a minimum. In order to minimise the effects of thermocurrents, balance should be obtained with the current in either direction.

Let $x$ be the resistance of that portion of the rod AC intercepted between the points $\mathrm{F}$ and $\mathrm{F}$, while $y$ is the resistance of the rod $\mathrm{CD}$ between the points $\mathrm{G}$ and $\mathrm{H}$. Further, let the resistance of the segments of the split resistances $\mathrm{KL}$ and IJ be called $r_{1}, r_{2}, r_{3}$, and $r_{4}$ respectively, these quantities to include the resistances of the leads to the points E, F, G, and $B$ respectively. Then the condition for no galvanometer deflection is

$$
\frac{x}{y}=\frac{r_{1}}{r_{2}}=\frac{r_{3}}{r_{4}}
$$

The theory of the method is most easily obtained graphically. Suppose that we plot a diagram on which the ordinates represent the potentials of the different points along the two rods ACD. Such a diagram is shown in Fig. $200(a)$, where the points are lettered to correspond with the points on Fig. 198. Next on a base of convenient length oQ (Fig. 200, b) let us plot as ordinates at $o$ the potentials of the points $E$ and $F$, and as ordinates at $Q$ the potentials of the points $\mathrm{G}$ and $\mathrm{H}$. Thus $\mathrm{OE}$ represents the potential of the point $E$, and $Q H$ that of the point $H$, while the straight line EH will represent the fall of the potential along the EKNLH (Fig. 198) if we suppose that abscissæ represent the resistance of this branch measured from the point $\mathbf{E}$. In the same way the straight line FG will represent the fall of potential along the branch FIMJG. Now the only point which is common to these two straight lines is the point $\mathbf{P}$ where they intersect, so that if the galvanometer is to have no deflection it must be connected between the points on the two branches which are represented by the point $z$. Now the two triangles EPF and HPG are similar, hence

$$
\frac{\overline{E F}}{\overline{G H}}=\frac{\overline{E P}}{\overline{P H}}=\frac{\overline{F P}}{\overline{P G}}=\frac{\overline{O Z}}{\overline{Z Q}} .
$$

Now EF represents the difference of potential between the points $\mathrm{E}$ and $\mathbf{F}$, while HG represents the difference of potential between the points $a$ and $\mathrm{H}$. But the current which flows through the portion of the one rod between $\mathrm{E}$ and $\mathrm{F}$ must be the same as the current which flows through 
that portion of the other rod included between $\mathrm{G}$ and $\mathrm{H}$, for no current passes across from $\mathrm{M}$ to $\mathrm{N}$ through the galvanometer. Hence

$$
\frac{\overline{E F}}{\overline{G H}}=\frac{x}{y}=\frac{\overline{O Z}}{\overline{Z Q}}
$$

Now $\overline{O Z} / \overline{Z Q}$ represents the ratio in which the point $P$ divides either of

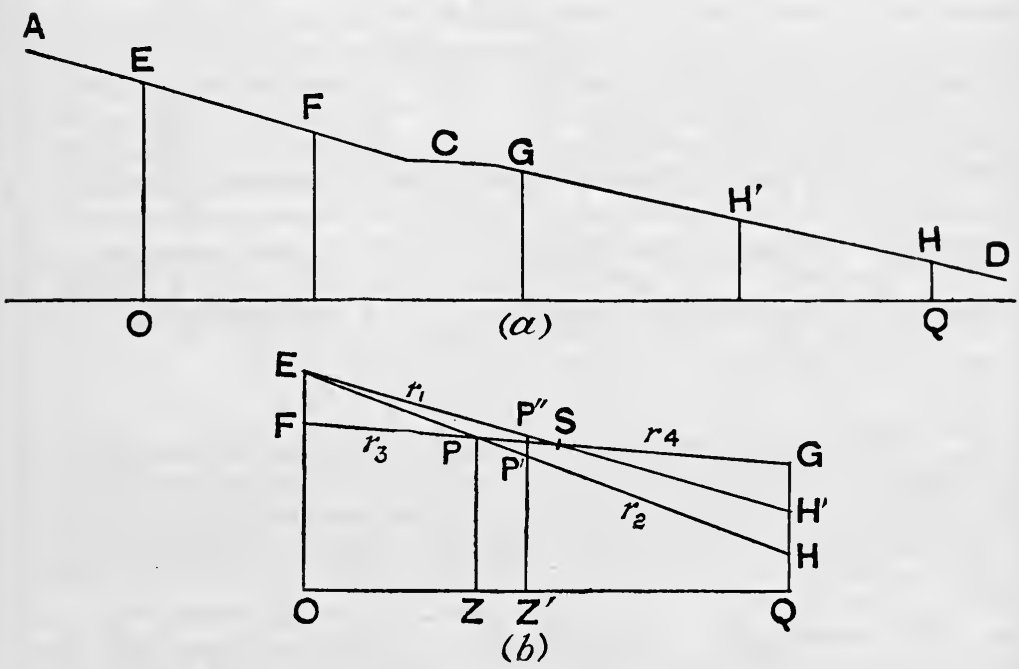

FIG. 200.

the branch resistances, and since this ratio is the same for either branch, we have

$$
\frac{\overline{O Z}}{\overline{Z Q}}=\frac{r_{1}}{r_{2}}=\frac{r_{3}}{r_{4}}
$$

that is, the condition that there should be no current through the galvanometer is

$$
\frac{x}{y}=\frac{r_{1}}{r_{2}}=\frac{r_{3}}{r_{4}}
$$

If $r_{1}=r_{2}$ and $r_{3}=r_{4}$, then $x=y$.

In general, the condition $r_{1} / r_{2}=r_{3} / r_{4}$ will not exactly be fulfilled, so that the question arises if $r_{1} / r_{2}$ is not exactly equal to $r_{3} / r_{4}$, which of these quantities, or what function of them, nust be equated to $x / y$. This can at once be answered by a study of Fig. $200(b)$. It is obvious that the slope of the line EH must always be greater than that of the line FG, 
for the difference of potential between $\mathrm{E}$ and $\mathrm{H}$ must be greater than that between $F$ and $G$. Suppose, then, that $r_{1}: r_{2}$ as $\overline{\mathrm{OZ}^{\prime}}: \overline{\mathrm{Z}^{\prime} \mathrm{Q}}$, and $r_{3}: r_{8}$ as $\overline{\mathrm{OZ}}: \overline{\mathrm{ZQ}}$, so that the points between which the galvanometer is connected are represented by $P$ and $\mathbf{P}^{\prime}$, evidently there would be quite a considerable difference of potential, and to obtain balance we should have to adjust, say, the contact $\mathrm{B}$ to a point where the potential was represented by $\mathrm{QH}^{\prime}$, so that the straight line joining $\mathrm{E}$ and $\mathrm{H}^{\prime}$ should pass through the point $\mathbf{P}^{\prime \prime}$ where $\mathbf{Z}^{\prime} \mathbf{P}^{\prime \prime}=\mathbf{Z P}$. Evidently, then, if $\mathbf{s}$ is the point where the lines $\mathbf{E H}^{\prime}$ and FG intersect, we have

$$
\frac{x}{y}=\frac{\overline{E F}}{\overline{G H}}=\frac{\overline{E S}}{\overline{S H}} .
$$

But from the figure, since the line FG is nearly horizontal, because the resistance between the points $F$ and $G$ (Fig. 198) is small, it follows that the ratio $\frac{\overline{E S}}{\overline{S H^{\prime}}}$ is nearly the same as $\frac{\overline{E^{\prime} P^{\prime}}}{\overline{P^{\prime \prime} H^{\prime}}}$ or $\frac{\overline{O Z^{\prime}}}{\overline{Z^{\prime} Q}}$, that is, as $r_{1} / r_{2}$. We thus see that even when the condition $r_{1} / r_{2}=r_{8} / r_{4}$ is not exactly fulfilled that, so long as the line FG is nearly horizontal, that is, that the resistance between the points where the contacts $F$ and $G$ touch the rods is small, we have.

$$
\frac{x}{y}=\frac{r_{1}}{r_{2}}
$$

The above investigation shows the importance of making the contacts $\mathrm{F}$ and $\mathrm{G}$ of the branch circuit as near the point of junction of the rods as possible.

By replacing one of the rods by a standard $0.01 \mathrm{ohm}$ coil the resistance of a certain length of the rod under experiment can be

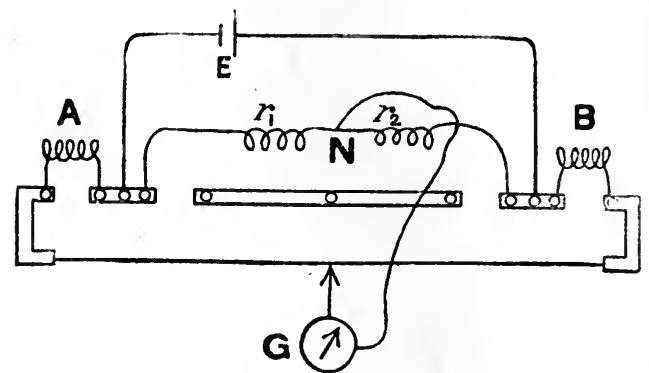

FIG. 201. determined in absolute measure, and hence the specific resistance of the material can be calculated if the crosssection of the rod is measured. The disadvantage of using a coil is that such coils are generally made of some alloy, such as manganin or constantan, which has a small temperature coefficient, while generally the material being measured is copper, which has a fairly large temperature coefficient. Thus if the temperature varies, the ratio of the 
resistance of a given length of the specimen to that of the coil varies also. If, however; a rod of copper is employed as the standard, then so long as the temperature of this standard and that of the specimen being tested vary together the ratio of their resistances remains the same.

The above investigation shows that particular care should be taken in measuring the ratio $r_{1} / r_{2}$, which must include not only the portions of the divided resistance, but also the wires used to connect the resistance to the contacts $\mathbf{E}$ and $\mathbf{F}$. This ratio can be determined by the method given in $\S 182$, the connections being made as shown in Fig. 201.

192. The Differential Galvanometer.-A differential galvanometer is one in which the coils are wound double, that is, two wires are wound on side by side, the ends of the two wires being brought to separate terminals. If the two circuits were exactly the same, then if a current is passed one way through one of the circuits and in the opposite direction through the other their effects will be exactly equal and opposite, and the needle will remain undeflected. In general, such equality is not exactly secured, and a small coil, which is in series with one of the circuits, is arranged so that it can be moved towards or away from one of the sets of magnets, and the position of this coil is adjusted till there is no deflection when the same current is passed through the two circuits in opposite directions. In many cases what is required is that there should be no deflection when the E.M.F. between the terminals of the two circuits is the same. To make the balance in such a case the two coils are connected in parallel, but in such a manner that the current in the two coils is in opposite directions, and the adjusting coil is moved till there is no deflection. It must be remembered that the adjustment, whether for equal currents or for equal potential differences, depends on the exact position of the magnet system with reference to the coils, and hence on the level of the galvanometer. For this reason each time the galvanometer is moved the adjustment must be repeated.

When the galvanometer has to be adjusted for equal potentials, Crawley ${ }^{1}$ has proposed to use an adjustable resistance placed in series with the coil which has the greater magnetic effect on the needle,

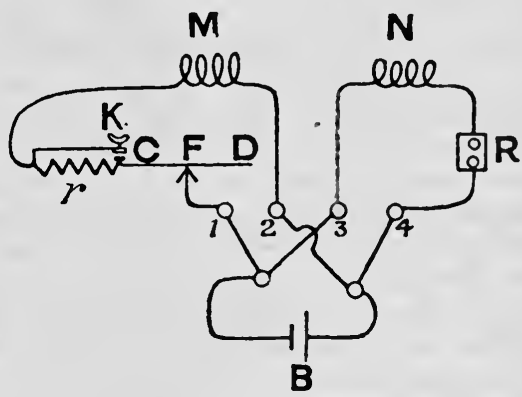

FIG. 202. this resistance being adjusted till when the coils are in parallel there is no deflection. The arrangement employed when balancing is shown in Fig. 202. $M$ and $\mathrm{N}$ are the two coils of the galvanometer which have very nearly the same resistance, a convenient value being 100 ohms each. The coil $M$ is connected to the terminal 1 on the base of the 
galvanometer through a resistance $r$ of $0.1 \mathrm{ohm}$, a stretched wire oD, and a sliding contact $\mathbf{F}$. A resistance $\mathbf{R}$ can, if necessary, be inserted in the circuit of the coil $\mathrm{N}$ so as to make it possible to balance on the slide wire. A key $\mathrm{K}$ when depressed short-circuits the resistance $r$, and hence alters the resistance of one of the circuits by a known amount, and the corresponding deflection serves to determine the value of a scale division of the galvanometer. It is advisable to have a short flexible lead permanently attached to each terminal of the coil so that these leads are included in the circuits when balancing.

193. Comparison of two Low Resistances with the Differential Galvanometer.-The differential galvanometer forms an easy, and at the same time fairly accurate, means of comparing two nearly equal resist-

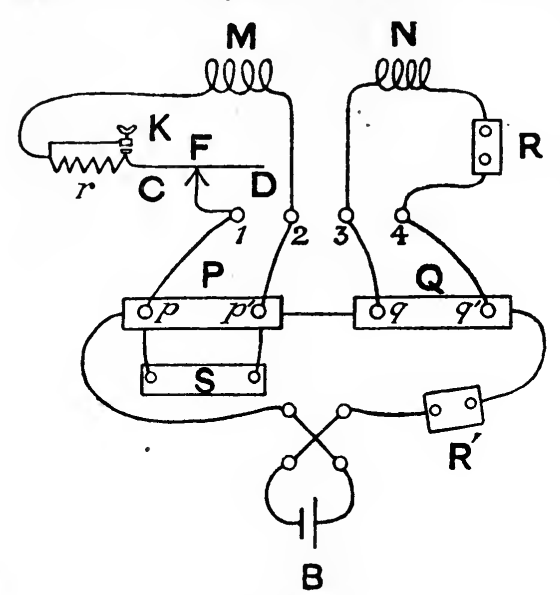

FIG. 203. ances, whether they are in the form of resistance coils with potential terminals, or rods with sliders. The manner in which the connections are made is shown in Fig. 203. The potential terminals of the two resistances $P$ and $Q$ which are to be compared are connected by means of the flexible leads with the four terminals of the differential galvanometer, and a current from a battery $B$ is passed through the resistances, which are placed in series. If the resistances are very nearly the same, or if they can be made the same, as in the case of rods in which the distance between the contacts on one of the rods can be altered, the galvanometer deflections are generally sufficient to determine the ratio of the resistances, since the resistance $r$ and the key $\mathbf{K}$ enable the resistance in one of the galvanometer circuits to be decreased by a known fraction of the resistance of the galvanometer coil.

If the difference between the resistance of $P$ and $\mathbf{Q}$ is such that a deflection of more than a few scale divisions is produced, then a resistance box $\mathrm{s}$ is placed as a shunt on whichever of the two resistances is the greater, and the value of $\mathbf{s}$ adjusted till there is no deflection. When this is the case we have

$$
Q=\frac{P S}{P+S}
$$

Although the effect of the two coils are equal on the needle, it does not follow that they have equal resistances. If this difference were very 
great, then, since the resistances $\mathrm{P}$ and $\mathrm{Q}$ are shunted by the galvanometer coils and a current is passed through these coils, the current through $\mathbf{P}$ would not be exactly the same as that through $Q$, and hence the above expression would not hold, for it has been obtained on the supposition that the same current flows through $P$ and $Q$. The correction to be applied in such a case can at once be obtained. In the absence of the shunt $\mathrm{s}$ the resistance between the points $p$ and $p^{\prime}$ is

$$
\frac{M P}{M+P},
$$

where $M$ is the resistance of the coil $M$ including the leads and adjusting resistance. Similarly, the resistance between the points $q$ and $q^{\prime}$ is $\frac{N Q}{N+Q}$. Now the differential galvanometer shows that these resistances are equal. Hence

or

$$
\begin{gathered}
\frac{M P}{M+P}=\frac{N Q}{N+Q}, \\
\frac{P}{Q}=1+\frac{P(N-M)}{N M} .
\end{gathered}
$$

Now $N-M$ is the difference between the resistance of the two galvanometer coils, and is always small compared to either $N$ or $\boldsymbol{M}$. Thus if $N$ and $M$ are each 100 ohms, and $N-M$ is even as much as $1 \mathrm{ohm}$, while $P$ and $Q$ are each about a tenth of an ohm, the correction factor $\frac{P(N-M)}{N M}$ has the value $\cdot 00001$, or the correction only amounts to 1 part in 100,000 . Thus as long as the difference $N-M$ is not very large, and care is taken to always have $M$ and $N$ large compared to $P$ and $Q$, no correction on account of this effect will be required. In any case in which the correction becomes appreciable it can immediately be calculated if the difference between the resistance of the two galvanometer circuits is measured.

194. The Shunt Potentiometer Method of Comparing two Low Resistances.-The method described in $\S 179$ for calibrating a wire may be applied to the comparison of two low resistances. ${ }^{1}$ Suppose that the two resistances to be compared, A and B (Fig. 204), are 0.1 and 0.01 $\mathrm{ohm}$, and that as usual they are provided with potential leads $a, a^{\prime}$ and $b, b^{\prime}$. The resistances $\mathrm{A}$ and $\mathrm{B}$ are connected as shown in series with a battery $B$ and an adjustable resistance $R_{1}$. A second circuit consists of two standard coils $P$ and $Q$, which in the case considered would have resistances of 10 and $1 \mathrm{ohm}$ respectively, connected in series through an adjustable resistance $R_{2}$ and a switch $C_{2}$ with a battery $B_{2}$. A resistance box $\mathrm{S}$ is arranged as a shunt on one or other of the resistances $P$ and $Q$

${ }^{1}$ Campbell, Proc. Physical Soc. of London (1903), xviii. 480. 
according as the ratio $\mathbf{A} / \mathbf{B}$ is greater or less than the ratio $\mathbf{P} / \mathbf{Q}$. A switch c is prepared, consisting of twelve mercury cups, which are connected in the manner shown to the potential leads on the resistances $A, B, P$, and $Q$, and the galvanometer $G$. The cups 1 and 2 are permanently connected together, and by means of a block of ebonite with four $U$-shaped pieces of copper, shown at $Y$, the two circuits can be connected in two separate manners. When the connector $\mathrm{x}$ is between the left-hand set of cups and the middle, the points $a$ and $p$ are connected together, and the points $a^{\prime}$ and $p^{\prime}$ are connected to the galvanometer. When, however, the

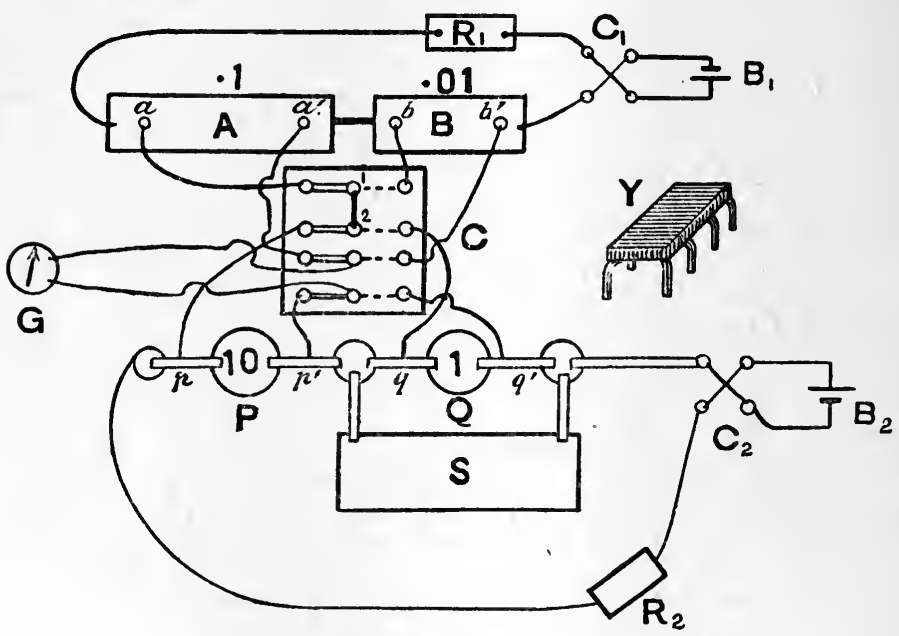

EIG. 204.

connector is moved into the right-hand position the points $b$ and $q$ are connected together, and the points $b^{\prime}$ and $q^{\prime}$ are connected to the galvanometer.

To make the measurement, the current in the circuit containing $\Delta$ and $\mathrm{B}$ is kept constant, and the resistance $R_{2}$ is adjusted till the galvanometer is undeflected when the connector is in the left-hand position. The connector is then placed in the right-hand position, and balance obtained by varying the shunt $\mathrm{s}$. Since a change in $\mathrm{s}$ will slightly alter the resistance in the circuit of the battery $\mathrm{B}_{2}$, the first adjustment will be slightly upset. $\mathrm{By}$, however, making the two adjustments alternately, a condition will soon be obtained in which there is balance in both positions. The effect of a change in $\mathrm{s}$ on the adjustment made when the connector is to the left will be decreased by making the E.M.F. of the battery $\mathbf{B}_{2}$ larger, and correspondingly increasing the resistance $\mathbf{R}_{2}$. This resistance may conveniently consist of a box of coils from a tenth of an ohm upwards and an adjustable carbon resistance, such as that shown 
in Fig. 205, placed in series. The carbon resistance consists of a number of plates of gas carbon about 7 centimetres square and 5 millimetres thick held in a frame. These plates can be compressed by turning the screw $\mathbf{A}$, and in this way the resistance between the copper plates BC is reduced. The carbon plates are insulated from the frame by vulcanised fibre tubes, which cover the rods by means of which the ends of the frame are connected. With fifty plates the resistance can be varied from about $0.3 \mathrm{ohm}$ to $3 \mathrm{ohms}$. This combination of a box of coils reading down to a tenth of an ohm and up to about $500 \mathrm{ohms}$ and a carbon resistance will be found of much use in many cases. The coils of the resistance box need not be carefully adjusted, but they ought to be wound with

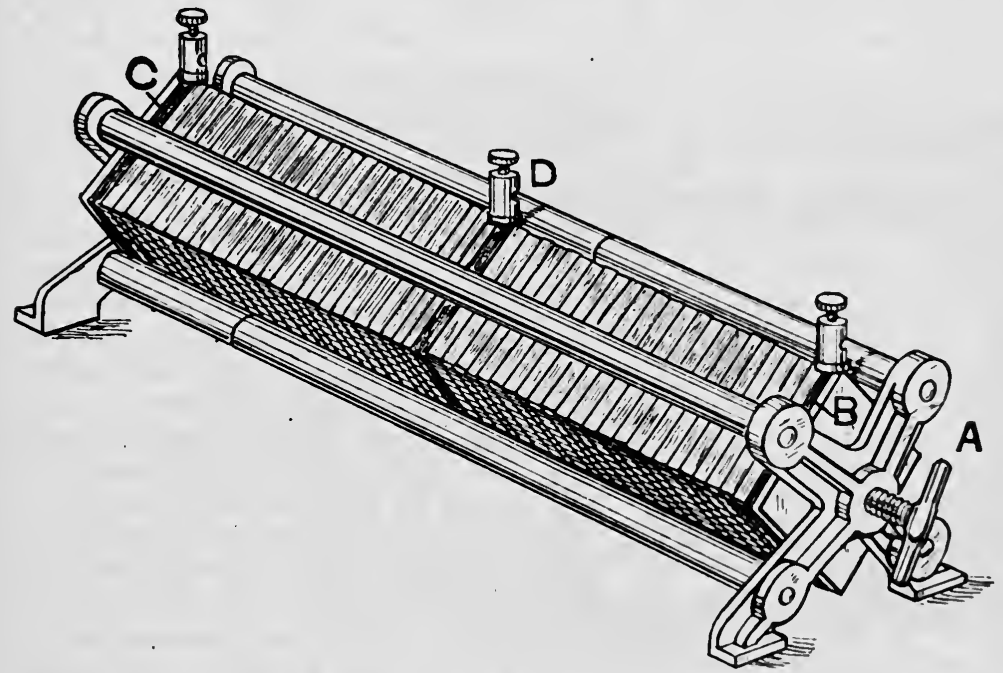

FTG. 205.

fairly thick wire, and to consist of some material, such as manganin, which has a very small temperature coefficient, so that the resistance of the coils shall not vary much owing to the heat developed by the current, and, further, a fairly strong current may be passed without fear of damaging the coils.

The balance is obtained with the batteries connected up in one direction, and then they are both reversed by means of the switches $c_{1}$ and $c_{2}$, and a balance is again obtained, the mean of the two values obtained for $\mathrm{s}$ being used in the calculation.

The resistance between the points $q$ and $q^{\prime}$ is

$$
\frac{Q S}{Q+S}
$$


Hence since the drop of potential between $a$ and $a^{\prime}$ is the same as that between $p$ and $p^{\prime}$, while that between $b$ and $b^{\prime}$ is the same as that between $q$ and $q^{\prime}$, if $c_{1}$ is the current through $\mathbf{A}$ and $\mathbf{B}$, while $c_{2}$ is the current through $\mathbf{P}$ and $Q$, we get

or

$$
\begin{aligned}
A c_{1} & =P c_{2} \\
B c_{1} & =\frac{Q S}{Q+S} c_{2} \\
A & =\frac{P(Q+S)}{Q S} .
\end{aligned}
$$

In the case where the resistances A and B (Fig. 206), which are to be compared, are nearly equal, a modification of the above method may be

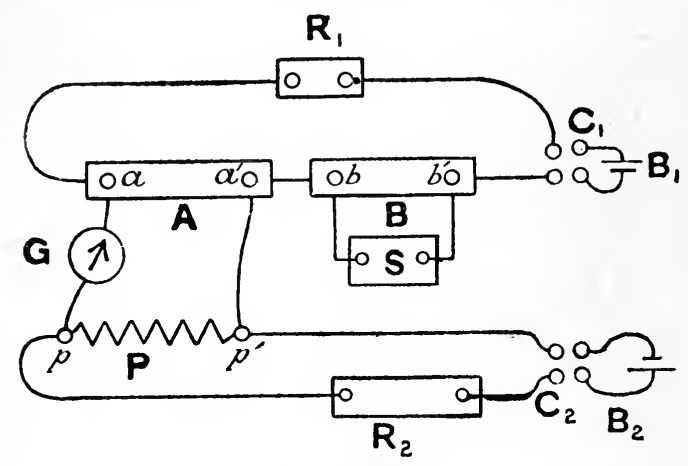

FIG. 206. employed. In this case the auxiliary resistance only contains a single resistance $P$, in addition to the variable resistance $R_{2}$, which is used to adjust the current in the auxiliary circuit. The resistance $P$ need not be known. A shunt $\mathbf{s}$ is placed between the terminals of whichever of the two resistances $A$ or $B$ is the larger. A switch similar to that shown in Fig. 205 is employed to join the galvanometer and a connecting wire between $a a^{\prime}$ and $p p^{\prime}$, or between $b b^{\prime}$ and $p p^{\prime}$. Balance is obtained by altering $\mathbf{s}$ and $\mathrm{R}_{2}$ as before, when

or

$$
\begin{aligned}
A & =\frac{B S}{B+S^{\prime}}, \\
\bar{B} & =S(1+S) .
\end{aligned}
$$

Of the various methods of comparing small resistance described, Mathiessen and Hockin's method is distinctly the least accurate, while the accuracy which can be attained with the others, when suitable precautions are taken, is about the same, and they are capable of showing a difference of about 1 in 100,000. For a discussion of methods of high precision for the comparison of resistances, see F. E. Smith, British Association Report, 1906, p. 106. 


\section{CHAPTER XXVII}

\section{THE RESISTANCE OF ELECTROLYTES}

195. Mance's Method of Measuring the Resistance of a Battery. -In this method the battery $\mathbf{E}$ to be tested is placed in one of the gaps of a Wheatstone's bridge, the position generally occupied by the battery being occupied by a make-and-break key $\mathrm{K}$, as shown in Fig. 207. The resistances $\mathbf{P}, \mathbf{Q}, \mathbf{R}$ are then adjusted, till completing the circuit at $\mathbf{R}$ does not alter the galvanometer deflection. When this is the case, the resistance of the battery is given by

$$
B=\frac{R Q}{P}
$$

This at once follows from the property of conjugate conductors; see p. 433.

The current which is passing through the galvanometer all the time will either produce a deflection right off the scale, or at any rate the deflection will be so great that the

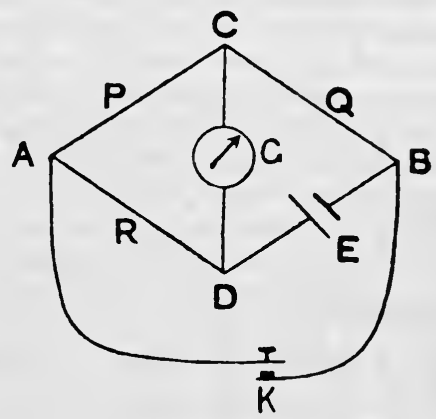

FIG. 207. galvanometer is not at all sensitive to small changes in the current, and hence considerable changes in the resistances in the branches can be made without appreciably altering the deflection. This difficulty can be got over by reducing the galvanometer deflection to zero by some external means. One method is to adjust the controlling magnet so that the needle occupies its central position when the current is passing. In many galvanometers, however, the controlling magnet is not sufficiently strong for this purpose, and an auxiliary magnet must be used. A better plan, however, is to reduce the deflection to zero by acting on the galvanometer needle by means of the field produced by an auxiliary current. If a differential galvanometer $(\$ 192)$ is available, one circuit can be used in connection with the bridge, while a current from an auxiliary battery is passed through the other circuit and an adjustable resistance, and the current in this circuit is so adjusted that the deflection is small. In the absence of a differential galvanometer, a Thomson galvanometer, in which the two sets of coils are brought to separate terminals, may be employed, 
one pair of coils being used for the bridge, and the other for the anxiliary current.

Another method of avoiding the difficulty, and one which avoids all chance of damaging the galvanometer by the passage of an excessively large current, has been recommended by Lodge. This consists in placing a condenser in series with the galvanometer. The presence of the condenser prevents any steady current passing through the galvanometer, which may therefore be made very sensitive, and should preferably have a high resistance. If, however, the difference of potential between the points $\mathbf{C}$ and $\mathrm{D}$ changes when the key $\mathrm{K}$ is closed, the charge in the condenser will alter, and this change in the charge of the condenser takes place through the galvanometer, which will therefore give a momentary kick. The experiment consists, therefore, in adjusting the resistances till there is no galvanometer kick when the key $\mathrm{K}$ is opened or closed. The condenser ought to have a capacity of about $\frac{1}{3}$ microfarad.

In the case of cells, such as Daniell cells, which have a fairly high resistance, and in which polarisation does not readily occur, the resistances used in the arms of the bridge need not be very much larger than the resistance of the battery being tested. If, however, a cell, such as a Léclanché, which polarises easily, is being tested, the resistances in the arms of the bridge should be kept large, otherwise polarisation of the cell will occur and interfere with the measurements.

196. Beetz's Method of Measuring the Resistance of a Battery. -In Mance's method of measuring the resistance of a cell it is necessary to allow the cell to send a current continuously while the measurement

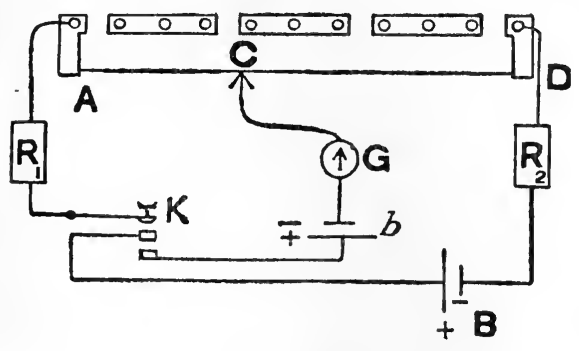

FIG. 208. is being made, and hence it is only applicable to so-called constant cells. In the case of inconstant cells, a method devised by Beetz, in which it is only necessary to close the battery circuit momentarily, can be employed. In addition to the cell $\mathbf{B}$ (Fig. 208), of which the resistance is required, this method requires a second cell $b$, which must of necessity have a lower E.M.F. than that of the cell B. These two cells are connected up with two resistance boxes $R_{1}$ and $R_{2}$, a stretched wire $A D$, which may be the wire of a metre bridge, a galvanometer $\mathrm{G}$, and a special form of key K. This key is so constructed that on pressing it down it first completes the circuit joining $R_{1}$ to $B$, and then immediately after completes the connection with the cell $b$. The experiment consists of adjusting the resistances $R_{1}$ and $R_{2}$ and the position of the slider $C$ on the wire, till on pressing the key the galvanometer remains undeflected.

If the E.M.F. of the cell $\mathrm{B}$ is $E$, since there is no current through the 
galvanometer, the current through the circuit BKADB is $E /\left(R_{1}+R_{2}+B+\rho\right)$, where $B$ is the resistance of the cell $\mathrm{B}$, and $\rho$ is the resistance of the whole bridge wire. Hence if $a$ is the resistance of the portion $\triangle \mathrm{C}$ of the bridge wire, the difference of potential between the points $K$ and $c$ of this circuit is

$$
\frac{E\left(R_{1}+a\right)}{R_{1}+R_{2}+B+\rho}
$$

But since there is no current through the galvanometer, the difference of potential between the ends of the branch KGC, due to the current in the other branch, must be equal and opposite to the E.M.F. $e$ of the cell $b$. Hence

$$
e=\frac{E\left(R_{1}+a\right)}{R_{1}+R_{2}+B+\rho} \text {. . . . . . }
$$

The resistances $R_{1}$ and $R_{2}$ are now altered, and a new position of balance is found. Using accented letters to indicate the values of the various resistances which occur when this second balance is being obtained, we have

$$
e=\frac{E\left(R_{1}^{\prime}+a^{\prime}\right)}{R_{1}^{\prime}+R_{2}^{\prime}+B+\rho} \text {. . . . . . }
$$

Hence from (1) and (2)

$$
B=\frac{\left(R_{1}^{\prime}+a^{\prime}\right)\left(R_{1}+R_{2}+\rho\right)-\left(R_{1}+a\right)\left(R_{1}^{\prime}+R_{2}^{\prime}+\rho\right)}{R_{1}-R_{1}^{\prime}+a-a^{\prime}},
$$

from which the resistance of the cell B can be obtained.

When making the adjustment, it will often be found that on pressing the key the galvanometer gives a sharp movement, and then on keeping the key down a slowly increasing deflection is obtained, which may be in the same or the opposite direction as that of the original kick. The first kick indicates that the balance is incomplete, while the slow drift is due to the fact that the E.M.F. of the cell $B$ is changing, due to the passage of the current. Hence in making the adjustment it is the initial kick which must be reduced to nothing, so that the galvanometer starts out on its slow drift without any preliminary waver.

197. Measurement of the Resistance of Electrolytes.-Owing to the phenomenon of polarisation which takes place at the surface of the electrodes used to connect an electrolytic resistance to the rest of the circuit, the measurement of the resistance of electrolytes is a matter of some little difficulty. Hence the methods used in measuring the resistance of metallic conductors have to be modified in the case of electrolytes in such a manner as to eliminate the effects of this polarisation. There are three methods commonly employed for this purpose, which depend on the following principles :-

(1) Balancing the polarisation in the cell containing the electrolyte 
being measured by the polarisation in an auxiliary cell with similar electrodes, and included in the opposite arm of the Wheatstone's bridge. At the same time by using high resistances in the arms of the bridge, and a large E.M.F. in the battery, swamping the residual effects of polarisation due to the two cells not being exactly alike.

(2) Using a potentiometer method, in which no current is allowed to pass through the electrodes used to connect the electrolyte to the measuring apparatus.

(3) Using a rapidly alternating current, in which case it is found that since it requires the passage of a certain quantity of electricity to develop the polarisation, the effect of polarisation may be reduced to an inappreciable amount if suitable precautions are taken to use electrodes of large area and not to have a large alternating current.

We shall proceed to describe a typical arrangement for carrying out an experiment by each of the above methods.

198. Measurement of Electrolytic Resistance by the Passage of a Direct Current.- The connections for this method of measuring the resistance of an electrolyte are shown in Fig. 209. The two arms A and B consist of two equal resistances of

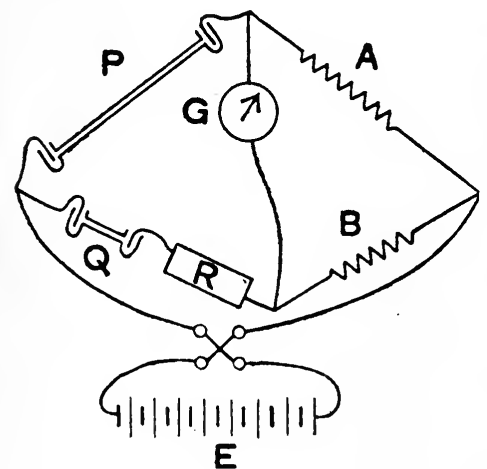

Fig. 209. about 1000 ohms, while in the other arms are placed two similar electrolytic cells, except that while in $P$ the tube containing the electrolyte is long, in the cell $Q$ the tube is short. An adjustable resistance $\mathbf{R}$ is placed in series with $\mathbf{Q}$, and the bridge is balanced by varying this resistance. The galvanometer G ought to be a high resistance one, and the battery $\mathrm{E}$ should have an electromotive force of from 10 to 30 volts.

The electrolytic cells consist of two stout-walled tubes $\mathbf{A}, \mathbf{A}^{\prime}$ (Fig. 210 ), with a side tubulure near the bottom of each, and a narrow-bore tube $\mathbf{B}$, which passes through corks in these tubulures. In the case of electrolytes which would be contaminated by contact with a cork, either wood or india-rubber, the end of the narrow tube can either be ground into the tubulure, or it can be fused into the side of the tube A. Each electrode consists of a plate of platinum foil $\mathrm{D}$, which is welded to a short length of platinum wire, this wire being fused through the bottom of a short length of glass tube c. By pouring mercury into this tube, connection between the cell and the rest of the bridge can be made by means of stout copper wires, the ends of which are amalgamated and dip into the mercury These electrodes are held in place by means of corks, through which the glass tubes c pass. 
The electrodes must be platinised by placing them in a solution of 3 parts of platinic chloride in 100 parts of water, to which a drop or two of lead acetate solution has been added ( 0.02 parts), and passing a current. The strength of the current must be so adjusted that a free but regular evolution of bubbles takes place, the direction of the current being reversed from time to time. The electrodes must then be well washed and allowed to soak in water for several hours.

The horizontal tubes B should be made from stout-bore thermometer tubing, a piece of tubing about $35 \mathrm{~cm}$. long being chosen, of which the bore is fairly uniform. To test the uniformity of the bore a thread of mercury 2 or $3 \mathrm{~cm}$. long is sucked into the tube and the length of the

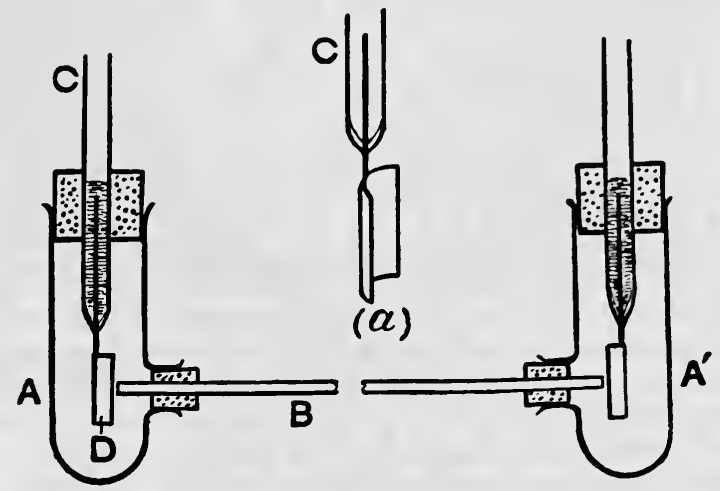

Fig. 210.

thread measured at different parts of the tube, just as was done in the case of the calibration of a mercury thermometer; see $\S 59$. A suitable piece of tubing having been found, $5 \mathrm{~cm}$. is cut off from nne end to form the tube of the smaller electrolytic cell.

If $L$ is the length of the tube $P$, while $l$ is that of $Q$, thes if they had exactly the same bore the resistance $R$ when balance is secured would be equal to the resistance of a column of the electrolyte of length $L-l$. In general, however, the bore is not quite uniform, and as a next approrimation we may suppose that while the bore of either $P$ or $Q$ is uniform, yet they are not exactly the same. If the radius of $\mathrm{P}$ is $A$, and that of $\mathrm{Q}$ is $a$, the volume of the bore of $\mathrm{P}$ is $\pi A^{2} L$, and that of $\mathrm{Q}$ is $\pi a^{2} l$. Hence if $W$ is the weight of mercury of density $\delta$, which will just fill the bore of $\mathbf{P}$, and $w$ is the corresponding quantity for $Q$, we have

$$
W=\delta \pi A^{2} L,
$$$$
w=\delta \pi a^{2} l \text {. }
$$ 
Suppose, now, that the two tubes are filled with an electrolyte of specific resistance $\rho$, then the resistance of the cell $P$ is given by

$$
P=\frac{L \rho}{\pi A^{2}}
$$

In the same way

Hence

$$
Q=\frac{l \rho}{\pi a^{2}}
$$

Hence

But

$$
P-Q=\rho\left\{\frac{L}{\pi A^{2}}-\frac{l}{\pi a^{2}}\right\}
$$

Hence

$$
\frac{L}{\pi A^{2}}=\frac{L^{2} \delta}{W} \text { and } \frac{l}{\pi a^{2}}=\frac{l^{2} \delta}{w} \text {. }
$$

$$
R=P-Q=\rho \delta\left\{\frac{L^{2}}{W}-\frac{l^{2}}{w}\right\},
$$

or

$$
\rho=R \div \delta\left\{\frac{L^{2}}{W}-\frac{l^{2}}{w}\right\}
$$

The factor by which $R$ is multiplied is a constant for any pair of tubes, and having been determined once for all, the specific resistance of the electrolyte is obtained by multiplying $R$ by this factor.

To determine the value of the reduction factor for a pair of tubes, the ends of the tubes must be ground off perpendicular to the axis of the tubes. The tubes having been well cleaned and dried, a piece of rubber tubing is attached to one end and mercury drawn up to the top of the tube. The lower end is then closed by pressing with the finger a small disc of glass against the ground end of the tube. The rubber tube is then removed and, if necessary, mercury added till the tube is quite full and a small globule of mercury stands above the end of the tube. A second glass plate is then slipped on so as to remove the excess mercury, and the mercury in the tube is allowed to flow into a weighed watch giass, and its weight is determined. The lengths of the tubes have to bo measured with some care, since they are involved to the second power. The best method to employ is the comparator method, described in $\S 19$.

In place of obtaining the values of the constant of the tubes by direct measurement, it may be obtained by filling the cells with an electrolyte of known conductivity and determining the corresponding value of $R$ for balance. In Table 25 will be found the specific resistance of solutions of certain salts for different concentrations as determined by Kohlrausch, and these may be employed to standardise the tubes. It is advisable not to use too weak a solution, otherwise the errors in making up the solution may have too much influence on the result.

To perform an experiment the cells are filled with the electrolyte, the electrodes placed in the end tubes, and the cells placed side by side in an. 
oil bath, the level of the oil being above the horizontal tubes. A thermometer is placed in the bath with its bulb alongside the horizontal tubes, and the bath must be kept well stirred during the measurements, as the conductivity of electrolytes varies considerably with the temperature. It is advisable to make settings with the current passing in either direction, for which purpose a commutator is placed in the battery circuit.

199. Comparison of the Specific Resistances of Electrolytes by the Potentiometer Method. - The theory of the method is the same as that used in the case of low resistances, described in $\S 190$. The solutions to be compared are contained in two tubes $\mathrm{x}$ and $\mathrm{y}$ (Fig. 211). The ends of these tubes are bent at right angles to the len:th of the tube and dip

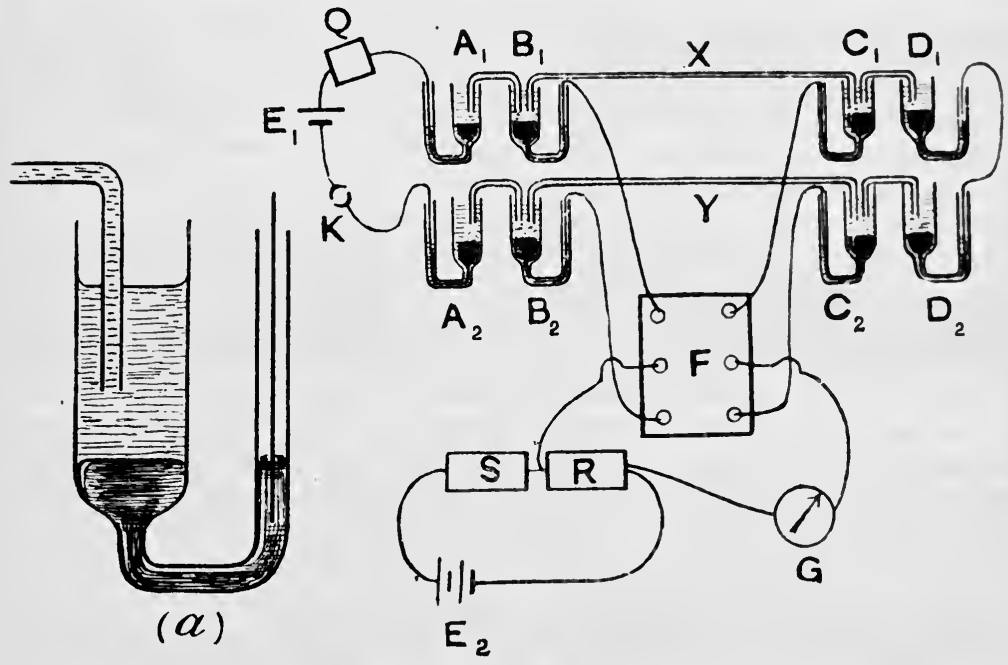

FiG. 211.

into two tubes B and c, forming mercury electrodes. The form of one of these tubes is shown in Fig. $211(a)$. They can be easily made by drawing down a piece of glass tube having a bore of about a centimetre, and then bending the drawn-down portion in the manner shown. At either end of each tube there is a second electrode vessel, which is connected with the first by means of a $U$ tube, which is filled with the same solution as the main tube. The battery $\mathbf{E}_{1}$, which is used to send a current through the tubes, is connected to $\mathbf{A}_{1}$ and $\mathbf{A}_{2}$, the connecting wires dipping into the drawn-down limbs of the vessels. The vessels $\mathbf{B}_{1}, \mathbf{C}_{1}, \mathbf{B}_{2}, \mathbf{C}_{2}$ are connected, by wires dipping into the mercury in the small side tubes, with a set of mercury cups $\mathbf{F}$. A second cell $\mathbf{E}_{2}$ is connected to two resistance boxes $S$ and $R$ placed in series, and wires are taken from the terminals of $\mathrm{R}$ through a high resistance galvanometer $G$ to the middle pair of mercury cups of $F$. The sum of the 
resistances $\mathbf{R}$ and $\mathrm{S}$ is kept constant, say, at 10,000 ohms, and the resistance in $\mathbf{R}$ required to give no deflection of the galvanometer determined when the secondary circuit is first connected to $\mathbf{B}_{1}$ and $\mathbf{C}_{1}$, and, secondly, when connected to $\mathbf{B}_{2}$ and $\mathrm{C}_{2}$.

The object of the additional electrode vessels is to reduce the effects of polarisation to a minimum, since, except when the point of balance is being sought, no current passes at the electrodes $\mathbf{B}_{1}, \mathbf{B}_{2}$ and $\mathbf{C}_{1}, \mathbf{C}_{2}$. Polarisation takes place at the electrodes $A_{1}, \Delta_{2}, D_{1}, D_{2}$, but the effect is only to slightly reduce the current in the circuit, an effect which, as it affects equally the current in the two tubes, does not influence the result.

If $R_{x}$ and $R_{y}$ are the resistances required for balance when the secondary circuit is connected to the ends of $X$ and $Y$ respectively, then

$$
\begin{aligned}
& X \\
& \bar{Y}
\end{aligned}=\begin{aligned}
& R_{x} \\
& R_{y}
\end{aligned}
$$

If $\rho_{x}$ is the specific resistance of the solution in $X$, the resistance of the solution in the tube will be given by $X=\rho_{x} x$, where $x$ is a constant depending on the length and bore of the tube. Similarly for the tube $\mathbf{X}$ we have $Y=\rho_{y} y$.

Hence

$$
\frac{\rho_{x}}{\rho_{y}}=\frac{X}{x} \cdot \frac{y}{Y}=\frac{R_{x}}{R_{y}} \cdot \frac{y}{x} .
$$

In order to determine the value of the factor $y / x$, an experiment must be made with the same solution in the two tubes. Let $r_{x}$ and $r_{y}$ be the values of $R$ required for balance. Then

or

$$
\begin{aligned}
& 1=\frac{r_{x}}{r_{y}} \cdot \frac{y}{x}, \\
& \frac{y}{x}=\frac{r_{y}}{r_{x}} .
\end{aligned}
$$

Thus the value of the factor required to obtain the ratio of the specific resistances of the two solutions is obtained, this factor being a constant as long as the same pair of tubes is employed. If the specific resistance of one of the solutions is known, then that of the other can immediately be calculated.

In the case of salts of the alkali metals, mercury electrodes such as described are quite satisfactory. The polarisation may, however, be reduced by covering the mercury surface in the electrode vessels with calomel (mercurous chloride) if the solutions being compared are chlorides, or with mercurous sulphate if they are sulphates. A little of the mercury salt is placed on the surface of the mercury before the solutions are poured in.

When performing the experiment the tubes and electrode vessels must be carefully cleaned and dried, filled with the solution, and the approximate value of $R$ required for balance determined. The tubes 
should then be left for some time, so that the temperature may become .uniform throughout, this temperature being read by means of a thermometer placed alongside. To assist in the temperature of the two tubes being the same, they ought to be placed close together. The current in the main circuit should be made as small as is consistent with sensitiveness, and should not be kept passing longer than is necessary for making a reading. It is often advisable to use a battery of larger E.M.F. than is absolutely required, and to reduce the current to the required value by means of a resistance $Q$. Since it is assumed that the current in both circuits remains constant throughout, the test balance should be made first with $X$ then with $Y$, and finally with $X$ again, the mean of the two values with $X$ being taken. In this way a steady change in the currents will not affect the results.

200. Measurement of Electrolytic Resistance by Kohlrausch's Method with Alternating Currents. - Kohlrausch has shown that the E.M.F. of polarisation in an electrolytic cell is proportional to the quantity of electricity which has passed through the cell, as long as the quantity of electricity which has passed is not too great. Thus the difference of potential between the electrodes of an electrolytic cell, through which a current $i$ is passing, when the resistance of the electrolyte is $R$, is given by

$$
E=R i+P \int i d t,
$$

where $P$ is a constant which depends on the area of the electrodes and on the nature of the electrodes and the electrolyte.

Suppose, now, that an alternating E.M.F. of frequency $p / 2 \pi$ is applied to the terminals of the cell. Then if the applied E.M.F. follows the simple harmonic law, it may be represented by $E_{0} \sin p t$, and as this must always be equal to the E.M.F. existing between the electrodes of the cell, we have

$$
R i+P \int i d t=E_{0} \sin p t .
$$

Differentiating this expression with respect to the time

$$
R \frac{d i}{d t}+I^{\prime} i=E_{0} p \cos p t .
$$

The integral of this equation is

$$
i=\frac{E_{0}}{R\left\{1+P^{2} / R^{2} p^{2}\right\}^{t}} \sin (p t+\theta),
$$

where the angle $\theta$ is such that

$$
\tan \theta=\frac{P}{R p}
$$


If the electrodes were such that there was no polarisation, so that $P=0$, the current would be given by

$$
i=\frac{E_{0}}{R} \sin p t
$$

Thus the effect of the polarisation is to change the phase of the current with reference to that of the applied E.M.F., and to decrease the amplitude. Both these effects vanish when the fraction $P / R p$ is vanishing small. Hence to decrease the effect of polarisation, $P$ should be small and $p$ great, that is, the area of the electrodes should be as great as is convenient, and they should be platinised, also the frequency of the alternations of the current should be great.

In order to supply the alternating currents, a small induction coil is used. Quite a small coil, which will only give a microscopic spark, is much the best, and it ought to be provided with a very rapid trembler. The terminals of the secondary of the coil are connected to the battery terminals of the Wheatstone's bridge, and the galvanometer is replaced by a telephone. It will be found a great convenience to use two telephones placed in series and fixed to a spring. This spring rests on the top of the observer's head and keeps one of the telephones pressed against either ear, thus allowing the hands to be free for manipulating the contact on the bridge. It is also a good thing to place the induction coil at some distance from the bridge, so that the noise of the interrupter is not heard.

If the resistance coils employed to balance the bridge have either self-induction or capacity, then there will be no arrangement of the bridge which will give complete silence in the telephone, and the point of minimum sound will be difficult to determine. Now, although the ordinary bifilar method of winding resistance coils almost completely avoids self-induction, yet coils wound in this way, especially those which contain very many turns of wire, have very appreciable capacity. To get over this difficulty, Chaperon has devised a form of winding in which the capacity is much reduced. Since, however, coils wound in this special way are seldom available, it is important to reduce the number of coils to a minimum, and not to use coils having a great number of turns, that is, coils of very high resistance. Hence the stretched wire form of bridge is better for use with this method than the Post Office form or dial form, and the resistances in the arms ought never to exceed $500 \mathrm{ohms}$.

If it is desired to measure the absolute specific resistance of an electrolyte, then the form of cell shown in Fig. 210 may be employed. It will, however, be advisable to use a fairly short and wide-bore tube, so that the resistance is not greater than $500 \mathrm{ohms}$. The electrodes ought to be platinised in the manner described on p. 479 .

When the relative specific resistances of two solutions has to be determined, or where a solution of known specific resistance is available 
for standardising the electrolytic cell, the forms of electrode described below may be employed.

In addition to the forms of cell described in the preceding sections, there are other forms which in special circumstances present advantages. A selection are shown in Fig. 212. The cell shown at $a$ (Fig. 212) will be found convenient when measuring the change of resistance with temperature of badly conducting solutions. The solution having been heated up to the highest temperature required, a series of measurements

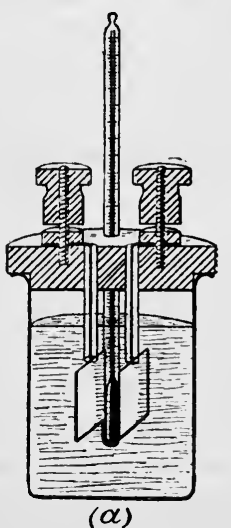

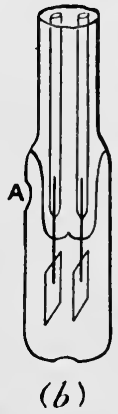

FIG. 212.
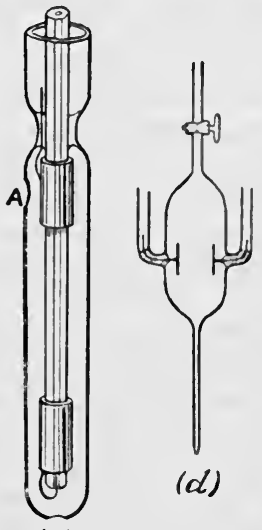

(d)

(c)

of resistance are made as the solution cools, the temperature being indicated by a thermometer with its bulb near the electrodes.

The cells shown at $(b)$ and $(c)$ are such that they can be introduced within the liquid contained in a bottle or flask, and so long as the liquid covers the upper hole $A$, the resistance is independent of the quantity of liquid outside the cell. In the case of bad conductors the form $(b)$ is used, while for fairly good conductors the form $(c)$ is better.

In the form of cell shown at $(d)$ the liquid is sucked up into the bulb, and then the tap is turned.

In the case of all these cells they may be standardised by using solutions of known conductivity (Table 25), the method of preparing such solutions being described in the following section.

201. The Preparation of Solutions for the Determination of Resistance.-In the preparation of solutions for conductivity determinations, particularly if dilute solutions are to be used, it is important that the water employed to make up the solutions should be as free from dissolved electrolytes as possible. Ordinary distilled water, particularly if it has stood about in glass bottles, has quite an appreciable conductivity. Its conductivity may be much reduced by placing some distilled water in 
a carefully cleaned beaker and boiling the water. The water is then thrown away and a fresh supply poured into the beaker, and the beaker is placed in a freezing mixture. The water in the beaker is not stirred, so that a coating of ice forms round the walls of the beaker. When about a quarter of the water has been frozen, the remaining water is poured away and the ice allowed to melt. The water produced from the melting of the ice will be found to have a much smaller conductivity than that of the original water. Such water must, however, be used at once, as if it is kept it will dissolve salts from the glass of the vessel in which it is kept. The rate at which water dissolves glass depends very considerably on the nature of the glass. The firm of Schott of Jena make a glass which is particularly resistant to the action of water, and can therefore be recommended for use when preparing solutions for resistance measurements and for the construction of the vessels in which the resistance of the solutions is measured.

The concentration of the salt used in the solutions is generally expressed in terms of the number of gram-molecules of the salt contained in a litre of the solution. By a gram-molecule is meant a number of grams of the salt equal to the molecular weight of the salt. Thus in the case of sodium chloride $(\mathrm{NaCl})$, of which the molecular weight is $58 \cdot 4$, the normal solution, that is, one containing 1 gram-molecule per litre, will contain 58.4 grams of the salt per litre. In the case of a salt, such as copper sulphate, the molecule of which contains five molecules of water of hydration, the molecular weight being $63.2+32+16 \times 4$ $+5(2+16)$, or $249 \cdot 2$, then the normal solution contains $249 \cdot 2$ grams of the hydrated salt per litre. 


\section{CHAPTER XXVIII}

\section{MEASUREMENT OF ELECTROMOTIVE FORCE}

202. The Construction of Standard Cadmium (Weston) Cells.The cell, consisting of an amalgam of cadmium as the positive pole, mercury as the negative pole, cadmium sulphate as electrolyte together with mercurous sulphate as a depolariser, has many advantages over the Clark cell, in which zinc replaces the cadmium. These advantages are a very much smaller temperature coefficient, greater permanence, and more rapid recovery from the effects of the passage of an excessive current.

The following description of the method of preparing cadmium cells is compiled from a specification prepared by Mr. F. E. Smith of the National Physical Laboratory. ${ }^{1}$ If the work for which the cells are required does not necessitate an accuracy greater than about 1 or 2 parts in 10,000, and the cells are not required to last for several years, then a simplified method of preparation may be employed, as in dicated below.

(A) Preparation of the Materials for a Standard Cadmium Cell.-(1) Mercury.-The mercury is allowed to fall in a very thin stream, obtained by attaching a very fine capillary to a separation funnel, through a long (1 metre) glass tube containing dilute nitric acid ( 1 of acid to 6 of water). A convenient form of tube is shown in Fig. 213. The lower part of the tube is drawn down and bent into an $S$ form, as shown. The mercury collects at the bottom of the tube, and then flows over into a bottle c. Having been passed twice through the acid, the mercury is passed through a column of water contained in a similar tube.

The mercury is then twice distilled in vacuo. There are a number of patterns of mercury still on the market, and that shown in Fig. 214, called the Clark still, may be taken as a type. At the same time, it is a pattern which the author. can recommend as requiring very little attention.

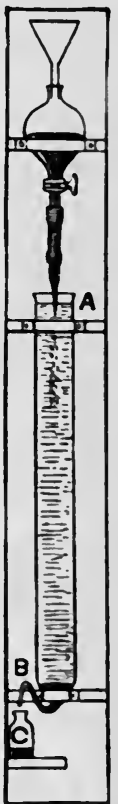

Fig. 213.

A fairly wide tube $A B$ has a bulb at the upper end, and a side tube $B$ near the bottom. Inside this tube is a second tube $\mathrm{AC}$, which is open at the top, and has an $S$ bend at the bottom. The side tube $B$ is connected by a length of thick-walled rubber tube with a Woolf's bottle 
by means of a glass $T$-piece DE. A funnel G, which serves for the introduction of the mercury, and a tube $\mathrm{H}$, fitted with a tap, pass through two well-fitting rubber corks in the upper openings of the bottle.

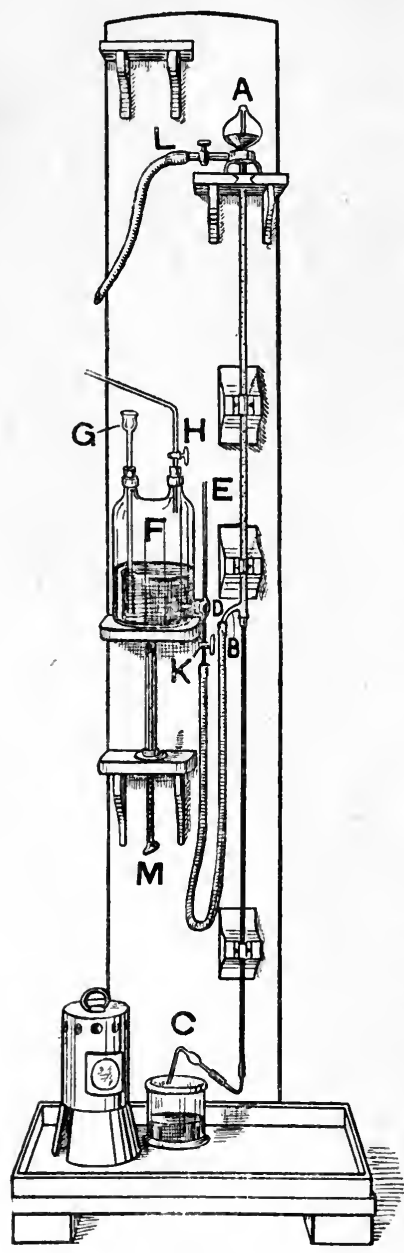

FIG. 214. A tap $K$ serves to stop the flow of mercury when adjusting the pump. A ring burner $\mathrm{L}$ below the bulb $\mathrm{A}$ serves to heat the mercury. The object of the tube $\mathrm{DE}$ is to keep the head of mercury constant. Thus after mercury has been introduced into the bottle, during which operation the cock $\mathrm{K}$ must be closed, air is sucked out through the tube $\mathrm{B}$ till bubbles enter through $D$. The tap $H$ is then closed, and on opening $\mathrm{K}$ the head of mercury is measured from $D$. As the mercury flows out of the bottle into the still, air enters through ED, but the level of the mercury at $\mathbf{D}$ remains unaltered.

To start such a still, supposing it empty, the bottle $\mathrm{F}$ having been filled with mercury, it is raised up till the mercury fills the tube $\mathbf{A B}$ and flows down the tube AC. The rate of flow of the mercury is adjusted so that the mercury passes down AC in separate short columns, which carry with them the air remaining in the bulb. The still during this operation acts as an ordinary Sprengel pump. When the exhaustion is complete, which is indicated by the sharp click heard as each mercury drop falls down the tube Ac, the bottle $\mathrm{F}$ is lowered on to a shelf provided, the height of which is adjusted so that the mercury about half fills the bulb $\mathbf{A}$. The burner $\mathbf{L}$ is then lighted, the gas at first being turned very low. When the mercury gets hot it boils, the temperature at which ebullition takes place being fairly low since the bulb is exhausted, and the vapour passes down the tube AC, where it becomes condensed by the cold mercury which is on its way up to the bulb. The first pound or so of mercury which is collected must be returned to the bottle $F$, since it contains the mercury which filled the bottom of the tube $\mathrm{AC}$, and has therefore not been distilled.

If an efficient shield is provided to protect the flame from draughts, 
and, further, if a regulator is placed to keep the gas pressure constant, such a still may be kept working day and night without more attention than is required to fill up the bottle $F$ when necessary. The mercury which is left in the tube AB contains all the metallic impurities, and hence must be run out from time to time.

The disadvantage of the Clark mercury still, and all stills of this kind, is that since zinc, cadmium, \&c., are all volatile, some of these impurities will be found in the distillate. It has, however, been shown by Hulett and Minchin ${ }^{1}$ that if air is passed through the mercury in the still, then the zinc, cadmium, and other more oxidisable impurities become oxidised, and since the oxides are not volatile, the distillate is free from these impurities. The form of still they employ is shown in Fig. 215 It consists of a distilling flask $\Delta$ with a round bottom, having a long neck. To the side tube B is fused a thin-walled tube $\mathbf{B O}$, about 2 centimetres in diameter and 50 centimetres long. The end of this tube is narrowed down, and passes through a wood cork in the receiving flask $D$. To a side tube (or if the flask has no side tube, to a tube passing through the cork) is attached a $T$-piece $\mathrm{E}$, having a cock $\mathrm{F}$ on one branch. The third branch of the $T$ is connected by thick-walled rubber tube to an aspirator (water pump). The cork in the flask $D$ may be made air-tight with sealing-wax.

Through the neck of the flask A passes a narrow glass tube I,

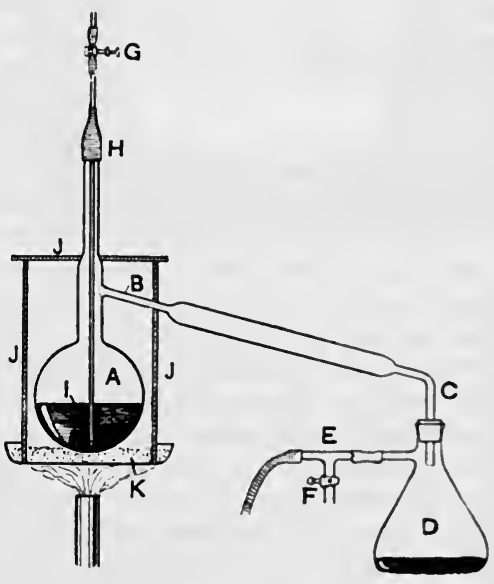

FIg. 215. drawn down to a fine capillary at the bottom. The joint between this tube and the flask is made air-tight by a short piece of rubber tubing, which slips tightly over both tubes. A short length of rubber tube is attached to the top of $\mathrm{I}$, and a pinch-cock on this tube serves to adjust the flow of air.

The flask A stands in a sand bath, and is surrounded by a shield $\mathrm{J}$, made of asbestos board.

The mercury having been introduced, the aspirator is started and the flask heated with a Bunsen burner, the pinch-cock $\mathrm{g}$ being so adjusted that the air bubbles through the mercury at the rate of one or two bubbles per second. If the distillation becomes too rapid, as it has a tendency to do just at first, it may be checked by admitting a little air through the tap $\mathbf{F}$. 
(2) Preparation of the Amalgam.-The amalgam used contains 12.5 per cent. of cadmium, and is prepared by electrolysis. To prepare this amalgam some of the pure distilled mercury is placed in a glass vessel, and above the mercury is poured a solution of cadmium sulphate, which has been acidified with a few drops of sulphuric acid. A glass tube $A$ (Fig. 216), with a platinum wire fused through the end, serves for making electrical connection with the mercury. The anode consists of a rod of pure commercial cadmium B, to which the positive pole of a battery is connected. The lower end of the cadmium rod rests in a

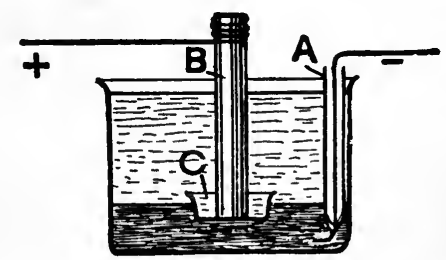

FIG. 216. small crystallising dish $\mathrm{c}$. The object of this dish is to prevent the slime which forms on the cadmium reaching the mercury. The resistance in the circuit is adjusted so that the fall of potential between the anode and cathode does not exceed 0.3 volt.

The cadmium anode is weighed before the electrolysis and also after, the difference of weight giving the weight of cadmium in the amalgam. The mercury also having been weighed, the quantity of mercury necessary to reduce the strength of the amalgam to 12.5 per cent. is added.

The amalgam is then heated over a water bath, the surface being still covered with a little of the cadmium sulphate solution, and well stirred. The amalgam having been cooled, the acid cadmium sulphate solution is removed, and neutral cadmium sulphate added. This neutral solution consists of the saturated solution prepared as described in (3), diluted with its own bulk of distilled water. The amalgam is now ready for use. It is entirely liquid at about $60^{\circ} \mathrm{C}$.

(3) Preparation of Cadmium Sulphate Crystals and Solution.-Commercially pure cadmium sulphate is dissolved in about 1.25 times its weight of distilled water, which will require continual agitation for some six hours, or periodical agitation for two or three days. This solution is filtered, and is then placed in crystallising dishes, whieh must be protected from dust, and allowed to slowly evaporate, the temperature not exceeding $35^{\circ} \mathrm{C}$. The crystals formed should be prevented from adhering together by removing the liquid to a fresh dish as soon as the crystals have grown to such an extent that they are mostly in contact. In this way about five-sixths of the liquid may be evaporated, and the mother liquid can be used for washing the mercurous sulphate, as described in (4).

The cadmium sulphate crystals must be washed with successive small quantities of distilled water, till the liquid that drains away shows no trace of acidity after testing for five minutes with congo red. The crystals while still moist must then be transferred to a stock bottle.

The saturated solution is prepared from the crystals by prolonged 
agitation with distilled water at a temperature of about $20^{\circ}$ to $25^{\circ} \mathrm{C}$.

(4) Preparation of the Mercurous Sulphate. - This is prepared by electrolysis, pure distilled mercury forming the anode, and a piece of platinum foil the cathode, the electrolyte being dilute sulphuric acid ( 1 volume of concentrated acid to 5 of water). The mercury is placed at the bottom of a large flat-bottomed glass dish and covered with about twenty times its volume of the dilute acid. The current is conveyed to the mercury as in the case of the preparation of the amalgam, and the platinum cathode is suspended in the upper portion of the acid. A current density of about 0.01 amperes per square centimetre of the mercury surface may be employed. During the electrolysis it is most important that the electrolyte near the surface of the mercury shall be kept in continual agitation. This may be done by using an L-shaped glass stirrer, with the horizontal bar near the mercury surface.

The salt obtained in this way ought to have a grey colour; if it is tinged yellowish, this shows that the stirring has not been sufficiently vigorous. Such yellowish salt must not be employed to construct cells.

The mercurous sulphate is agitated with dilute sulphuric acid (1 volume of acid to 6 of water) and distilled mercury, and is then filtered, and the greater part of the mercury is removed. The salt is then washed with small quantities of cadmium sulphate solution till free from acid, the mother liquid being first used, but the final washings being made with the neutral solution prepared from the crystals.

(5) Preparation of the Mercurous Sulphate Paste.-Some cadmium sulphate crystals are ground in an agate mortar with a little cadmium sulphate solution, and about one quarter the bulk of distilled mercury and twice the bulk of mercurous sulphate. The whole must be well

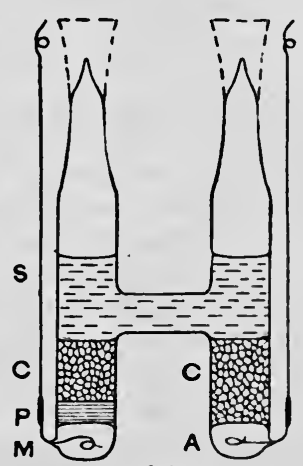

(a)

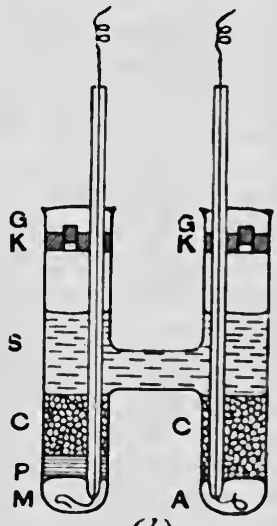

(b)

FIG. 217. ground together, the quantity of cadmium sulphate solution being so adjusted that a thin paste is formed.

(B) Filling the Cell.-In Fig. 217 are shown two types of cell. That on the left $(a)$ is hermetically sealed, while that on the right $(b)$ is 
closed by means of corks and marine glue. The glass tube for the (b) form is rather simpler to make, since the platinum wires have not to be fused through the sides. This type is, however, slightly more difficult to fill, since the glass tubes which protect the wires leading down to the amalgam and mercury respectively are apt to become displaced during the process of filling. In either figure $M$ is mercury, $\mathbf{A}$ the amalgam, $\mathrm{P}$ the mercurous sulphate paste, $\mathrm{c}$ cadmium sulphate crystals, and $\mathbf{s}$ saturated solution of cadmium sulphate.

When setting up the cells, the first operation is to amalgamate the platinum wires used to make contact with the mercury and amalgam. In the case of the $(b)$ form this can be done by heating the platinum red hot, and then dipping it in mercury. In the $(a)$ form the amalgamation can be performed by electrolysing a solution of mercuric nitrate.

The corks $\mathrm{K}$, used to close the (b) pattern tubes, must be well boiled in water, and then soaked in the cadmium sulphate solution. In addition to the hole bored to allow the passage of the electrodes, a second hole must be bored through which the paste, crystals, \&c., can be introduced.

The amalgam having been melted over a water bath, the surface being covered with some dilute cadmium sulphate solution, some of the amalgam is sucked into a heated pipette. The end of the pipette being introduced into the limb of the $\mathrm{H}$ tube, the amalgam is allowed to flow down into the tube, sufficient being introduced to well cover the platinum wire. After the amalgam has solidified, the limb containing it should be washed out with a little cadmium sulphate solution. Into the other limb is then introduced, first, the mercury, and then the paste, a small pipette made by drawing down a piece of glass tube being used, care being taken that the paste does not spread up the side of the tube. Next a layer of finely powdered cadmium sulphate crystals is placed in each limb, and, finally, some saturated cadmium sulphate solution is added.

The cells are then to be stored in a warm room for a week or more, to allow some of the liquid to evaporate, the new crystals deposited serving to bind the original crystals together. In the case of the $(a)$ type the glass tubes are sealed off before the blow-pipe, care being taken not to heat the contents of the cell. In the cells of type (b), hot marine glue is poured on the top of the corks, the small central holes having been closed by means of little pieces of cork.

When in use, cells of type (a) should be immersed in an oil bath, while cells of type $(b)$ may be partly immersed either in an oil or water bath.

In many cases a cell which is correct to about one part in two thousand is amply accurate enough, in which circumstances a very much simplified method of preparation may be adopted. The mercury used should be distilled in vacuo as described, but the amalgam may be prepared by dissolving commercially pure cadmium in the mercury. The cadmium sulphate solution may be prepared by simply dissolving the bought crystals in distilled water, while the crystals themselves may be 
used in the cell. Bought mercurous sulphate is more apt to produce differences ; if, however, Karlbaum's is obtainable, no very serious difference will be produced by using the salt as supplied. If this salt is unobtainable, then it will be advisable to prepare the salt by the method described above.

The E.M.F. at a temperature $t$ of a cadmium cell, prepared as described in the specification given above, may be taken ${ }^{1}$ as given by

$$
E_{t}=1.0186-3.8 \times 10^{-5}(t-20)-0.065 \times 10^{-5}(t-20)^{2}
$$

between the limits of $10^{\circ}$ and $30^{\circ}$. It is not advisable to use the cell at temperatures outside these limits. In Table 26 will be found the value of the E.M.F. of the cadmium cell at different temperatures calculated from the above formula.

203. The Construction of the Standard Clark Cell.-This cell differs from the cadmium cell in that the cadmium is replaced by zinc. Thus the positive pole consists of an amalgam containing 10 per cent. of zinc, while the cadmium sulphate crystals and solution are replaced by zinc sulphate crystals and solution.

The procedure described with reference to the cadmium cell may mutatis mutandis be applied to the construction of the Clark cell, and hence need hardly be repeated. This is particularly the case, since it is likely that shortly the Clark cell will be entirely replaced as a legal standard by the cadmium cell.

The E.M.F. of a Clark cell, at a temperature $t$, is given in international volts by ${ }^{2}$

$$
E_{t}=1.4328-119 \times 10^{-5}(t-15)-0.7 \times 10^{-5}(t-15)^{2} .
$$

204. Comparison of E.M.F.'s with the Potentiometer.-By fas the most accurate and convenient method of comparing the E.M.F. of cells is the potentiometer. There are various arrangements for carrying out the method, which will be described in the next section, but the principle of all is the same. Suppose that a cell $\mathbf{E}$ (Fig. 218), of which the E.M.F. is larger than that of the cells to be compared, sends a current through a stretched wire $\mathbf{A B}$, and to the end $\Delta$ of this wire, which is connected to the positive pole of $\mathbf{E}$, is connected the positive pole of one of the cells $e_{1}$, which are to be compared. Then a wire connected to the other pole of the cell $e_{1}$ will be at a lower potential than the potential at $\mathbf{A}$, and as by supposition the cell $\mathbf{E}$ has a larger E.M.F. than that of $e_{1}$, there will be some point along the wire $\Delta B$ at which the potential, owing to the flow of the current in the wire, is as much below that at $\mathbf{A}$ as is the lead attached to the negative pole of $e_{1}$. Hence if we bring the lead in contact with this point on the stretched

1 Jaeger and Kahle, Wied. Annalen (1898), 1xv. 942. The E.M.F. is expressed in international volts. The E.M.F. of a cadmium cell in true volts $\left(10^{8} \mathrm{cog} . \mathrm{s}\right.$. units) is probably 1.0184 at $20^{\circ}$.

2 Jaeger and Kahle, Wied.' Annalen (1898), lxv. p. 941. 
wire, since they are at the same potential, no current will pass from the lead to the stretched wire; in other words, a galvanometer $G$ will show no deflection. Hence if $C$ is the current in the wire $\mathbf{A B}$, and the resistance of the portion of this wire between $A$ and $C$ is $r_{1}$, we have that the E.M.F. of the cell $e_{1}$ is given by

$$
e_{1}=r_{1} C \text {. }
$$

If, now, a second cell, of which the E.M.F. is $e_{2}$, is used in place of $e_{1}$, and the resistance of the portion of the wire between $A$ and the new position $\sigma^{\prime}$, for which the galvanometer indicates no current, is $r_{2}$, then

Hence

$$
e_{2}=r_{2} C \text {. }
$$

$$
\frac{e_{1}}{e_{2}}=\frac{r_{1}}{r_{2}}
$$

-If the wire is uniform, and the distances of the points $\mathbf{C}$ and $\mathbf{c}^{\prime}$ from $\mathbf{A}$ are $l_{1}$ and $l_{2}$ respectively, this result may be written

$$
\frac{e_{1}}{e_{2}}=\frac{l_{1}}{l_{2}}
$$

Such a potentiometer may be made direct reading by using a standard cell, say, a cadmium cell, the E.M.F. of which at the temperature of the observation is known. Thus suppose that the temperature is $20^{\circ} \mathrm{C}$., so that the E.M.F. is 1.0184 volts. If the wire is divided into 2000 divisions, the movable contact is placed at the reading $1018 \cdot 4$, counting from the end $A$, and an adjustable resistance $R^{\prime}$ in the circuit of the cell $\mathbf{E}$ is adjusted till the galvanometer is undeflected. When this is the case, we know that the difference of potential between $A$ and the 1018.4 division is 1.0184 volts, and hence, if the wire is uniform, that the difference of potential between the point $A$ and the 1000th division is 1 volt. If, then, keeping the resistance $R^{\prime}$ constant we replace the cadmium cell by a cell of unknown E.M.F., and the point of balance is at the $m$ th division, the E.M.F. of the cell will be $m / 1000$ volts. Of course this relation will only hold so long as the current flowing through the wire remains constant. Hence it is necessary to test this by replacing the standard cell from time to time, and if necessary readjust the resistance $R^{\prime}$. If the battery $E$ consists of one or two accumulators of a capacity of about twenty ampere hours, and, after fully charging, they are partly discharged and allowed to send a current through the wire for a little time before the final adjustment is made, it will be found that the changes in the current are extremely slow, so that the adjustment remains satisfactory for a very considerable time.

205. Potentiometers. - (1) Stretched Wire Potentiometer. - A stretched wire potentiometer, such as that shown diagrammatically in 
Fig. 218, may be made up by means of a metre bridge. The cell $\mathbf{x}$ ought to be one or more accumulators, and the two cells to be compared may conveniently be connected to a commutator, such as that

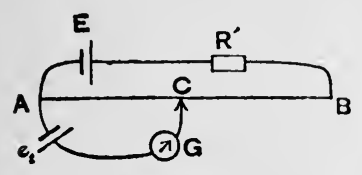

FIG. 218. shown at c (Fig. 219), by means of which the change from one to the other may be rapidly effected. It is advisable to use a high resistance galvanometer, so that when the position of balance is being sought only a small current should pass through the cell, a matter of considerable importance when using standard cells, such as Clark cells or cadmium cells. For this same reason it is advisable to insert a resistance of about $10,000 \mathrm{ohms}$ in series with the galvanometer, a key being arranged to short-circuit this resistance when balance has nearly been secured.

(2) Rayleigh's Form of Potentiometer.-Lord Rayleigh, in his work on Clark cells, used a form of potentiometer which is very convenient, and, where the utmost accuracy is not desired, does not involve the use of any elaborate apparatus. The arrangement is shown in Fig. 219. Here the stretched wire is replaced by two resistance boxes $P$ and $Q$, each capable of giving a resistance of 10,000 ohms, and when in use the sum of the two resistances $P$ and $Q$ is kept constant and equal to 10,000 ohms. The positive poles of the two cells $e_{1}$ and $e_{2}$, which are being compared, are connected to one end of the resistance $P$ and the galvanometer $G$ to the other end, while by means of a commutator $\mathrm{c}$ either cell can be connected to the galvanometer at will.

If $P_{1}$ and $P_{2}$ are the resistances

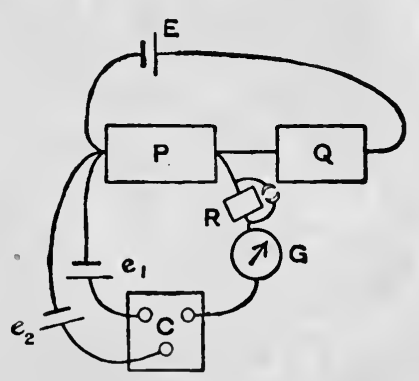

Fig. 219. which have to be unplugged in $P$ to produce balance when first one cell and then the other are introduced, we have

$$
\frac{e_{1}}{e_{2}}=\frac{P_{1}}{P_{2}}
$$

This arrangement may be used to give the E.M.F. of a cell being tested direct by making the resistance of $P 1000$ times the E.M.F. of a standard cell, and then adjusting $Q$ till, when the standard is in circuit, the galvanometer is undeflected. The resistance of $P$, when any other cell is used, will then be numerically equal to the E.M.F. of the cell multiplied by 1000 . Of course the sum of the resistances $P$ and $Q$ must be kept constant after the adjustment with the standard cell has been made, and this adjustment must be tested from time to time 
to allow for changes in the current through the resistance boxes. It is advisable to have a resistance $R$ of about $10,000 \mathrm{ohms}$ in series with the galvanometer when making the preliminary adjustments, and to cut this resistance out of the circuit for the final adjustments.

(3) The Kelvin and Varley Slide.-The objection to the slide wire form of potentiometer is that, in the first place; to obtain accuracy of reading the wire must be long, and it must be calibrated. Further, owing to the wire being exposed throughout its whole length and the action of the sliding contact it is liable to damage, which will necessitate recalibration. In Rayleigh's form of potentiometer these objections are

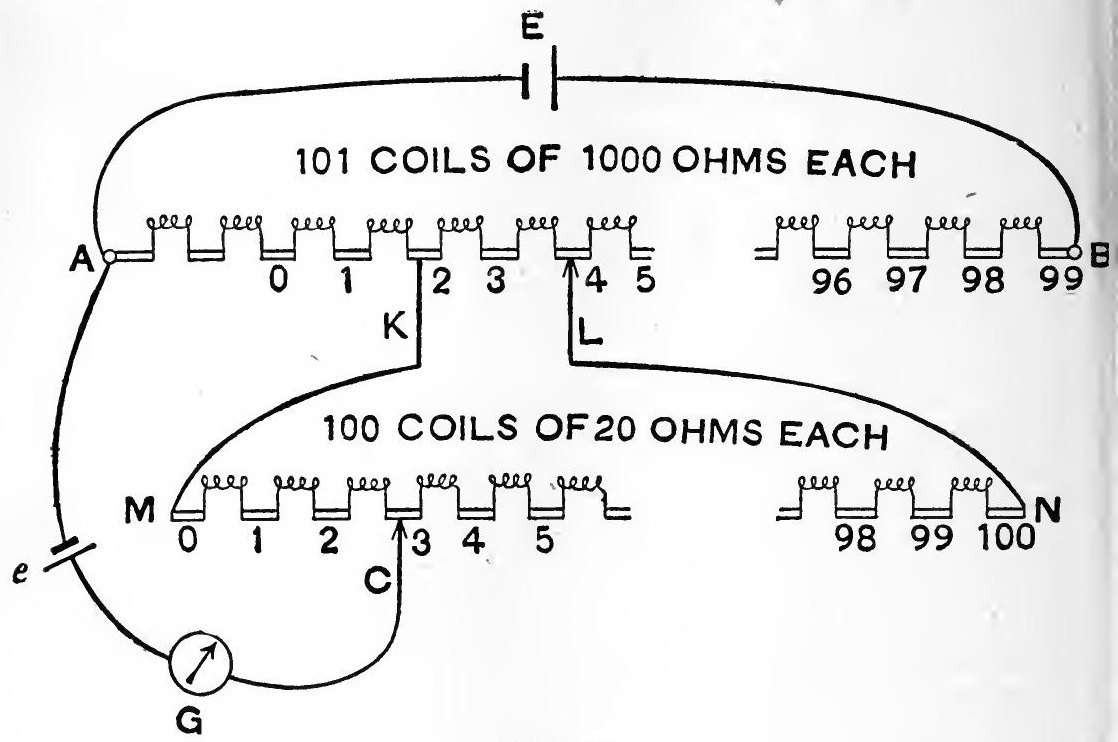

F1G. 220.

removed, but where great accuracy is required the resistance boxes require to be carefully calibrated, an operation in the usual form of box which involves very considerable labour. Further, it is important that the coils in the two boxes should have the same actual values, so that removing one coil from one box and introducing a coil of the same nominal value in the other should not alter the total resistance in the circuit. An arrangement of resistances called the Kelvin and Varley slide overcomes these objections. The plan on which the slide works is shown diagrammatically in Fig. 220. A hundred and one coils AB, each having a resistance of $1000 \mathrm{ohms}$, are connected in series with a contact stud between each of the coils. A hundred equal coils MN, each having a resistance of $20 \mathrm{ohms}$, are also connected in series with contact studs between the 
coils. The terminals of this second set of coils are connected to two sliding contacts $\mathrm{KL}$, which are fixed at such a distance apart that they always bridge over two coils of the first set in the manner shown in the figure. Now when the sliders are in the position shown in the figure the resistance from $A$ to $K$ is $4000 \mathrm{ohms}$, and the resistance from $\mathrm{L}$ to $\mathrm{B}$ is $95,000 \mathrm{ohms}$. The resistance from $\mathrm{K}$ to $\mathrm{L}$ is $1000 \mathrm{ohms}$, since the coils MN, which have a combined resistance of $2000 \mathrm{ohms}$, are in parallel with two of the coils of the $\mathrm{AB}$ series. Hence the combined resistance between $\mathbf{A}$ and B, whatever the position of the sliders $\mathrm{KL}$, is $100,000 \mathrm{ohms}$.

A single slider moves over the contacts of the 100 coils of 20 ohms each, and to this contact is connected the galvanometer and one pole of the cell $e$, which is being tested. Suppose, now, that the other pole of this cell is connected to $\Delta$, while a battery $\mathbf{E}$ is connected to $\Delta$ and $\mathrm{B}$, and that by first moving the double slider $\mathrm{KL}$ approximate balance is

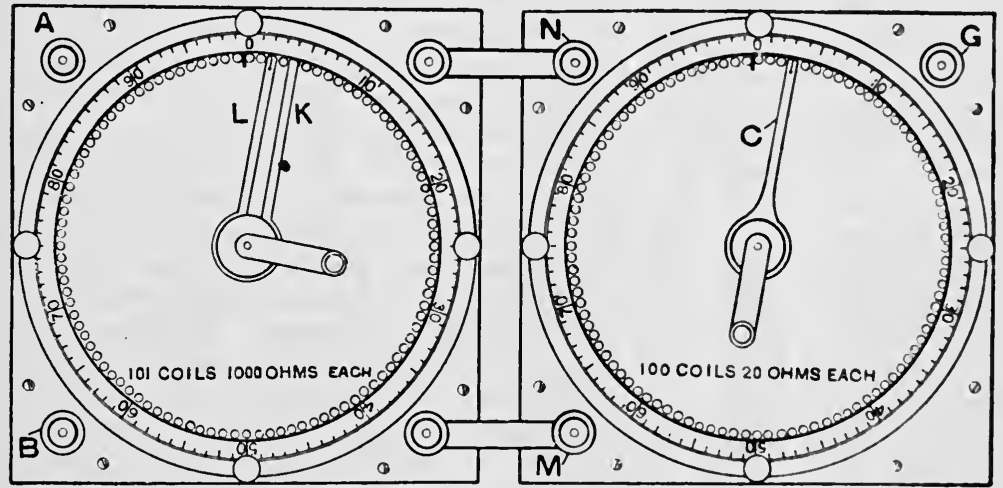

FIG. 221.

obtained, and that final balance is obtained by moving the slider c, the position of balance being as shown in the figure. If, now, we call the whole drop of potential between $A$ and $B$ 100, the drop of potential between $\mathrm{A}$ and $\mathrm{K}$ will be 4 . Also the drop of potential between $\mathrm{K}$ and $\mathrm{L}$ will be 1, that is, the drop of potential between $\mathrm{M}$ and $\mathrm{N}$ will be 1 . The drop of potential between $M$ and $c$ will, therefore, be $3 / 100$ of 1 , or 0.03 . Hence the whole drop of potential between $A$ and $c$ will be $4+.03$, or $4 \cdot 03$, which, it will be noticed, is at once given by the numbers opposite the sliders $\mathrm{L}$ and $\mathrm{c}$. If, now, another cell is used, and the sliders moved till the galvanometer is again undeflected, the readings corresponding to $\mathrm{L}$ and $\mathrm{C}$ will give the drop of potential equal to the E.M.F. of this cell in terms of the drop of potential between $\mathrm{A}$ and $\mathbf{B}$. Hence if this drop of potential has remained constant, that is, if the E.M.F. of the cell $\mathbf{E}$ has remained constant, the ratio of the E.M.F.'s of the two cells will be equal to the ratio of the readings of the two slides in the two cases. 
It will be noticed that the arrangement virtually amounts to a long stretched wire, in which in place of being able to make contact at every part we are restricted to making contact at $100 \times 100$, or ten thousand points, which are distributed evenly along the wire.

The usual form which the Kelvin and Varley slide takes is shown in Fig. 221, and the arrangement will be at once clear by comparison with the diagram in Fig. 220. When using the slide to compare E.M.F.'s the connections are similar to those shown in Fig. 220, and the method of conducting the experiment is in every way the same.

Owing to the fact that all the coils in each dial have the same resistance, the adjustment and testing of such a set of resistances is particularly easy.

206. The Capillary Electrometer.-A convenient form of capillary

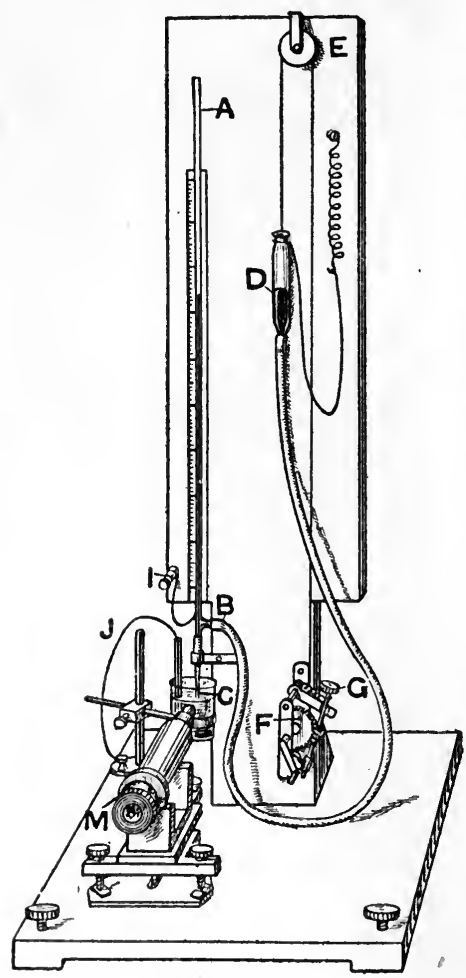

FIG. 222. electrometer is shown in Fig. 222. It consists of a vertical upright tube $\Delta B$, alongside which is fixed a scale, while to a side tube $B$ is connected a mercury reservoir $\mathrm{D}$ by an india-rubber tube. This reservoir is supported by a string, which passes over a pulley $\mathrm{E}$ on to a drum $\mathrm{F}$. The drum can be rotated rapidly for the rough adjustment by a small handle, and slowly, for the final adjustment, by the tangent screw $\mathrm{G}$. To the lower end of $A B$ is attached by a short sleeve of rubber tube a piece of glass tube drawn down at the end to a fine capillary. The end of the capillary dips beneath the surface of an electrolyte contained in a glass vessel, the lower part of the vessel being filled with mercury. A platinum wire I, fused through the side of the tube $\mathbf{A B}$, and a second wire $\mathrm{J}$, enclosed in a piece of glass tube, serve to join the electrometer to the circuit. The position of the mercury meniscus in the capillary is observed by means of a microscope $\mathbf{M}$, which is fitted with an eye-piece scale.

The position of the vessel containing the electrolyte should be so adjusted that the capillary is near the wall next the microscope, otherwise a clear image of the meniscus will not be obtained. The end of the eye-piece scale should always be brought to coincide with the end of the 
capillary, so that if the microscope is accidentally displaced it may bo put back into its original position. The mercury meniscus, when the wires $I$ and $J$ are joined together, so that no difference of potential is applied to the meniscus, should be near the middle of the scale, and by altering the pressure, by raising or lowering $\mathrm{D}$, the meniscus should be brought to coincidence with one of the long divisions of the scale. If the meniscus is always brought to this division, then the changes in the reading for the upper end of the mercury column in $\triangle B$ will give the variation in the surface tension.

The electrolyte generally used is a dilute solution of sulphuric acid containing 1 volume of acid to 7 of water. The solution is saturated with mercurous sulphate by placing a little of this salt on the top of the mercury.

The capillary employed should be of such a bore that the surface tension supports a head of mercury of about $20 \mathrm{~cm}$. With such a capillary the application of $10^{-4}$ volt will cause the meniscus to move appreciably when a microscope magnifying fifty diameters is used.

For a description of the theory of the capillary electrometer, the reader may consult Watson's Physics, $\$ 552$.

207. To Examine the Relation between the Surface Tension of Mercury and an Electrolyte and the E.M.F. acting across the Surface. -Connect a single secondary cell E (Fig. 223) to two resistance boxes $R$ and $s$, the sum of the two resistances in circuit being always kept equal to 10,000 ohms. The two ends of the resistance $\mathrm{s}$ are connected to two mercury cups, 1 and 2 , in a block of paraffin. The terminal I of the capillary electrometer is connected to 2, and the terminal $\mathrm{J}$ to a third cup 3 .

By altering the resistance $\mathrm{s}$, any difference of potential may be applied to the terminals of the electrometer. Thus if $E$ is the E.M.F. of the battery, and $S$ is the resistance unplugged in s, $R+S$ being 10,000 , the applied E.M.F. is

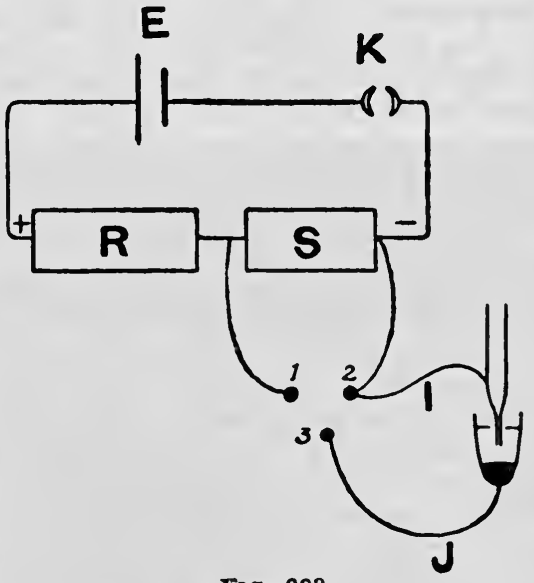

Fia. 223. $S E / 10,000$ volts.

First join the cups 2 and 3, and adjust the position of the meniscus in the capillary, so that it coincides with some fixed division of the eyepiece scale, and read the height of the mercury column. Then starting with the resistance $S$ low, and having connected the cups 1 and 3 , take the readings of the heights of the mercury column required to bring the 
meniscus back to the fixed mark for different values of $S$. If the resistance $S$ be increased beyond a certain value, bubbles of hydrogen will appear in the capillary, and it will be found impossible to proceed beyona this point.

By reversing the battery, so as to make the mercury in the capillary positive, it will be found possible to obtain a few more points. As, however, $S$ gets above $100 \mathrm{ohms}$, the mercury surface becomes sluggish, owing to oxidation of the mercury, and the experiment must be stopped. The oxidised surface may be got rid of by raising the mercury reservoir, so.as to cause some of the mercury to flow out of the capillary.

Plot a curve showing the connection between the surface tension and the applied E.M.F. The scale readings for the top of the mercury column may be taken as ordinates, and the values of $S$ as abscissæ. The curve will be found to have a maximum for an applied E.M.F. of about 1 volt. To determine the E.M.F. corresponding to the maximum surface tension, which probably represents the contact electromotive force between mercury and the electrolyte (see Watson's Physics, § 552), it is necessary to measure the E.M.F. of the cell E. This may best be performed by comparing it with a standard Clark or cadmium cell by the method given in $\S 204$, in which comparison the capillary electrometer may be used in place of the galvanometer. To obtain the exact point of the curve corresponding to the maximum, choose points in the two sides of the maximum at which the surface tension is the same, and join each pair of points. If each of these lines be bisected, the middle points will be found to lie very nearly on a vertical straight line, and the point where this line cuts the curve will give the maximum surface tension, and the abscissa of this point is the E.M.F. required. 


\section{CHAPTER XXIX •}

\section{RESISTANCE THERMOMETERS AND THERMO-JUNCTIONS}

208. Determination of the Constants of a Platinum Thermometer. -It has been found that the resistance of a wire of pure platinum at a temperature $t$ is given in terms of the resistance $K_{0}$ at $0^{\circ}$ by an expression of the form

$$
R_{t}=R_{0}\left(1+a t+b t^{2}\right),
$$

where $a$ and $b$ are constants. This formula is found to hold over a very large range of temperatures ; it is, however, not a convenient one to use, since to deduce $t$ from the values of $R_{t}$ and $R_{0}$ involves the solution of a quadratic equation. Professor Callendar has shown that if the resistance of the wire at $100^{\circ}$ is $R_{1}$, and we define the platinum temperature $p t$, corresponding to the resistance $R_{t}$, by

$$
p t=100 \frac{R_{t}-R_{0}}{R_{1}-R_{0}} \quad \text {. . . . . . . }
$$

then the difference between the temperature on the air scale and that on the platinum scale is given by

$$
t-p t=\delta\left\{\frac{t}{100}-1\right\} \frac{t}{100} \quad . \quad . \quad . \quad . \quad .
$$

where $\delta$ is a constant for any particular sample of wire. Thus the righthand side of equation (2) gives the correction which has to be applied to the platinum temperature, deduced from the resistance measurements by means of equation (1), to obtain the corresponding temperature on the air thernometer scale. The increase of resistance between $0^{\circ} \mathrm{C}$. and $100^{\circ} \mathrm{C}$., viz. $R_{1}-R_{0}$, is known as the Fundamental Interval.

It will be seen that the correction involves $t$, the temperature on the air thermometer, i.e. the quantity which we wish to obtain. This difficulty may be removed, in the case of fairly low temperatures, by calculating an approximate value of $t$ by replacing $t$ in the right-hand side by $p t$, and then using this approximate value of $t$ to calculate a more accurate value of the correction. Griffiths has described another method of avoiding this difficulty, which depends on the observation that if very pure platinum is used, then the value of $\delta$ does not differ much from 1.5 . A table, such as that given below, is first constructed, giving the value 
of the correction, assuming that $\delta$ has the value of 1.5 for different values of $p t$, and these corrections are plotted on a large scale on a curve.

Relation between Platinum Temperatures and Air Thermometer Temperatures when $\delta=1 \cdot 5$.

\begin{tabular}{|c|c|c|c|c|c|}
\hline $\begin{array}{l}\text { Platinum } \\
\text { Scale. }\end{array}$ & Correction. & $\begin{array}{l}\text { Air Thermo- } \\
\text { meter Scale. }\end{array}$ & $\begin{array}{l}\text { Platinum } \\
\text { Scale. }\end{array}$ & Correction. & $\begin{array}{l}\text { Air Thermo- } \\
\text { meter Scale. }\end{array}$ \\
\hline $\begin{array}{r}-100 \\
-50 \\
0 \\
+50 \\
100 \\
150 \\
200 \\
250 \\
300 \\
350\end{array}$ & $\begin{array}{r}+2.9 \\
+1 \cdot 1 \\
0.0 \\
-0.4 \\
0.0 \\
+1 \cdot 2 \\
+3.1 \\
+6.0 \\
+9.8 \\
+14.5\end{array}$ & $\begin{array}{r}\circ 9 \\
-97 \cdot 2 \\
-48 \cdot 9 \\
0 \cdot 0 \\
49 \cdot 6 \\
100 \cdot 0 \\
151 \cdot 2 \\
203 \cdot 1 \\
256 \cdot 0 \\
309 \cdot 8 \\
364 \cdot 5\end{array}$ & $\begin{array}{r}4000 \\
450 \\
500 \\
550 \\
600 \\
650 \\
700 \\
750 \\
800 \\
900 \\
1000\end{array}$ & $\begin{array}{r}+20.2 \\
+27.0 \\
+34.9 \\
+44.0 \\
+54.4 \\
+66.2 \\
+79.4 \\
+94.2 \\
+110.7 \\
+149.4 \\
+197.0\end{array}$ & $\begin{array}{r}4200 \cdot 2 \\
477 \cdot 0 \\
534 \cdot 9 \\
594 \cdot 0 \\
654 \cdot 4 \\
716 \cdot 2 \\
779 \cdot 4 \\
844 \cdot 2 \\
910 \cdot 7 \\
1049 \cdot 4 \\
1197 \cdot 0\end{array}$ \\
\hline
\end{tabular}

If, then, the value of $p t$ has been determined with a thermometer for which the coefficient $\delta$ has the value $\delta^{\prime}$, ascertain from the curve the value of the correction $d$ corresponding to the platinum temperature $p t$, then the correction in the case of the actual thermometer is

$$
\frac{\delta^{\prime}}{1 \cdot 5} \times d
$$

so that

$$
t=p t+\frac{\delta^{\prime}}{1 \cdot 5} d
$$

In order to determine the value of $\delta$ for any particular thermometer the resistance must be measured at three known temperatures, the three chosen being usually the melting point of ice, the boiling point of water, and the boiling point of sulphur.

If the height of the barometer when the boiling point of sulphur is determined is $760+h$ millimetres, the temperature of the vapour over boiling sulphur is given by

$$
t_{s}=444 \cdot 53+0 \cdot 082 h
$$

provided $h$ is small.

Substituting this value of $t_{s}$ in the expression $\left(\frac{t}{100}-1\right) \frac{t}{100}$, we get

$$
15 \cdot 32+0 \cdot 0065 h \text {. }
$$


Hence if $p t$, is the temperature of the sulphur vapour in the platinum scale, we have

or

$$
t_{s}-p t_{s}=\delta\{15 \cdot 32+0.0065 h\},
$$

$$
\delta=\frac{444 \cdot 53+0 \cdot 082 h-p t_{s}}{15 \cdot 32+0 \cdot 0065 h}
$$

The most convenient form of bridge to use with a platinum thermometer is that described in $\S 186$. The leads connected to the thermometer are attached to the binding screws $P_{1}$ and $P_{2}$, while the compensating leads are attached to $C_{1}$ and $c_{2}$. Since the passage of the current will of necessity heat the thermometer wire, it is necessary to use as small a current as possible, and hence it is advisable to place a resistance of from 50 to $100 \mathrm{ohms}$ in the battery circuit, a single accumulator being used to send the current.

A convenient form of platinum thermometer is shown 1n Fig. 224. The wire is wound in a double spiral on a mica frame, and the leads, as well as the compensating leads, which are connected together at the lower end, are kept apart by being threaded through a number of small mica discs. For temperatures up to about $300^{\circ} \mathrm{C}$., the thermometer can be enclosed in a glass tube; for higher temperatures, a glazed porcelain tube must be employed.

When determining the resistance in ice and steam the apparatus used in obtaining the fixed points of a mercury thermometer is employed, the precautions given in $\$ \$ 59$, 60 being observed.

To determine the resistance in sulphur vapour, the piece of apparatus shown in Fig. 225 may be employed. It consists of a glass ${ }^{1}$ tube $\mathbf{A B}$, with a bulb at the bottom, such as is used with Victor Meyer's vapour density apparatus. The bulb fits tightly in a hole in a piece of asbestos board CD, while a tube of asbestos board $F$ sur-

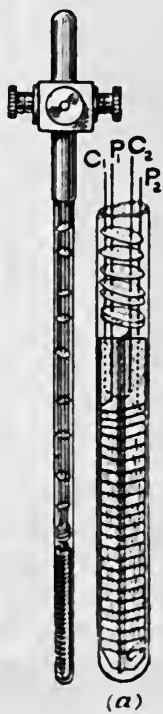

Frg. 224. rounds the upper part of the glass tube, the intervening space being packed with asbestos wool. A cone of asbestos board $\mathrm{F}$ protects the lower part of the apparatus from draughts, and the top of the tube is closed by a sheet of asbestos, through which the thermometer passes. A cylindrical screen $G$, made of tinned iron, surrounds the "bulb" of the thermometer, and serves to protect it from the loss of heat by radiation. This screen is shown developed at $\mathrm{H}$. A piece of thin tinned iron having been cut into this shape, it is bent into a cylinder, and the three upper tags are bound to the thermometer stem with iron wire. The lower tags are bent to meet under the thermometer.

1 This glass tube may be replaced by a piece of thin steel tube, closed at the bottom by an iron cap. Thick iron gas-pipe is not suitable, as too much beat is conducted along the walls. 
Enough sulphur is introduced to fill the bulb to about three centimetres above the board CD, and the apparatus is heated over a Bunsen burner, the flame being adjusted so that the vapour reaches about half-way

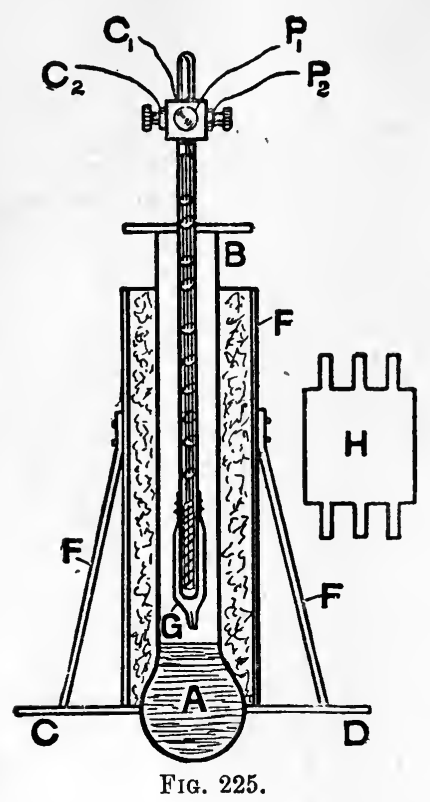

up the part of the tube left exposed at the top. After use, the apparatus should be laid on its side at a small inclination and the sulphur allowed to solidify in that position. If the sulphur solidifies in a mass at the bottom and is afterwards heated from the bottom, there is a possibility of bursting of the bulb owing to the melting of the lower layers while the upper are still solid.

When the resistance of the thermometer has become constant it must be noted, as also the height of the barometer. If $R_{0}$ is the resistance in ice, and $R_{x}$ the resistance in steam, the temperature of which deduced from the barometer pressure is $t_{1}$, then the change of resistance for a rise of temperature from $0^{\circ}$ to $100^{\circ}$, which is called the fundamental interval $(F I)$, is given by

$$
R_{1}-R_{0}=F I=100\left(R_{x}-R_{0}\right) / p t_{1}
$$

where $p t_{1}=t_{1}-\delta\left\{\frac{t_{1}}{100}-1\right\} \frac{t_{1}}{100}$.

As $t_{1}$ will always be very nearly $100^{\circ}$ C., the correction to be applied to obtain $p t_{1}$ will be very small, and may be obtained with sufficient accuracy by assuming that $\delta=1 \cdot 5$. Then if $R_{s}$ is the resistance in sulphur

$$
p t_{s}=100\left(R_{s}-R_{0}\right) / F I \text {. }
$$

Finally, the correct value of $\delta$ for the thermometer used is obtained by equation (3) given above. Knowing the fundamental interval and the value of $\delta$, we have the data for deducing the temperature from measurements of the resistance of the thermometer.

209. Measurement of the E.M.F. of a Thermocouple.-The most satisfactory way of measuring the E.M.F. of a thermocouple is the potentiometer method, described in $\S \S 204,205$. A suitable arrangement is shown in Fig. 226. The main circuit consists of the accumulator $\mathbf{E}$, a wire $\mathbf{A B}$ stretched alongside a scale and calibrated, a resistance box $\mathbf{R}$ capable of giving a resistance up to $1100 \mathrm{ohms}$, and a resistance box $r$, which is adjustable by tenths of an ohm. In the absence of such a box, one adjustable by ohms, together with a carbon variable resistance placed in series, may be used.

One lead from the hot junction $B$ is taken to $A$, the lead from the cold junction $c$ being brought to the mercury cup 3 . The galvanometer 
G, which ought to have a low resistance, is connected to a movable contact on the wire and to the mercury cup 1. A standard cadmium cell $(\$ 202)$ is connected to the cup 2 and to the point $\mathbf{r}$.

The wire AB ought to be at least a metre long, and it is a convenience if its resistance is exactly $1 \mathrm{ohm}$. If the resistance of the wire is a little over an ohm, then a resistance $\mathrm{s}$ may be connected in parallel with the wire, and adjusted so that the resistance of the two in parallel is $1 \mathrm{ohm}$ (see p. 457). Suppose that the temperature of the cadnium cell is $15^{\circ}$, so that its E.M.F. is $1 \cdot 0188$, then the resistance $R$ is made 1018 ohms, so that the resistance between $A$ and $F$ is

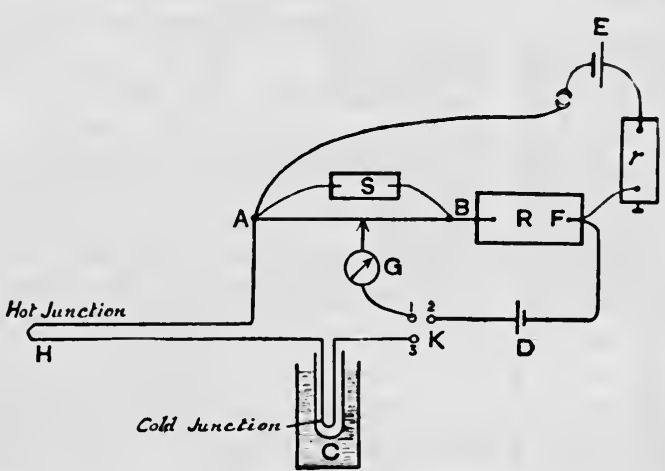

FIG. 226. 1019 ohms. The mercury cups 1 and 3 are then connected together, and the galvanometer contact moved to the end $\boldsymbol{\Delta}$ of the wire. The resistance $r$ is then adjusted till the galvanometer is undeflected. When this is the case, the difference of potential between $\Delta$ and $F$ is 1.019 volt, and hence the difference of potential between the ends of the wire is 001 volt, and each millimetre of the wire corresponds to a microvolt. Hence if, when the mercury cups 1 and 3 are connected, the galvanometer contact has to be made at a point $n$ millimetres from $A$, so that there shall be no galvanometer deflection, then the E.M.F. of the thermocouple is $n$ microvolts.

210. Standardisation of a Thermocouple.-For high temperatures $\left(300^{\circ}\right.$ to $\left.1000^{\circ}\right)$ the most satisfactory thermocouple is one consisting of pure platinum and platinum containing 10 per cent. of rhodium, the wire being about $6 \mathrm{~mm}$. in diameter. If one junction of such a thermocouple is kept at $0^{\circ} \mathrm{C}$., and the other is heated to a temperature $t$, the E.M.F. developed in the circuit can be expressed by a formula of the form

$$
E_{t}=a+b t+c t^{2} .
$$

It is found, however, that such an expression only holds at high tempera tures, $300^{\circ} \mathrm{C}$. and over.

In the case of the Reichsanstalt standard $P t-P t R$ junction, the E.M.F. is given in microvolts $\left(10^{-6}\right)$ by

$$
E_{t}=-310+8.048 t+0.00172 t^{2},
$$


and it is at once evident that such a formula cannot hold at low temperatures; for instance, at $0^{\circ}$, i.e. when the two junctions are at the same temperature, it gives an E.M.F. of -310 microvolts. Over the range $300^{\circ} \mathrm{C}$. to $1100^{\circ} \mathrm{C}$. the formula, however, gives good results.

Another type of expression which is found to hold over a considerable range of temperature is

$$
\log E_{t}=A \log t+B
$$

where $A$ and $B$ are constants. Some values of these constants are given below:-

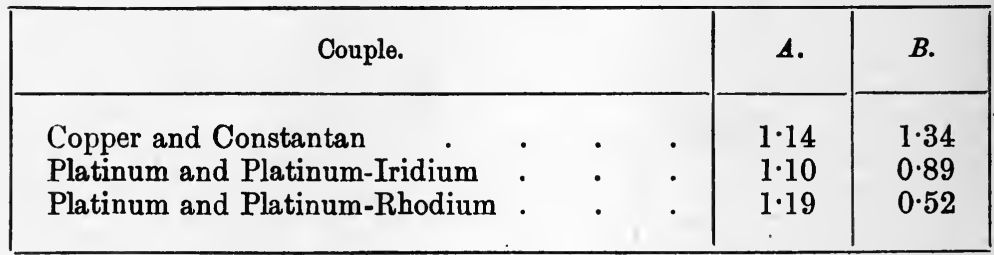

In standardising such a thermocouple, observations of the melting points of various metals are employed, the following being frequently used :-

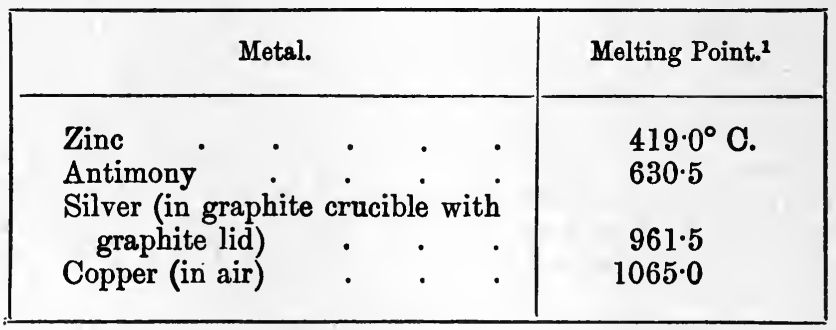

The form of oven used by Holborn and Day for standardising thermocouples is shown in Fig. 227. It consists of a graphite crucible B, in which the metal is heated, and in the case of silver, where it is necessary to protect the molten metal from the air, this is covered by a second crucible $\mathbf{D}$ through a hole in which passes a thin-walled porcelain tube. The heat is supplied by a coil of uncovered nickel wire wound on a fireclay $^{2}$ tube. The wire has a diameter of about $1 \mathrm{~mm}$., and when the oven has to be heated to a temperature of $1000^{\circ}$, about 600 watts must be supplied to the circuit. The heating coil is packed round with asbestos, and the whole enclosed by thick-walled fireclay slabs. The thermoelement wires are threaded down thin porcelain tubes, which are contained within an outside porcelain tube $5 \mathrm{~mm}$. in diameter, having walls

1 Holborn and Day, Drude Annalen (1905), ii. 505.

2 Such tubes are sold under the trade name of "Morganite." 
$1.5 \mathrm{~mm}$. thick. The end of this tube dips about $4 \mathrm{~cm}$. below the surface of the molten metal.

When performing an experiment the metal is melted and then the heating current is slightly reduced, so that the temperature slowly falls, and the E.M.F. of the thermo-element is noted at equal short intervals of time. When solidification takes place the rate of cooling is reduced, and the E.M.F. at the melting point is obtained by plotting the E.M.F.'s observed against the time, as was done in $\S 104$.

For comparatively low temperatures, say, below $300^{\circ}$, the platinum-platinum-rhodium thermocouple is unsuited, the thermo-electromotive force being so small as to make it impossible to measure temperatures with the accuracy that is generally desired at these temperatures. In such a case a junction consisting of pure iron and pure nickel will be found suitable. Such a couple gives about 30 microvolts per degree, so that even this couple is comparatively insensitive, as it is difficult to measure the E.M.F. to much less than 1 microvolt, and in most cases a platinum thermometer would be more suitable. If, however, such a thermocouple is

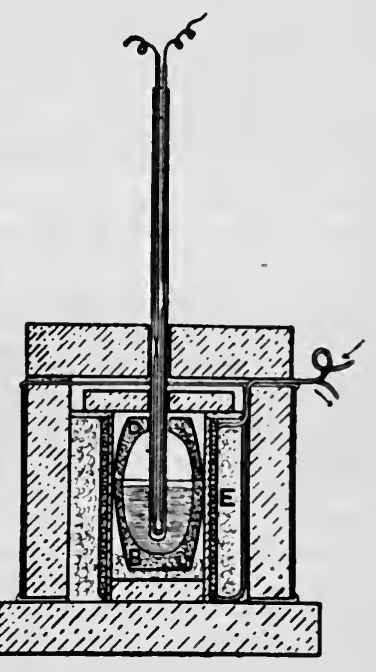

FIG. 227. used, then it may be standardised by heating to known temperatures, such as those given in $\S 98$, and a curve may be plotted showing the connection between E.M.F. and temperature by means of which the temperature corresponding to any particular E.M.F may be read off. 


\section{CHAPTER XXX}

\section{MEASUREMENT OF CURRENT}

211. The Tangent Galvanometer.-The magnetic field $F$ at the centre of a circular coil of mean-radius $R$, and containing $n$ turns, when traversed by a current of $A$ amperes, the cross-section of the coil being very small compared to $R$, is given by

$$
F=\frac{\pi n A}{5 R} \text { gausses. }^{1}
$$

Hence if such a coil is placed with its plane in the magnetic meridian at a place where the horizontal component of the earth's field is $H$, a freely suspended magnetic needle of very small length will set itself inclined at an angle $\theta$ to the meridian such that

$$
A=\frac{5 r H}{\pi n} \tan \theta \quad . \quad . \quad . \quad . \quad . \quad .
$$

Hence if the dimensions $R$ and $n$ of the coil are known, and $H$ and the angle $\theta$ are measured, we can obtain the value of the current which traverses the coil.

The above expression only holds within the limitations mentioned; namely, that the cross-section of the coil is small compared to the radius, and that the needle is very short. If these conditions are not fulfilled, then the expression connecting $F$ and $R$ requires modification. If the section of the coil is a rectangle, the axial breadth, that is, the dimension parallel to the axis of the coil being $b$, and the radial depth being $d$, and the distance ${ }^{2}$ between the poles of the needle $l$, then the value of the current is given by (see Maxwell's Electricity and Magnetism, vol. ii. chapter $\mathbf{x v}$.)

$$
A=\frac{5 r H}{\pi n}\left\{1+\frac{1}{8} \frac{b^{2}}{r^{2}}-\frac{1}{12} \frac{d_{2}}{r^{2}}-\frac{3}{16} \frac{l^{2}}{r^{2}}\left(1-5 \sin ^{2} \theta\right)\right\} \tan \theta .
$$

1 The unit of magnetic force on the c.g.s. system is called a gauss.

2 As an approximation the distance between the poles may be taken as equal to $2 / 3$ of the length of the needle. 
By suitably choosing the dimensions of the cross-section the term $\frac{b^{2}}{8 r^{2}}-\frac{d^{2}}{12 r^{2}}$ can be made to vanish. This occurs when

$$
\frac{b^{2}}{8}=\frac{d^{2}}{12}
$$

that is, when

$$
\stackrel{b}{d}=\frac{\sqrt{2}}{\sqrt{3}}
$$

The value of the term $\frac{3}{16} \frac{l^{2}}{r^{2}}\left(1-5 \sin ^{2} \theta\right)$, since it depends on the angle $\theta$ through which the needle is deflected, varies with the deflection. The following table shows the value of the factor $\left(1-5 \sin ^{2} \theta\right)$ for some values of $\theta:-$

\begin{tabular}{|c|c|}
\hline$\theta$ & $1-5 \sin ^{2} \theta$ \\
\cline { 2 - 2 } $10^{\circ}$ & +0.85 \\
$20^{\circ}$ & +0.42 \\
$26^{\circ} 34^{\prime}$ & \pm 0.00 \\
$30^{\circ}$ & -0.25 \\
$40^{\circ}$ & -1.07 \\
$50^{\circ}$ & -1.93 \\
\hline
\end{tabular}

The value of the correction factor, and also the magnitude of the error produced by any want of adjustment of the needle to the centre of the coil, can be materially reduced by employing a double coil. This arrangement, which is due to Helmholtz, consists of two equal coils placed coaxially and with their planes at a distance apart equal to the radius of either, the needle being placed on the common axis half-way between the coils.

If $r$ is the mean radius of the coils, $2 x$ the distance between their mean planes, and the total number of turns on the two coils is $N$, then the axial component of the field at a point in the plane perpendicular to the common axis half-way between the mean planes of the coils, at a distance $y$ from the axis, is given by

$$
\begin{array}{rl}
F=2 \pi N & C\left\{\frac{r^{2}}{a^{3}}+\frac{b^{2} r^{2}}{2 a^{7}}\left(4 x^{2}-r^{2}\right)+\frac{d^{2}}{6 a^{7}}\left(2 x^{4}-11 x^{2} r^{2}+2 r^{4}\right)\right. \\
& +\frac{3 r^{2}}{4 a^{7}}\left(4 x^{2}-r^{2}\right) y^{2}-\frac{15}{8} \frac{b^{2} r^{2}}{a^{11}}\left(8 x^{4}-12 x^{2} r^{2}+r^{4}\right) y^{2} \\
& \left.-\frac{d^{2}}{8 a^{11}}\left(8 x^{6}-136 x^{4} r^{2}+159 x^{2} r^{4}-12 r^{6}\right) y^{2}+d c .\right\} . .
\end{array}
$$


where $b$ is the axial breadth of the cross-section of each coil, $d$ is the radial depth, and $a^{2}=r^{2}+x^{2}$, and $C$ is the current in c.g.s. units.

If $2 y$ is the distance between the poles of the needle suspended at the centre of the double coil, then the above expression can be used to calculate the couple acting on the needle, and hence if the deflection $\theta$ is measured, and $H$ is known, to calculate the value of the current traversing the coils. In general, it will be found that the terms within the brackets, except the first, are excessively small, and may be neglected. Thus if $r=30 \mathrm{~cm}$., $x=15 \mathrm{~cm}$., $b=\cdot 6 \mathrm{~cm}$., $d=\cdot 35$, we have

$$
F=2 \pi N C\left\{0.02365-0.0_{7} 5+0 \cdot 0_{6} 11 y^{2}-\right\} .
$$

212. Adjustment of a Tangent Galvanometer.-Before a tangent galvanometer can be used to measure a current, it is necessary that the following adjustments be made :-

(1) The plane of the coil must be vertical.

(2) The needle must be at the centre of the coil.

(3) The axis of the needle, when no current is passing, must be parallel to the plane of the coils.

(4) The scale must be parallel to the plane of the mirror when the needle is undeflected.

In order to make the first adjustment, a plumb-line may be set up near the instrument, and nearly in the same plane as the coil. The levelling screws are then adjusted till the face of the coil is parallel to the plumb-line. The coil is generally provided with marks, by means of which we may see when the needle is at the centre of the coil, and it is important to verify this adjustment.

To adjust the axis of the needle parallel to the plane of the coil, a cell, preferably a secondary cell, must be connected to the galvanometer through a resistance and a reversing key. The

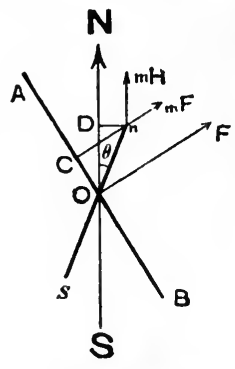

FIG. 228. E.M.F. of the cell must be so large that a measurable deflection is produced when the added resistance is at least $10 \mathrm{ohms}$, otherwise changes in the resistance of the commutator contacts may produce considerable errors. The coils must then be turned about a vertical axis till the deflection obtained is the same whichever way the current is passing. The theory of this adjustment is as follows:Suppose that when no current is passing the needle makes an angle $\alpha$ with the plane of the coil, and let $F$ be the strength of the magnetic field at the centre produced when a current $C$ traverses the coil, the deflection of the needle produced being $\theta$. In Fig. 228 let $N S$ be the magnetic meridian, that is, the direction of the undeflected needle, $A B$ the plane of the coil, and $s n$ the needle when deflected. Then the magnetic field $F$, due to the coil, is along $O F$. Hence if $m$ is the strength of the pole $n$ of the needle, we have a force $m H$ parallel to $O N$, and a 
force $m F$ parallel to $O F$. The moment of the first of these is $m H \cdot \overline{D n}$ and that of the second is $m F \cdot \overline{O C}$, and since there is equilibrium we have

$$
m H \cdot \overrightarrow{D n}=\dot{m F} \cdot \overrightarrow{O C} \text {. }
$$

But if $l$ is half the length of the needle,

$$
\overline{D n}=l \sin \theta, \text { and } \overline{O C}=l \cos (\theta+\alpha) .
$$

Hence

$$
H \sin \theta=F \cos (\theta+a) .
$$

Next suppose that the current is reversed, the deflection being now $\theta^{\circ}$, we should have by exactly the same reasoning

$$
H \sin \theta^{\prime}=F^{\prime} \cos \left(\theta^{\prime}-a\right) .
$$

Hence if the deflections are the same, i.e. if $\theta=\theta^{\prime}$, we get

$$
\cos (\theta+a)=\cos (\theta-a),
$$

which only holds if $a=0$, that is, if the plane of the coil coincides with the axis of the needle in its undeflected position.

The manner in which the fourth adjustment is made has already been described in $\S 172$.

213. The Sine Galvanometer.-If a galvanometer similar to a tangent galvanometer, that is, one with a coil the radius of which is large compared to the size of the needle, is so mounted that it can be turned about a vertical axis, and the angle through which it is turned can be accurately measured, then a very convenient method of working is as follows:-The current being passed in one direction, the whole galvanometer is turned till the needle lies in the plane of the coils, a mirror attached to the needle, and a telescope and scale attached to, and turning with, the coils being used to tell when this adjustment is complete. The azimuth reading having been noted, the current is reversed, and another azimuth reading is obtained when the needle is again in the plane of the coils. If $2 \theta$ is the difference between the azimuth readings, then using the same notation as in $\S 211$, the current $A$ (in amperes) is given by

$$
A=\frac{5 r H}{\pi n}\left\{1+\frac{1}{8} \frac{b^{2}}{r^{2}}-\frac{1}{12} \frac{d^{2}}{r^{2}}+\frac{3}{16} \frac{l^{2}}{r^{2}}\right\} \text {. }
$$

It will be observed that the correction depending on the length of the needle is the same for all values of $\theta$; this is owing to the fact that when taking a reading the axis of the needle is always at right angles to the field due to the coil. Further, since the needle and coil have always the same relative positions, the torsion of the suspension fibre does not come in. The correction for the size of the needle can be reduced by adopting the Helmholtz arrangement for the coiis. 
214. Measurement of a Current by the Deposition of Copper.Where a current has to be measured, or a current measuring instrument calibrated, and an accuracy of about 1 part in 500 is sufficient, this can most conveniently be done by the electrolysis of a copper sulphate solution between copper electrodes. The conditions necessary for satisfactory working have been determined by T. Gray, ${ }^{1}$ and the following method is based on his results :-

A convenient form of electrolytic cell for use with currents up to a few amperes is shown in Fig. 229. The anode consists of two copper

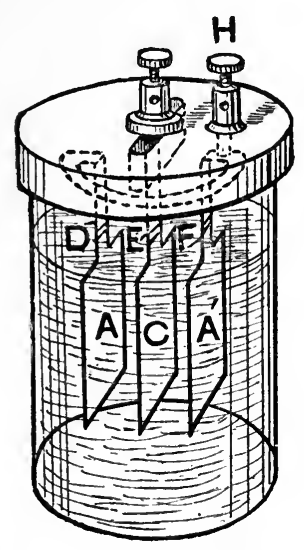

FIG. 229. plates $\mathbf{A}$ and $\mathbf{A}^{\prime}$, which are held in clips fixed to a circular - disc of wood, the wood having been soaked in hot paraffin wax. The two clips are connected together on the upper side of the disc, and a binding screw $\mathrm{H}$ serves to attach the connecting wire. The cathode a consists of a similar plate of copper, which fits in a clip, to which is attached a binding screw $G$. A slit in the wooden disc allows of the cathode being removed for weighing. The disc rests on the top of a beaker, in which copper sulphate is placed. The electrode plates are made from

high-conductivity sheet copper, the cathode being about 0.3 millimetre thick (No. 30 standard wire-gauge), and the anode plates about a millimetre thick (No. 19 standard wire-gauge). The area of the cathode should be such that there are at least 50 square centimetres of surface per ampere of current passing through the cell. The edges and corners of the electrodes should be carefully rounded, otherwise there is a tendency for the deposit to be very thick and rough at the edges.

The solution consists of copper sulphate dissolved in water till the density of the solution is between $1 \cdot 15$ and $1 \cdot 18$, that is, contains between 20 and 25 grams of crystallised copper sulphate to 80 grams of water. To this solution is added 1 per cent. of strong sulphuric acid.

When performing an electrolysis the cathode plate is removed and well polished with sand or sand-paper (not emery paper), washed in water, and dipped for a few minutes in a bath of water containing a little sulphuric acid, the object of the acid being to remove any oxide not removed by the sand-paper. Having been rinsed in pure water, the plate is placed in the electrolytic cell, and a current passed for a short time. While this

1 Phil. Mag., October 1886. 
current is passing, the resistance of the circuit can be adjusted so that the current which passes is of the desired magnitude. The plate is then removed and well rinsed in pure water, allowed to drain on to a piece of white blotting-paper, and then dried by heating the tag with a spirit lamp. This heating must be rery carefully performed, otherwise the copper will oxidise. The electrode having cooled, it is weighed, and only placed in the electrolytic cell immediately before the current is passed, since copper is slightly soluble in copper sulphate solution. After the electrolysis is completed, the plate is removed, rinsed in water, to which a few drops of sulphuric acid has been added; then well rinsed in water free from acid, allowed to drain on to a sheet of clean white blotting-paper, and dried as before, and then weighed. The increase in weight in grams divided by the time the current passed and by 0.0003294 , gives the current in amperes.

215. Calibration of an Ammeter by Copper Deposition.-A circuit is arranged as shown in Fig. 230. The copper voltameter $\mathrm{v}$ is connected to a switch c, by means of which either the voltameter or a resistance $r$ can be placed in circuit. The circuit also contains the battery $\mathbf{E}$, the ammeter $\Delta, \mathbf{a}$ resistance box $\mathbf{B}$, and an adjustable carbon resistance $\mathbf{P}$. In order that changes in the resistance of the voltameter may not produce much variation in the strength of the current, it is advisable to use a battery having an E.M.F. considerably greater than is absolutely necessary to send the desired current and to include resistance in the circuit.

The cathode having been cleaned and placed in the cell, the current is allowed to pass and the resistance $R$ adjusted till the desired deflection of the ammeter is obtained. The switch is then turned over so as

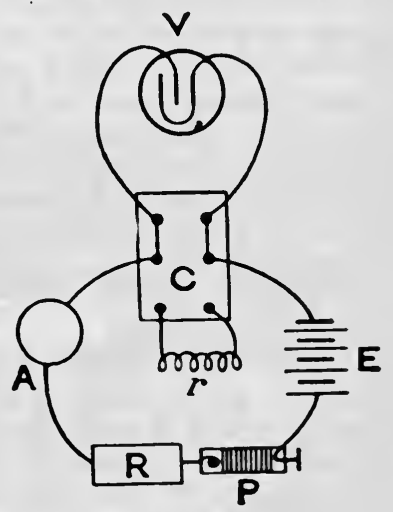

Fig. 230. to place $r$ in the circuit, and this resistance is adjusted till, whether the voltameter or the resistance are in circuit, the current is the same. The resistance $r$ may conveniently consist of a length of uncovered manganine or platinoid wire, the resistance being adjusted by drawing the wire through the binding screws of the commutator.

The adjustment of $r$ being complete, the cathode is removed, washed, dried, and weighed. During this opecation the current can be allowed to pass through the resistance $r$. The cathode is then replaced, and at a noted time, either on a chronometer or a clock, the current is switched over into the voltameter. The reading of the ammeter is noted, and the current kept constant, by adjusting the resistances $\mathbf{R}$ and $P$, for from half-an-hour to an hour, and then at a noted time the current is broken, the cathode removed, washed, and dried as before, and again weighed. 
If $w$ is the increase in weight of the cathode in grams, and the current flowed for $t$ seconds, the current in amperes is given by

$$
A=\frac{w}{0.0003294 t}
$$

Readings ought to be made at three or four points on the scale of the ammeter approximately equally distributed throughout the range of the instrument. In the case of the lower readings, if the instrument is a long range one, the current must be passed for a longer time than for the upper points. Since there is a small loss of weight when a copper plate is allowed to stand in copper sulphate solution, and this loss will be much more important at the lower current readings, a correction ought to be applied. It has been found that with a solution of the strength recommended above the loss amounts to about 0.005 milligrams per square centimetre of the cathode surface per hour. Hence if $S$ is the area of the cathode, and the electrolysis has lasted for $x$ hours, the observed increase of weight must be increased by $0.000005 S x$ grams.

216. The Silver Voltameter.-If suitable precautions are taken, a current can be measured by means of the deposition of silver to within 1 or 2 parts in 10,000. The most convenient form of voltameter is

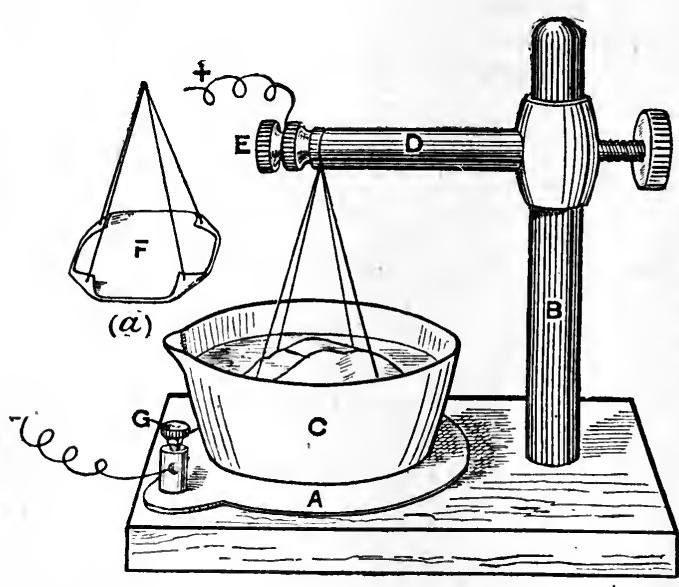

FIG. 231. that used by Rayleigh. In it the anode consists of a plate or rod of puresilverwrapped in Swedish filter paper, and the cathode consists of a platinum bowl or crucible. The platinum dish c (Fig. 231) rests in a hole in a copper plate $A$, which is itself supported on a wooden base, the wood having been treated with hot paraffin. A binding screw $G$ serves for the attachment of the negative terminal of the circuit. An upright ebonite rod B carries a horizonta] rod $D$, the height of which can be adjusted with a set screw. The anode $F$ is suspended from a binding screw attached to the end of the arm by four platinum wires, and the positive wire of the circuit is also attached to $\mathrm{E}$. 
The anode $\mathbf{F}$ consists of a square plate of pure silver, of which the corners have been turned up and holes have been drilled for the suspension wires. This anode is thoroughly cleaned by rubbing with seasand and washing, first in ordinary water, and finally in distilled water, and is then wrapped round with a piece of filter paper, so that the black powder of disintegrated silver which forms at the anode may not fall on the deposited silver in the bowl.

The platinum bowl which is to form the cathode is well cleaned, rinsed in distilled water, dried by heating over a spirit flame, and placed in a desiccator to cool. It is then weighed.

The silver nitrate solution is obtained by dissolving from 15 to 20 grams of pure recrystallised silver nitrate in 100 c.c. of distilled water. The solution ought to be neutral to litmus paper. If acid, digestion for some time with silver oxide will remove the acidity.

The method of conducting the electrolysis is similar to that described in the preceding section. After the deposition is complete, the solution is carefully poured off into a clean beaker and examined for specks of silver which may have become detached. If any are discovered, they must be replaced in the bowl. The bowl is then rinsed out by filling up with distilled water twice, the water in each case being poured into a clean beaker so that any detached particles of silver may be discovered. Finally the bowl is filled with water and allowed to stand for an hour. This water having been poured off, the bowl is heated over a small spirit flame till quite hot, then placed in a desiccator to cool, and finally weighed.

The current used ought not to exceed 0.03 ampere per square centimetre of cathode surface.

If the solution used in the electrolysis has been freshly prepared, or at any rate there has not been deposited from it more than 3 grains of silver per 100 c.c. of the solution, the electro-chemical equivalent may be taken as 0.001118 . Hence if $w$ is the weight of silver deposited in $t$ seconds, the current is given in international amperes ${ }^{1}$ by

$$
A=\frac{v}{0.001118 t}
$$

If the same solution is used for some time, it is found that the weight of silver deposited by a given quantity of electricity gradually increases, the increase amounting finally to as much as 1 part in 1000 .

Where great accuracy is aimed at, it must be remembered that it will probably be necessary to correct $w$ for the errors in the weights, and, further, that it is the absolute errors that are required, and not, as is often

1 The international ampere is defined as the current which in one second will deposit 0.001118 grams of silver. A true ampere, i.e. a tenth of a c.g.s. unit current, is probably slightly larger than the international ampere, the weight of silver which it will deposit in one second being 0.0011183 grams (see Phil. Trams. Royal Society (1902), vol. crcviii. p. 456). 
the case, simply the relative errors. For this reason it is advisable, where possible, to so adjust the time of deposition or the current, or both, that the weight of silver deposited amounts to very nearly an integral number of grams, so that as few separate weights as possible may be used when determining the weight of silver. It is also advisable to counterpoise the empty bowl by means of a separate set of weights, these weights remaining unchanged throughout the weighings. Allowance must also be made for want of equality in the balance arms (see § 26), and for buoyancy of the air (see $\S 28$ ).

217. The Potentiometer Method of Measuring a Current.-When a cell of which the E.M.F. is accurately known is available, then the most convenient and accurate method of measuring a current is to balance the difference of potential produced between the terminals of a known resistance when traversed by the current against the E.M.F. of the cell. The most convenient cell to use for

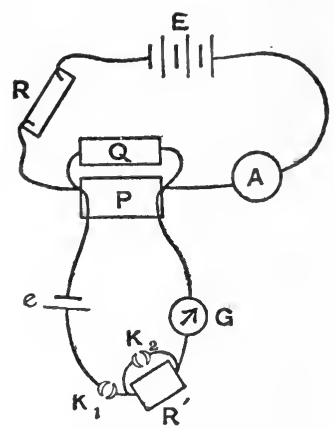

FIG. 232. this purpose is a cadmium cell, the construction of which is described in $\S 202$.

The arrangement of connections is shown in Fig. 232. Suppose that the graduations of an ammeter A are to be tested. A battery $\mathrm{E}$, preferably of accumulators, is connected so as to send a current through the ammeter and two adjustable resistances $\mathbf{R}$ and $\mathbf{P}$. Of these the absolute values of the resistances in the box P must be known, while since $\mathbf{R}$ is only used to adjust the current to the desired value, the absolute values are in this case immaterial. The standard cell $e$ is connected directly to one terminal of the resistance $P$ and to the other terminal through a key $K_{1}$, a resistance of about 100,000 ohms $\mathbf{R}^{\prime}$, and a high resistance galvanometer $\mathrm{G}$. A key $\mathrm{K}_{2}$ allows of the resistance $\mathbf{R}^{\prime}$ being cut out when the adjustment is nearly complete. The object of this resistance is to prevent any but very minute currents being passed through the standard cell. Since the E.M.F. of the cell $e$, particularly if it is a Clark cell, depends on the temperature, this cell should be placed in a water or oil bath, the temperature of which can be obtained by means of a thermometer.

The key $\mathrm{K}_{1}$ being open, the current in the main circuit is adjusted till the ammeter shows the deflection required. The key $K_{1}$ is then closed, $\mathrm{K}_{2}$ being open, and the resistance $\mathrm{P}$ is adjusted till the galvanometer is deflected in opposite directions by the change of $\mathbf{P}$ by the smallest step which the box allows. The higher of these two resistances being unplugged in $P, Q$ is adjusted till, even when the key $K_{2}$ is shut, the galvanometer is undeflected or, if it is impossible to obtain perfect balance, the resistance which would have to be removed from $\mathbf{Q}$ to produce balance is obtained by galvanometer deflections. 
If $A$ is the current in the main circuit, the difference of potential in volts between the terminals of the resistance $P$ is given by

$$
\frac{P Q}{P+Q} A,
$$

and this is equal to the E.M.F. of the cell $e$. Hence if $e_{t}$ is the E.M.F. of this cell ${ }^{1}$ at the temperature of the observation,

$$
A=\frac{P+Q}{P Q} e_{t} \text {. }
$$

Where the utmost accuracy is required, and the exact value of the current is immaterial, the resistance $P$ can consist of a single standard resistance coil, such as that shown in Fig. 197, the terminals of the standard cell circuit being connected to the binding screws $\mathrm{J}$ and $\mathrm{H}$. In this case, in addition to the resistance $R$ used to roughly adjust the current in the main circuit, there must be included one or more adjustable carbon resistances by means of which the current can be adjusted so that the galvanometer shows no deflection. When this is the case, the current $A$ is given by

$$
A=e_{t} / P .
$$

By using one or more standard cells in the auxilinry circuit and different resistances $P$, it is possible to apply this method for measuring both small and large currents. Thus if a standard $0.01 \mathrm{ohm}$ resistance is used, currents from a little over 100 amperes up to 1000 amperes can be measured by using one to ten cadmium cells in the auxiliary circuit.

1 The E.M.F.'s of Clark and cadmium cells at different temperatures are given in Table 26. 


\section{CHAPTER XXXI}

\section{THE BALLISTIC GALVANOMETER AND MEASUREMENT OF CAPACITY}

218. The Ballistic Galvanometer.-A ballistic galvanometer is one which is used for measuring the quantity of electricity which passes, the whole duration of the current being small compared to the time of swing of the galvanometer needle. The conditions which a ballistic galvanometer has to fulfil are that the time of vibration of the needle shall be fairly long, and that the damping shall not be very great. Subject to these conditions, any form of galvanometer can be used ballistically.

When a transient current is sent through a galvanometer, the duration of the current being such that the needle has not had time to move appreciably before the current has stopped, then it can be shown that the quantity of electricity $Q$ which has passed through the galvanometer is given by

$$
Q=\frac{H T}{\pi G}\left(1+\frac{\lambda}{2}\right) \sin \frac{\alpha}{2} \quad . \quad . \quad . \quad .
$$

where $a$ is the amplitude of the first swing of the needle, $T$ is the time of a complete vibration of the needle, $G$ is the field produced at the centre of the coils when they are traversed by unit current, and hence is a constant for any particular galvanometer, $\lambda$ is the logarithmic decrement of the oscillations of the needle, and $H$ is the strength of the magnetic field in which the needle swings. If $G$ is the field produced by a current of 1 ampere (10-1 c.g.s. units), then $Q$ is given in the above formula in coulombs (10-1 c.g.s. units of quantity).

First suppose that the damping is zero. Then if $M$ is the magnetic moment of the needle and $I$ its moment of inertia, the couple acting on the needle when the galvanometer is traversed by a current $C$, the needle being in its undeflected position is $M G C$. The impulse due to the couple acting during the time $d t$ is MGCdt. Hence if the whole of the transient current passes before the needle has appreciably moved from its position of rest, the total momentum communicated to the needle is $M G \int C d t$, or $M G Q$. The angular velocity with which the needle starts moving is $\frac{d \alpha}{d t}$, and the momentum it possesses owing to this angular velocity is $I_{\overline{d t}}^{d a}$. Hence equating the two expressions 
we have obtained for the momentum, we get

or

$$
\begin{aligned}
& I_{\frac{d \alpha}{d t}}=M G Q \\
& \frac{d a}{d t}=\frac{M G Q}{I} \quad \cdot \quad \cdot \quad \cdot \quad \cdot \quad \cdot \quad \cdot
\end{aligned}
$$

The kinetic energy possessed by the needle at the start of its swing is $\frac{1}{2} I\left(\begin{array}{l}d \alpha \\ d t\end{array}\right)^{2}$, and as the needle swings round this kinetic energy is converted into potential energy, due to the work done against the magnetic field in which the needle moves. When the deflection is $a$, the couple due to the field $H$ is $M H \sin a$. The work done while $a$ increases to $\alpha+d \alpha$ is therefore $M H \sin \alpha . d \alpha$, and the total work done up to the elongation $a$ is $M H \int_{0}^{a} \sin a . d a$, or $M H(1-\cos a)$.

Equating the kinetic energy at the start to the work done against the field up to the instant when the needle comes to rest at the elongation, we get

$$
\frac{1}{2} I\left(\frac{d a}{d t}\right)^{2}=M H(1-\cos a)
$$

Substituting for $\frac{d \alpha}{d t}$ from (2), and making use of the relation $1-\cos a$ $=2 \sin ^{2} \frac{\alpha}{\overline{2}}$

$$
\frac{M M^{2} G^{2} Q^{2}}{2 I}=2 M H \sin ^{2} \frac{a}{2} \quad \cdot \quad \cdot \quad \cdot \quad \cdot \quad \cdot .
$$

Now if $T$ is the period of the galvanometer needle, we hare (see Watson's Physics, § 430)

$$
T=2 \pi \sqrt{\frac{I}{M H}} .
$$

Substituting the value of $\frac{I}{M}$ obtained from this expression in (3), we get

$$
Q=\frac{H T \sin _{2}^{\alpha}}{\pi G} \text {. . . . . . . }
$$

When the motion of the needle of the galvanometer is damped, the logarithmic decrement being $\lambda$, then it can be shown that the righthand side of (4) must be multiplied by $(I+\lambda / 2)$ (see Maxwell's Electricity and Magnetism, vol. ii. p. 356).

If the throw is measured by means of a telescope, or lamp, and scale, 
the deflection being $d$ divisions of the scale, and the distance of the mirror from the scale $D$ divisions of the scale, then if $\alpha$ is small we may to a near approximation take

$$
\begin{gathered}
\sin \frac{\alpha}{2}=\alpha / 2, \text { and } \frac{d}{D}=\tan 2 \alpha=2 \alpha, \\
\sin \frac{\alpha}{2}=\frac{d}{4 D} .
\end{gathered}
$$

or

Hence

$$
Q=\frac{H T d}{4 \pi G D}\left(1+\frac{\lambda}{2}\right)=K d\left(1+\frac{\lambda}{2}\right)
$$

where $K$ is a constant so long as the conditions under which the galvanometer is set up are not changed.

If the throw $d$ is not small, then, as is shown in $\S 170$, we have

$$
\sin \frac{\alpha}{2}=\frac{d}{4 D}(1-\delta)
$$

where $\delta$ is a quantity which depends on the value of $d / D$, and can be obtained from Table 24 . In this case

$$
\begin{aligned}
Q & =\frac{H T d}{4 \pi G D}\left(1+\frac{\lambda}{2}\right)(1-\delta), \\
& =K d\left(1+\frac{\lambda}{2}\right)(1-\delta) .
\end{aligned}
$$

For ordinary measurements with the ballistic galvanometer it will in general be sufficient if the period $T$ is between ten and twenty seconds. When, however, the transient currents are produced by the change in induction in electromagnets and such like, where the time constant is very large, it will be always necessary to assure oneself that the whole current is over in a time small compared to the period of the needle, and it may be necessary to load the needle and so increase its period.

One of the assumptions made in deducing the formula for the ballistic galvanometer must be carefully borne in mind, namely, that the axis of the needle before the passage of the electricity must be parallel to the plane of the galvanometer coils. It is essential when using a ballistic galvanometer that this adjustment be carefully attended to at the start and verified periodically during the course of the measurements. The method by which the needle is adjusted parallel to the plane of the coils is given on pp. 426, 510 .

219. Determination of the Constant of a Ballistic Galvanometer.-Arrange a cell $\mathrm{E}$ (Fig. 233), preferably an accumulator, to send a current through the galvanometer $G$, and a resistance $R$ of at least 
100,000 ohms, the galvanometer being shunted by a resistance $s$. The value of $\mathbf{s}$ is adjusted till a deflection nearly to the end of the scale is obtained. This deflection having been noted, the current is reversed by the commutator $c$, and the deflection again observed. Let $d^{\prime}$ be the mean of the two deflections, the scale distance being $D$. Then, if the deflections are small, we have, if $\theta$ is the angle through which the needle has been deflected, $\theta=d^{\prime} / 2 D$; or more nearly $\theta=\frac{d^{\prime}}{2 D}(1-\delta)$, where the value of $\delta$ is obtained from Table 24. If $\Gamma$ is the resistance of the galvanometer, and $S$ and $R$ the resistances of the shunt and the resistance in the box $\mathrm{R}$ respectively, $E$ is the E.M.F. of the cell and $B$ its resistance. We have that the current sent by the battery is

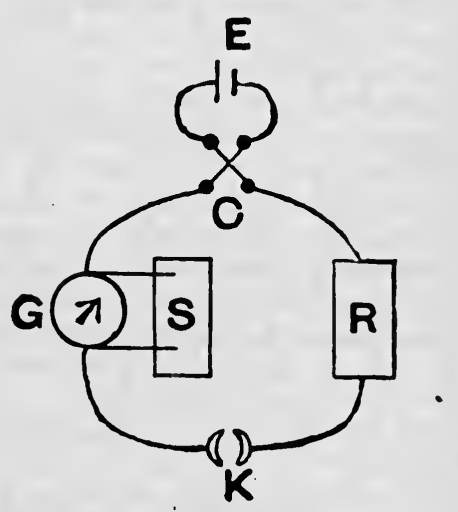

FIG. 233.

$$
E \div\left\{B+R+\frac{\Gamma S}{\Gamma+S}\right\}
$$

and the current through the galvanometer is

$$
\frac{E}{B+R+\frac{\Gamma S}{\Gamma+S}} \cdot \frac{S}{\Gamma+S} \text { or } \frac{E S}{(B+R)(\Gamma+S)+\Gamma S}
$$

But the current through the galvanometer is also given by ${ }^{1} \frac{H}{G} \tan \theta$. Hence 2

$$
\frac{H d}{2 G D}=\frac{E S}{(B+R)(\Gamma+S)+\Gamma S}
$$

The period $T$ is obtained by observing the time of a number of oscillations with a stop-watch or chronometer in the manner described

1 The expression $\frac{H}{\bar{G}} \tan \theta$ for the current through the galvanometer only holds so long as the field due to the current in the galvanometer coils is uniform throughout the space through which the needle moves. Whether it is justifiable to use this expression can be determined br making a calibration of the scale as described in $\$ 174$, and seeing whether over the range employed the carrent is proportional to the tangent of the angle of deflection.

${ }^{2}$ If $E$ is measured in volts and the resistances in ohms, then $G$ corresponds to the field produced at the centre of the coil when traversed by 1 ampere. 
in $\S 43$. The logarithmic decrement $\lambda$ is obtained by observing the amplitude of successive elongations in the manner described in $\S 56$.

The resistance of the battery, if an accumulator is used, will be negligibly small compared to $R$, and hence may be neglected. If, however, a cell having considerable resistance is used, its resistance must be measured. The E.M.F. must be measured by comparison with a standard cell.

Another way of proceeding is to use a standard cadmium cell in place of $E$, in which case the E.M.F. will be known ; but since the resistance of such cells is considerable, the value of $B$ will have to be determined, which is best done by the alternating current method. Since, however, if a single cell were placed in one arm of the bridge it would be able to send an appreciable current, the resistance of the arms not being very great, and as such a current would affect the E.M.F. of the cell, it is best to place two cells in series with like poles connected, so that their E.M.F.'s are opposed, and to measure the sum of their resistances. When making the deflection experiment with the ballistic galvanometer the two cells are used in series, but now with unlike poles connected.

220. Standardisation of a Ballistic Galvanometer by means of a Solenoid Inductor.- Suppose that a long, straight cylinder uniformly wound with insulated wire, the number of turns per centimetre being $n$, is traversed by a current of $A$ amperes, then, neglecting a small correction for the effect of the ends, the strength of the magnetic field near the centre of the coil is given by (Watson's Physics, § 516)

$$
F=0 \cdot 4 \pi n A \text { gausses.1 }
$$

If $F$ is the field strength at any point on the axis of the coil, then it can be shown that, making allowance for the effects of the ends,

$$
F=0 \cdot 2 \pi n A\left(\cos \psi_{1}+\cos \psi_{2}\right),
$$

where $\psi_{1}$ and $\psi_{2}$ are the angles subtended at the given point by a radius of the coil at either end. Hence if $r$ is the radius of the coil, and $2 l$ the length, we have for a point at the centre of the coil

$$
F=0.4 \pi n A \cdot \frac{i}{\sqrt{l^{2}+r^{2}}},
$$

or approximately,

$$
F=0 \cdot 4 \pi n A\left(1-\frac{1}{2} r^{2}\right)
$$

If the length of the coil is $100 \mathrm{~cm}$., so that $l=50 \mathrm{~cm}$., then the following

1 The name gauss has been given to the c.g.s. unit of magnetic force. 
table shows the value of the correcting factor $\left(1-\frac{1}{2} \frac{r^{2}}{l^{2}}\right)$ for different values of $r:-$

\begin{tabular}{|c|c|}
\hline Radius of Coll. & Value of $\left(1-\frac{1}{2} \frac{r^{2}}{l^{2}}\right)$ for $l=50 \mathrm{~cm}$. \\
\hline $5 \mathrm{~cm}$. & 0.9950 \\
4 & 0.9968 \\
3 & 0.9982 \\
2 & 0.9992 \\
1 & 0.9998 \\
\hline
\end{tabular}

It will be seen that for a coil 100 centimetres long and 4 centimetres in diameter the correction amounts to 0.08 per cent., that is, less than 1 part in 10,000, an amount which can in general be neglected. In what follows we shall suppose that the coil is so long compared with its diameter that, at or near the centre, the correction for the ends can be neglected. In any case, where a correction may be necessary it is quite easy to apply it.

'The total induction through a cross-section of the coil taken near the centre is $F \cdot \pi r^{2}$ or

\section{$0.4 \pi^{2} r^{2} n A$ maxwells. ${ }^{1}$}

Hence, since there is no field outside such a coil, at any rate if the coil is very long, the induction through a loop of wire which surrounds the coil near the centre will be

$$
0.4 \pi^{2} r^{2} n A \text {. }
$$

Thus if a secondary coil having $N$ turns be wound round the centre of the long coil, the total induction through this secondary will be

$$
0.4 \pi^{2} r^{2} n A N \text {. }
$$

If, now, the current $A$ in the primary be either started or stopped, the change in induction in the secondary will have the value given above, or if the current is simply reversed, the change in induction in the secondary will be

$$
0 \cdot 8 \pi^{2},{ }^{2} n A N
$$

Herice if $R$ is the resistance of the secondary and any conductors, such as leads and a ballistic galvanometer, included in the secondary circuit, the

1 The name maxwell is given to the unit of magnetic induction or flux in the c.g.s. system of electromagnetic units. 
quantity of electricity which will traverse the secondary when a current of $A$ amperes is reversed in the primary will be given by

$$
\begin{aligned}
Q & =\frac{0.8 \pi^{2} r^{2} n A N}{R} \text { c.g.s. units } \\
& =\frac{8 \pi^{2} r^{2} n A N}{R} \text { coulombs, }
\end{aligned}
$$

and if $d$ is the throw of the galvanometer, the scale distance being $D$, and the logarithmic decrement $\lambda$, we get

$$
Q=\frac{H T d}{4 \pi G D}\left(1+\frac{\lambda}{2}\right)=K d\left(1+\frac{\lambda}{2}\right)=\frac{8 \pi^{2} r^{2} n A N}{R} \text { coulombs, }
$$

where the throw is supposed small, and $K$ is the constant $(\S 218)$ of the ballistic galvanometer. This equation allows of the ballistic constant $K$ of the galvanometer being calculated.

A convenient form of coil is one 100 centimetres long with a diameter of 4 centimetres, formed by winding two layers of number 20 S.W.G. double-silk covered copper wire on a length of glass tube. The object of having two layers is that the two ends of the coil should be at the same end. If for any reason a single layer is used, then the wire should be brought along the axis of the coil, the two terminals being placed close together at one end. Each layer after being wound must be given a coating of shellac varnish. The number of turns must be counted, and the mean diameter over each layer measured. The mean radius of the wire can be obtained by dividing the length of the coil by the number of turns, and the mean radius of each layer of the coil is the external radius less the radius of the wire. The secondary consists of two layers containing 100 turns each

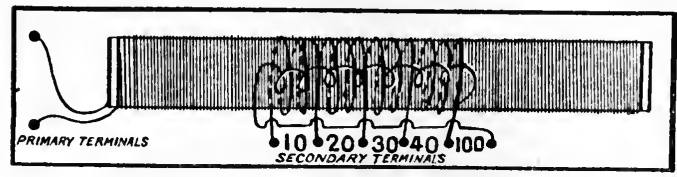

FrG. 234. of number 28 S.W.G. double-silk covered wire, wound uniformly over the middle part of the primary. It is convenient to wind the secondary in separate sections, the lower layer of 100 turns forming one section, and the upper layer being divided into sections of $10,20,30$, and 40 turns respectively, the points between the ends of the sections being brought to binking screws attached to the base on which the coil is fixed, as shown diagrammatically in Fig. 234. In this way it is possible to use a secondary containing various numbers of turns according to the quantity of electricity it is required to send through the galvanometer. 
221. To Compare the Capacities of two Condensers with the Ballistic Galvanometer.-When making experiments on condensers a discharge key, such as that shown in Fig. 235, will be found of much use. The metal arm $\mathbf{A B}$ is pivoted to $\mathbf{A}$, and there is a spring which tends to lift the arm up. At the end B there are two steps, such that when the catch on the top of the arm $\mathrm{c}$ is in the lower step, then the arm rests against a stop $\mathrm{E}$, while when the catch is in the middle step the arm does not touch either $\mathbf{D}$ or $\mathbf{E}$. If the catch is held back so as to miss the lower step, then the spring forces the arm $A B$ against the stop D. Suppose that one pole of a battery $x$ (Fig. 235, a) is connected to one plate of a condenser $c$, and the other pole to the stop $\mathbf{E}$ of the key, while the same plate of the condenser is joined through the galvanometer

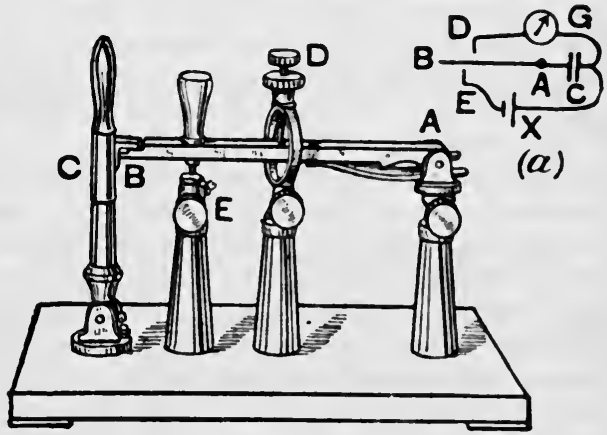

FIG. 235 . G to the stop $D$, the arm $\triangle B$ being connected to the other plate of the condenser. Then on pressing the arm $\mathbf{A B}$ down, the plates of the condenser are connected to the opposite poles of the battery, and hence the condenser is changed. On moving the catch so that $\triangle B$ is in its central position, the charged condenser is disconnected from the battery, while if the catch is released the arm $A B$ strikes against the stop $D$, and the condenser discharges through the galvanometer.

Each of the condensers which have to be compared is connected to the key in turn, and the throw of the ballistic galvanometer is obtained when the condenser is discharged after being charged by the same battery. If $E$ is the E.M.F. of the battery, a quantity which it is not necessary to know so long as it remains constant, and $d_{1}$ and $d_{2}$ are the throws produced by the discharge of two condensers of capacity $C_{1}$ and $C_{2}$ then the charges of the condensers are $E C_{1}$ and $E C_{2}$ respectively, and since the throws of the galvanometer are proportional to the charges, we have, if the throws are small,

$$
\frac{C_{1}}{C_{2}}=\frac{d_{1}}{d_{2}} .
$$

A nearer approximation is obtained by using the correction factors for $\sin \theta / 2$, given in Table 24, when

$$
\frac{C_{1}}{C_{2}}=\begin{aligned}
& d_{1}\left(1-\delta_{1}\right) \\
& d_{2}\left(1-\delta_{2}\right)
\end{aligned}
$$


If the capacity of one condenser is very much greater than that of the other, so that if the sensitiveness of the galvanometer or the E.M.F. of the charging battery is made large enough to give a measurable kick with the smaller condenser, the kick obtained with the larger is off the scale, a modification will be necessary.

In such a case the simplest, and at the same time the most accurate, method of procedure is to use a number of standard cells to charge the smaller condenser, and a single cell to charge the larger.

In the absence of standard cells, other cells or accumulators may be used, and the E.M.F.'s used with the two condensers can be compared by one of the methods given in $\S 204,205$.

Where a ballistic galvanometer of very long period is available, instead of using two batteries of different E.M.F.'s we may shunt the galvanometer when using the condenser of larger capacity. If the shunt is adjusted so that the kicks with the two galvanometers are exactly the same, then the ratio of the capacities is equal to $(G+S) / S$, where $G$ is the resistance of the galvanometer, and $S$ the resistance of the shunt.

When a discharge; such as that of a condenser, is passed through a shunted ballistic galvanometer, owing to the fact that the shunt is generally wound non-inductively, while the coils of the galvanometer must necessarily be wound inductively, the currents in the galvanometer and shunt do not bear a constant ratio to one another throughout the passage of the discharge. While the current is increasing, owing to the induction of the galvanometer, the fraction of the current passing through the shunt is greater than would be the case if the current were steady. While the current is decreasing, on the other hand, a larger proportion of the current passes through the galvanometer than when the current is steady. It can be shown, however, that the total quantity of the discharge which passes through the galvanometer bears the same ratio to the total discharge as does the fraction of the current which passes in the case of steady currents. If, however, the needle has time to appreciably move during the discharge, since any movement will decrease the impulse due to the passage of a given quantity of electricity, the effect of the delay in the current in the galvanometer, due to the induction of the coils, is to reduce the deflection produced by the fraction of the discharge which passes through the galvanometer. When the period of the needle is so great compared to the time occupied by the discharge that the whole discharge has passed through before the needle has moved, there is no error produced on this account. This effect is particularly likely to cause errors when the condenser employed is one in which there is much residual charge, so that the discharge is prolonged by this soakage effect, that is, with condensers with paraffined paper as the dielectric, or when measuring the capacity of a telegraph cable, in which case other methods must be employed; see $\$ 226$.

222. Comparison of Electromotive Forces with the Ballistic Galvanometer.-The E.M.F.'s of two cells or batteries can be compared by using each in turn to charge the same condenser, and then obtaining the 
throws when the discharge is passed through the ballistic galvanometer. The E.M.F.'s are to one another as the throws produced, if the deflections are small. When the throws are not small, then they must be corrected by means of the corrections for $\sin \theta / 2$, given in Table 24 .

It is advisable to take the throw with the cell of smaller E.M.F. first, as otherwise, if the condenser employed gives any residual charge, the residual charge left after the charge to the higher potential may appreciably affect the result obtained with the lower E.M.F. When using a condenser, it is always advisable to keep the armatures in metallic connection except when actually making a measurement, so as to allow any residual charge to escape.

223. Measurement of a Magnetic Field with the Earth Inductor. -If a coil is placed with its plane perpendicular to the lines of force of a magnetic field, and is then rotated through an angle of $180^{\circ}$ about an axis in its own plane, the quantity of electricity in c.g.s. units which will flow through a circuit connected to the coil is given by

$$
Q=\frac{2 a H}{R},
$$

where $a$ is the inductive area of the coil, that is, the sum of the areas enclosed by all the turns of wire on the coil, $R$ is the total resistance in c.g.s. units of the circuit in which the coil is included, and $H$ is the strength of the magnetic field. If $R$ is measured in ohms $\left(10^{9}\right.$ c.g.s. units), then

$$
Q=\frac{2 a H}{R} 10^{-\rightarrow}
$$

If a ballistic galvanometer is included in the coil circuit, so that $R$ includes the resistance of the coil, the leads and the galvanometer, then if $d$ is the throw when the coil is reversed, we have, if $d$ is small, 1

Hence

$$
\begin{aligned}
& Q=K d(1+\lambda / 2) . \\
& H=\frac{K d R}{2 a}\left(1+\frac{\lambda}{2}\right) \cdot . . . \cdot .
\end{aligned}
$$

from which, if we know the constant $K(\$ 218)$ of the ballistic galvanometer, the value of the strength of the field can be obtiined.

A form of coil suitable for applying this method to the measurement of the earth's field is shown in Fig. 236. It consists of a square coil $\mathbf{A B}$ containing a number of turns of insulated wire, the area included by the turns on each layer having been measured while the coil was being wound. This coil can be rotated about an axis ss', two stops limiting the rotation

1 If $d$ is not small, then we must take $Q=K d(1-\delta)$, the value of $\delta$ being ob. tained from Table 24 corresponding to $\sin \theta / 2$. 
to exactly $180^{\circ}$. The ends of the coil are connected by short lengths of flexible wire with the binding screws $C$ and $D$, by means of which the coil

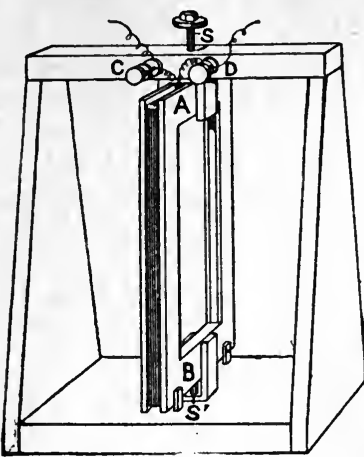

FIG. 236. is connected to the rest of the circuit. It will be seen that the coil stand which carries the coil can be placed either with the axis about which the rotation takes place vertical or horizontal.

By means of a prismatic compass or long suspended magnetic needle draw a line on the table magnetic east and west, and arrange the coil so that when against one of the stops the plane of the coil is at right angles to the magnetic meridian. Then determine the throw $d_{1}$ of the ballistic galvanometer when the coil is rotated through $180^{\circ}$. Next place the stand on its side so that the axis about which the coil can turn is horizontal, and starting with the coil in the horizontal plane determine the throw $d_{2}$ when the coil is rotated through $180^{\circ}$.

Now if $H$ and $V$ are the horizontal and the vertical components respectively of the earth's field at the spot where the coil is placed, we have

$$
H=\frac{K d_{1} R}{2 a} \text { and } V=\frac{K d_{2} R}{2 a}
$$

Hence if $\theta$ is the magnetic dip,

$$
\tan \theta=\frac{V}{H}=\frac{d_{2}}{d_{1}} \text {. . . . . . . . }
$$

If the value of the horizontal component of the earth's field is known, then an earth inductor forms a convenient means of determining the constant $K$ of a ballistic galvanometer, for if we know $H$, then the equation (1) above will allow of $K$ being calculated.

When measuring the strength of a magnetic field, the value of the constant $K$ may with advantage be determined by including in the galvanometer circuit the secondary of the solenoid inductor $(\S 220)$, and taking throws with the earth inductor and the solenoid inductor alternately.

1'ace the secondary of the solenoid inductor in series with the earth inductor and a ballistic galvanometer, care being taken to place the solenoid at such a distance from the inductor and the galvanometer that the solenoid when traversed by a current has no appreciable direct magnetic effect on either. This can easily be tested by breaking the galvanometer circuit and cutting out the secondary by joining together the ends of the leads which come from the galvanometer and earth 
inductor, and seeing whether reversing the current in the primary of the solenoid produces any kick of the galvanometer. If only a very slight kick is produced, this can often be reduced to zero by turning the solenoid round into some definite azimuth, which must be found by trial. When this adjustment is being made, the earth inductor must occupy the position it will occupy either at the commencement or end of its movement.

The throw of the galvanometer must then be determined, (1) when the earth inductor is rotated through $180^{\circ}$, and (2) when a current of $A$ amperes is reversed in the primary of the solenoid inductor. If $H$ is the field in which the earth inductor is turned, $a$ is the inductive area of the earth inductor, and $d_{1}$ and $d_{2}$ are the throws,

then

$$
H=\frac{K d_{1} R}{2 a}(1+\lambda / 2)
$$

and

$$
K=\frac{0 \cdot 8 \pi^{2} r^{2} n A N}{R d_{2}\left(1+\frac{\lambda}{2}\right)},
$$

where $R$ is measured in c.g.s. units $\left(10^{9} \mathrm{ohms}\right)$. From these equations $K$ can be eliminated, and hence $H$ may be calculated.

The current $A$, or the number of turns in the secondary $N$, should be so adjusted that $d_{2}$ is nearly the same as $d_{1}$. When this condition is fulfilled, it is not necessary to apply a correction to reduce to the sine of half the angle of throw, since the correction would affect $d_{1}$ and $l_{2}$ in the same ratio.

The current $A$ must be measured either with a sensitive ammeter, or by the potentiometer method described in $\$ 217$. For reversing the current a Pohl commutator, such as that shown in Fig. 179, will be found most convenient. Since it is important that the current should not vary between the time when it is measured and the instant when the throw is taken, it is advisable to use accumulators, and to use a higher E.M.F. than is absolutely necessary, reducing the current to the desired amount by means of an added resistance.

224. Measurement of the Strength of a Magnetic Field with an Exploring Coil.-The principle of the earth inductor may be employed to measure other magnetic fields besides that due to the earth. As most such fields which have to be measured are not uniform, the coil, by the rotation of which the induced current is obtained, is made much sumaller than that used in the earth inductor. The induction coil used for exploring the magnetic fields near electromagnets is attacher to a handle, and is made narrow, for the coil has often to be introduced into narrow gaps, the necessary inductive area heing obtained by using a large number of turns of very fine wire. 'The throw can be caused in two ways. Where there is sufficient room, the coil can be turned through $180^{\circ}$. For this 
purpose there is fitted a spring and catch, and on releasing the catch and holding the handle firm the coil is rapidly rotated through $180^{\circ}$ by the spring. If the space in which the field is to be measured is confined, then the kick is measured when the coil is suddenly removed from the given position to some other position where the field is negligible com. pared to that being measured.

As an exercise in this method, the strength of the field between the pole-pieces of an electromagnet for different currents in the filed coils and for different distances between and shapes of the pole-pieces may be measured.

Such an exploring coil may be standardised by using two equal solenoids placed with their ends close together, only just allowing space for the insertion of the exploring coil, and determining the throw when a known current is reversed in the solenoids. If, at the same time, a throw is taken with a secondary wound on one of the solenoids, so that the current in the two cases is the same, it is possible to calculate the inductive area of the inductor coil, making use of the expressions given in the preceding sections.

225. Comparison of Capacities by de Sauty's Method.-The two condensers to be compared, $\mathrm{c}_{1}$ and $\mathrm{c}_{2}$, are placed in the adjacent arms of a Wheatstone bridge (see Fig. 237). The re-

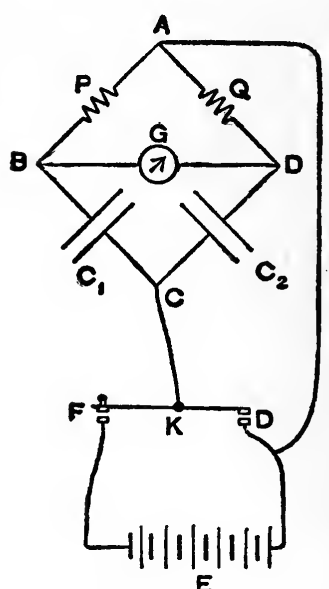

E

Frg. 237. maining arms consist of the two non-inductive resistances $P$ and $Q$. A high resistance galvanometer $\mathrm{G}$ is connected between $\mathrm{B}$ and $\mathrm{D}$, while a battery, which ought to have a rather high E.M.F., but since very little current is taken need not have the power of sending a large current, is connected to $A$ and to a key k. This key is so arranged that when the knob is pressed it makes contact at $F$, and the condensers are charged to the E.M.F. $\mathbf{E}$ of the battery. When the key is released, contact is made at $D$, and the condensers discharge.

The resistances $\mathbf{P}$ and $\mathbf{Q}$ are adjusted till there is no galvanometer kick when the key is depressed or released. When this condition is fulfilled we have

$$
\frac{C_{1}}{C_{2}}=\frac{Q}{P}
$$

The resistances $P$ and $Q$ should be large, and the E.M.F. of the battery be increased till sufficient sensitiveness is obtained. This method is not applicable when the absorption of one of the condensers is very different from that of the other.

The charges of the condensers, when the key makes contact at $F$, are $C_{1} E$ and $C_{2} E, E$ being the E.M.F. of the battery. The portion of the 
charge of $C_{1}$ which passes through the galvanometer when the key makes contact at $\mathrm{D}$ is

$$
\frac{P}{P+Q+G} C_{1} E
$$

In the same way, the portion of the charge of $C_{2}$ which passes through the galvanometer is

$$
\frac{Q}{P+Q+G} C_{2} E \text {. }
$$

Hence, since these discharges pass through the galvanometer in opposite directions, and the galvanometer is undeflected, we have

or

$$
\begin{gathered}
P C_{1}=Q C_{2}, \\
\frac{C_{1}}{C_{2}}=\frac{Q}{P} .
\end{gathered}
$$

226. Comparison of Capacities by the Method of Mixtures.-One coating of either condenser is connected to the middle pair of mercury cups of a Pohl commutator $\mathrm{k}$ (Fig. 238 ), from which the cross connectors between the end cups have been removed. The other coatings of the condensers are connected to one terminal of a high resistance galvanometer G, and also to earth. The testing battery $\mathbf{E}$ is connected to the terminals of two adjustable high resistance boxes $P$ and $Q$. The points $A$ and $B$ are connected to the one set of outside mercury cups of the Pohl commutator, while the other pair are connected through a key $k$ to the galvanometer. A wire is also run between the point $B$ and that terminal of the galvanometer to which the condensers are directly connected.

When the key $\mathbf{r}$ is placed so as to connect the mercury eujs 1 to 3

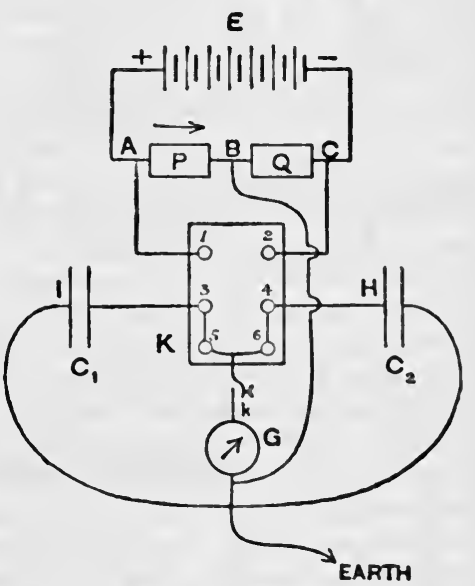

FIG. 238. and 2 to 4 , the condensers are charged. If the current passing throngh $P$ and $Q$ is $i$, the charge of $C_{1}$ will be $i P C_{1}$ for the difference of potential between $\mathrm{A}$ and $\mathrm{B}$, and therefore also between the terminals of the condenser $\mathrm{c}$ is $i P$. In the same way the charge of $C_{2}$ is $i\left(C_{2}\right.$. When the key $\mathrm{K}$ is placed so as to connect 3 to 5 and 4 to 6 , the charges in the condensers can mix, and the resulting charge in the two is equal to 
$i\left(P C_{1}-Q C_{2}\right)$. When the key $k$ is closed, this charge passes through the galvanometer. Hence if on closing $k$ there is no galvanometer. kick, we conclude that there was no charge left after the condenscrs hare been connected.

Hence

$$
\begin{gathered}
P C_{1}-Q C_{2}=0 . \\
\frac{C_{1}}{C_{2}}=\frac{Q}{P}
\end{gathered}
$$

or

Thus the experiment consists in altering the resistances $\mathbf{P}$ and $\mathbf{Q}$ till on mixing and depressing the key $k$ there is no galvanometer deflection.

When applying this method, particular care must be taken that the battery is well insulated.

A modification of this method, described by Young, ${ }^{1}$ is particularly applicable in the case of long telegraphic cables in which there is appreciable dielectric conductivity, and where al'so the great length of the core

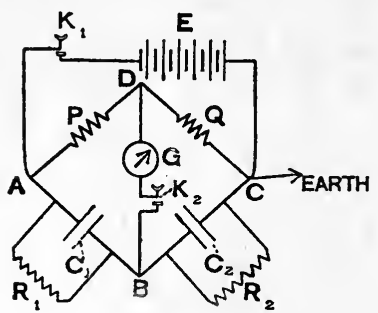

FIG. 239. causes a considerable time to elapse before the cable becomes charged. The connections are represented in Fig. 239, where $o_{1}$ is the condenser to be tested, between the coatings of which there is leakage, which in the figure is represented by a resistance $R_{1}$, placed in parallel with the condenser. The standard condenser $\mathrm{C}_{2}$ is supposed to have a very high dielectric resistance, and an adjustable high resistance $\mathbf{R}_{2}$ is placed across its terminals. The approximate values of $\mathbf{P}$ and $\mathbf{Q}$ for $\mathbf{a}$ balance having been found by the method of mixtures as in the previous method, the key $\mathrm{k}_{2}$ is kept depressed and the resistance $R_{2}$ is adjusted so that on making the battery circuit by depressing the key $\mathrm{k}_{1}$, and keeping the current on for, say, one minute, there is no steady galvanometer deflection. The absence of this steady deflection indicates that the bridge is balanced for steady currents, so that $R_{1} / R_{2}=P / Q$.

The resistances $P$ and $Q$ are then finally adjusted, the procedure being as follows :-The key $K_{2}$ being closed, the battery key $K_{1}$ is closed for one minute. The key $\mathrm{K}_{2}$ is then opened, and then the battery key $\mathrm{K}_{1}$. The charges of the condensers are allowed to mix for from fifteen to thirty seconds, and then the key $\mathrm{K}_{2}$ is closed and the galvanometer observed. When $P$ and $Q$ are so adjusted that there is no galvanometer kick, the following relation holds, as in the previous method

$$
\frac{C_{1}}{C_{2}}=\frac{Q}{P}
$$

1 Journal of the Institution of Electrical Engineers (1889), vol. xxviii. p. 485. 
227. Measurement of a Capacity in Electromagnetic Units in terms of a Resistance and a Time.-A condenser c (Fig. 240) is arranged so that one armature is permanently connected with one corner of a Wheatstone bridge, while by means of a commutator the other armature is alternately connected to the points $a$ and $b$, so that the condenser is alternately charged and discharged. The commutator is shown at $(a), 1$ and consists of an ebonite cylinder having two stepped pieces of brass on the cylindrical surface. The brushes $a, b$, and $c$ press against the brass, and as the commutator rotates the brush $(c)$ is
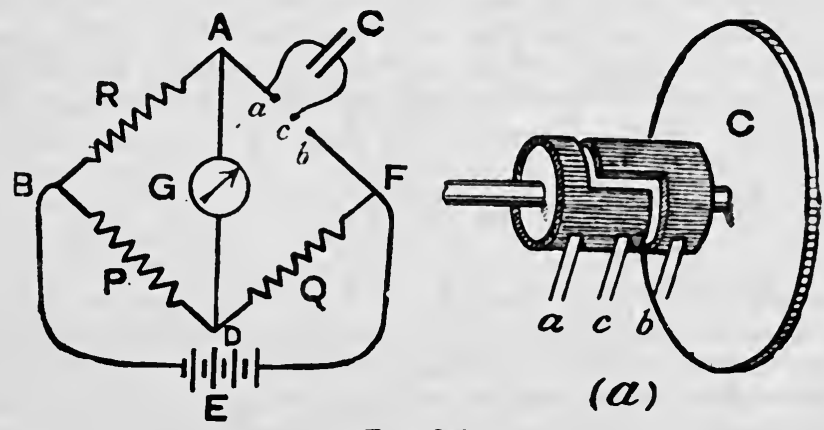

FIt. 240.

alternately connected to $a$ and to $b$. The commutator is driven by a motor, and the speed is measured by means of a tuning-fork and a stroboscopic disc c (see $\$ 109$ ).

If $C$ is the capacity of the condenser, and the commutator charges and discharges the condenser $n$ times per second, while the resistances of the arms $P, Q$, and $R$ are so adjusted that the galvanometer $G$ is undeflected, we have (see J. J. Thomson's Electricity and Magnetism, $\S 260)$.

$$
\frac{1}{n C}=\frac{R(P+Q+B)+B P}{P(Q+B)+(G+R)(P+Q+B)}\left\{G+Q+\frac{Q}{P}(G+R)\right\},
$$

where $G$ is the resistance of the galvanometer arm, and $B$ that of the battery arm. Generally the resistance of the battery is so small compared to the other resistances that it can be neglected, so that the expression reduces to

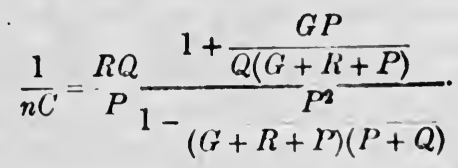

1 A description of a good form of commutator will be fonnd in a paper by Fleming and Clinton, Philosophical Magazine (1903), v. $\mu$. 
If the capacity of the condenser is small, then $R$ will have to be taken very large, and hence $Q$ will also be very large compared to $P$. In this case the expression reduces to

$$
\frac{1}{n C}=\frac{R Q}{P}
$$

and it will be noticed that the condenser and commutator produce the same result as if they were replaced by a resistance $1 / n C$.

If the resistances are expressed in ohms, then the capacity $C$ will be expressed in farads $\left(10^{-9}\right.$ c.g.s. units). To obtain the capacity in microfarads, the capacity expressed in farads must be divided by $10^{6}$.

When measuring a fairly large capacity, say, of the order of a microfarad, a Post Office bridge may be used, the ratio arms $P$ and $Q$ being 10 to 1000 . When very small capacities are being measured, it is necessary to employ large resistances, and a battery having a large E.M.F., say, 50 or 100 volts. For an air condenser having plates of about $30 \mathrm{~cm}$. in diameter at a distance of about a millimetre, $R$ may be a megohm $(1,000,000 \mathrm{ohms})$ and $Q$ about $300,000 \mathrm{ohms}$, balance being obtained by using a Post Office or equivalent resistance box for $P$. When using high resistances and high E.M.F.'s, it is very important to carefully insulate the various wires, while one point, preferably one terminal of the galvanometer, may be connected to earth by being connected to the water or gas pipes.

By determining by this method the capacity in electromagnetic units of a condenser of such a shape that the capacity in electrostatic units can be calculated from the dimensions, the ratio of the units, $V$, may be determined. A suitable condenser can be built up of two silvered sheets of plate glass, the silver being removed for about $2 \mathrm{~cm}$. round the edge. The plates are placed with their silvered sides towards one another, and separated by three small pieces of ebonite or glass, the distance between the plates being obtained by measuring the thickness of these distance pieces with a screw gauge. In order to make contact, a thin strip of the silvering is left on each plate leading up to one of the corners, a piece of the glass at one of the other corners being cut off, and the plates are so arranged that the silver strip on one plate is opposite the trimmed corner of the other. Contact may be made by clamping a wire down on the silver, a few thicknesses of thin tin-foil being interposed to ensure good contact.

If $A$ is the area of either of the plates, and $d$ is the distance between them, then, neglecting the effect of the edges, the capacity in electrostatic units is $A / 4 \pi d$.

Another method of conducting the experiment, which eliminates the effect of the edge of the condenser plates, is to have two cylindrical condensers of the same diameter but different lengths, say, one twice as long as the other. The capacities of these condensers are measured, and let us suppose the numbers obtained are $C_{1}$ and $C_{2}$, the lengths being 
$l_{1}$ and $l_{2}$ respectively, and the radius of the outer surface of the inner tube being $r_{1}$, and that of the inner surface of the outer tube being $r_{2}$. Then since, neglecting the effect of the ends, the capacity per unit length of such a condenser is

we have

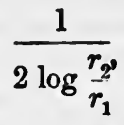

$$
C_{2}-C_{1}=\frac{l_{2}-l_{1}}{2 \log \frac{r_{2}}{r_{1}}}
$$

Cylindrical condensers suitable for this experiment may be made up out of "triblet" drawn brass tube, $r_{1}$ being about $2.5 \mathrm{~cm}$., and $r_{2}=2 \cdot 6$, the lengths being $15 \mathrm{~cm}$. and $30 \mathrm{~cm}$. The brass tubes have their ends turned off square, and are fitted at the ends into shallow grooves cut in discs of ebonite. On one of the discs are screwed two binding screws connected by wires with the two tubes. 


\section{CHAP'TER XXXII}

\section{MEASUREMENT OF SELF AND MUTUAL INDUCTION}

228. Measurement of the Coefficient of Self-Induction of a Coil by Rayleigh's Method. - The coefficient of self-induction of a coil which does not contain any magnetisable material, and which has a fairly large

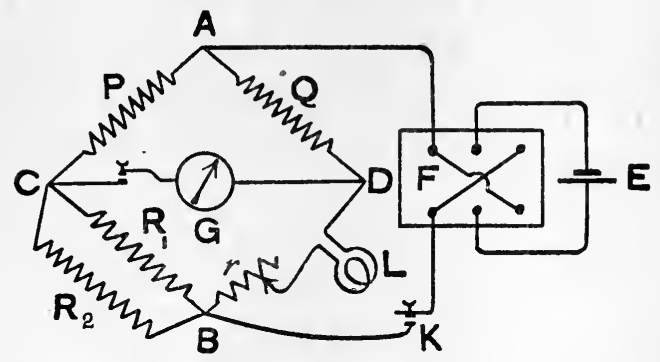

FIG. 241. coefficient $\left(10^{7} \mathrm{~cm}.\right)$, can be measured by a method described by Maxwell and modified by Rayleigh. The coil L (Fig. 241) is placed in one arm of a Wheatstone's bridge, and the bridge having been balanced for steady currents, the kick $\alpha$ of a ballistic galvanometer $G$ obtained when the cur-

rent is reversed is noted. Next the resistance of the arm CB is altered by an amount $d R$, and the steady deflection $\theta$ of the galvanometer produced, owing to the want of balance, is noted. If, then, $T$ is the period of the galvanometer needle, $\lambda$ its logarithmic decrement, and $C_{1}$ and $C_{2}$ the currents traversing the arms CB and BD respectively when the balance is upset by the change of resistance, it can be shown ${ }^{1}$ that the coefficient of self-induction $L$ of the coil is given by

$$
L=\frac{T \delta R C_{1}}{2 \pi C_{2}}\left(1+\frac{\lambda}{2}\right) \frac{\sin \alpha / 2}{\tan \theta} \text { henrys.2 }^{2}
$$

In the practice of this method it will be found advantageous to make the two ratio coils $P$ and $Q$ equal, and each as nearly equal in resistance to the coil as possible. Then in order to be able to obtain the exact balance required when making the observations of throw, an adjustable resistance $r$ is placed in series with the coil, and the branch CB consists of two resistance boxes placed in parallel. The adjustable resistance $r$

1 J. J. Thomson, Electricity and Magnetism, § 248.

8 A henry is $10^{9} \mathrm{~cm}$., and the value obtained for $L$ is given in henrys if $\delta R$ ia measured in ohms. 
may either consist of a short length of uncovered German silver or manganine wire, the length of which is adjusted by slipping it through the binding screw by which the coil is connected to the bridge, or by an adjustable carbon resistance. So long as the carbon resistance is kept fairly tightly screwed up, it will be found quite satisfactory and very much more easily adjusted than the wire. In addition to the commutator $F$, it is advisable to have a key in both the battery and the galvanometer circuits. If a Post Office bridge is used, these keys are provided. The object of the keys is to allow of the battery being connected only for as short a time as possible. If the current is allowed to flow continuously, the balance will be found to be continually altering. This is due to the change in temperature of the resistances, particularly that of the coil, for the coil generally consists of copper wire, for which the temperature coefficient is very much greater than is the case for the alloys used to construct the resistance coils.

In order that it may be possible to adjust the resistance of the arm CB to the exact value required for balance, it is necessary that one of the resistances $R_{1}$ and $R_{2}$ shall be large, that is, the other resistance must only slightly exceed the resistance in the arm BD. Hence the resistance, $R_{2}$ say, being infinite, $R_{1}$ is adjusted till it is the nearest unit higher than the resistance of the coil $L_{n}$. The adjustable resistance $r$ is then altered till balance is very nearly secured, care being taken, however, to have $B_{1}$ a little too great. The final balance is then obtained by altering $\mathbf{R}_{2}$. Immediately balance for steady currents has been obtained, the throw obtained by reversing the current with the commutator $F$ is observed, the battery and galvanometer keys being of course kept closed. The resistance $R_{2}$ is then altered by such an amount that the steady deflection is approximately the same as the throw and the deflection is noted. Balance for steady currents is then again secured, and the whole series of operations repeated several times, the interval between the several observations being made as small as possible.

The time of vibration of the galvanometer must be determined, as also the logarithmic decrement. When determining the logarithmic decrement the galvanometer key must be kept closed, for the decrement on open circuit is not as great as that on closed circuit, owing to the currents induced in the coils by the motion of the needle when the circuit is closed.

The value of the factor $C_{1} / C_{2}$ can generally be calculated on the assumption that none of the current goes through the galvanometer, since with a sensitive galvanometer the current required to produce the steady deflection is very small. Hence since $R+\delta R$ is the resistance of the branch $\mathrm{CB}$ when the steady deflection is being obtained, and the resistance of the branch $\mathrm{BD}$ is the same as the resistance $R$ of the branch OB when balanced for steady currents, since $P=Q$, we have

$$
\frac{C_{1}}{C_{8}}=\frac{R+Q}{R+\delta R+P}
$$


In this case, as in all others where a ballistic galvanometer is used, an auxiliary coil should be placed near the galvanometer with a cell and tapping key connected, so that by momentarily passing a current through this coil the observer may be able to check the swing of the galvanometer needle.

229. Use of a Rotating Commutator when making Measurements of Induction (Ayrton and Perry's Secohmmeter).-In the case of coils having small coefficients of self-induction, the method described in the last section is not sufficiently sensitive to give accurate results. In order to increase the sensitiveness by sending a rapid succession of induction currents through the galvanometer, Ayrton and Perry ${ }^{1}$ have designed a commutator which they call a secohmmeter. This commutator performs two functions. In the first place, it makes the battery circuit, the

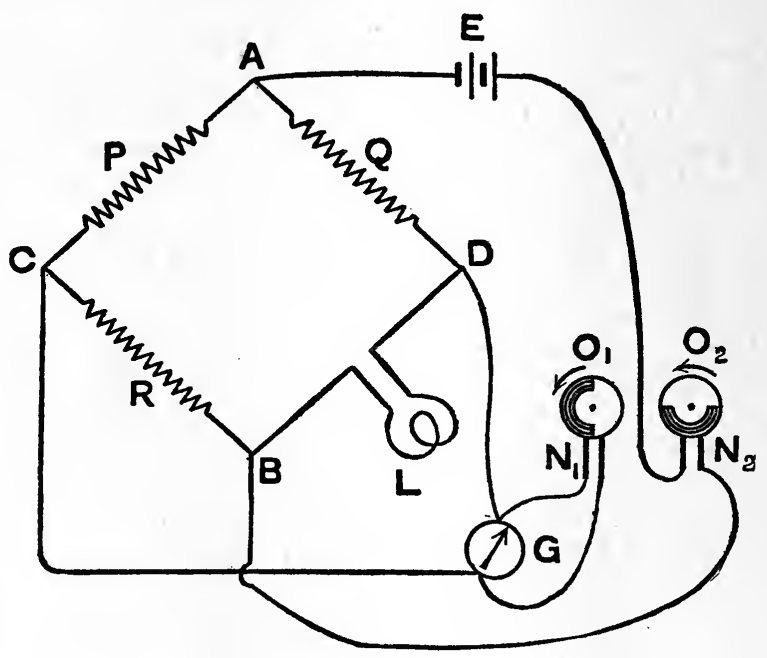

FIG. 242.

galvanometer being short-circuited. Next it removes the short-circuit from the galvanometer, and then breaks the battery circuit, so that the induction current can pass through the galvanometer. Next shortcircuits the galvanometer and afterwards makes the battery circuit, the short-circuit on the galvanometer preventing any of the make induced current passing through the galvanometer. This cycle of operations is repeated at a speed which is only limited by the necessity that the current should have attained its final state before the galvanometer is made or broken. The arrangement is shown diagrammatically in Fig. 242.

1 Journal of the Society of Telegraph Engineers (1887), vol. xvi. p. 292. 
The commutator consists of two parts, one $o_{1}$, being connected to the galvanometer $\mathrm{G}$, and the other, $\mathrm{o}_{2}$, to the battery $\mathrm{E}$. In reality these two parts would be fixed to the same axle, so that they rotate at the same speed. The shaded parts of the commutators are supposed to be metal, and the unshaded parts non-conducting, so that when the shaded portion is opposite the brushes $\mathrm{N}_{1}$ or $\mathrm{N}_{2}$ the galvanometer is short-circuited, or the battery circuit completed, as the case may be, while when the unshaded portion of the cylinder is opposite the brushes, the galvanometer is in circuit or the battery circuit broken. One form of commu. tator is shown in Fig. 243, the arrangement of which will be obvious from the drawing. In another form the brushes are lifted up to break the circuits by means of cams which are attached to a rotating shaft. Since it is necessary to know the speed at which the commutator is rotating, some form of speed indicator must be attached. In the absence of a direct reading speed indicator a stroboscopic disc may be attached to the commutator and the speed of rotation ad-

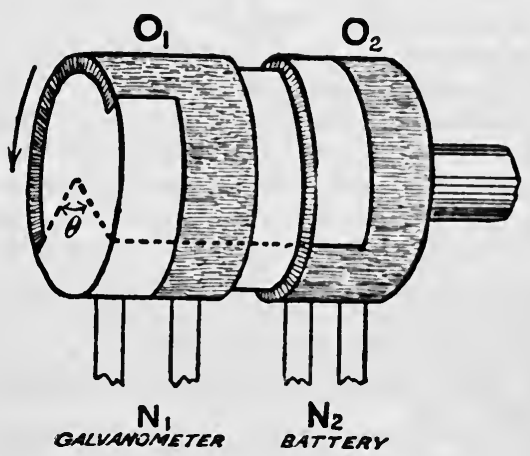

FIG. 243. justed, so that when viewed through a pair of slits attached to the prongs of a tuning-fork one of the rows of dots appears stationary, in the manner described in $\S 109$.

The method of using the secohmmeter is as follows:-Using the bridge in the ordinary manner, balance for steady currents. Then rotate the commutator at a speed of $n_{1}$ revolution per second, and adjust the resistance $R$ till the galvanometer is undeflected, and let the change in $R$ necessary be $\delta R_{1}$. Next rotate the commutator at some other speed, $n_{2}$, and again determine the change in $R$, say, $\delta R_{2}$, from the value for balance with steady currents necessary to restore balance. Then if, within the limits of accuracy of the settings, we have

$$
\frac{\delta R_{1}}{n_{1}}=\frac{\delta R_{2}}{n_{2}}
$$

it shows that the frequency of the commutator has not been so great that the steady state has not had time to become established before the galvanometer is either put into circuit or cut out. If, however, the ratios are different, then a slower speed of the commutator must be tried, till a constant value for the ratio is obtained. 
A value of $\delta R / n$ which does not vary with the speed having been obtained, the coefficient of self-induction of the coil is given by ${ }^{2}$

$$
L=\frac{K \delta R}{n} \text { henrys, }
$$

where $K$ is a constant depending on the setting of the two portions of the commutator with reference to one another. This constant is the ratio of the angle $\theta$ through which the commutator turns between the instant when the battery circuit is closed and the instant when the galvanometer is short-circuited to $360^{\circ}$. The angle $\theta$ (Fig. 243) may be determined experimentally by throwing the bridge out of adjustment, and having attached a divided circle to the commutator, rotating this latter by hand and noting the angle through which it must be rotated between the production of a deflection, owing to the closing of the battery circuit, and the return of the needle to zero owing to the shortcircuiting of the galvanometer. The most satisfactory method of determining $K$ is, however, to use the commutator to measure a known self-induction, and to deduce the value of $K$ from the equation

$$
K=\frac{n L}{\delta R} \text {. }
$$

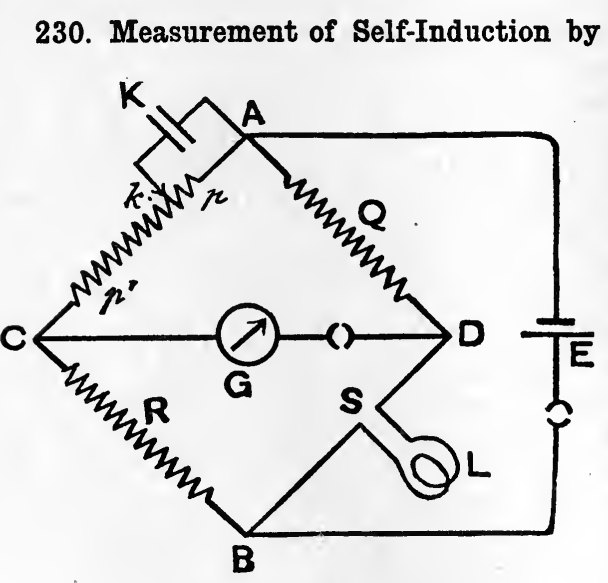

FIG. 244. cit 8 i m in ton's Method 2): - A Wheatstone's bridge is arranged with the coil in one of the branches, and balance for steady currents is obtained. A condenser $\mathbf{K}$ is then arranged as a shunt on part of the resistance of the arm AC (Fig. 244), which is conjugate to the arm containing the coil. The ratio of the resistances $p$ and $p^{\prime}$, into which the point $k$, where one armature of the condenser is connected, divides the resistance forming the arm AC, is varied, till on making or breaking the battery circuit there is no galvanometer kick. When this is the case, the coefficient of selfinduction of the coil is given by

1 For a proof of this formula the reader must refer to the paper by Professors Ayrton and Perry, loc. cit.

\footnotetext{
2 Philosophical Magazine (1887), xxiv. p. 54.
} 


$$
L=K p^{2} S /\left(p+p^{\prime}\right) \text { henrys, }
$$

where $K$ is the capacity of the condenser in farads, and $S$ is the resistance of the arm BD in which the coil is placed.

If $x$ is the current fowing through the arm BD, i.e. through the coil, and $y$ the current flowing through the arm $\mathbf{A C}$ when the currents are steady, then the charge in the condenser is $K p y$, and on breaking the battery circuit the quantity of electricity which traverses the coil owing to self-induction is $L x / X$, where $X$ is the resistance of the arm BD together with the resistance of the remainder of the network between the points $B$ and $D$ when the battery circuit is broken. ${ }^{1}$ Thus

$$
X=R+S+\frac{(P+Q) G}{P+Q+G},
$$

where $P$ is written for the resistance of the arm $\mathbf{A C}$, and $G$ is the resistance of the galvanometer. Hence the quantity of electricity which traverses the galvanometer due to the self-induction of the coil is

$$
\frac{L x}{R+S+\frac{(P+Q) G}{P+Q+G}} \times \frac{P+Q}{P+Q+G} \text { or } \frac{L x P}{P(R+S)+C(R+P)},
$$

since $P S=R Q$ as the bridge is balanced for steady currents. ${ }^{2}$

In the same way the quantity of electricity which traverses the galvanometer (in the reverse direction) due to the discharge of the condenser is

$$
K p y \frac{p}{P+Q+\frac{\left(R+S^{\prime}\right) G}{R+S+G}} \times \frac{R+S}{R+S+G} \text { or } \frac{K \prime^{2} y R}{P(R+S)+G(R+\bar{P})} .
$$

Since the galvanometer is unaffected, these two quantities must be equal. Hence

or

$$
\begin{aligned}
L x P & =K p^{2} y R, \\
L & =\frac{K p^{2} R}{P} \cdot \frac{y}{x} .
\end{aligned}
$$

But

so that

$$
\frac{y}{x}=\frac{S}{R}
$$

$$
L=\frac{K \nu^{2} S}{P}
$$

1 Since the bridge is balanced for steady currents, the hattery and galvano. meter arms are conjugate. Hence the current in the galvanometer produced by the E.M.F. of induction is independent of the resistance of the battery. Thus the expression obtained when the battery arm is broken will also hold when the battery is connected to the network (see $\$ 176$ ).

2 The fact that one of the arms has self.induction does not affect the total quantity of electricity which traverses the arms, although it does affect the ratio of the currents in the different arms at any instant during the variable ptase. 
The arrangement of resistance boxes used in practice is shown in Fig. 245. The sum of the two resistances $p$ and $p^{\prime}$ being made equal to $1000 \mathrm{ohms}$, the values of the resistances $Q$ and $R$ are adjusted so that there is balance for steady currents, an adjustable resistance $r$ in series with the coil being used if necessary for the final adjustment. The resistance $p$ is then adjusted, care being taken to keep the sum $p+p^{\prime}$

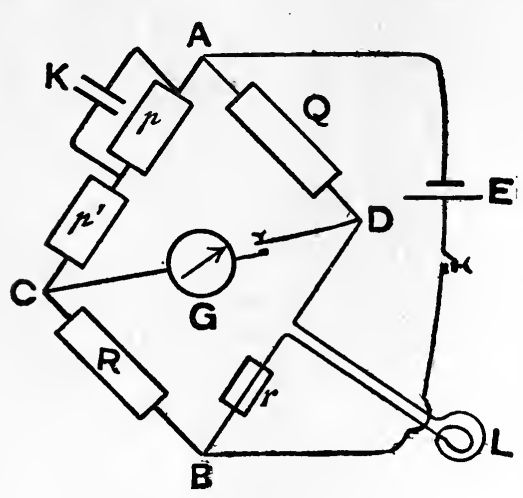

FIG. 245. constant, till there is no inductive kick of the galvanometer on making or breaking the battery circuit.

The sensitiveness of the arrangement can be much increased by the use of a commutator, such that the battery circuit is rapidly reversed, while between the battery reversals the galvanometer connections are also reversed, so that any residual current due to imperfect balance passes through the galvanometer in the same direction. In this case, as in all cases where a commutator is used to increase the sensitiveness of a zero method, it is not necessary to know either the number of rotations per second or the lead of the brushes. It is, however, always advisable to observe with the commutator running at different speeds, so as to make certain that the speed used is not too great. If the commutator is running to fast, it generally happens that the discharge due to the condenser is over before the reversal of the galvanometer, while the inductive discharge due to the coil may not have finished. This is particularly the case when using coils with iron cores. In the case of circuits having large time constants it is impossible to obtain balance, since the inductive discharge lasts so much longer than the condenser discharge, so that although the correct balance may be attained such that the quantities of electricity which pass through the galvanometer due to these two effects may be equal, yet a small kick is first obtained in one direction due to the condenser, and then there is a kick in the other direction due to the self-induction of the coil. In such a case the condenser is removed, and the bridge having been balanced for steady currents, the kick $d_{1}$ obtained when the current is reversed is observed. The coil is then replaced by two resistance boxes in series, with a condenser of capacity $\mathrm{K}$ placed as a shunt on one of them. The resistances unplugged from these boxes are adjusted till the bridge is again balanced for steady currents, showing that the sum of these resistances, $p+p^{\prime}$, is equal to the resistance of the same arm when the coil was in place. The resistance $p$ in parallel with the condenser is adjusted till the throw of 
the galvanometer when the battery is reversed is about the same as with the coil, and the value of this throw $d_{2}$ is observed. Then

$$
\frac{L}{K p^{2}}=\frac{d_{1}}{d_{2}} \text {. }
$$

Since it is assumed that the E.M.F. and resistance of the battery remain constant, it is advisable to use accumulators, and to repeat the observations several times, alternating the kicks with the coil and the condenser.

231. Measurement of the Self-Induction of a Coil by Comparison with a Capacity (Anderson's Method). ${ }^{1}$-A Wheatstone's bridge is built up, as shown in Fig. 246, P, Q, and $\mathrm{R}$ being non-inductive resistances, and $\mathrm{L}$ the coil of which the self-induction is to be measured. The condenser $\mathrm{K}_{1}$ is connected to the point $B$ and to one terminal of a galvanometer $G$ and telephone $\mathrm{T}$, and the three wires are joined to the point $\mathrm{A}$ through an adjustable non-inductive resistance $r$. The other terminals of the galvanometer and telephone are connected to a two-way switch $k$, by means of which either can be put in communication with $\mathrm{c}$. The battery $\mathbf{E}$ is connected to a key $k_{2}$ and a buzzer $z$. This latter consists of a small electromagnet, the armature forming a break, such as is usually fitted to an induction coil.

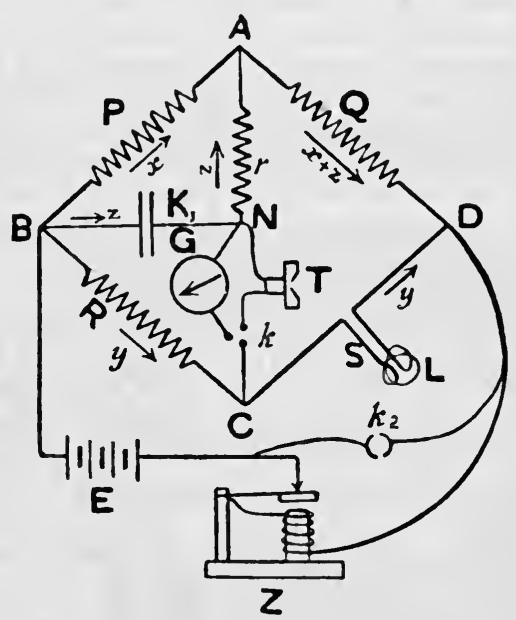

FIG. 246.

The bridge is first balanced for steady currents, using the galvanometer $G$, the key $k_{2}$ being closed.

The key $k_{2}$ is then opened so that the buzzer works, ${ }^{2}$ producing a rapidly interrupted current in the network of conductors, and the telephone is used in place of the galvanometer. The resistance $r$ is then adjusted till the sound in the telephone is a minimum. When this adjustment is complete we have

$$
L=K\{r(R+S)+R Q\},
$$

where if $K$ is measured in farads and the resistances in ohms, the selfinduction is obtained in henrys.

1 Philosophical Magazine (1891), xxxi. p. 334. See also Fleming. Philosophical Magazine (1904), vii. p. 5*6.

2 In place of the buzzer and telephone a rocking commutator or secohm. meter can be employed with the galvanometer only. Fleming, however, has shown that the buzzer and telephone vely materially increase the sensitivenese of the arrangement. 
We may suppose that the potential of the point $\mathrm{N}$ is always equal to that of the point $\mathrm{c}$, and let $x, y$, and $z$ be the total quantities of electricity which have traversed the arms BA, BC, and BK respectively, from the starting of the currents up to the time $t$. Since the current in the arm BA at the time $t$ is $\frac{d x}{d t}$ and so on, we have the difference of potential between $\mathrm{B}$ and $\mathrm{A}$ measured along the arm $\mathrm{P}$ is $P \overline{d x}$, and the difference of potential between these same points measured along BNA is $\frac{z}{\bar{K}}+r \frac{d r}{d t}$, for the difference of potential between the poles of the condenser $\mathrm{K}$ is equal to the charge $z$ divided by the capacity $K$. Hence equating the two expressions for the difference of potential between $B$ and $\mathbf{A}$ we get

$$
P \frac{d x}{d t}=\frac{z}{K}+r \frac{d z}{d t} \cdot \text {. . . . . . . }
$$

Similarly, since $\mathrm{N}$ and $\mathrm{c}$ are at the same potential

$$
\frac{z}{K}=R \frac{d y}{d t}
$$

The difference of potential between $\mathrm{C}$ and $\mathrm{D}$ is equal to the sum of the differences of potential due to the steady current, viz. $S \frac{d y}{d t}$, and that due to the self-induction, viz. $L \frac{d}{d t}\left(\begin{array}{l}d y \\ d t\end{array}\right)$.

The difference of potential between $\mathrm{c}$ and $\mathrm{A}$ reckoned along CNAD is

$$
r_{d t}^{d z}+Q \frac{d}{d t}(x+z)
$$

Hence

$$
r \frac{d z}{d t}+Q\left(\frac{d x}{d t}+\frac{d z}{d t}\right)=S \frac{d y}{d t}+L \frac{d^{2} y}{d t^{2}} \quad \text {. . . . . }
$$

Substituting in (3) the values for $\frac{d^{2} y}{d t^{2}}, \frac{d y}{d t}$, and $\frac{d x}{d t}$, deduced from (1) and (2), we get

$$
\left\{r+Q+\frac{Q r}{P}-\frac{L}{R K}\right\} \frac{d z}{d \bar{t}}-\left(\frac{S}{R}-\frac{Q}{P}\right) \frac{z}{K}=0 . . .
$$

Now, if there is no steady deflection the coefficient of $z$ in (4) must be zero, which gives the usual condition

$$
\frac{S}{\bar{R}}=\frac{Q}{P}
$$


If, further, there is to be no variable current, then the coetticient of $\frac{d z}{d t}$ must be zero, that is,

$$
r+Q+\frac{Q r}{P}-\frac{L}{R K}=0,
$$

which gives, since $\frac{R}{P}=\frac{S}{Q}$,

$$
L=K\{r(R+S)+R Q\} .
$$

When performing the experiment, the resistance $\mathbf{P}, \mathbf{Q}$, and $\mathbf{R}$ may consist of the arms of an ordinary Post Office Wheatstone's bridge, in which a resistance box is placed in series with the telephone or galvanometer in the galvanometer gap, the condenser being connected as shown in Fig. 246.

232. Measurement of Self-Induction by means of a Variable Standard of Self-Induction.-Where a variable standard of self-induction is available, of which the maximum value is at least as great as that of the coil to be measured, the following method, which is a modification of one devised by Maxwell, is convenient. The coil to be measured, L (Fig. 247), and the adjustable standard $N$, are placed in adjacents arms of a Wheatstone's bridge, the other arms being formed by non-inductive resistances. It will generally be found convenient to place an adjustable resistance, such as a bare wire or a carbon resistance $r$, in series with either $\mathrm{L}$ or $\mathrm{N}$ to allow of the final adjustment for steady currents being made.

The bridge having been adjusted for steady currents, the standard of self-induction is adjusted till there is

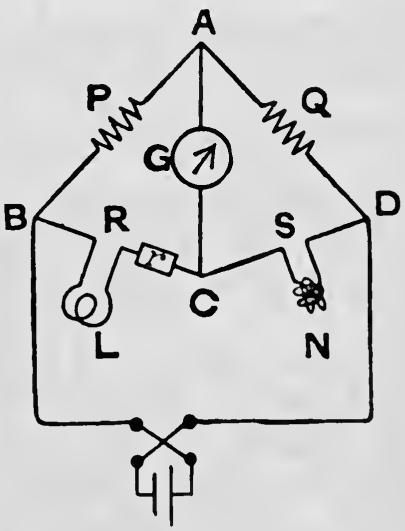

E

F1c. 247. no galvanometer kick on reversal of the battery current. When this is the case we have, if $N$ is the value of the self-induction of the standard.

$$
\frac{L}{N}=\frac{R}{S}=\frac{P}{Q} .
$$

In the absence of a variable standard of self-induction, or when comparing two fixed self-inductions, the above method is inapplicable. Maxwell has shown that when two coils having self-induction are arranged as are $\mathrm{L}$ and $\mathrm{N}$ in Fig. 247 , then the bridge will be balanced both for 
steady currents, and also during the time when the current is varying, if the following relation between the resistances of the arms holds,

$$
\frac{L}{N}=\frac{R}{S}=\frac{P}{Q} .
$$

The application of this method is, however, so very tedious, since it involves a double adjustment, as to render it unpractical. A modification of the method has been devised by Niven, ${ }^{1}$ in which the necessity for the double adjustment is avoided. The method is not, however, a very convenient one. A fairly rapid way of making the comparison, but one involving the use of a ballistic galvanometer, is as follows :-Arrange the two coils having self-induction as in Fig. 247, and having balanced for steady currents note the swing $d_{1}$ when the current is reversed. Next remove one of the coils, say $L$, and substitute a non-inductive resistance, and having adjusted till the bridge is again balanced for steady currents, measure the throw $d_{2}$ when the current is again reversed.

When the two coils are used we have, if $k$ is a constant depending on the galvanometer, $Q L-P N=k d_{1}$.

When the coil $\mathrm{N}$ only is included, we have, in the same way,

Hence

$$
\begin{gathered}
P N=k d_{2} . \\
L=\frac{P}{\bar{Q}}\left(1+\frac{d_{1}}{d_{2}}\right) .
\end{gathered}
$$

Since it is assumed that the current remains constant throughout, it

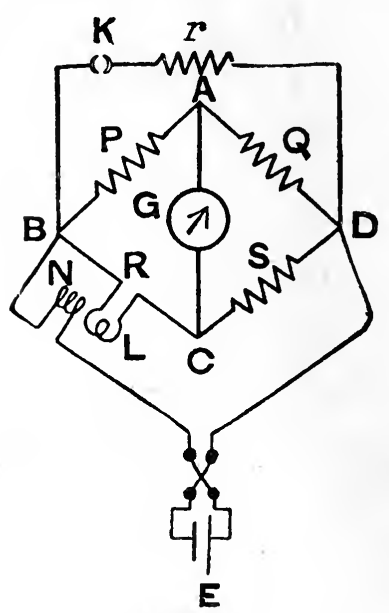

Fig. 248. is important to use accumulators, and to alternate the two measurements several times.

233. To Compare the MutualInduction between two Coils with the Self-Induction of one of them.Suppose the coil $\mathrm{I}$ is that of which the coefficient of self-induction $\mathrm{L}$ has to be compared with the coefficient of mutual-induction $M$ between this coil and another coil $\mathrm{N}$. The coil $\mathrm{L}$ is included in one arm of the Wheatstone's bridge, and the coil $\mathrm{N}$ is placed in the battery circuit, as shown in Fig. 248. The bridge having been adjusted for steady currents, during which adjustment the key $\mathrm{K}$ may be open, this key is closed, and the resistance $r$ is adjusted till there is no kick on reversing the battery current. This adjustment is only possible if the coefficient of selfinduction of $\mathbf{L}$ is greater than the coefficient of mutual-induction be-

1 Philosophical Magazine (1887), vol. xxiv. p. 227. 
tween the two coils. If this condition is not fulfilled, then the modification given below must be employed. When the adjustment for both steady and variable currents is complete, we have

$$
\frac{M}{L}=-\frac{P r}{r(P+R)+P(S+R)},
$$

the minus sign indicating that the ooil $\mathrm{N}$ must be so connected up as to give an opposite induced E.M.F. in the branch $\mathrm{BC}$ to that produced by $\mathrm{L}$.

The fact that the galvanometer gives no kick when the battery is reversed shows that the integral E.M.F. induced in the arm BC, due to the reversal of the current in the coil $\mathrm{N}$, is equal and opposite to the integral F.M.F. induced in this arm, due to the self-induction of the coil $\mathrm{L}$. Hence if $x$ is the steady current in $x$, and $y$ that in $L$, we have

or

$$
\begin{aligned}
& x M+y L=0, \\
& \frac{M}{L}=-\frac{y}{x} .
\end{aligned}
$$

But $x$ is the total current in the battery branch, and the ratio of the current in the arm $\mathrm{BC}$ to the total current is given by

$$
\frac{y}{x}=\frac{\frac{(P+Q) r}{P+Q+r}}{\frac{(P+Q) r}{P+Q+r}+R+S}=\frac{(P+Q) r}{(P+Q) r+(R+S)(P+Q+r)} .
$$

But $Q=\frac{S}{R} P$, hence making this substitution

$\frac{y}{x}=\frac{P\left(1+\frac{S}{R}\right) r}{P\left(1+\frac{S}{R}\right) r+R\left(1+\frac{S}{R}\right)\left(P+\frac{S P}{R}+r\right)}=\frac{\operatorname{Pr}}{r(P+R)+P(S+R)}$.

Substituting this value for $\frac{y}{x}$ in (1), we get

$$
\frac{M}{L}=-\frac{P r}{r(P+R)}+P(S+R) .
$$

When $M$ is greater than $L$, the coil s can be included in the branch $r$ in place of being in the battery arm. If $r^{\prime}$ is the value of the total resistance in the $r$ branch when there is balance for variable currents, we have

$$
\frac{M}{L}=-\frac{r^{\prime}}{R+S} \text {. }
$$

Both the above methods allow of the use of a rotating commutator, such as the secohmmeter, by means of which the sensitiveness may be very materially increased.

234. Measurement of the Coefficient of Mutual-Induction between two Coils by means of a Variable Standard of Self-Iuduction.-The two coils $L_{1}$ and $L_{2}$, of which the cofticient of mutual-induction $x$ is to be determined, are placed in one arm of a Wheatstone bridge, as shown 
in Fig. 249, and the variable standard of self-induction $\mathrm{N}$ is placed in the adjacent arm. The bridge having been balanced for steady currents, the standard $\mathrm{N}$ is adjusted till there is no kick on reversing the battery current. Suppose that the coils $L_{1}$ and $L_{2}$ are so connected that the mutual-in-

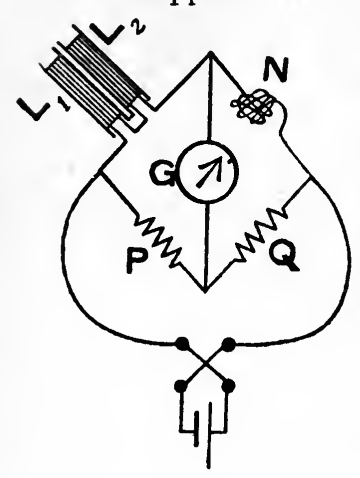

E duction assists the self-induction, and that the value of the standard when the adjustment for variable currents is complete is $N_{1}$. Then

$$
L_{1}+L_{2}+2 M=N_{1} \text {. }
$$

If, now, the connections of one of the coils $\mathrm{L}_{1}$ or $\mathbf{L}_{2}$ is reversed, so that the effect of the mutualinduction is opposed to the effects of the self-inductions of the separate coils, and $N_{2}$ is the new value of the variable self-induction when the bridge is balanced for variable currents, we have

Hence

$$
\begin{gathered}
L_{1}+L_{2}-2 M=N_{2} . \\
M=\frac{1}{4}\left(N_{1}-N_{2}\right) .
\end{gathered}
$$

234a. Measurement of a Mutual-Induction

FIG. 249. . by comparison with a Capacity. Carey Foster's Method.-Of the two coils between which the mutual-induction has to be measured, L, Fig. 249A, is connected to a battery $\mathrm{k}$, through a non-inductive resistance $r$ and a makeand-break key $\mathbf{A}$. One pole of the condenser $\mathbf{K}$ is connected direct to one end of the resistance $r$, and the other pole connected through a galvanometer $\mathrm{G}$ to the other end of this resistance. The other coil $\mathrm{M}$ is connected through the adjustable non-inductive resistance $\mathbf{R}$ as a shunt on the gal-

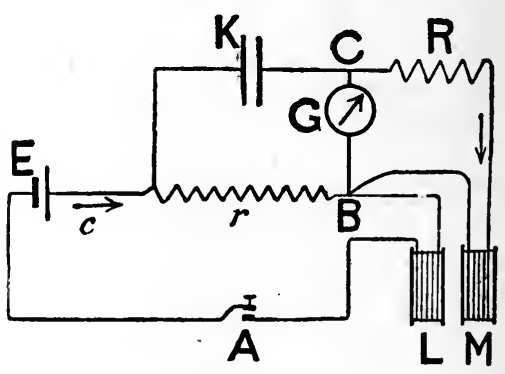

FIG. 249A. vanometer, as shown. The resistance $R$ is adjusted till on closing or opening the key $A$ the galvanometer gives no kick. When this is the case the coefficient of mutualinductance $\mathrm{M}$ is given by

$$
M=r R K,
$$

where $K$ is the capacity of the condenser, and $R$ is the resistance of $M$ and $R$.

Suppose that the key is closed, and that when the current has reached the steady state the current passing through the resistance $r$ is $c$, so that the difference of potential between the ends of the resistance $r$ is $r c$, and hence the charge on the condenser is $r c K$.

Since the galvanometer is undeflected on the average the current pass ing must be zero, and hence the average difference of potential between the 
points $B$ and $\mathbf{C}$ must be zero. Hence the charge received by the condenser must be equal to the total quantity of electricity which traverses the branch containing $\mathbf{M}$ and $\mathbf{R}$. If $c$ is the instantaneous value of the current in the coil $L$, the induced E.M.F. in the coil $M$ at any given instant is $M_{\frac{d c}{d t}}$, and the quantity of electricity displaced through $M$ and $\mathbf{R}$ in a time $d t$ due to this E.M.F. is $\frac{M}{R} \frac{d c}{d t} \cdot d t$. Thus the total quantity displaced from the instant when the key is elosed (or opened), up to the time when the steady state is reached is $\frac{M}{R} \int d c$ or $\frac{M c}{R}$. Since this quantity is equal to the charge in the condenser we have

$$
\begin{aligned}
r c K & =\frac{M c}{R} \\
\text { or } \quad M & =r R K
\end{aligned}
$$

235. The Comparison of two Mutual-Inductances. - The two pairs of coils are arranged, as shown in Fig. 250, one coil of each pair being in the battery circuit. The other two coils are connected to a galvanometer through the resistances $\mathbf{P}$ and Q. The values of $P$ and $Q$ are then adjusted till there is no galvanometer kick on reversing the current in the battery circuit. When this adjustment is complete, we have, if $s_{1}$ and $s_{2}$ are the resistances of the coils connected to the galvanometer,

$$
\frac{M_{1}}{M_{2}}=\frac{S_{1}+P}{S_{2}+Q}
$$

\section{The Measurement of Self-Induction}

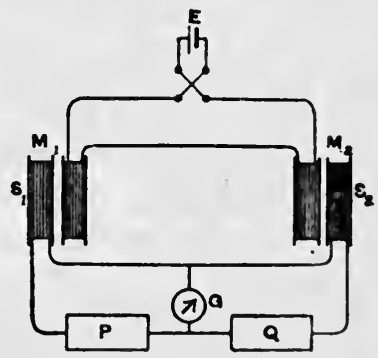

FIG. 250 . with Alternating Currents (Wien-Dolezalek Method).-A very accurate method of measuring self-induction, particularly small inductances, has been described by Wien, ${ }^{1}$ in which an alternating current is employed. Two coils, $\mathrm{L}_{1}$ and $\mathrm{L}_{2}$ (Fig. 251), having self-induction are placed in adjacent arms of a Wheatstone's bridge. One of these coils may conveniently have its self-induction adjustable, though the value of the selfinduction need not be known. A non-inductive resistance $r^{\prime}$ is placed as a shunt on the coil $\mathrm{L}_{1}$, and another non-inductive resistance $s$ is placed in series with $\mathrm{L}_{2}$.

The two remaining resistances, $P$ and $Q$, may be formed by a bridge wire. The source of current is a small induction coil $\mathrm{M}$, the make-andbreak of which consists of a microphone attached to a telephone diaphragm. This method of obtaining a sinusoidal current is due to Dolezalek. The arrangement used, called a hummer, is shown in Fig. 252. 
It consists of an iron disc $\mathrm{AB}$, to the centre of which is attached a small carbon disc $\mathbf{c}$. To this disc is attached a small silk bag $\mathbf{D}$ filled with carbon grains, forming the microphone contact. One terminal of a battery $\mathrm{E}$ is attached to $\mathrm{AB}$, and the other to the primary $\mathbf{P}$ of a small induction coil, the other terminal of the primary being connected to $\mathrm{D}$ by a fine flexible wire. A hollow cylindrical steel magnet $\mathrm{G}$ is placed near the disc $\mathrm{AB}$, and on the outside of this magnet is wound a coil $\mathrm{F}$, which is in series with the secondary $s$ of the induction coil. The terminals $a$ and $b$ are connected to the bridge. When the diaphragm $\mathrm{AB}$ has been set in vibration, the microphone contacts at $D$ vary in resistance, and hence the primary current varies in intensity. Hence there is an induced secondary current produced, and this current passing through the

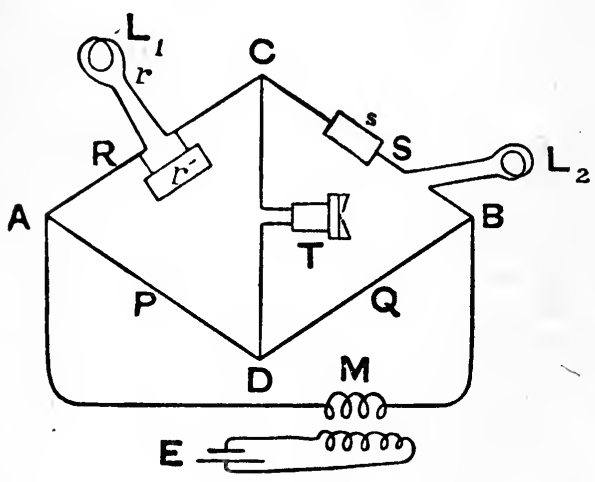

FIG. 251.

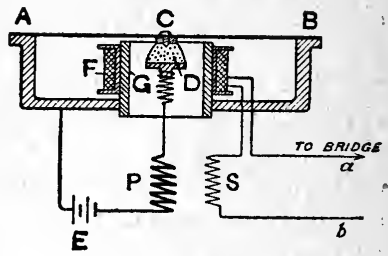

FIG. 252.

coil $\mathrm{F}$ causes the magnetism of the magnet $\mathrm{G}$ to vary, and in this way keeps up the vibrations of the disc. In place of the galvanometer a special telephone is employed, which is so adjusted that its diaphragm has the same natural period as that of the diaphragm of the hummer. The result is that the telephone practically only responds to alternating currents of the one period, and the results. are the same as if a simple sine-form alternating current was used in the bridge.

The resistance $r^{\prime}$ (Fig. 251) being given some value about equal to that of the coil $L_{1},{ }^{1}$ the resistances $s, P$ and $Q$ are adjusted till there is no sound in the telephone $\mathrm{T}$.

Since we are dealing with simple sine-form alternating currents, it can be shown (see Wien, loc. cit.) that we may apply the ordinary condition for steady current balance of the bridge if we replace the resist-

${ }^{1}$ Since it is desirable to have the point of balance near the centre of the wire, i.e. $P=Q$, it follows from (2) below that $r^{\prime}$ must always be greater than $S$. If this condition is not satisfied, no point of balance will be found. 
ances ${ }^{1}$ of the arms by the operator $r+i p L$, where $r$ is the resistance, and $L$ the self-induction of the arm, $p$ is $2 \pi$ times the frequency of the alternating current, and $i$ is $\sqrt{ }-1$.

In the case of two conductors in series, or parallel, the value of the operator $r+i p L$ for the combination is obtained from the values for the individual conductors by the same rules as is the resistance of two conductors in series or in parallel, as the case may be.

Hence, since the resistance $r^{\prime}$ is non-inductive (i.e. $L=0$ ), the operator in the case of the arm $\mathrm{Ac}$ (Fig. 251) becomes

$$
\frac{\left(r+i p L_{1}\right) r^{\prime}}{r+i p L_{1}+r^{\prime}}
$$

The condition for balance of the bridge is

$$
\frac{\left(r+i p L_{1}\right) r^{\prime}}{\frac{r+i p L_{1}+r^{\prime}}{S+i p L_{2}}}=\frac{P}{Q},
$$

where $S$ is the resistance of the arm cв. By multiplying across, the above equation reduces to

$$
r^{\prime}\left(r+i p L_{1}\right) Q=P\left\{S\left(r+r^{\prime}\right)-p^{2} L_{1} L_{2}+i\left[S p L_{1}+\left(r+r^{\prime}\right) p L_{2}\right]\right\} .
$$

Equating the real parts of this equation, we get

$$
\begin{gathered}
r r^{\prime} Q=P\left\{S\left(r+r^{\prime}\right)-p^{2} L_{1} L_{2}\right\}, \\
L_{1} L_{2}=\frac{P S\left(r+r^{\prime}\right)-r r^{\prime} Q}{P p^{2}}=\frac{S\left(r+r^{\prime}\right)-r r^{\prime} Q}{p^{2}} \quad \text {. . . }
\end{gathered}
$$

or

In the same way, equating the imaginary parts, we get

or

$$
\begin{aligned}
p r^{\prime} Q L_{1}= & S P p L_{1}+P p\left(r+r^{\prime}\right) L_{2}, \\
& \frac{L_{1}}{L_{2}}=\frac{P\left(r+r^{\prime}\right)}{r^{\prime} Q-S P}
\end{aligned}
$$

Equations (1) and (2) enable us to calculate both $L_{1}$ and $L_{2}$.

To obtain the quantity $p$ ( $2 \pi$ times the frequency), the frequency of the alternating current must be measured. This quantity may be obtained by comparing the pitch of the note given by the telephone with that of a tuning-fork of known pitch. The comparison can be made either by means of a monochord ( $\$ 110)$, or by using a resonance column (\$112).

Several measurements ought to be made, using different values for the shunt resistance $r^{\prime}$.

In place of the microphone and telephone a stretched wire interrupter and a vibration galvanometer may be used. See Camplell, I'hilosophical Ma!jazine [6], vol. xiv. p. 494, Uctober 1907.

1 If the arm consints of an inductive resistance and a condenser of capacity $C$ in series, then the operator has the form $r+i p L-i \frac{1}{p C}$, and this operator can be used to replace the resistance in obtaining the conditions of halance of a bridge in which condensers are included when alternating currents are employed. 


\section{CHAPTER XXXIII}

PERMEABILITY

237. Measurement of the Permeability of Iron by the Magnetometer Method.-If a rod of magnetic material, such as iron, is placed with its length parallel to the lines of force of a magnetic field of strength $F$, the rod becomes magnetised by induction. If the strength of the poles induced is $m$, and the distance between them is $l$, the magnetic moment of the rod is $\mathrm{ml}$. If the volume of the $\operatorname{rod}$ is $v$, the intensity of magnetisation $I$ of the iron is given by

$$
I=\frac{m l}{v} \text {. }
$$

In general, the intensity of magnetisation is not quite uniform throughout the whole length of the rod, there being a falling off near the ends. If, however, the rod is very long compared to its cross-section $s$, the effects of the ends is very small, and then the distance between the poles may be taken as equal to the length of the rod without appreciable error, and we have

$$
I=\frac{m}{8}
$$

The number of lines of force which proceed from a pole of strength $m$ being $4 \pi m$, the number of lines which leave the north pole of the rod and enter the south pole are $4 \pi m$, and hence the number which cross unit area of the cross-section of the iron is $4 \pi \mathrm{m} / \mathrm{s}$. Since in addition to the lines due to the magnetism induced in the rod we have the lines due to the magnetising field, the total number of lines which cross unit area is $4 \pi m / s+F$. The total number of lines which cross unit area is called the induction $B$, hence since $I=m / s$,

$$
B=F+4 \pi I \text {. }
$$

The ratio of the induction $B$ to the inducing field $F$ is called the permeability $\mu$, so that $\mu=B / F$. The ratio of the intensity of magnetisation $I$ to the inducing field is called the magnetic susceptibility $k$, so that $k=I / F$, and hence

$$
\mu=1+4 \pi k \text {. }
$$

In the above relations the magnetising field $F$ is the strength of the 
field within the iron itself, and this is not in general equal to the strength of the field before the introduction of the iron, owing to the fact that the poles induced at the ends of the rod produce a field which is in the opposite direction to the original field. If, however, the rod is very long and thin the poles are weak, and are at such a great distance from the greater part of the iron that the demagnetising field due to the poles may be neglected, so that in such a case we may take the field which exists before the introduction of the iron as the magnetising field $F$. The method by which, in the case of a short cylinder, the effect of the demagnetising field due to the ends may be allowed for is described later $(\S 238)$.

In the magnetometer method of measuring the permeability of iron the material is taken in the form of a long thin wire DG (Fig. 253), and is magnetised by being placed within a long solenoid $\mathrm{BO}$, through which

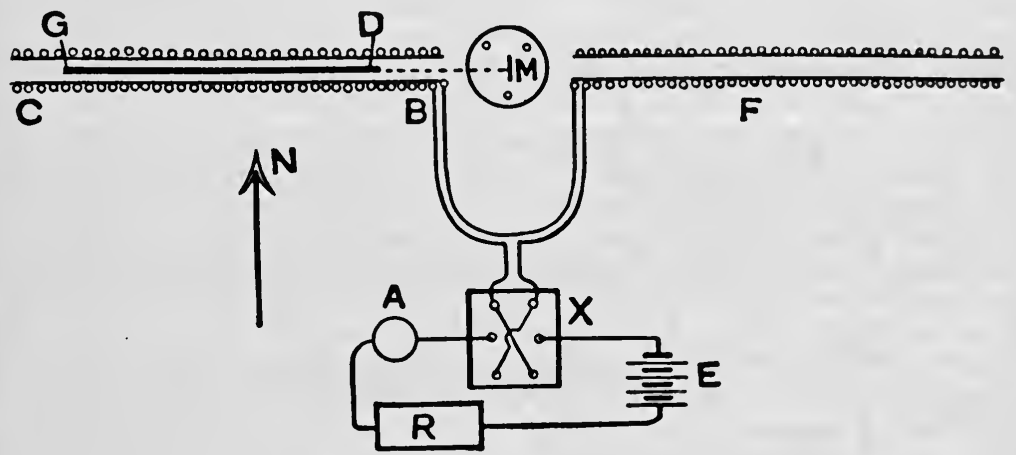

FIG. 253.

a current is passed. The induced magnetism is then measured by noting the deflection which the iron causes to a magnetometer needle a placed in the neighbourhood, the magnetic field produced at the needle of the magnetometer by the direct action of the solenoid being compensated by means of an auxiliary coil $F$, through which the current which passes through the solenoid is also passed.

If the solenoid is placed with its axis $\mathrm{E}$. and W. (magnetic), the earth's field will have no component parallel to the length of the wire, and when long thin wires are used, the effect of the transverse magnetisation due to the earth's field may be neglected.

Let the magnetising solenoid $\mathrm{BC}$ be placed in the magnetic east and west position, with its axis passing through the needle of the magnetometer, the mid-point of the wire being at a distance I) from the needle, while the length of the wire is $2 L$, and its cross-section is $s$. If the intensity of magnetisation of the iron is $I$, the magnetic moment of the iron is $2 s L I$. If $M^{\prime}$ is the moment of the magnetometer neerle, the 
couple, exerted by the iron on the needle, if we suppose the poles to be at the ends of the wire, when the needle has been deflected through an angle $\theta$ from the magnetic meridian is ${ }^{1}$

$$
\frac{4 M^{\prime} s L I D}{\left(D^{2}-L^{2}\right)^{2}} \cos \theta
$$

If $H$ is the value of the horizontal component of the field in which the magnetometer needle hangs, the couple acting on the needle tending to bring it back into the meridian is $M^{\prime} H \sin \theta$. When the needle is in equilibrium, these two couples must be equal, and hence

$$
\begin{gathered}
\frac{4 M^{\prime} s L I D}{\left(D^{2}-L^{2}\right)^{2}} \cos \theta=M^{\prime} H \sin \theta, \\
\text { or } \quad I=\frac{H\left(D^{2}-L^{2}\right)^{2}}{4 s L D} \tan \theta .
\end{gathered}
$$

If $\frac{L^{2}}{D^{2}}$ is small compared to unity, then this expression may be.written

$$
I=\frac{H D^{3}\left(1-2 \frac{L^{2}}{D^{2}}\right)}{4 s L}
$$

where the second term within the brackets is often so small as to be negligible. Hence if $H$ and $D$ are known, $I$ can be calculated from the magnetometer deflection.

If the magnetising solenoid contains $n$ turns per centimetre, and is traversed by a current of $C$ c.g.s. units, the field at the centre of a long solenoid is equal to $4 \pi n C$. Hence if the iron wire is so long that we may neglect the demagnetising effects of the field due to the poles, the magnetising field $F$ acting on the iron is given by

$$
F=4 \pi n C \text { gausses. }
$$

If the current is $A$ amperes, then, since an ampere is one-tenth of a c.g.s. unit,

$$
F=\frac{2 \pi n A}{5} \text { gausses. }
$$

The current is obtained from the battery E, which may with advantag c consist of accumulators, and passes through a variable resistance $R$, an ammeter $\mathbf{A}$, and a commutator $\mathbf{x}$.

A suitable magnetising coil can be made by winding No. 22 (standard wire-gauge) double-silk covered copper wire on a tube $50 \mathrm{~cm}$. long and $1 \mathrm{~cm}$. outside diameter. If the coil is only to be used for

1 Watson's Text-Book of Physics, § 429. 
magnetometer experiments, then it may be wound on a brass tube. When, however, it is to be used in addition for ballistic experiments, it is better wound on a non-conducting tube, such as glass or vulcanised fibre. If eight layers of wire are wound on the tube, the end of each alternate layer being taken to binding screws attached to the base on which the coil is mounted, then by using different numbers of layers, varying magnetising forces can be obtained without using a very great range of currents. The wire must be wound on very regularly, and the number of turns in each layer noted. It is also a good thing to note the mean diameter of each layer, and to give each layer a coating of shellac varnish. Each layer of such a coil will have a resistance of about 2 ohms, and with a current of 2 amperes the whole coil will give a magnetising field of about 360 c.g.s. units.

In order to vary the magnetising field when carrying the iron round a cycle, some means of varying the current by varying the resistance in the circuit will have to be adopted. This adjustable resistance must be such that it can be either increased or decreased by suitable steps in a perfectly regular manner. Thus when the current is being increased, the resistance must never, even temporarily, be decreased, and vice vers $\alpha$, or owing to hysteresis the curve obtained will be irregular. The steps by which the resistance is altered should not be equal ones, but should get greater and greater as the resistance in the circuit increases, 80 that the changes in the current, and hence also in the magnetising field, are approximately equal. If $r$ is the resistance of the whole circuit when the largest current is passing, and it is required to obtain $n$ steps, then the resistances which have to be added to $r$ are

$$
\frac{n r}{n(n-1)} ; \frac{n r}{(n-1)(n-2)} ; \frac{n r}{(n-2)(n-3),} \text { \&c. }
$$

Thus if the value of $r$ is 9 ohms and we require ten steps, the resistances to be added to $r$ are

$$
1 ; 2 \cdot 25 ; 3.86 ; 6 ; 8 ; 13.5 ; 21 ; 36 ; 81 \text { ohms. }
$$

In general, it will be found convenient to prepare a special set of resist. ances to suit any given coil, for they need not be adjusted at all carefully, and in fact the required lengths of wire can be measured off with sufficient accuracy, the resistance of a metre of the wire having been measured.

In place of the usual plug contact, which is expensive to make and difficult to keep in proper condition, the arrangement shown in Fig. 254, which has been designed by Professor Callendar, may be used. A strip of 
copper or brass is taken, and a number of equally spaced holes about $0.6 \mathrm{~cm}$. in diameter are bored. This strip is fastened to a piece of wood by two screws between each pair of holes, this piece of wood forming the top of the box in which the coils will be contained. The strip of copper is then removed and sawn across through each of the large holes, and the separate pieces again attached to the wood. An ordinary binding screw terminal $\mathbf{A}$, the screw-down portion of which is of greater diameter than the holes, is then fastened, as shown, at the centre of each of the holes. The ends of the wires are brought up through the wood and soldered to the pieces of copper, the wire itself being coiled in the form of a double spiral, either loose or round a wooden peg. When the binding screws are screwed down they make contact with the copper strips on either side, and the coil which is attached to these strips is short-circuited. When the binding screw is raised the current must, however, pass through the coil. This form of contact is easy to make, and can be cleaned without difficulty.

The apparatus is set up in the following manner:-The magnetometer having been set up and levelled, the magnetising coil and compensating coils are placed with their axes in the magnetic east and west positions, and at such a height that their axes pass through the needle of the magnetometer; and the ammeter, adjustable resistance, and a battery are connected up, as shown in Fig. 253. A current is then passed through the circuit, no iron being in the coils, and the position of the compensating coil is adjusted till the magnetometer is undeflected when the current is reversed. The iron, in the form of a wire about $30 \mathrm{~cm}$. long and 1 to $2 \mathrm{~mm}$. in diameter, is straightened and demagnetised by one of the methods described in $\S 240$. In the case of fine wire it will be found convenient to thread it through a fine bore glass tube, fixing it in position with a little sealing-wax. This glass tube is then supported axially within the magnetising coil by means of two corks, and is adjusted so that the wire lies symmetrically with respect to the coil. If the glass tube is made so long that it projects beyond the magnetising coil, and the distance of the end of the wire is measured from the end of the tube, the longitudinal adjustment may be made by measuring from the end of the tube.

Having inserted the largest resistance in the circuit, the current is made, and the deflected reading of the magnetometer and the value of the current are read and recorded. The resistance is then decreased and a new reading obtained, and so on till the maximum current is reached. In this way the magnetisation curve for an unmagnetised piece of iron is obtained.

Before proceeding to take a complete cycle of magnetisation so as to deduce the hysteresis, it will be found ad visable to take the iron ten or twenty times round the proposed cycle. Then starting with the current at its maximum value in one direction, the current is reduced by inserting the resistances in the circuit, and the values of the current and the magnetometer readings are recorded. When all the resistance is in the circuit the current is 
broken, and the magnetometer reading taken with no magnetising force. The current is then reversed, and the readings corresponding to gradually increasing currents in this reverse direction are obtained. Next the current is gradually reduced to zero, reversed and increased to the maximum value in the original direction, readings of the ammeter and magnetometer being taken at each step.

To reduce the results the dimensions of the iron will be required, as well as the distance of the iron from the magnetometer needle and the value of the horizontal component of the earth's magnetic field at the place where the magnetometer needle hangs. This latter can most conveniently be obtained by deflecting the magnetometer by passing a measured current through a coil of known dimensions. If the dimensions of the magnetising coil and of the compensating coil are the same, the best way to proceed is to reverse the connections on one of them, so that they now tend to deflect the needle in the same direction, and to read the current which must be passed to obtain a deflection nearly up to the end of the scale. If $\theta$ is the angle through which the magnetometer needle is deflected when the coils are traversed by a current $C$ c.g.s. units, each of the coils containing $n$ turns of wire per centimetre, the mean diameter of the windings being $r$, and the length of each coil $2 L$, while the distances of the mid-points of the coils from the magnetometer needle is $D$, then

$$
H=\frac{8 \pi n r^{2} L C}{\left(D^{2}-L^{2}\right)^{2}} \cot \theta
$$

since the magnetic moment of each coil is $2 \pi n r^{2} L C$.

If the dimensions of the magnetising coil are alone known, then the earth compensating coil must be cut out of circuit, and the magnetising coil alone used. The expression for calculating $H$ is half that given above. The advantage of using the two coils is, that if we measure the distance between the centres of the coils, and take half this distance for $D$, then, so long as the magnetometer needle is placed fairly accurately half-way between the coils, errors due to the difficulty of accurately measuring to the magnetometer needle will be practically eliminated.

The results of the experiments with the iron must be plotted, taking the magnetising force $F$ as abscissa, and either the magnetic induction or the intensity of magnetisation as ordinate. In the case of the long thin cylinder, such as is used in this experiment, the magnetising force may be taken as equal to the magnetic field within the coil before the introduction of the iron, the demagnetising field due to the poles at the ends of the wire being neglected.

As will be seen by a study of Fig. 255, both the induction and the intensity of magnetisation increase very rapidly during the portion $\mathbf{A B}$ of the curve, and hence if the deflection of the magnetometer is to be kept on the scale, it is necessary to have the magnetometer at such a distance that the deflections produced, when small values of the magnetising field are used, are small, and hence the accuracy with which the 
corresponding B or I can be determined is small. This difficulty may be met by placing the compensating coil nearer the magnetometer than the position where it exactly neutralises the field produced by the magnetising coil. The magnetometer deflections then give the difference between the effect of the iron and the excess of magnetic action of the compensating coil, and this excess can be determined by making a series of readings of the magnetometer deflection produced when no iron is contained in the magnetising coil.

Another method of arranging the specimen is to place the magnetising coil in the magnetic east and west direction, with its centre on the

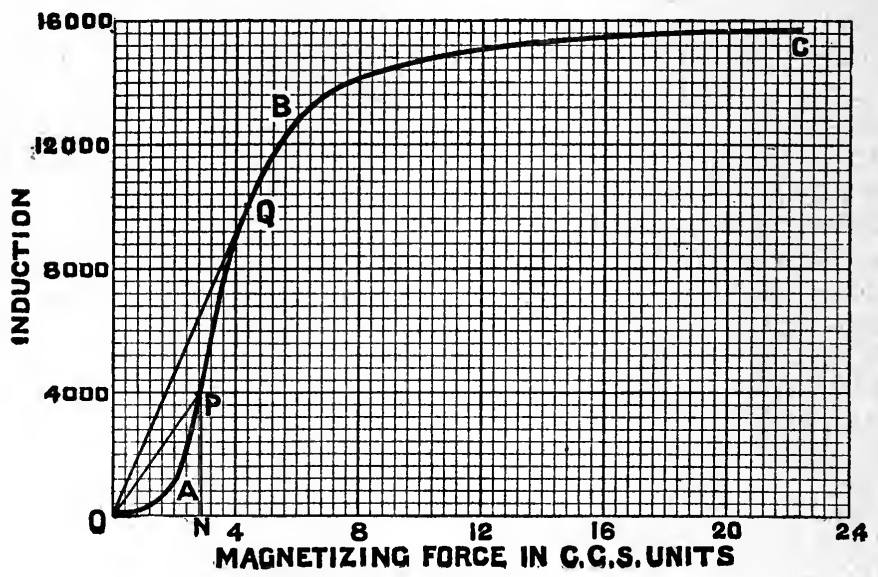

FIG. 255.

magnetic meridian passing through the magnetometer, as shown in Fig 256 , the compensating coil $\mathrm{F}$ being placed in a symmetrical position on the other side of the magnetometer.

If $\mathbf{D}$ is the perpendicular distance of the magnetometer needle $\mathrm{M}$ from the iron wire $\mathrm{GD}$, the couple acting on the magnetometer needle when the deflection is $\theta$ is

$$
\frac{M^{\prime} s L I}{\left(L^{2}+D^{2}\right)^{\frac{3}{3}}} \cos \theta
$$

Hence

or

$$
\begin{aligned}
\frac{M^{\prime} s L I}{\left(L^{2}+D^{2}\right)^{\frac{3}{2}}} \cos \theta & =M^{\prime} H \sin \theta, \\
I & =\frac{H\left(L^{2}+D^{2}\right)^{\frac{3}{2}}}{s L} \tan \theta .
\end{aligned}
$$

The advantage of this position over the end-on position lies in the fact that the uncertainty as to the location of the poles of the magnetised 
iron produces less error than in the case where one end of the specimen is comparatively close to the magnetometer. Further, in the end-on position a displacement of the pole

$\mathrm{F}$

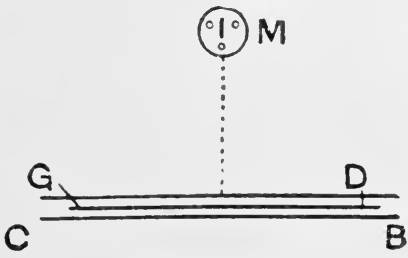

FIG. 256. along the specimen, since it directly alters the distance between the pole and the magnetometer, and the deflection varies inversely as the cube of this distance, may produce a very considerable error. In the side on position, however, if the poles are displaced, as one pole approaches the magnetometer the other pole recedes from the magnetometer, and hence for small displacements these two effects will compensate for one another.

Both the above methods of ob. servation, however, suffer from the serious objection that we have to assume a knowledge of the position of the poles in the iron, and in deducing the formulæ we have supposed that the poles are at the ends of the wire. There is no doubt that this assumption is wrong, and a somewhat better result will be obtained if we assume that the distance between the poles is $2 / 3$ of the length of the wire.

In this case the formula in the first case, when the axis of the iron passes through the magnetometer, becomes

$$
I=\frac{H\left(D^{2}-\frac{4}{9} L^{2}\right)^{2}}{4 s L D} \tan \theta,
$$

and in the second case

$$
I=\frac{H\left(\frac{4}{9} L^{2}+D^{2}\right)}{s L} \tan \theta .
$$

This uncertainty as to the position of the poles can in a measure be removed by adopt ing a third arrangement, which is shown diagrammatically in Fig. 257 . The magnetis-

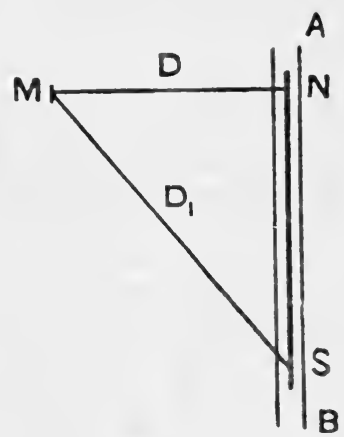

F16. 257. ing coil is here placed vertical, and due east or west of the magnetrmeter needle $\mathrm{M}$, and the iron is adjusted so that the upper pole is in the same horizontal plame as the needle. This adjustment can be made by passing a current throun the magnetising coil, and then moving the iron wire up or down till the deflectun of the magnetometer is a maximum. In this way the position of the pole $\mathrm{s}$, with reference to the wire, is found with sutficient accuracy, and since the 
wire is symmetrical, we therefore know the position of the pole $\mathrm{s}$ and the distance between the poles. Thus the distances $\mathrm{D}$ and $\mathrm{D}_{\mathbf{1}}$ of the poles from the magnetometer needle can be calculated. If the strength of either of the poles is $m$, then the horizontal component of the field at $M$ due to the two poles is

or

$$
\begin{gathered}
\frac{m}{D^{2}}-\frac{m}{D_{1}^{2}} \cos N M s, \\
m\left\{\frac{1}{D^{2}}-\frac{D}{D_{1}^{3}}\right\} . \\
m=I s .
\end{gathered}
$$

But

Hence the horizontal component is

$$
\text { Is }\left\{\frac{1}{D^{2}}-\frac{D}{D_{1}^{3}}\right\}
$$

and if $\theta$ is the deflection of the magnetometer,

$$
I s\left\{\frac{1}{D^{2}}-\frac{D}{D_{1}^{3}}\right\} \cos \theta=H \sin \theta,
$$

or

$$
I=\frac{H \tan \theta}{s\left\{\frac{1}{D^{2}}-\frac{D}{D_{1}^{3}}\right\}}=\frac{H D^{2}}{s\left\{1-\frac{D^{3}}{D_{1}^{3}}\right\}} \tan \theta .
$$

The disadvantage of this method is that in addition to the magnetising field of the coil in which the iron wire is placed, there is the vertical component of the earth's field acting on the iron. It is possible to neutralise the earth's field within the coil if an additional layer of wire is wound and a current of suitable amount is passed through this winding. The adjustment is, however, troublesome, and, further, since it is impossible to introduce the wire into the coil without getting it magnetised, it becomes necessary to demagnetise the iron in place. In general, it will be found most convenient not to attempt to neutralise the earth's field, but to apply a correction afterwards. This correction will only be appreciable in the case of soft iron, in which material the permeability for small fields is fairly large.

Suppose that Fig. 258 represents the result of plotting B against the magnetising field, on the supposition that the wire is at the start unmagnetised, and the magnetising field $\mathrm{F}$ is that deduced from the current in the magnetising coil, i.e. we neglect the effect of the vertical component of the earth's field. It will be seen that the curve cuts the axis of $\mathrm{F}$ at two points $a$ and $c$, which are not symmetrical with reference to the origin 0 ; the points of intersection corresponding to the values of $F+1 \cdot 3$ and $-2 \cdot 2$. Hence if the origin is moved to the point $-0 \cdot 45$, the curve 
will be symmetrical about the new axis of $B$, the quantity +0.45 representing the value of the vertical component of the earth's field. In the same way the points $b$ and $d$, where the curve cuts the axis of $B$, or the points $e f$, where $B$ is a maximum for the fields used, are not symmetrical, and to obtain symmetry we must move the origin down to the point -200 ,

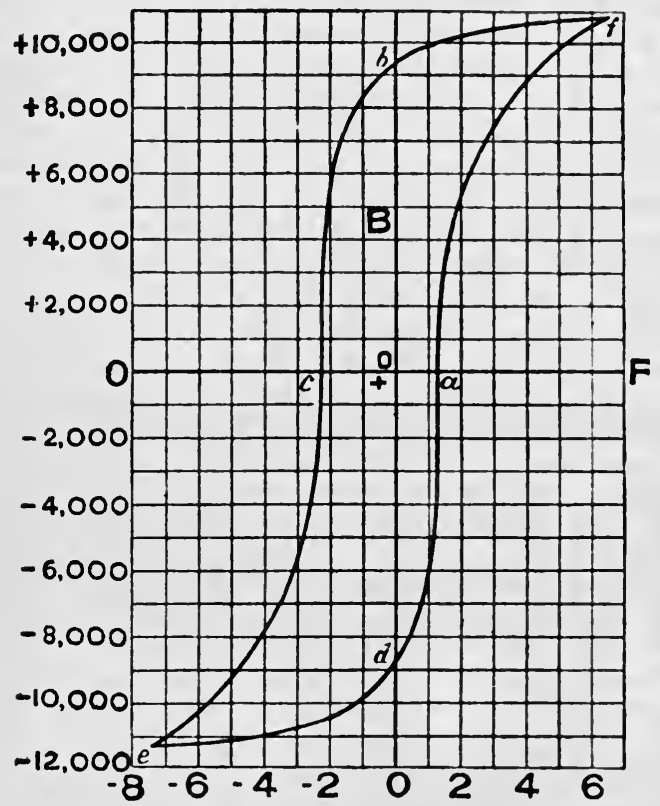

FIG. 258.

the value $B=200$ representing the induction produced in the iron by the earth's field. Hence by using these new axes we obtain a curve in which both $B$ and $C$ represent the actual values of these quantities, and the effect of the earth's ficld has been taken into account.

238. Correction on Account of the Finite Length of the Speci. men.-In the magnetometer method we assume that the magnetisation throughout the rod is uniform, and that the magnetising field is also uniform. Now this is certainly not true, for the magnetising field is not exactly the same as the field which would exist if the iron were removed, owing to the field produced by the magnetisation developed in the specimen. Now, although this demagnetising effect of the induced magnetisation can be calculated in the ease of an ellipsold, such a shape is not practicable, and although a very long and thin cylinder may, as a first approximation, be taken as being equivalent to an ellipsoid, yet 
experiment has shown that even in such a case the magnetisation is very far from uniform. ${ }^{1}$ Hence where accurate measurements of the magnetic properties of a sample of iron are required, it is necessary to employ some other method in which the iron is magnetised uniformly. Such a method is described in the next section.

Experiments have, however, been made to obtain the correction which has to be applied on account of the demagnetising field due to the poles developed at the ends of the specimen, and the results obtained may be applied to obtain an approximate correction in such cases where only comparatively short cylindrical rods of the iron are available.

If $F^{\prime}$ is the field at any point of a cylinder of iron due to the magnetism induced in the iron, then this quantity varies from one part of the bar to another, being a minimum at the centre of the bar. The mean value of $F^{\prime}$, however, is proportional to the mean intensity of magnetisation $I$, and the ratio $F^{\prime} / I$ is called the demagnetising factor $N$ for the bar. The value of this factor $N$ depends on the dimension ratio $m$ of the bar, that is, on the ratio of the length to the diameter. The following table gives the values of $N$ for different values of $m$ :-

Demagnetising Factors for Cylindrical Rods.

\begin{tabular}{|c|c||c|c|}
\hline$m$ & $N$ & $m$ & $N$ \\
\hline 10 & $0 \cdot 2160$ & 80 & 0.0069 \\
20 & 0775 & 100 & 0045 \\
30 & 0393 & 150 & 0020 \\
40 & 0228 & 200 & 0011 \\
50 & 00162 & 300 & 0005 \\
60 & 0118 & 500 & .00018 \\
& & 1000 & .00005 \\
\hline
\end{tabular}

Assuming that we know the demagnetising factor for a particular specimen, the easiest way of applying the correction is as follows :Plot the results of the measurements of $I$ and $F$, on the assumption that the demagnetising field is zero, thus obtaining the curve oA (Fig. 259). We have now to reduce the values of $F$ by an amount $F^{\prime}$ or $N I$, where $N$ is the demagnetising factor for the specimen. To do this draw the straight line oc, making an angle $\phi$ with the $Y$ axis, where

$$
\tan \phi=N \text {. }
$$

Then if we take the line oc as a new axis of $Y$, the values of the magnetising field measured from this new axis will be corrected for the demagnetising field. For consider a point $P$ on the curve. The intensity of magnetisation is $\mathrm{OM}$, and the demagnetising field is therefore $\mathrm{N} . \mathrm{OM}$. But $\overline{\mathrm{NM}}=\overline{\mathrm{OM}} \tan \phi=\mathrm{N} . \overrightarrow{\mathrm{OM}}$. Hence the demagnetising field is repre-

1 See Lamb, Philosophical Magazine, September 1899. 
sented by $\overline{N M}$, and the actual magnetising field acting on the iron is $\overline{O Q}-\overline{N M}$, that is, represented by the line NP. Thus to obtain the true

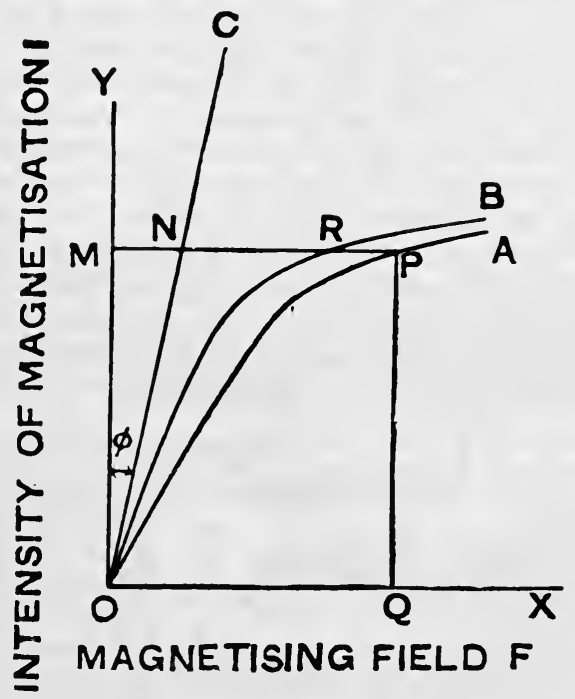

Fia. 259.

curve $\mathbf{O B}$, we decrease the abscissa of every point on the observed curve $\mathbf{O A}$ by an amount equal to the intersept between the line oc and the axis of $Y$.

239. The Ballistic Method of Measuring Permeability. - If a: ring of iron of which the dimensions are indicated by the symbols shown in Fig. 260 is wrapped round with a secondary coil containing $N$ turns, and over this is wound a magnetising coil of $M$ turns, while the magnetising coil is traversed by a current $C$. Then the mean magnetising field over the cross-section of the iron is ${ }^{1} F=2 M C / r$. The field at the inner edge of the ring is greater than this mean and that near the

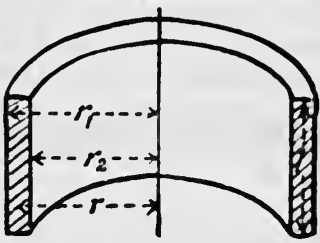

FIG. 260. outer is less, but if the radial width of the iron $r_{1}-r_{2}$ is kept small compared to the mean radius $r$, the

1 If we suppose the iron removed without disturbing the primary coil, and then carried a unit magnetic pole once round the anchor ring thus left, the work done would be $4 \pi M C$. For we should have carried the pole once round $M /$ circuits, through each of which a current $C$ is flowing. (See Watson's Physics, \$ 478.) If $F$ is the field along the middle of the ring, i.e. along a circle of ralius $r$, the work done when the unit pole is carried once round this circle is $2 \pi r P$. Hence equating the two expressions for the work, we get

$$
2 \pi r P=4 \pi M C \text {. }
$$


magnetising field may be assumed to be uniform and equal to the mean value. ${ }^{1}$

If $B_{1}$ is the,induction within the iron when the magnetising current is $C$, then the total induction, or the number of tubes of force which cross any cross-section of the iron, is $s B_{1}$, where $s$ is the cross-section of the iron. Hence since there are $N$ turns in the secondary, the total induction through the secondary is $s B_{1} N$. If the secondary is connected to a ballistic galvanometer, the resistance of the secondary circuit being $R$, and the magnetising current is changed to $C_{2}$, the magnetic induction in the iron becoming $B_{2}$, the quantity of electricity which flows through the secondary circuit will be given by

$$
Q=\frac{s N\left(B_{2}-B_{1}\right)}{R} \text {. }
$$

Hence if the throw of the ballistic galvanometer is $a$, and the constant of the galvanometer is $K$, we have

$$
\begin{gathered}
\frac{s N\left(B_{2}-B_{1}\right)}{R}=K \sin \frac{a}{2} \cdot\left(1+\frac{\lambda}{2}\right), \\
B_{2}-B_{1}=\frac{R K}{s N} \sin \frac{a}{2} \cdot\left(1+\frac{\lambda}{2}\right) .
\end{gathered}
$$

Thus a change of the magnetising field from $2 M C_{1} / r$ to $2 M C_{2} / r$ has caused a change in the induction 'of $B_{1}-B_{2}{ }^{2}$ which can immediately be calculated from the throw of the ballistic galvanometer if the constant of the instrument is known.

The general arrangement of the apparatus is shown in Fig. 261. The secondary coil of the iron anchor ring is connected to a ballistic galvanometer $\mathrm{G}$ and to a secondary coil $\mathrm{D}$ wound on a long solenoid FH. The object of this coil $D$ is to determine the constant of the galvanometer by noting the throw when a known current in the primary is

1 The errors produced in the case of iron by assuming that the field is uniform throughout the cross-section for different values of the external radius $r_{2}$, the width of the ring, namely, $r_{2}-r_{1}$, being unity, are as follows :-

\begin{tabular}{|c|c|c|c|c|}
\hline \multirow{2}{*}{ Value of $r_{2}-r_{1}}$. & \multicolumn{3}{|c|}{ Values of $r_{2}$ for the Error to be } \\
\cline { 2 - 5 } & $1 \%$. & $2 \%$ & $.4 \%$ & $1.0 \%$ \\
\hline 1.0 & 20 & 10 & 8 & 6.7 \\
\hline
\end{tabular}

2 Where $B_{2}-B_{1}$ is given in maxwells if $R$ is measured in c.g.s. units (10 $\left.10^{-9} \mathrm{ohms}\right)$. 
reversed (see $\S 220$ ). The primary coil of the anchor ring is connected through a two-way key $\mathbf{k}_{2}$ to a commutator $c$. This commutator is one designed by Ewing for use in such measurements, and may consist of a Pohl commutator, in which the connection between the cups 3 and 4 has been removed. To the cups 1 and 4 are connected an ammeter J, a battery $E$, and an adjustable resistance $R_{1}$. To the cups 3 and 4 are connected a key $k_{1}$ and a resistance box $R_{2}$, joined in parallel. The primary of the solenoid FH is connected to one cup of the two-way key $\mathrm{K}_{2}$ and to the cup 5 of the commutator $\mathrm{c}$, as shown in the figure.

When the key $\mathbf{K}_{1}$ is closed, then throwing over the commutator $\mathbf{c}$ simply reverses the current in the primary of the ring, without altering the magnitude of the current. When, however, the key $K_{1}$ is open, then putting the commutator over to the left not only reverses the current, but also reduces it, since the additional resistance $R_{2}$ is now inserted in the battery circuit.

When the constant of the ballistic galvanometer is to be determined, key $\mathrm{K}_{2}$ is turned to the right and the key $\mathrm{K}_{1}$. is closed, and the throw of the galvanometer noted when the commutator $\mathbf{c}$ is put across. The method of deducing the constant is that given in $\S 220$.

The key $\mathrm{K}_{1}$ remains closed while the mag-

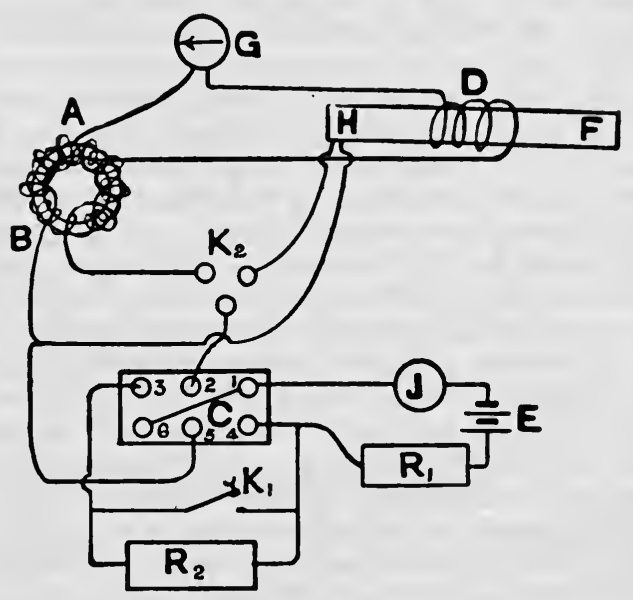

FIG. 261. netisation curve is obtained. The ring having been demagnetised by reversals $(\$ 240)$, the resistance $R_{1}$ is adjusted to give the minimum current. The commutator is then reversed twenty times to establish a cyclical state in the iron. The current is then noted and half the galvanometer kick when the current is reversed is obtained. The eurrent is then increased by reducing $\mathrm{K}_{1}$, and the procelure repeated. This is continued until the maximum current is reachel. The cycle of magnetisation is obtained by finding the change in induction when the current is reluced from its maximum value to various lower values and when changed to currents of different values in the reverse direction. The resistance $R_{1}$ is retained at that which gives the maximum current and the key $\boldsymbol{k}_{1}$ being closed, the commutator $\mathrm{c}$ is worked backwards and forwaris some twenty times, so as to establish a cyclical state in the iron. The 
commutator then being to the left, the current passing is noted and the key $\mathbf{K}_{1}$ is opened, thus producing a drop in the magnetising force, and the kick of the galvanometer is noted, as well as the new value of the current. We thus obtain the change in $\mathrm{B}$ produced by a certain drop in $\mathbf{F}$.

The key $\mathbf{K}_{1}$ is then closed and the commutator $\mathbf{c}$ worked for about twenty times to re-establish the initial conditions, and $\mathrm{R}_{2}$ is given a larger value. The current having been read, the key $\mathbf{K}_{1}$ is opened and the throw noted, as well as the new value of the current. In this way the change in B corresponding to a greater change in $\mathrm{F}$ is obtained. In this way a number of points are obtained, the final one corresponding to the resistance $\mathbf{R}_{2}$ being infinite, i.e. the kick corresponding to the change in induction when the magnetising field $\mathrm{F}$ changes from its maximum value to zero.

To obtain the portion of the curve corresponding to negative values of $F$, the key $K_{1}$ is closed and the commutator reversed twenty times, being finally left to the right. $R_{2}$ having been given a large value, the key $\mathrm{K}_{1}$ is opened and the commutator $\mathbf{c}$ moved to the left. In this way the current is reversed, and at the same time is reduced to a small value, the throw corresponding to a change of $F$ from its maximum positive value to a small negative value. Proceeding in this way $R_{2}$ is gradually decreased till it becomes zero, when the throw corresponds to the change in B, produced by reversing the maximum magnetising field. Half this change in $B$ gives the value of $\mathbf{B}$ corresponding to the maximum value of $\mathrm{F}$, and hence having plotted this point the other points in the curve can at once be set out, for we know the difference between the value of $\mathbf{B}$ for each of the steps in $F$ and the value of $B$ for the maximum value of $\mathbf{F}$.

It is unnecessary to observe the other half of the cycle, since the curve obtained will be similar to the first half.

By changing the value of the resistance $R_{1}$ cycles with different maximum values of $\mathrm{B}$ can be obtained.

Having plotted the $B-F$ curve, the hysteresis may be calculated by measuring the area of the loop enclosed by the curve. If $A$ is the area of the loop, then the energy dissipated per cycle in each cubic centimetre of the iron is $A / 4 \pi$ (see Watson's Physics, $\S 509$ ). If the $I-F$ loop is plotted, then the hysteresis loss per cubic centimetre per cycle is numerically equal to the area of the loop.

When calculating the hysteresis, it must be remembered that the area in square centimetres will only represent the hysteresis numerically if 1 centimetre represents unity in both B and F. Since B is in general enormously greater than $\mathrm{F}$, the scale adopted for $\mathrm{B}$ must be taken much smaller than that taken for $\mathrm{F}$. If $1 \mathrm{~cm}$. on the curve represents $f$ units of $\mathrm{F}$ and $b$ units of $\mathrm{B}$, then 1 square centimetre of the loop represents $f b$ ergs.

The advantage of the anchor ring is that since there are no free poles developed there is no demagnetising field, and hence the magnetising 
force is the same as the magnetic field which would exist inside the magnetising coil supposing the iron were absent. There is, however, a practical objection to this form in that every sample of iron wiich is to be tested requuires winding with a primary and a secondary coil, and since the winding has perforce to be done by hand, the process is one which takes some time. The ballistic method may be applied to the case of samples in the form of long rods by using as the magnetising coil a helix similar to that used in $\S 220$, and surrounding the centre of the specimen with a secondary coil, which is connected up to a ballistic galvanometer, as in the case of the anchor ring. This secondary coil may either be wound direct on the iron, or it can be wound on a glass or vulcanised fibre tube, which is slipped over the iron. If the secondary is wound on a tube, the diameter of the tube should be as small as possible, so that the space between the secondary coil and the iron may be as small as possible. A correction will have to be applied for the effect of the lines of force which thread through the secondary coil between the coil and the iron. If the cross-section of the iron is $s$, and that of the secondary coil is $\sigma$, while there are $N$ turns in the secondary, then when the magnetising field is $F$ the induction through the secondary, due to the lines of force of the magnetising field which are included between the secondary and the iron, is $(\sigma-s) N F$. Hence when the magnetising field is changed by an amount $\delta F$, the change in induction through the secondary, due to the field between the secondary eircuit and the iron, will be $(\sigma-s) N \delta F$, and hence the measured induction, as deduced from the galvanometer throw, will have to be reduced by this amount to give the change in induction through the iron. The advantage this method of testing a long thin rod has over the magnetometer method is that we are only here concerned with the intensity of magnetisation of the iron near the middle of the rod, which, if the rod is long and thin, is very nearly independent of the effect of the ends.

240. To Demagnetise a Specimen of Iron.--Since the induction in a sample of iron at any given instant depends not only on the magnetisi: $g$ force which exists at the time, but also to some extent on the previous Inagnetic history of the specimen, it is of importance to be able to reduce the iron to some definite magnetic condition which shall be independent of all previous magnetisation. A specimen of iron to which this process has been applied is said to be demagnetised.

In the case of thin wires, perhaps the most convenient method of demagnetisation is to heat the wires to redness. This can be done by slowly passing the wire through a Bunsen tlame, eare being taken to hold the wire horizontal and in an east and west direction, so that it is unacted upon by the earth's field.

In many cases, however, as, for example, with an anchor ring wound with its coils, heating to redness is impossible. 'The procedure here adopted is to pass an alteruating current through the primary winding, and to gralually reduce the strength of this alternating current to zero. It is of importance to stirt with a sufficiently strong current, 
otherwise the effects of previous magnetisations may not entirely be removed. The current employed should be such as to give an intensity of magnetisation considerably greater than that corresponding to the point where the $I-F$ curve bends sharply over, i.e. the field should be sufficient to magnetise the iron to saturation. In the case of soft iron, if the magnetising field at the start amounts to about 10 c.g.s. units (gausses), the demagnetisation will be complete.

If an alternating current is available, then this may be used to produce the demagnetisation, otherwise a small commutator driven by a small motor must be used to convert a direct current into an alternating one. A resistance, consisting of a trough about $50 \mathrm{cms}$. long and 5 $\mathrm{cm}$. wide and $8 \mathrm{~cm}$. deep, filled with zinc sulphate solution, should be included in the circuit. The electrodes consist of two amalgamated plates of zinc. One of these plates is fixed, and the other is movable. The trough is tilted, so that the depth of the solution at the end farthest from the fixed electrode is only about a centimetre. Starting with the movable electrode close to the fixed one, the movable electrode is slowly moved to the shallow end of the trough, by which means the current is gradually reduced to zero. 


\section{CHAPTER XXXIV}

\section{THE QUADRANT ELECTROMETER}

241. The Quadrant Electrometer. - The original Kelvin pattern quadrant electrometer was a very elaborate instrument, troublesome to set up, and difficult to keep in adjustment. Of recent years, however, a more simple form of quadrant electrometer has been found just as accurate, and very much easier to use. The best known pattern of quadrant electrometer of this more simple form is that designed by Dolezalek, ${ }^{1}$ and is shown in Fig. 262. The needle is made of two pieces of silvered paper of the form shown at (a) attached to a thin stem of aluminium, which also carries a mirror. The needle system is suspended by a thin strip of phosphor bronze or a quartz fibre, rendered conducting by being smeared

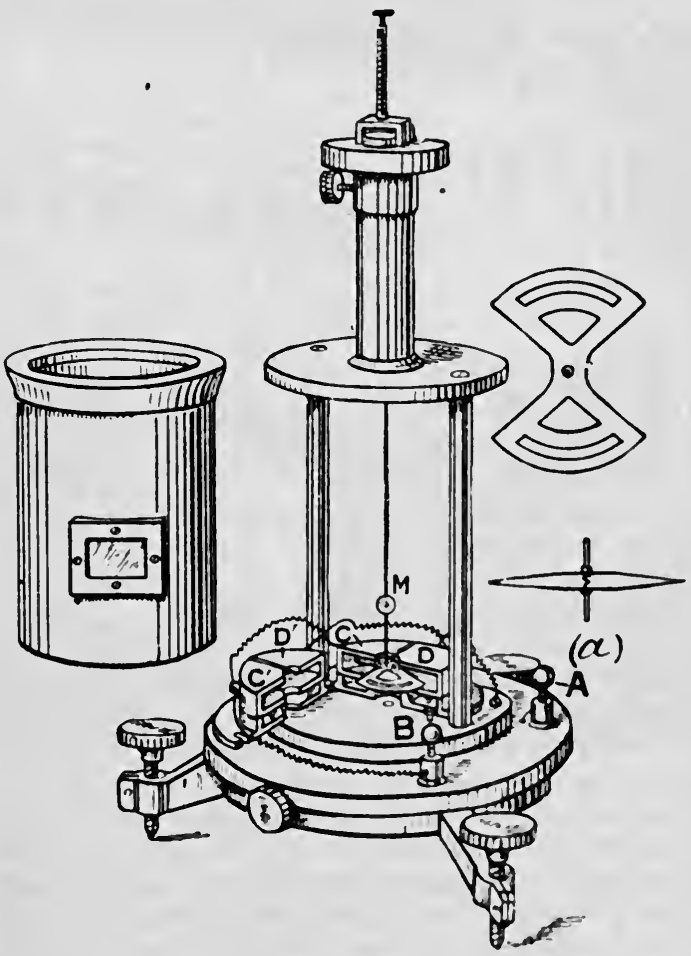

FI(3. 262. with a solution of calcium chloride, or coated with a thin layer of platinum.

The needle should be charged to between 100 and 200 volts, and for 
this purpose it is connected to one pole of a battery of about eighty small accumulators, the other pole of the battery, as well as the outside case of the instrument, being earthed by being joined to the gas or water pipes.

A convenient form of battery of small accumulators for use with an electrometer is shown in Fig. 263. The cells consist of glass tubes having a diameter of about 1 inch, and a length of 6 inches. Forty of these glass tubes are imbedded in paraffin wax contained in a shallow wooden tray, the upper inch of the tubes also being coated with paraffin wax to prevent the acid creeping. This coating is easily performed after the tubes are fixed in the tray by dipping them into some melted wax contained in a shallow dish. The electrodes consist of strips of lead bent into a $U$

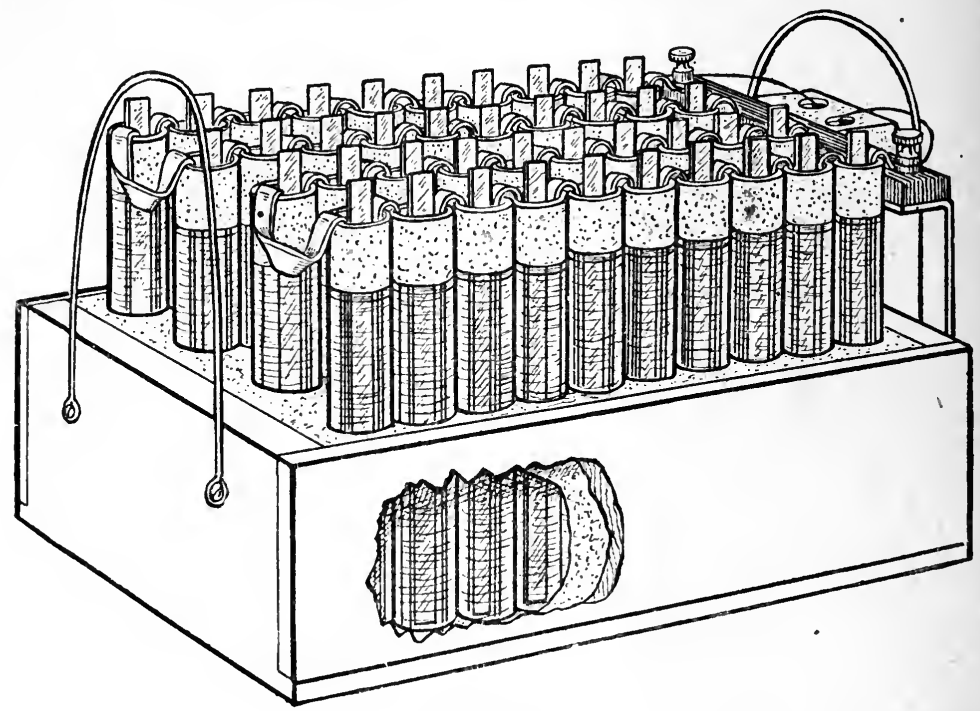

FTG. 263.

shape, each strip dipping into two adjacent glass tubes. A small strip of glass is placed between the lead plates in each tube, so as to prevent shortcircuiting by the lead plates coming in contact. The glass tubes are filled to within about an inch of the top with a solution of sulphuric acid, made by adding 1 part of pure sulphuric acid to 8 parts of distilled water.

If such a box containing forty cells is connected to a 100 volt lighting circuit through an ordinary 25 candle-power 100 volt incandescent lamp, the cells will be charged at the right rate. If the voltage of the electric supply is 200 , then two such boxes may be charged in series. When the cells are first set up, it will be necessary to charge them first in one direction and then in the other some twenty or thirty times, so as to "form" the plates. After the plates have been formed in this way, the 
cells should receive a good charging in one direction, and then care must be taken to always charge them in this direction.

It will be found advantageous to enclose the trays containing the cells in wooden boxes fitted with lids, as in this way the cells are protected from dust. The leads from the cells may be taken through holes in the sides of these boxes, the holes being bushed with paraffin wax.

Two boxes containing forty cells in each can be used in series to charge the needle of the electrometer, the boxes being charged by being connected in parallel if the lighting circuit is at 100 volts.

One pair of quadrants of the electrometer is permanently connected to earth by being joined to the case of the instrument. The other pair is connected to the apparatus being employed, and to a key by which these quadrants also may be earthed when required. A simple form of key for this purpose is shown in Fig. 264. It consists of a block of paraffin wax $\mathbf{A}$, in which is scooped a mercury cup $B$, the mercury being connected to the electrometer by the wire c. A piece of clock spring D is screwed to the base, and to the side of this spring is soldered a piece of wire $\mathrm{E}$. The spring is connected to earth through a wire $\mathrm{G}$, and when the spring is bent by pulling on a string $\mathrm{F}$, the point of $\mathrm{E}$ dips into the mercury, and the

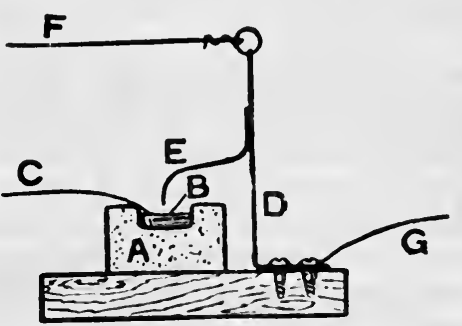

FIG. 264. electrometer is earthed.

Having set up the electrometer, and adjusted a teleseope and scale to measure the movement of the needle, connect the needle and both sets of quadrants to earth. Having made a note of the scale reading, charge the needle, kecping the quadrants earthed. If there is a change in the reading, it shows that the needle is not symmetrical with respect to the quadrants. In some forms of electrometer one of the quadrants is made adjustable, and in this ease this quadrant must be moved till the needle comes back to the position it occupied before it was charged. In most cases, however, it is very much easier to adjust the needle by tilting the whole instrument by means of the levelling screws. The scale reading having been brought back to its original value by tilting the instrument, it will be advisable to discharge the needle and make certain that the "uncharged" reading has not altered.

When the needle has bcen adjusted to a symmetrical position in this way, the deflections on the two sides of the zero should, for equal charges, be the same, also the position of the zero will not alter if the potential of the needle changes

242. Determination of the Capacity of a Quadrant Electrometer. -For many experiments it is necessary to know the capacity of a quadrant electrometer, for often this capacity is comparable in magnitude 
with the capacity of the condensers, \&c., with which the instrument is connected.

The electrometer A (Fig. 265) is connected to the key F, which is of the pattern shown in Fig. 264, and to a small mercury cup $\mathrm{G}$ in a block of paraffin. A second mercury cup in this block is connected to one coating of a small condenser. This condenser consists of two concentric brass tubes. The outer tube $D$ is connected with earth, while the inner

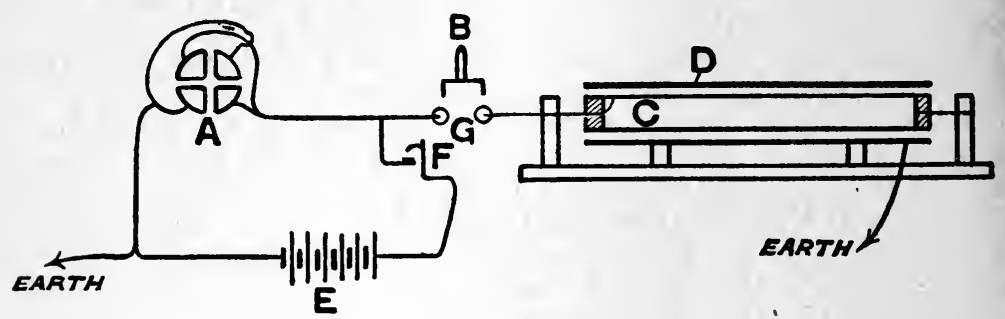

Fra. 265.

tube is insulated by being supported on two small ebonite blocks. If $l$ is the length of the tubes, $a$ the external diameter of the inner tube, and $b$ the internal diameter of the outer tube, the capacity is given in electrostatic units by

$$
C=\frac{l}{2 \log _{\epsilon} \frac{b}{a}}
$$

This expression neglects the effect of the ends, but if the space between the tubes is small, the error produced on this account will not be sufficient to invalidate the results.

The condenser being disconnected from the electrometer, the battery $\mathbf{E}$ is joined to the electrometer by closing the key $F$, and the deflection $d_{1}$ is observed. The key $\mathrm{F}$ having been opened, the electrometer is caused to share its charge with the condenser by inserting the small copper bridge B in the mercury cups $\mathrm{G}$. If the deflection is now $d_{2}$, we have, if $c$ is the capacity of the electrometer,

$$
c=C \frac{d_{2}}{d_{1}-d_{2}}
$$

For if we assume that the deflection is proportional to the potential, the original charge on the electrometer is $a c d_{1}$, where $a$ is a constant factor, while the charge on the electrometer and condenser is $\alpha(c+C) d_{2}$. Hence, since these charges are equal,

$$
c d_{1}=(c+C) d_{2}
$$

from which the formula given above at once follows. 
243. Determination of the Saturation Current. - If a layer of some radio-active substance, such as uranium oxide, is placed on an insulated metal plate c (Fig. 266), and this plate is connected to one pole of a battery $\mathbf{E}$, the other pole being earthed, then an electrometer $\mathbf{A}$ connected to a second insulated plate $\mathrm{B}$ will gradually be deflected, showing that a current is passing through the gas between the plates $\mathrm{C}$ and $\mathrm{B}$. If the difference of potential between the plates $\mathrm{B}$ and $\mathrm{C}$ is gradually increased, then the current which passes also gradually increases. This increase is at first rapid, but becomes slower and slower, till finally a fairly moderate further increase of E.M.F. produces no further increase in the current. This nlaximum current is called the saturation

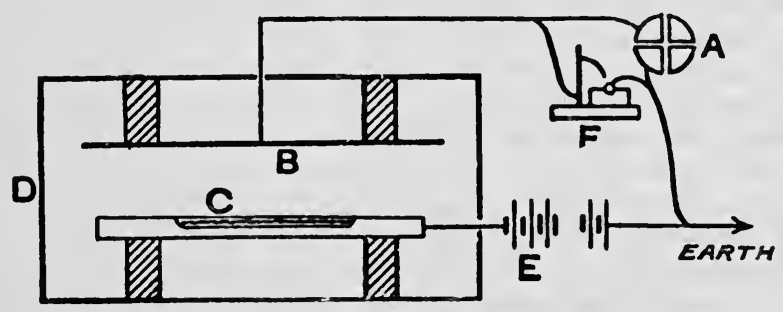

FIG. 266.

current, and is such that the ions being produced between the plates due to the radio-active material are being removed by this current as fast as they are being produced (see Watson's Physics, § 566).

The metal plates B and c (Fig. 266) are supported on ebonite pillars inside a metal box $\mathrm{D}$, and the radio-active substance is spread on the plate $\mathrm{c}$. The distance between the plates ought to be about a centimetre, when, with uranium oxide, the saturation current will be obtained with between 100 and 200 volts.

If $c$ is the capacity in electrostatic units of the electrometer and its connections, including the plate B (this quantity can be determined in the manner described in the last section), $d$ the number of scale divisions passed over in a second when the needle has settled down to its uniform speed of rotation, and $D$ the deflection produced by 1 rolt, that is, $1 / 300$ electrostatic units, then the rise in potential in one second is

$$
\frac{d}{300 D} \text { electrostatic units. }
$$

The charge on the electrometer and plate $\mathrm{B}$ has therefore increased in one second by $\frac{c d}{300 D}$ electrostatic units. 
But the increase in the charge in one second is equal to the current: Hence the current is

$$
\begin{aligned}
& \frac{c d}{300 D} \text { electrostatic units, } \\
& \frac{c d}{9 D} \times 10^{-11} \text { amperes }
\end{aligned}
$$

or

To perform the experiment, the small accumulators described in $\S 241$ are used, and starting with a few cells, the rate at which the electrometer deflection increases when the earthing key F (Fig. 266) is opened is determined. To obtain this rate, the time taken for the deflection to increase by 100 scale divisions is noted on a stop-watch, care being taken not to start observing till the needle has moved some little distance from its position of rest, so that its motion has had time to become uniform. When using an electrometer in this way to measure a current, it is important that the needle should be almost dead-beat. If the damping is too small, then the needle will move with an oscillatory movement superimposed on the steady movement. If, however, the damping is too great, the needle will take some time before its motion becomes uniform.

If the rate of movement of the needle is too great to be accurately observed, then a condenser may be put in parallel with the electrometer, in which case the quantity $c$ in (1) is the sum of the capacities of the condenser and the electrometer.

Determine the current passing between the plates $\mathrm{C}$ and $\mathrm{B}$ for different E.M.F.'s by the above method, and plot your results in the form of a curve, taking the current as ordinate, and the applied E.M.F. as abscissa. If you have been able to apply a sufficiently high E.M.F. to obtain the saturation current, the curve will be almost horizontal for the higher E.M.F.'s, and the current corresponding to this horizontal portion of the curve will be the saturation current.

Alter the distance between the plates $\mathrm{B}$ and $\mathrm{c}$, and repeat the observations. In this way prove that as the distance between the plates increases the current produced by a given difference of potential increases, in this respect differing from what occurs when an E.M.F. is applied to an electrolyte.

244. Determination of an Insulation Resistance.-The electrical resistance of an insulating material or the value of any very high resistance may be found by the leak of the charge of a condenser through the material. The difference of potential between the plates of the condenser is measured by a quadrant electrometer connected to the two plates. A preliminary experiment is first performed to see whether there is any leak through the condenser itself or through the electrometer. The condenser is first charged by connecting the terminals to a suitable battery, and the connection is then broken, and readings are taken of the time and deflections of the electrometer as the potential 
difference falls. The experiment is then repeated with the insulating material under test permauently connected to the two terminals of the condenser. The capacity of the condenser should be such as to ensure a satisfactory rate of leak, and, in the case of an extremely high resistance, the capacity of the electrometer itself may be sufficient. Let the capacity of the condenser and electrometer in parallel be $C$ and the potential difference between the plates at any moment be $V$. Then the quantity of electricity on them at any moment is $C V$ and the current flowing through the combination is $d C V / d t$. If $R_{1}$ be the resistance of the electrometer and condenser in parallel, we shall have in the preliminary experiment

$$
\begin{gathered}
-\frac{d C V}{d t}=\frac{V}{R_{1}} \\
-C R_{1} \frac{d V}{V}=d t \\
C R_{1} \log _{\epsilon} \frac{V_{0}}{V_{1}}=t_{1}-t_{0} \\
C R_{1} \log _{\epsilon} \frac{d_{0}}{d_{1}}=t_{1}-t_{0}
\end{gathered}
$$

where $d_{0}$ and $d_{1}$ are the deflections of the electrometer at times $t_{0}$ and $t_{1}$. In the second experiment, if the unknown resistance is $R_{2}$, we have $R_{1}$ and $R_{2}$ in parallel, and

$$
C \frac{R_{1} R_{2}}{R_{1}+R_{2}} \log _{\epsilon} \frac{d_{0}^{\prime}}{d_{1}^{\prime}}=t_{1}^{\prime}-t_{0}^{\prime}
$$

where $d_{0}^{\prime}, d_{1}^{\prime}, t_{0}^{\prime}$, and $t_{1}^{\prime}$ are the corresponding ohservations. From the two equations, the two unknown quantities $R_{1}$ and $R_{2}$ can be obtained.

If a known high resistance $R_{2}$ is available, the experiment nay be used to measure the resistance and capacity of an unknown condenser. A condenser may be made of a slab of ebonite with tin foil fixed over the two large surfaces. Its capacity having been found by the above experiment, the dielectric constant of the ebonite may be obtained by calculating the capacity the condenser would have if the ebonite was replaced by air, and finding the ratio of the two capacities. The specitic resistance of the ebonite can also be calculated from the value of $R_{1}$ obtained.

The experiment can also be performed by the method of $\$ 221$. The condenser, to the terminals of which the resistance is connected, is charged and immediately discharged through a ballistic galvanometer and the kick noted. This is repeated with various short intervals between the time of charging and discharging, the condenser being insulated for these periods except for the high resistance. In this way the curve showing the rate of discharge can be obtained. 


\section{CHAPTER XXXV}

\section{THE MECHANICAL EQUIVALENT OF HEAT}

245. Measurement of Joule's Equivalent in Mechanical Units.-The apparatus for this method has been designed by Professor Callendar, and is manufactured by the Cambridge Scientific Instrument Co. It is shown in Fig. 267. The heat is developed by the friction between a stationary silk belt and a revolving drum round which it passes, and

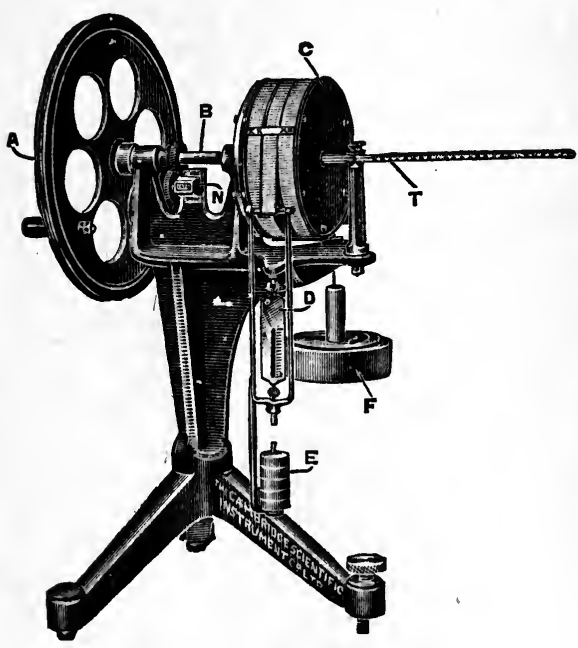

Fig. 267. it is measured by the rise in temperature of the drum and the water it contains. The calorimeter drum $\mathrm{c}$ is made of thin brass, and is suitably mounted for rotation about a horizontal axis B. The driving wheel A can either be turned by hand or may be driven by a small electric motor. The drum is fixed to and kept apart from a disc at the end of the axis by means of six studs of ivory to prevent heat transference from the drum. An axial hole in the outer end of the drum serves for the introduction of water into the lower half and the insertion of a thermometer which has a bulb bent round to dip into the water. The swirling of the water during the rotation of the drum ensures efficient mixing and uniformity of temperature. The number of revolutions of the drum is recorded by the counter $\mathrm{N}$. The silk belt passes round the drum for one and a half complete turns. For half this distance the belt is composed of two widths in parallel, with an equal space between them, and affixed to one end is a single width which passes round the drum for the other half of the distance, lying between the double portion for half a turn. The free end of the double belt supports the large weight $F$, while the single belt supports 
the smaller weight $\mathbf{E}$. The weight $\mathbf{E}$ is also partly supported by a spring balance $D$ attached to the frame of the apparatus. When the drum is rotated, the friction causes $F$ to rise, and the weights should be adjusted so that $\mathrm{F}$ and $\mathrm{E}$ remain stationary in floating equilibrium. The surface of the brass drum should be kept smooth and bright, and the silk belt should be clean and dry. Before making a determination, the drum may be lightly polished. The presence of dirt or moisture will cause the friction to be erratic and render it difficult to obtain steady equilibrium. Such variation as may be present is eliminated to a large extent by the action of the spring $D$. If for any reason the friction becomes less, $F$ will tend to fall and $\mathrm{E}$ to rise, a smaller proportion of the weight $\mathrm{E}$ being then taken by the spring balance and more by the belt. The increased tension of the belt will then increase the friction again and tend to restore the original balance. The torque on the drum is measured by the radius multiplied by the difference between the tension on the belt at the two ends, i.e. $F$ at one end and $\mathrm{E}$ less the force on the spring balance at the other. The work done is equal to the torque multiplied by the radians turned through, i.e. by $2 \pi n$ where $n$ is the number of revolutions.

A determination is conducted as follows: A weighed quantity of water nearly sufficient to fill the lower half of the drum is inserted by a pipette, and the thermometer is placed in position. A few turns of the drum are made and the weights are adjusted so that the weight $\mathbf{P}$ is carried up into floating equilibrium, and the pointer of the balance is about midway on its scale. The weights required are practically independent of the speed of rotation. The turning is then continued at a rate of about 100 revolutions per minute and the temperature is noted at every 100 revolutions. The time is also noted and the readings of the spring balance. When a rise of 5 or 6 degrees has been obtained, the rotation is discontinued and the calorimeter allowed to cool, a time-temperature curve of cooling being obtained. From this a correction for the heat lost by radiation during the experiment can be obtained, as in the specific heat experiment (\$ 82). If $M$ is the mass of water including the water equivalent of the drum, and $\theta$ is the average rise in temperature for $n$ revolutions, corrected for radiation loss, then the heat developed during $n$ revolutions is $M \theta$. If $D$ is the average reading of the spring balance and the radius of the drum is $R$, the work done in ergs is $2 \pi n R(F-F+D) g$. We therefore have where $J$ is Joule's equivalent in ergs per calorie

$$
J M \theta=2 \pi n R g(F-E+D) .
$$

246. Measurement of Joule's Equivalent in Electrical Units.-If a current $C$ is passed through a wire of resistance $R$, the difference of potential between the ends being $E=C K R$, then the heat developed in $t$ seconds being $H$,

$$
J H=E C t=C^{2} R t .
$$


$J$ is the value of Joule's Equivalent and if $C$ and $K$ are measured in amperes and volts respectively, $J$ will be obtained in joules per calorie.

As the resistance of the wire will usually vary as it becomes heated, it is most convenient to use the former formula and to measure $E$ and $C$ at frequent intervals during the experiment. The heat is used to warm a quantity of liquid and is measured by the rise in

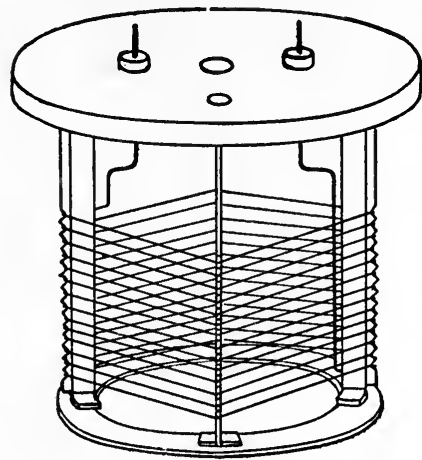

Fig. 268. temperature produced. A calorimeter and water jacket similar to that described in $\S 82$ may be used in the determination, the calorimeter being about $6 \mathrm{~cm}$. diameter and $8 \mathrm{~cm}$. high. A light framework is attached to the under side of the lid of the calorimeter in order to carry the wire through which the current will flow. This framework, Fig. 268, consists of four uprights fixed into the lid at the top and to a light copper ring at the bottom. The uprights are made of thin copper foil, bent double down their length and holding between the two parts a strip of thin mica. The mica projects beyond the foil, and has a serrated edge directed outward diagonally from the frame. Bare wire can thus be passed round and round the frame horizontally and will be held in the serrations of the mica. The whole should be as light as possible in order to reduce its water equivalent. Two thick copper wires of negligible resistance are passed through insulating plugs of ebonite in the lid and terminate about $2 \mathrm{~cm}$. below it. The resistance coil is made of manganin, and is soldered to one of the copper wires, then passed round and round the frame. Care must be taken that it lies evenly and taut in the serrations of the mica, so that there is no possibility of one turn coming into contact with an adjacent one. When the coil is wholely wound, the wire is passed upwards inside the frame and soldered on to the second copper wire. The arrangement is completed by a stirrer and thermometer which is graduated in tenths of $\mathrm{a}^{\circ} \mathrm{C}$., passing through the holes shown in the lid.

The electrical connections are shown in Fig. 269. The current is obtained from the battery $\mathbf{B}$ and regulated by the rheostat $\mathbf{R}$. $\mathbf{K}$ is a key, s a standard 1-ohm coil, and $\mathrm{A}$ an ammeter for preliminary adjustment of the current. The potential difference at the ends of the calorimeter coil is measured with a Rayleigh potentiometer, $\S 205$, by balancing it against that of the standard cells w. The current is similarly obtained by measuring the potential difference between the terminals of the standard 1-ohm coil. The experiment is conducted as follows: The calorimeter having been filled with water to a depth 
of $1 \mathrm{~cm}$. above the coil, it is weighed and placed in the water-jacket. The key $K$ is then inserted and the current adjusted to the approximate value desired, by the rheostat and ammeter. The readings of the potentiometer for balance of the potential differences across the calori. meter wire and across the standard coil are found and noted. The current is then broken and the water allowed to cool. These preliminary readings will enable the values to be obtained rapidly during the experiment proper. When the calorimeter has cooled to atmospheric temperature or thereabouts, readings are taken of the time and temperature for some minutes. The current is then switched on, and

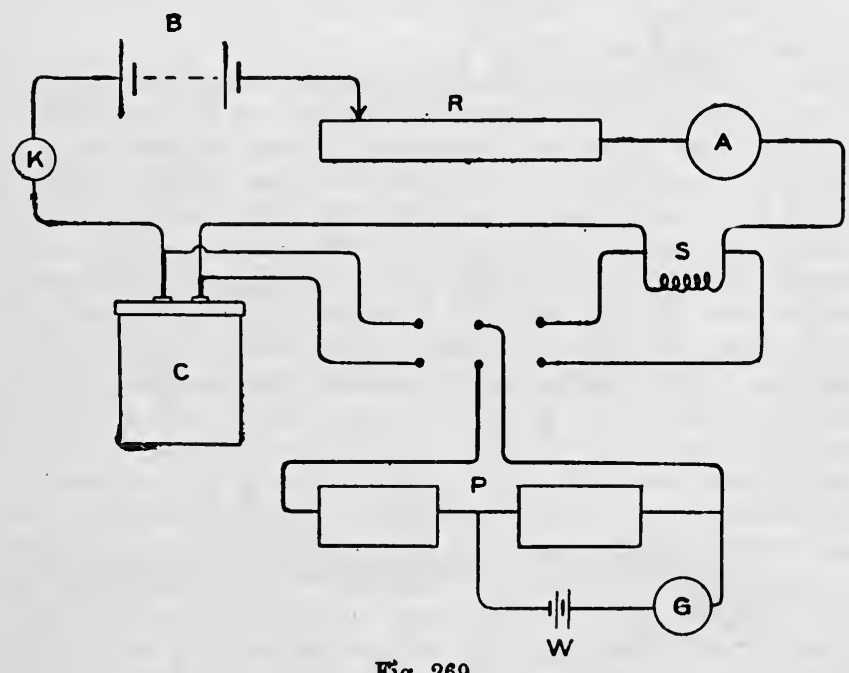

Fig. 269.

readings of the time, temperature, and the two potential differences are taken every half-minute. After the experiment has continued for about three minutes with a rise of temperature of about $5^{\circ} \mathrm{C}$., the current is broken and readings of time and temperature taken as the calorimeter cools. During the whole experiment the water should be stirred vigorously in order to equalise the temperature throughout.

From the time-temperature curve a correction can be made for the heat lost by radiation as in the case of specific heat experiments by the method of mixtures. Owing to the time taken to reach the maximum temperature, this correction is much greater than in the specific heat determinations. In order that it shall not be too great, the duration of the experiment must be limited to a very few minutes, and the current and resistance of the wire adjusted to enable this time to produce a reasonable increase of temperature of the water required to immerse the wire. In apparatus of the size described carrying about 200 grams of 
water, a coil of 3 ohms carrying 3 amperes is suitable. The principal cause of error in the determination is the water equivalent of the apparatus which is greater and less definite than in the specific heat measurements, owing to the mass of the wire and frame and the use of mica and ebonite. If $M$ is the mass of water including the water equivalent, and $\theta$ is the rise in temperature corrected for radiation loss,

$$
\begin{aligned}
J H=J M \theta & =C E t \\
J & =C E t / M \theta .
\end{aligned}
$$

247. Measurement of Joule's Equivalent by the Continuous-Flow Calorimeter. - A method has been devised by Callendar in which the radiation correction can be eliminated and the water equivalent correction does not occur. A steady flow of water is maintained through a tube in which it is heated by an electric current passing through a strip of manganin. After some time a steady state is reached and the apparatus takes up a temperature distribution which remains constant. Except for the heat lost by radiation, the energy is now used solely to heat the stream of water, and none is used to heat the apparatus, whose water equivalent is thus not required. The radiation correction is eliminated by taking two currents of water of different magnitude, and applying in each case sufficient electrical energy to give the same temperature rise and distribution, and hence the same radiation loss. The difference in the electrical energies in the two cases is the energy required to heat a stream of water equal to the difference of the two streams used. If the rise in temperature is not exactly the same in the two determinations, it can be assumed that the radiation loss is proportional to the average difference of temperature between the calorimeter and its surroundings.

The general arrangement of the apparatus is shown in Fig. 270. The Marriott bottle $\mathrm{H}$ supplies the vessel $\mathrm{K}$ with distilled water. In $\mathrm{K}$ a constant head is maintained, the overflow running down the central tube. The water passes from $\mathbf{k}$ through a tube of capillary bore to the jacket of the calorimeter c. The viscous resistance of this capillary tube is much greater than that of the calorimeter, and, as it is surrounded by water at an approximately constant temperature, its viscous resistance and consequently the rate of flow of the water remains very nearly constant. The water after passing through the jacket enters the central tube in which it is heated. It thus always enters the calorimeter at the temperature of the jacket. The heater is of manganin, and is made in the form of a twisted strip which serves the double purpose of rendering the surface exposed as great as possible, and also of causing the water to circulate in its flow. Its resistance is about $1 \mathrm{ohm}$.

The construction of the calorimeter is obvious from the diagram, but the insertion of the twisted strip and its end connections presents certain difficulties. A section of one end is shown in Fig. $270(a)$, in which $a b$ represents the end of the calorimeter glass tube and $c d$ a 
concentric tube of very thin metal closed at the end $d$. A flat strip of metal is soldered on the outside of $c d$, forming a spiral, and compelling the water to flow round $c d$ in the concentric space between $a b$ and $c d$. A thermometer placed within $c d$ measures the temperature of the water. The twisted strip of manganin in which the heat is developed is fixed to the end $d$ of the metal tube, and a tab $e$ serves to connect the current and potential leads. The twisted strip can be fixed to one metal tube before insertion into the apparatus, but at the other end it must be

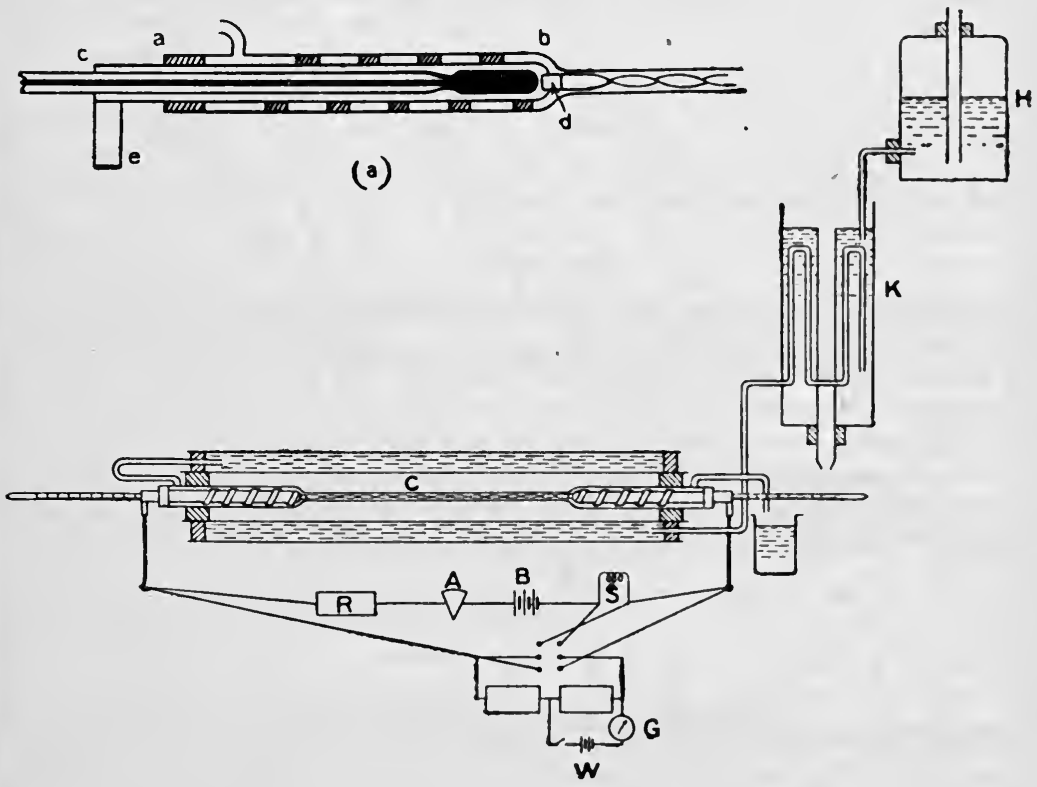

Fig. 270.

attached in situ. This is done by having a headless screw soldered on to the end of the strip and a corresponding threaded screw-hole in $d$. The strip is held by a fine screw-driver passing down the inside of cd, which can then be screwed on to the strip. A small portion of solder placed within the tube at $d$ and fused by a red-hot metal wire placed in contact, completes the altachment and renlers it watertight.

The electrical connections are similar to those of the preceding experiment. The potential difference between the ends of the heating: strip is foumd by balancing it against that due to standard cells $v$. For the slow flow the current should be adjusted to about 1.5 amperes, and the height of $\mathrm{K}$ ahove the calorimeter should be such as to give a flow of water whose temperature rise is about $5^{\circ} \mathrm{C}$. For the quick flow, 
the current should be about $2 \cdot 2$ amperes, and the height of $\mathrm{K}$ increased so that the rise in temperature is approximately the same as before. The flow in the second case will be about double that in the first. An ammeter $\mathrm{A}$ is placed in circuit so that the current may be approximately adjusted without resource to the potentiometer circuit.

To avoid parallax, the thermometers may be read by telescopes placed some distance away. After the temperatures have become steady, the two thermometers are read, and the water is allowed to flow into a weighed beaker until about 100 grams have been collected. The temperature rise is again noted, and the mean value taken. While the water is flowing into the beaker, the electrical measurements may be made. The whole operation is then repeated three times.

Let $C_{1}, E_{1}, Q_{1}$ and $\theta_{1}$ be the current, potential difference, quantity of water per second, and temperature rise for the slow flow. The central tube is at a mean temperature of $\frac{1}{2} \theta_{1}$ above that of the jacket. Hence the radiation loss will be proportional to $\frac{1}{2} \theta_{1}$. Let it be (say) $h \theta_{1}$ calories per second. If $s$ is the specific heat of water,

$$
C_{1} E_{1}=J s Q_{1} \theta_{1}+h \theta_{1}
$$

Four such equations will be obtained from the four series of observations with the slow flow, and an average may be obtained by addition. If $C_{2}, E_{2}, Q_{2}$ and $\theta_{2}$ are the corresponding observations for the quick flow,

$$
C_{2} E_{2}=J s Q_{2} \theta_{1}+h \theta_{2}
$$

From the two equations $h$ can be eliminated and $J s$ determined. If $s$ be taken as unity, $J$ is known.

The two thermometers used in the experiments should be compared by placing them in a bath of water at the temperatures of the observations. 


\section{A P P E N D I X}

1. Glass Blowing.-The art of performing simple glass blowing opera tions is so essential, that although it is almost impossible to impart it by description, yet it may be worth while to devote a few pages ${ }^{1}$ to giving a few hints. In glass blowing success can only be obtained after much practice, and wherever possible the student is advised to obtain some lessons from a competent worker.

The blow-pipe to be used is an important adjunct, but the exact pattern is not of very much importance. There are many forms of blowpipe on the market, some of which are complicated and expensive. The student is, however, recommended to use a simple pattern, having a single tap on the gas-supply, and a joint by which the inclination of the jet can be varied. The chief fault of most blow-pipes as supplied by the makers is that the nipple at the end of the gas-supply pipe is much too fine. The bore of the hole in the nipple ought to be between 0.5 and $2 \mathrm{~mm}$., a very useful size being $1.4 \mathrm{~mm}$. A foot bellows of ample size will be required to supply the air, and this bellows must be fastened to the blow-pipe table, so that when in use it cannot move away from the operator's foot, as invariably occurs at a critical moment if it simply rests on the floor alongside the table. Fletcher's universal blow-pipe, number 3, on a stand, with a tap to the gas-supply, and his foot blower, number 3 or 5 , will be found suitable for all but the heaviest glass blowing required in an ordinary physical laboratory.

Since soda-glass is almost invariably used for making physical apparatus, and, further, its manipulation is simpler than that of lead-glass, the following notes only apply to the former :-The glass used should be of a good quality, and should bear heating in the blow-pipe flame for some considerable time without showing any signs of becoming frosted.

Other accessories which will be required in addition to the blow-pipe and bellows are: (1) some small triangular files for cutting off glass tube; (2) some pieces of wood charcoal, sharpened at the end into conical points of different sizes; (3) a wire nail, about 2 inches long, fitted in a handle : the top of the nail is cut off, and this end is forced into the handle so that the point projects for about an inch; (4) a small mouth-piece made by first expanding the end of a piece of glass tube of

1 A small book in which there are many hints on glass blowing is Shenstone's The Methods of Glass Blowing (Longmans). Many hints on glass blowing and many other matters of interest to the worker in a physical laboratory will bo found in Laboratory Arts, by Threlfall (Macmillan). 
about $7 \mathrm{~mm}$. diameter, and then, when the glass is still hot, pinching the tube together near the end. This mouth-piece is attached to about a metre of small-bore rubber tubing, and is used for blowing into pieces of apparatus which cannot conveniently be brought directly to the mouth, or where pressure has to be exerted on the work while being heated in the flame.

(a) Bending Glass Tube.-Glass tube up to about a centimetre in diameter can most conveniently be bent by heating the place where the bend is to occur over an ordinary bat's-wing burner. While the tube is being heated it must be continually rotated, being supported by the hands placed as nearly as possible at the centre of gravity of the two portions of the tube on opposite sides of the heated portion. When it is felt that the tube has become quite bendable, remove it from the flame, and slowly and steadily 'bend the two sides upwards. Throughout the bending operation the tube should be kept in a vertical plane, otherwise the weight of the heated portion of the tube will cause the bend to be on one side.

To be nd tube having a diameter greater than a centimetre without reducing the bore is a difficult operation. One end of the tube must be stopped with a cork, and in the other is placed a cork, through which passes a short length of quill tubing. This piece of tube is connected to the rubber-tube attached to the mouth-piece described above. The tube to be bent is heated in a very large blow-pipe flame, and as the glass softens the hands are very slightly pressed together, so as to slightly thicken the glass. If the glass during this operation shows a tendency to con. strict the bore, it must be removed from the flame, and must be gently blown out. When about 4 or $5 \mathrm{~cm}$. of the tube has been thickened in this way, having thoroughly and uniformly heated the thickened portion, remove the tube from the flame and gently draw it out, and immediately after, or rather almost during the drawing out, bend the tube into the required shape. During the drawing out and bending blow gently into the tube, so as to prevent the sides from collapsing.

If constricting the tube at the bend is no disadvantage, then the following method is quite easy:- Heat the tube in a moderate sized flame, and gradually thicken the glass by very gently moving the hands towards one another. When about $4 \mathrm{~cm}$. of the tube has been thickened, so that the bore is about two-thirds of its original size without the outside being much reduced in size, remove the glass from the flame, and very slowly draw the ends apart. When the drawn-down portion is about $8 \mathrm{~cm}$. long, bend the two ends upwards. The glass at the drawndown portion being thick will retain enough heat to remain flexible for some considerable time. Hence it will be easy to adjust the bend to the desired shape. The thickness of the walls also prevents the sides of the tube collapsing at the bend.

(b) To Close the End of a Tube.-Hold the end of the tube in the blow-pipe flame, and when the edge of the glass is soft, take a small piece of waste glass and push the walls of the tube together. Having bunched the end of the tube together, ailow the waste piece of glass to 
adhere, and then heat the tube a little beyond the bunched in portion with a small flame. The tube must be kept in continuous rotation during this operation, and a gentle outward strain kept on the piece of glass which was fixed on the end. In this way a narrow neck is produced, and by continuing the heating this neck is gradually closed, and the end piece of glass is drawn off. The tube will thus be closed, but the closed end will be conical, ending in a sharp point where the glass was drawn off. The conical end is then heated pretty strongly, and after removal from the flame, the end is rounded by blowing gently into the tube. To successfully round off the tube, the heating and blowing require the exercise of some judgment. If, however, the following is kept in mind, success will be obtained after a little practice :-When the tube is in the flame the thinner portions get hottest, and hence if you blow into the tube immediately after its removal from the flame, these hot portions will be most blown out, and hence the thin parts will become thinner. If, however, you wait a little after removing the glass from the flame, the thin parts will cool most rapidly, and hence if you then blow into the tube you will blow out the thicker portions, since these will have retained the heat. In this way the thickness of the glass is gradually made more uniform. It will in general be necessary to heat up the end two or three times, blowing it out between each heating, before a nicely rounded end, having a uniform thickness, is obtained.

(c) To Blow a Bulb on the End of a Tube.-Having closed the end of the tube in the manner described above, hold the tube in the right hand, with the closed end slightly higher than the open end, and so that the flame plays on the tube a little inside the end. Keep the tube rotating backwards and forwards by rolling it with the thumb over the extended fingers. When the glass has thickened and the bore been reduced to about half, start heating a little farther along the tube. The rate of turning must be so adjusted that it just counteracts the tendency of the heated portion to droop under the action of gravity. If the heated portion becomes very irregular, remove the tube from the flame, and gently blow into the tube. By alternate heating and blowing obtain a pear-shaped mass of glass, the bore of the tube, however, running almost to the end. It is most important that the bore should extend almost to the end of the lump of glass, and this was the reason why the first heating was made just inside the end. When the pearshaped mass of glass has been obtained it must be heated to a bright red, the heating being as uniform as possible. Then remove the gliss from the flame, and holding the tube as nearly vertical as possible, the heated end being downwards, very gently blow into the tube. It is advisable to keep the tube slowly rotating while you are blowing out the bulb. If the bulb shows a marked inclination to bulge out in one direction, hold the tube horizontal with the bulge turned downwards. The uprush of cold air produced by convection will in this position slightly cool the lower portion of the bulb, and hence this portion will not be blown out so easily. 
If the first attempt results in a very irregular bulb, by turning on the gas full and using a large flame with little air the bulb may slowly be reduced in size. Whenever the surface becomes crinkled, gently blow into the tube. This reducing process must be continued till the pearshaped mass of glass, with a small hole down the centre, is again obtained. It is useless to attempt to again blow up the bulb after only slightly reducing its size.

(d) To Blow a Bulb in the Middle of a Tube.-Having closed one end of the tube, either by drawing it out in the flame or by means of a cork, hold the tube on the two sides of the place where it is desired to make the bulb in the two hands. The two hands must be held in the same way, either with the tube held on the outstretched fingers by means of the thumbs, the backs of the hands being downwards, or rolling on the thumb and first finger, the back of the hands being turned upwards. Unless both hands are held in the same way, it will be found very difficult to turn the two halves of the tube at exactly the same rate. Heat the tube in a moderately large flame, keeping up a to-and-fro rotatory motion, and slowly press
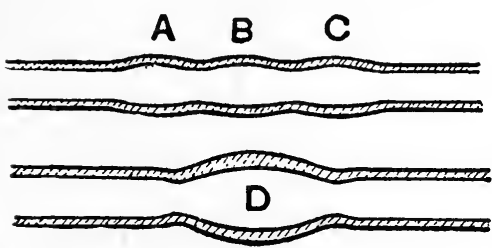

FIG. 271. the two halves together. When the glass has thickened, blow out the mass slightly as at A (Fig. 271). Then heat the tube alongside the thickened portion, and having thickened the glass, slightly blow it out. Repeat the process again if a large bulb is required, so that the tube has the form shown in the figure. Next using a large flame, heat the thickened portion, and by alternately heating and gently blowing, work the glass into the form shown at $\mathrm{D}$. Finally, heat the collected glass to a uniform bright red, and then removing the tube from the flame, blow the bulb of the required size. The bulb must be blown slowly, and any tendency to a bulge on one side checked by turning this side down. If an oval bulb is required, the two portions of the tube must be pulled apart as the bulb is reaching its full size.

(e) To Join together Two Pieces of Tube.-If the two pieces of tube have the same diameter, then having seen that the ends to be joined are both smooth and at right angles to the axis of the tube, close the other end of one piece. Then using a fairly large flame, heat the extreme ends of the tubes, and when the glass is quite hot and plastic remove from the flame, and immediately press the two ends together, taking care that they are in contact all round. If the tubes are of small diameter, turn the flame down and thoroughly heat the joint all round till the glass has fallen together enough to appreciably reduce the bore. Remove the tube from the flame and gently blow into the open tube, and at the same time draw the two halves of the tube apart. The pressure exerted and the 
amount of traction must be so adjusted that the diameter of the tube at the joint becomes the same as that of the rest of the tube, while the thickness of the glass at the joint is also the same as that of the rest of the tube. The joint must then be well annealed by rotating it over a small flame, obtained without using any air in the blow-pipe. In this smoky flame the glass will become coated with soot. After heating the glass for a minute or two over the smoky flame, wrap the joint round with cotton wool, and set it aside to cool.

If the tubes have a diameter greater than about $7 \mathrm{~mm}$., then after making them adhere as described above, turn the flame down so that a very small pointed jet is obtained. With the point of this jet heat a small portion of the joint till the glass is quite fluid. Then remove the glass from the flame, and by gently blowing into the tube blow out the heated portion of glass which will have caved in under the action of the flame. Next heat in the same manner the adjacent portion of the joint, and proceeding in this way gradually work completely round the joint. $\mathrm{By}$ this procedure the glass at the joint will be thoroughly fused together, and during the process the portions of the joint, which are not being strongly heated, will be rigid, and so will assist in preventing the joint becoming irregular. Having welded the glass well together by the above method, the whole joint must be heated by rotating the tube in a slightly larger flame, and then by blowing and gentle traction the joint must be brought to the same diameter as the rest of the tube. Finally, the joint must be annealed as described above.

If the tubes to be joined are of small diameter, it will be found of advantage to enlarge them at the ends to be joined. This can be done by heating the end of the tube and then inserting the iron nail fitted to a handle and rotating the tube, the nail being pressed against the inside of the tube.

If the tubes to be joined are of different diameters, then the larger tube must be drawn down till the drawn-down portion is of the same diameter as the smaller tube. The junction between this drawn-down portion and the other tube is then effected as in the case of two tubes of equal diameter.

(f) To Ma'se a T-piece.-To make a T-piece, close one end of the glass $A$, which is to form the top of the $T$, and also one end of the glass B, which is to form the stem. Then using a very small pointed flame, heat a single spot on the tube $\Delta$ at the point where the side tube is to be attached. During this heating the glass must not be rotated. When the spot of glass is red-hot, blow gently into the tube, and thus raise a small pimple on the glass. The diameter of this pimple ought to be the same as the diameter of the tube B. Next strongly heat the extreme top of the pimple, and blow strongly into the tube. In this way a large kidneyshaped bulb of very thin glass will be produced. This bulb is broken away, leaving a hole in the side of the tube $\Delta$ with a raised edge, the diameter of the hole being approximately the same as the diameter of the tube $\mathbf{B}$. 
Taking the tube $\mathbf{A}$ in the left hand and the tube $\mathbf{B}$ in the right, heat the hole in $\mathbf{A}$ and the end of $\mathbf{B}$ in a fairly large flame, and when the edges are fairly fluid bring them in contact, being careful that no gaps are left.

Next attach the rubber tube and mouth-piece to the open end of the tube $\Delta$, and using a very small jet heat a single point of the joint till the glass flows together, after which gently blow out this heated portion. Proceeding bit by bit, heat

FIG. 272. all round the joint in this way, being particularly careful to round off the corners A, B (Fig. 272). Finally, give the whole joint, including the portion $\mathbf{c}$ of the tube $\mathbf{A}, \mathbf{a}$ good general heating in a fairly large flame, and having carefully annealed in a smoky flame, pack the joint in cotton wool and allow it to cool.

(g) To Seal a Platinum Wire through the Side of a Glass Tube.-To make the hole in the glass tube through which the platinum wire is to pass, heat a small spot on the tube, and when the glass is red-hot, touch the place with a piece of platinum wire. The glass will adhere to the end of the wire, and by quickly drawing the wire away the glass will be drawn out into a fine capillary tube. Break off this capillary near the surface of the tube and, if necessary, trim the end flush with the side of the tube with a file. Draw down a piece of the same length of glass tube as that into which the wire is to pass to a capillary with walls about a millimetre thick, and of such a bore that the platinum wire fits fairly closely. Cut off about a centimetre of this capillary and thread it on the platinum wire, and then heat one end of the capillary so that it flows round the wire. By means of another portion of the drawndown tube wind a little glass round the fused end of the capillary so as to form a little bead of glass, as shown at $(a)$, Fig. 273. Pass the wire and the capillary through the hole in the tube, and with a small pointed flame strongly heat the

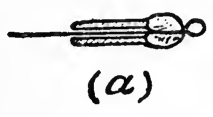

FIG. 273.

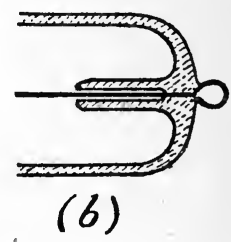

(6) bead of glass and the portion of the tube in the immediate vicinity. During the heating move the wire about a little by means of a pair of small forceps, and occasionally blow down the tube. In this way the seal is to be brought to the form shown at (b), Fig. 273. Such a seal must be very carefully annealed by being heated for several minutes in a smoky flame, and then being packed round with cotton wool.

Wherever possible, a platinum wire should be sealed through the end of a glass tube, rather than through the side. In fact it is quite worth while, in the case where an electrode is required to pass through the side of a glass tube, to seal on a small side tube, the wire passing through the end of this tube. 
'I'he disadvantage of the form of electrode shown in Fig. 273 is that the loop of platinum on the outside is apt to get broken off. A form of

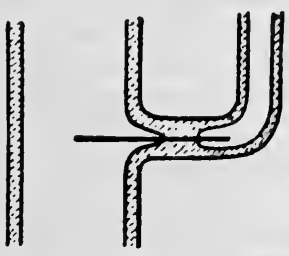

FIG. 274. electrode which will often be found better is shown in Fig. 274. A short length of narrow tubing has its bore contracted, and is then attached to the side of the tube, forming a T-piece. The platinum wire is then inserted, and the thickened part of the side tube is heated till the glass flows round the middle of the wire. Finally, if necessary, the side tube is bent round at right angles. When in use a little mercury is placed in the side tube, and the connecting wire dips into this mercury.

(h) To Cut Large Bore Glass Tube.-Glass tube up to about $2 \mathrm{~cm}$. in diameter can be cut satisfactorily by means of a file; in the case of larger tubes, however, the only really satisfactory method of making a neat square cut is by making a circular scratch on the inside of the tube with a diamond. The instrument used to make such a scratch is shown in Fig. 275. It consists of a steel rod $\triangle B$, having a small cutting diamond let in at $\mathbf{A}$. A metal disc $\mathrm{C}$ attached to a collar can be clamped by means of a screw at any desired point along the rod $\mathbf{A B}$. The disc $\mathbf{c}$ having been set so that the distance between it and the diamond point is equal to the length of tube to be cut off, the diamond is lightly pressed against the inside of the tube, while the tube is rotated. In this way a very fine scratch will be made round the tube. If

- in place of a fine

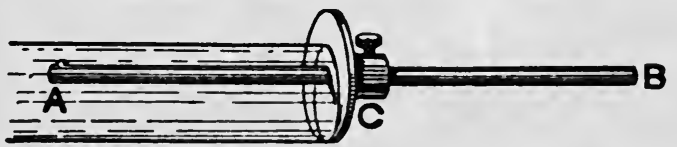

FIG. 275. scratch a wide rough scratch is obtained, it shows that the diamond is not acting properly, and generally means that too much pressure is being exerted. Having made a satisfactory scratch, try whether the tube will break when you apply a fairly strong longitudinal pull to the tube. If such a pull will not cause the tube to break, bring the scratched part quickly into a sinall flame, rotating the tube the while. The sudden heating will cause the tube to crack at the place where the diamond made the scratch.

2. Working Fused Silica-Manufacture of Quartz Fibres.Amorphous silica, ohtained by the fusion of pure quartz, has many properties which render it very valuable in the physical laboratory. The chief of these are, first, that its coefficient of thermal expansion is excessively small (about 0.0000001 ), so that a piece of the material may be suddenly introduced into the oxy-hydrogen flame. or when red-hot plunged into cold water, without cracking. Secondly, fibres of this material are not only very strong for their cross-section, but what is very 
much more valuable, they are almost entirely free from torsional elastic fatigue. It is this property which makes quartz fibres so very valuable for supporting galvanometer and electrometer needles, for it is only with this material that the position of the zero does not move after the needle has been deflected. With all other materials there is always a movement of the zero, owing to the imperfect recovery of the fibre from the effects of the torsion to which it has been subjected.

Since the manipulation necessary to make and mount such quartz fibres as are ordinarily required for suspension purposes in the laboratory is easily acquired, it is hoped that the following hints will encourage all students to familiarise themselves with the processes :-

Since quartz only fuses at a temperature higher than that attainable with the ordinary air-gas blow-pipe, it is necessary to use either an oxygen and coal-gas or oxygen and hydrogen jet. Personally, the author prefers hydrogen to coal-gas, since a smaller and hotter flame can be obtained. The coal-gas, however, has cheapness to recommend it. The

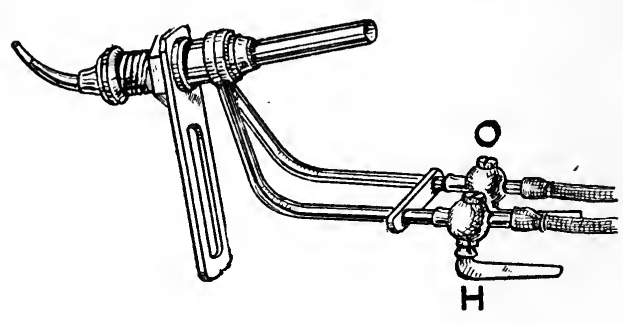

FIG. 276. blow-pipe to be used is such as is employed for the limelight, and what is called a mixed jet is to be preferred. In this type of jet the gases are mixed in a small chamber before they reach the jet. A suitable form of jet is shown in Fig. 276. The bottle containing the oxygen, also the one containing the hydrogen, if hydrogen is used, should be fitted with a pressure regulator, so that the amount of gas in the flame can be regulated by the taps on the jet. If no pressure regulators are used, then the adjustment must be performed by means of the cocks on the bottles, for if the taps on the jet are used, the rubber tubing will burst.

The supply of the gases must be so adjusted that a narrow flame about $20 \mathrm{~cm}$. long is obtained which emits only a very slight noise so long as the jet is at rest, but gives a roar when the jet is moved fairly rapidly from side to side.

A pair of very dark smoked spectacles will be required, since the hot quartz is much too bright to be looked at with the naked eye. It is advisable to remember that although the flame is quite invisible through such glasses, yet it is still there, and a very nasty burn will result if the fingers are inadvertently placed in the flame.

The most troublesome process when working fused silica is to fuse the quartz into sticks, since when a crystal of quartz is heated it immediately flies into small fragments. By far the cheapest method of obtaining sticks of fused quartz is to buy them from Messrs. Baird and 
Tatlock of 14 Cross Street, Hatton Garden, London, since the cost in gas of preparing such sticks will probably amount to more than the price. ${ }^{1}$ If, however, it is desired to prepare the sticks of fused quartz, the following method, described by Shenstone, will be found fairly easy :Small pieces of pure clear quartz are placed in a platinum crucible, and heated over an ordinary blow-pipe to a bright red. The quartz is then tipped into a large beaker containing cold water, and, as a result of the innumerable small cracks produced by the sudden cooling, acquires a milk white colour. Two fairly large pieces of this milky quartz are held in two small forceps, and the edges are heated in oxy-hydrogen flame and brought into contact. They adhere together, and then holding one piece in the forceps the other is melted, or at any rate partly melted. A third lump is then added, and this is melted, and so on. When about an inch of very rough rod has been built up in this way, heat the rod in the middle strongly, and then slowly draw it out, forming a fairly uniform rod having a diameter of about $2 \mathrm{~mm}$. Cut this rod in half, and then using these smooth portions as handles, bring the rough ends together in the flame, and work the quartz, by alternate heating and drawing down, to a uniform rod. Next cut this rod in half, and fuse a fresh lump of milky quartz to one end of one half, and then fuse on the other half of the rod and work up the fresh quartz. Proceeding in this way, with a little practice very fairly uniform rods of the fused silica can be built up. Having once got started, always add fresh quartz by cutting the stick and inserting the new material in the middle. In this way the portions of the stick already prepared serve as handles.

Before starting to make quartz fibres it will be necessary to prepare some frames on which to store the fibres. A convenient form of frame is shown in Fig. 277, and for ordinary purposes may conveniently be about 30 centi-

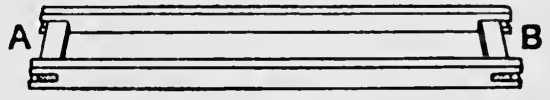

Frg. 277. metres long. Four or five of these frames may be stored together in a wooden box, into which they fit, each frame resting on the one below, the raised sides of the frames keeping the ends, to which the fibres are attached by shellac varnish, from touching.

The bow and arrow used to draw out the fibre are shown in Fig. 275. The support A for the arrow consists of a piece of wood 25 centimetres long, with a narrow groove running along its upper surface. The bow itself, BC, consists of a piece of straight-grained yellow pinc 108 centimetres long. The section at the middle is a square of $1 \cdot 2$ centimetre side, and the section gradually tapers down to a circle of about 0.8 centi. metre in diameter at the ends. The string consists of a piece of ordinary thin twine, and is of such a length that when the bow is unbent,

1 The present price is about 20 s. per ounce. A quarter of an ounce will make all the fibres required in a laboratory for very many years. 
the string is almost tight. The trigger $D$ consists of a $U$-shaped piece of brass wire, the limbs of the $U$ passing through two small holes in the end of $\Delta$, as shown in the section at $(a)$. This trigger is attached by a piece of string to a treadle board $\mathrm{E}$, so that by pressing the foot on $\mathrm{E}$ the trigger is pulled down, and the string of the bow,

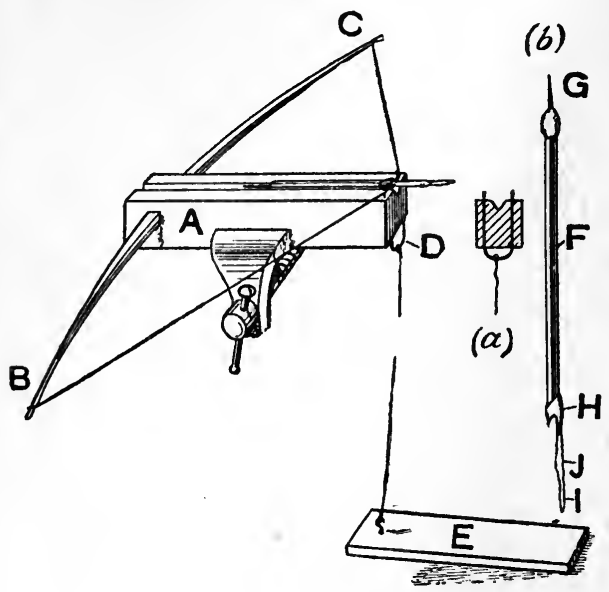

FIG. 278. which was held by the portions of the $U$ which project above $A$, is released.

The arrow $(b)$ consists of a piece of straw $F$ about 13 centimetres long, having an ordinary sewing-needle a cemented into one end by means of sealing-wax. The other end $\mathrm{H}$ is plugged with a little sealing-wax, and a notch is burnt to receive the string of the bow. A piece of the fused quartz I, about. 8 centimetres long and 1 to 2 millimetres in diameter, is cemented to the arrow with sealing - wax, as shown.

When shooting an arrow, the base $A$ of the bow is held in a small vice fixed to a table, and the trigger $\mathbf{D}$ being in place, the bow is bent. The arrow is then placed in the groove with the notch against the string between the two uprights of the trigger. Then holding the end I of the quartz in the left hand, and the oxy-hydrogen jet in the right, direct the flame on the point $J$ of the quartz. The jet ought to be nearly horizontal and at right angles to the length of the quartz. When the quartz is seen to have become quite soft, press down the treadle $\mathrm{E}$, at the same instant raising the flame and tightening your grasp on the end of the quartz. If the operations have been correctly timed, the arrow will be shot out, and the heated portion of the quartz will be drawn out into a fine fibre.

The thickness of the fibre depends in a measure on the thickness of the original quartz rod, but chiefly on the temperature to which the quartz was raised when the arrow was fired. Practice will soon indicate when to fire in order to obtain fibres of any desired thickness.

Having produced a fibre, its collection and mounting presents some little difficulty. To assist in the collection it will be found advisable to set up a board at about 6 to 8 metres from the bow, into which board the arrow will embed itself. Then between the bow and the board place a number of chairs in pairs, each pair to be back to back, and separated 
by about a metre. Between the backs of each pair of chairs place a clean length of glass tubing, the tube being at right angles to the direction of motion of the arrow, and about a foot below the path along which the arrow will travel. These glass tubes serve to keep the fibre off the floor.

If a fairly thick fibre has been produced, it will probably stretch from the arrow to the piece of quartz left in the hand. In such a case break off the fibre from the quartz in your hand, and fix a small piece of gummed paper to the end of the fibre. This can be performed by folding a piece of gummed paper, and having placed the fibre in the fold, pressing the sides together. Having in this way marked one end of the fibre, take one of the frames (Fig. 277) and coat the ends $A$ and B with shellac varnish. Then going to the arrow, break off the fibre, and place the end against the varnish near one side of the frame. The frame must now be slowly turned so as to wind on the fibre, during which process the paper attached at the free end will serve as a weight to keep the fibre taut.

If the fibre is fine, it will probably have broken near the bow, and a somewhat different procedure is necessary. Take a piece of glass tube or rod about 30 centimetres long, and starting at the arrow, allow the fibre to run over the rod as you move slowly towards the bow. Watch the fibre carefully so as to see when you get near the end. When the glass rod is about a foot from the end, take a folded piece of gummed paper between the finger and thumb of the left hand, and catch the fibre near the end in the fold. Having attached the paper, drop it, and starting at the arrow, proceed as described above to wind the fibre on a frame.

To attach a quartz fibre to, say, a galvanometer needle system, first measure the length the fibre has to be, and then draw a line on a clean sheet of paper, and on this line make two transverse marks, showing the length the fibre is to have. Having selected a frame containing a fibre of suitable diameter, attach a piece of gummed paper to one end, and then break the fibre between this paper and the end. Next grasp the fibre near the fixed end between the finger and thumb of the left hand, and pull it away from the frame. Take the pin to which the top of the fibre is to be attached and put a spot of shellac varnish on the end, placing the pin at the edge of the table, with the point projecting over the edge of the table. The above must be done without letting go the fibre, or may be done before the fibre is detached from the frame. Next taking a sewing-needle in the right hand, guide the end of the fibre, which will be seen projecting above the thimb and finger of the left hand, with the needle so that it touches the shellac. The shellac varnish must now be heated by holding a piece of heated copler wire below, the heating being continued till the varnish froths up. When the heating is complete, release the fibre by opening the fingers, so that the piece of paper at the end hangs down. Raise the pin to which the fibre is now attached, and gently draw the paper at the free end over the sheet of 
paper on which the length of the suspension fibre has been marked. Stop when the end of the pin is opposite the further mark, the fibre lying along the longitudinal line. Now draw a sharp knife firmly across the fibre at about half a centimetre beyond the mark which shows the length of the fibre. Having placed a drop of shellac varnish on the end of the wire which carries the magnets and mirror, support this system with the end projecting over the edge of the table, and taking the pin to which the fibre is attached in the left hand, cause the end of the fibre to touch the varnish. A needle held in the right hand may be used to direct the fibre. Then boil off the alcohol from the varnish, as was done before.

Having attached the fibre, prop the needle system up almost vertical before attempting to lift it by means of the fibre, for quartz fibres will not support a bending stress.

If difficulty is found in seeing the fibres, it will be advisable to either work over a piece of clean mirror, or to work at a table before a window, the table being fitted with a drawer. If the drawer is opened a few inches and a piece of black velvet is placed in it, a dark background will be provided against which it will be found easy to see the fibres.

For ordinary galvanometer suspensions, fibres having diameters from .002 to .006 millimetre will be found suitable.

For further particulars of the methods of manipulating quartz fibres the reader must be referred to the following:-On Laboratory Arts, Threlfall ; Boys, Philosophical Magazine, p. 498, June 1887, and p. 463, 1894.

3. Making Divided Scales.-Divided scales on glass can best be made by etching with hydrofluoric acid. The surface of the glass must be protected with some kind of varnish, the division lines being drawn through the coating of varnish. One of the most satisfactory varnishes is obtained by diluting Brunswick black with its own volume of benzene. The glass must be thoroughly cleaned and the varnish poured on the surface, being made to flow evenly all over by suitably tilting the glass. When every part of the surface has been coated, allow as much of the varnish as will drain off in half a minute to flow back into the bottle, and then lay the glass on a horizontal surface. After from ten to twenty minutes the varnish will have become tacky, and the drawing of the division lines may be proceeded with. The varnish will remain sufficiently soft for several hours. If, however, for any reason the division lines are not drawn within an hour or two of coating the glass, it will be advisable to dissolve off the varnish with benzene and give the glass a fresh coating.

In the case of a tube, the varnish must be applied by means of a fairly large soft camel's hair brush.

- Having made the division lines by one of the methods described below, cut a piece of clean white blotting-paper a little larger than the divided portion of the surface, and lay this paper over the divisions. Then by means of a straw moisten the paper with hydrofluoric acid, 
taking care that no air bubbles are enclosed between the paper and the glass. The exclusion of air bubbles will be facilitated if the hydrofluoric acid is applied regularly, starting from one end of the paper. The acid must be left on from one to two minutes, the exact time depending on the strength of the acid and the character of the division lines required. It is always advisable to make a trial on a waste piece of glass, for which purpose several groups of three or four division lines may be ruled, the acid being left on one group for half a minute, on the next for one minute, and so on.

To make the division lines and figures visible, rub over with some black paint, to which a little varnish has been added. Allow the paint to remain on for a few minutes, and then rub off the excess with ordinary writing paper. When removing the excess of paint, only rub in a direction perpendicular to the division lines.

A similar process can be employed to etch scales on brass, the hydrofluoric acid being replaced by dilute nitric acid.

In place of using the Brunswick black, beeswax may be used; it will, however, be found very much more difficult to obtain a uniform coating with the wax. The same remark applies even more to paraffin wax, so that on the whole it will be found to save time in the long run to use Brunswick black.

If very fine division lines are required on glass, then it will be advisable to rule them. A splinter of diamond is generally employed for the purpose, but a small crystal of carborundum (carborundum crystals only cost a few shillings the pound) will be found to give much better lines. With a diamond the edges of the lines are very apt to be splintered. If a very light pressure is used with carborundum, the edges of the lines will be quite sharp and free from all splintering.

If a dividing engine is available, then making the graduations presents no difficulty. The tool used to make the scratches on the varnish is a sharp point of steel, fairly light pressure being used to press the point against the work.

In the absence of the dividing engine, a screw-cutting lathe may be employed to make scales which are correct to about a twentieth of a millimetre, so long as the leading screw of the lathe is a good one. It will be advisable to fit the traversing carriage of the lathe with an arrangement to carry the tracing point by which the length of the divisions can be adjusted, and the fifth and tenth divisions are automatically made longer than the other graduations. With a leading screw having eight threads to the inch, the following change wheels give a very close approximation to a millimetre per turn :-Headstock, 28; stud, 32,36 ; screw, 100 .

A scale may be copied by hand by means of the arrangement shown in Fig. 279. The scale to be copied, $\Delta B$, must be one with fairly deep division marks, preferably of steel. It is fixed to a block of wood about $2 \mathrm{~cm}$. high, this block being fixed to a base board c about $130 \mathrm{~cm}$. long. The glass to be divided, DE, is cemented to the base board, or held down 
by clips, so that the line along which the graduations are to be made is a prolongation of the scale AB. To transfer the graduations from the scale to the glass, a beam compass FG is employed. This beam compass consists of a piece of light brass tube about $70 \mathrm{~cm}$. long and $1.5 \mathrm{~cm}$. in diameter. At either end is soldered a small tube at right angles to the axis of $\mathrm{FG}$, and through these cross tubes pass two steel rods, the rods being held in place by set screws. Both the steel rods are pointed at the lower end, the rod $\mathrm{H}$ serving as a scriber to make the graduations on the glass. The end of the rod $\mathrm{I}$ is placed in the division lines of the scale $\mathbf{A B}$. Attached to the tube FG, and alongside the tube which carries $I$, is a brass rod $\mathrm{KL}$, bent as shown. The side of this rod rests against the edge of the scale, and thus ensures that the point of I always occupies the same relative position along the division lines. The end $\mathrm{L}$ of the rod is always allowed to rest against the top of the base board $c$, and $I$ is so adjusted that when

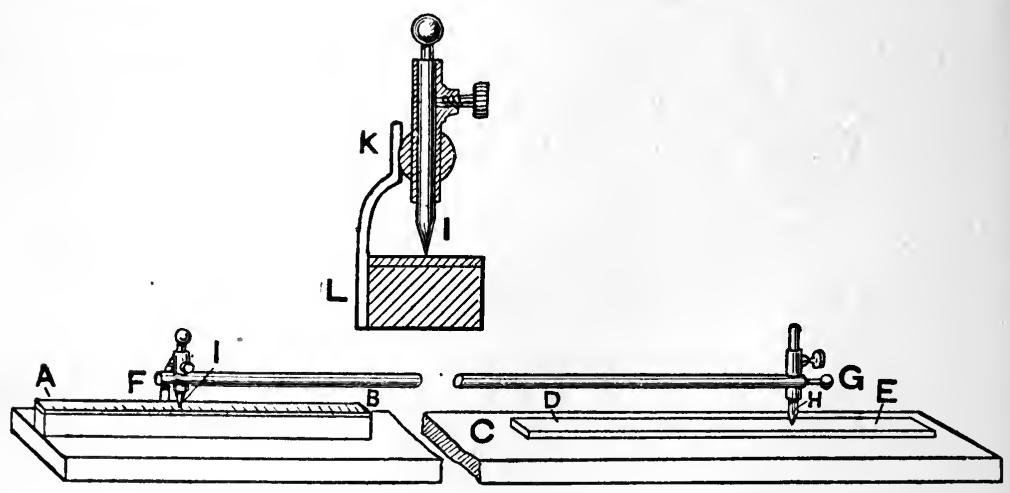

FIG. 279.

its point is on the scale, and $I$ rests on the base, the tracing point II is vertical. It will be found of assistance to have a knob on the top of $I$, and to hold this knob in the left hand. A knob $\mathrm{G}$ is fixed to the end of the tube FG and serves as a handle, by means of which the scribing point. is guided with the right hand.

Starting with the point at a whole centimetre division near the lefthand extremity of the scale, a division line is made by lightly drawing $G$ towards one. The point $I$ is then moved to the next division to the right, $\mathrm{H}$ being lifted off the glass, and a second division line is made, and so on. Some little practice will be required before the lines will be obtained of uniform width, and also equally spaced. A very light pressure must be exerted with either hand, and no attempt must be made to guide the point. It will be advisable to fix the base board $\mathrm{c}$ at about $20 \mathrm{~cm}$. from the edge of a table, and to rest the elbows of both arms on the table top. 
An arrangement suitable for dividing short scales, and which can easily be fitted up in the laboratory, is shown in Fig. 280 . A round steel $\operatorname{rod} A$ is fixed about $1 \mathrm{~cm}$. above a base board, and there are two carriages, $B$ and $c$, which can slide on this rod, the rod passing through a split tube D (Fig. 280, a). This split tube can be pinched together by the milled-headed screw $\mathrm{E}$, so that the carriage is clamped to the rod A. A point $\mathrm{F}$ on each carriage rests on a piece of plate glass $\mathrm{G}$, so that the upper surfaces of the carriages are horizontal. On the top of carriage $\mathrm{C}$ is clamped a micrometer screw-gauge $\mathrm{n}$, while on $\mathrm{B}$ is screwed an arm $\mathrm{K}$, which has a steel sphere $\mathrm{J}$ at the end, this sphere coming between the jaws of the screw-gauge. The plate on which the scale is to
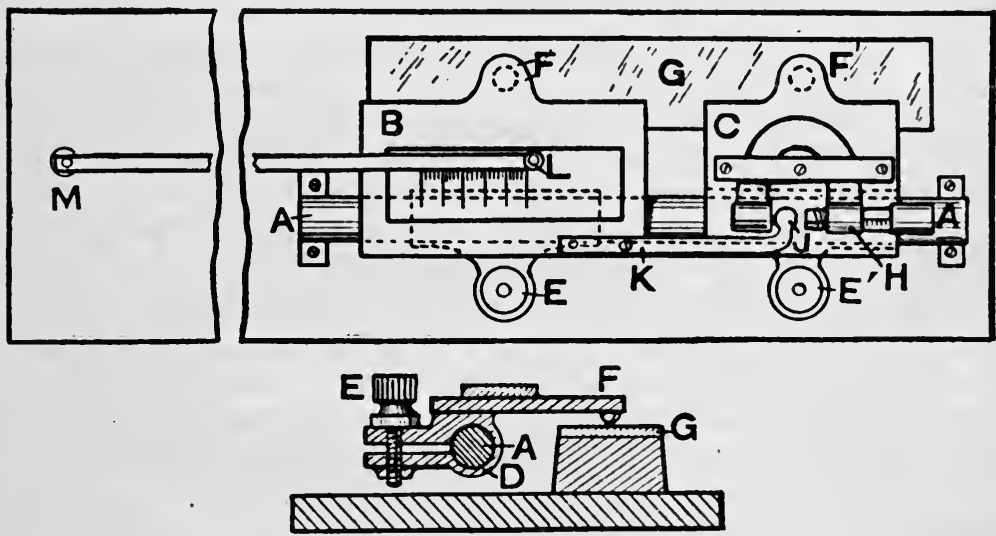

(a)

FIG. 280 .

be divided is fixed to the carriage B, while the scriber $\mathbf{L}$, used to make the division lines, consists of a pointed steel rod attached to the end of a long rod which is pivoted at $\mathbf{M}$.

To use this instrument the carriages are adjusted so that the sphere $\mathbf{J}$ is in contact with the left-hand jaw of $\mathrm{H}$, and the screw is brought up to touch the sphere. The jaws are then opened by an amount equal to the distance between the division lines on the scale which is to be divided. Starting with both carriages in such a position that the seriber is at the left-hand end of the plate on which the scale is to be divided, clamp carriage $\mathrm{B}$, and bring carriage $\mathrm{C}$ as far to the left as the right-hand jaw of the micrometer will allow, and clamp. Then rule the first graduation line with the scriber. Next unclamp B and move it to the left as far as the left-hand jaw of the micrometer will allow, and clamp. Rule the second line. Unclamp $\mathrm{c}$ and move to the left, then clamp. Unclamp B, move to the left, clamp and rule a division line, and so on. In this way 
the scale is produced by a step by step process, the length of the step being regulated by the distance through which the sphere $J$ can travel between the jaws of the micrometer.

Such a dividing engine is unsuited for any but quite short scales, and it is more difficult to produce uniform results than would perhaps at first sight be imagined. It will, however, be found of considerable service when making verniers, the scale on which the vernier is to be used being preferably made by the beam-compass method.

4. Silvering Glass.-There are a number of methods of silvering glass, each of which in practised hands is capable of giving good results. The following method, due to Brasheur, will, however, probably be found to give the most consistent results, and has the further advantage that some of the solutions employed will keep, and hence a stock can be made at one time.

The following solutions will be required :-

A. The reducing solution-

Loaf sugar or sugar candy .

Strong, pure nitric acid (sp. gr. $1 \cdot 22) \cdot . \quad$. 40 gran

Alcohol (absolute) . . . . . . 175 c.c.

Distilled water . $\quad . \quad$. $\quad . \quad 1000$ c.c.

The sugar is dissolved in the water, and then the alcohol and nitric acid are added. The solution will require to be kept for at least a week before it is used, and must be kept in a stoppered bottle.

$B$. Silver solution-

Silver nitrate

Distilled water :

C. Ammonia solution-

Ammonia (sp. gr. $\cdot 880$ )

Distilled water .

D. Potash solution-

Caustic potash (prepared by alcohol)
Distilled water $. \quad . \quad . \quad 0.5$ gram.

Solutions $B$ and $C$ will keep for some months if contained in well stoppered bottles and kept in the dark. Solution $D$ will only keep for a few weeks.

The above amounts will suffice for silvering a mirror having an area of about 50 square $\mathrm{cm}$. if used in a dish such that most of the silver is deposited on the mirror, and not on the sides of the dish. In smaller or larger mirrors, proportional quantities of the ingredients can be taken.

To prepare the solution for silvering, take about four-fifths of the silver solution $B$ and place it in a beaker which has been washed out with strong nitric acid and then with distilled water, and add the ammonia solution $C$ drop by drop, stirring all the while, till the pre- 
cipitate is partly dissolved, the solution being a light brown colour. Then very carefully add drop after drop of the ammonia, stirring for nearly a minute between each drop, till the solution attains a light straw colour. If by accident too much ammonia is added, so that after stirring for some time the solution becomes colourless, add a drop or two of the silver nitrate solution. It is esseritial to success that no excess of ammonia is present.

Next add the potash solution $D$, and then having diluted the remainder of the ammonia to about twice its bulk, add this solution drop by drop, till the precipitate formed when the potash was added is nearly all dissolved. A few particles of the precipitate may refuse to dissolve; these should be neglected, and the addition of ammonia stopped when the bulk of the liquid becomes colourless. Now add some of the reserve silver nitrate solution drop by drop till the solution, even after stirring for two or three minutes, does not become clear, but remains of a light brown or yellow colour. The solution may then be filtered, and is ready for use.

The glass to be silvered must be cleaned in the following manner. Since to obtain a satisfactory deposit it is essential that the glass surface should be perfectly clean, the student is advised to follow out the following directions implicitly :-

1. Remove any wax or oil on the glass by means of turpentine.

2. Dissolve off any pre-existing silver with nitric acid, and then rinse in water.

3. Immerse in a strong solution of caustic potash, and rub the surface to be coated with a swab of cotton wool tied to the end of a glass rod. Care must be taken not to scratch the surface with the glass rod. The cotton wool should be carefully tied over the end of the rod. Rinse in water.

4. Immerse in strong nitric acid, and again rub the surface with a swab, as in 3 .

5. Wash thoroughly with distilled water, giving the surface a rub with the swab during the first few washings.

6. Leave the objects covered with distilled water till they are wanted for silvering.

The objects to be silvered can either be placed at the bottom of a glass dish, the surfaces to be silvered being turned upwards, or they can be attached by a speck of wax at the back to a plate of glass, the glass resting on the top of the vessel containing the solution, so that the surfaces to be coated dip below the surface of the solution. In this case the silver is deposited upwards. The adrartage of this latter arrangement is that any solid specks in the solution do not settle on surfaces being silvered, and hence do not produce holes in the film, as occurs when the surfaces are at the bottom.

The glass having been cleaned, take the solution prepared as described above and add 6 c.c. of the reducing solution $A$ for each gram of silver nitrate taken, and having well mixed the solutions, pour over tine 
surfaces to be silvered. The solution will first turn almost black, and then the silver will start depositing, and the solution will gradually turn first to a light brown, and then after fifteen to thirty minutes it will become almost clear, when the deposition is complete. The rate at which the deposition goes on depends in a great measure on the temperature, and it is an advantage to work in a room at a temperature of about $20^{\circ} \mathrm{C}$. If the temperature is much below $20^{\circ}$, it is advisable to heat the solution to $20^{\circ}$ before adding the reduced solution.

After the deposition is complete remove the glass, being careful not to touch the silvered surface, and thoroughly wash with distilled water. Then stand the mirror on its edge on a piece of clean filter paper, and allow the film to become thoroughly dry.

If the mirror is to be used with the silvering on the back of the glass, the film of silver will require protecting by a coat of varnish. The best varnish to use is gold lacquer, as thin a coating as possible being employed.

If the silver film has to be polished, so as to obtain reflection direct from the silver, as will be the case with the mirrors used in Michelson's interferometer, prepare two or three pads by stuffing some pieces of clean soft chamois leather with cotton wool. First lightly rub the silver film with one of the pads, using circular strokes. Then place a piece of rouge about the size of a pea on a clean sheet of paper, and rub one of the pads on the paper, so as to work the rouge well into the leather. The mirror is then polished with this pad, using light circular strokes.

When silvering, always remember (1) an excess of ammonia must be avoided, and (2) the surfaces to be silvered must be absolutely clean.

5. Mounting Cross-wires in Telescopes and Microscopes.-An operation which occurs with some frequency in a physical laboratory is the replacement of broken cross-wires in the eye-pieces of microscopes and telescopes. In the case of low power eye-pieces, and where it is not necessary to read very accurately, such as for use with the telescope and scale method of measuring rotations, cross-wires made of a single thread of silk are quite satisfactory.

The ring on which the cross-wires are mounted having been removed from the telescope, the remains of the cement used to attach the old cross-wires is dissolved off with alcohol or amyl-acetate. In general, two lines at right angles, used to define the positions of the wires, will be found scribed on the ring. If no such

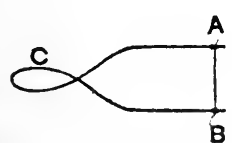

FIG. 281. lines exist, it will be advisable to put them on, using a sharp needle as a scriber.

The satisfactory attachment of the fibre will be very much facilitated if a piece of brass wire is bent into the shape shown in Fig. 281, the distance between the arms $\mathbf{A B}$ being about twice the diameter of the ring on which the fibre is to be mounted. Having selected a fibre of unspun silk, place it on a piece of black velvet, or on a piece of mirror glass, and by means of a needle and a pair of forceps, proceed to split 
it into the two fibres of which each single thread generally consists. Having separated a sufficient length of fibre, coat the end of one of the prongs of the bent wire with a little solution of celluloid in amyl-acetate. Such solution is sold as a transparent varnish for coating silver goods, and is known as silico enamel. In a few seconds the varuish will become tacky, when the end of the wire may be pressed against the end of the silk fibre. The fibre having become attached to the cement, give the wire a turn or two, so as to wind the fibre round the prong. Next coat the other prong with the varnish, and having slightly pressed the two prongs together with the left hand, wind the silk fibre round the second prong. After a few seconds, when the varnish has dried, the pressure on the prongs may be released, when the spring of the wires will keep the fibre taut.

Next gently lay the fibre in place on the ring, allowing the end $\mathrm{c}$ of the wire frame to rest on the table, the other end being supported by the fibre as it rests on the top of the rirg. Then with a pin or very small brush place a small drop of the varnish at either end of the fibre, and allow the arrangement to remain till the varnish is quite hard. When the varnish has set, the superfluous fibre can be cut away with a sharp pair of scissors.

The second cross-wire is cemented in place in exactly the same way, the lines ruled on the ring being used as a guide to ensure that the two fibres are at right angles.

In place of silk, very fine nickel wire may be used for cross-wires. Such wire, having a diameter of 0.02 millimetre, can be obtained from Messrs. Moul \& Co., 76-78 York Street, Westminster, London, and costs one shilling per metre.

For use in the eye-piece of a micrometer, silk cross-wires are in general too coarse, and no better material seems to have been discovered than spider web. The most suitable web is that produced by the Diadema spider, a spider with a large body and comparatively short legs. In the absence of the Diadema, any spider can be made to give fairly satisfactory threads.

Having obtained a spider, he can generally be persuaded to make a thread by gently tilting him off a piece of card, the thread being left attached to the card as the spider falls. This thread is then wound on a light wire frame of the shape shown in Fig. 281. In the case of a fresh spun web, it will be unnecessary to use any varnish to attach the thread to the wire. Having obtained the thread on the wire, the attachment to the ring is performed in exactly the same manner as in the case of the silk.

Quartz fibres are quite unsuited for cross-wires, as it is almost impossible to keep them sufficiently tight and yet not break them when replacing the ring in the microscopt.

6. On the Use of Manganin Wire for the Construction of Resistance Coils. - The alloy of copper, nickel, and manganes', called manganin, is very suitable for the construction of resistance coils, since it has an extremely small temperature coetficient, and is fairly cheap. 
In order that a resistance made of manganin may retain its resistance unaltered, the following must be attended to :-

1. The wire must be attached to the terminals by means of silver solder. The tin in ordinary soft solder appears to exert an influence on the wire, causing its resistance to alter.

2. The wire, after being wound in the coil, must be annealed at a temperature of $140^{\circ} \mathrm{C}$. for at least twenty-four hours.

3. The wire must be protected from the air by being coated with shellac.

The following table gives suitable sizes of -wire for coils of various resistance :-

\begin{tabular}{|c|c|c|}
\hline $\begin{array}{c}\text { Resistance, } \\
\text { Ohms. }\end{array}$ & $\begin{array}{c}\text { Size of Wire. } \\
\text { Standard Wire-Gaugo. }\end{array}$ & $\begin{array}{c}\text { Maximum Current. } \\
\text { Amperes. }\end{array}$ \\
\hline $0 \cdot 1$ & Two of 16 in parallel. & 32 \\
1 & 19 & 10 \\
10 & 25 & $3 \cdot 2$ \\
50 & 27 & $1 \cdot 5$ \\
100 & 33 & $1 \cdot 0$ \\
500 & 37 & $0 \cdot 4$ \\
1000 & 38 & $0 \cdot 1$ \\
\hline
\end{tabular}

In the last column are given the maximum currents which ought to be passed through the coils if of the form described below, when they are

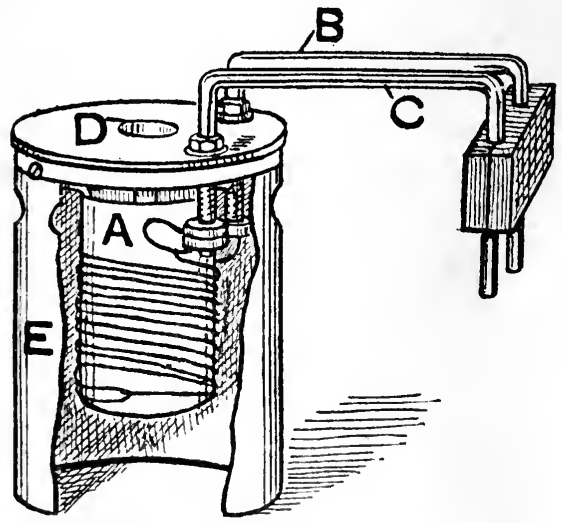

FIG. 282. immersed in an oil bath. Where measurements of precision are made, the currents ought, however, not to exceed a tenth of the values given.

The form of coil recommended by the Reichsanstalt is shown in Fig. 282. The wire, double-silk covered, is wound on a brass cylinder A, which is covered with a layer of silk, the silk having been thoroughly coated with shellac varnish before the wire is wound on. The ends of the wire are silver soldered to small copper 'discs, and these discs are attached by screws and soft solder to the ends of the thick copper rods $\mathbf{B}$ and $\mathbf{c}$. The rods are fastened to a thick disc of ebonite, in which a central hole $\mathrm{D}$ is pierced for the passage 
of a thermometer. A length of brass tube $\mathrm{E}$, open at the bottom, serves to protect the coil from blows.

When making a coil, sufficient wire is cut off to give a resistance about 4 per cent. more than the resistance it is wished to construct,' and the silk having been removed for about 4 centimetres from one end, the wire is thoroughly cleaned with sand-paper. A small copper disc, 1 centimetre diameter and about 4 millimetres thick, has a central hole bored, and a V notch filed across one face. The notched face is covered with a paste of borax and water, as is also the end of the wire. The disc is placed on a block of charcoal, a small piece of silver solder (silver 2 parts, brass 1 part) is put in the notch, and the disc is heated with a blow-pipe till the solder is quite fluid. The end of the wire is then placed in the notch, and almost simultaneously the flame is removed. The disc must be made sufficiently hot before the wire is put in place, so that it is not necessary to continue heating with the flame. If the wire is heated in the flame the manganese will be burnt out, and the properties of the wire will be altered. Immediately the solder has solidified, plunge the disc in water. Then clean off the borax, and polish up the side opposite to that on which the wire is attached, and give it a coating of soft solder with an ordinary soldering iron. The wire must then be doubled on itself and wound uniformly on the brass cylinder $A$, being held in place by a few turns of silk. Next give the wire a thorough coating of shellac varnish, made by dissolving selected shellac in absolute alcohol, and place the coil in an air bath heated to $140^{\circ} \mathrm{C}$., leaving it there for twenty-four hours. During the annealing it is advisable to give the coil two or three additional coats of varnish.

The coil having been annealed, its resistance must be adjusted to the desired value. Screw the copper disc which is attached to one end of the coil to one of the copper connectors ( $B$ or $C$ ), and then heat the junction with a soldering iron, and run a little soft solder between the disc and the rod, care being taken to get no solder on the face of the dise to which the manganin wire is attached.

The other end of the wire, having been well cleaned with sand-paper, is clamped between two copper discs, which are screwed to the end of the other connector, and the resistance of the coil is compared with a standard coil. The wire is adjusted between the copper dises till the resistance is a little greater than the desired value. The position is marked, which may most conveniently be done by sharply bending the wire at the edge of the discs. This end of the wire is then soldered to a copper disc exactly as was the other end, and the resistance of the coil is carefully measured, and ought to be a very little too great. Next calculate by what len!th the coil would have to be reduced to give the correct resistance, and then measure off three times this length from one end, and having bared the wire at the point, solder another piece of the same wire at this point. This wire must be attached with silver solder, and the

1 The resistance of a manganin wire decreases from 2 to 3 per cent. when the wire is annealed. 
best method of procedure is as follows:-Having carefully cleaned the portions of wire to be joined, give the additional piece two turns round the other wire, and place a little powdered borax on the joint. Then heat a small bead of solder, together with a little borax, on a piece of charcoal, and when the solder is heated well above the melting point, place the junction on the bead, removing the flame immediately the solder flows. A very small flame ought to be used when making this joint. Next adjust the length of the added wire so that the resistance of the whole coil, with this wire shunting the end portion of the main wire, is a very little too small, and then attach a copper disc to the added wire with silver solder. The two copper discs must now be screwed to the connector, and the junctions between the discs made good with soft solder. If the operations have been successfully performed, the resistance of the coil will now be slightly too small, and the added wire is then reduced in diameter by careful filing till the resistance is correct.

In the case of coils wound with fine wire, it will be found easiest to attach a portion of thick wire in series with the fine wire, and then having adjusted the length till the coil has a slightly too low resistance, to adjust finally by filing the thick wire.

The above descriptions apply to the construction of a manganin resistance coil where the utmost accuracy in adjustment and permanence is desired. For many purposes in the laboratory such refinement is unnecessary. In such a case, having cut off a length slightly in excess of that required to give the desired resistance, coil the wire into a double coil, coat it with shellac, and anneal at $140^{\circ}$. The annealing even may be omitted, in which case the coils will slowly change in resistance, and a fairly frequent recalibration will be necessary. The coils are then attached to the copper connectors or the contact blocks, as the case may be, with soft solder, and adjusted by altering the length; for which purpose the wire is diawn through the solder, which is made fluid. by placing a hot soldering iron in the neighbourhood. 
TABLE I.

Reduction of Barometer Readings to $0^{\circ} \mathrm{C}$.

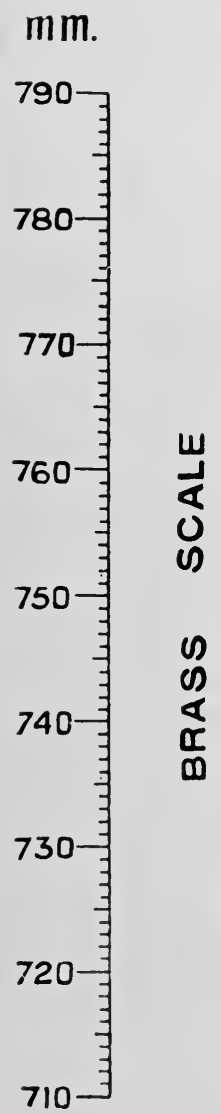

$\mathrm{mm}$.
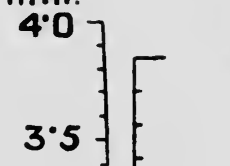

C

$\mathbf{m} \mathbf{m}$.

$\mathrm{mm}$.
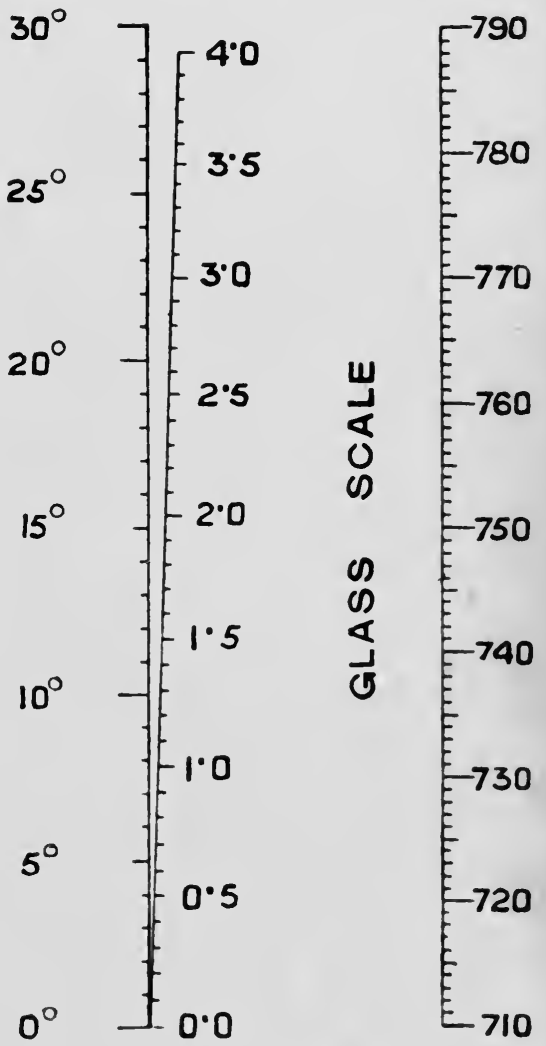



\section{TABLE II.}

Approximate Formulos.

If $a, b, c$, and $d$ are small compared to unity, then to a first approximation we have

1. $(1 \pm a)(1 \pm b)(1 \pm c) \ldots=1 \pm a \pm b \pm c \pm \ldots$

2. $(1 \pm a)^{2}=1 \pm 2 a$.

3. $(1 \pm a)^{n}=1 \pm n a$.

4. $\sqrt{ }(1 \pm a)=1 \pm \frac{a}{2}$.

5. $\sqrt{ } a b=\frac{1}{2}(a+b)$.

6. $\frac{1}{1 \pm a}=1 \mp a$.

7. $\frac{1}{(1 \pm a)^{\frac{1}{t}}}=1 \mp \frac{a}{2}$.

8. $\frac{1}{(1 \pm a)^{n}}=1 \mp n a$.

9. $\frac{(1 \pm a)(1 \pm b)}{(1 \pm c)(1 \pm d)}=1 \pm a \pm b \mp c \mp d$.

10. $e^{a}=1+a$.

11. $x^{a}=1+a \log _{e} x$.

12. $\log _{e}(1+a)=a-\frac{a^{2}}{2}$.

13. $\sin (\theta \pm a)=\sin \theta \pm a \cos \theta$.

14. $\cos (\theta \pm a)=\cos \theta \mp a \sin \theta$.

In 13 and $14 a$ is measured in radians, and if it does not exceed $2^{\circ}$, the above expressions are correct to three decimal places, and if a does not exceed $30^{\prime}$, to four places. 


\section{TABLE III.}

\section{Reduction of Weighings to Vacuo.}

If $D$ is the density of the body, and $\delta$ is that of the weights, and $\sigma$ the density of the air, the correction to the weight of the body is $w \sigma\left(\frac{1}{D}-\frac{1}{\delta}\right)$, where $w$ is the apparent weight of the body. The following table gives the values of the factors $\sigma\left(\frac{1}{D}-\frac{1}{\delta}\right)$ calculated by using the mean value 0012 for $\sigma$. The tabulated number multiplied by $w$ in grams gives the correction in milligrams, and is to be added to the apparent weight if the sign is positive :-

\begin{tabular}{|c|c|c|c|}
\hline \multirow[b]{2}{*}{$\begin{array}{c}\text { Density of Body. } \\
D .\end{array}$} & \multicolumn{3}{|c|}{ Correction in Milligrams. } \\
\hline & $\begin{array}{c}\text { Brass Ẃ eights. } \\
\delta=\mathbf{8} 4 .\end{array}$ & $\begin{array}{l}\text { Platinum Weights. } \\
\delta=21 \cdot 5 \text {. }\end{array}$ & $\begin{array}{l}\text { Quartz or Aluminium } \\
\text { Weights. } \\
\delta=2 \cdot 65 \text {. }\end{array}$ \\
\hline $\begin{array}{r}.50 \\
.55 \\
.60 \\
.65 \\
.70 \\
.75 \\
.80 \\
.85 \\
.90 \\
.95 \\
1.0 \\
1.1 \\
1 \cdot 2 \\
1.3 \\
1.4 \\
1.5 \\
1.6 \\
1.7 \\
1.8 \\
1.9 \\
2.0 \\
2.5 \\
3.0 \\
3.5 \\
4.0 \\
5.0 \\
6.0 \\
8.0 \\
10.0 \\
15.0 \\
20.0\end{array}$ & $\begin{array}{r}+2 \cdot 26 \\
2 \cdot 04 \\
1 \cdot 86 \\
1 \cdot 70 \\
1 \cdot 57 \\
1 \cdot 46 \\
1 \cdot 36 \\
1 \cdot 27 \\
1 \cdot 19 \\
1 \cdot 12 \\
1 \cdot 06 \\
\cdot 95 \\
\cdot 86 \\
\cdot 78 \\
\cdot 71 \\
.66 \\
.61 \\
.56 \\
.52 \\
\cdot 49 \\
\cdot 46 \\
.34 \\
\cdot 26 \\
.20 \\
\cdot 16 \\
\cdot 10 \\
.06 \\
+\cdot 01 \\
-\cdot 02 \\
.06 \\
-.08\end{array}$ & $\begin{array}{c}+2 \cdot 34 \\
2 \cdot 13 \\
1 \cdot 94 \\
1 \cdot 79 \\
1 \cdot 66 \\
1 \cdot 55 \\
1 \cdot 44 \\
1 \cdot 36 \\
1 \cdot 28 \\
1 \cdot 21 \\
1 \cdot 14 \\
1 \cdot 04 \\
\cdot 94 \\
\cdot 87 \\
\cdot 80 \\
\cdot 75 \\
\cdot 69 \\
\cdot 65 \\
\cdot 62 \\
\cdot 58 \\
\cdot 54 \\
\cdot 43 \\
\cdot 34 \\
\cdot 29 \\
\cdot 24 \\
\cdot 19 \\
\cdot 14 \\
\cdot 09 \\
\cdot 06 \\
\cdot 03 \\
\cdot 004\end{array}$ & 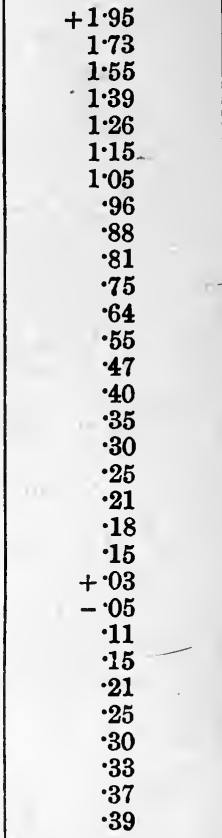 \\
\hline
\end{tabular}


TABLE IV.

Density of Dry Air at different Temperatures and Pressures.

\begin{tabular}{|c|c|c|c|c|c|c|c|c|}
\hline \multirow{2}{*}{$\begin{array}{c}\text { Tempera- } \\
\text { ture. }\end{array}$} & \multicolumn{6}{|c|}{ Pressure in Millimetres. } & \multirow{2}{*}{\multicolumn{2}{|c|}{ Proportional Parte. }} \\
\hline & 720 & 730 & 740 & 750 & 760 & 770 & & \\
\hline & 0.00 & 0.00 & 0.00 & 0.00 & 0.00 & 0.00 & \multirow{5}{*}{$\begin{array}{ll}17 & \\
1 \mathrm{~mm} & =2 \\
2 & =3 \\
3 & =5 \\
4 & =7 \\
5 & =8 \\
6 & =10 \\
7 & =12 \\
8 & =14 \\
9 & =15\end{array}$} & \multirow{5}{*}{$\begin{array}{c}4 \\
0 \circ \cdot 1=0 \\
\cdot 2=1 \\
\cdot 3=1 \\
\cdot 4=2 \\
\cdot 5=2 \\
\cdot 6=2 \\
\cdot 7=3 \\
\cdot 8=3 \\
\cdot 9=4\end{array}$} \\
\hline $0^{\circ}$ & 1225 & 1242 & 1259 & 1276 & 1293 & 1310 & & \\
\hline $\begin{array}{l}1 \\
2 \\
3 \\
4\end{array}$ & $\begin{array}{l}1221 \\
1216 \\
1212 \\
1207\end{array}$ & $\begin{array}{l}1237 \\
1233 \\
1228 \\
1224\end{array}$ & $\begin{array}{l}1254 \\
1250 \\
1245 \\
1241\end{array}$ & $\begin{array}{l}1271 \\
1267 \\
1262 \\
1258\end{array}$ & $\begin{array}{l}1288 \\
1284 \\
1279 \\
1274\end{array}$ & $\begin{array}{l}1305 \\
1301 \\
1296 \\
1291\end{array}$ & & \\
\hline 5 & 1203 & 1220 & 1236 & 1253 & 1270 & 1286 & & \\
\hline $\begin{array}{l}6 \\
7 \\
8 \\
9\end{array}$ & $\begin{array}{l}1199 \\
1194 \\
1190 \\
1186\end{array}$ & $\begin{array}{l}1215 \\
1211 \\
1207 \\
1202\end{array}$ & $\begin{array}{l}1232 \\
1227 \\
1223 \\
1219\end{array}$ & $\begin{array}{l}1249 \\
1244 \\
1240 \\
1235\end{array}$ & $\begin{array}{l}1265 \\
1261 \\
1256 \\
1252\end{array}$ & $\begin{array}{l}1282 \\
1277 \\
1273 \\
1268\end{array}$ & & \\
\hline 10 & 1181 & 1198 & 1214 & 1231 & 1247 & 1264 & \multirow{4}{*}{ 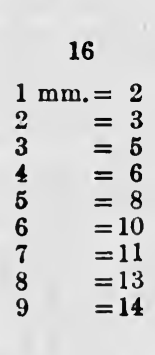 } & \multirow{9}{*}{$\begin{array}{c}5 \\
0^{\circ} \cdot 1=1 \\
\cdot 2=1 \\
\cdot 3=2 \\
\cdot 4=2 \\
\cdot 5=3 \\
\cdot 6=3 \\
\cdot 7=4 \\
\cdot 8=4 \\
\cdot 9=5\end{array}$} \\
\hline $\begin{array}{l}11 \\
12 \\
13 \\
14\end{array}$ & $\begin{array}{l}1177 \\
1173 \\
1169 \\
1165\end{array}$ & $\begin{array}{l}1194 \\
-1190 \\
1185 \\
1181\end{array}$ & $\begin{array}{l}1210 \\
1206 \\
1202 \\
1198\end{array}$ & $\begin{array}{l}1227 \\
1222 \\
1218 \\
1214\end{array}$ & $\begin{array}{l}1243 \\
1238 \\
1234 \\
1230\end{array}$ & $\begin{array}{l}1259 \\
1255 \\
1250 \\
1246\end{array}$ & & \\
\hline 15 & 1161 & 1177 & 1193 & 1209 & 1226 & 1242 & & \\
\hline $\begin{array}{l}16 \\
17 \\
18 \\
19\end{array}$ & $\begin{array}{l}1157 \\
1153 \\
1149 \\
1145\end{array}$ & $\begin{array}{l}1173 \\
1169 \\
1165 \\
1161\end{array}$ & $\begin{array}{l}1189 \\
1185 \\
1181 \\
1177\end{array}$ & $\begin{array}{l}1205 \\
1201 \\
1197 \\
1193\end{array}$ & $\begin{array}{l}1221 \\
1217 \\
1213 \\
1209\end{array}$ & $\begin{array}{l}1237 \\
1233 \\
1229 \\
1225\end{array}$ & & \\
\hline 20 & 1141 & 1157 & 1173 & 1189 & 1205 & 1220 & \multirow{5}{*}{$\begin{array}{ll}15 & \\
1 \mathrm{~mm} . & =1 \\
2 & =3 \\
3 & =4 \\
4 & =6 \\
5 & =8 \\
6 & =9 \\
7 & =11 \\
8 & =12 \\
9 & =13\end{array}$} & \\
\hline $\begin{array}{l}21 \\
22 \\
23 \\
24\end{array}$ & $\begin{array}{l}1137 \\
1133 \\
1130 \\
1126\end{array}$ & $\begin{array}{l}1153 \\
1149 \\
1145 \\
1141\end{array}$ & $\begin{array}{l}1169 \\
1165 \\
1161 \\
1157\end{array}$ & $\begin{array}{l}1185 \\
1181 \\
1177 \\
1173\end{array}$ & $\begin{array}{l}1200 \\
1196 \\
1192 \\
1188\end{array}$ & $\begin{array}{l}1216 \\
1212 \\
1208 \\
1204\end{array}$ & & \\
\hline 25 & 1122 & 1138 & 1153 & 1169 & 1184 & 1200 & & \\
\hline $\begin{array}{l}26 \\
27 \\
28 \\
29\end{array}$ & $\begin{array}{l}1118 \\
1115 \\
1111 \\
1107\end{array}$ & $\begin{array}{l}1134 \\
1130 \\
1126^{\circ} \\
1123\end{array}$ & $\begin{array}{l}1149 \\
1146 \\
1142 \\
1138\end{array}$ & $\begin{array}{l}1165 \\
1161 \\
1157 \\
1153\end{array}$ & $\begin{array}{l}1180 \\
1176 \\
1173 \\
116 ! !\end{array}$ & $\begin{array}{l}1196 \\
1192 \\
1188 \\
1184\end{array}$ & & \\
\hline 30 & 1103 & 1119 & 1134 & 1149 & 1165 & 1180 & & \\
\hline
\end{tabular}


TABLE $\mathrm{V}$.

(1) Density of Water at various Temperatures measured on the Hydrogen Scale.

\begin{tabular}{|c|c|c|c|c|c|c|c|c|c|c|}
\hline $\begin{array}{c}\text { Tempera- } \\
\text { ture. }\end{array}$ & 0 & 1 & 2 & 3 & 4 & 5 & 6 & 7 & 8 & 9 \\
\hline $\begin{array}{l}0^{\circ} \mathrm{C} . \\
10 \\
20 \\
30\end{array}$ & $\begin{array}{r}0.99987 \\
973 \\
823 \\
567\end{array}$ & $\begin{array}{l}993 \\
963 \\
802 \\
537\end{array}$ & $\begin{array}{l}997 \\
953 \\
780 \\
505\end{array}$ & $\begin{array}{l}999 \\
940 \\
756 \\
473\end{array}$ & $\begin{array}{r}1 \\
927 \\
732 \\
440\end{array}$ & $\begin{array}{l}999 \\
913 \\
707 \\
406\end{array}$ & $\begin{array}{l}997 \\
897 \\
681 \\
371\end{array}$ & $\begin{array}{l}993- \\
880 \\
654 \\
336\end{array}$ & $\begin{array}{l}988 \\
862 \\
626 \\
299\end{array}$ & $\begin{array}{l}981 \\
843 \\
597 \\
262\end{array}$ \\
\hline $\begin{array}{l}40 \\
50\end{array}$ & $\begin{array}{r}224 \\
0.98807\end{array}$ & $\begin{array}{l}186 \\
762\end{array}$ & $\begin{array}{l}147 \\
715\end{array}$ & $\begin{array}{l}107 \\
669\end{array}$ & $\begin{array}{l}066 \\
621\end{array}$ & $\begin{array}{l}025 \\
573\end{array}$ & $\begin{array}{l}\overline{982} \\
525\end{array}$ & $\begin{array}{l}\overline{940} \\
475\end{array}$ & $\begin{array}{l}\overline{896} \\
425 \\
\end{array}$ & $\begin{array}{l}\overline{852} \\
375\end{array}$ \\
\hline $\begin{array}{l}60 \\
70\end{array}$ & $\begin{array}{r}324 \\
0.97781\end{array}$ & $\begin{array}{l}272 \\
723\end{array}$ & $\begin{array}{l}220 \\
666\end{array}$ & $\begin{array}{l}167 \\
607\end{array}$ & $\begin{array}{l}113 \\
548\end{array}$ & $\begin{array}{l}059 \\
489\end{array}$ & $\begin{array}{l}005 \\
429\end{array}$ & $\begin{array}{l}\overline{950} \\
368\end{array}$ & $\begin{array}{l}\overline{894} \\
307\end{array}$ & $\begin{array}{l}\overline{838} \\
245\end{array}$ \\
\hline 80 & 183 & 121 & 057 & $\overline{994}$ & $\overline{930}$ & $\overline{865}$ & $\overline{800}$ & $\overline{734}$ & $\overline{668}$ & $\overline{601}$ \\
\hline $\begin{array}{r}90 \\
100\end{array}$ & $\begin{array}{l}0.96534 \\
0.95838\end{array}$ & $\begin{array}{l}467 \\
765\end{array}$ & $\begin{array}{l}399 \\
693\end{array}$ & 330 & 261 & 192 & 122 & 051 & $\overline{981}$ & 909 \\
\hline
\end{tabular}

(2) Volume of one Gram of Water.

\begin{tabular}{|c|c|c|c|c|c|c|c|c|c|c|}
\hline $\begin{array}{c}\text { Tempera- } \\
\text { ture. }\end{array}$ & 0 & 1 & 2 & 3 & 4 & 5 & 6 & 7 & 8 & 9 \\
\hline 0 & $1 \cdot 00013$ & 007 & 003 & 001 & 000 & 001 & 003 & 007 & 012 & 019 \\
\hline 10 & 027 & 037 & 048 & 060 & 073 & 087 & 103 & 120 & 138 & 157 \\
\hline 20 & 177 & 199 & 221 & 244 & 268 & 294 & 320 & 347 & 376 & 405 \\
\hline 30 & 435 & 466 & 497 & 530 & 563 & 598 & 633 & 669 & 706 & 743 \\
\hline 40 & 782 & 821 & 861 & 901 & 943 & 985 & $\overline{028}$ & $\overline{072}$ & $\overline{116}$ & $\overline{162}$ \\
\hline 50 & 1.01207 & 254 & 301 & 349 & 398 & 448 & 498 & 548 & 600 & 652 \\
\hline 60 & 705 & 758 & 812 & 867 & 923 & 979 & $\overline{036}$ & $\overline{093}$ & $\overline{151}$ & $\overline{210}$ \\
\hline 70 & 1.02270 & 330 & 390 & 452 & 514 & 576 & 639 & 703 & 768 & 833 \\
\hline 80 & 899 & 965 & $\overline{032}$ & $\overline{099}$ & $\overline{168}$ & $\overline{237}$ & $\overline{306}$ & $\overline{376}$ & $\overline{447}$ & $\overline{518}$ \\
\hline 90 & 1.03590 & 663 & 736 & 810 & 884 & 959 & $\overline{035}$ & $\overline{111}$ & $\overline{188}$ & $\overline{265}$ \\
\hline 100 & 1.04343 & 422 & 501 & & & & & & & \\
\hline
\end{tabular}


TABLE VI.-Correction for Buoyancy in Density Measurements.

If $D^{\prime}$ is the density uncorrected for buoyancy, then the corrected density is $D^{\prime}+\sigma\left(1-D^{\prime}\right)$, and in the following table are given the values of the correction term $\sigma\left(1-D^{\prime}\right)$ for various values of $D^{\prime}$, the mean value 0012 being assumed for 0 .

\begin{tabular}{|c|c|c|c|c|}
\hline$D$ & Correction. & $D$ & Correction. & $\begin{array}{l}\text { Proportlonal } \\
\text { Parte. }\end{array}$ \\
\hline $\begin{array}{r}\cdot 5 \\
1 \cdot 0 \\
2 \cdot 0 \\
3 \cdot 0 \\
4 \cdot 0 \\
5 \cdot 0 \\
6 \cdot 0 \\
7 \cdot 0 \\
8 \cdot 0 \\
9 \cdot 0 \\
10 \cdot 0\end{array}$ & $\begin{array}{l}+\cdot 0006 \\
-0012 \\
-\cdot 0024 \\
-\cdot 0036 \\
-\cdot 0048 \\
-\cdot 0060 \\
-\cdot 0072 \\
-\cdot 0084 \\
-.0096 \\
-\cdot 0108\end{array}$ & $\begin{array}{l}12 \cdot 0 \\
14 \cdot 0 \\
16 \cdot 0 \\
18 \cdot 0 \\
20 \cdot 0\end{array}$ & $\begin{array}{l}-0132 \\
-.0156 \\
-.0180 \\
-.0201 \\
-0228\end{array}$ & $\begin{aligned} & 0012 \\
& \cdot 1=0001 \\
& \cdot 2=0002 \\
& \cdot 3=0004 \\
& \cdot 4=0005 \\
& \cdot 5=0006 \\
& \cdot 6=0007 \\
& \cdot 7=0008 \\
& \cdot 8=0010 \\
& \cdot 9=0011\end{aligned}$ \\
\hline
\end{tabular}

TABLE VII.-Moments of Inertia.

The following table contains the squares of the radii of gyration, and these numbers multiplied by the mass of the body will give the moment of inertia :-

\begin{tabular}{|c|c|c|c|c|}
\hline Shape of Body. & & $\begin{array}{l}\text { Position of Axis about } \\
\text { which Rotation takes } \\
\text { place. }\end{array}$ & $\begin{array}{l}\text { Square of } \\
\text { Radius } \\
\text { of Gyration. }\end{array}$ & \\
\hline Solid cylinder & - & $\begin{array}{l}\text { Perpendicular to axis } \\
\text { of cylinder through } \\
\text { centre of gravity }\end{array}$ & $\frac{l^{2}}{12}+\frac{r^{2}}{4}$ & $\begin{array}{l}r=\text { radius. } \\
l=\text { length. }\end{array}$ \\
\hline Solid cylinder & - & $\begin{array}{l}\text { Coincident with axis } \\
\text { of cylinder }\end{array}$ & $\frac{r^{2}}{2}$ & $r=$ radius. \\
\hline Hollow cylinder & & $\begin{array}{l}\text { Coincident with axis } \\
\text { of cylinder }\end{array}$ & $\frac{R^{2}+r^{2}}{2}$ & $R=$ external radius. \\
\hline Hollow cylinder . & - & $\begin{array}{l}\text { Through centre of } \\
\text { gravity and per- } \\
\text { pendicular to axis } \\
\text { of cylinder }\end{array}$ & $\frac{R^{2}+r^{2}}{4}+\frac{h^{2}}{12}$ & $\begin{array}{l}r=\text { internal radias. } \\
h=\text { lengtb. }\end{array}$ \\
\hline Sphere . & - & Through centre & $\frac{2 r^{2}}{5}$ & $r=$ radius. \\
\hline Hollow sphere & - & Through centre & $\begin{array}{l}2 R^{3}-r \\
3 h^{3}-r^{3}\end{array}$ & $\begin{array}{l}R=\text { external malius. } \\
r=\text { internal radius. }\end{array}$ \\
\hline Rectangular bar. & . & $\begin{array}{l}\text { Through centre of } \\
\text { two opposite faces }\end{array}$ & $\frac{a^{2}+b^{3}}{12}$ & $\begin{array}{l}a \text { and } b \text { are aljacent } \\
\text { edpes of the faces } \\
\text { through which } \\
\text { the axis paraes. }\end{array}$ \\
\hline Anchor ring & - & About a diameter & $\frac{K^{2}}{2}+\frac{5 r^{2}}{x}$ & $\begin{array}{l}R=\text { radius of anchor } \\
\text { ring. }\end{array}$ \\
\hline Anchor ring & - & $\begin{array}{l}\text { Through centre, and } \\
\text { perpendicular to } \\
\text { plane of ring }\end{array}$ & $k^{n}+3^{3}$ & $\begin{array}{l}r \text { radius of circu. } \\
\text { lar cross section. }\end{array}$ \\
\hline
\end{tabular}

If the square of the rathus of gyration about agwn axis is to then the aquare of the radius of gyration about a parallel axis at a distance $d$ is $1 \beta^{2}+d^{2}$. 
TABLE VIII.-Reduction of Periodic Time to infinitely small Arc, and Correction on Account of Chronometer Rate.

Reduction to infinitely small Arc.-If $\theta$ and $\theta^{\prime}$ are the initial and final amplitudes of the vibration, the correction is $\frac{\theta \theta}{16}$.

\begin{tabular}{|c|c|c|c|c|c|c|}
\hline \multirow{2}{*}{$\begin{array}{l}\text { Amplitude at } \\
\text { Commencement } \\
\text { in Minutes } \\
\text { of Arc. }\end{array}$} & \multicolumn{6}{|c|}{ Amplitude at End in Mintutes of Arc. } \\
\hline & $80^{\prime}$ & $70^{\prime}$ & $60^{\prime}$ & $50^{\prime}$ & $40^{\prime}$ & $30^{\prime}$ \\
\hline $\begin{array}{c}100^{\prime} \\
90 \\
80 \\
70 \\
60 \\
50\end{array}$ & $\begin{array}{c}0.00004 \\
.00004 \\
.00003 \\
.\end{array}$ & $\begin{array}{r}0.00004 \\
.00003 \\
.00003 \\
.00003\end{array}$ & $\begin{array}{r}0.00003 \\
\cdot 00003 \\
\cdot 00003 \\
\cdot 00002 \\
\cdot 00002\end{array}$ & $\begin{array}{r}0.00003 \\
.00002 \\
.00002 \\
.00002 \\
.00002 \\
.00001\end{array}$ & $\begin{array}{r}0.00002 \\
.00002 \\
.00002 \\
.00001 \\
.00001 \\
\cdot 00001 \\
\cdot 00001\end{array}$ & $\begin{array}{r}0.00002 \\
.00001 \\
.00001 \\
.00001 \\
.00001 \\
.00001 \\
.00001\end{array}$ \\
\hline
\end{tabular}

Correction for Chronometer Rate.-If $s$ is the daily rate of the chronometer, the factor required is $1-\frac{s}{86400}$.

\begin{tabular}{|c|c|c|}
\hline $\begin{array}{c}\text { Daily Rate, } \\
\text { Seconds. }\end{array}$ & $\begin{array}{c}\text { Chronometer } \\
\text { Gaining. }\end{array}$ & $\begin{array}{c}\text { Chronometer } \\
\text { Losing. }\end{array}$ \\
\hline 5 & 0.99994 & $1 \cdot 00006$ \\
10 & 88 & 12 \\
15 & 83 & 17 \\
20 & 77 & 23 \\
25 & 71 & 29 \\
30 & 65 & 35 \\
35 & 59 & 41 \\
40 & 54 & 46 \\
45 & 48 & 52 \\
50 & 42 & 58 \\
\hline
\end{tabular}

TABLE IX.-Correction of the Sun's or a Star's Apparent Altitude to allow for Parallax and Refraction.

The correction is to be subtracted, and is the difference between the refraction and the parallax.

\begin{tabular}{|c|c|c|c|}
\hline $\begin{array}{l}\text { Apparent } \\
\text { Altitude. }\end{array}$ & Correction. & $\begin{array}{l}\text { Apparent } \\
\text { Altitude. }\end{array}$ & Correction. \\
\hline $\begin{array}{l}20^{\circ} \\
21 \\
22 \\
23 \\
24 \\
25 \\
26 \\
27 \\
28 \\
29 \\
30\end{array}$ & $\begin{array}{lr}2 & 30^{\prime \prime} \\
2 & 22 \\
2 & 15 \\
2 & 8 \\
2 & 2 \\
1 & 56 \\
1 & 51 \\
1 & 46 \\
1 & 41 \\
1 & 37 \\
1 & 33\end{array}$ & $\begin{array}{l}32^{\circ} \\
34 \\
36 \\
38 \\
40 \\
45 \\
50 \\
55 \\
60 \\
70 \\
80\end{array}$ & $\begin{array}{lr}1^{\prime} & 2^{\prime \prime} \\
1 & 19 \\
1 & 13 \\
1 & 7 \\
1 & 3 \\
0 & 52 \\
0 & 43 \\
0 & 36 \\
0 & 29 \\
0 & 18 \\
0 & 9\end{array}$ \\
\hline
\end{tabular}




\section{AP.PENDIX}

TABLE X.-Reduction of Barometer Readings to $0^{\circ} \mathrm{C}$.

In the following table are given the corrections in millimetres to be applied to reduce the measured height of the barometer to what it would be if the mercury and scale were both at $0^{\circ} \mathrm{C}$., that is, the values of the quantity $\frac{(\delta-\alpha) t}{1+\delta t} h_{t}$, the correction in every case being subtractive :-

\begin{tabular}{|c|c|c|c|c|c|c|c|c|c|c|c|}
\hline \multirow{3}{*}{ 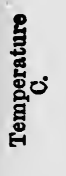 } & \multicolumn{5}{|c|}{ Glass Scale. } & \multicolumn{5}{|c|}{ Brass Scale. } & \multirow{3}{*}{ 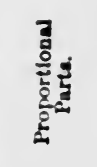 } \\
\hline & \multicolumn{5}{|c|}{ Uncorrected Height. } & \multicolumn{5}{|c|}{ Uncorrected Height. } & \\
\hline & 740 & 750 & 760 & 770 & 780 & 740 & 760 & 760 & 770 & 780 & \\
\hline $\begin{array}{l}1 \\
2 \\
3 \\
4\end{array}$ & $\begin{array}{c}\text { mm. } \\
\cdot 13 \\
\cdot 26 \\
\cdot 38 \\
\cdot 51\end{array}$ & $\begin{array}{r}\mathrm{mm} . \\
\cdot 13 \\
26 \\
\cdot 39 \\
\cdot 52\end{array}$ & $\begin{array}{l}\mathrm{mm} . \\
\cdot 13 \\
\cdot 26 \\
\cdot 39 \\
\cdot 53\end{array}$ & $\begin{array}{r}\mathrm{mm} . \\
\cdot 13 \\
\cdot 27 \\
\cdot 40 \\
\cdot 53\end{array}$ & $\begin{array}{c}\mathrm{mm} . \\
\cdot 13 \\
-27 \\
\cdot 41 \\
\cdot 54\end{array}$ & $\begin{array}{c}\mathrm{mm} . \\
\cdot 12 \\
-24 \\
\cdot 36 \\
\cdot 48\end{array}$ & $\begin{array}{r}\text { mm. } \\
\cdot 12 \\
.25 \\
\cdot 37 \\
\cdot 49\end{array}$ & $\begin{array}{r}\mathrm{mm} . \\
\cdot 12 \\
-25 \\
\cdot 37 \\
\cdot 50\end{array}$ & $\begin{array}{r}\mathrm{mm} . \\
\cdot 13 \\
-25 \\
\cdot 38 \\
\cdot 50\end{array}$ & $\begin{array}{r}\mathrm{mm} . \\
\cdot 13 \\
\cdot 2.5 \\
\cdot 38 \\
\cdot 51\end{array}$ & \multirow{14}{*}{$\begin{aligned} & \cdot 12 \\
& 0 \cdot 1=\cdot 01 \\
& \cdot 2=02 \\
& 3=03 \\
& \cdot 4=05 \\
& \cdot 5=06 \\
& \cdot 6=\cdot 07 \\
& 7=08 \\
& \cdot 8=\cdot 10 \\
& \cdot 9=\cdot 11\end{aligned}$} \\
\hline 5 & 64 & $\cdot 65$ & $\cdot 66$ & $\cdot 67$ & $\cdot 68$ & $\cdot 60$ & $\cdot 61$ & $\cdot 62$ & 63 & 64 & \\
\hline $\begin{array}{l}6 \\
7 \\
8 \\
9\end{array}$ & $\begin{array}{r}\cdot 77 \\
.90 \\
1.02 \\
1 \cdot 15\end{array}$ & $\begin{array}{r}\cdot 78 \\
.91 \\
1 \cdot 04 \\
1 \cdot 17\end{array}$ & $\begin{array}{r}\cdot 79 \\
\cdot 92 \\
1 \cdot 05 \\
1 \cdot 18\end{array}$ & $\begin{array}{r}\cdot 80 \\
\cdot 93 \\
1 \cdot 07 \\
1 \cdot 20\end{array}$ & $\begin{array}{r}.81 \\
.95 \\
1.08 \\
1.21\end{array}$ & $\begin{array}{r}72 \\
.85 \\
.97 \\
1.09\end{array}$ & $\begin{array}{r}73 \\
.86 \\
.98 \\
1 \cdot 10\end{array}$ & $\begin{array}{r}74 \\
87 \\
.99 \\
1 \cdot 12\end{array}$ & $\begin{array}{r}75 \\
.88 \\
1.01 \\
1 \cdot 13\end{array}$ & $\begin{array}{r}76 \\
.89 \\
1.02 \\
1 \cdot 15\end{array}$ & \\
\hline 10 & $1 \cdot 28$ & $1 \cdot 30$ & $1 \cdot 31$ & $1 \cdot 33$ & $1 \cdot 35$ & $1 \cdot 21$ & $1 \cdot 22$ & $1 \cdot 24$ & $1 \cdot 26$ & 1.27 & \\
\hline $\begin{array}{l}11 \\
12 \\
13 \\
14\end{array}$ & $\begin{array}{l}1.41 \\
1.53 \\
1.66 \\
1.79\end{array}$ & $\begin{array}{l}1 \cdot 43 \\
1.56 \\
1.69 \\
1.81\end{array}$ & $\begin{array}{l}1.45 \\
1.58 \\
1.71 \\
1.84\end{array}$ & $\begin{array}{l}1 \cdot 46 \\
1 \cdot 60 \\
1 \cdot 73 \\
1 \cdot 86\end{array}$ & $\begin{array}{l}1.48 \\
1.62 \\
1.75 \\
1.89\end{array}$ & $\begin{array}{l}1.33 \\
1.45 \\
1.57 \\
1.69\end{array}$ & $\begin{array}{l}1 \cdot 35 \\
1 \cdot 47 \\
1 \cdot 59 \\
1 \cdot 71\end{array}$ & $\begin{array}{l}1 \cdot 36 \\
1 \cdot 49 \\
1 \cdot 61 \\
1 \cdot 73\end{array}$ & $\begin{array}{l}1.38 \\
1.51 \\
1.63 \\
1.76\end{array}$ & $\begin{array}{l}1.40 \\
1.53 \\
1.65 \\
1.78\end{array}$ & \\
\hline 15 & 1.92 & $1 \cdot 94$ & 1.97 & $2 \cdot 00$ & $2 \cdot 02$ & $1 \cdot 81$ & $1 \cdot 83$ & $1 \cdot 86$ & $1 \cdot 88$ & 1.91 & \\
\hline $\begin{array}{l}16 \\
17 \\
18 \\
19\end{array}$ & $\begin{array}{l}2 \cdot 05 \\
2 \cdot 17 \\
2 \cdot 30 \\
2 \cdot 43\end{array}$ & $\begin{array}{l}2 \cdot 07 \\
2 \cdot 20 \\
2 \cdot 33 \\
2 \cdot 46\end{array}$ & $\begin{array}{l}2 \cdot 10 \\
2 \cdot 23 \\
2 \cdot 36 \\
2 \cdot 49\end{array}$ & $\begin{array}{l}2 \cdot 13 \\
2 \cdot 26 \\
2 \cdot 39 \\
2 \cdot 53\end{array}$ & $\begin{array}{l}2 \cdot 16 \\
2 \cdot 29 \\
2 \cdot 43 \\
2 \cdot 56\end{array}$ & $\begin{array}{l}1 \cdot 93 \\
2 \cdot 05 \\
2 \cdot 17 \\
2 \cdot 29\end{array}$ & $\begin{array}{l}1 \cdot 96 \\
2 \cdot 08 \\
2 \cdot 20 \\
2 \cdot 32\end{array}$ & $\begin{array}{l}1 \cdot 98 \\
2 \cdot 10 \\
2 \cdot 23 \\
2 \cdot 35\end{array}$ & $\begin{array}{l}2 \cdot 01 \\
2 \cdot 13 \\
2 \cdot 26 \\
2 \cdot 38\end{array}$ & $\begin{array}{l}2 \cdot 03 \\
2 \cdot 16 \\
2 \cdot 9 \\
2 \cdot 41\end{array}$ & \\
\hline 20 & $2 \cdot 56$ & $2 \cdot 59$ & $2 \cdot 62$ & $2 \cdot 66$ & $2 \cdot 69$ & $2 \cdot 41$ & $2 \cdot 44$ & $2 \cdot 47$ & $2 \cdot 51$ & $2 \cdot 54$ & \\
\hline $\begin{array}{l}21 \\
22 \\
23 \\
24\end{array}$ & $\begin{array}{l}2 \cdot 68 \\
2 \cdot 81 \\
2 \cdot 94 \\
3 \cdot 06\end{array}$ & $\begin{array}{l}2 \cdot 72 \\
2 \cdot 85 \\
2 \cdot 98 \\
3 \cdot 11\end{array}$ & $\begin{array}{l}2 \cdot 76 \\
2 \cdot 89 \\
3 \cdot 02 \\
3 \cdot 15\end{array}$ & $\begin{array}{l}2 \cdot 79 \\
2 \cdot 92 \\
3 \cdot 06 \\
3 \cdot 19\end{array}$ & $\begin{array}{l}2 \cdot 83 \\
2 \cdot 96 \\
3 \cdot 10 \\
3 \cdot 23\end{array}$ & $\begin{array}{l}2 \cdot 53 \\
2 \cdot 65 \\
2 \cdot 77 \\
2 \cdot 89\end{array}$ & $\begin{array}{l}2 \cdot 56 \\
2 \cdot 69 \\
2 \cdot 81 \\
2 \cdot 93\end{array}$ & $\begin{array}{l}2 \cdot 60 \\
2 \cdot 72 \\
2 \cdot 84 \\
2 \cdot 97\end{array}$ & $\begin{array}{l}2 \cdot 63 \\
2 \cdot 76 \\
2 \cdot 88 \\
3.01\end{array}$ & $\begin{array}{l}2 \cdot 67 \\
2 \cdot 79 \\
2 \cdot 92 \\
3 \cdot 05\end{array}$ & \\
\hline 25 & $3 \cdot 19$ & $3 \cdot 23$ & $3 \cdot 28$ & $3 \cdot 32$ & $3 \cdot 36$ & 3.01 & 3.05 & .309 & $3 \cdot 13$ & $3 \cdot 17$ & \\
\hline $\begin{array}{l}26 \\
27 \\
28 \\
29\end{array}$ & $\begin{array}{l}3 \cdot 32 \\
3 \cdot 45 \\
3 \cdot 57 \\
3 \cdot 70\end{array}$ & $\begin{array}{l}3 \cdot 36 \\
3 \cdot 49 \\
3 \cdot 62 \\
3 \cdot 75\end{array}$ & $\begin{array}{l}3 \cdot 41 \\
3 \cdot 54 \\
3 \cdot 67 \\
3 \cdot 80\end{array}$ & $\begin{array}{l}3 \cdot 45 \\
3.59 \\
3 \cdot 72 \\
3.85\end{array}$ & $\begin{array}{l}3 \cdot 50 \\
3 \cdot 63 \\
3 \cdot 77 \\
3 \cdot 90\end{array}$ & $\begin{array}{l}3 \cdot 13 \\
3 \cdot 25 \\
3 \cdot 37 \\
3 \cdot 49\end{array}$ & $\begin{array}{l}3 \cdot 17 \\
3 \cdot 29 \\
3 \cdot 41 \\
3 \cdot 54\end{array}$ & $\begin{array}{l}3 \cdot 21 \\
3 \cdot 34 \\
3 \cdot 46 \\
3 \cdot 58\end{array}$ & $\begin{array}{l}3 \cdot 26 \\
3 \cdot 38 \\
3 \cdot 51 \\
3 \cdot 63\end{array}$ & $\begin{array}{l}3 \cdot 30 \\
3 \cdot 4: 2 \\
3 \cdot 55 \\
3 \cdot+3\end{array}$ & \\
\hline 30 & $3 \cdot 83$ & $3 \cdot 88$ & 3.93 & 3.98 & 4.03 & $3 \cdot 61$ & $3 \cdot 66$ & $3 \cdot 71$ & $3 \cdot 75$ & $3 \cdot 80$ & \\
\hline $\begin{array}{l}31 \\
32 \\
33 \\
34\end{array}$ & $\begin{array}{l}3 \cdot 95 \\
4 \cdot 08 \\
4 \cdot 21 \\
4 \cdot 33\end{array}$ & $\begin{array}{l}4 \cdot 01 \\
4 \cdot 14 \\
4 \cdot 26 \\
4 \cdot 39\end{array}$ & $\begin{array}{l}4 \cdot 06 \\
4 \cdot 19 \\
4 \cdot 32 \\
4 \cdot 45\end{array}$ & $\begin{array}{l}4 \cdot 11 \\
4 \cdot 25 \\
4 \cdot 38 \\
4 \cdot 51\end{array}$ & $\begin{array}{l}4 \cdot 17 \\
4 \cdot 30 \\
4 \cdot 43 \\
4 \cdot 57\end{array}$ & $\begin{array}{l}3 \cdot 73 \\
3 \cdot 85 \\
3 \cdot 97 \\
4 \cdot 09\end{array}$ & $\begin{array}{l}3 \cdot 78 \\
3 \cdot 100 \\
4 \cdot 02 \\
4 \cdot 14\end{array}$ & $\begin{array}{l}3.83 \\
3.95 \\
4.07 \\
4.20\end{array}$ & $\begin{array}{l}3 \cdot 88 \\
4 \cdot 00 \\
4 \cdot 13 \\
4 \cdot 25\end{array}$ & $\begin{array}{l}3 \cdot 03 \\
4 \cdot 05 \\
4 \cdot 18 \\
4 \cdot 31\end{array}$ & \\
\hline 35 & $4 \cdot 46$ & $4 \cdot 52$ & $4 \cdot 58$ & $4 \cdot 65$ & $4 \cdot 71$ & $4 \cdot 21$ & $4 \cdot 26$ & $4 \cdot 32$ & $4 \cdot 38$ & 443 & \\
\hline
\end{tabular}




\section{TABLE XI.}

Correction to the Height of the Barometer to allow for the effect of Capillarity.

The correction, which is given in millimetres, has always to be added to the observed height.

\begin{tabular}{|c|c|c|c|c|c|c|c|c|}
\hline \multirow{2}{*}{$\begin{array}{l}\text { Diameter } \\
\text { of Tube, } \\
\text { mm. }\end{array}$} & \multicolumn{8}{|c|}{ Height of the Meniscus in Millimetres. } \\
\hline & $\cdot 4$ & $\cdot 6$ & $\cdot 8$ & $1 \cdot 0$ & $1 \cdot 2$ & $1 \cdot 4$ & $1 \cdot 6$ & $1 \cdot 8$ \\
\hline 4 & $\cdot 83$ & $1 \cdot 22$ & $1 \cdot 54$ & $1 \cdot 98$ & $2 \cdot 37$ & & & \\
\hline 5 & $\cdot 47$ & $\cdot 65$ & $\cdot 86$ & $1 \cdot 19$ & $1 \cdot 45$ & $1 \cdot 80$ & & \\
\hline 6 & $\cdot 27$ & $\cdot 41$ & $\cdot 56$ & $\cdot 78$ & .98 & $1 \cdot 21$ & $1 \cdot 43$ & \\
\hline 7 & $\cdot 18$ & $\cdot 28$ & $\cdot 40$ & $\cdot 53$ & $\cdot 67$ & $\cdot 82$ & $\cdot 97$ & $1 \cdot 13$ \\
\hline 8 & & $\cdot 20$ & $\cdot 29$ & $\cdot 38$ & $\cdot 46$ & $\cdot 56$ & $\cdot 65$ & $\cdot 77$ \\
\hline 9 & & $\cdot 15$ & $\cdot 21$ & $\cdot 28$ & $\cdot 33$ & $\cdot 40$ & $\cdot 46$ & $\cdot 52$ \\
\hline 10 & & & $\cdot 15$ & $\cdot 20$ & $\cdot 25$ & $\cdot 29$ & $\cdot 33$ & $\cdot 37$ \\
\hline 11 & & & $\cdot 10$ & $\cdot 14$ & $\cdot 18$ & $\cdot 21$ & $\cdot 24$ & $\cdot 27$ \\
\hline 12 & & & 07 & $\cdot 10$ & $\cdot 13$ & $\cdot 15$ & $\cdot 18$ & $\cdot 19$ \\
\hline 13 & & & & 07 & $\cdot 10$ & $\cdot 12$ & $\cdot 13$ & $\cdot 14$ \\
\hline
\end{tabular}




\section{APPENDIX}

TABLE XII.

Reduction of the Volume of a Gas to Standard Pressure.

The table gires the values of the logarithms of the factors $\frac{B}{760}$ for different values of $B$, the height of the barometer at the time the volume of the gas was measured.

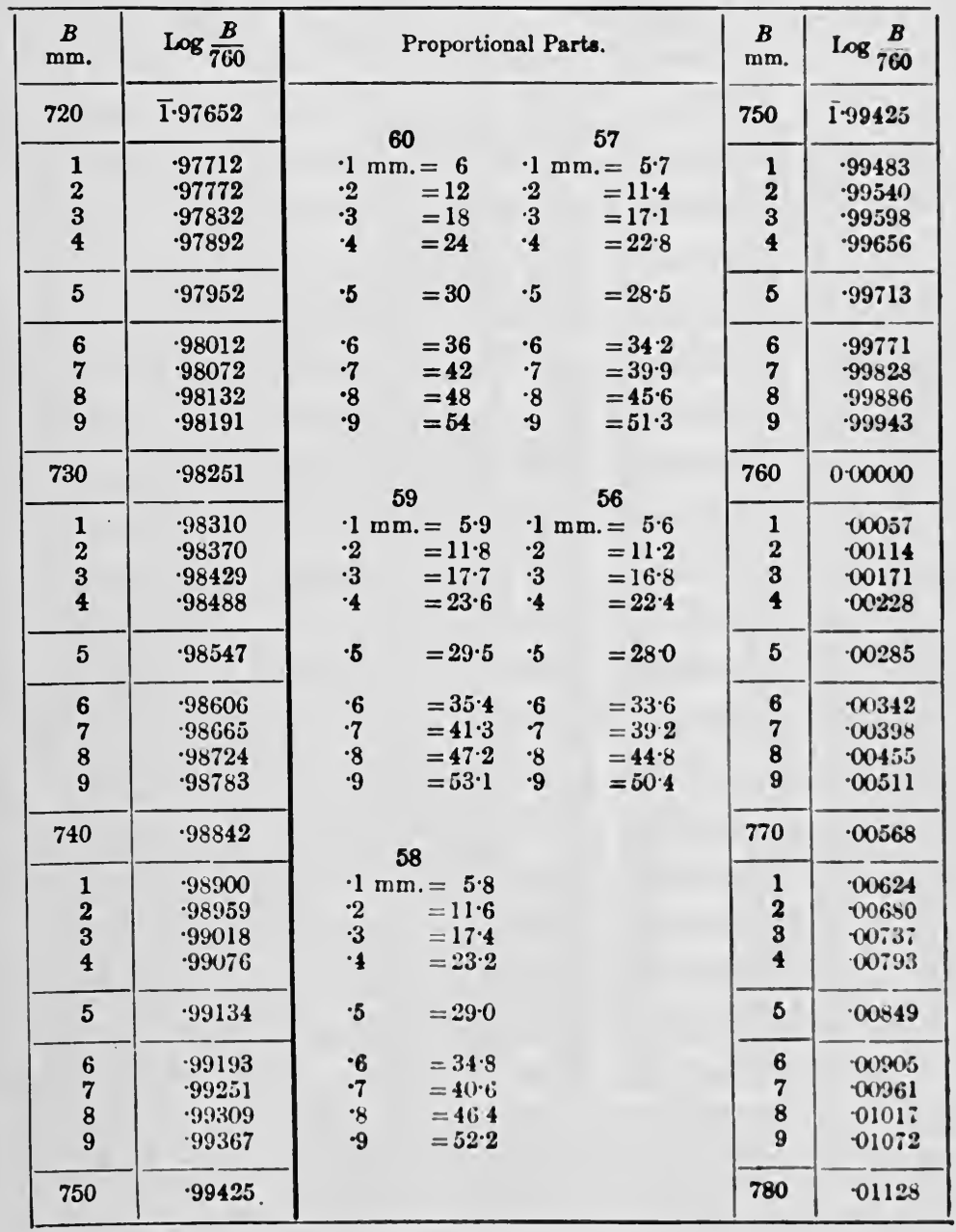




\section{Table XIII.}

Values of $(1+a t)$ for reducing the Volume of Gases to Standard Temperature for Values of $t$ from $0^{\circ}$ to $40^{\circ} \mathrm{C} .(\alpha=0.003670)$.

\begin{tabular}{|c|c|c|c|c|}
\hline Temperature. & $1+a t$ & Temperature. & $1+a t$. & $\begin{array}{l}\text { Proportional } \\
\text { Parts. }\end{array}$ \\
\hline 1 & $1 \cdot 00367$ & 21 & $1 \cdot 07707$ & 367 \\
\hline 2 & $1 \cdot 00734$ & 22 & $1 \cdot 08074$ & $0^{\circ} \cdot 1=36 \cdot 7$ \\
\hline 3 & 101101 & 23 & $1 \cdot 08441$ & $\cdot 2=73 \cdot 4$ \\
\hline 4 & $1 \cdot 01468$ & 24 & $1 \cdot 08808$ & $\cdot 3=110 \cdot 1$ \\
\hline 5 & $1 \cdot 01835$ & 25 & $1 \cdot 09175$ & $\cdot 4=146 \cdot 8$ \\
\hline 6 & $1 \cdot 02202$ & 26 & $1 \cdot 09542$ & $\cdot 5=183 \cdot 5$ \\
\hline 7 & $1 \cdot 02569$ & 27 & 1.09909 & $\cdot 6=220 \cdot 2$ \\
\hline 8 & $1 \cdot 02936$ & 28 & $1 \cdot 10276$ & $\cdot 7=256 \cdot 9$ \\
\hline 9 & $1 \cdot 03303$ & 29 & $1 \cdot 10643$ & $\cdot 8=293 \cdot 6$ \\
\hline 10 & $1 \cdot 03670$ & 30 & $1 \cdot 11010$ & $\cdot 9=330 \cdot 3$ \\
\hline 11 & $1 \cdot 04037$ & 81 & $1 \cdot 11377$ & 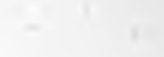 \\
\hline 12 & $1 \cdot 04404$ & 32 & $1 \cdot 11744$ & \\
\hline 13 & $1 \cdot 04771$ & 33 & $1 \cdot 12111$ & \\
\hline 14 & 1.05138 & 34 & $1 \cdot 12478$ & \\
\hline 15 & 1.05505 & 35 & $1 \cdot 12845$ & \\
\hline 16 & $1 \cdot 05872$ & 36 & $1 \cdot 13212$ & \\
\hline 17 & $1 \cdot 06239$ & 37 & $1 \cdot 13579$ & \\
\hline 18 & $1 \cdot 06606$ & 38 & $1 \cdot 13946$ & \\
\hline 19 & $1 \cdot 06973$ & 39 & $1 \cdot 14313$ & \\
\hline 20 & 1.07340 & 40 & $1 \cdot 14680$ & \\
\hline
\end{tabular}




\section{TABLE XIV.}

Tension of Water Vapour and Mass of Water in a Culic Metre of Saturated Air.

(1) Vapour Tension of Water for every Degree between $0^{\circ} \mathrm{C}$. and $100^{\circ} \mathrm{C}$. For further values in the neighbourhood of $100^{\circ} \mathrm{C}$., see Table $X V$.

\begin{tabular}{|c|c|c|c|c|c|c|c|c|c|c|}
\hline \multirow{2}{*}{ 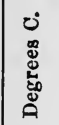 } & \multicolumn{10}{|c|}{ Vapour Tension in Millimetres in Mercury. } \\
\hline & $0^{\circ}$ & 1 & 2 & 3 & 4 & 5 & 6 & 7 & 8 & 9 \\
\hline $0^{\circ}$ & $4 \cdot 58$ & $4 \cdot 93$ & $5 \cdot 29$ & $5 \cdot 68$ & $6 \cdot 10$ & $6 \cdot 54$ & $7 \cdot 01$ & $7 \cdot 51$ & $8 \cdot 05$ & $8 \cdot 61$ \\
\hline 10 & $9 \cdot 21$ & $9 \cdot 84$ & $10 \cdot 52$ & $11 \cdot 23$ & $11 \cdot 99$ & $12 \cdot 79$ & $13 \cdot 64$ & $14 \cdot 53$ & $15 \cdot 48$ & $16 \cdot 48$ \\
\hline 20 & $17 \cdot 54$ & $18 \cdot 65$ & $19 \cdot 83$ & $21 \cdot 07$ & $22 \cdot 38$ & $23 \cdot 76$ & $25 \cdot 22$ & $26 \cdot 75$ & $28 \cdot 36$ & 30.05 \\
\hline 30 & $31 \cdot 83$ & $33 \cdot 71$ & $35 \cdot 67$ & $37 \cdot 74$ & $39 \cdot 91$ & $42 \cdot 19$ & $44 \cdot 58$ & $47 \cdot 08$ & $49 \cdot 71$ & $52 \cdot 46$ \\
\hline 40 & $55 \cdot 34$ & $58 \cdot 36$ & $61 \cdot 52$ & $64 \cdot 82$ & $68 \cdot 28$ & $71 \cdot 90$ & $75 \cdot 67$ & 79.62 & 83.74 & 88.05 \\
\hline 50 & $92 \cdot 54$ & $97 \cdot 24$ & $102 \cdot 1$ & $107 \cdot 2$ & $112 \cdot 6$ & $118 \cdot 1$ & $123 \cdot 9$ & 129.9 & $136 \cdot 2$ & $142 \cdot 7$ \\
\hline 60 & 149.5 & 156.5 & $163 \cdot 8$ & $171 \cdot 5$ & $179 \cdot 4$ & $187 \cdot 6$ & $196 \cdot 2$ & $205 \cdot 1$ & $214^{\circ} 3$ & $223 \cdot 9$ \\
\hline 70 & 233.8 & $244 \cdot 1$ & $254 \cdot 8$ & $265 \cdot 9$ & $277 \cdot 4$ & $289 \cdot 3$ & $301 \cdot 6$ & $314 \cdot 4$ & $327 \cdot 6$ & $341 \cdot 3$ \\
\hline 80 & $355 \cdot 5$ & $370 \cdot 1$ & $385 \cdot 2$ & $400 \cdot 9$ & $417 \cdot 1$ & $433 \cdot 8$ & $451 \cdot 1$ & $468 \cdot 9$ & $487 \cdot 3$ & $506 \cdot 4$ \\
\hline 90 & 526.0 & $546 \cdot 3$ & $567 \cdot 2$ & 588.8 & $611^{\circ} 0$ & $634 \cdot 0$ & $657 \cdot 7$ & $682 \cdot 1$ & $707 \cdot 3$ & $733 \cdot 2$ \\
\hline 100 & 760.0 & $787 \cdot 6$ & & & & & & & & \\
\hline
\end{tabular}


TABLE XIV. (continued)-(2) Mass of Water contained in a Cubic Metre of Air at a Pressure of $76 \mathrm{~cm}$. of Mercury, and Saturated at various Temperatures between $0^{\circ}$ and $35^{\circ} \mathrm{C}$.

\begin{tabular}{|c|c|c|c|c|c|c|c|c|c|c|}
\hline \multirow{3}{*}{ Degrees. } & \multicolumn{10}{|c|}{ Mass of Water in Grams. } \\
\hline & & \multicolumn{4}{|c|}{ Tenths. } & \multirow[b]{2}{*}{7} & \multirow[b]{2}{*}{$\cdot 8$} & \multirow[b]{2}{*}{.9} \\
\hline & $\cdot 0$ & $\cdot 1$ & $\cdot 2$ & 3 & $\cdot 4$ & $\cdot 5$ & $\cdot 6$ & & & \\
\hline $\mathbf{0}$ & $4 \cdot 84$ & $4 \cdot 87$ & $4 \cdot 90$ & 4.93 & $4 \cdot 97$ & $5 \cdot 00$ & $5 \cdot 04$ & $5 \cdot 07$ & $5 \cdot 11$ & $5 \cdot 14$ \\
\hline $\begin{array}{l}1 \\
2 \\
3 \\
4\end{array}$ & $\begin{array}{l}5 \cdot 18 \\
5 \cdot 54 \\
5 \cdot 92 \\
6 \cdot 33\end{array}$ & $\begin{array}{l}5 \cdot 21 \\
5 \cdot 57 \\
5 \cdot 96 \\
6 \cdot 37\end{array}$ & $\begin{array}{l}5 \cdot 25 \\
5 \cdot 61 \\
6 \cdot 00 \\
6 \cdot 41\end{array}$ & $\begin{array}{l}5 \cdot 28 \\
5 \cdot 65 \\
6 \cdot 04 \\
6 \cdot 46\end{array}$ & $\begin{array}{l}5 \cdot 32 \\
5 \cdot 69 \\
6 \cdot 08 \\
6 \cdot 50\end{array}$ & $\begin{array}{l}5 \cdot 35 \\
5 \cdot 73 \\
6 \cdot 12 \\
6 \cdot 54\end{array}$ & $\begin{array}{l}5 \cdot 39 \\
5 \cdot 77 \\
6 \cdot 16 \\
6 \cdot 59\end{array}$ & $\begin{array}{l}5 \cdot 43 \\
5 \cdot 80 \\
6 \cdot 20 \\
6 \cdot 63\end{array}$ & $\begin{array}{l}5 \cdot 46 \\
5 \cdot 84 \\
6 \cdot 25 \\
6 \cdot 67\end{array}$ & $\begin{array}{l}5 \cdot 50 \\
5 \cdot 88 \\
6 \cdot 29 \\
6 \cdot 72\end{array}$ \\
\hline 5 & $6 \cdot 76$ & $6 \cdot 81$ & $6 \cdot 85$ & $6 \cdot 90$ & $6 \cdot 94$ & $6 \cdot 99$ & $7 \cdot 03$ & $7 \cdot 08$ & $7 \cdot 12$ & $7 \cdot 17$ \\
\hline $\begin{array}{l}6 \\
7 \\
8 \\
9\end{array}$ & $\begin{array}{l}7 \cdot 22 \\
7 \cdot 70 \\
8 \cdot 21 \\
8 \cdot 76\end{array}$ & $\begin{array}{l}7 \cdot 27 \\
7 \cdot 75 \\
8 \cdot 27 \\
8 \cdot 81\end{array}$ & $\begin{array}{l}7 \cdot 31 \\
7 \cdot 80 \\
8 \cdot 32 \\
8 \cdot 87\end{array}$ & $\begin{array}{l}7 \cdot 36 \\
7 \cdot 85 \\
8 \cdot 37 \\
8 \cdot 92\end{array}$ & $\begin{array}{l}7 \cdot 41 \\
7 \cdot 90 \\
8 \cdot 43 \\
8 \cdot 98\end{array}$ & $\begin{array}{l}7 \cdot 46 \\
7 \cdot 95 \\
8 \cdot 48 \\
9 \cdot 04\end{array}$ & $\begin{array}{l}7 \cdot 51 \\
8 \cdot 01 \\
8 \cdot 54 \\
9 \cdot 10\end{array}$ & $\begin{array}{l}7 \cdot 55 \\
8 \cdot 06 \\
8 \cdot 59 \\
9 \cdot 15\end{array}$ & $\begin{array}{l}7 \cdot 60 \\
8 \cdot 11 \\
8 \cdot 65 \\
9 \cdot 21\end{array}$ & $\begin{array}{l}7 \cdot 65 \\
8 \cdot 16 \\
8 \cdot 70 \\
9 \cdot 27\end{array}$ \\
\hline 10 & $9 \cdot 33$ & $9 \cdot 39$ & $9 \cdot 45$ & $9 \cdot 51$ & $9 \cdot 57$ & $9 \cdot 63$ & $9 \cdot 69$ & $9 \cdot 75$ & $9 \cdot 81$ & $9 \cdot 87$ \\
\hline $\begin{array}{l}11 \\
12 \\
13 \\
14\end{array}$ & $\begin{array}{r}9.93 \\
10.57 \\
11.25 \\
11.96\end{array}$ & $\begin{array}{l}10 \cdot 00 \\
10 \cdot 64 \\
11 \cdot 32 \\
12 \cdot 03\end{array}$ & $\begin{array}{l}10 \cdot 06 \\
10 \cdot 71 \\
11 \cdot 39 \\
12 \cdot 11\end{array}$ & $\begin{array}{l}10 \cdot 12 \\
10 \cdot 77 \\
11 \cdot 46 \\
12 \cdot 18\end{array}$ & $\begin{array}{l}10 \cdot 19 \\
10 \cdot 84 \\
11 \cdot 53 \\
12 \cdot 26\end{array}$ & $\begin{array}{l}10 \cdot 25 \\
10.91 \\
11 \cdot 60 \\
12 \cdot 33\end{array}$ & $\begin{array}{l}10 \cdot 31 \\
10 \cdot 97 \\
11 \cdot 67 \\
12 \cdot 41\end{array}$ & $\begin{array}{l}10 \cdot 38 \\
11 \cdot 04 \\
11 \cdot 74 \\
12 \cdot 48\end{array}$ & $\begin{array}{l}10 \cdot 44 \\
11 \cdot 11 \\
11 \cdot 82 \\
12 \cdot 56\end{array}$ & $\begin{array}{l}10 \cdot 51 \\
11 \cdot 18 \\
11 \cdot 89 \\
12 \cdot 64\end{array}$ \\
\hline 15 & $12 \cdot 71$ & $12 \cdot 79$ & $12 \cdot 87$ & $12 \cdot 95$ & $13 \cdot 02$ & $13 \cdot 10$ & $13 \cdot 18$ & $13 \cdot 26$ & $13 \cdot 34$ & $13 \cdot 42$ \\
\hline $\begin{array}{l}16 \\
17 \\
18 \\
19\end{array}$ & $\begin{array}{l}13 \cdot 50 \\
14 \cdot 34 \\
15 \cdot 22 \\
16 \cdot 14\end{array}$ & $\begin{array}{l}13 \cdot 58 \\
14 \cdot 42 \\
15 \cdot 31 \\
16 \cdot 24\end{array}$ & $\begin{array}{l}13 \cdot 67 \\
14 \cdot 51 \\
15 \cdot 40 \\
16 \cdot 33\end{array}$ & $\begin{array}{l}13 \cdot 75 \\
14 \cdot 60 \\
15 \cdot 49 \\
16 \cdot 43\end{array}$ & $\begin{array}{l}13 \cdot 83 \\
14 \cdot 68 \\
15 \cdot 58 \\
16 \cdot 53\end{array}$ & $\begin{array}{l}13 \cdot 92 \\
14 \cdot 77 \\
15 \cdot 67 \\
16 \cdot 62\end{array}$ & $\begin{array}{l}14 \cdot 00 \\
14 \cdot 86 \\
15 \cdot 77 \\
16 \cdot 72\end{array}$ & $\begin{array}{l}14 \cdot 08 \\
14 \cdot 95 \\
15 \cdot 86 \\
16 \cdot 82\end{array}$ & \begin{tabular}{|l|}
$14 \cdot 17$ \\
$15 \cdot 04$ \\
$15 \cdot 95$ \\
$16 \cdot 92$
\end{tabular} & $\begin{array}{l}14 \cdot 25 \\
15 \cdot 13 \\
16 \cdot 05 \\
17 \cdot 02\end{array}$ \\
\hline 20 & $17 \cdot 12$ & $17 \cdot 22$ & $17 \cdot 32$ & $17 \cdot 42$ & $17 \cdot 52$ & $17 \cdot 62$ & $17 \cdot 73$ & $17 \cdot 83$ & $17 \cdot 93$ & $18 \cdot 04$ \\
\hline $\begin{array}{l}21 \\
22 \\
23 \\
24\end{array}$ & $\begin{array}{l}18 \cdot 14 \\
19 \cdot 22 \\
20 \cdot 35 \\
21 \cdot 54\end{array}$ & $\begin{array}{l}18 \cdot 25 \\
19 \cdot 33 \\
20 \cdot 47 \\
21 \cdot 67\end{array}$ & $\begin{array}{l}18 \cdot 35 \\
19 \cdot 44 \\
20 \cdot 59 \\
21 \cdot 79\end{array}$ & $\begin{array}{l}18 \cdot 46 \\
19 \cdot 55 \\
20 \cdot 70 \\
21 \cdot 91\end{array}$ & $\begin{array}{l}18 \cdot 57 \\
19 \cdot 67 \\
20 \cdot 82 \\
22 \cdot 04\end{array}$ & $\begin{array}{l}18 \cdot 67 \\
19 \cdot 78 \\
20 \cdot 94 \\
22 \cdot 16\end{array}$ & $\begin{array}{l}18 \cdot 78 \\
19 \cdot 89 \\
21 \cdot 06 \\
22 \cdot 29\end{array}$ & $\begin{array}{l}18 \cdot 89 \\
20 \cdot 01 \\
21 \cdot 18 \\
22 \cdot 41\end{array}$ & \begin{tabular}{|l|}
$19 \cdot 00$ \\
$20 \cdot 12$ \\
$21 \cdot 30$ \\
$22 \cdot 54$
\end{tabular} & $\begin{array}{l}19 \cdot 11 \\
20 \cdot 24 \\
21 \cdot 42 \\
22 \cdot 67\end{array}$ \\
\hline 25 & $22 \cdot 80$ & $22 \cdot 92$ & $23 \cdot 05$ & $23 \cdot 18$ & $23 \cdot 31$ & $23 \cdot 44$ & $23 \cdot 58$ & $23 \cdot 71$ & $23 \cdot 84$ & $23 \cdot 97$ \\
\hline $\begin{array}{l}26 \\
27 \\
28 \\
29\end{array}$ & $\begin{array}{l}24 \cdot 11 \\
25 \cdot 49 \\
26 \cdot 93 \\
28 \cdot 45\end{array}$ & $\begin{array}{l}24 \cdot 24 \\
25 \cdot 63 \\
27 \cdot 08 \\
28 \cdot 60\end{array}$ & $\begin{array}{l}24 \cdot 38 \\
25 \cdot 77 \\
27 \cdot 23 \\
28 \cdot 76\end{array}$ & $\begin{array}{l}24 \cdot 51 \\
25 \cdot 91 \\
27 \cdot 38 \\
28 \cdot 92\end{array}$ & $\begin{array}{l}24 \cdot 65 \\
26 \cdot 06 \\
27 \cdot 53 \\
29 \cdot 07\end{array}$ & $\begin{array}{l}24 \cdot 79 \\
26 \cdot 20 \\
27 \cdot 68 \\
29 \cdot 23\end{array}$ & $\begin{array}{l}24 \cdot 93 \\
26 \cdot 35 \\
27 \cdot 83 \\
29 \cdot 39\end{array}$ & $\begin{array}{l}25 \cdot 07 \\
26 \cdot 49 \\
27 \cdot 99 \\
29 \cdot 55\end{array}$ & $\left|\begin{array}{l}25 \cdot 21 \\
26 \cdot 64 \\
28 \cdot 14 \\
29 \cdot 71\end{array}\right|$ & $\begin{array}{l}25 \cdot 35 \\
26 \cdot 78 \\
28 \cdot 29 \\
29 \cdot 87\end{array}$ \\
\hline 30 & $30 \cdot 04$ & $30 \cdot 20$ & $30 \cdot 36$ & $30 \cdot 53$ & $30 \cdot 69$ & $30 \cdot 86$ & $31 \cdot 03$ & $31 \cdot 19$ & $31 \cdot 36$ & $31 \cdot 53$ \\
\hline $\begin{array}{l}31 \\
32 \\
33 \\
34\end{array}$ & $\begin{array}{l}31 \cdot 70 \\
33 \cdot 45 \\
35 \cdot 27 \\
37 \cdot 18\end{array}$ & $\begin{array}{l}31 \cdot 87 \\
33 \cdot 63 \\
35 \cdot 46 \\
37 \cdot 38\end{array}$ & $\begin{array}{l}32 \cdot 04 \\
33 \cdot 81 \\
35 \cdot 65 \\
37 \cdot 58\end{array}$ & $\begin{array}{l}32 \cdot 22 \\
33 \cdot 99 \\
35 \cdot 84 \\
37 \cdot 77\end{array}$ & \begin{tabular}{|l|}
$32 \cdot 39$ \\
$34 \cdot 17$ \\
$36 \cdot 03$ \\
$37 \cdot 97$
\end{tabular} & $\begin{array}{l}32 \cdot 56 \\
34 \cdot 35 \\
36 \cdot 22 \\
38 \cdot 17\end{array}$ & \begin{tabular}{|l|}
$32 \cdot 74$ \\
$34 \cdot 53$ \\
$36 \cdot 41$ \\
$38 \cdot 37$
\end{tabular} & $\begin{array}{l}32 \cdot 91 \\
34 \cdot 72 \\
36 \cdot 60 \\
38 \cdot 57\end{array}$ & \begin{tabular}{|l|}
$33 \cdot 09$ \\
$34 \cdot 90$ \\
$36 \cdot 79$ \\
$38 \cdot 78$
\end{tabular} & $\begin{array}{l}33 \cdot 27 \\
35 \cdot 09 \\
36 \cdot 99 \\
38 \cdot 98\end{array}$ \\
\hline 35 & $39 \cdot 18$ & $39 \cdot 39$ & $39 \cdot 59$ & $39 \cdot 80$ & $40^{\circ} 01$ & $40 \cdot 22$ & 40.43 & $40 \cdot 64$ & $40^{\circ} 85$ & 41.06 \\
\hline & 0 & $\cdot 1$ & $\cdot 2$ & $\cdot 3$ & 4 & $\cdot 5$ & $\cdot 6$ & $\cdot 7$ & 8 & $\cdot 9$ \\
\hline
\end{tabular}




\section{TabLe XV.-Boiling Point of Water.}

The following table contains the boiling point of water under different pressures between $700 \mathrm{~mm}$. and $799 \mathrm{~mm}$. The numbers given are due to Wieb (Zeitschrift für Instrumentenkunde (1893), xiii. 329 ; see also Landolt \& Börnstein's Tabellen).

\begin{tabular}{|c|c|c|c|c|c|c|c|c|c|c|}
\hline \multirow{2}{*}{ 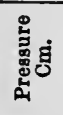 } & \multicolumn{10}{|c|}{ Millimetres. } \\
\hline & 0 & 1 & 2 & 3 & 4 & 5 & 6 & 7 & 8 & 9 \\
\hline 70 & $97 \cdot 714$ & $\cdot 753$ & $\cdot 792$ & $\cdot 832$ & $\cdot 871$ & $\cdot 910$ & $\cdot 949$ & .989 & $\overline{028}$ & $\overline{067}$ \\
\hline 71 & $98 \cdot 106$ & $\cdot 145$ & $\cdot 184$ & $\cdot 223$ & $\cdot 261$ & $\cdot 300$ & $\cdot 339$ & $\cdot 378$ & $\cdot 416$ & $\cdot 455$ \\
\hline 72 & $98 \cdot 493$ & $\cdot 532$ & .570 & $\cdot 609$ & $\cdot 647$ & $\cdot 686$ & 724 & 762 & $\cdot 800$ & $\cdot 838$ \\
\hline 73 & $98 \cdot 877$ & $\cdot 915$ & .953 & .991 & $\overline{.029}$ & $\overline{067}$ & $\overline{104}$ & $\overline{142}$ & $\overline{\cdot 180}$ & $\overline{218}$ \\
\hline 74 & $99 \cdot 255$ & 293 & $\cdot 331$ & 368 & $\cdot 406$ & $\cdot 443$ & $\cdot 481$ & $\cdot 518$ & $\cdot 555$ & $\cdot 592$ \\
\hline 75 & $99 \cdot 630$ & 667 & $\cdot 704$ & $\cdot 741$ & $\cdot 778$ & 815 & $\cdot 852$ & $\cdot 889$ & .926 & $\cdot 963$ \\
\hline 76 & $100 \cdot 000$ & .037 & .074 & $\cdot 110$ & $\cdot 147$ & $\cdot 184$ & $\cdot 220$ & $\cdot 257$ & $\cdot 293$ & $\cdot 330$ \\
\hline 77 & $100 \cdot 366$ & $\cdot 403$ & $\cdot 439$ & $\cdot 475$ & .511 & $\cdot 548$ & $\cdot 584$ & 620 & $\cdot 656$ & 692 \\
\hline 78 & $100 \cdot 728$ & $\cdot 764$ & .800 & $\cdot 836$ & $\cdot 872$ & .908 & $\cdot 944$ & $\cdot 979$ & $\overline{015}$ & $\overline{051}$ \\
\hline \multirow[t]{2}{*}{79} & $101 \cdot 087$ & $\cdot 122$ & $\cdot 158$ & $\cdot 193$ & $\cdot 229$ & $\cdot 264$ & $\cdot 300$ & $\cdot 335$ & $\cdot 370$ & -406 \\
\hline & 0 & 1 & 2 & 3 & 4 & 5 & 6 & 7 & 8 & 9 \\
\hline
\end{tabular}

Propontional Parts.

\begin{tabular}{|l|l|l|l|l|l|}
\hline $\mathrm{mm}$. & .039 & .038 & .037 & .036 & .035 \\
\hline 0.1 & .004 & .004 & .004 & .004 & 004 \\
0.2 & .008 & .008 & .007 & .007 & 007 \\
0.3 & .012 & .011 & .011 & .011 & .011 \\
0.4 & .016 & 015 & 015 & .014 & 014 \\
0.5 & .020 & .019 & .019 & .018 & .018 \\
0.6 & .023 & .023 & .022 & .022 & .021 \\
0.7 & .027 & .027 & .026 & .025 & 025 \\
0.8 & .031 & .030 & .030 & .029 & .028 \\
0.9 & .035 & .034 & .033 & .032 & .032 \\
\hline
\end{tabular}


TabLe XVI._Vapour Pressure of Liquids suitable for Use in Vapour Jackets.

\begin{tabular}{|c|c|c|c|c|c|}
\hline \multicolumn{2}{|c|}{ Carbon Bisulphide. } & \multicolumn{2}{|c|}{ Ethyl Alcohol. } & \multicolumn{2}{|c|}{ Chlorobenzene. } \\
\hline Temperature. & $\begin{array}{c}\text { Vapour Pressure } \\
\text { mm. }\end{array}$ & Temperature. & $\begin{array}{c}\text { Vapour Pressure } \\
\text { mm. }\end{array}$ & Temperature. & $\begin{array}{c}\text { Vapour Pressure } \\
\text { mm. }\end{array}$ \\
\hline $\begin{array}{l}10^{\circ} \\
11 \\
12 \\
13 \\
14 \\
15 \\
16 \\
17 \\
18 \\
19 \\
20 \\
21 \\
22 \\
23 \\
24 \\
25 \\
26 \\
27 \\
28 \\
29 \\
30 \\
31 \\
32 \\
33 \\
34 \\
35 \\
36 \\
37 \\
38 \\
39 \\
40 \\
41 \\
42 \\
43 \\
44 \\
45 \\
46 \\
47 \\
48 \\
49 \\
50\end{array}$ & $\begin{array}{l}198 \cdot 5 \\
207 \cdot 0 \\
215 \cdot 8 \\
225 \cdot 0 \\
234 \cdot 4 \\
244 \cdot 2 \\
254 \cdot 3 \\
265 \cdot 7 \\
275 \cdot 4 \\
286 \cdot 6 \\
298 \cdot 1 \\
309 \cdot 9 \\
322 \cdot 1 \\
334 \cdot 7 \\
347 \cdot 7 \\
361 \cdot 1 \\
375 \cdot 0 \\
389 \cdot 2 \\
403 \cdot 9 \\
409 \cdot 0 \\
434 \cdot 6 \\
450 \cdot 7 \\
467 \cdot 2 \\
484 \cdot 2 \\
501 \cdot 7 \\
519 \cdot 7 \\
538 \cdot 2 \\
557 \cdot 2 \\
576 \cdot 8 \\
596 \cdot 9 \\
617 \cdot 5 \\
638 \cdot 7 \\
660 \cdot 5 \\
682 \cdot 9 \\
705 \cdot 9 \\
729 \cdot 5 \\
753 \cdot 8 \\
778 \cdot 6 \\
804 \cdot 1 \\
830 \cdot 3 \\
857 \cdot 1\end{array}$ & $\begin{array}{l}\mathbf{4 5} \\
\mathbf{4 6} \\
\mathbf{4 7} \\
48 \\
49 \\
50 \\
51 \\
52 \\
53 \\
\mathbf{5 4} \\
\mathbf{5 5} \\
\mathbf{5 6} \\
\mathbf{5 7} \\
\mathbf{5 8} \\
\mathbf{5 9} \\
\mathbf{6 0} \\
\mathbf{6 1} \\
\mathbf{6 2} \\
\mathbf{6 3} \\
\mathbf{6 4} \\
\mathbf{6 5} \\
\mathbf{6 6} \\
\mathbf{6 7} \\
\mathbf{6 8} \\
\mathbf{6 9} \\
\mathbf{7 0} \\
\mathbf{7 1} \\
\mathbf{7 2} \\
\mathbf{7 3} \\
\mathbf{7 4} \\
\mathbf{7 5} \\
\mathbf{7 6} \\
\mathbf{7 7} \\
78 \\
\mathbf{7 9}\end{array}$ & $\begin{array}{l}172 \cdot 2 \\
181 \cdot 0 \\
190 \cdot 1 \\
199 \cdot 2 \\
209 \cdot 6 \\
220 \cdot 0 \\
230 \cdot 8 \\
242 \cdot 1 \\
253 \cdot 8 \\
265 \cdot 9 \\
278 \cdot 6 \\
291 \cdot 9 \\
305 \cdot 7 \\
320 \cdot 0 \\
334 \cdot 9 \\
350 \cdot 3 \\
356 \cdot 4 \\
383 \cdot 1 \\
400 \cdot 4 \\
418 \cdot 4 \\
437 \cdot 0 \\
456 \cdot 4 \\
476 \cdot 5 \\
497 \cdot 3 \\
518 \cdot 9 \\
541 \cdot 2 \\
564 \cdot 4 \\
588 \cdot 4 \\
613 \cdot 2 \\
639 \cdot 0 \\
665 \cdot 6 \\
693 \cdot 1 \\
721 \cdot 6 \\
751 \cdot 0 \\
781 \cdot 5\end{array}$ & $\begin{array}{r}75 \\
76 \\
77 \\
78 \\
79 \\
80 \\
81 \\
82 \\
83 \\
84 \\
85 \\
86 \\
87 \\
88 \\
89 \\
90 \\
91 \\
92 \\
93 \\
94 \\
95 \\
96 \\
97 \\
98 \\
99 \\
100 \\
101 \\
102 \\
103 \\
104 \\
105 \\
106 \\
107 \\
108 \\
109 \\
110 \\
111 \\
112 \\
113 \\
114 \\
115 \\
116 \\
117 \\
118 \\
119 \\
120 \\
121 \\
122 \\
123 \\
124 \\
125 \\
126 \\
127 \\
128 \\
129 \\
130 \\
131 \\
132\end{array}$ & $\begin{array}{l}119 \cdot 5 \\
124 \cdot 2 \\
129 \cdot 1 \\
134 \cdot 2 \\
139 \cdot 4 \\
144 \cdot 8 \\
150 \cdot 3 \\
156 \cdot 1 \\
162 \cdot 0 \\
168 \cdot 0 \\
174 \cdot 3 \\
181 \cdot 7 \\
187 \cdot 3 \\
194 \cdot 1 \\
201 \cdot 2 \\
208 \cdot 4 \\
215 \cdot 8 \\
223 \cdot 5 \\
221 \cdot 3 \\
239 \cdot 4 \\
247 \cdot 7 \\
256 \cdot 2 \\
265 \cdot 0 \\
274 \cdot 0 \\
283 \cdot 3 \\
292 \cdot 8 \\
302 \cdot 5 \\
312 \cdot 5 \\
322 \cdot 8 \\
333 \cdot 4 \\
344 \cdot 2 \\
355 \cdot 3 \\
366 \cdot 7 \\
378 \cdot 3 \\
390 \cdot 3 \\
402 \cdot 6 \\
415 \cdot 1 \\
428 \cdot 0 \\
441 \cdot 2 \\
456 \cdot 7 \\
468 \cdot 5 \\
482 \cdot 7 \\
497 \cdot 2 \\
512 \cdot 1 \\
527 \cdot 3 \\
542 \cdot 8 \\
558 \cdot 7 \\
575 \cdot 1 \\
591 \cdot 7 \\
608 \cdot 8 \\
626 \cdot 2 \\
644 \cdot 0 \\
662 \cdot 2 \\
680 \cdot 8 \\
699 \cdot 7 \\
719 \cdot 0 \\
738 \cdot 7 \\
758 \cdot 8\end{array}$ \\
\hline
\end{tabular}




\section{TABLE XVII.}

\section{Vapour Pressure of Mercury.}

The numbers in the following table were obtained by Knudsen ( $A n n$. d. Physik [iv.], vol. xxix. p. 179, 1909).

\begin{tabular}{|c|c|c|c|}
\hline Temperature. & $\begin{array}{c}\text { Vapour Pressure. } \\
\text { mm. }\end{array}$ & Temperature. & $\begin{array}{c}\text { Vapour Pressure. } \\
\text { mm. }\end{array}$ \\
\hline $\begin{array}{l}40^{\circ} \\
50 \\
60 \\
70 \\
80 \\
90 \\
100 \\
110 \\
120 \\
130 \\
140 \\
150 \\
160 \\
170\end{array}$ & $\begin{array}{l}0.006 \\
0.013 \\
0.025 \\
0.048 \\
0.088 \\
0 \cdot 157 \\
0.271 \\
0.455 \\
0.742 \\
1 \cdot 181 \\
1 \cdot 837 \\
2.797 \\
4 \cdot 174 \\
6 \cdot 114\end{array}$ & $\begin{array}{l}180^{\circ} \\
190 \\
200 \\
210 \\
220 \\
230 \\
240 \\
250 \\
260 \\
270 \\
280 \\
290 \\
300\end{array}$ & $\begin{array}{c}8 \cdot 803 \\
12 \cdot 47 \\
17 \cdot 41 \\
23 \cdot 96 \\
32 \cdot 52 \\
43 \cdot 60 \\
57 \cdot 78 \\
75 \cdot 75 \\
98 \cdot 24 \\
126 \cdot 2 \\
160 \cdot 5 \\
202 \cdot 4 \\
253 \cdot 1\end{array}$ \\
\hline
\end{tabular}

\section{TABLE XVIII.}

\section{Depression of Zero.}

The numbers for verre dur are the means of those obtained by Guillaume, Thiesen, and Schloesser; those for $16^{\prime \prime \prime}$ the means of those obtained by Thiesen, Schloesser, and Böttcher; those for 59"' were obtained by Thiesen.

\begin{tabular}{|c|c|c|c|}
\hline Temperature. & Verre dur. & Jena $16^{\prime \prime \prime}$. & Jena $59^{\prime \prime \prime}$. \\
\hline $\begin{array}{r}10^{\circ} \\
20 \\
30 \\
40 \\
50 \\
60 \\
70 \\
80 \\
90 \\
100\end{array}$ & $\begin{array}{r}0^{\circ} \cdot 008 \\
.017 \\
.027 \\
.037 \\
.048 \\
.060 \\
.071 \\
.084 \\
.097 \\
\cdot 111\end{array}$ & $\begin{array}{r}0^{\circ} .005 \\
.011 \\
.017 \\
.024 \\
.031 \\
.039 \\
.048 \\
.057 \\
.066 \\
.077\end{array}$ & $\begin{array}{r}0^{2} 005 \\
.009 \\
014 \\
017 \\
.021 \\
.024 \\
.027 \\
030 \\
.033 \\
035\end{array}$ \\
\hline
\end{tabular}




\section{TABle XIX.}

Corrections to reduce Readings on Mercury-in-Glass Thermometer to the Hydrogen or Air Thermometer.

The numbers for verre dur are from the results published by the Bureau International; those for the Jena glass from tables published by Gritzmacher in Wied. Annalen (1899), lxviii. p. 769. The original numbers are, in most cases, given to an additional place. Since, however, different observers have obtained numbers which differ by as much as $0^{\circ} \cdot 01$, it seems unnecessary to include the third place.

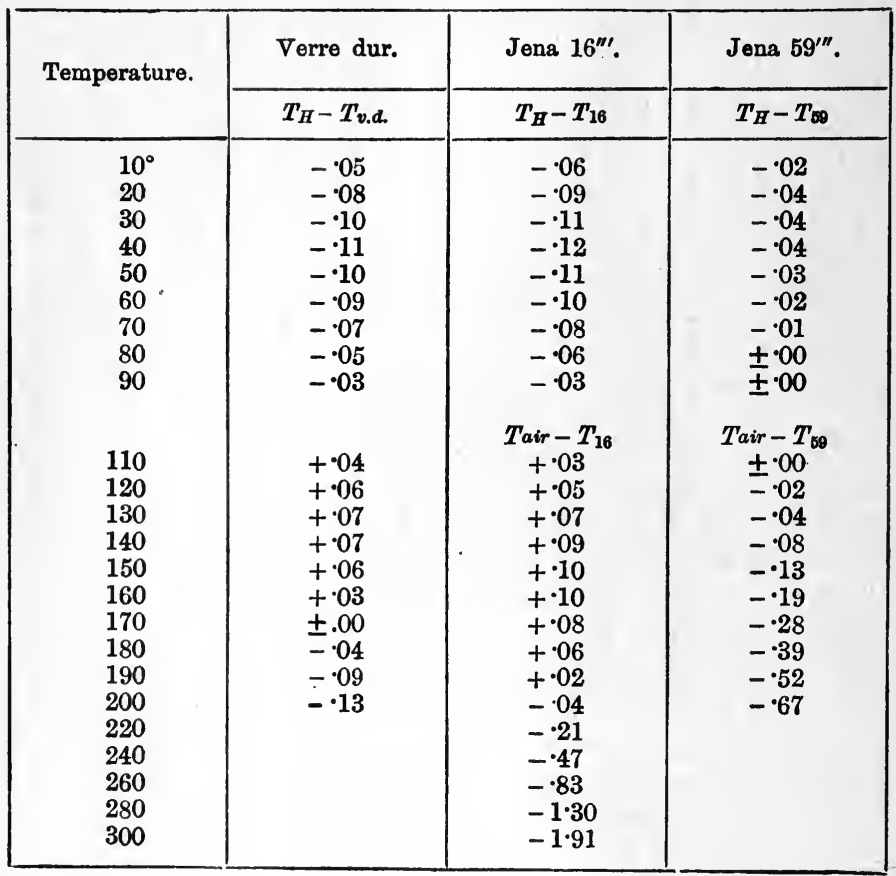


TABLE XX.

(1) Density of Mercury at various Temperatures measured on the Hydrogen Scale.

\begin{tabular}{|c|c|c|c|c|c|c|c|c|c|c|}
\hline $\begin{array}{c}\text { Tempera- } \\
\text { ture. }\end{array}$ & 0 & 1 & 2 & 3 & 4 & 5 & 6 & 7 & 8 & 9 \\
\hline $\begin{array}{l}0^{\circ} \mathrm{C} . \\
10 \\
20\end{array}$ & $\begin{array}{r}13.5955 \\
708 \\
462\end{array}$ & $\begin{array}{l}930 \\
683 \\
437\end{array}$ & $\begin{array}{l}905 \\
659 \\
413\end{array}$ & $\begin{array}{l}880 \\
634 \\
388\end{array}$ & $\begin{array}{l}856 \\
609 \\
364\end{array}$ & $\begin{array}{l}831 \\
585 \\
339\end{array}$ & $\begin{array}{l}806 \\
560 \\
315\end{array}$ & $\begin{array}{l}782 \\
536 \\
290\end{array}$ & $\begin{array}{l}757 \\
511 \\
266\end{array}$ & $\begin{array}{l}733 \\
487 \\
241\end{array}$ \\
\hline $\begin{array}{l}30 \\
40 \\
50 \\
60 \\
70\end{array}$ & $\begin{array}{r}217 \\
13.4972 \\
728 \\
485 \\
242\end{array}$ & $\begin{array}{l}192 \\
948 \\
704 \\
460 \\
218\end{array}$ & $\begin{array}{l}168 \\
923 \\
679 \\
436 \\
194\end{array}$ & $\begin{array}{l}143 \\
899 \\
655 \\
412 \\
169\end{array}$ & $\begin{array}{l}119 \\
874 \\
631 \\
388 \\
145 \\
\end{array}$ & $\begin{array}{l}094 \\
850 \\
606 \\
363 \\
121\end{array}$ & $\begin{array}{l}070 \\
826 \\
582 \\
339 \\
097\end{array}$ & $\begin{array}{l}045 \\
801 \\
553 \\
315 \\
073\end{array}$ & $\begin{array}{l}021 \\
777 \\
533 \\
291 \\
048 \\
\end{array}$ & $\begin{array}{l}\overline{996} \\
752 \\
509 \\
266 \\
024\end{array}$ \\
\hline $\begin{array}{r}80 \\
90 \\
100\end{array}$ & $\begin{array}{r}13 \cdot 4000 \\
13.3759 \\
518\end{array}$ & $\begin{array}{l}\overline{976} \\
735\end{array}$ & $\begin{array}{l}\overline{952} \\
711\end{array}$ & $\begin{array}{l}928 \\
687\end{array}$ & $\begin{array}{l}\overline{904} \\
662\end{array}$ & $\begin{array}{l}\overline{879} \\
638\end{array}$ & $\begin{array}{l}\overline{855} \\
614\end{array}$ & $\begin{array}{l}\overline{831} \\
590\end{array}$ & $\begin{array}{l}\overline{807} \\
566\end{array}$ & $\begin{array}{l}782 \\
542\end{array}$ \\
\hline
\end{tabular}

(2) Volume of one Gram of Mercury.

\begin{tabular}{|r|r|r|c|c|c|c|c|c|c|c|c|}
\hline $\begin{array}{c}\text { Tempers- } \\
\text { ture. }\end{array}$ & \multicolumn{1}{|c|}{0} & 1 & 2 & 3 & 4 & 5 & 6 & 7 & 8 & 9 \\
\hline 0 & 073554 & 567 & 581 & 594 & 608 & 621 & 634 & 648 & 661 & 674 \\
10 & 688 & 701 & 714 & 728 & 741 & 755 & 768 & 781 & 795 & 808 \\
20 & 821 & 835 & 848 & 862 & 875 & 888 & 902 & 915 & 928 & 942 \\
30 & 955 & 969 & 982 & 995 & $\overline{009}$ & 022 & 035 & 049 & 062 & 076 \\
40 & 074089 & 102 & 116 & 129 & 143 & 156 & 169 & 183 & 196 & 210 \\
50 & 223 & 236 & 250 & 263 & 277 & 290 & 304 & 317 & 330 & 344 \\
60 & 357 & 371 & 384 & 398 & 411 & 424 & 438 & 452 & 465 & 478 \\
70 & 492 & 505 & 519 & 532 & 545 & 559 & 572 & 586 & 599 & 613 \\
80 & 626 & 640 & 653 & 667 & 680 & 694 & 707 & 721 & 734 & 748 \\
90 & 761 & 775 & 788 & 802 & 815 & 829 & 842 & 856 & 869 & 883 \\
100 & 896 & & & & & & & & & \\
\hline
\end{tabular}




\section{TABLE XXI.}

Specific Heat of Water.

The numbers in this table are due to Callendar (Phil. Trans. Royal Society (1912), vol. 212 (A), p. 30, and are expressed in terms of the calorie at $20^{\circ} \mathrm{C}$.

\begin{tabular}{|c|c|c|c|}
\hline Temperature. & Specific Heat. & Temperature. & - Specific Heat. \\
\hline $\begin{array}{r}0 \\
5 \\
10 \\
15 \\
20 \\
25 \\
30 \\
35 \\
40 \\
45\end{array}$ & $\begin{array}{r}1 \cdot 0106 \\
1 \cdot 0060 \\
1 \cdot 0031 \\
1 \cdot 0012 \\
1 \cdot 0000 \\
\cdot 9992 \\
\cdot 9988 \\
\cdot 9986 \\
\cdot 9986 \\
\cdot 9987\end{array}$ & $\begin{array}{r}\mathbf{5 0} \\
\mathbf{5 5} \\
60 \\
65 \\
70 \\
75 \\
80 \\
85 \\
90 \\
95 \\
100\end{array}$ & $\begin{array}{r}\cdot 9990 \\
.9994 \\
.9999 \\
1.0006 \\
1.0013 \\
1.0020 \\
1.0029 \\
1.0038 \\
1.0048 \\
1.0058 \\
1.0070\end{array}$ \\
\hline
\end{tabular}

\section{TabLe XXII.}

Refractive Indices for Sodium Light $(\lambda=589 \mu \mu)$.

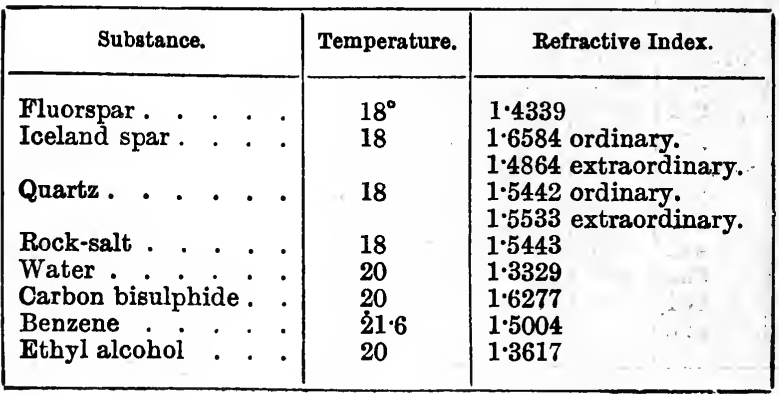




\section{TABLE XXIII.}

\section{Wave-lengths.}

The following wave-lengths are in air at a temperature of $15^{\circ} \mathrm{C}$ and a pressure of $760 \mathrm{~mm}$.:-

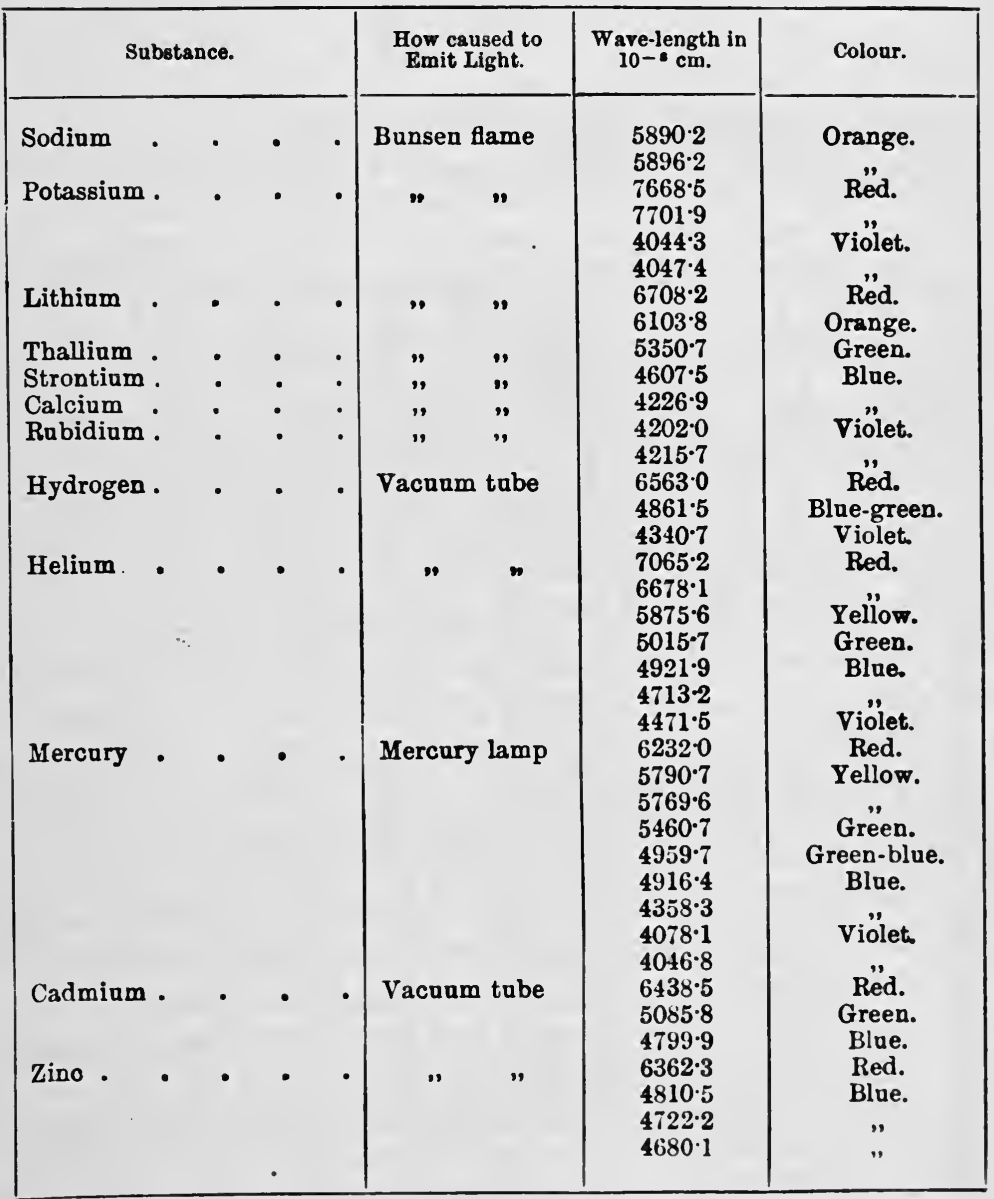




\section{TABLE XXIV.}

Correction to Scale Readings when a Mirror and Scale is used to Measure a Rotation.

If $d$ is the deflection read off on a scale at a distance $D$, and $\theta$ is the angle through which the mirror has turned, then we have to a first approximation

$$
\begin{aligned}
\theta=\sin \theta=\tan \theta & =\frac{d}{2 D}, \\
\sin \theta / 2 & =\frac{d}{4 D} .
\end{aligned}
$$

In the following table are given for values of $d / D$ between $\cdot 01$ and $\cdot 3$ a quantity $\delta$, such that to within terms in $d^{5} / D^{5}$, we have

\begin{tabular}{|c|c|c|c|c|c|c|c|c|c|c|}
\hline Function. & $d / D=\cdot 01$ & $\cdot 02$ & $\therefore 03$ & $\cdot 04$ & .05 & .06 & $\cdot 07$ & .08 & .09 & $\cdot 10$ \\
\hline$\theta$ & .00003 & $\cdot 00013$ & $\cdot 00030$ & .00053 & $\cdot 00083$ & .00120 & $\cdot 00163$ & .00212 & .00269 & .00331 \\
\hline $\operatorname{Sin} \theta$ & .00004 & $\cdot 00015$ & .00034 & $\cdot 00060$ & $\cdot 00094$ & $.0013 \tilde{~}$ & .00184 & .00239 & .00302 & .00373 \\
\hline $\operatorname{Sin} \theta / 2$ & .00003 & $\cdot 00014$ & $\cdot 00031$ & .00055 & $\cdot 00086$ & $\cdot 00124$ & .00168 & .00219 & .00277 & .00342 \\
\hline $\operatorname{Tan} \theta$ & .00002 & $\cdot 00010$ & $\cdot 00022$ & .00040 & .00062 & .00090 & .00122 & .00160 & .00201 & .00249 \\
\hline & $d / D=\cdot 11$ & $\cdot 12$ & $\cdot 13$ & $\cdot 14$ & $\cdot 15$ & $\cdot 16$ & $\cdot 17$ & $\cdot 18$ & $\cdot 19$ & $\cdot 20$ \\
\hline$\theta$ & .00400 & .00476 & $\cdot 00557$ & $\cdot 00645$ & .00740 & $\cdot 00840$ & .00946 & $\cdot 01059$ & .01177 & $\cdot 01301$ \\
\hline $\operatorname{Sin} \theta$. & .00450 & .00535 & .00627 & .00726 & .00832 & .00944 & .01064 & .01190 & .01323 & .01461 \\
\hline $\operatorname{Sin} \theta / 2$ & .00413 & $\cdot 00491$ & .00575 & $\cdot 00666$ & .00763 & .00867 & .00976 & .01092 & $\cdot 01214$ & $\cdot 01341$ \\
\hline $\operatorname{Tan} \theta$ & .00300 & .00357 & .00418 & .00485 & .00556 & .00632 & .00712 & .00797 & .00886 & .00980 \\
\hline & $d / D=\cdot 21$ & 22 & $\cdot 23$ & $\cdot 24$ & $\cdot 25$ & $\cdot 26$ & $\cdot 27$ & $\cdot 28$ & $\cdot 29$ & $\cdot 30$ \\
\hline$\theta$ & .01432 & .01567 & .01709 & 01857 & .02008 & .02166 & .02329 & .02497 & .02671 & .02848 \\
\hline $\operatorname{Sin} \theta$. & .01607 & .01761 & .01918 & .02084 & .02253 & .02431 & .02612 & .02801 & .02994 & .03194 \\
\hline $\operatorname{Sin} \theta / 2$ & .01475 & .01616 & .01761 & .01913 & .02069 & .02232 & .02399 & .02572 & .02749 & .02933 \\
\hline $\operatorname{Tan} \theta$. & $\cdot 01079$ & .01182 & .01288 & .01400 & 01515 & .01635 & .01759 & .01887 & .02018 & .02154 \\
\hline
\end{tabular}

$$
\begin{aligned}
\theta & =\frac{d}{2 D}\left(1-\delta_{1}\right), & \sin \frac{\theta}{2} & =\frac{d}{4 D}\left(1-\delta_{3}\right), \\
\sin \theta & =\frac{d}{2 D}\left(1-\delta_{2}\right), & \tan \theta & =\frac{d}{2 D}\left(1-\delta_{4}\right):
\end{aligned}
$$




\section{TABLE XXV.}

\section{Conductivity of Electrolytes.}

In the table $p$ is the weight of the anhydrous electrolyte in 100 grams of the solution, $m$ is the number of gram molecules of the electrolyte in a litre of the solution, $d$ is the density of the solution at $18^{\circ} \mathrm{C}$, and $\sigma$ is the conductivity of the solution in ohm ${ }^{-1}$ centimetre ${ }^{-1}$ at $18^{\circ} \mathrm{C}$.

\begin{tabular}{|c|c|c|c|c|c|c|c|c|c|}
\hline Substance. & $\boldsymbol{p}$ & $m$ & $d$ & $\sigma \times 10^{4}$ & Substance. & $p$ & $m$ & $d$ & $\sigma \times 10^{4}$ \\
\hline $\mathrm{KCl}$ & $\begin{array}{r}5 \\
10 \\
15 \\
20\end{array}$ & $\begin{array}{l}0.691 \\
1 \cdot 427 \\
2 \cdot 208 \\
3 \cdot 039\end{array}$ & $\begin{array}{l}1 \cdot 0308 \\
1 \cdot 0638 \\
1 \cdot 0978 \\
1 \cdot 1335\end{array}$ & $\begin{array}{r}690 \\
1359 \\
2020 \\
2677\end{array}$ & $\mathrm{ZnSO}_{4}$ & $\begin{array}{l}15 \\
20 \\
25 \\
30\end{array}$ & $\begin{array}{l}2 \cdot 169 \\
3 \cdot 053 \\
4 \cdot 040 \\
5 \cdot 124\end{array}$ & $\begin{array}{l}1 \cdot 1675 \\
1 \cdot 2323 \\
1 \cdot 2045 \\
1 \cdot 3788\end{array}$ & $\begin{array}{l}415 \\
468 \\
480 \\
444\end{array}$ \\
\hline $\mathrm{NaCl}$ & $\begin{array}{r}5 \\
10 \\
15 \\
20 \\
0\end{array}$ & $\begin{array}{l}0.884 \\
1.830 \\
2.843 \\
3.924 \\
5.085\end{array}$ & $\begin{array}{l}1 \cdot 0345 \\
1.0707 \\
1 \cdot 1087 \\
1 \cdot 1477 \\
1.1898\end{array}$ & $\begin{array}{r}672 \\
1211 \\
1642 \\
1957\end{array}$ & $\mathrm{CuSO}_{4}$ & $\begin{array}{r}5 \\
10 \\
15\end{array}$ & $\begin{array}{l}0 \cdot 655 \\
1 \cdot 387 \\
2 \cdot 194\end{array}$ & $\begin{array}{l}1 \cdot 0531 \\
1 \cdot 1073 \\
1 \cdot 1675\end{array}$ & $\begin{array}{l}189 \\
320 \\
421\end{array}$ \\
\hline $\mathrm{CuCl}_{2}$ & $\begin{array}{r}5 \\
10 \\
15 \\
20 \\
25 \\
30\end{array}$ & $\begin{array}{l}0.938 \\
1.957 \\
3.059 \\
4.253 \\
5.545 \\
6 \cdot 945\end{array}$ & $\begin{array}{l}1.0409 \\
1.0852 \\
1 \cdot 1311 \\
1 \cdot 1794 \\
1.2305 \\
1 \cdot 2841\end{array}$ & $\begin{array}{r}643 \\
1141 \\
1505 \\
1728 \\
1781 \\
1658\end{array}$ & $\mathrm{CdSO}_{4}$ & $\begin{array}{r}5 \\
10 \\
15 \\
20 \\
25 \\
30\end{array}$ & $\begin{array}{l}0 \cdot 504 \\
1 \cdot 060 \\
1.674 \\
2 \cdot 354 \\
3 \cdot 112 \\
3.958\end{array}$ & $\begin{array}{l}1 \cdot 0486 \\
1 \cdot 1026 \\
1 \cdot 1607 \\
1 \cdot 2245 \\
1 \cdot 2950 \\
1 \cdot 3725\end{array}$ & $\begin{array}{l}146 \\
247 \\
325 \\
388 \\
430 \\
436\end{array}$ \\
\hline & - & 8468 & 1.3420 & 1306 & $\mathrm{H}_{2} \mathrm{SO}_{4}$ & 5 & 1.053 & 1.0331 & 2085 \\
\hline $\mathrm{KNO}_{3}$ & $\begin{array}{r}5 \\
10 \\
15 \\
20\end{array}$ & $\begin{array}{l}0.509 \\
1.051 \\
1.626 \\
2.240\end{array}$ & $\begin{array}{l}1 \cdot 031 \\
1.063 \\
1.097 \\
1 \cdot 113\end{array}$ & $\begin{array}{r}454 \\
839 \\
1186 \\
1505\end{array}$ & & $\begin{array}{l}10 \\
15 \\
20 \\
25 \\
30 \\
35\end{array}$ & $\begin{array}{l}2 \cdot 1.6 \\
3 \cdot 376 \\
4 \cdot 655 \\
6.019 \\
7 \cdot 468 \\
9 \cdot 011\end{array}$ & $\begin{array}{l}1 \cdot 0673 \\
1 \cdot 1036 \\
1 \cdot 1414 \\
1 \cdot 1807 \\
1.2207 \\
1.2625\end{array}$ & $\begin{array}{l}3915 \\
5432 \\
6527 \\
7171 \\
7388 \\
7243\end{array}$ \\
\hline $\mathrm{ZnSO}_{4}$ & $\begin{array}{r}5 \\
10\end{array}$ & $\begin{array}{l}0.651 \\
1.371\end{array}$ & $\begin{array}{l}1 \cdot 0509 \\
1 \cdot 1069\end{array}$ & $\begin{array}{l}191 \\
321\end{array}$ & & $\begin{array}{l}40 \\
45 \\
50\end{array}$ & $\begin{array}{l}10 \cdot 649 \\
12 \cdot 396 \\
14 \cdot 258\end{array}$ & $\begin{array}{l}1 \cdot 3056 \\
1 \cdot 3508 \\
1 \cdot 3954\end{array}$ & $\begin{array}{l}6500 \\
6164 \\
5405\end{array}$ \\
\hline
\end{tabular}


TABLE XXVI.

E.M.F. of Clark and Cadmium Cells at Different Temperatures.

The values in the following table were calculated by Marck (Drude Anu. (1900), i. p. 617) from the formulæ

Clark cell . . . . $E_{t}=1 \cdot 4328-11 \cdot 9(t-15) 10^{-4}-0.07(t-15)^{2} 10^{-4}$,

Cadmium cell . . $E_{t}=1 \cdot 0186-38 \cdot 0(t-20) 10^{-6}-0 \cdot 65(t-20)^{2} 10^{-6}$, the E.M.F. being expressed in International volts.

E.M.F. of Clark Cell.

\begin{tabular}{|c|c|c|c|c|c|c|c|c|c|c|}
\hline \multirow{2}{*}{ Degrees C. } & \multicolumn{10}{|c|}{ Tenths. } \\
\hline & 0 & 1 & 2 & 3 & 4 & 5 & 6 & 7 & 8 & 9 \\
\hline $\begin{array}{l}0^{\circ} \\
1 \\
2 \\
3 \\
4 \\
5 \\
6 \\
7 \\
8 \\
9 \\
10 \\
11 \\
12 \\
13 \\
14 \\
15 \\
16 \\
17 \\
18 \\
19 \\
20 \\
21 \\
22 \\
23 \\
24 \\
25 \\
26 \\
27 \\
28 \\
29 \\
30\end{array}$ & $\begin{array}{r}1 \cdot 4491 \\
81 \\
70 \\
60 \\
50 \\
40 \\
29 \\
18 \\
07 \\
1 \cdot 4397 \\
86 \\
74 \\
63 \\
51 \\
40 \\
28 \\
16 \\
04 \\
1 \cdot 4291 \\
79 \\
67 \\
54 \\
41 \\
28 \\
15 \\
02 \\
1 \cdot 4188 \\
75 \\
61 \\
47 \\
1 * 4134\end{array}$ & $\begin{array}{l}90 \\
80 \\
69 \\
59 \\
49 \\
39 \\
28 \\
17 \\
06 \\
96 \\
85 \\
73 \\
61 \\
50 \\
38 \\
27 \\
15 \\
02 \\
90 \\
78 \\
65 \\
53 \\
40 \\
27 \\
14 \\
01 \\
87 \\
73 \\
60 \\
46 \\
32\end{array}$ & $\begin{array}{l}89 \\
79 \\
68 \\
58 \\
48 \\
38 \\
27 \\
16 \\
05 \\
94 \\
83 \\
72 \\
60 \\
49 \\
37 \\
26 \\
13 \\
01 \\
89 \\
77 \\
64 \\
51 \\
38 \\
25 \\
12 \\
99 \\
86 \\
72 \\
58 \\
45 \\
31\end{array}$ & $\begin{array}{l}88 \\
78 \\
67 \\
57 \\
47 \\
37 \\
26 \\
15 \\
04 \\
93 \\
82 \\
71 \\
59 \\
48 \\
36 \\
24 \\
12 \\
00 \\
88 \\
75 \\
63 \\
50 \\
37 \\
24 \\
11 \\
98 \\
84 \\
71 \\
57 \\
43 \\
29\end{array}$ & $\begin{array}{l}87 \\
77 \\
66 \\
56 \\
46 \\
36 \\
25 \\
14 \\
03 \\
92 \\
81 \\
70 \\
58 \\
46 \\
35 \\
23 \\
11 \\
99 \\
86 \\
74 \\
62 \\
49 \\
36 \\
23 \\
10 \\
97 \\
83 \\
69 \\
56 \\
42 \\
28\end{array}$ & $\begin{array}{l}86 \\
76 \\
65 \\
55 \\
45 \\
35 \\
24 \\
13 \\
02 \\
91 \\
80 \\
68 \\
57 \\
45 \\
34 \\
22 \\
10 \\
97 \\
85 \\
73 \\
60 \\
47 \\
34 \\
21 \\
08 \\
95 \\
82 \\
68 \\
54 \\
41 \\
27\end{array}$ & $\begin{array}{l}85 \\
75 \\
64 \\
54 \\
44 \\
33 \\
23 \\
12 \\
01 \\
90 \\
79 \\
67 \\
56 \\
44 \\
33 \\
21 \\
08 \\
96 \\
84 \\
72 \\
59 \\
46 \\
33 \\
20 \\
07 \\
94 \\
80 \\
67 \\
53 \\
39 \\
25\end{array}$ & $\begin{array}{l}84 \\
73 \\
63 \\
53 \\
43 \\
32 \\
22 \\
11 \\
00 \\
89 \\
78 \\
66 \\
55 \\
43 \\
31 \\
19 \\
07 \\
95 \\
83 \\
70 \\
58 \\
45 \\
32 \\
19 \\
06 \\
92 \\
79 \\
65 \\
51 \\
38 \\
24\end{array}$ & $\begin{array}{l}83 \\
72 \\
62 \\
52 \\
42 \\
31 \\
20 \\
10 \\
99 \\
88 \\
77 \\
65 \\
53 \\
42 \\
30 \\
18 \\
06 \\
94 \\
81 \\
69 \\
56 \\
43 \\
30 \\
18 \\
05 \\
91 \\
77 \\
64 \\
50 \\
36 \\
22\end{array}$ & $\begin{array}{l}82 \\
71 \\
61 \\
51 \\
41 \\
30 \\
19 \\
09 \\
98 \\
87 \\
75 \\
64 \\
52 \\
41 \\
29 \\
17 \\
05 \\
92 \\
80 \\
68 \\
55 \\
42 \\
29 \\
16 \\
03 \\
90 \\
76 \\
62 \\
49 \\
35 \\
21\end{array}$ \\
\hline
\end{tabular}

E.M.F. of Cadmiun Cell.

\begin{tabular}{|c|r|r|r|r|r|}
\hline Temperature. & E.M.F. & Temperature. & E.M.F. & Temperature. & E.M.F. \\
\cline { 2 - 6 } & 1.0189 & $17^{\circ}$ & 1.0187 & $24^{\circ}$ & 1.0184 \\
\cline { 3 - 6 } $10^{\circ}$ & 9 & 18 & 7 & 25 & 4 \\
12 & 9 & 19 & 6 & 26 & 3 \\
13 & 8 & 20 & 6 & 27 & 3 \\
14 & 8 & 21 & 6 & 2 & 3 \\
15 & 8 & 22 & 5 & 29 & 2 \\
16 & 7 & 23 & 5 & & \\
\hline
\end{tabular}


TABLE XXVII.-Connection between the Units in the C.G.S. System and the Practical S'ystem. (See Walson's “Physics,” §§ 539, 540, 541.)

\begin{tabular}{|c|c|c|c|c|c|c|}
\hline \multicolumn{3}{|c|}{ Quantity. } & $\begin{array}{l}\text { Name of Unit in } \\
\text { C.G.S. System. }\end{array}$ & Dimensions. & $\begin{array}{l}\text { Namo of Unit in } \\
\text { Practical System. }\end{array}$ & $\begin{array}{l}\text { Value of Practical } \\
\text { Unit in C.G.S. }\end{array}$ \\
\hline Length & $\therefore$ & . . & Centimetre & $L$ & Quadrant & $10^{9} \mathrm{~cm}$. \\
\hline Mass . & - & . . & Gram & $M$ & & $10^{-11}$ gram. \\
\hline Time . & . & . & Second & $T$ & Second & 1 second. \\
\hline Force . & - & - & Dyne & $L . M T^{-2}$ & - & $\cdot$ \\
\hline Work . & - & . & Erg & $L^{2} B T^{-2}$ & Joule & $10^{7}$ ergs. \\
\hline Power . & . & - . & Erg per sec. & $L^{2} M T^{-3}$ & Watt & $10^{7}$ ergs per sec. \\
\hline Resistance & & . . & & $L T^{-1} \mu$ & Ohm & $10^{9} \mathrm{~cm} \cdot / \mathrm{sec}$. \\
\hline Current & . & . . & & $L^{\frac{1}{2}} M^{\frac{1}{3}} \tau^{-1} \mu^{-\frac{1}{2}}$ & Ampere & $10^{-1}$ \\
\hline Quantity & . & . & & $L^{\frac{1}{2}} M^{\frac{1}{3} \mu-\frac{1}{2}}$ & Coulomb & $10^{-1}$ \\
\hline E.M.F. . & . & . . & & $\left.L^{3} M^{3} T^{-2} \mu\right\}$ & Volt & $10^{y}$ \\
\hline Magnetic & fiel & ld & Gauss & $L^{-\frac{1}{2}} M^{\frac{1}{2}} T^{-1} \mu^{-\frac{1}{2}}$ & None & $10^{-10}$. \\
\hline $\begin{array}{l}\text { Magnetic } \\
\text { density } \\
\text { duction }\end{array}$ & or & $\begin{array}{c}\text { flax } \\
\text { in- }\end{array}$ & Maxwell & $L^{-\frac{1}{b}} M^{\frac{1}{3}} T^{-1} \mu^{\frac{1}{b}}$ & None & $10^{-10}$ \\
\hline Inductanc & & . . & Centimetre & $L_{\mu}$ & Henry & $10^{9}$. \\
\hline Capacity & - & - & & $L^{-1} T^{2} \mu^{-1}$ & $\begin{array}{l}\text { Farad } \\
\text { Microfarad }\end{array}$ & $\begin{array}{l}10^{-9} \\
10^{-13}\end{array}$ \\
\hline
\end{tabular}

Ratios of Electrostatic Units to Electromannetic Units.

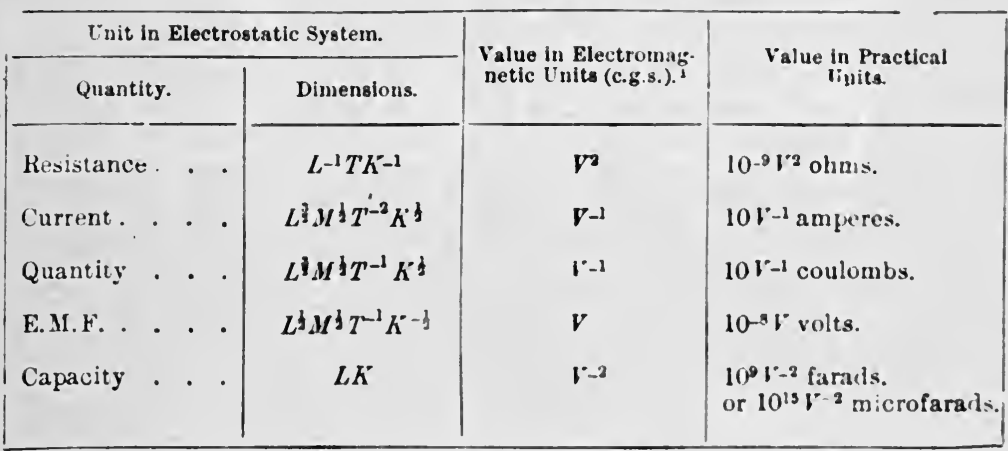




\section{TABLe XXVIII.}

Density.

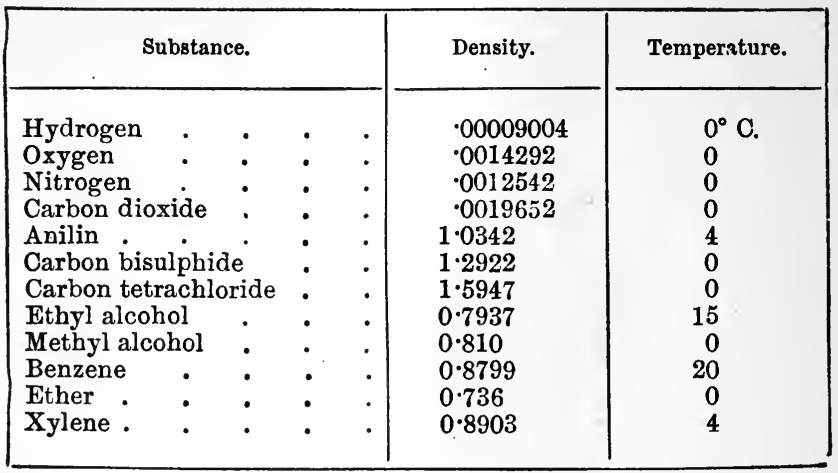

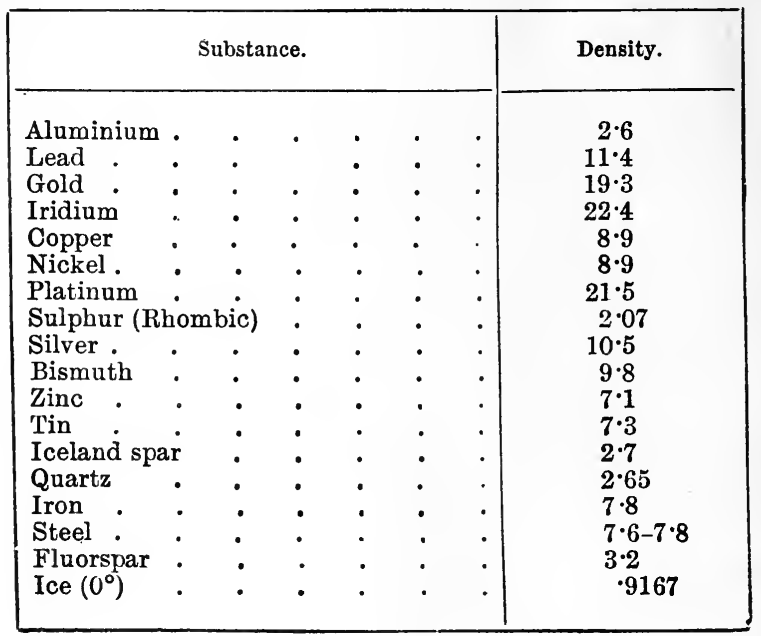




\section{TABLE XXIX.}

\section{Specific Resistance.}

\begin{tabular}{|c|c|c|c|c|}
\hline \multicolumn{3}{|c|}{ Substance. } & $\begin{array}{c}\text { Specific Resistance. } \\
\text { at } 18^{\circ} \mathrm{C} \text {. }\end{array}$ & Temperature \\
\hline $\begin{array}{l}\text { Aluminium } \\
\text { Copper : } \\
\text { Gold } \\
\text { Iron : } \\
\text { Steel : } \\
\text { Mercury : } \\
\text { Nickel : } \\
\text { Platinum } \\
\text { Silver : } \\
\text { Tin : } \\
\text { Zine : }\end{array}$ & 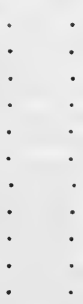 & 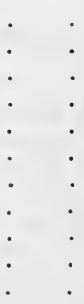 & $\begin{array}{c}2 \cdot 94 \times 10^{-6} \\
1 \cdot 78 \\
2 \cdot 42 \\
12 \\
20 \\
\left(20^{\circ}\right) 95 \cdot 76 \\
11 \cdot 8 \\
11 \cdot 0 \\
1 \cdot 66 \\
11 \cdot 3 \\
6.1\end{array}$ & $\begin{array}{l}38 \times 10^{-4} \\
42 \cdot 8 \\
40^{\circ} \\
62 \\
16-42 \\
9 \cdot 0 \\
49 \\
38 \\
40 \\
45 \\
37\end{array}$ \\
\hline $\begin{array}{l}\text { Brass } \\
\text { Constantan } \\
\text { German Silve } \\
\text { Manganin }\end{array}$ & $\dot{.}$ & 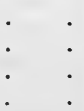 & $\begin{array}{c}6 \text { to } 9 \\
49 \\
16 \text { to } 40 \\
44\end{array}$ & $\begin{array}{l}10 \\
0-0 \cdot 5 \\
2 \cdot 3-6 \\
0-0 \cdot 5\end{array}$ \\
\hline
\end{tabular}

\section{TABLE XXX.}

\section{British Standard Wire Gauge.}

\begin{tabular}{|c|c|c|c|c|c|}
\hline S.W.G. & $\begin{array}{l}\text { Diameter } \\
\text { in mm. }\end{array}$ & $\begin{array}{c}\text { Ohms per } \\
\text { Sfetre (Copper). }\end{array}$ & S.W.G. & $\begin{array}{l}\text { Diameter } \\
\text { in mn. }\end{array}$ & $\begin{array}{c}\text { Ohms per } \\
\text { Jetre (Copper). }\end{array}$ \\
\hline 12 & $2 \cdot 64$ & $\cdot 0032$ & 26 & 457 & $\cdot 105$ \\
\hline 14 & $2 \cdot 03$ & .0054 & 23 & 376 & - 155 \\
\hline 16 & $1 \cdot 63$ & $\cdot 0083$ & 30 & $\cdot 315$ & $\because 2 \cdot 2$ \\
\hline 18 & $1 \cdot 22$ & .0148 & 32 & 274 & $\cdot 293$ \\
\hline 20 & $\cdot 914$ & $\cdot 0260$ & 34 & 234 & $-40\}$ \\
\hline 22 & 711 & $\cdot 0435$ & 36 & $\cdot 193$ & $\because 90$ \\
\hline 24 & $\cdot 559$ & $0 \div 0$ & 38 & 122 & .950 \\
\hline
\end{tabular}




\section{IN DEX}

A

Absorption hygrometer, 249

Absorpition of light, 390

Accumulators, small, 570

Adjustable carbon resistance, 473

Air, density of, 77, 599

Air thermometer, 203

constant pressure, 209

constant volume, 204

reduction of readings of mercury

thermometer to, 182, and Table XIX.

Alyebraical expression, to find an, to represent a series of results, 10

Alternating current method of measuring self-induction, 548

Alternating current method of measuring the resistance of electrolytes, 483

Ammeter, calibration of, 513, 516

Analysis, harmonic, 35

Anderson's method of measuring selfinduction, $\mathbf{5 4 3}$

Angle, measurement of, of prism, 290

Apparent expansion of a liquid, 191, $201^{\circ}$

Approximate formulæ, 597

A rea, measurement of, 32

A rithmometer, 29

Aspirator, 249

Atmospheric pressure, measurement of, 155

Ayrton and Perry's secohmmeter, 538

B

\section{Balance, 63}

\section{- ratio of arms of, 68}

Ballistic galvanometer, 518, 520, 522

Ballistic method of measuring permea. bility, 563

Barometer, 155

- corrections to readings, 157,611 , 612 , and Table I.

Battery resistance, Beetz's method, 476

- Mance's method, 475

Beats of two notes, 277,281
Beckmann's apparatus for measuring the boiling point, 264

- apparatus for measuring the freezing point, 258

- thermometer, 262

Beetz's method of measuring the resistance of a cell, 476

Berthelot's method of measuring latent heat, 237

Biprism, Fresnel's, 316, 320

Bird call for producing high note, 286

Brasheur's method of silvering glass, 590 .

Bunsen photometer, 383

Bunsen's ice calorimeter, 230

Buoyancy, correction for, 76, 79, 609

\section{C}

Cable, measurement of insulation resistance of, 461

Cadmium cell, 487

- E.M.F. of, 626

Calculating machines, 29

Calibration of ammeter, 513,516

- of a Callendar and Griffiths bridge, 457

- of a dial-pattern Wheatstone's bridge, 452

- of a spectroscope, 311, 312

- of $\approx$ wire, $437,440,441$

of tube of thermometer, 162

Callendar and Griffiths pattern Wheatstone's bridge, 455

Calorimeter, 214

Calorimetry, 213

Candle-power of incandescent lamp, 387

Capacity, absolute measurement of, 533 - comparison of, by de Sauty's method, 530

comparison of, by method of mixture, 531

- comparison of, with ballistic galvanometer, 525

Capillarity, 135 
Capillary electrometer, 498

Carbon resistance, adjustable, 473

Carey Foster's method of calibrating a wire, 437

\section{2}

method of comparing resistances,

- method of measuring a mutualinduction, 548

Cathetometer, 53

Chemical (absorption) hygrometer, 249

Claek cell, E.M.F. of, 493,618

Clement and l)esormes' method of measuring the ratio of the specific heats, 267

Clock, determination of rate of, 127, 131

Coincidence, method of, when determining a period, 120

Collimation, adjustment for, 54

Colour-blindness, 394

Colour mixtures, 391

top, 392

Commutator, Pohl's, 426 .

Comparator, 51

Comparison of thermometers, 174

Comptometer, 32

Condensers, comparison of capacity of, $525,530,531,571$

Conductivity for heat, 270,272

- of electrolytes, 475,625

Continuous-flow calorimeter, 580

Cool column, correction of thermometer readings for, 180

Cooling, specific heat by the method of, 224

Copper, deposition of, for current measurement, 512

- voltameter, 512

Corrections to observed quantities, 3

Cross-wires, mounting, 592

Curres, application of, to reduction of observations, 5

_ drawing, with spline, 9

D

I)('clination, 396

Demagnetisation of iron, 567

l)emagnetising factor, 562

Density, 79

- correction for buoyancy of air, 74,609

of air; $7 \pi$, Table IV.

of ice, 228

of liquids, 87,89

of solids, 8:2, 84,85

- of various substances, 628

I lepression of zero in mercury thermometers, 182, 185, 619
Depression of freezing point, 258, 261

de Sauty's method of comparing cajas. city, 530

Dew point, 247

Dial-pattern Wheatstone's bridge, 450 , 452

Differential galvanometer, 469

Diffraction fringes, 322

- grating, 314

- through a slit, 335

Dilatometer, volume, 197

weight, 193

Dip, 415

Discharge key, 525

Dispersion, 305

Dividing scales on glass, 586

Dolezalek's method of measuring self. induction, 549

- quadrant electrometer, 569

Double bridge for measuring low resistances, 465

E

Earth inductor, 527

Electrolytes, conductivity of, 625

- resistance of, 475

Electrometer, cajillary, 498

- quadrant, 569

Elliptically polarised light, 378, 380

Emergent column, correction of thermometer readings for, 180

E. M.F. of Clark and cadmium cells, 626 standards of, 487

E.M.F.'s, comparison of, 493,526

- comparison of, by galvanometer deflections, 430

Expransion, apparent, of a liquid, 191, 201

- cubical, 191

- cubical, with hydrostatic balance, 200

193

cubical, with weight thermometer,

cubical, with volume dilatometer, 197

- linear, 187, 188

Exploring coil, 529

Eve and ear method of measuring a period, 102

Eye-piece, adjustment of, 49,289

-, Gauss, 290

$\mathbf{F}$

Figure of merit of galvanometer, 428

Filler for specific gravity bottles and dilatometers, 90

Fixed points, auxiliary, 178 
Fixed points of thermometer, 169, 172

Flatness, test of, 356

Flicker photometer, 386

Focal length of lenses, 348, 359

length of mirrors, 352

Fourier analysis, 35

Freezing point of solution, 258

Frequency of tuning fork, 275, 277, 279, 281

Fresnel's biprism, 316, 320

Fundamental interval, correction for, 173

— of platinum thermometer, 504

G

" $g$, " determination of, by Borda's pendulum, 118

- by Kater's pendulum, 124

Galvanometer, ballistic, 518 differential, 469

- figure of merit of, 428 sine, 511

- suspended coil pattern, 427

- suspended needle pattern, 425

- tangent, 508

Gas, reduction of volume of, 613,614

Gauss eye-piece, 290

Geographical meridian, 397

Glass blowing, 583

Grating, resolving power of, 341

- measurement of wave-length with, 314

\section{$\mathrm{H}$}

Harmonic anslysis, 35

Heat, conductivity for, 270,272

— of solution, 241

Heater for calorimeter, 215, 233

High note, wave-length of, 286

High resistance, measurement of, 460

Holmgren's test wools, 394

Horizontal component of the earth's field, 403, 406, 407

Hydrogen scale, reduction of readings of mercury thermometer to, 182, and Table XIX.

Hydrostatic balance, measurement of cubical expansion with, 200

Hygrometer, 247, 248, 249

\section{I}

Ice, density of, 228

latent heat of, 233

Ice calorimeter (Bunsen's), 230

Illumination of divisions of standard scale, 50
Incandescent lamp, candle-power of, 387

Inertia, moments of, 102, 609

Insulation resistance, 464, 574

Interference fringes, localisation of, 324

Interferometer, Michelson's, 327

Rayleigh's, 343

Iron, permeability of, 552

\section{$\mathrm{J}$}

Jolly photometer, 385

Joly's steam calorimeter, 239

Joule's equivalent, $\mathbf{5 7 6}$

$\mathrm{K}$

Kelvin and Varley slide, 496

Kelvin's double bridge, 465

Key, discharge, 525

for electrometer, 571

Pohl's, 426

Kohlrausch's method of measuring the resistance of electrolytes, 483

Kundt's method of measuring the velo. city of sound, 284

\section{L}

Lag of thermometer, correction for, 220

Latent heat, by Berthelot's method, 237 of ice, 233

of steam, 235

Length, correction of measurements of, to allow for effects of temperature, 52 measurement of, 41

- standards of, 41

Lenses and mirrors, 348

Light, sources of, for dispersion measurements, 305

- standards of, 382

Light filter, 310

Liquid, specific heat of, 223, 224

Localisation of interference fringes, 324

Logarithmic decrement, 149

- paper, 11

Low resistances, measurement of, 462, $465,470,471$

Luminosity of pigments, 394

Lummer-Brodhun photometer, 384

\section{M}

Magnet, temperature coefficient of, 414

Magnetic field, measurement of, with earth inductor, 527

- measurement of, with exploring coil, 529

Magnetic meridian, $390^{\circ}$ 
Magnetometer, 404, 407

method of measuring permeability, 553

II agnifying power of microscope, 368

\section{- telescope, 367}

Mance's method of measuring the re. sistance of a battery, 475

Manganin wire resistances, 601

Manometer for very small pressures, 140

Mathematical tables, 25

Mathiessen and Hockin's method of measuring low resistances, 462

Nelting point by the method of cooling, 267

Melting point of metals, 506

Mercury arc, 308

- still, 487, 489

- vapour pressure of, 619

Metals, melting point of, 506

Metastatic thermometer, 262

Michelson's interferometer, 327

Micrometer microscope, 48

- screw, 44

Microscope, magnifying power of, 368

Mirror and scale for measuring rotations, 420,624

Mirrors, radius of, curvature of, 352

Mixture, method of, for comparing capacity, 531

Vixture, method of. in calorimetry, 213

Molecular weight from the depression of the freezing point of solutions, 261

Iloments of inertia, comparison of, 102 table of, 609

Movable zero method of using a mercury thermometer, 183

II utual induction, $546,548,549$

\section{$\mathrm{N}$}

Newton's rings, 332

Normal equations, 21

0

Optical bench, 317,348

lever, 59

\section{$\mathbf{P}$}

Parallax of sun or star, 610

Pendulum, Borila's, 122

compound, 126

effect of arc on period, 114, and

Table VIII.

__ Kater's reversible, 124

simple, 114

Period, determination of, by method of coincidence, 120
Period, measurement of, by eye and ear method, 102

Permeability, 552

— by the ballistic method, 563

by the magnetometer method, 553

Photographic lens, test of, 363

Photometers, 383

Photoped, 385

Pigments, luminosity of, 394

Pitch comparison of, of two forks, 281

- of tuning fork, $275,277,279$

Planimeter, 33

Platinum thermometer, 501

Pohl commutator, 426

Poisson's ratio, 101

Polarisation by reflection, 370

- rotation of the plane of, 370

Potentiometer method of messuring current, 516

Potentiometers, 494

Poynting's polariscope, 374

Prism, measurement of angle of, 290

Pyknometer, 85

Q

Quadrant electrometer, 569

Q capacity of, 571

Quartz fibres, 599

\section{$\mathbf{R}$}

Radius of curvature of mirrors, 352

- of curvature with optical lever, 61 of curvature with spherometer, 46

Rato of clock, 127, 131, 610

Ratio of specific heats (Clement and Desorme's method), 26-

- of specific heats from velocity of sound, 286

Rayleigh's method of measuring selfinduction, 536

refractometer, $\mathbf{3 4 3}$

Reduction of barometer readings, 157, and Tables I., X., and XI.

Refraction of sun or star, 610

Refractive index by total reflection. 297, 299, 303

_- index of gases, 345

— index of prism, 295, 302

- index of various substances, 622

Refractometer, Rayleigh's, $3 \$ 3$

Regnault's hygronieter, 247

Resistance coils, construction of, 601

- high, 460 low, $452,465,470,471$

617 
Resistances, comparison of, by Carey Foster's method, 442

- comparison of, with the differential galvanometer, 470

determination of ratio of two nearly equal, 446

Resolution, limit of, of telescope, 335

Resolving power of spectroscope, 338

Rigidity, by Searle's method, 111

- simple, 111

Rimington's method of measuring selfinduction, 540

Ritchie photometer, 386

Rotation of the plane of polarisation, 371

Rotatory power, measurement of, 376

Rumford photometer, 385

\section{S}

Saccharimetry, 377

Saturation current, 573

Scales, dividing, on glass, 594

Screw gauge, 47

- micrometer, 44

Secohmmeter, 538

Self-induction, by Anderson's method, 543

- by comparison with a variable standard, 545

- by Rimington's method, 540 548

by the Wien-Dolezalek method,

comparison of, with a mutual induction, 546

- Rayleigh's method, 536

- with secohmmeter, 538

Semi-logarithmic paper, 16

Series of results, representation of, $\tau$, 10

Sextant, 127

Shunt potentiometer method of comparing low resistances, 471

Silica, fused, 589

Silver voltameter, 514

Silvering glass, 598

Simple rigidity, 111

Simpson's rule, 32

Sine galvanometer, 511

Slide rule, 25

Soap bubble, measurement of pressure inside, 139

Sodium sulphate, transition temperature of, 179

Solenoid inductor, 522

Soliel's compensator, 376

Solution, heat of, 241
Solutions, preparation of, for electrolytic resistance measurements, 485

Sound, velocity of, 282,284

Specific gravity bottle, 85,89

- gravity bottles, filler for, 90

heat by the method of cooling, 224 heat by the method of mixture,

$213,222,223$

heat of water, 622

- heats, ratio of, 267,286

Specific resistance, 629

Spectrometer, adjustment of, 290

Spectro-photometer, 388

Spectroscope, adjustment of, 290

calibration of, 311,312

resolving power of, 338

Spherometer, 45

Splines, 9

Standard metre scale, 42 scale, illumination of, 50

Standard wire gauge, 629

Steam, calorimeter (Joly's), 239

- latent, heat of, 235

Stirrer for calorimeter, 214

Stroboscopic disc, 277

Strohul and Barus's method of calibrating a wire, 441

Sugar, rotation of the plane of polarisation by, 371, 377

Sulphur, boiling point of, 502, 504

Surface, tension by capillary tube, 139

- tension, effeet of E.M. F. on, 499

tension of soap film, 139

\section{$\mathrm{T}$}

Tables, application of, to the reduction of observations, 5

mathematical, 25

Tangent galvanometer, 508

Telescope, adjustment of, for parallel rays, 289,291

_ magnifying power of, 367

— resolving power of, 335

Temperature coefficient of magnet, 414

Tension of water vapour, 615,617

Thermocouple, 504

Thermometer, air, 203

- air, constant pressure, 209

— air, constant volume, 204

- auxiliary fixed points, 178

Beckmann's, 262

calibration of tube, 162

174 comparison of a, with standard,

correction for emergent column, 
Thermometer correction for fundamental interval, 173

- depression of mercury, 185

172

- lag, correction for, 220

- mercury, reduction of readings to the gas scale, 620

- movable zero method of using, 183

- reduction of readings of mercury, to air or hydrogen scales, 182, and Table XIX.

Thermometers, platinum resistance, 501

Thomson and Varley slide, 496

'Thomson's double bridge, 465

Time, determination of, with sextant, 131

Transition temperatures, $1 ; 8$

'Truvelling microscope, 48

Tuning fork, pitch of, $275,277,279$

\section{U}

Units, connection between, of various systems, 627

\section{V}

Vacuo, reduction of weights to, 76 , 606

Vacuum tubes as sources of light, $300^{\circ}$

Vapour density (Victor Meyer's method), 253

- (Hofiniun's method), 255

Viapour pressure, 245

- of mercury, 619

of various liquids, 618

of water, 615 , 511

Velocity of suund by resonance, 28:

- - by liundt's method, '284

Veruier, 43

Viscusity, measurement of. by thw through capillary tube, $11: 2$

Viscosity of a gas, 146

_ measurement of, by oscillating disc (Meyer's methird), 153

Voltaneter, copper, $51:$

- silver, 514

Volume lilatometer, 197
W

Water, boiling point of, 617

density of, 608

specific heat of, 622

- vapour, uass of, in 1 cubic metre of saturated air, 616

vapour, tension of, 615

Wave-length of a high note, 286

Wave-length of light, with diffraction fringes, 322

- with diffraction grating, 314

— with Fresnel's biprism, 316, 320

with Newton's rings, 334

with spectroscope, 311

Wave-lengths, table of, 623

Weighing by substitution (Borda's method), 69

- by the methorl of oscillation, $66^{\circ}$

- methorl of double (Gauss's methor). 69

Weighings, reduction of, to vacuo, i6, 606

Weight thermometer, 193

Weights, caliliation of a set of, 71

Wet and dry bulb hygrometer, 243

Wheatstone's bridge, 432

- Callendar and Gritfiths pattern, $45 j$

- dial-pattern, 450

- metre form, 43\%

- Post Ollice form, 448

Wien's method of measuring self-induction, 549

Wire, calibration of, $437,4 \$ 0,4+1$

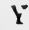

Young's molulus by bendin:, 97, 98

modulus by searle's methud, $9 \pi$.

111

- modulus by stretching, 93

532

method of comparing capacities,

\section{Z}

Zero, depression of, in mercury ther mometers. 18:. 18j, 619

- movable, 18:3 




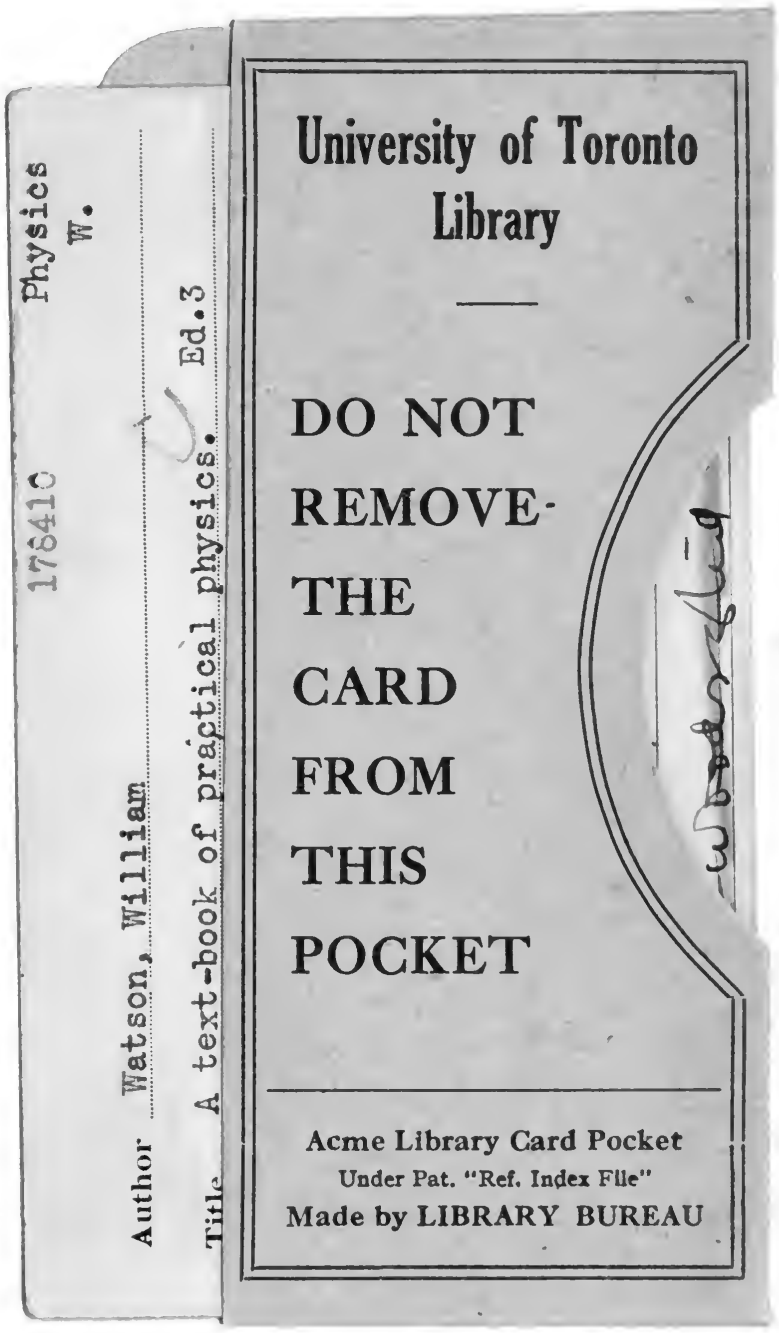


